Fall 2007

\title{
2007 Miracle Yearbook
}

Cedarville University

Follow this and additional works at: https://digitalcommons.cedarville.edu/yearbooks

Part of the Higher Education Commons, Organizational Communication Commons, and the Public Relations and Advertising Commons

\section{Recommended Citation}

Cedarville University, "2007 Miracle Yearbook" (2007). Yearbooks. 1.

https://digitalcommons.cedarville.edu/yearbooks/1

This Book is brought to you for free and open access by DigitalCommons@Cedarville, a service of the Centennial Library. It has been accepted for inclusion in Yearbooks by an authorized administrator of DigitalCommons@Cedarville. For more information, please contact digitalcommons@cedarville.edu. 



\section{Table of Contents}

\begin{tabular}{|c|c|c|c|c|}
\hline & & Juniors & 98 & Organizations \\
\hline Opening & 1 & Sophomores & 112 & Spring \\
\hline Dedication & 8 & Freshmen & 130 & Memory \\
\hline Fall & 10 & Activities & 148 & Miracle Staff \\
\hline Faculty \& Staff & 28 & Athletics & 174 & Index \\
\hline Seniors & 60 & Ministries & 208 & Closing \\
\hline
\end{tabular}



Minacle 


\section{Stoviting Oili}

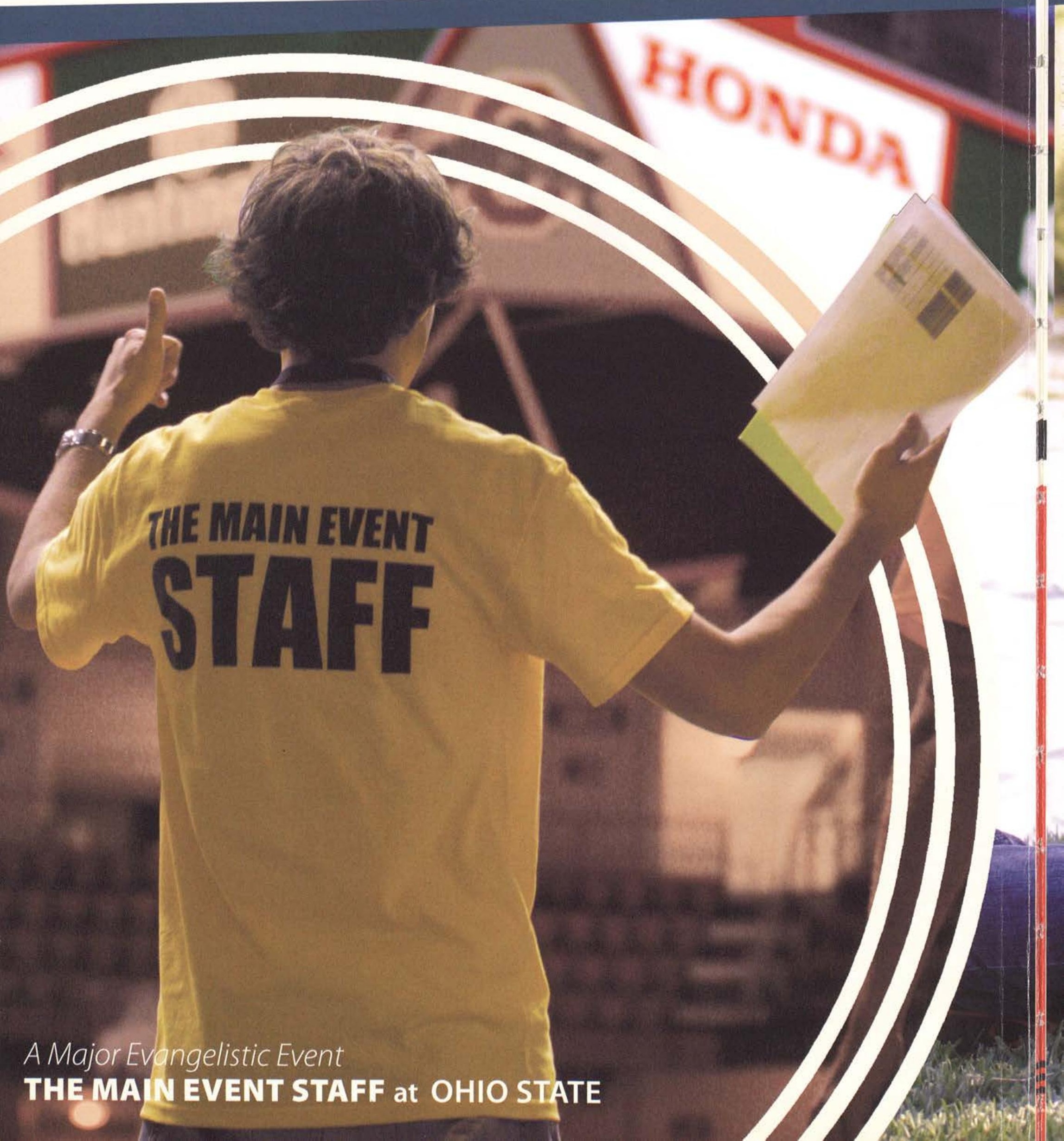




\section{Wiah

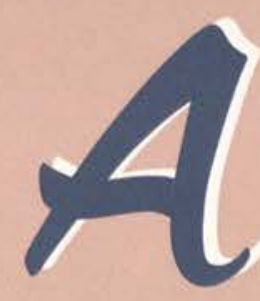 \\ Bangy!}

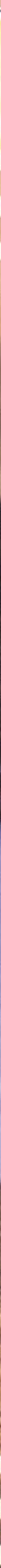

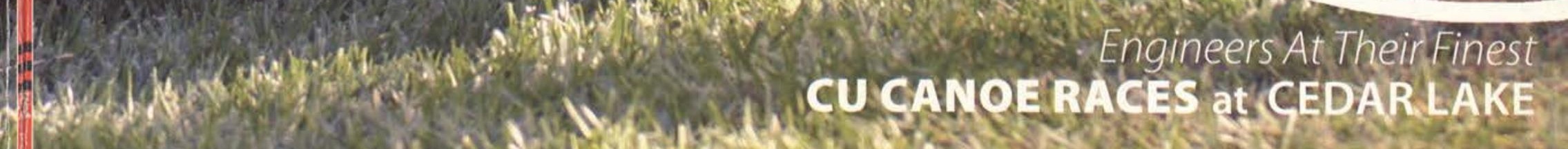




\section{Come 4 and}

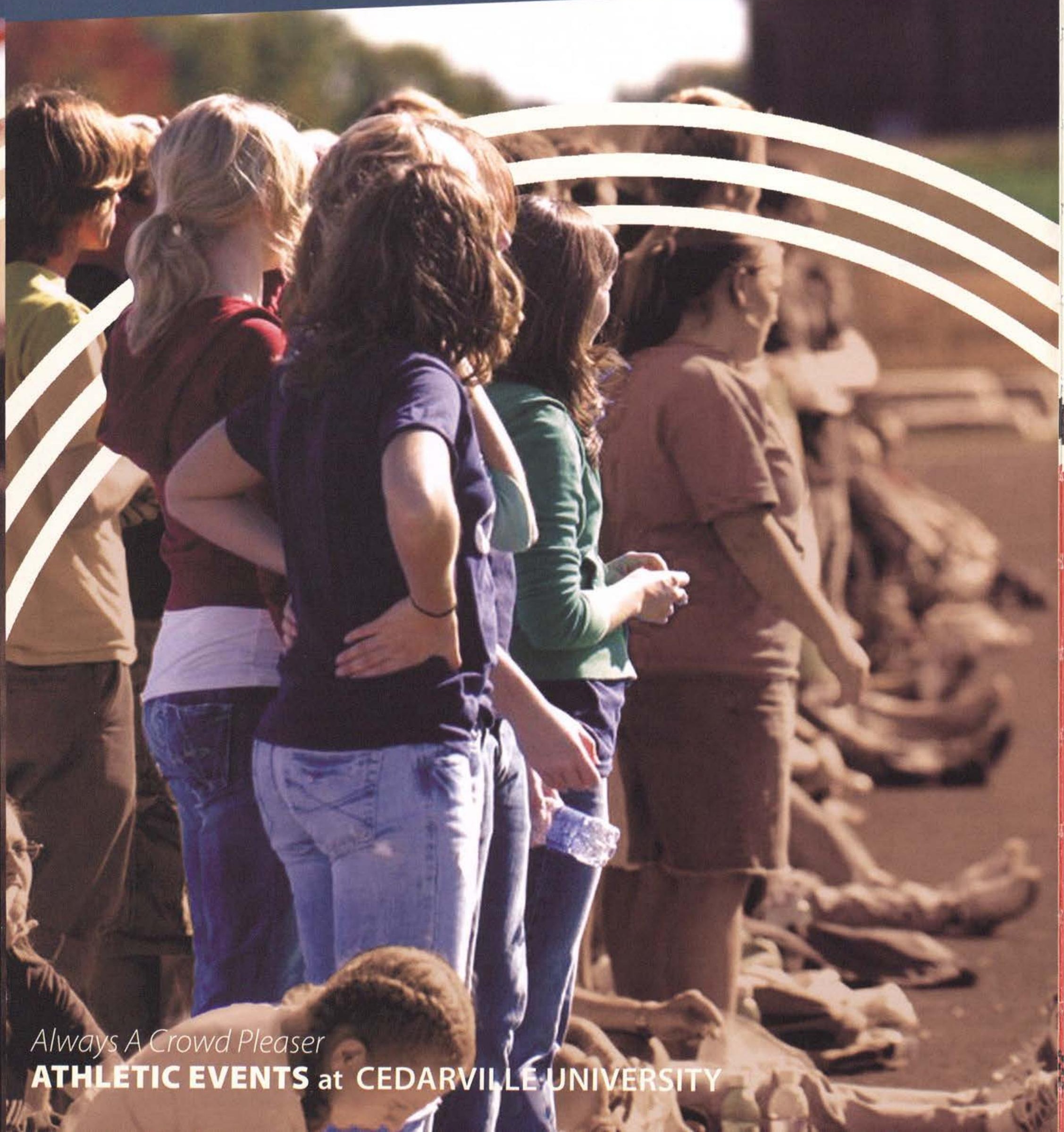




\section{Be Entertanined!}

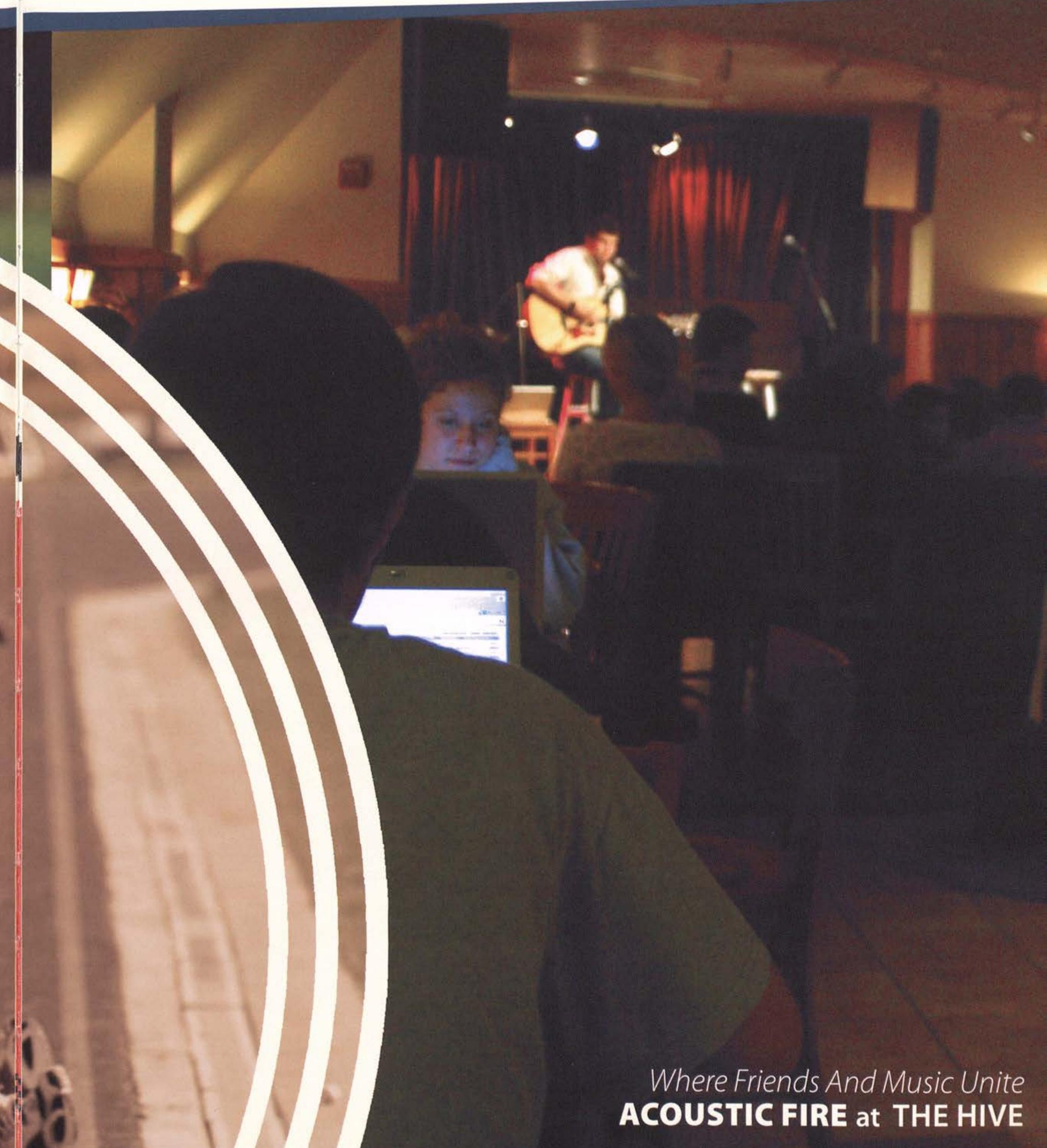



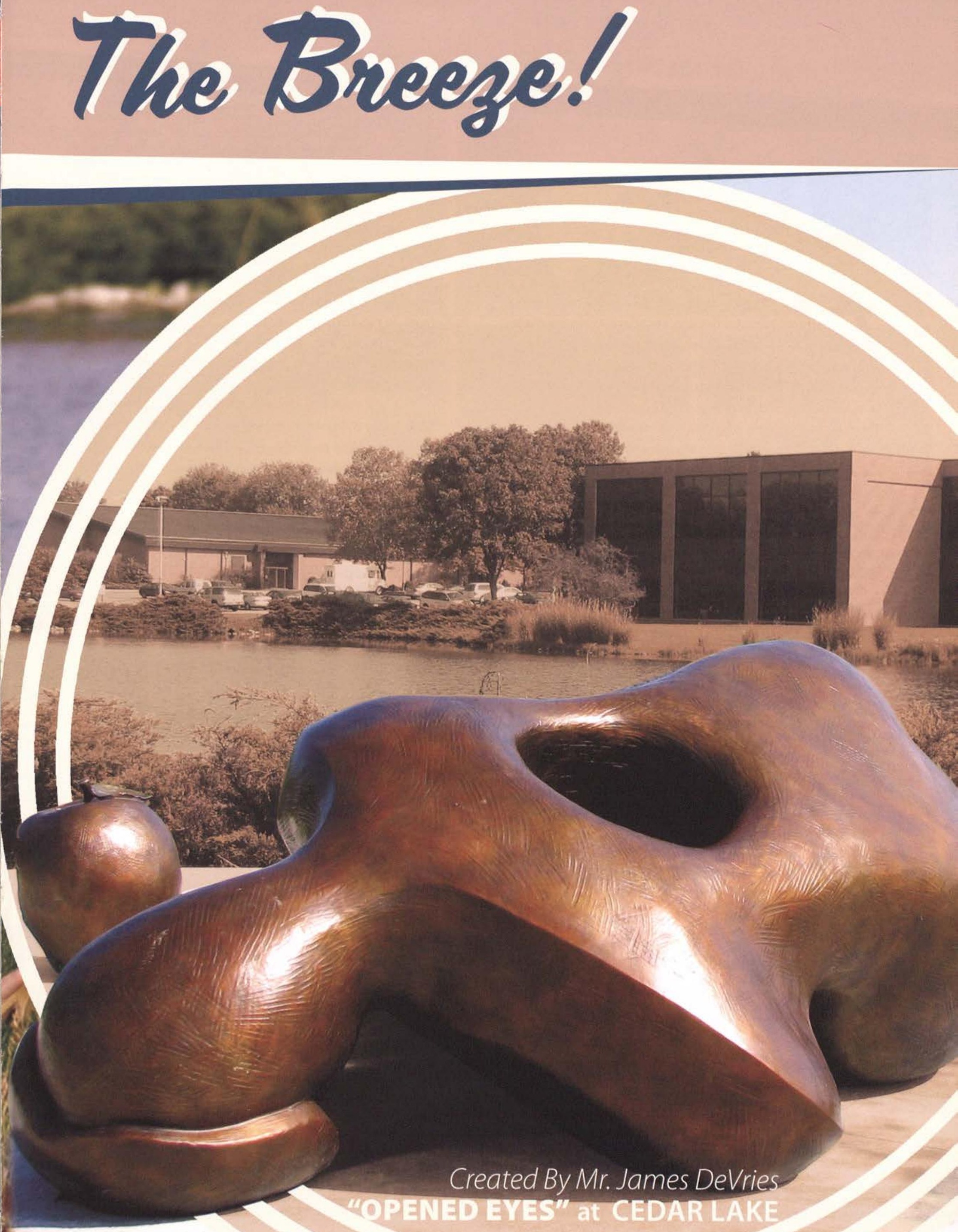

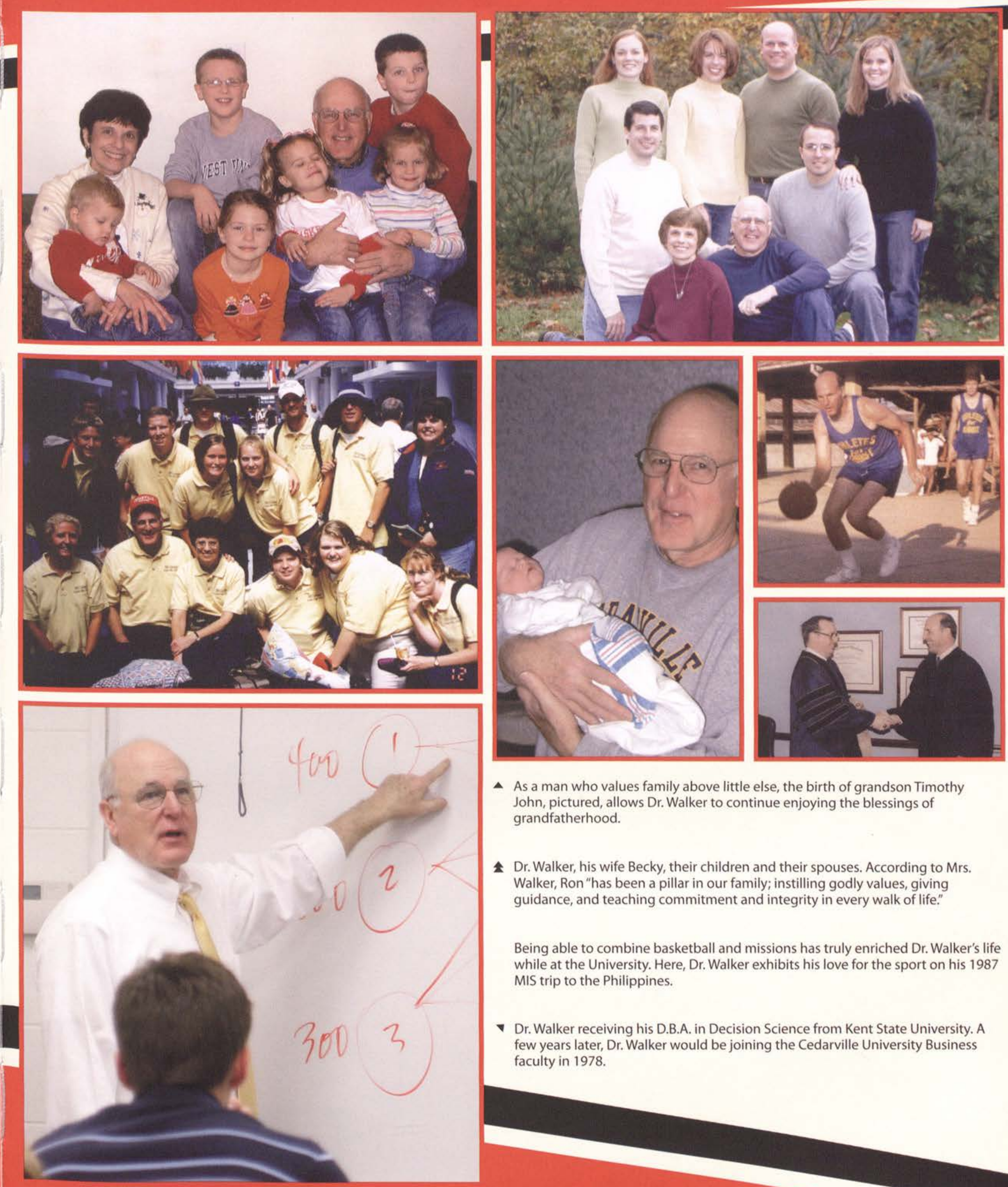

- As a man who values family above little else, the birth of grandson Timothy John, pictured, allows Dr. Walker to continue enjoying the blessings of grandfatherhood.

- Dr. Walker, his wife Becky, their children and their spouses. According to Mrs. Walker, Ron "has been a pillar in our family; instilling godly values, giving guidance, and teaching commitment and integrity in every walk of life."

Being able to combine basketball and missions has truly enriched Dr. Walker's life while at the University. Here, Dr. Walker exhibits his love for the sport on his 1987 MIS trip to the Philippines.

- Dr. Walker receiving his D.B.A. in Decision Science from Kent State University. A few years later, Dr. Walker would be joining the Cedarville University Business faculty in 1978.

- Dr. Walker contributes greatly to the Business department, imparting his skills in teaching statistics and Operation Analysis classes. Senior Daryl Sando has always enjoyed the style and format of Dr. Walker's courses. She said, "His classes were hard but they really showed me what I needed to do work hard and excel." 


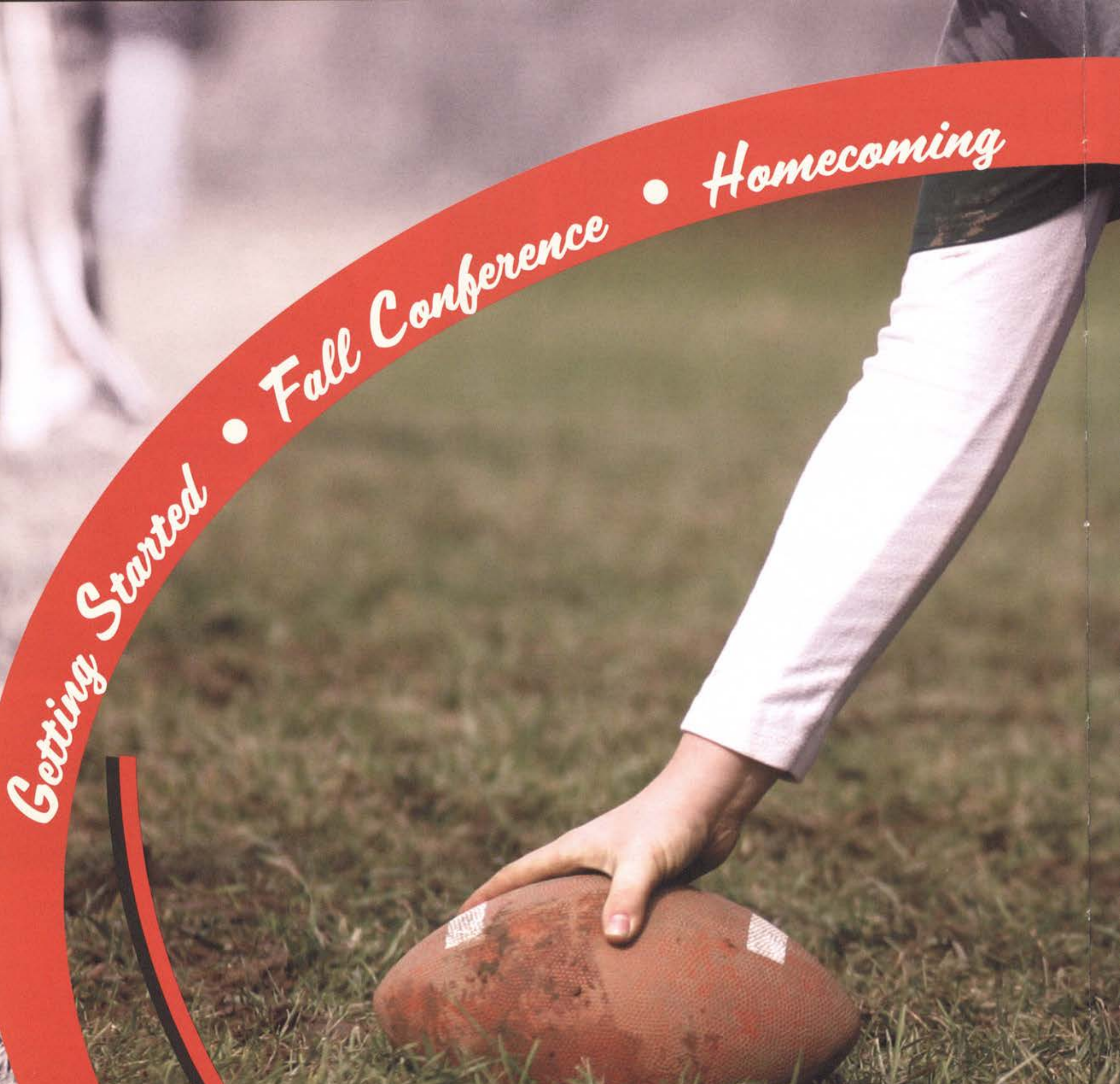
29 \%

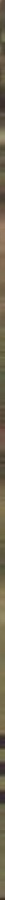


"I t was so much more than I expected," reflected freshman student Sarah Early on the weekend that seems a million miles away now: Getting Started. The freshman class of 2006 arrived earlier than returning students in order to have the campus to themselves for a special orientation time. Friday through Sunday was a bustle of activity between moving in, student and parent orientation sessions, and bonding time for all new students. Enthusiasm spread like wildfire across campus as music blared from dorm parking lots, students busily set up for the next nine months of their lives, and parents saw their children growing up right in front of their eyes.

The faculty and staff of Cedarville planned many orientation sessions for both students and parents to acclimate them to life as a member of the Cedarville family. Many parents especially appreciated the time and thought that Cedarville put into making sure they knew how to stay connected to their child both academically and emotionally. "I was surprised at how much I didn't know and how much I could still be involved in my child's life and education through Cedarville," expressed parent Sharon Martin.

The highlight of Getting Started Weekend for most new students was the Early Arrival Party. With free food, games, and random entertainment, the party provided a great atmosphere for meeting new people and getting a glimpse of college life at Cedarville. New student Kortni Hasselbach added, "The whole weekend was really effective for meeting people - the party, small groups, dorm units - everything."

Getting Started Weekend exceeded the expectations of both students and parents as they were "oriented" to this new aspect of their lives. It gave them a chance to experience with their families the exciting changes that were taking place. While it was the end for some things, it was the beginning and the "getting started" of something bigger than any freshman could imagine.

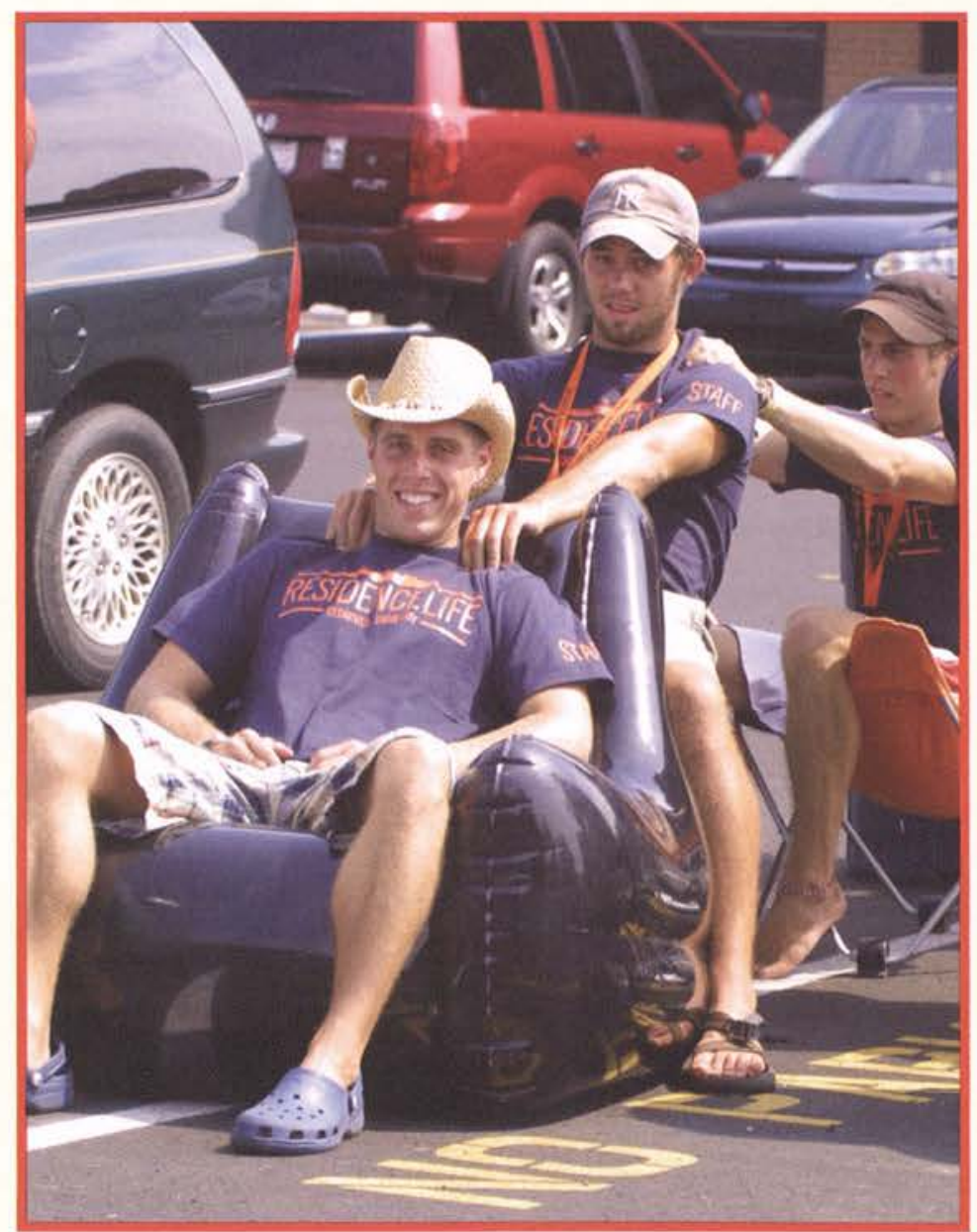

- Lawlor RAs Justin McCoy, Tim Miller, and Dan Lagan take a break from helping students move in. "Getting Started weekend is a blast. I especially like the look on the freshman parents' faces when they realize this is who they're leaving their son with," said Justin.

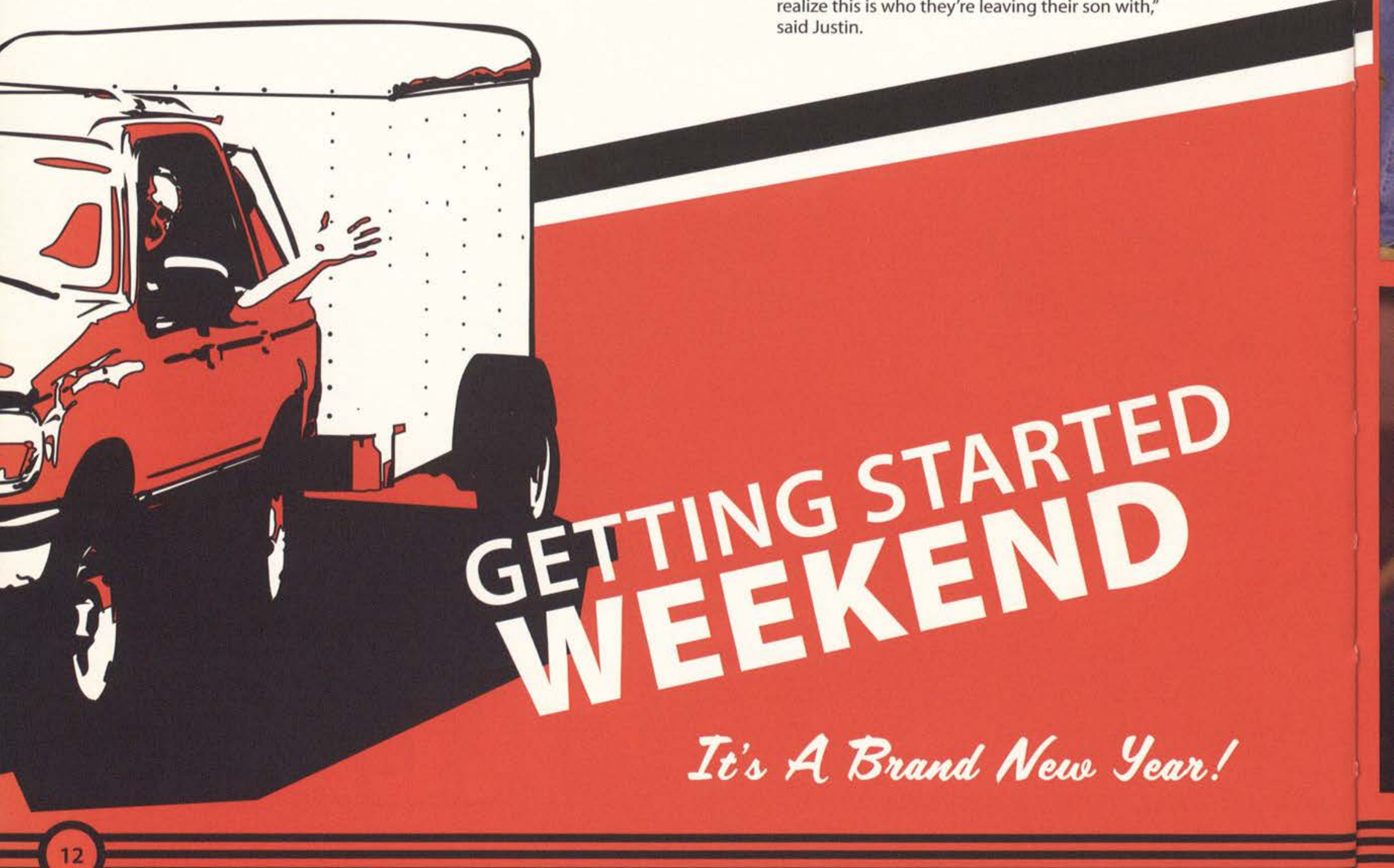




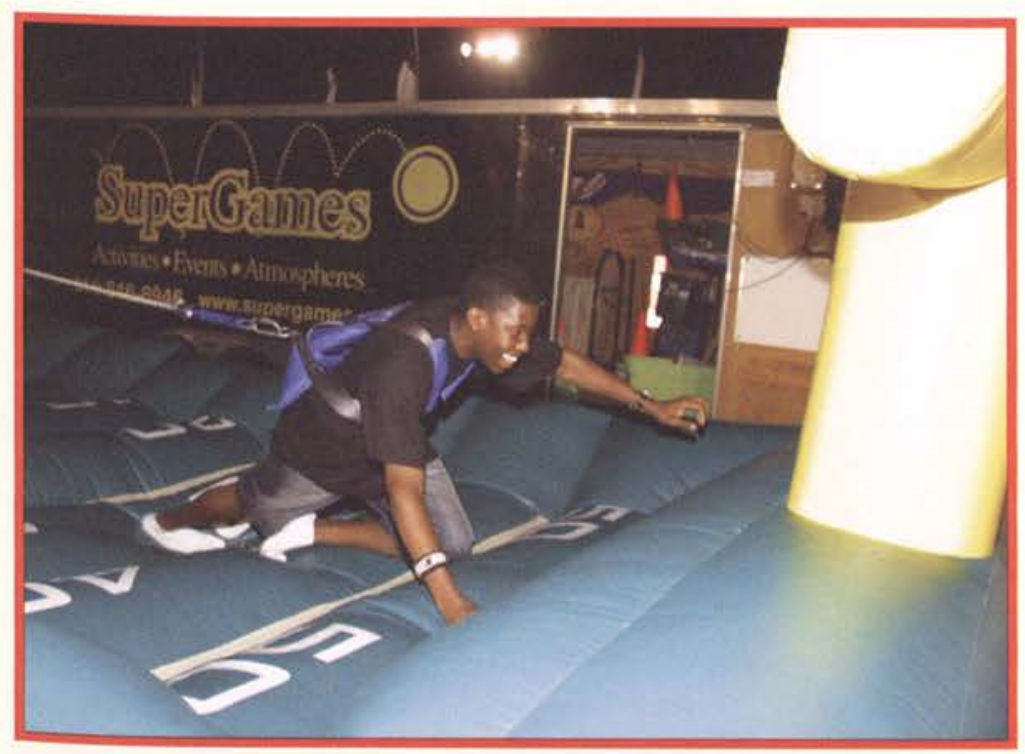

- The Freshman Early Arrival Party proves to be a challenge to Stephen Okoro as he takes on one of the many inflatable games of the night.

- Sporting their new SGA t-shirts, Christy Zike and her family enjoy the weekend together and take advantage of all there is to do.

Alberto Carrion worships God as the Class of 2010 comes together for a special welcome session and praise and worship time. "Getting started in a new school far away from home can be a challenge, but in the midst of that, one must seek God's guidance," remembers Alberto.

- "This is the closest you'll ever get to dancing at Cedarville!" said freshman Robert Wynalda as he, Darrin Carrier, and Matt Krogstad participate in the traditional 500 Miles Dance.

- Getting Started crew members Elizabeth Studebaker and Caleb Hensley help freshmen and their families move into the dorms.
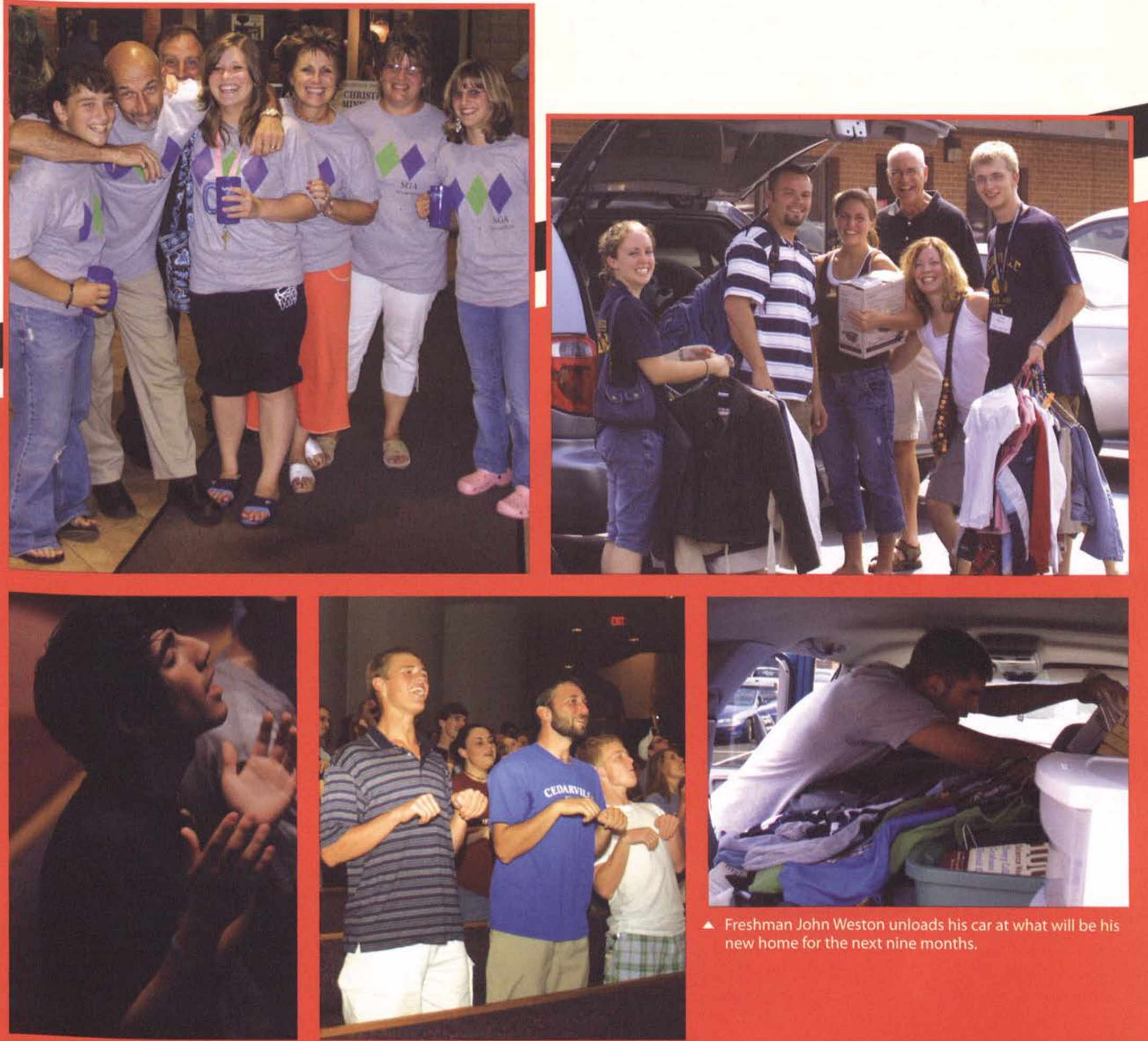

A. Freshman John Weston unloads his car at what will be his new home for the next nine months. 
$c$ edarville University prides itself on starting the school year off with the Fall Bible Conference. What better way to commence a new year than by easing a few chapels and night services in before classes? "It gave us a fresh perspective on what we believe and why, and how we should act upon our belief," sophomore Kaitlin Dunnevant reflected. On August 21, 2006, President Bill Brown set the 2006-2007 school year in motion by introducing our theme: "Go and Make Disciples" from the Great Commission of Matthew 28:17-20. Those who experienced hard circumstances over the summer were lifted up in prayer-a powerful reminder of what over three thousand students plus faculty and staff can bear through the support of their brothers and sisters in Christ. Students also enjoyed a new worship leader, Matt Rexford, and innovative praise songs. The following day, Dr. Brown introduced the Bible Conference speaker: inspirational, lively, and challenging Dr. Voddie Baucham. Though returning students were disappointed with not being able to sing the traditional Lion of Judah, Dr. Baucham brought his own lion to the stage in his messages about contending for the faith. Senior Sarah Ensslen was especially appreciative of his topic for the week. She said, "Contending for the faith was a great topic for our last Fall Bible Conference as we will soon leave Cedarville and be immersed in secular society every day."

No student was caught sleeping as Baucham began by placing Jeremiah 29:11 back into context and answered our worldview questions with

Colossians 1:15-23. Sophomore Corey Reeder appreciated the passion he held for his chosen topic. "[His] passion was evident in how sound his doctrine was and how he solidly stood on the things he knew." His genuine desire to touch the lost, oppressed, and hurting world inspired many as he urged students to rise up and share the gospel. His compelling gestures, compassionate tears, and urgent voice made every student feel the depth of his ultimate challenge: "Eternity is at stake, Cedarville!"

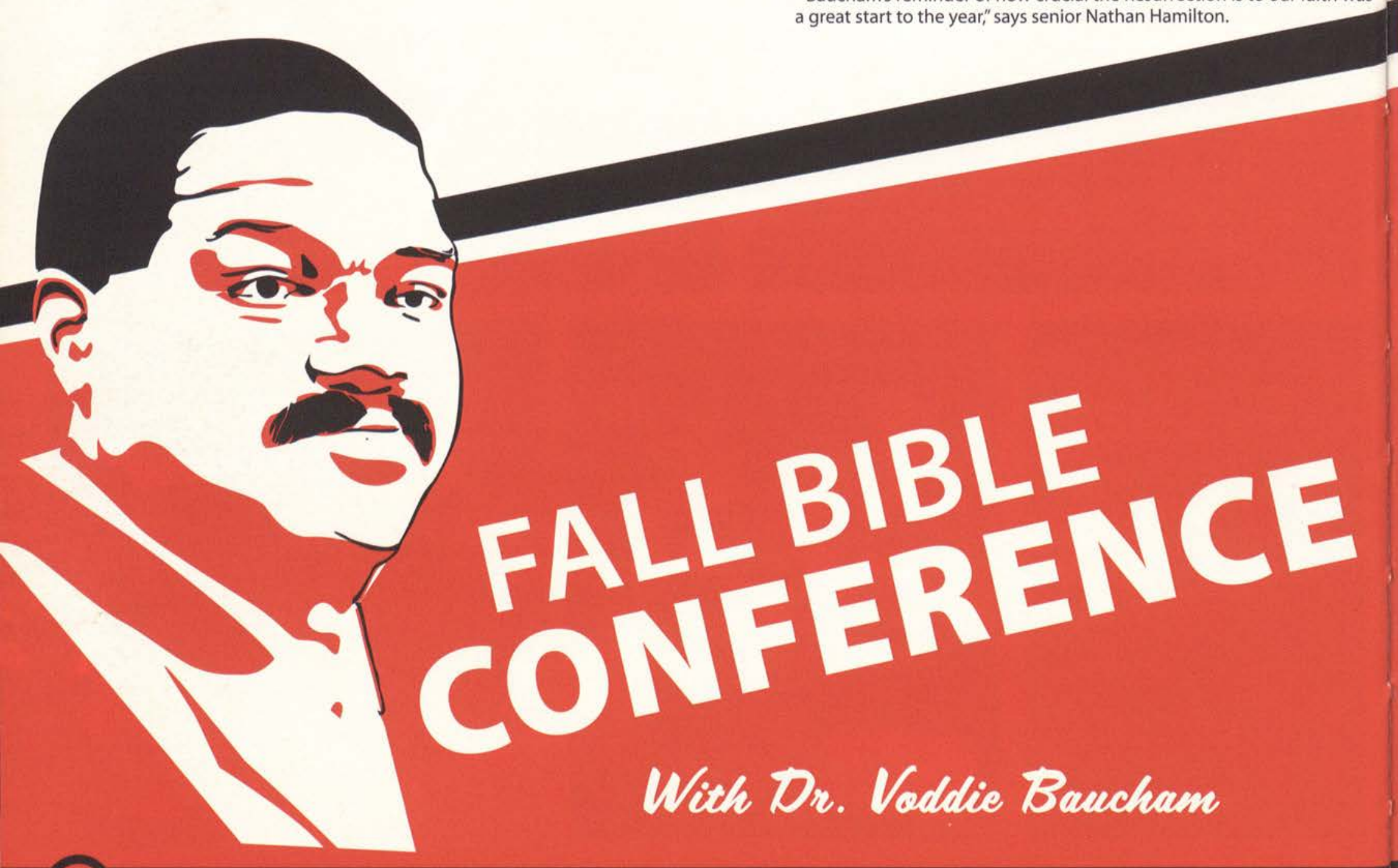




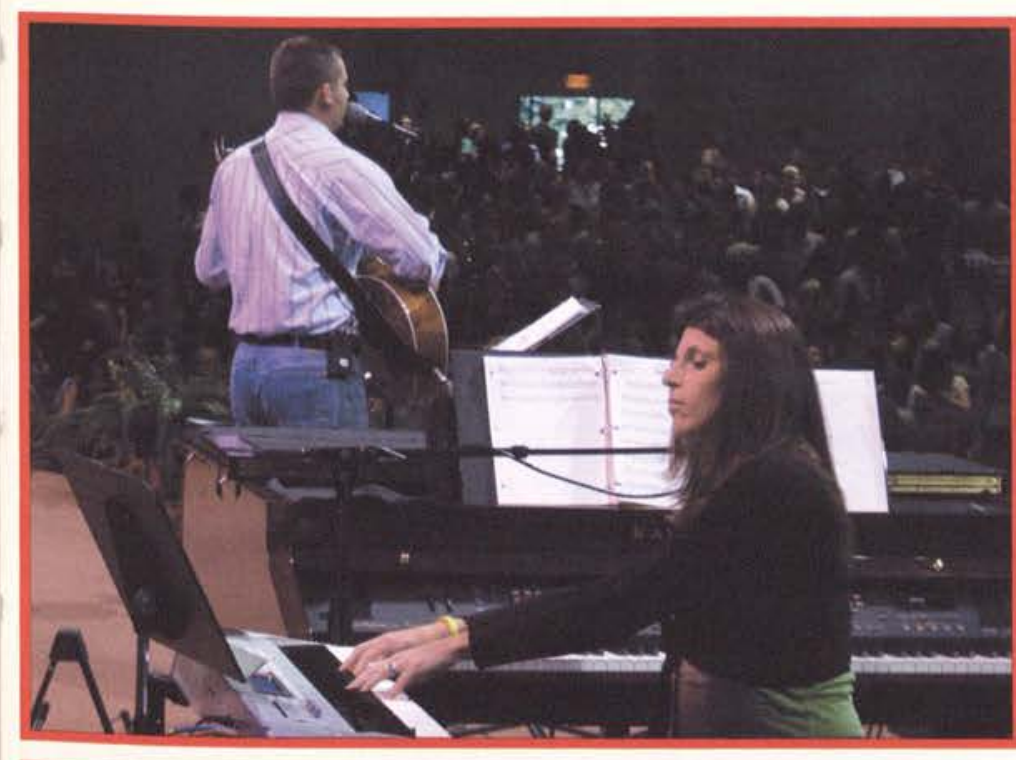

- Matt and Whitney Rexford start the school year off with innovative songs and lively worship. Although this was a change from Mr. DiCuirci's usual hymns at the beginning of each semester, many students appreciated the shift in music and the way that God used Matt to lead the student body in worship.

- Cedarville President Dr. Bill Brown commences the school year with the focus of "Go and Make Disciples," a prayer for the upcoming year, and a welcoming smile for the freshmen.

Matt introduces us to another new song during the week of Bible Conference, "Hosanna," which has quickly become a favorite around campus.

Music Professor Connie Anderson proudly shows off her graduation garb for another Commencement. Seeing the accomplished teachers sitting on the stage inspires the students to take advantage of their education and accomplish great tasks.

- Junior John Hawkins appreciates how the commencement worship "gave students the opportunity to see that they can be worship leaders just by showing up and rejoicing in the love of God." Sophomore Michelle Kropf also enjoys the new worship songs which gave her a "glimpse into what Heaven will be like, all joining together as one glorifying God."
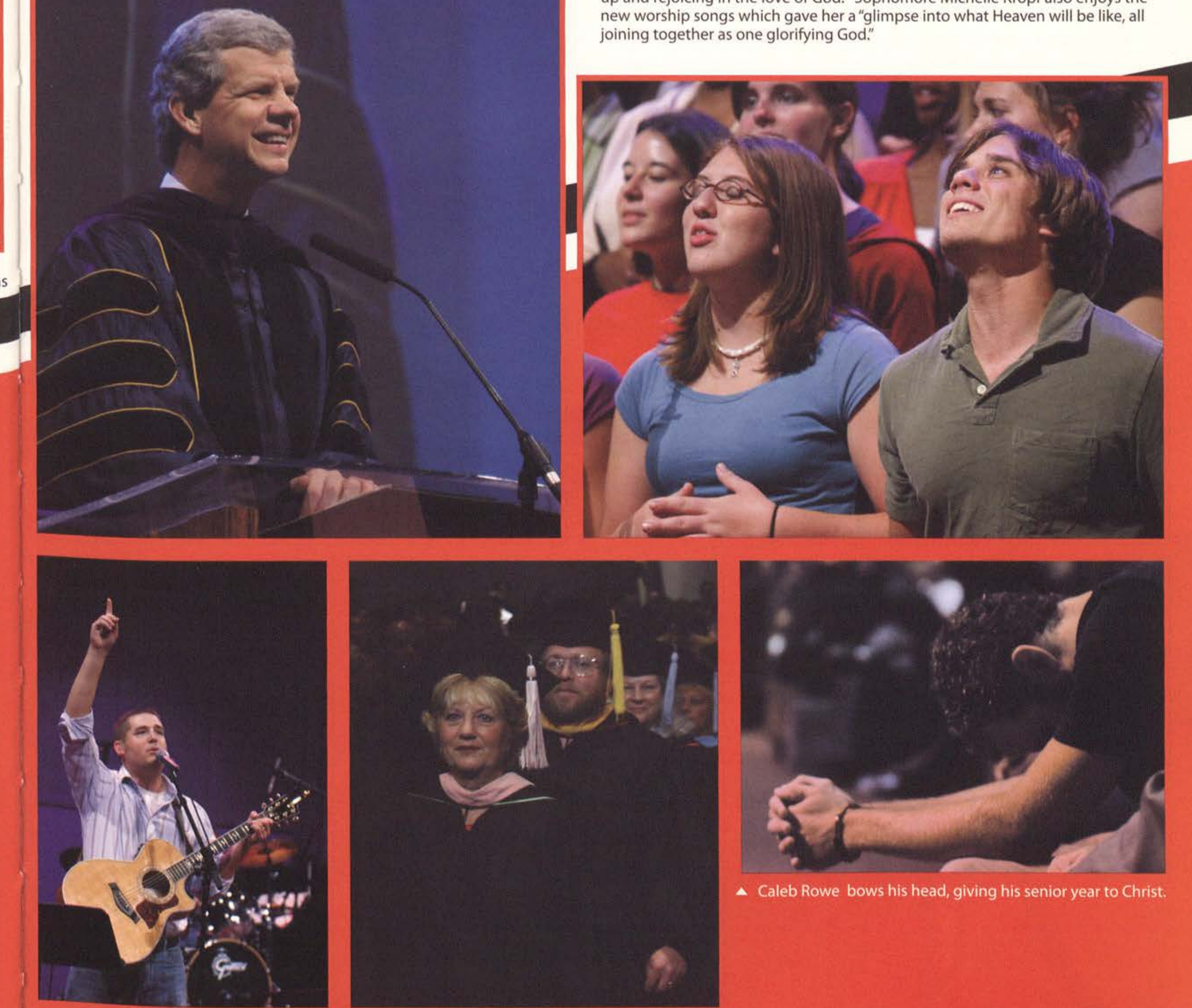

- Caleb Rowe bows his head, giving his senior year to Christ. 


\section{$S$} unlight slanted through a blue-and-yellow-tinted Main Street, speckling the pavement already crowded with students, alumni, and town residents, setting an October mood for Homecoming 2006 - festivities began with a bang. Cedarville police led the pack at the Homecoming Parade, followed by Grand Marshall Lew Gibbs and winners of the annual alumni awards. Following them was a whole host of familiar characters - the Addam's Family, Batman and the Joker, Gilligan, the Beverly Hillbillies, Ross and Rachel, Lucy, even Big Bird, all making appearances on behalf of a particular organization or dormitory. Root beer floats, tootsie rolls, and old-fashioned crackerjack boxes completed the scene, parade staples that reminded everyone Homecoming was just beginning.

That evening, the air, thick with spirit and excitement, rose to contain the screams of the more than 1,200 Yellow Jacket fans, many of them returning alumni, as they watched a rookie on the men's soccer team, Jason Buckley, blast the winning goal. Fellow teammate, senior Elliot Moore, described the experience as "one of those games that you want to win so bad because you want alumni to walk away with a smile on their faces. It's great playing, especially when you know there are so many people out there."

At half-time, the new cross-country track was unveiled and senior Jason Briggs became Cedarville's first Homecoming King in years. Fellow senior Nikki Carroll was crowned Homecoming Queen. "I was stunned to be nominated for queen, so when it was announced, I was absolutely shocked!" she said. "In my book, it's not important who won or didn't. It's that each of the nominees, and all of us, strive to be a reflection of Christ."

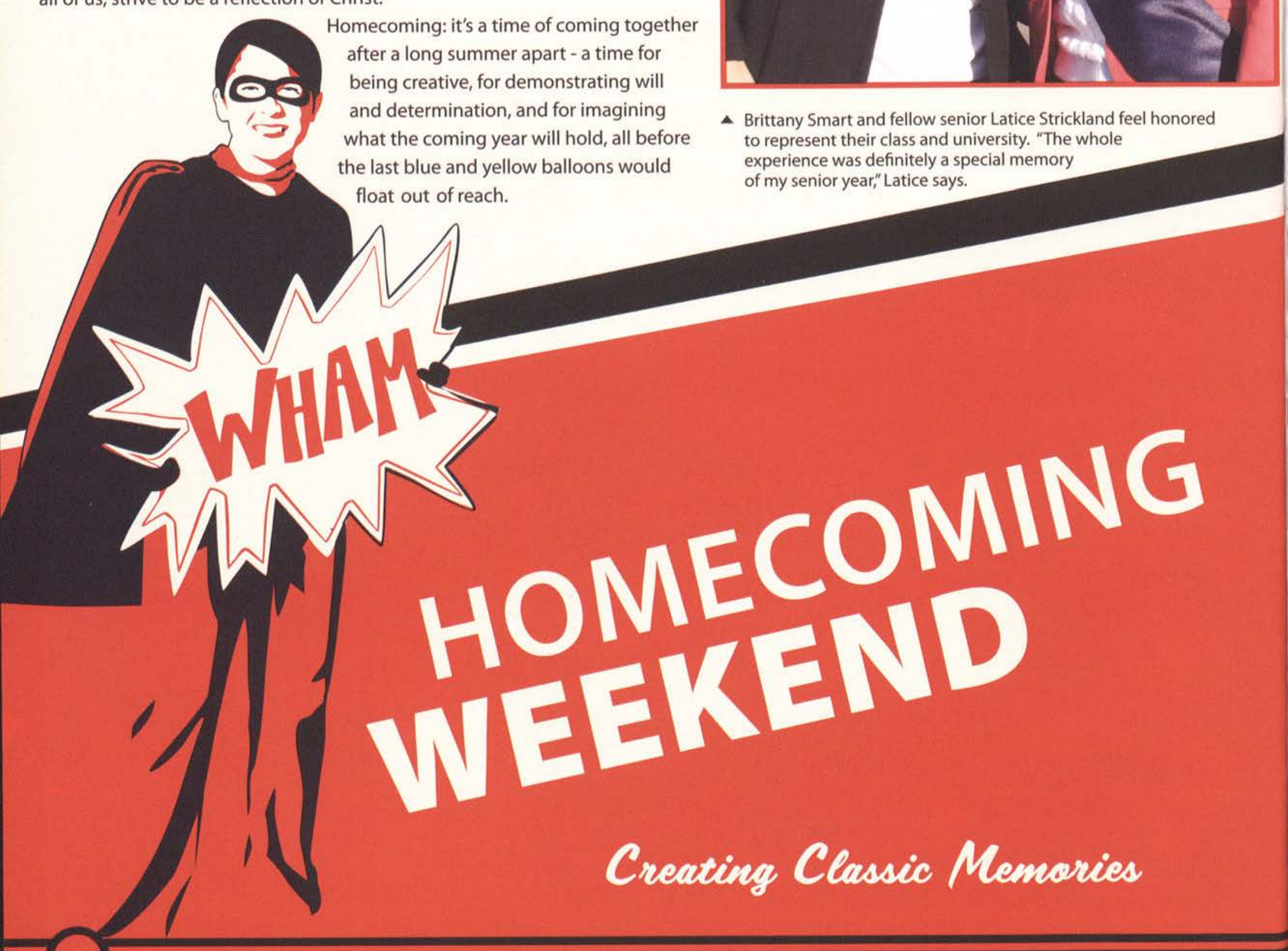

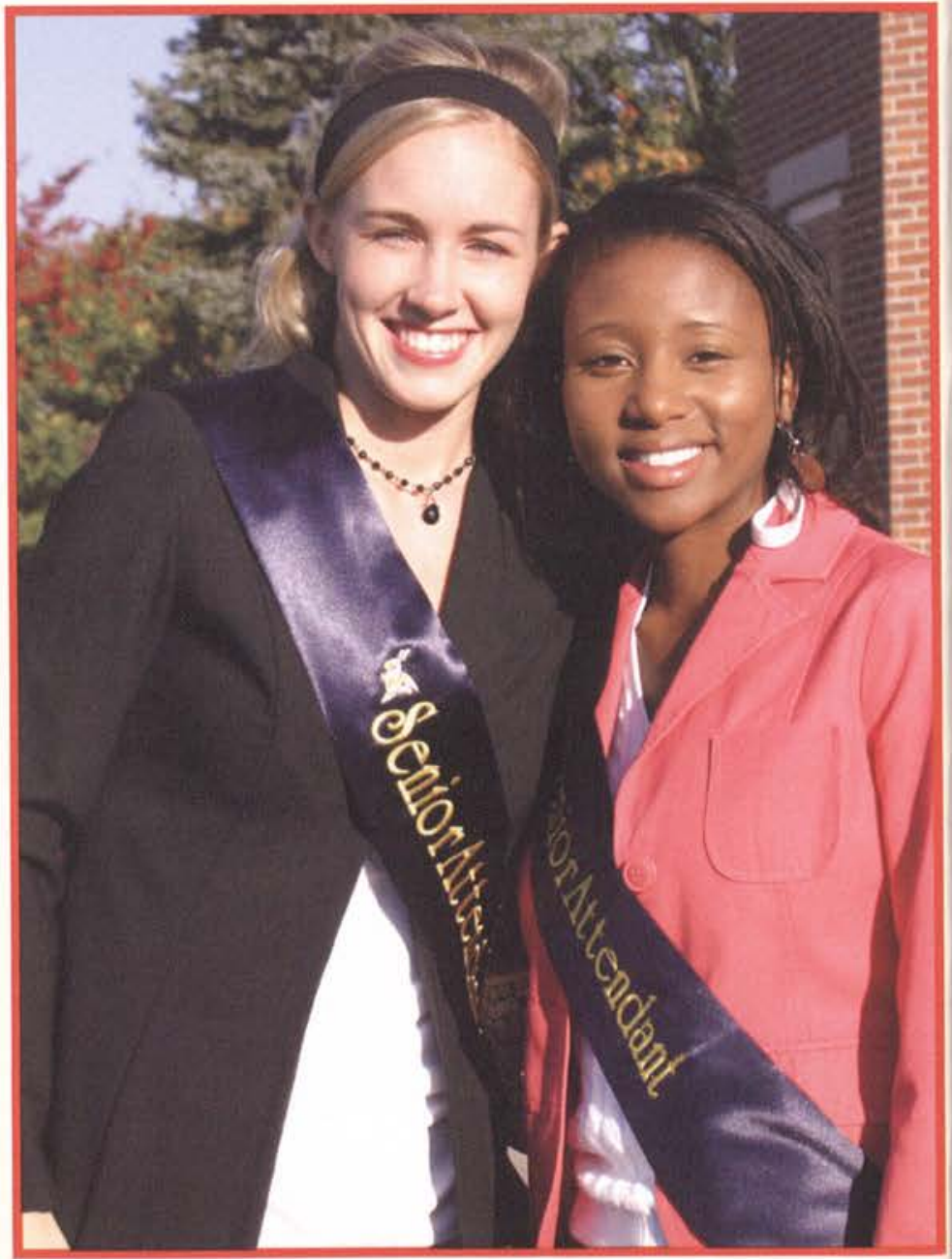

Brittany Smart and fellow senior Latice Strickland feel honored Brepresent their class and university. "The whole experience was definitely a special memory of my senior year," Latice says. 

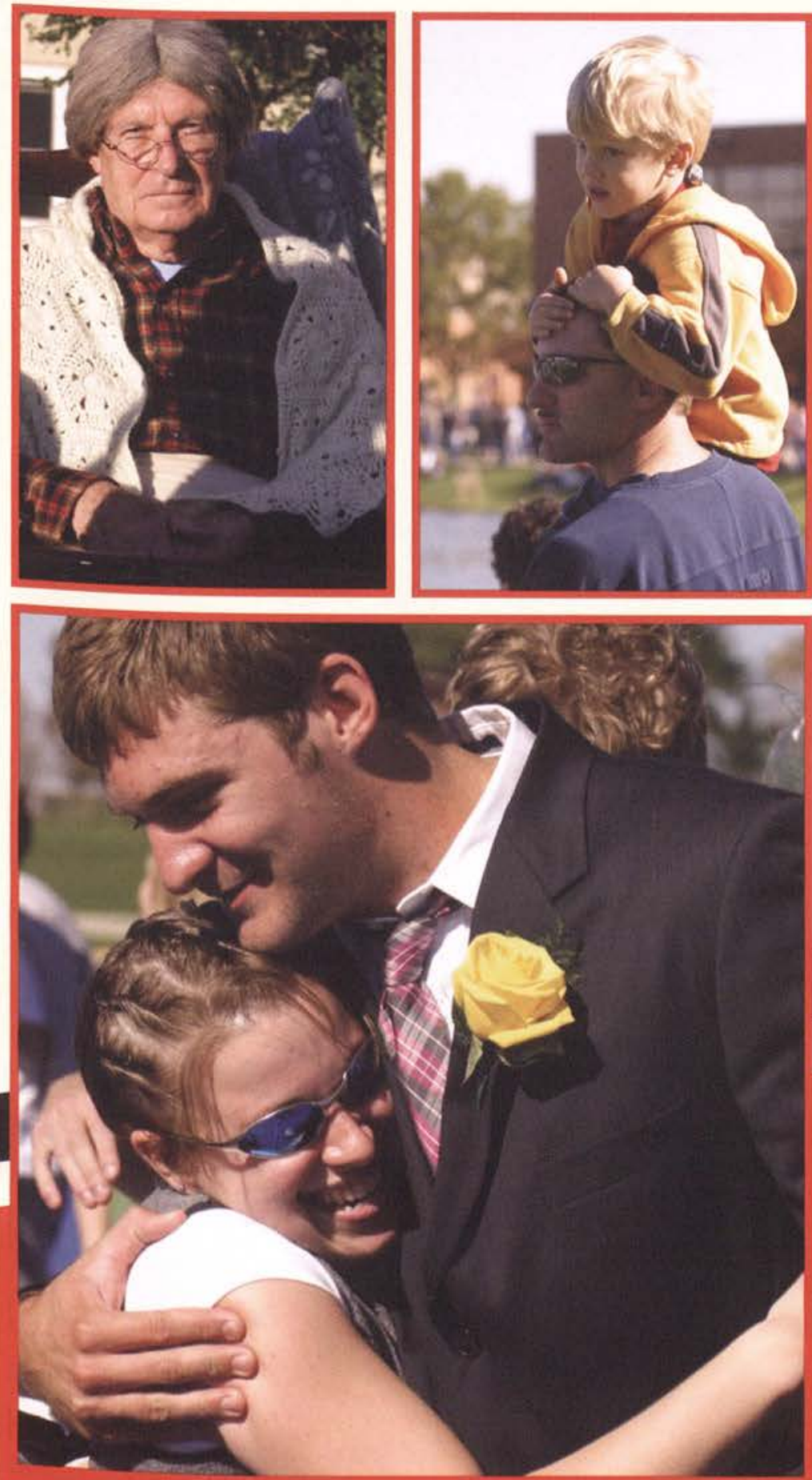

列

carts down Main Street to the tunes of yesteryear, these ladies hold

everyone transfixed.

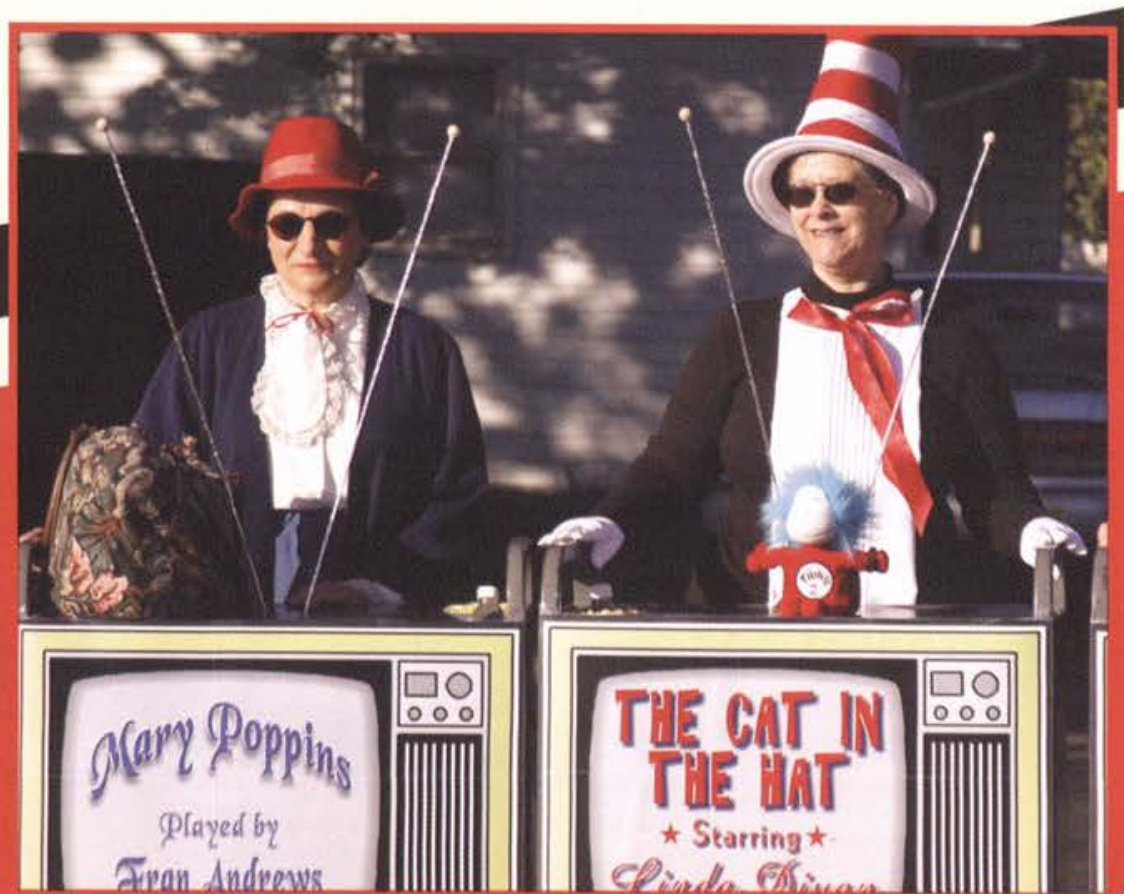

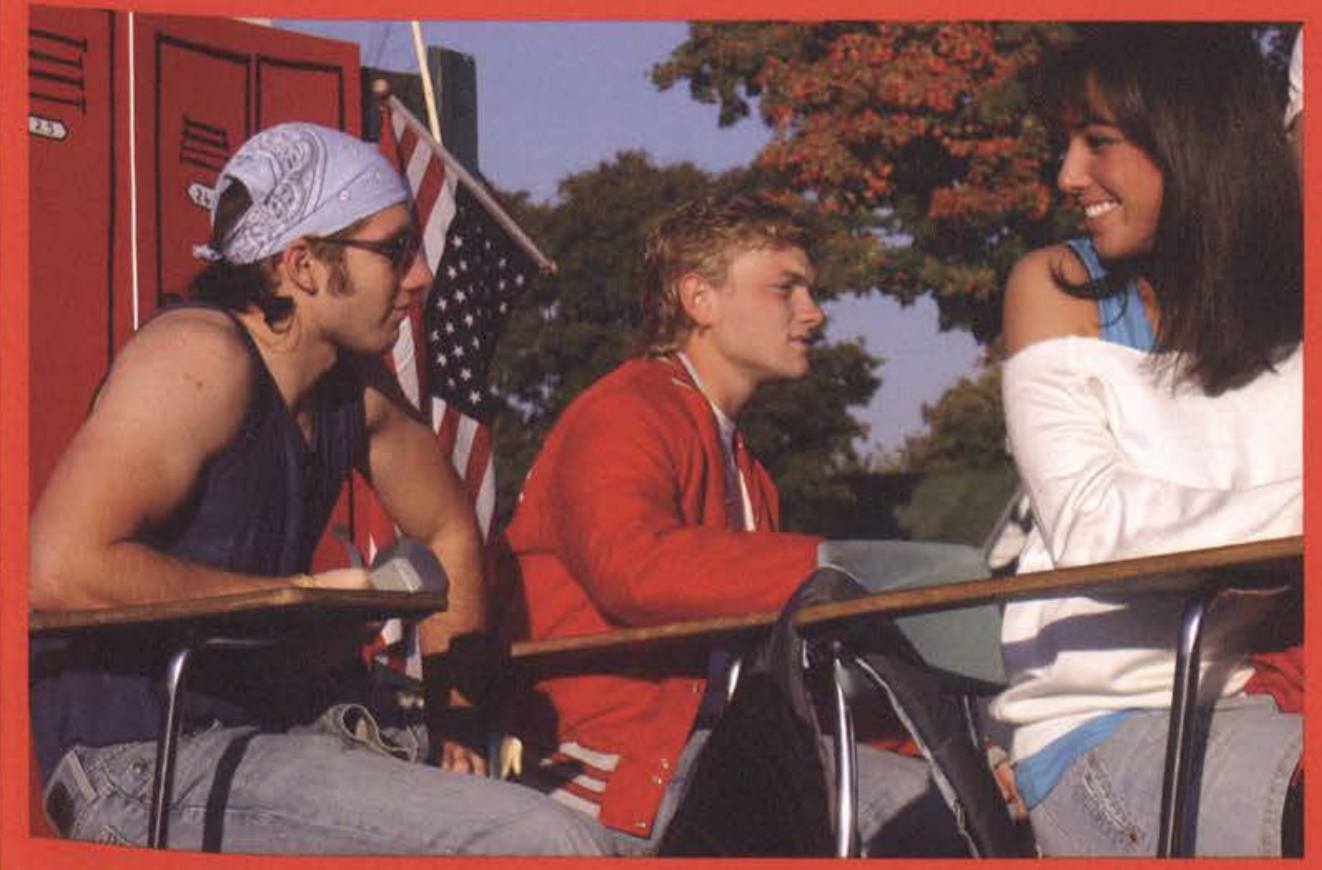

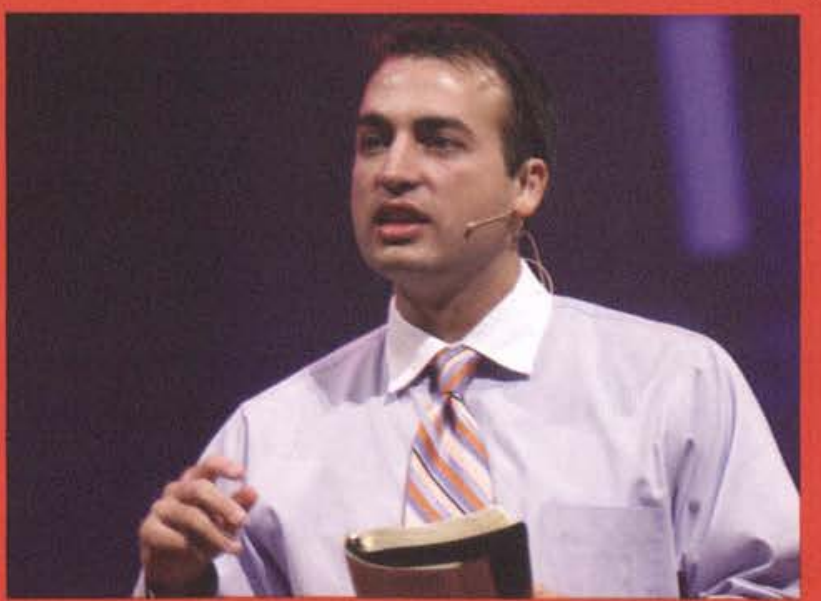

Alumnus Scott Lehr's heartfelt testimony of trust through life's darkest moments inspires the entire university community to ask a big God for small miracles. 
c lear skies and steady waters conflicted with rattled emotions and less-than-sturdy canoe designs at this year's cardboard canoe race. Having returned to the standard race format after a treacherous new course was attempted last year, several freshman engineers made it safely to the other side of Cedar Lake with little damage to their precious creations. While a number of competitors did an impressive job of paddling through the turbulent waters, this year's winners held the spectators' attention with a unique design and break-neck time.

Freshman Michael Conyers, a member of the winning team, described the special design of their canoe. As opposed to the typical canoe design, Conyers and his team built a canoe that was 11 feet long and 2 feet wide, allowing smooth movement across the water. However, according to advisor and senior Ryan Prins, having Conyers on the team enabled their victory simply because "he's a stud."

Another surprising factor that clenched the prized TI-89's for these freshman engineers was their passenger, junior Amanda Cole. According to teammate Nathan Ciolek, "She was yelling what to do to our guys while riding across the lake. If she wasn't allowed to help paddle in any way, at least she could keep the engineers

\section{help paddle in any way, at le}
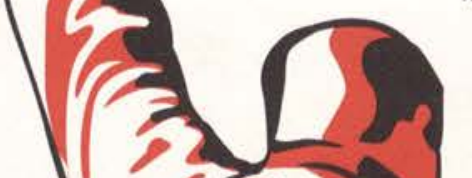

Engineering students weren't the only victors on canoe race day, however. In the departmental races, the Centennial Library staff's canoe barely snatched the coveted trophy from the Student

Campus Activities Board in the last seconds of the race. Junior paddler Linnea Norris explained the most difficult part of the race was "jumping out of the canoe at the end into squishy lake muck that encapsulated my legs."

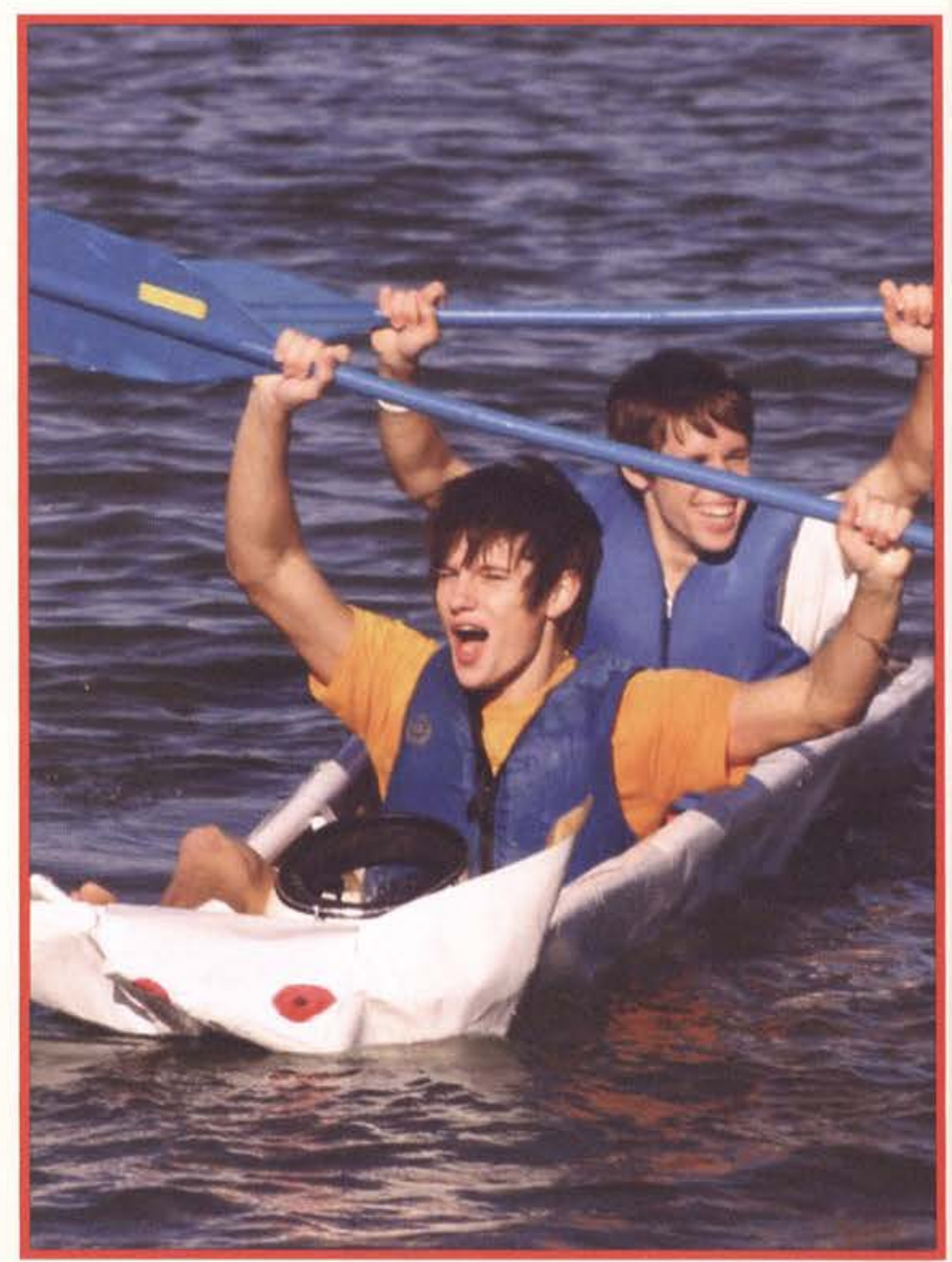

- Trey Johnston and Kyle Spivey (back) celebrate their demolition derby win. According to Spivey, "after the first [brace] broke..I

thought we were dead, but in the end The Gremlin prevailed"

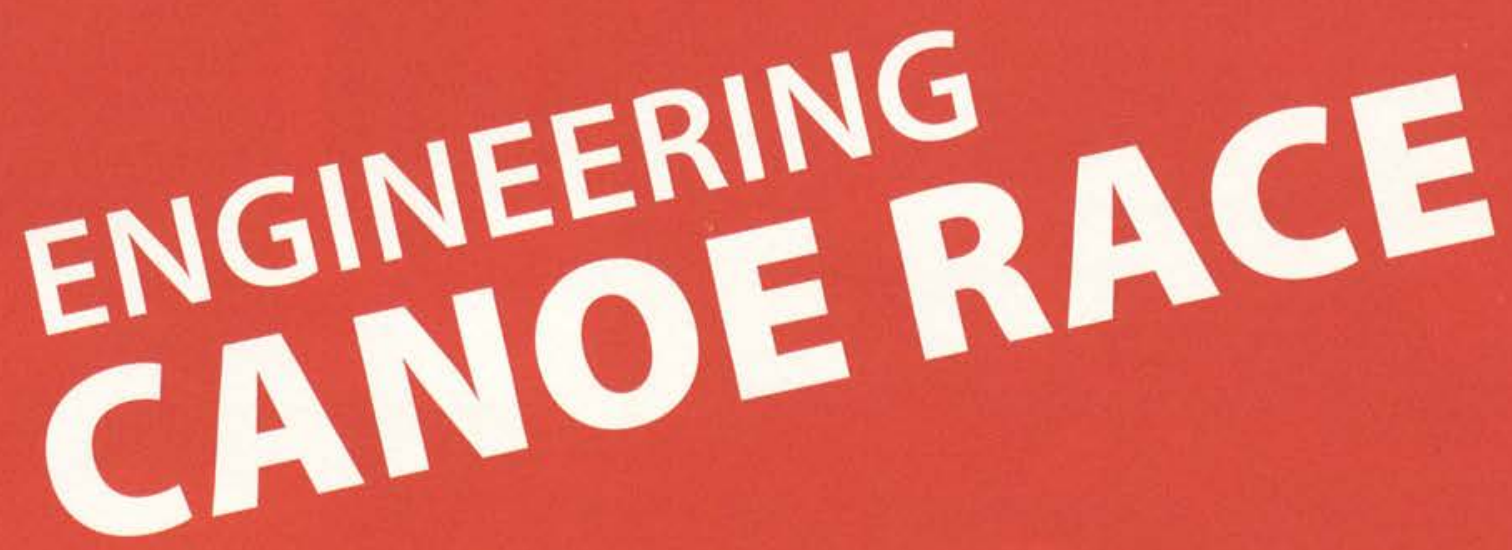

And... They'n OBS! 


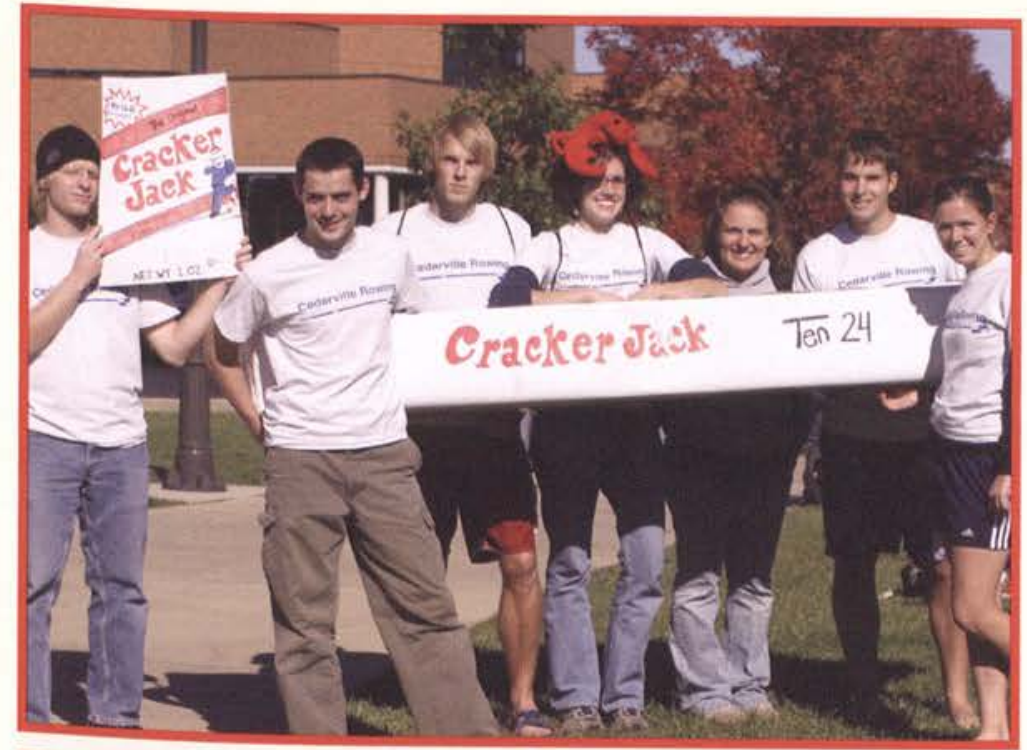

4 Members of Ten24 (formerly $C A B$ ) pose in front of their departmental canoe entry. Senior Courtney Brott explained that "last year the boat sunk right away, so having a competitive finish was a lot of fun for all of us."

- John Hagen (back) describes the harrowing journey: "We paddled across the whole lake as fast as we could, leaking and slowly sinking the entire way. Finally, just as we reached the bank of the finish, we went under. But thanks to the graciousness of Dr. Chaznov, we still counted as a finish!"

- The Canoe race consistently attracts a healthy crowd every year. Junior Bob Thalman, one of the spectators, recalls that "It was completely nerve-racking to watch. The demolition derby was incredible; I'm a big fan. I'm so glad I didn't have to swim around in Cedar Lake; l'd probably mutate."

F Sophomore Rachel Gredy, bringing up the rear, leaves the canoe race experience with nothing but positivity. She explains, "It was fun getting to know the girls and figuring out ways of not sinking in the lake, which was all that mattered to me at the time!"
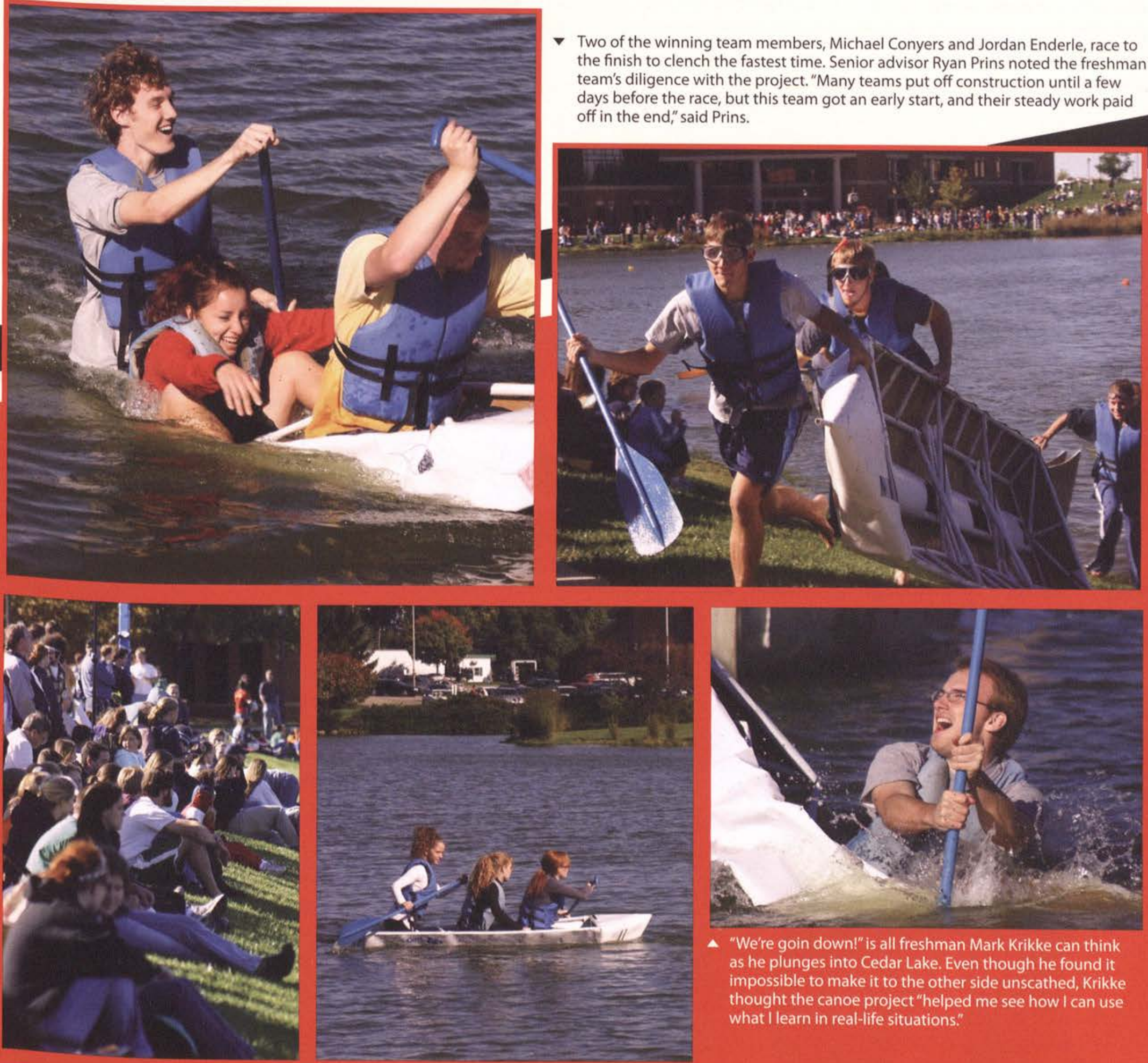

- "We're goin down!" is all freshman Mark Krikke can think as he plunges into Cedar Lake. Even though he found it impossible to make it to the other side unscathed, Krikke thought the canoe project "helped me see how I can use what I learn in real-life situations." 


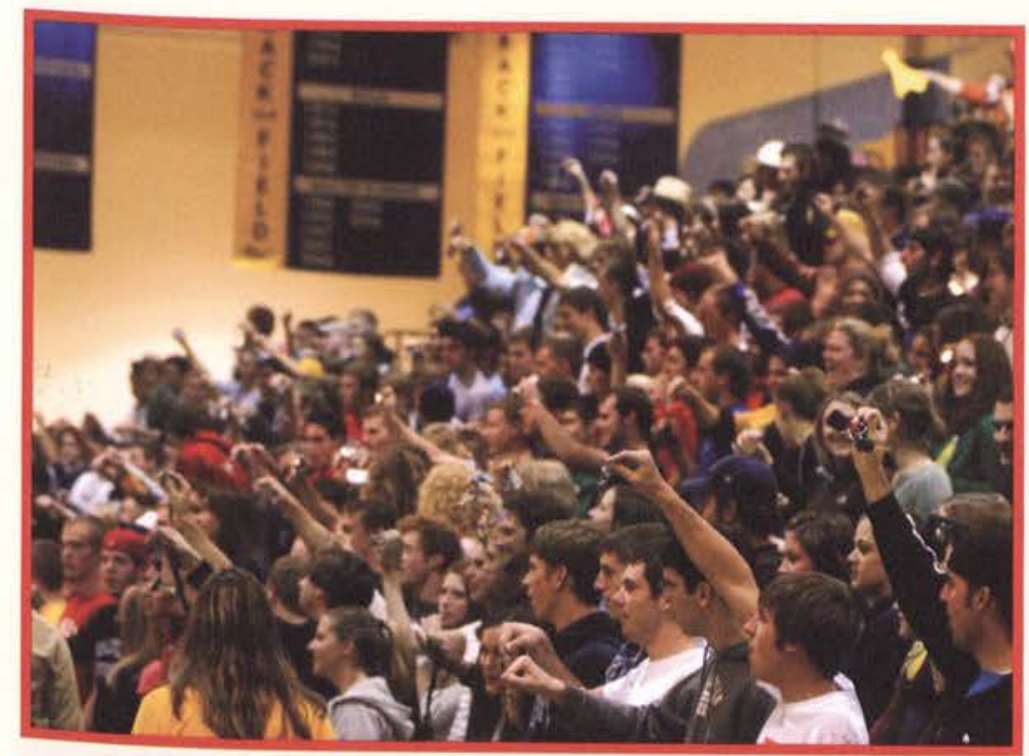

The crowd encourages the teams with its energy and enthusiasm. At Rod's instruction, fans jingle their keys, wave their CU Rally Towels, and shout, "Let's go Jackets!

- Rod Bradley starts the evening off by educating the fans, teaching us the basic cheers. The crowd quickly learned to stomp and shout "Defense!" and make some noise for their favorite team.

- Cheerleading co-captain Heather Kelton is being held in a half extension. Heather says, "It's always fun to get the crowd yelling 'Blue and gold' with us."

Greg Briggs shows his support for the Jackets by competing in a halftime relay race while struggling to hold up a larger player's jersey.

4 "Moonlight Madness is one of the greatest times of my Cedarville experience," says Maicol Venter, who is pictured during the tip-off with rival Chris Walker.

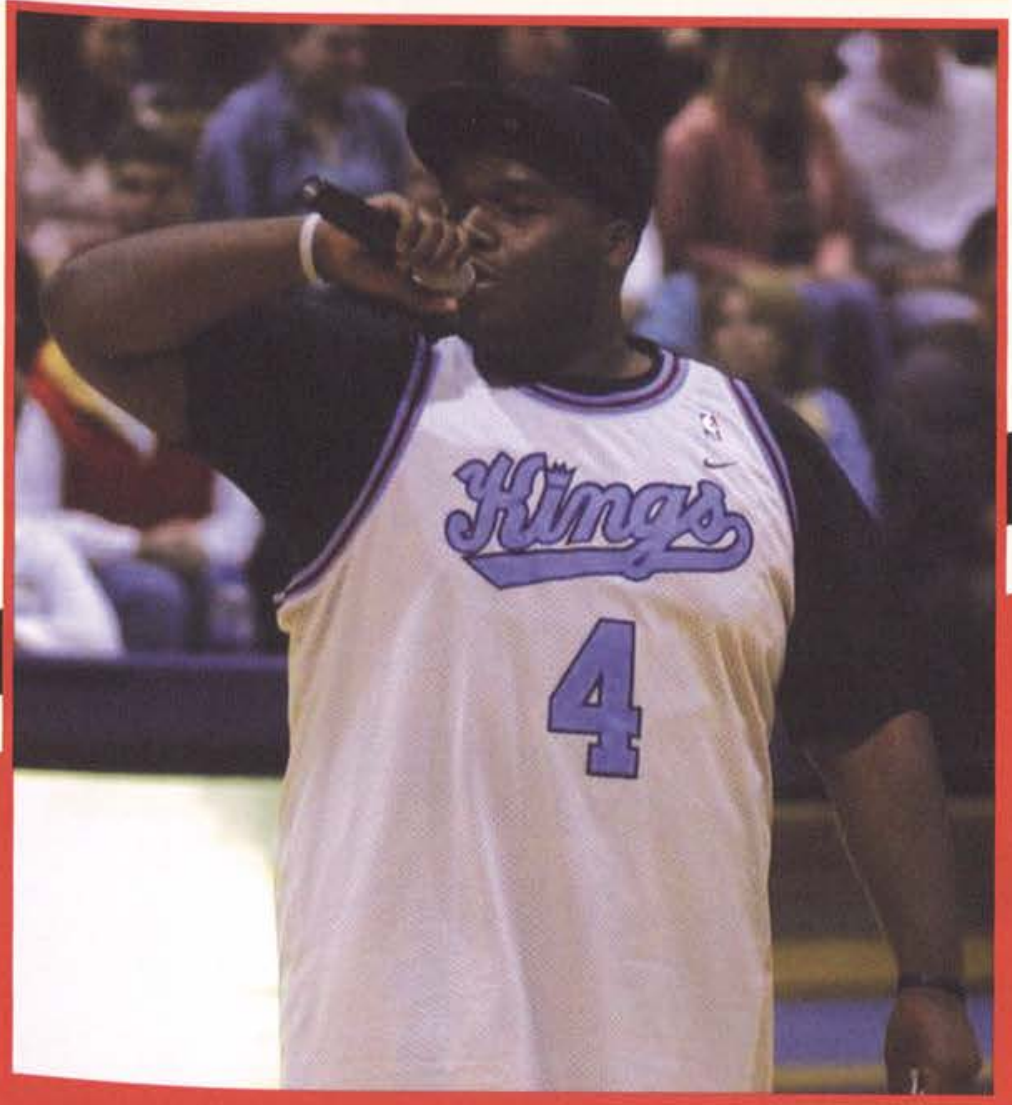

- "I had so much fun cheering this year. It was really great to see so much school spirit thanks to the amazing fans," says cheerleading co-captain Ashley Coale.
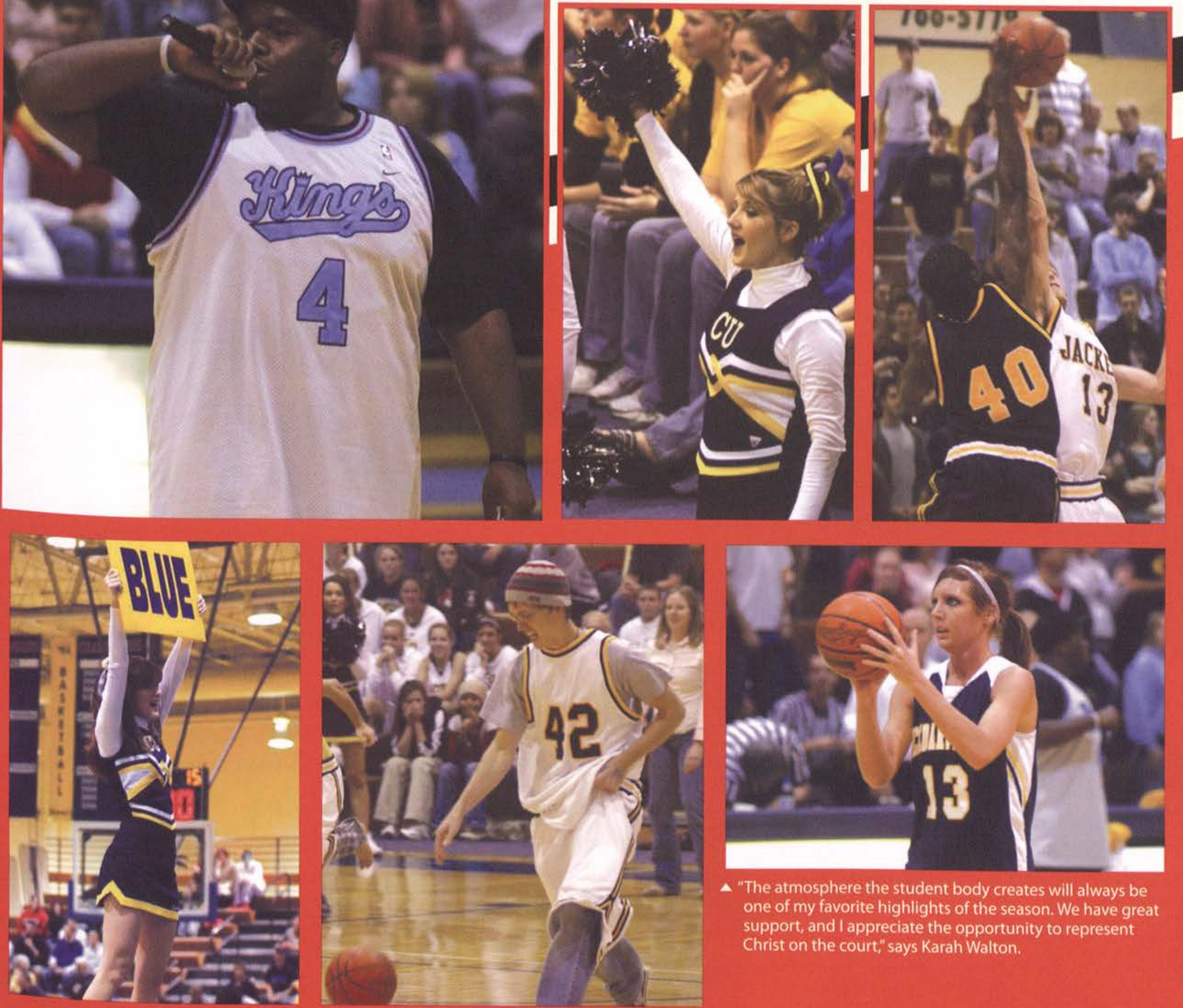

A "The atmosphere the student body creates will always be one of my favorite highlights of the season. We have great support, and I appreciate the opportunity to represent Christ on the court," says Karah Walton. 
$S$ nap! From the thriller pantomime until the revelation, every audience member asked "whodunit?" as Cedarville's actors performed Agatha Christie's classic play, The Mousetrap. Each audience member was handed a black card and asked to vote during intermission for whom they suspected was the murderer. For those unfamiliar with the play, the suspect was constantly shifting from one character to another. The mystery increased as the unfortunate Mrs. Boyle, played by senior Rebecca Wolff, was killed. Rebecca agreed the audience probably was not too sad to see this cynical woman go, yet she had fun "pretending to be strangled and actually making it sound realistic."

Paravicini, the "unexpected guest" played by Grant Hansen, caused raised eyebrows as Grant enjoyed "identifying the minute mannerisms and quirks of [Paravicini, which] transformed the black and white pages of the script into a believable character." After her interview with Detective Sergeant Trotter, Miss Casewell, played by senior Jaimie Teekell, exclaimed that the inspector has solved the case! Jaimie admits, "The best part about playing Miss Casewell was her cynicism!" At last, the cloak was thrown off to reveal Trotter, played by sophomore Daniel Zimmerman, as the murderer. Daniel had played the part of Paravicini in a high school performance of The Mousetrap and wanted to play the murderer this time. His wish was granted as he surprised everyone who had not already been familiar with Agatha Christie's works. His challenge was "acting as an actor within another actor." Senior Bonnie Wright, an avid Agatha Christi reader, enjoyed the performance. Bonnie noted that while the audience was given an opportunity to vote for the murderer, Trotter was not on the ballot, which "added to the whole deception of the play."

Bravo to the actors, crews, and technicians for their ability to master another classic performance of one of the longest running

plays in history.
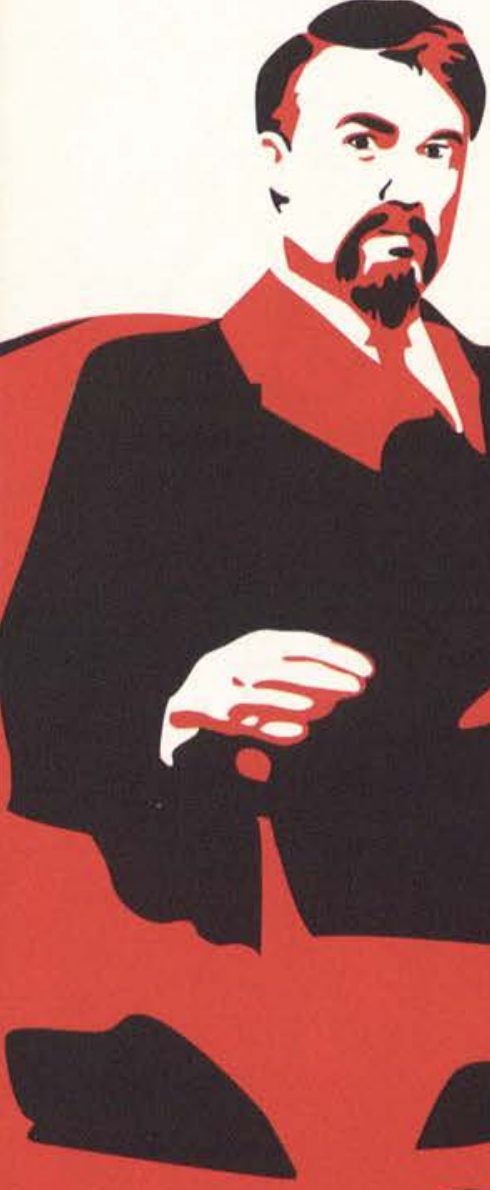

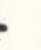
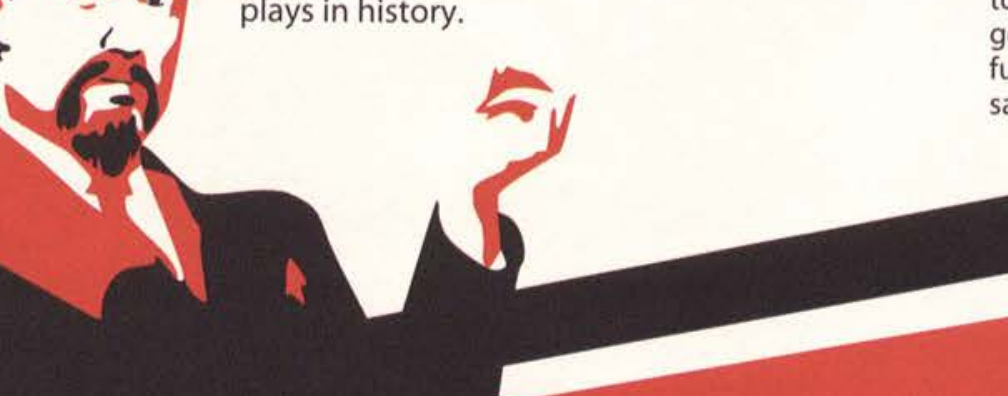

\footnotetext{
(1)
}

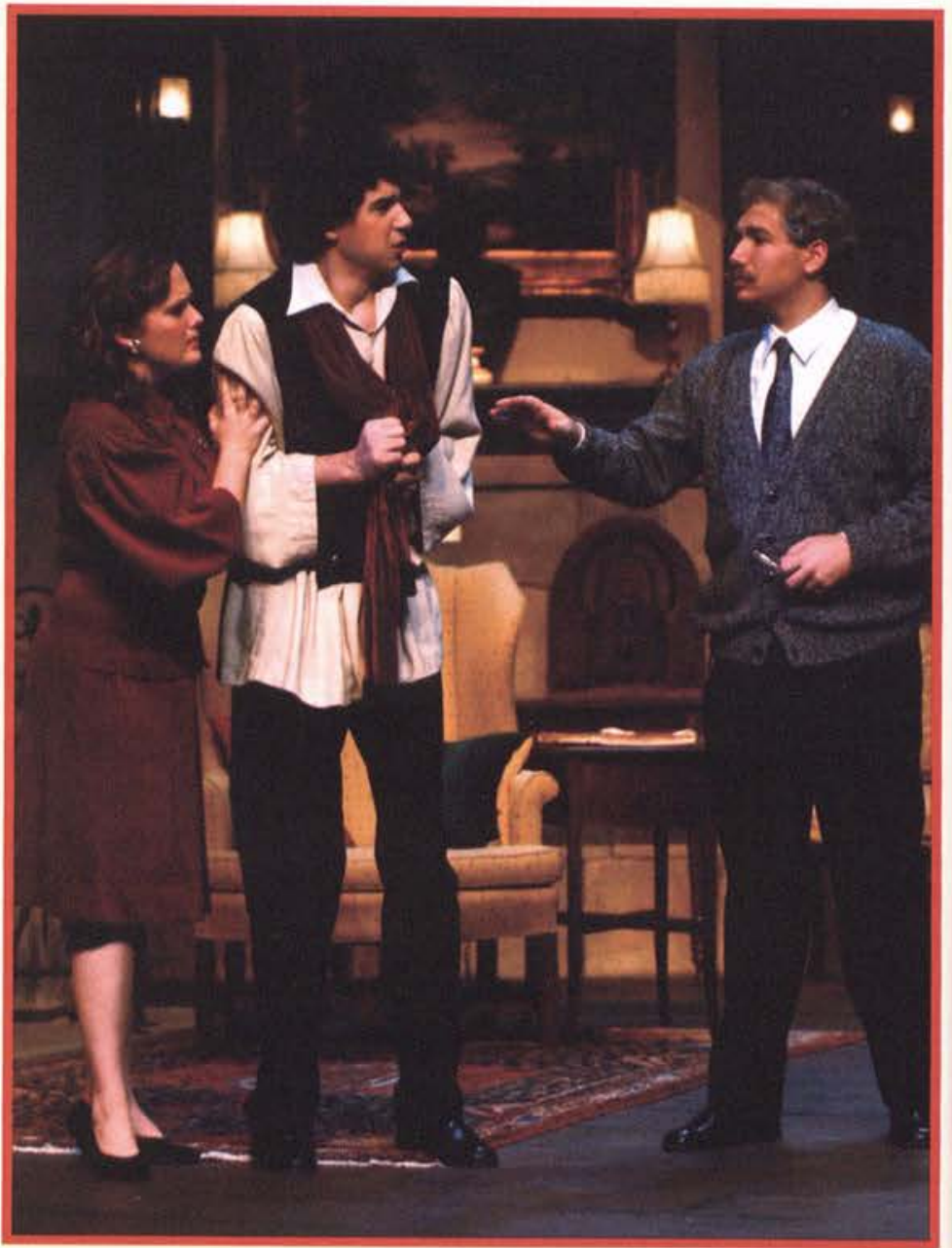

- Major Metchalf, played by senior Eric Mishne, reminds Mollie and Christopher that murder is not a game. "No one knew really what was going on until Metcalf told all in the end, and I had fun tying all the ends together," says Mishne. 


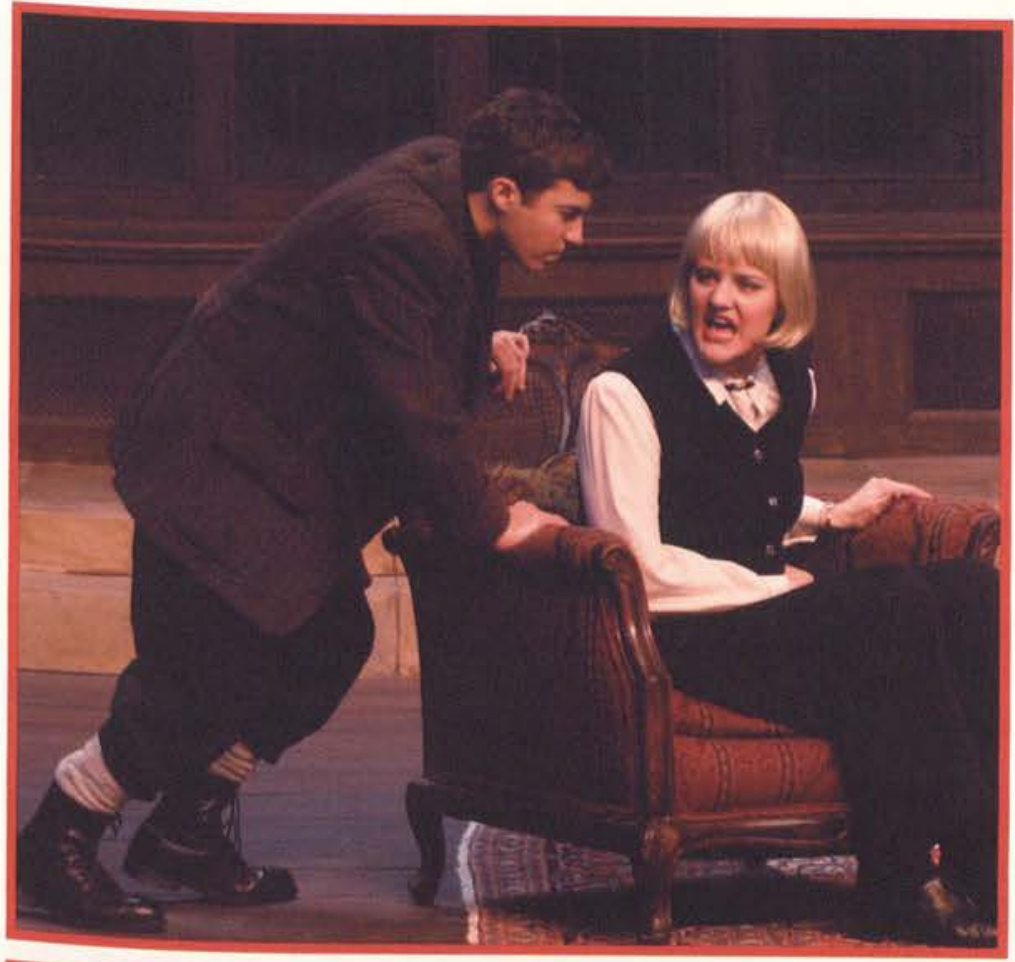

4 The Whodunit begins to unravel as Detective Sergeant Trotter, played by sophomore Daniel Zimmerman, leans in to interrogate Miss Casewell, played by senior Jaimie Teekell.

- Christopher Wren playfully chokes the snooty Mrs. Boyle, while singing "Three Blind Mice" with what seems like innocent fun-or was it? Rebecca Wolff loved knowing the audience was guessing the entire time who was going to be the victim: "The whole experience was a blast...including pretending to be strangled and actually making it sound realistic."

The audience and cast alike are astonished by Mr. Paravicini, played by sophomore Grant Hansen. Grant enjoyed becoming Paravicini through "the fastidious dress (including manicure) and unnerving habits of a less than reputable character." As the 'unexpected guest,' he "had fun being mysterious and creepy throughout the show."

4 Stage manager senior Victoria E. Duff, Director Robert L. Clements, and senior Assistant Stage Manager Phil McLeod proudly sit back and smile at a job well done. Director Robert Clements compliments the students as "a wonderful group of young adults who worked hard to strive for excellence and to bring honor and glory to our Lord."

- Mollie tries to calm her husband Giles down as he threatens Christopher Wren to stay away from Mollie. "What I liked most about playing Christopher Wren," says senior Colin Luther, "was having the ability to make the audience laugh at his child-like enthusiasm while simultaneously irritating all of the other characters."
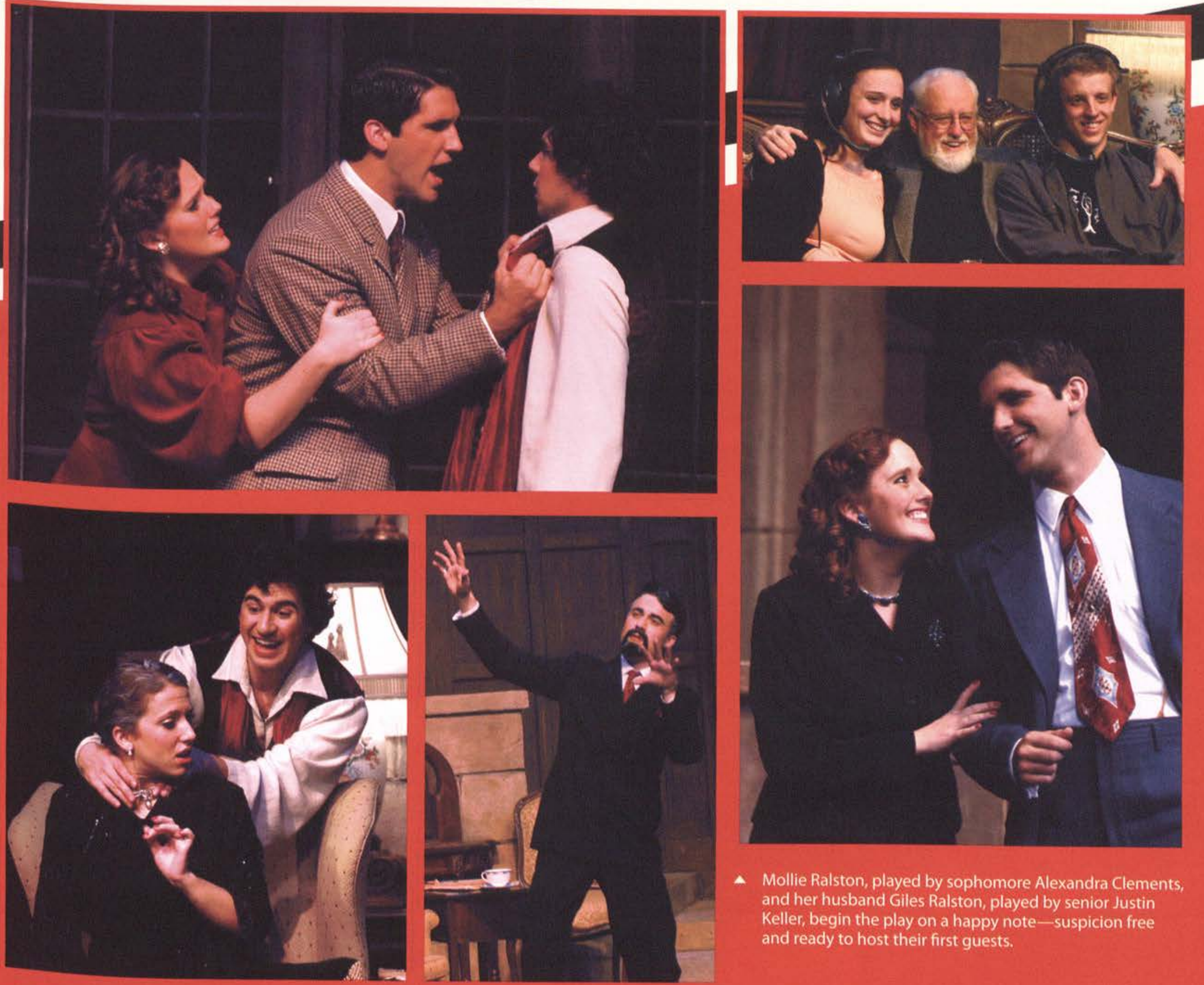

A Mollie Ralston, played by sophomore Alexandra Clements, and her husband Giles Ralston, played by senior Justin Keller, begin the play on a happy note-suspicion free and ready to host their first guests.

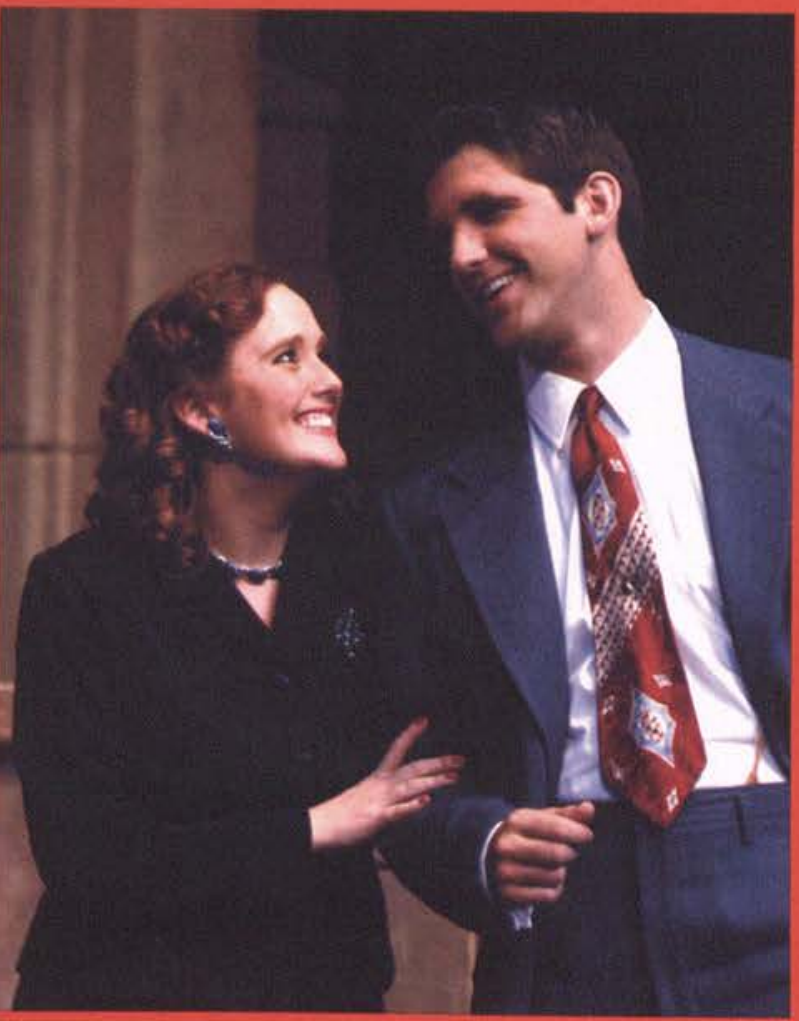



needed sleep or neglected homework. However, for those who took the occasion seriously, Cedarville's annual Day of Prayer proved to be an effective and refreshing way to reconnect with God amidst the stress of college and everyday life.

A few distinctive qualities separated this year's Day of Prayer from previous years. Instead of having a sermon that related to prayer, students Luke Seelye, Kevin Gulley, and Elliot Moore led the three different sections of the service: a time of repentance, a time for praise, and finally a time for prayer. Junior Sarah Newman especially enjoyed the corporate prayer at the end of chapel. She said, "I liked hearing everyone praying at once as a united student body."

Senior Elliot Moore, one of the student leaders of the service, explained his perspective from onstage:"I noticed that many people stayed afterwards and prayed in groups or just talked with each other. Pastor Rohm even commented on how this year's Day of Prayer seemed to be different in the attitude and spirit of the students." Clearly, the new format added a refreshing change and a push in the right direction in the prayer lives of the students.

Another defining change in this year's Day of Prayer was the use of three separate rooms designated for the prayer needs of unsaved family members, those in the mission field, and miscellaneous concerns of the students. Moore explained that "a number of people came through the rooms even hours after the chapel service," which illustrated the deep love and concern the students of Cedarville shared for one another. Senior Andrea Martin, a participant in the prayer rooms, said that praying over one's individual concerns "reminded [her] that God holds each of our requests in His hands." Hopefully, Cedarville's Day of Prayer 2006 left a permanent reminder of God's power and the ability we all have to come to Him in sacred prayer.
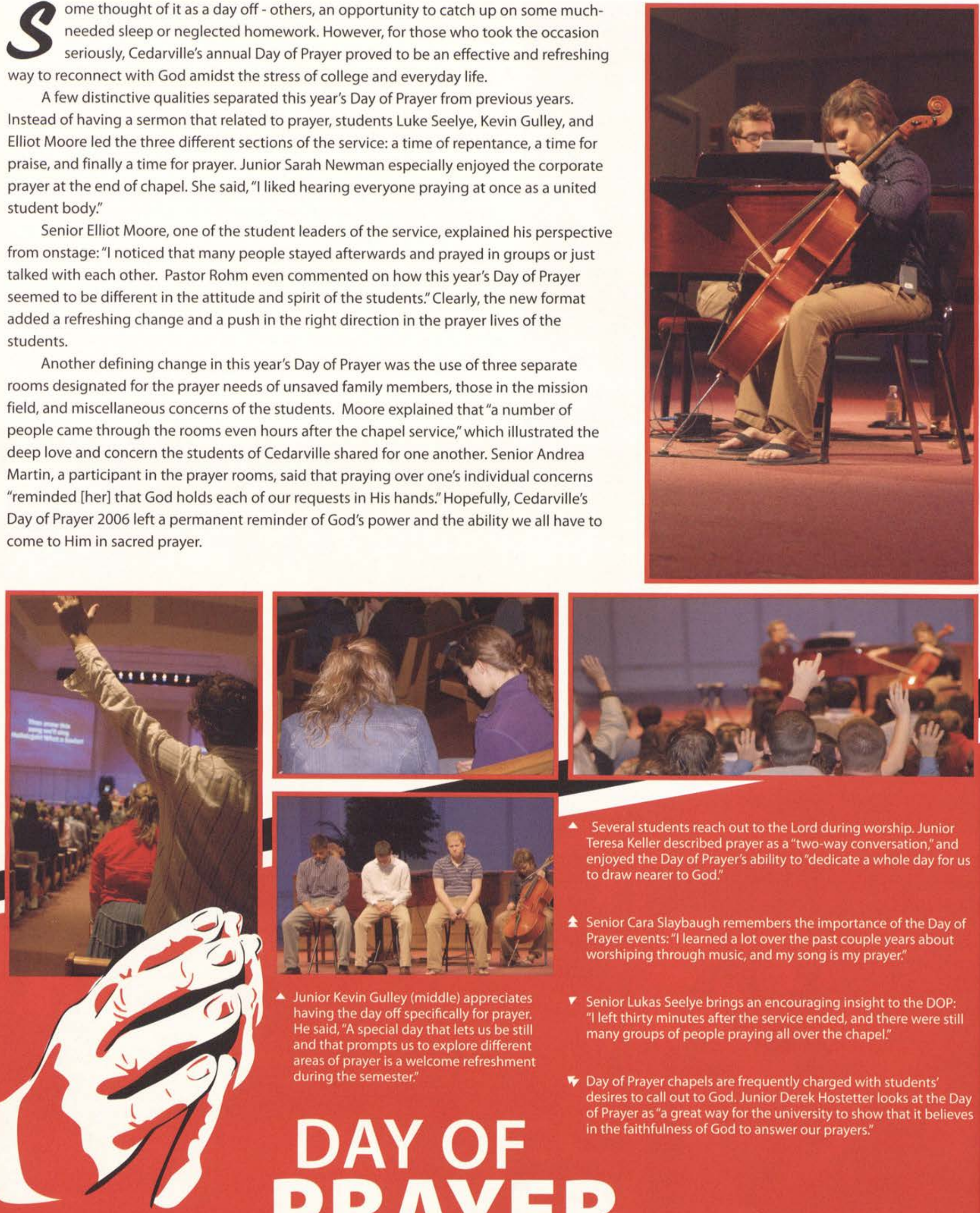

- Several students reach out to the Lord during worship. Junior Teresa Keller described prayer as a "two-way conversation," and enjoyed the Day of Prayer's ability to "dedicate a whole day for us to draw nearer to God".

A Senior Cara Slaybaugh remembers the importance of the Day of Prayer events: "I learned a lot over the past couple years about worshiping through music, and my song is my prayer."

A Junior Kevin Gulley (middle) appreciates having the day off specifically for prayer. He said, "A special day that lets us be still and that prompts us to explore different areas of prayer is a welcome refreshment during the semester.

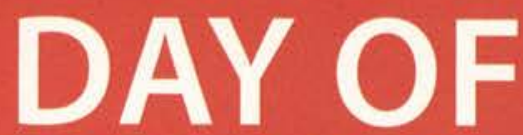

Senior Lukas Seelye brings an encouraging insight to the DOP: "I left thirty minutes after the service ended, and there were still many groups of people praying all over the chapel."

Day of Prayer chapels are frequently charged with students desires to call out to God. Junior Derek Hostetter looks at the Day of Prayer as "a great way for the university to show that it believes in the faithfulness of God to answer our prayers."

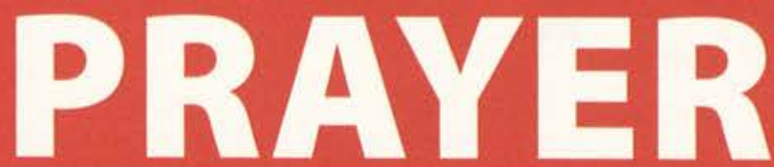



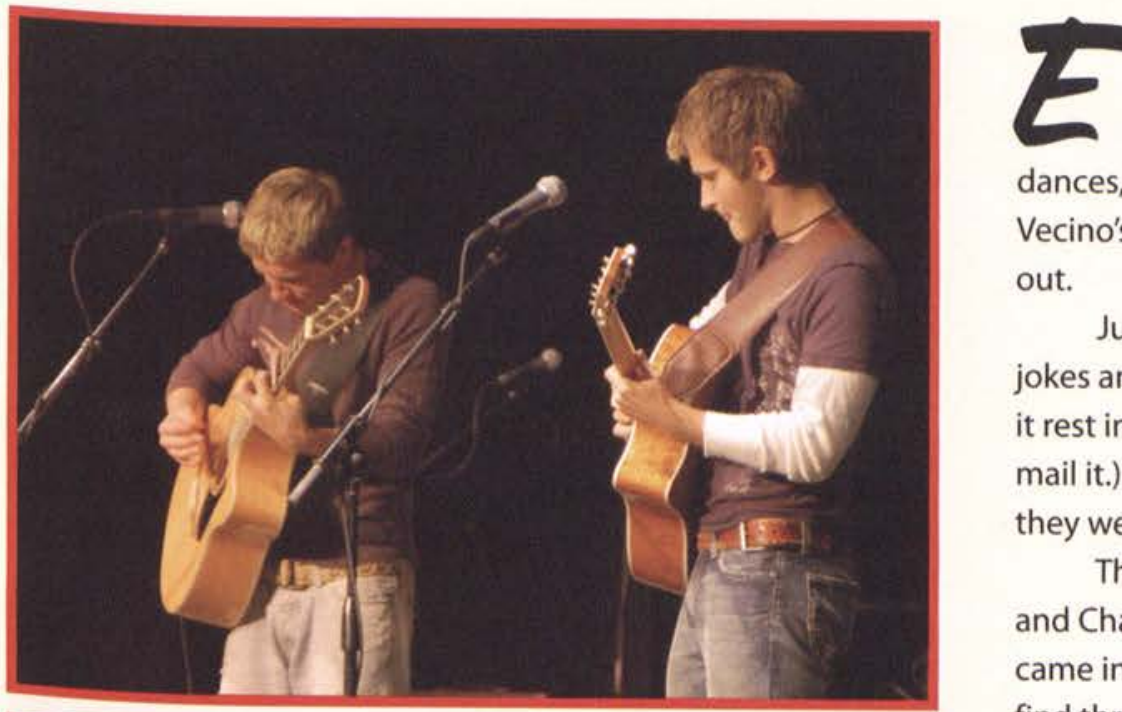

veryone knows that college students are low on funds. For some, spending the $\$ 5$ go to the New Student Talent Show, which showcased freshmen performing skits, songs, and interpretive dances, might have been a sacrifice of vending machine runs, mochas at Vecino's, or next semester's tuition. The 11 p.m. show, however, quickly sold out.

Junior Wade McComas, host of the NSTS, kept things lively with his jokes and David Letterman-style Top 10 List asking what the Black Savvy (may it rest in peace) really was (\#1:The abyss that our tuition falls into after we mail it.). He said, "Just getting to make some people laugh or a least act like they were laughing was really cool."

The group "The Webs" (Derek Stockwell, Stephen Eslick, Justin Willetts, and Chase Baldwin) performed a dance seen in an "Ok Go" music video and came in first during both shows. Derek Stockwell said, "I decided to try and find three other guys that were brave enough to take on the task. I really

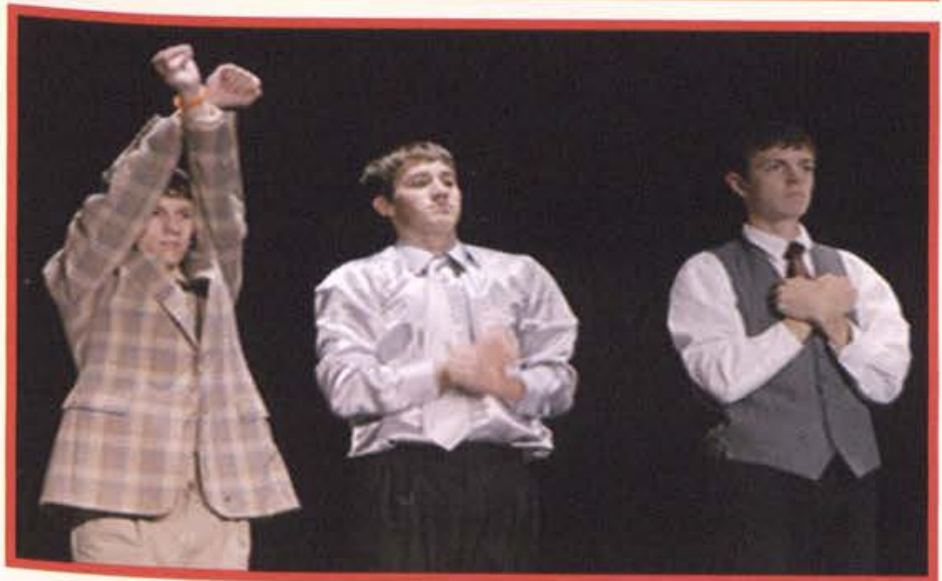

- The serious faces on "The Webs" comprised of Justin Willets, Chase Baldwin, Stephen Eslick, and Derek Stockwell (not pictured) didn't last long. Derek Stockwell says, "I was blind sided by the unexpected request for an encore, but more than happy to oblige."

- Freshmen Zach Seelye and Nate Keller play Damien Rice's "Cannonball." don't think I could have found three better guys for the part." The audience voted singer-songwriter Jen Greer runner-up at the 11 o' clock show, and at 1 it was Zach Seelye and Nate Keller with Damien Rice's "Cannonball."

Speaking about his experience in the NSTS, Stephen Eslick said, "My high point was right after we got off stage and knew that we nailed it... keeping a straight face out there wasn't easy to do, so once we were behind the curtain the joy spilled over and we went nuts." The crowd went nuts too, and judging by the energy in the auditorium that night, the general consensus was that although lots of things might not be worth quite five dollars, the New Student Talent Show definitely was.

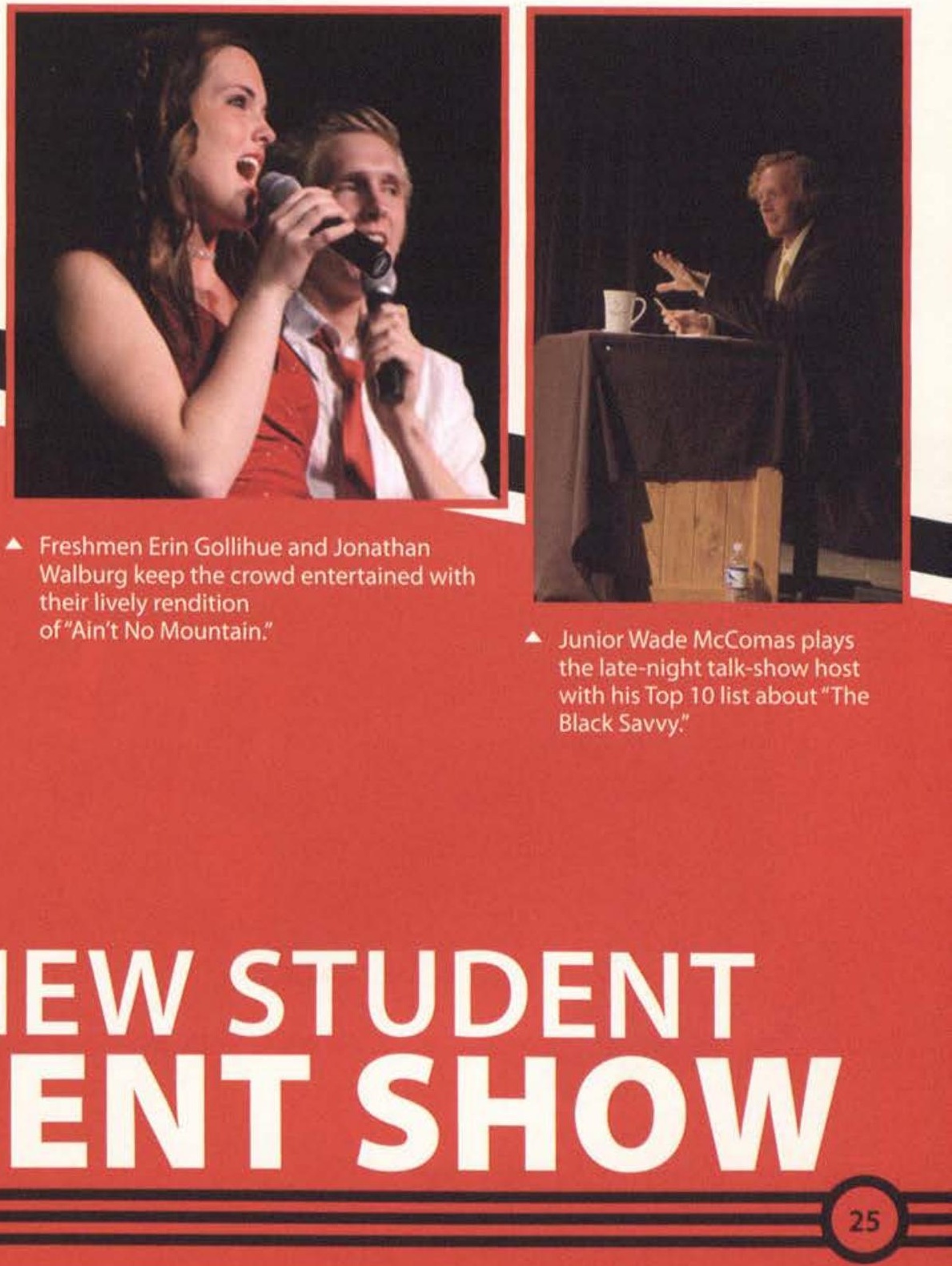




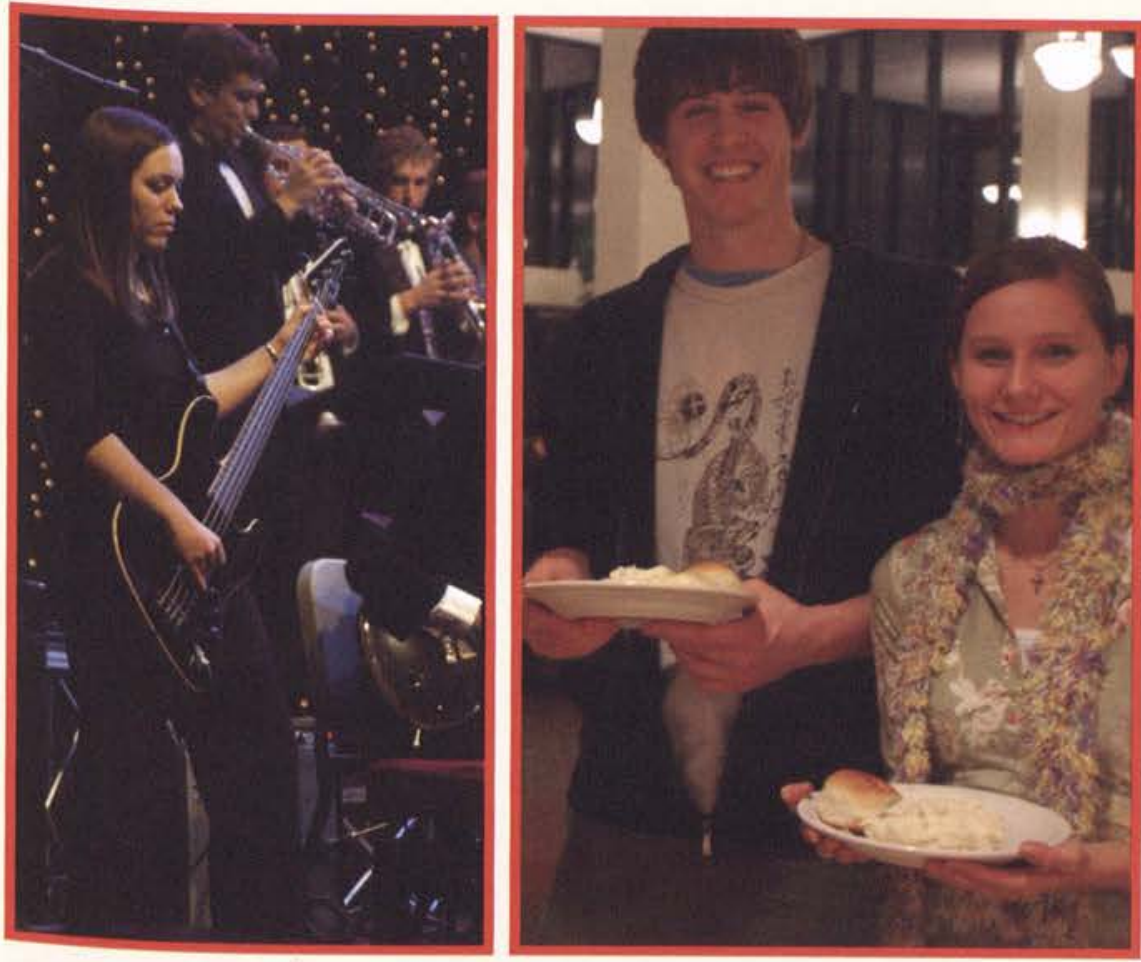

4 Sophomores Rick Bricker and Felicia Lustig help themselves to some mashed potatoes during a special holiday meal at Chuck's.

4 Sophomore Bekah Adams, senior Aaron Hollopeter, and sophomore Dan Lewis get into the Christmas spirit while playing thier instruments in the Jazz Band for Cedarville's annual community Christmas Concert.

- Sophomores Abby Douglas and Jennifer Powell work on creating a gingerbreadhouse for open dorms. The girls transformed their room into a candy shop, passing out chocolate dipped oreos and pretzels, candy canes, and rock candy as a part of their dorm theme in Willets North Back - a street in New York!

In light of the holidays, senior Dan Jaquery won't say no to a kiss under the mistletoe! Seniors Sarah Baczek and Amanda Oliver pucker up.

4 How many university presidents are willing to read bedtime stories to their students? Dr. Brown wraps up the Campus Christmas night with a reading from "How the Grinch Stole Christmas."

- Performing for a packed house, members of Denver and the Mile High Orchestra form a chorus line as they share their own unique reenactment of "How the Grinch Stole Christmas".
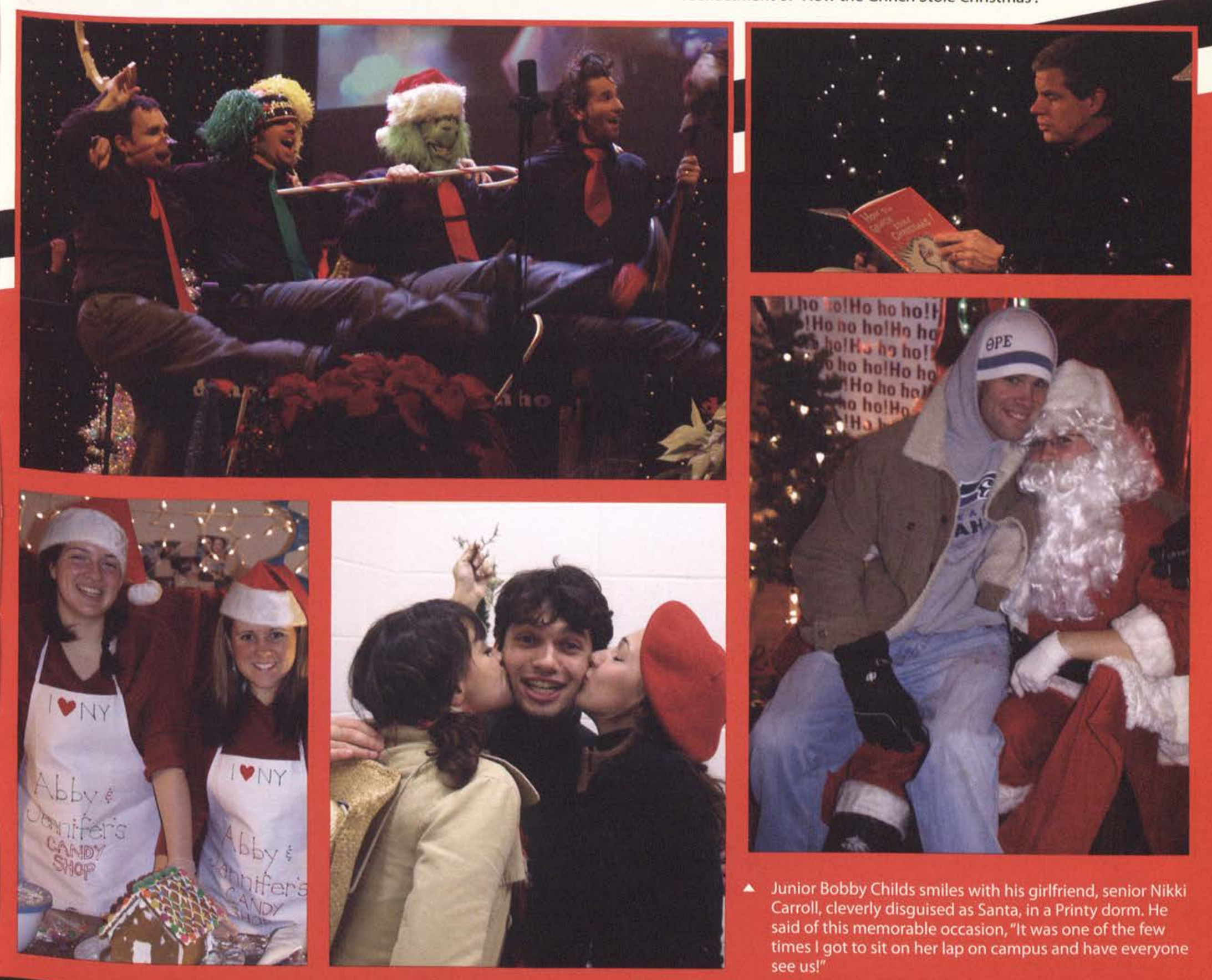

- Junior Bobby Childs smiles with his girlfriend, senior Nikki Carroll, cleverly disguised as Santa, in a Printy dorm. He said of this memorable occasion," It was one of the few times I got to sit on her lap on campus and have everyone see us!" 

Ex 



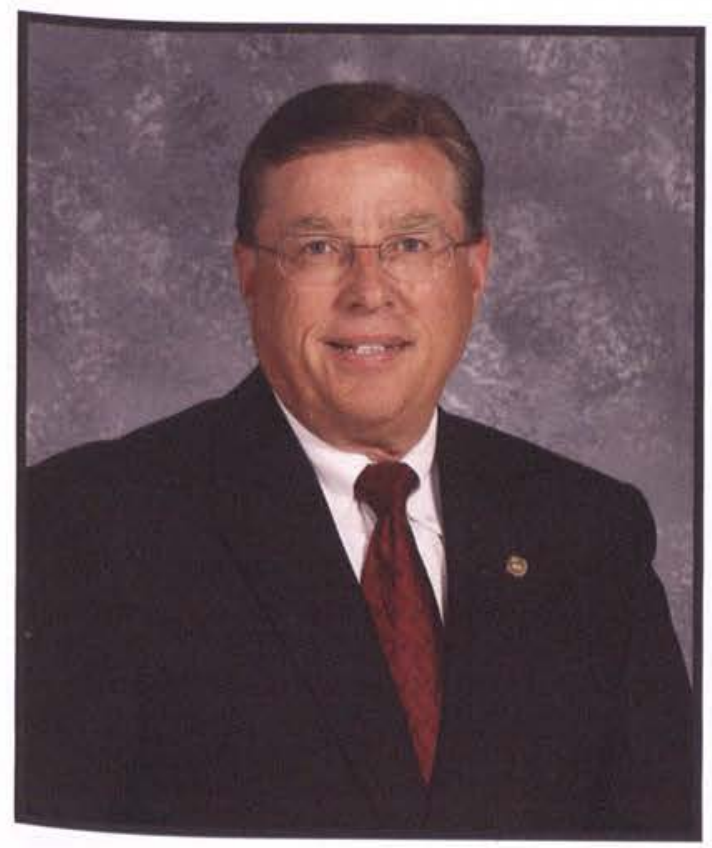

Dr. John Anglea

Vice President for Business

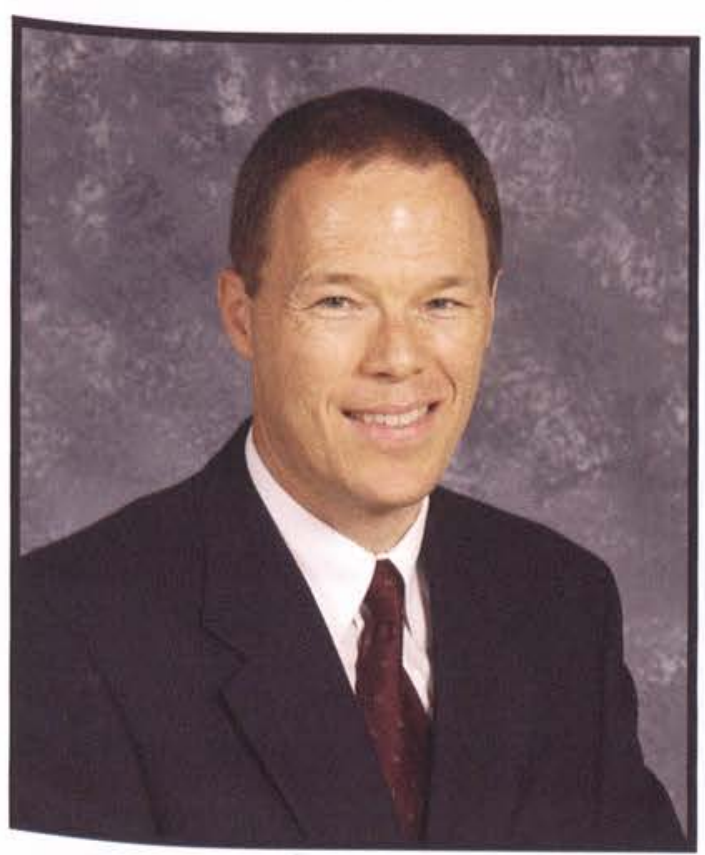

Mr. Dave Ormsbee

Vice President for Advancement

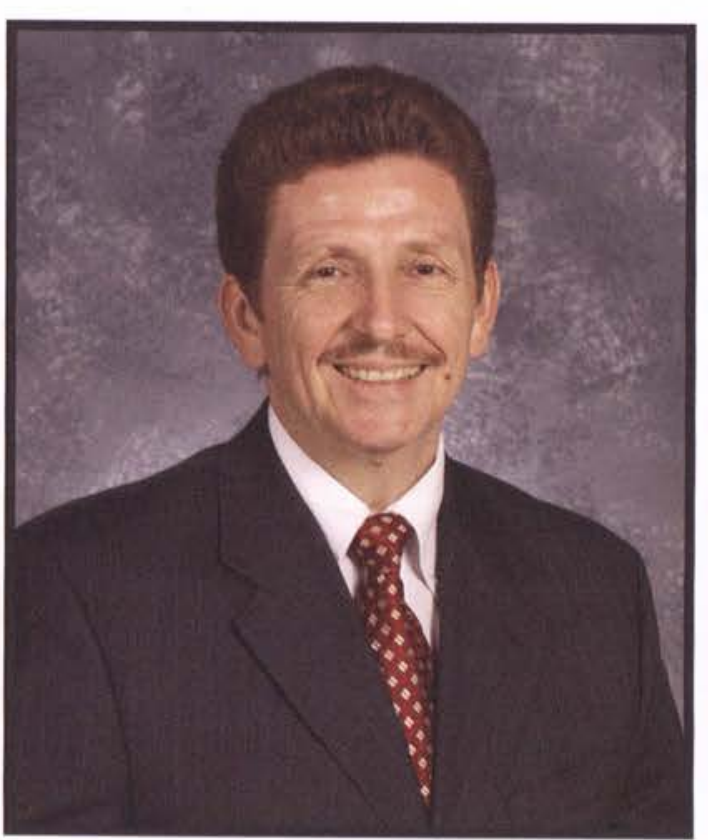

Dr. John Gredy

Vice President for Enrollment Management

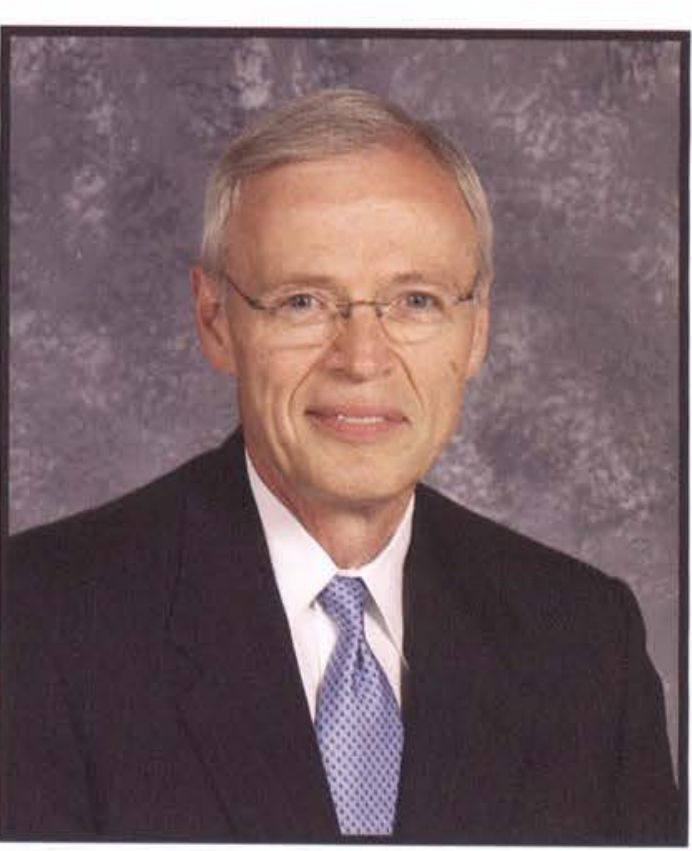

Mr. Bob Rohm

Vice President of Christian Ministries

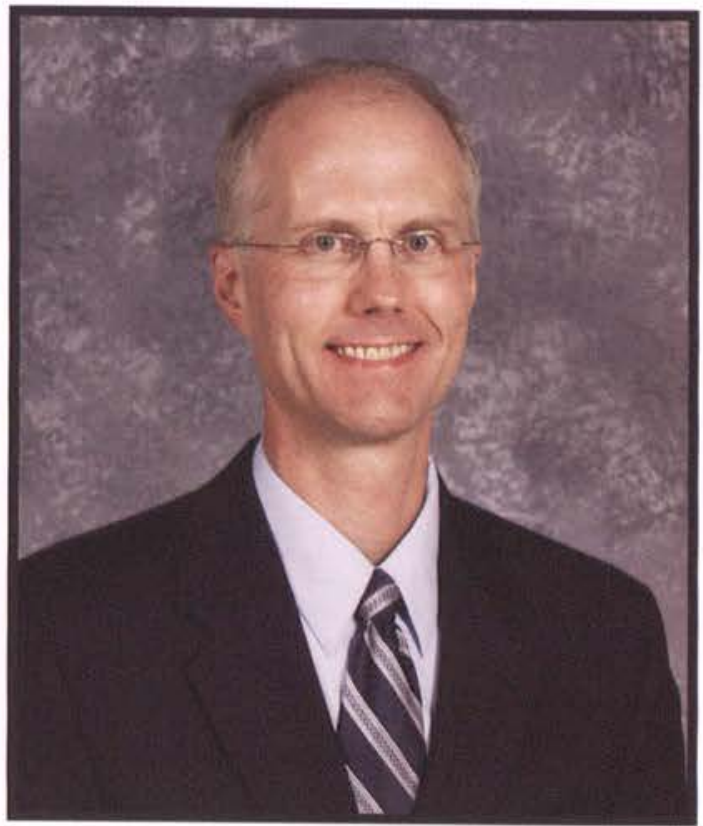

Dr. Bob Milliman Academic Vice President

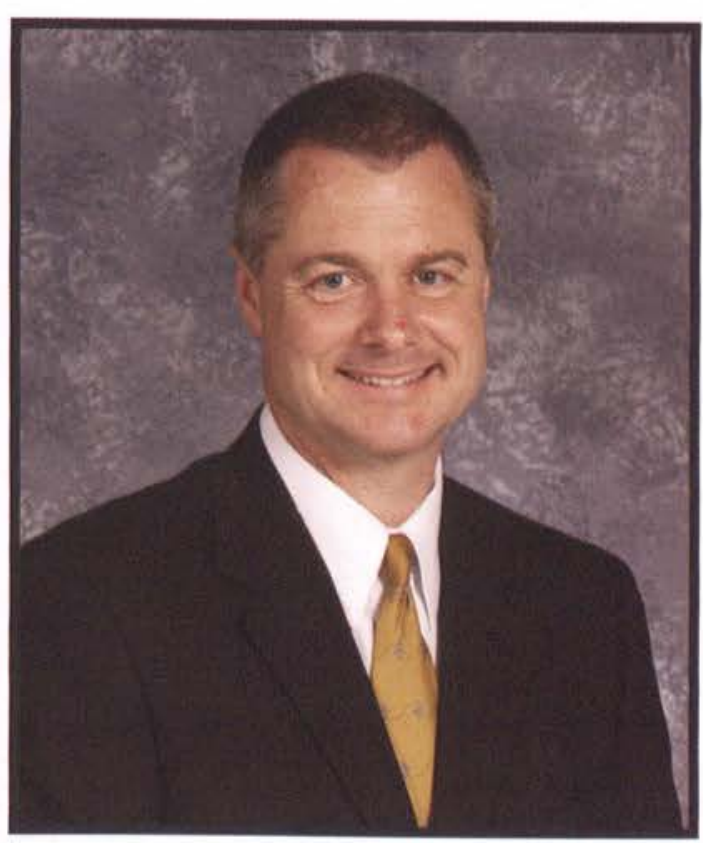

Dr. Carl Ruby

Vice President of Student Life 

of Humanities,

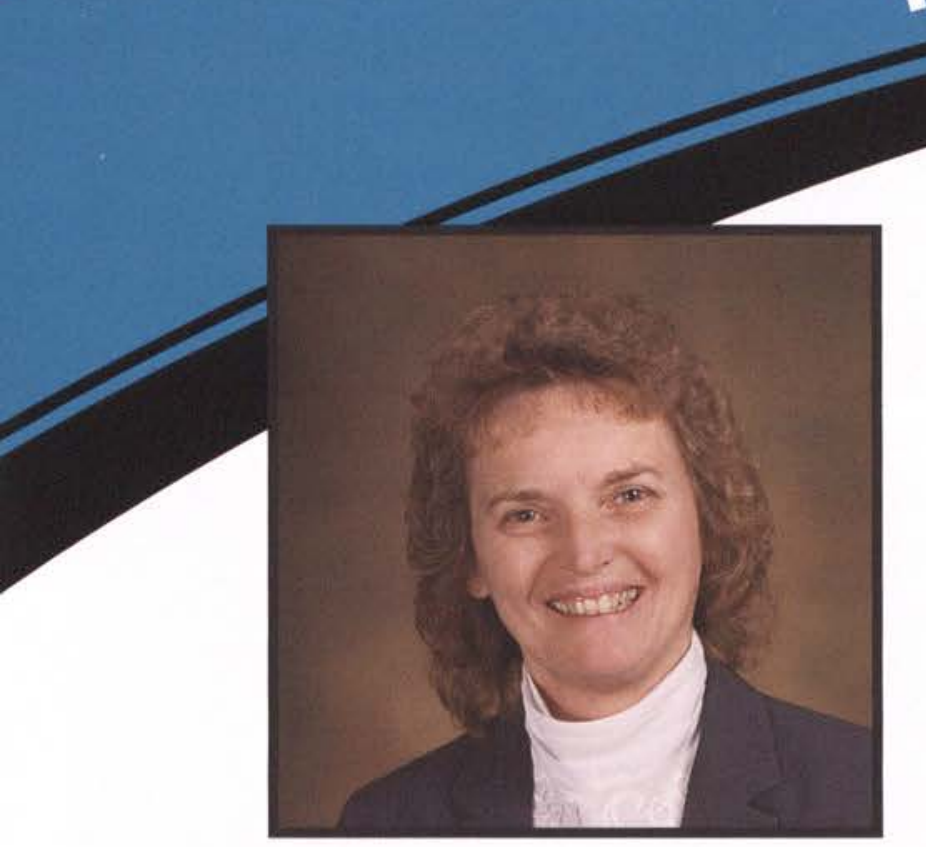

Dr. Pamela Johnson Acting Dean
The Humanities, Fine Arts and Bible department at Cedarville this year experienced the most success in their individual extra-curricular endeavors.

A highlight for the Bible department was the completion of Dr. Brown's re:View worldview DVD curriculum, which won several accolades, including the Silver Telly Award, which beat out 15,000 other competitors. The series won seven other awards before the end of the fall semester.

The debate and forensics teams, representing the Communication Arts department, both performed impressively. Debate won several competitions, including a first place at the John G. Fee memorial tournament, and forensics also finished strong with a third place standing overall against sixty student competitors at the Heart of Michigan Classic.

The Language and Literature department found success in their Foreign Film Series, the national publication of the Cedarville Review, and the acquisition of a new staff member, Nellie Haack. Also, the department was fortunate to host two conferences: the Conference of Christian Literature in the fall semester and an undergraduate conference in the spring semester.

The Music and Art department saw growth in the addition of Laura Ferranti, who enriched the department with her help in music education courses.

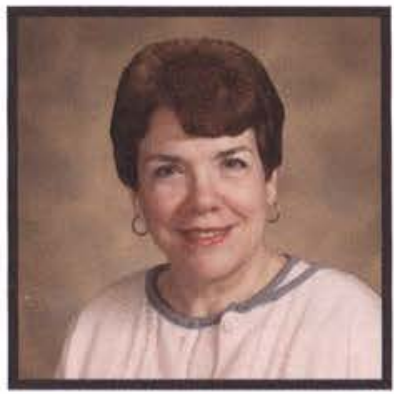

Sherrie Wood Dean's Assistant

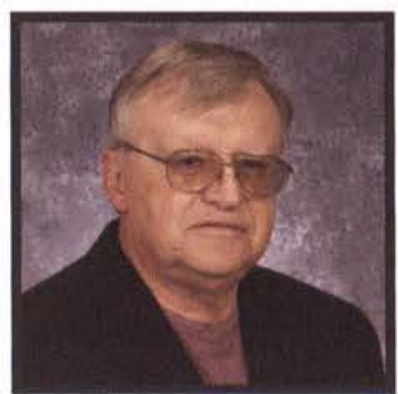

Dave Bastress Electronic Media Tech.

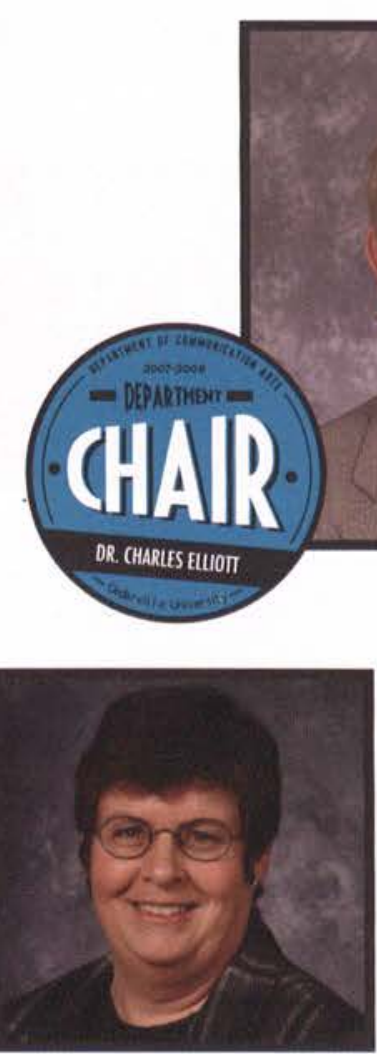

Deborah Bisset Administrative Assistant

\section{Department of Communication Arts}

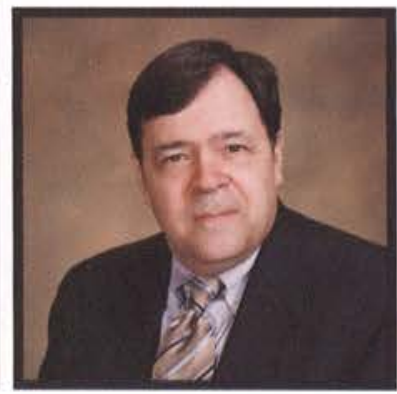

Dr. Wesley J. Baker Distinguished Professor

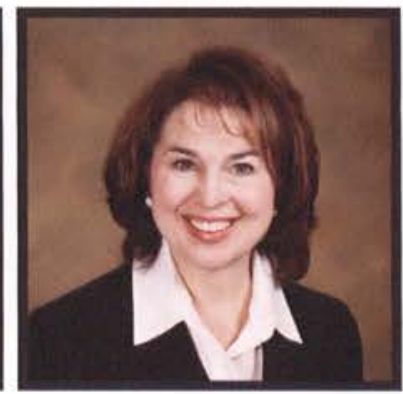

Rebecca Baker Assistant Professor

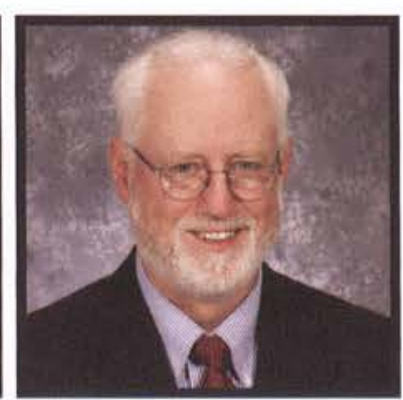

Bob Clements Assistant Professor

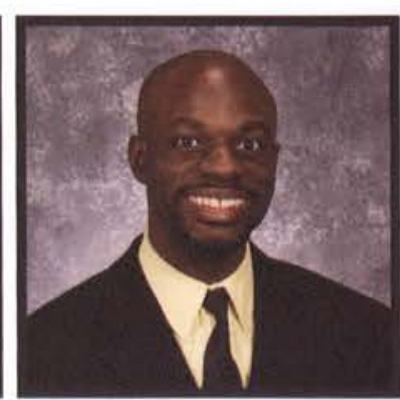

Derek Green Assistant Professor

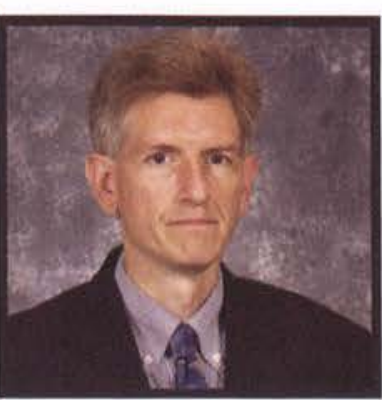

Dr. Clark Greer Associate Professor 


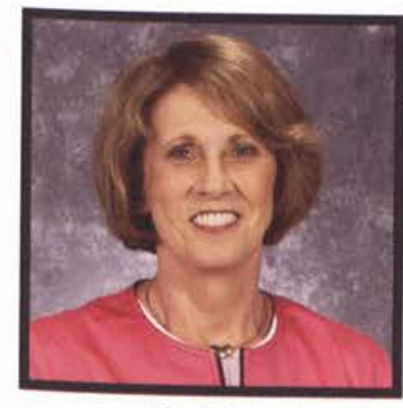

Dr. Deborah Haffey Professor

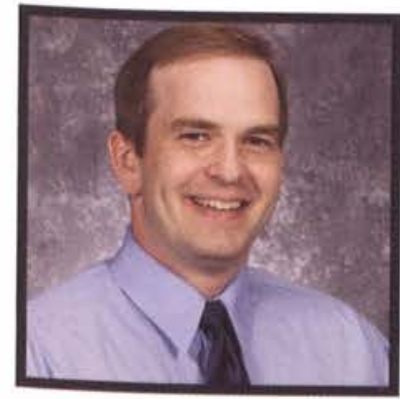

Matt Moore

Assistant Professor

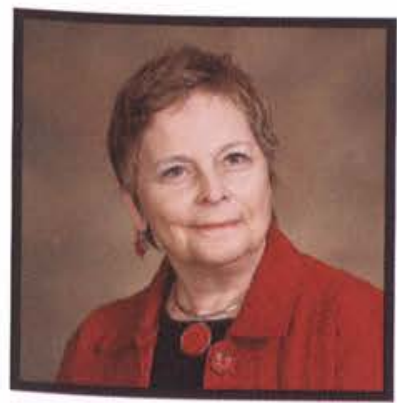

Dr. Margaret Wheeler Assistant Professor

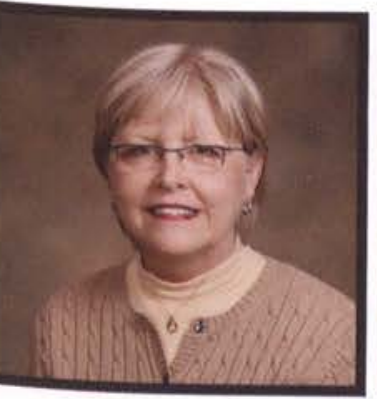

Pam Bromer Administrative Assistant

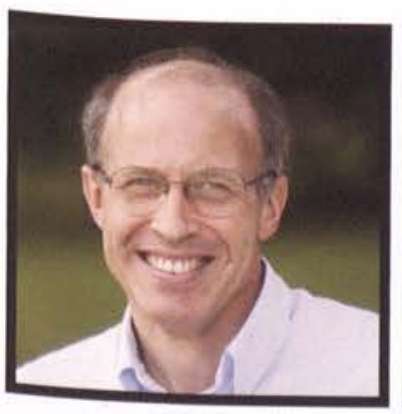

Dr. Dan Estes

Distinguished Professor

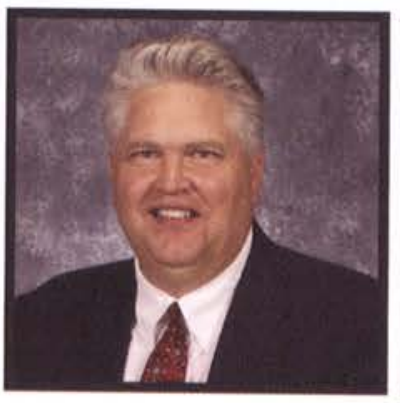

James Kragel Associate Professor

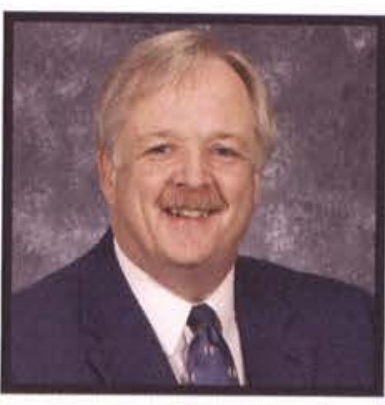

Kurt Moreland

Associate Professor

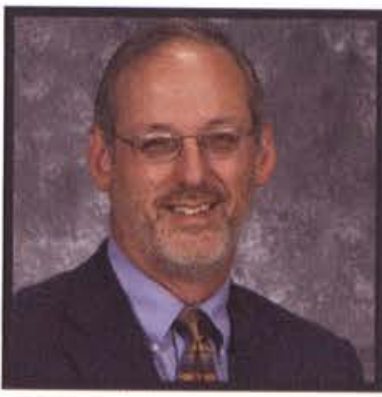

Dr. Mike Lopez

Professor

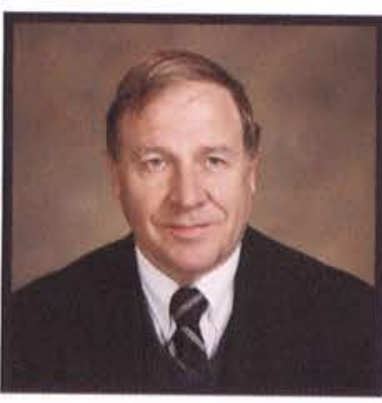

Dr. Jim Phipps

Professor

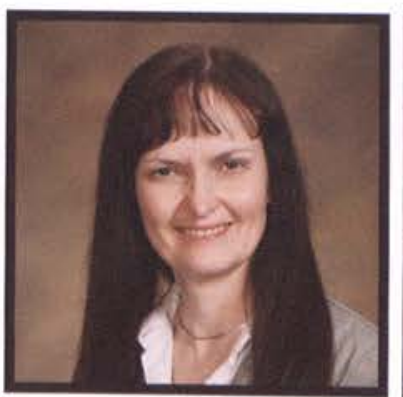

Mischelle Mclntosh Assistant Professor

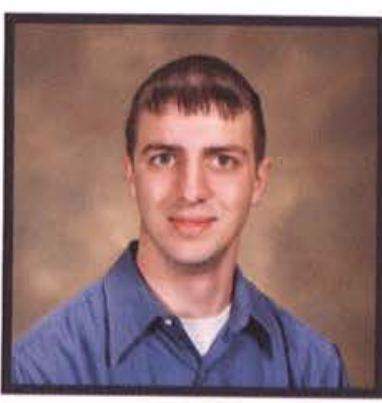

Timothy Phipps Asst. Technical Director

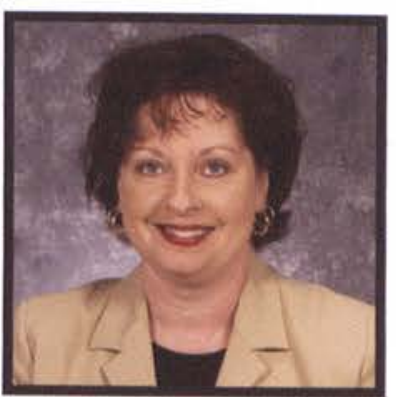

Dr. Diane Merchant Professor

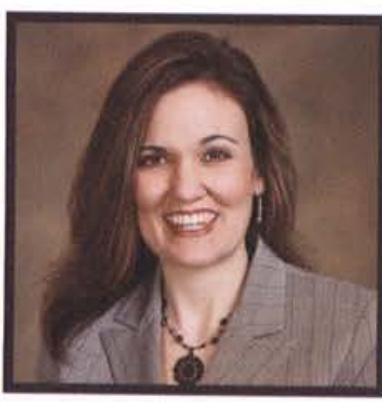

Dr. Rebecca Sietman Assistant Professor

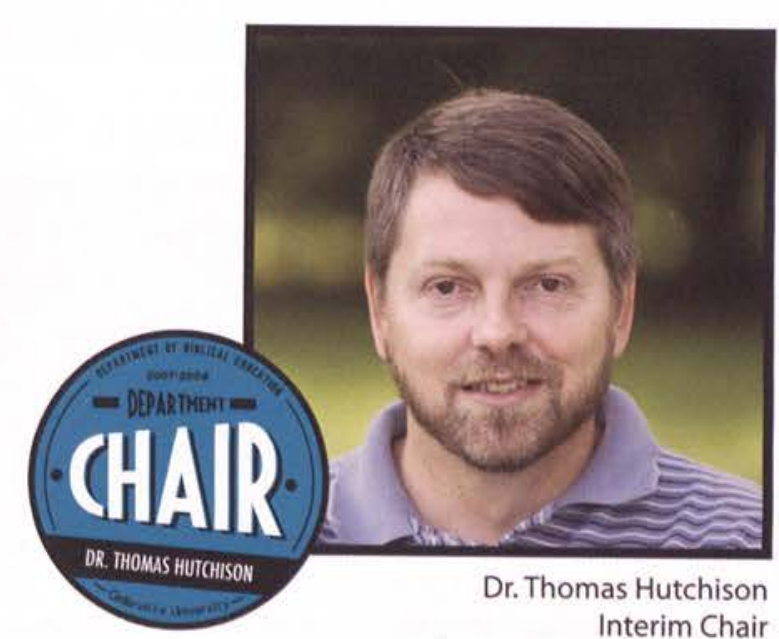

\section{Department of Biblical Education}

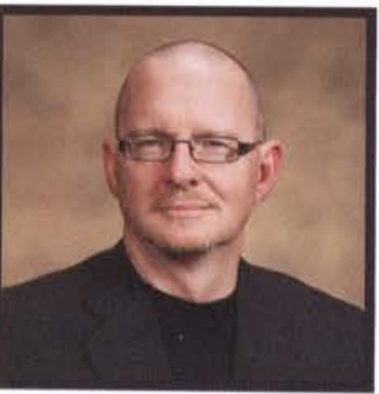

Dr. Jeff Cook Associate Professor

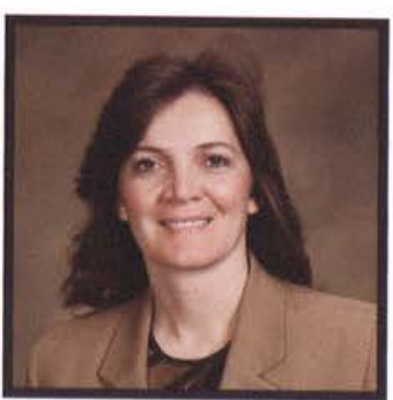

Dr. Joy R. Fagan Assistant Professor

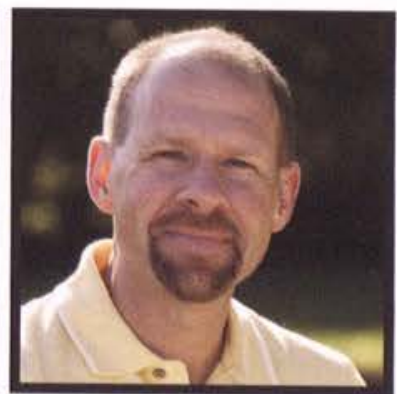

Dr. Gregory Couser Professor

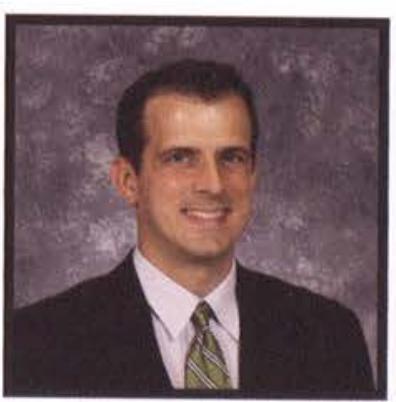

Dr. Timothy Gombis Assistant Professor

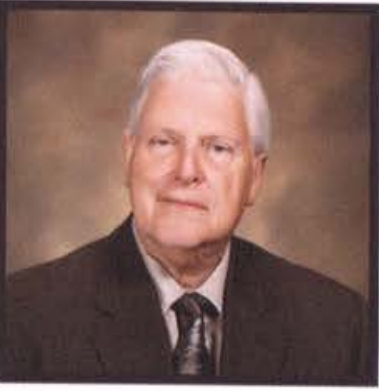

Dr. James Bjornstad Professor

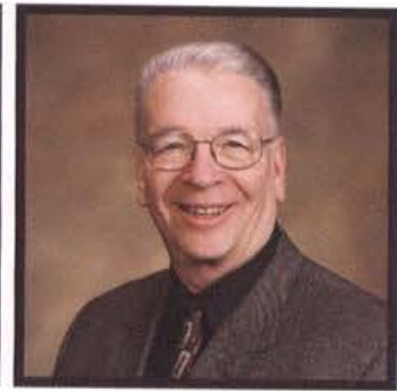

Dr. Richard Blumenstock Associate Professor

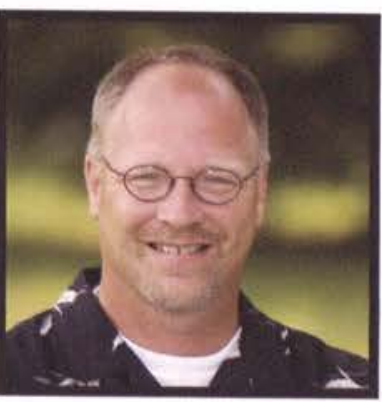

Dr. Scott Dixon Associate Professor

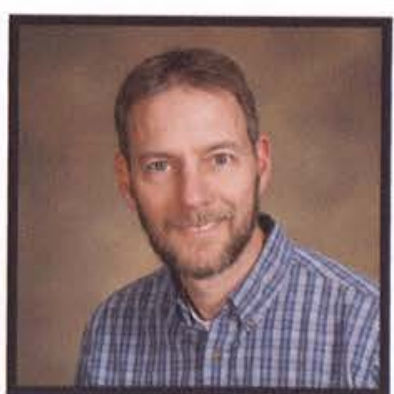

Dr. Donald Grigorenko Assistant Professor

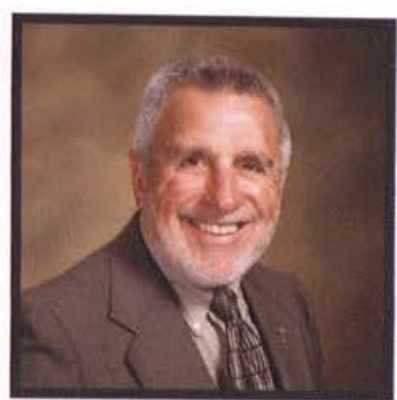

Dr. David Drullinger Professor

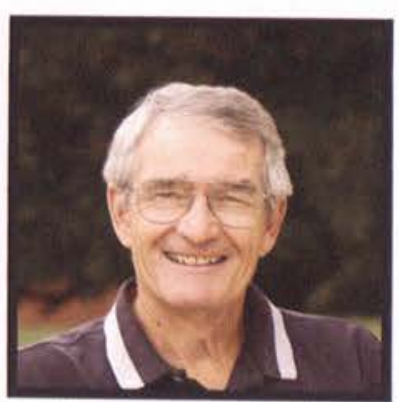

Dr. Bob Gromacki Distinguished Professor 


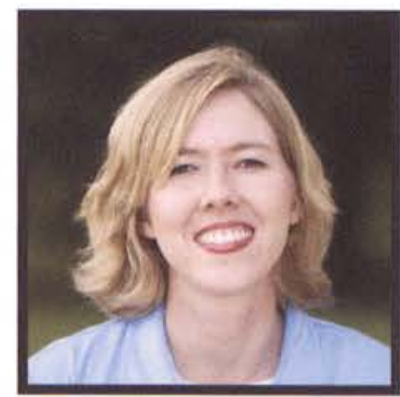

Amy-Hope Guisleman Assistant Professor

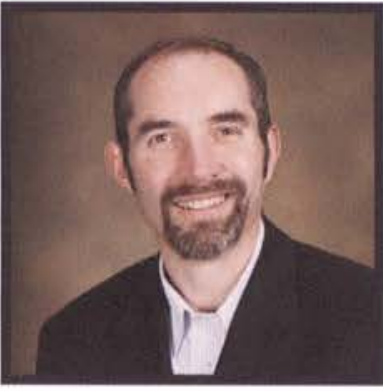

Dr. Chris Miller Professor

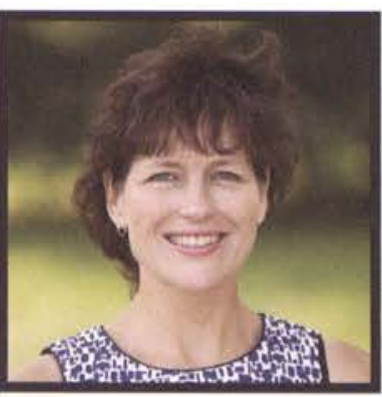

Kelly Hellwig Secretary

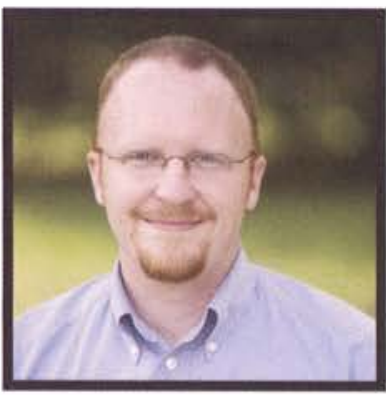

Dr. David Mills Associate Professor

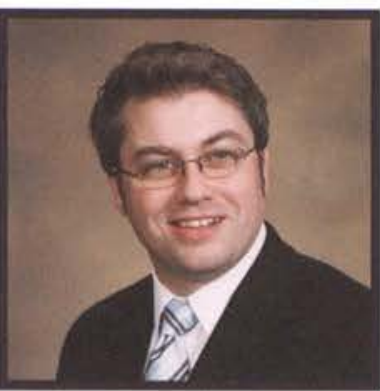

Dr. David M. Hoffeditz Associate Professor

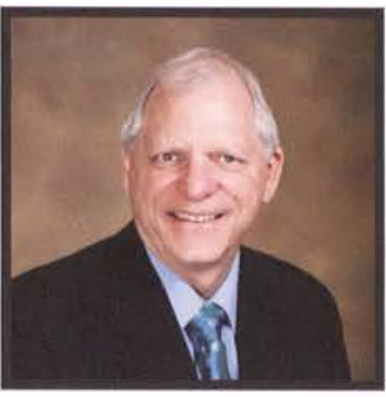

Mike Parrott

Assistant Professor

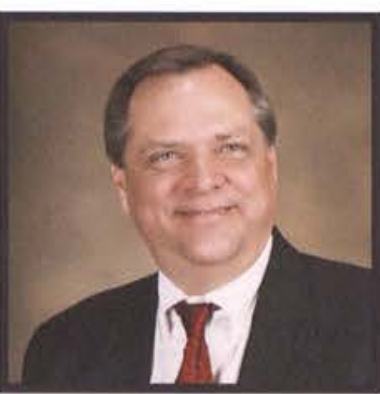

Dr. Steve Janssen Assistant Professor

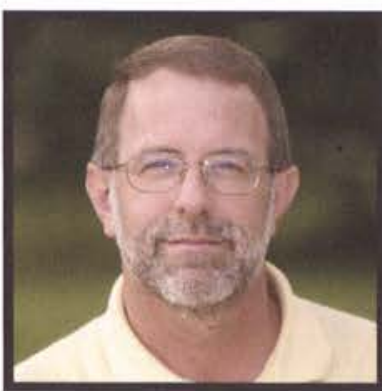

Dr. David Mappes Associate Professor

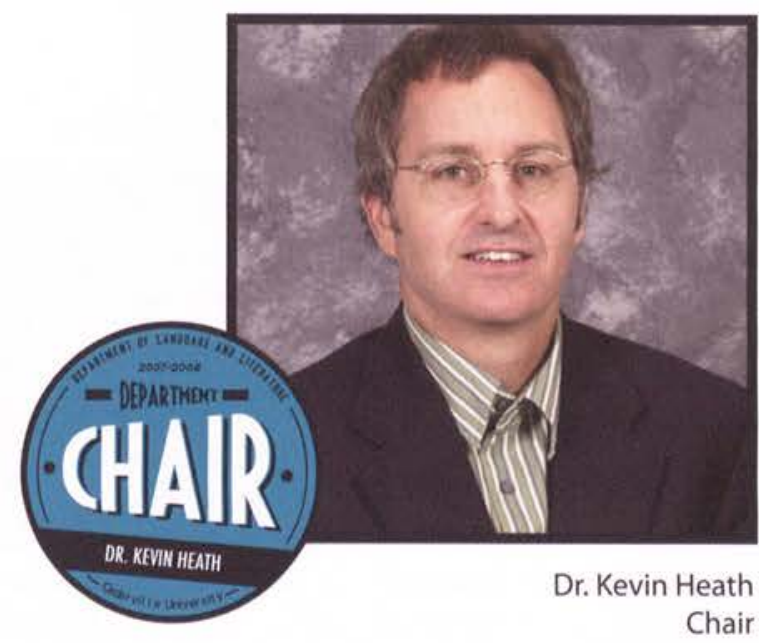

Department of Language \& Literature

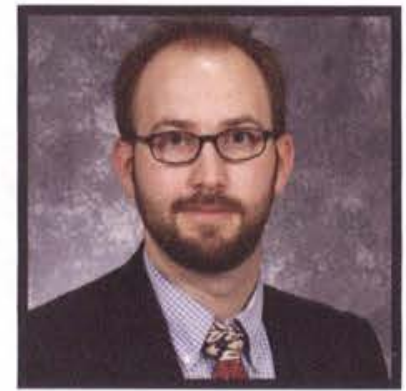

Ryan Futrell Assistant Professor

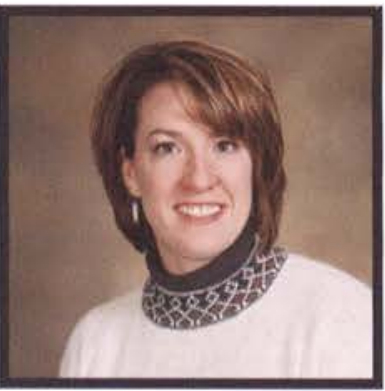

Cynthia Messer Assistant Professor

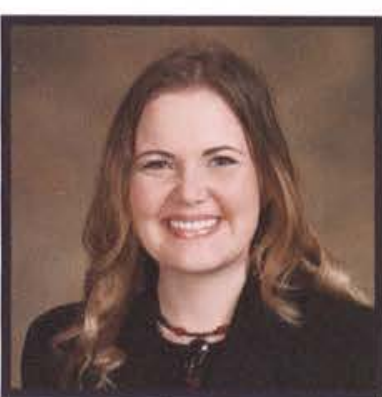

Nellie Corder Haack Assistant Professor

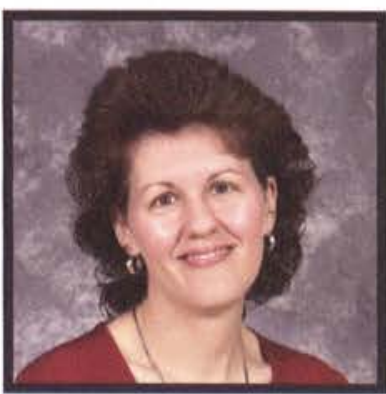

Julie Moore Assistant Professor

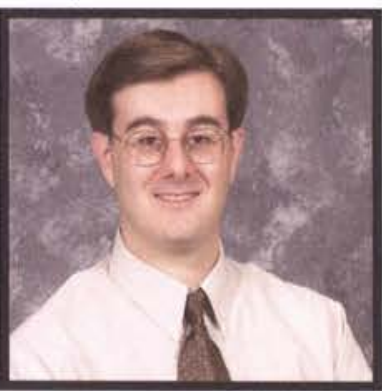

Dr. Scott Calhoun Associate Professor

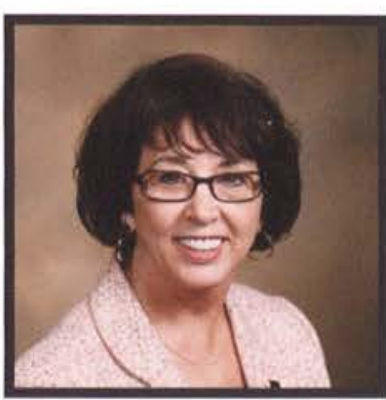

Sandi Harner Professor

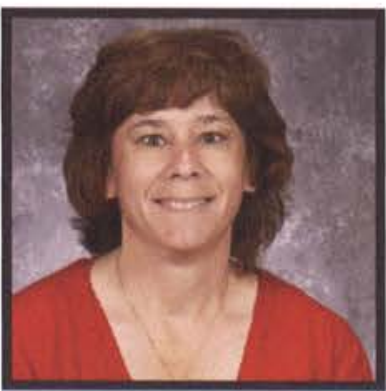

Monique Muncy Administrative Assistant

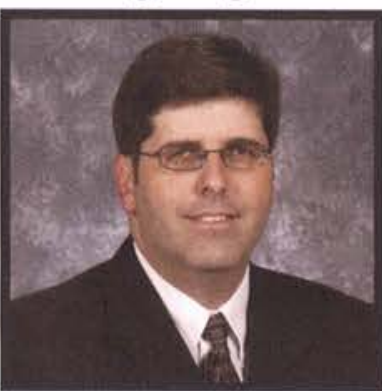

Daniel Clark Assistant Professor

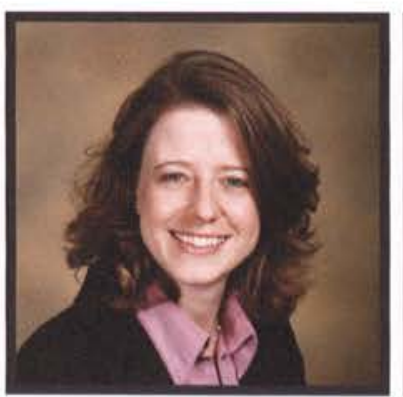

Shannah Hogue Assistant Professo

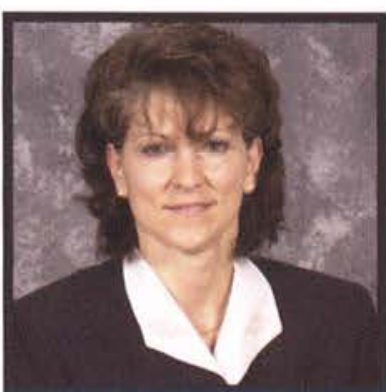

Karen Powe Associate Professor

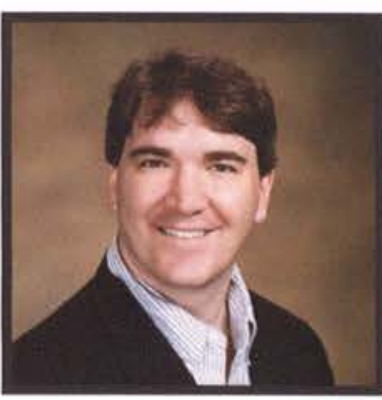

Dr. Donald Deardorff Associate Professor

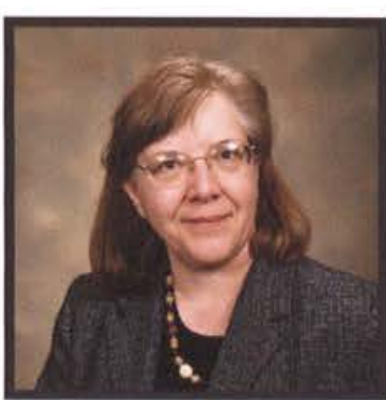

Dr. Barbara Loach Professor

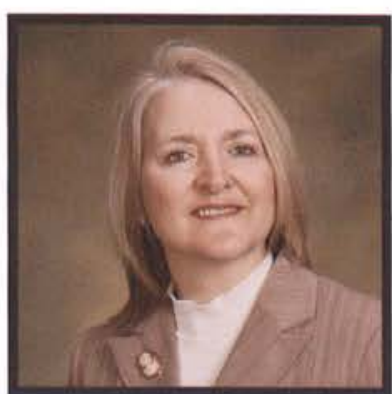

Dr. Annis N. Shaver Assistant Professor 


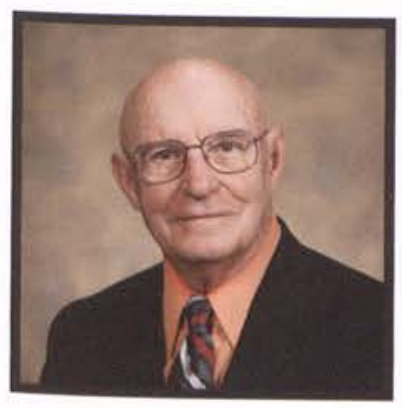

Edward Spencer Professor

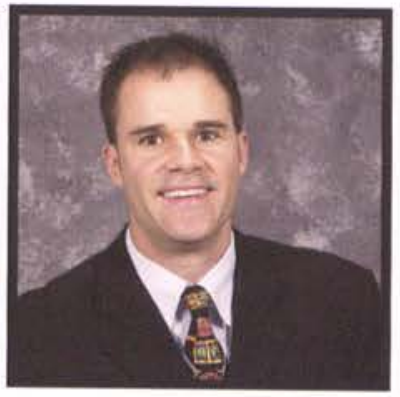

Dr. Andrew Wiseman Assistant Professor

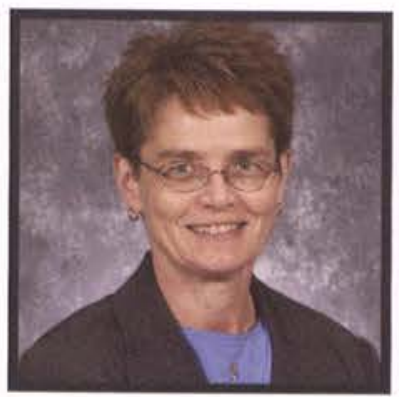

Dr. Peggy Wilfong Professor

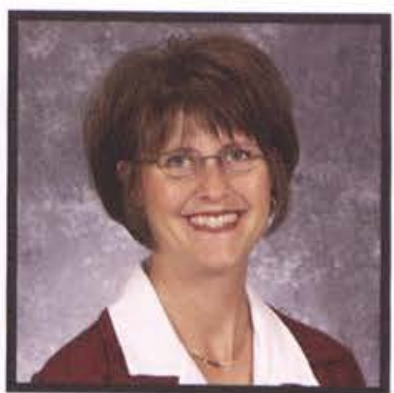

Michelle Wood Assistant Professor

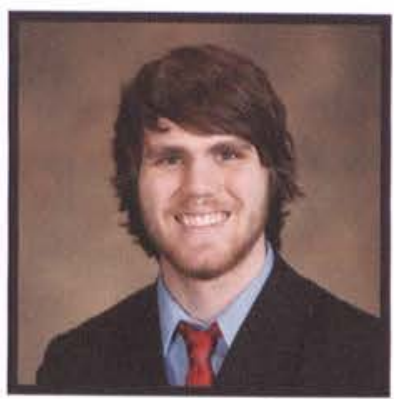

Alan Tuttle Adjunct Instructor

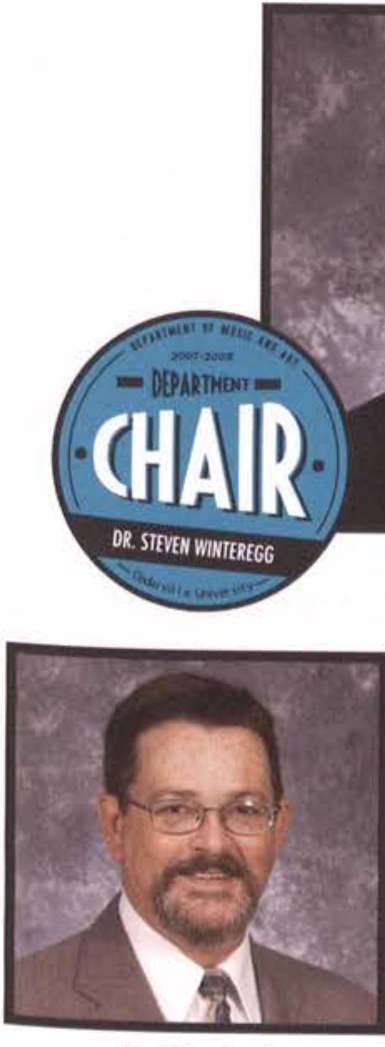

Dr. Chuck Clevenger Professor

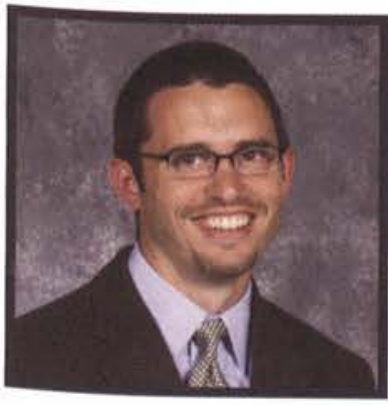

Aaron Gosser Instructor

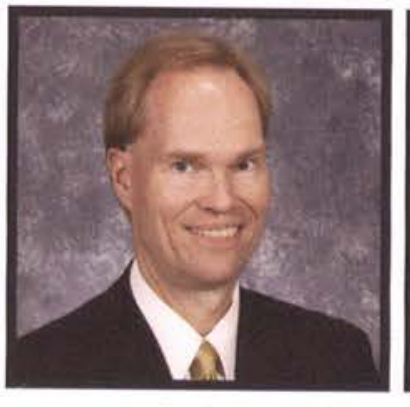

Dr. Bruce Curlette Professor

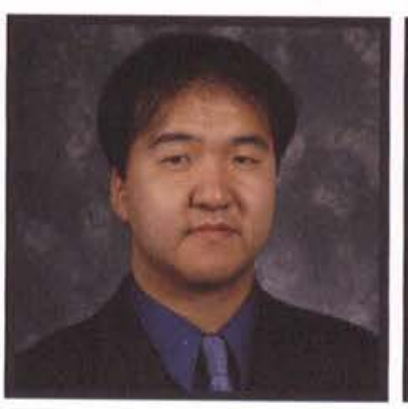

Jun Kim Assistant Professor

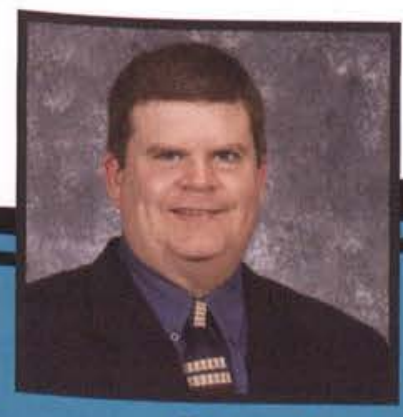

Dr. Roger O'Neel Assistant Professor

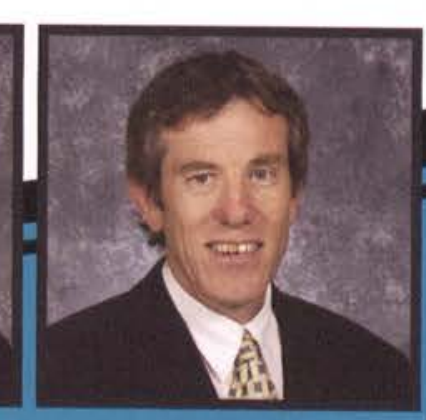

Charles Pagnard Professor

Department of Music \& Ant
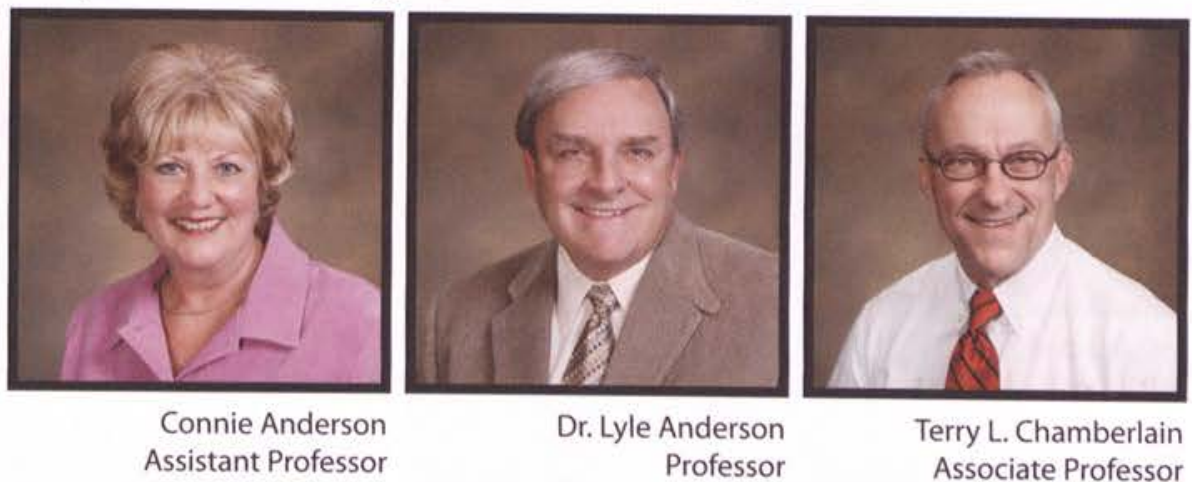

Terry L. Chamberlain Associate Professor

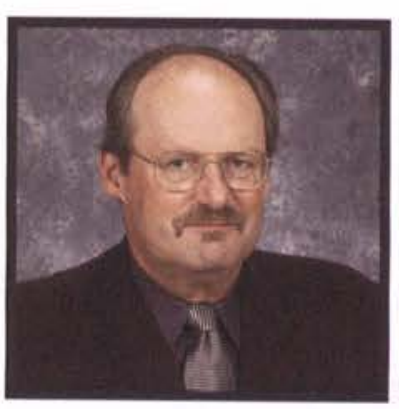

Mike DiCuirci Professor

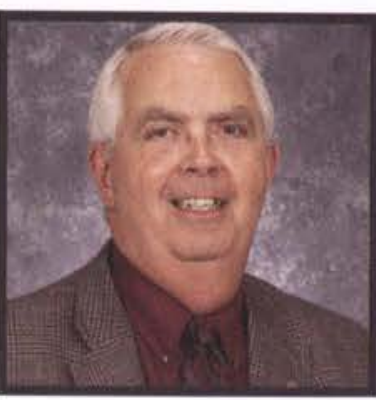

Dr. Dave Matson Professor

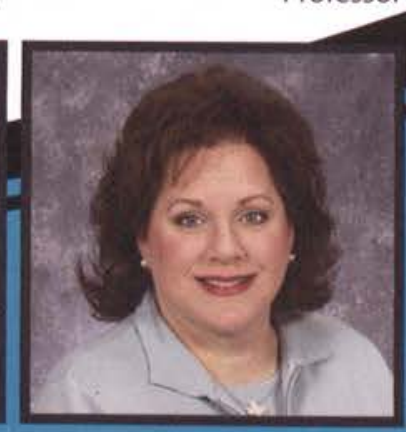

Beth Porter Associate Professor

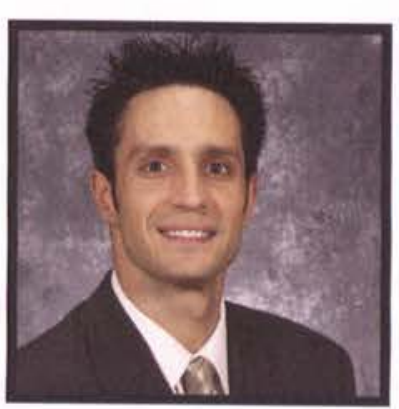

Dr. Taylor Ferranti Assistant Professor

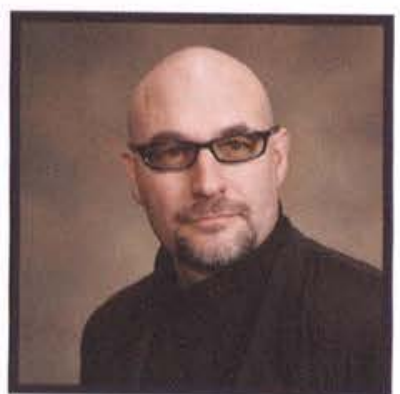

Timothy Frame Instructor
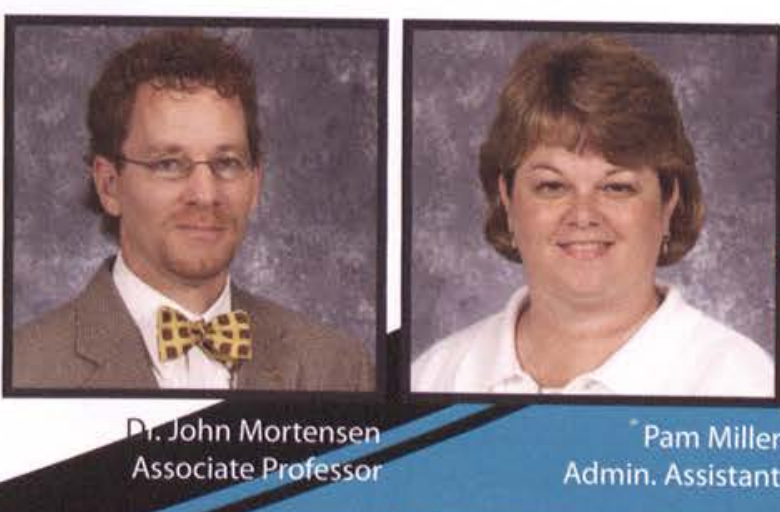

Pam Miller Admin. Assistant

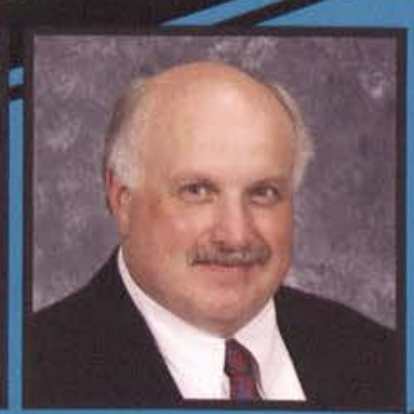

Dr. Mark Spencer Associate Professor

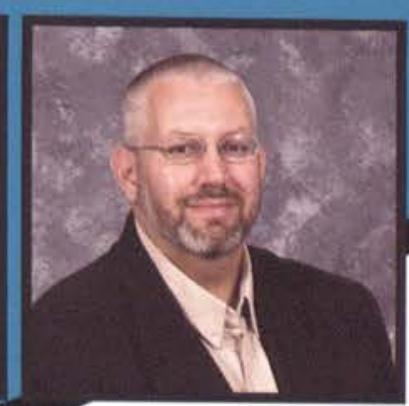

Daniel Sternsher Assistant Professor 



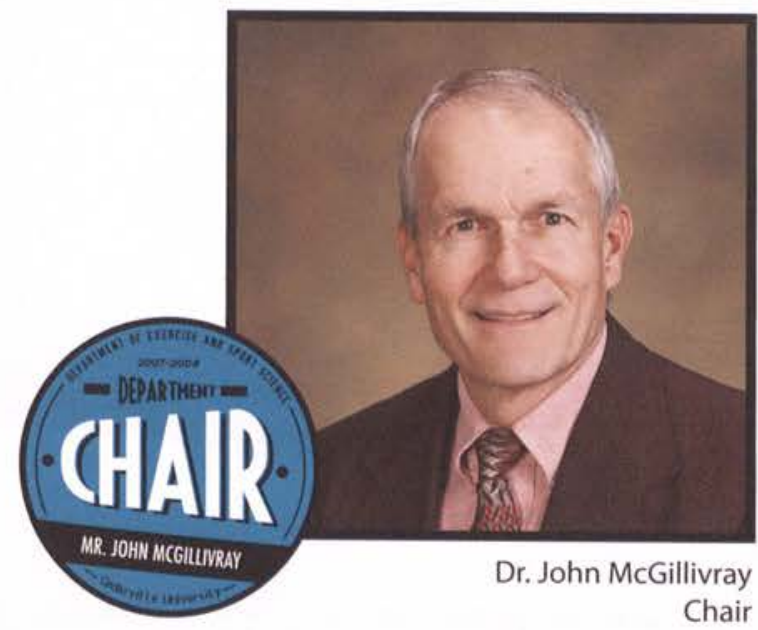

\section{Department of Exercise and Sports Science}

Dr. April Crommett Assistant Professor

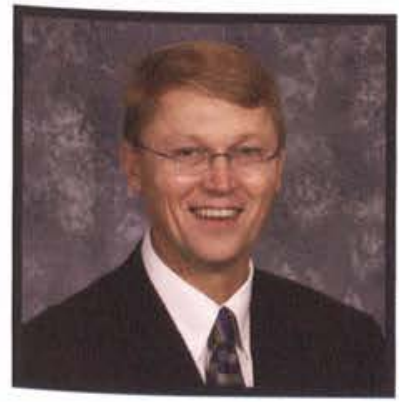

Kirk Martin Assistant Professor

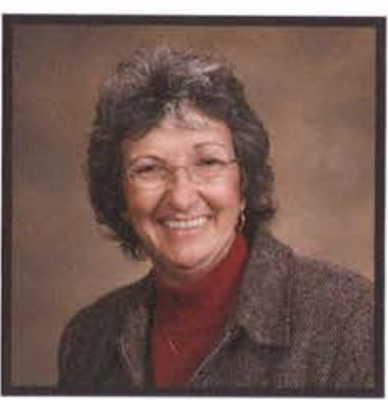

Kathy Freese Associate Professor

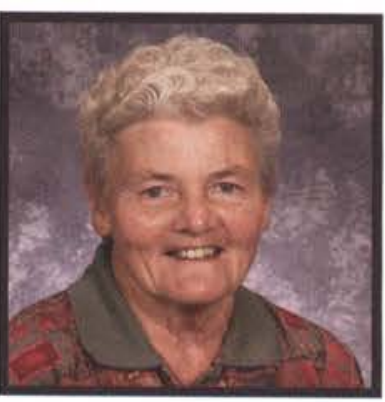

Dr. Dee Morris Adjunct Instructor

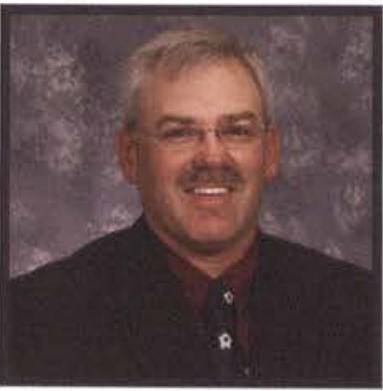

Ben Belleman Assistant Professor

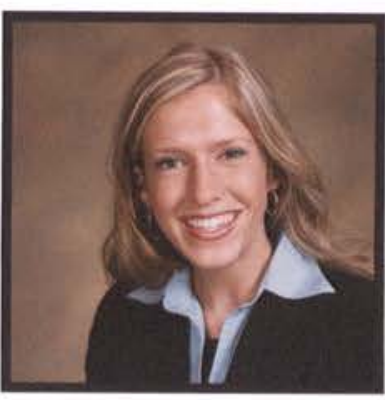

Dr. Alan Geist Assistant Professor

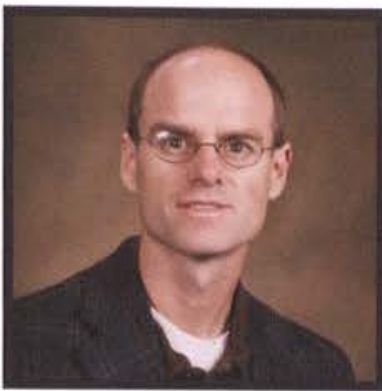

Jeff Bolender

Assistant Professor

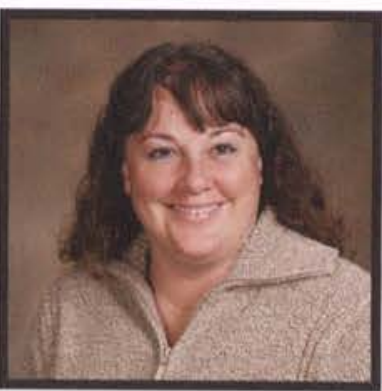

Elvin King

Associate Professor

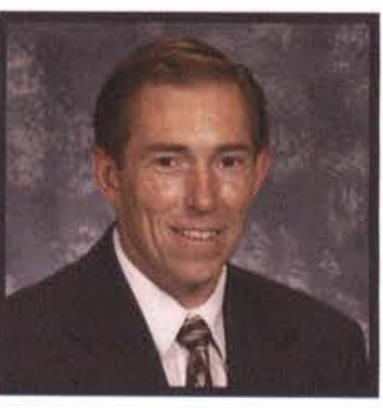

Paul Orchard Assistant Professor

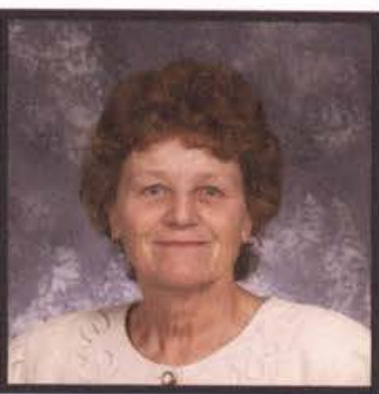

Libby Shearer Instructor

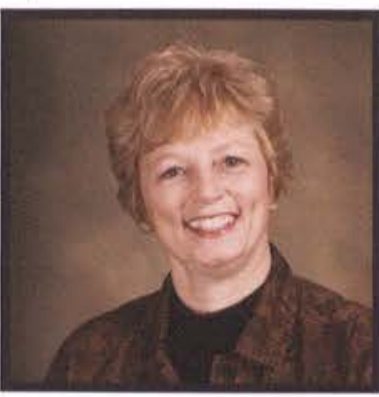

Teresa Clark Associate Professor

\section{Department of Athletics \& Campus Recreation}

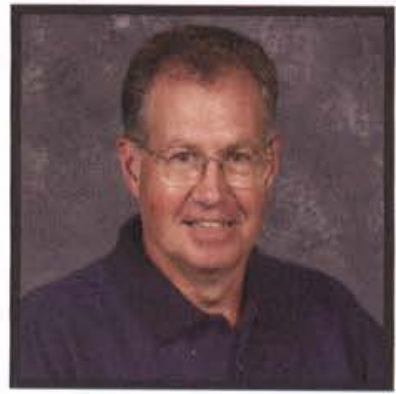

Jim Clark

Asst. Sports Info. Dir.

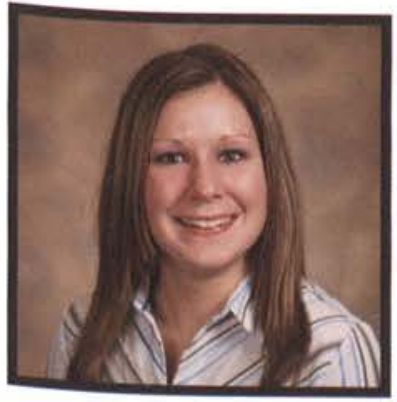

Lynsey Fabian

Assistant Women's Basketball Coach

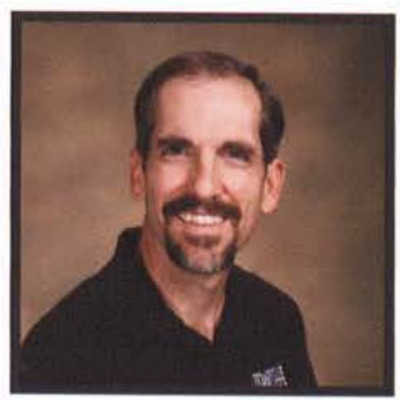

Mark Matthews Director of Campus Recreation

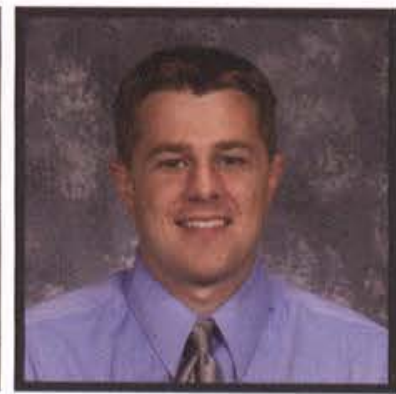

Pat Estepp Assistant Coach

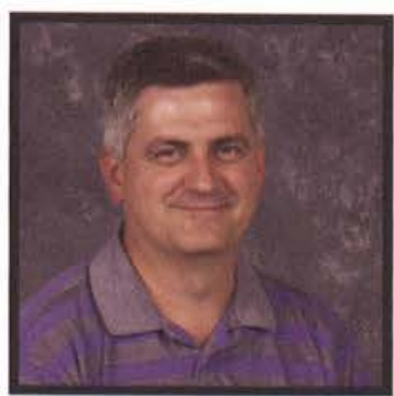

Mark Womack Sports Information

Director

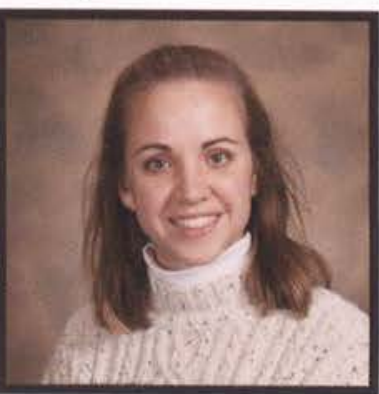

Melissa Hartman Head Volleyball Coach

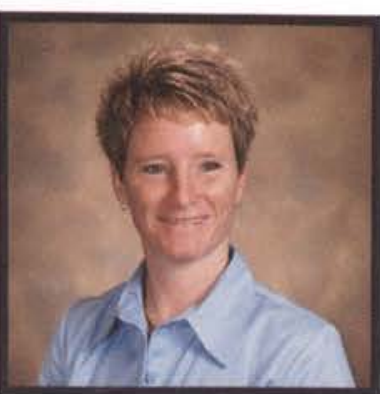

Lori J. Huckaby Assistant Women's Basketball Coach 



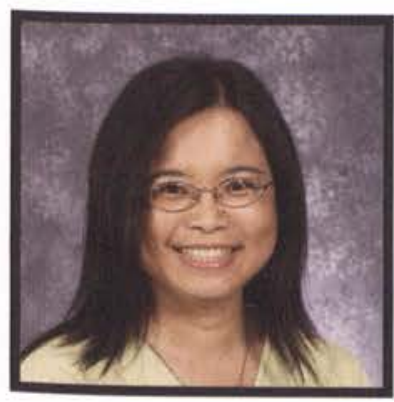

Chu-Yu Huang Assistant Professor

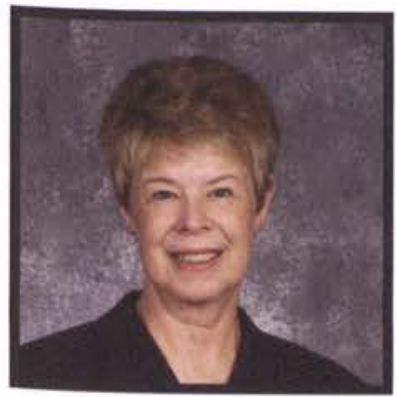

Dr. Susan Salladay Professor

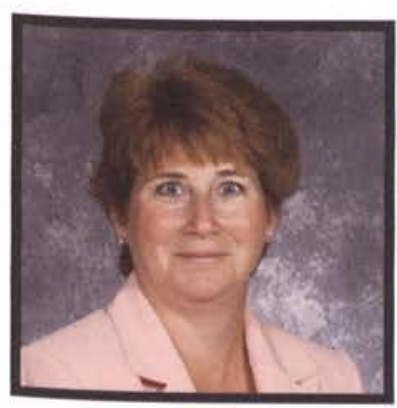

Amy Voris Instructor

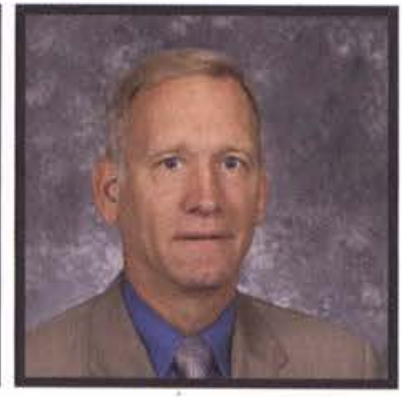

Mark Klimek Associate Professor

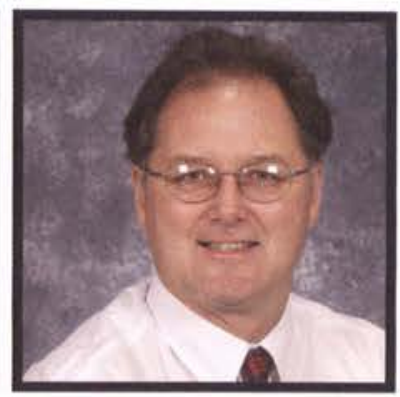

Dr. David Sharp Professor

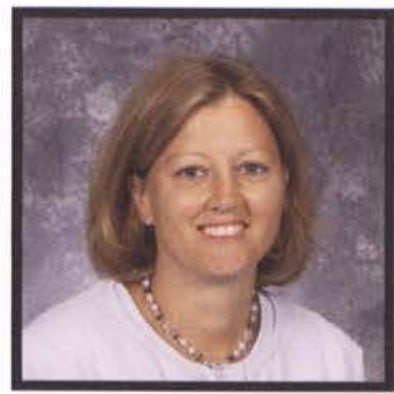

Suzanne Leafever Assistant Professor

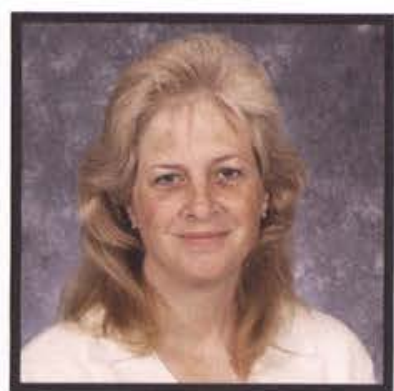

Dr. Kimberly Sharp Associate Professor

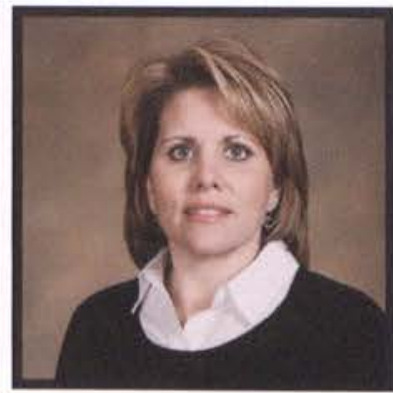

Denise Martin Adjunct Professor

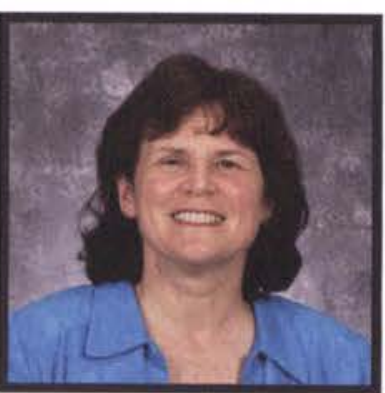

Dr. Sandra Shortt Adjunct Instructor

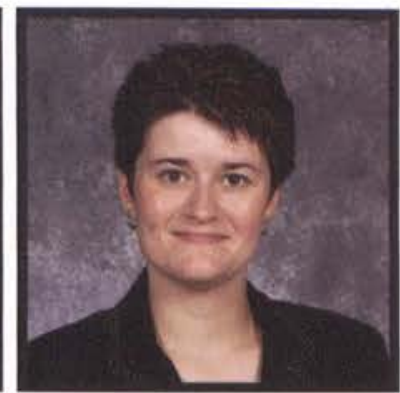

Rachel Parrill Instructor

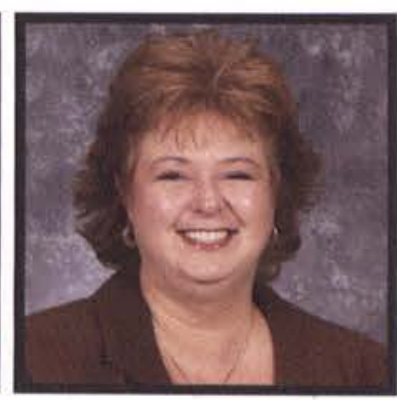

Rhonda Smith Adjunct Instructor

\section{Department of}

\section{Engineering \& Computer Science}

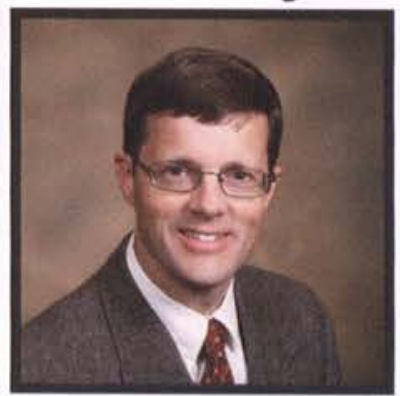

Dr. Gerry Brown Assistant Professor

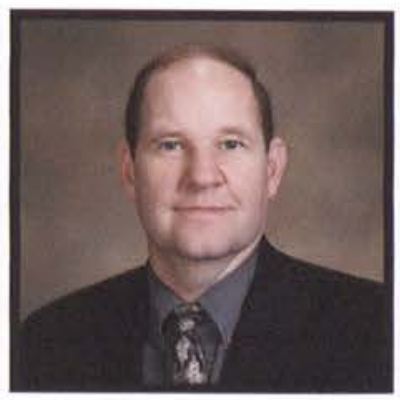

Dr. David Gallagher Professor

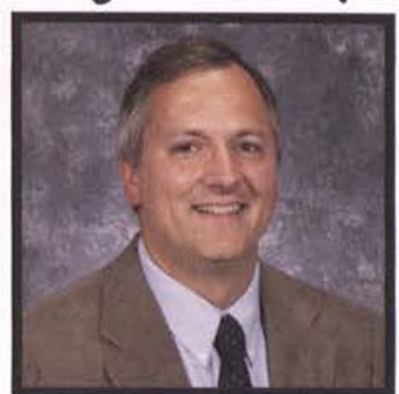

Dr. Pete Burban Associate Professor

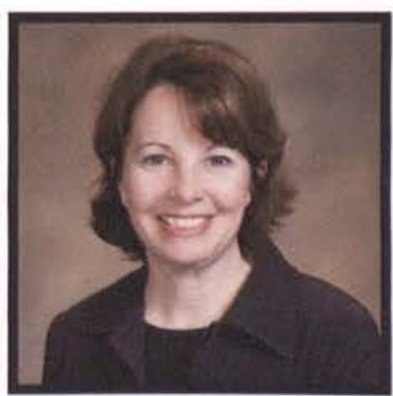

Cheryl L. Hadra Department Secretary

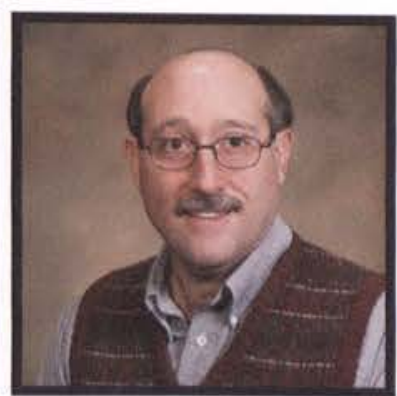

Dr. Robert Chaznov Assistant Chair

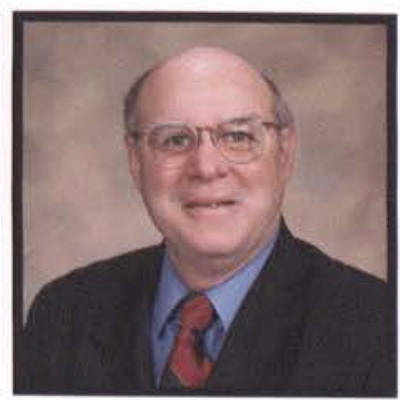

Dr. Harwood Hegna Professor 


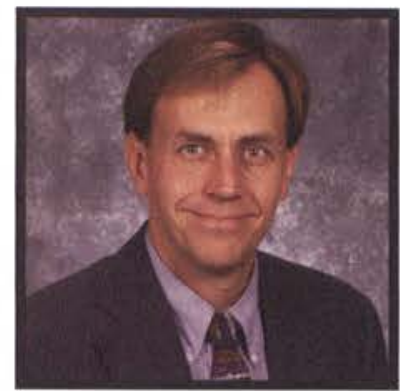

Jay Kinsinger

Assistant Professor

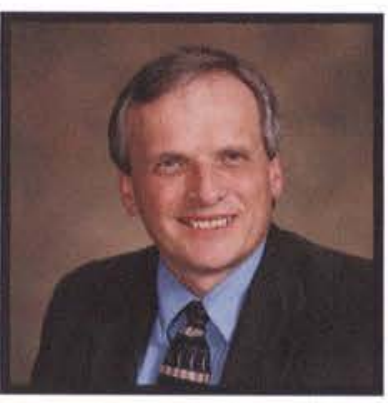

Dr. D. Jeffrey Shortt

Associate Professor

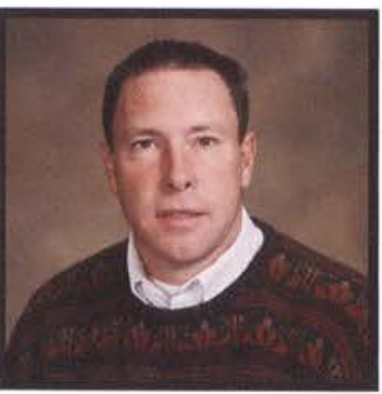

Jim Landers Technician

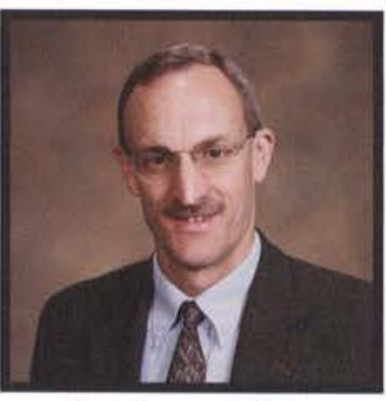

Dr. Thomas Thompson Associate Professor

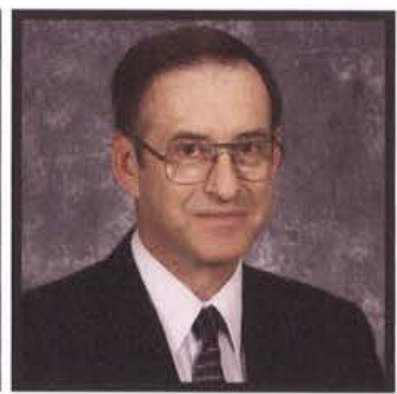

Robert Laramore Professor

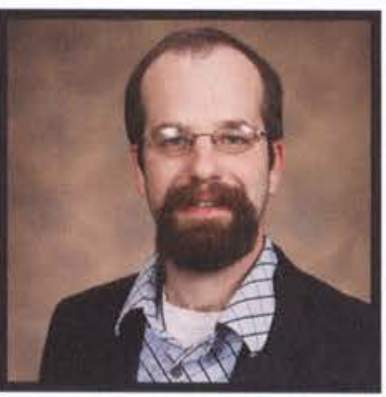

Timothy R. Tuinstra Assistant Professor

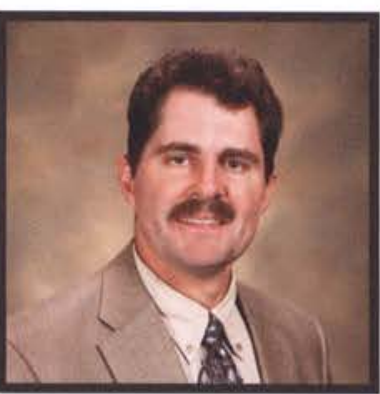

Dr. Timothy Norman Professor

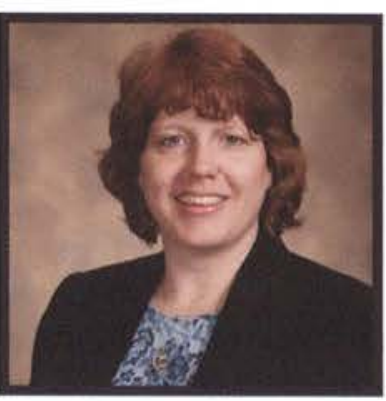

Jean Weyandt Administrative Assistant

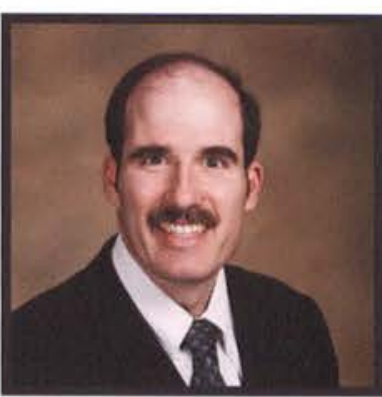

Dr. Keith Shomper Associate Professor

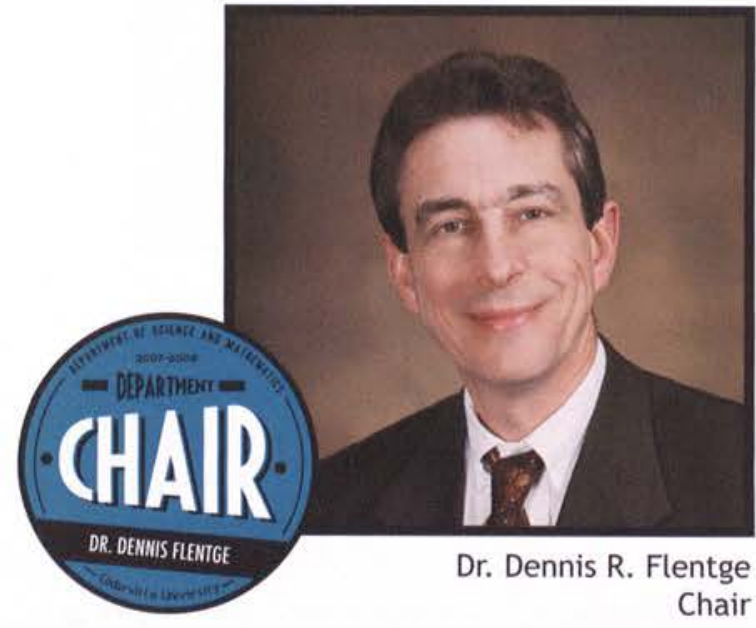

\section{Department of Science \& Mathematics}

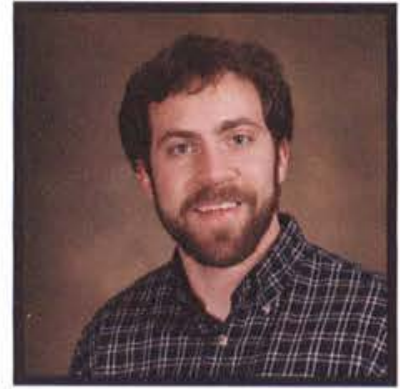

Aaron Costerisan Center for Bioethics Fellow

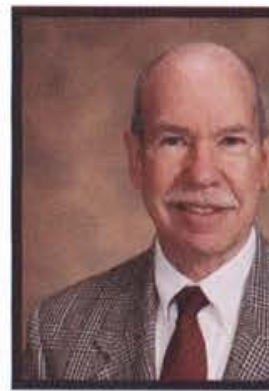

Dr. Larry Helmick Professor

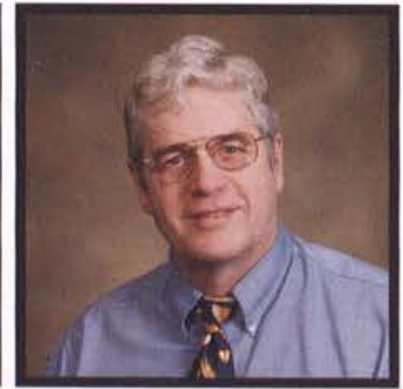

Dr. Lee Eimers Professor

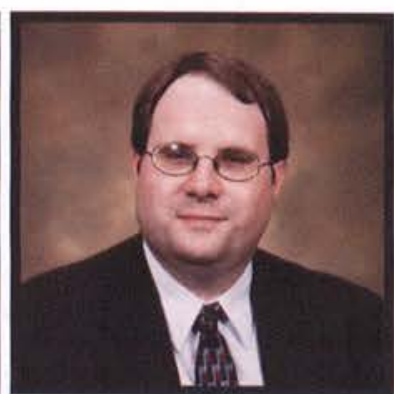

Aaron Hutchinson Assistant Professor

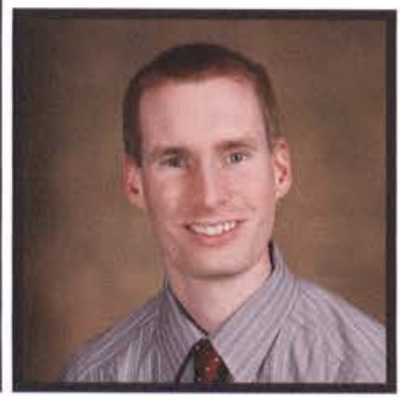

Andrew Black Lab Instructor

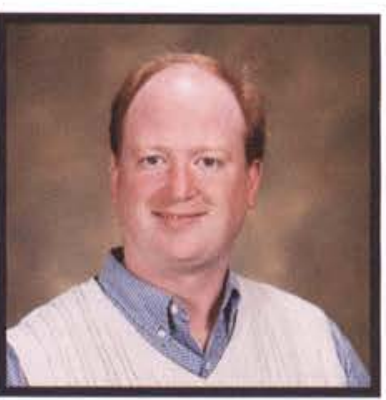

Dr. Darrin Frey Associate Professor

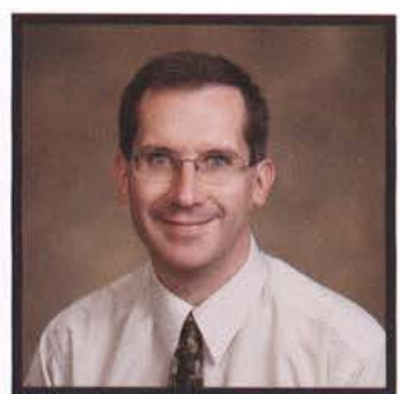

Eric L. Johnson Lab Technician

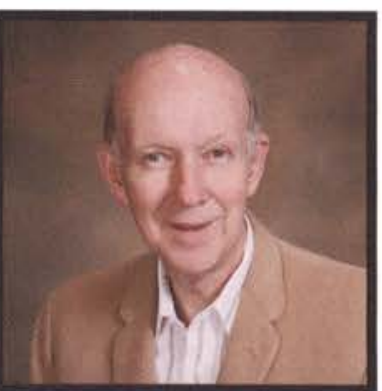

Dr. Donald Baumann Professor

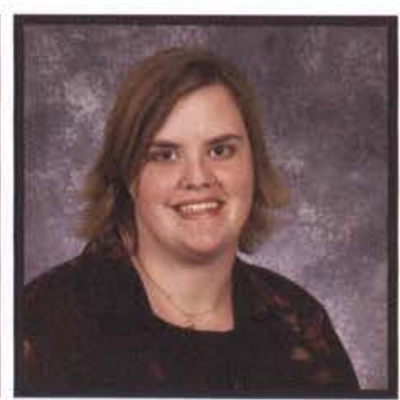

Sarah Gilchrist Instructor

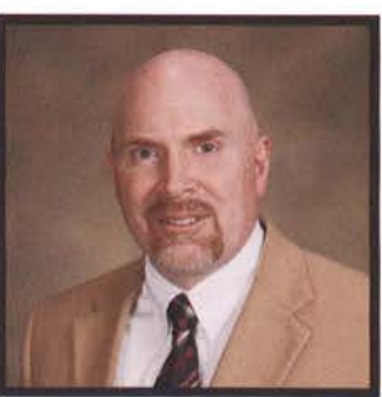

William I. Jones Assistant Professor

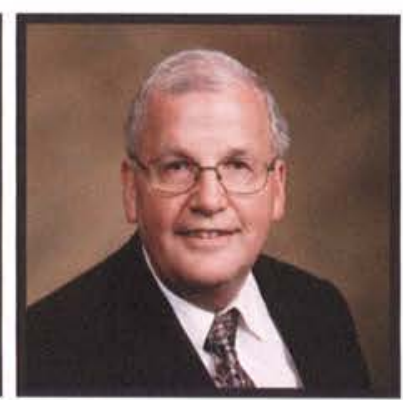

Dr. Edwin Braithwaite Professor

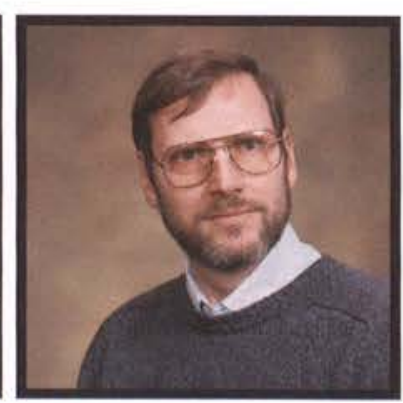

Dr. Steven Gollmer Associate Professor

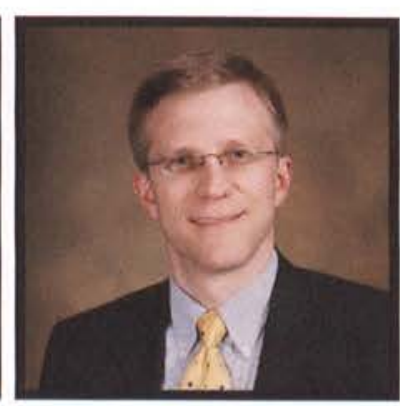

Dr. Mark McClain Associate Professor 


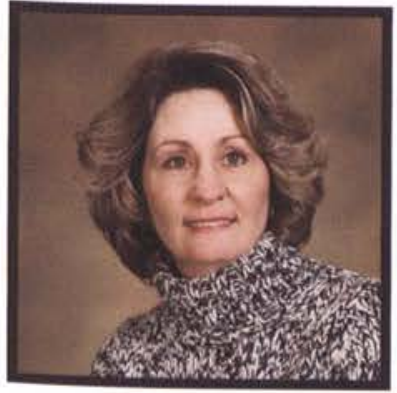

Shelly Nutter Administrative Assistant

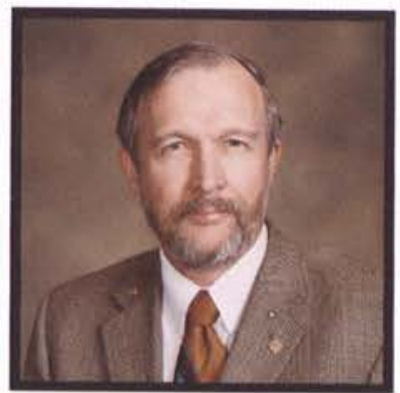

Robert M. Shumacher Assistant Professor

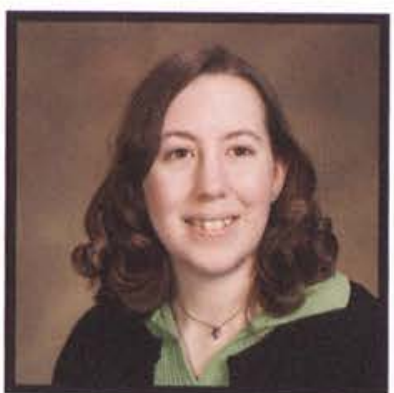

Jennifer Secor Instructor

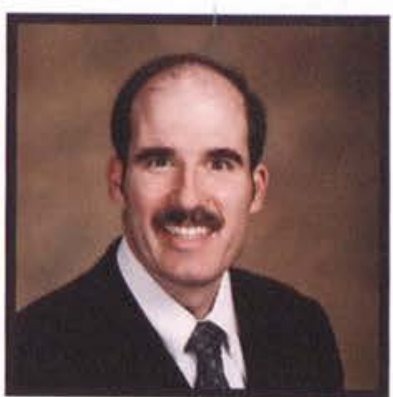

Dr. Keith Shomper Associate Professor

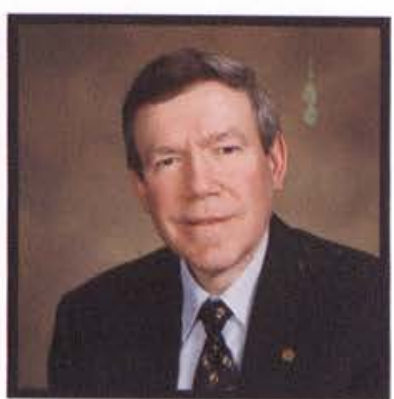

Dr. John Silvius Senior Professor

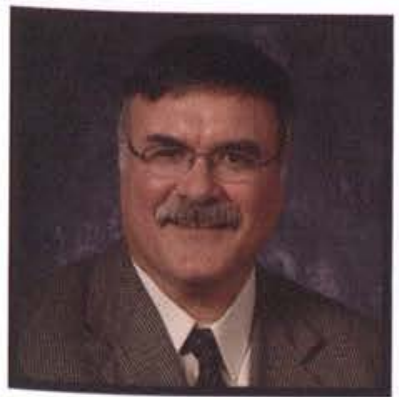

Dr. Dennis Sullivan Professor

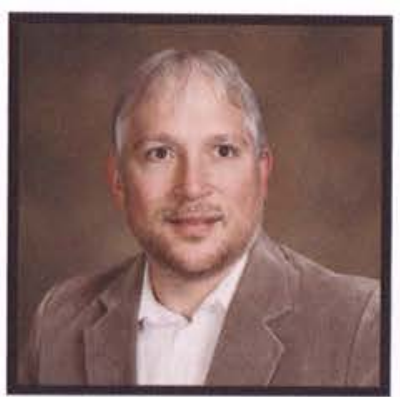

Dr. John Whitmore Associate Professor

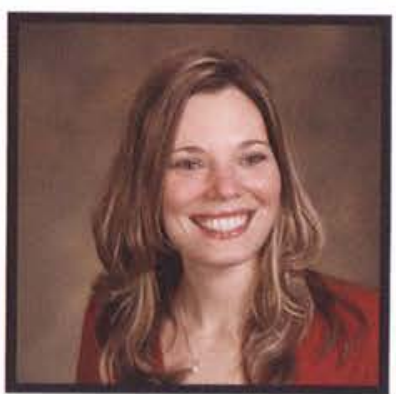

Cynthia Wingert Intructor

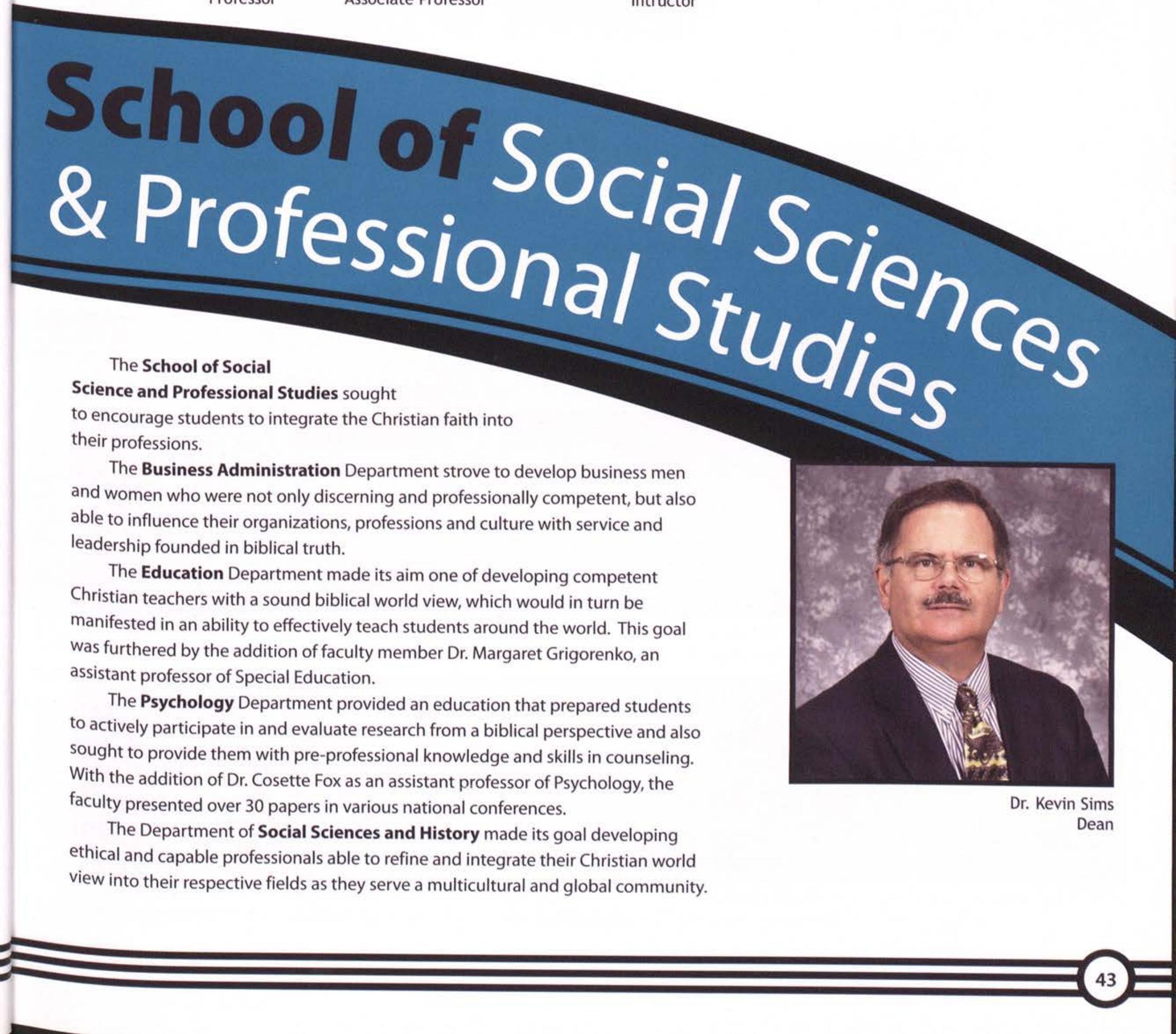




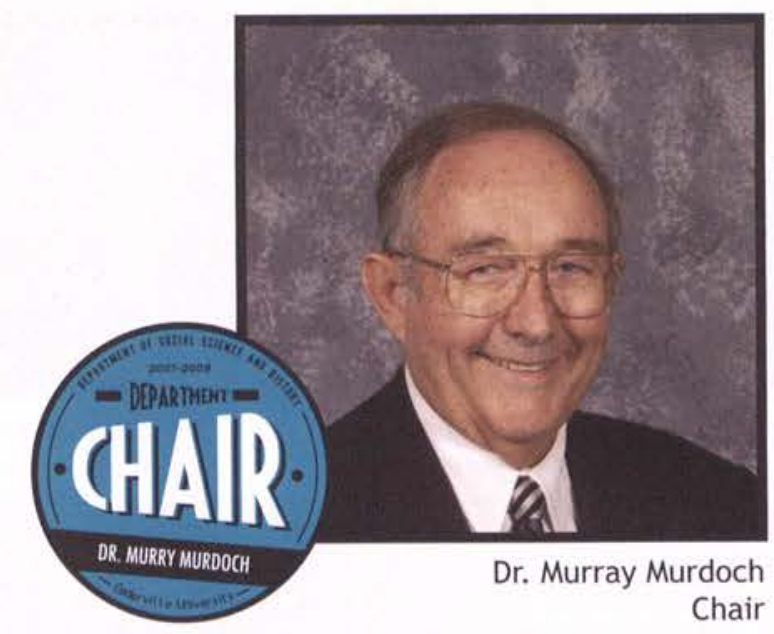

\section{Department of Social Science \& History}

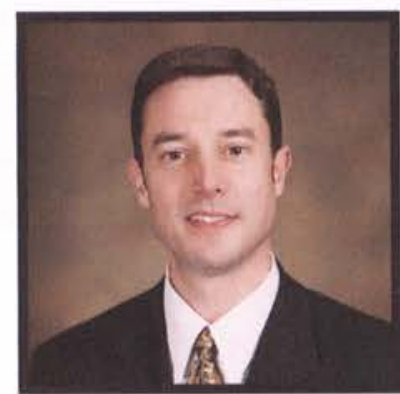

Dr. Tom Mach Assistant Professor

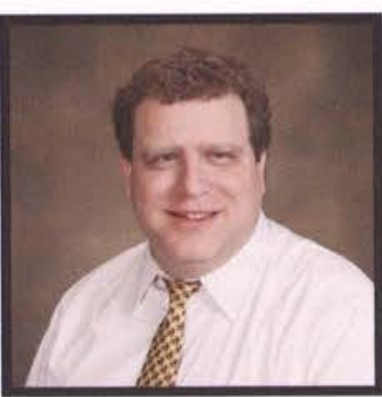

David Meyer Assistant Professor

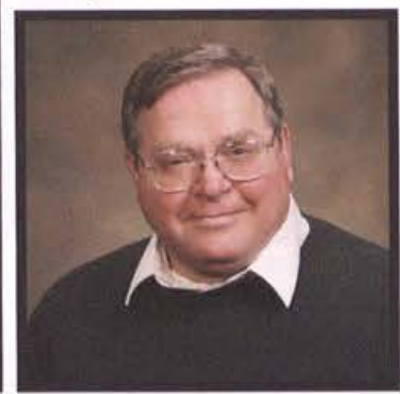

Dr. Nelson Henning Associate Professor

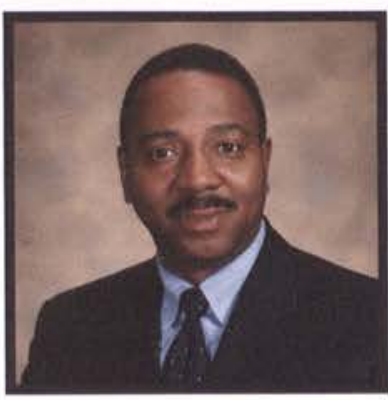

Patrick Oliver Assistant Professor

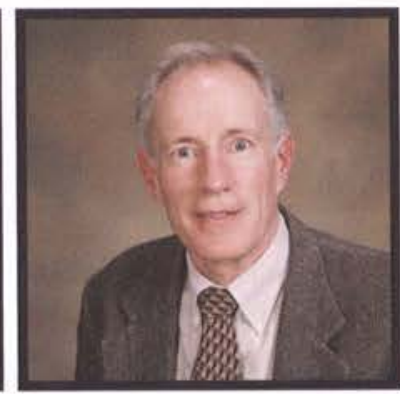

George Huff Assistant Professor

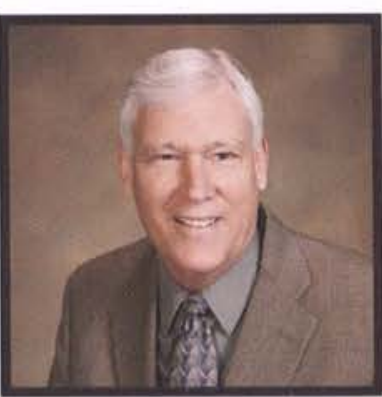

Dr. Robert G. Parr Professor

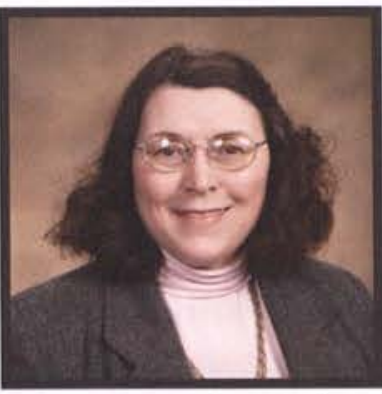

Esther Lanham Administrative Assistant

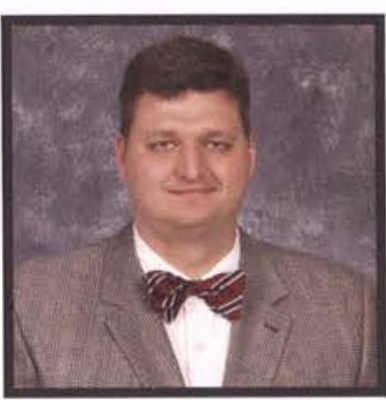

Dr. Mark Smith Assistant Professor

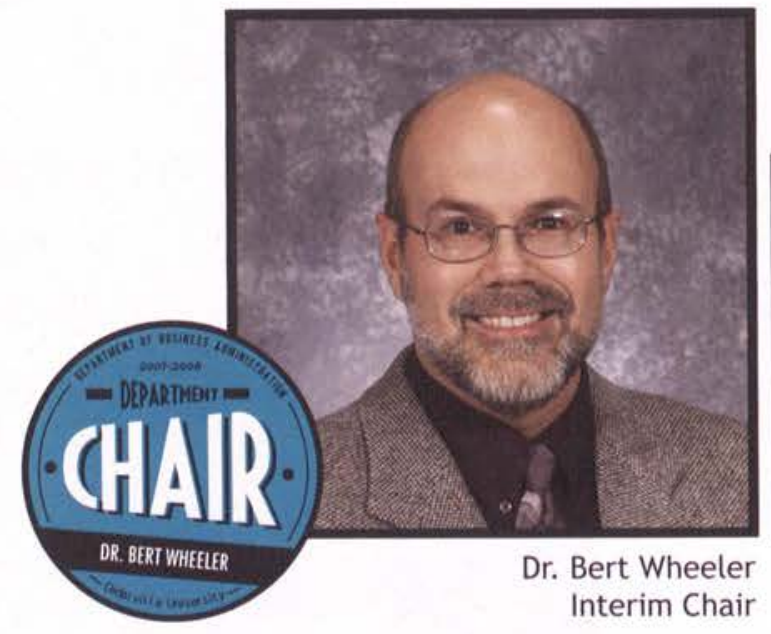

\section{Department of Business Administration}

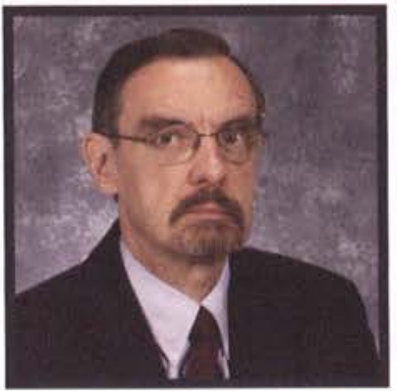

Harry Anderson Assistant Professor

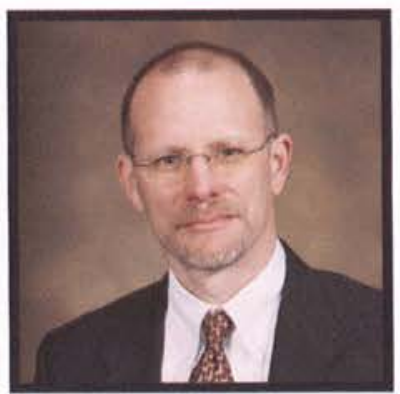

Jeff Guernsey Assistant Professor

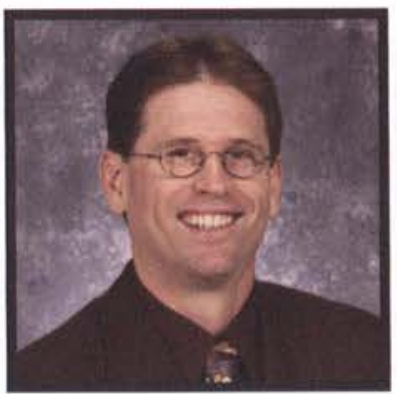

Dr. Jon Austin Associate Professor

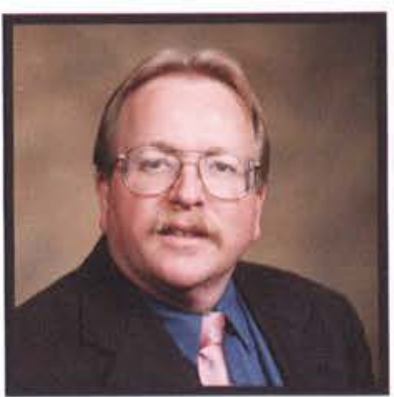

Tim Hansell Assistant Professor

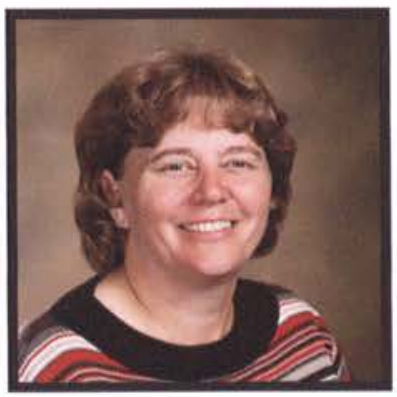

Vicki Edem Administrative Assistant

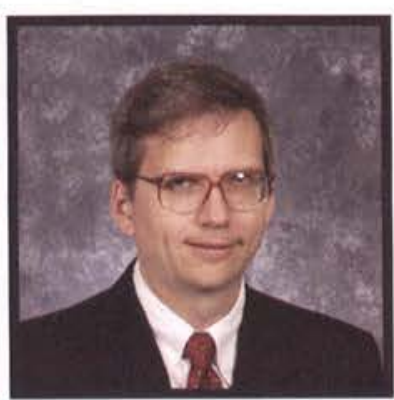

Charles Hartman Associate Professor
Dr. Franco Gandolfi Associate Professor
Professor 


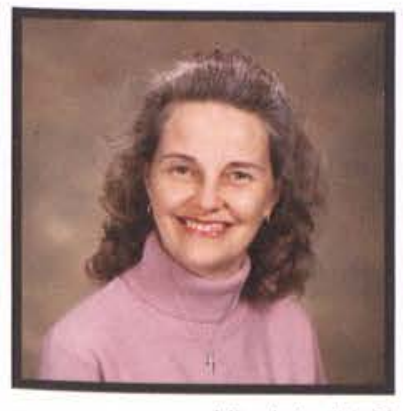

Virginia Huff Secretary

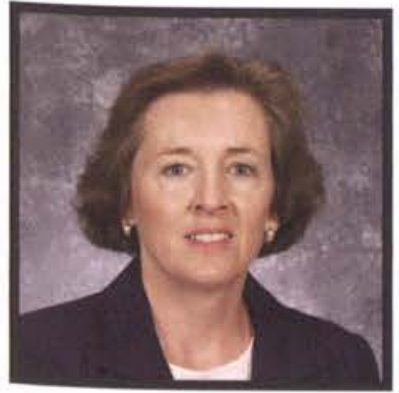

Anne Rich Assistant Professor

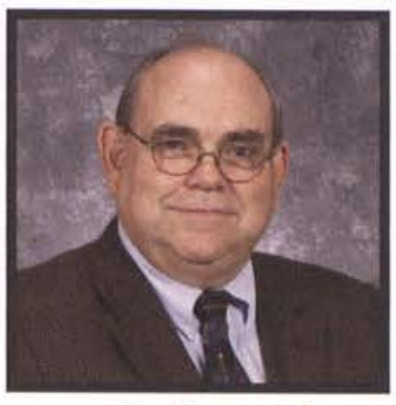

Dr. Sharon Johnson

Professor

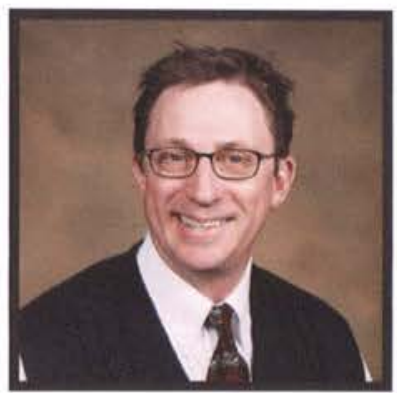

Dr. Dave Schmidt Associate Professor

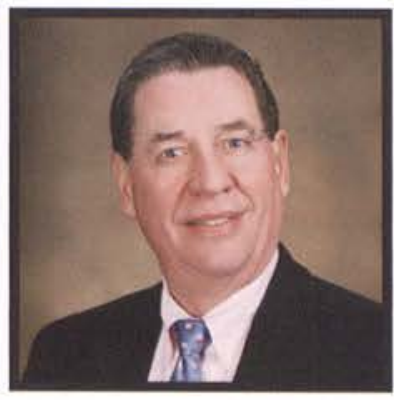

John LeBlanc Associate Professor

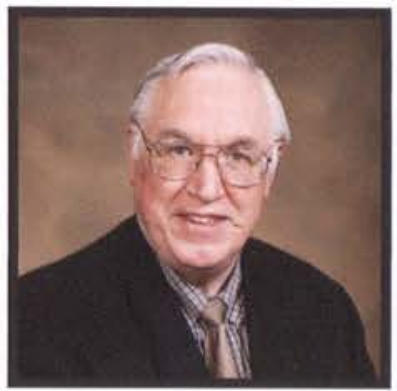

Dr. Galen Smith Professor

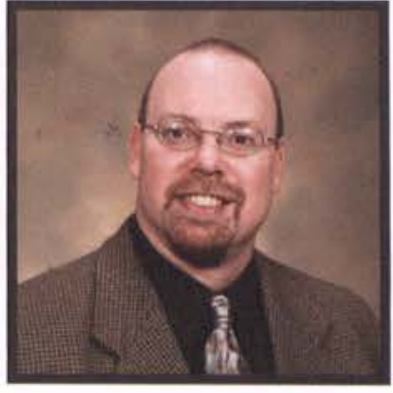

Dr. Richard Martinez Associate Professor

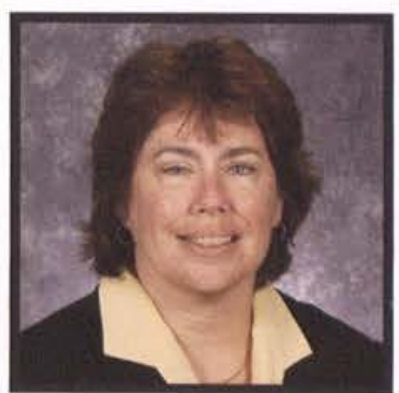

Dr. Sarah Smith Associate Professor

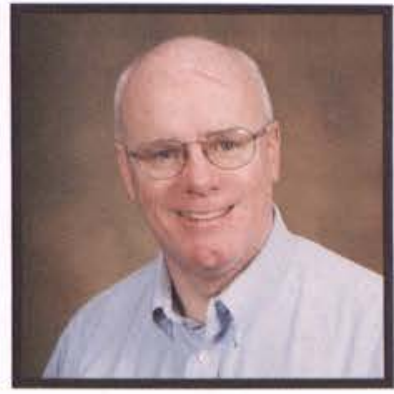

Dr. William Ragle Associate Professor

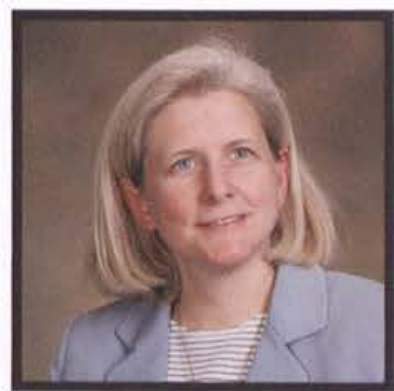

Susan Terkelsen Assistant Professor

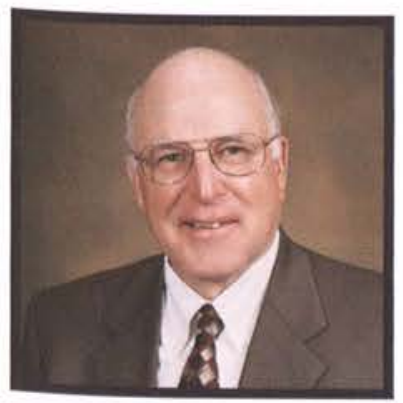

Dr. Ronald Walker Professor

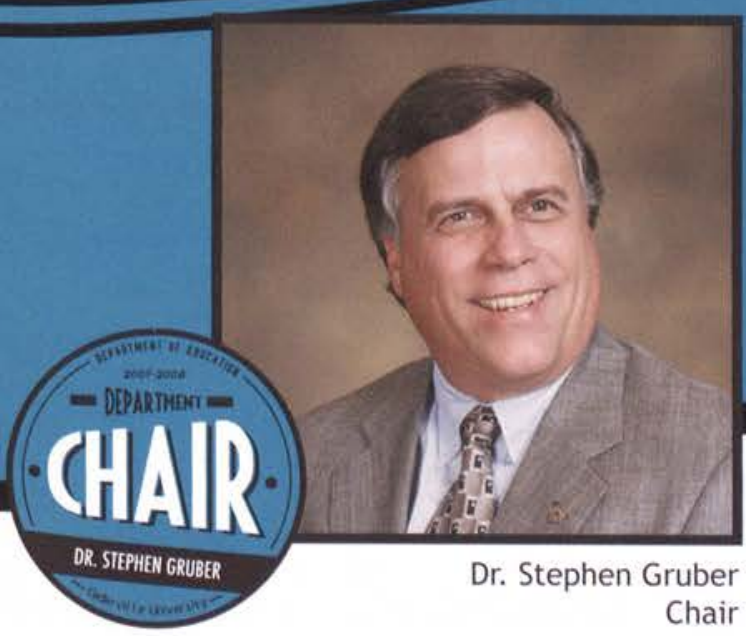

Departincent of Education

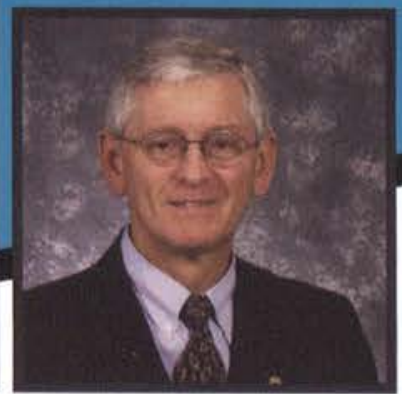

Dr. Merlin Ager Professor

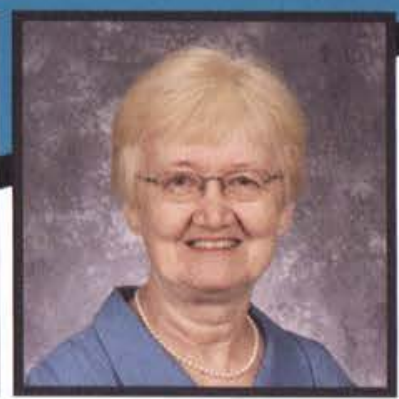

Dr. Sue Baker Professor

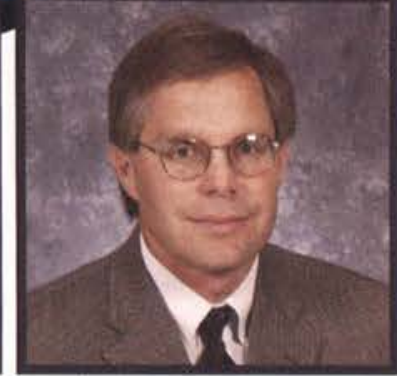

Dr. Eddie Bauman Professor

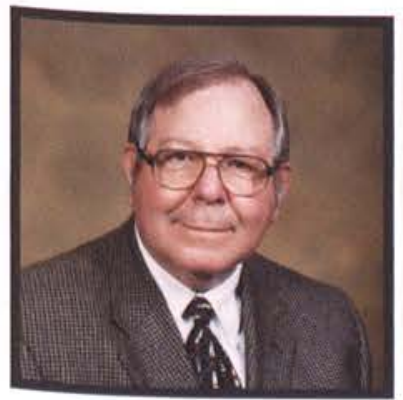

Omer Bonenberger Associate Professor

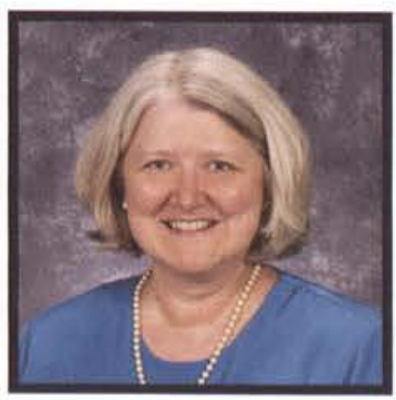

Terry Comers Instructor

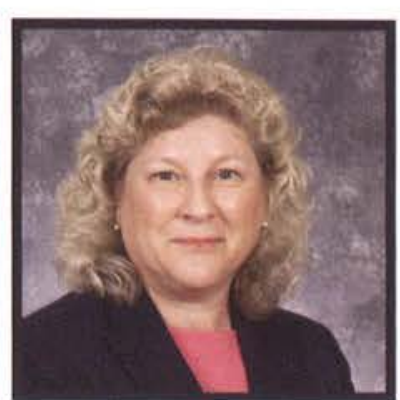

Carol Estes Assistant Professor

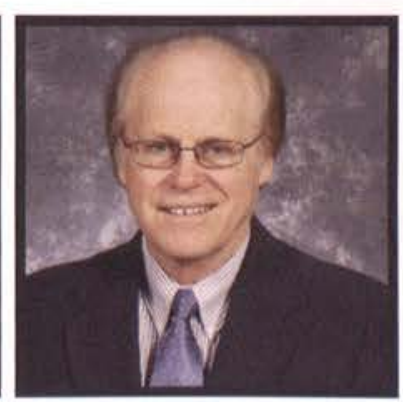

Dr. Dwayne Frank Professor Emeritus

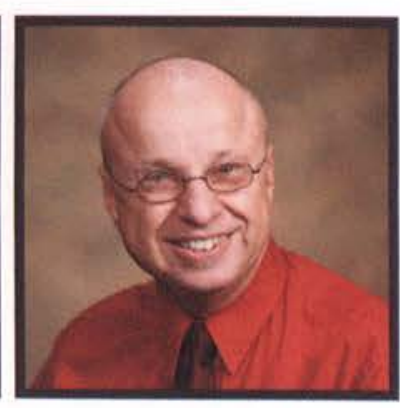

Dr. Tim Heaton Associate Professor 


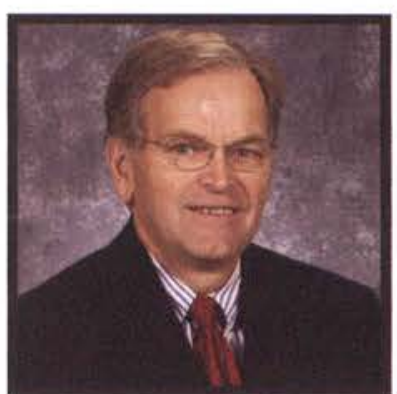

John Hess

Director

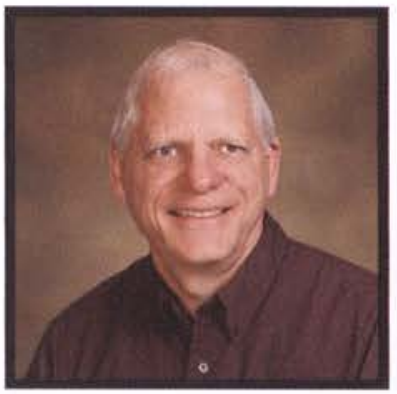

Mike Parrot Assistant Professor

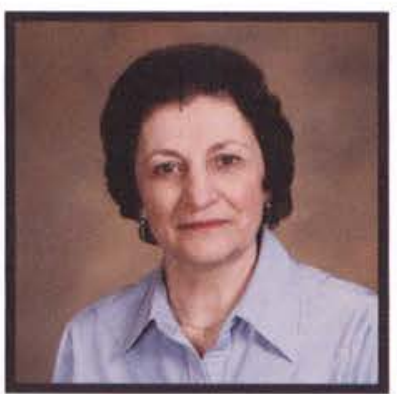

Dr. Cheryl Irish Associate Professor

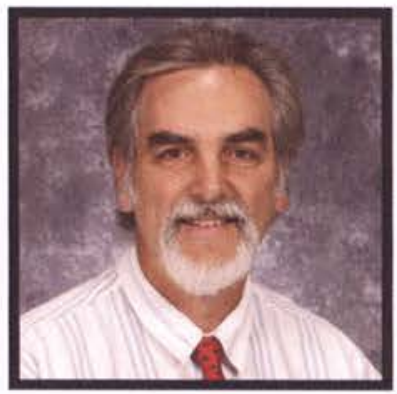

Dr.Tom Sweigard Assistant Professor

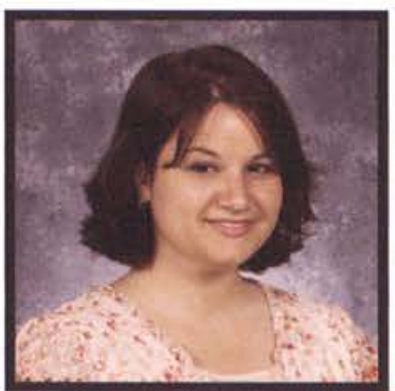

Laura Jolly License \& Testing Cord

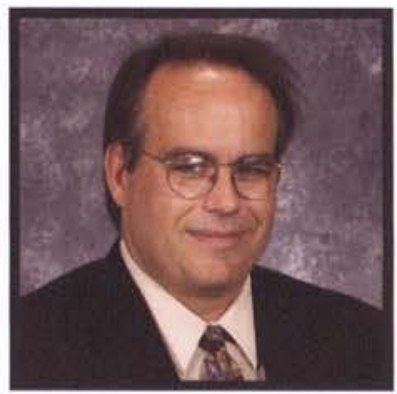

Kevin Winslow Assistant Professor

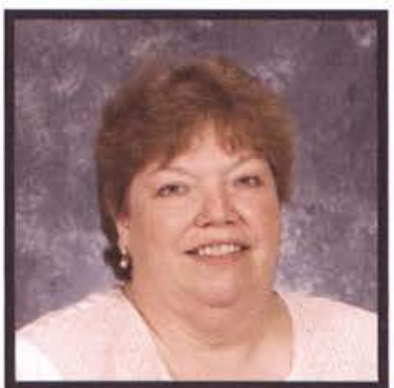

Toi King Academic Dept. Secretary

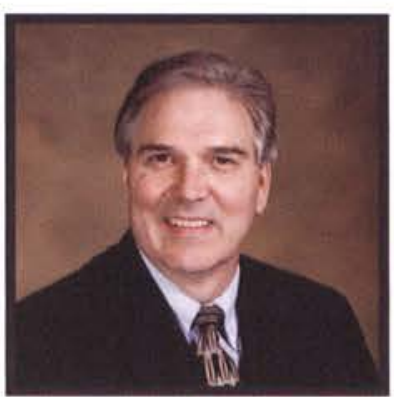

Dr. Bryan Moore Assistant Professor

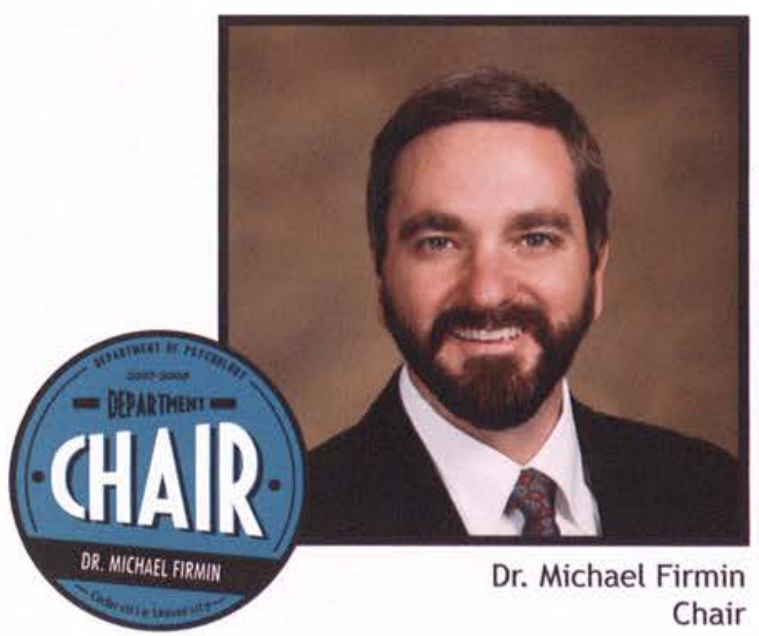

\section{Department of Psychalagy}

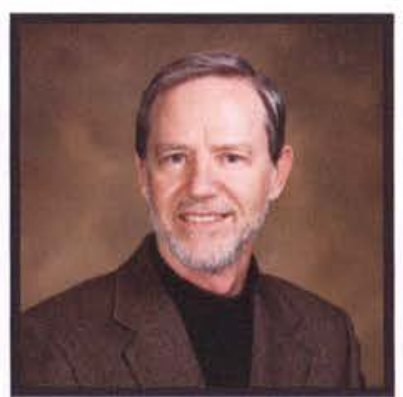

Dr. Milt Becknell Associate Professor

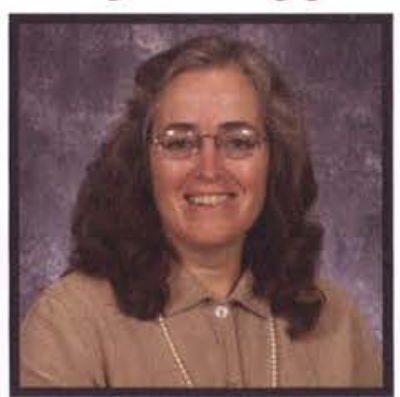

Kay McFarlane Secretary

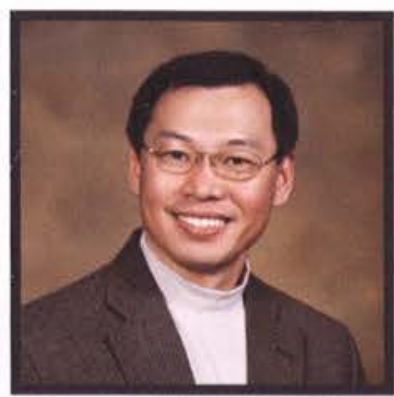

Dr. Luke Tse Assistant Professor 
Academic

Cedarville's Academic Division, a small department composed of Academic Assistance,

Computer Services, the Library, and the Registrar's Office, expanded beyond measure this year.

Computer Services dramatically expanded wireless network coverage to include access in the bedroom areas of the residence halls, whereas previously access was mainly restricted to academic buildings and the grounds. On a typical afternoon, approximately 700 students now connect to the network in this manner.

Academic Assistance, more commonly known around campus simply as "The Cove," expanded its influence through its mission to help Cedarville students become even more successful in their academic pursuits. The office served between fifteen and thirty students a day, up 80 percent from the previous year. The Cove also hosted numerous events aimed at promoting the services available to students who visit the office. A spectacular event they called "Lemon Breeze" attracted 700 students and this year also saw the highest number ever attend the 153/13 event for freshmen and undeclared majors. Furthermore, the office's unique Academic Probation course, MAP, and nationally certified tutoring program were the anticipated topics of their presentations to the National Academic Advising Association.

Through these and other areas too many to mention, the Academic Division has continued to do what it can to support the work of Cedarville University.

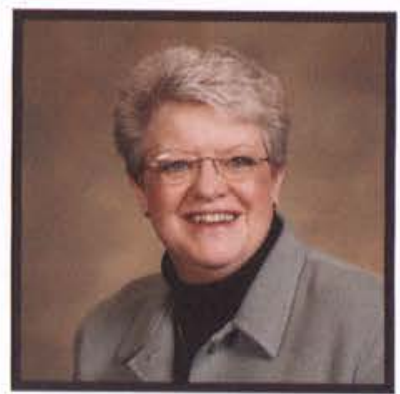

Fran Campbell Registrar

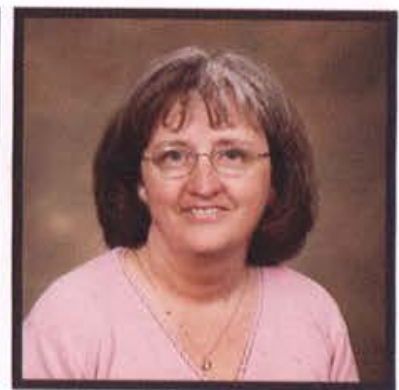

Cynthia Davis Assistant to the Registrar

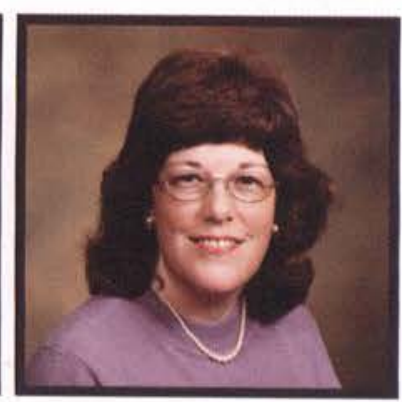

Linda Chrystal Administrative Assistant

\section{Assistants \& Secretaries}

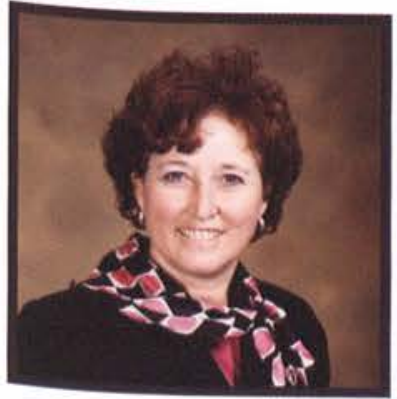

Carol George

Executive Admin. Asst. for the Office of the President

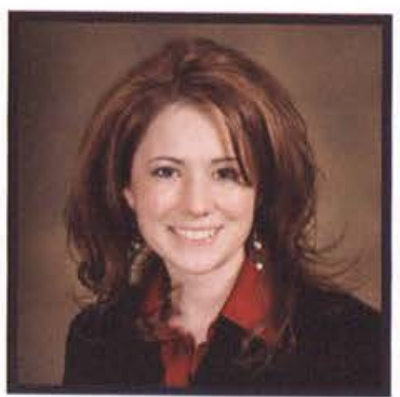

Teressa Mahl Secretary for the Office of the President

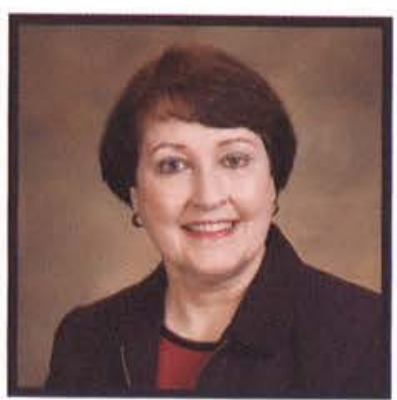

Lynn Rohm Admininistrative Assistant to the Chancelor

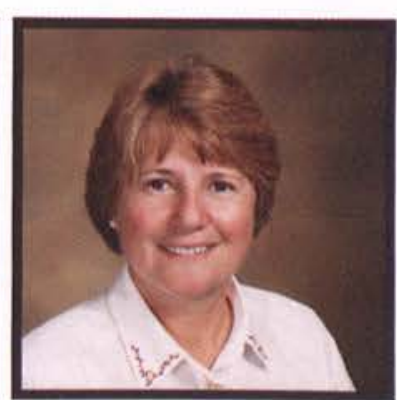

Joy Williams

Administrative Assistant
Dr. Duane Wood Executive Director 


\section{Library Faculty}

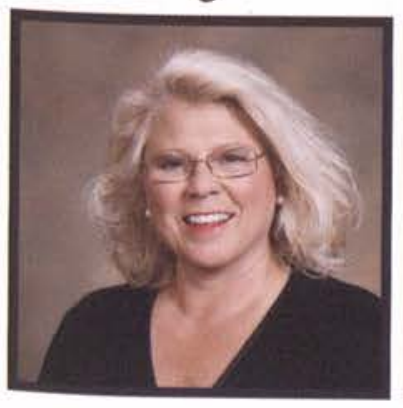

Janice Bosma Associate Director

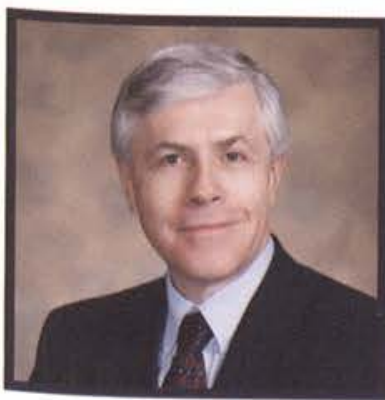

Greg Martin Assistant Professor

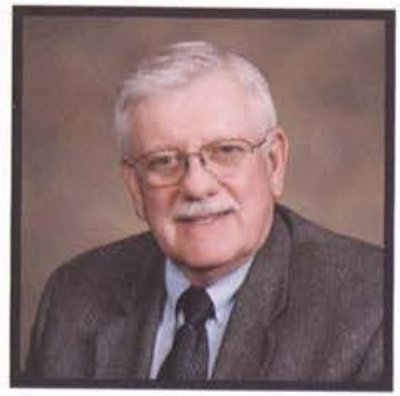

Carl Brandon Assistant Director

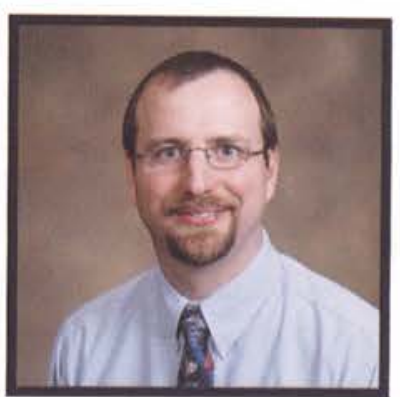

Rory Patterson

Digital Services Librarian

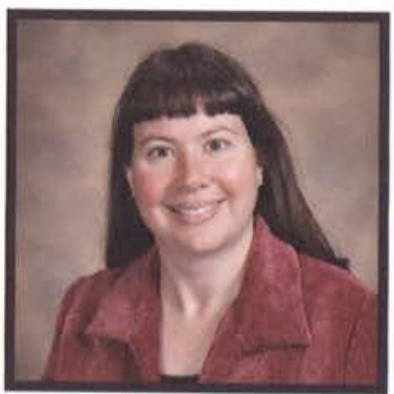

Julie Deardorff Assistant Director

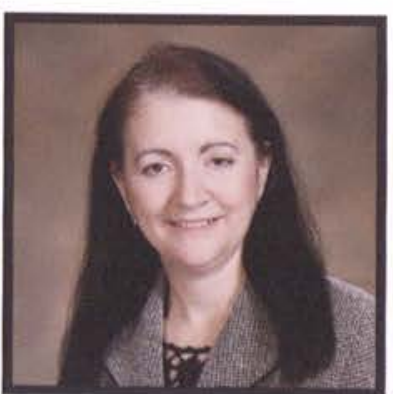

Tonya Fawcett Assistant Director

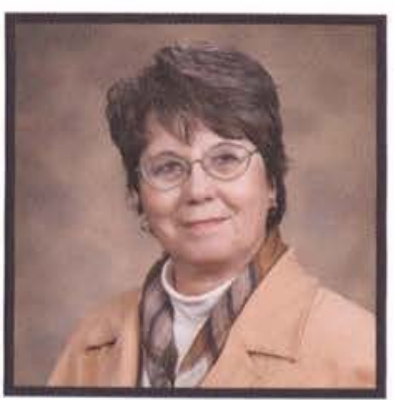

Lynne Funtik

Senior Reference Librarian

\section{Library Stabl}

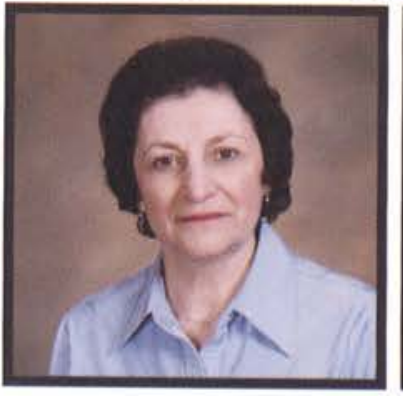

Fran Andrews Office Manager

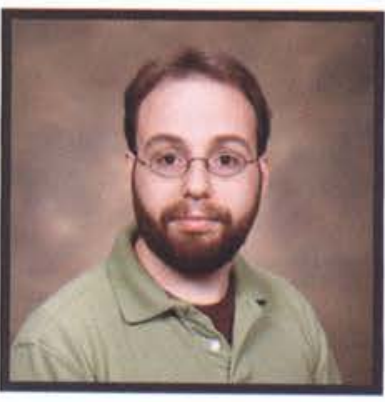

Josh Heinrich

AV Services Technician

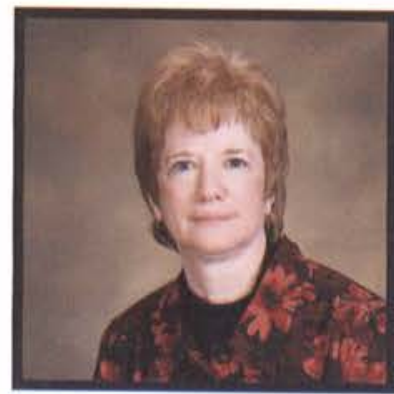

Luann Nicholas Interlibrary Services Coordinator

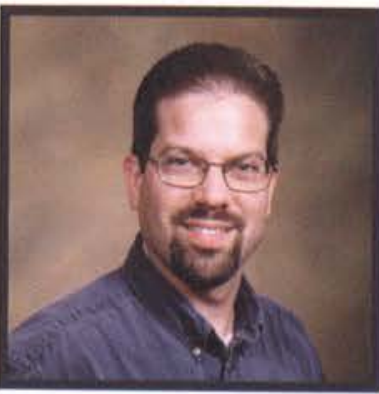

Scott Deetz AV Services Manager

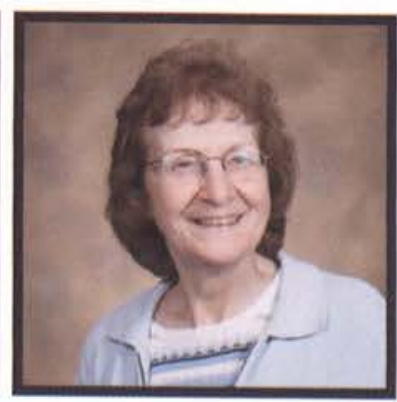

Rachel Johnson Collection Tech. Assistant

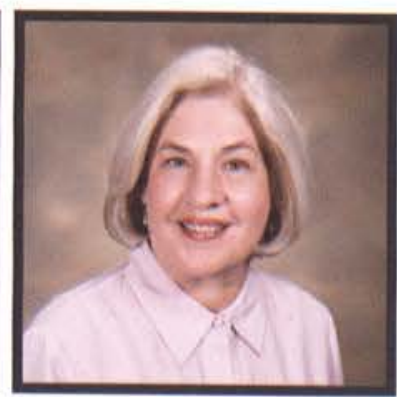

Dianne Seals Graphic Tech. Assistant

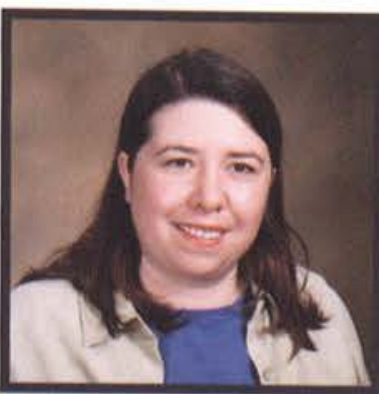

Dara Fraley Educational Tech. Analyst

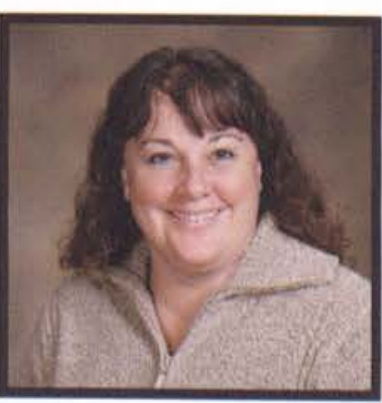

Laura LeMaster Serials Coordinator

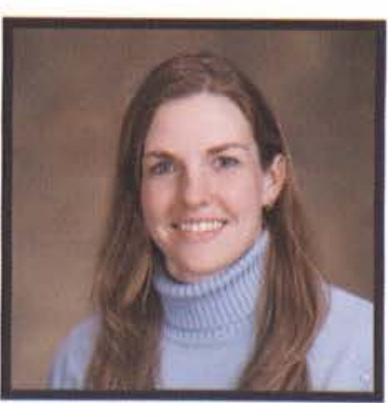

Tricia Walker

Technical Coordinator, Reader Services

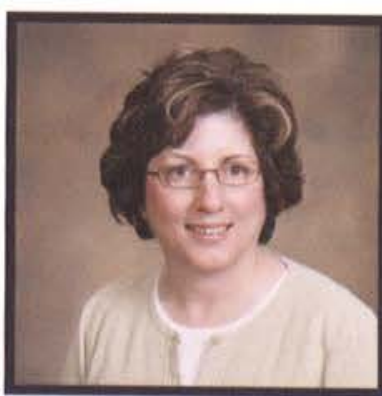

Becky Hayes Circulation Manager

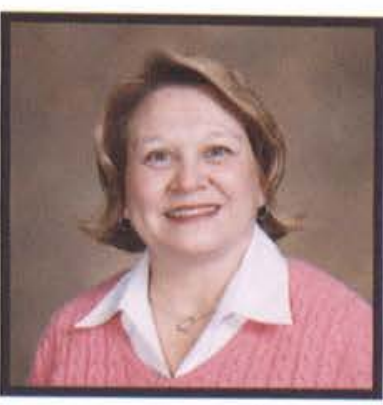

Andi Mounts Assistant Circulation Services Manager 



\section{Counseling Services}

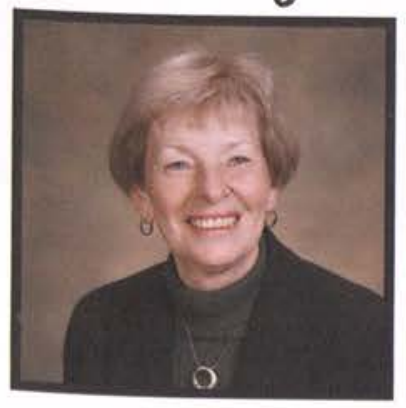

Helen Blumenstock Secretary

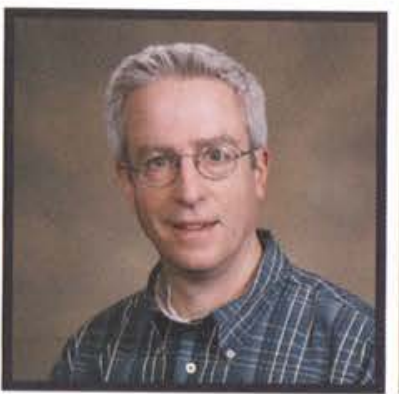

Jeffrey Fulmer Counselor

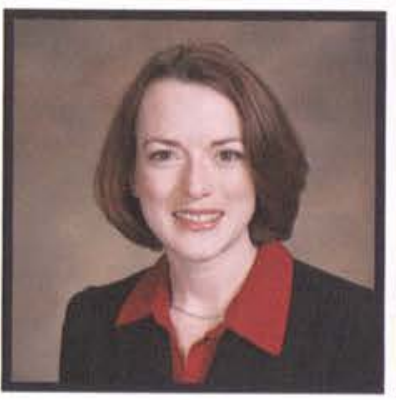

Rachel Heffield

Counselor

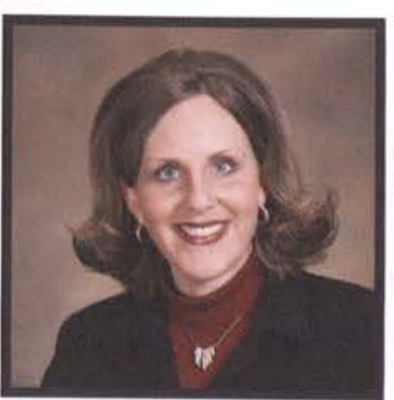

Lori Hoffeditz

Counselor

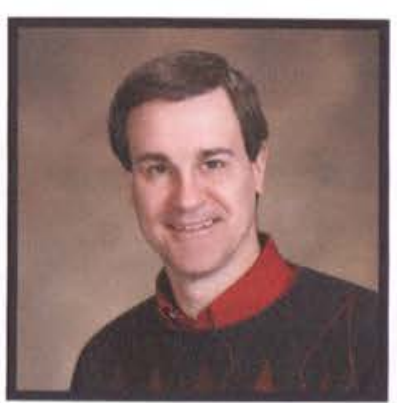

John Potter Director

\section{Resident Directars}

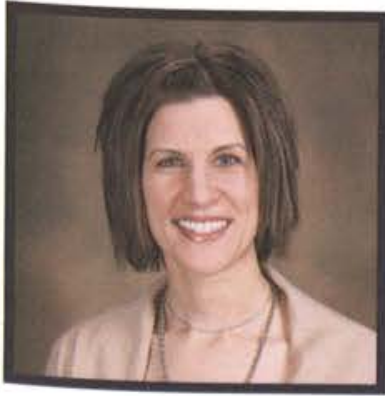

Bonnie Kuvshinikov Murphy Hall

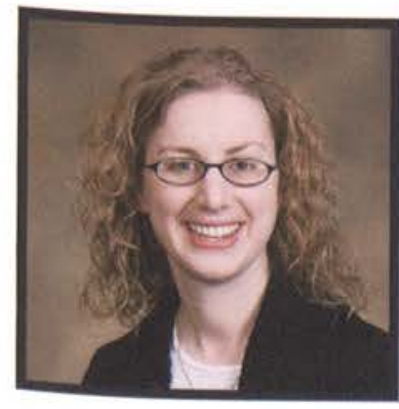

Becky Stowers

Faith Hall

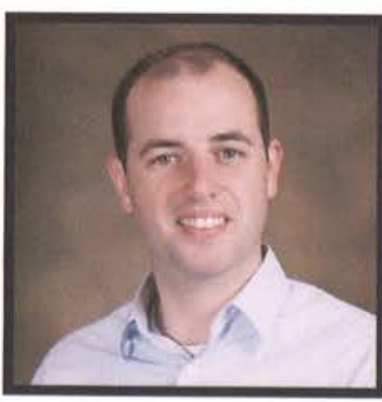

Bob Lutz McChesney Hall

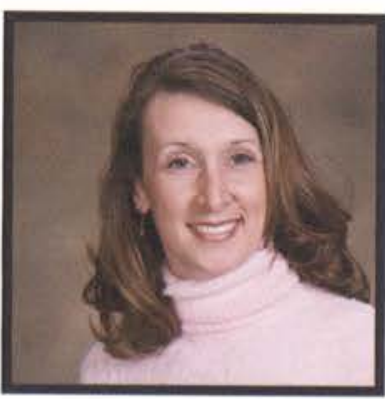

Susan West McKinney $\mathrm{Hal}$

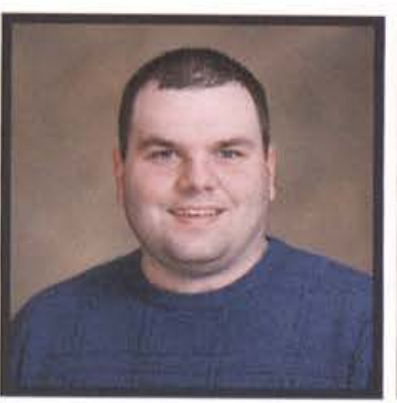

Adam T. McCune The Hill

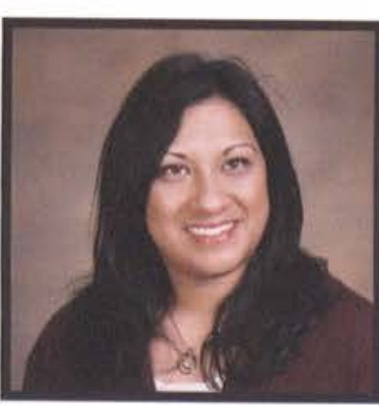

Rachel Rodriguez Willetts Hall

\section{University Medical Services}

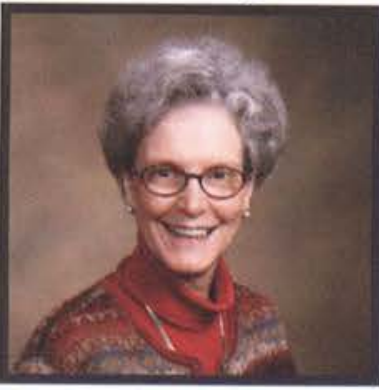

Martha Kaercher Student Insurance Coordinator

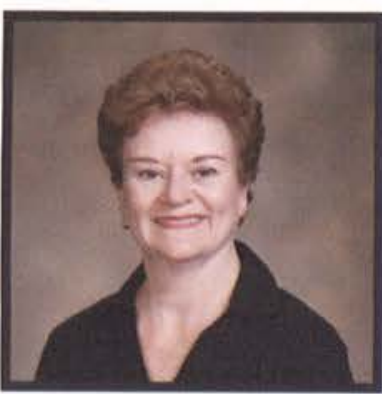

Beverly Robey Nursing Supervisor

\section{Campus Activities}

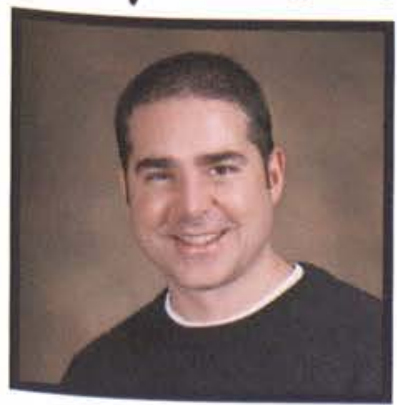

Jeff Beste

Associate Dean for Student Leadership Development

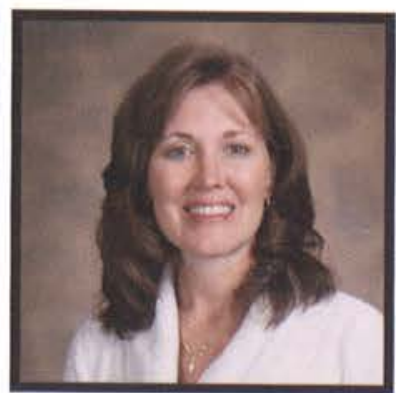

LeAnn Hill Secretary

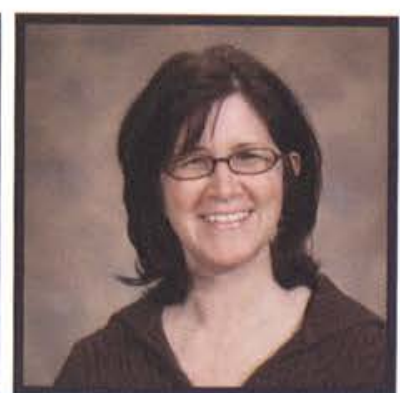

Elaine Keller Administrative Assistant

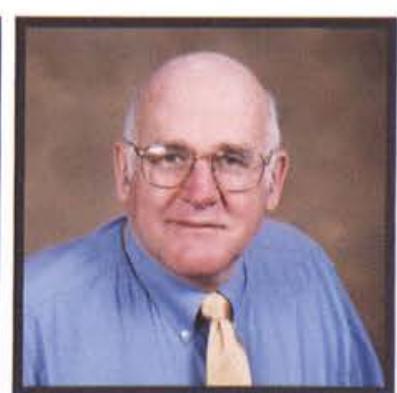

Dick Walker

Dean for Community \& Family Programs 



\section{Marketing Services}

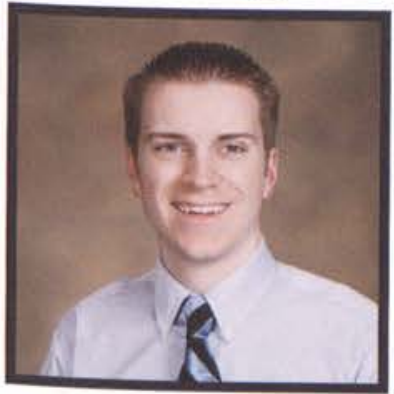

Jon Collins Assistant Director

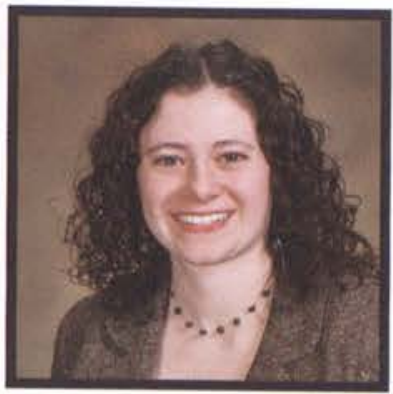

Chrissy Faulkner Assistant Director

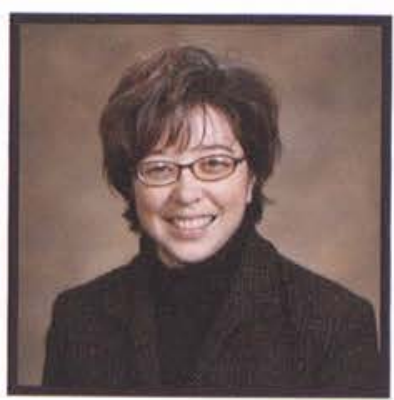

Yukiko Johnson Marketing Assistant

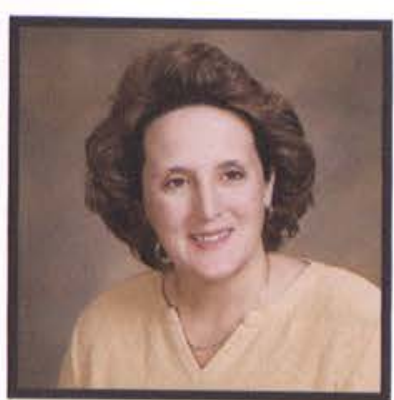

Joan Wilson Church \& School Relations Coordinator

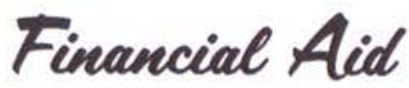

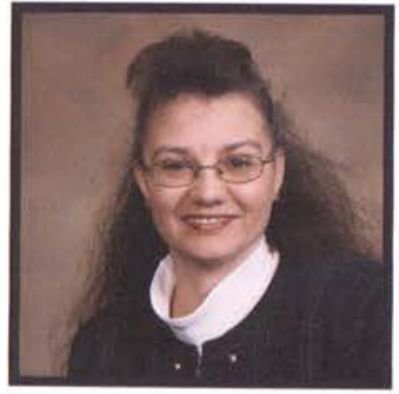

Tonya J. Bailey Financial Compliance Manager

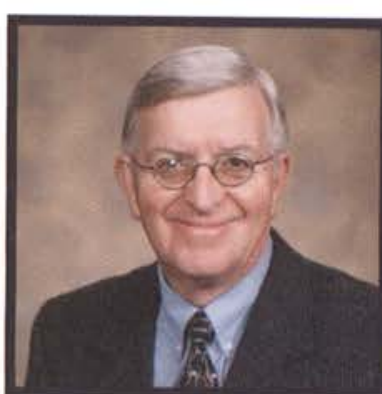

Ken Rotrof Assistant Director

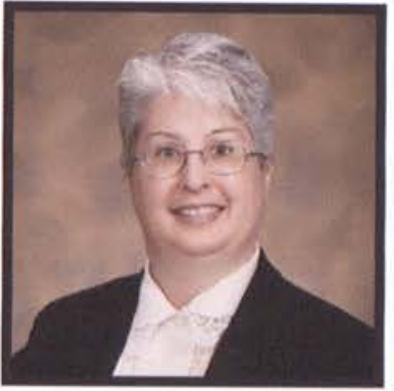

Pam Flippin Loan Processing Manager

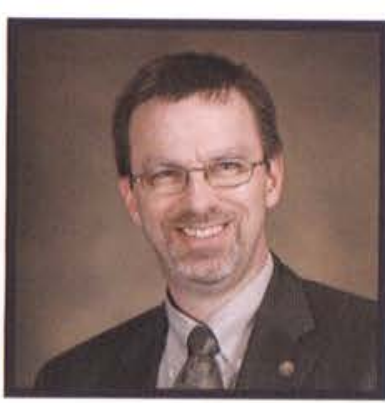

Steve Winey Assistant Director

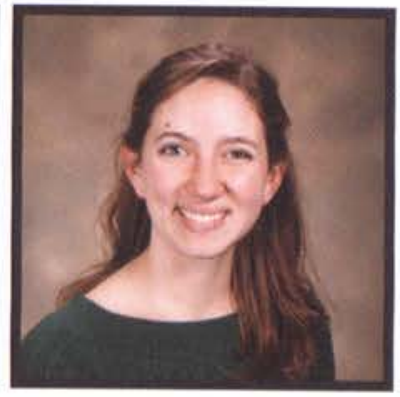

Jaime Hawkins Student Loan Coordinator, Disbursement Manager

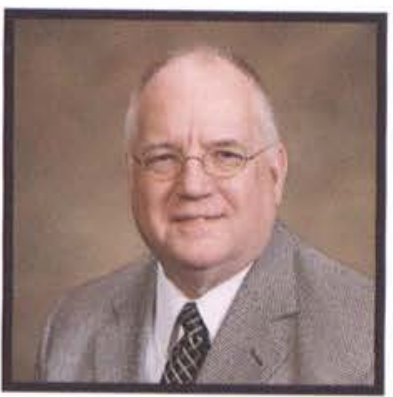

Fred Merritt Director

\section{Management Stabs}

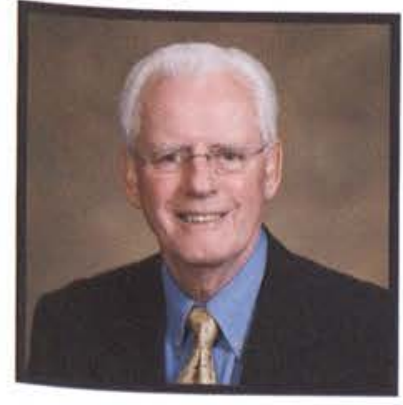

Harold R. Green

University Representative

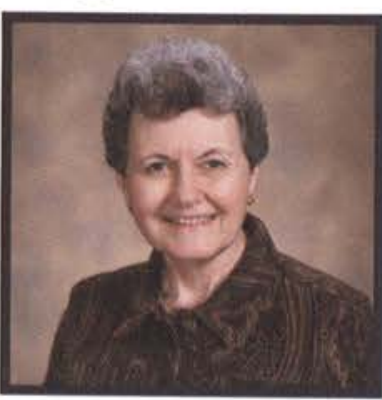

Margaret Green University Representative

\section{Public Relations}

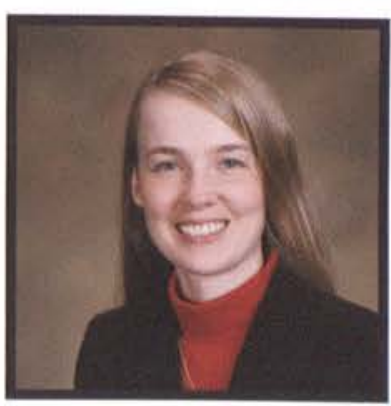

Kara DeMusz Associate Director

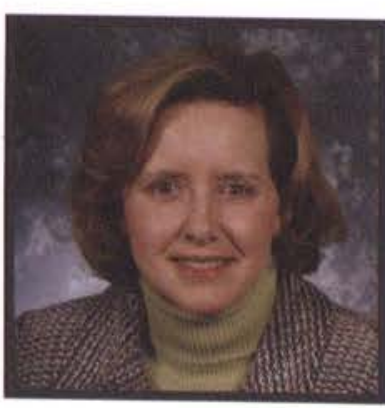

Cessna Winslow Public Relations Writer 


\section{University Bookstare}

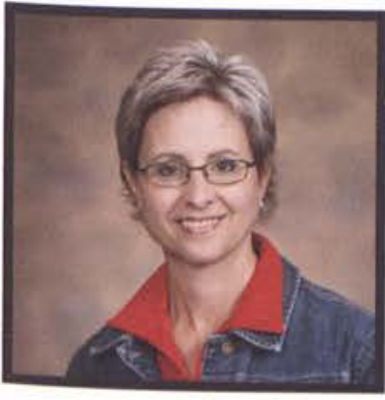

Ann DeLange

General Staff Buyer

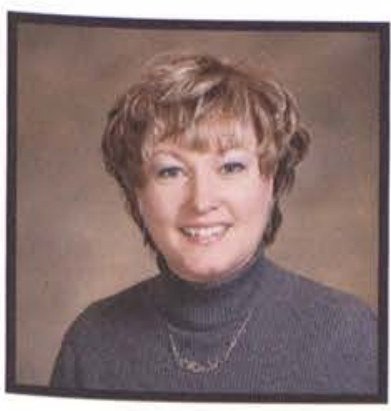

Sarah Young

General Staff Clerk

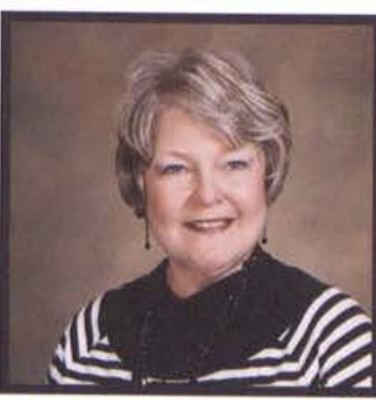

Jeanie LeBlanc Gift and Card Buyer

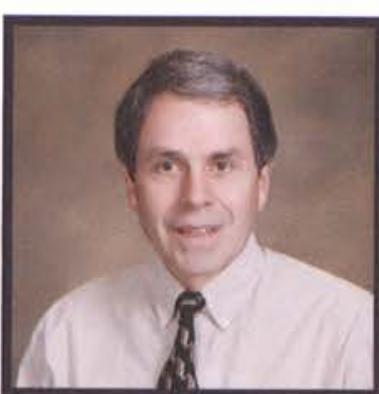

Lee Mattson Supervisor

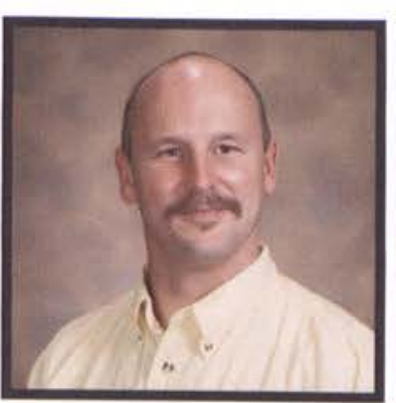

Rill Thompson Textbook \& Back Office Supervisor

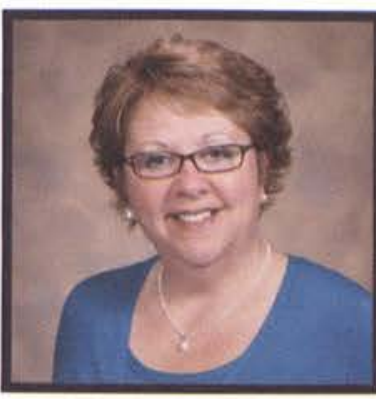

Nellie Tourney Assistant to the Supervisor

\section{Building \& Grounds}

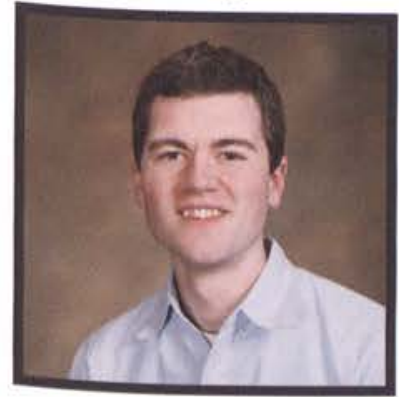

Johnathan Bowers Work Order Process Supervisor

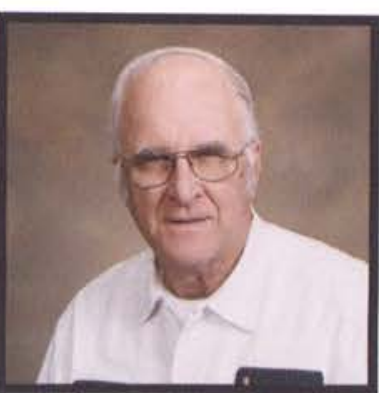

Richard Cughan General Maintenance

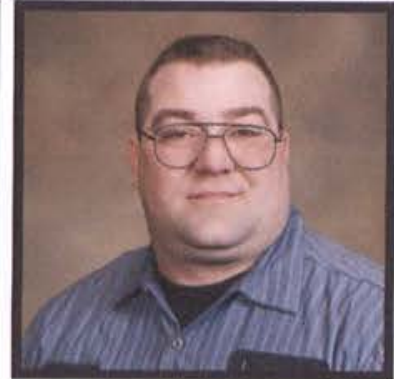

Steve Prether Groundskeeper

\section{Cashier's Obsice}

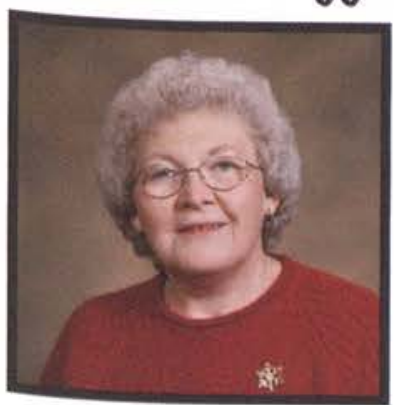

Margaret Burrichter Cashier
Human Resources

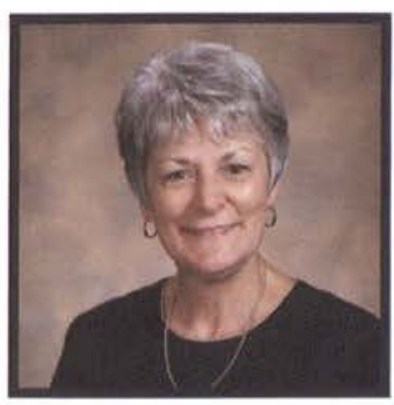

Trish Huber Administrative Assistant
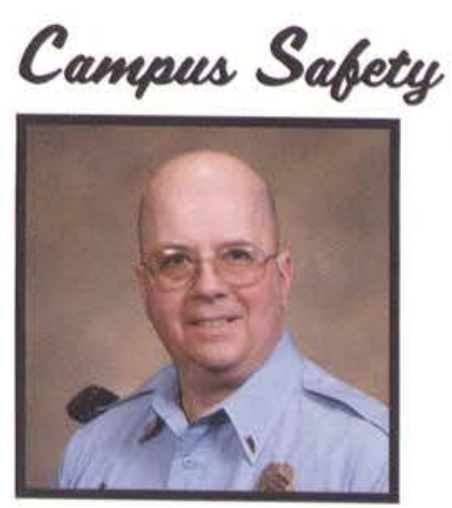

James Bowersox Supervisor 


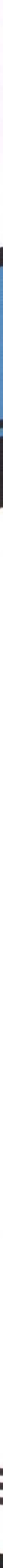




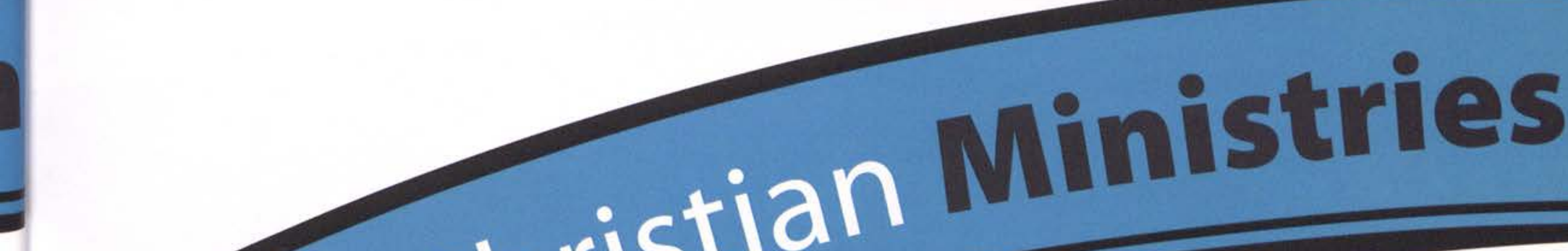
Christian Ministries

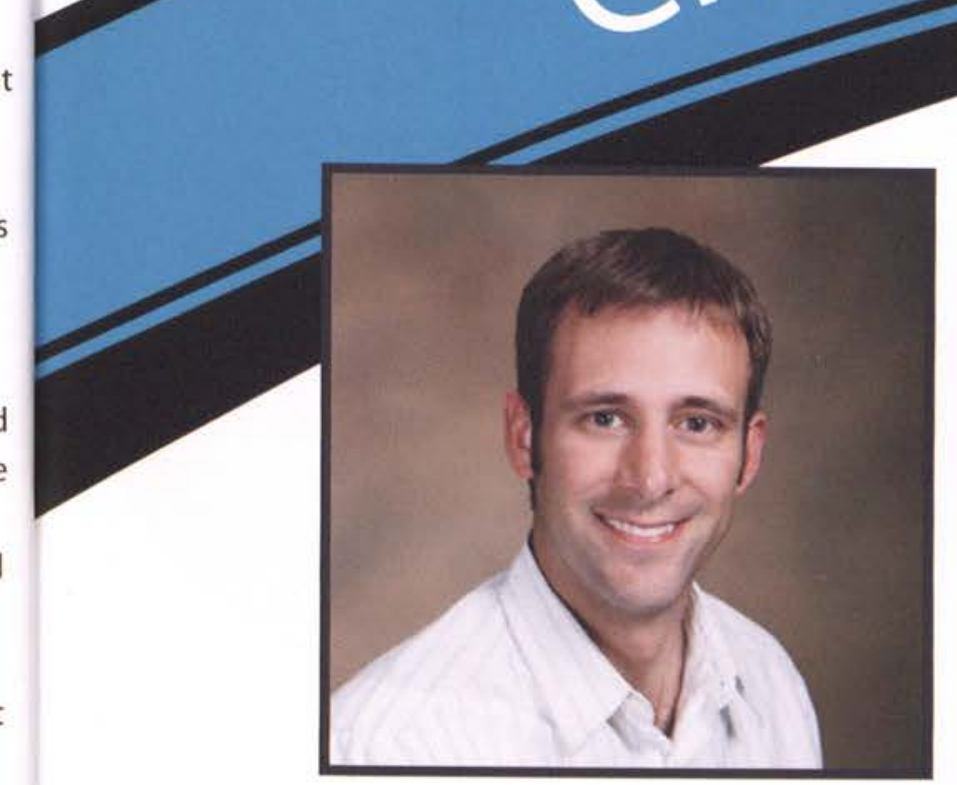

Mark Irving Director
The Christian Ministries Division has an everbroadening and diverse arena in which to minister to both students and those outside Cedarville. Christian Ministries consisted of Touring Teams, Community Ministry groups, Missions, and Discipleship Groups. The Touring Teams division this year consisted of 3 Drama Teams - the Lifeline Players and two Masters' Puppets Teams while HeartSong has 3 touring teams. In August, the HeartSong Touring Teams Department doubled in size, hiring Jessica Waggoner as the new Administrative Assistant and Justin Spann as the Assistant to the Director of HeartSong. The Community Ministries division had connections with more than 60 ministries and agencies including prison ministries, outreach ministries for urban communities, and abstinence programs. Also, the Missions subdivision of Christian Ministries sent hundreds of students out into the world to do missions work through the various MIS teams over spring break, Christmas break, and over the summer. This summer students headed to 21 different countries throughout the world to minister using sports, music, and teaching. While the first three divisions focused on those outside Cedarville, the Discipleship Groups minister to Cedarville students and included Bible study groups, prayer groups such as Elijah's Fire, and the pre-marital counseling program Fit to Be Tied. Clearly, Christian Ministries is an indispensable part of Cedarville University.

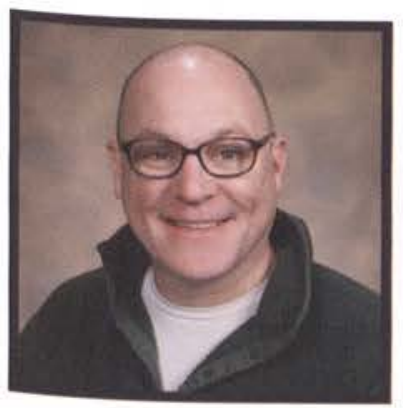

Jim Cato Heartsong Director

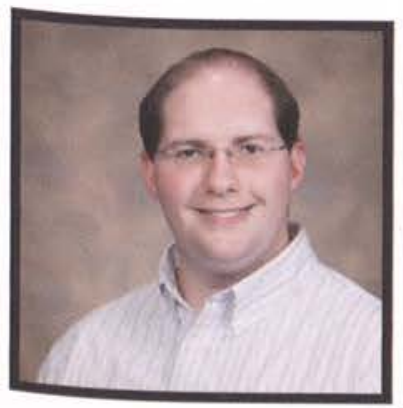

Joel Tomkinson Assistant Director of Heartsong

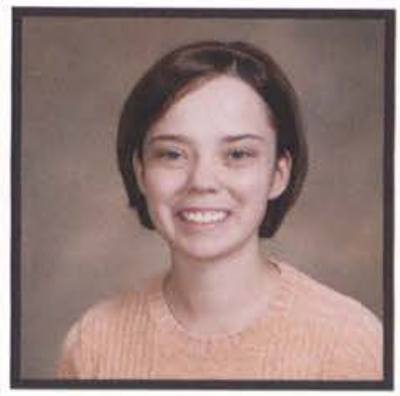

Brooke Colon Secretary

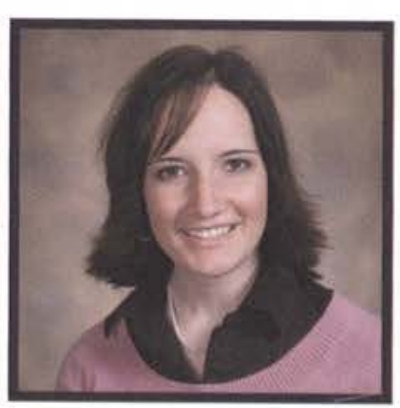

Jessica Waggoner Administrative Assistant

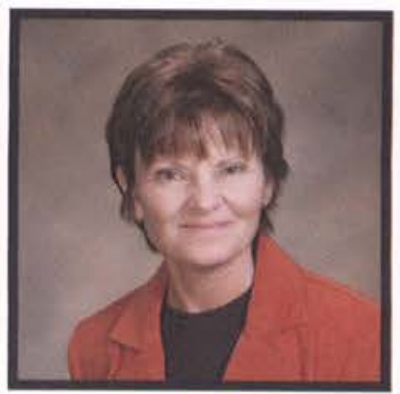

Vicky L. Hines Admin. Assistant

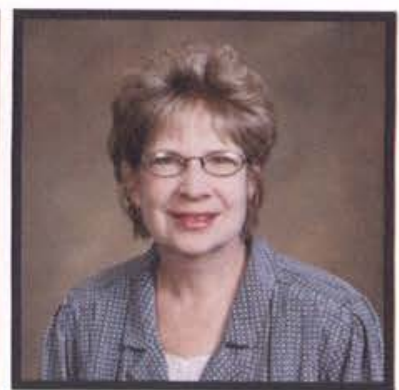

Jackie Pyles Receptionist

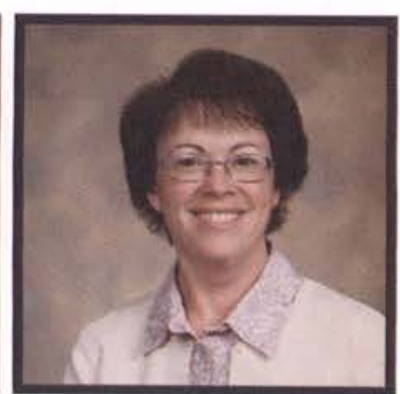

Cheryl Shupe Executive Secretary 


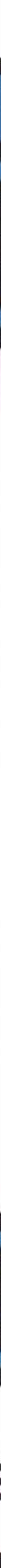




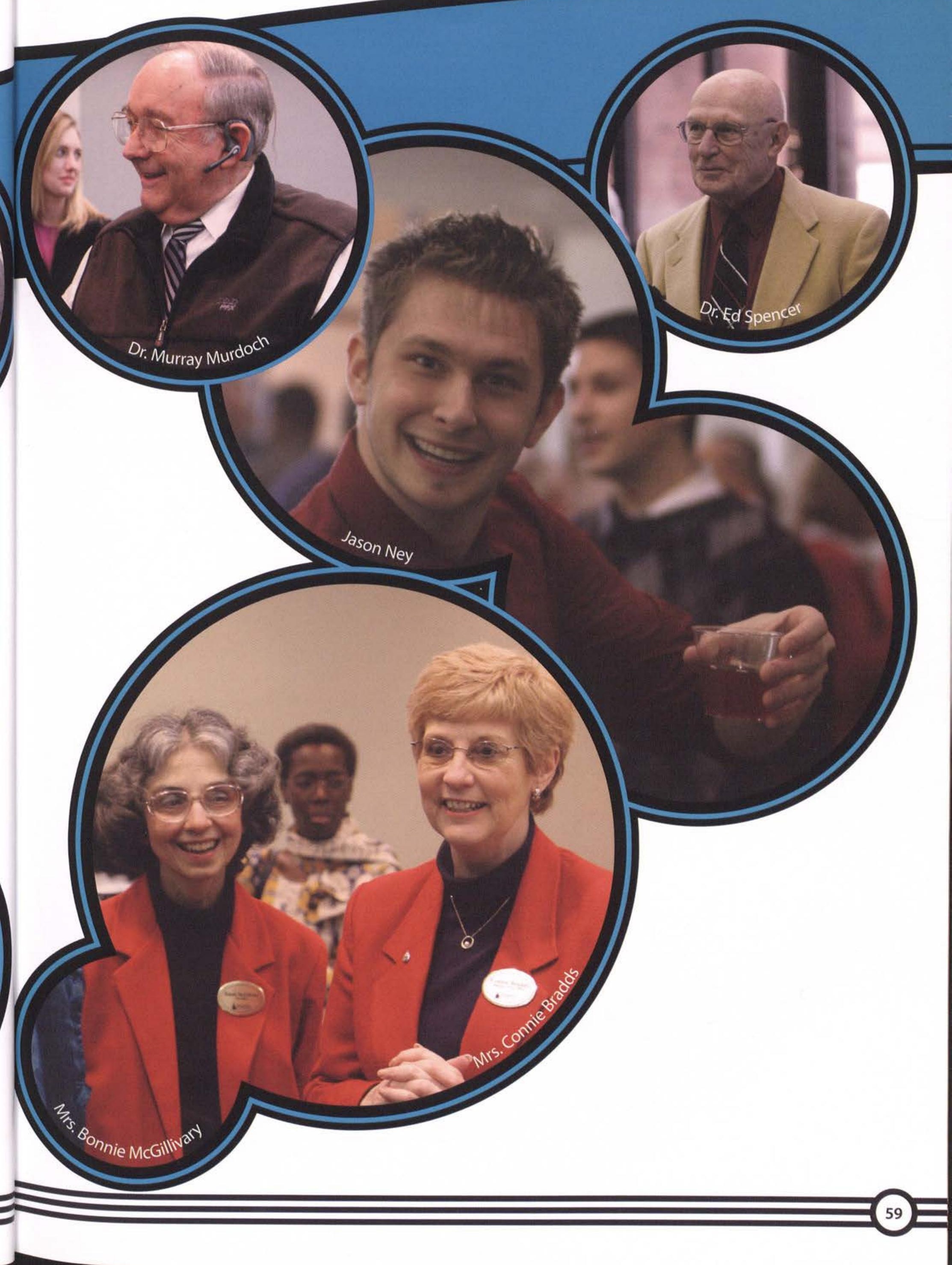




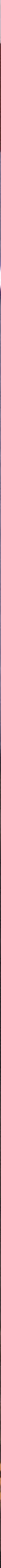




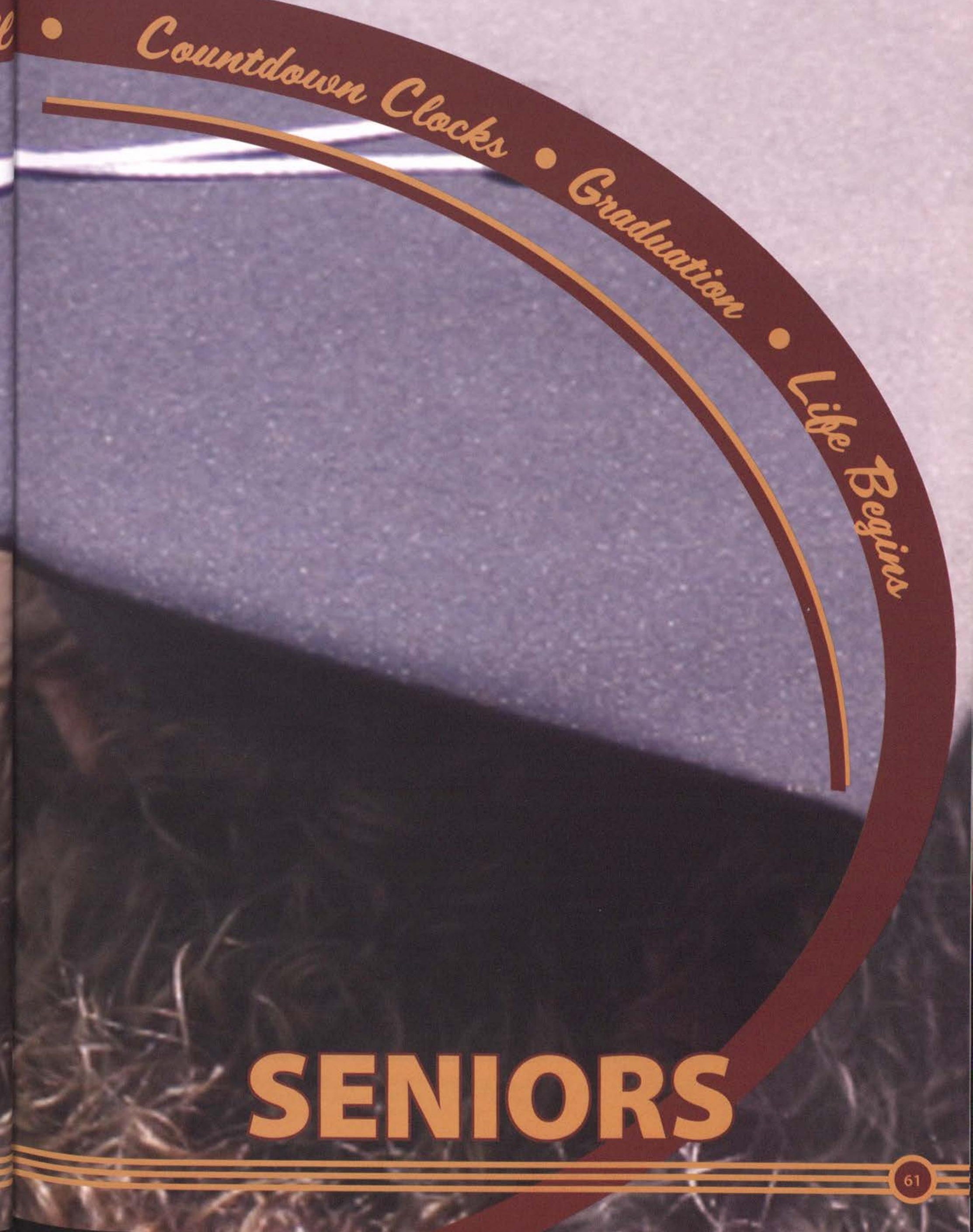



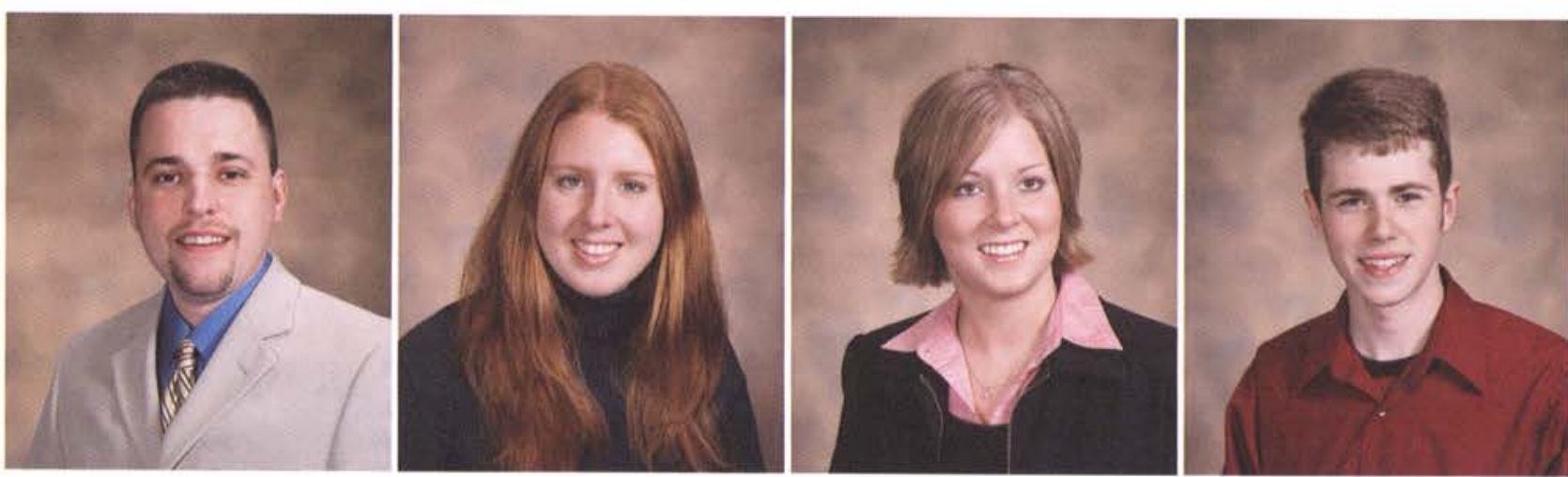

Timothy Kevin Abe Pastoral Studies

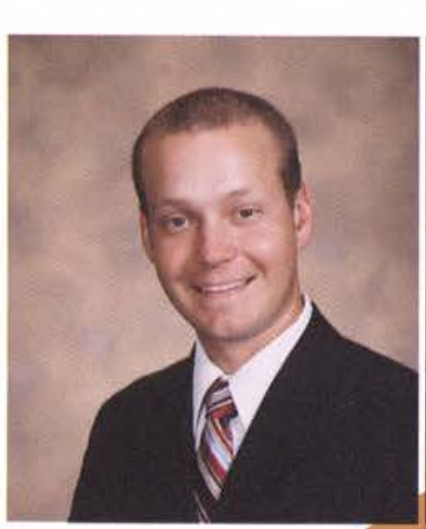

Scott Michael Aker History

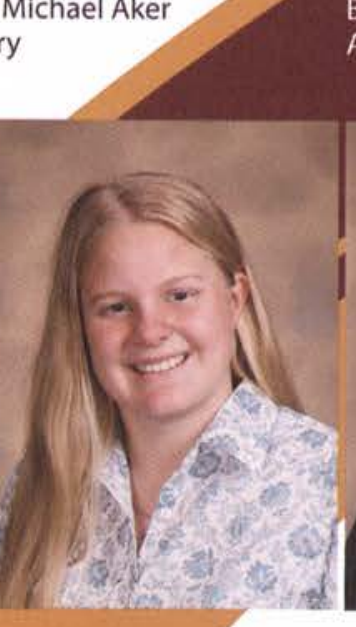

Sarah Brittany Anderson AYA Integrated Science .

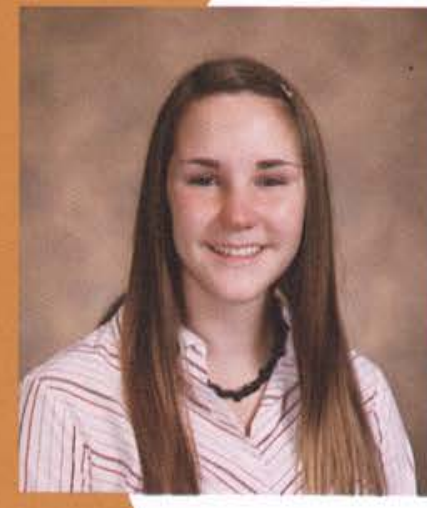

Jessica Lynn Annable Middle Childhood Education
Amanda R. Acker

AYA Social Studies Education \& History

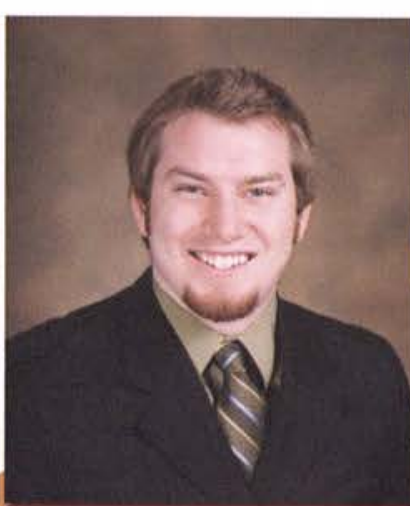

Brian Joshua Alburger Athletic Training

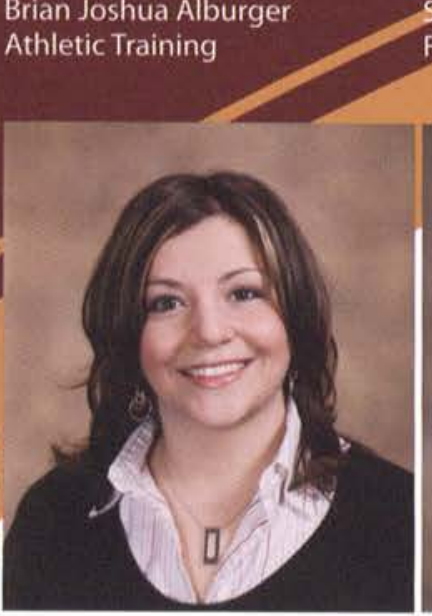

Jamie Lynn Anderson Nursing

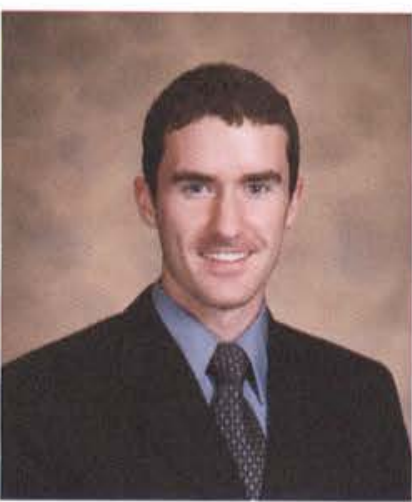

Daniel Arnold Chemistry
Christin Adams

Multi-Age Special Education
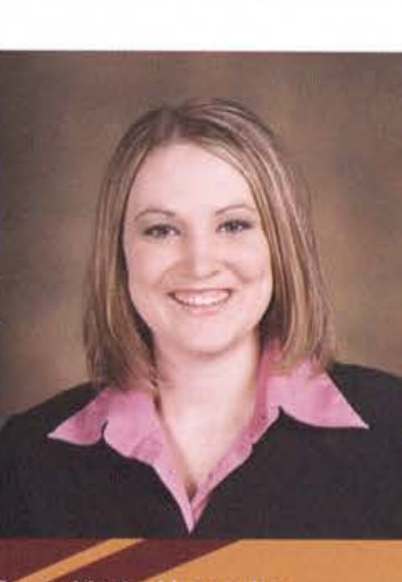

Stacy Marie Alexander Finance \& Marketing
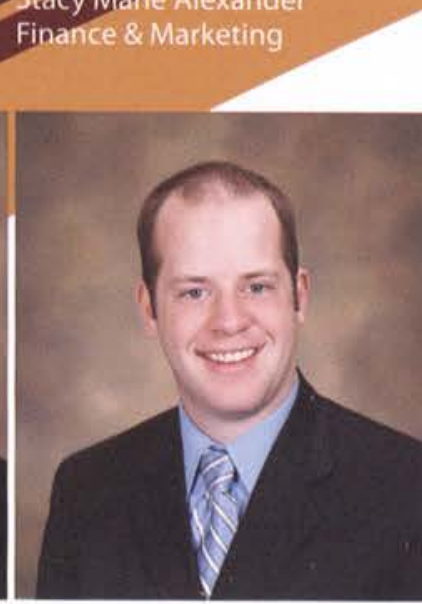

Tyler Anderson

Management \& Youth Ministries

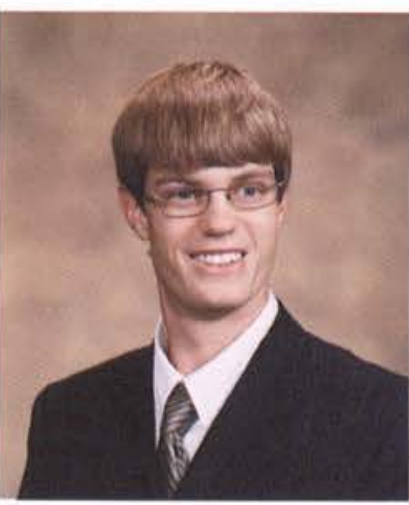

Matthew J. Averbeck History
Theodore Adams '08

Multi-Age Music Education Nursing

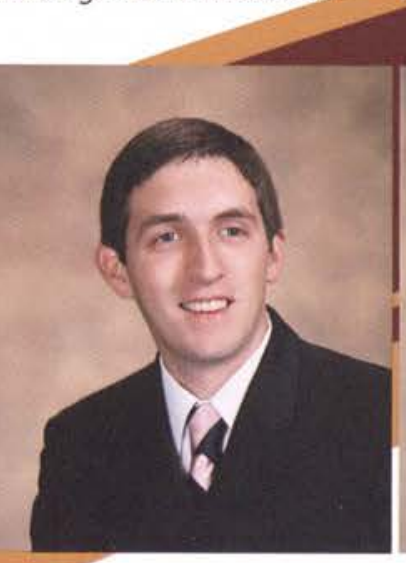

Andrew Stephen Althouse Graphic Design
Bryan C. Amerine Marketing
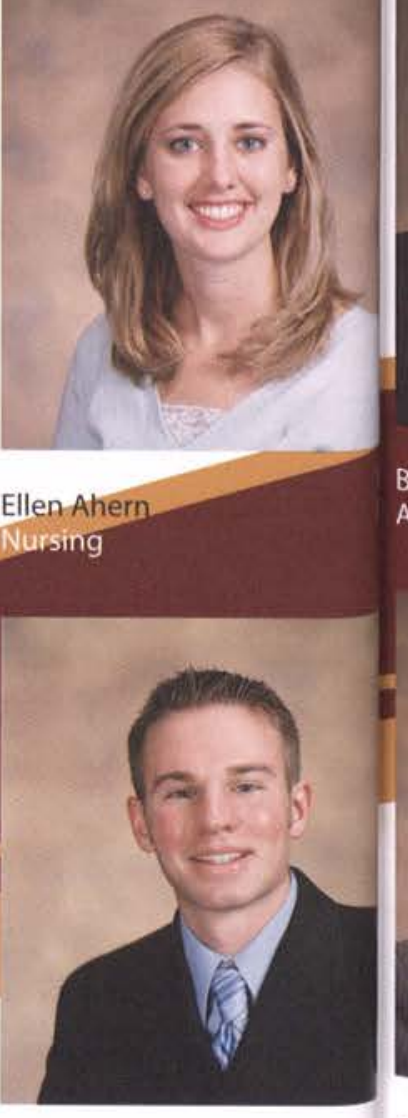

D
$Y_{0}$
$E_{0}$

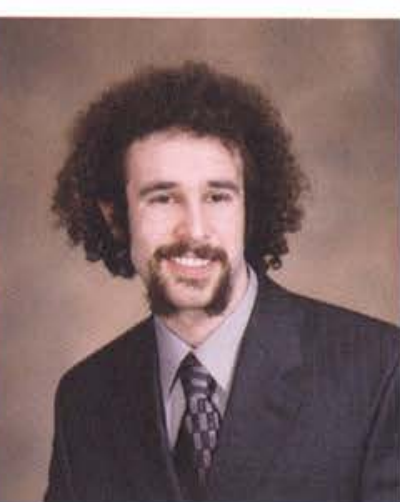

Michael P. Anfang

Electrical Engineering

Tammy L. Angelini

Psychology
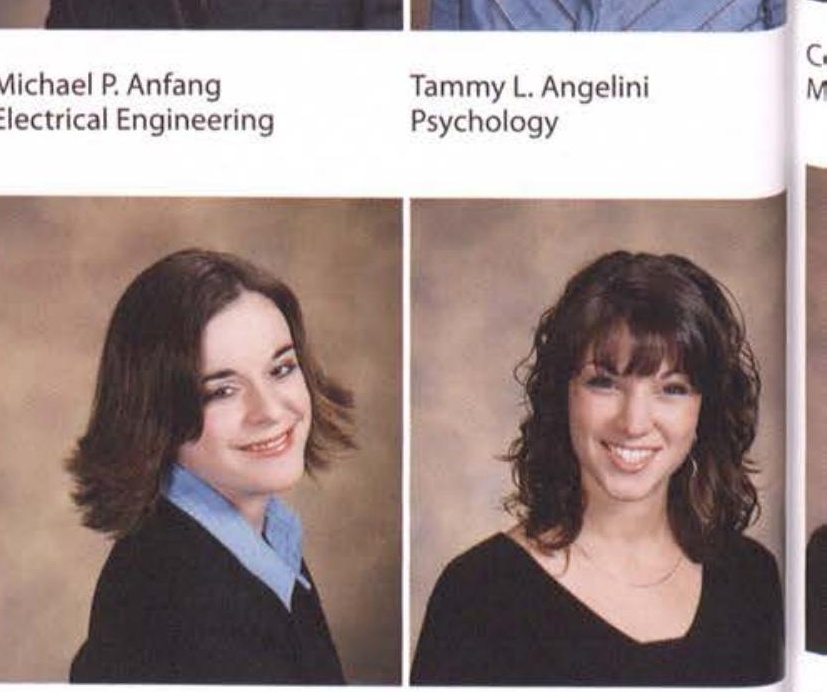

Angela Christine Bacon English

Sarah Elizabeth Baczek Graphic Design 


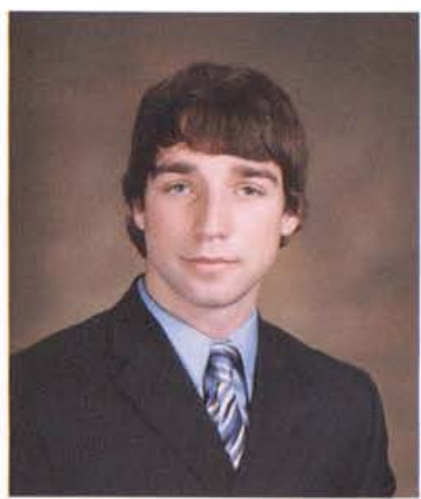

Jacob Paul Bezeck Management

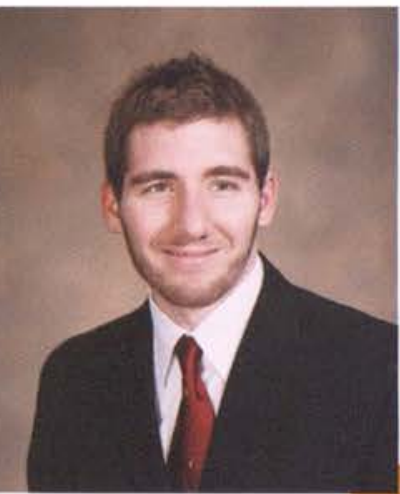

Brant Timothy Bloem Criminal Justice
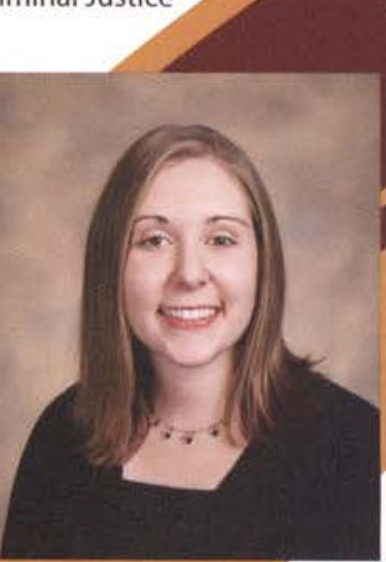

Melissa Jeanne Borton Psychology

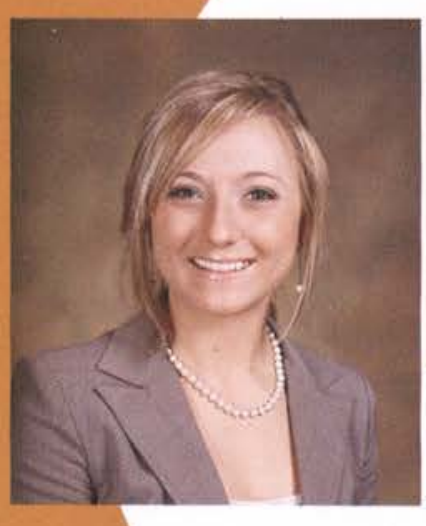

Chelsea Bowman

Early Childhood Education

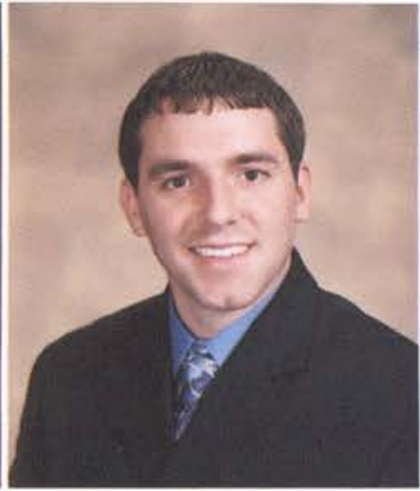

Jordan Joseph Biga Psychology

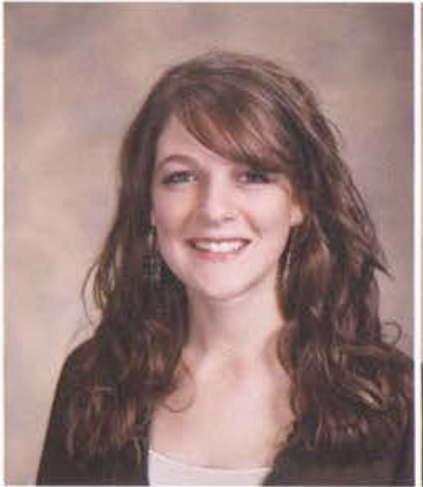

Debra Joy Birch Social Work

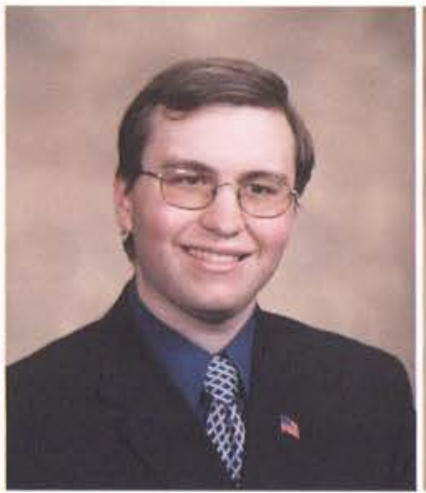

Joshua Paul Blackburn Electrical Engineering

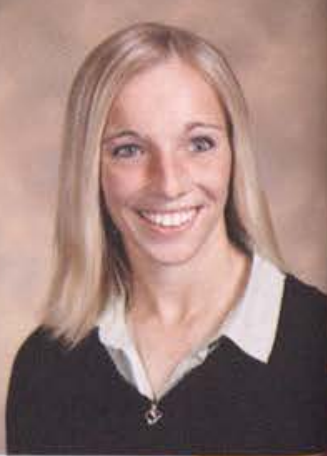

lle Brackbur Lisa Michelle Biackburn Multi-Age Physical Education

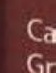
encing
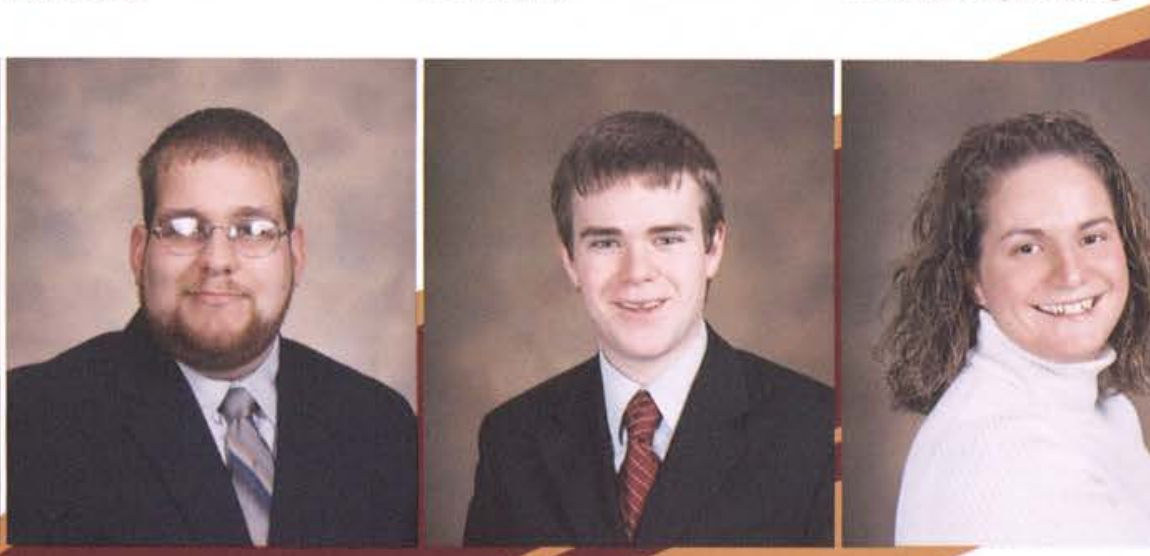

Bachar A. Boland Psychology
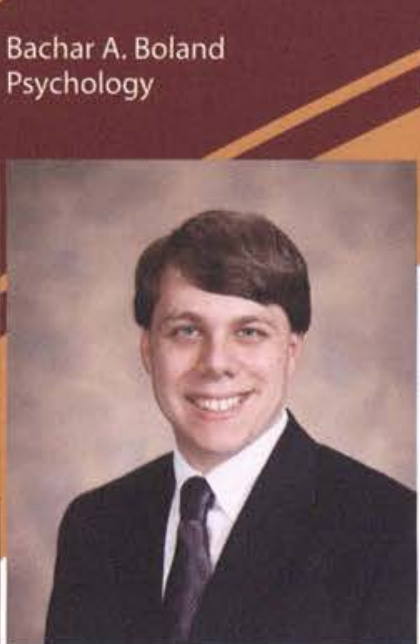

Jonathan David Boston Mechanical Engineering

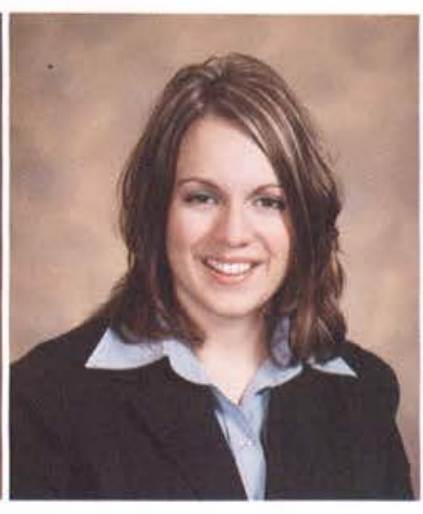

Emilee Nicole Bowman Music Education
Julia Katrin Bradley

Marketing
Susan Marie Bosworth

Nursing

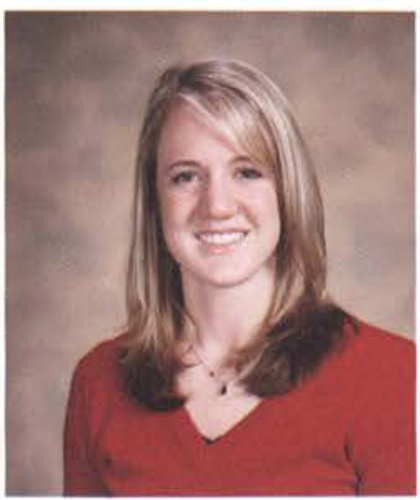

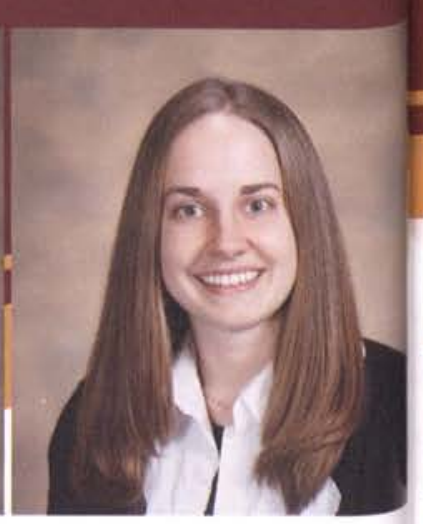

Tarah Elizabeth Boone Youth Ministry \& Christian Education
Rebekah Ruth Bole Nursing
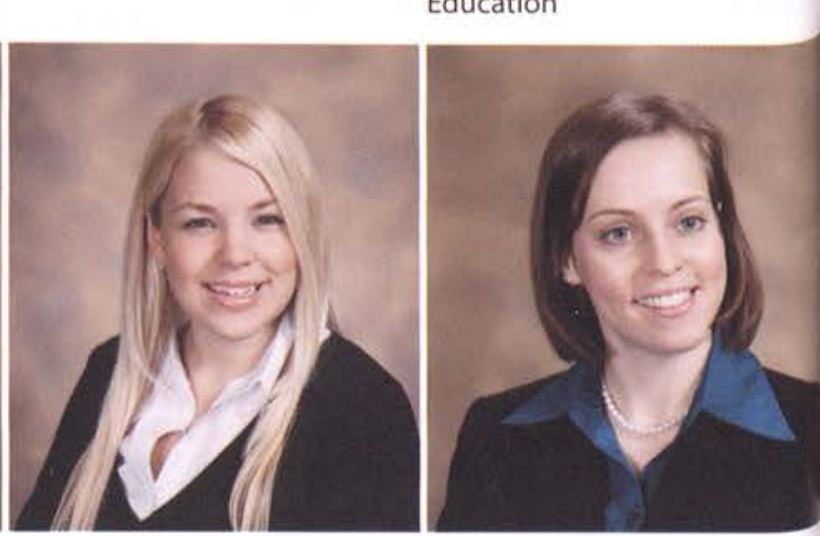

Jenniver Michelle Boulet Middle Childhood Education
Brittney Elizabeth Bowman Early Childhood Education

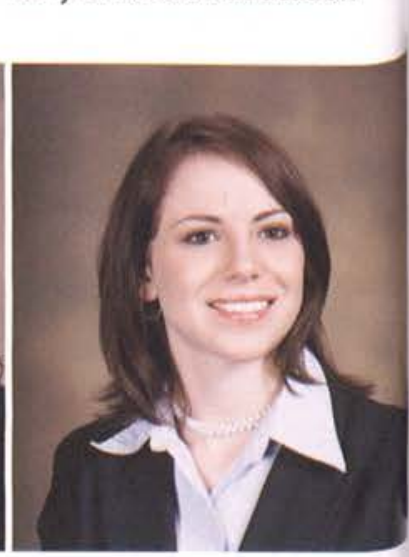

Sarah Mary Grace Brahler Criminal Justice
Melanie Lyn Brady Sociology

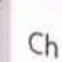

$\mathrm{Ch}$

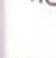

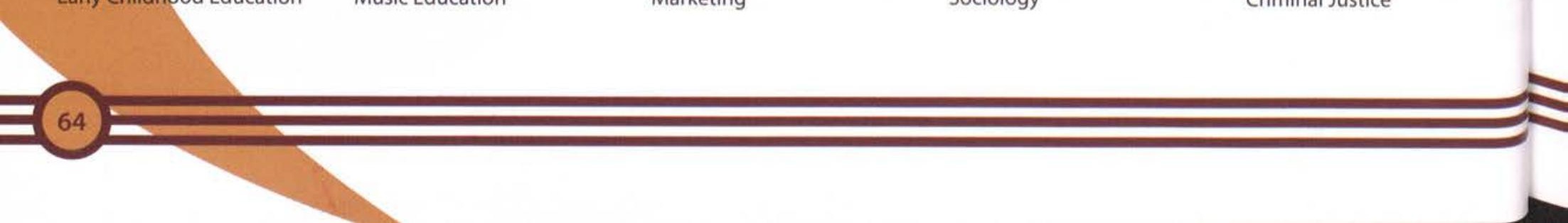



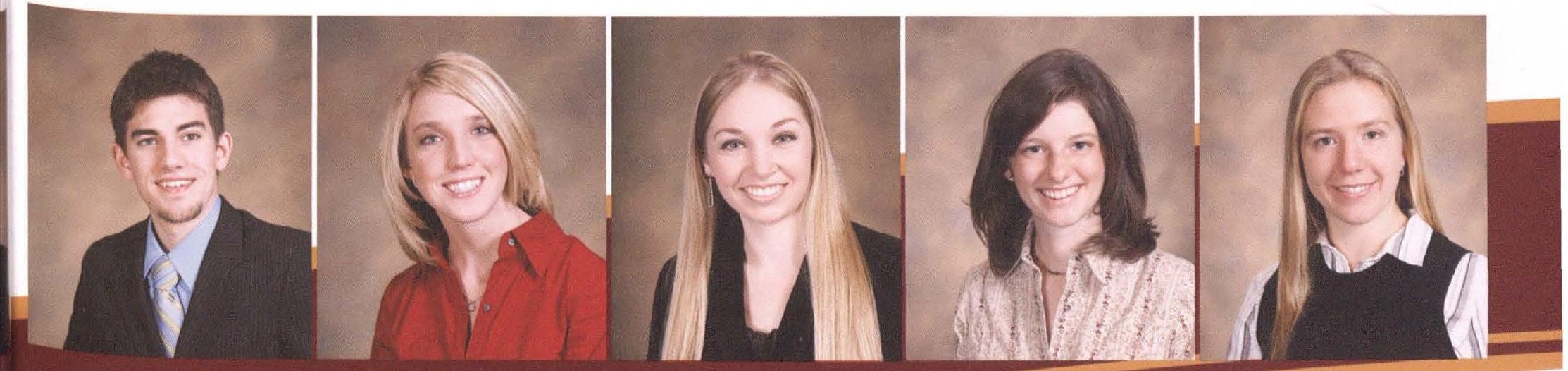

Cameron Paul Braun

Graphic Design
Mindy C. Braun Management
Gena M. Bravick Spanish-\&integrater
Carla Jean Brenneman Susan Summer Brewster Psychology \&World Misisons Nursing

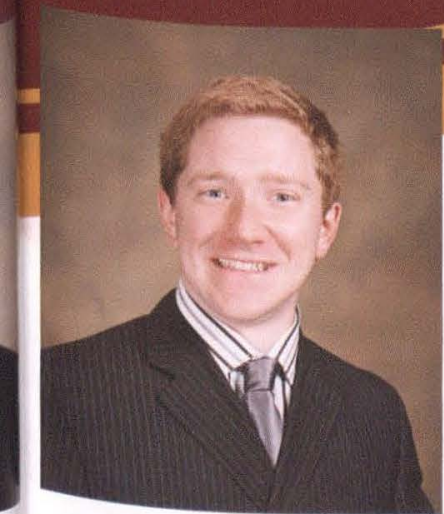

Charles Aaron Briggs

Mechanical Engineering

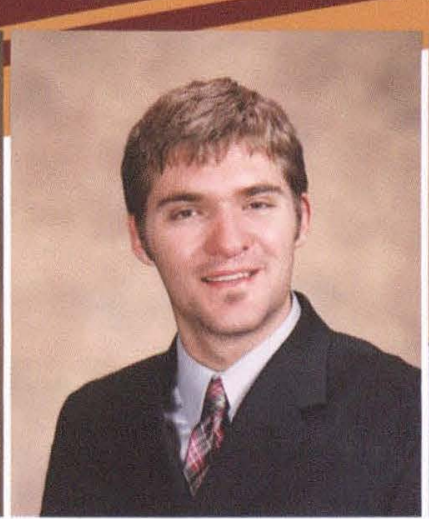

Jason Paul Briggs

Mechanical Engineering

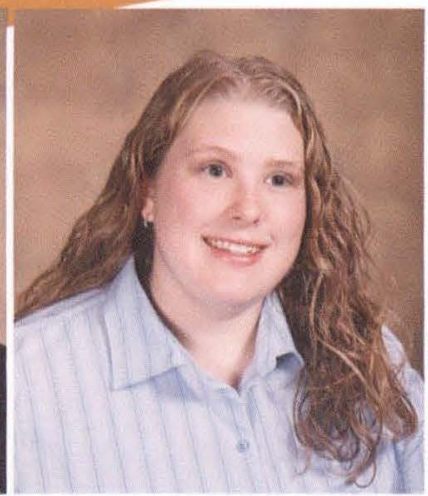

Sarah Elizabeth Britton Middle Childhood Education

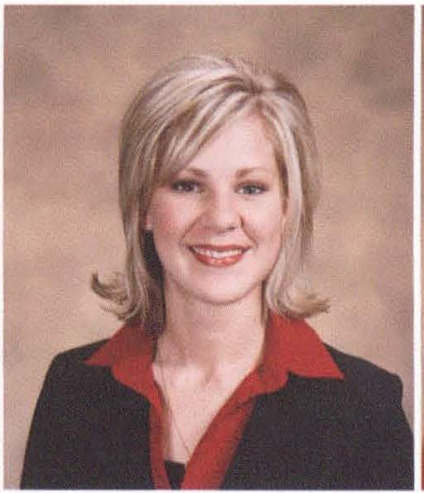

Hilary Elizabeth Bromley Chemistry

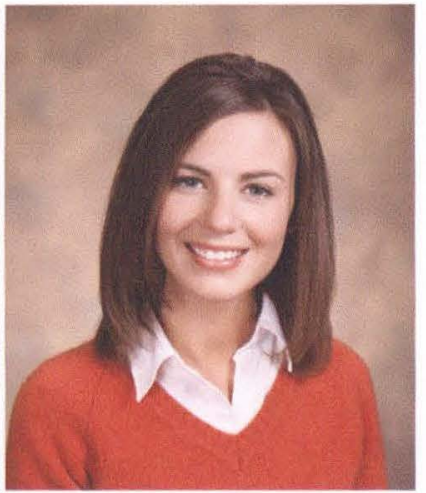

Melissa D. Brorein

Multi-Age Music Education
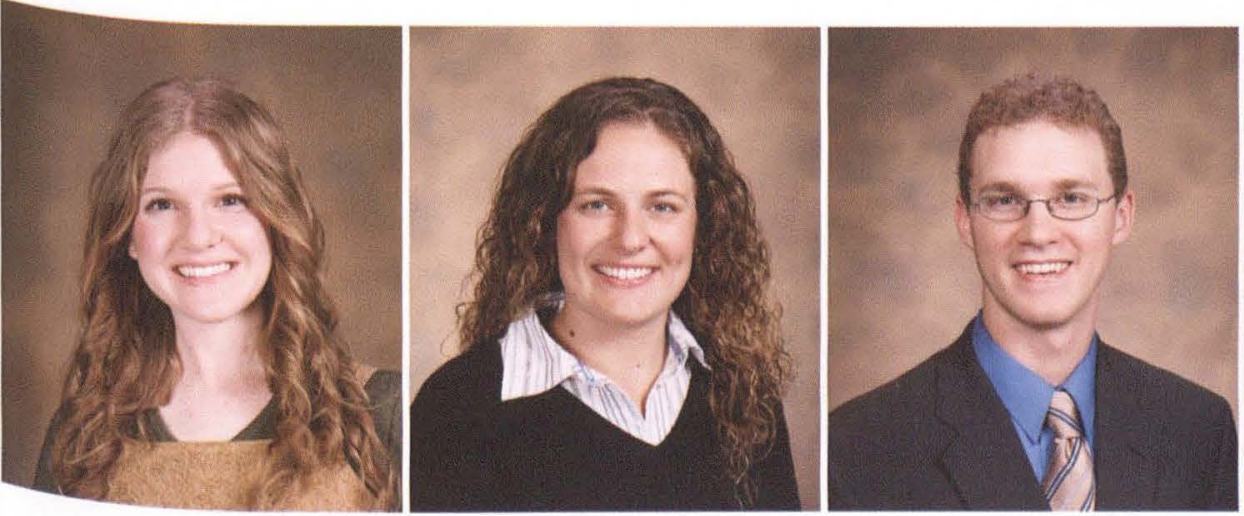

Courtney J. Brott

Comrehensive Bible, Youth

Andrew Douglas Brown

Integrated Social Studies \&

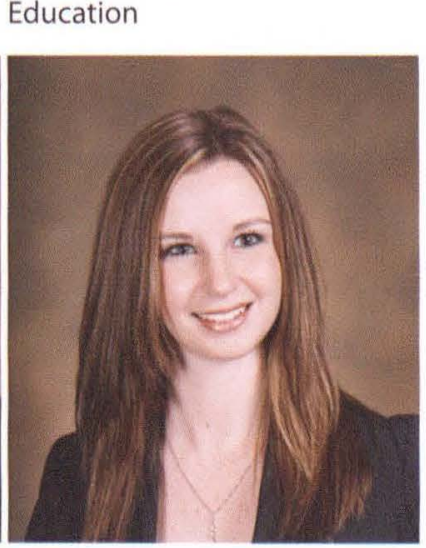

Kimber

ey

ie Brown Nursing

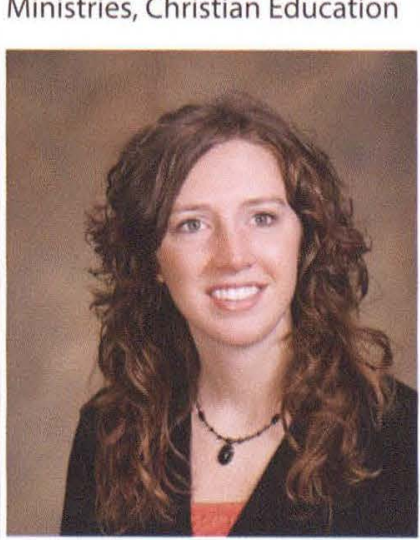

Suzanne Leigh Browning
Carol Ann Brownle Early Childhood Education
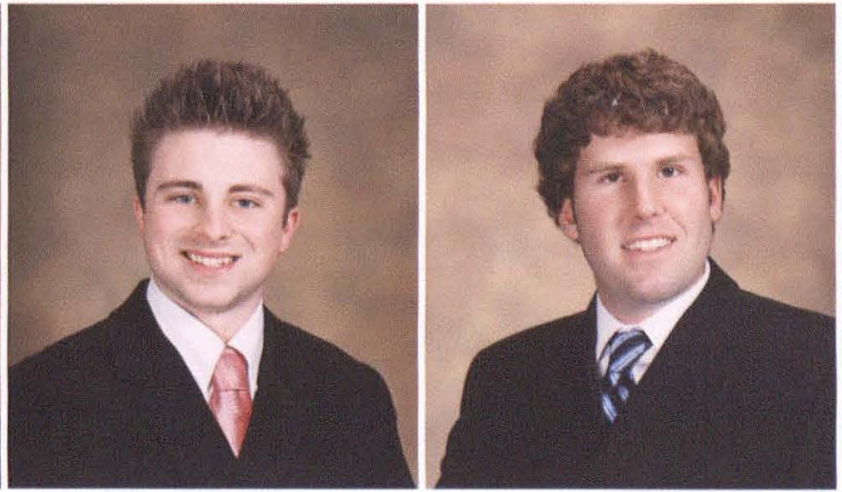

David Charles Brown Pre-Law

Kevin D. Brown

Psychology \& Criminal Justice

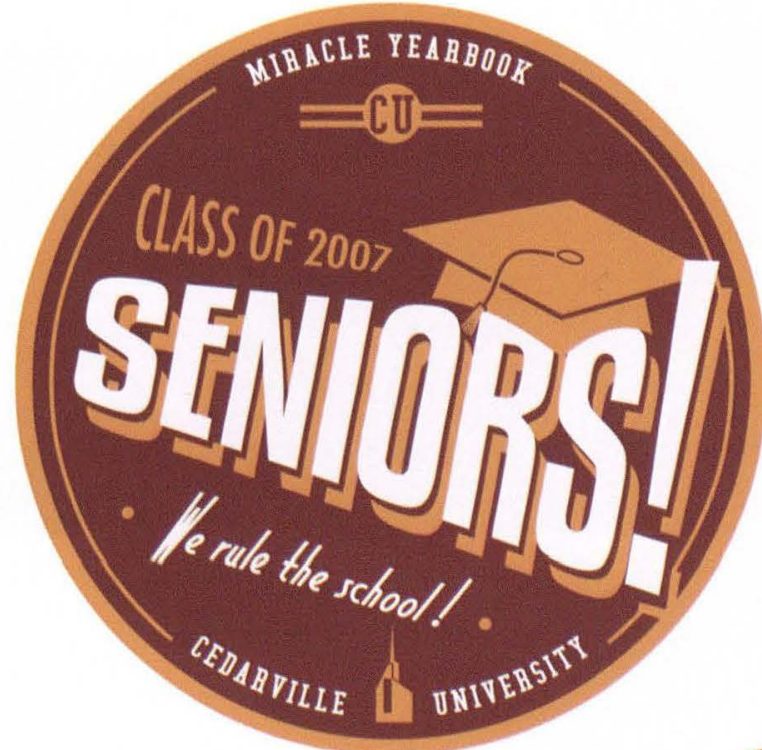




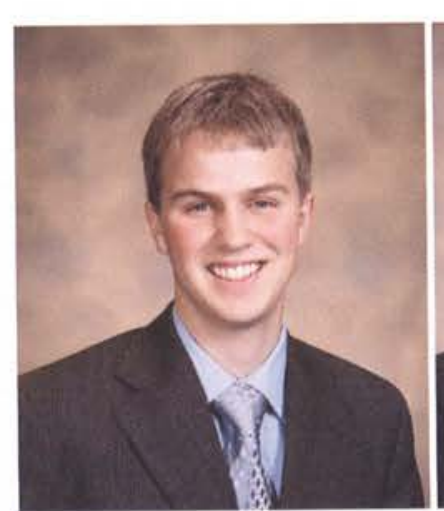

Andrew Graham Bruder Sports Management

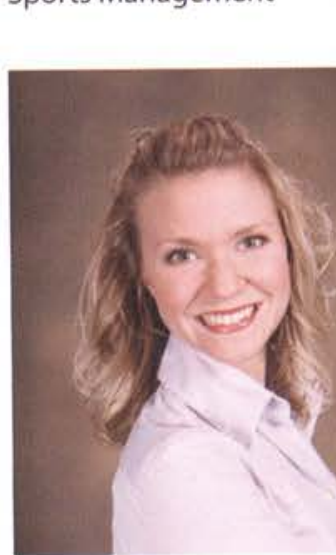

Andrea Nicole Bungart Nursing

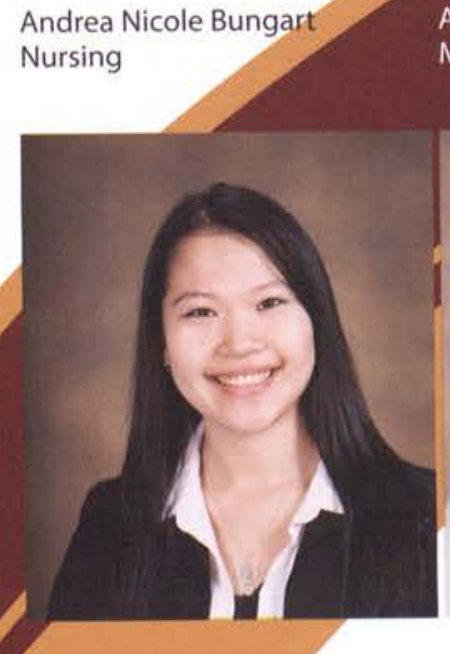

Naomi Rose Burks Nursing
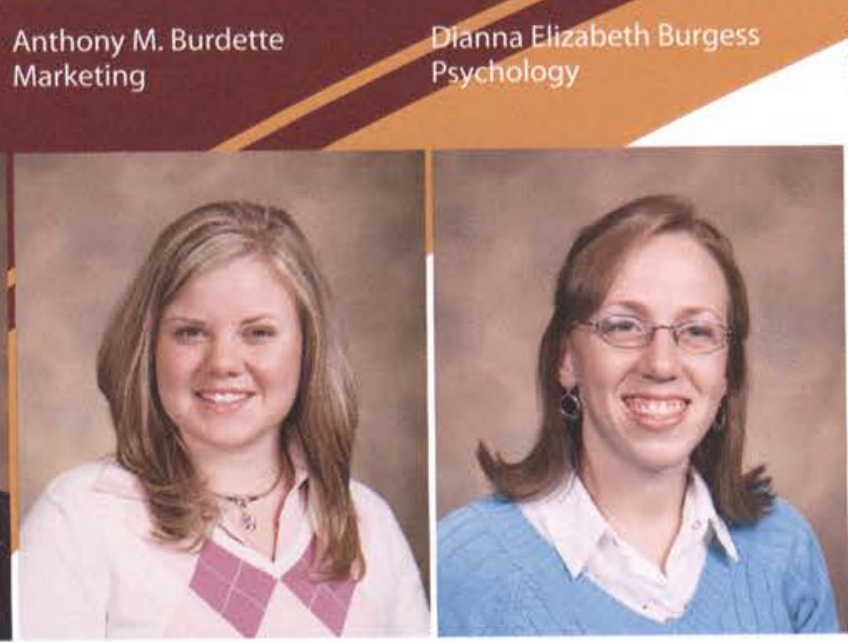

Ashley Burnett

Accounting \& Finance

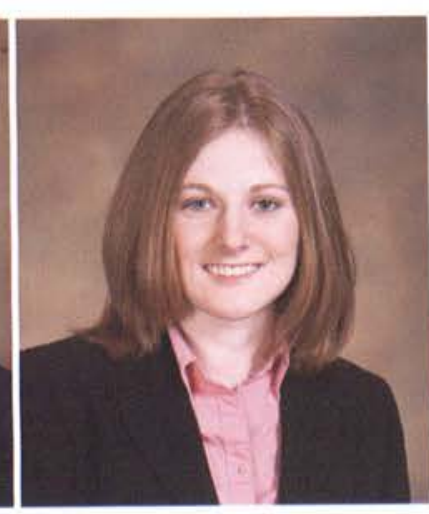

Emily Nicole Cain Marketing

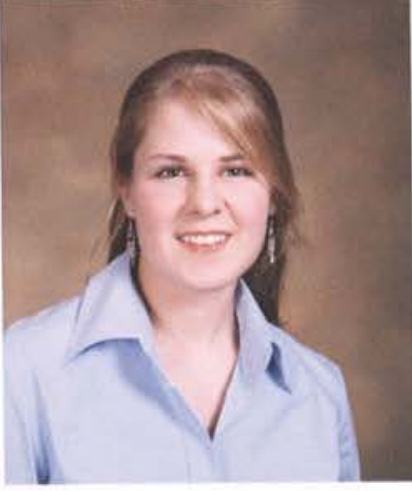

Sarah Rosanne Buchheit Psychology

Preseminary Bible \&

Psychology

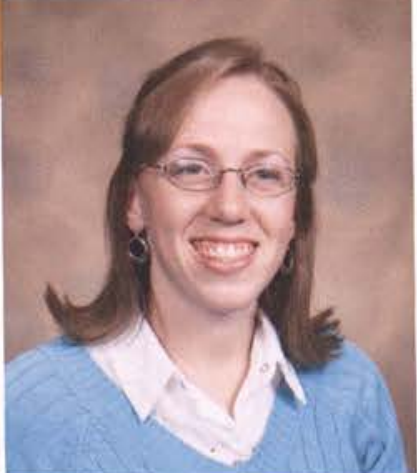

Lindsey G. Burns

Early Childhood Education

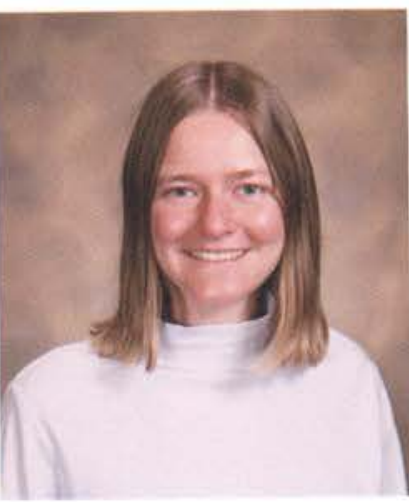

Julie R. Campbell Accounting
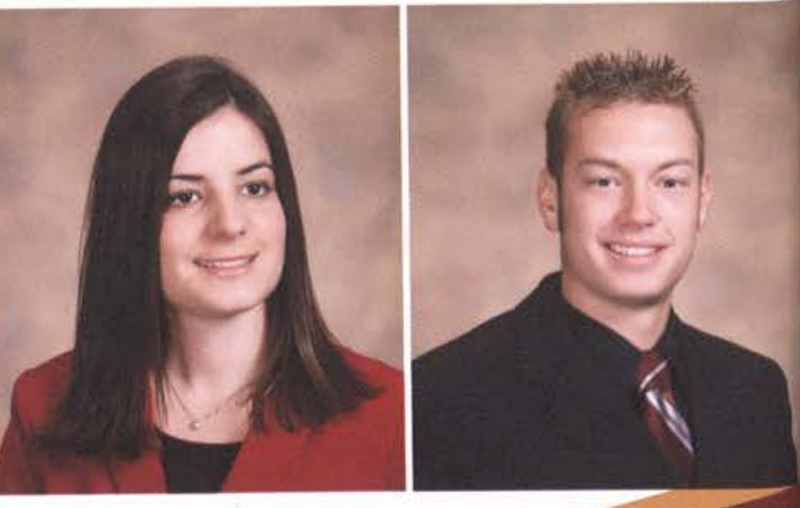

Ashley Michele Buehler Middle Childhood Education Nursing

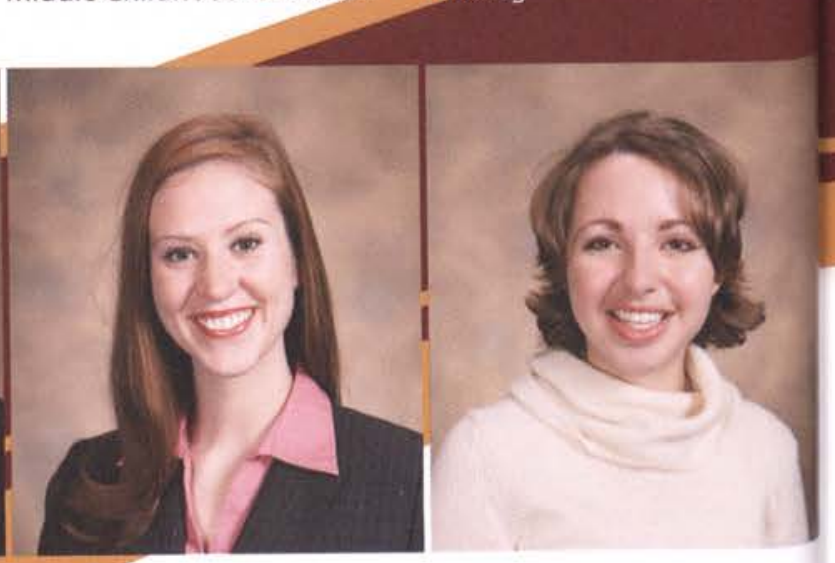

arah Rayann Burke Early Childhood Education

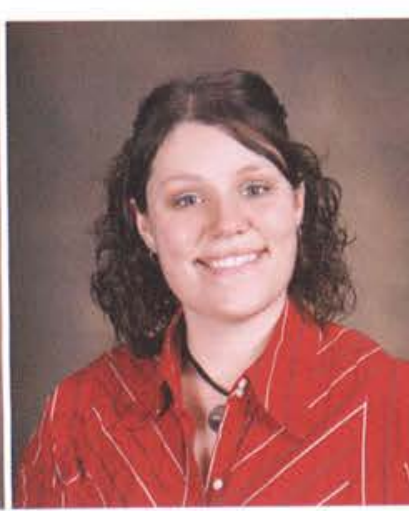

Elizabeth Grace Burkett Spanish Education

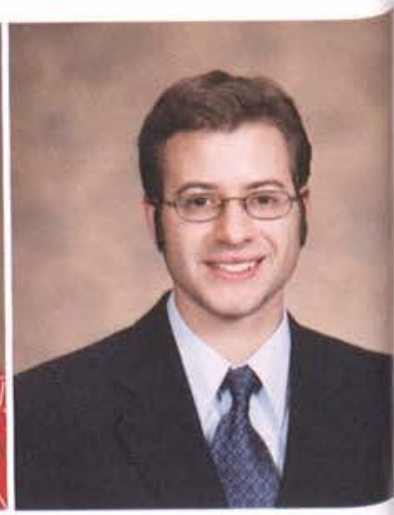

Nursing

Gregory K. Butz Integrated Social Studies \& Education

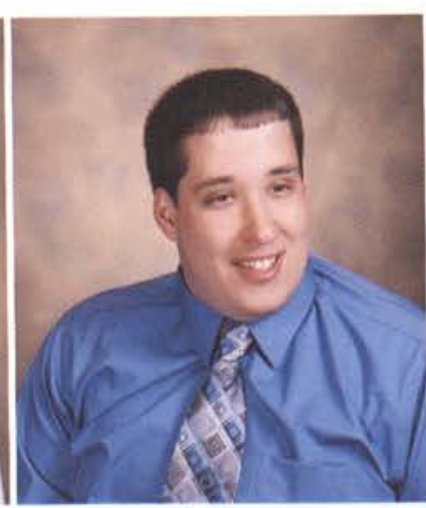

Nathan Prentice Campbell Communication Arts

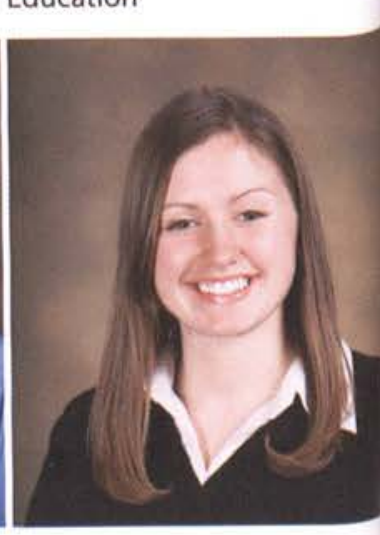

Nikki L. Carroll Youth Ministry, Christian Education, Pre-Seminary Bibl 


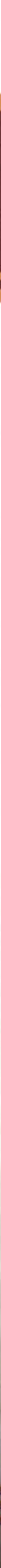




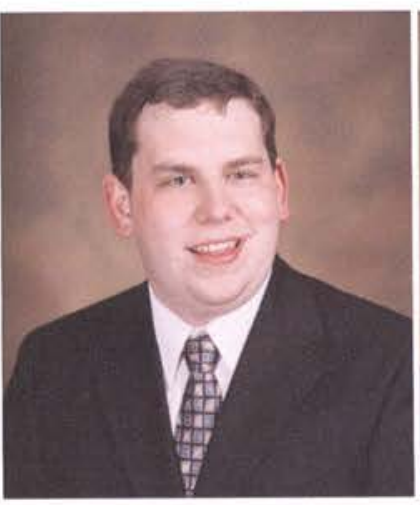
Mechanical Engineering
Douglas S. Conway

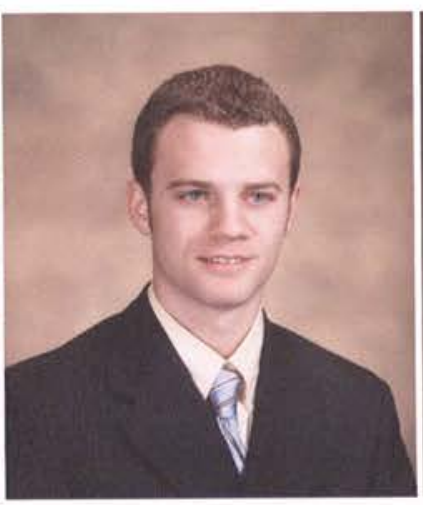

Jeremy Ross Conyers Mechanical Engineering

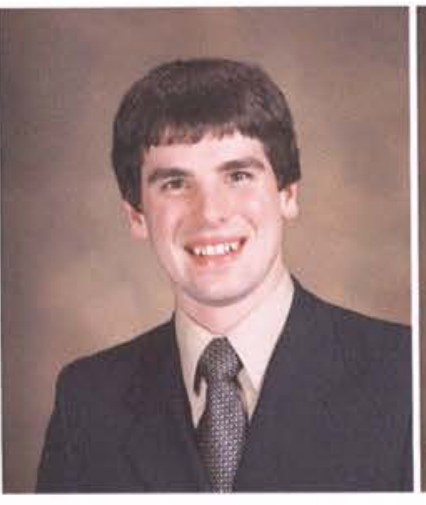

Aaron M. Cook Pastoral Studies

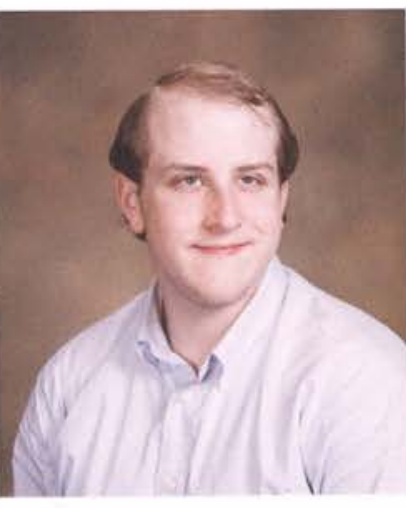

Robert N. Cook Electronic Media

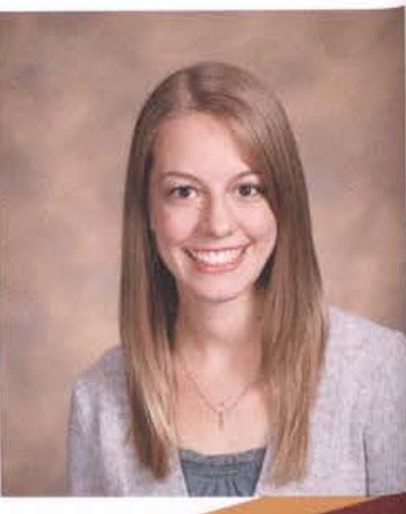

Shannon Kav-Cookson Early Childhood Education \& Special Education

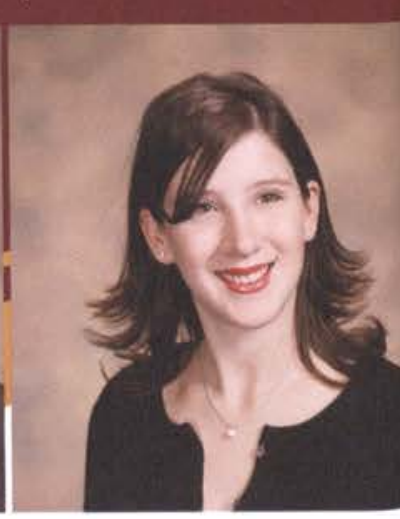

Christi Marie Coulter Middle Childhood Education

Kimberly Gail Cowell Early Childhood Education

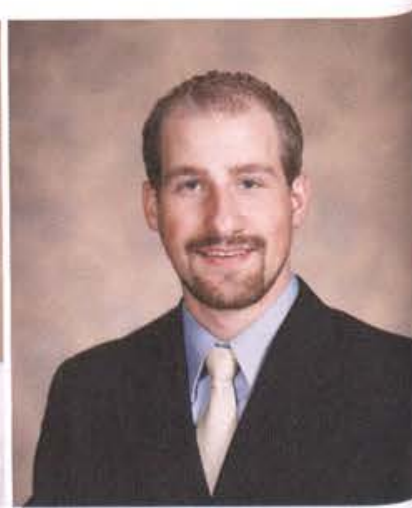

Lori Beth Crain Communication Arts .

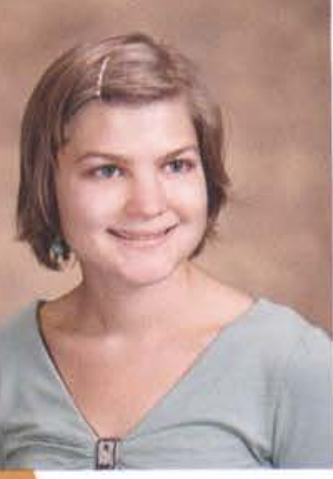
Nursing
Ruth Esther Daniel
Shelby Ruth Corley Communication Arts

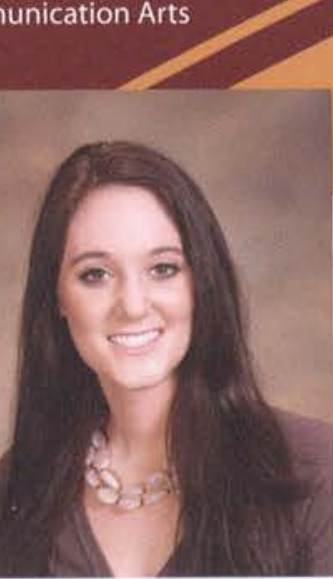

Christina Lynn Cronshaw Nursing

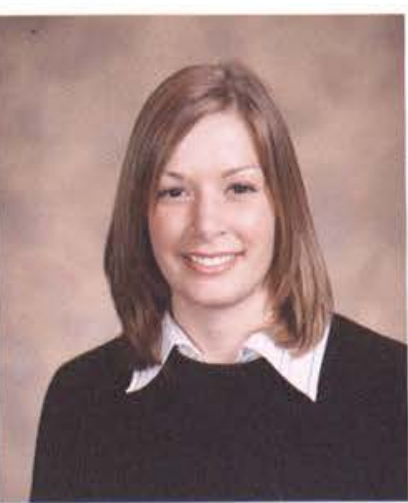

Cathy Ann Daniels Middle Childhood Education

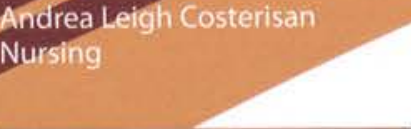

Nursine

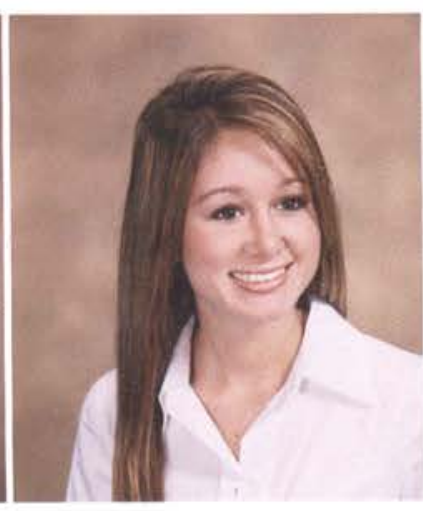

Erin Leigh Cunningham Nursing
Johnathan Mark Daiello Graphic Design
Erica Lynn Cunningham Criminal Justice

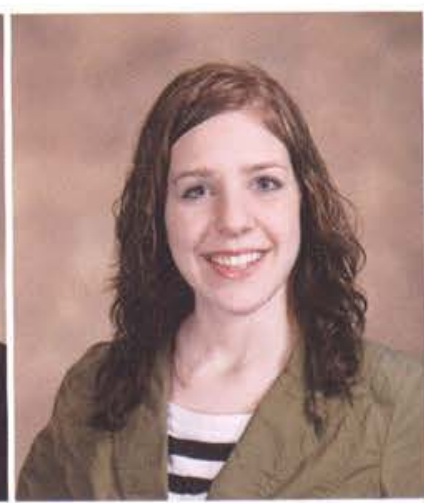

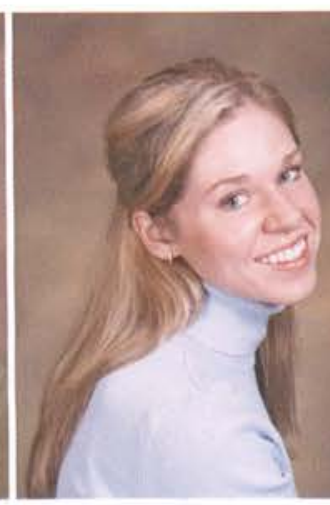

Kelli Marie Daugherty International Studies

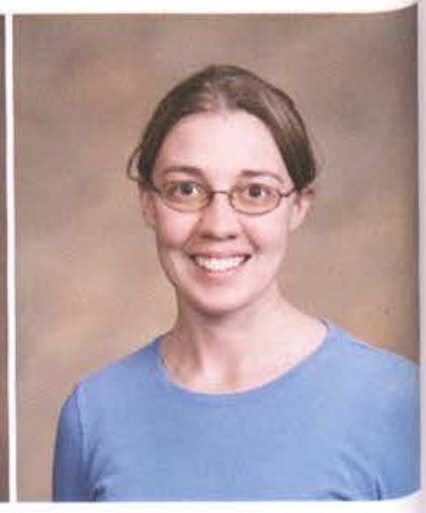

Bethany Davies Nursing
Andrea M. Dankert Nursing

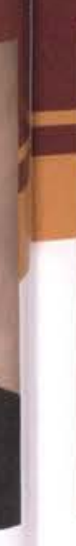



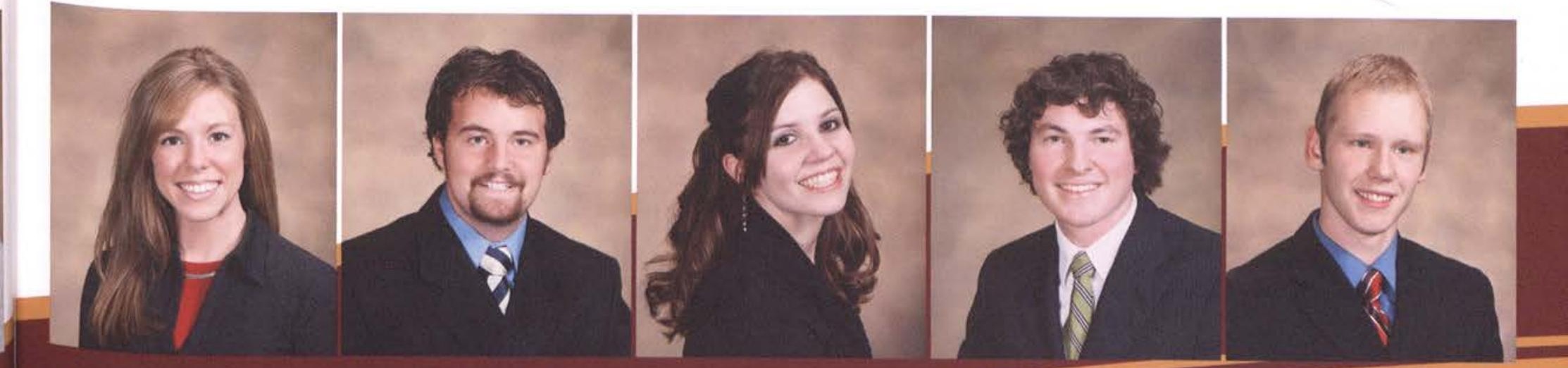

Ellen E. Davis

Middle Childhood Education
Nathanael Philip Davis Environmental Biology
Melanie Marie Day _ Matthew-Deakin

Timothy Allan DeKoninck Political Science \& International Biology

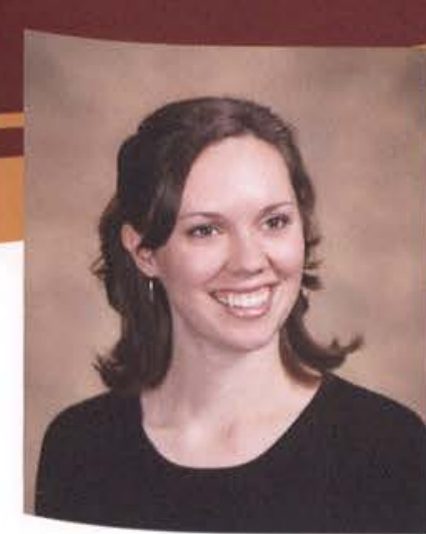

Abby Katherine DeLange Multi-Age Spanish Education \& Spanish

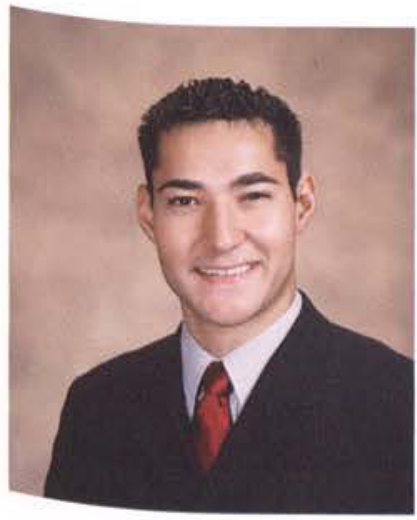

Michael Andrew Dotson Mechanical Engineering

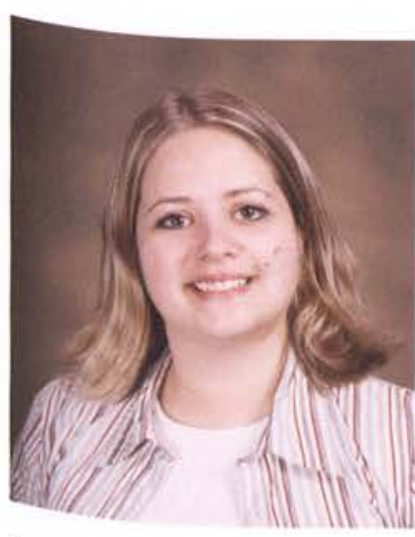

Kristin Anne Dudreck Music Performance

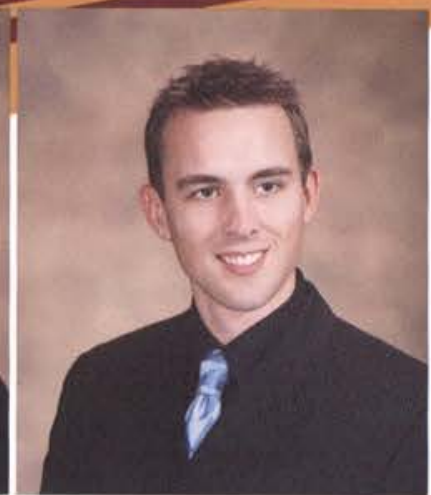

Chris Jacob DeLange Criminal Justice

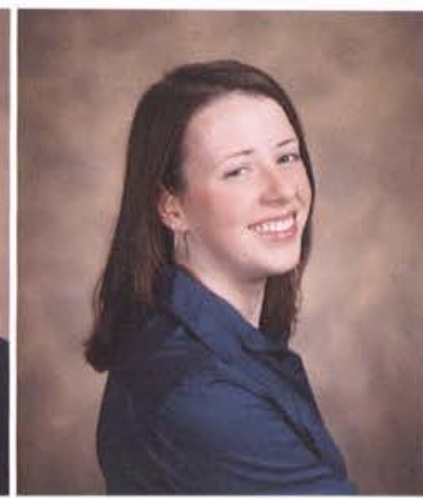

Sara Nicole Douglas Marketing

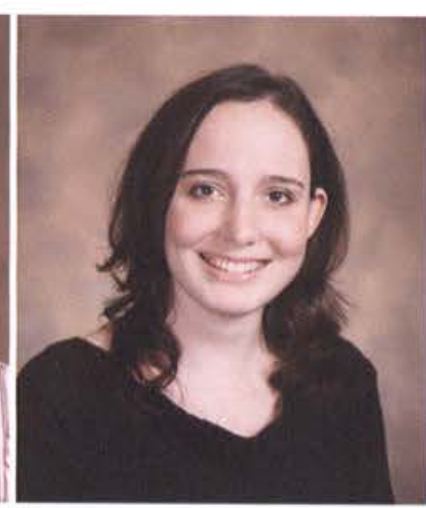

Victoria Evelyn Duff Theatre

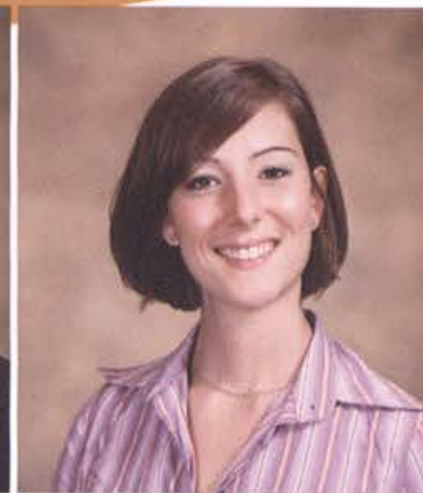

Lori E. Dennis

Nursing

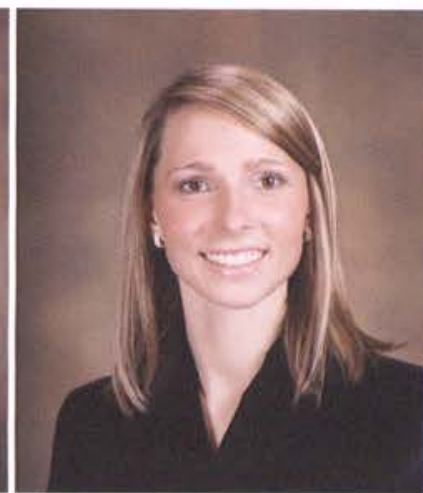

Hannah Marie Draxler Athletic Training \& PrePhysician Assistant Studies

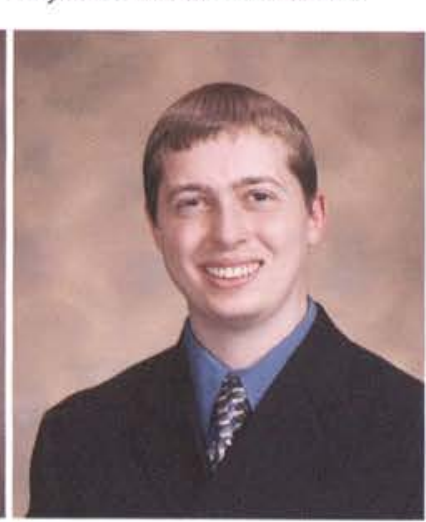

Benjamin I. Dungan Chemistry

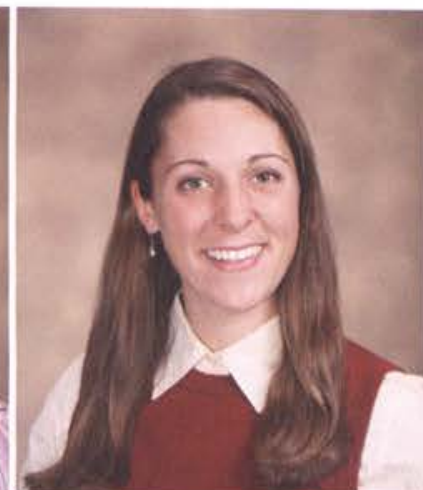

Colleen R. Derry

Social Science
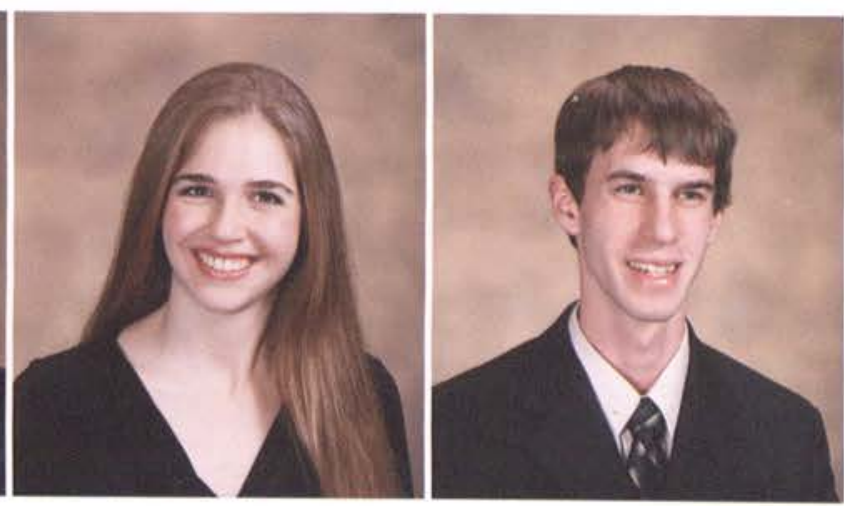

Rachel Michelle Dremann Philip G. Dudley Piano Performance \& Keyboard International Studies \& Social Pedagogy Science

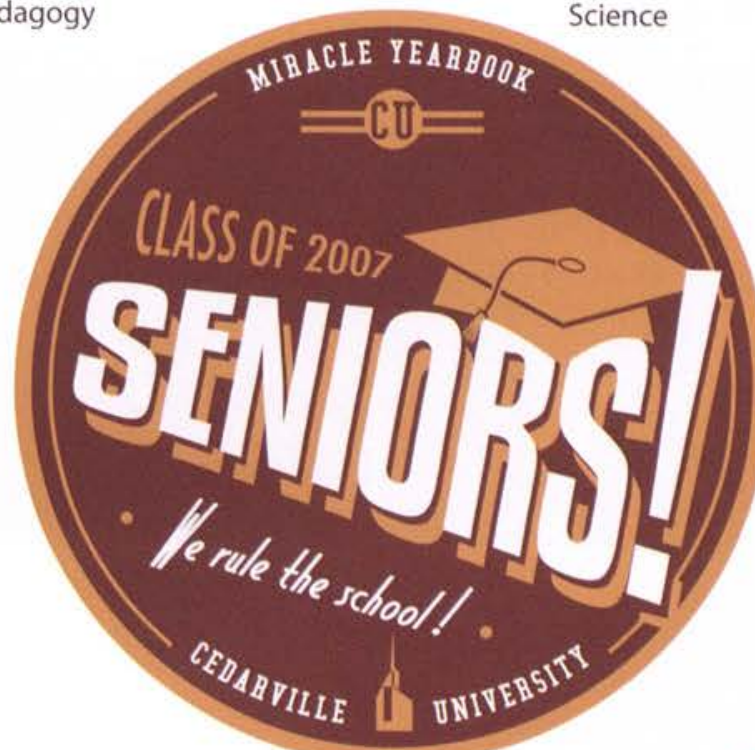




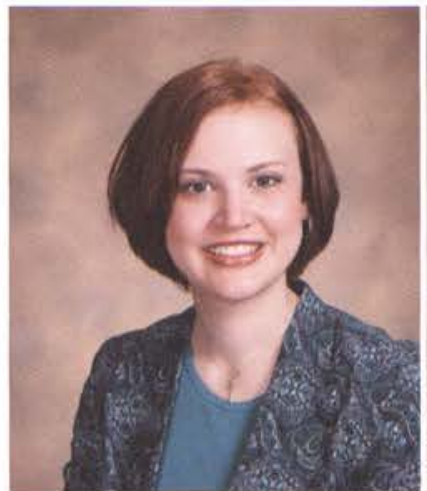

Deborah Susan Dunteman Integrated Social Studies \& Education

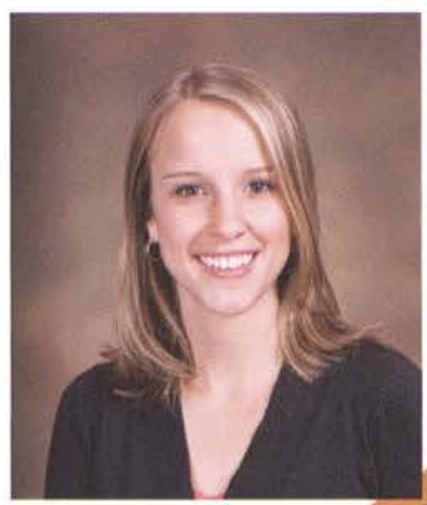

Megan Leigh Eddy Nursing

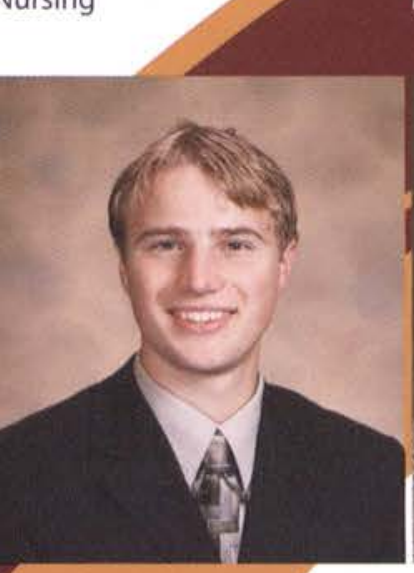

Joseph James Eisentrager Electrical Engineering

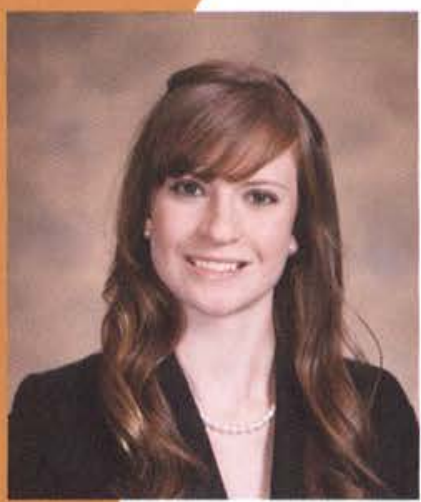

Abby Ellis

Electrical Engineering

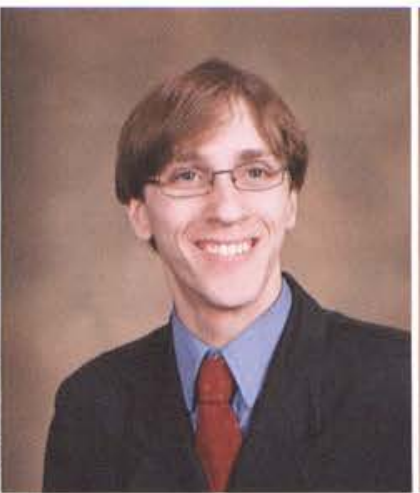

John N. Durkee

Sociology

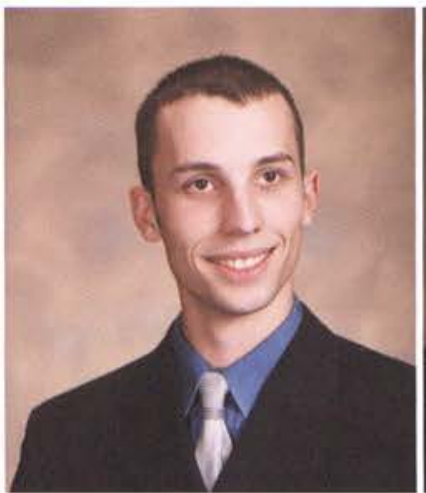

Zachary R. Eagle

Electronic Media-Audio

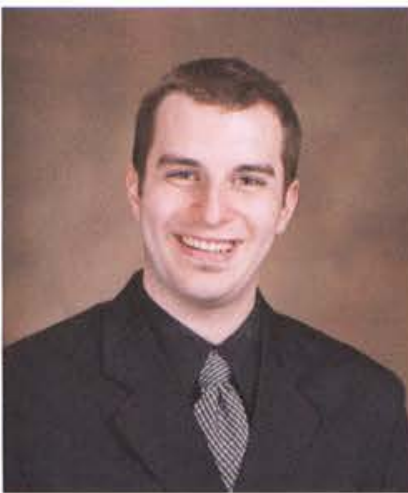

Seth Daniel Eaton International Studies

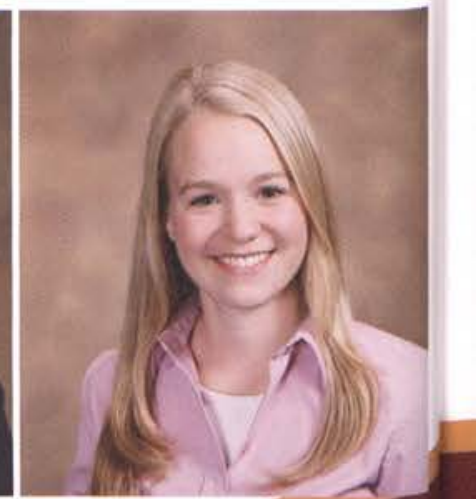

Jenny Rae Eby English 

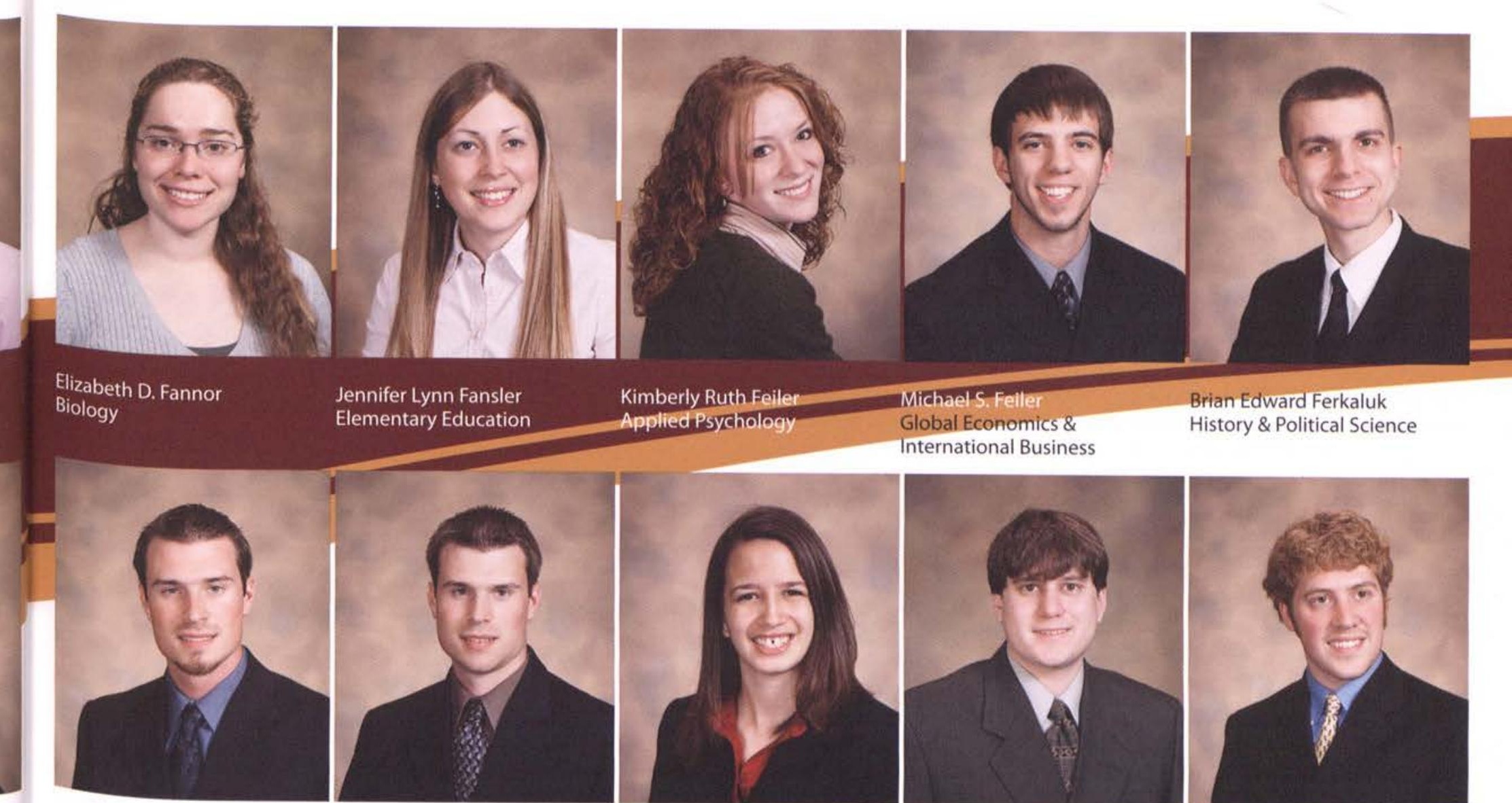

Kimberly Ruth Feiler
Applied Psychology

Michael S. Feiler

Global Economics \&

Brian Edward Ferkaluk

International Business

History \& Political Science
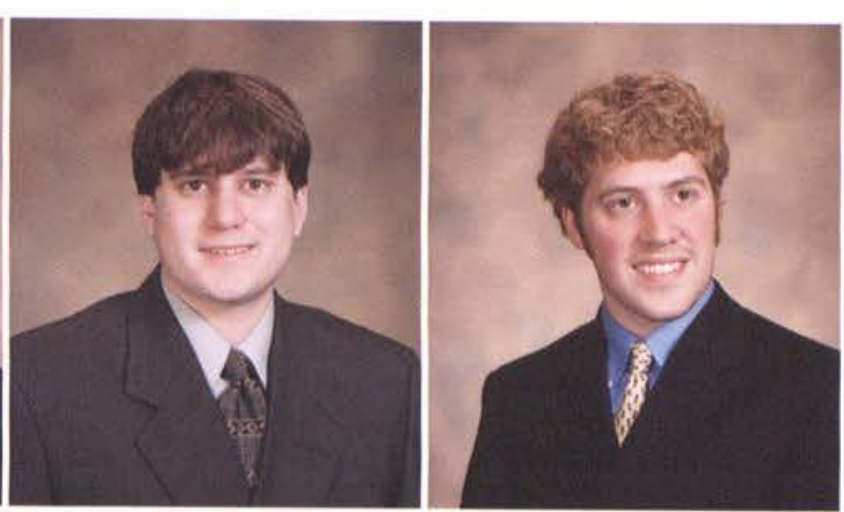

Christopher Allen Few

Physics Education

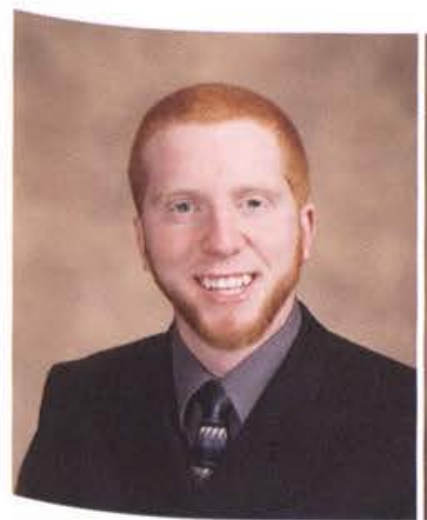

Caleb Lee Fletcher Electronic Media

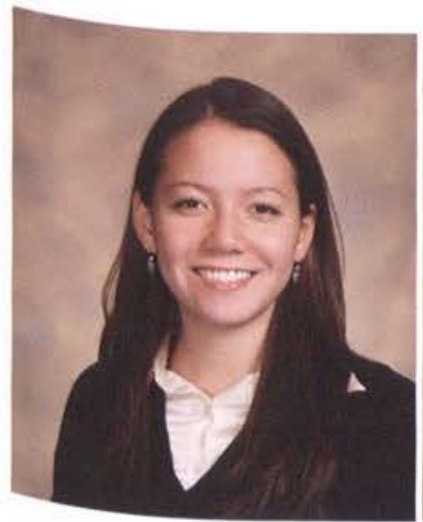

Jannette Diane Foy Mathematics
David Bryan Few

AYA Physics

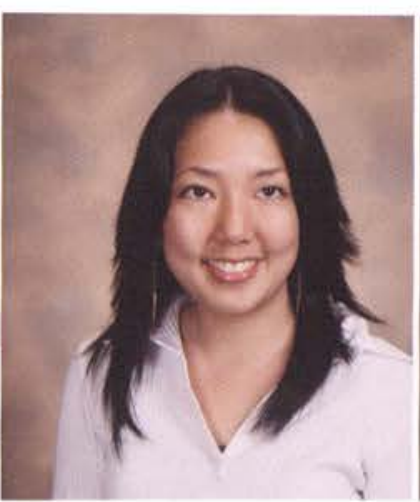

Jamie C. Foo Li Mei

Exercise Science

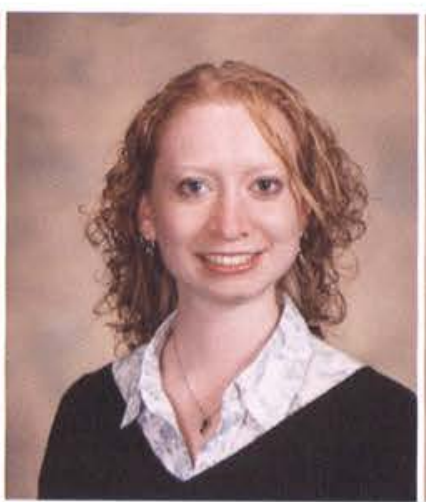

Melanie Faith Frageorgia English
Victoria T. Fiorelli

Applied Psychology

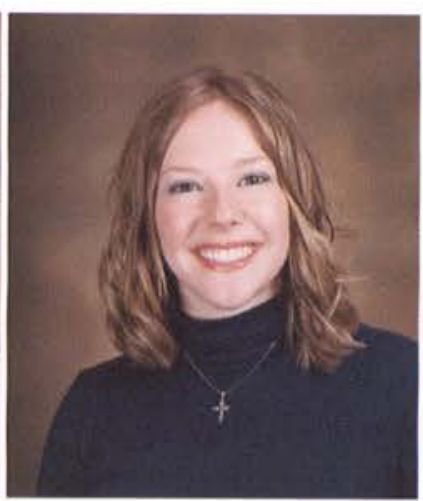

Jessica C. Forsythe

Nursing

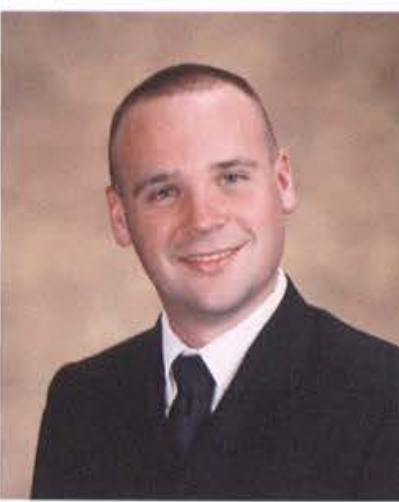

Michael Benjamin Francis International Studies
Steven Karl Fladda English

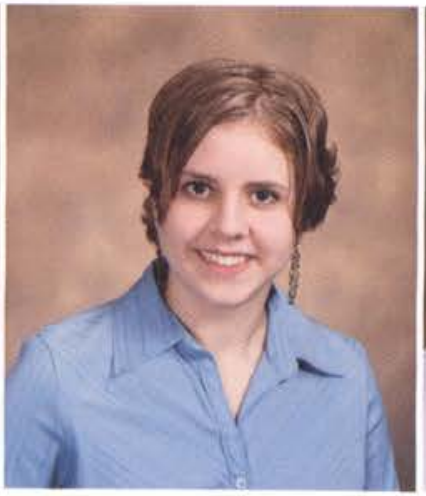

Janna Beth Foster

Psychology
Adam Ross Flenar

Computer Science

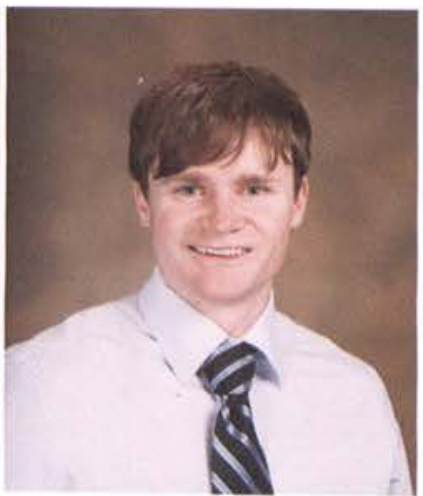

Jesse Fox

Psychology

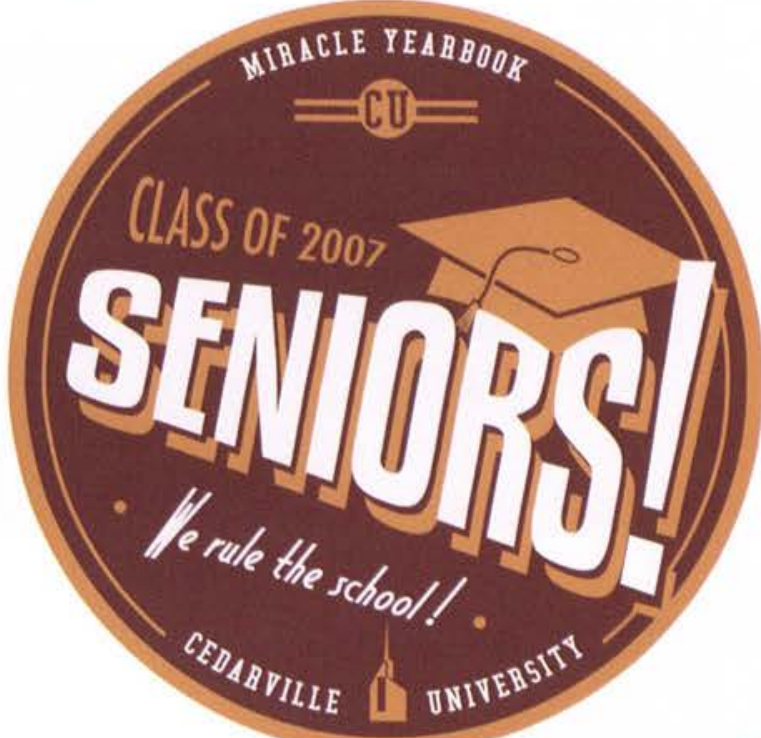




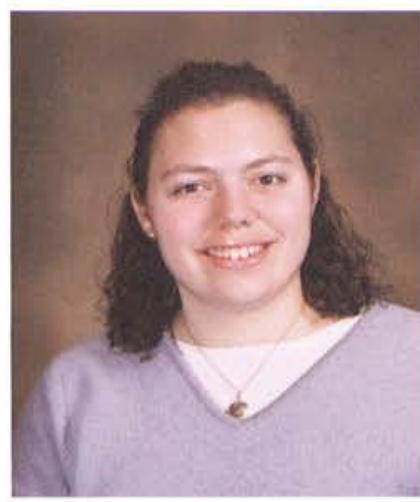

Rachel Mae Freswick Nursing

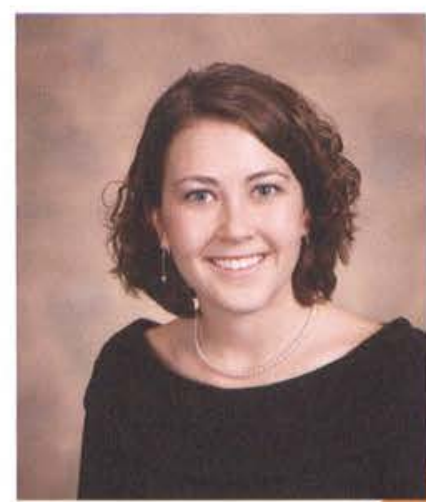

Natalie Rose Gamache Communication Arts 2

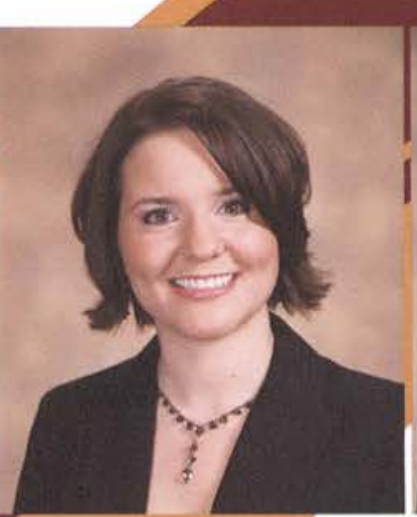

Kelly Lauren German Early Childhood Elementary

Education

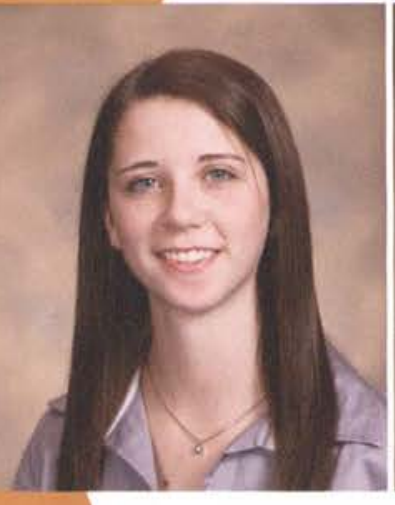

Shelley E. Gilbert Early Childhood Education

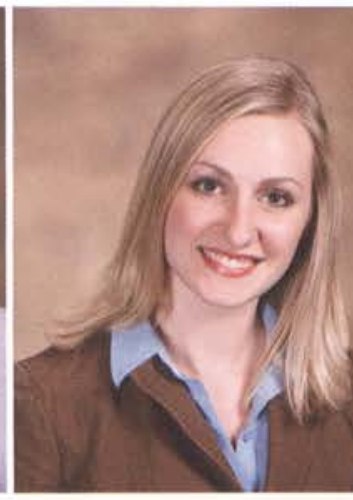

Michal D. Friend Marketing

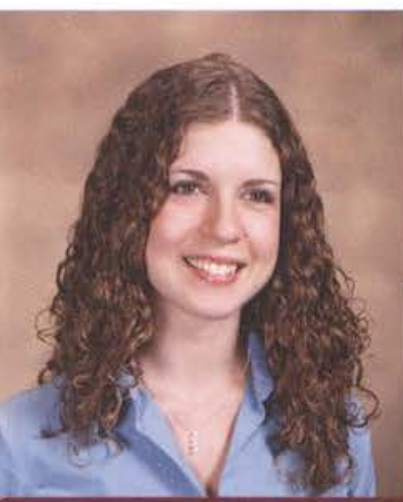
$\begin{array}{ll}\text { Kari Ann Gannon } & \text { Julla Ruth Geber } \\ \text { Multi-Age Music Education } & \text { Nursing }\end{array}$ Multi-Age Music Education Nursing

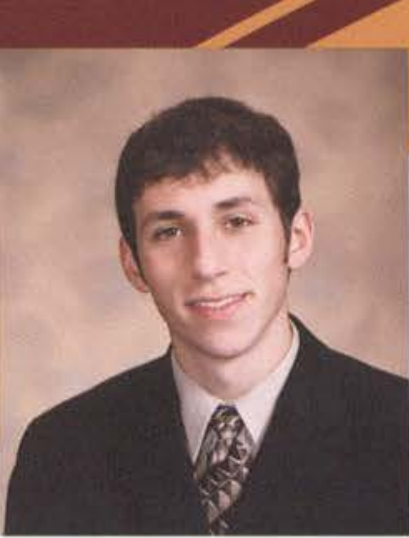

Michael B. Gerringer Environmental Biology

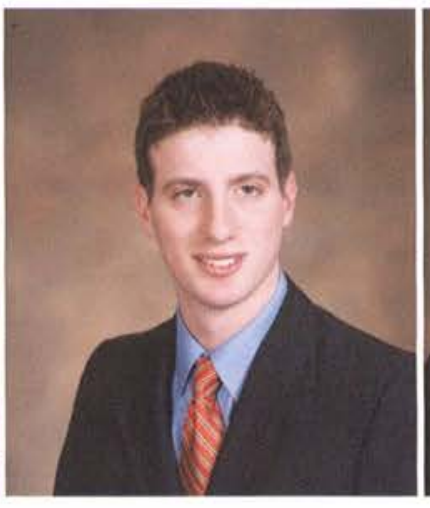

Justin Paul Gildow Nursing
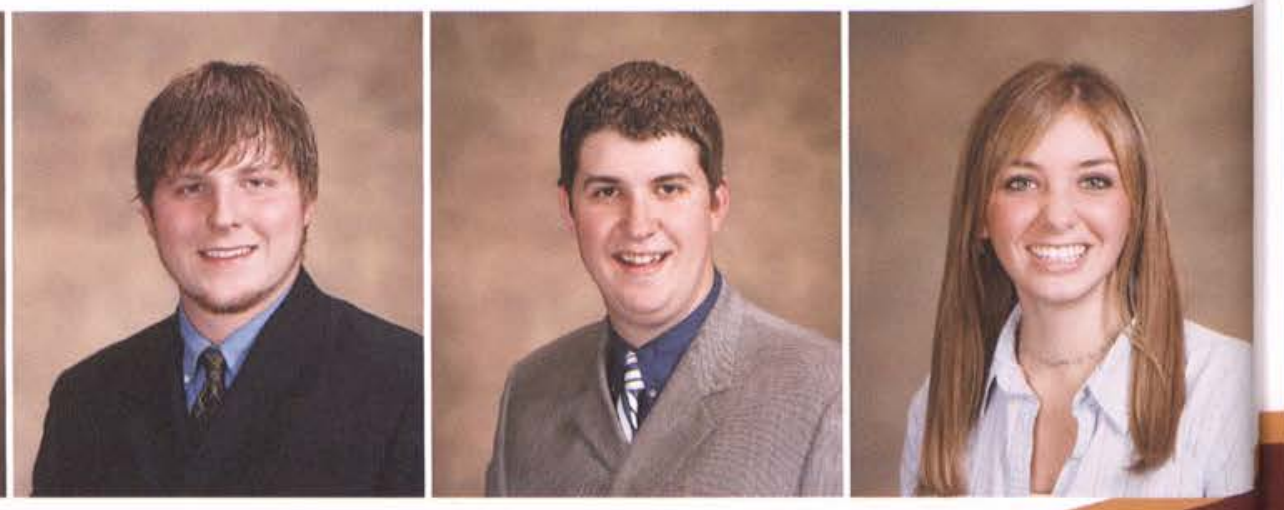

David James Fuls Management

Mark Edward Gainer Early Childhood Education Psychology
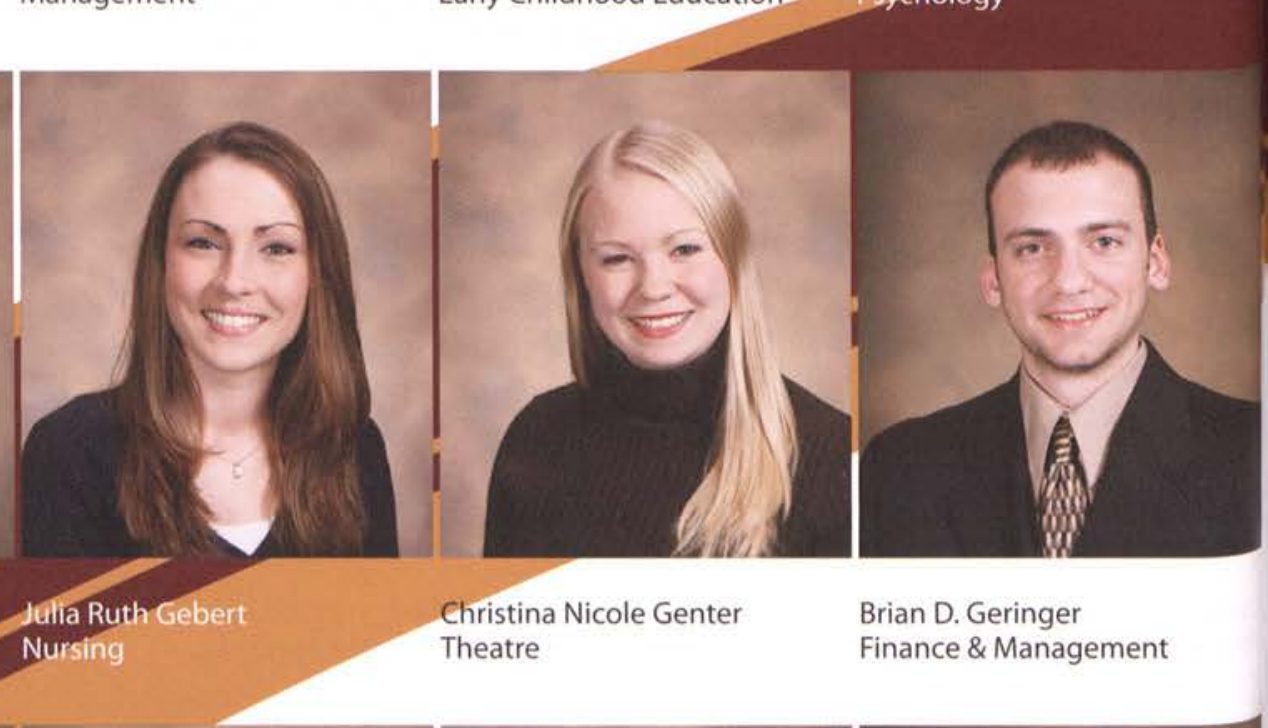

Brian D. Geringer

Finance \& Management
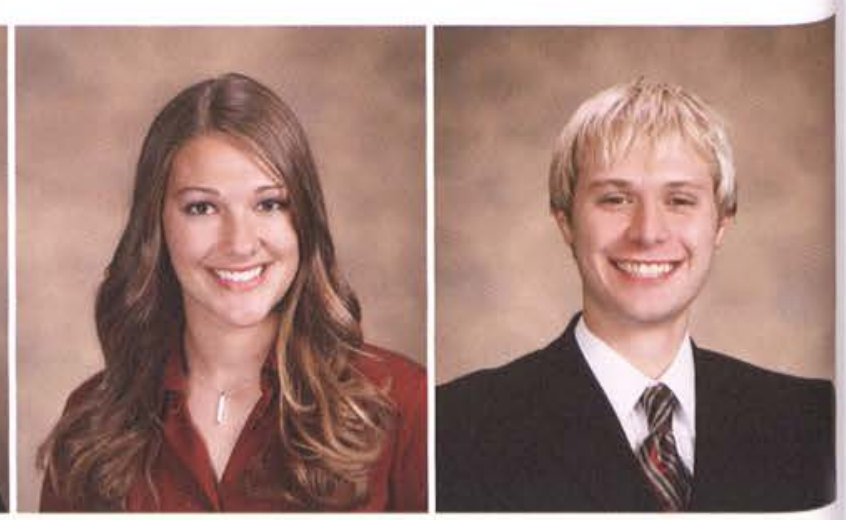

Rebekah Lee Gibson Early Childhood Education \& Special Education

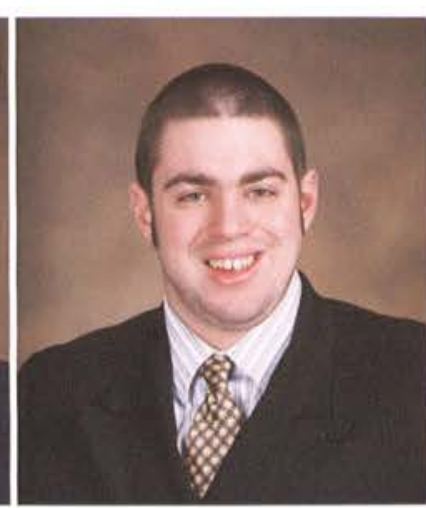

Daniel J. Giles

Mechanical Engineering
Sarah Christine Gibson Vocal Performance

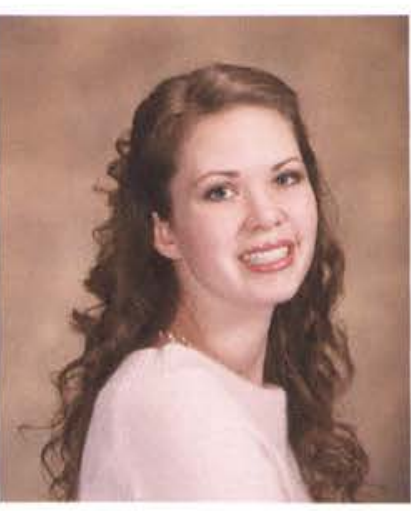

April D. Gilgillen

Early Childhood Education
Zachary Kiser Gibson Finance

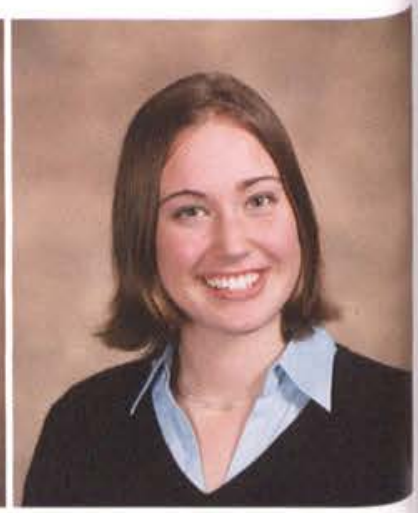

Bethany Ruth Gilmour English 

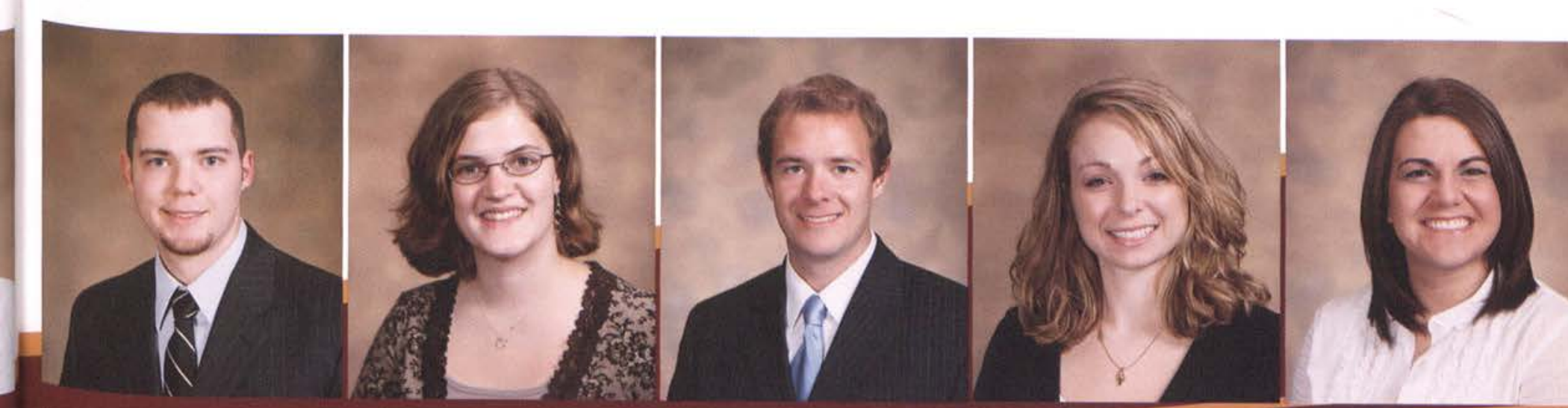
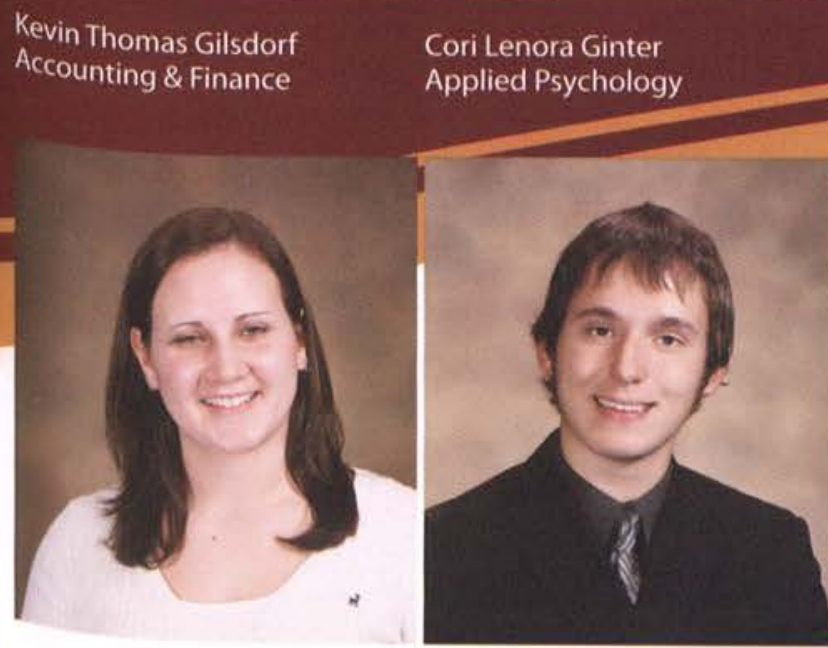

Monica Renee Godinez
Nursing

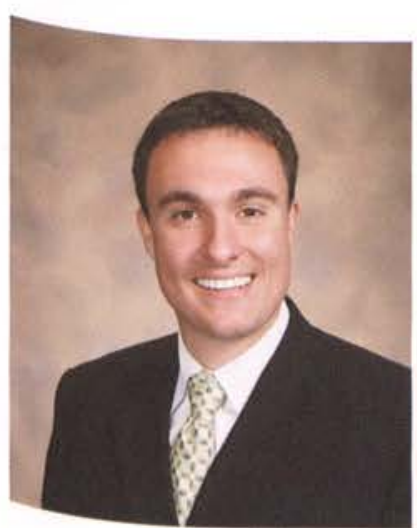

Joel David Green

Marketing

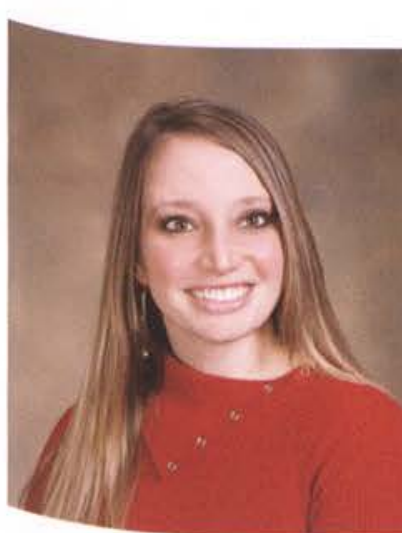

Abigail Christine Guiler Spanish
Peter Dale Gohdes

Mechanical Engineering

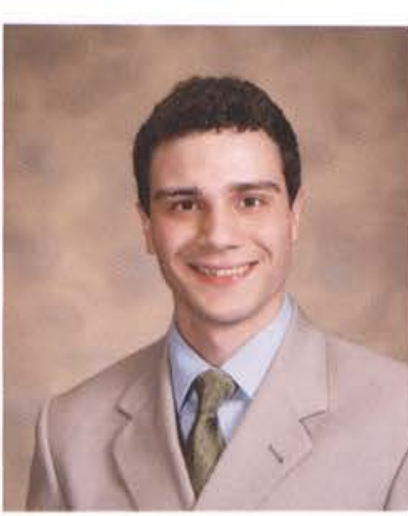

Shawn Christopher Green

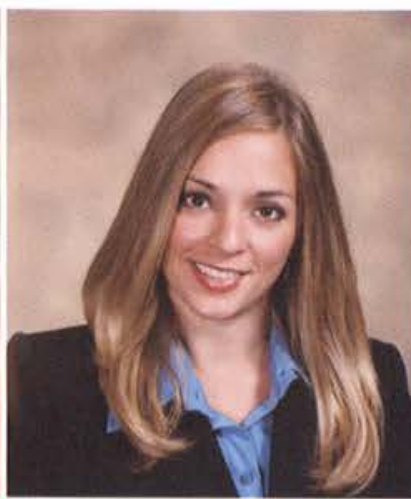

Carrie J. Gumm English Communication Arts
Earl Aldege Girouard Jessica Rachel Glass

Mechanicall Engineering

Organizational

Communications

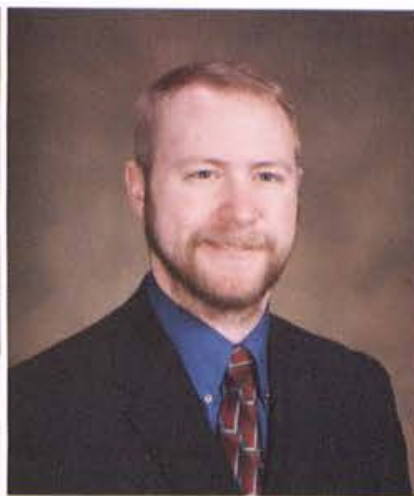

Nicholas A. Gray

Youth Ministry

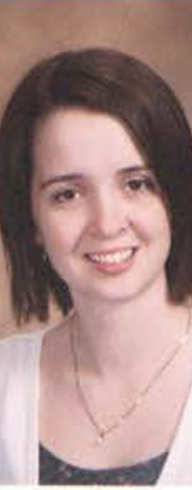
Marketing
Catherine Marie Gooch
Ashley Marie Gochenaur

Comprehensive

Communications

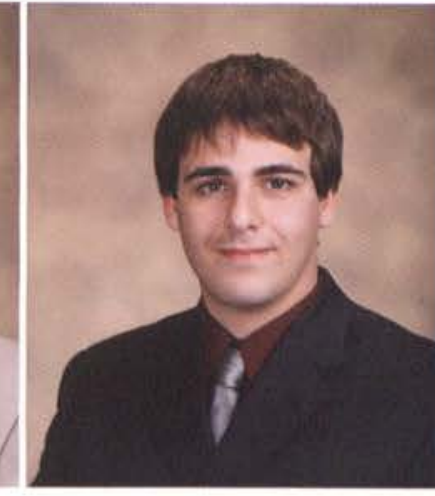

Jedediah L. Gregory

Organizational

Communications

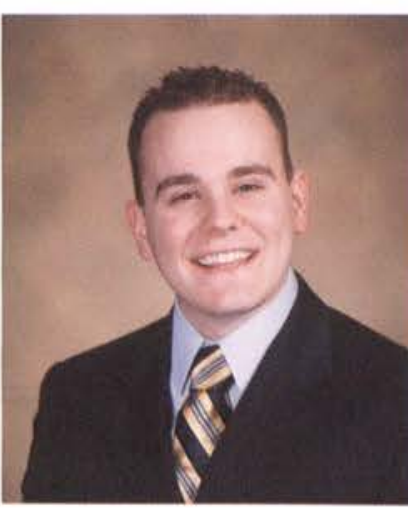

Michael E. Gumprecht

Criminal Justice \& Pre-Law

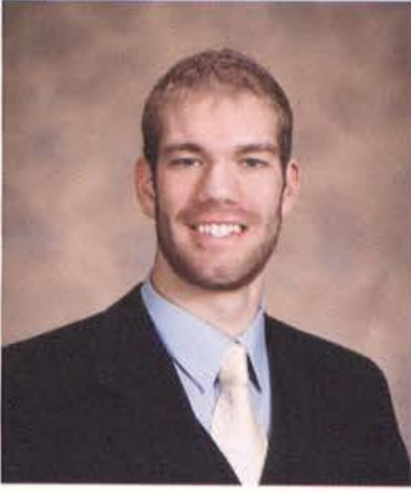

Erin Ashley Green

Communication Arts

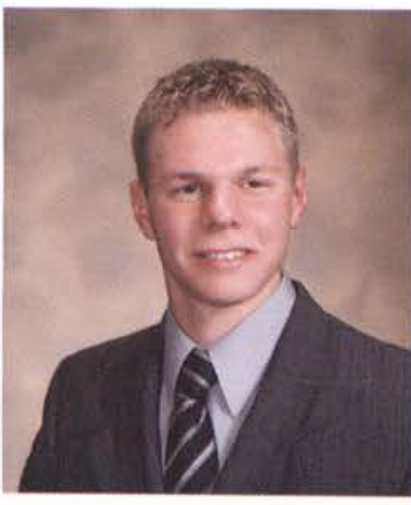

Joshua K. Greve Pre-Seminary Bible

Daniel Grant Groseclose Criminal Justice

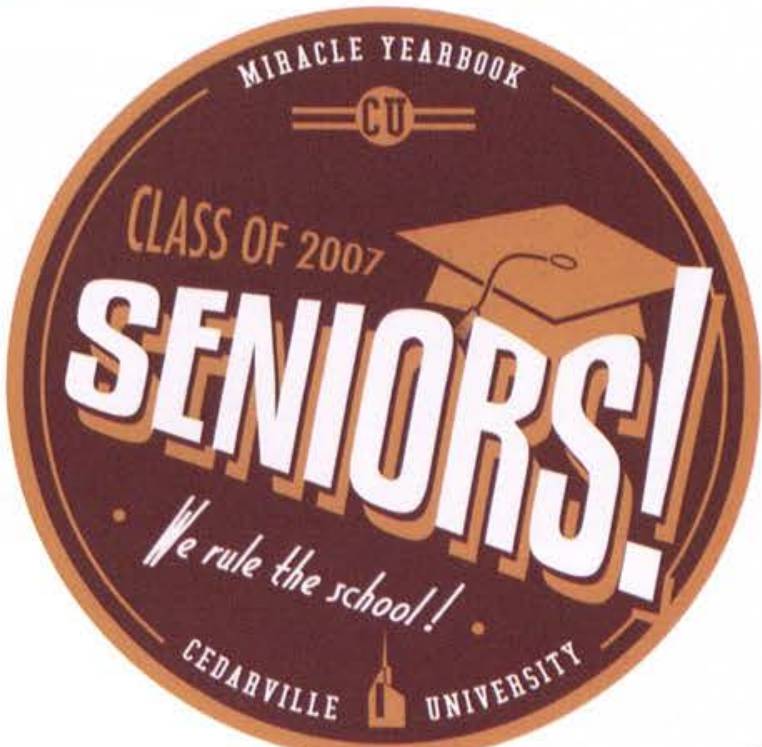



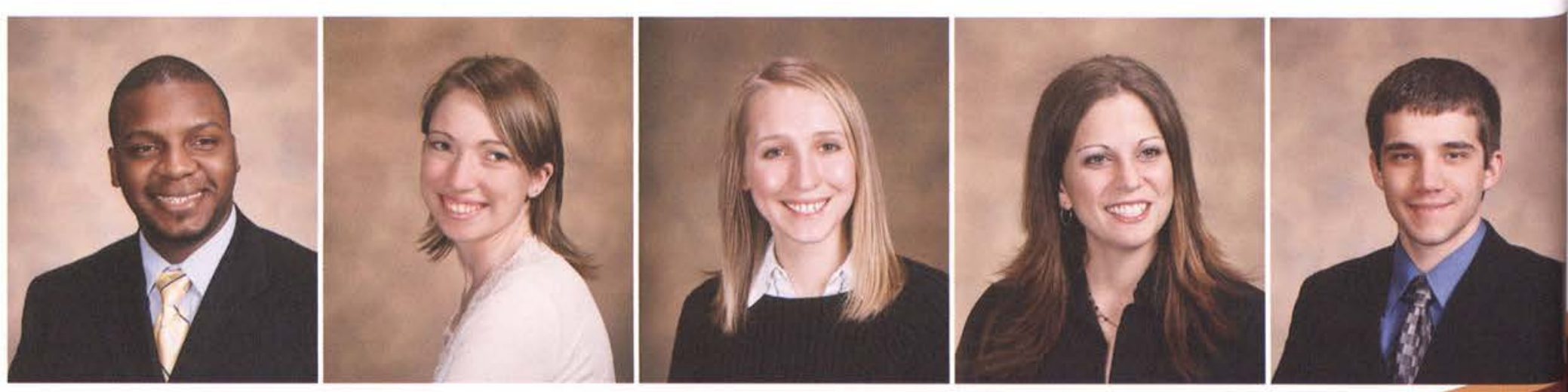

Kyle J. Gunn

Pre-Seminary Bible

Angela M. Hagensen Technical \& Professional

Communication

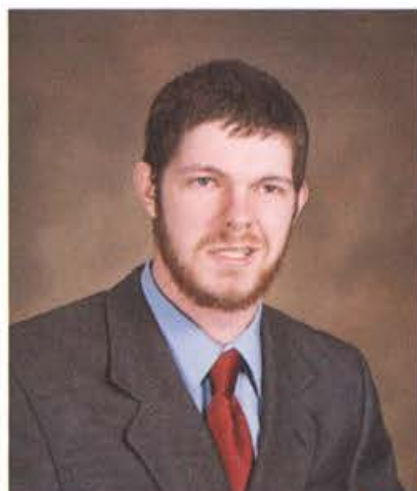

Michael David Hand Jr. Computer Engineerin
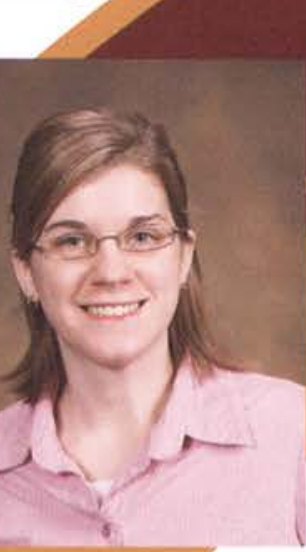

Janie R. Harriman Psychology

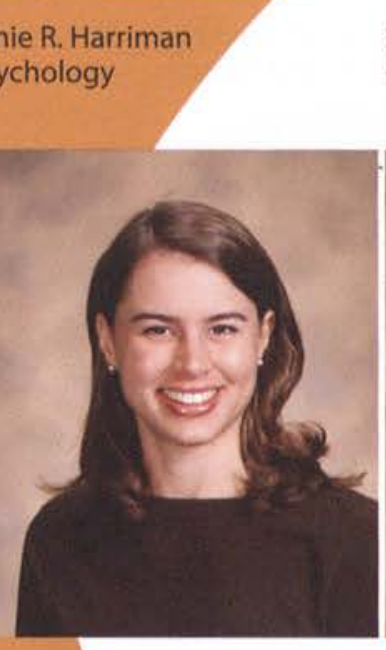

Marie Heinig Elizabeth
Biology

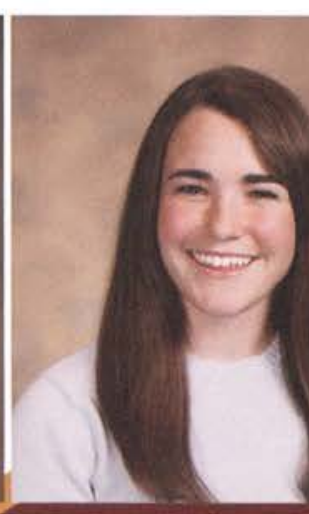

Megan Annette Hanes Biology

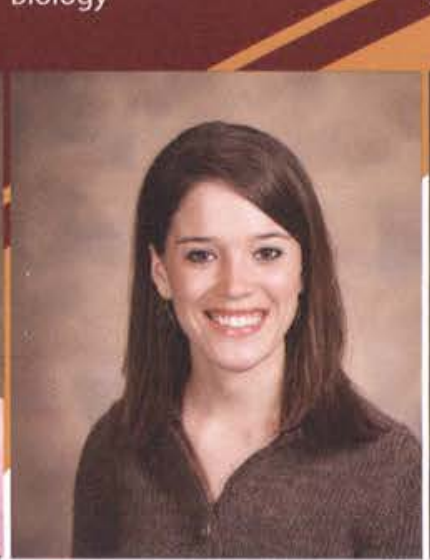

Erin Ashley Harrison Nursing

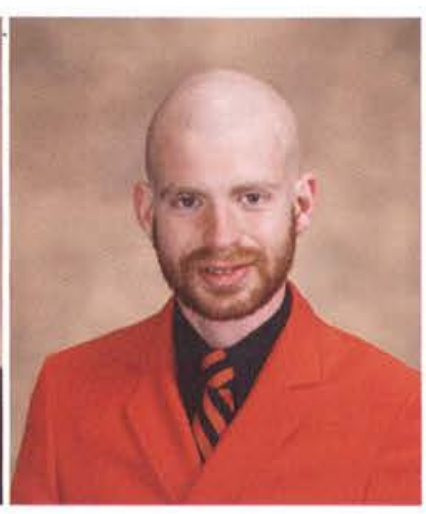

Daniel Christian Hekel History
Ruthann Hager

Social Work
Sarah Leslie Hall

Exercise \& Sport Science
Nathan Lee Hamilton

Computer Science

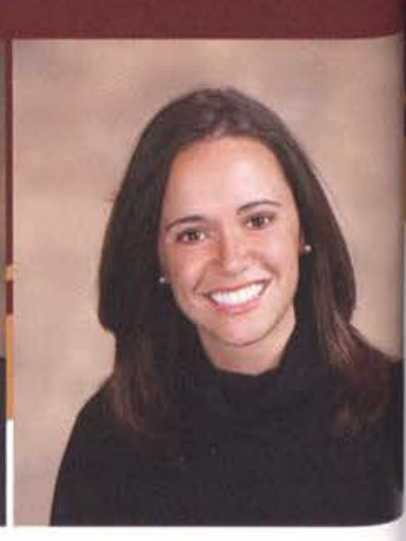

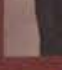

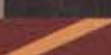

Micah Robert Harding Sport Management
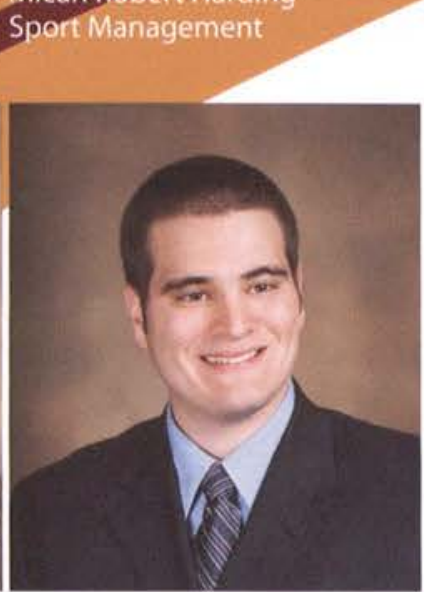

Nathan Bradley Harro Computer Science

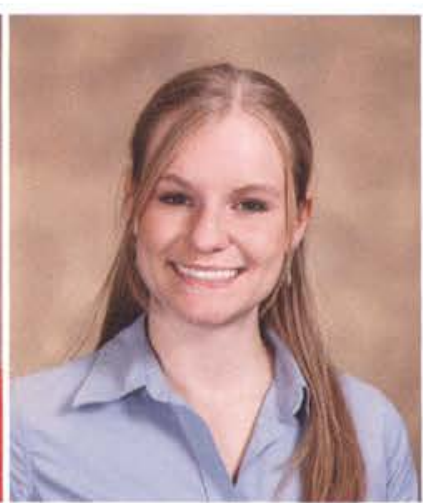

Angela Jean Henker Early Childhood Education

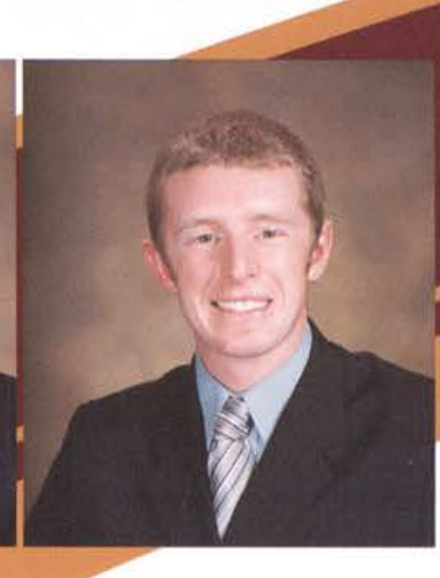

Christopher James Harmon Middle Childhood Education

Ivey Rebecca Harrell Nursing
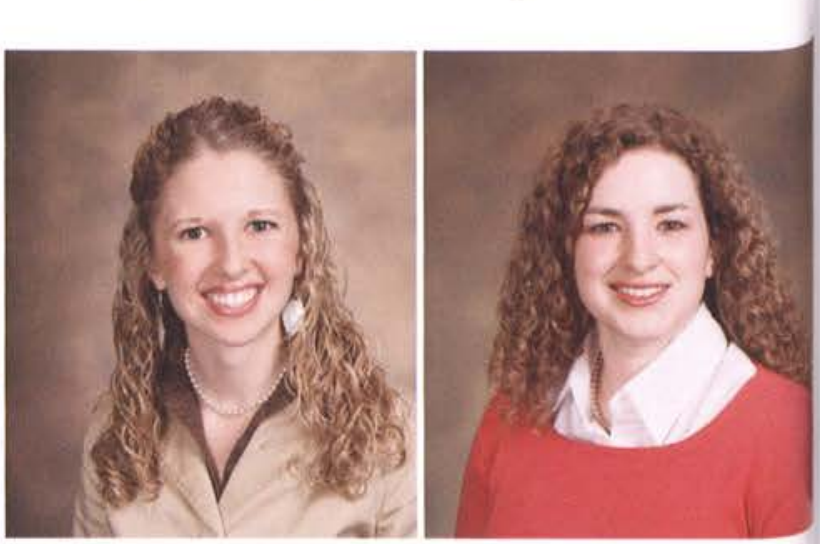

Taylor Laine Hawkins Early Childhood Education

Anna Grace Hayes Technical \& Professional

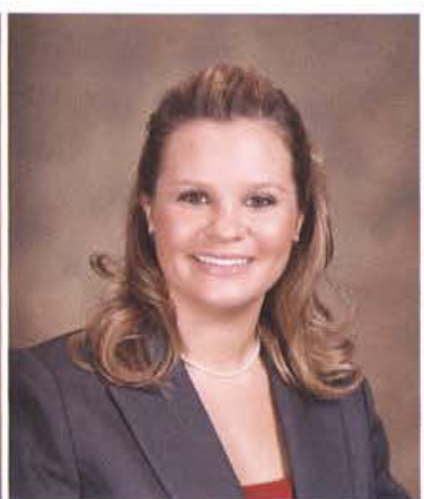

Stacey Ashlynn Henness Biology Communication

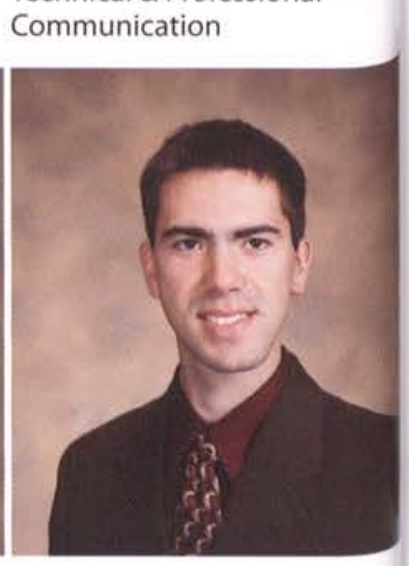

Ethan Scott Hennessey Electrical Engineering 

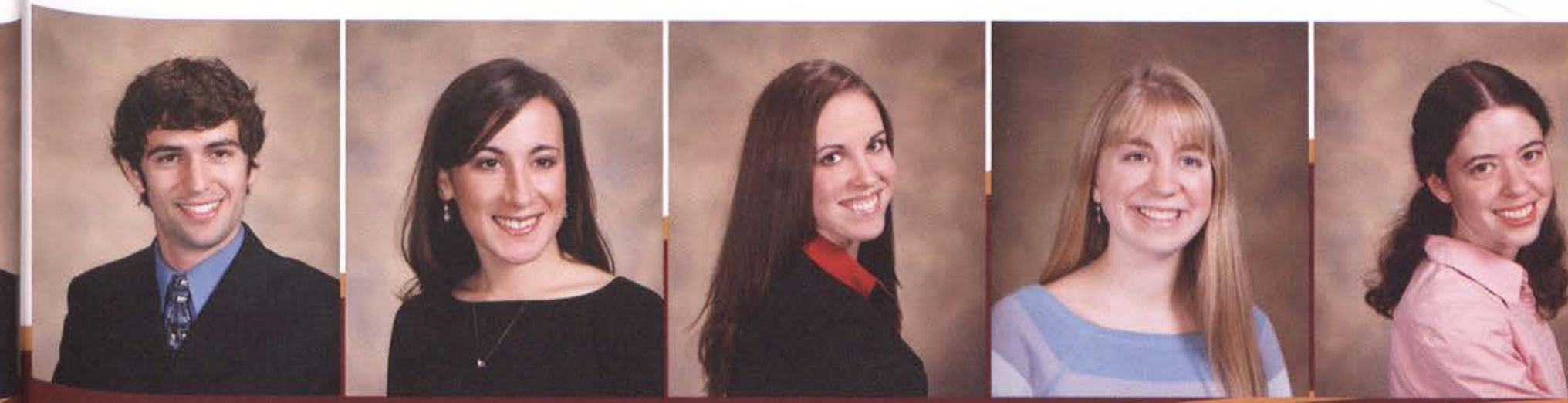

Mark Charles Hershey Pre-Seminary Bible

Alison Christine Hess Early Childhood Education
Kristin Laurel Hecsler Integrated Social Studies Education

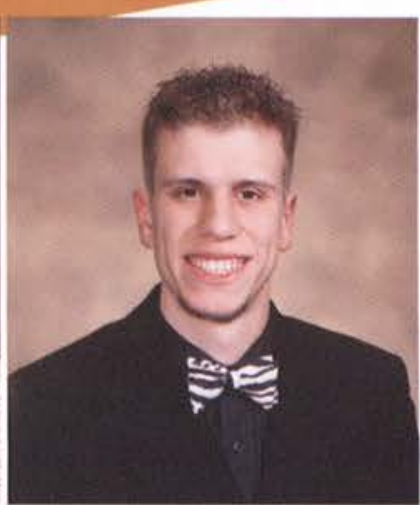

Aaron Mark Hollopeter Music
Tricia Lynn Hine Spanish \& Comprehensive Bible

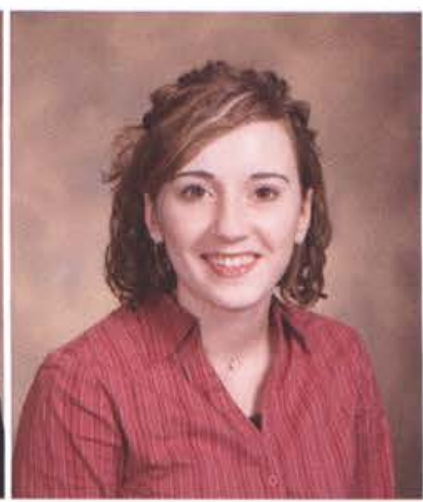

Sara Jane Hoover English
Elizabeth Ashley Hinks Early Childhood Education

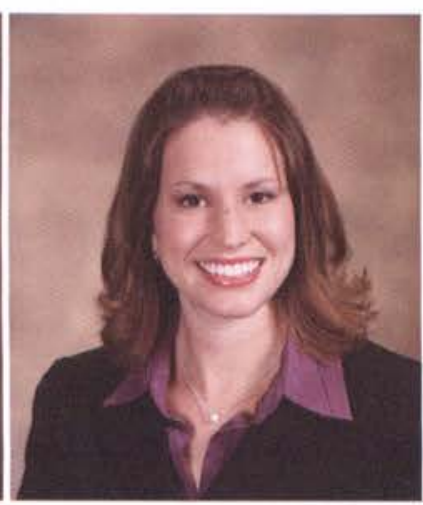

Emily Jeanette Hose Applied Psychology

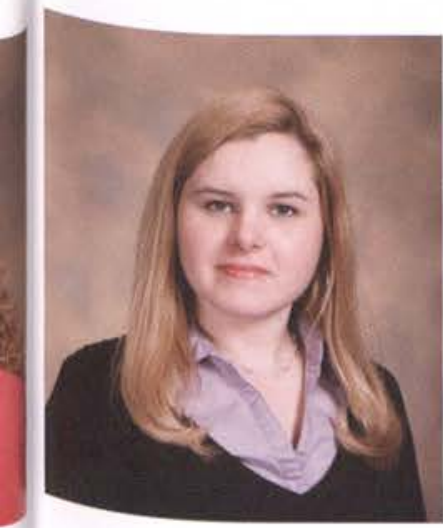

Leighana Lynn Hosford Middle Childhood Education

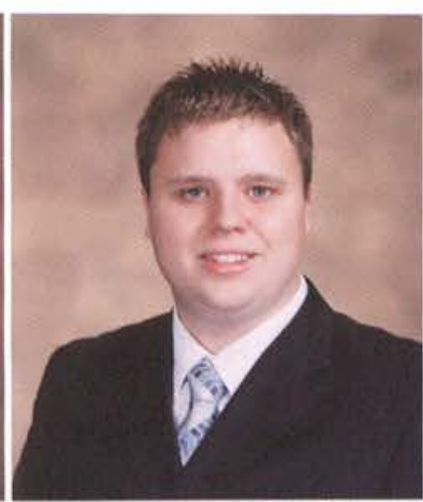

Benjamin Gordon Houghton Accounting

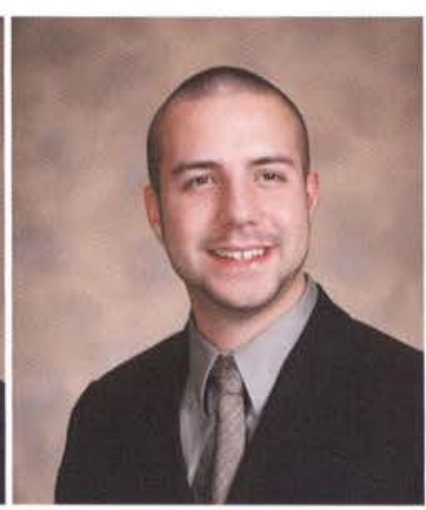

Joel Mark Hoffman

History \& Political Science

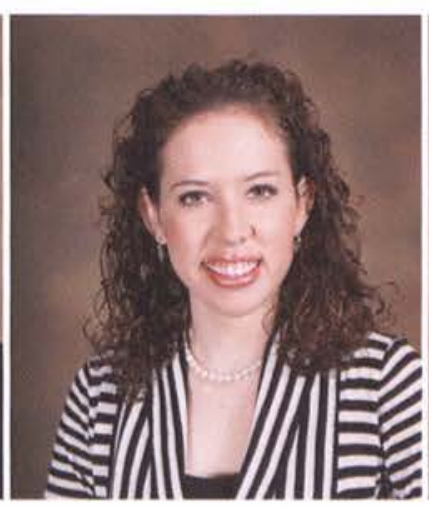

Christina Joy Hovestol Nursing

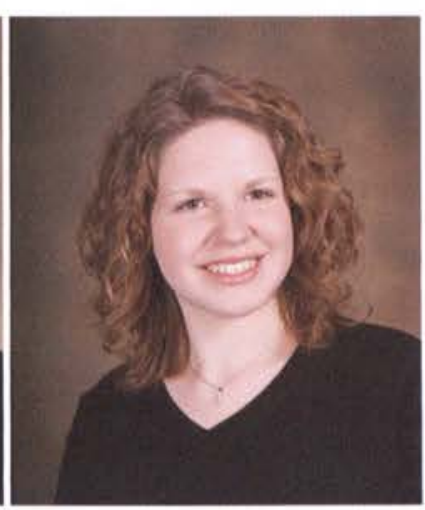

Julie Kirsten Hull Management

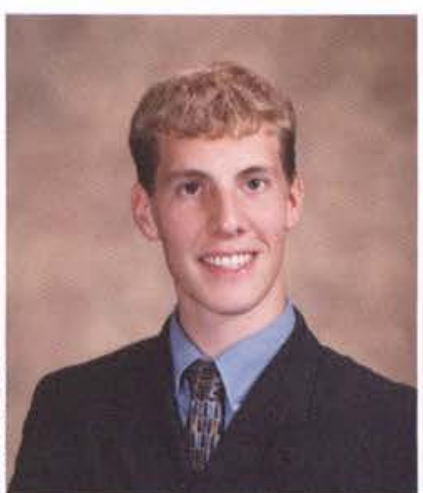

Michael Robert Hubenthal Mechanical Engineering

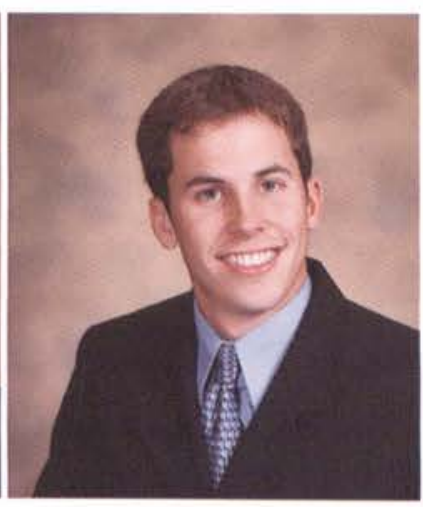

Timothy James Hubler Management
A

Aaron Charles Huffman phic Design

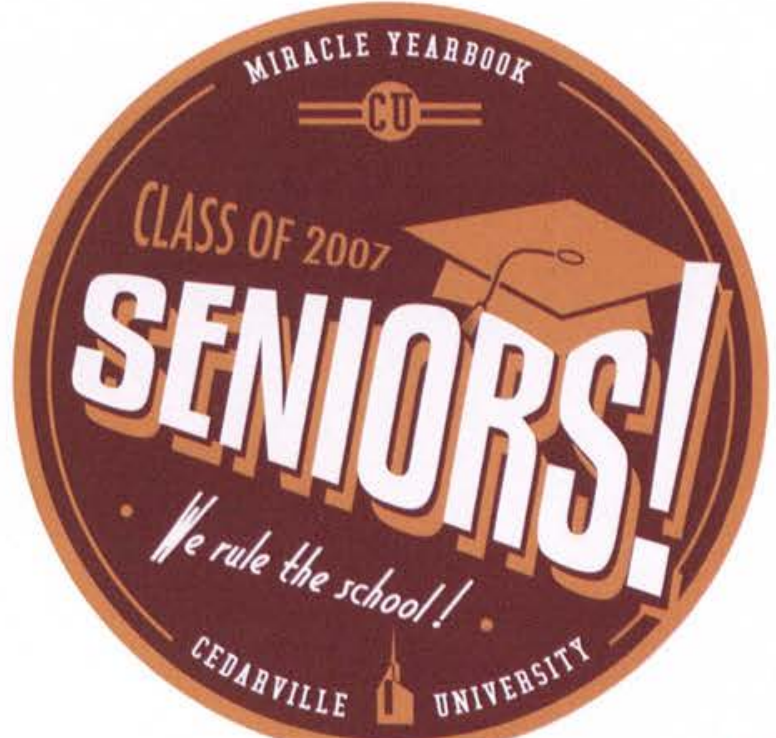




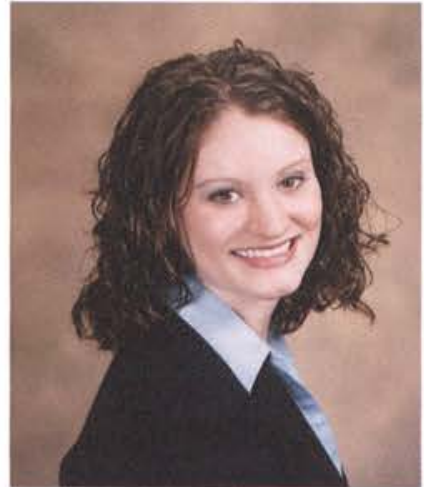

Ashley Lauren Hume Communication Arts

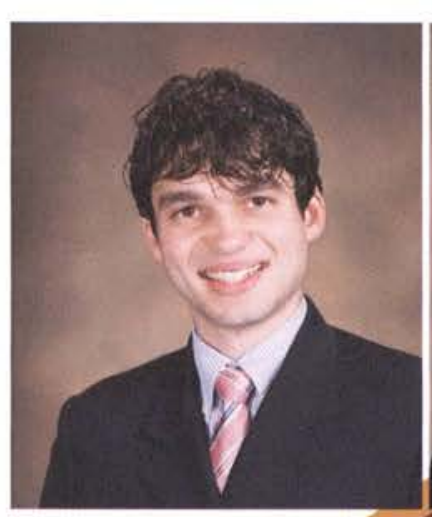

Daniel Tubandt Jaquery Finance \& Managemea 2

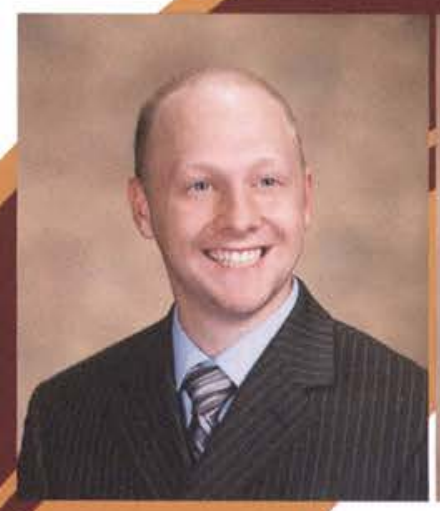

Eric Michael Johnson Mechanical Engineering

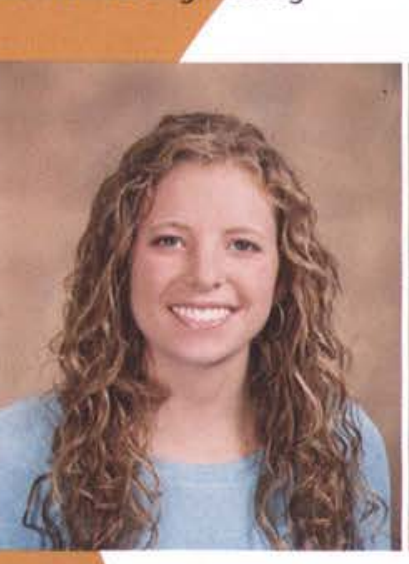

Leah Renee Jones Graphic Design

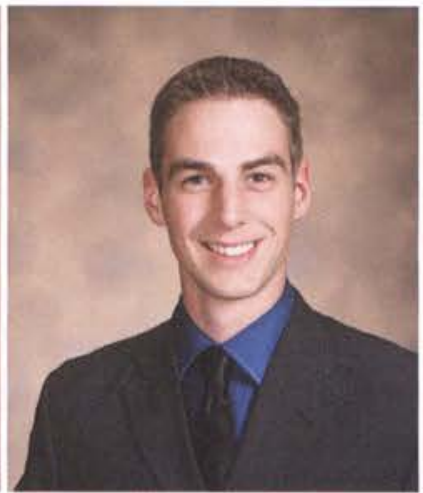

John M. Hummitzsch Sport Management
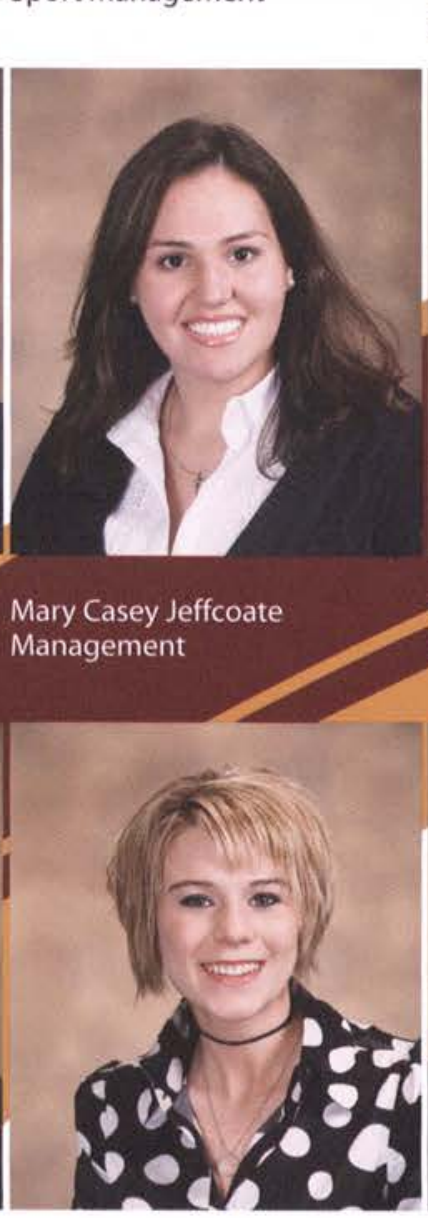

Holly Lynn Johnson Graphic Design

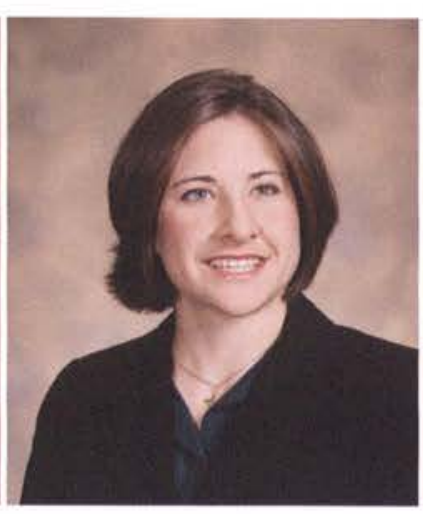

Amanda-Jo Adele Joswig Biology

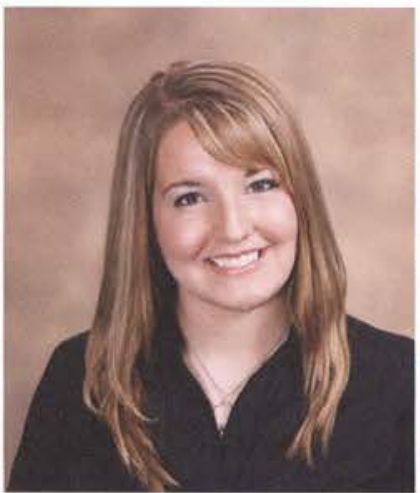

Ashley Diane Hunt

Early Childhood Education \& Special Education

Ashley Beth Hunter Organizational Communications

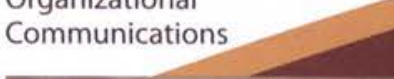

Jordan M. Jacobsen

Comprehensive

Communications

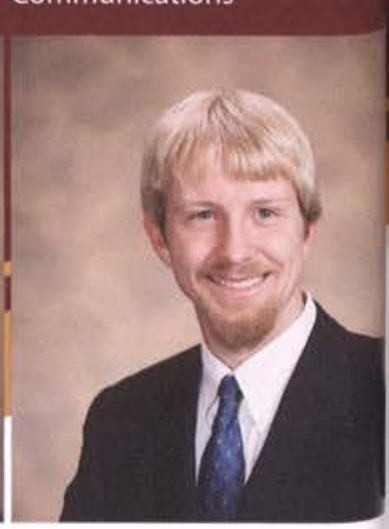

Christopher D. Johnson Marketing

Clinten J. Johnson Mechanical Engineering
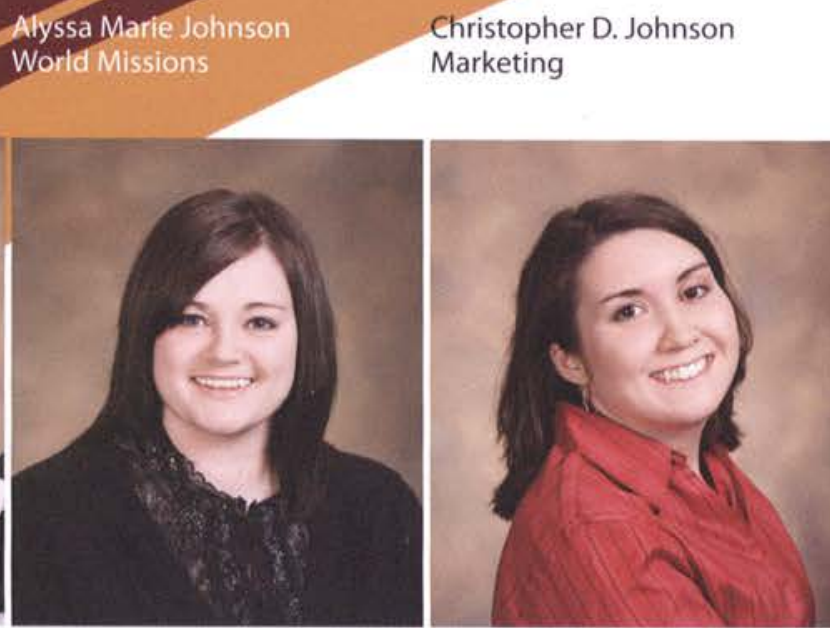

Melissa Anne Johnson Biology

Sarah Elizabeth Johnson Applied Psychology

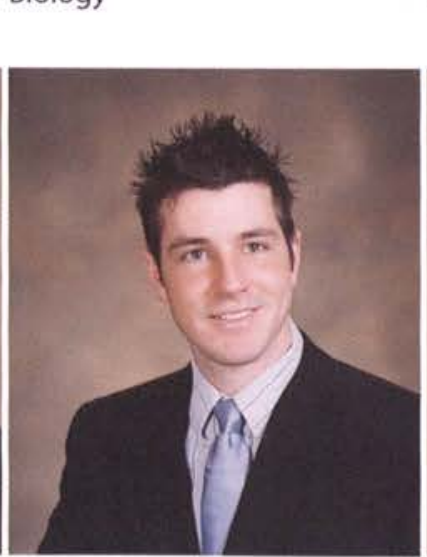
Athletic Training

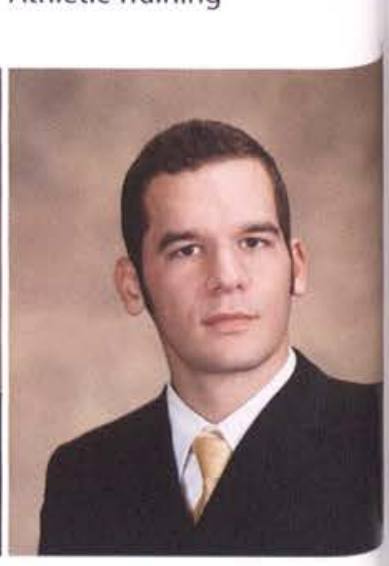

Caleb "Bucky" Foster Kauffman Jack E. Kehl III Communication Arts Biology Pre-Med 


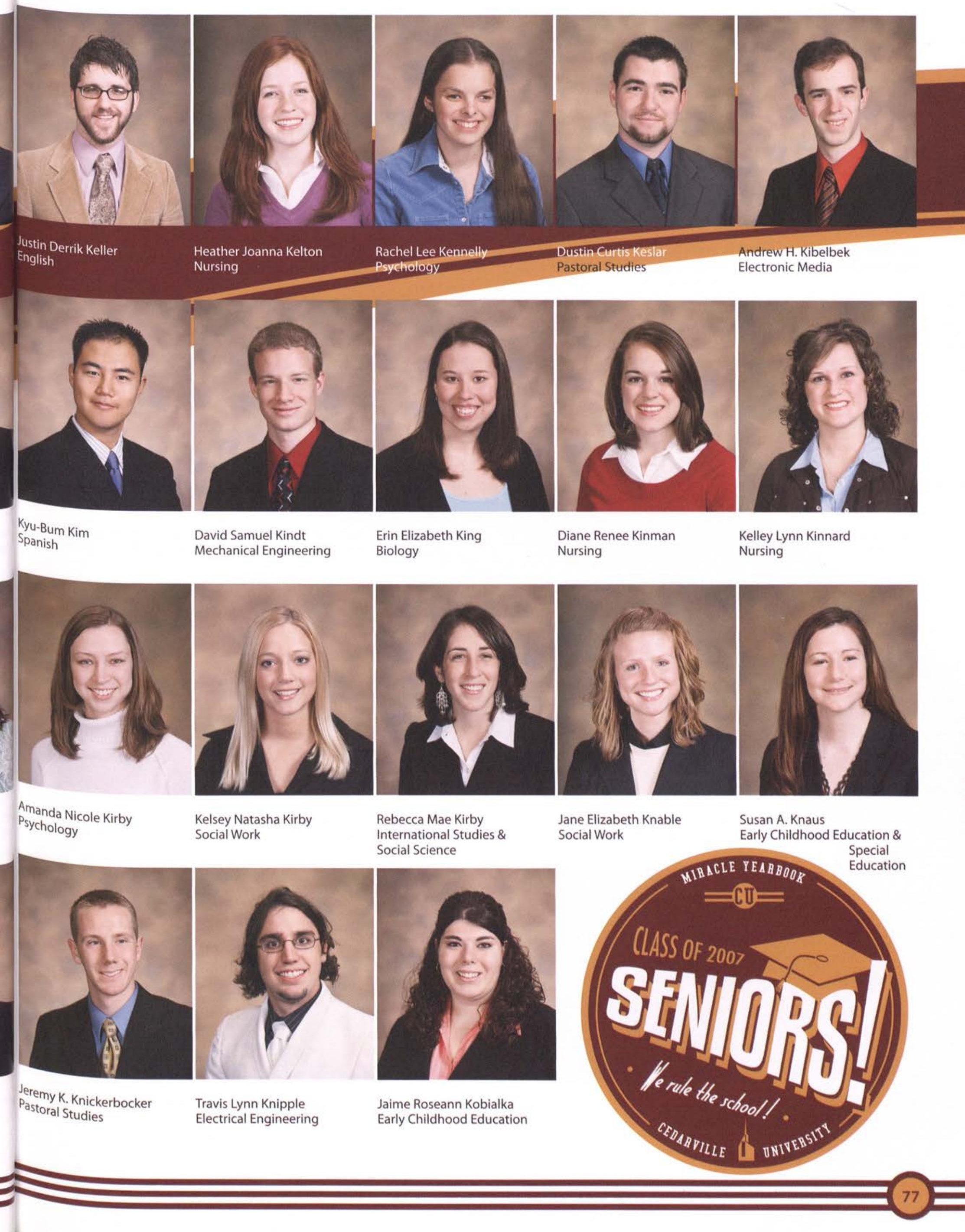




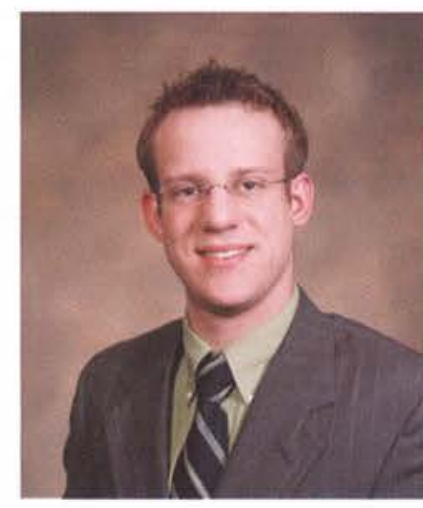

Benjamin Ferriss Kouba Electronic Media

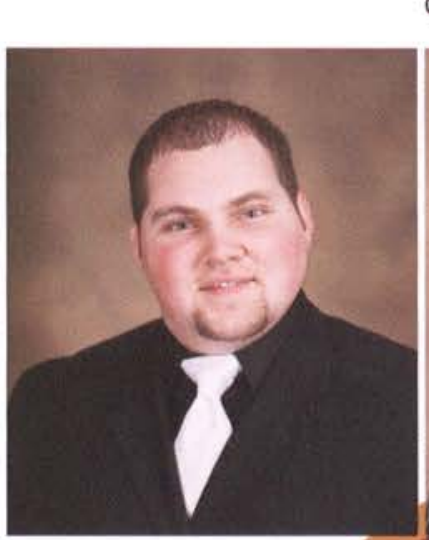

Loren Michael Kuhn Sport \& Exercise Scien
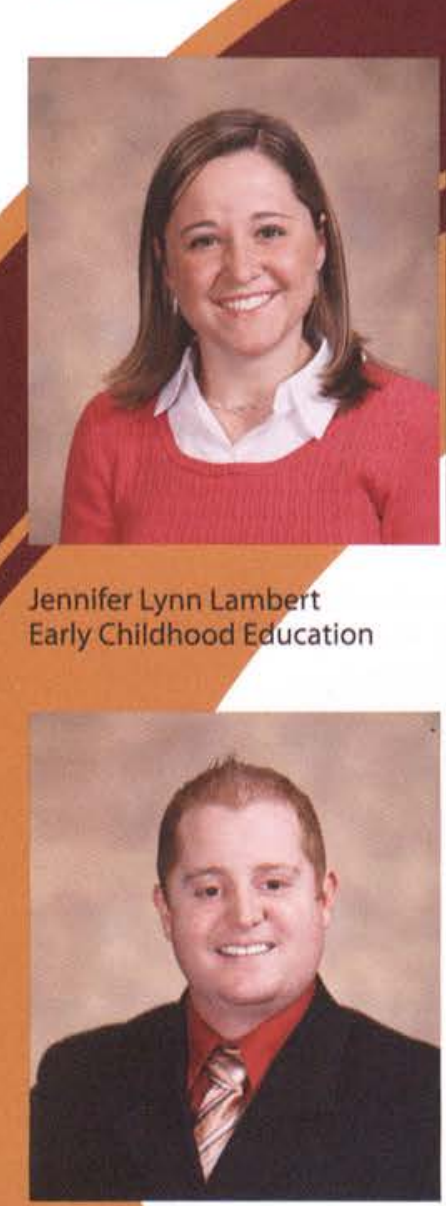

Jonathan R. Lawson

Youth Ministries \& Christian

Education

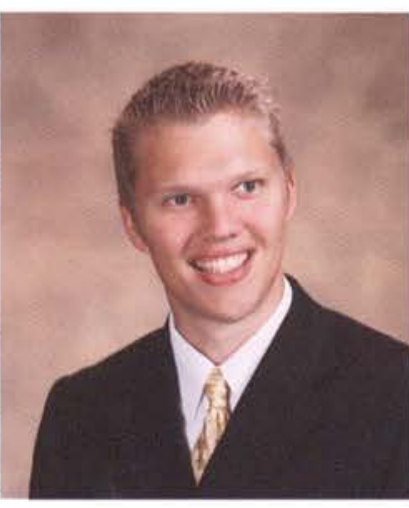

Jonathan R. Kragel

Comprehensive

Communications

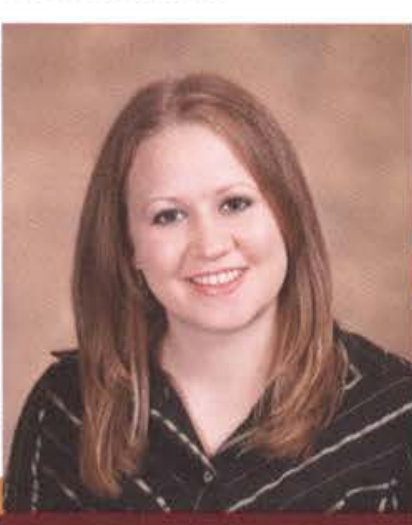

Amanda Ruth Kuntz Management

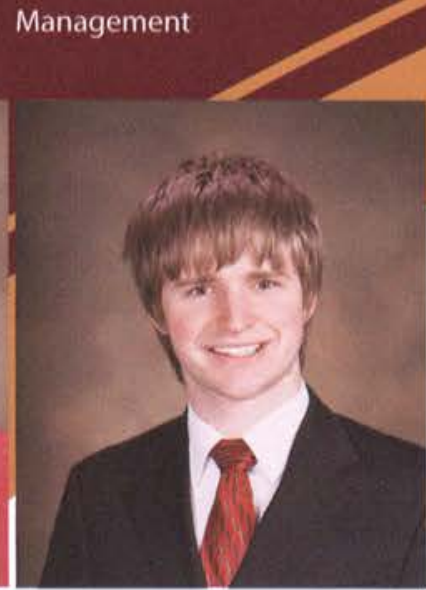

Nathan R. Landis

Computer Engineering

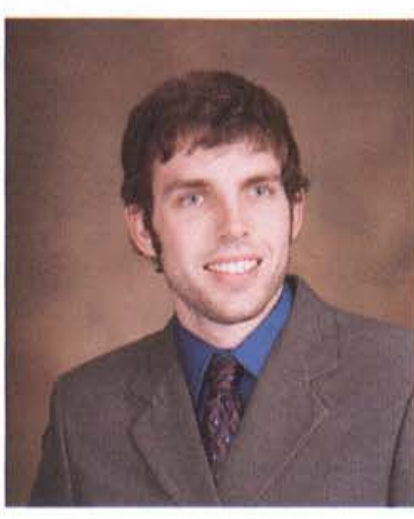

Evan A. Lee

Psychology

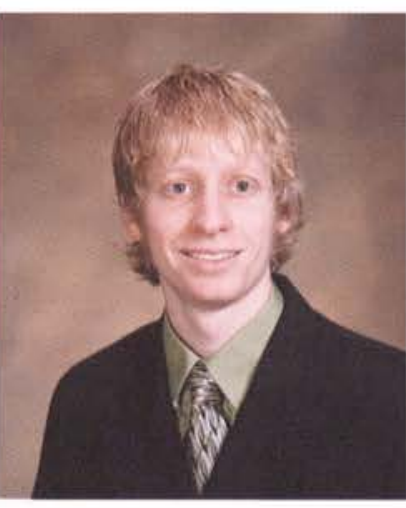

Derek M. Kreider

Middle Childhood Education Jennifer Bethany Krier
Studio Art
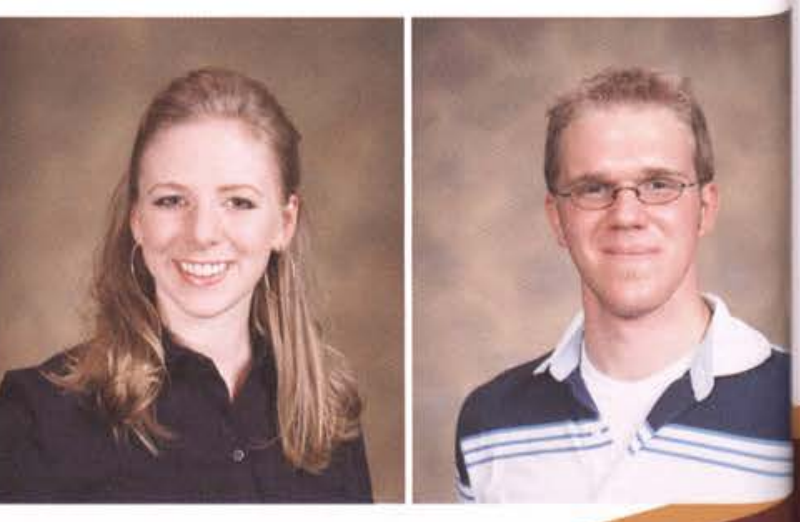

Devin Michan/Krupka

Finance, Accounting \& Politic Science

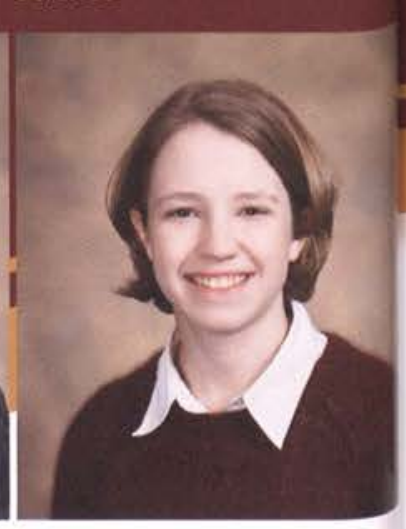

Kathryn Elizabeth Lakomy Philosophy

Mark Andrew Kwast David Michael Lagan Electronic Media
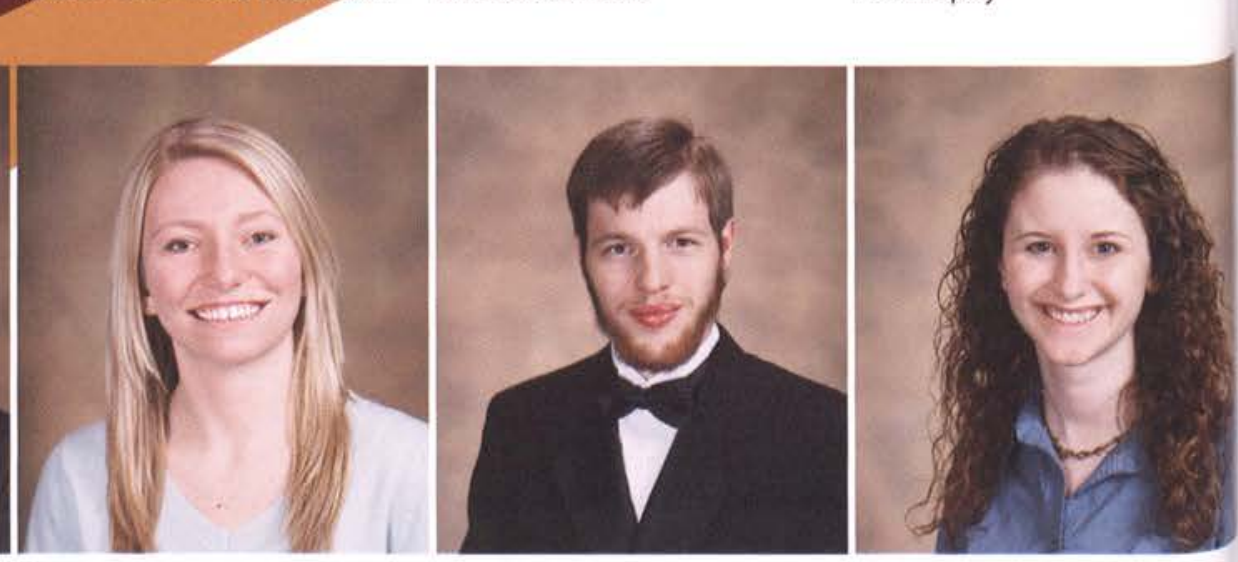

Stephanie Sue Langley Accounting

Joshua Edward Lansford Computer Engineering

Jessica Lynn LaPorte Early Childhood Education

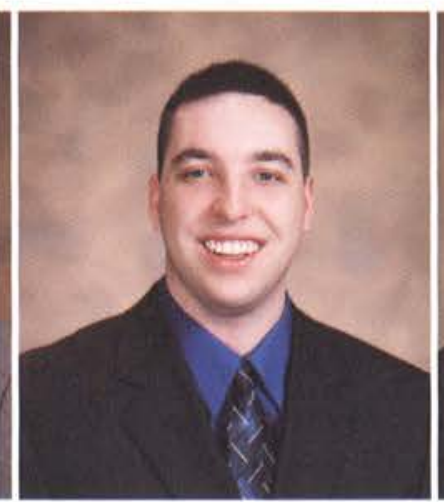

Andrew James Leeds Management Information Systems

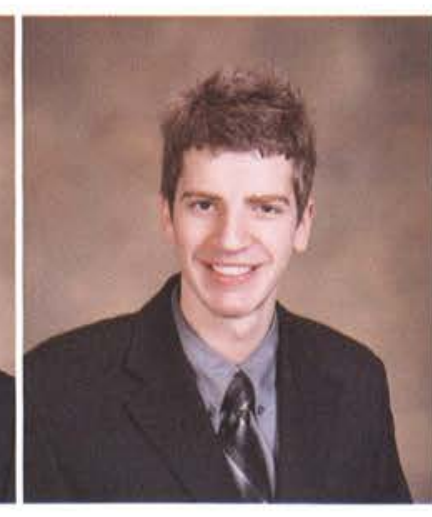

Grant Mitchell Letizia Communication Arts

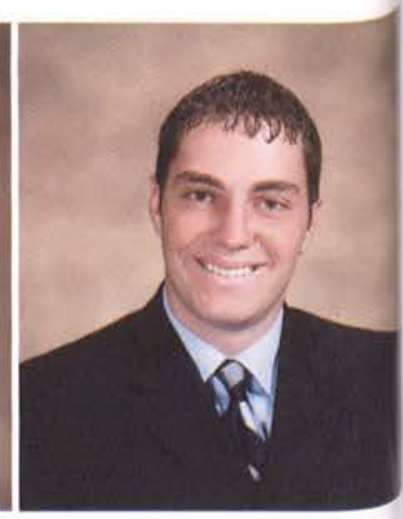

Jacob Wayne Lewis Finance \& Management 

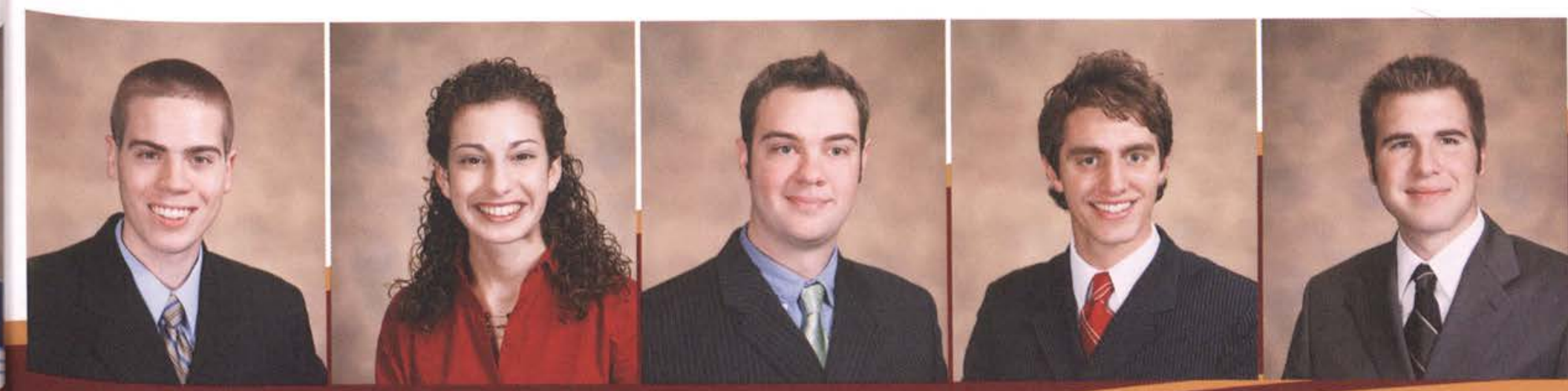

\section{David Alton Lindholm Chemistry \& Pre-Med}

Lindsay Allison LoDico Accounting
Peter Phillip Loeser MechanicallEngineering
David Samuel Logan Individualized Studies
Justin Lawrence Logan Finance \& Accounting

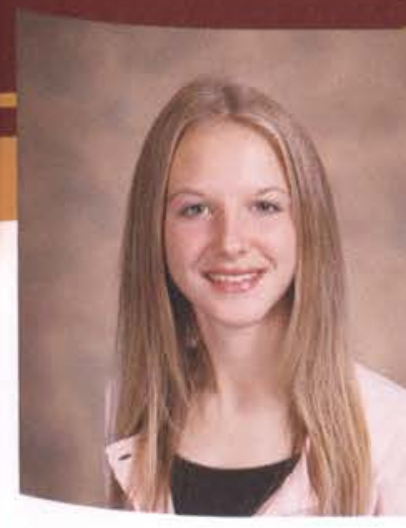

Jillian Susanna Losee Psychology \& Spanish

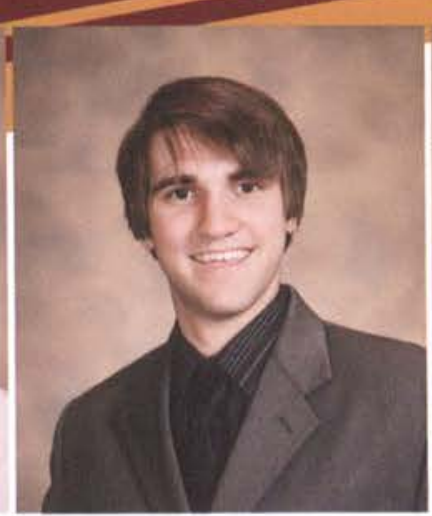

Trevor Macdonald Love Chemistry \& Biology

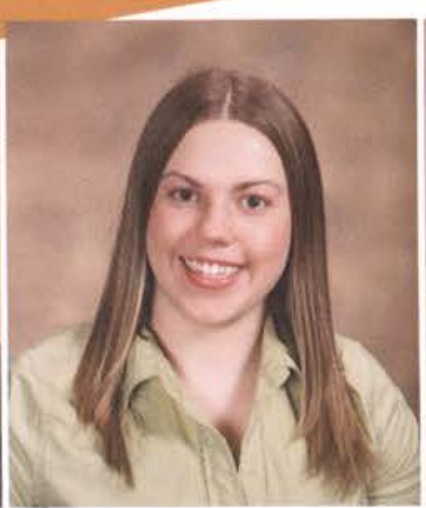

Christine Faith Lowry Nursing

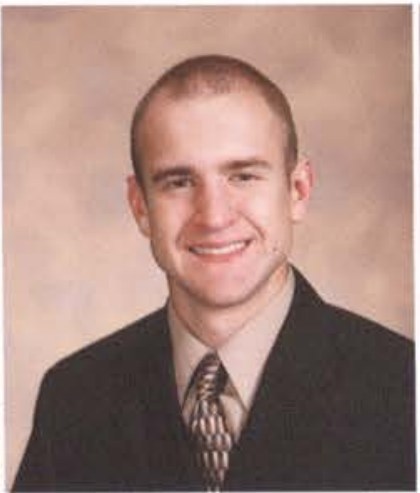

Timothy Ryan Luety Accounting

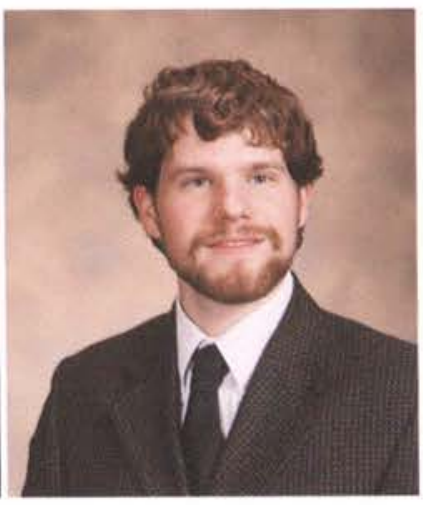

Dennis Alan Lunt Philosophy

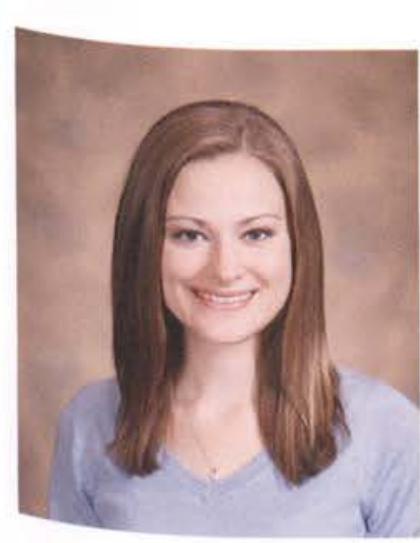

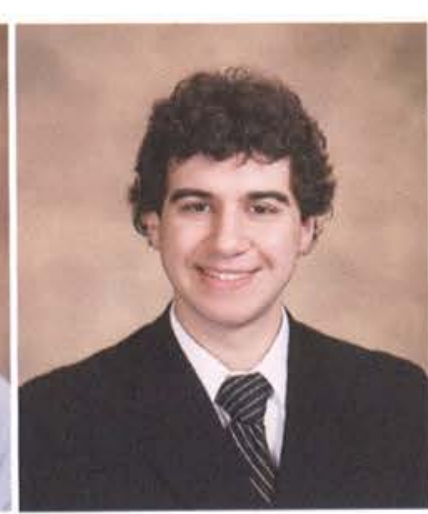

Colin L. Luther Electronic Media

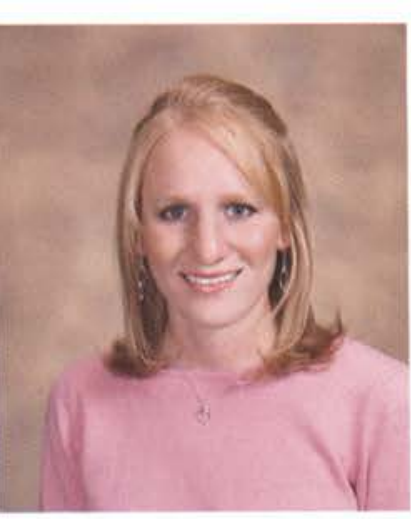

Kristen Marie Malpass Spanish

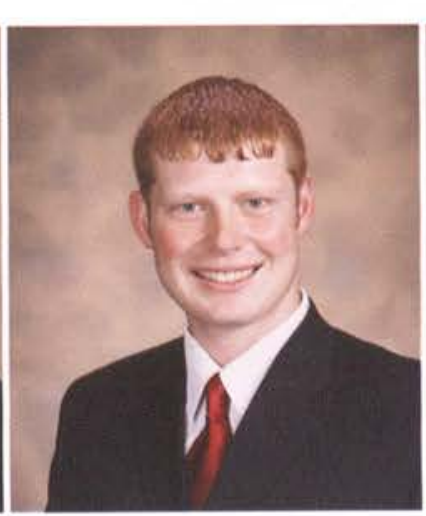

Justin M. Lyon Criminal Justice

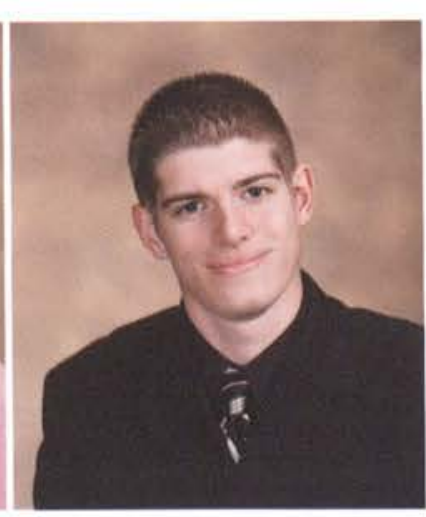

Jonathan A. Manchester History \& Political Science

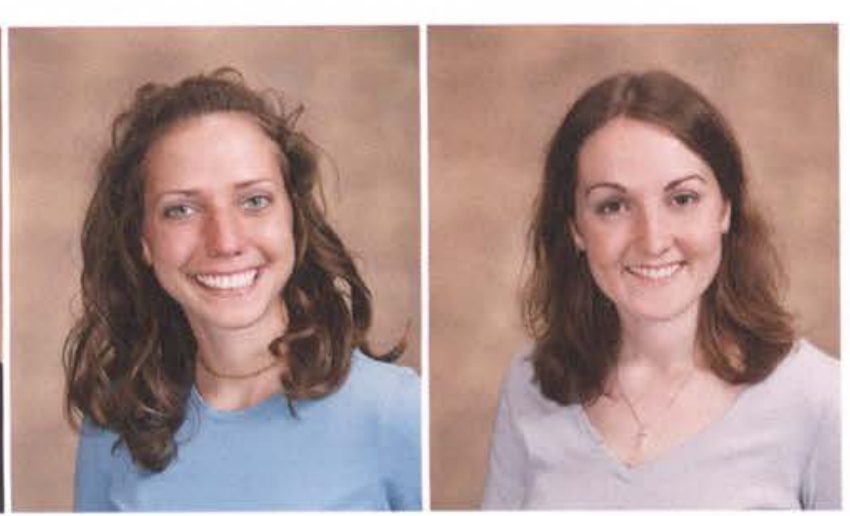

Samantha Maat

Kathleen Marie MacNeil Biology \& Pre-Physical Therapy Music Education
Jennifer Joy Magin
Social Work 


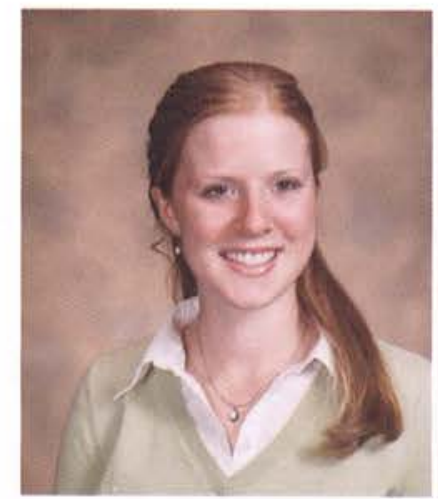

Adrienne R. Mansker Education

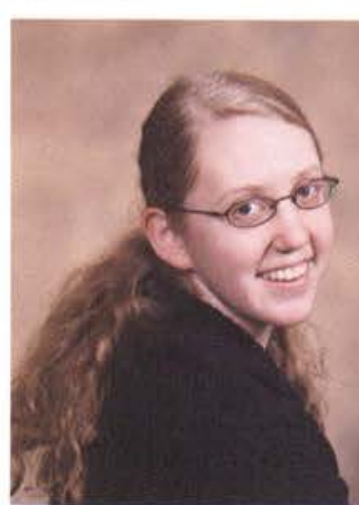

Andrea P. Martin Pre-Law

r

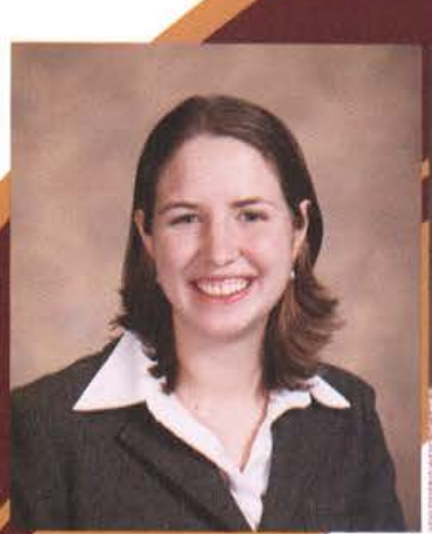

Lisa Virginia Mathews Mathematics
Brittany L. Marietta

Communications
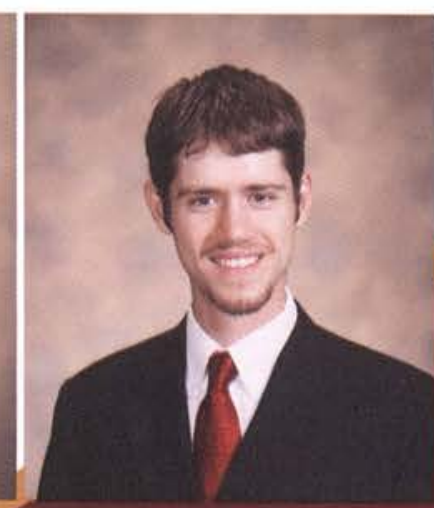

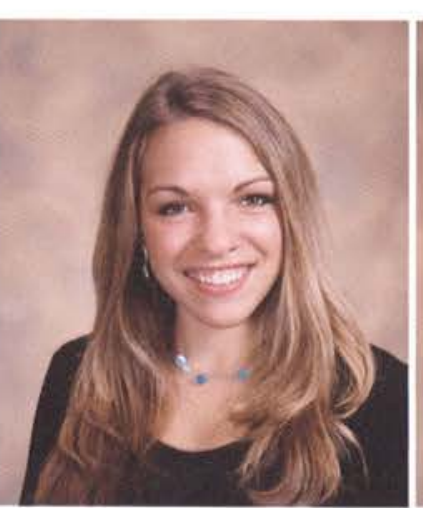

Brook Elyse Marietta

Organizational

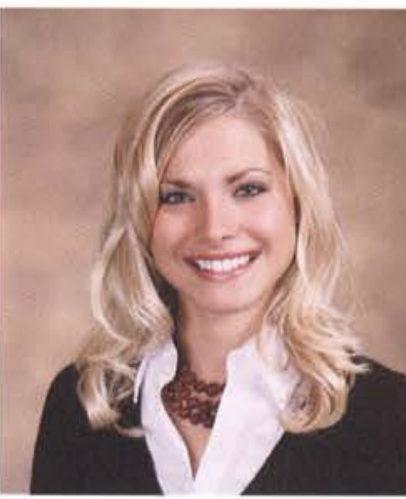

Stephanie L. Marnitz

Todd J.Marquardt

Multi-Age Special Education Management \& Marketing

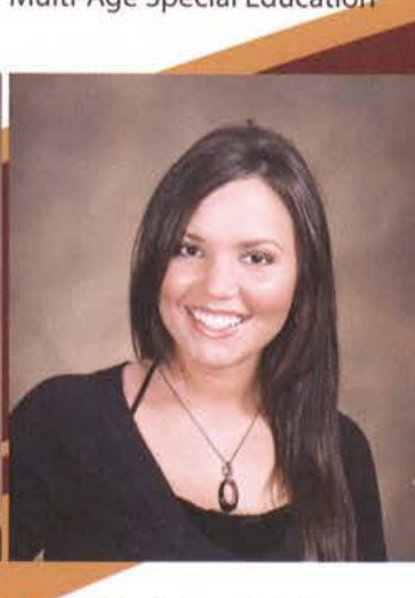

Rachel Marie Martindale Nursing

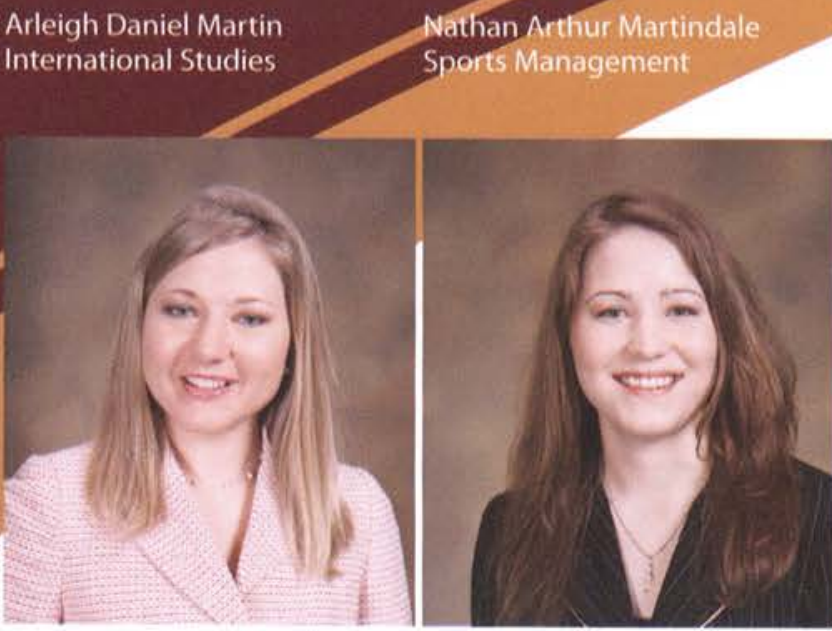

Joy Lynn Mathisen

Middle Childhood Education

Me

Nursing

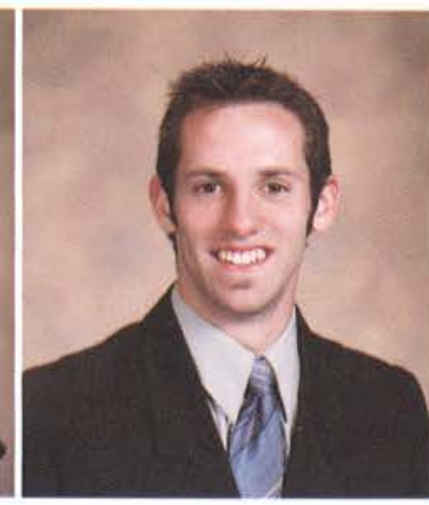

Joel William McCloskey
Kathryn Lambert McCay Communication Arts

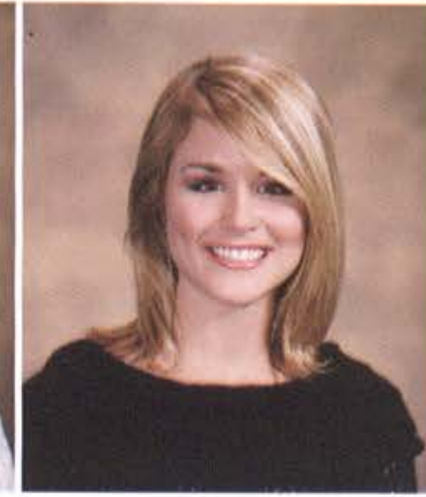

Finance

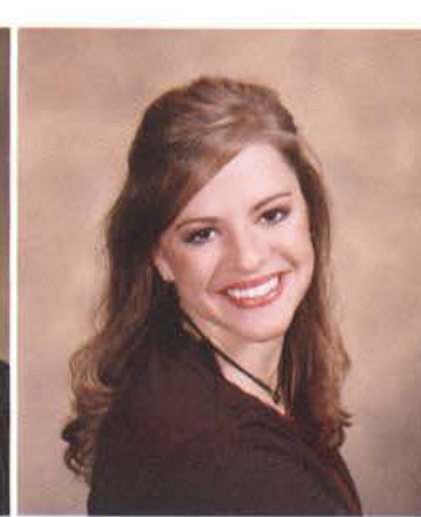

Hannah Christine May Christian Education \& Youth Ministry

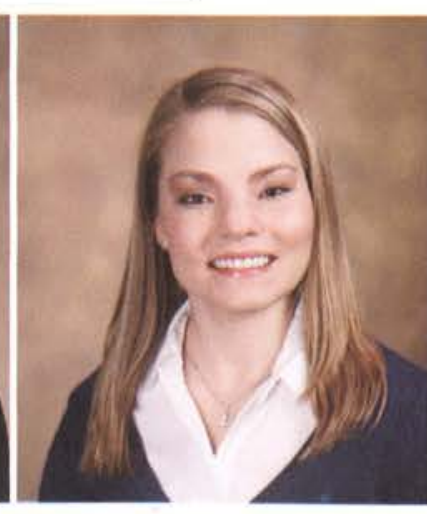

Laura Beth McClure Pre-Law

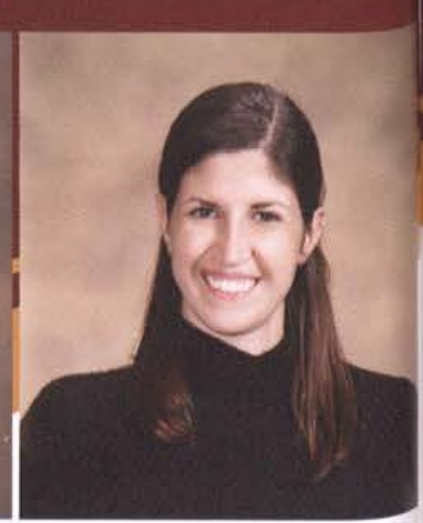

Amy Elizabeth Masterson Graphic Design

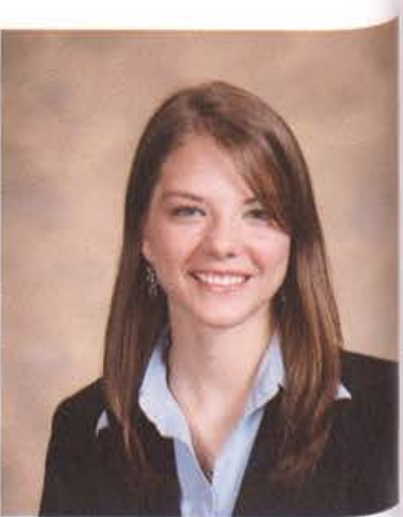

Elizabeth Anne Maynard \& Special Education

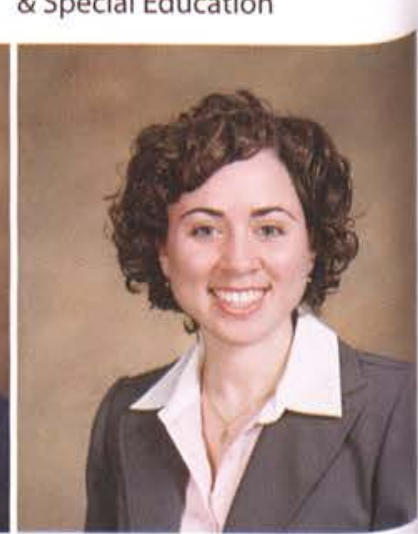

Amy Allyson McCormack Early Childhood Education Early Childhood Education 

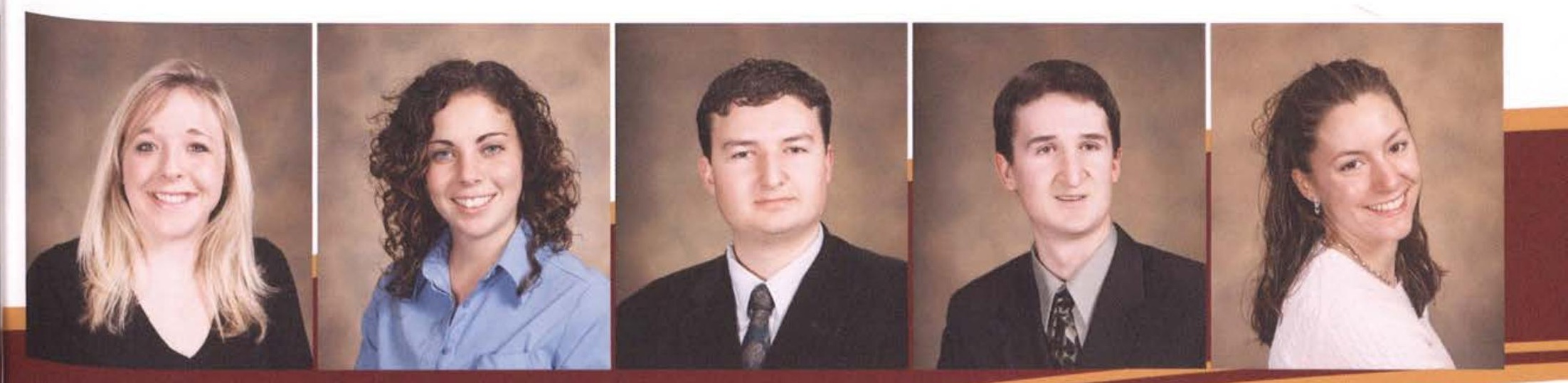

Elizabeth Katie McCrorey Psychology

Laura Ann McCully Marketing
David P. McCune YouthMinistry
Stephen-Andrew McCune Bethany Ann McDaniel Music Middle Childhood Education

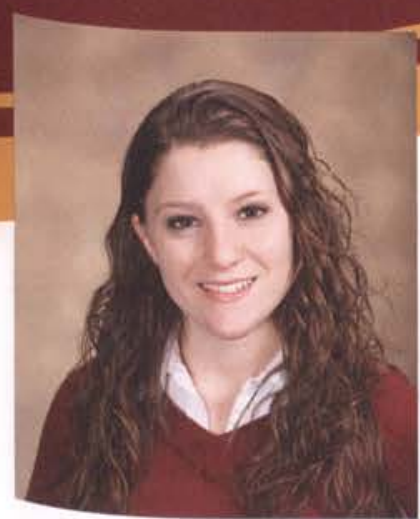

Kaitlin Amber McDaniel Integrated Language Arts Education

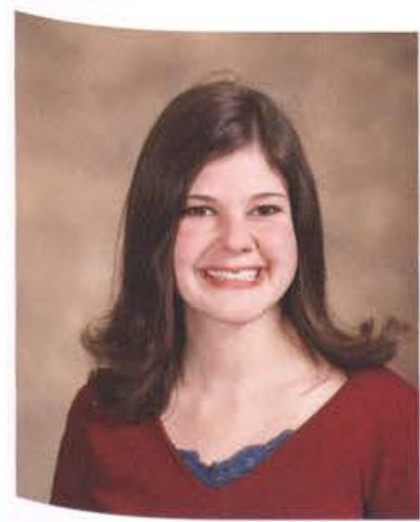

Brittany E. McNichols Nursing

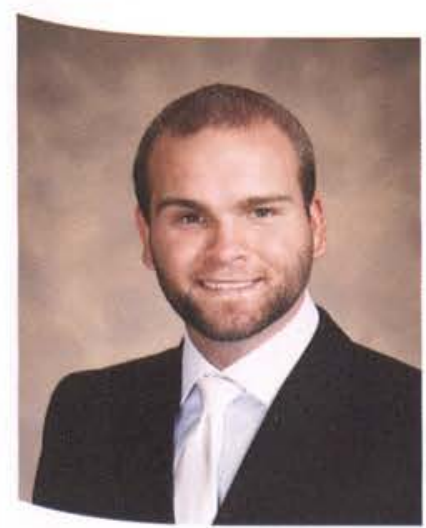

Griffin Paul Meyers Mechanical Engineering

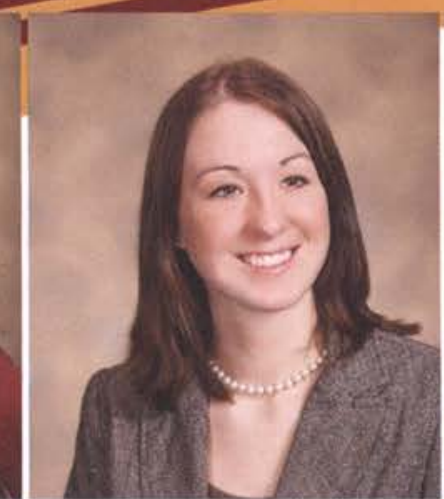

Naomi R. McDivitt

Early Childhood Education

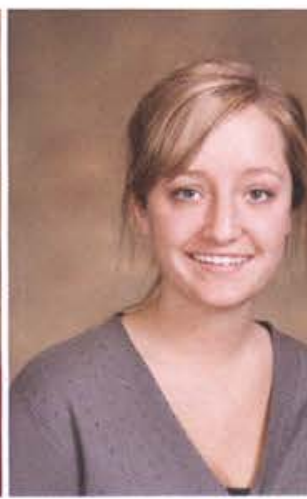

Tiffanie Renee Meissner Nursing

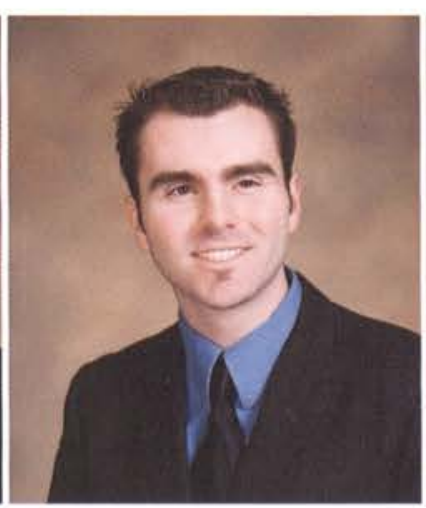

Derek T. Micucci Communication Arts

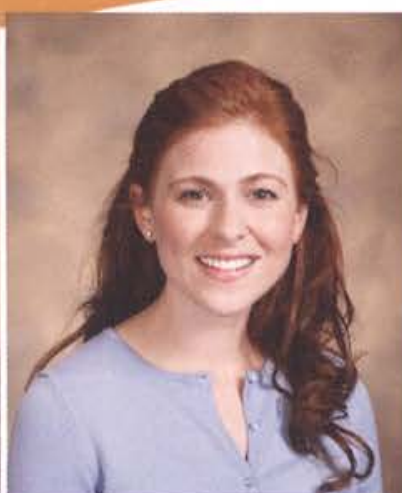

Lauren Elizabeth McGrew Nursing

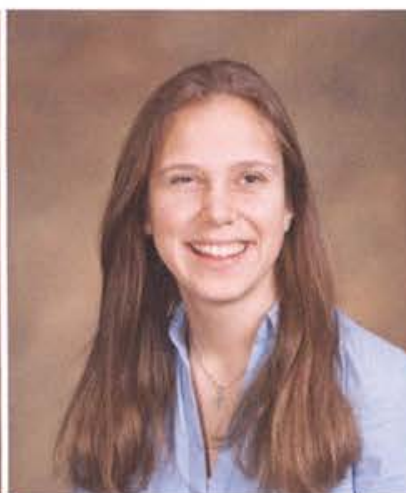

Jennifer Lynn McKellar Exercise Science

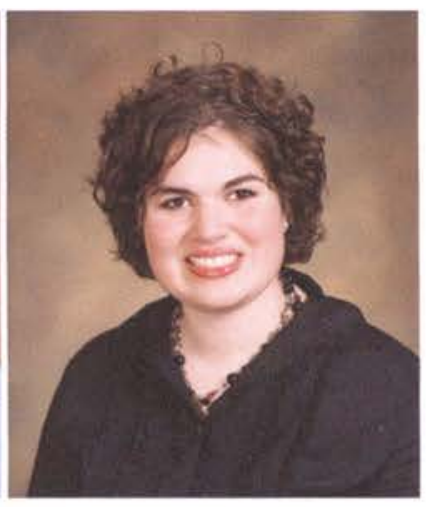

Amanda Lynn McKinley Political Communications

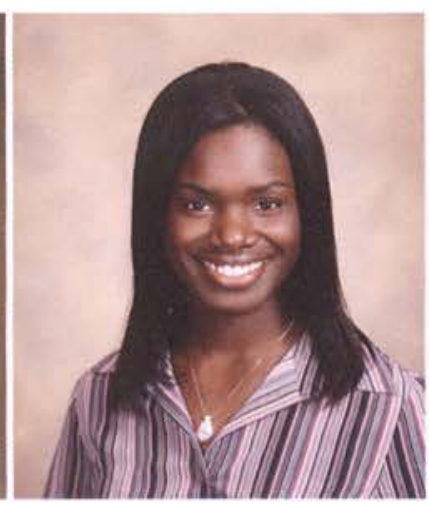

Vashti Terese Mensah Biology

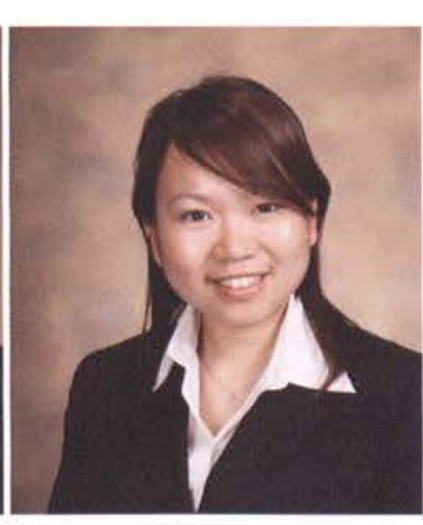

Aya Midorikawa

Middle Childhood Education

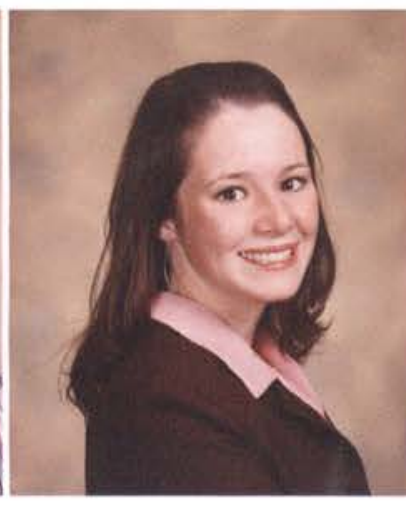

Michelle Nicole Merkh Early Childhood Education

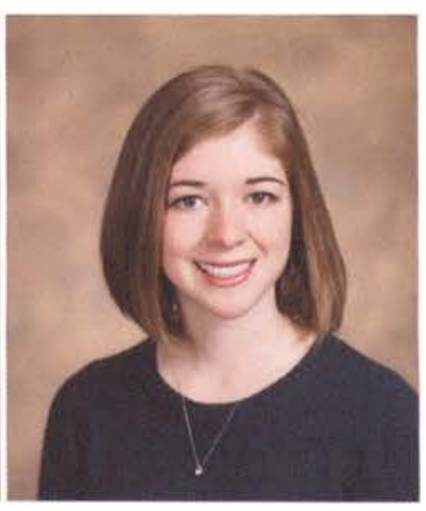

Kara R. Merrick Graphic Design

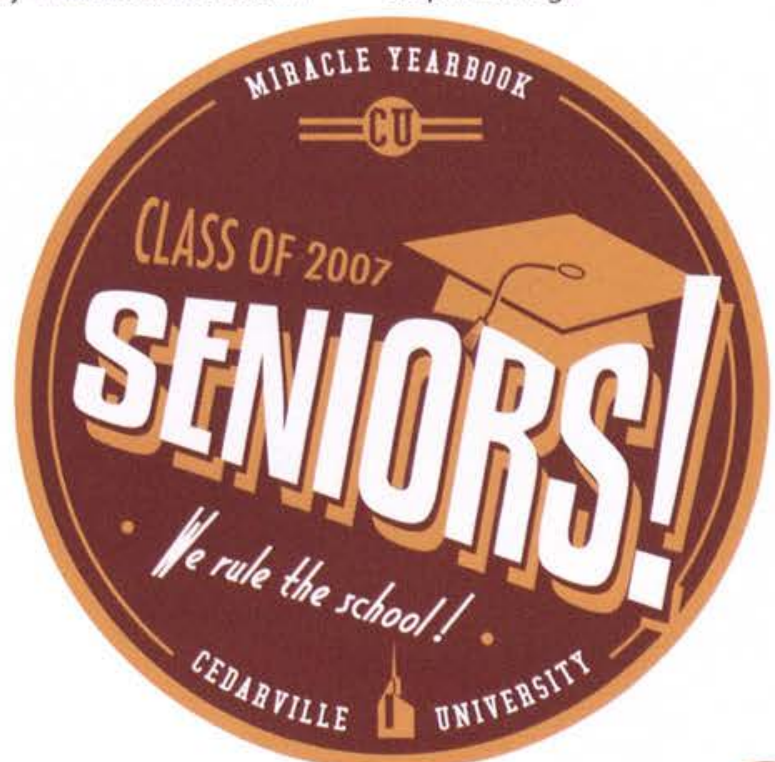




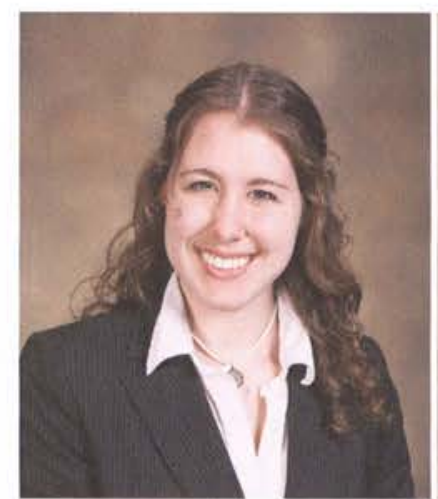

Hannah Pearl Mingonet Exercise \& Sport Science

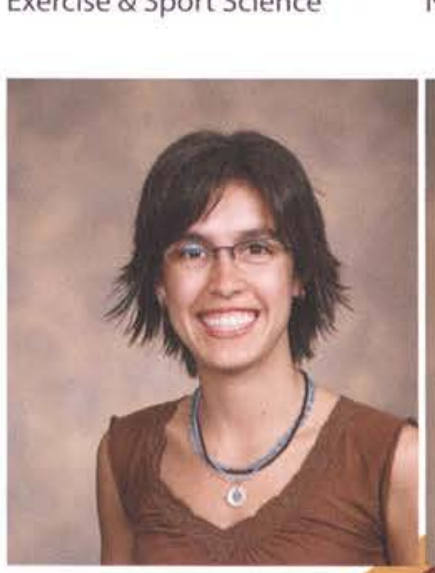

Loren Nicole Montes

Nursing

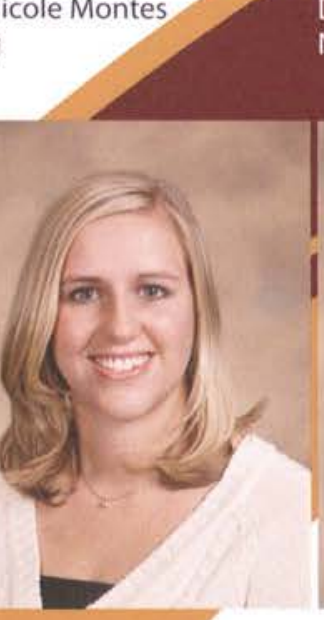

Nichole Marie Mouw Nursing

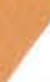

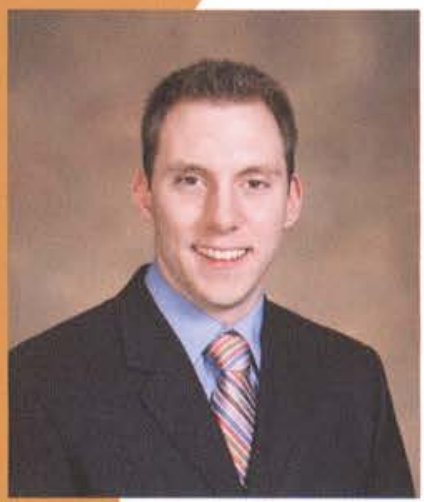

Timothy Scott Neetz Pre-Seminary Bible
Amanda Nicole Minor

Nursing

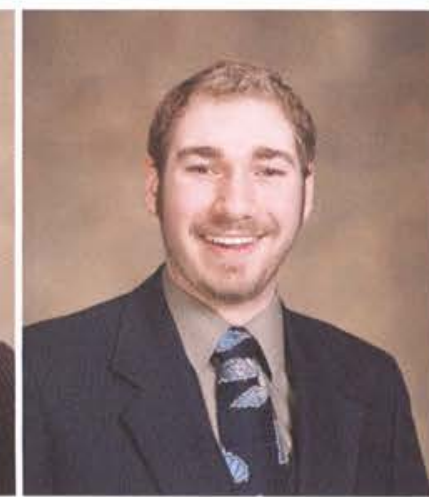

Eric W. Mishne

Theatre

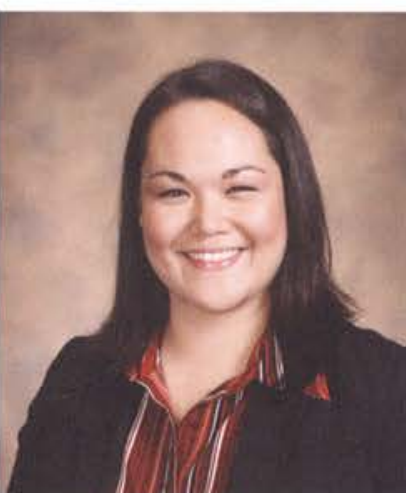

Amy Miura Social Work

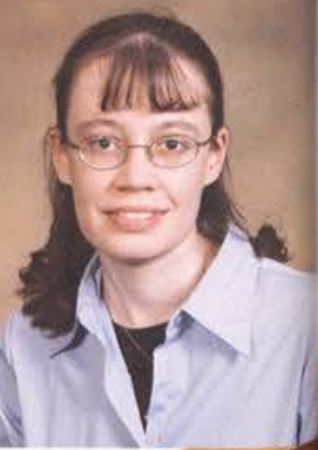

Andrea bawn Moeller

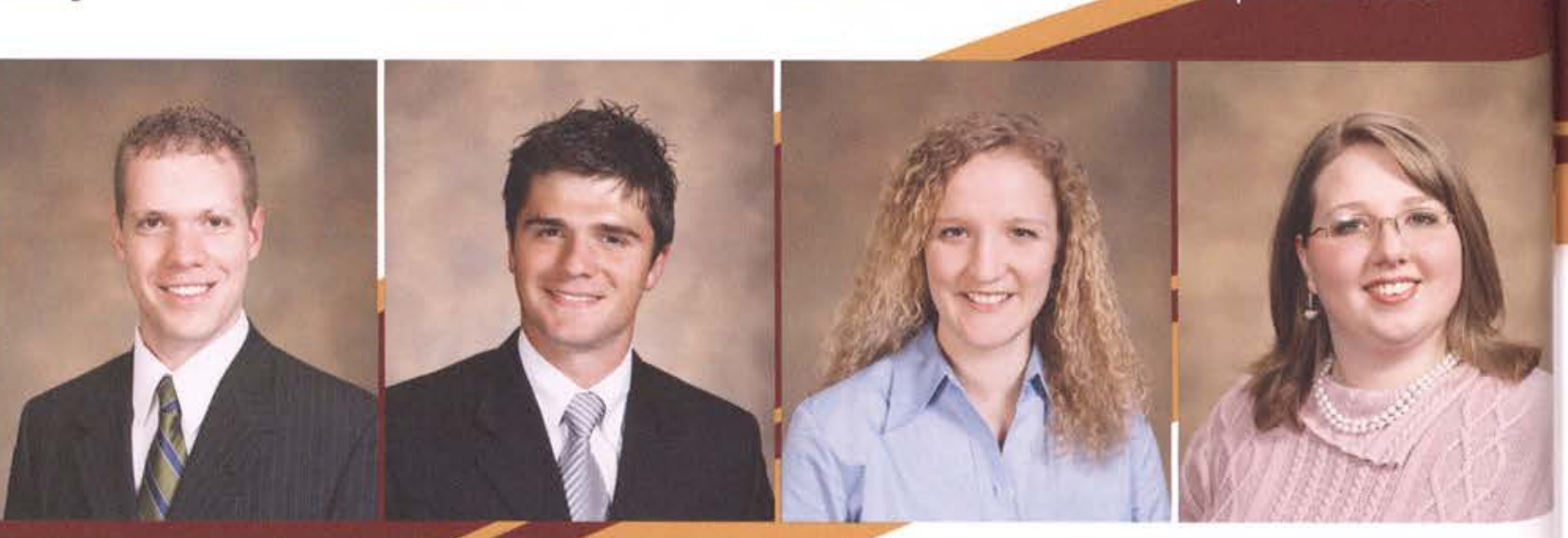

Kristina Sue Morgan Social Work

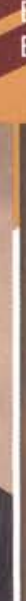

Elliot Robert Moore Exercise Science Ashley
Music
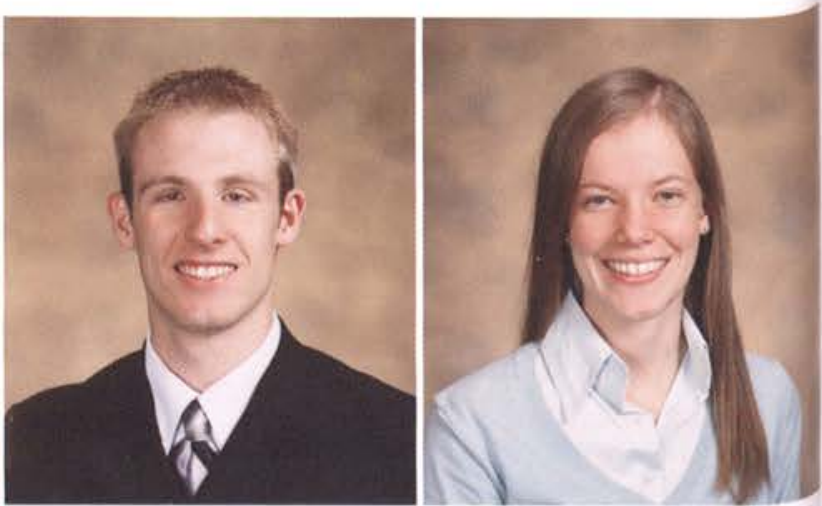

Jason Andrew Myers Comprehensive Bible
Kelly L. Myers Nursing
Brian Thomas Napp Accounting
Rebekah Sue Nearhoof Accounting

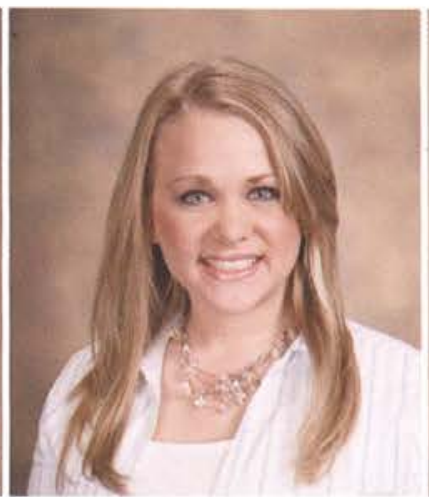

Kathryn A. Nicholl Social Work

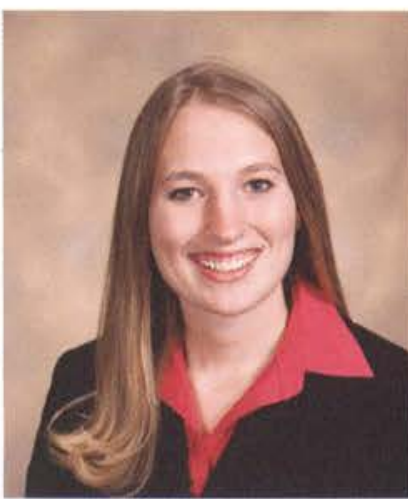

Tiffany E. Nickum International Studies \& Social Science

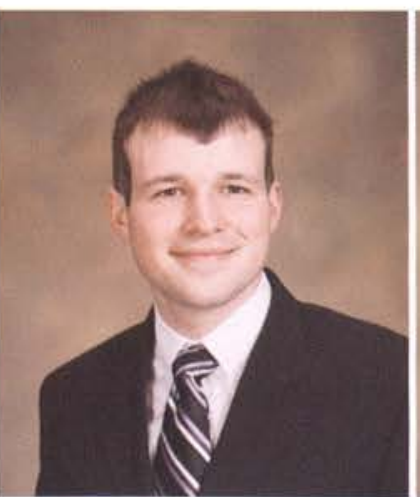

Daniel M. Nikitin Global Economics \& International Business

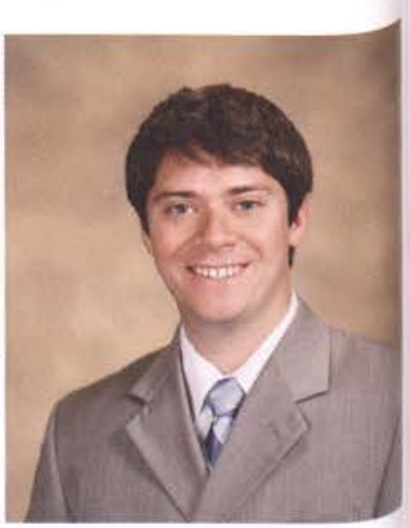

Aaron John Niznick Athletic Training 


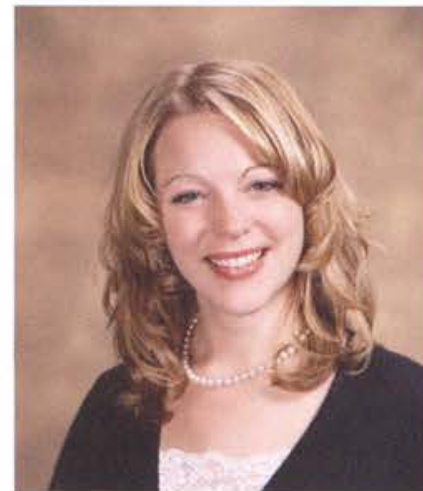

Kaitlin Joy Pankratz Applied Psychology

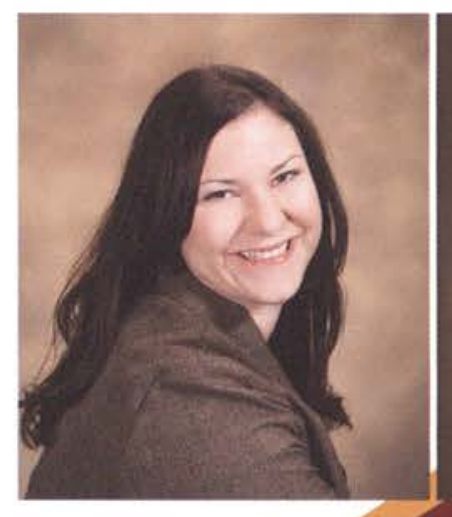

Emily Jean Peacock Psychology \& Criminal Justice
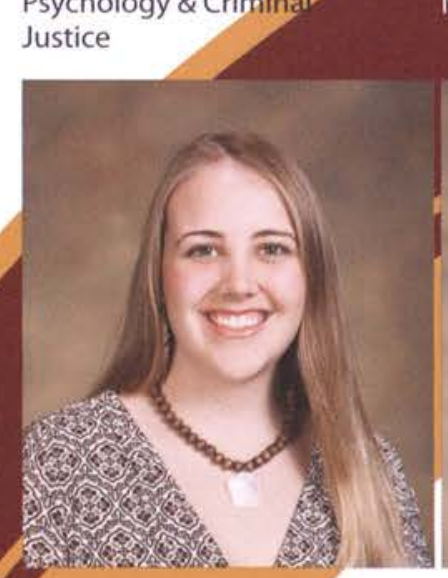

Maribeth Anne Phillips Nursing Pritips

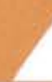

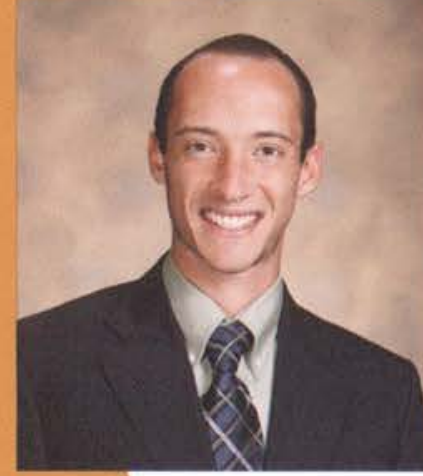

Bryan Stanley Pittman Management

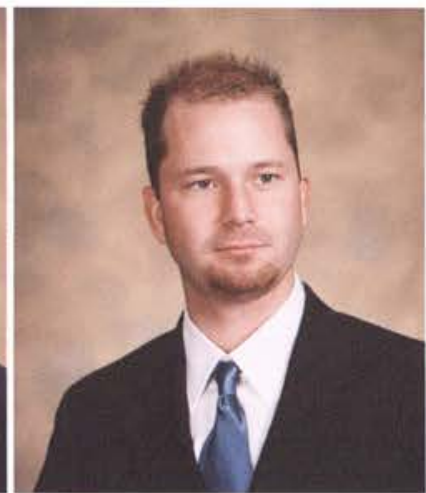

Michael Joel Parido Mechanical Engineering

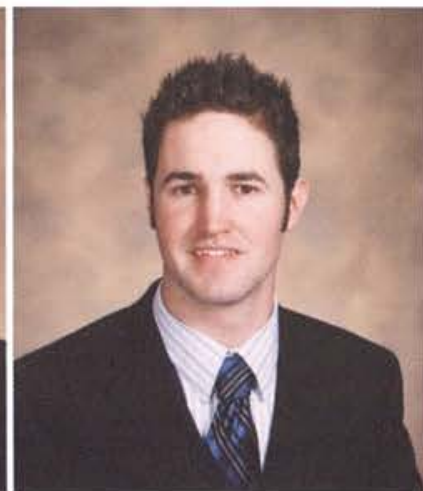

Charles Andrew Parks Mechanical Engineering

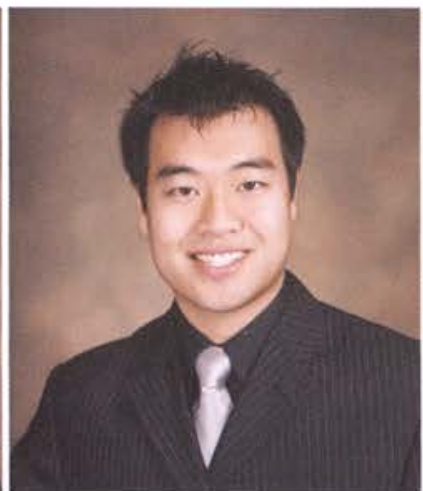

Neil Chan Patnaude Management Information Integrated Language Arts Systems

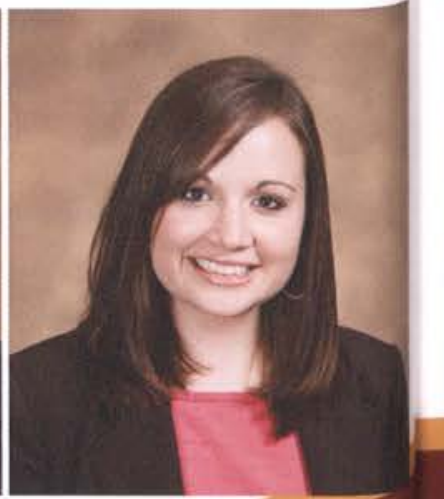

Education

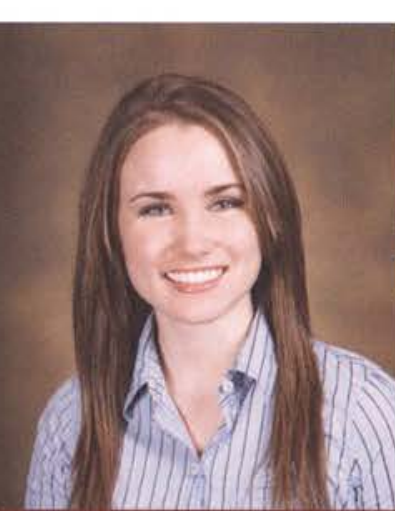

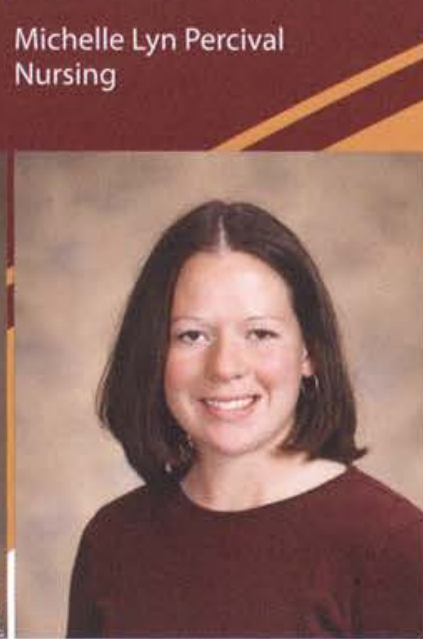

Rebecca L. Phillips Athletic Training
Kathleen Denise Picard Psychology

\section{aura Danae Perrien} Intercultural Communications Nursing r

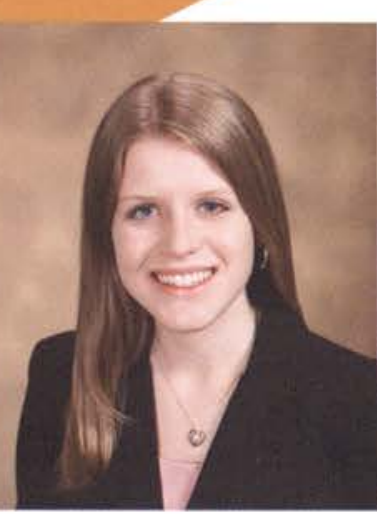

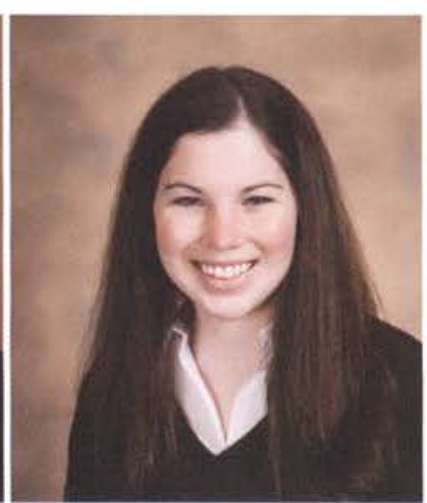

Bethany Marie Pifer English

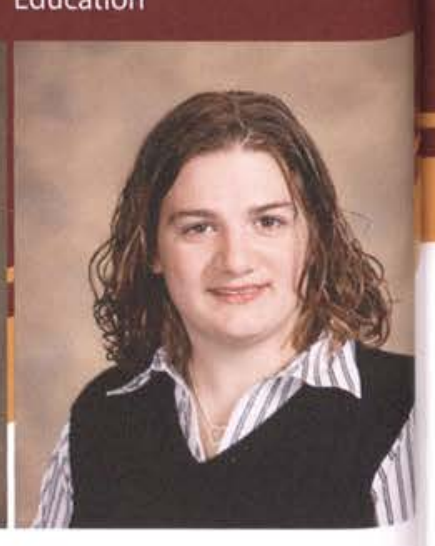

Tabitha L. Pfoutz

Youth Ministries \& Christian Education

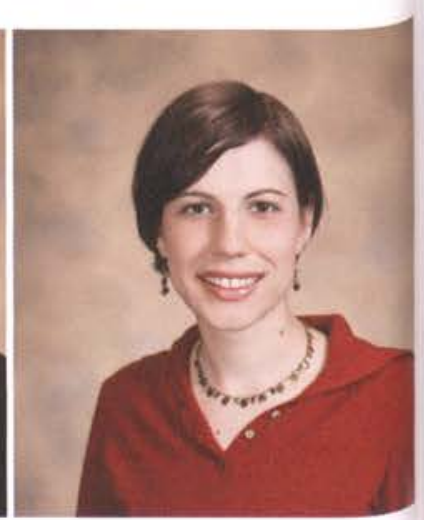

Hannah C. Pilgrim Nursing

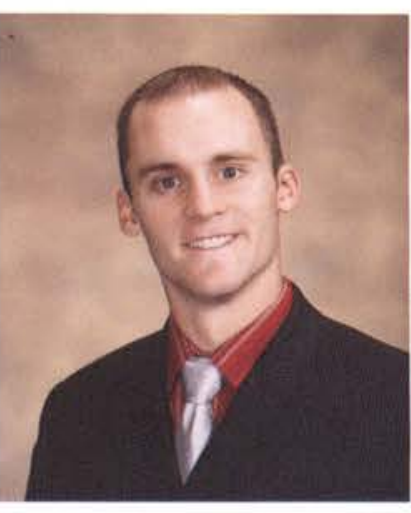

Adam Harold Ploeg Athletic Training

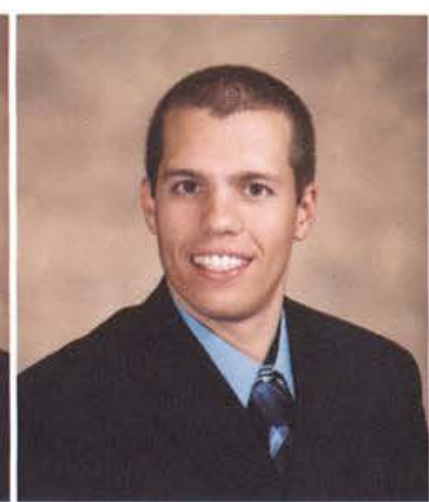

Christopher Steven Plume Mechanical Engineering

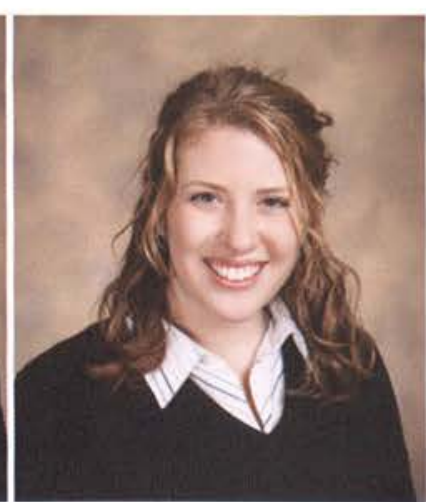

Karen Lynn Poch Social Work

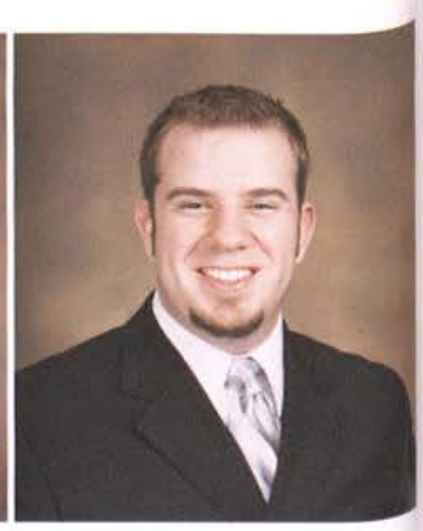

Joseph John Poelzer Psychology 


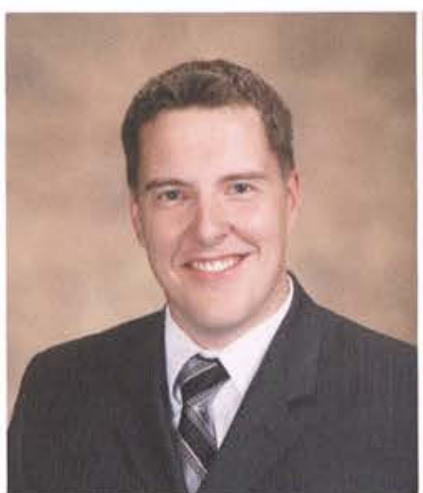

Michael Lee Rhoads Finance

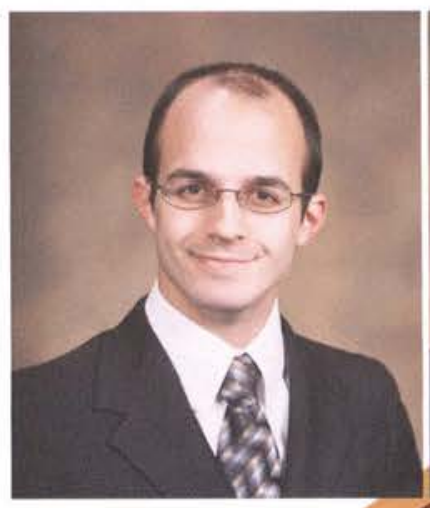

Timothy Seth Ritchey Management Informa: Systems
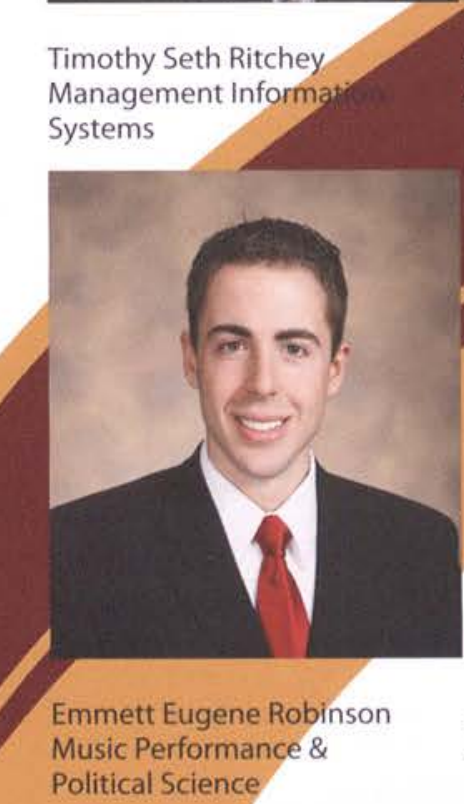

Emmett Eugene Robinson Music Performance \&

Political Science

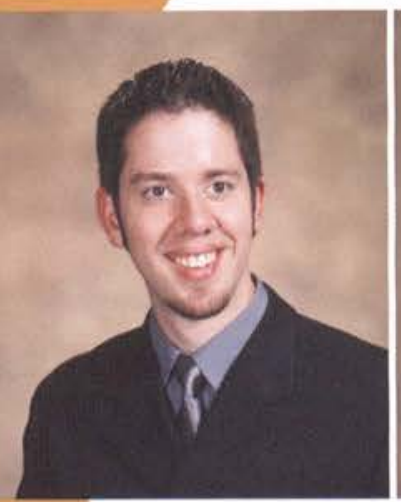

Ryan James Rotman Church Music Ministries

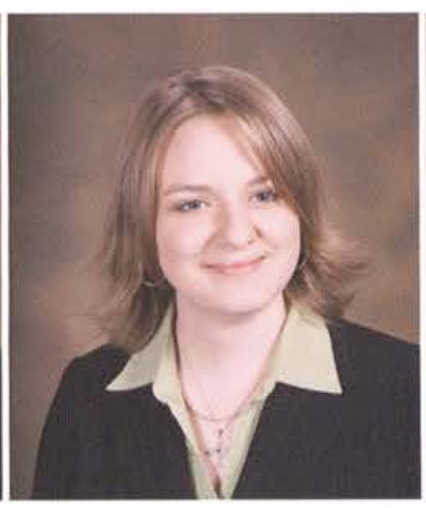

Courtney M. Rice Church Music Ministries

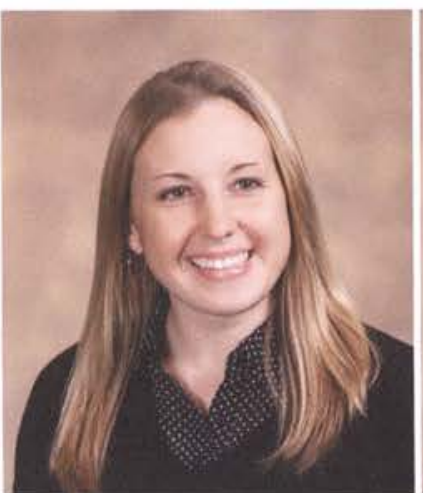

Regan Rachelle Rice Multi-Age Special Education \& Accounting \& Finance Early Childhood Education

Karen Lynn Rieck

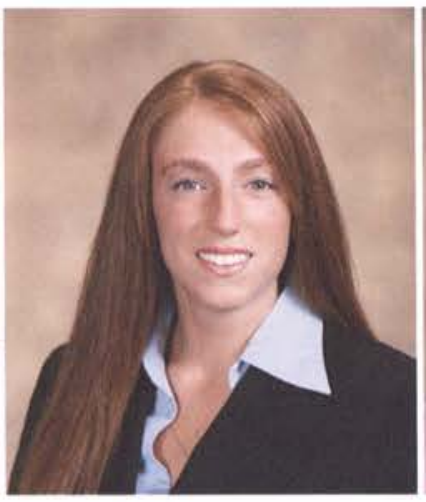
Accounting \& Finance Integrated Language Arts
Education

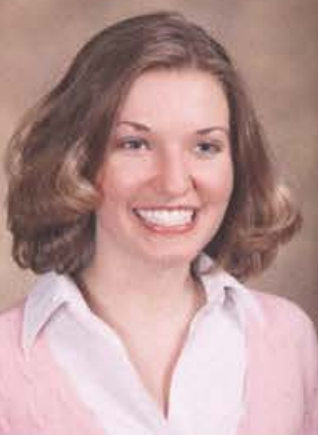

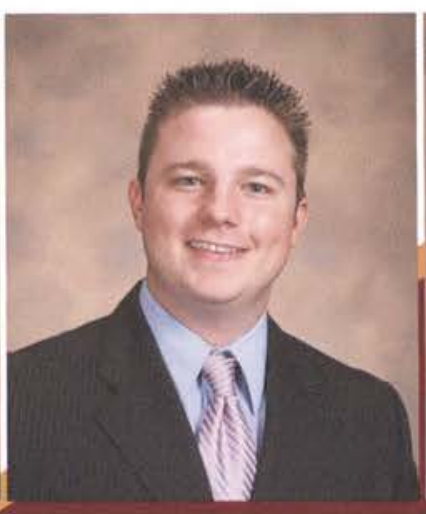
Aaron J. Riznick
Athletic Training

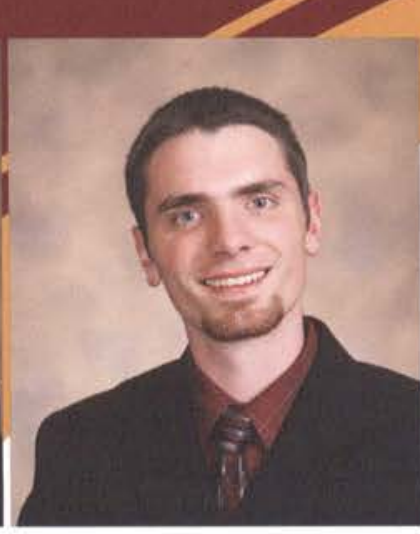

Jordan Timothy Root Applied Psychology

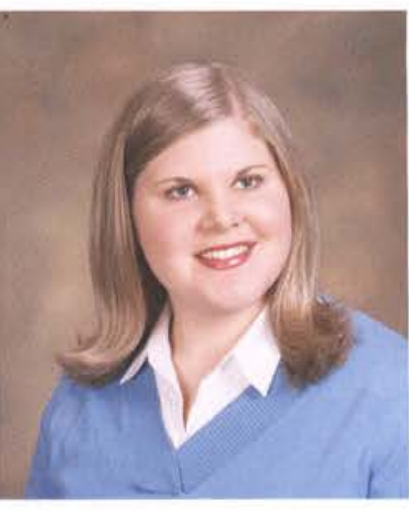

Cristine Lynn Rozelle Biology
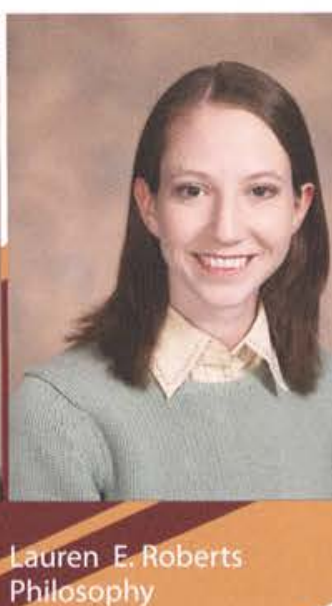

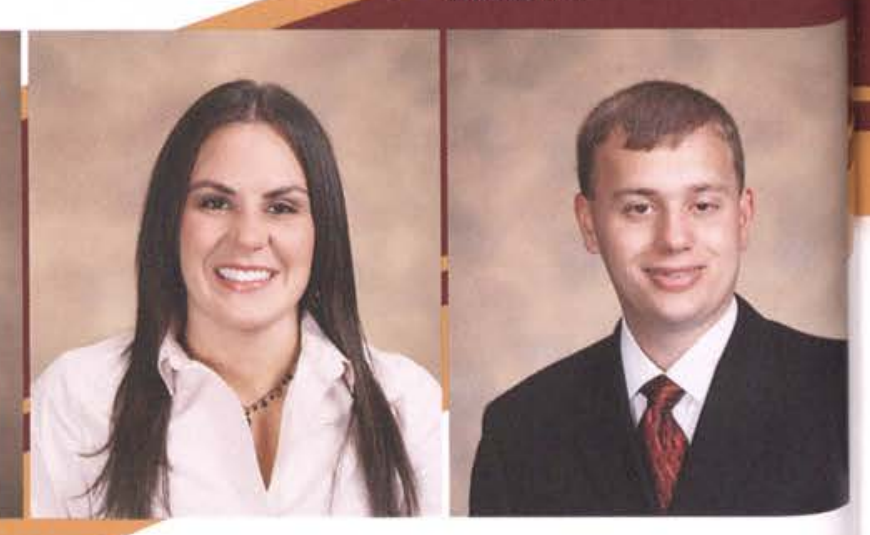

Eric D. Robinette Biology Kara Lynn Robertson
Nursing

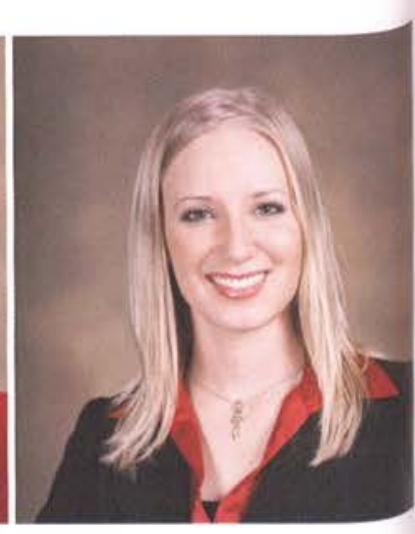

Adam Mark Rose Mechanical Engineering

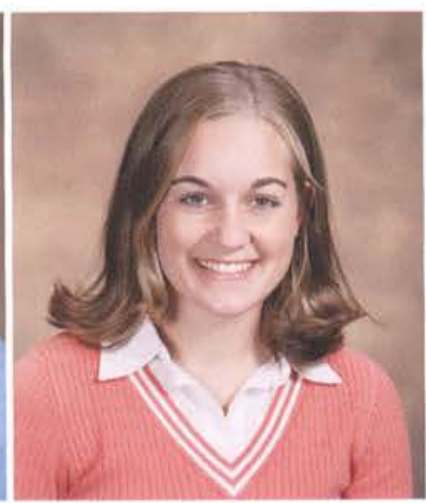

Karen A. Ruhlman Early Childhood Education

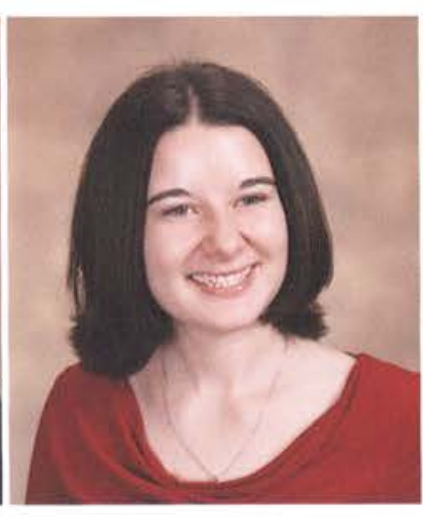

Ashley L. Ross

Comprehensive Bible

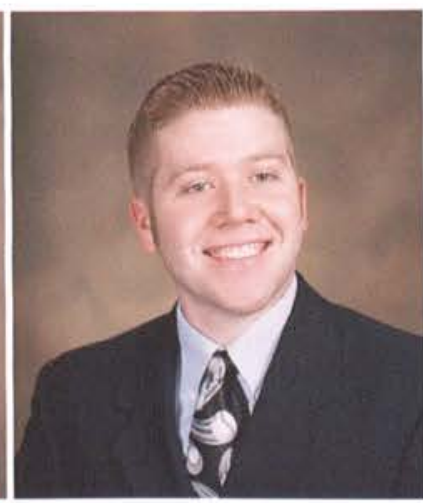

Richard J. Russell Sport Management
Jennifer Lauren Rost Organizational Communications

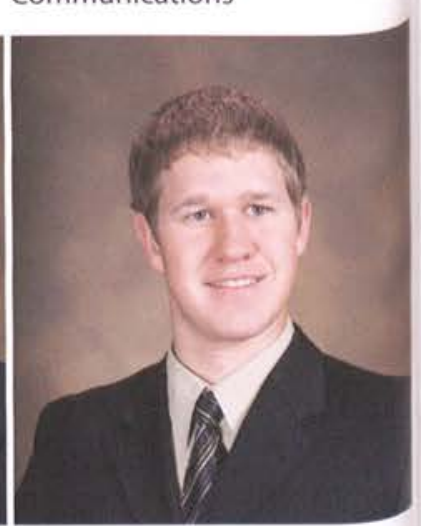

Gary Anthony Rutrough Management 


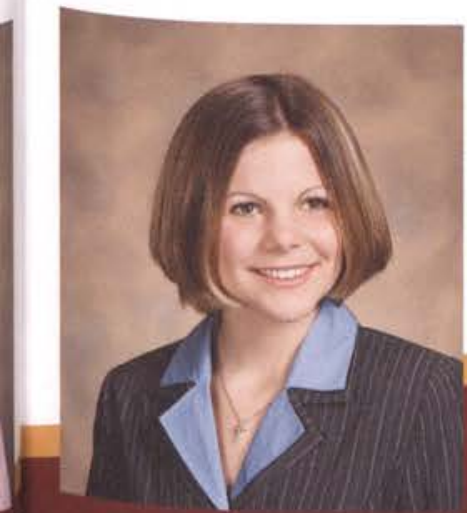

Kelsey Denise Salisbury Early Childhood Education

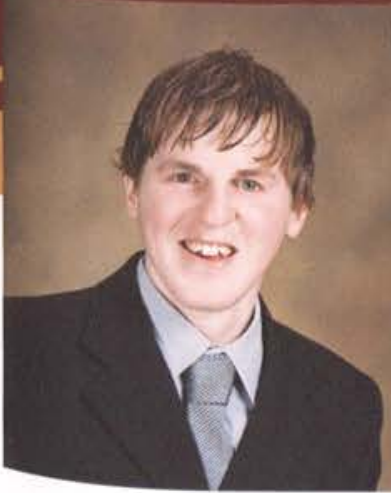

Timothy C. Sandlund Management

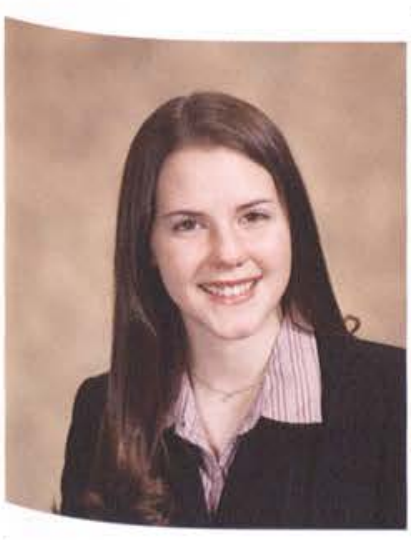
Karen Lynne Schanely
Music

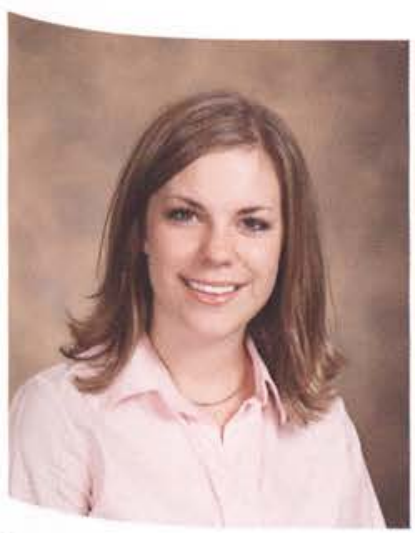

Teresa Lyn Schmidt Marketing

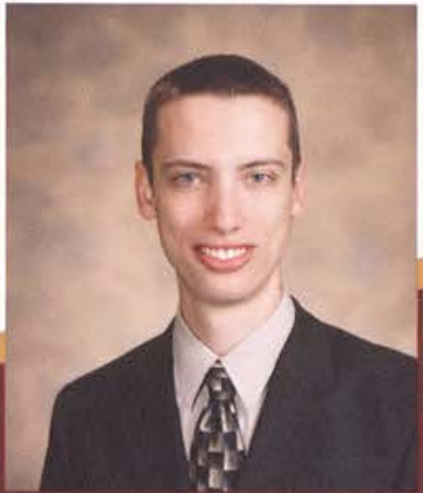

Brian L. Sallee

Global Economics

\& International Business

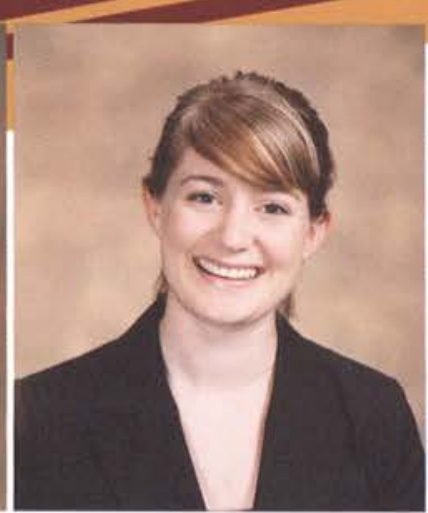

Daryl Anne Sando

Global Economics \&

International Business

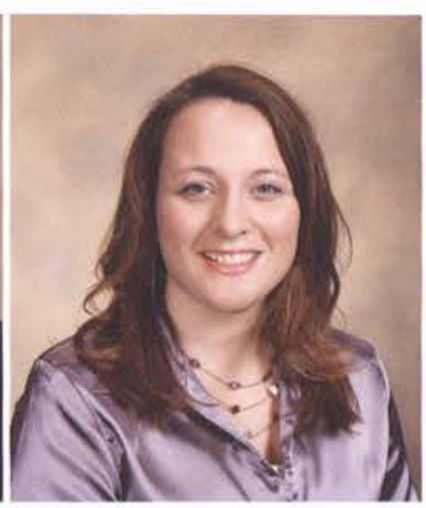

Jessica Erin Scheidt

Early Childhood Education

Joshua Rae Schenk

Management and Finance
Prycholo Sammons

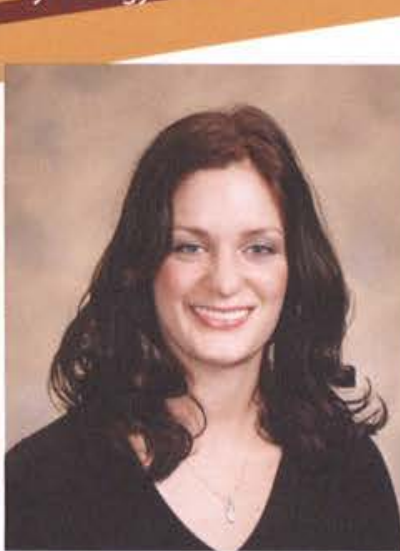

Kaitlin Christine Sands Communication Arts

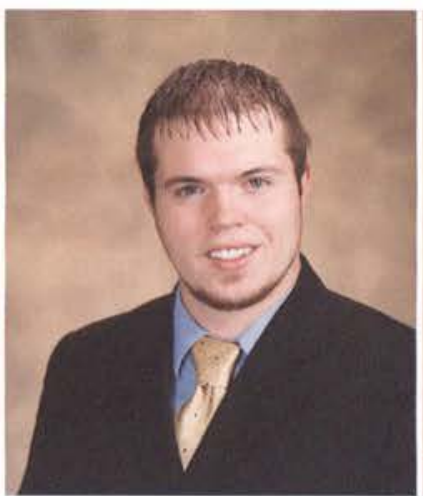

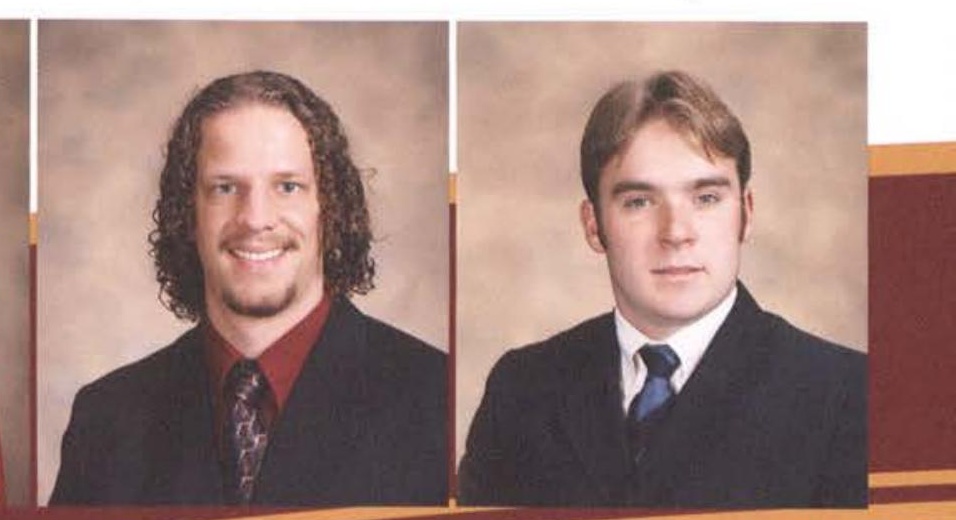

Aubrey Craig Samuelsen AYA Integrated Mathematics Management Education

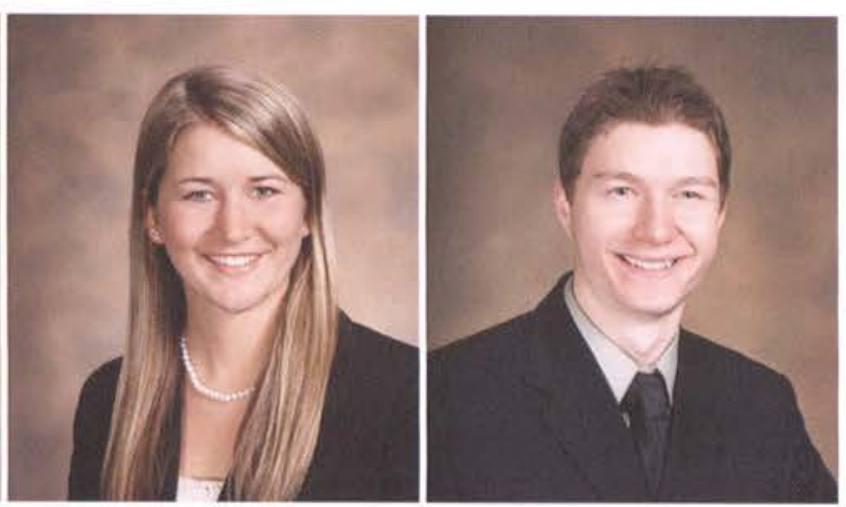

Katherine Diane Sawdon Organizational Communications

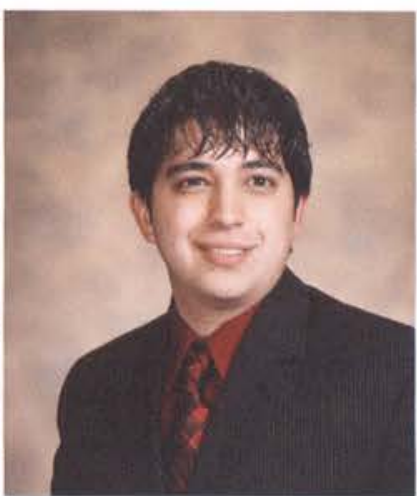

Matthew Lee Schermer
James Clifford Sawin Sports Management

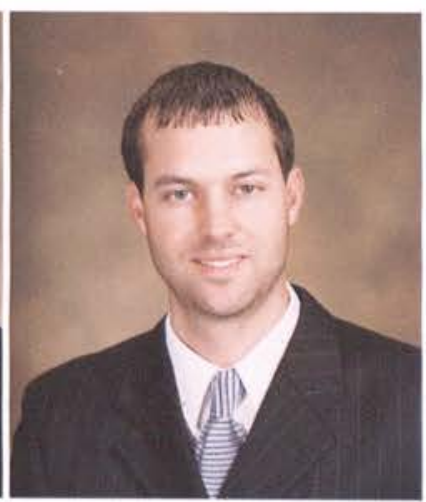
Sports Management

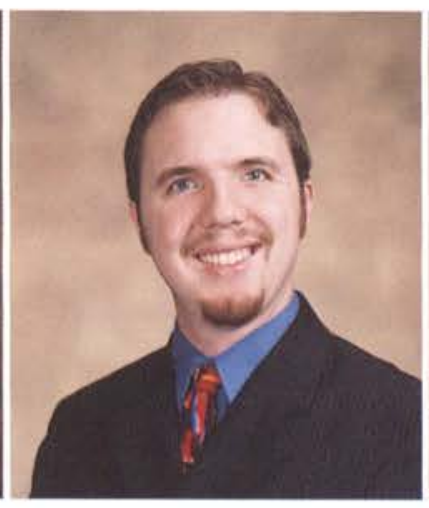

Bradley David Schmoyer Management Information Systems

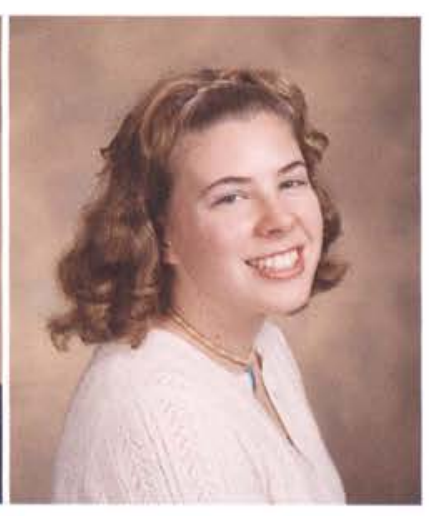

Rebecca Gail Schrank Nursing

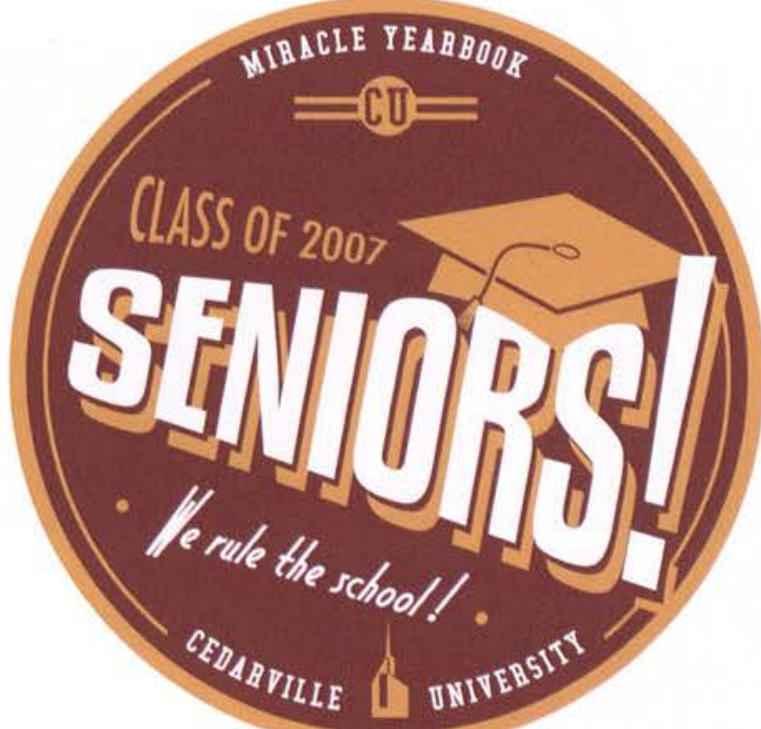




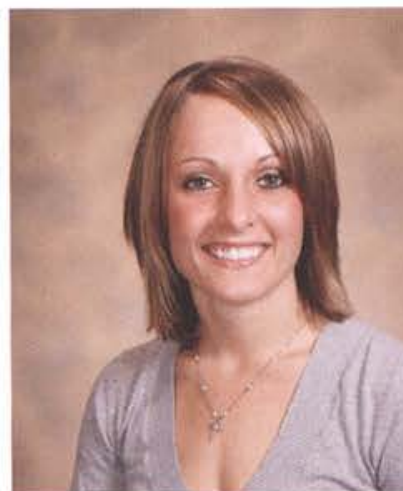

Karah Lee Schroeder Nursing

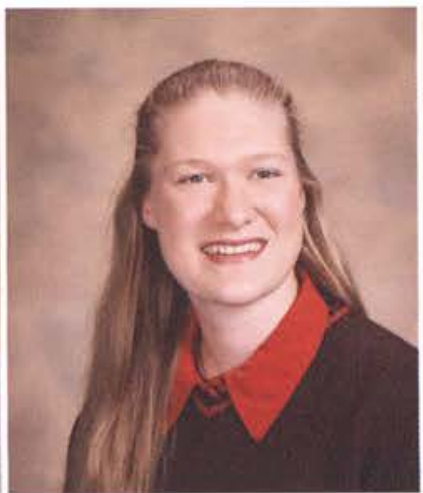

Marie Jewell Schumacher Middle Childhood Education

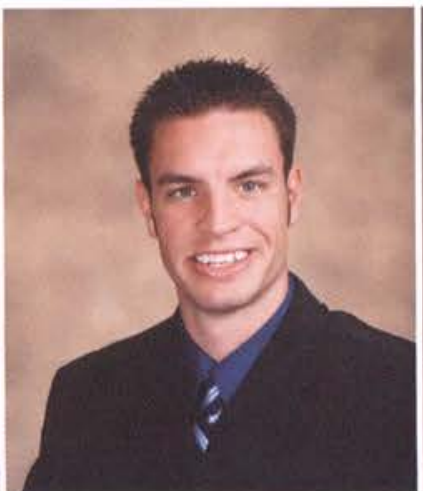

Tyler Kent Schumacher Mechanical Engineering
Brittny Leigh Scott Middle Childhood Education

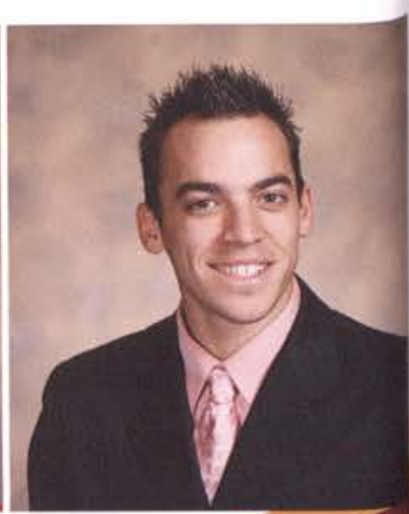

Jason Farnswent Scott Sports and Exercise Studies

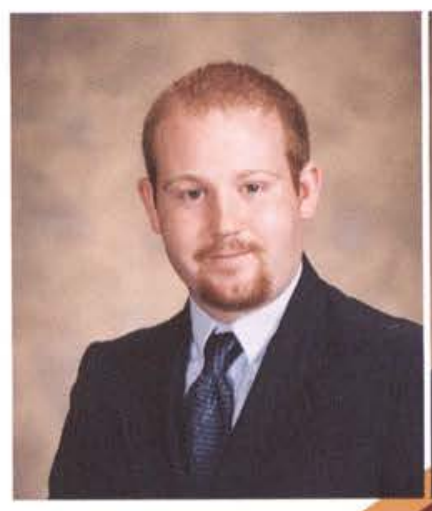

Todd Andrew Scott Criminal Justice

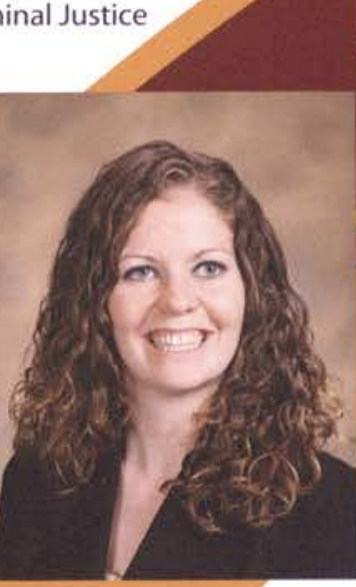

Stephanie Rene Shank Exercise Science

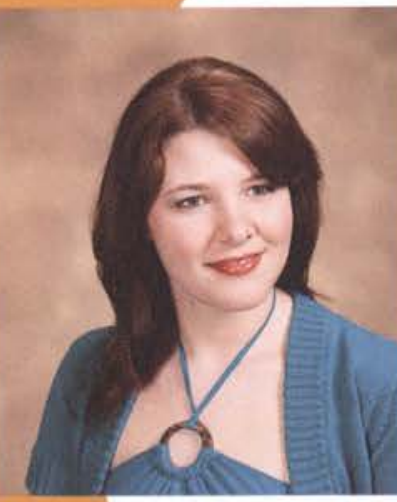

Bethany Grace Sibbitt Biology

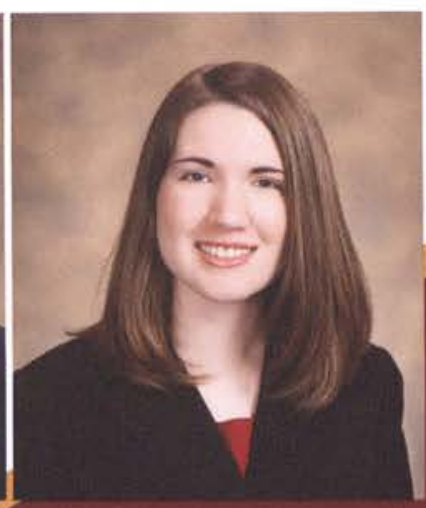

Kathleen Anne Scull Biology \& AYA Life Science Education

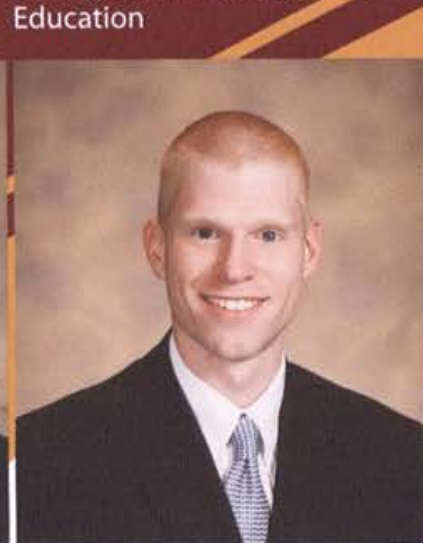

Peter A. Shellabarge Nursing

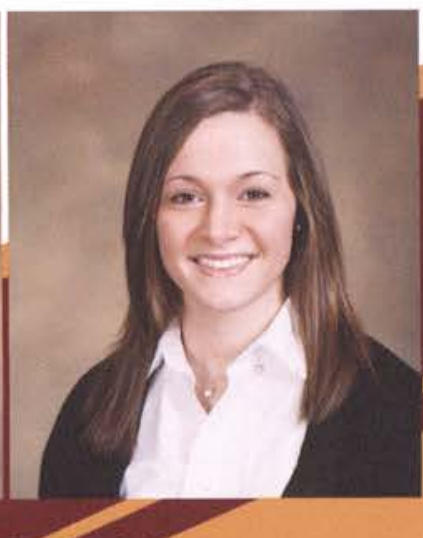

Sarah J. Searles Sociology

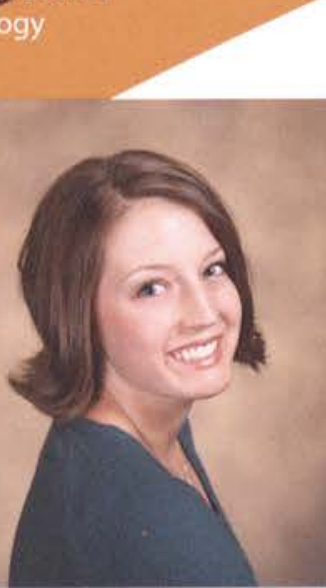

Alicia Dawn Sheppard Middle Childhood Education \& Science Education

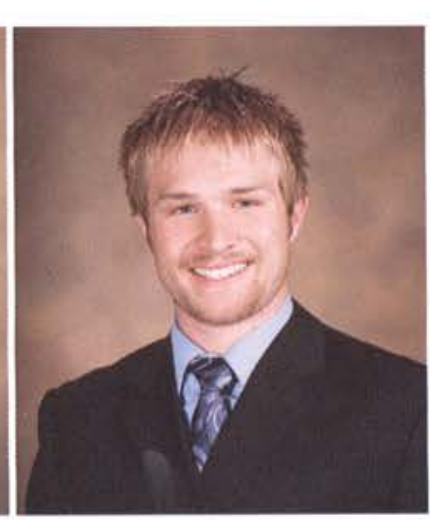

Philip Ryan Shimer World Missions

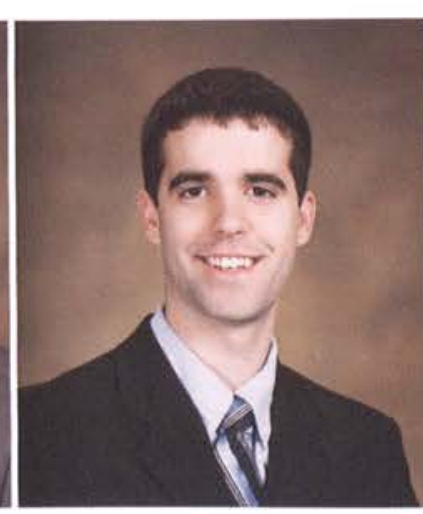

Eric David Simonaire Computer Science

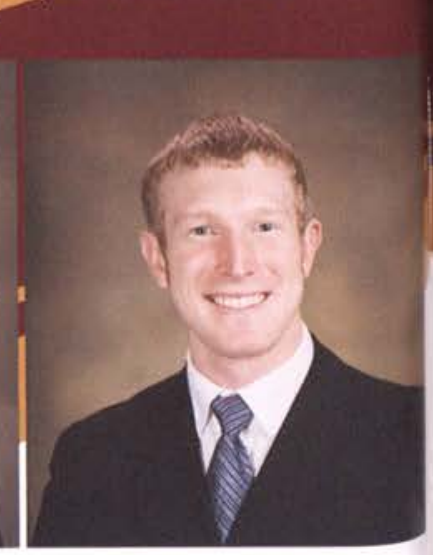

Jason Edward Shaeffer Mechanical Engineering

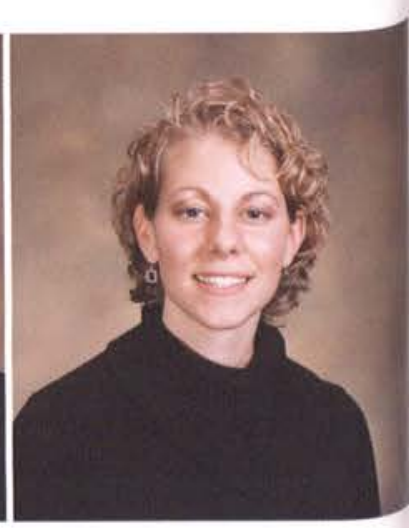

Rebekah A. Shipper English

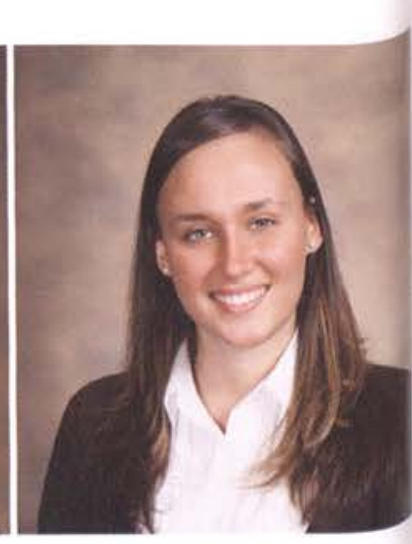

Tasha M. Simons Music Education
Keith Michael Sider Church Music Ministries
Daniel Robert Sietman Communication Arts

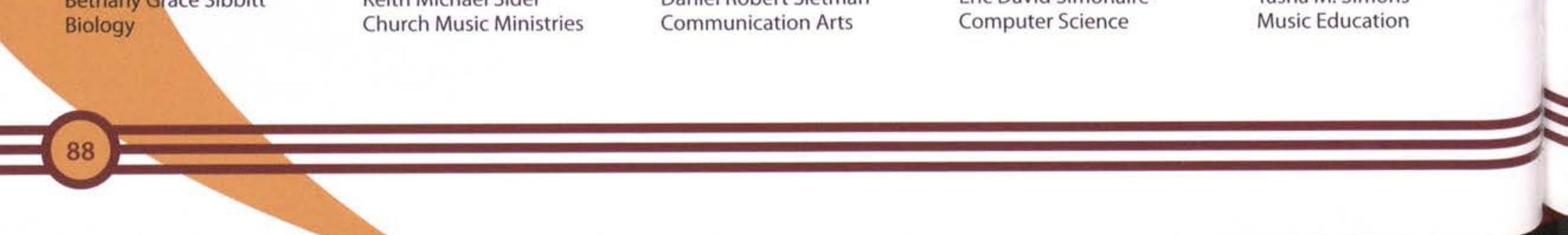



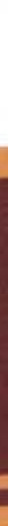

Cara Ann Slaybaugh

Brittany M. Smart

Sports Exercise Studies
Anna Grace Smith Multi-Age Spanish Education
Brandon Joseph Smith Criminal Justice
Candace Patrice Smith Early Childhood Education

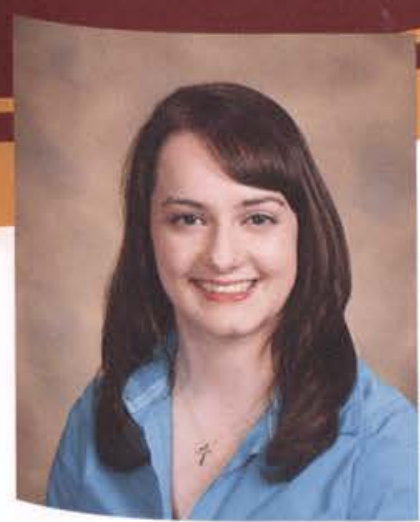

Elizabeth Richelle Smith Marketing \& Finance

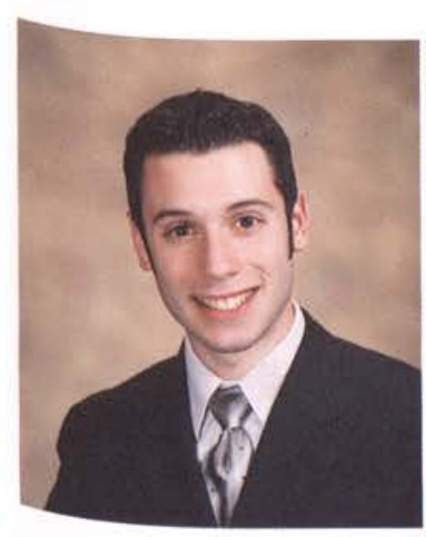

Robert K. Stahl

Accounting \& Finance

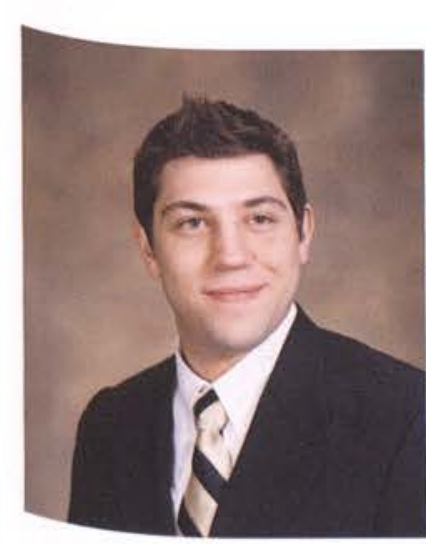

Andrew Wesley Steury

Accounting \& Marketing

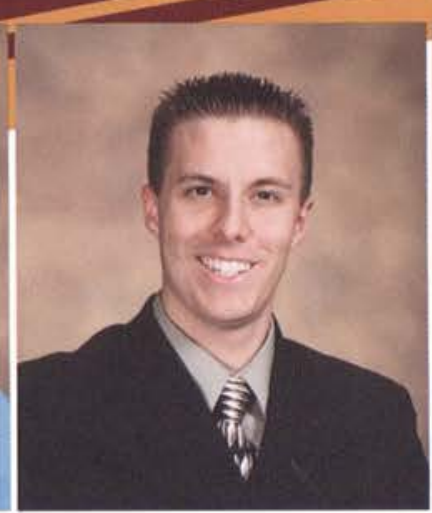

Luke Andrew Snider Electrical Engineering

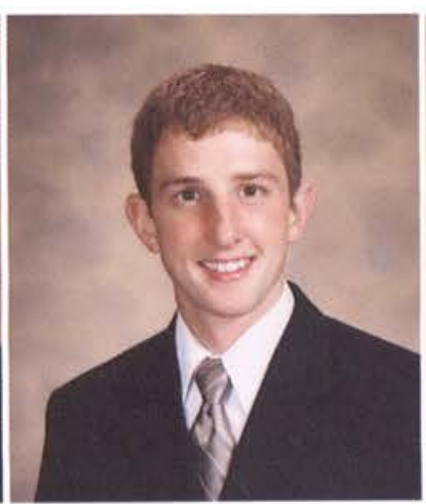

Derek Carlton Stambaugh Management

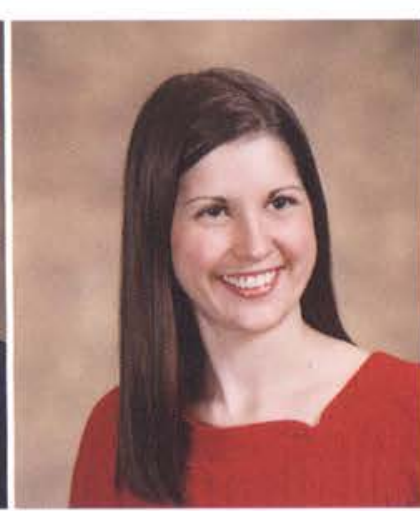

Christin Renee Stock Nursing

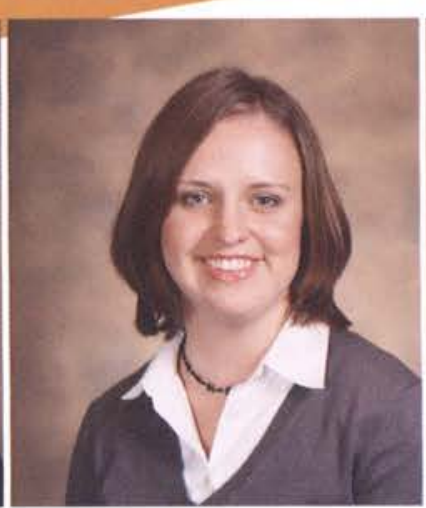

Dana Marie Snyder

Middle Childhood Education

Eric James Spahr
Mechanical Engineering

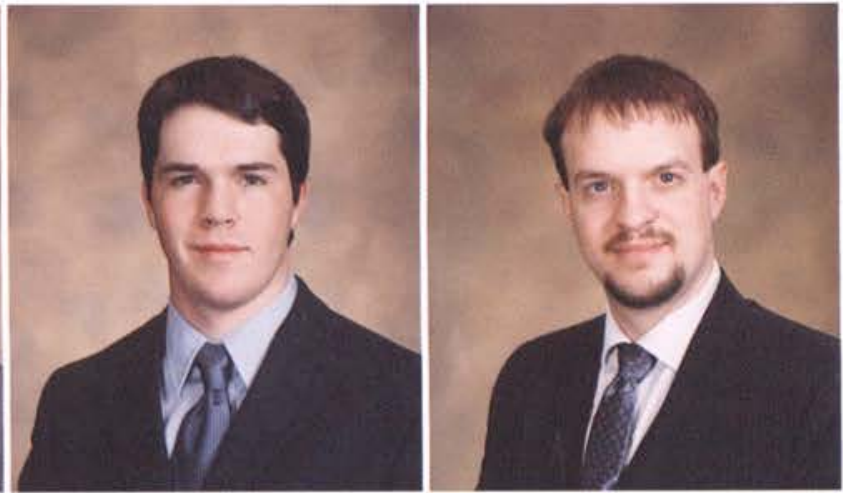

Paul Michael Squires

Youth Ministries \& Christian Education

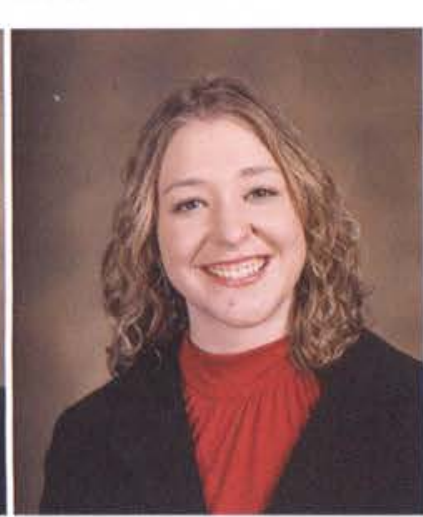

Michelle Charlene Stanton Psychology

Shelly Lynn Sterner Integrated Language Arts Nursing

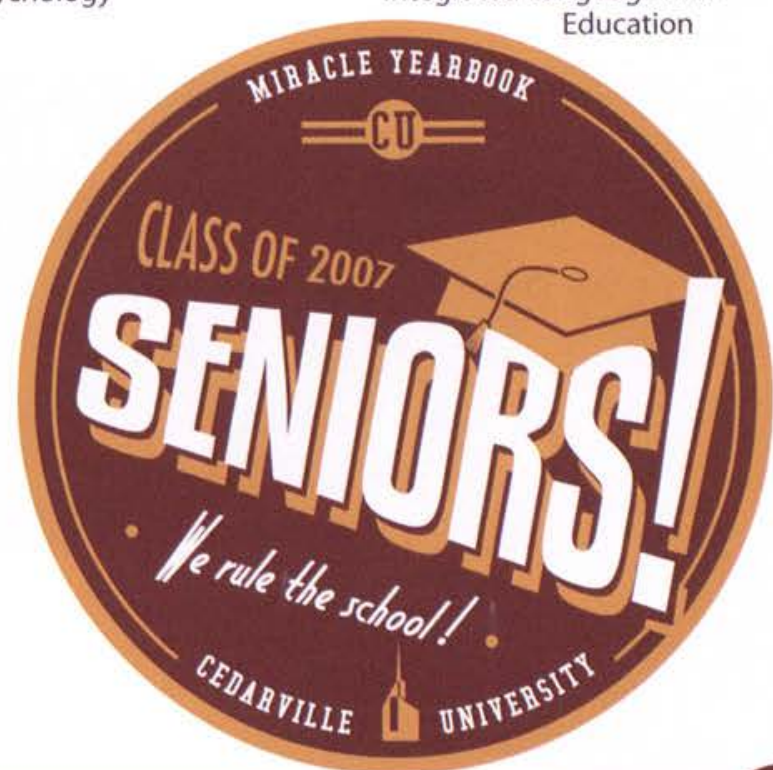




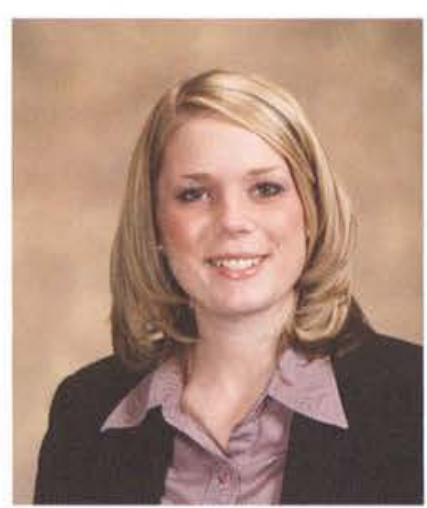

Lindsay L. Stone

Social Work

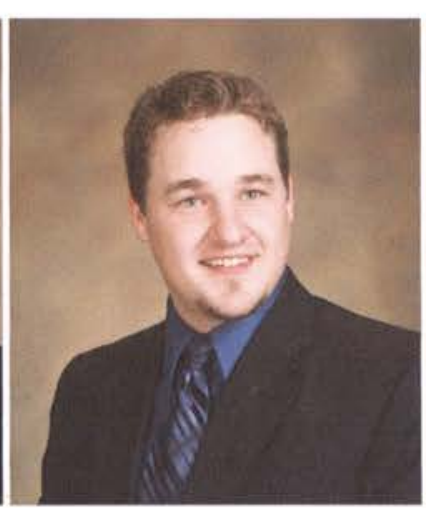

Jacob R. Stout

Youth Ministries

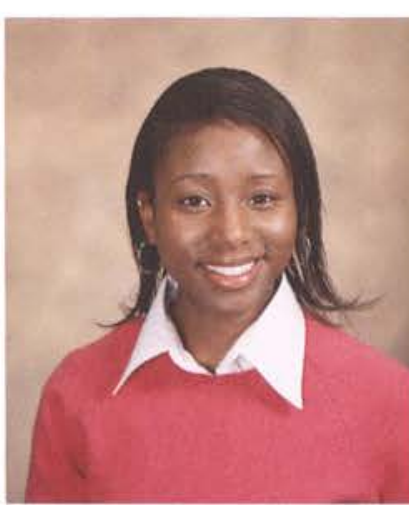

Latice L. Strickland

Technical \& Professional

Communication

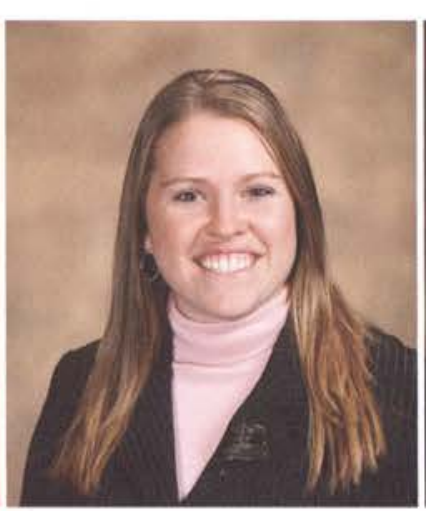

Rachel Sturgis

Criminal Justice

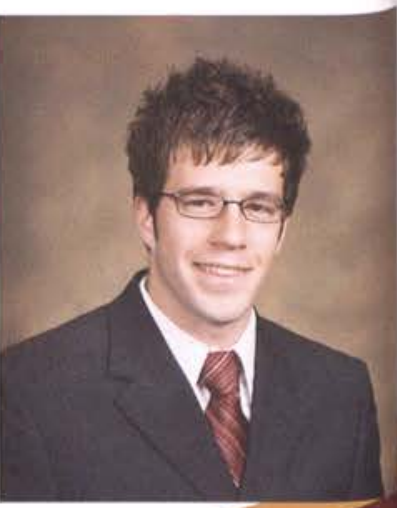

Ryan Andrew Stutzman

Ma
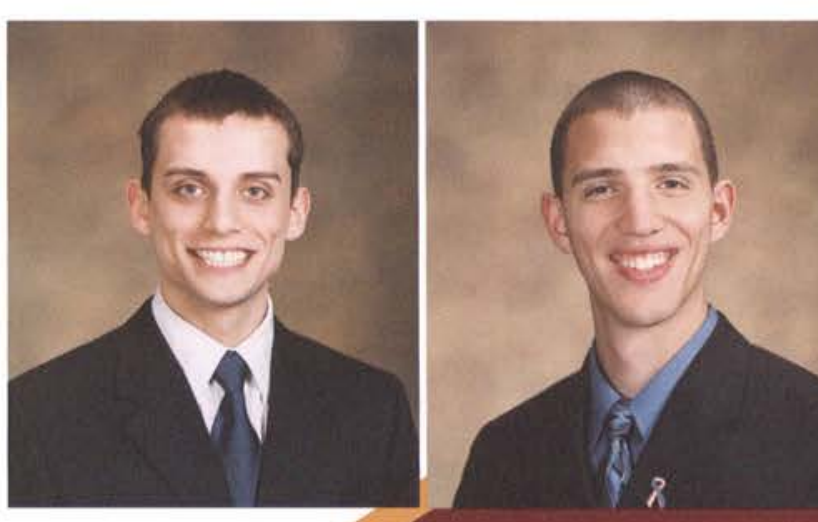

Artur Suchorzewski International Studies 2

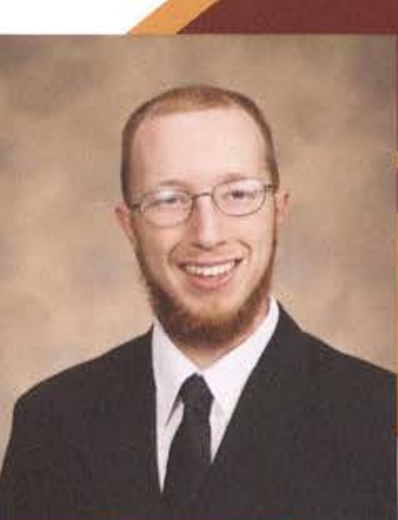

Daniel Lee Tate History

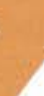

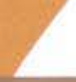

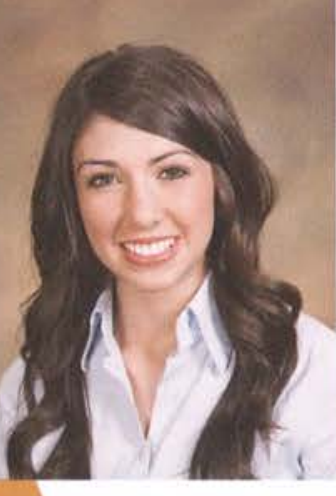

Rachel Fairle Teat Biology

\section{Jeremy Lee Sutphen Comprehensive Bible}

\section{Bible}

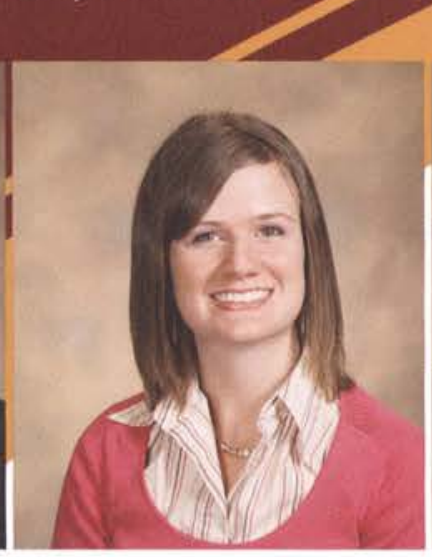

Bethany Joy Taylor

Middle Childhood Education Communication Arts

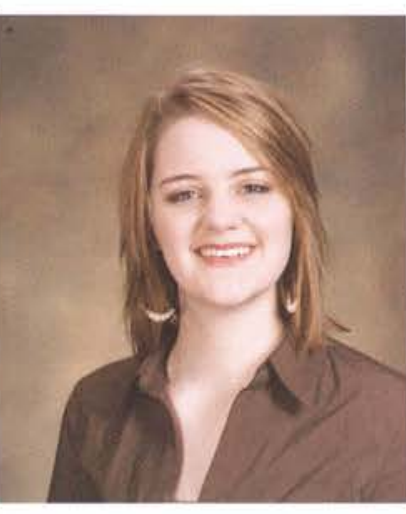

Jaimie Lynne Teekell

Comprehensive

Communications

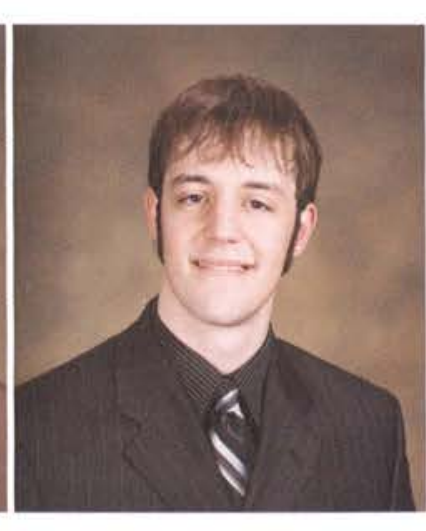

Joshua A. Teekell

Mechanical Engineering

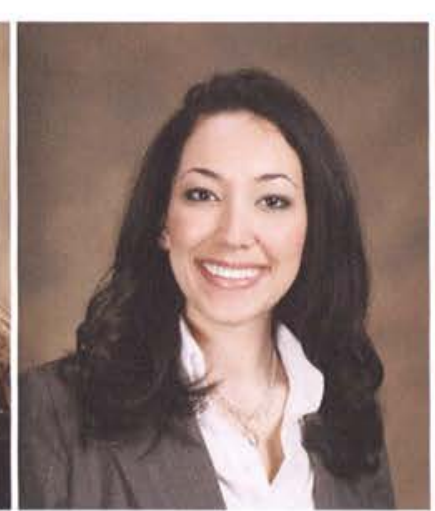

Hannah Elizabeth Taylor

Organizational

Communications

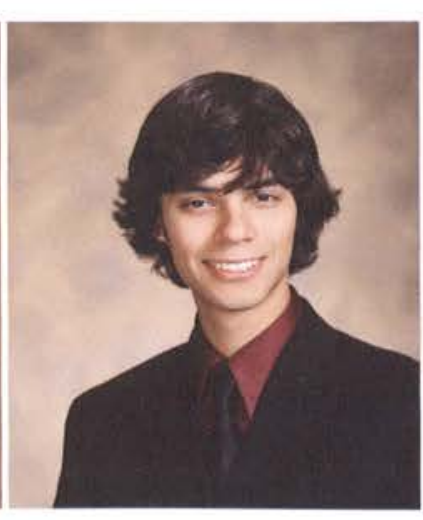

Daniel Eric Tello

Graphic Design
Laura Elizabeth Teague

Early Childhood Education

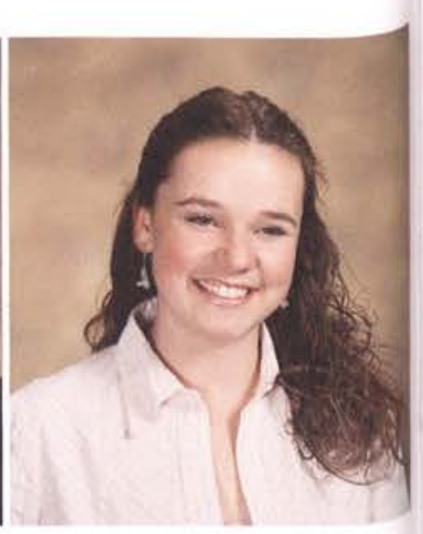

Mary Grace Thengvall

Comprehensive

Communications
Phillip Aaron Sweeten

Athletic Training

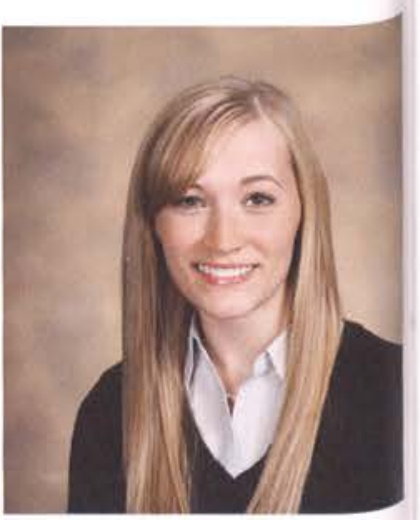

Donna Marie Sweede

English

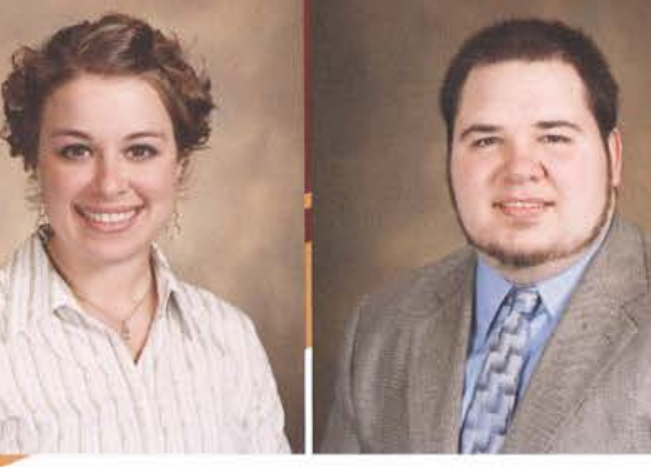

1)

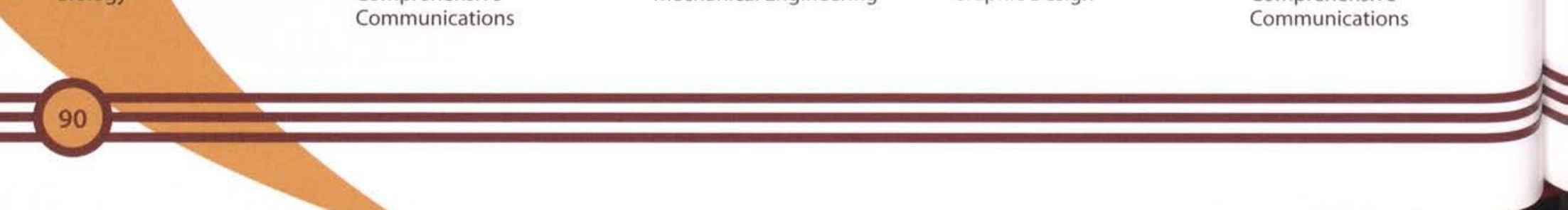




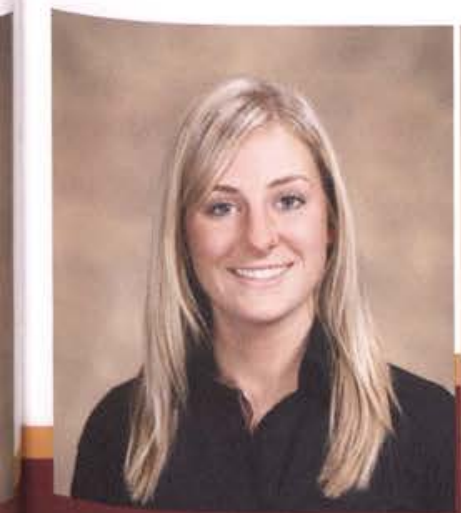

Jessica E. Thomas Social Work

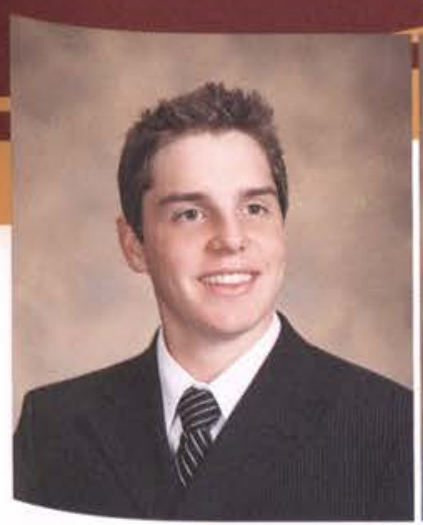

Andrew Paul Tripp Organizational Communications

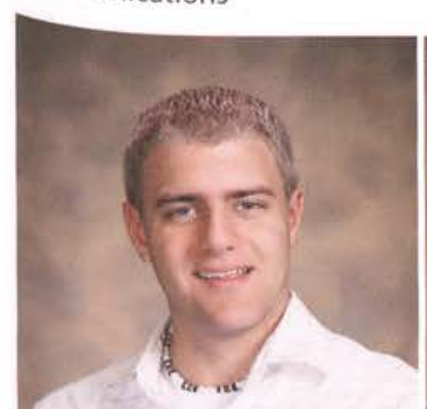

David Van Schepen

Mechanical Engineering

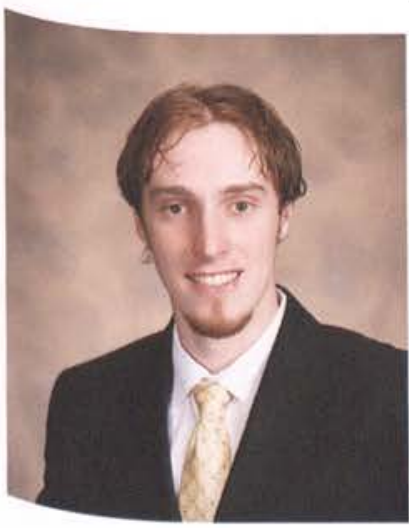

Maicol Venter

Pre-Seminary Bible

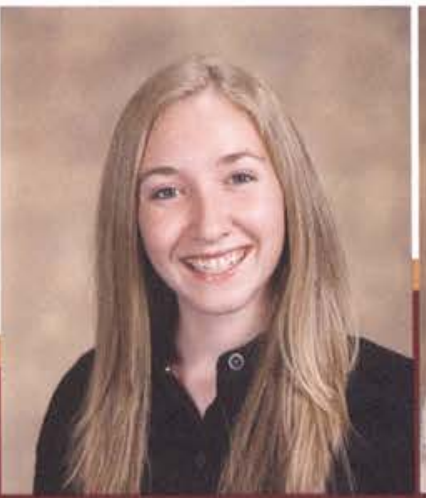

Crystal Marie Thompson Christian Education \& Youth Ministry

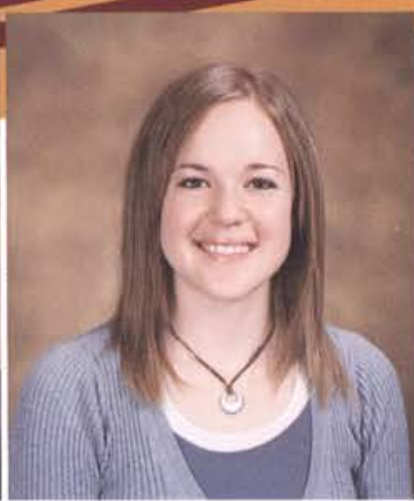

Julie Suzanne Tullett Electronic Media

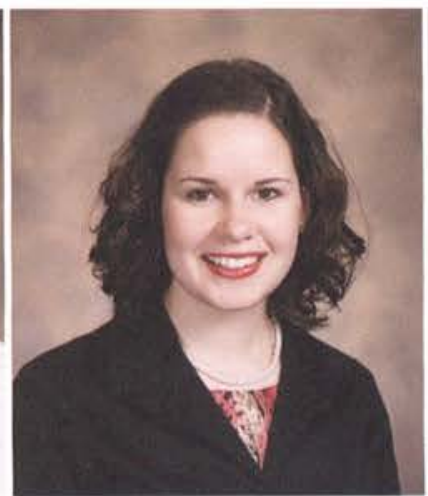

Emily Michelle Van Vliet Electrical Engineering \& Communication Arts

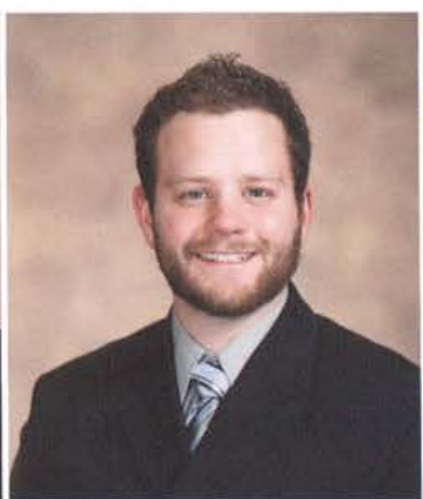

Timothy J. Vernon Electronic Media
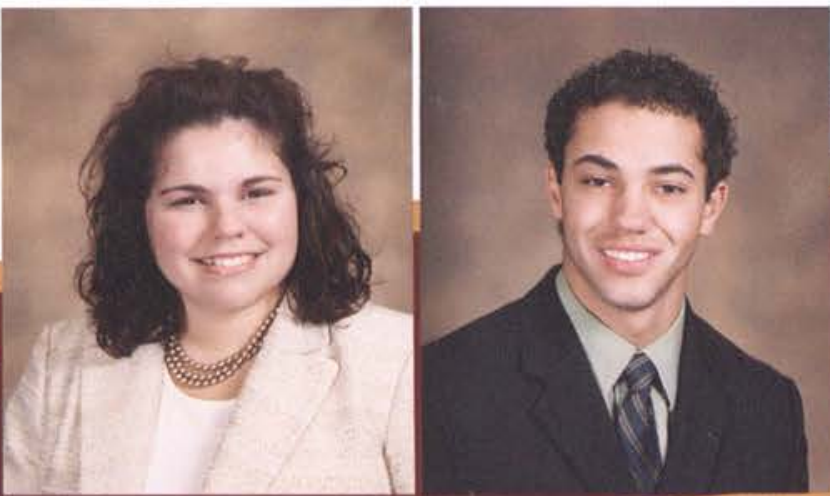

Kristen MichelleTisdale Political Science

Justin C. Traucht

Global Economics \&

International Business
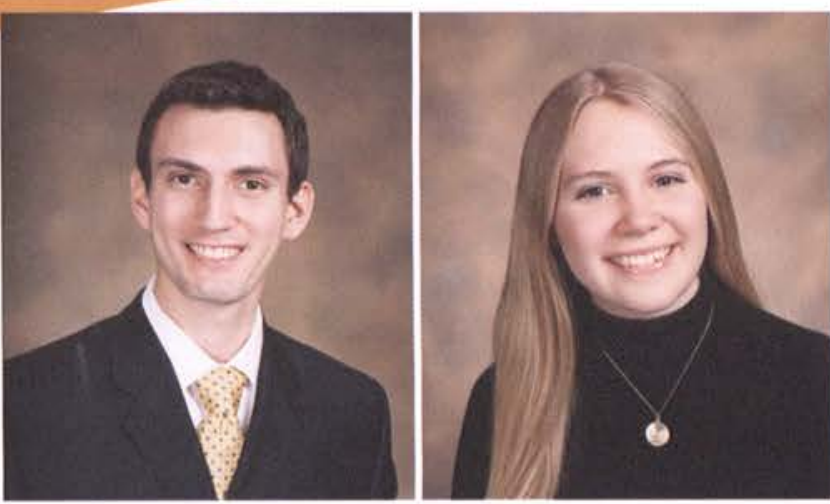

Physical Education

Mark William Urman Electronic Media

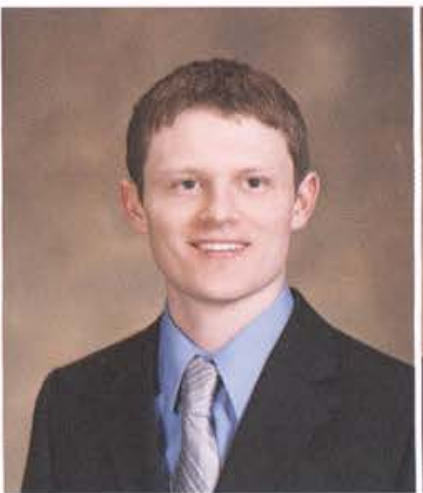

Christopher Vande Lune Marketing

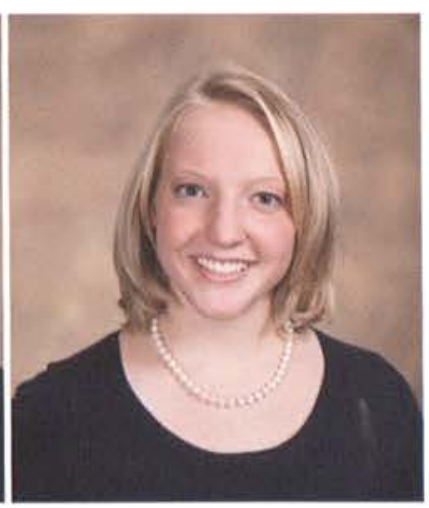

Elizabeth Jean Verwys Marketing
Jennifer Marie Van Dyke Pre-Law

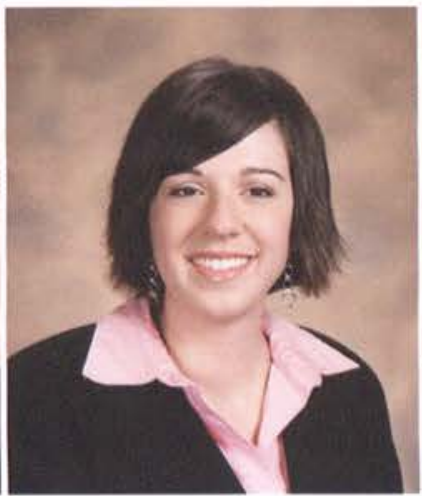

Christina N. VanderSchuur Nursing

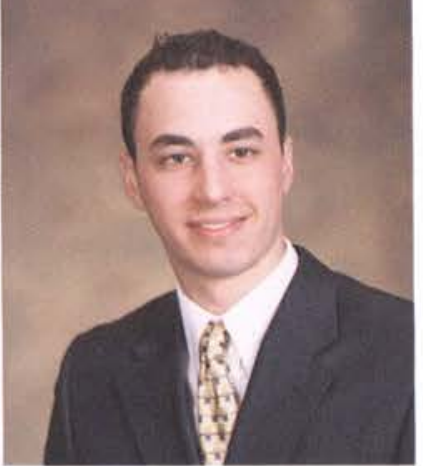

Howard W. Van Matre IV International Studies

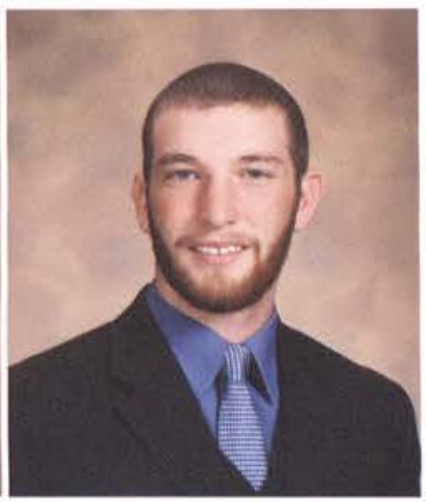

Matthew P. Vanlier Sport Management

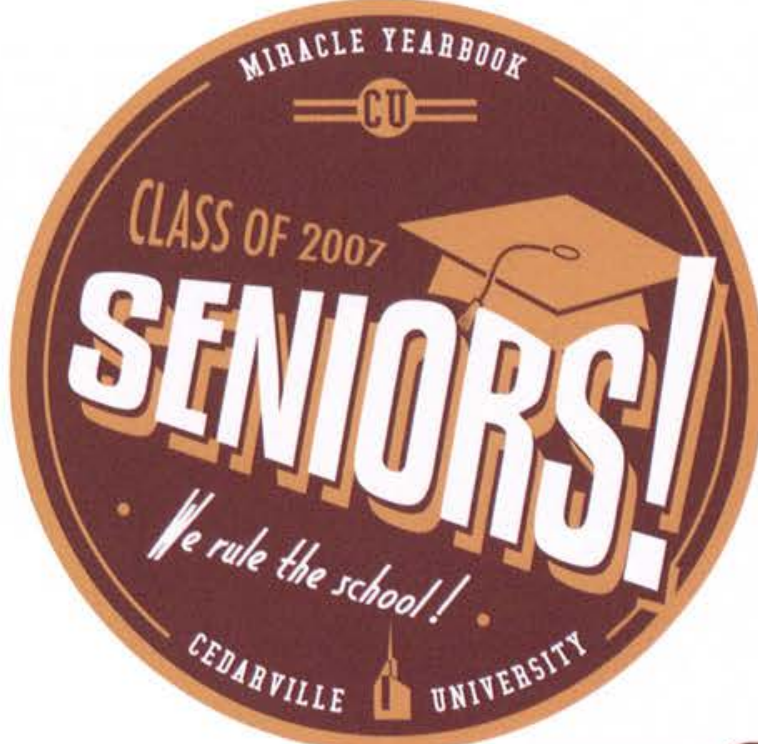




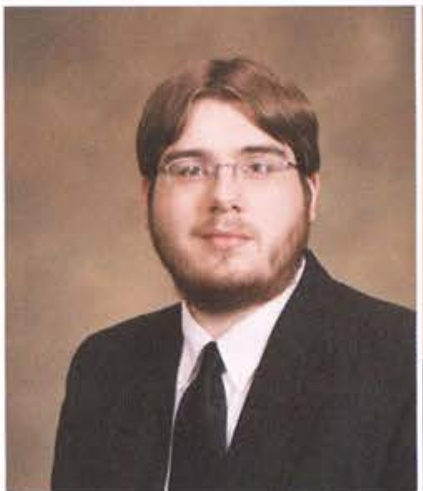

Craig Henry Vinson

Electronic Media

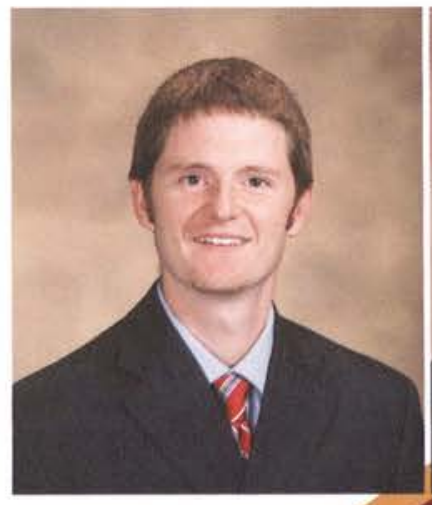

Paul Bennett Walker Youth Ministries \& Christian Education

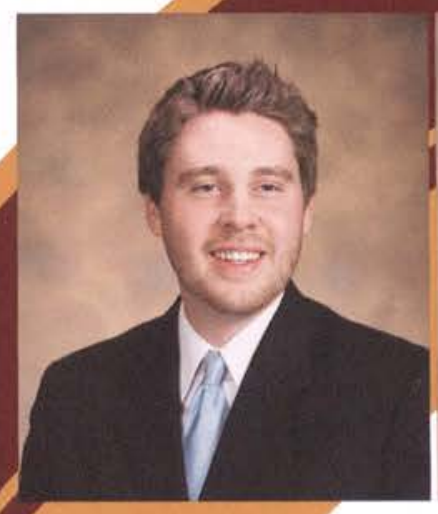

Timothy Joseph Warren Biology Pre-Med

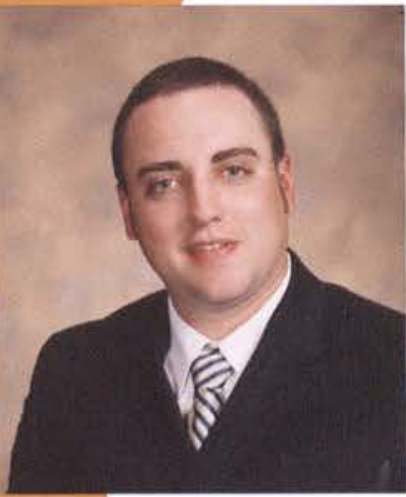

Philip A. Westenbarger Pre-Seminary Bible

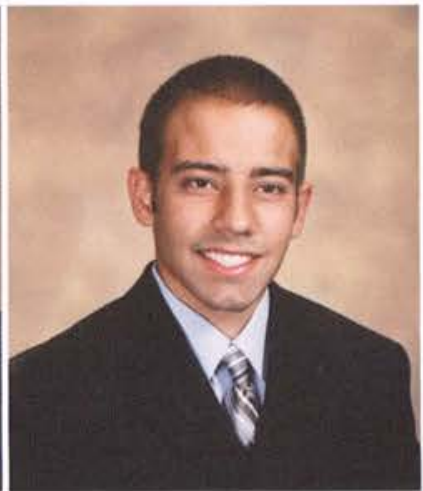

Benjamin P. Volpe Criminal Justice

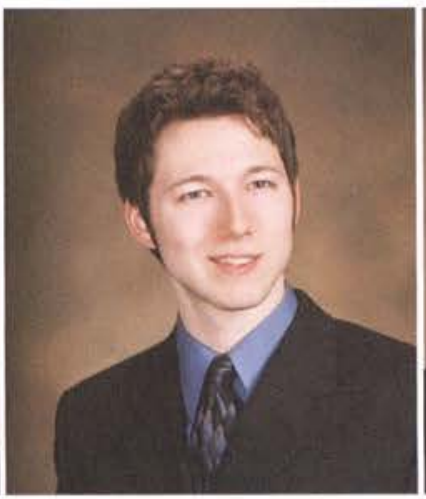

Jeffrey R. Wagner

Management Information Systems

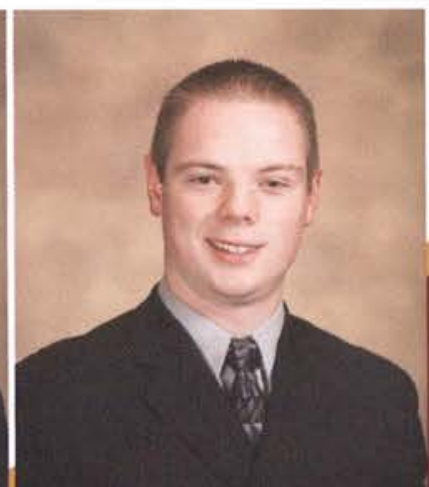
Joel D. Wallen
Pastoral Studies

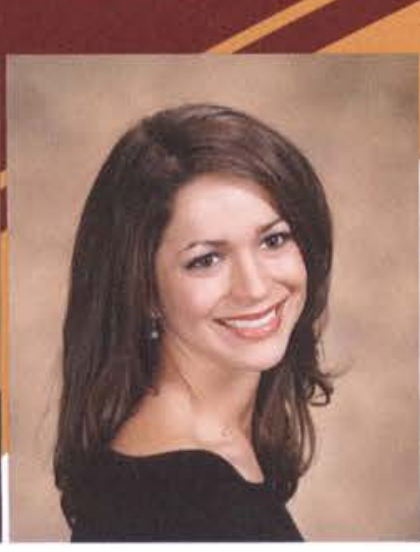

Ashley J. Watson Nursing

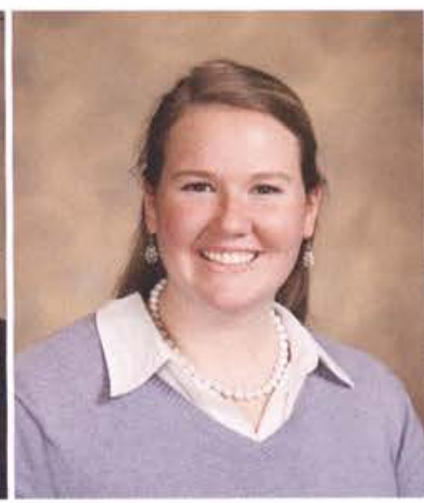

Katie Joy Wheeler Comprehensive Bible
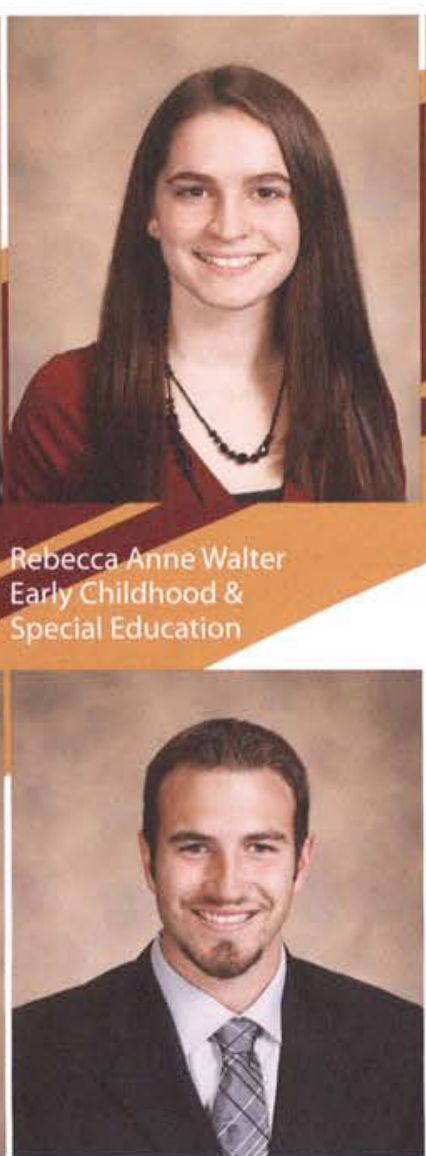

Matthew Eugene Watson Pre-Seminary Bible \& Communication Arts

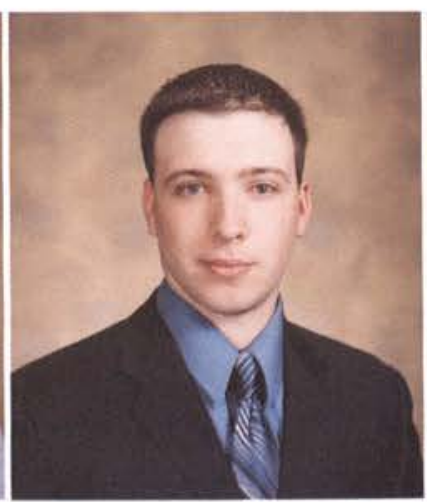

Daniel J. White Technical \& Professional Communication

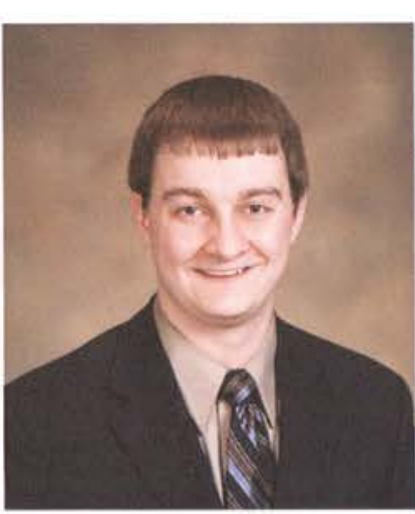

Andrew Josiah Wailes Computer Engineering 12

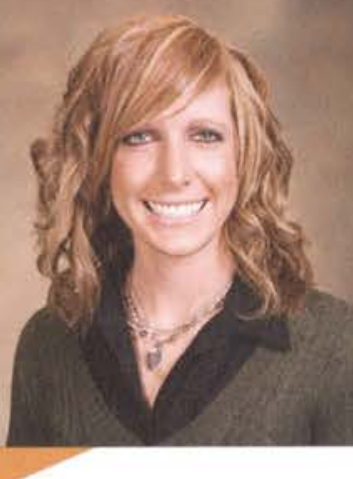

Karah Beth Walton

Exercise \& Sport Science

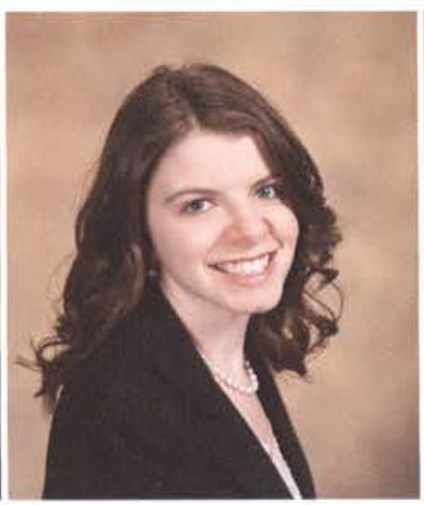

Sarah Christine Welch Accounting

Gina Marie Wells Communication Arts

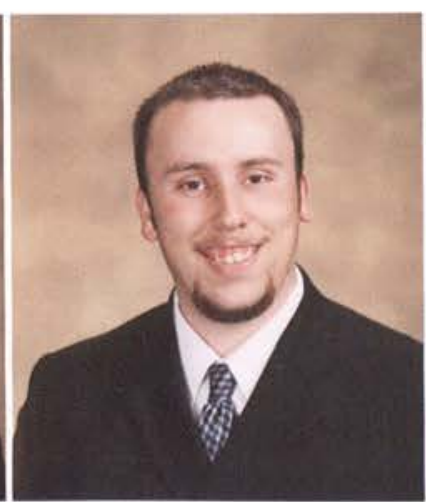

Joshua D. Wilcox Graphic Design
Shawna Lynn-Waldo
Athletic Training

Katie Ann Warndahl

Comprehensive Bible

Katie Elizabeth Willaman Mathematics
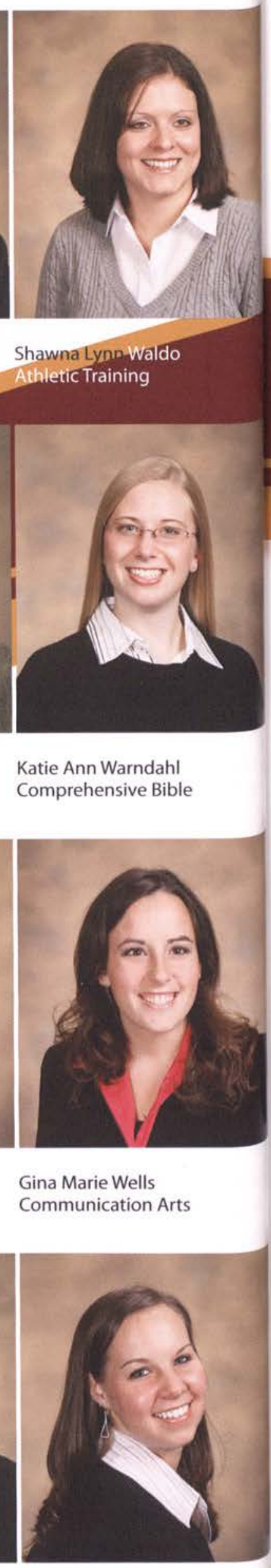


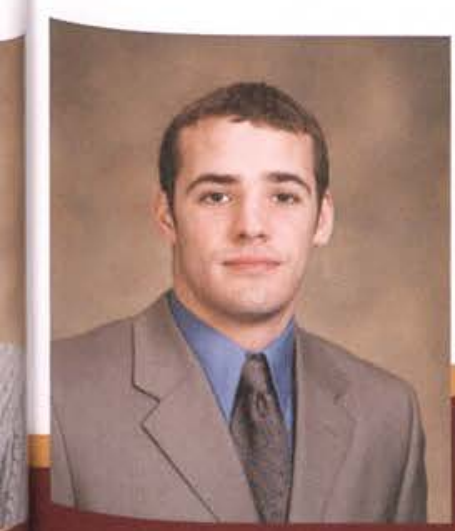

Jordan Michael Williams World Missions

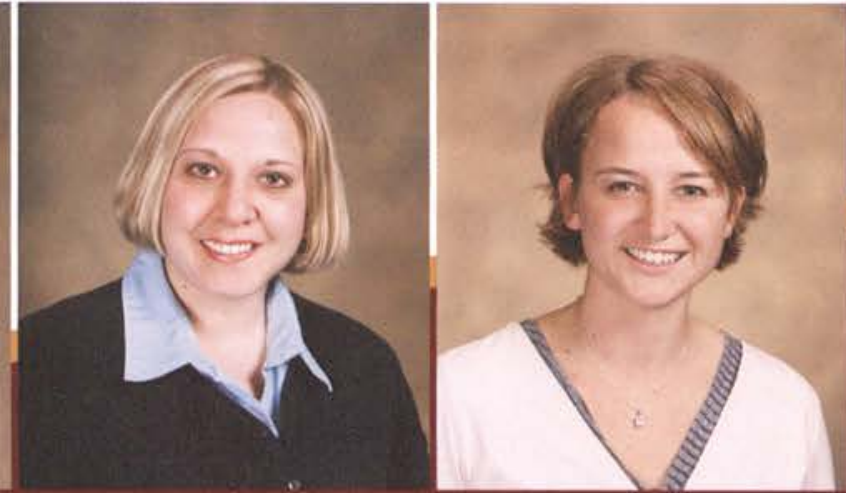

Sara M. Williams Mathematics
Rebekah B. Williamson Nursing

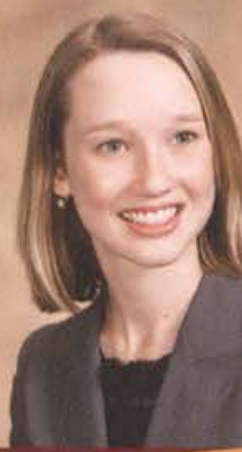

risten L. Wills

AYA Integrated Language Arts Education

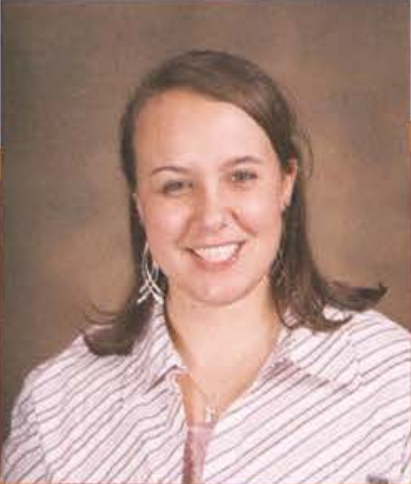

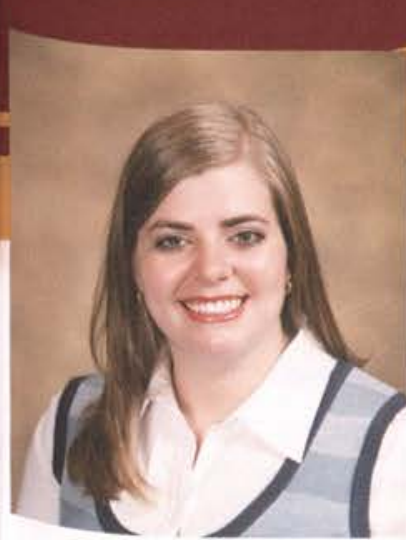

Natalie Renee Winder Music Education

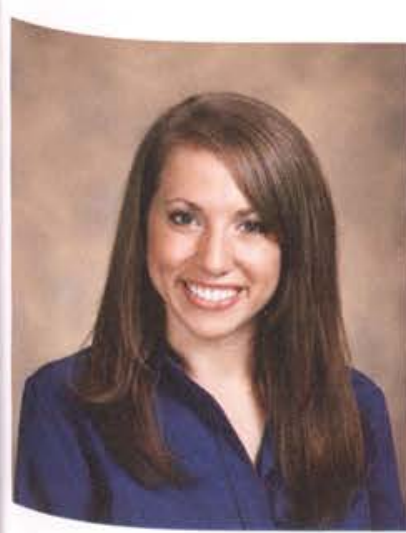

Re

Rebecca Nora Wolff

heatre \& Communication Arts Organizational

Communication

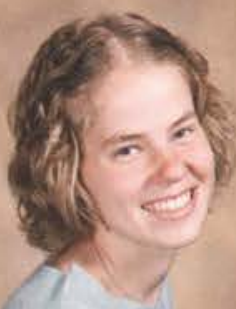

Eannie J. Wright

Early Childhood \&

Special Education

Nursing

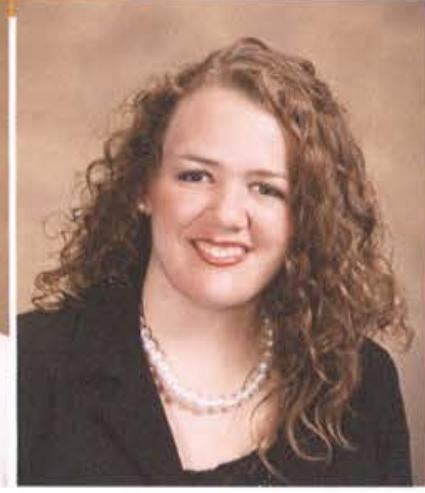

Elizabeth Ann Winner

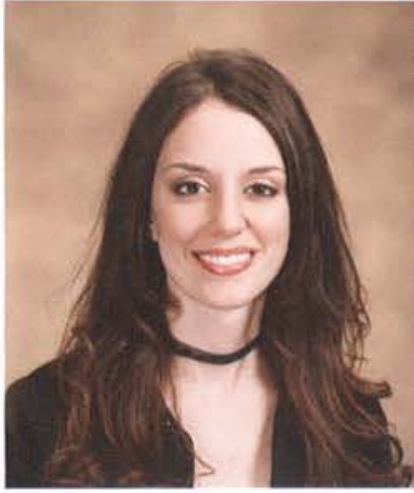

Lindsay Marie Wirt

Social Work

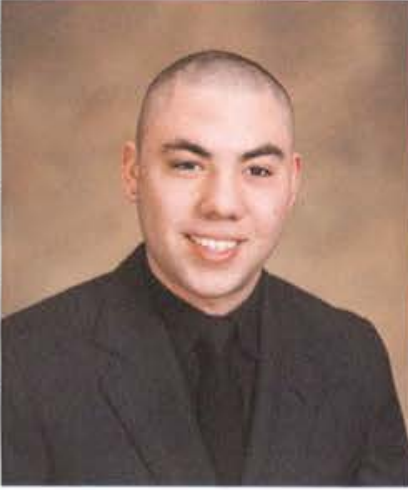

Andrew Leonard Wong

Criminal Justice

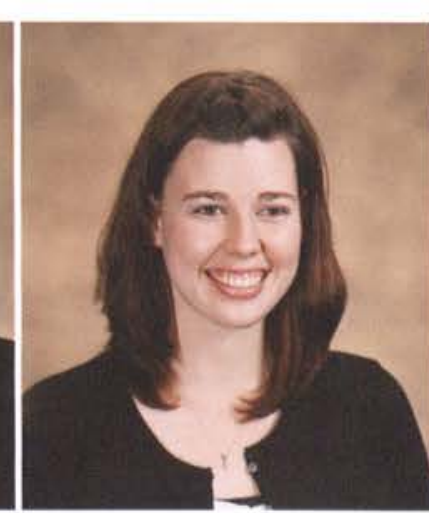

Tiffany Rae Wyant

AYA Social Studies Education

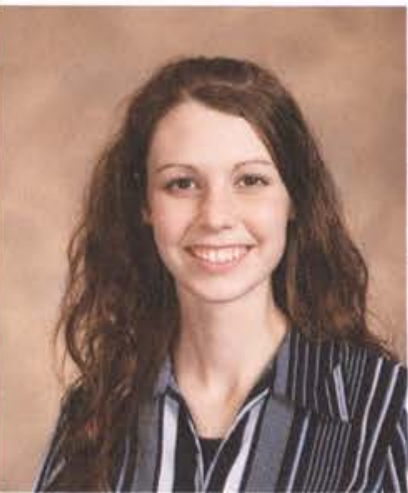

Tiffany Renee Wise

Music Education

Kelly Ann Witte

History

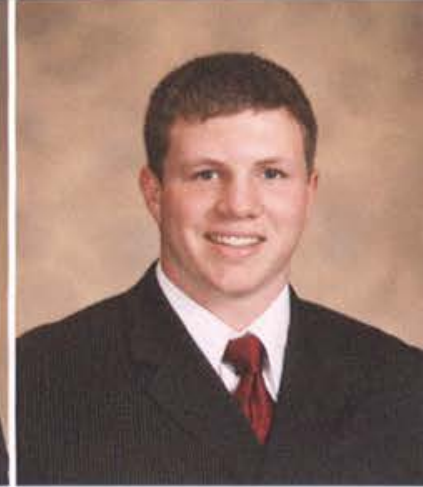

Philip Andrew Woodbury Accounting \& Finance

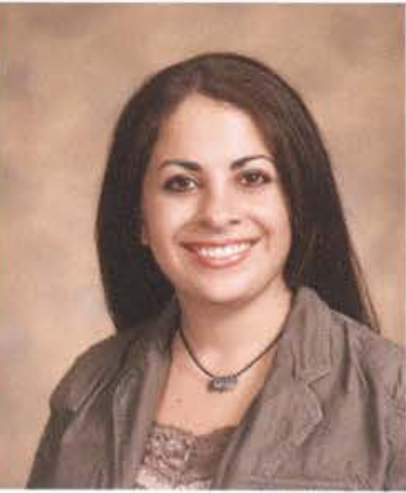

Amy R. Wright Criminal Justice

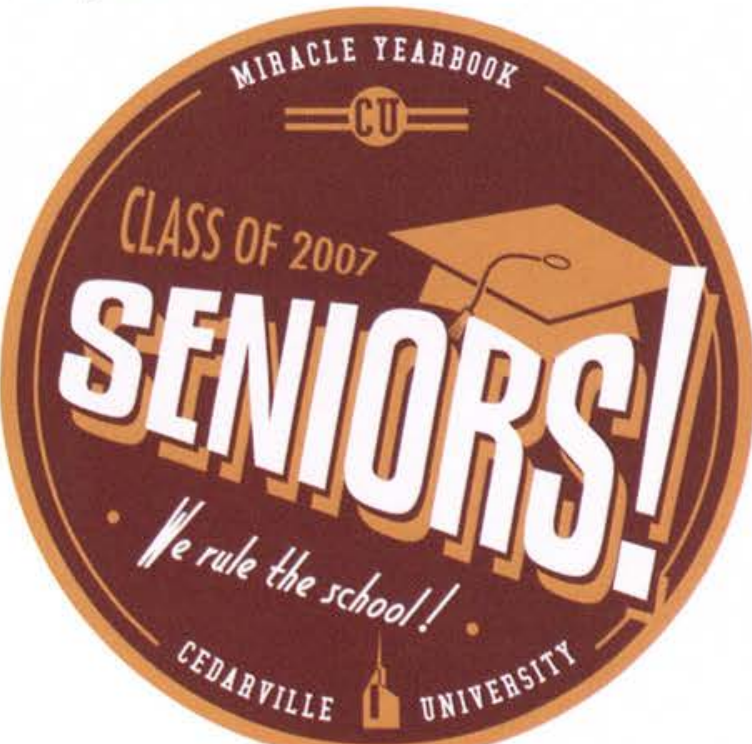




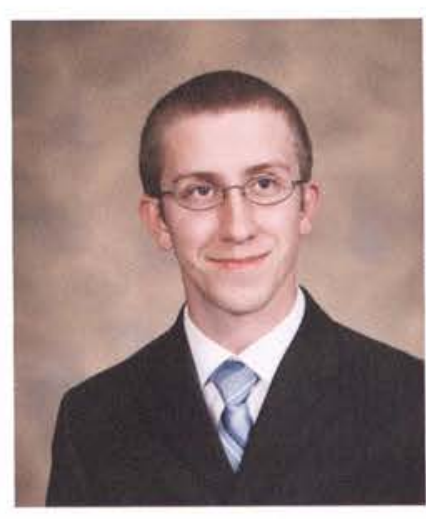

Steven Charles Wynn Early Childhood Education
Melissa Sue Wysong Marketing

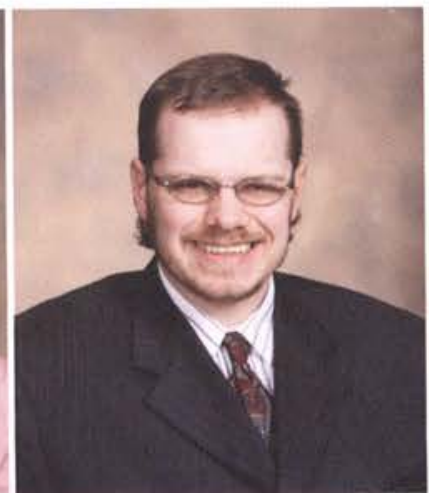

Louis Edward Yaklich III History

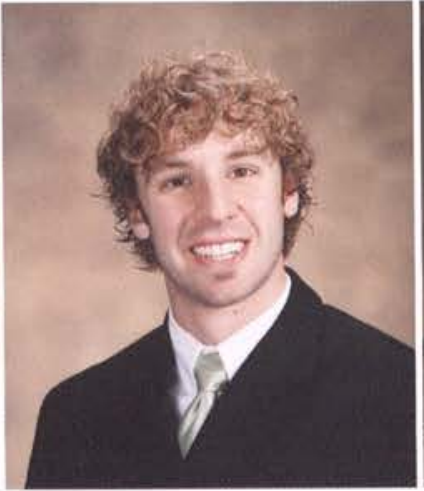

Tyler Russell Yoder History \& Pre-Seminary
Sarah Elizabeth York Social Work
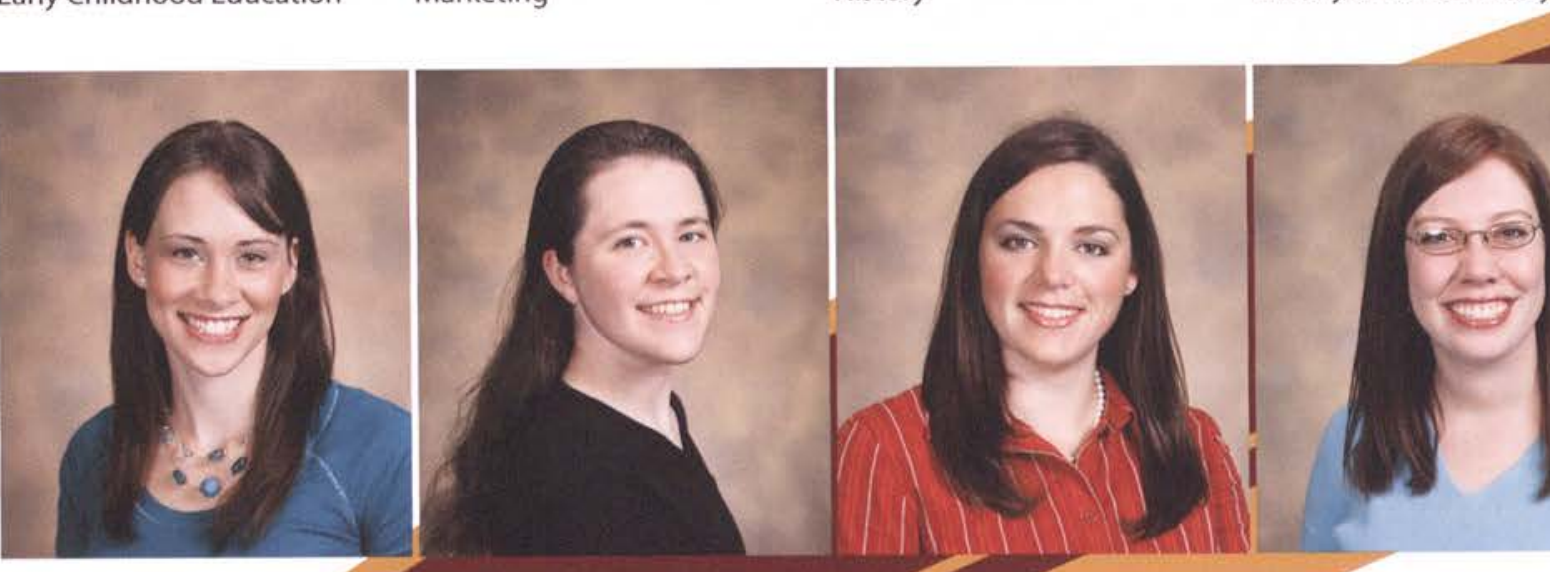



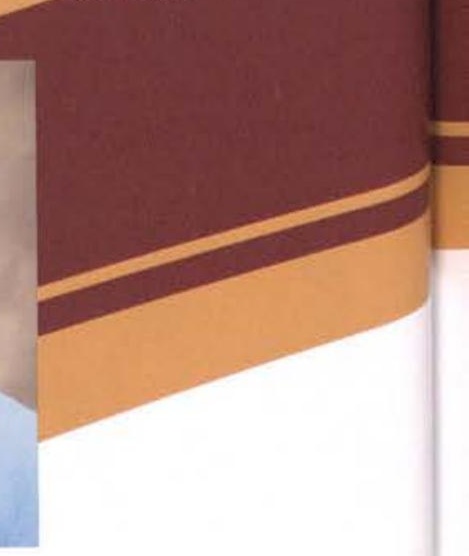

Alathea Grace Young Christian Education

Deborah Kaye Young Sociology \& History
Rachel Lymne Young Early Childhood \&
Kristen Michelle Zion

Pre-Seminary Bible

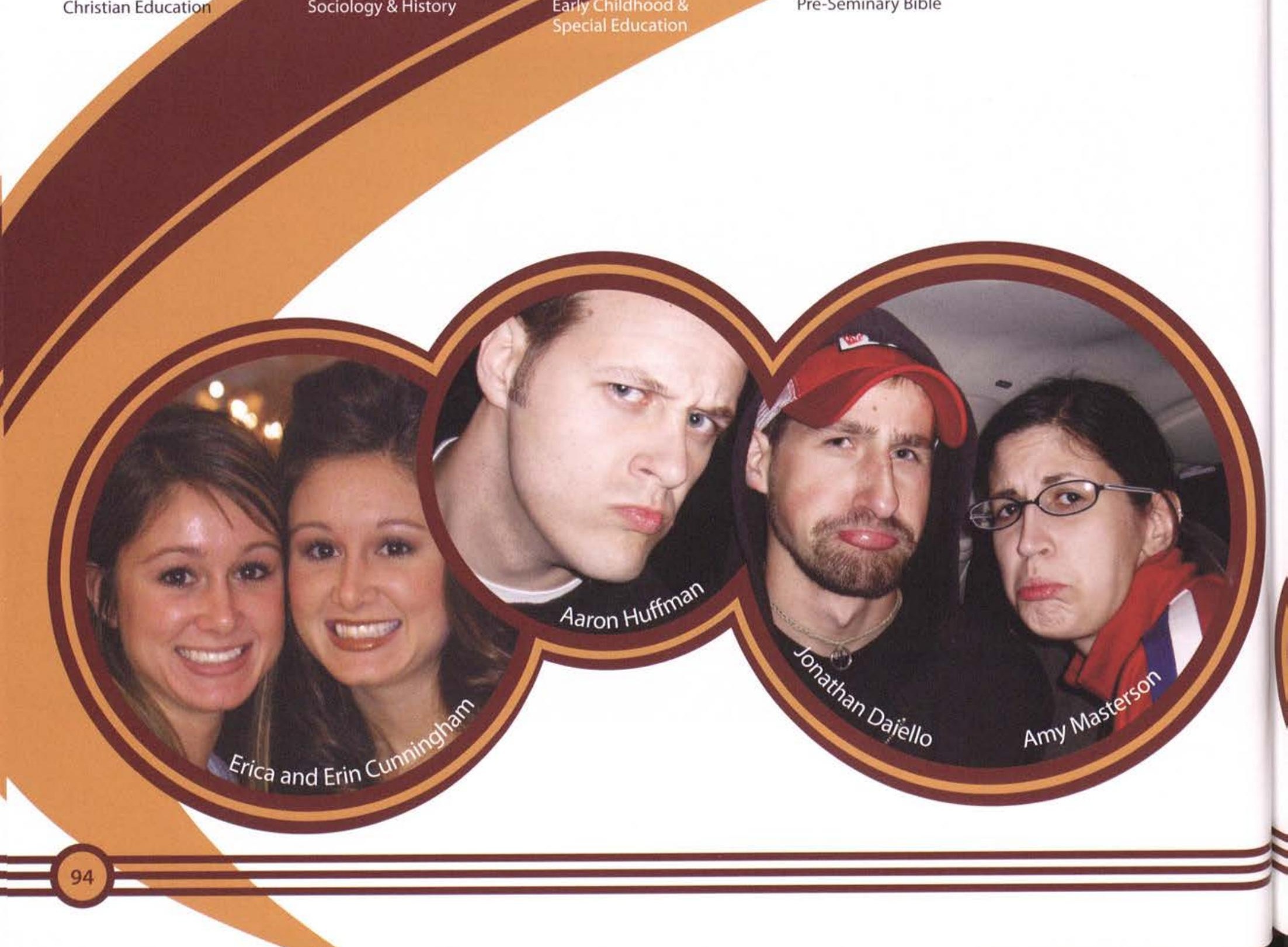




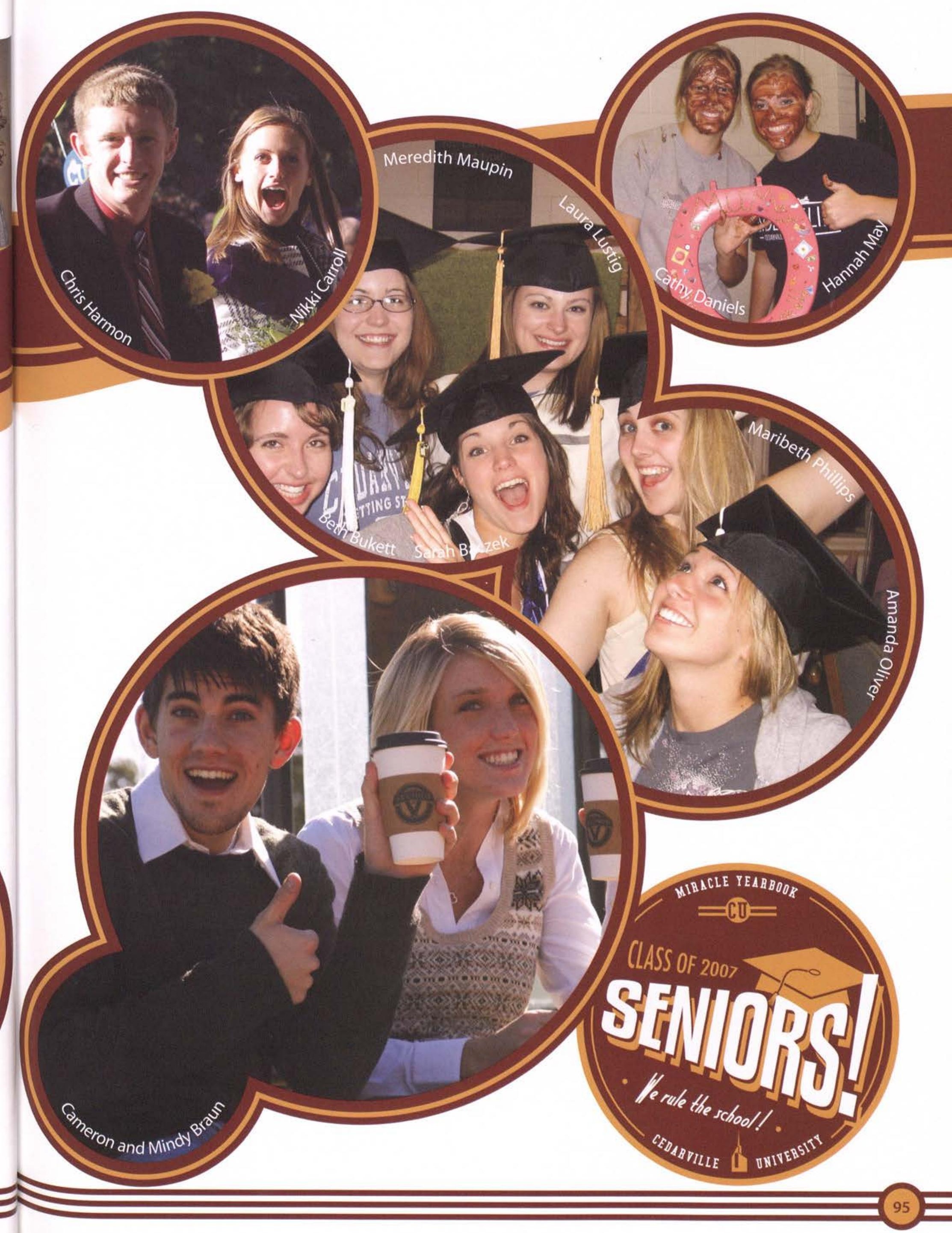




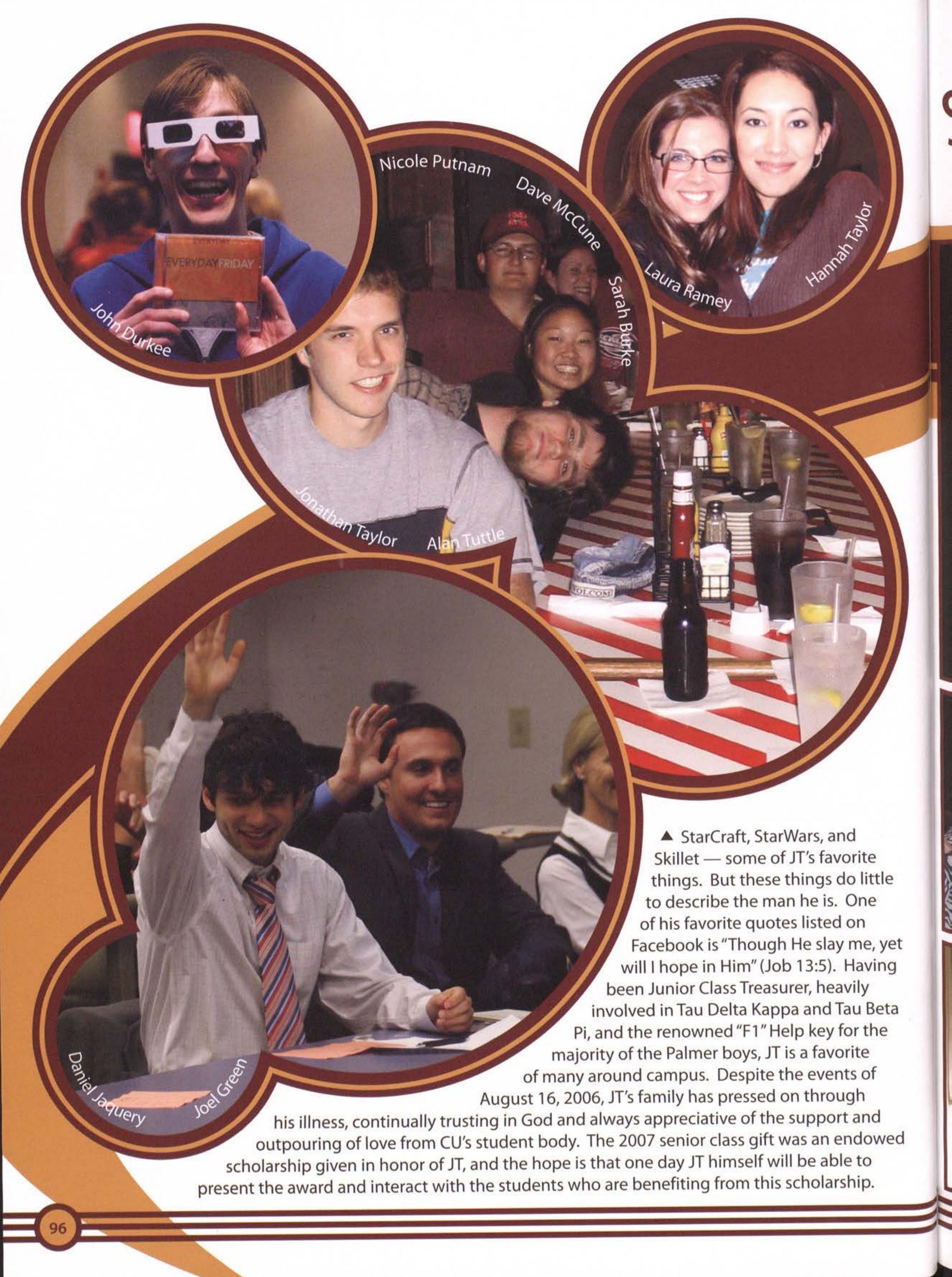




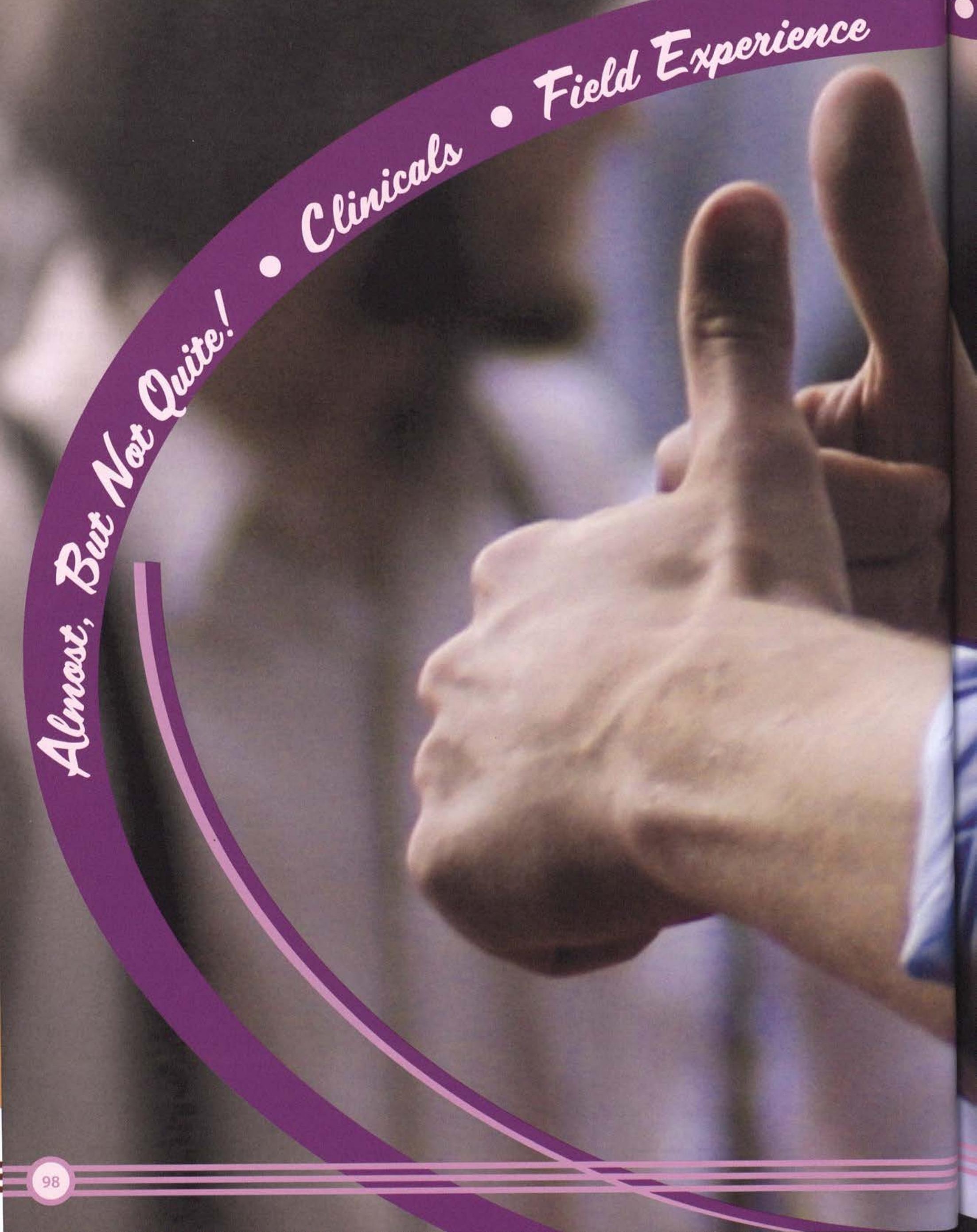




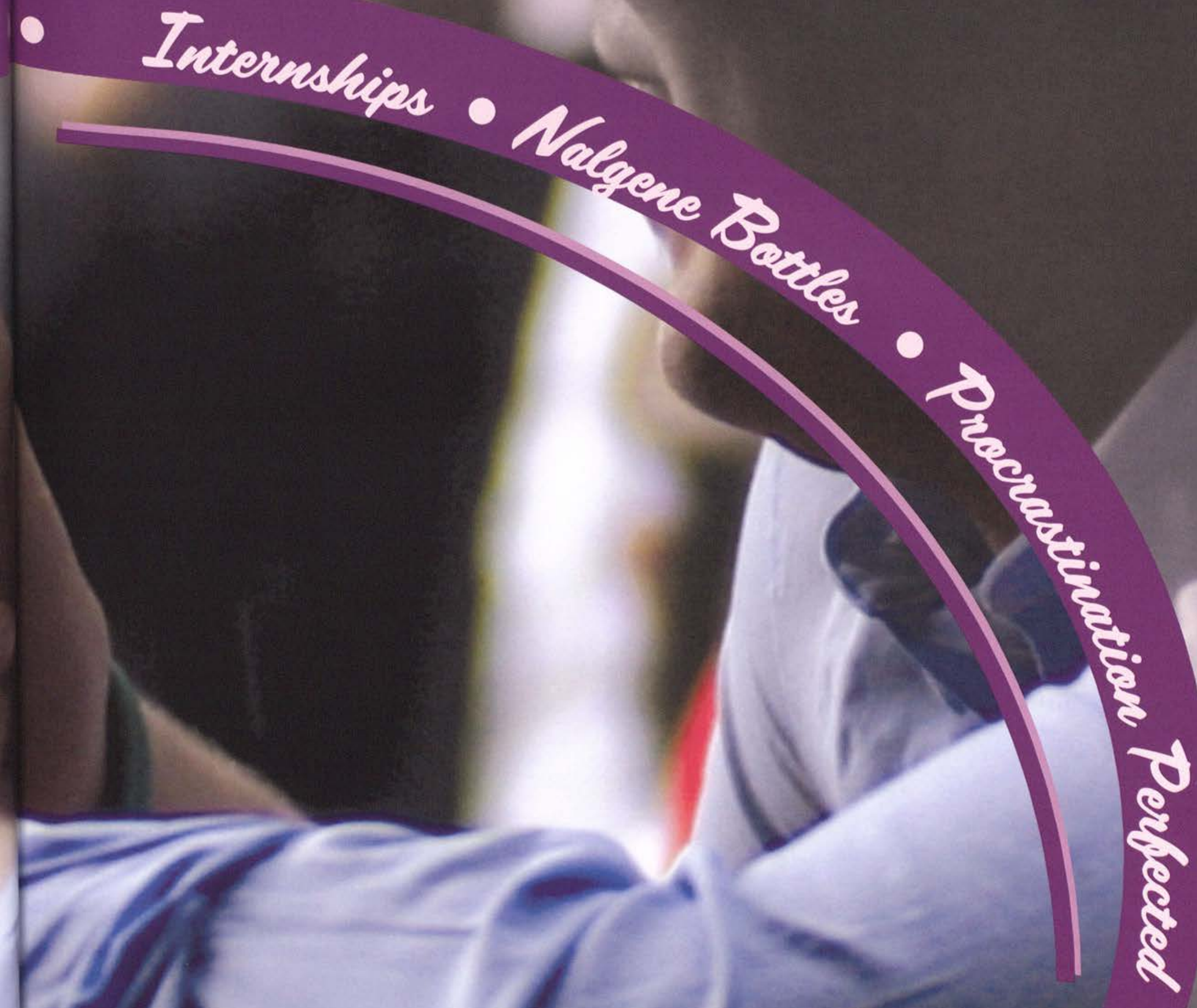

\section{JUNORS}




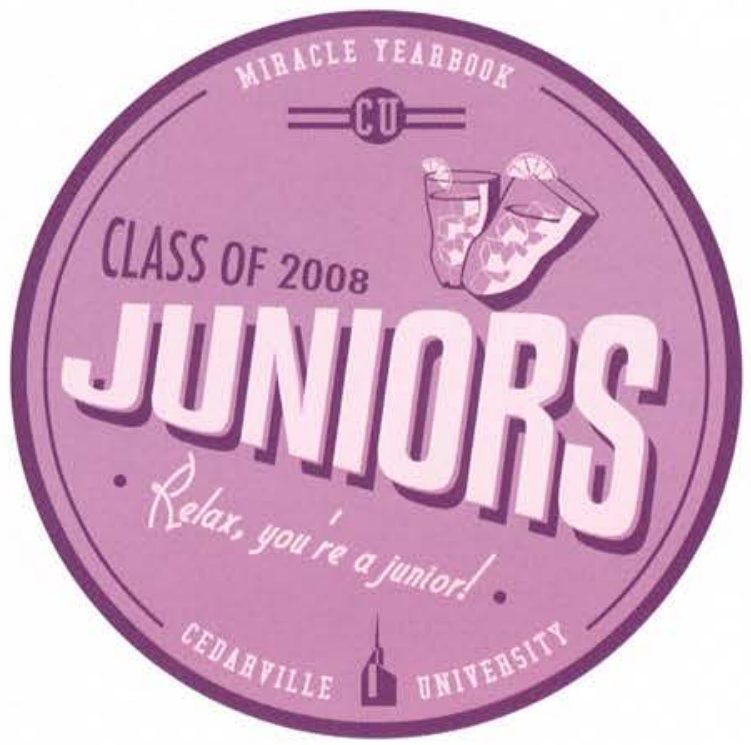

Matthew Abraham

Alyssa Adams

Emily Alexander
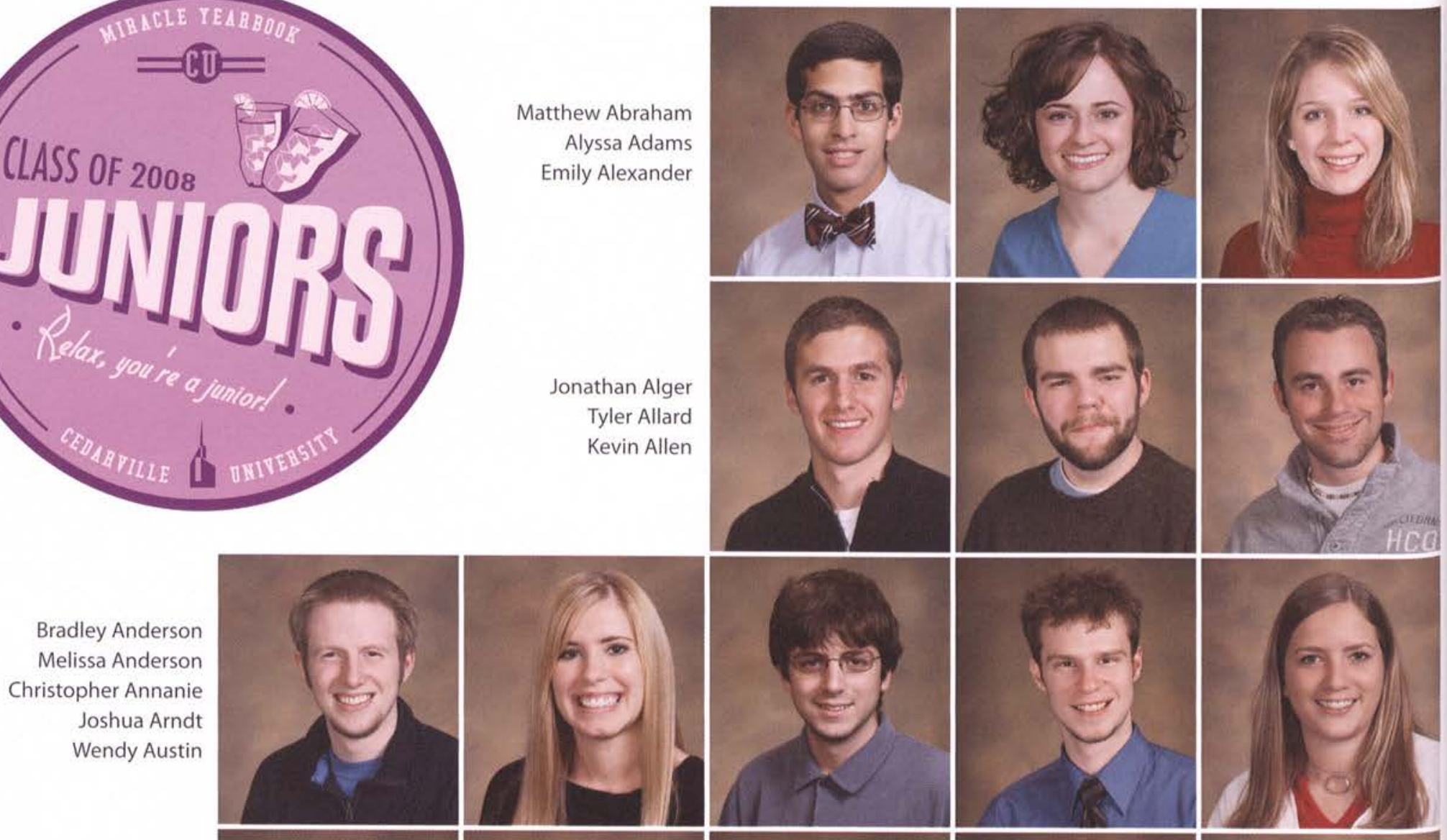

Nathan Averbech

Amy Babbit

Maria Balch

Daniel Ballard Jonathan Banks
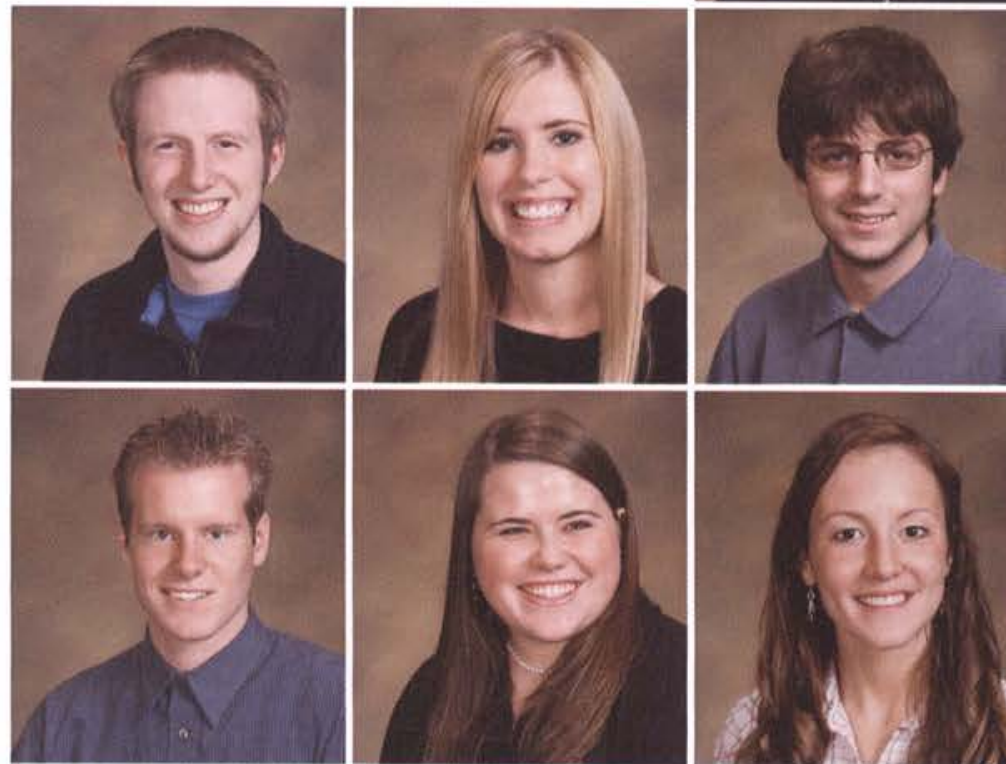

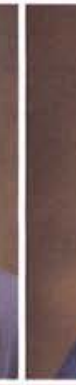
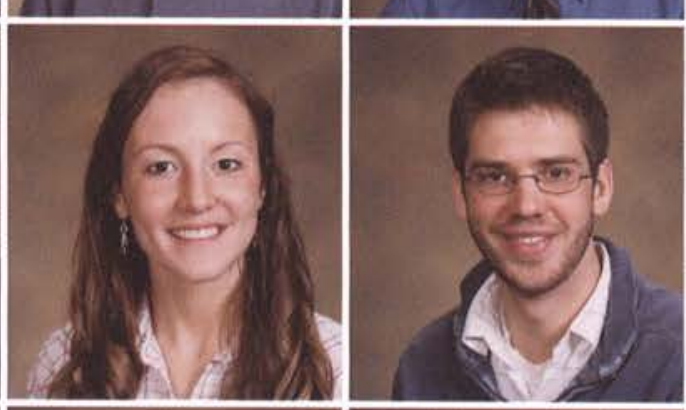

Andrew Barber

Julia Basford

Sarah Behn

David Bennett

Kathryn Biehl

Renae Bonde

Thomas Borck Jr.

Greg Briggs

Zack Brochu

Emily Broersma

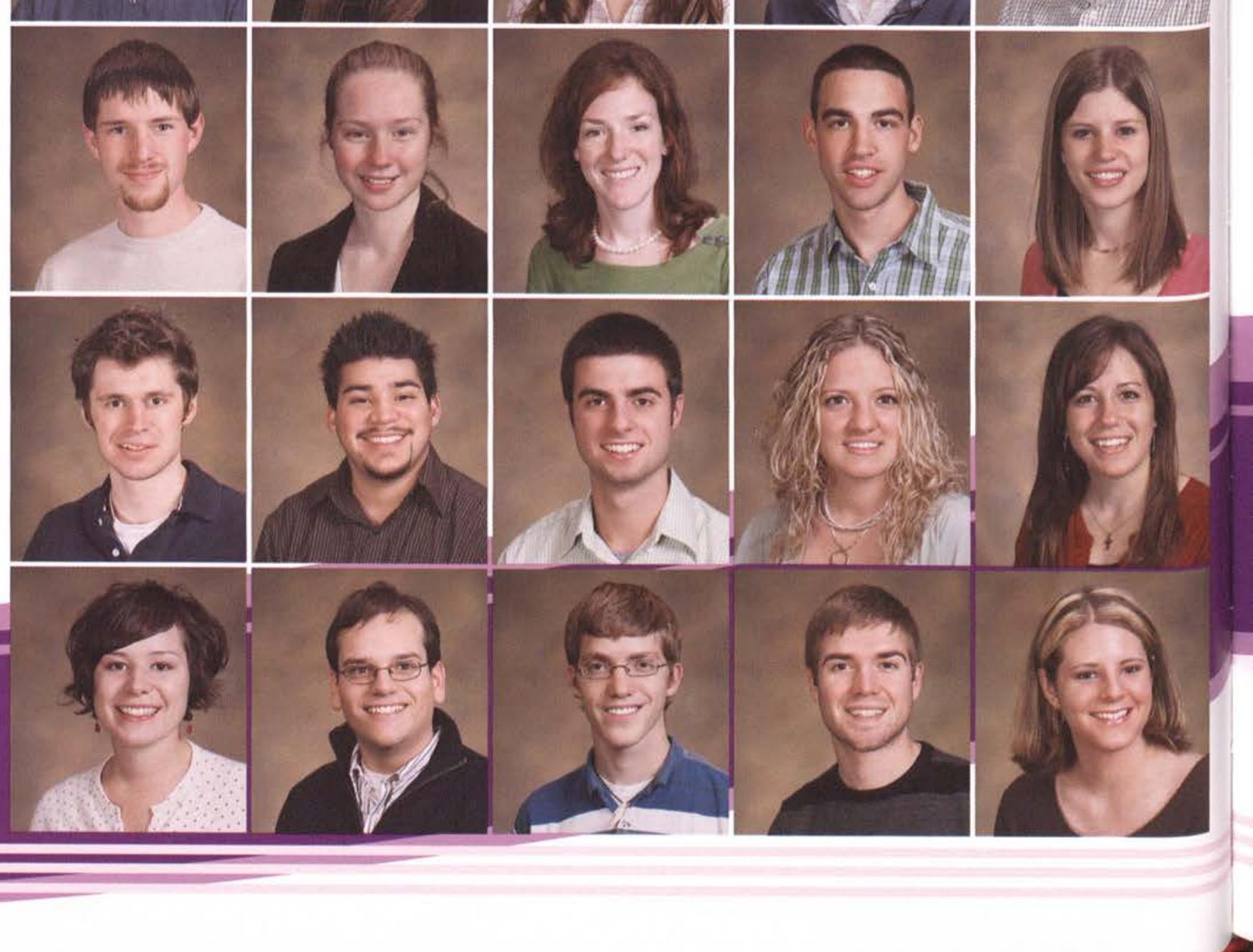




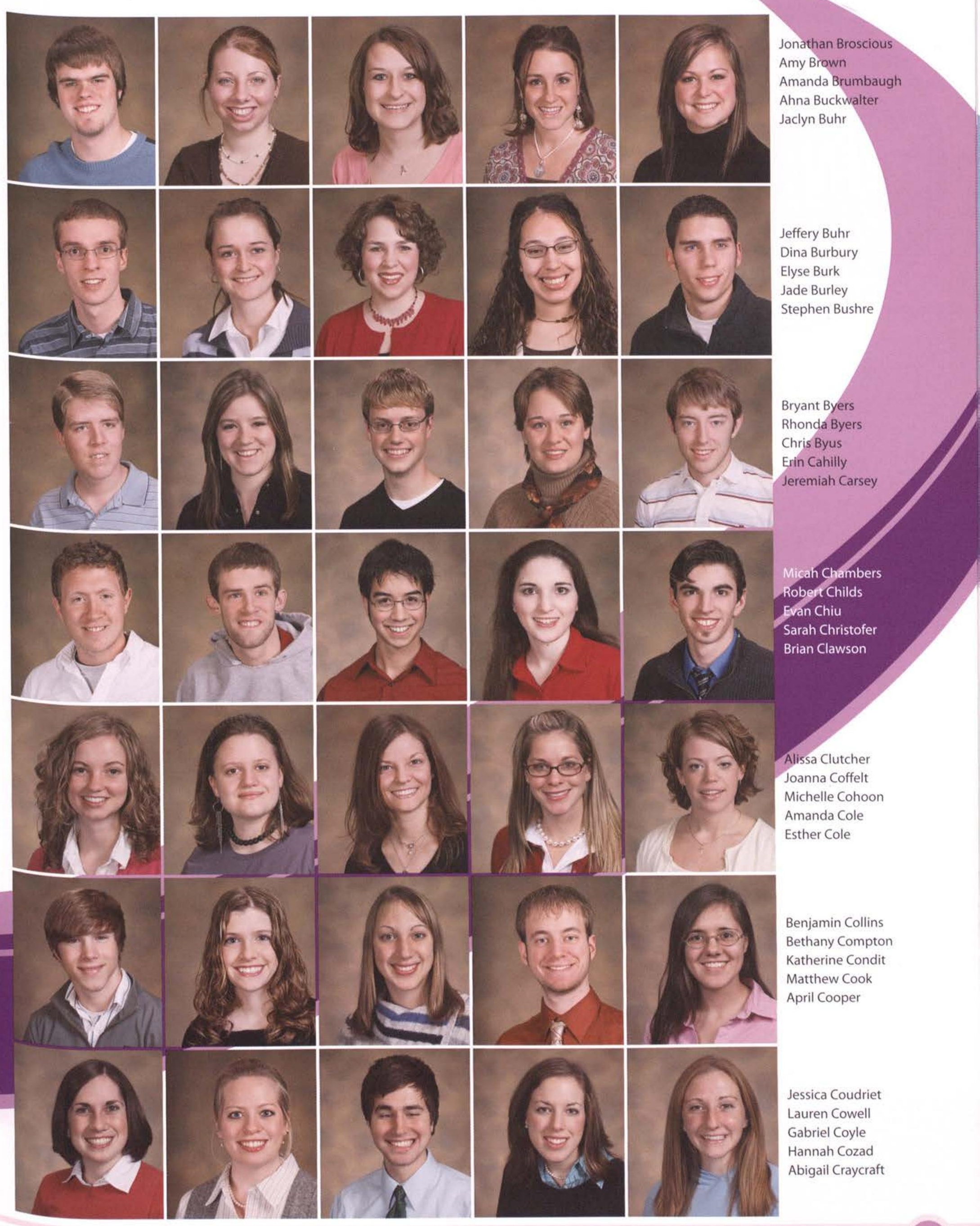



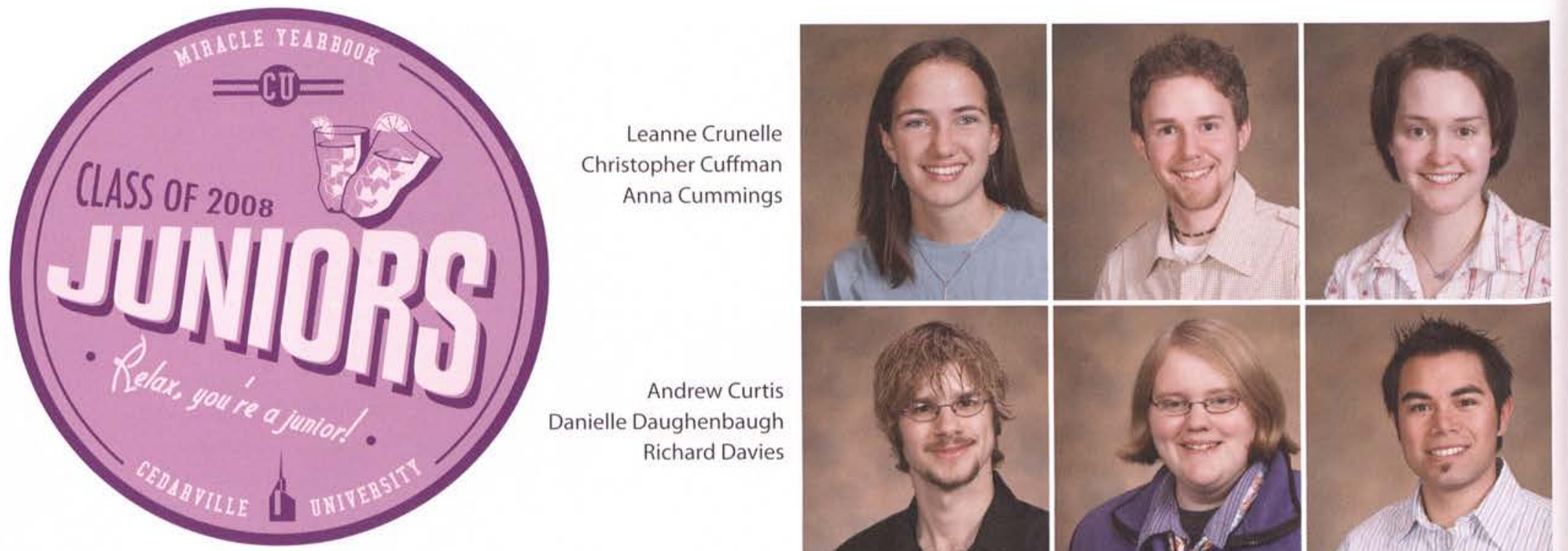

Jennifer Davis

Kenneth Davis

Matthew Davis

Michelle Davis

Lindsay Didonato

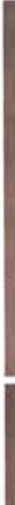

Jennifer Dixon

Justin Dodson

Brittany Donald

Emily Doot

Arielle Dorsey

Brandon Doyle

Rebekah Drum

Lani Duell

David Duerrwaechter

Courtney Edmonds

Caleb Ernst

Tiffany Erspamer

Rebecca Erwin

Pamela Eustace

Jonathan Evans
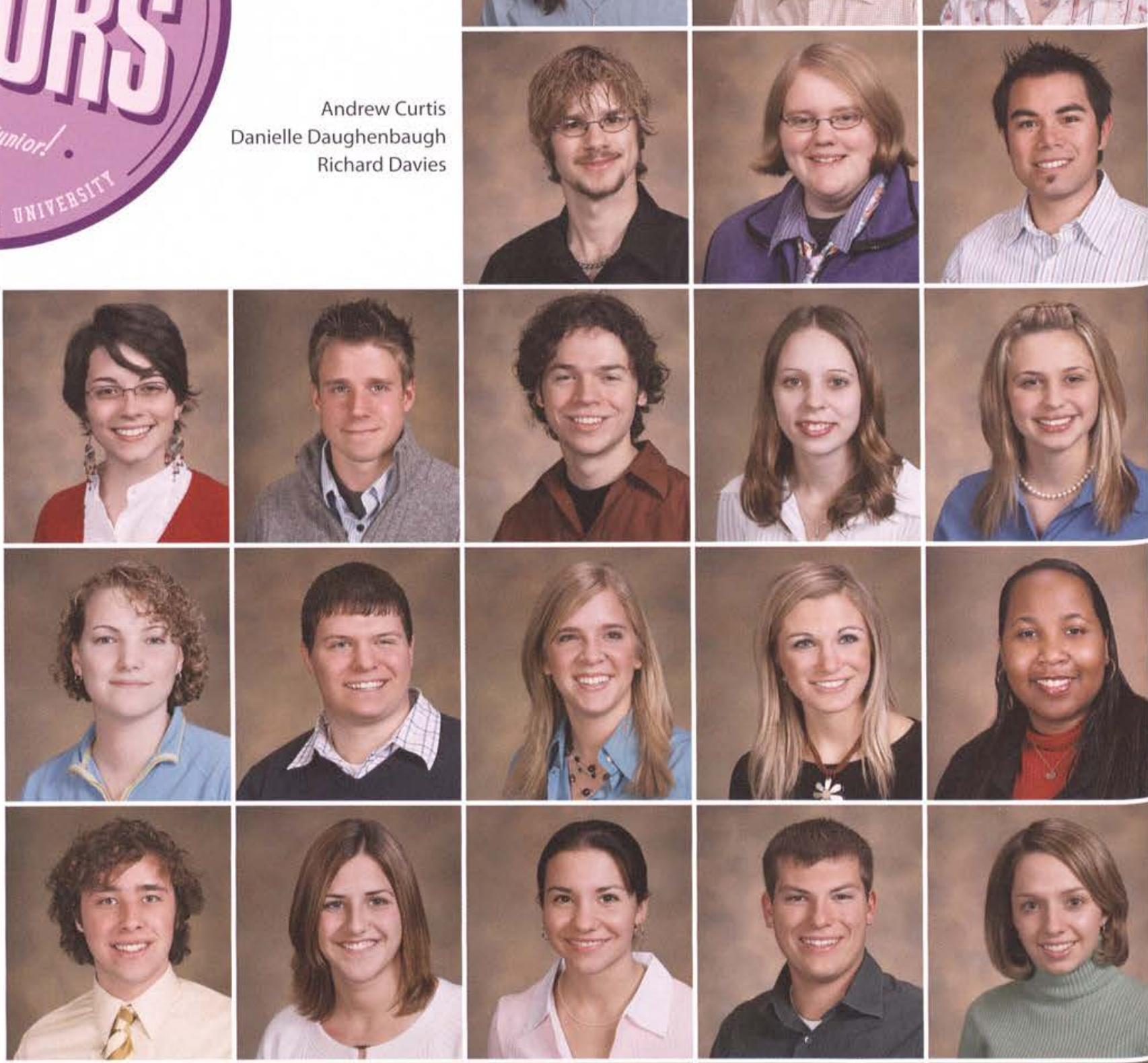

Jorns
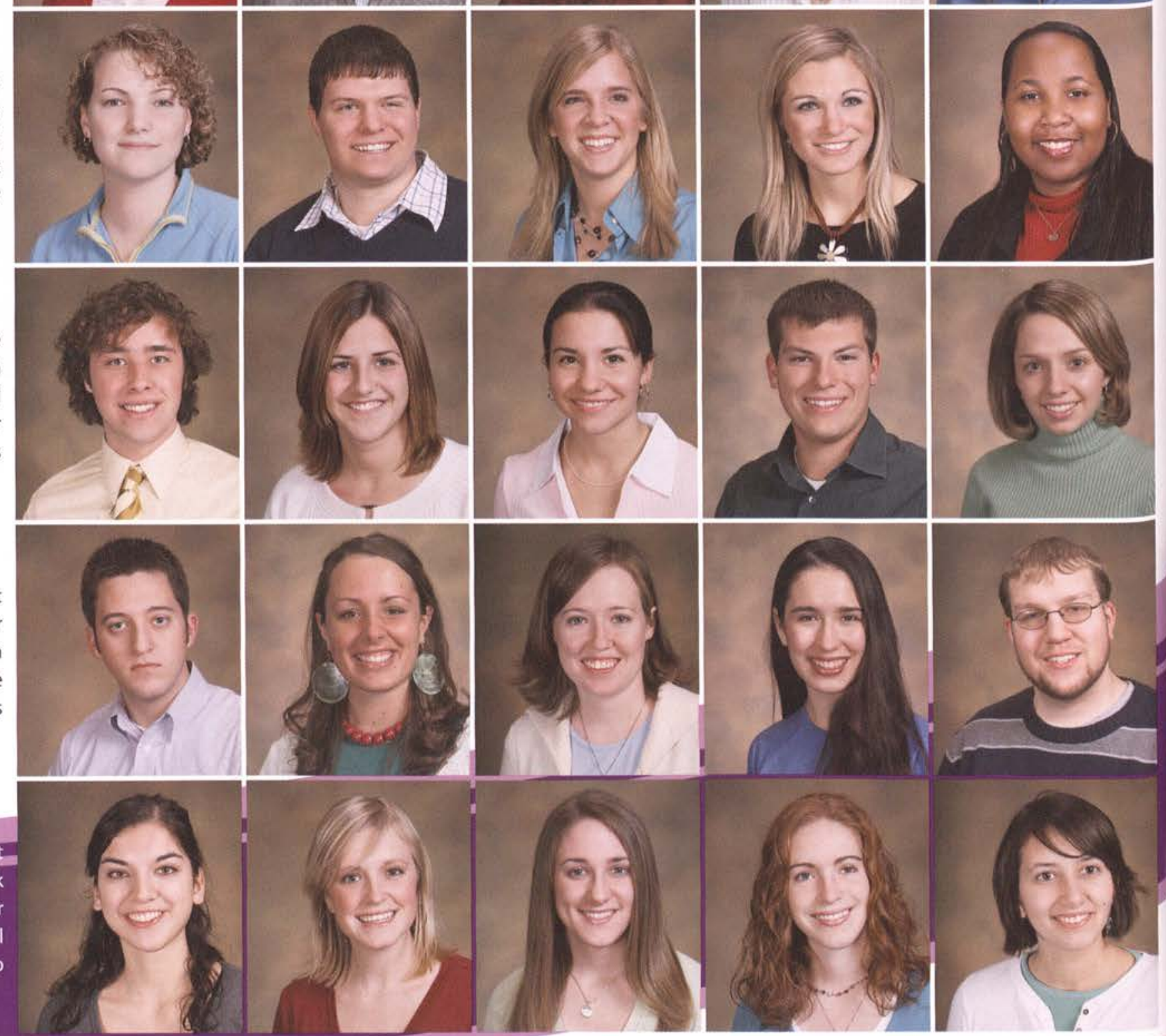

Abby Evereti Katelyn Farrar

Katherine Farrell

Kristie Febo 


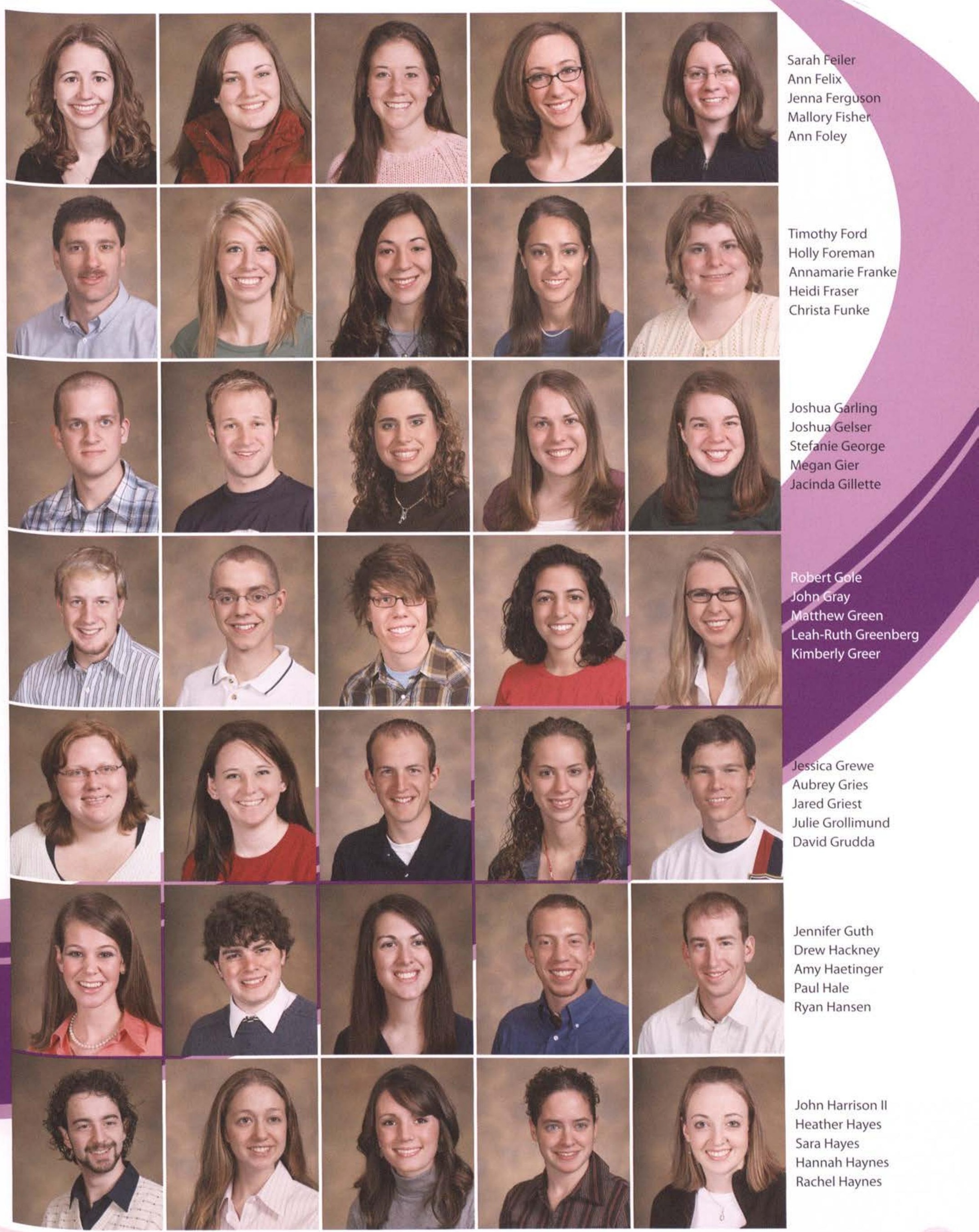



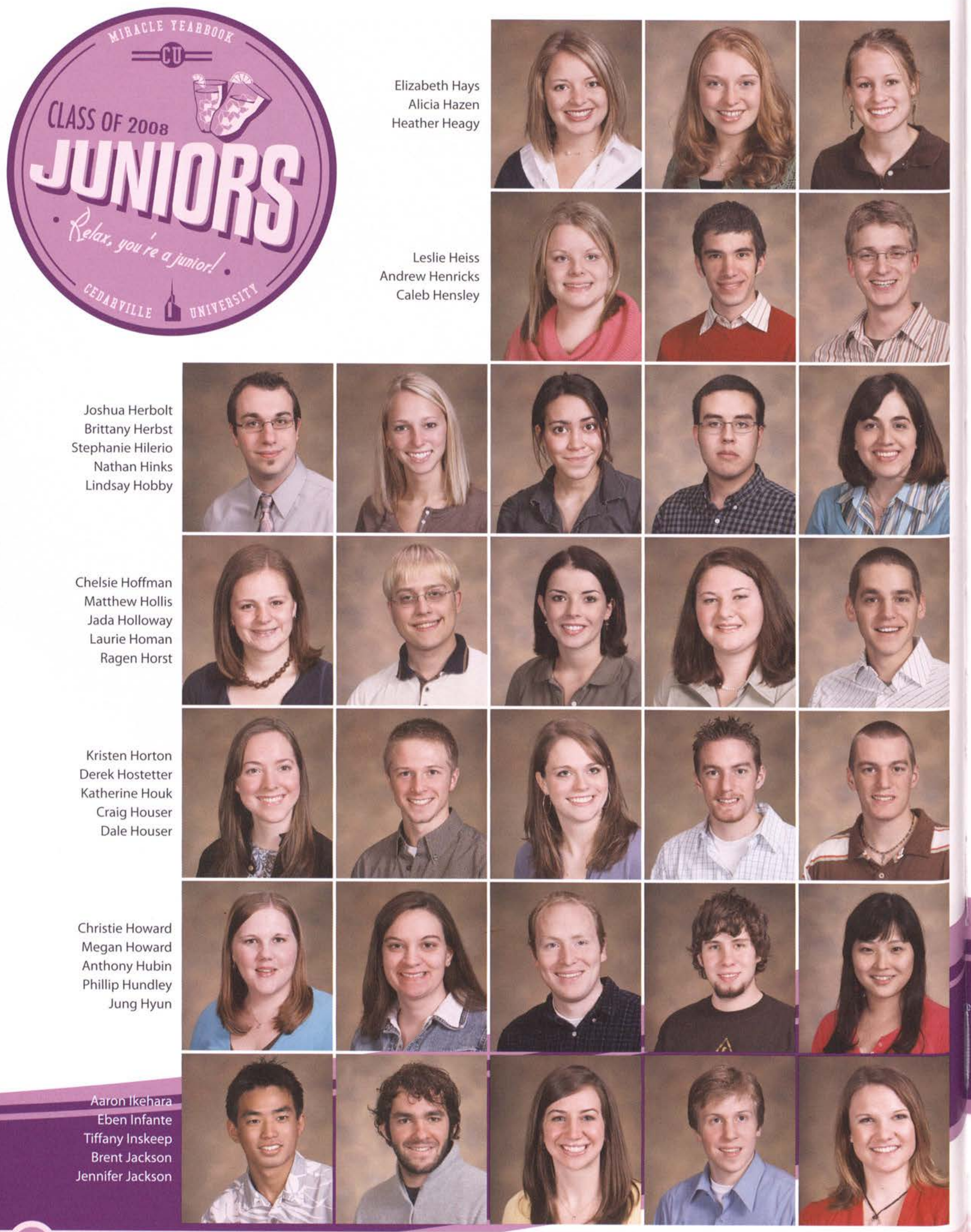

Christie Howard Megan Howard Anthony Hubin Phillip Hundley Jung Hyun

Aaronlikehara Eben Infante Tiffany Inskeep Brent Jackson Jennifer Jackson

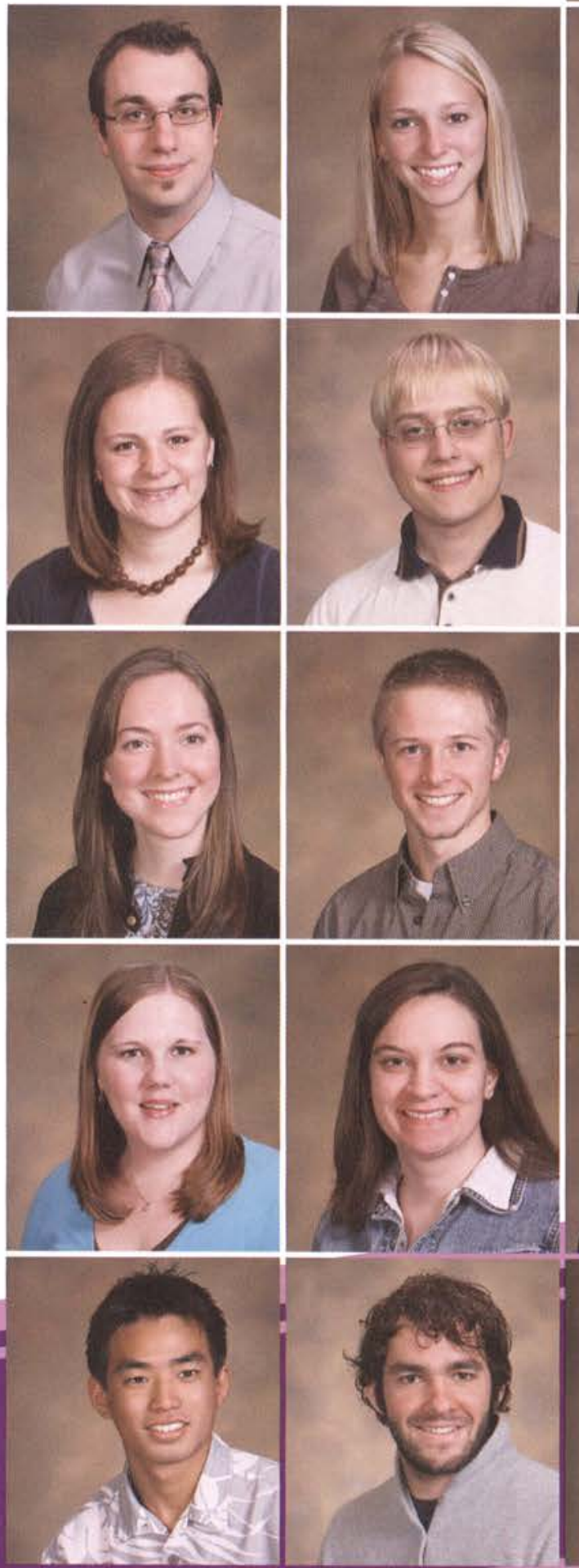

Elizabeth Hays

Alicia Hazen

Heather Heagy

Caleb Hensley

Brittany Herbst

Nathan Hinks

Lindsay Hobby

Chelsie Hoffman

Matthew Hollis

Jada Holloway

Laurie Homan

Ragen Horst

Kristen Horton

Derek Hostetter

Katherine Houk

Craig Houser 


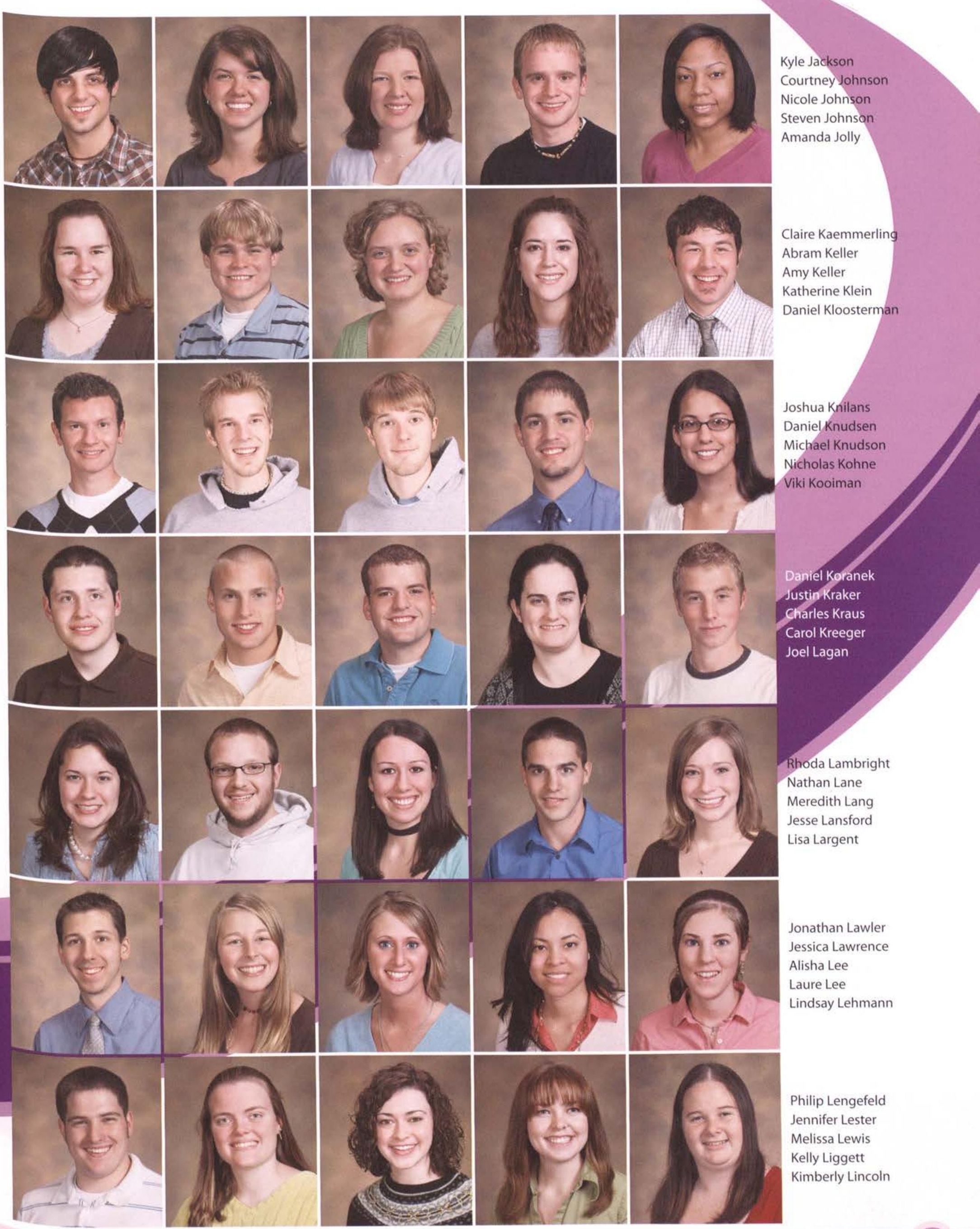



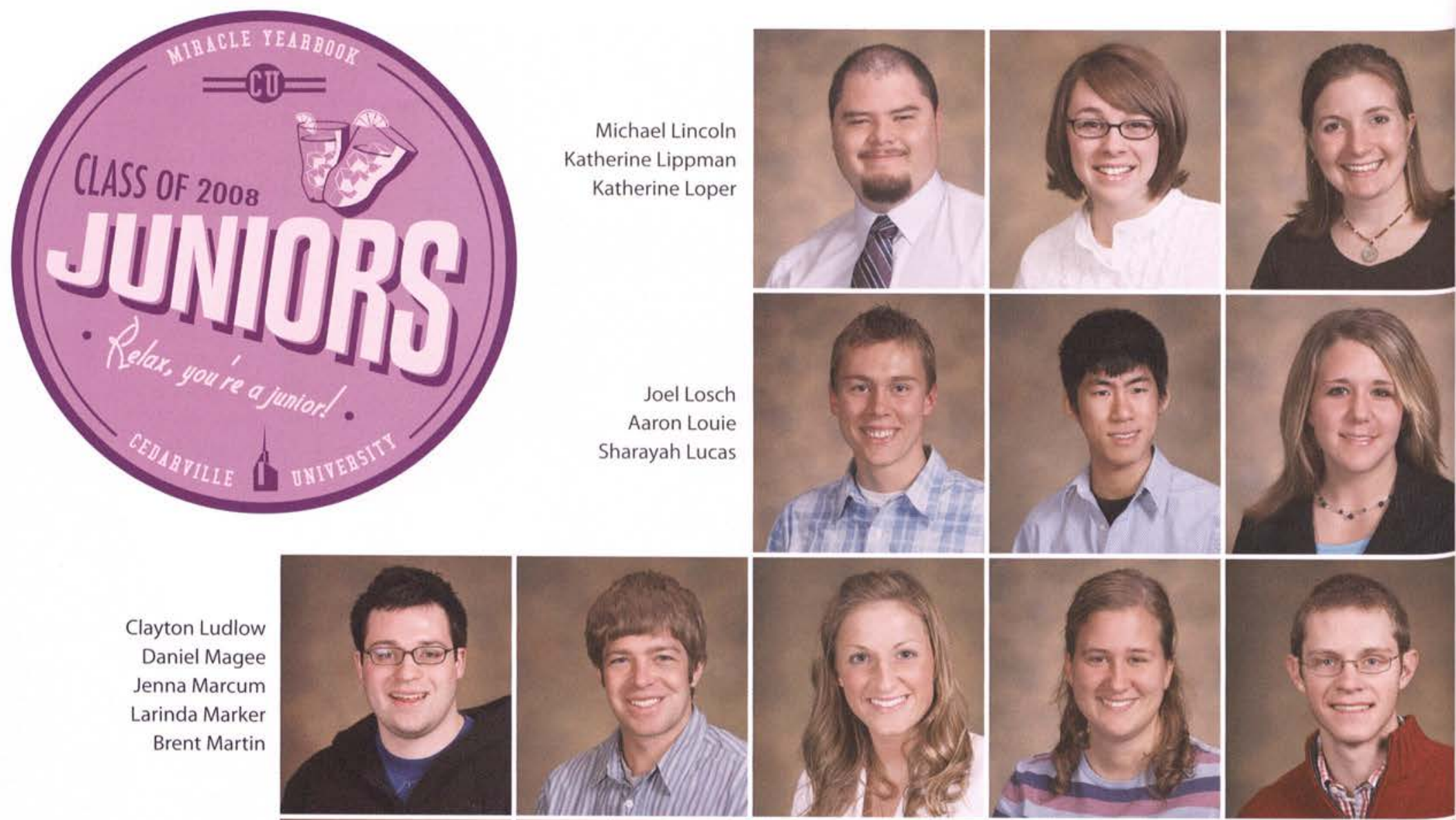

Daniel Magee

Jenna Marcum

Larinda Marker

Brent Martin

Michael Lincoln

Katherine Lippman

Katherine Loper
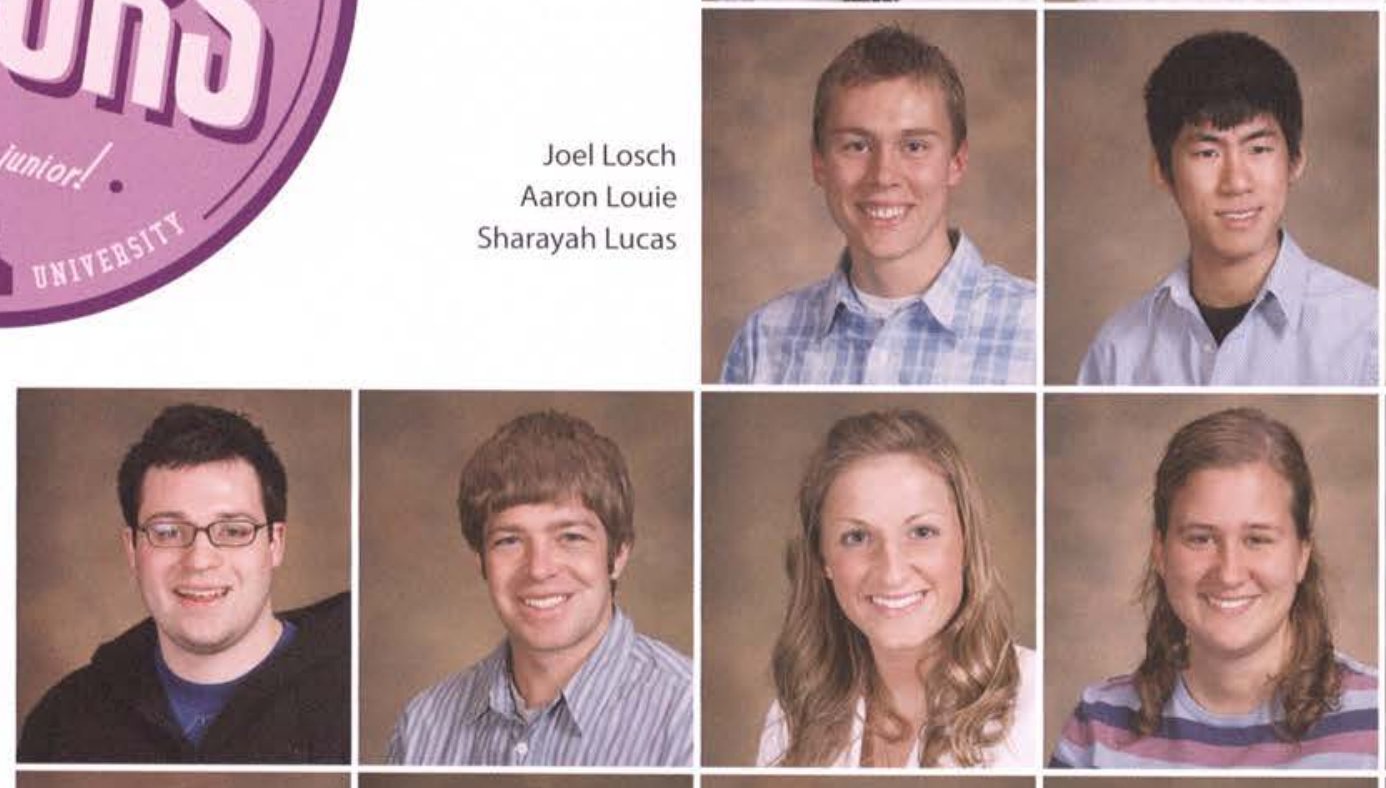

Elizabeth Martin

Micah Martin

Julie Martz

Melissa Mathews

Kendra Mattson

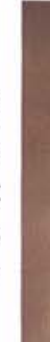
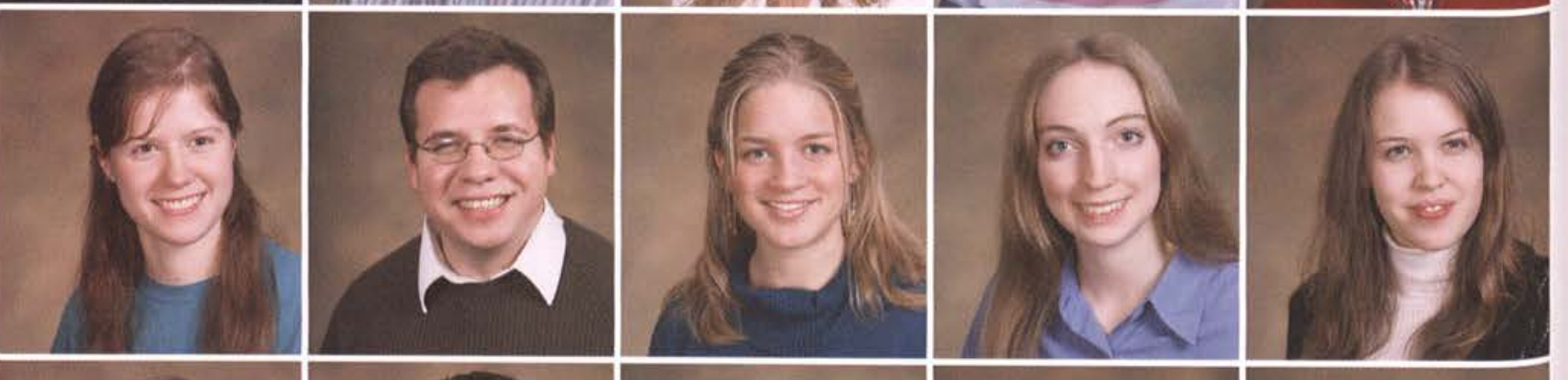

Michele Mayer

James McClenahan

John McClure

Amanda McCollim

Wade McComas

Ustin McCoy

Amanda McKinley

Julie McQueen

Benjamin McRae

Kena Mena
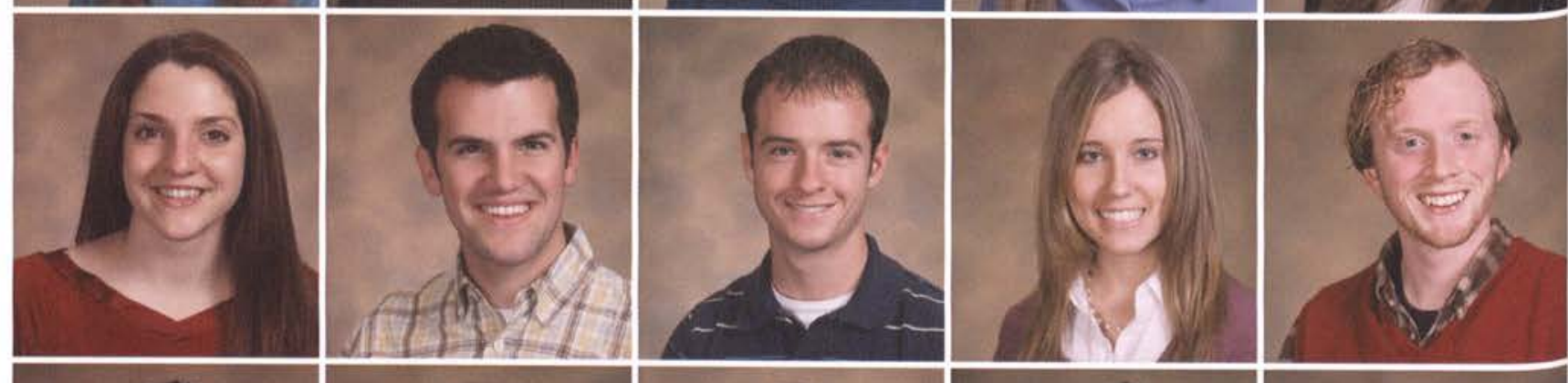

Kristin Merkel

Marguerite Mikolon

Jeremy Miller

Laura Miller

Scott Miller
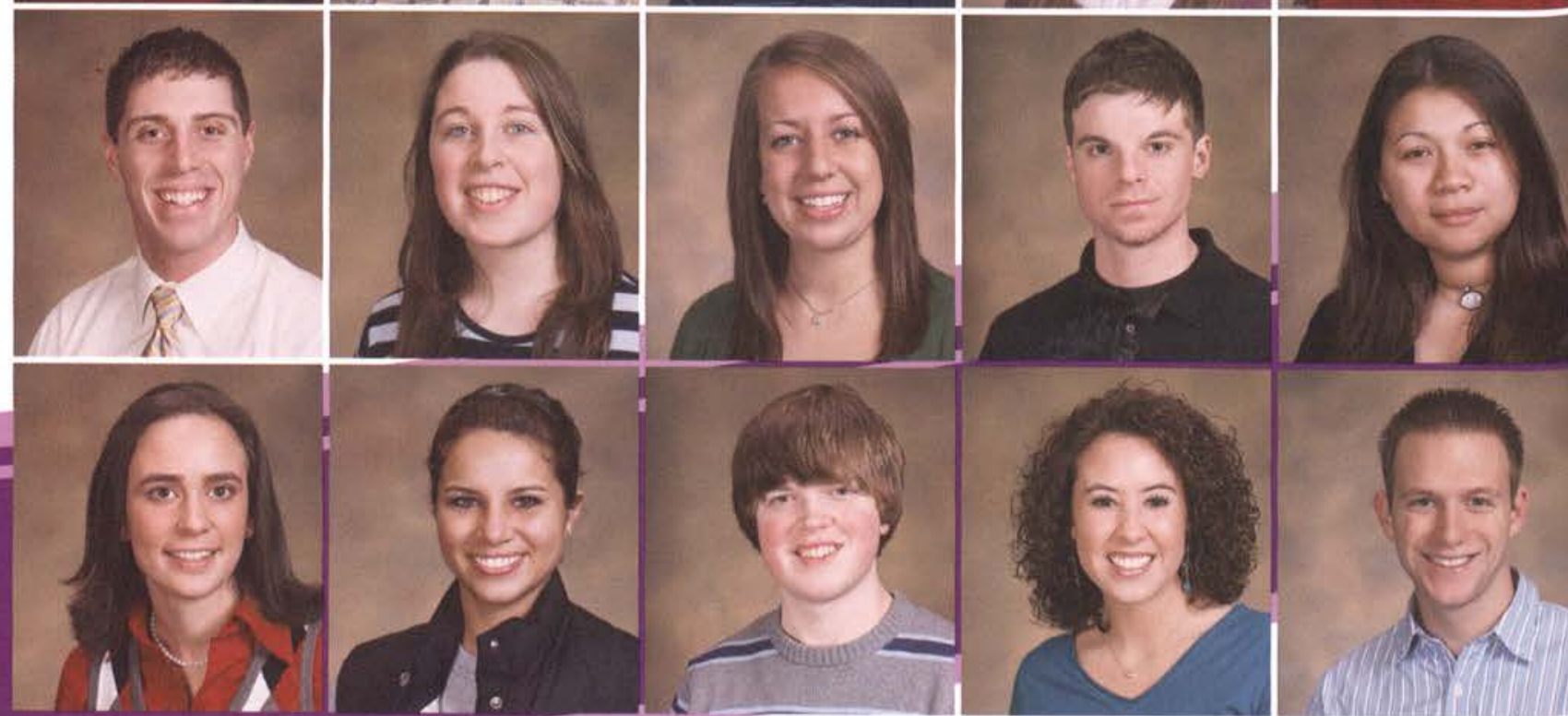


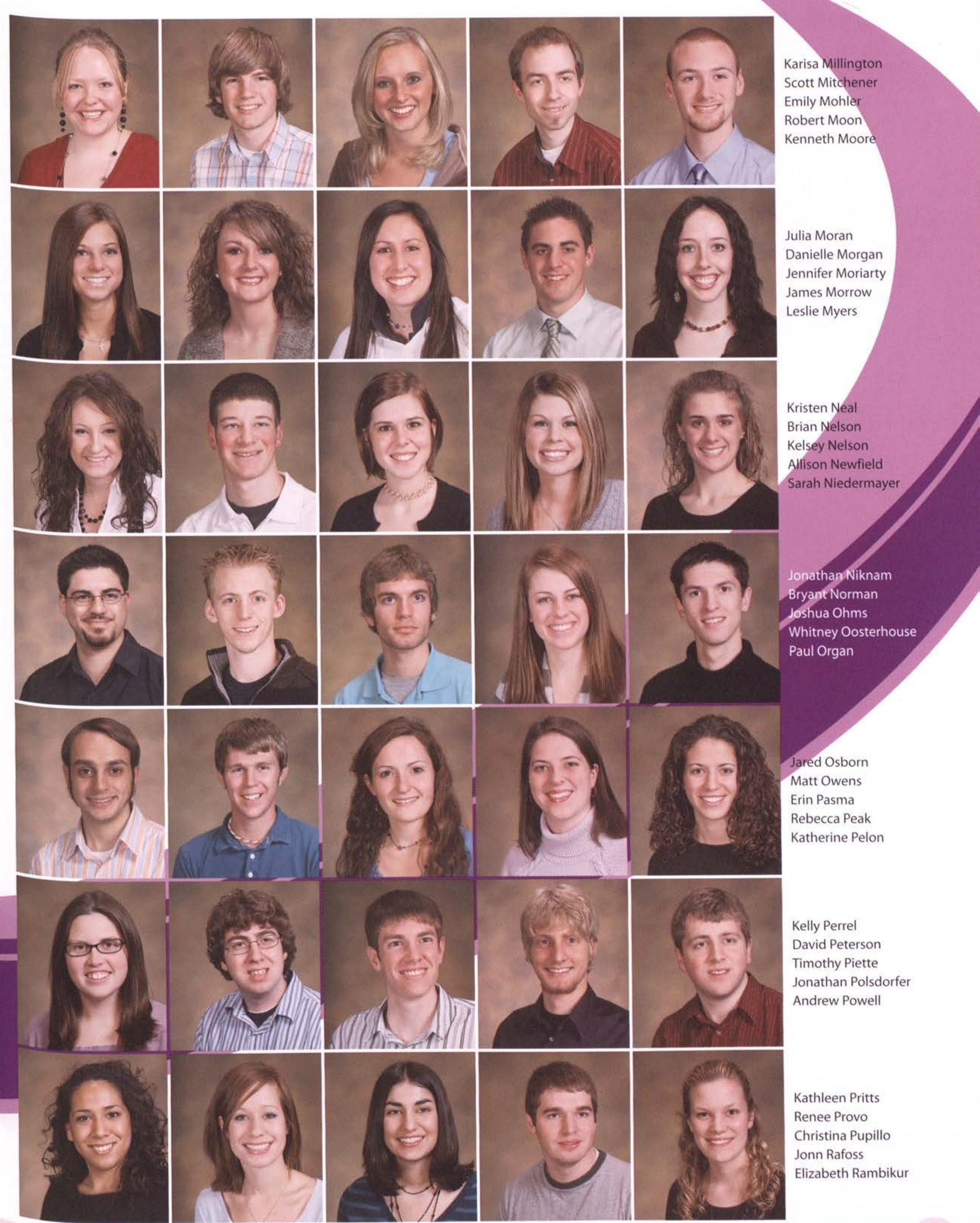



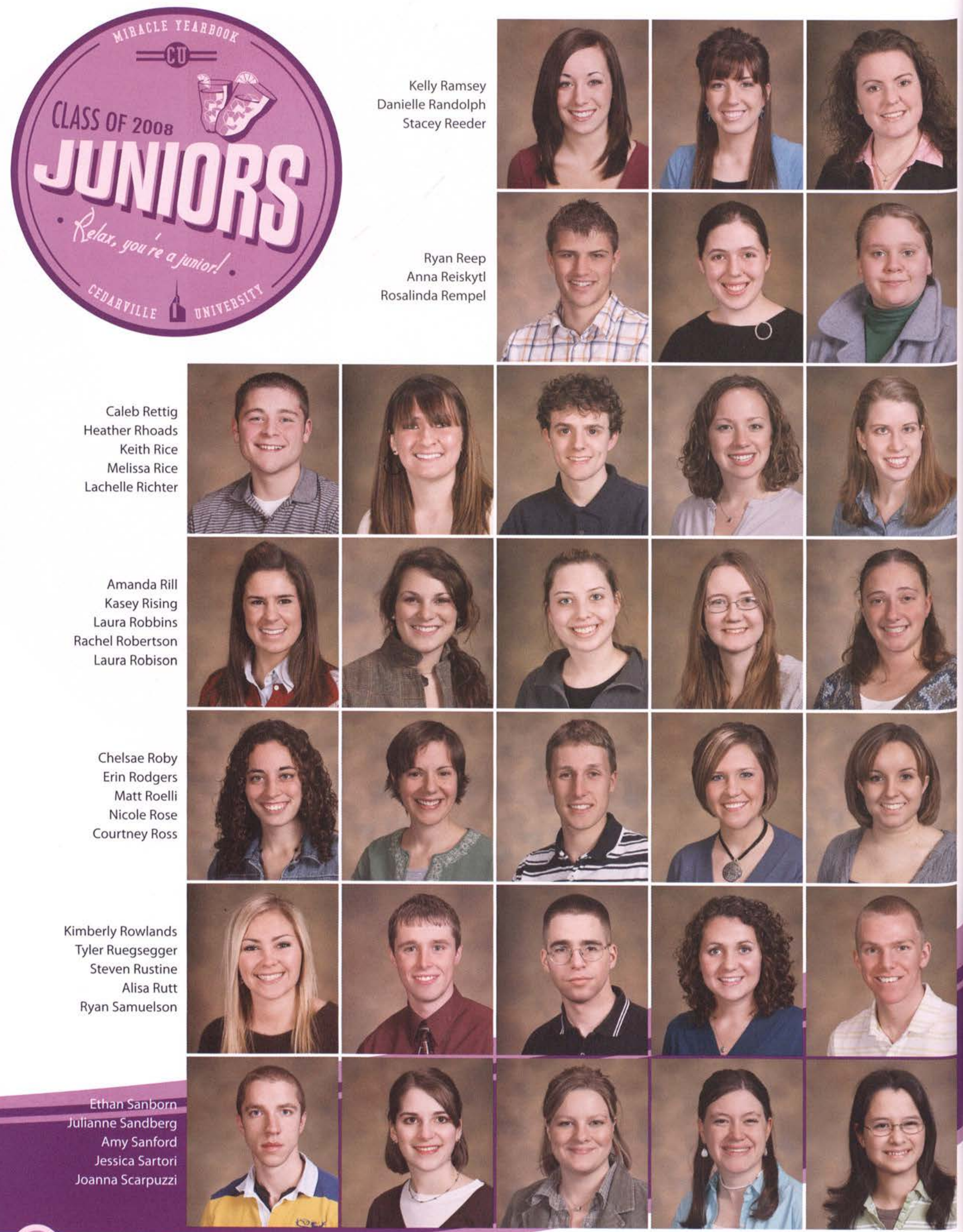

Kimberly Rowlands

Tyler Ruegsegger

Steven Rustine

Alisa Rutt

Ryan Samuelson

Ethan Sanborn

Julianne Sandberg

Amy Sanford

Jessica Sartori

Joanna Scarpuzzi

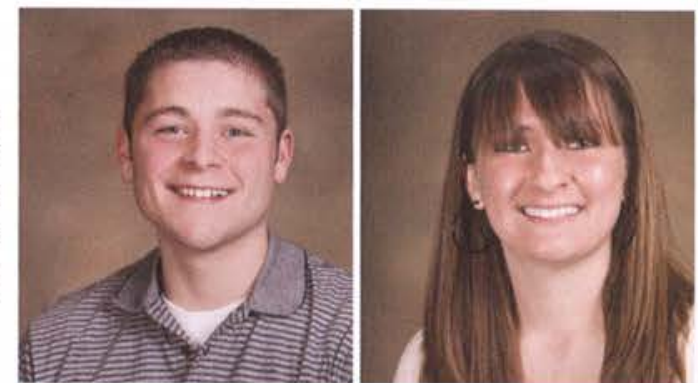

Danielle Randolph

Stacey Reeder
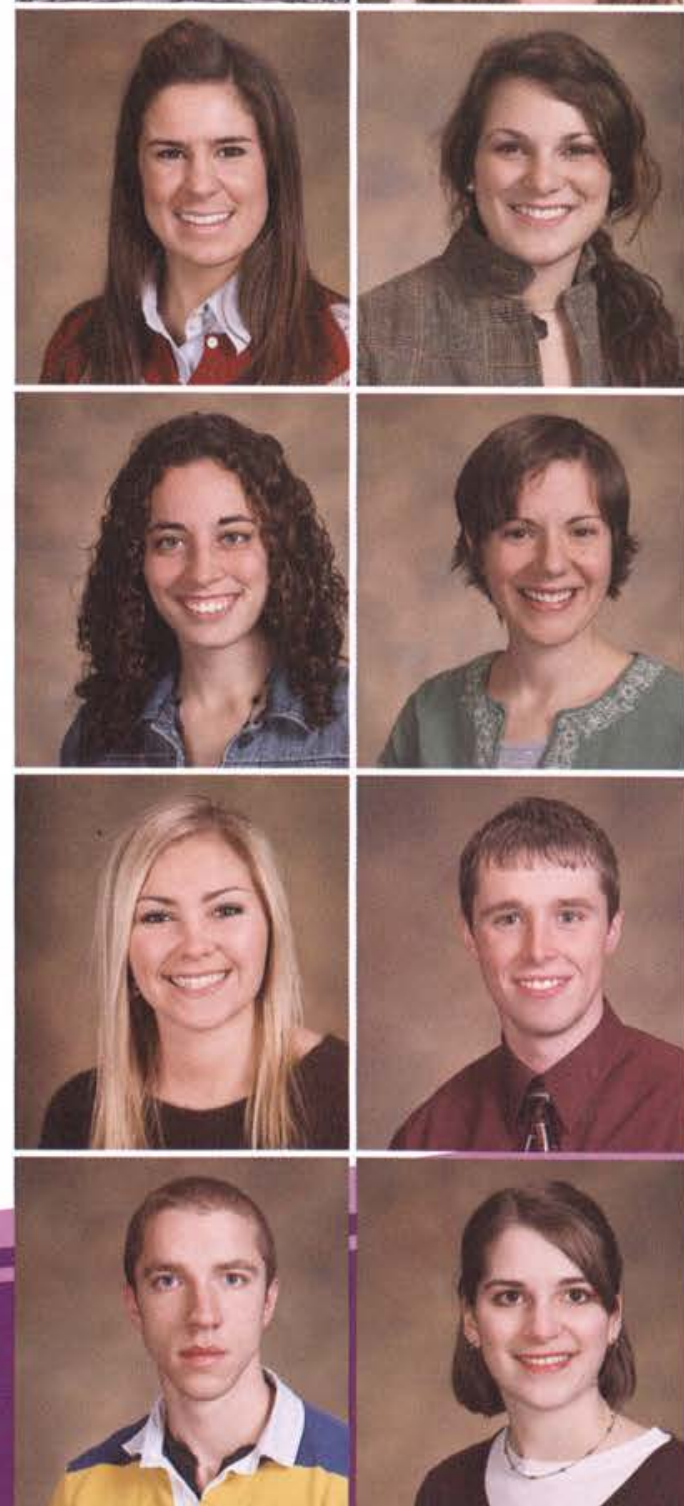

cossurs

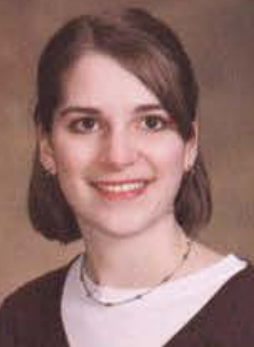




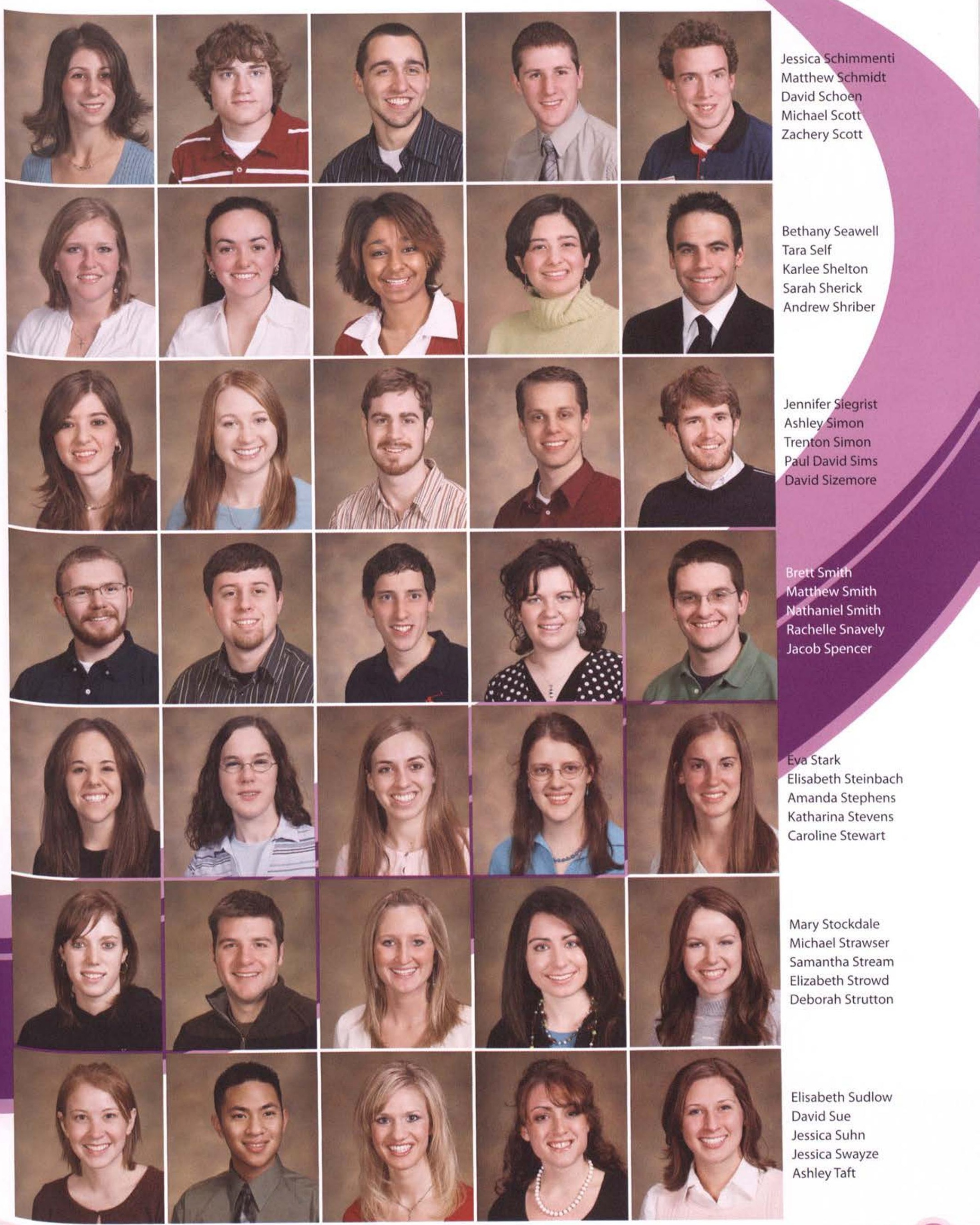



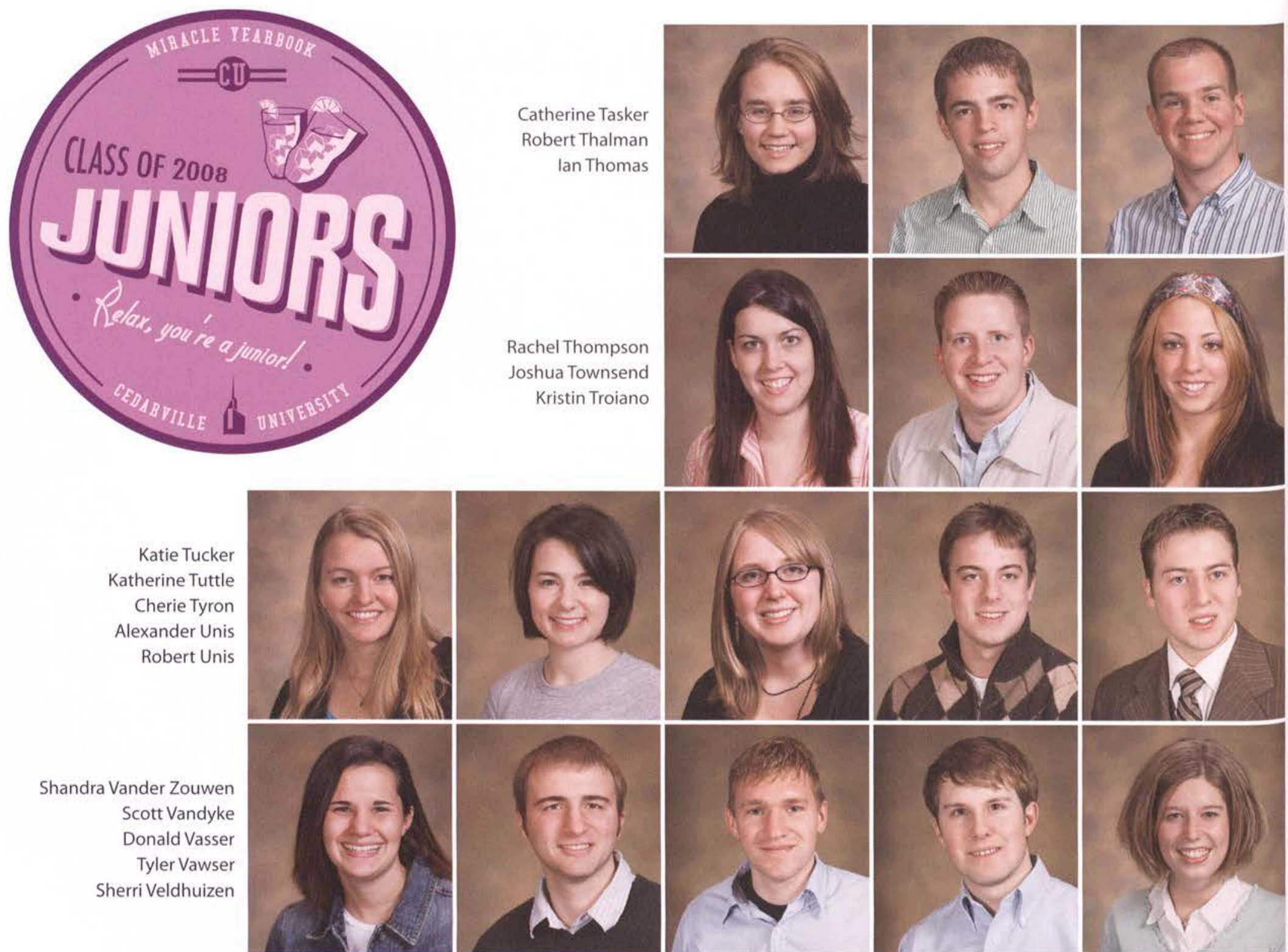

Shandra Vander Zouwen Scott Vandyke Donald Vasser Tyler Vawser Sherri Veldhuizen
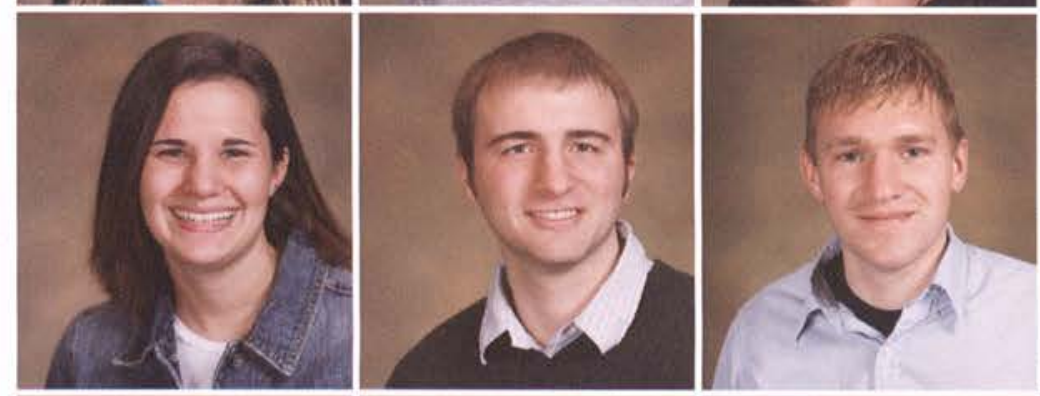

oshua Townsend
Kristin Troiano
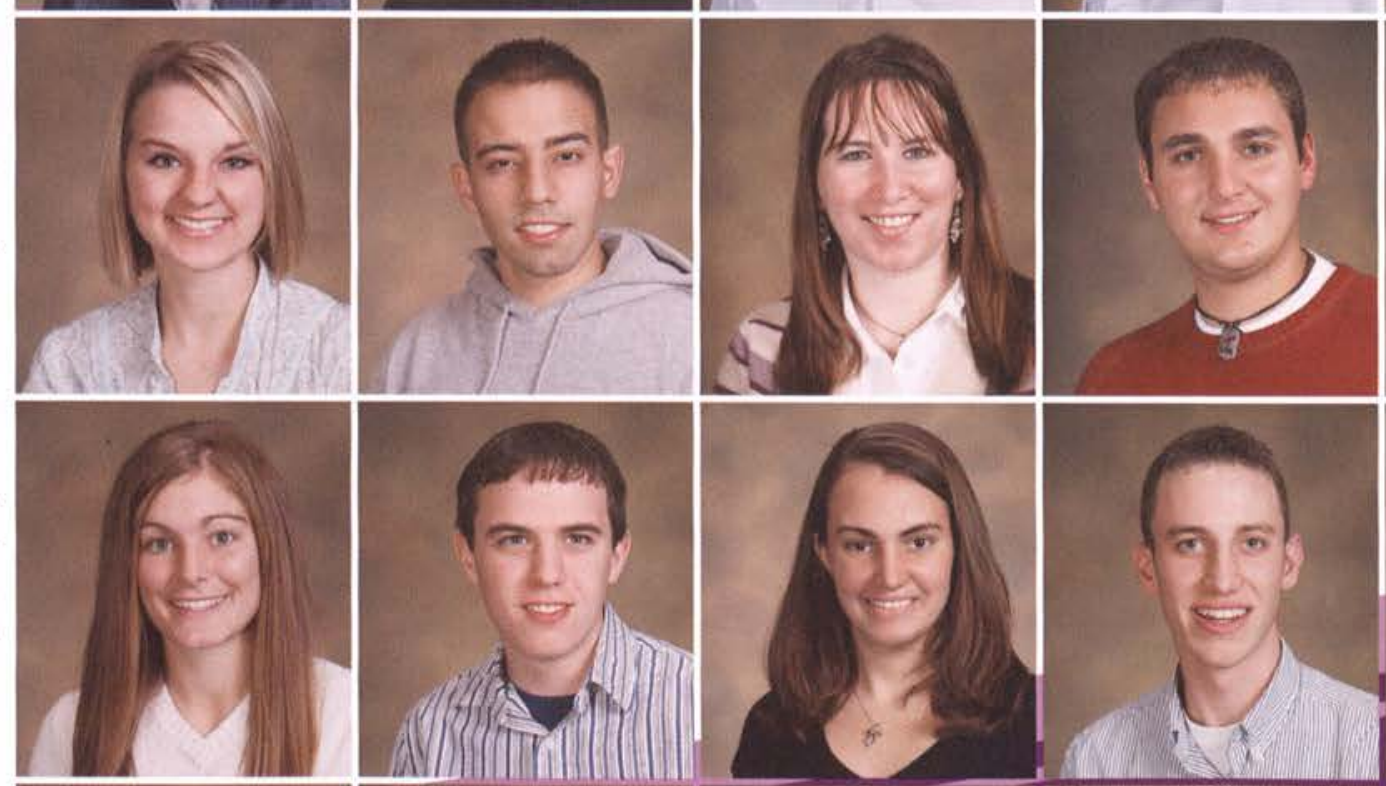

Andrea Walker Joshua Walther Christina Weathers Marcus Weaver Tricia Wegman

Cassie Weideman Kendall Welker Nathan Wexler Alicia Whitman Joshua Willetts
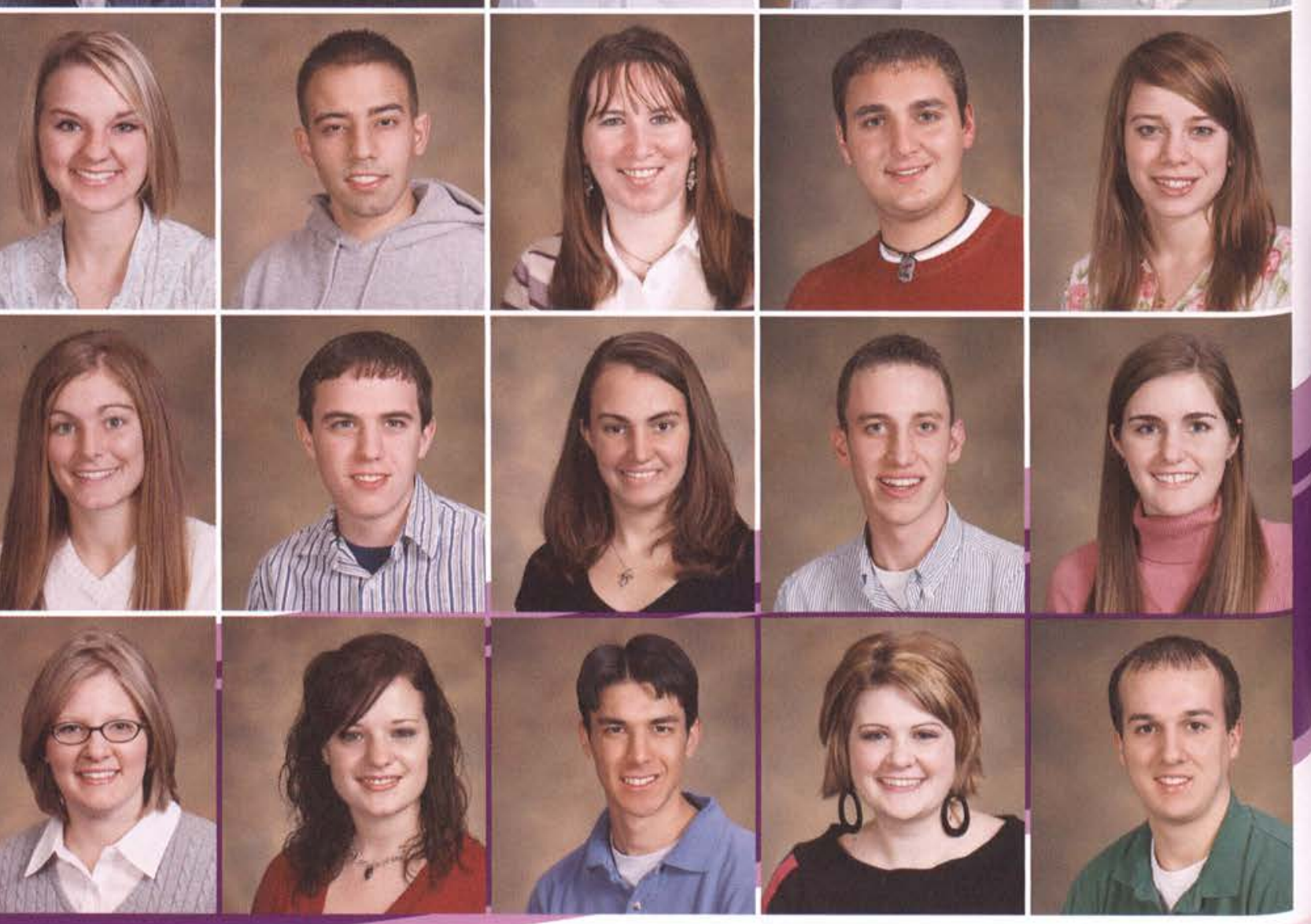

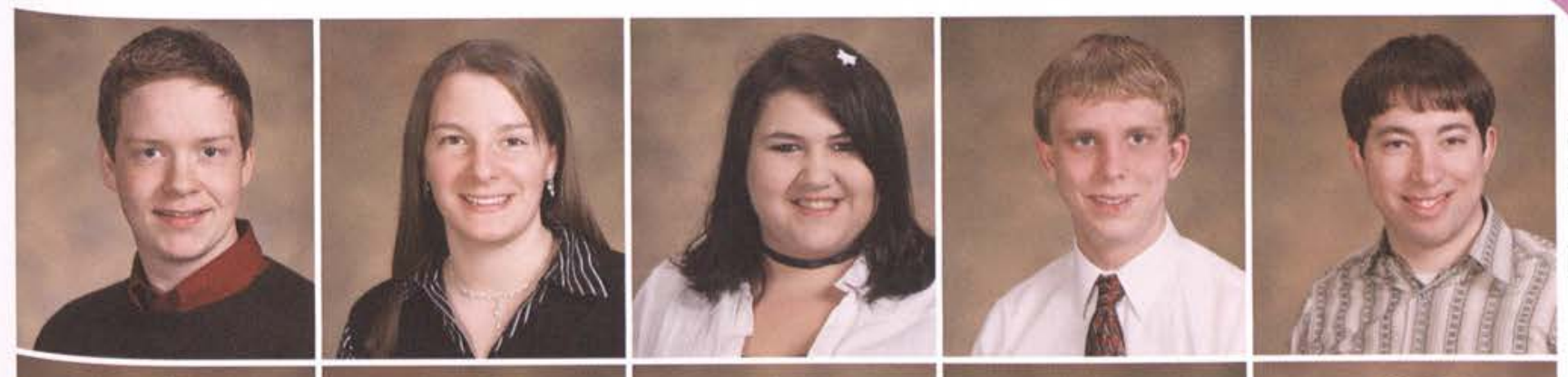

Garrett Williams

Michelle Williams

Suzanne Wilson

Thomas Woltman

Nicholas Wood
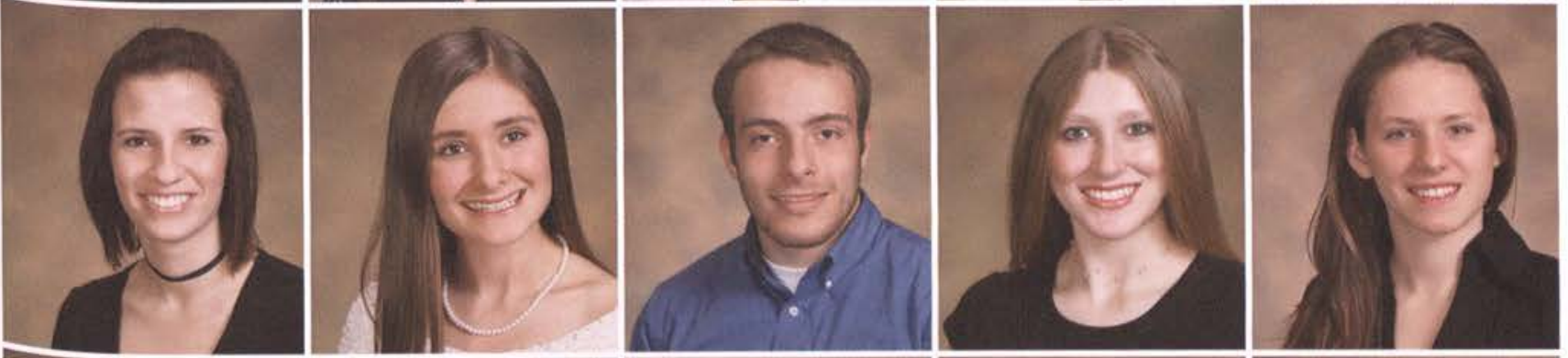

Alissa Woodwyk

Melinda Workman

Craig Wright

Elisa Yanega

Kati Yosinski
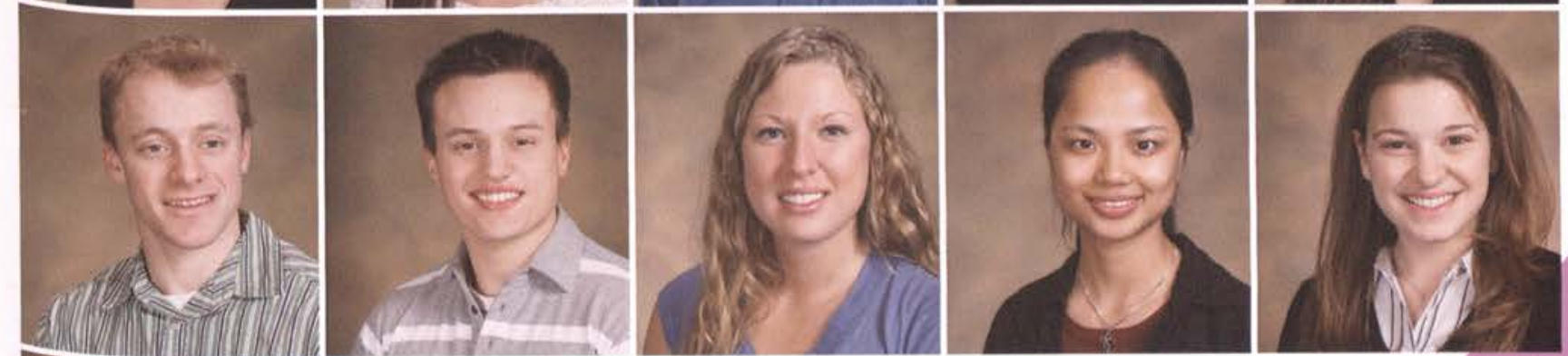

Richard Young Jason Zastrow Sarah Zeltman Bingjia Zhang Laura Ziegenfus
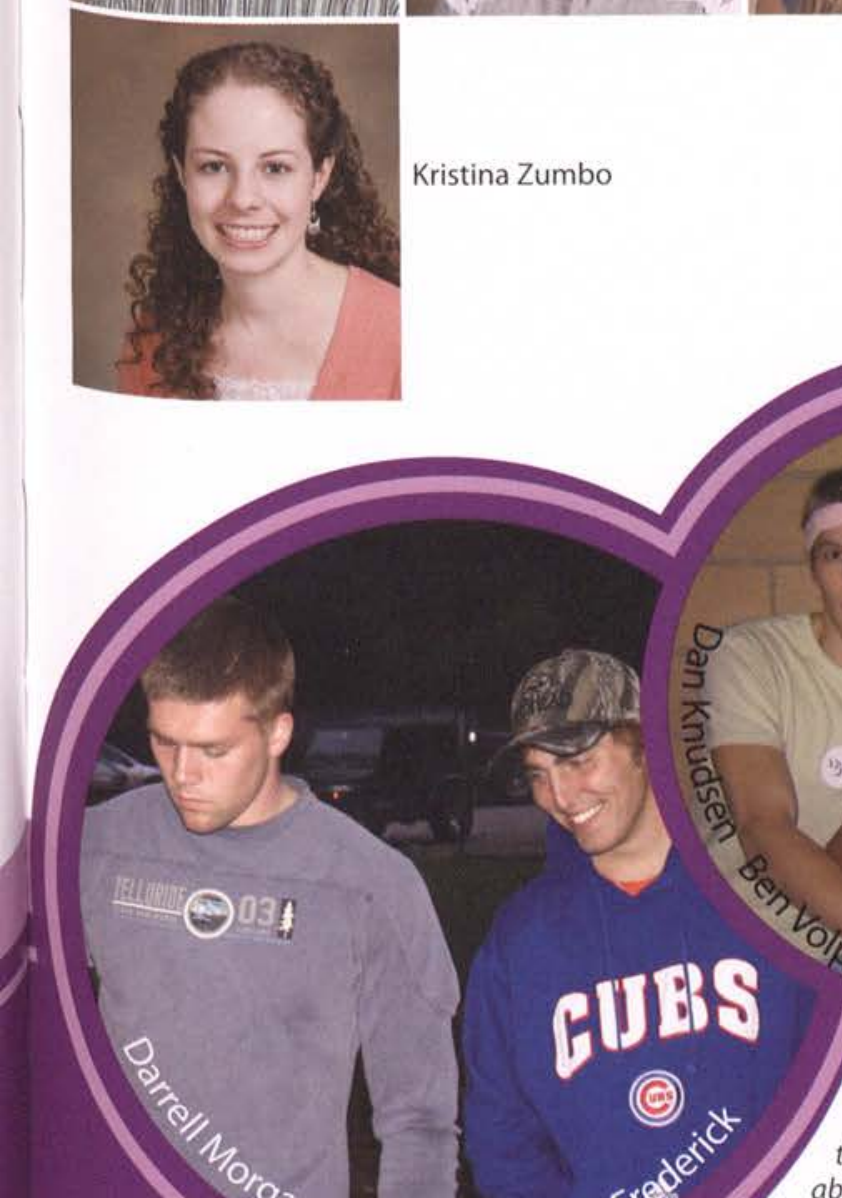
A What changes one phone
call can make. It's that one that all of us parents have heard about, but silently dread getting. So starts the journal "ChecklnOnDan.

blogspot.com" when it covers the latest updates

on junior Dan Knudson. While on a spring break MIS

trip to Mexico, Dan landed on his neck when attempting to do a

back flip. After several discouraging weeks of total paralysis, Dan has slowly regained feeling. Although he remains in a wheelchair for the time being, he is back to eating and drinking on his own, and each daily improvement is more encouraging than the next. However, there is far more to Dan than what has been happening these past two months. He is a pre-law major, an OPE-ite, and involved in ten 24; he loves ultimate Frisbee, hunting, and his two new twin nieces. Overall, Dan is a fun-loving guy who is sorely missed on the $\mathrm{CU}$ campus, but who through his strength and determination has been a source of encouragement to us all. 


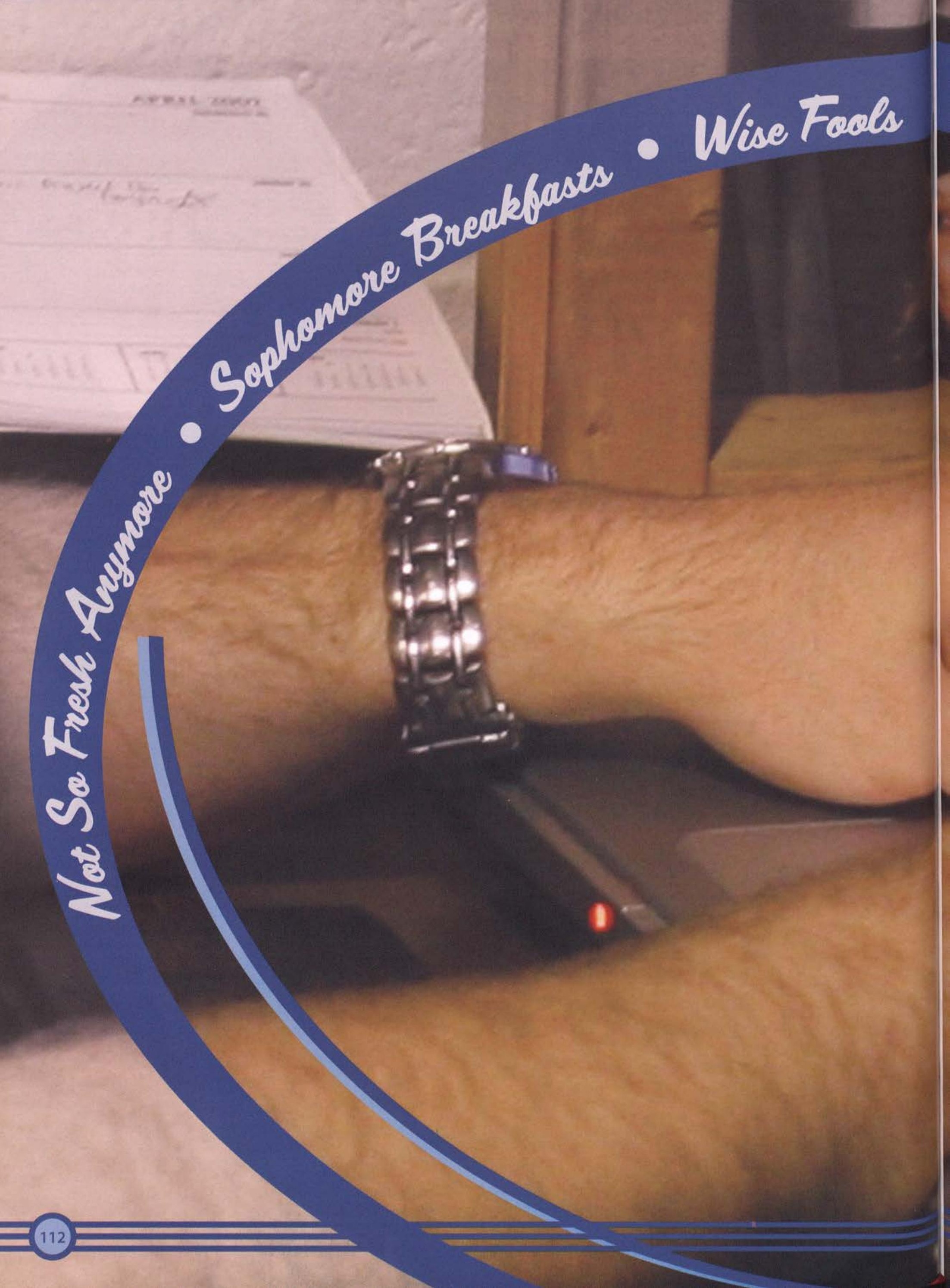


- Bay Meox 6 !'

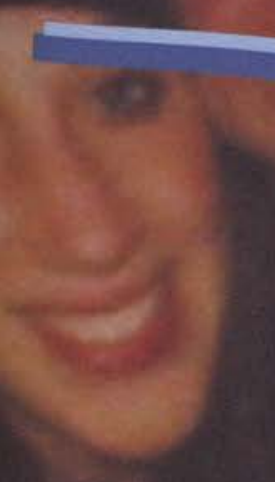

$\infty$
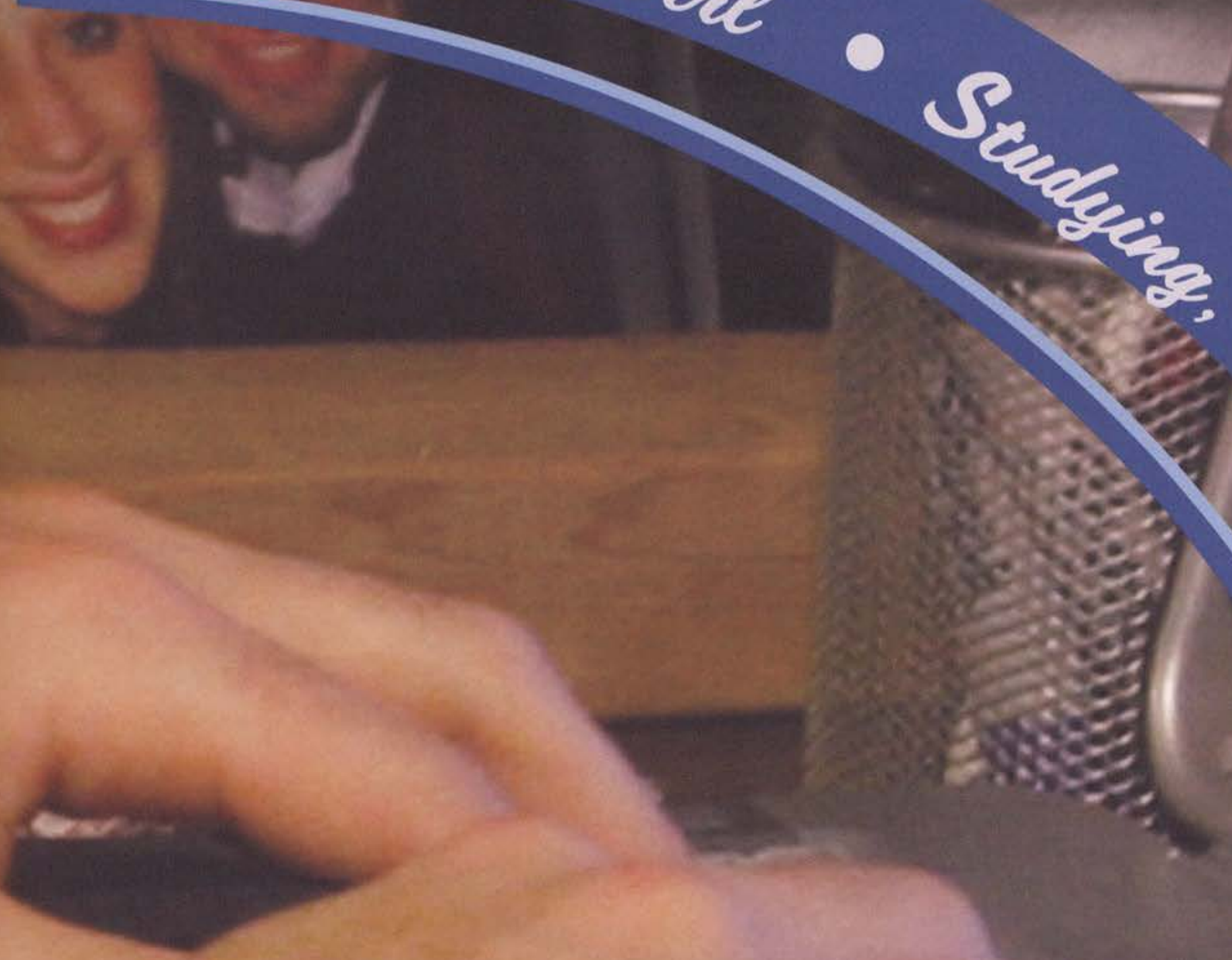

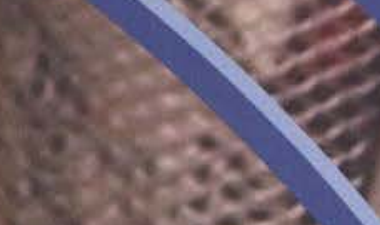

$3 x+2=2=$

$3 x^{2}+2=0$

are is:

4.5.

exs

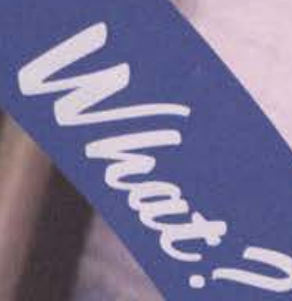

(
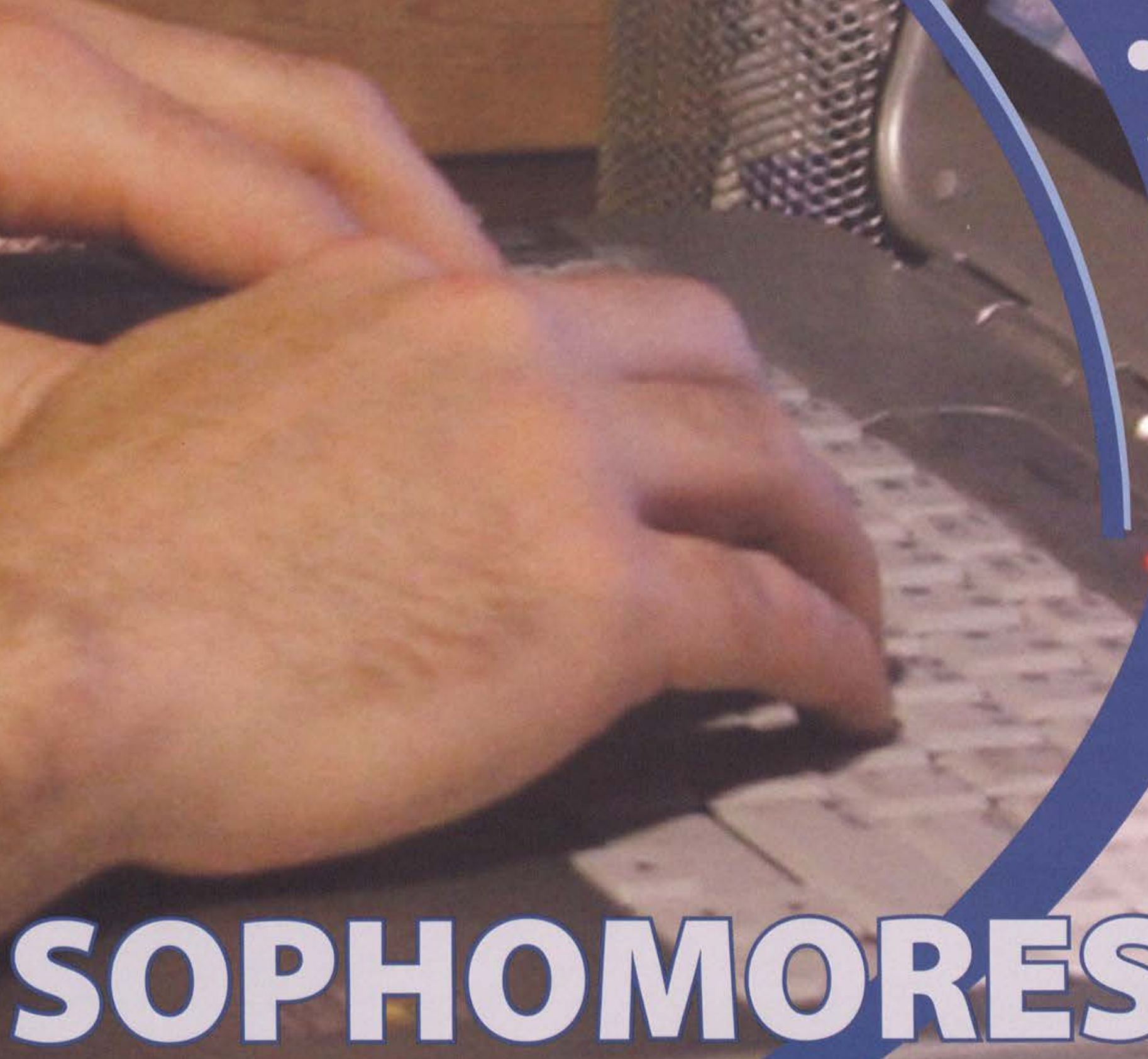

SOMnOM

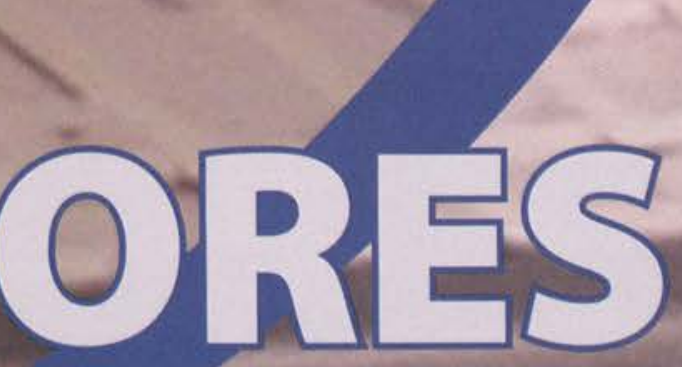

(113) $=$ 

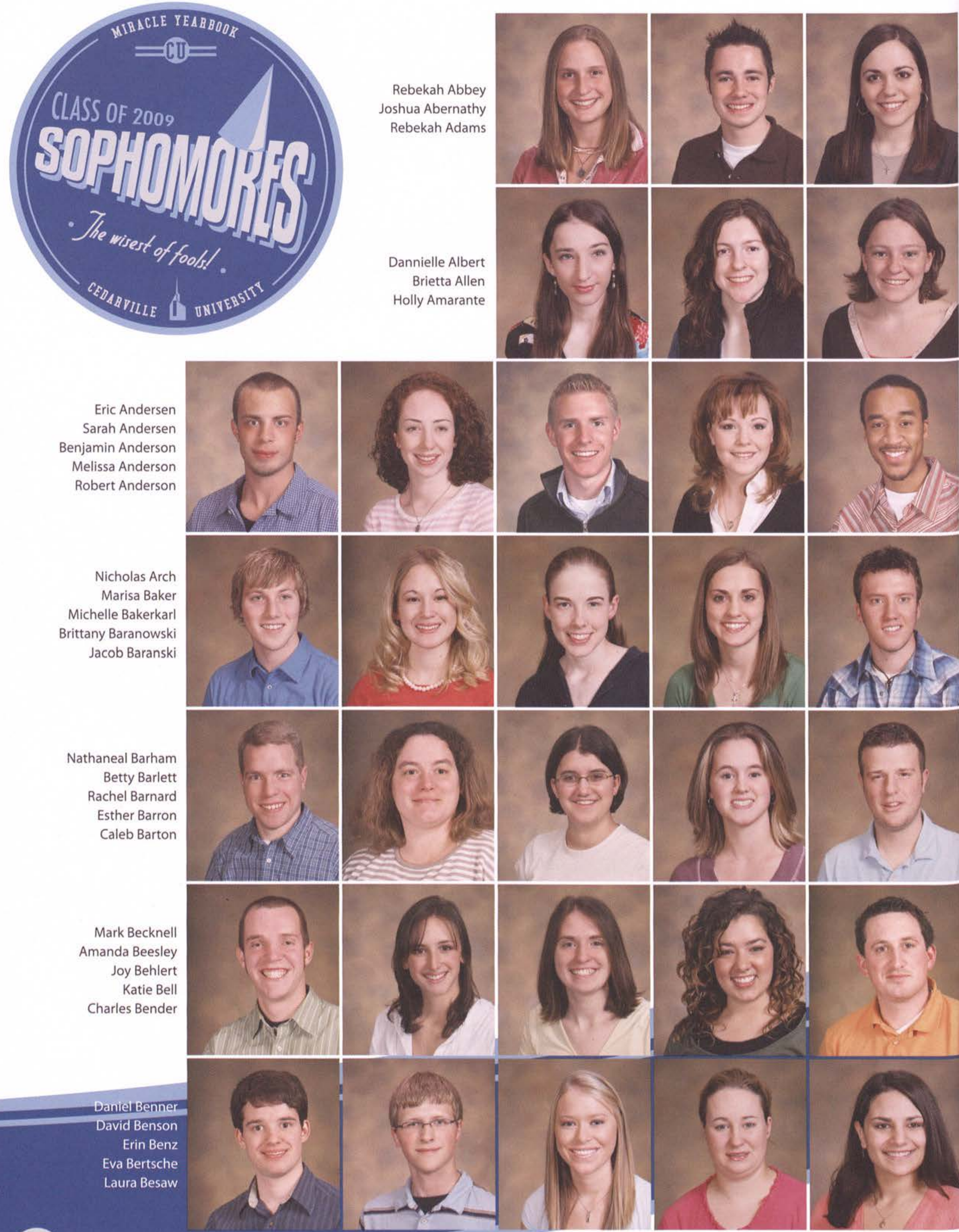

Amanda Beesley

Joy Behlert

Katie Bell

Charles Bender
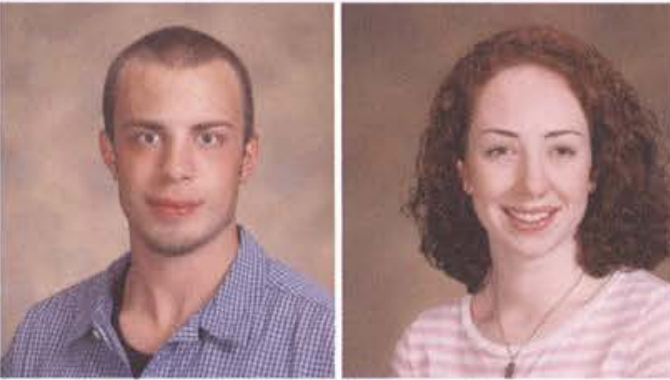

Nicholas Arch

Marisa Baker

Michelle Bakerkarl

作

Nathaneal Barham

Rachel Barnard

Esther Barron

Caleb Barton

Daniel Benner David Benson

Erin Benz

Eva Bertsche

Laura Besaw

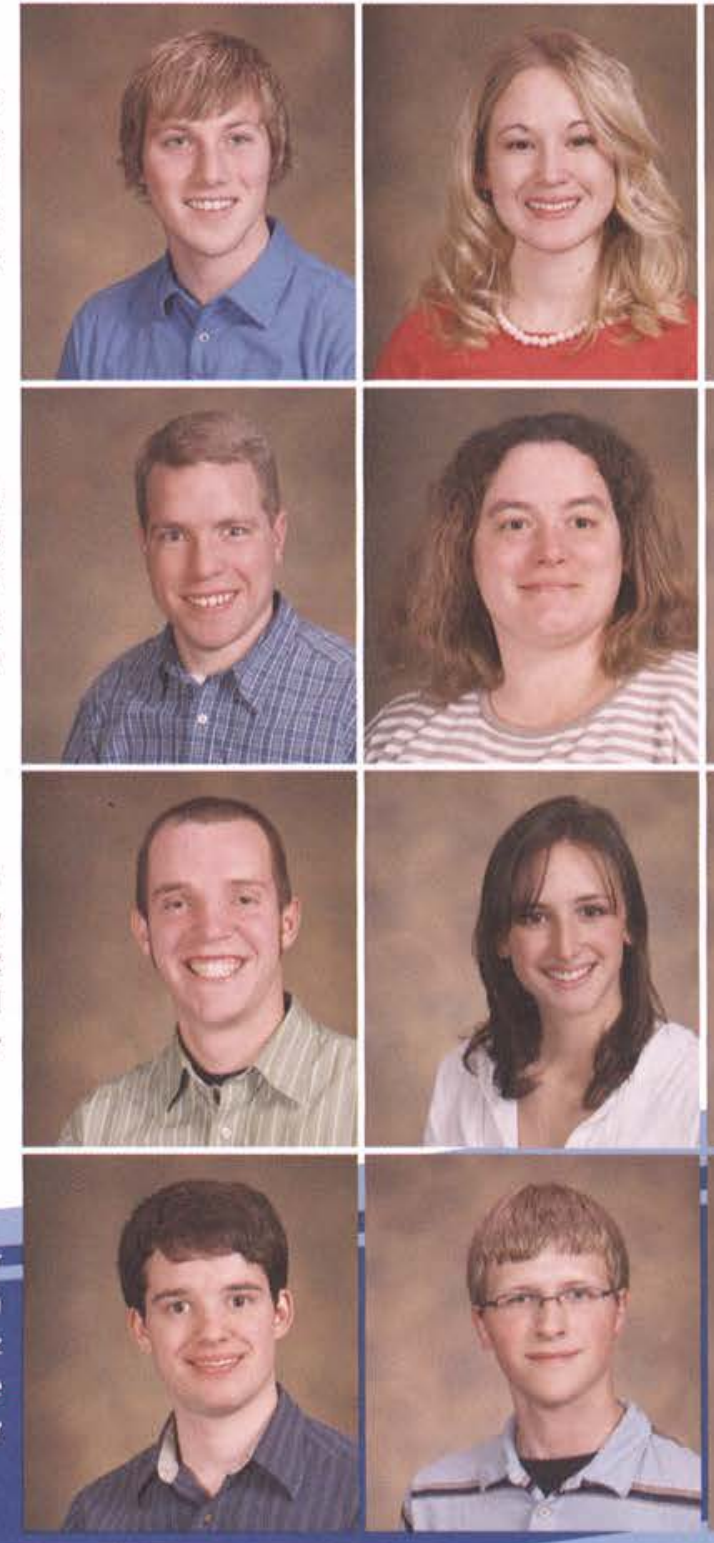




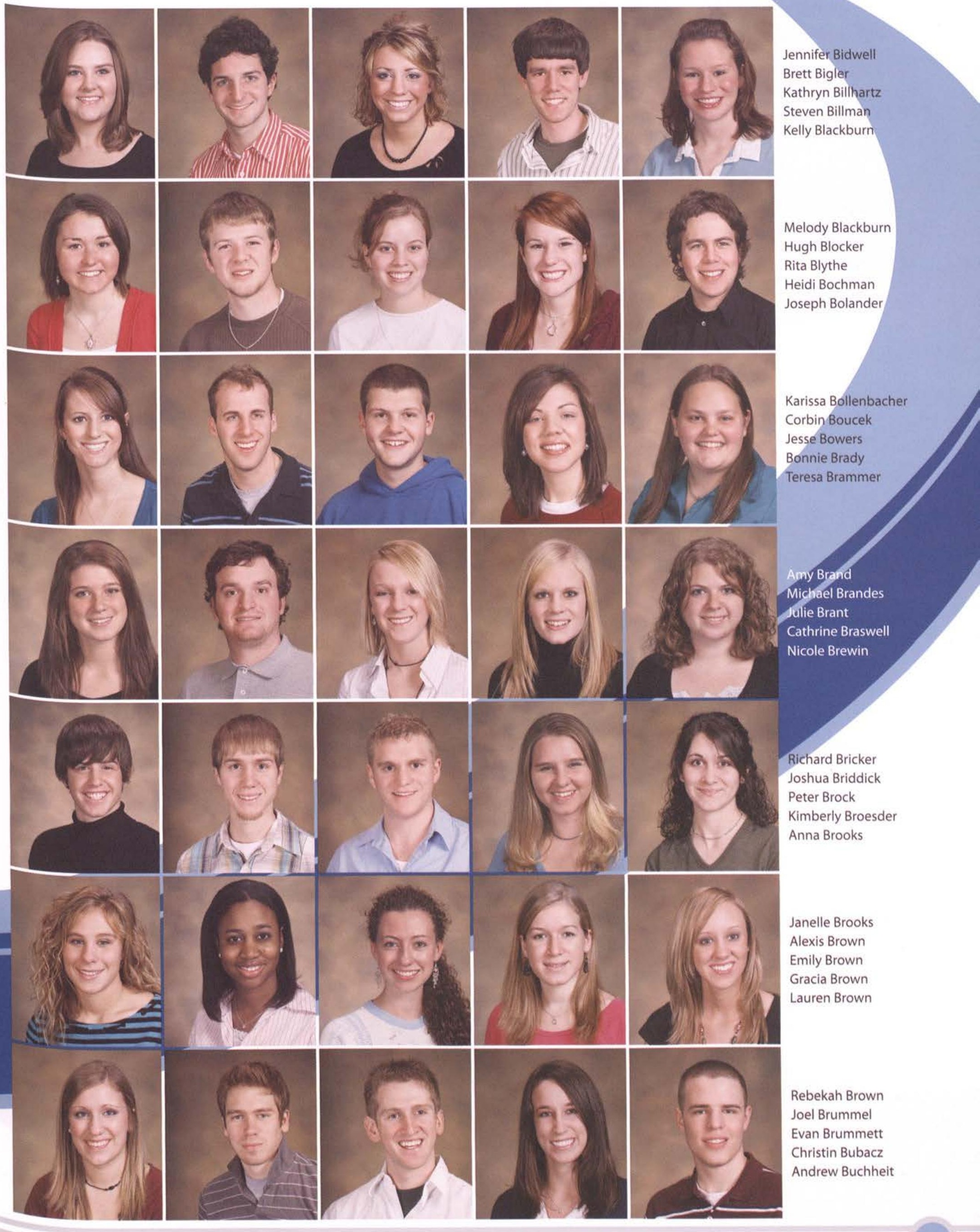



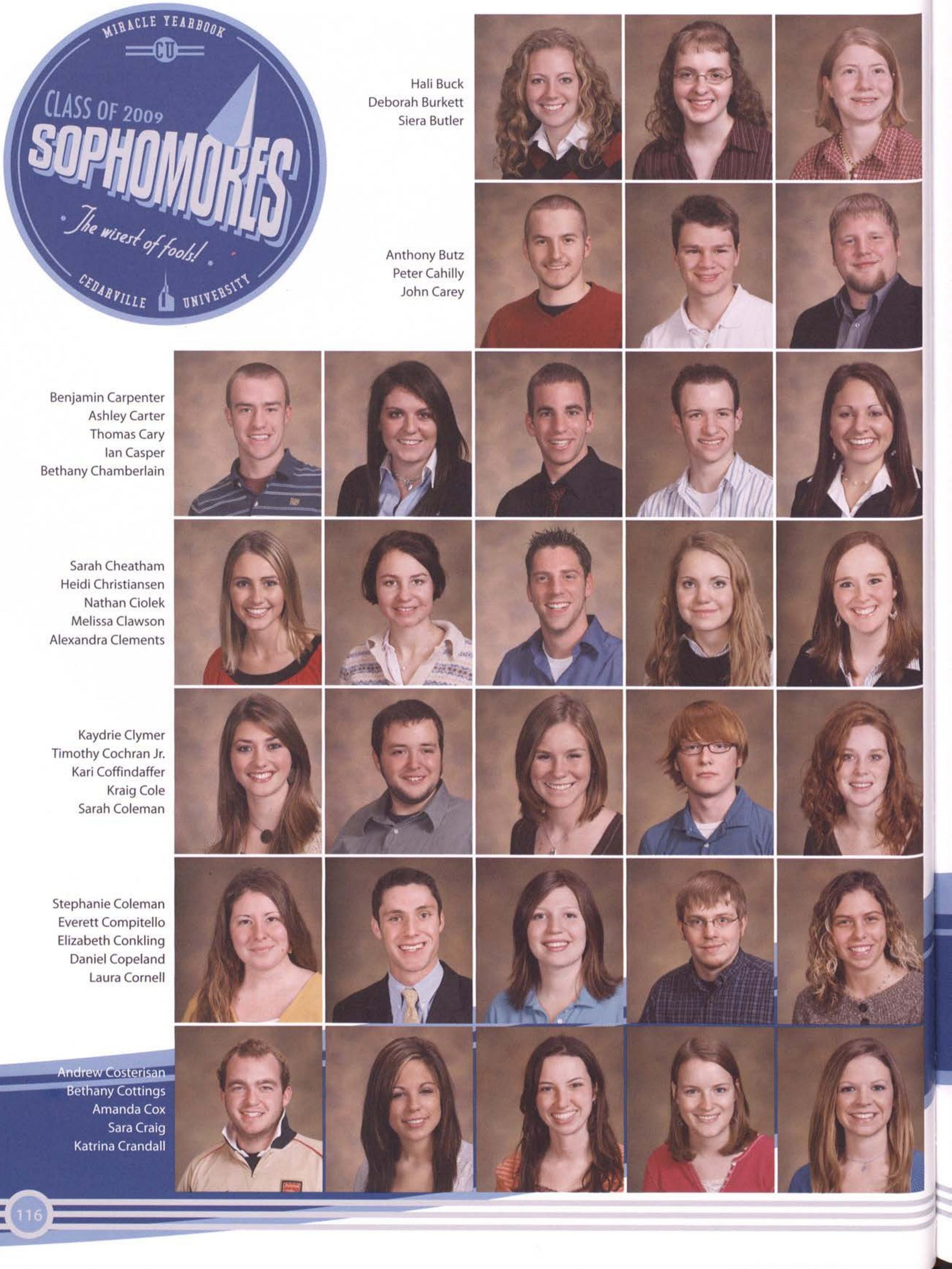


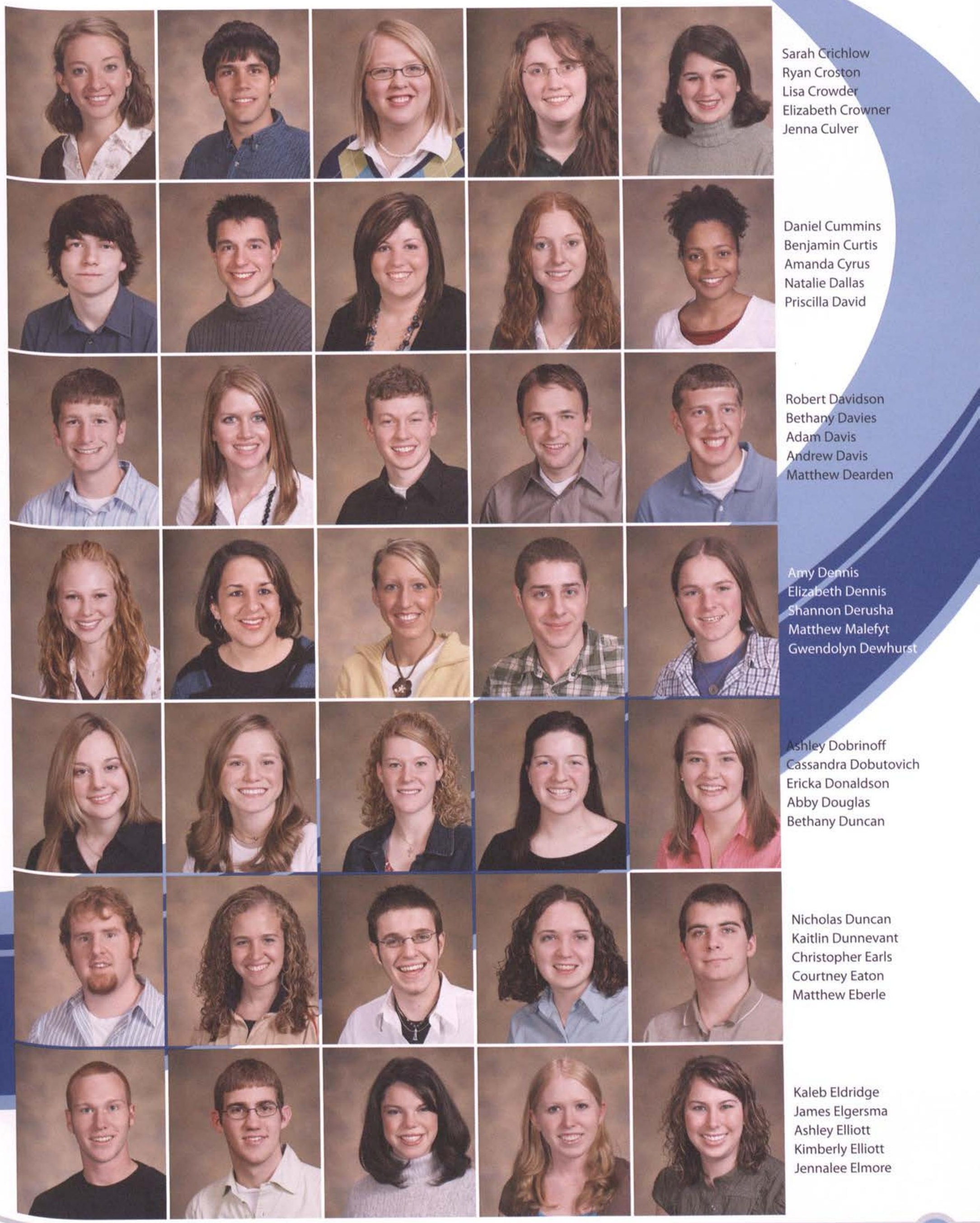



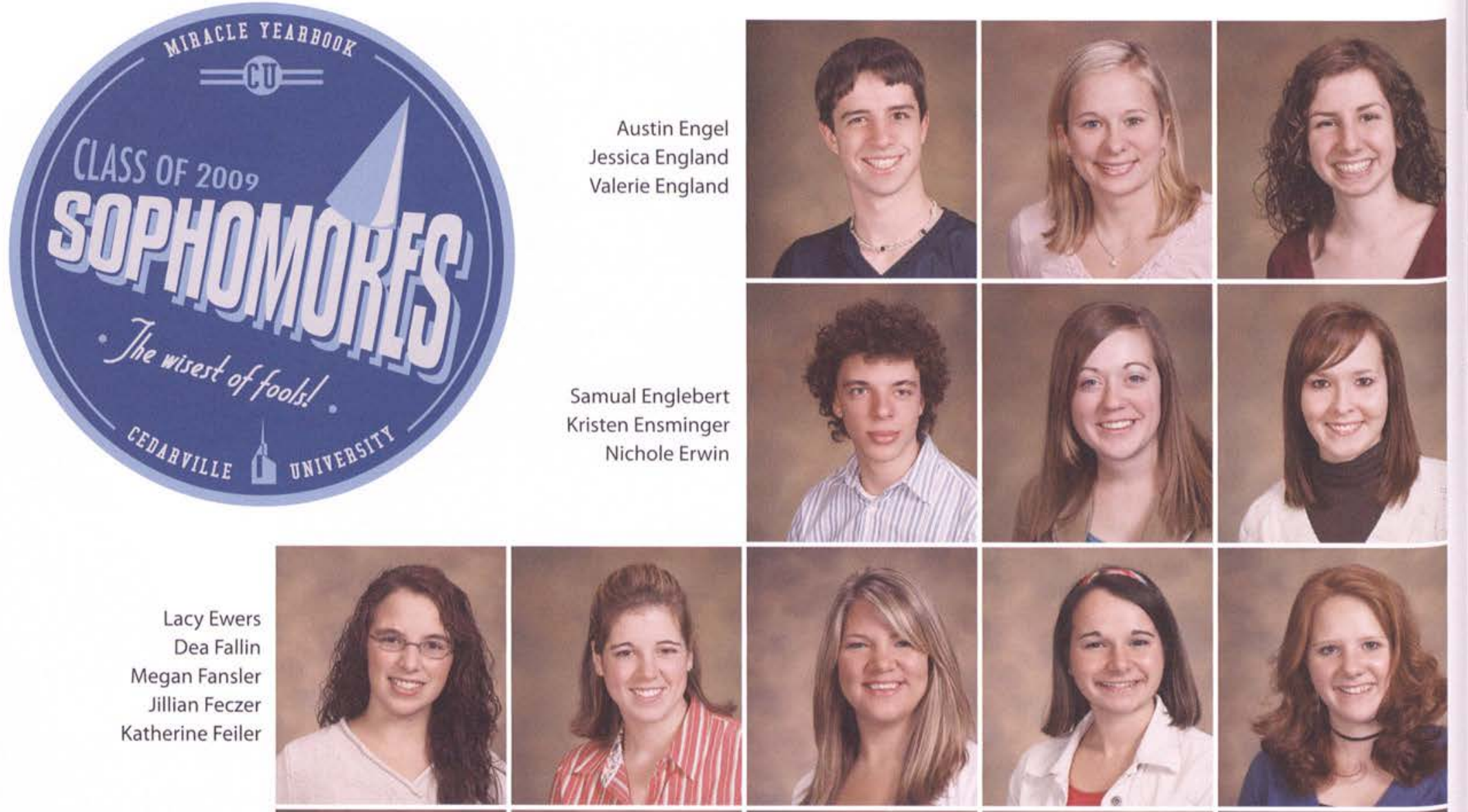

Kristen Ferguson

Talitha Ferrell

Jonathan Fleetwood

Crystal Flippin

Elizabeth Flow
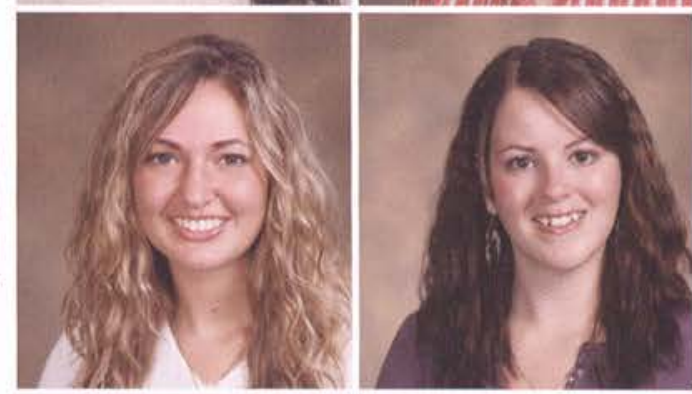

Matthew Focht

Justin Francis

Richard Freed

Kaitlyn French

Johanna Frichtl
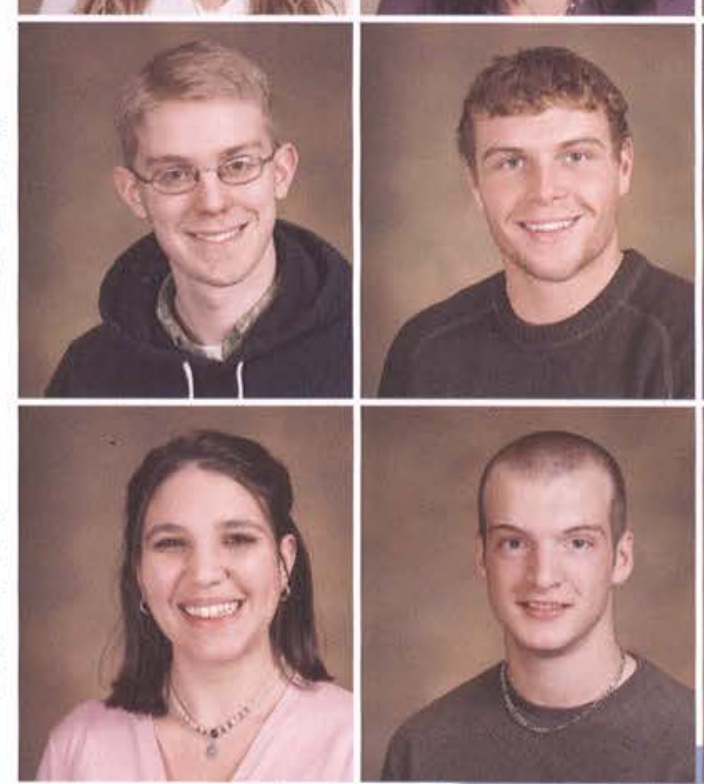

Elsye Fye
Jacob Garrison

Amber Geringer

Rachel Gibson

Josh Gigliotti

Keisey Gilsdorf

Ashley Gleichauf

Terri Goodman

Emilie Gouch

Michelle Graeff
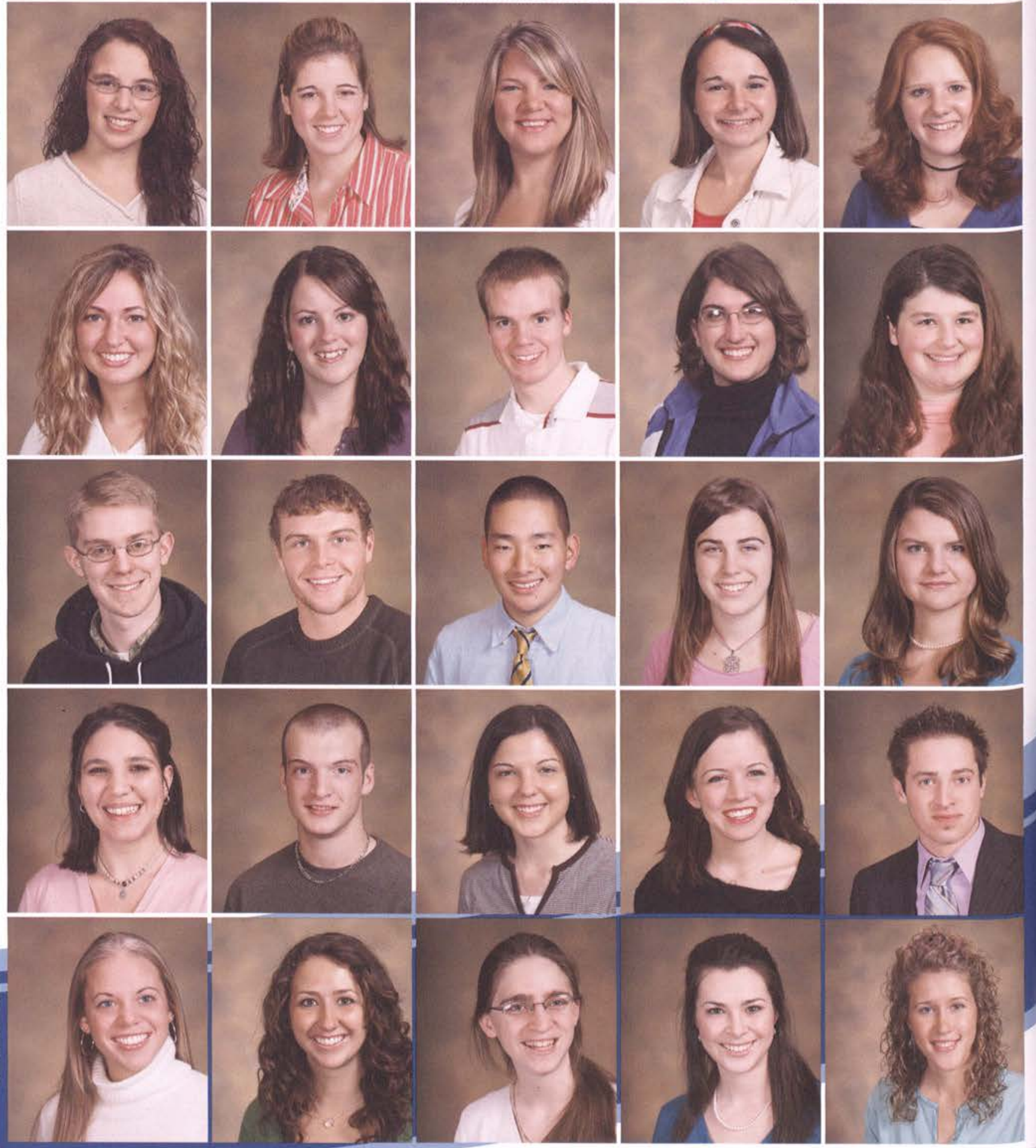


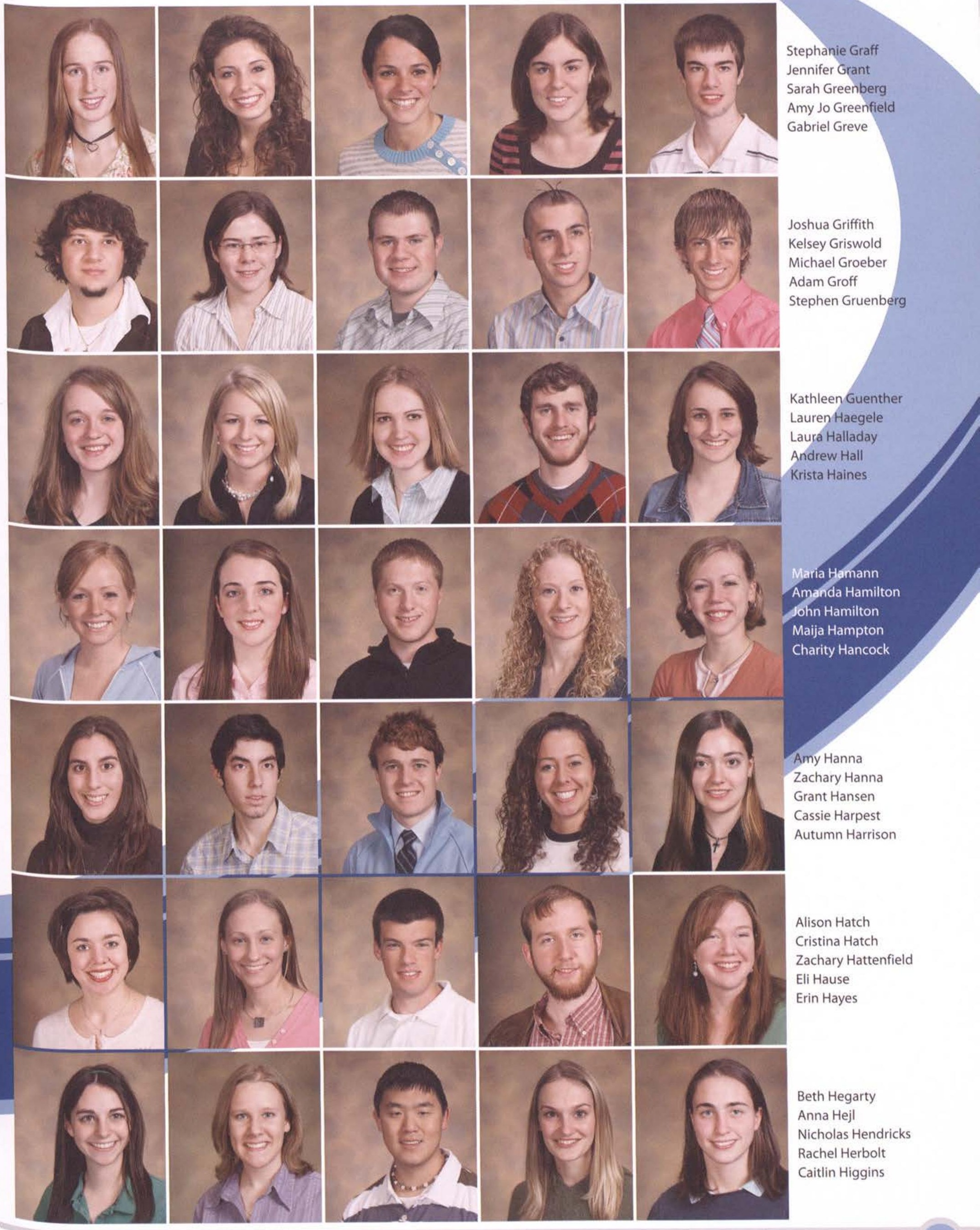




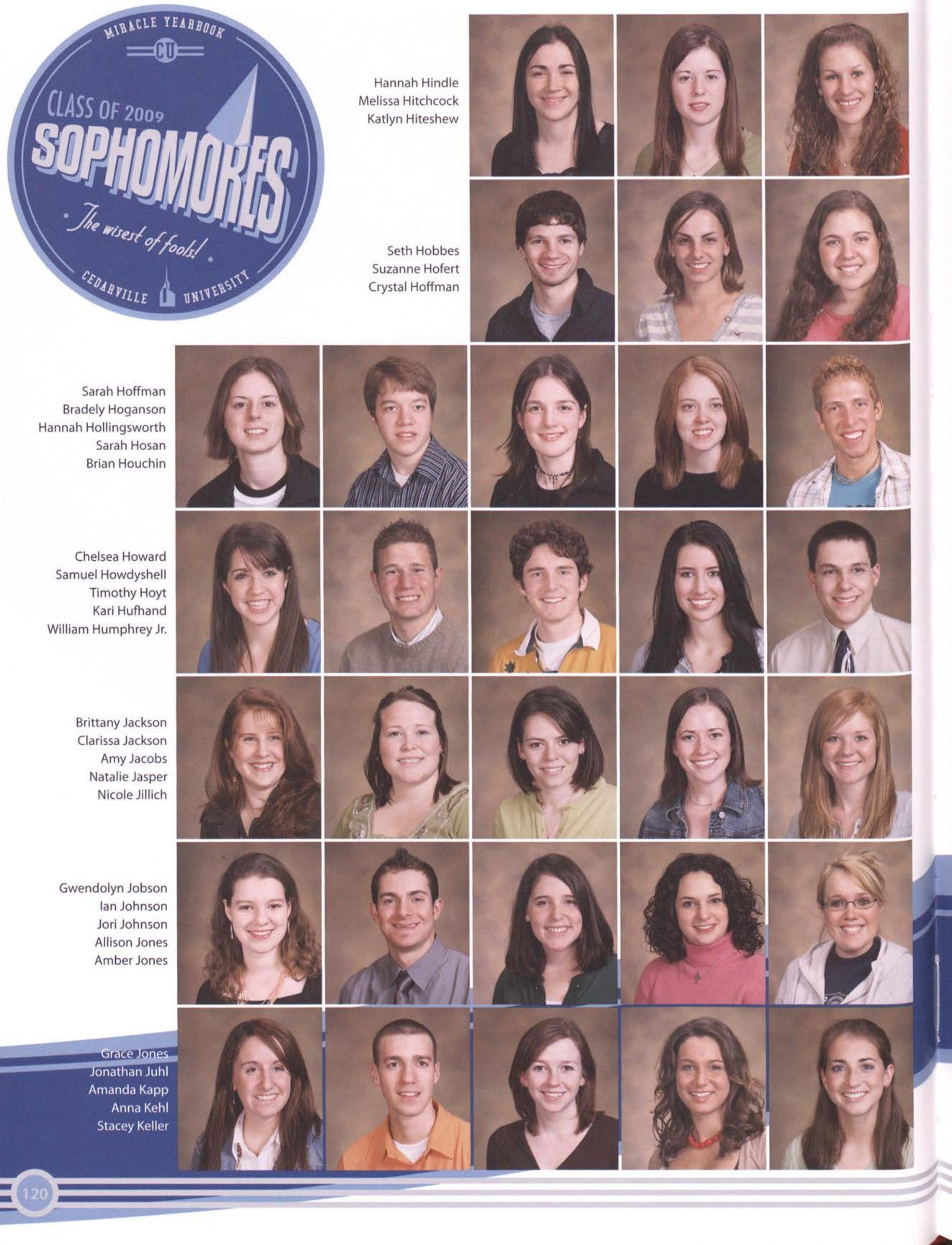




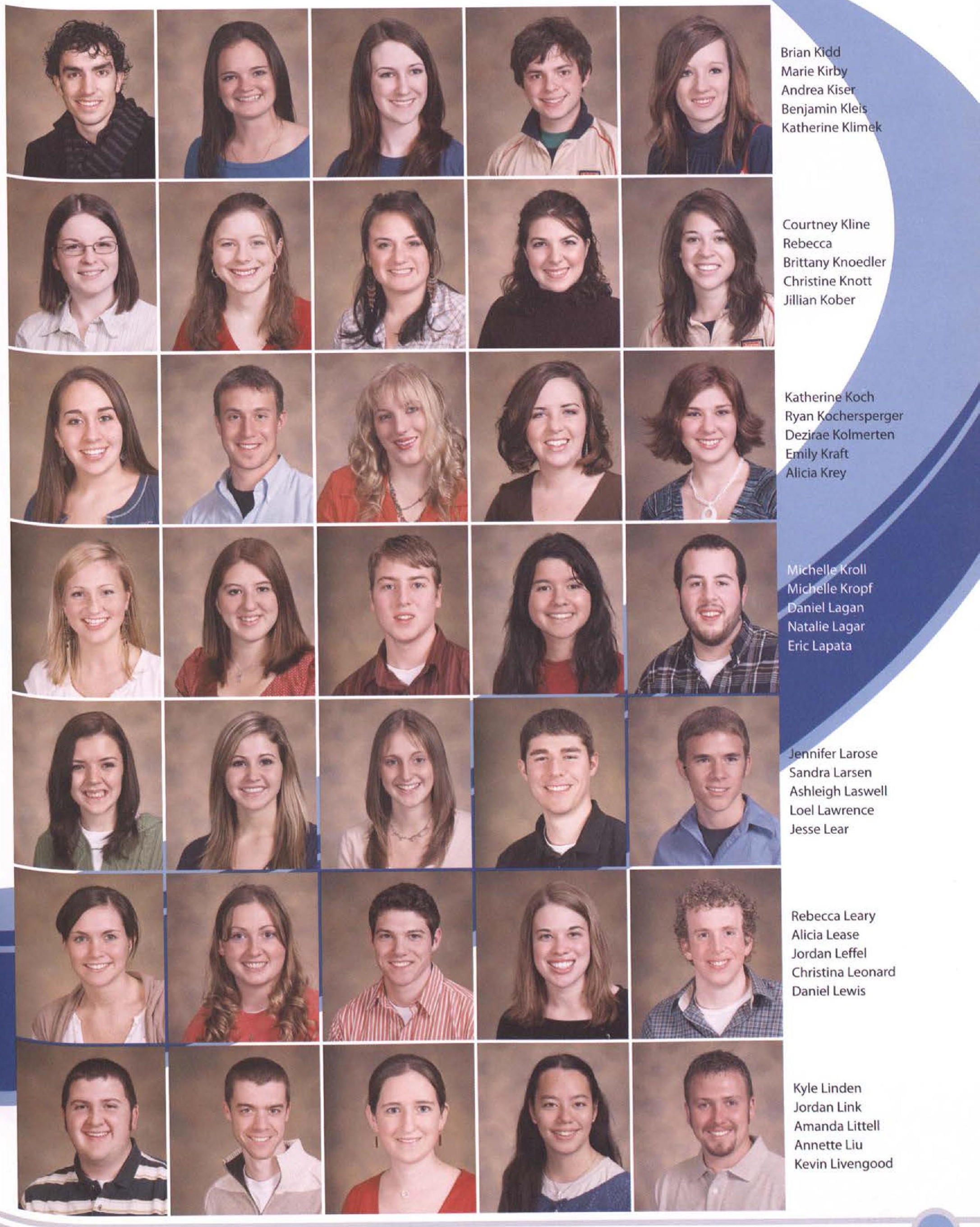




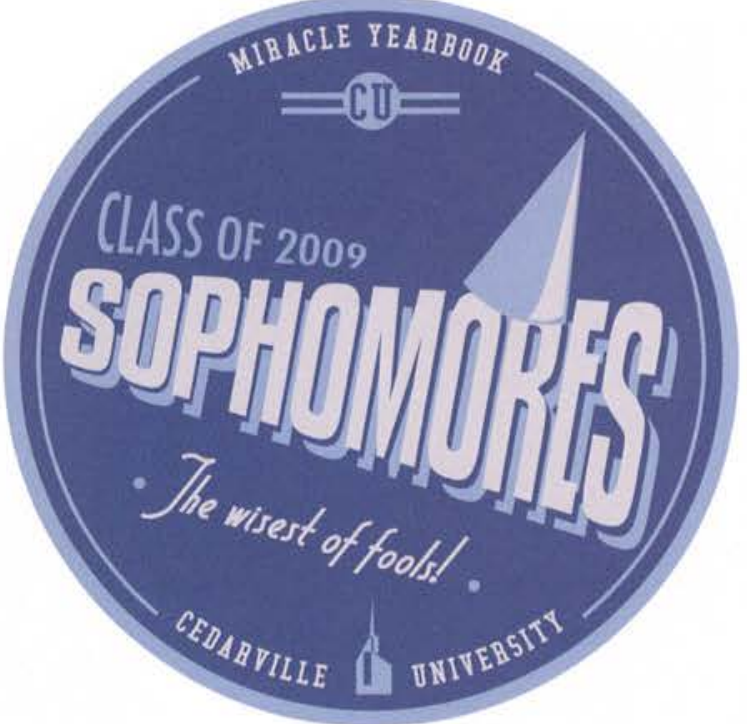

Racquel London

Grace Looney

Michael Loosa

Kailee Lorenzen

Felicia Lustig

Kathryn Lutz

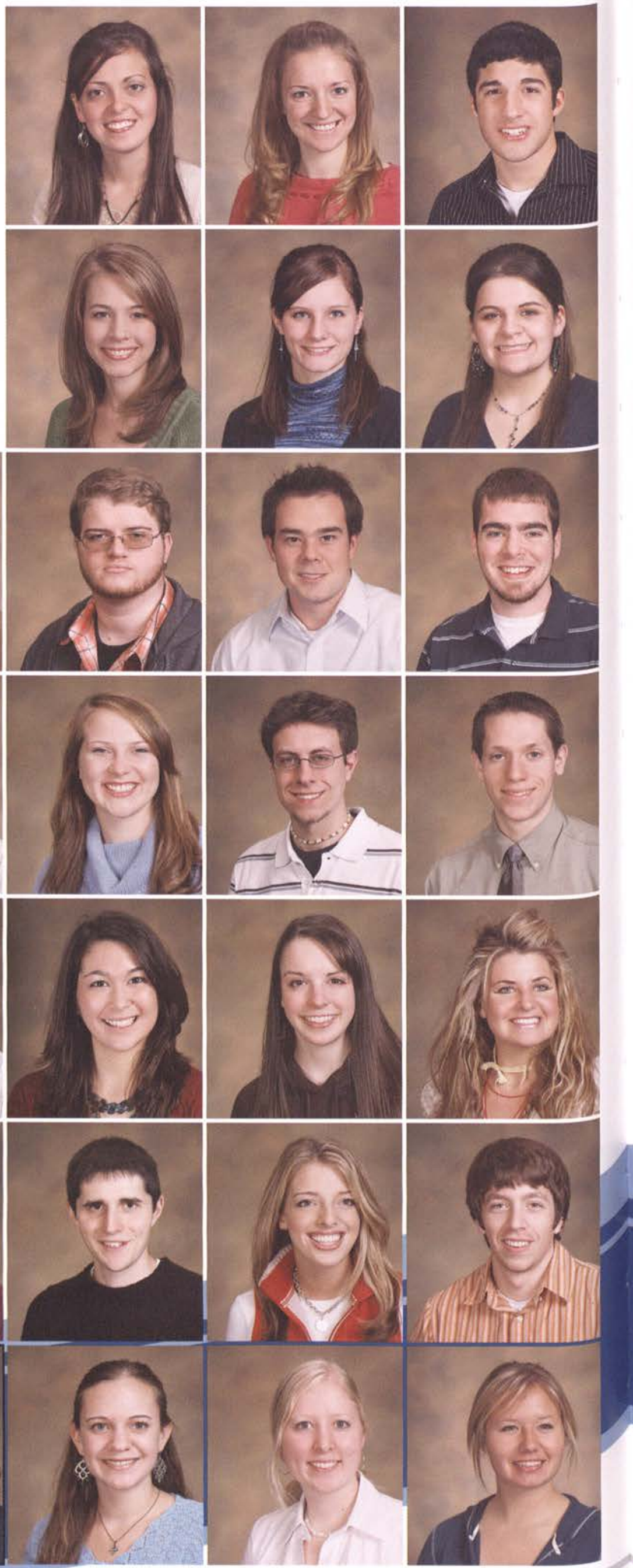

Heather McGee

Kristine McGunnigal

Nathan McKanna

Kate McKee

Nathan McLeese

AnjuinMehra Faith Meitzler

Angeline Melendez

Lyne Mellum

Heather Merrell
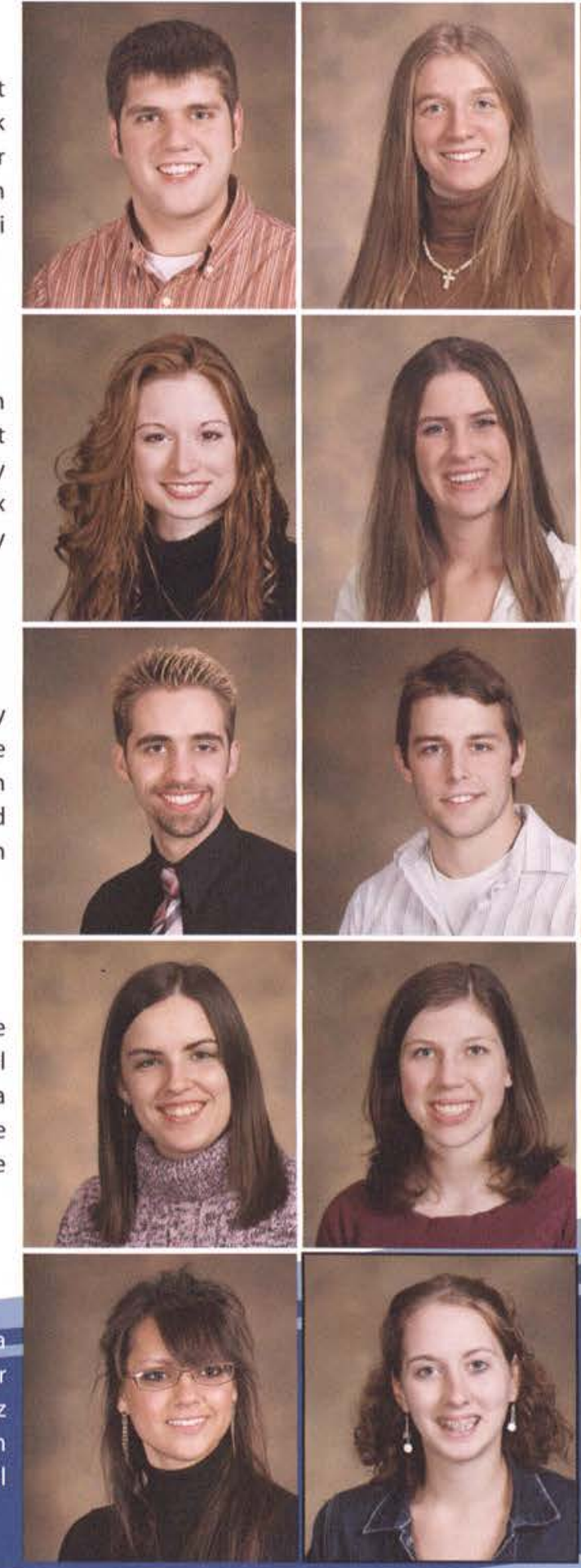


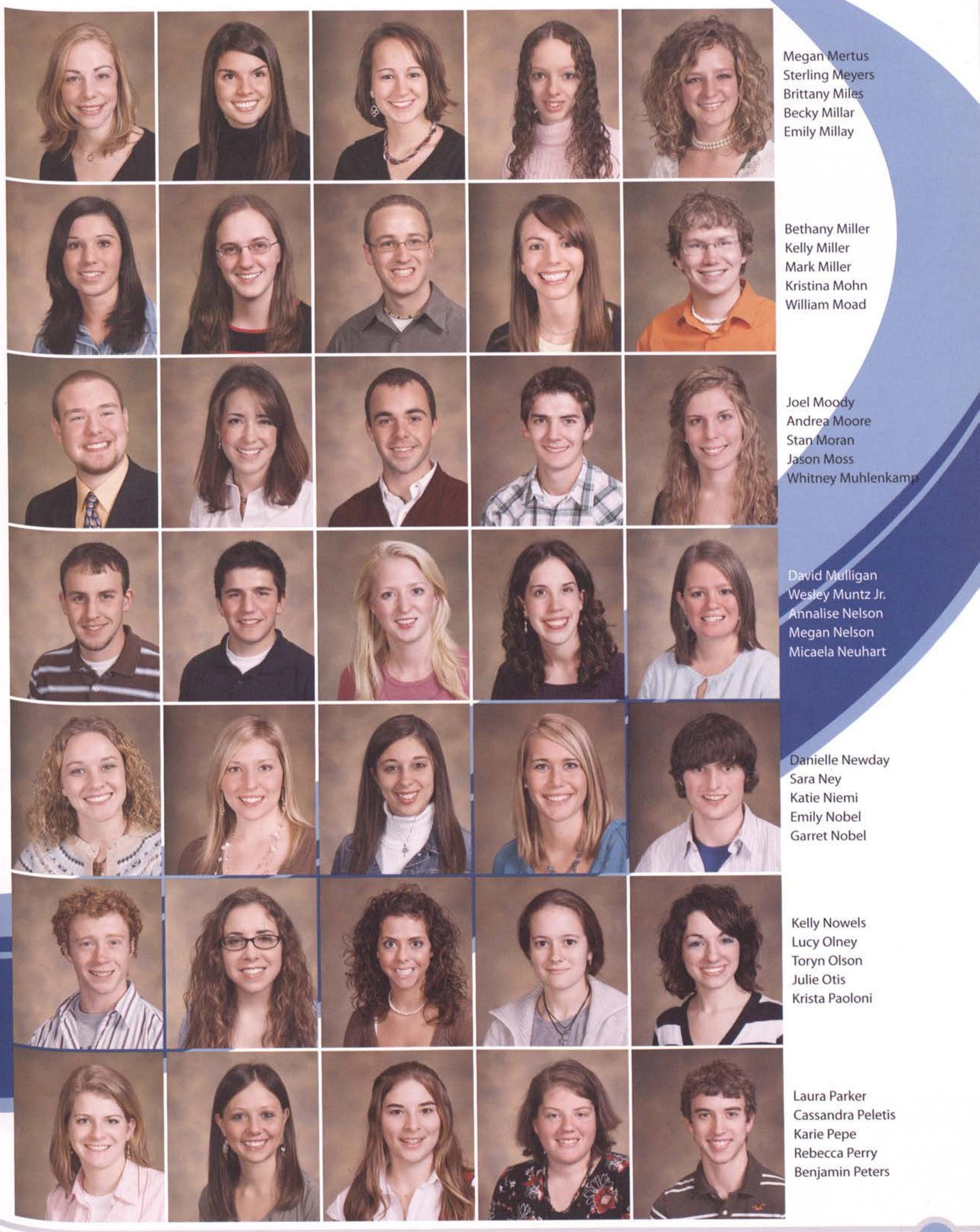



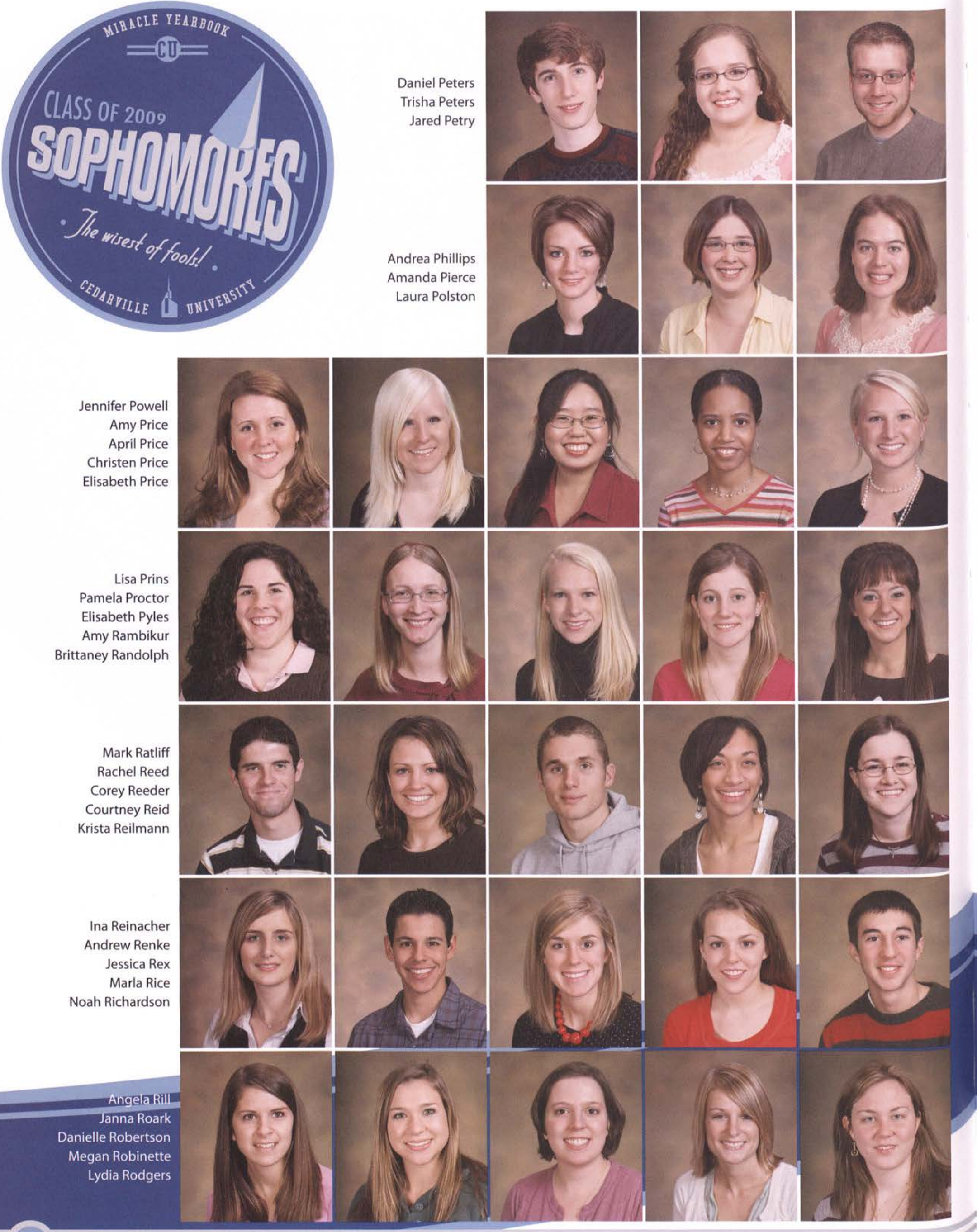

Amy Price

Christen Price

Elisabeth Price

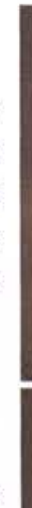

Pamela Proctor

Elisabeth Pyles

Amy Rambikur

Brittaney Randolph
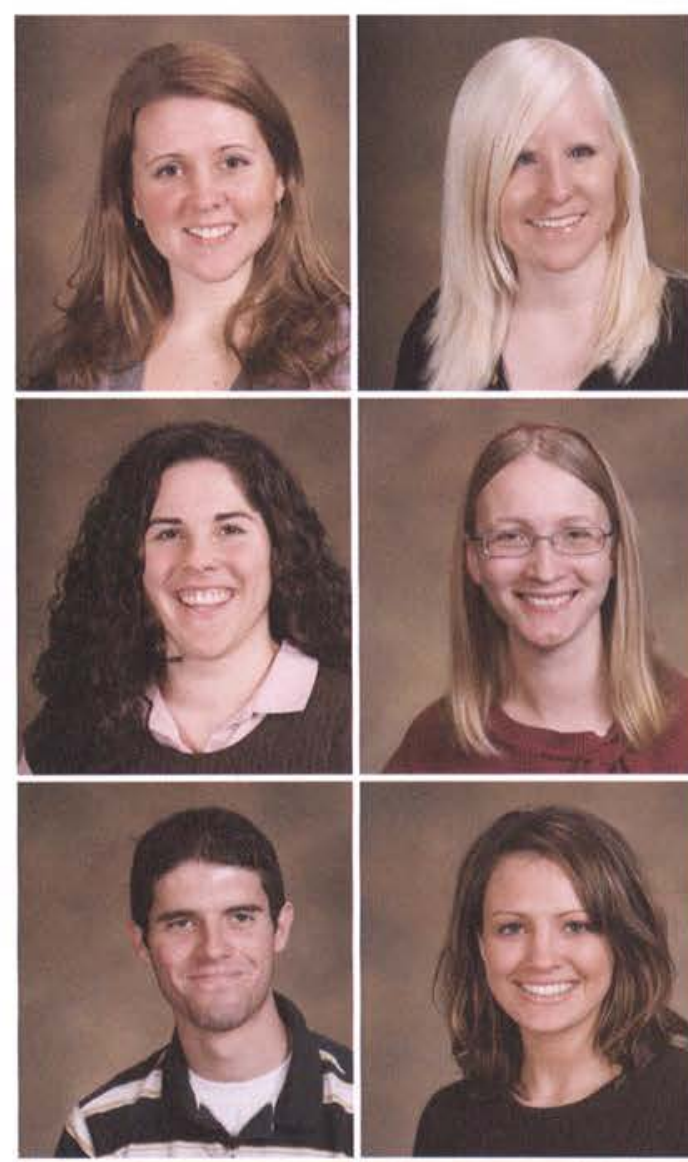

Mark Ratliff

Rachel Reed

Corey Reeder

Courtney Reid Krista Reilmann

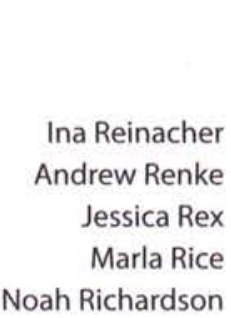

Noah Richardson
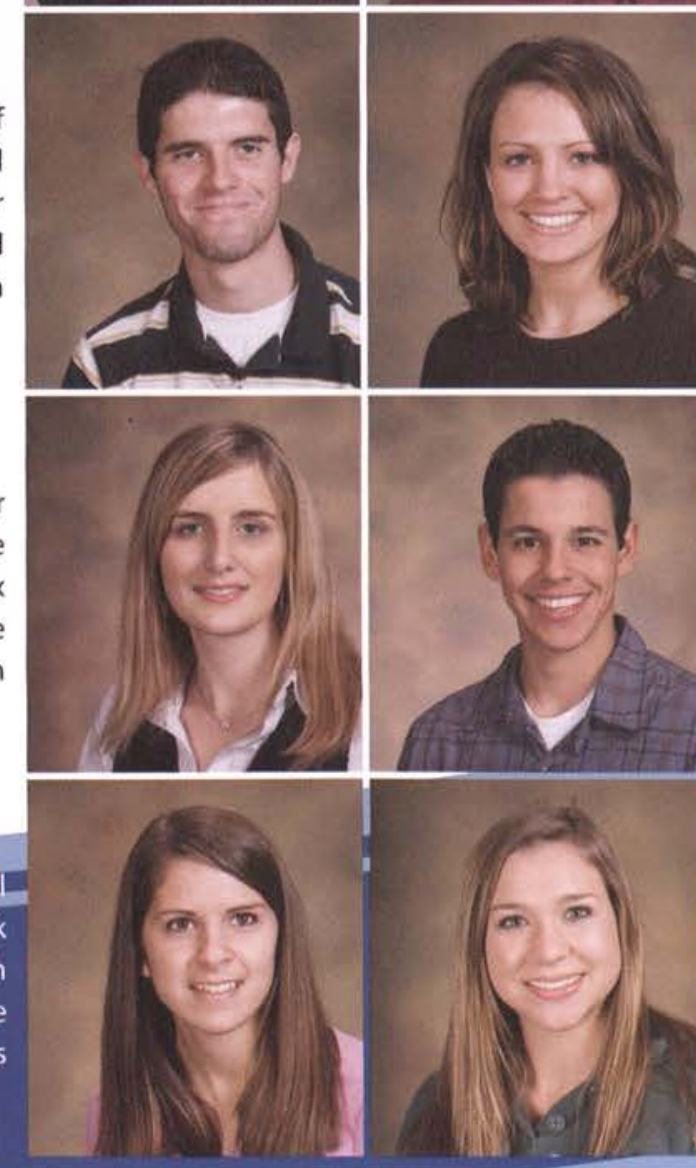

Angela Rill Janna Roark

Danielle Robertson

Megan Robinette

Lydia Rodgers

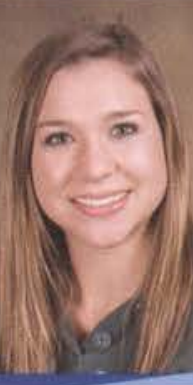




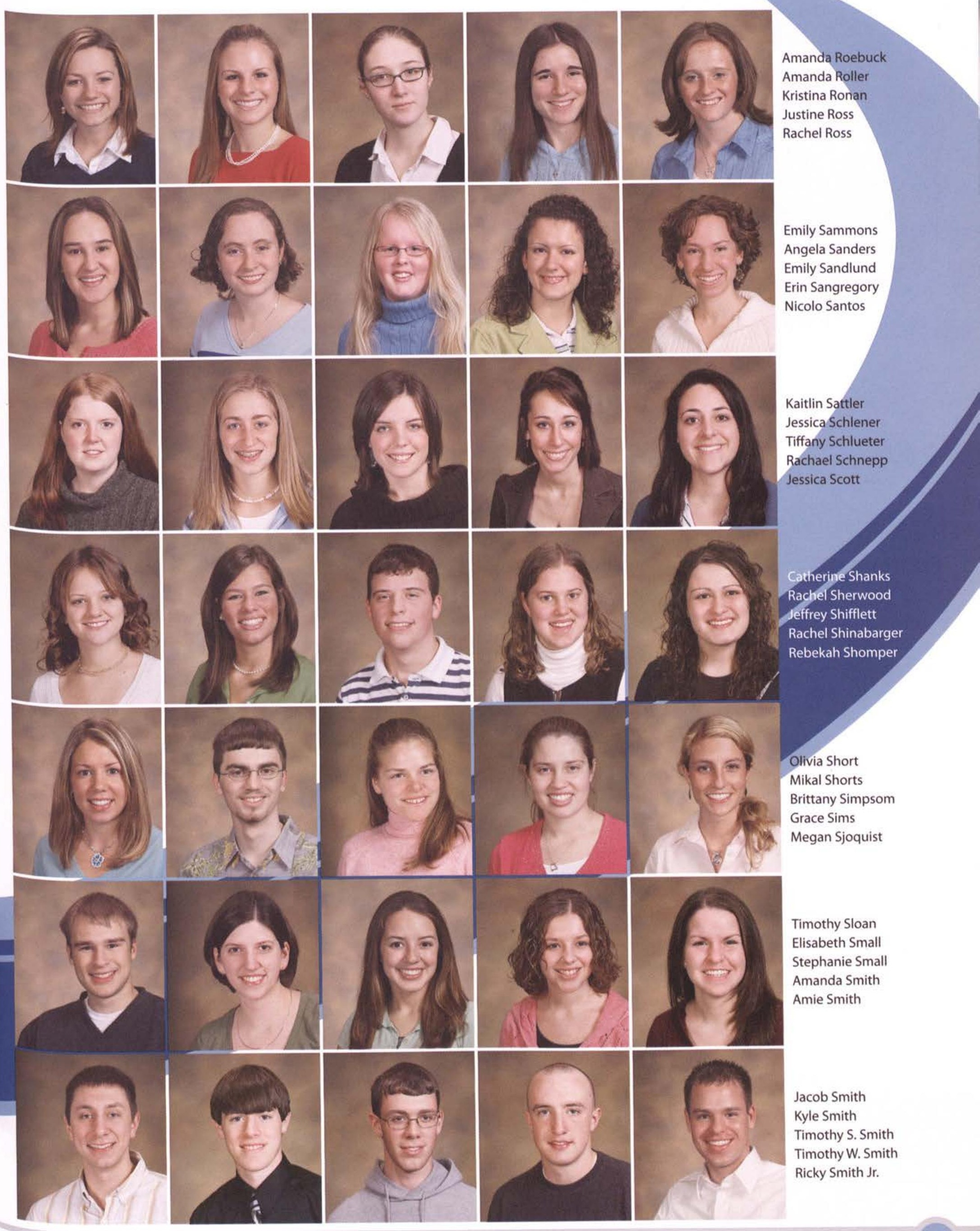



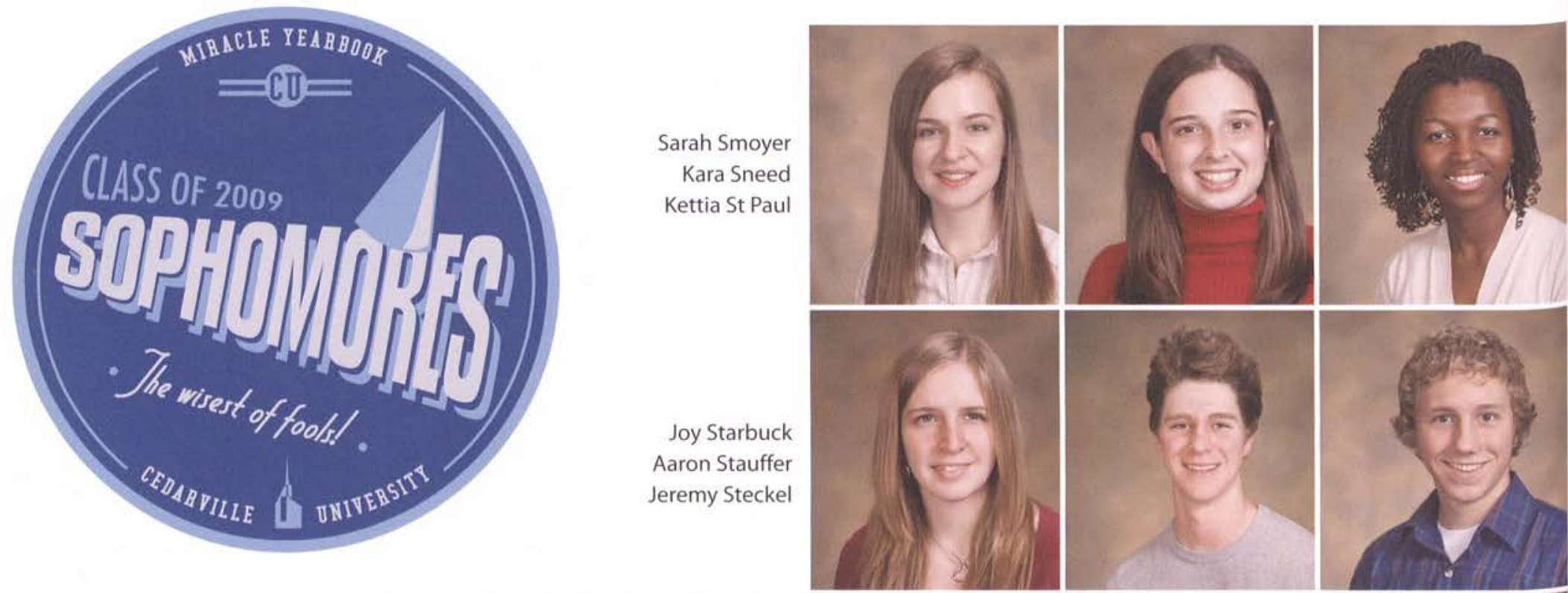

Meghan Steele

Michael Steele

Jarrod Steinmetz

Jessica Steinmetz

David Stephens
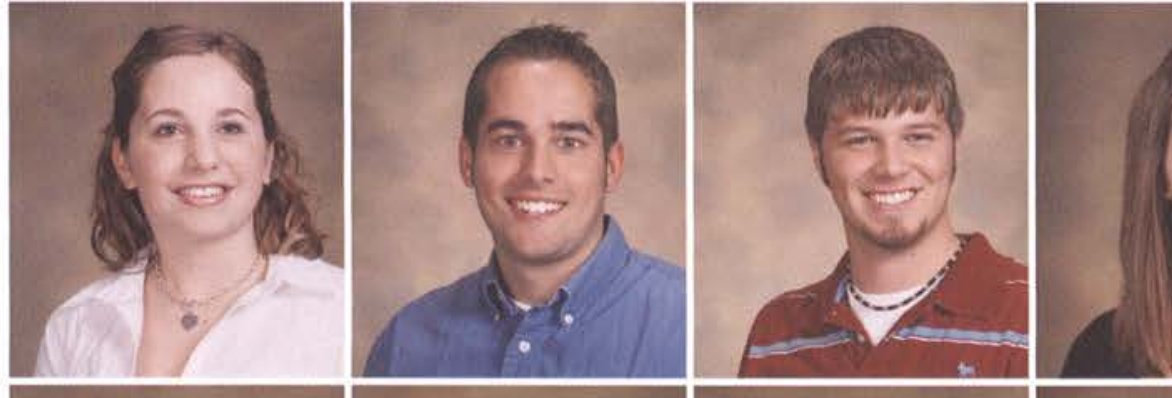

Jennifer Stevens

Ashleigh Stewart

Amanda Stock

Taylor Storey

Macy Straton
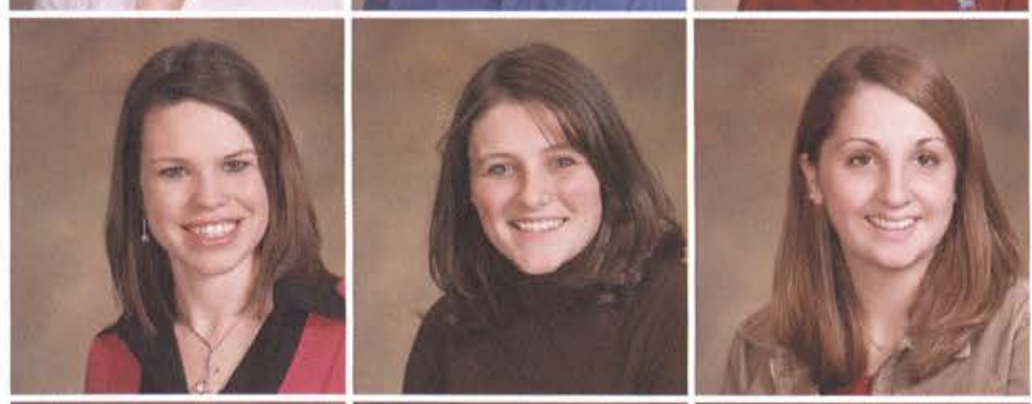

Anna Strayhorn

Elizabeth Studebaker

Caleb Svendsen

Kelly Teague

Michaela Thompson
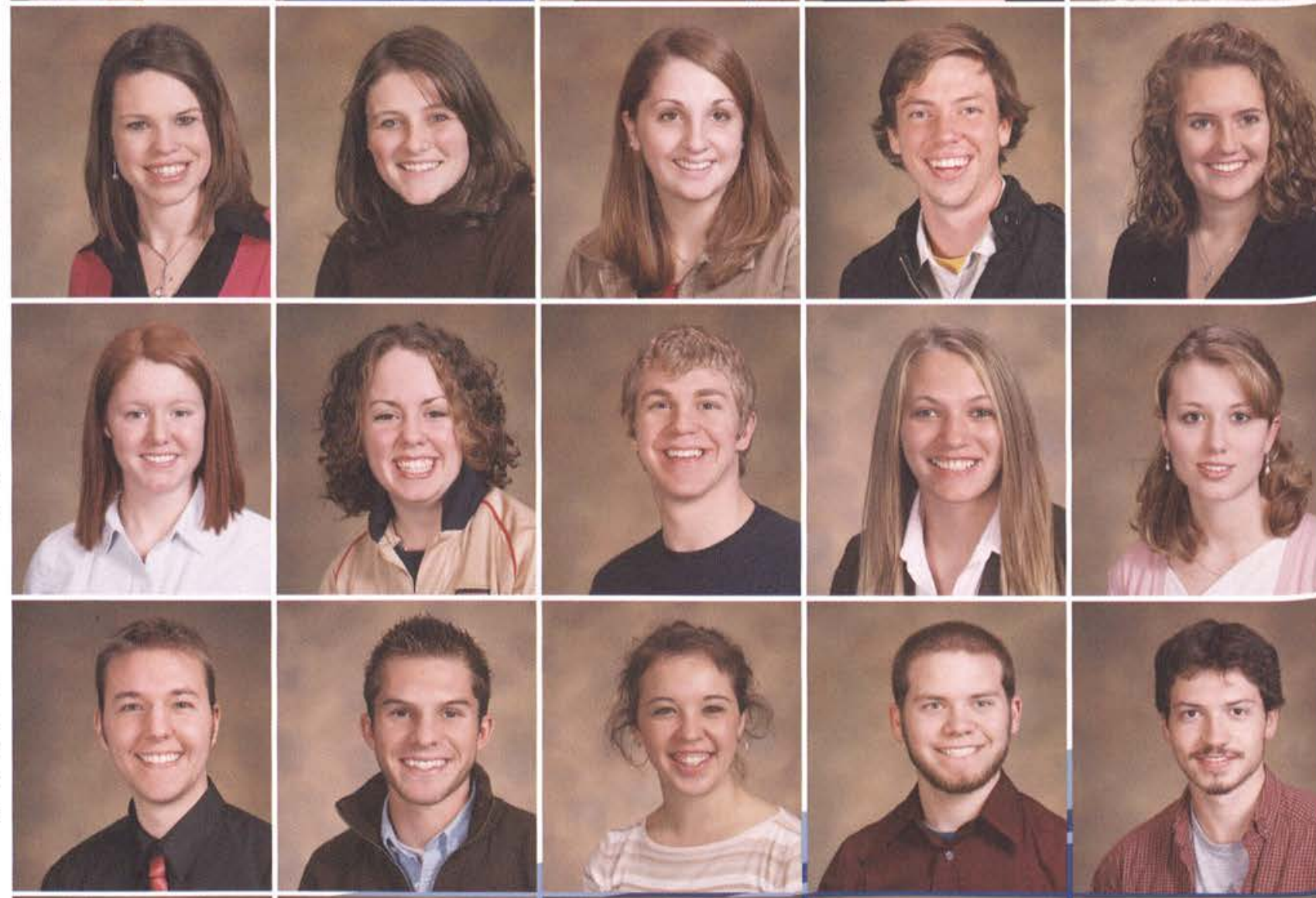

Stephen Tormey

Christopher Travis

Mallory Travis

Matthew Trego

Brent Trowbridge

John Van DerWal Anna Van Vliet

Emily Vandette

Ethan Vandette

Emily Vanmatre

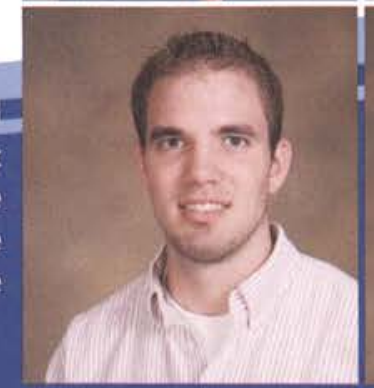




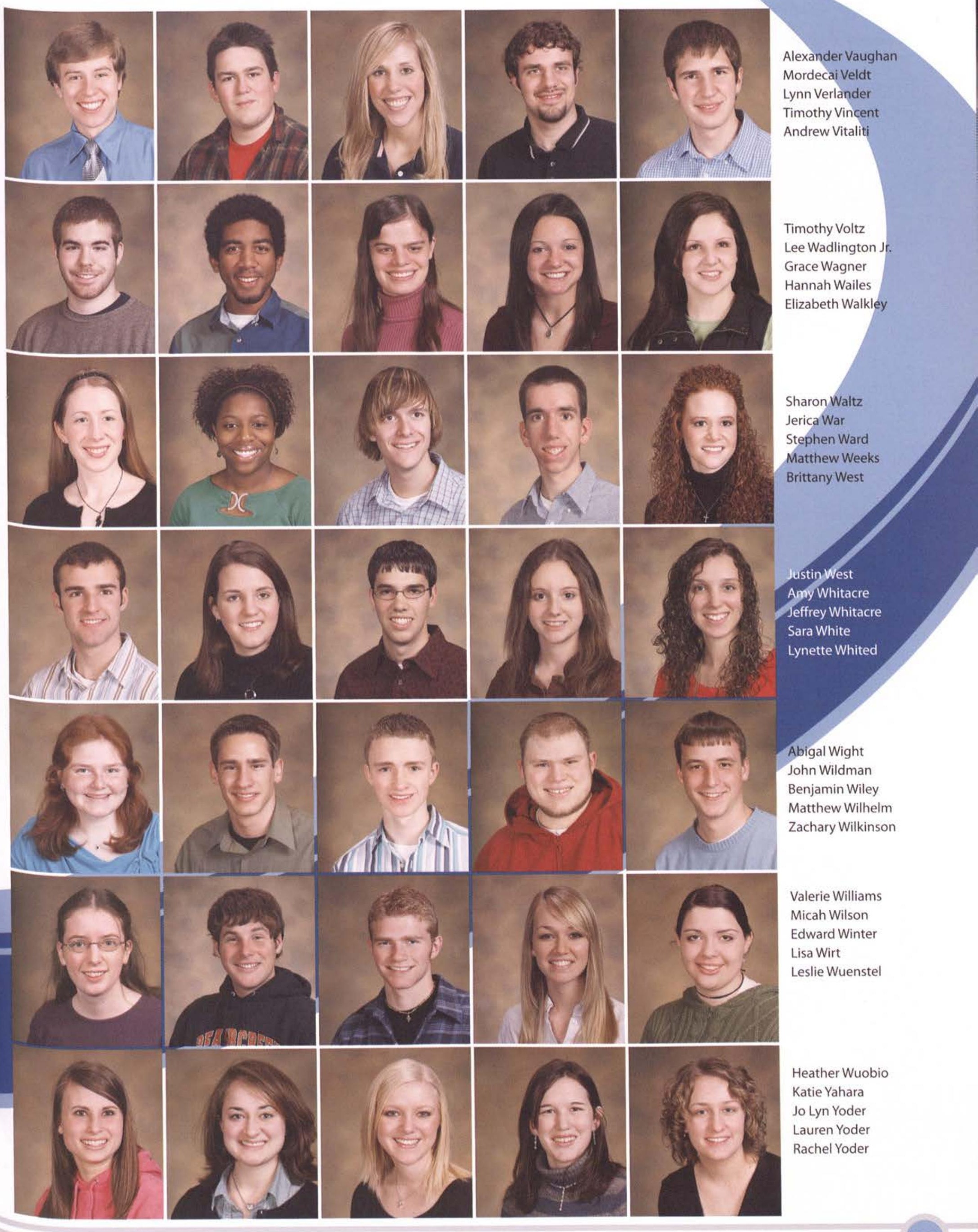



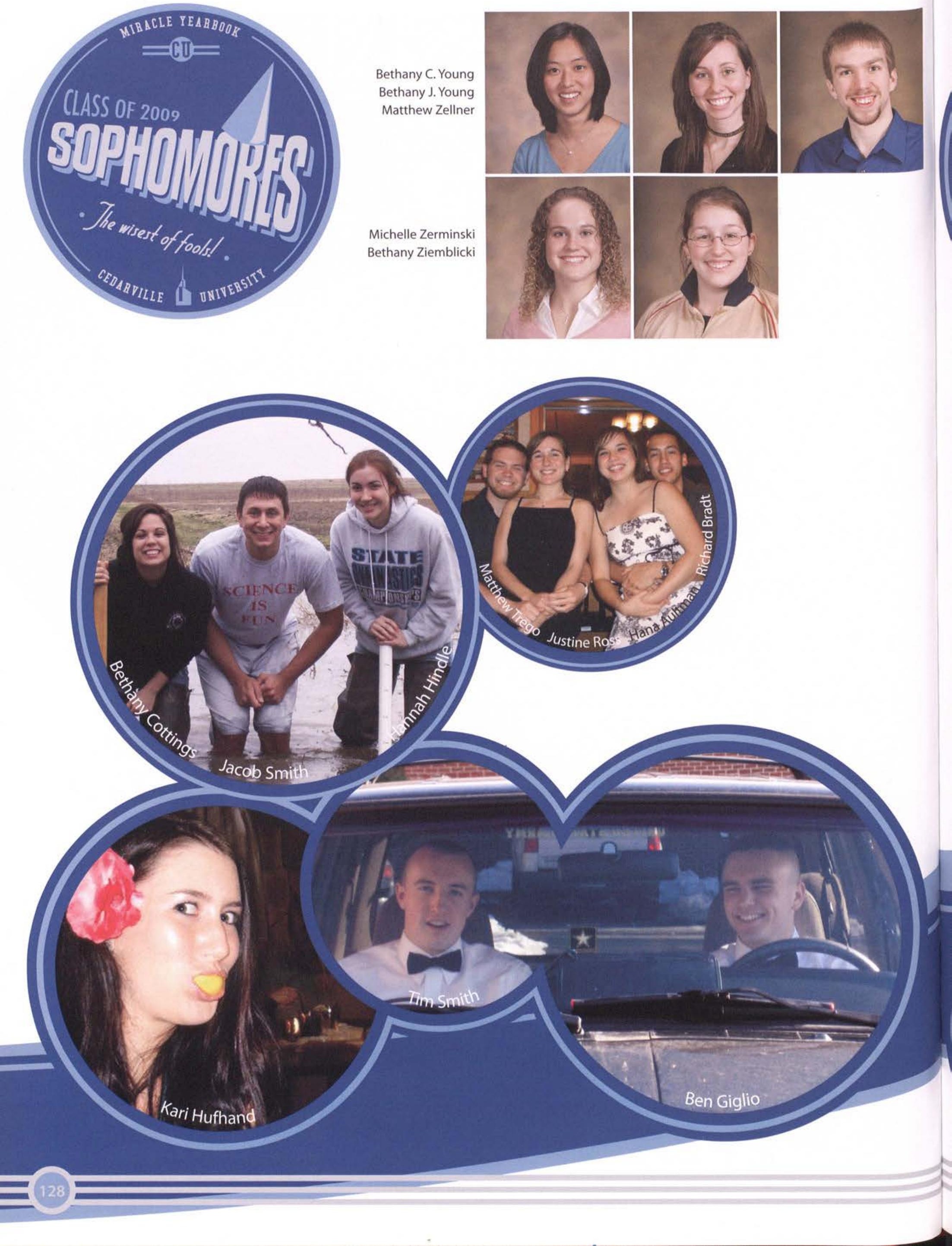


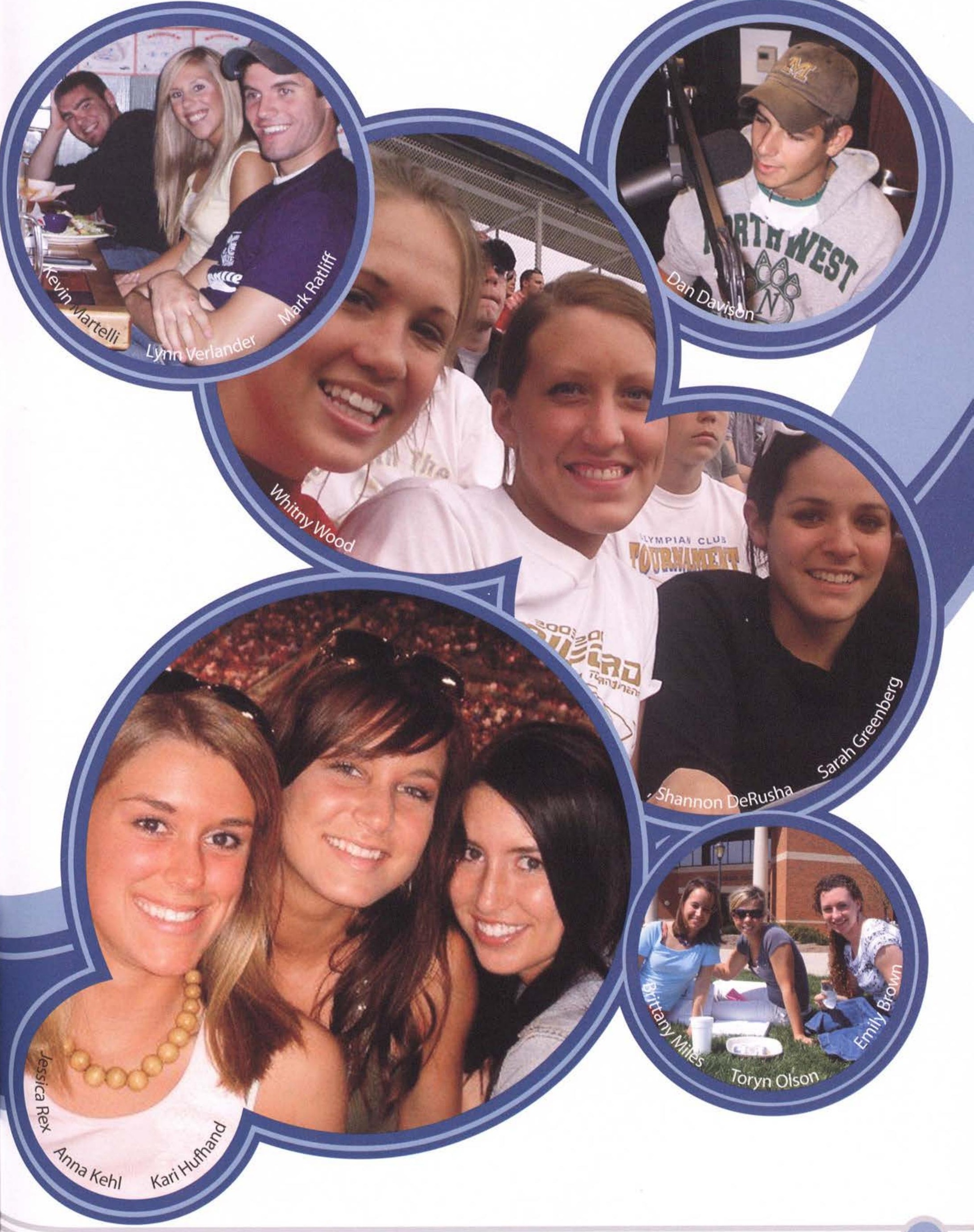




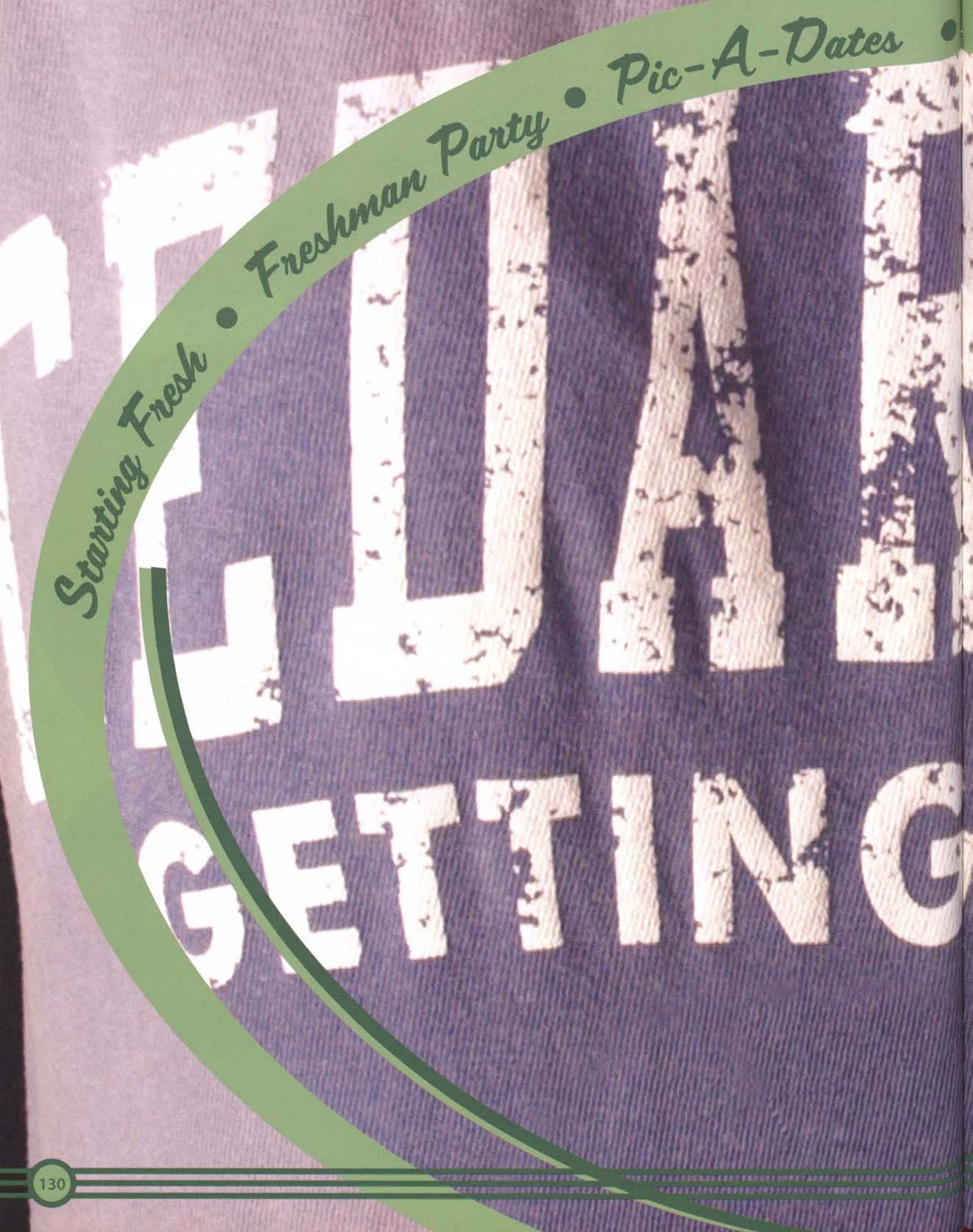




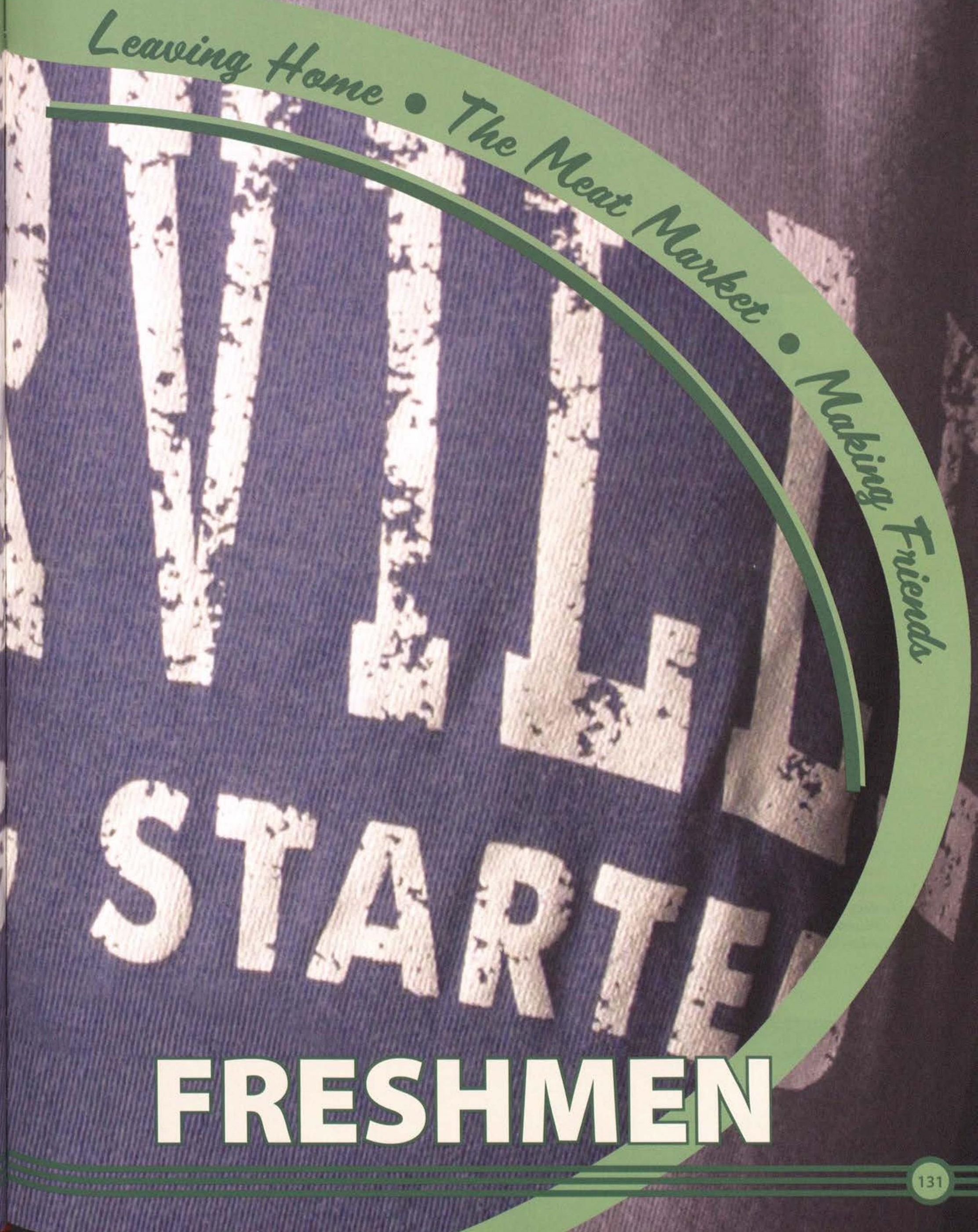



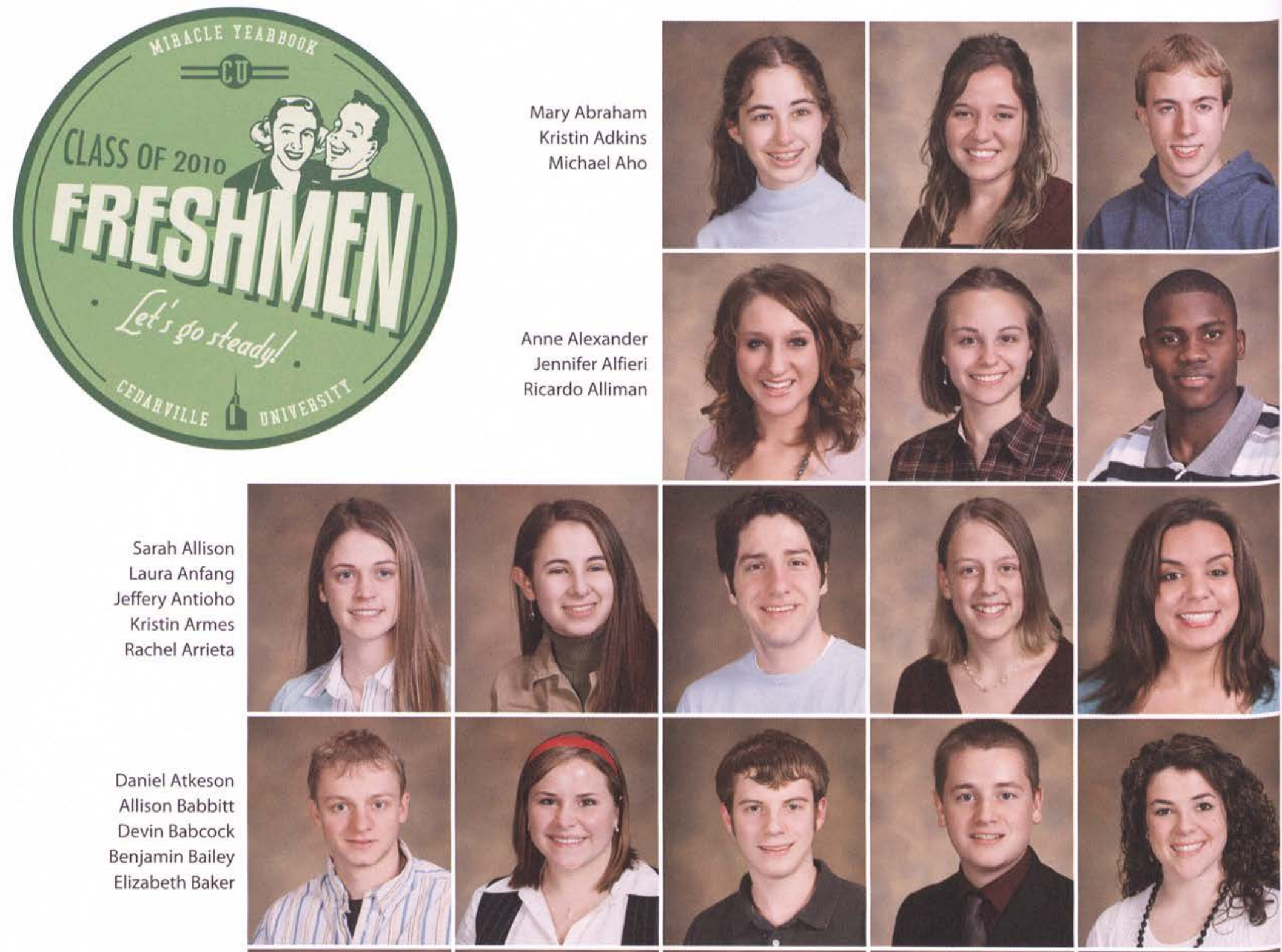

Daniel Atkeson Allison Babbitt Devin Babcock Benjamin Bailey Elizabeth Baker

Sarah Allison
Laura Anfang Jeffery Antioho Kristin Armes Rachel Arrieta

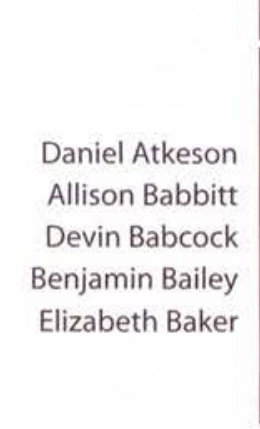

Chase Baldwin Jared Banz Elizabeth Barkas Brianne Barnes Diana Basford Leanna Baumer
Eric Bell
Chelsea Belleman
Robert Bentley
Stacey Bernard
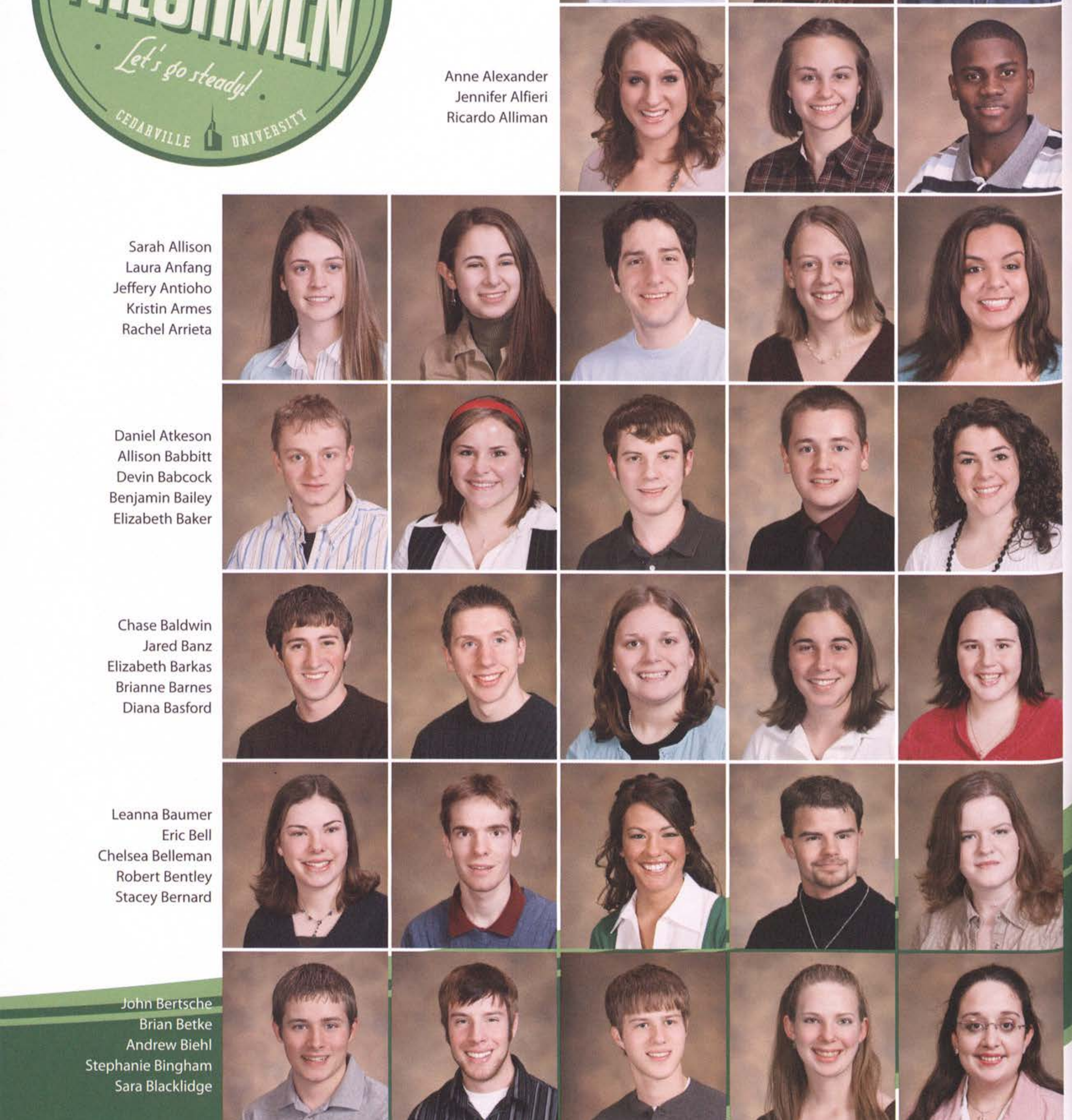

John Bertsche Brian Betke Andrew Biehl Stephanie Bingham Sara Blacklidge
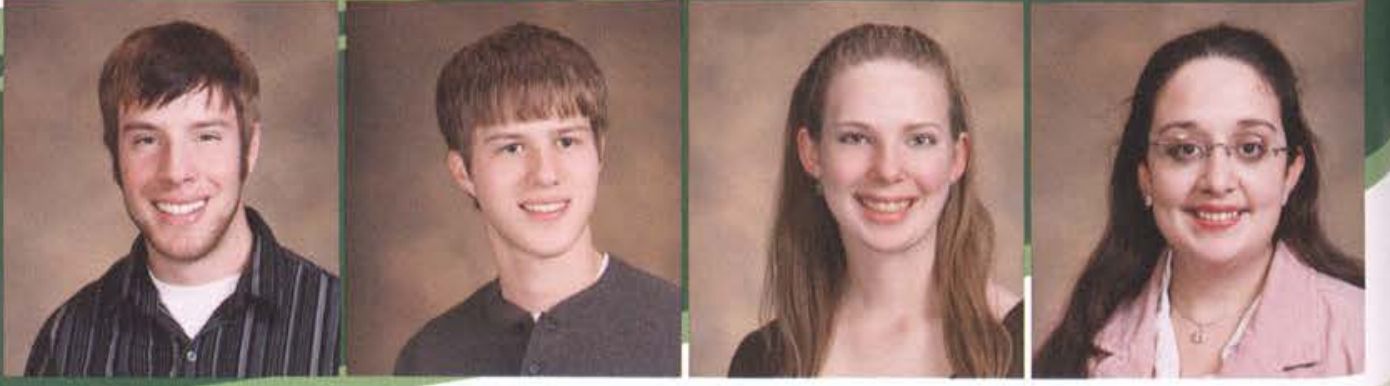


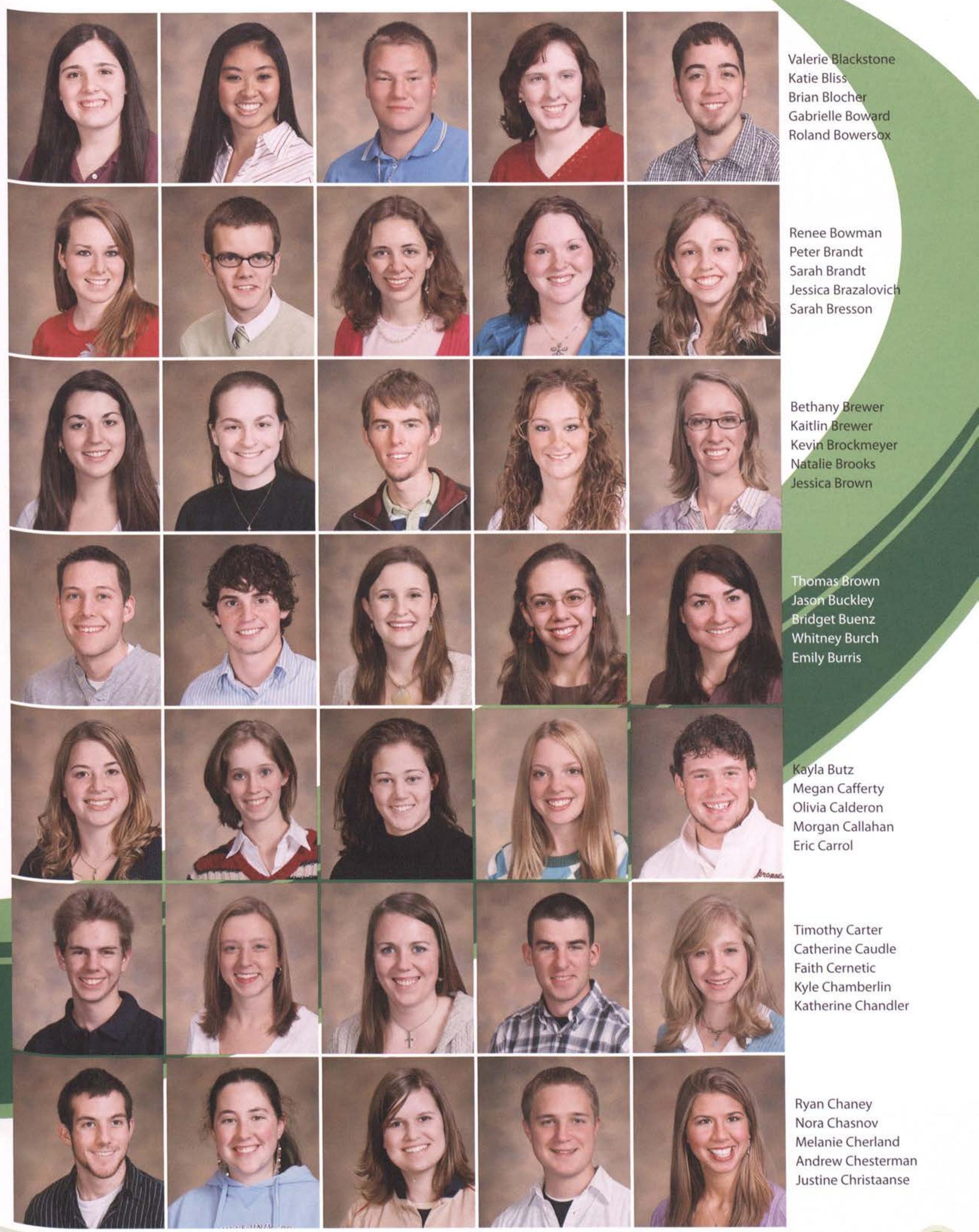



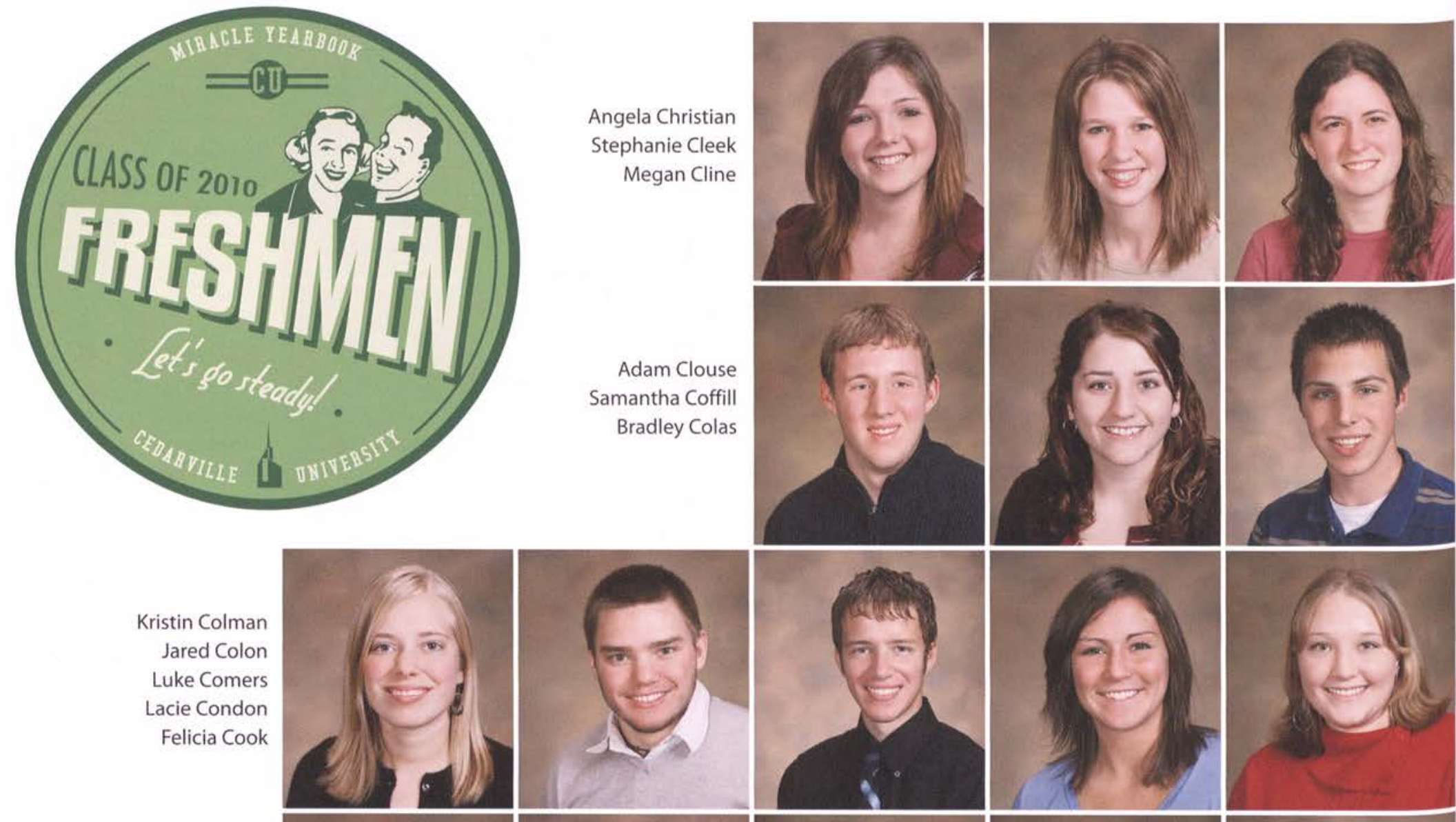

Angela Christian

Stephanie Cleek

Megan Cline

mantha Coffill

Bradley Colas
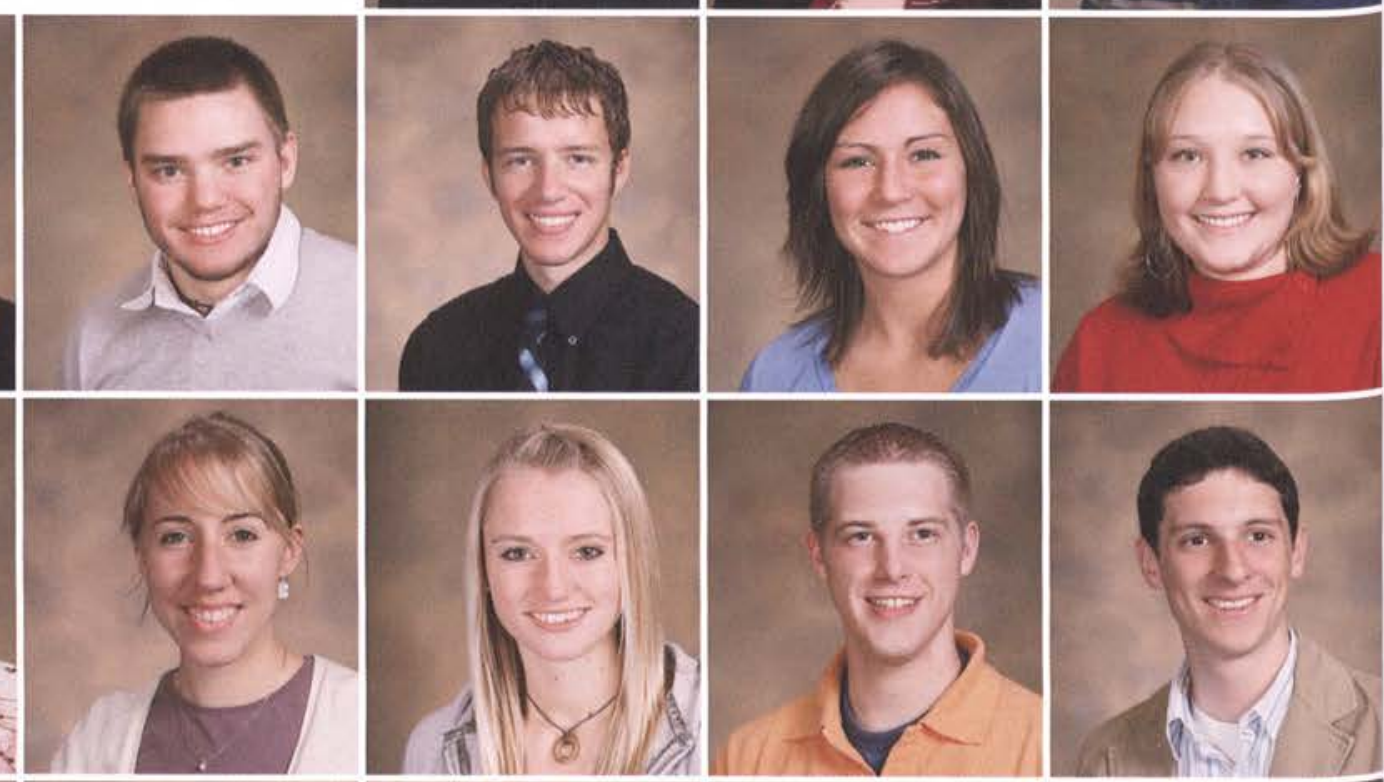

Jazmine Cook

Kaitlyn Cook

Lisa Cook

Shaun Cookson

John Cooper

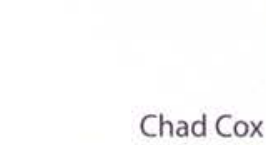

Gregory Craft

Matthew Crissman

Lisbeth Cummings

Evelyn Cunliffe

rine Daeschner

Ashley Dasuqi

Nicholas Davey

Courtney Davis
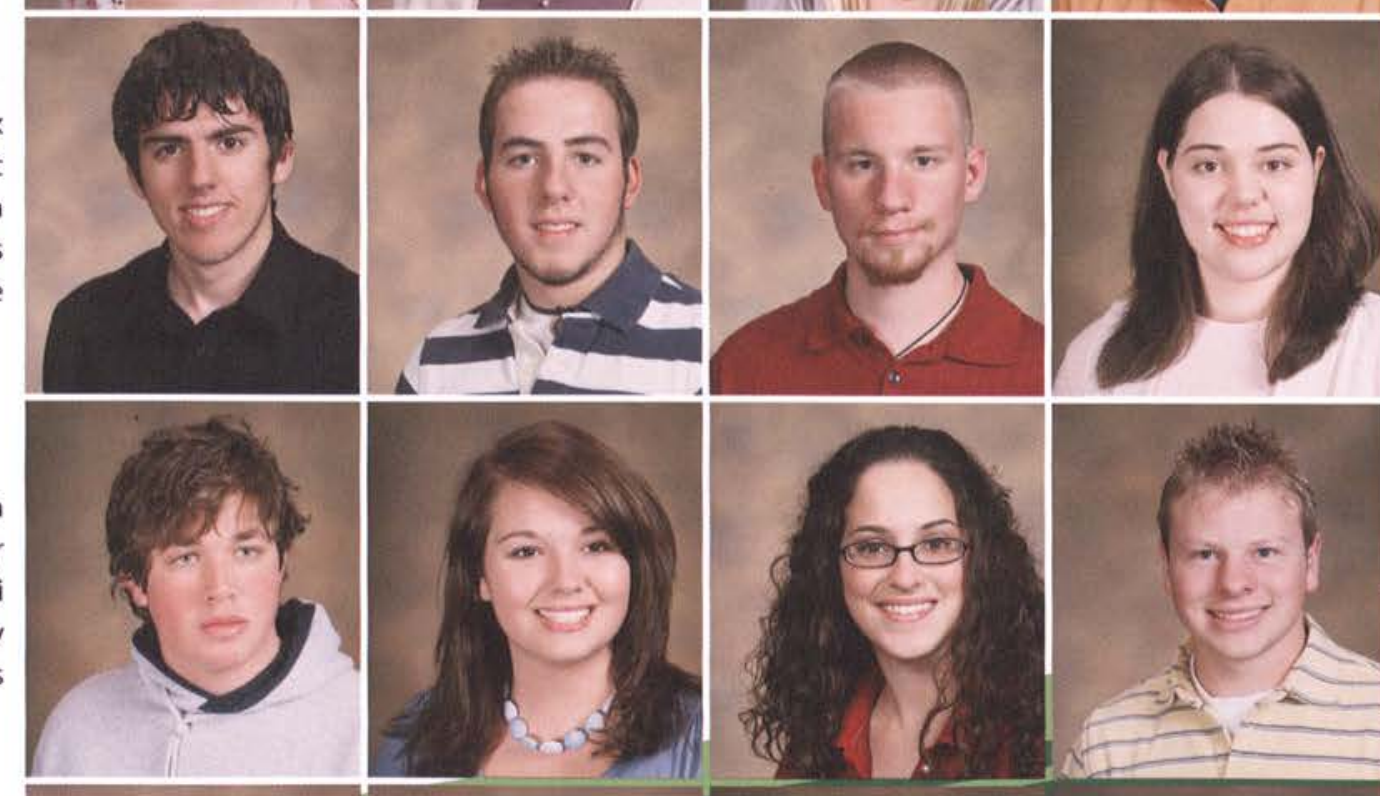

Diana Deakin Carolyn Deemer

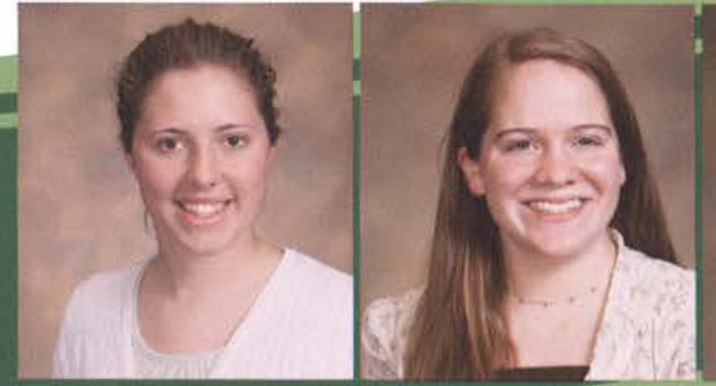

Jennifer Degroft

Tricia Dekorne

Joshua Deschaine
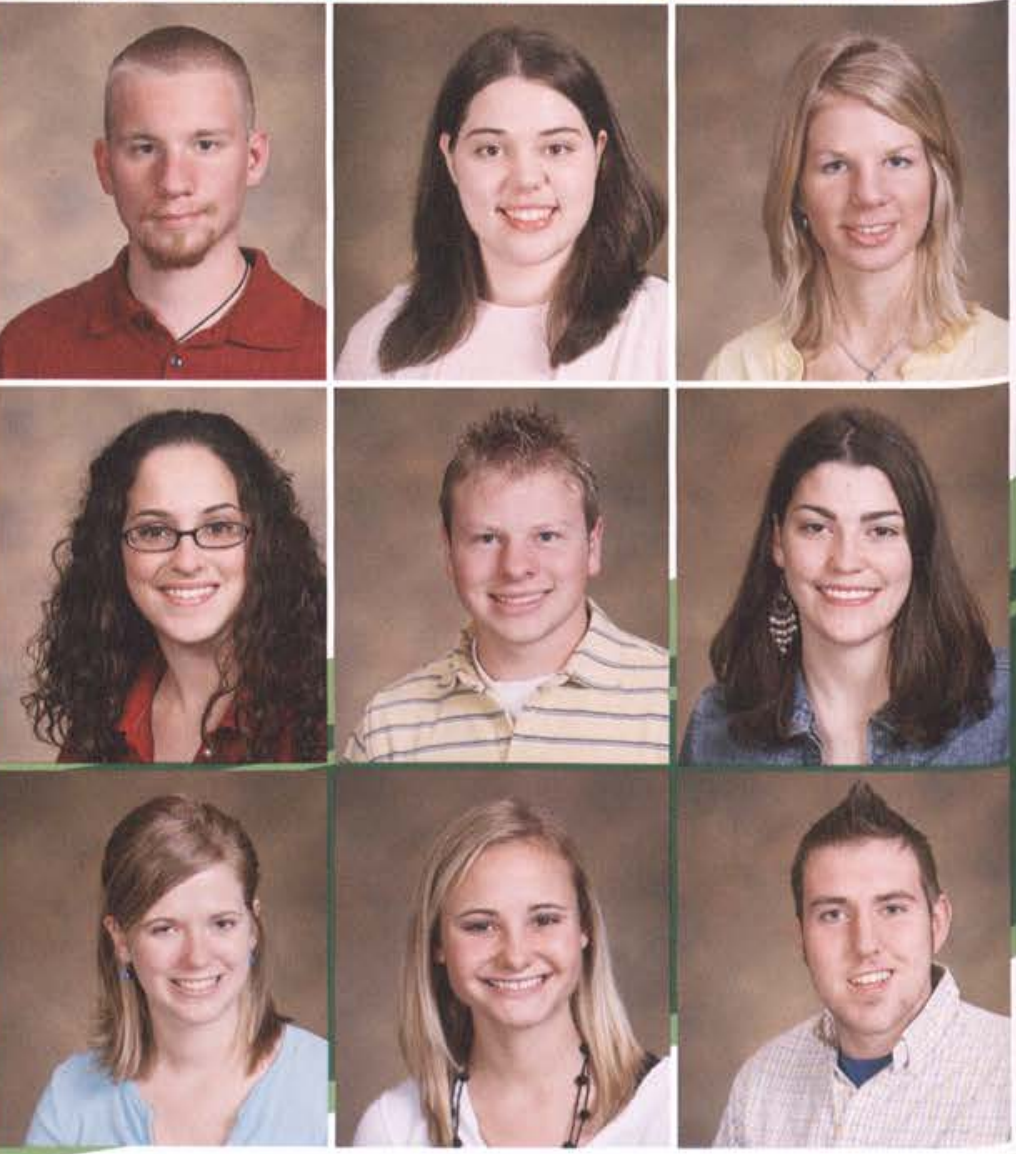

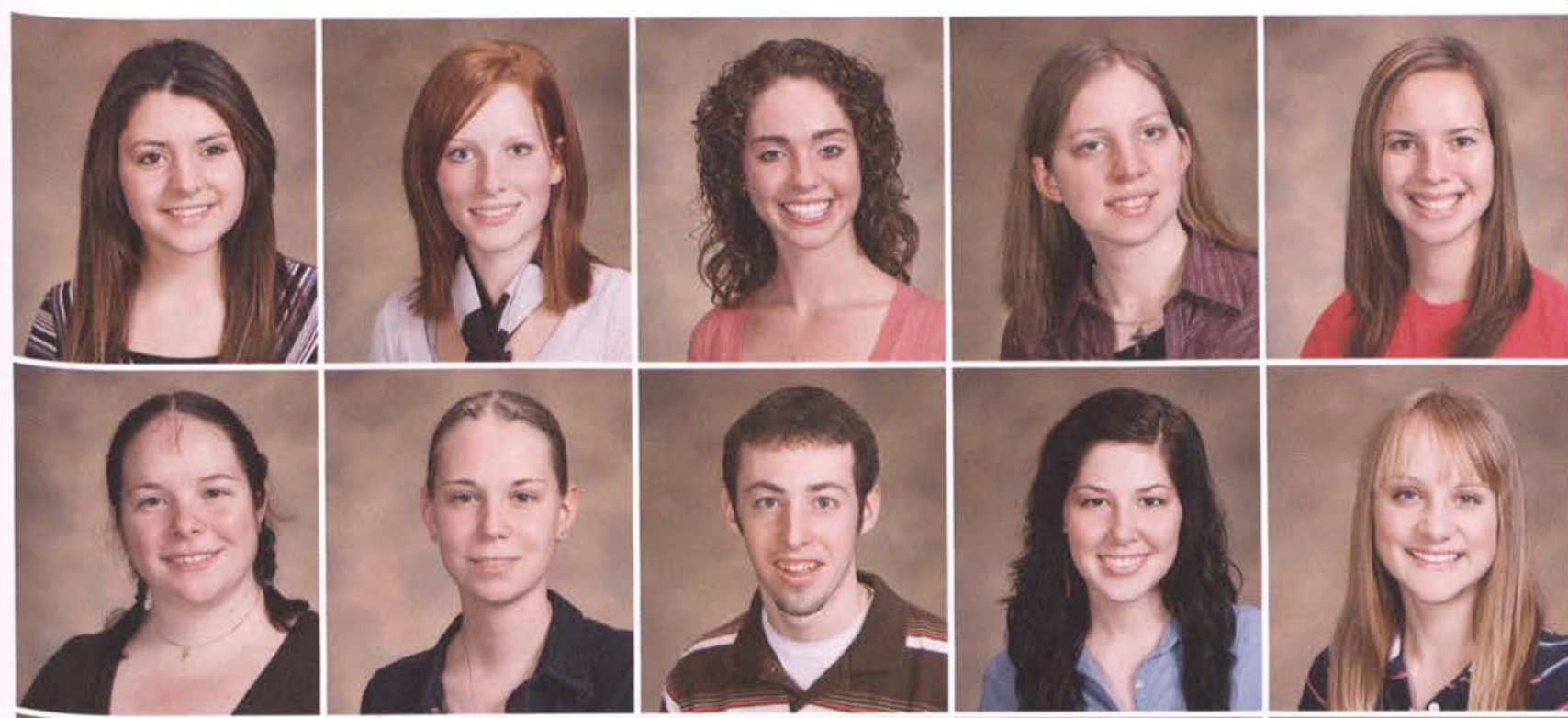

Kimberly Deseno

Katherine Devault

Jenna Develbiss

Barbara Dickhoner

Kelly Donohue
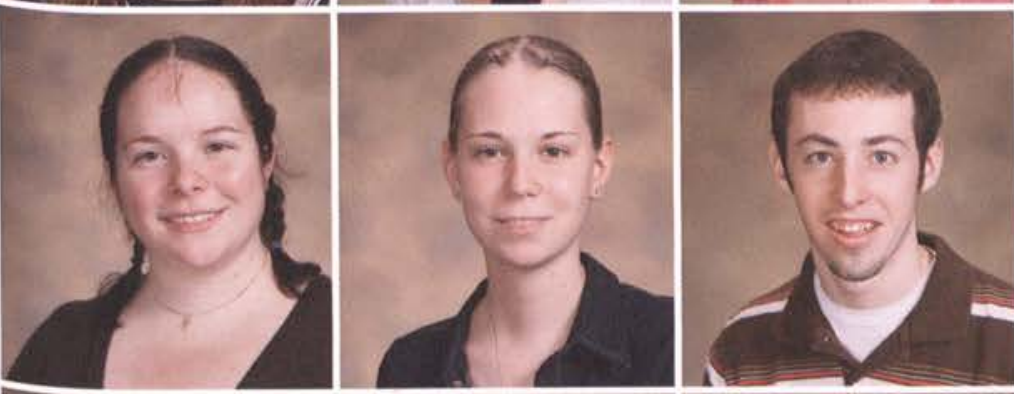

Emily Driscoll

Stephanie Driscoll

David Duarte

Rachel Duarte

Monica Duffell
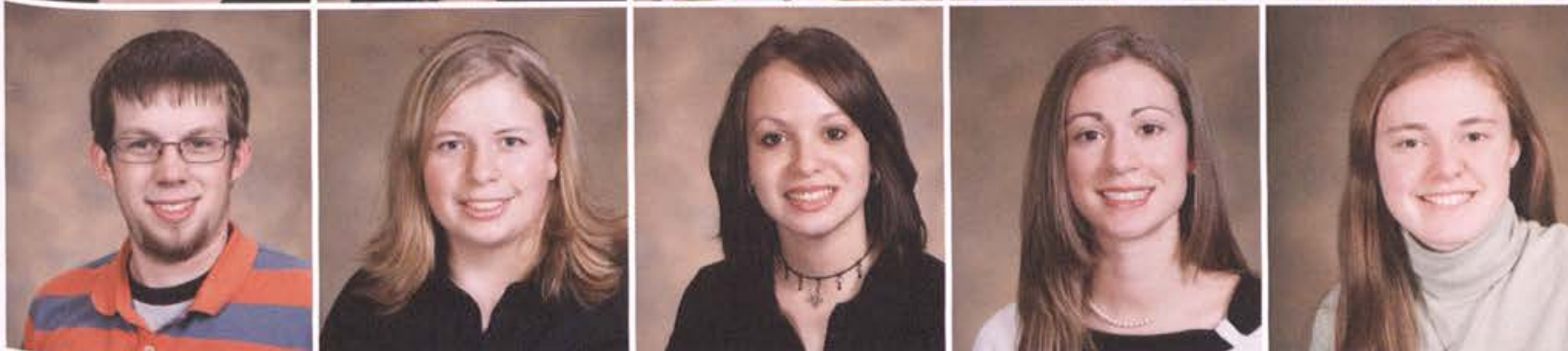

John Dunning

Sarah Early

Dorianne Eaves

Meredith Edwards

Sarah Egenreider
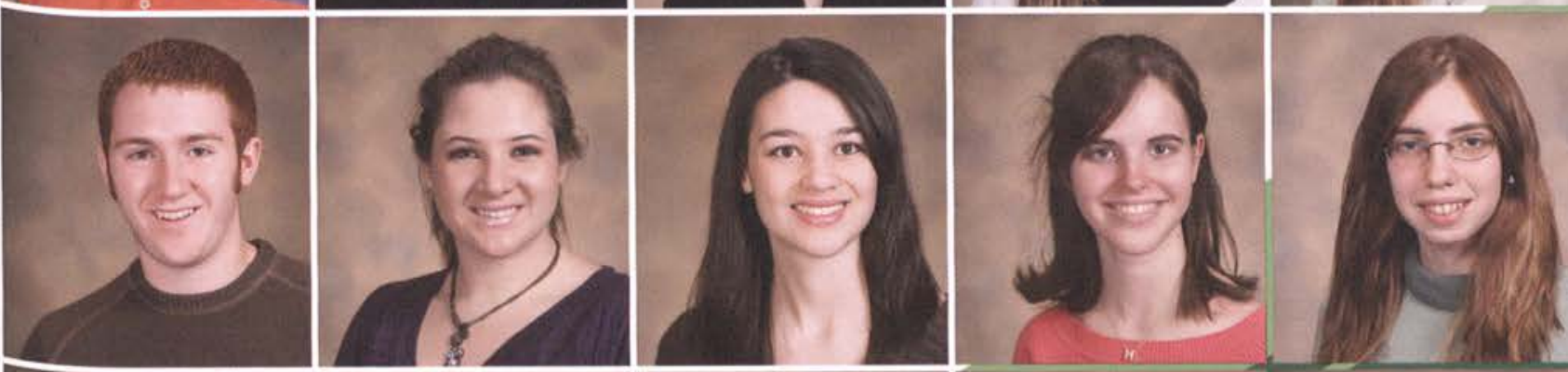

acob Eldridge

Sara Elliott

Annamarie Elmore

Haznnah Endres

Amy Farnham
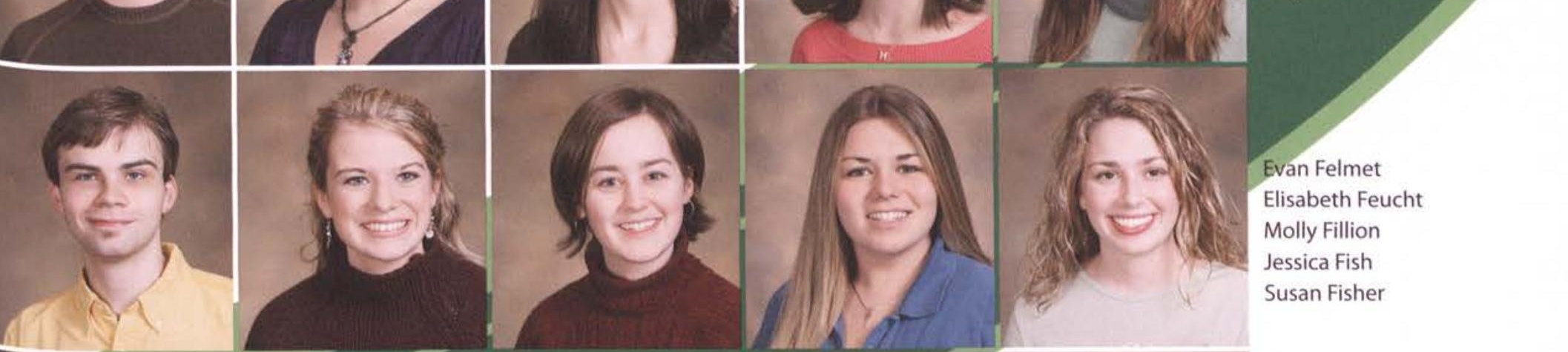

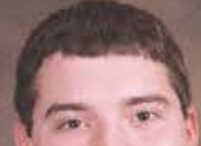

1

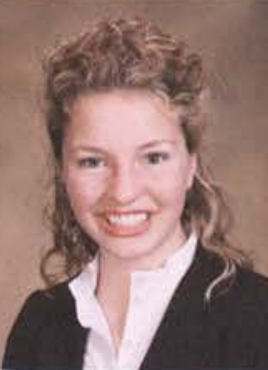

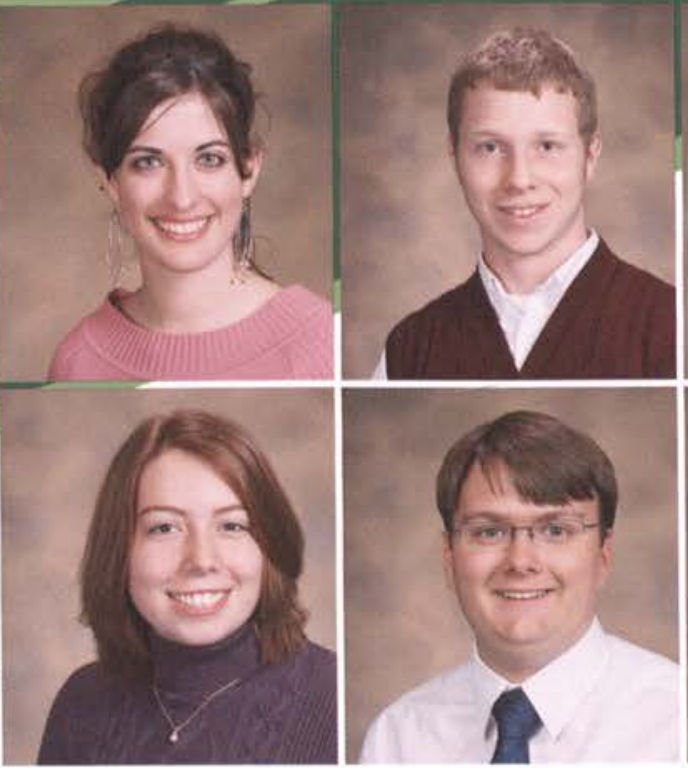
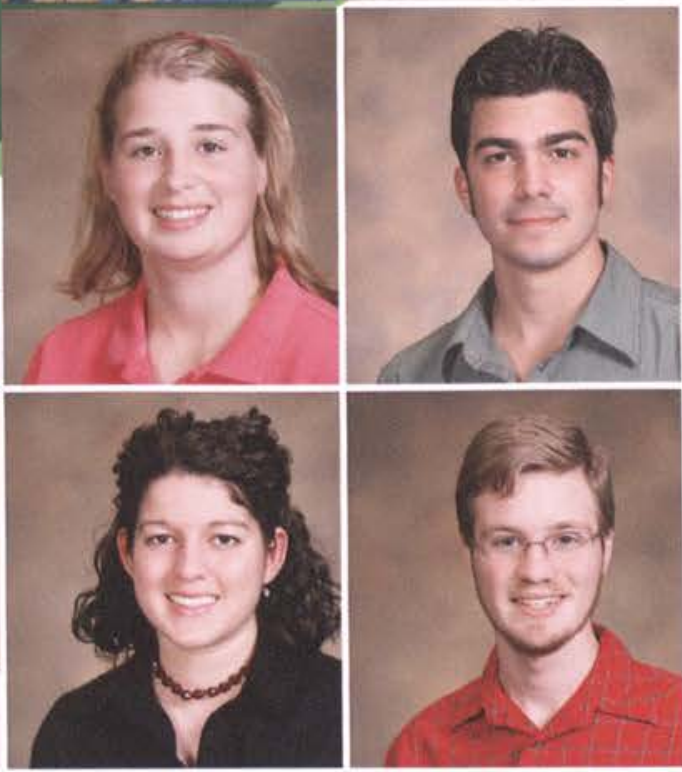

Justin Flatt

Heather Flippin

Daniel Floren

Martha Flow

Matthew Foote

Jill Forsberg

Kristie Frasier

Brent Fugate

Rachael Fuls

Dalen Fultz 

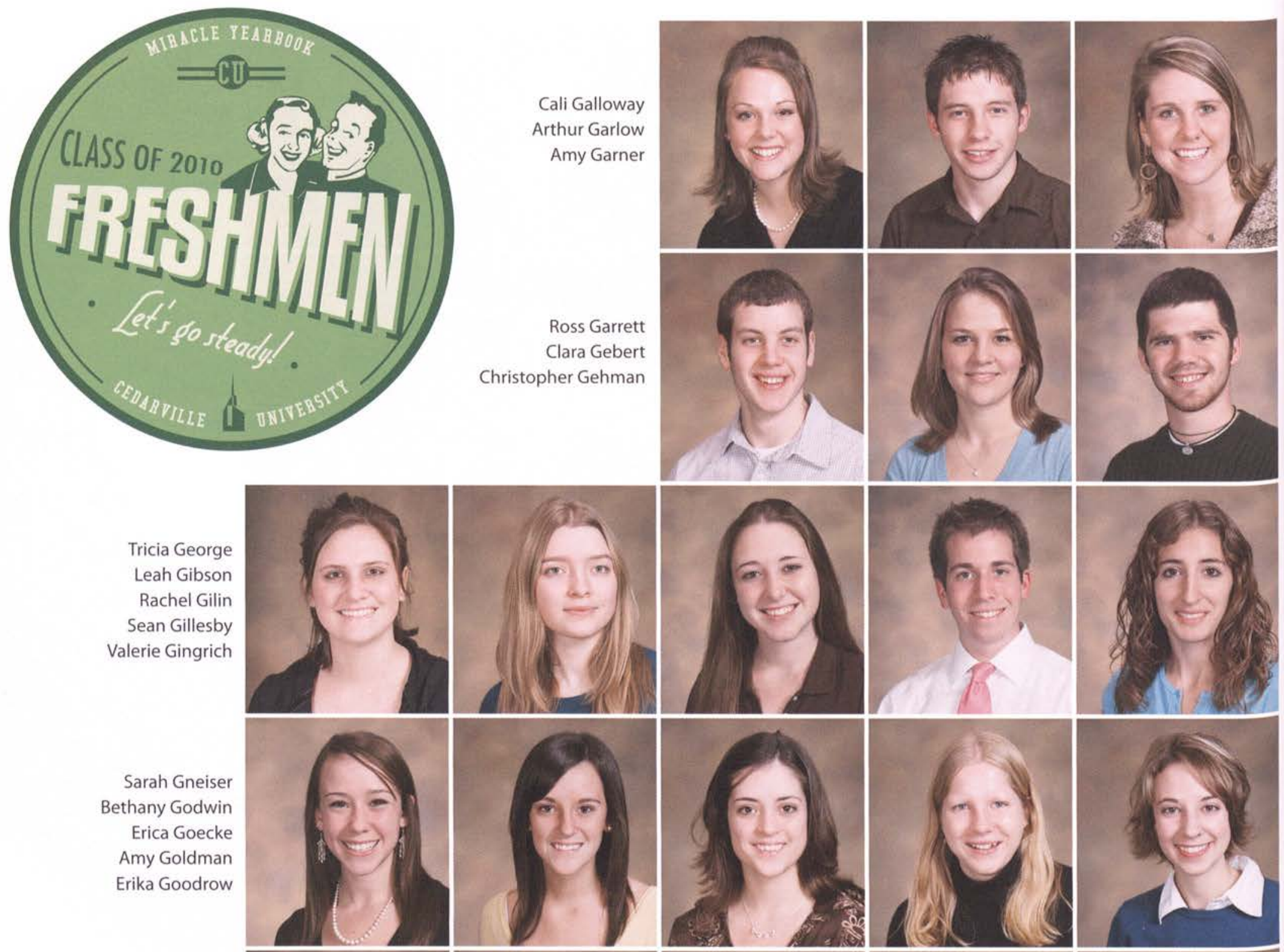

Sarah Gneise

Bethany Godwin

Erica Goecke

Amy Goldman

Erika Goodrow

Jonathan Gorrel

Evan Graham

Amanda Graves

Lisa Gray

Jennifer Greer
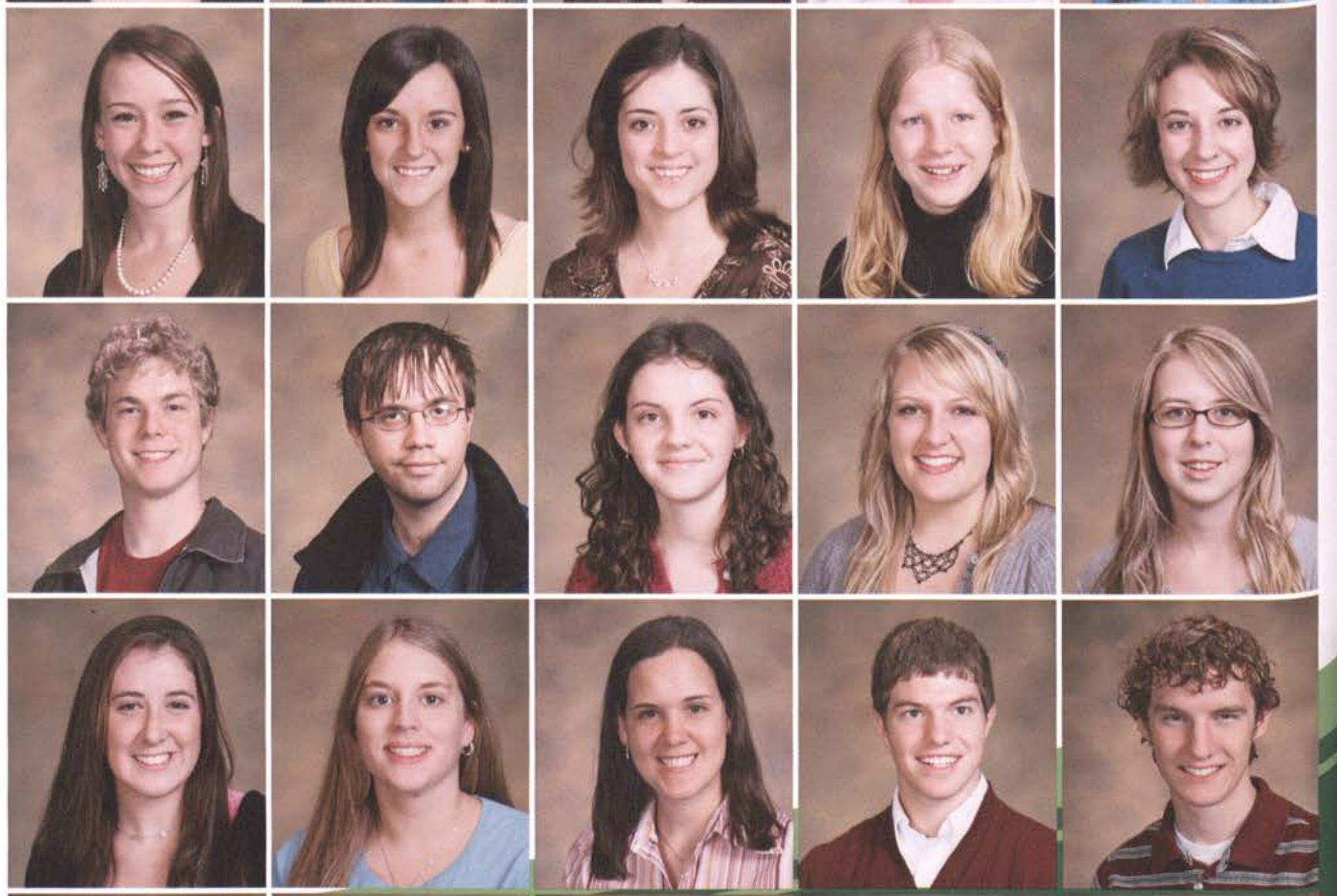

Rebecca Gregg Ashley Groce

Deborah Groen

Kurt Groman

John Hagen

Regina Hager

Stephen Halwes

Kristen Hamminga

Jacob Hand

Emily Haney
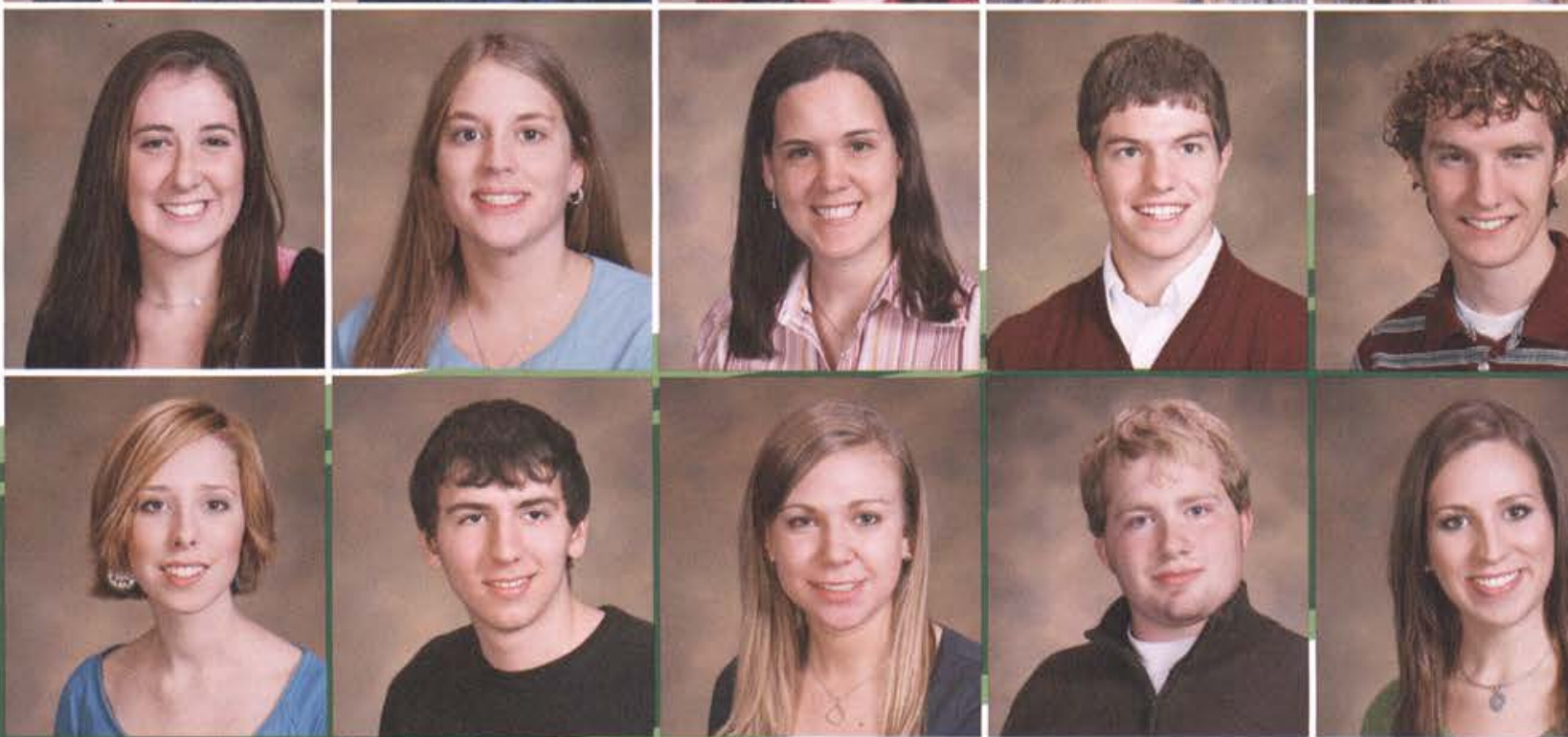


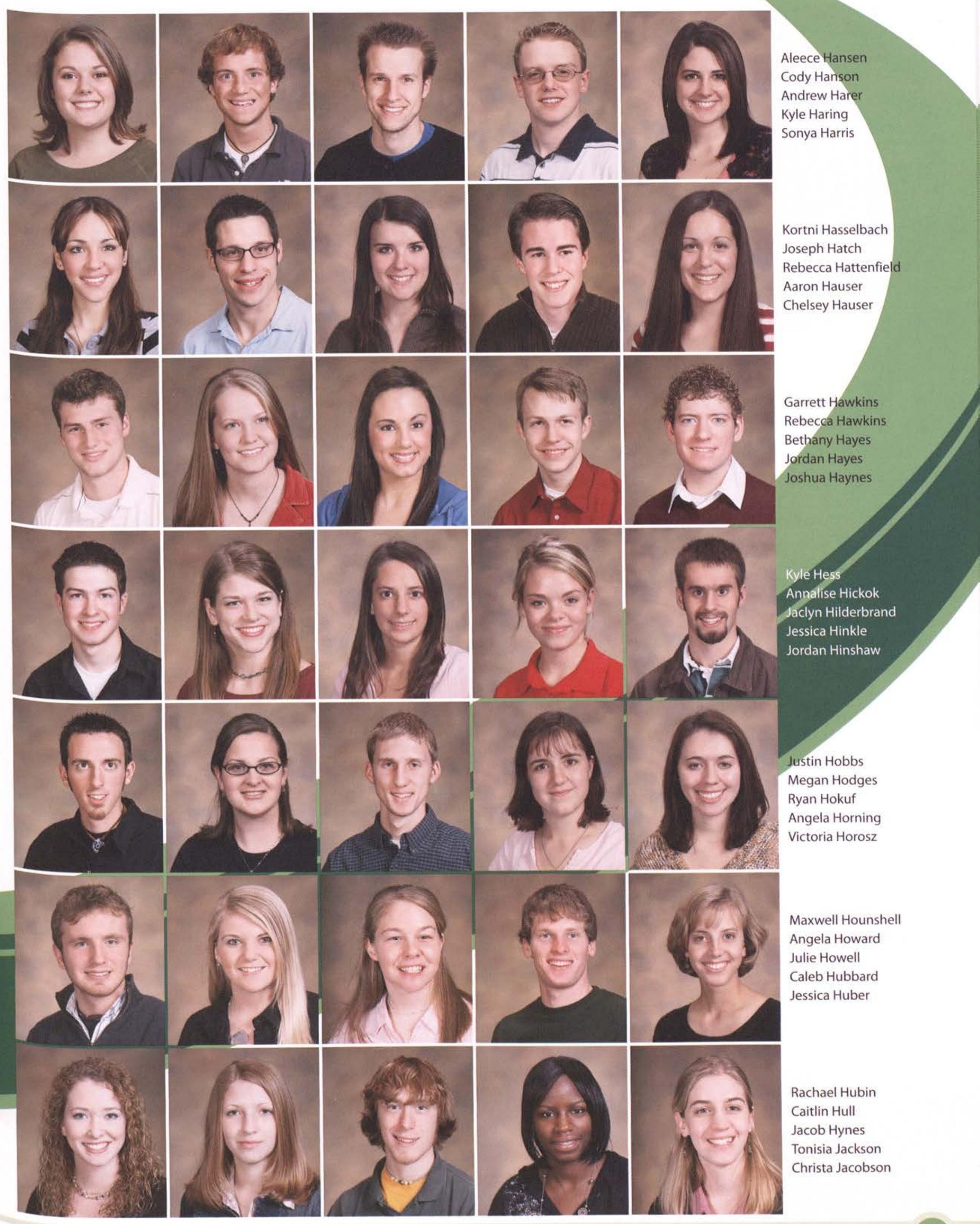



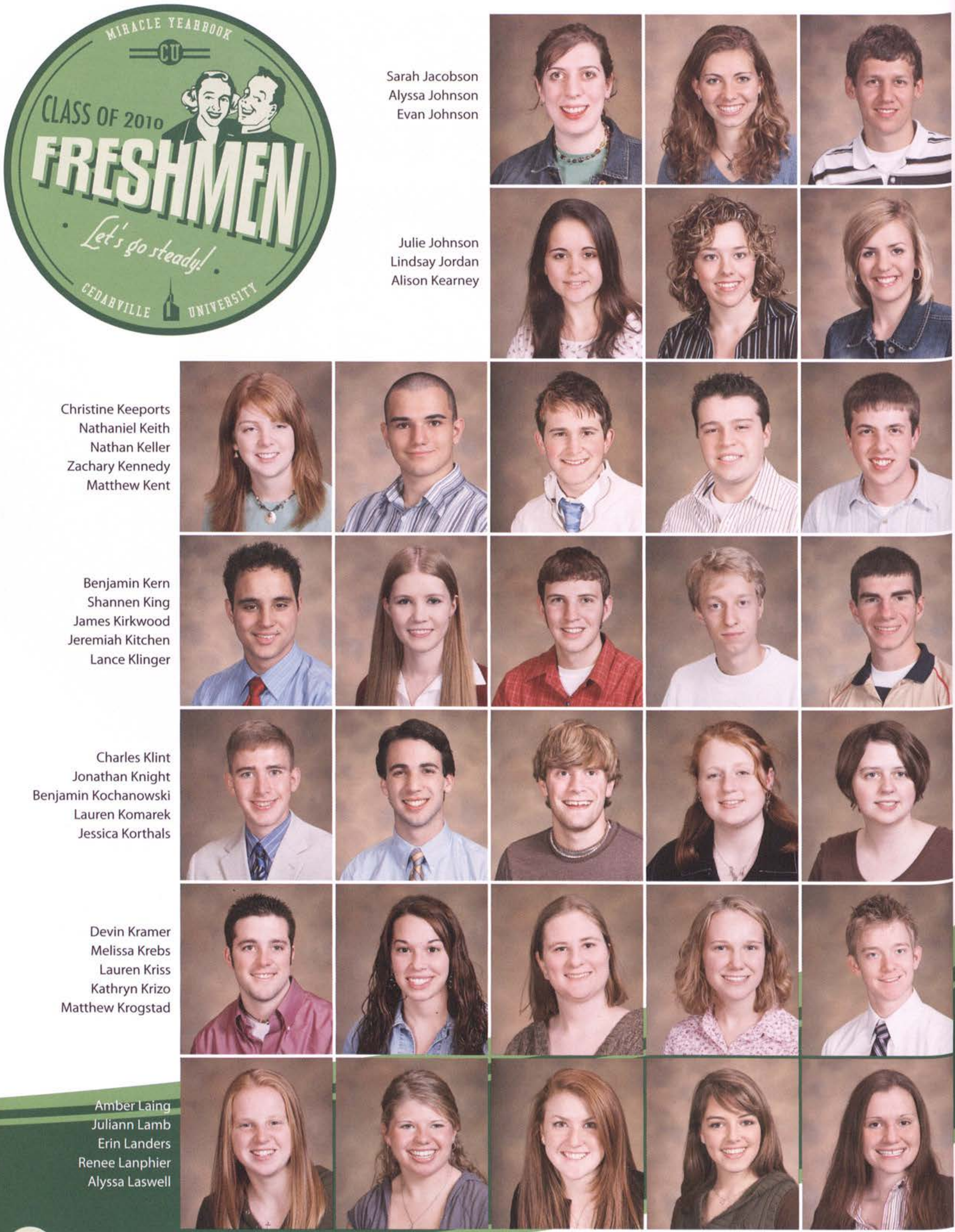

Sarah Jacobson

Alyssa Johnson

Evan Johnson

Julie Johnson

Lindsay Jordan

Alison Kearney

Christine Keeports Nathaniel Keith Nathan Keller

Zachary Kennedy Matthew Kent

Benjamin Kern

Shannen King

James Kirkwood

Jeremiah Kitchen

Lance Klinger
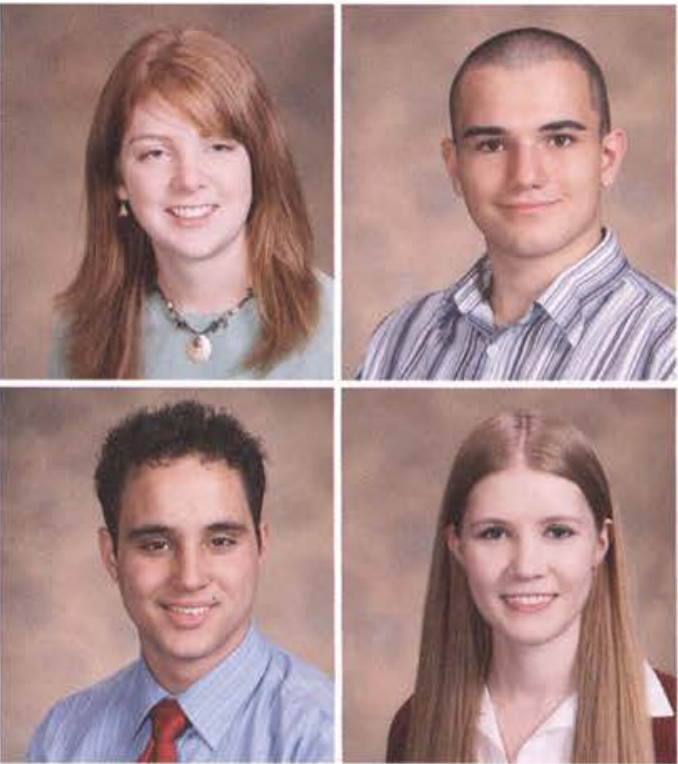

Charles Klint

Jonathan Knight

Benjamin Kochanowski

Lauren Komarek

Jessica Korthals

Melissa Krebs

Lauren Kriss

Kathryn Krizo

Matthew Krogstad
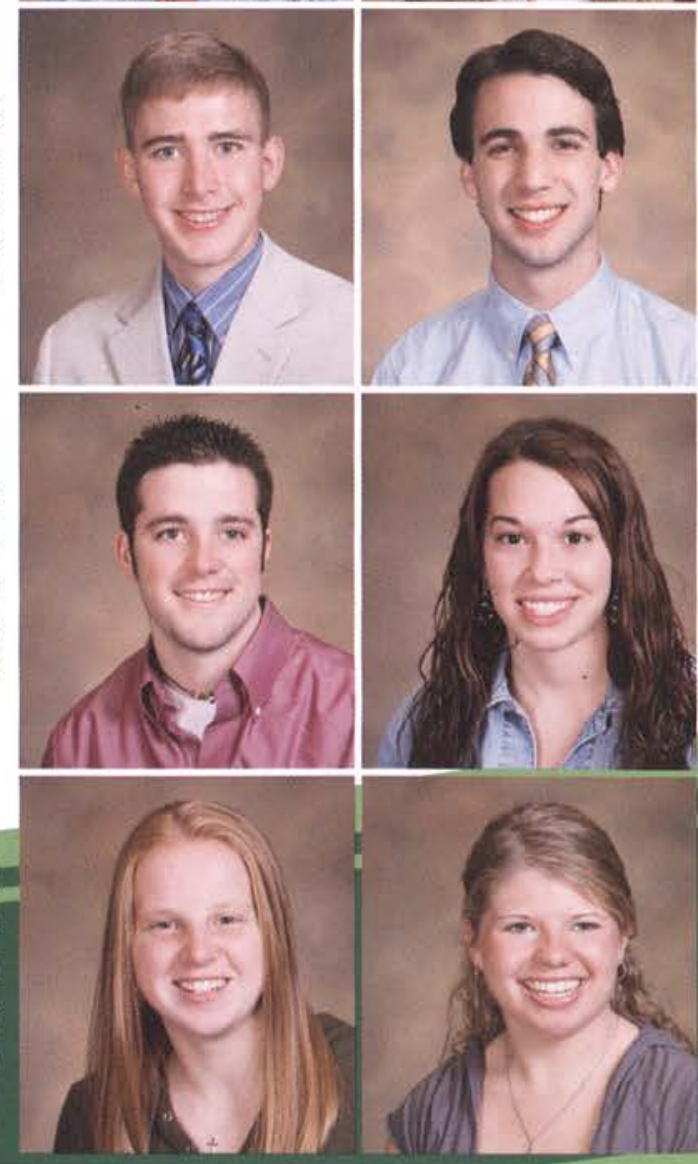
Juliann Lamb Erin Landers Renee Lanphier Alyssa Laswell

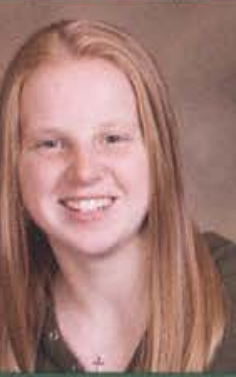




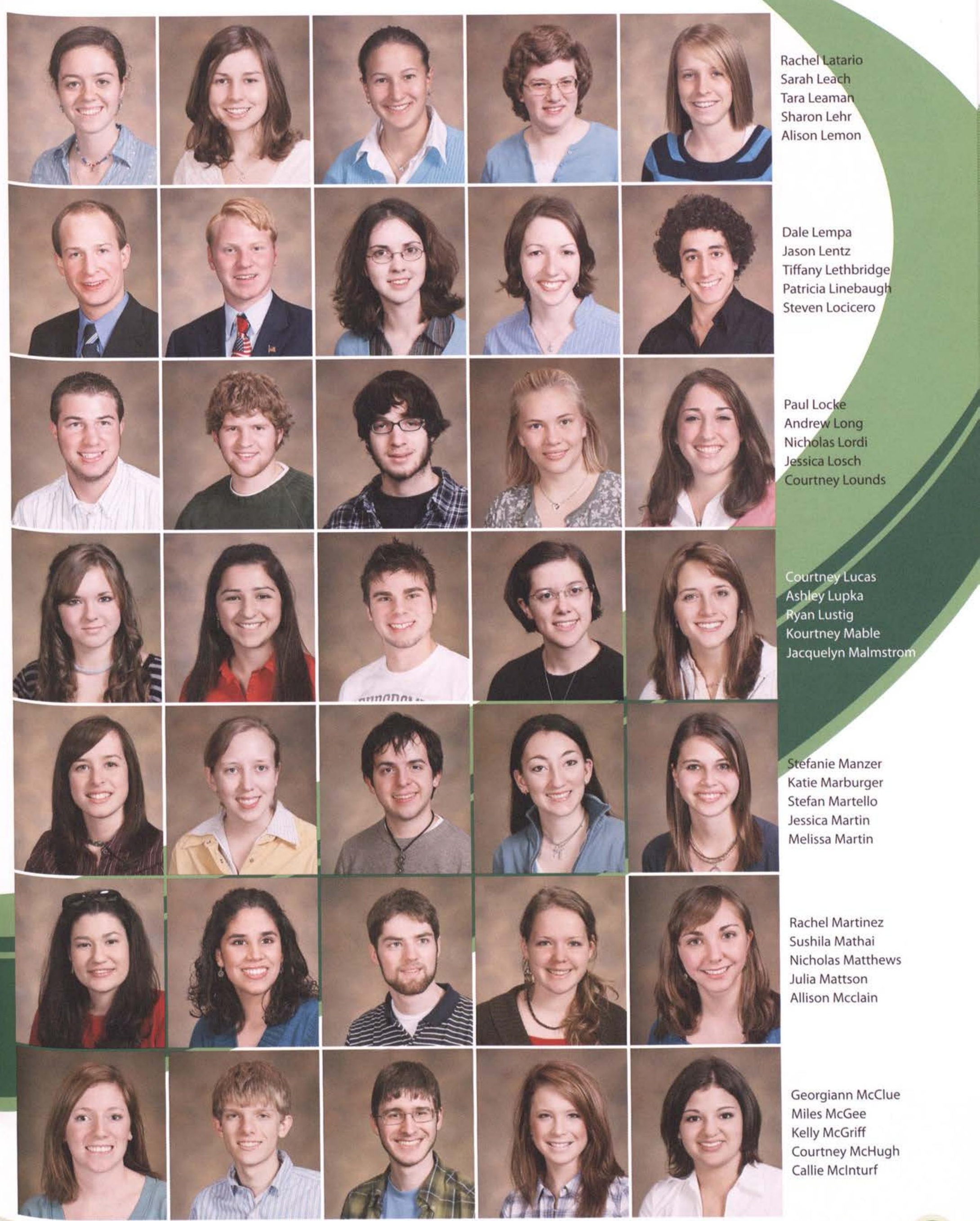



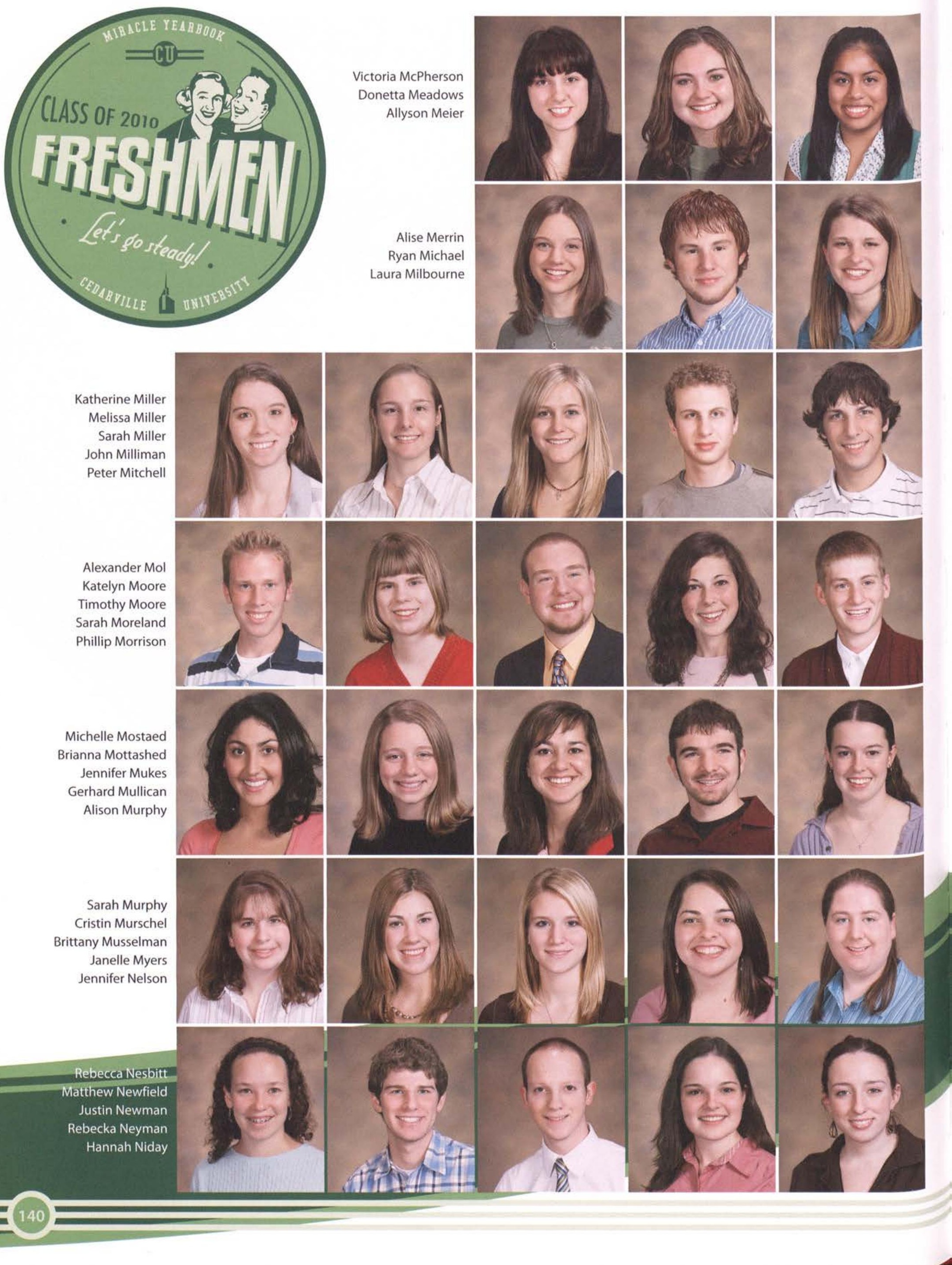

Victoria McPherson

Donetta Meadows

Allyson Meier
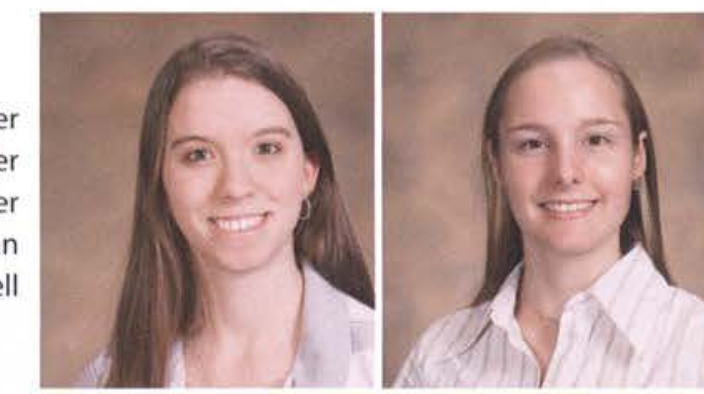

Alise Merrin

Ryan Michael Laura Milbourne

Katherine Miller Melissa Miller

Sarah Miller John Milliman

Peter Mitchell

Alexander Mol

Katelyn Moore

Timothy Moore

Sarah Moreland

Phillip Morrison
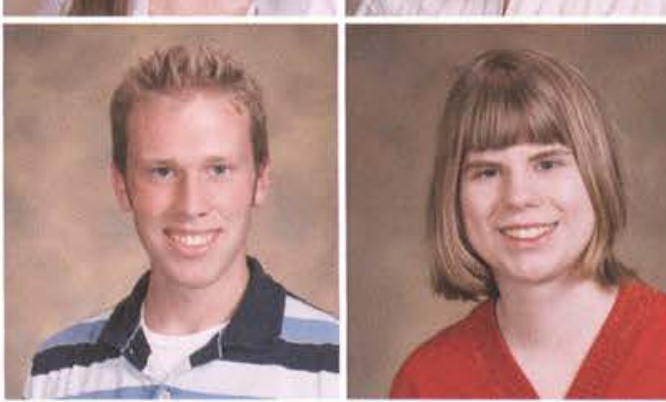

Michelle Mostaed

Brianna Mottashed

Jennifer Mukes

Gerhard Mullican

Alison Murphy

Sarah Murphy

Cristin Murschel

Brittany Musselman

Janelle Myers

Jennifer Nelson
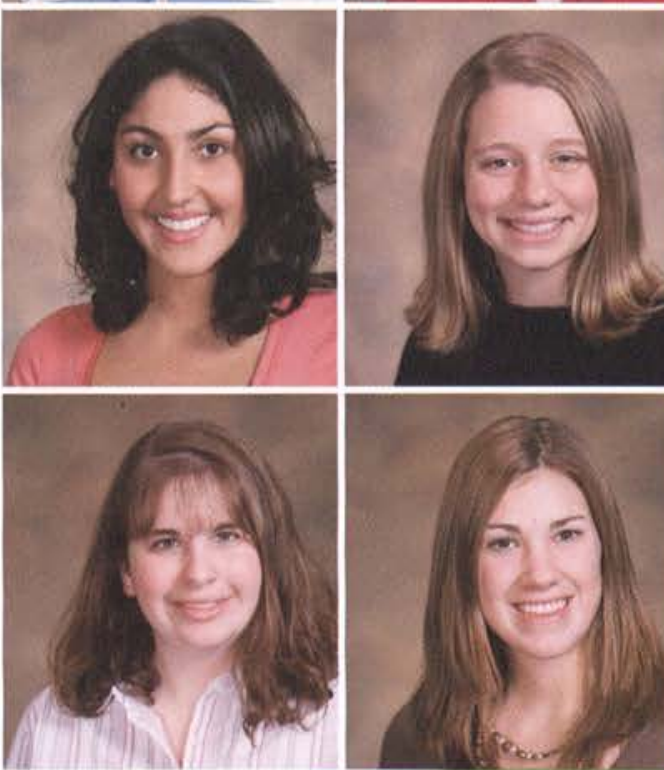

Rebecca Nesbitt Matthew Newfield Justin Newman Rebecka Neyman Hannah Niday

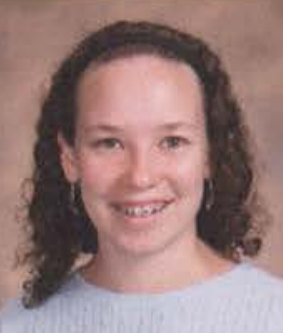




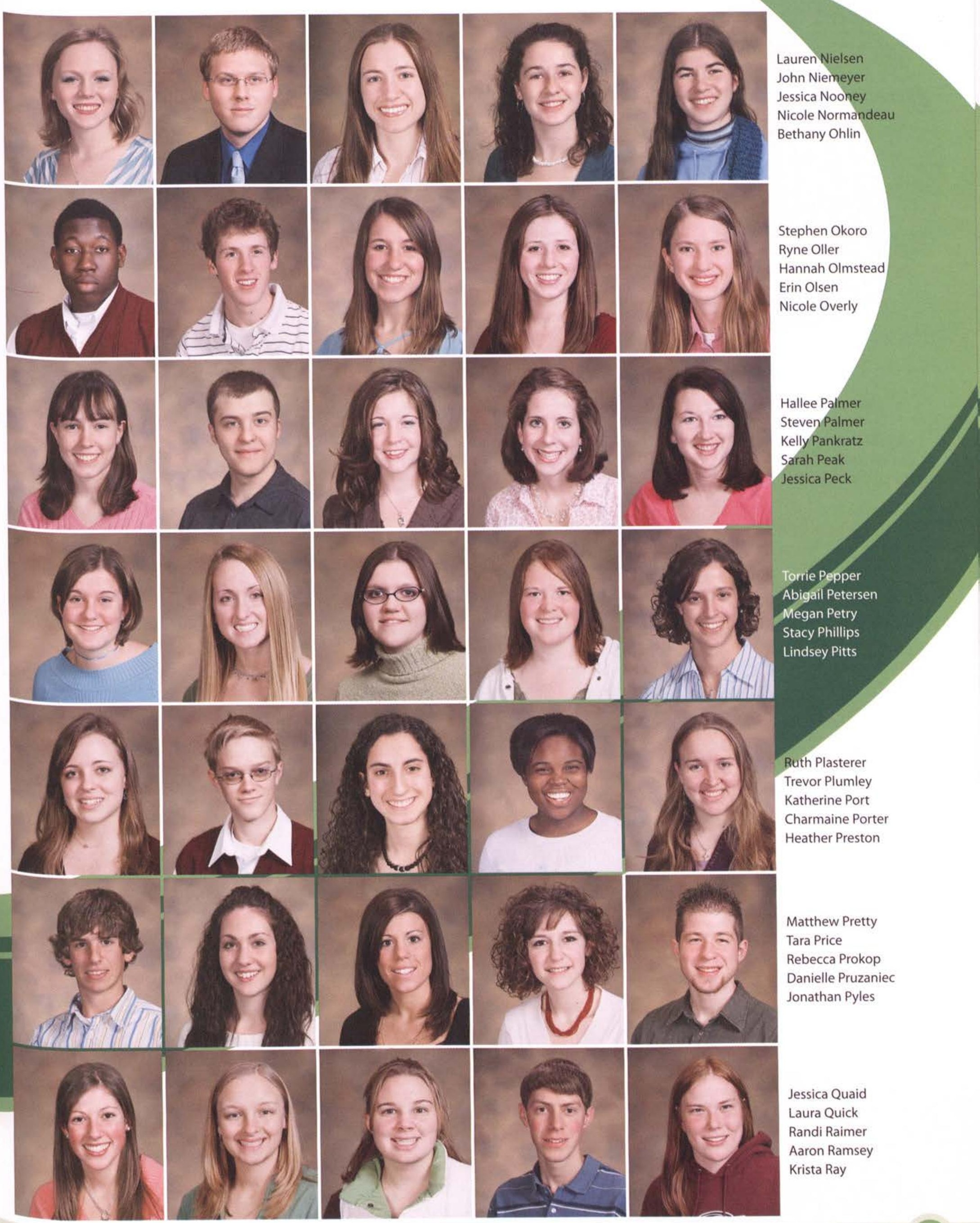



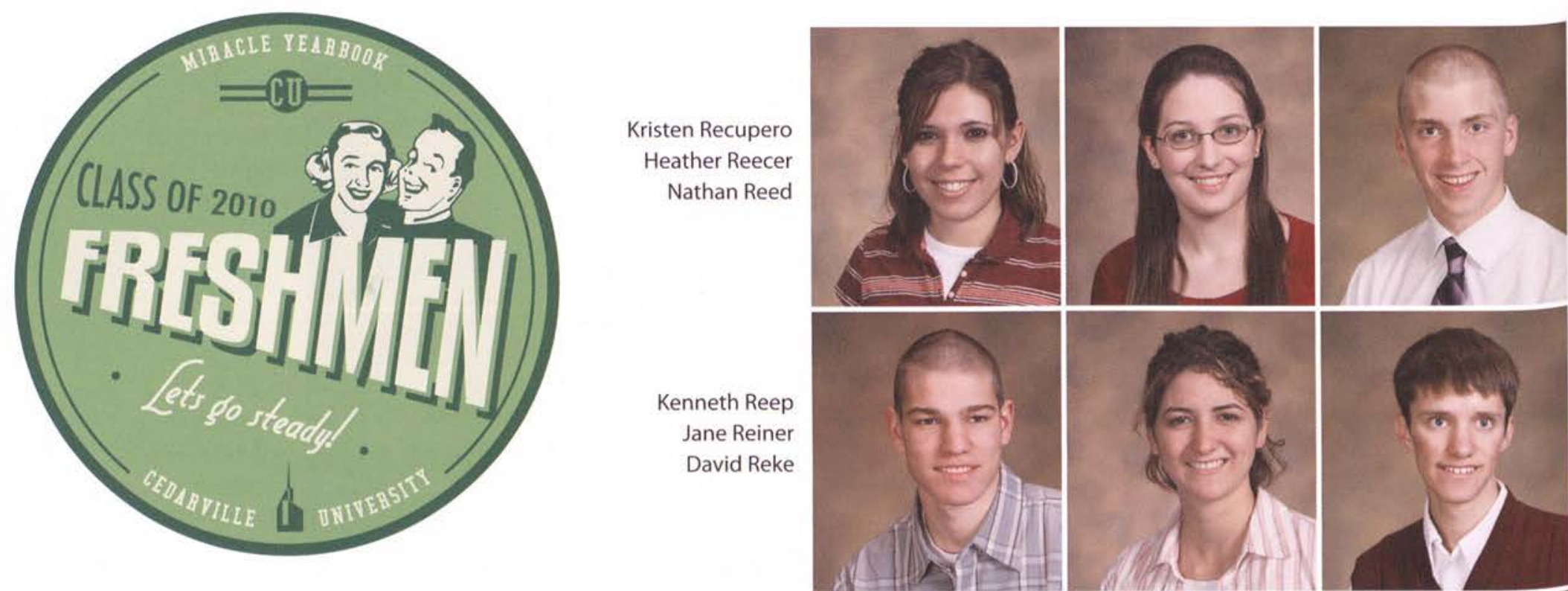

Katelyn Reuther Heather Rice Meredith Richard Lindsey Richardson Kristin Rintelman

Sarah Robbins E. Samuel Roberto Amanda Roberts John C. Roberts John F. Roberts

Keith Robinette Stefanie Rodgers Nevin Rosner Lisa Rothacker Stacie Rudd
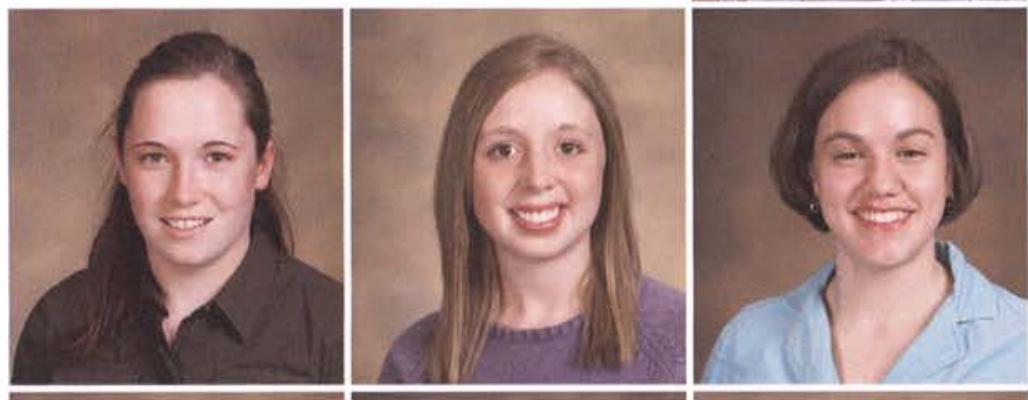

Kenneth Reep Jane Reiner David Reke

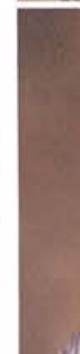

Nathan Reed
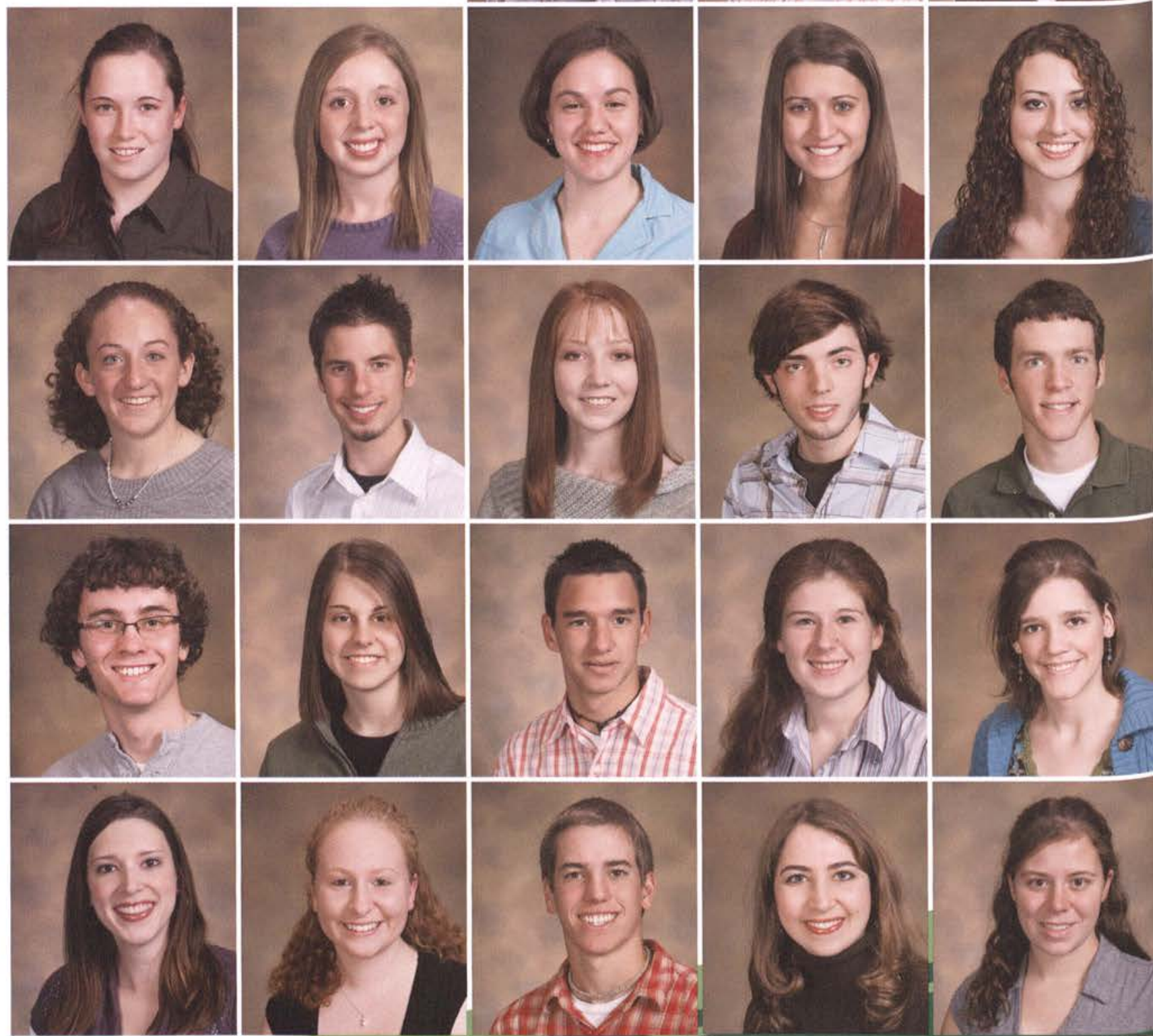
Emily Ruffner
Katy Russell
Brandon Salsbury
Janaye Sandberg
Karyn Sandefur
Emily Ruffner
Katy Russell
Brandon Salsbury
Janaye Sandberg
Karyn Sandefur
Emily Ruffner
Katy Russell
Brandon Salsbury
Janaye Sandberg
Karyn Sandefur
Emily Ruffner
Katy Russell
Brandon Salsbury
Janaye Sandberg
Karyn Sandefur
Emily Ruffner
Katy Russell
Brandon Salsbury
Janaye Sandberg
Karyn Sandefur

Timothy Sanford Angela Sangregory Sarah Schaszberger Genelle Schedlbauer Sean Scheerschmidt
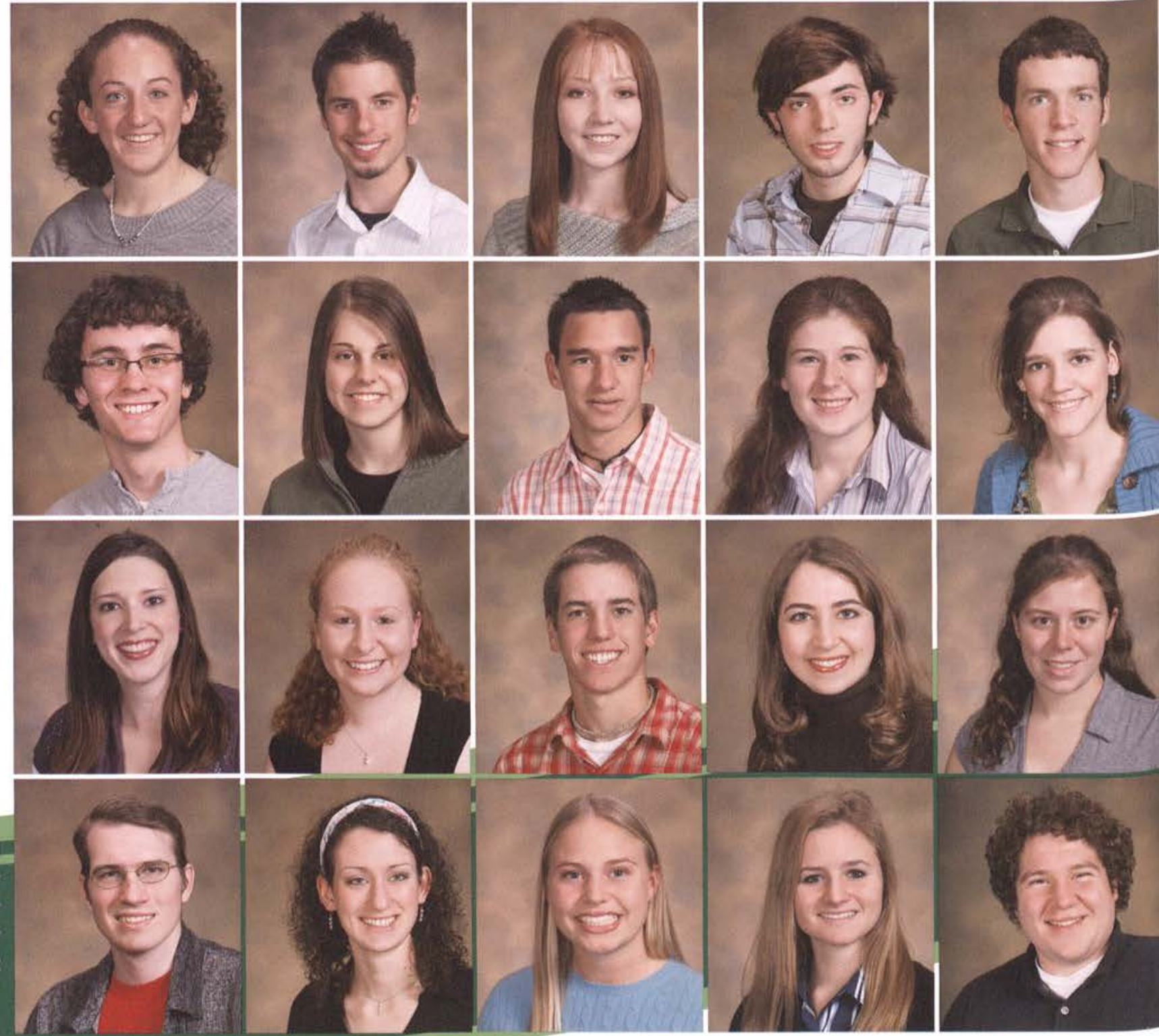


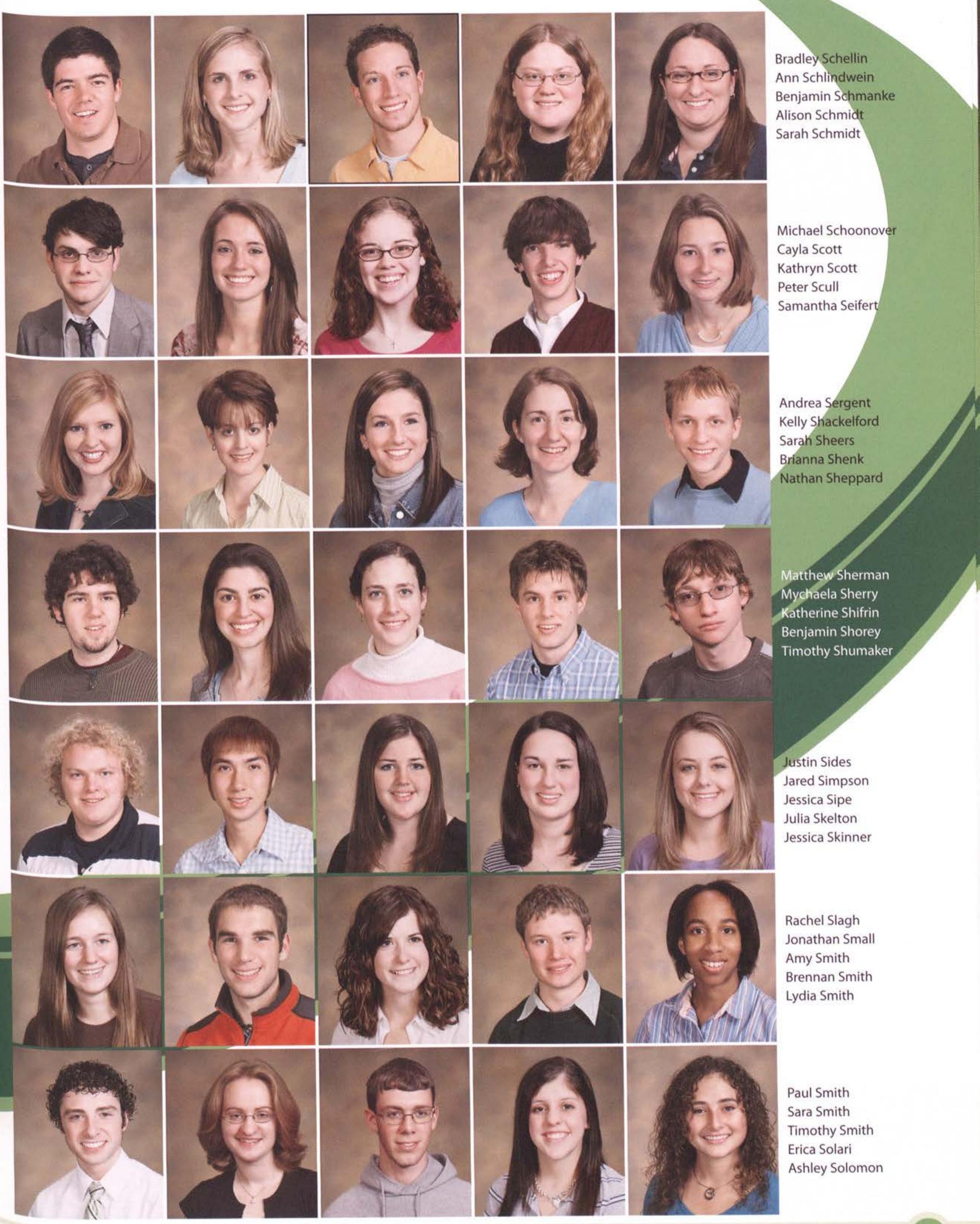




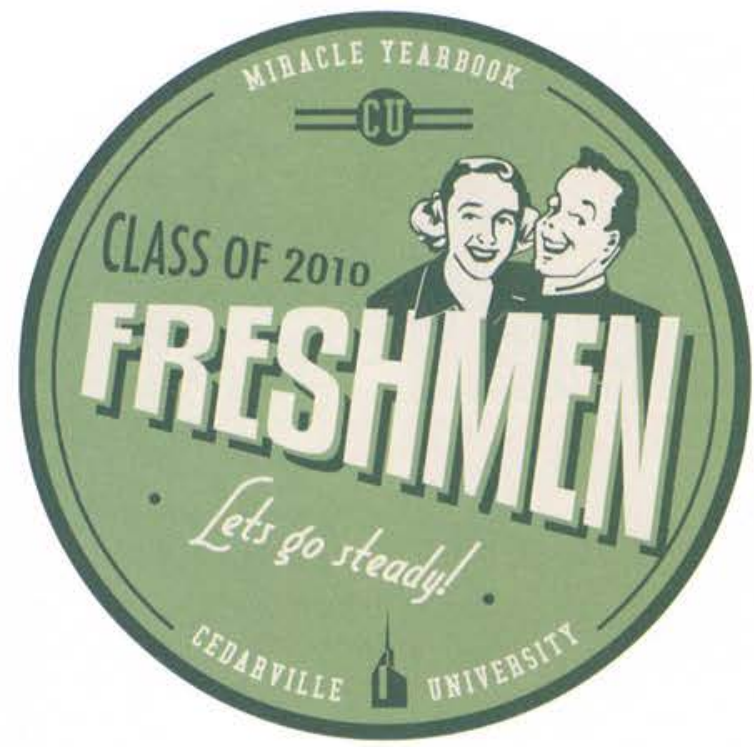

Lauren Sosey

Caleb Speicher

Kyle Spivey

Crystal States

Jennifer Steckel

Mary Steinbach

Levi Sterling

Derek Stockwell
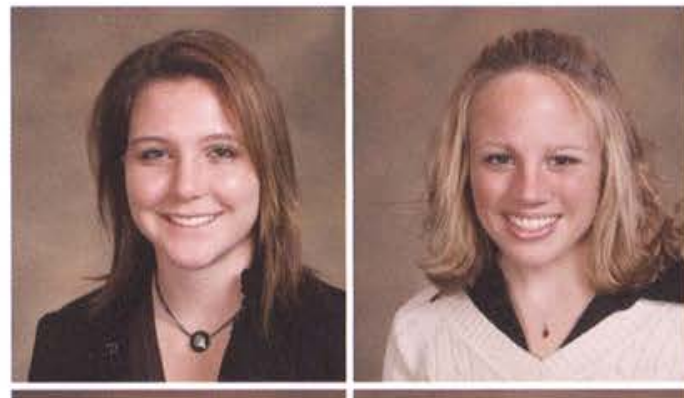

Stephanie Stahl

Joshua Stands

Samuel Starr

Sonja Storhaug

Heather Stout

Amy Streitmatter

Michelle Stringfellow

Elizabeth Strode
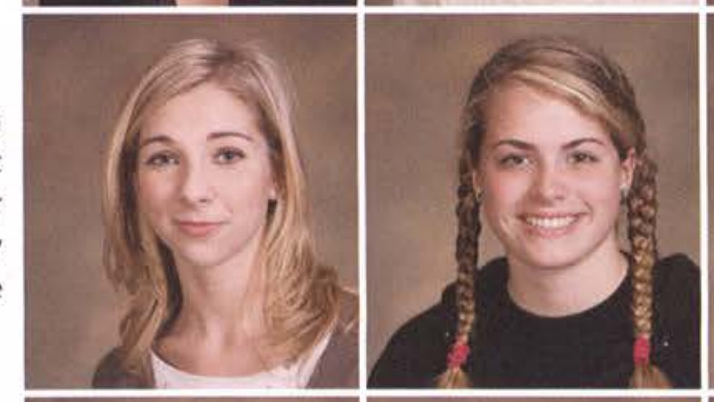

Sari Stroud-Lusk

Jessica Stroup

Nathaniel Studebaker

Amanda Sugg

Zachary Surant

Abby Swaney Elizabeth Sweeney

Brittany Sylvester

Valerie Talbott

Nathan Terra
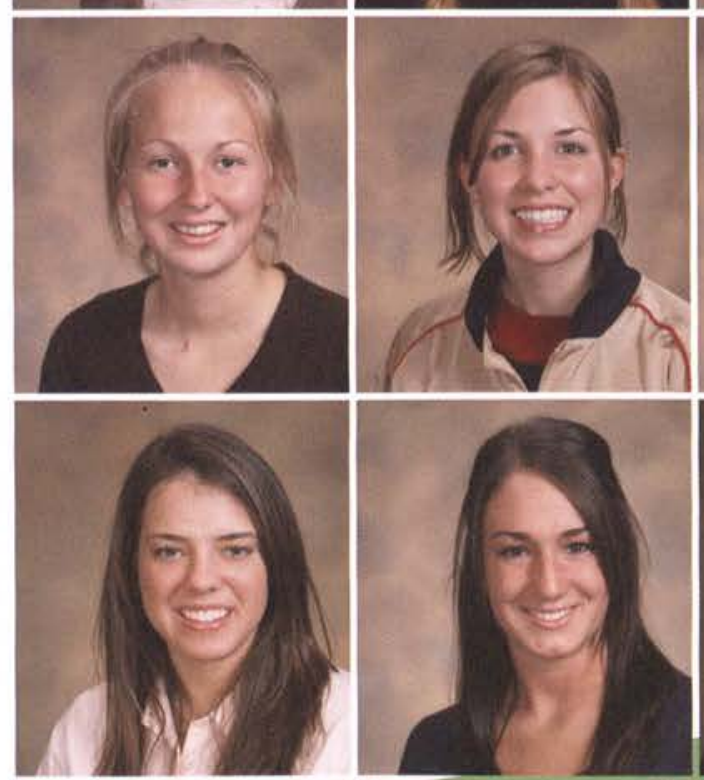

Benjamin Thacker Laura Thayer

Daniel Thomas

Katie Thomas

William Thompson
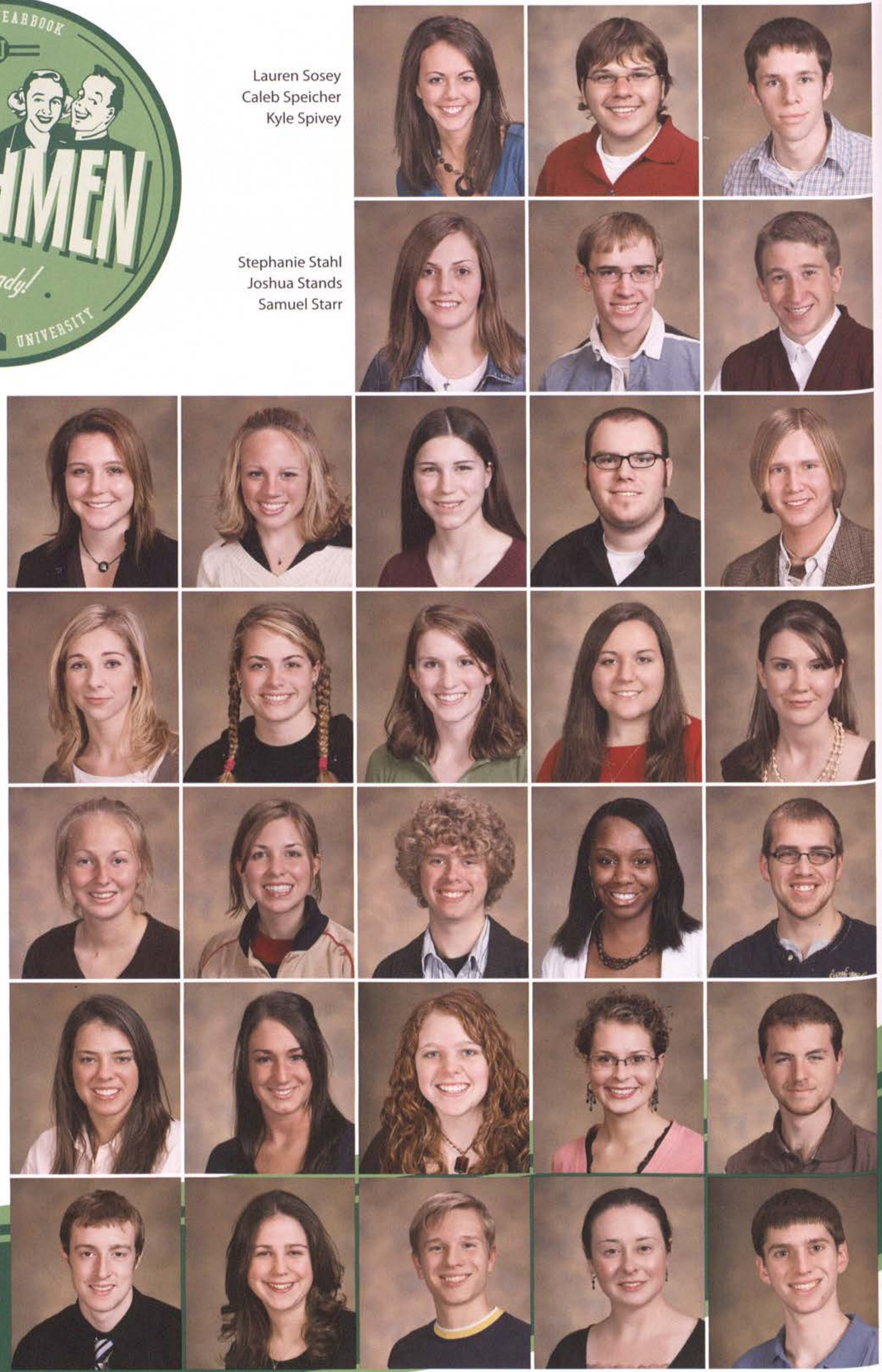

144 


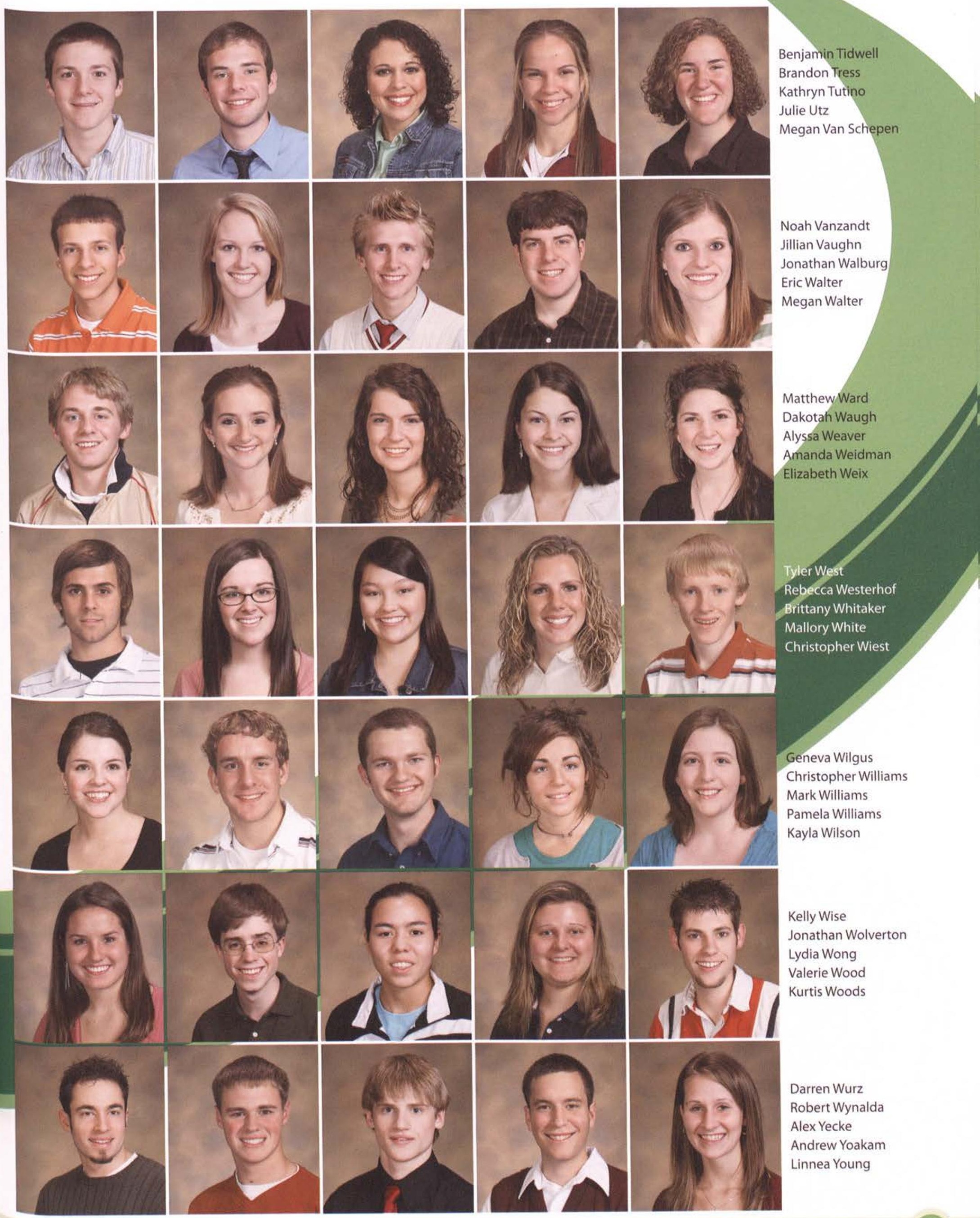



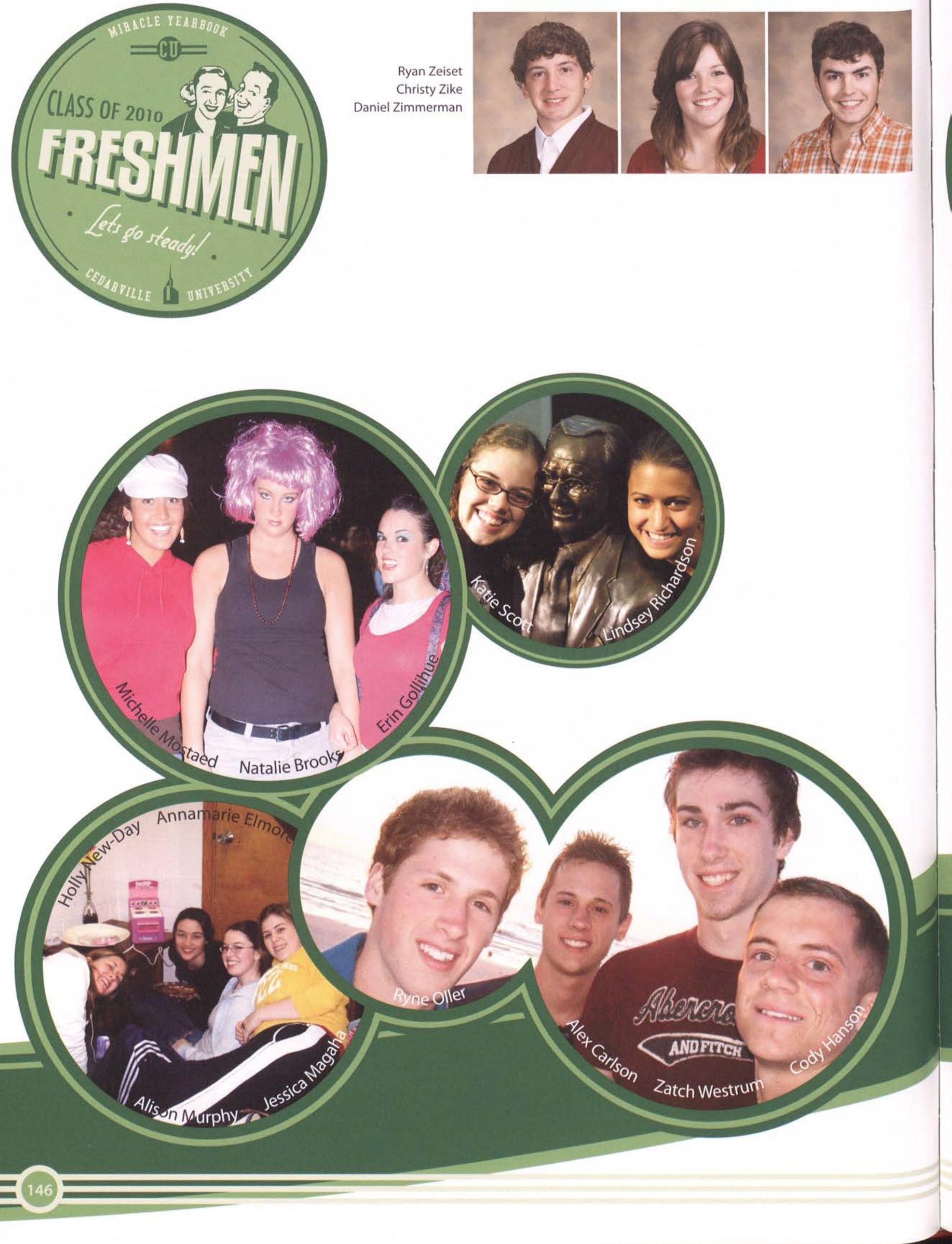


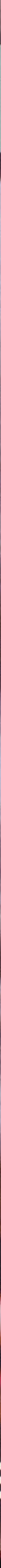




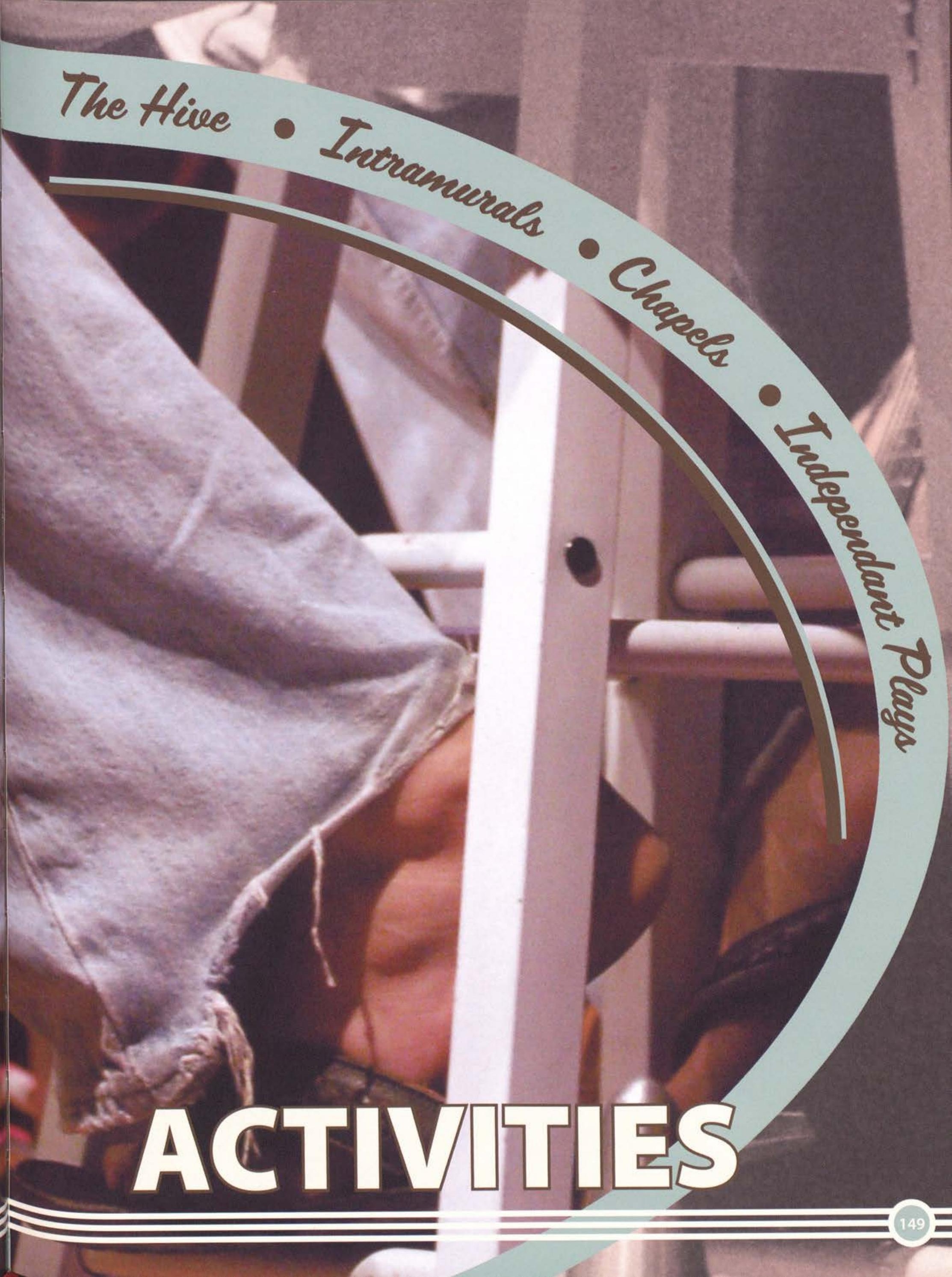


$+$
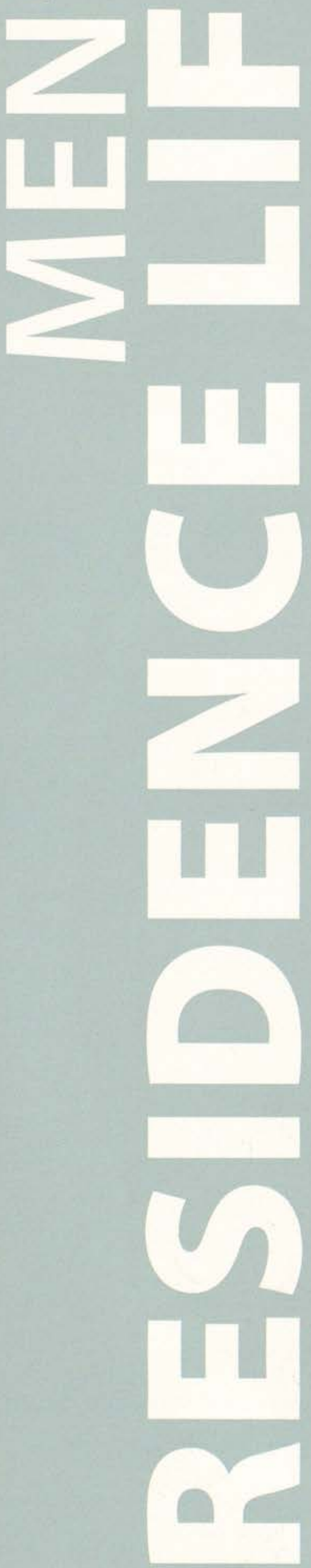

across campus." This year, Lawlor residents could make an even better impression on their female guests thanks to their newly renovated lounge. However, not all male residents rated their dorms based on their appeal to Cedarville girls. Brock residents seemed to appreciate the chaos of dorm life. Wade McComas said, "If there is one place where the simple-minded can find rest, it is most assuredly here, in Brock Hall." Ryan Bower agreed, saying, "The brotherhood, the fun, and of course, the idiocy of this dorm is something I won't ever forget. In Brock, the inmates run the asylum, and I wouldn't want it any other way." The men of the Hill also supported their dorms. Carr resident Nathan Chester championed his beloved Hill, saying, "While the rest of the dorms try to act cool, the guys at the Hill, though slandered and put down, are cool?"

McChesney also provided its residents with incredible friends and fun times. Andrew Costerisan said, "Having R.A.s looking out for your well-being, whether it's by reminding you to eat or asking how your spiritual walk is going, is quite encouraging. Everyone keeps an eye out for each other." However, Brian Nelson favored McChesney for different reasons. "My favorite times are when we play pranks on each other, especially when it involves alarm clocks in the air conditioning vent."

Similarly, Ed Dunning described life in Rickard as "adventurous, thought-provoking, and sometimes all-out goofy. Whether it be sitting in the hall with the guys talking theology or climbing up the stairs old school with blankets tied to the railing... we just like hanging out with each other."

Faced with eccentric unit-mates and outrageous pranks, Cedarville men took everything in stride. They bonded not only from hall Bible studies, but also from the chaos of dorm life.

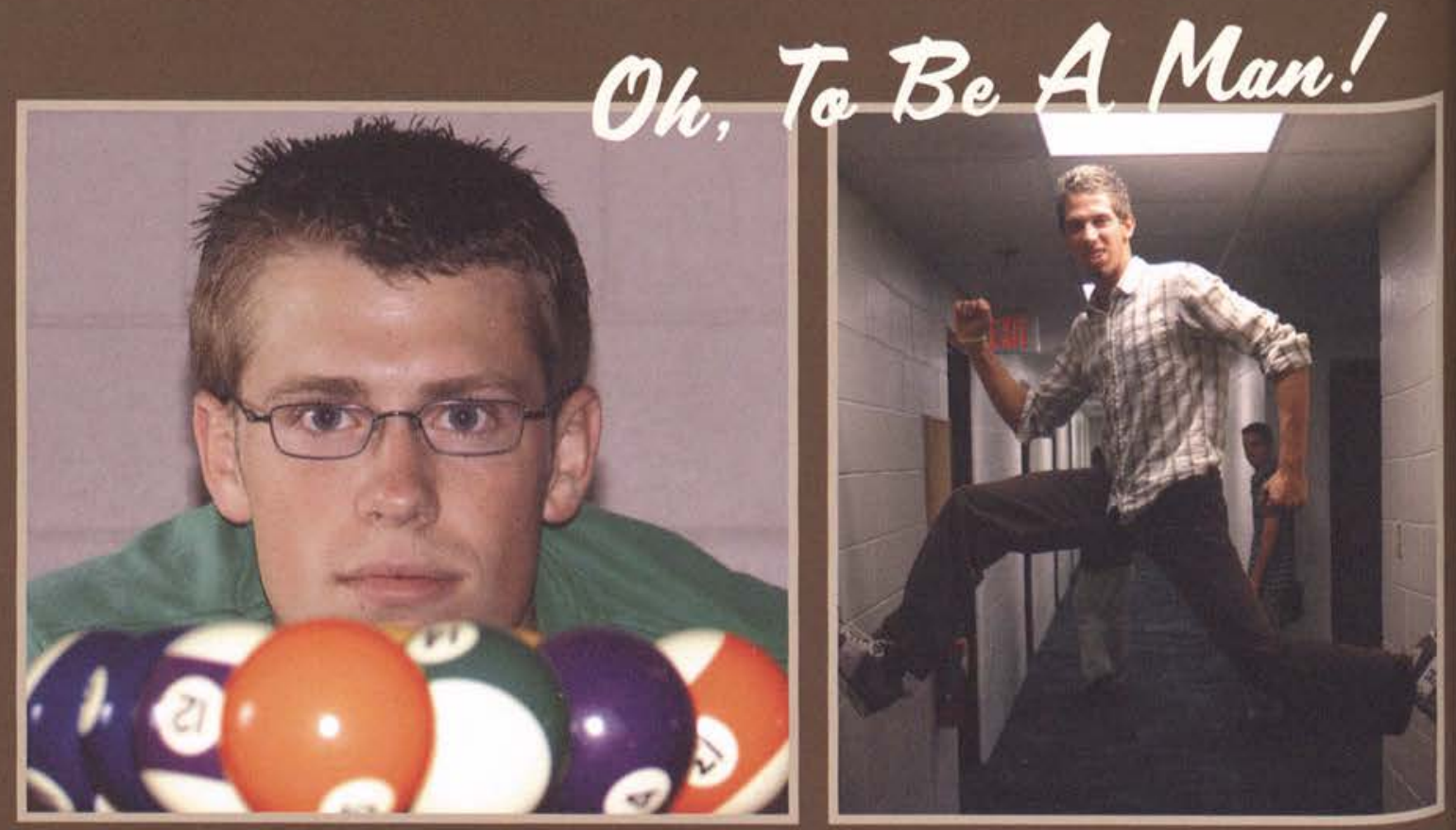

- "Lawlor forces immature, proud boys to live with each other, sharpening each other to become mature, humble men of God," said Evan Carter, pictured playing pool in the Miter Center.

- "Living in Brock Hall can be dangerous. One must always be ready for action. It is no place for the faint of heart," said Brian Houchen.

- "A few of us boys popped in to say hello to the guys on the second floor," said John Bardakjy aided by Chester Dudick and Derek Kreider.

W Mike Loosa and Jeff Antioho are enjoying girls' open dorms in Mike's sister's dorm room.
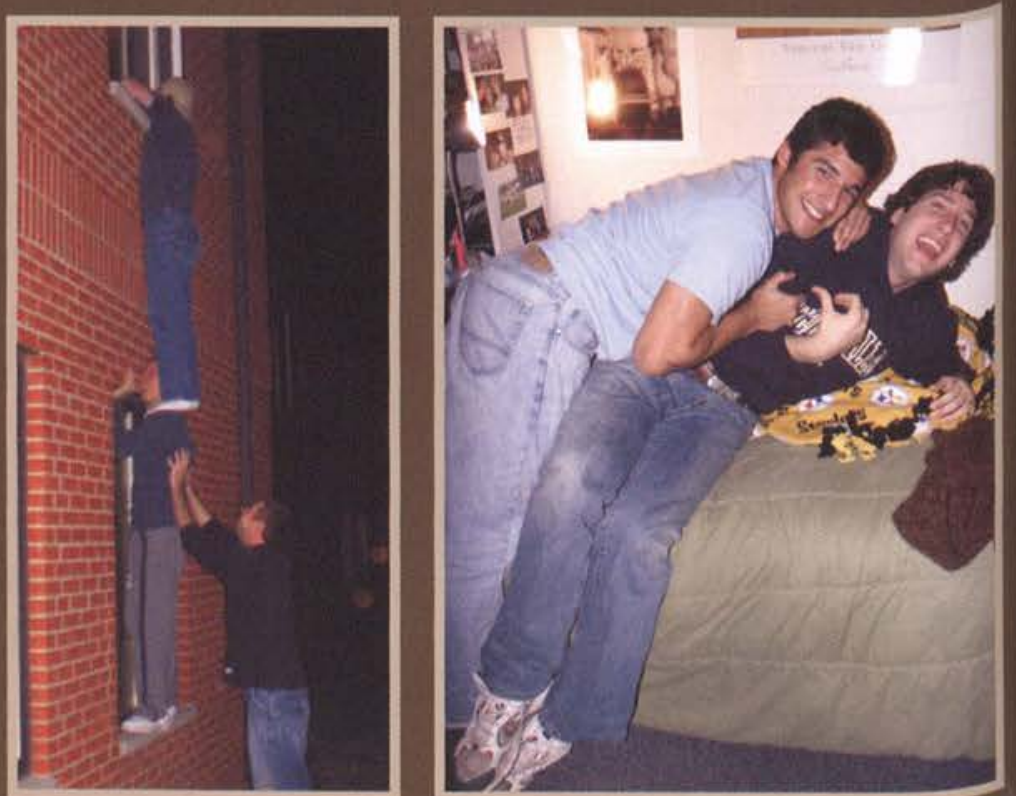


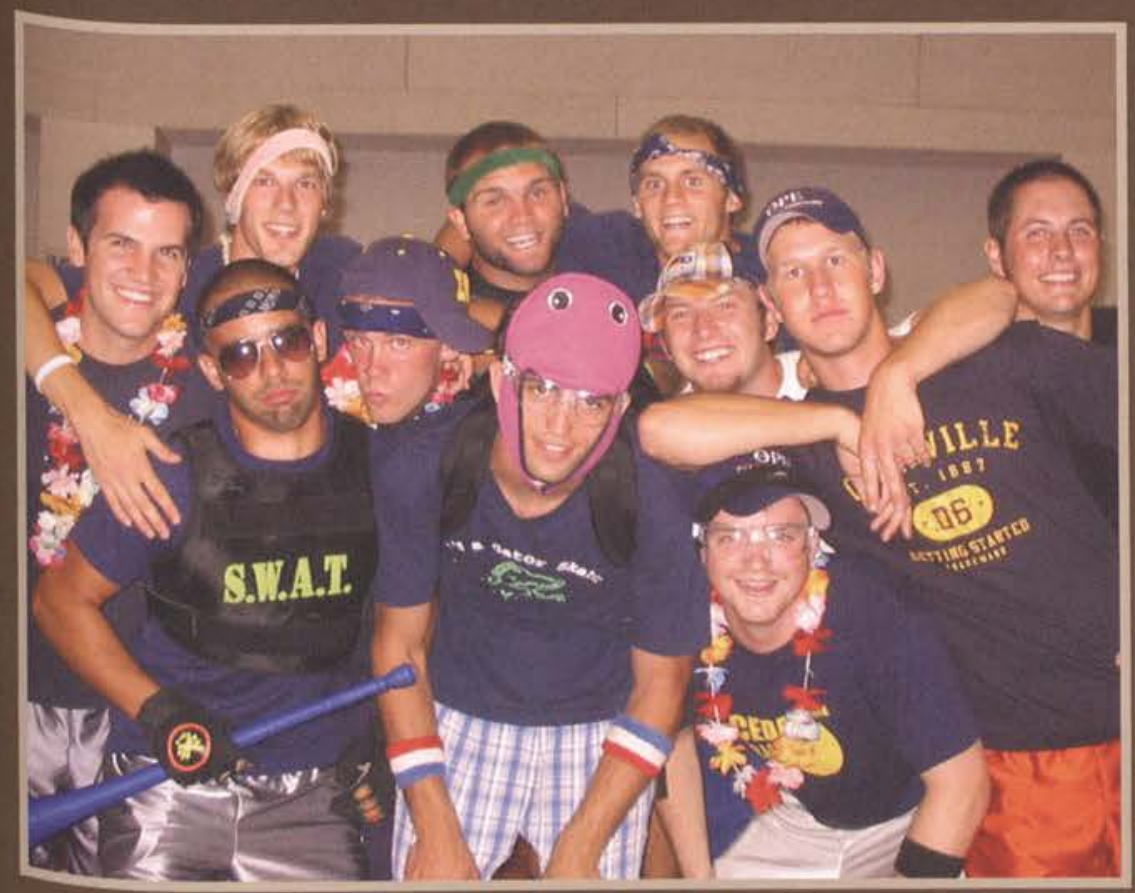

4 Hallmates James McClenahan, Dan Knudsen, Griffin Meyers, Adam Ploeg, Ben Volpe, Bobby Childs, Mark Hershey, Brian Alburger,

Anthony Rutrough, Dan Johnson, and Josh Hueni are enjoying their afternoon at the Beachball Volleyball event.

- The men of Lawlor and the Hill face off in their annual football game. Lawlor won again this year, leaving the score at Lawlor-4, Hill-1.

- Matt de Waal Malefyt and others from Rickard paint the rock after curfew. Matt said, "We later found out that our sister hall ratted us out and called Campus Safety."

Fuys often bond quickly with their hallmates, whether through trips to Springfield or hall dinners. However, some of the guys find unusual ways to bond. This St. Clair hall dresses up for a snowball fight with friends from Printy: Steven LiCicero, Kyle Hess, Chucky Klint, Ben Bertsche, Dalen Fultz, John Stevenson, Jay Coulter, and Bryant Beyers

- Shane Flannery makes fun of Eric Kempton's Earth Science rocks while taking a Lawlor Unit 38 picture. "We are a very close unit," said Chris Beals, also pictured.
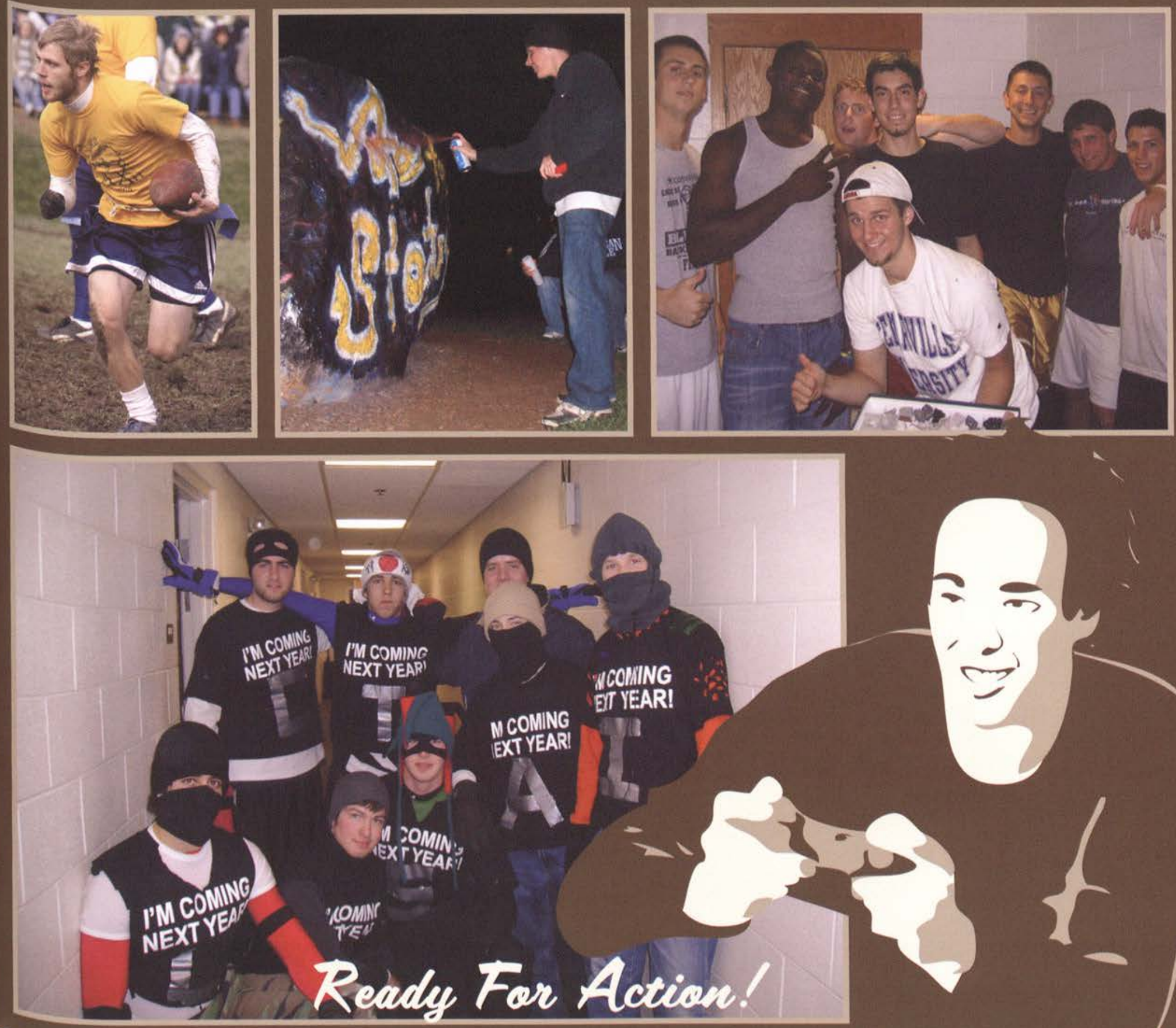

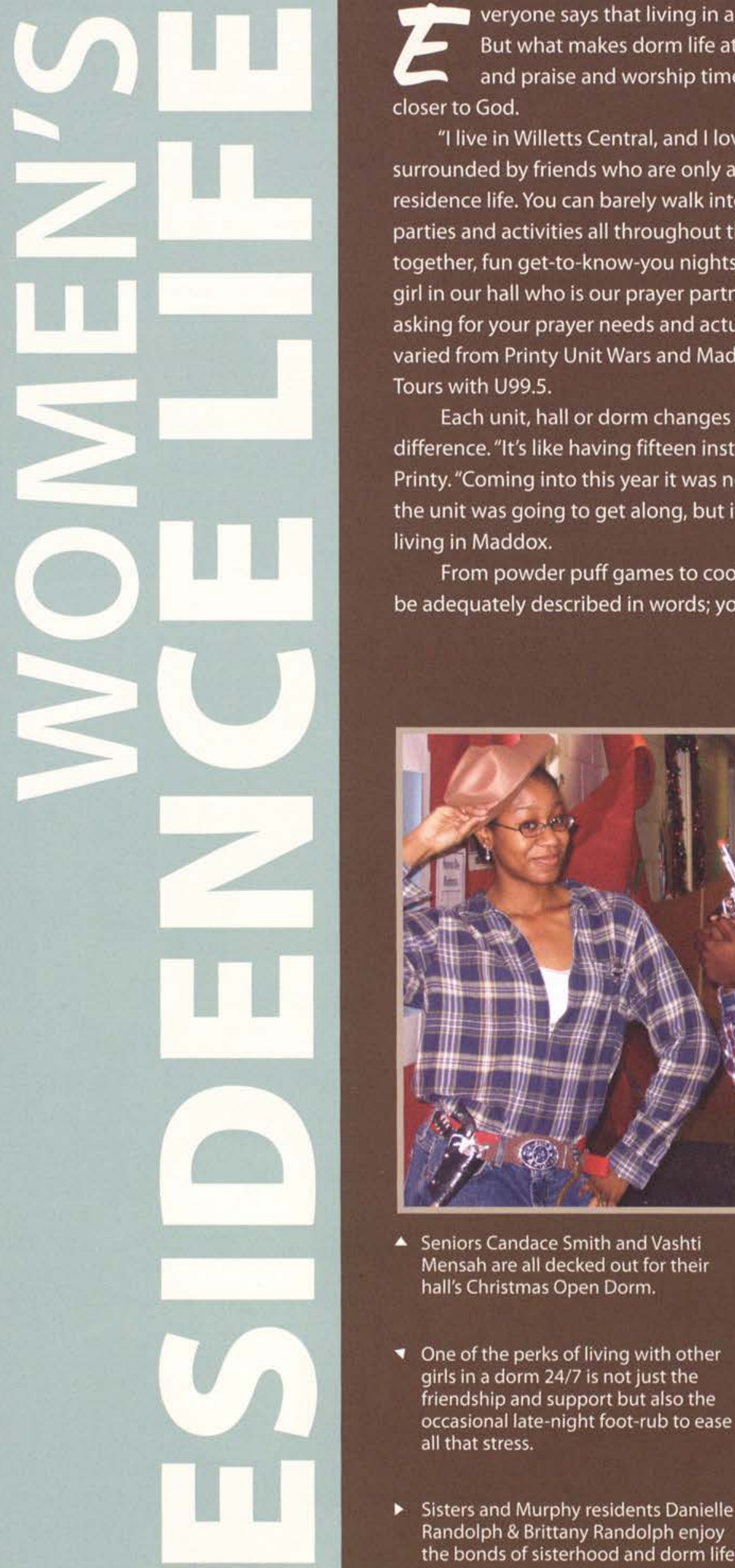

- Seniors Candace Smith and Vashti Mensah are all decked out for their hall's Christmas Open Dorm.

- One of the perks of living with other girls in a dorm 24/7 is not just the friendship and support but also the occasional late-night foot-rub to ease all that stress.

- Sisters and Murphy residents Danielle Randolph \& Brittany Randolph enjoy the bonds of sisterhood and dorm life.

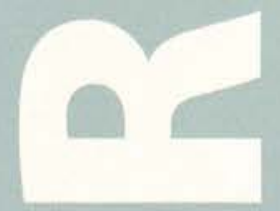

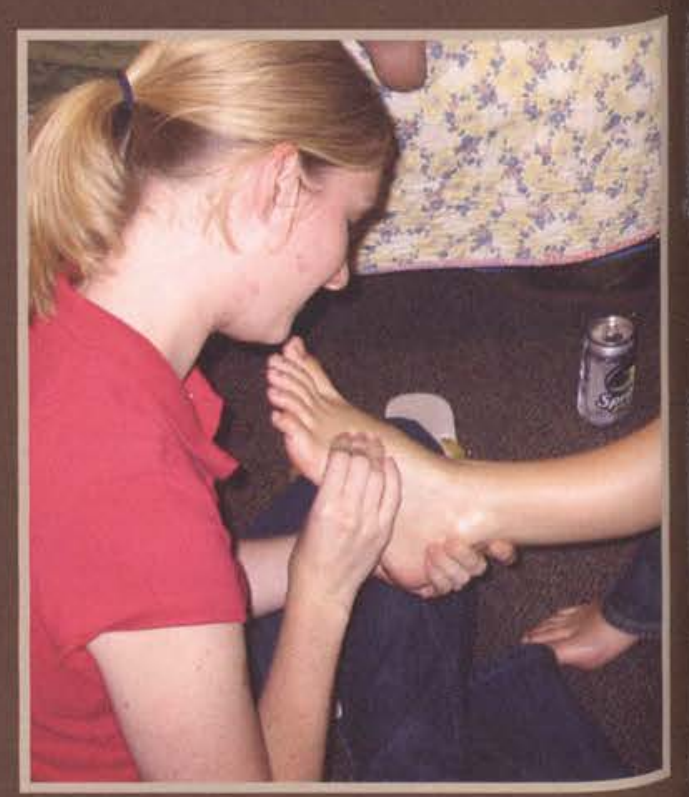

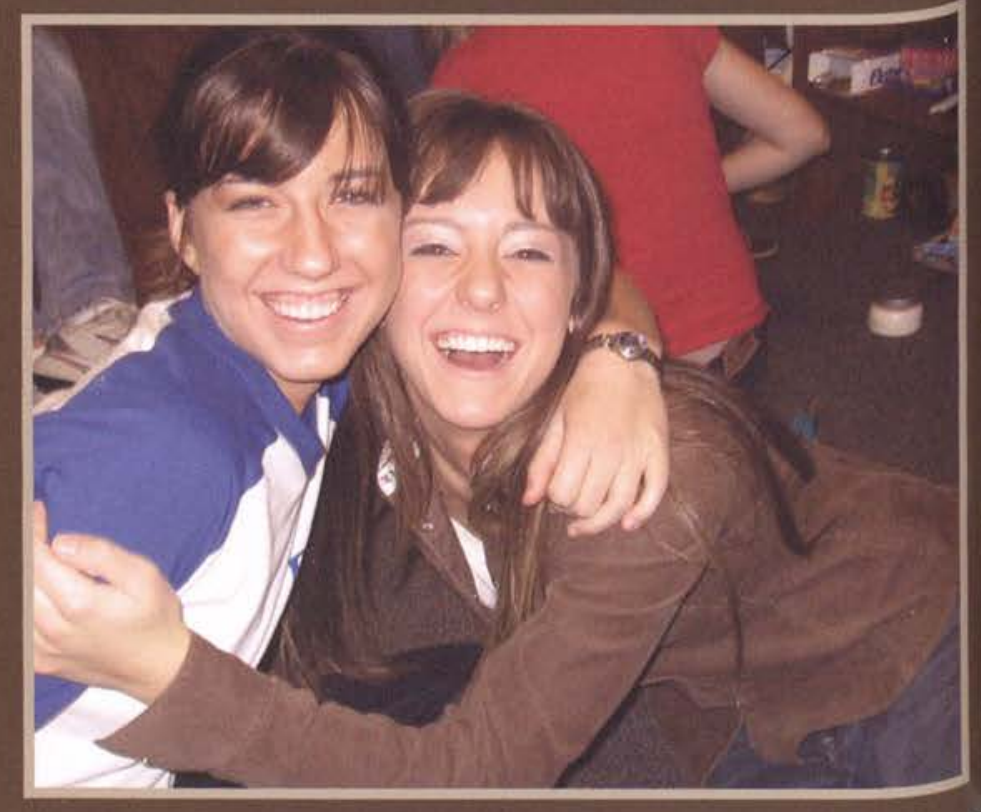




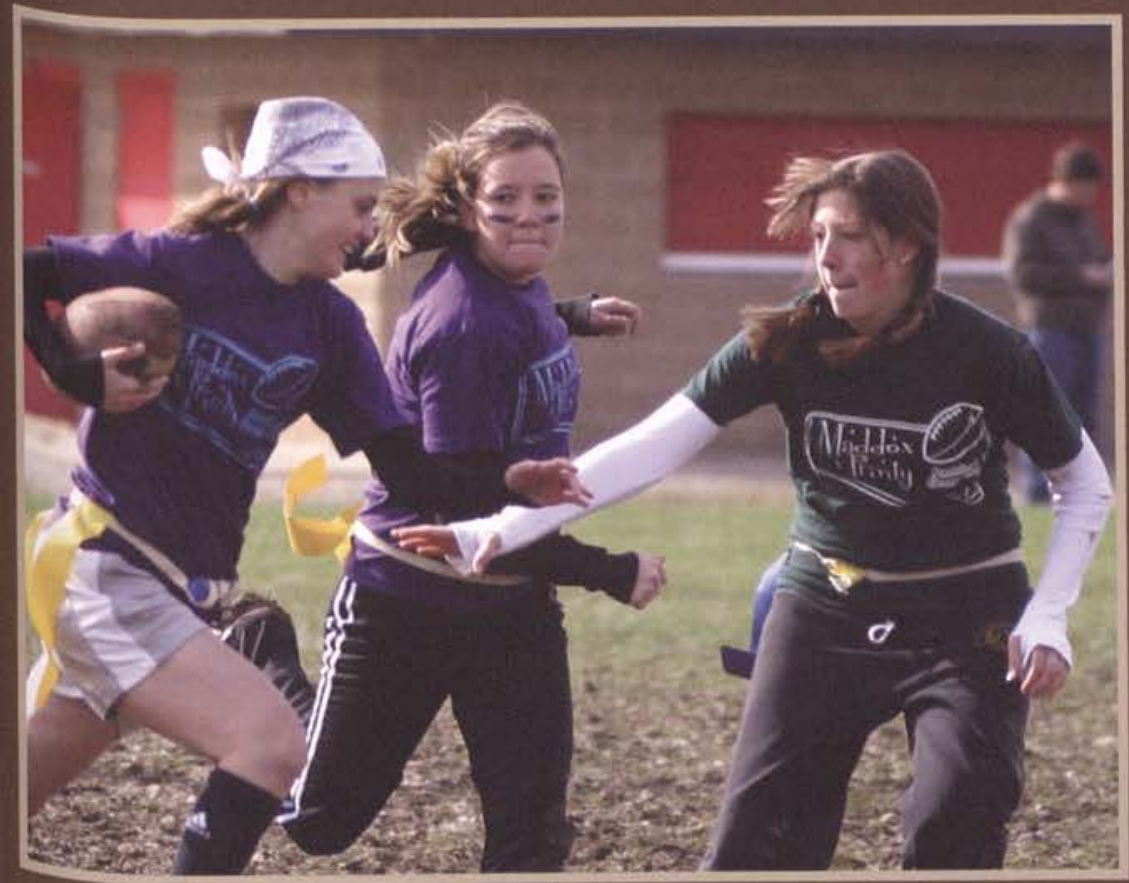

4. Freshman Jessica Quaid takes on the Maddox girls in the annual Printy/ Maddox Powder Puff football game.

- Melody Miller decides to take a power nap before joining the party again - because Faith girls love to party hardy!

4 Over in McKinney, Becky Erwin turns dorm life upside down as the life of the party.

7. McKinney girls Sara Villanyi, Katie Biehl, Julie Moran, and Ahna Buckwalter contemplate their exciting weekend plans while taking time from their busy lives to pose for the camera.

- The girls of Printy Units 26 and 28 , Chelsea Belleman, Kelly Wise and Rachel Gilin take time for a smile during a long night of scuffling for shoes, random food scarfing, ring pops, and syrup at the annual Printy Wars, where units 18 and 20 emerged victorious.
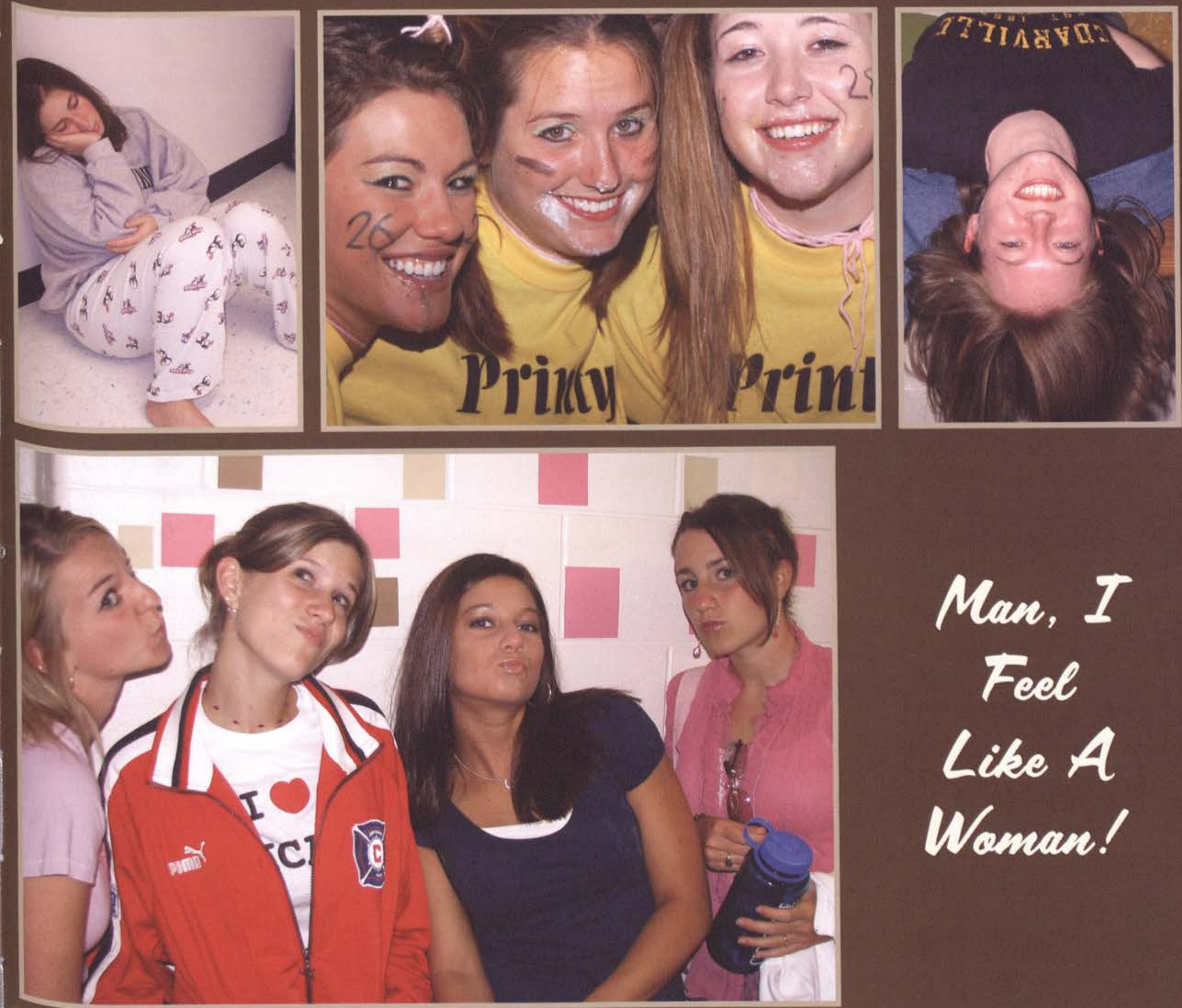


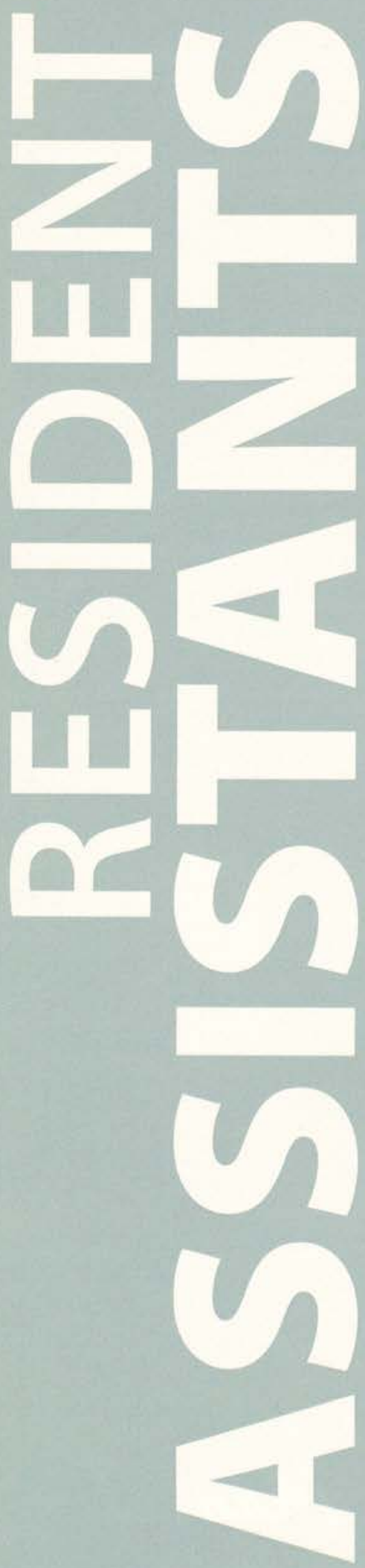

hy do Resident Assistants choose their job? Willetts' junior Jada Holloway said it was "the
people you can impact and share the experience with." Printy's senior Michelle Evington
countered, "The chance to build into freshman girls in a unique way." Lawlor's junior Justin McCoy added his two cents by saying he was an RA because of "The relationships I've gained with the guys in my unit." Lawlor's junior Daniel Lagan finished out with saying that he liked "the opportunity I have on a daily basis to positively impact people's lives." Money was never a reason; all-in checks, buzzer duty, meetings, confrontations, and emergency room trips make the job tedious enough to sift out any selfish reason for being an RA. "The hardest part of being an RA is dealing with misconceptions that surround us," Lawlor's junior David Sizemore admited. A little known fact: confrontation lies at the bottom of every RA's agenda, yet it is a required part of the lives those RAs lead. Lawlor's senior Nathanael Davis shared his heart: "The hardest part is watching a guy I care about consciously fail, knowing the consequences he puts upon himself...having helped them once, yet no longer able to help them again." The entire week before residents come to campus, RAs and RDs train, receive information about the coming year, and learn the importance of working together as a team. "We have the best time of getting to know each other," Brock's junior Rod Bradley stated. "During the school year I do not get to see these guys a lot, so it is fun building the camaraderie before the school year starts." RAs overwhelmingly agreed the Resident Director was a reason they chose their dorms. Printy and Lawlor RAs also chose the excitement of loving and mentoring freshmen. Through one of the hardest jobs on campus, RAs learn responsibility, love, selflessness, respect, and the importance of relying on God.

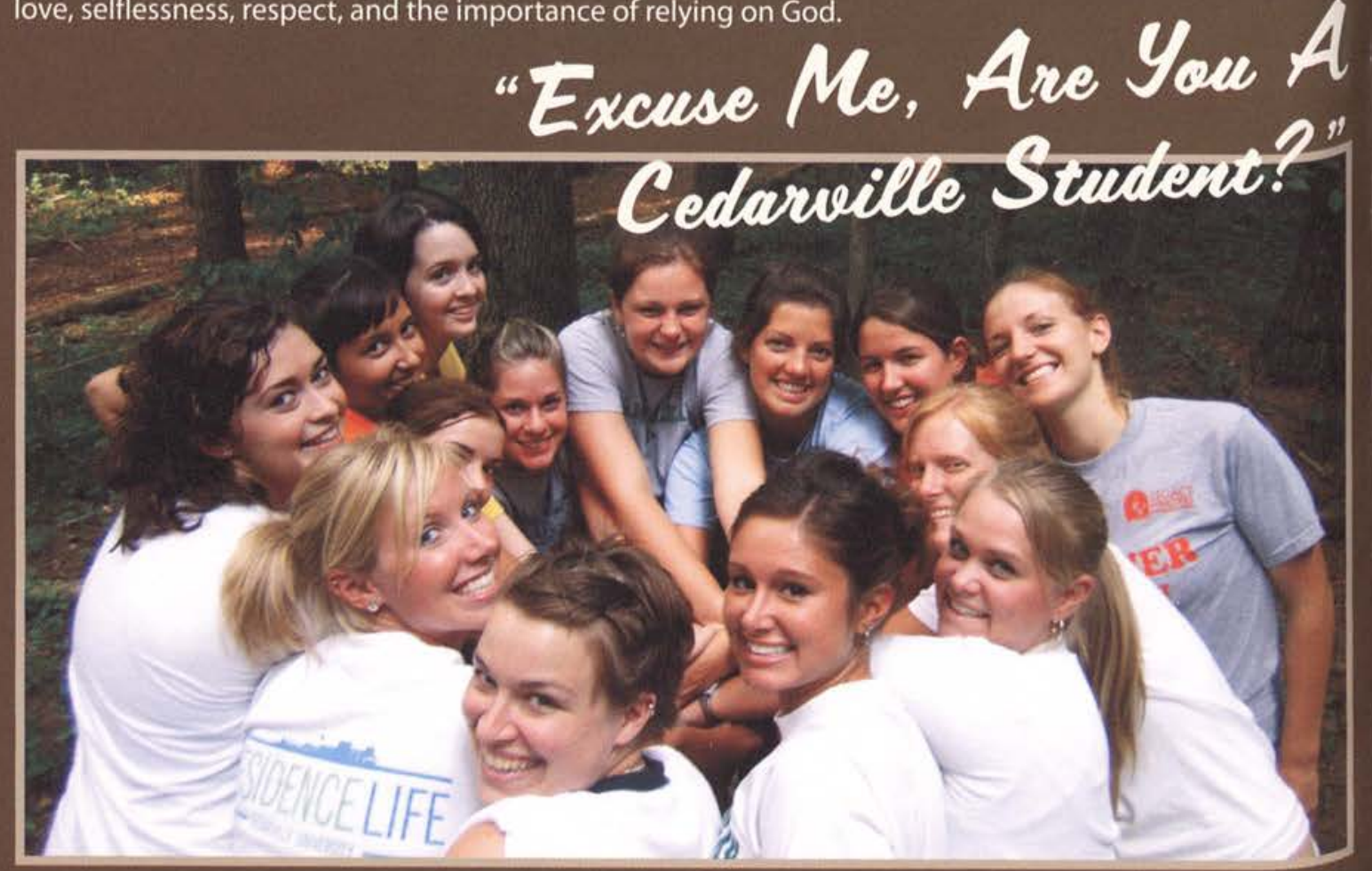

- Willetts RAs pull together for a team-building activity during RA Training. Throughout the year, they draw strength from the lessons, and Resident Director Rachel Rodriguez believes, "My RAs learned to be a great team, and that made this year so fun working together!"

- Left to right, St. Clair RAs Tim Neetz and John Hummitzsch, and McChesney RA Justin Dodson practice"looking tough and showing their gangsta faces" during RA Training week. "Don't mess with these RAs," Neezt warns.

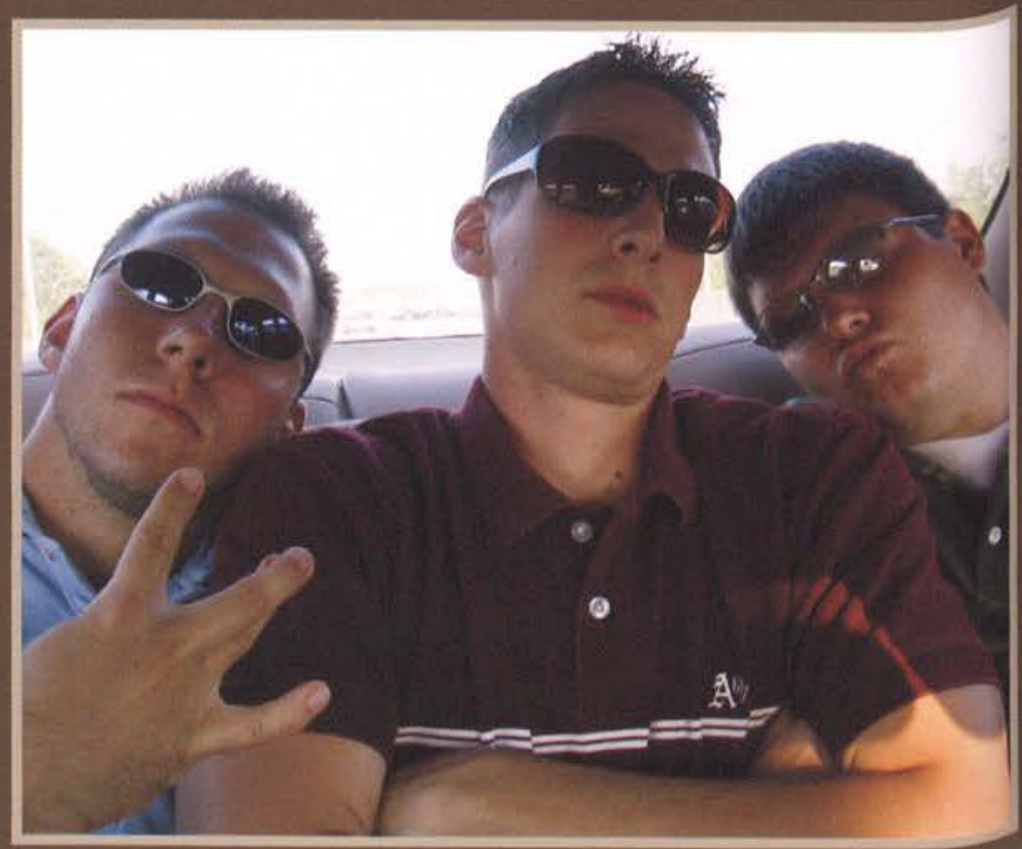




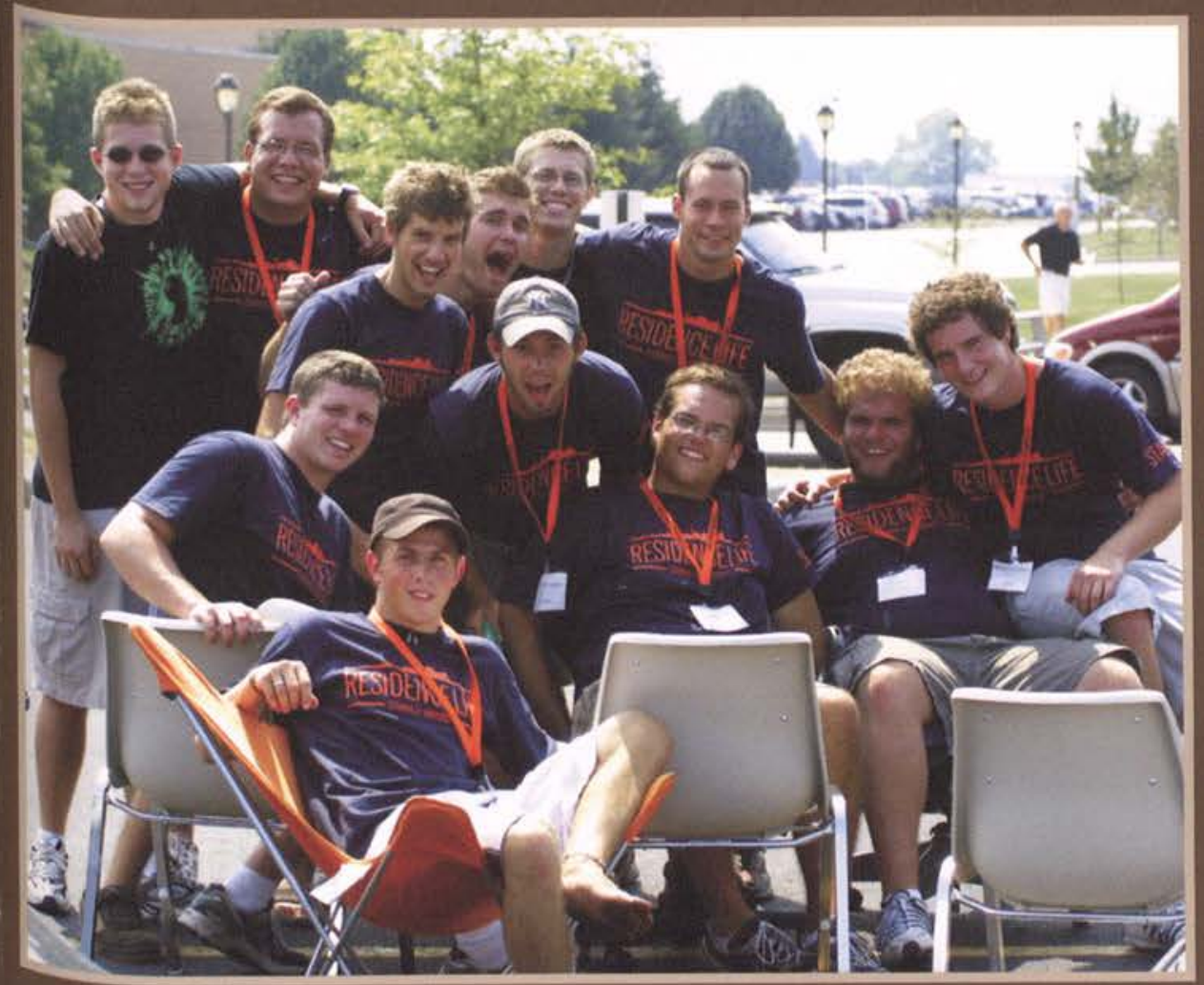

4. The RAs of Lawlor Hall enjoy each other's company as they anticipate students arriving during Getting Started weekend. RD Brian Burns appreciates his assistants and has cherished his last year. "I have loved being an RD here in Lawlor and serving with the best RAs on campus."

- Senior Joe Poelzer recalls, "RA training set the tone that carried over into our halls to make a lasting impression. John Hummitzsch and I have been RAs in St. Clair for the past two years. I consider him a great friend."

- The RAs of Murphy - Rachel Sturgis, Kimberly Rowlands, Ashley Hunter, Beth Hays, Michal Friend, and Anna Smith - relax at Bravo for an end-of-the-year bash.

4 Jessica Dodson loves getting her Printy RAs involved in fun and creative activities. "We cheered our Printy powderpuff team to their second consecutive victory. GO GREEN!"

4 Senior Joel Green, St. Clair RA, appreciates the time he is able to spend with both St. Clair and McChesney RAs under Bob Lutz' leadership: "These guys are crazy, but serving with them as an RA has been the most rewarding thing that I have done here at Cedarville."

- "Printy RAs never cease to surprise me with their creativity. Sarah Behn and Kristen Doles arrived at our Halloween RA meeting dressed as 'trees,"' describes their RD Jessica Dodson.
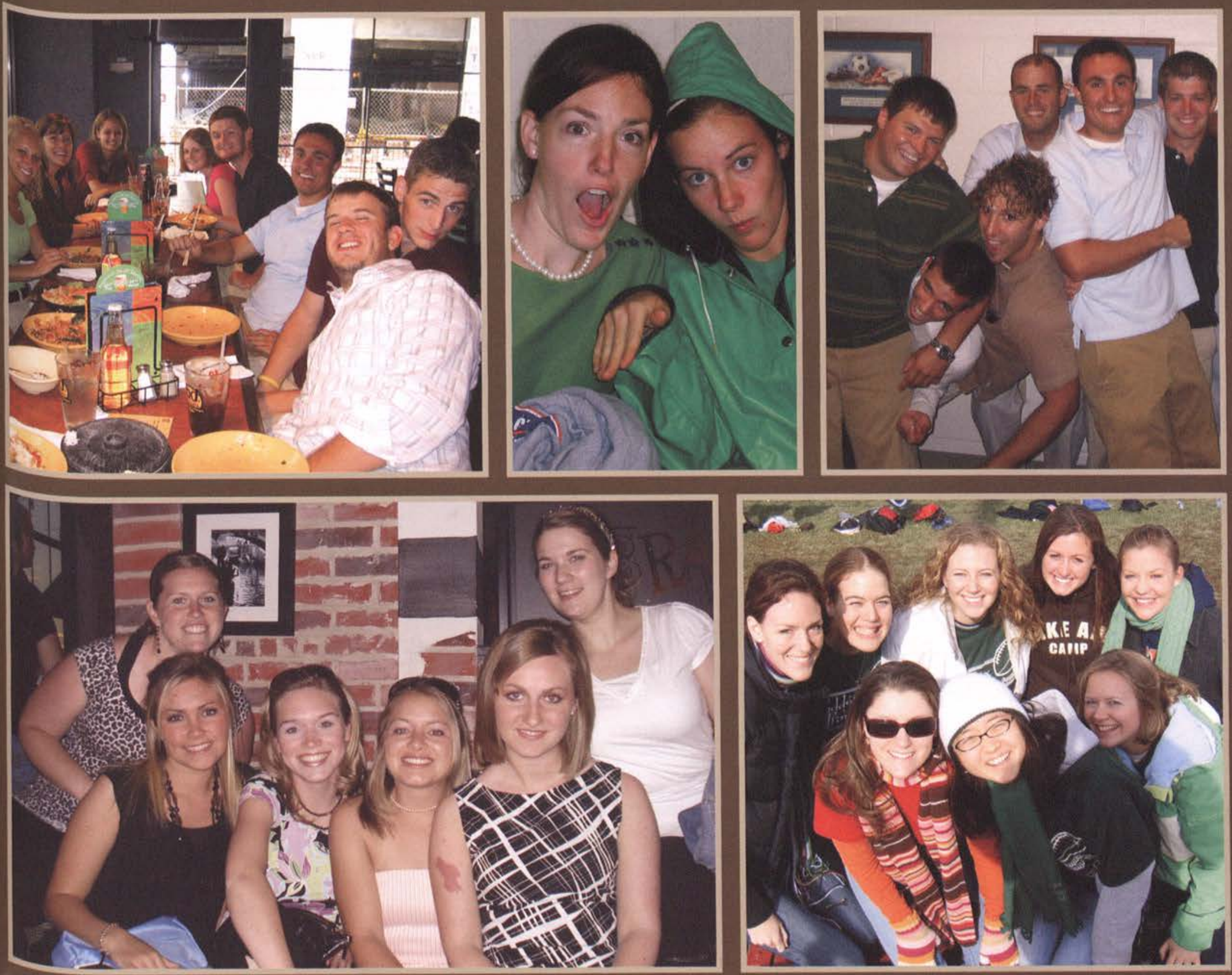


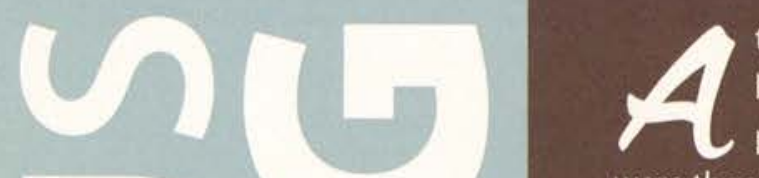

t 1:00 A.M. on Saturday nights, the students would be compelled to head back to their dorm to be tucked in by their beloved RAs., but a few select and often envied students maintained the privilege to stay out past curfew and clean their own bathrooms. These lucky men and women were those who arranged to live off campus during the school year.

It was generally known around Cedarville that getting the administration to grant permission to live off campus was not always an easy task. Many students who chose to step away from dorm life did so for financial reasons--though the appeal of a little more freedom didn't deter students either. Those who were granted permission got a little taste of what life might be like after graduation, only with friends and shared costs to help take away the shock. "I loved living off campus," senior Leah Jones said. "It definitely gave me more freedom and a more realistic life than living in a dorm with RAs and RDs constantly making sure I followed the rules."

Off-campus living was definitely an appealing alternative for many. Cooking their own meals, not worrying about whether a particular night was "open-dorm" night, and enjoying the fact that there were fewer school loans to be taken out for the year were all perks to leaving dorm life behind. However, off campus students admitted that with their privilege came considerable added responsibility. Senior Heid Charlton noted, "The hardest part about living off campus was definitely time management. Some things you don't think about when you're thinking of living off campus... it was a bit of an adjustment learning how to balance cleaning/cooking/homework and a social life!" There may have been some rocky times, but for most off-campus students, the experience was well worth the extra cleaning and cooking duty.
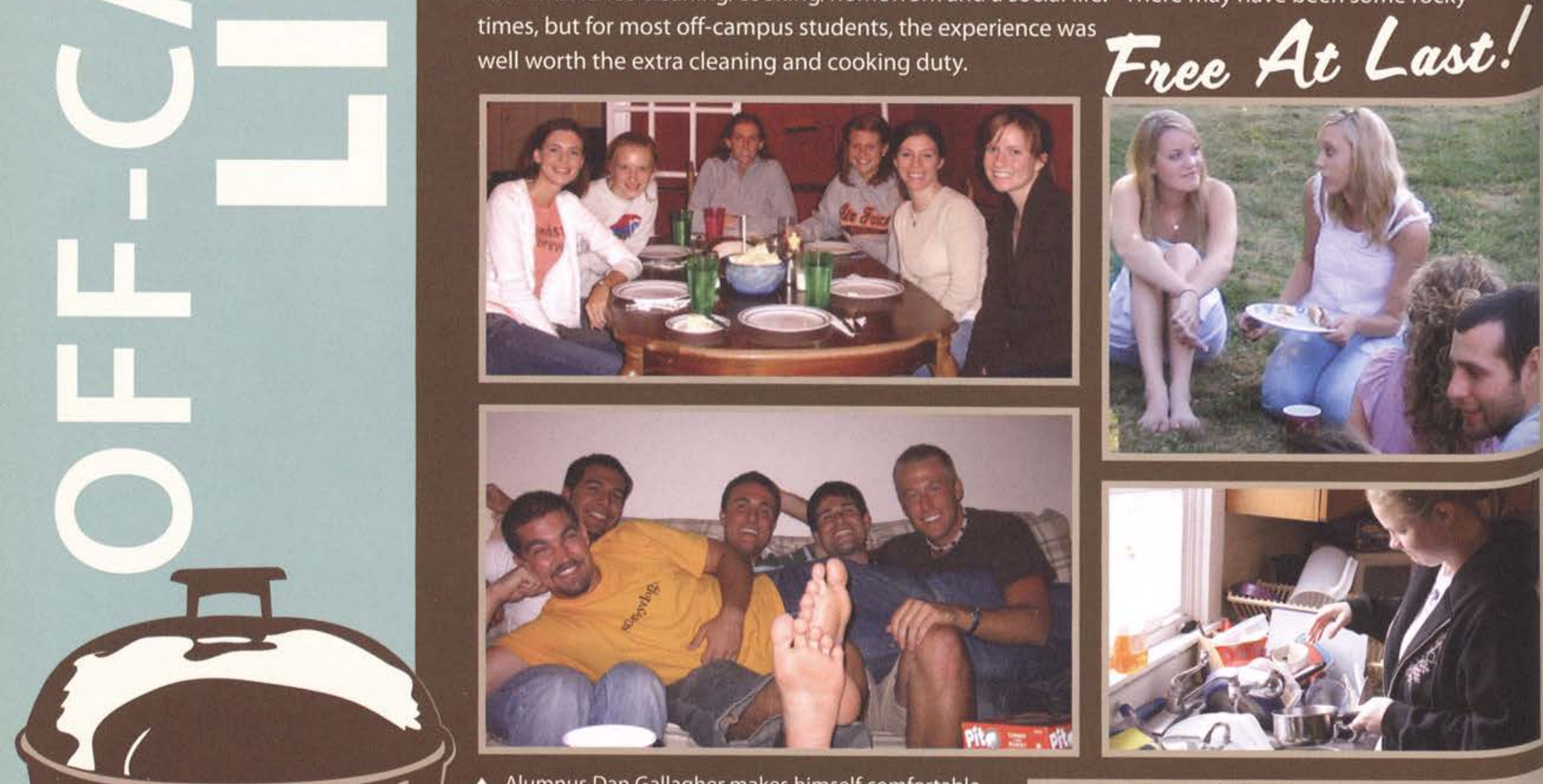

- Alumnus Dan Gallagher makes himself comfortable on top of seniors Michael Pritts, Joel Green, Nathan Walter, and Nate Weeber.

A Melissa Brorein, Jillian Losee, Karen Rieck, Jane Knable, Kim Ehlers, and Abby Ellis get together for a Thanksgiving dinner.

- Sophomores Jessica Bashore and Lauren Brown and senior Ricky Normandeau enjoy their time at a cookout in the beginning of the year at Andrew Wong, Colin Luther, and Dan Tello's house.

- Senior Sarah Andersen washes a dish for lunch. Senior Amy Masterson commented, "It's the not-soglamourous side of off-campus living."

- Sara Vilanyi, Ahna Buckwalter, Katie Biehl, and Julie Moran treat each other to backrubs at Brittany Herbst's house while "watching" the Ohio State/ Michigan game. Sara comments, "All I know is that OSU won:

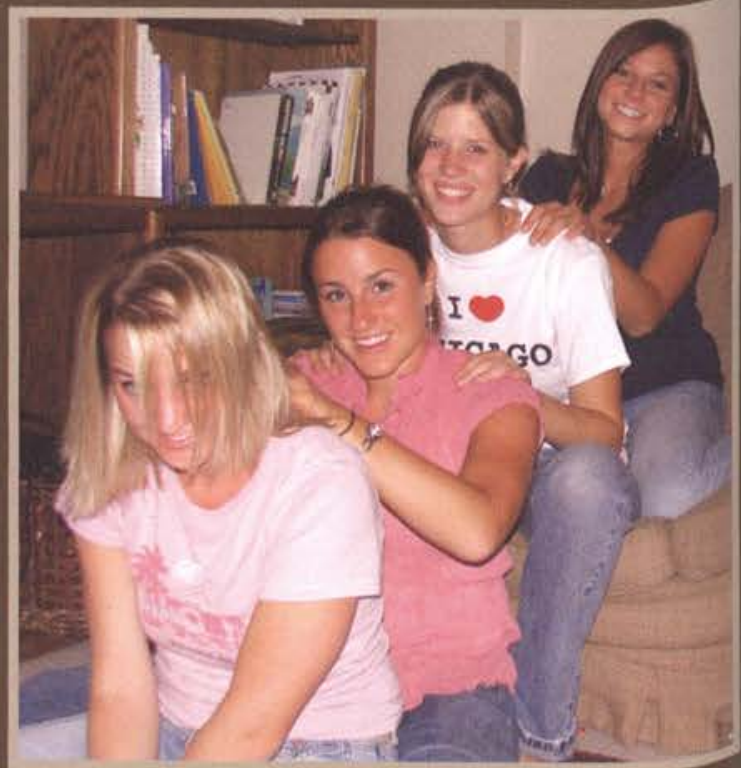




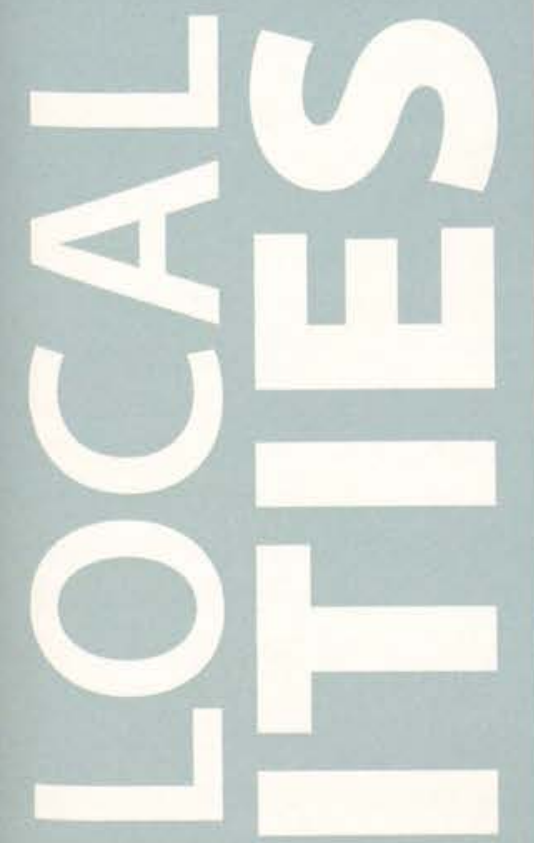

he first memory many have of their time at Cedarville is the road here. From cities and towns across the country, everyone makes the initial drive down Route 72 past cornfields and

soybeans, with a loaded trunk and far more gadgets than they'll ever have time to use once that

\section{first 8 a.m. class hits.}

Small towns have many charms. For college towns like Cedarville, these charms are town and college favorites. Music and coffee at Beans' $n$ ' Cream, pizza parties at Colonial's, daily six-inch specials from Subway, and ice cream cravings satisfied by Mom \& Dad's -- the town has a lot of character to add to the Cedarville experience. This year, Beans ' $n$ ' Cream started "Acoustic Nights," featuring local talent and serving as a respite from the ever-present need to study.

Further out, students enjoyed the shade of rocks, trees, and wildlife at the Cedarville Gorge and John Bryan State Park. Hiking to the Fort Ancient Indian mounds, disc golfing at Wittenburg and Antioch, perusing the shops down Main Street in Yellow Springs, and enjoying the relaxing nature of Young's Dairy are special activities everyone loves to enjoy - even if it's during that first awkward pick-a-date. Junior Erin Cahilly jokingly sums up her trips to Young's: "pestering cows, pretending to golf, plenty of fun, permanent memories."

For movies and fine dining, one usually heads to the cluster of theaters thirty minutes or so from campus. For senior Jessica Annable, "A movie in Beavercreek with some Target snacks snuck in? I don't think a Friday could get any better."

As is true with all campuses, no matter where students go after they turn down Main Street, the same road that leads in, leads out, and many take it, just as long as that means getting back by curfew.
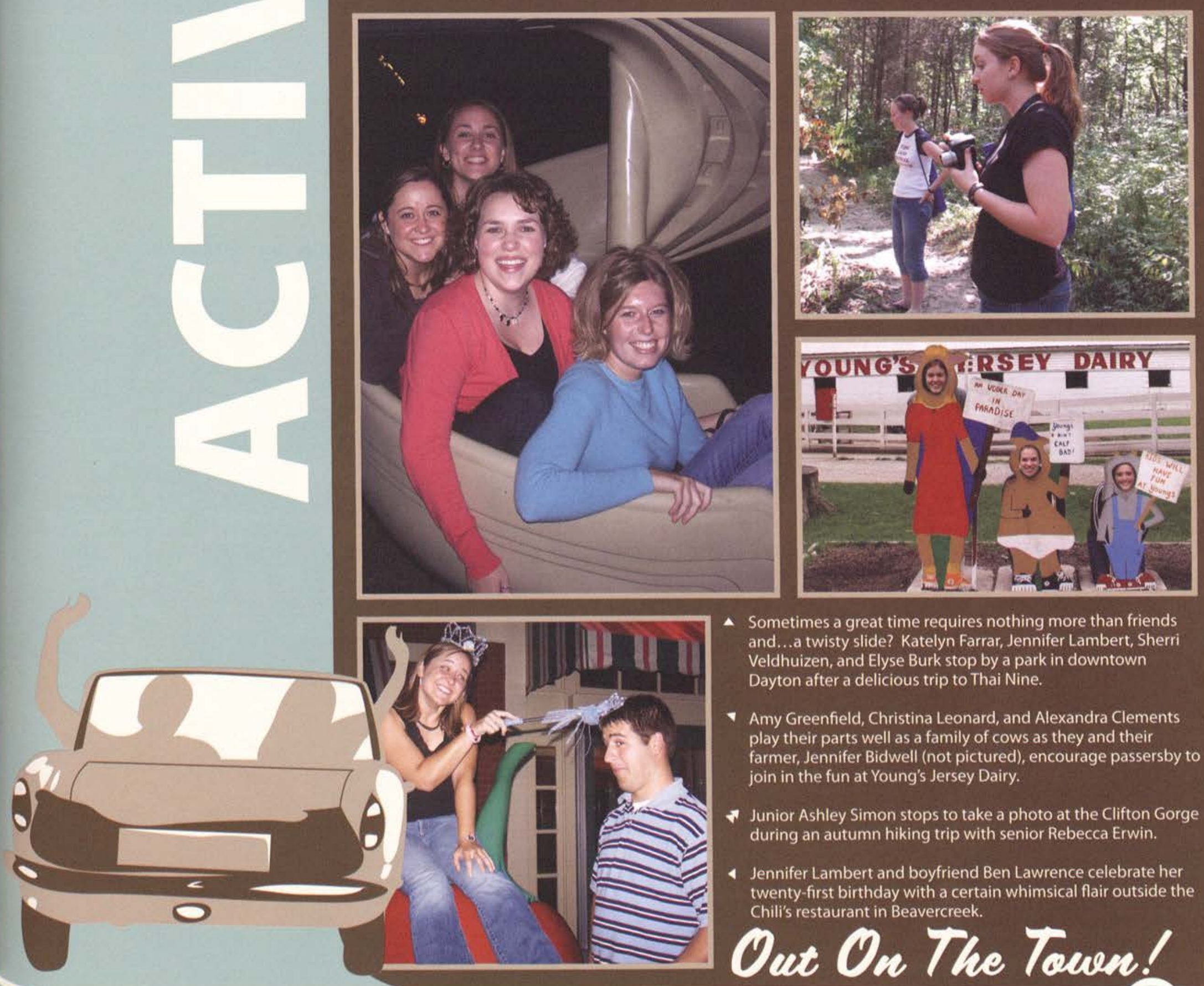

- Sometimes a great time requires nothing more than friends and.... a twisty slide? Katelyn Farrar, Jennifer Lambert, Sherri Veldhuizen, and Elyse Burk stop by a park in downtown Dayton after a delicious trip to Thai Nine.

- Amy Greenfield, Christina Leonard, and Alexandra Clements play their parts well as a family of cows as they and their farmer, Jennifer Bidwell (not pictured), encourage passersby to join in the fun at Young's Jersey Dairy.

7 Junior Ashley Simon stops to take a photo at the Clifton Gorge during an autumn hiking trip with senior Rebecca Erwin.

4 Jennifer Lambert and boyfriend Ben Lawrence celebrate her twenty-first birthday with a certain whimsical flair outside the Chili's restaurant in Beavercreek.

\section{Out On The Towen!}




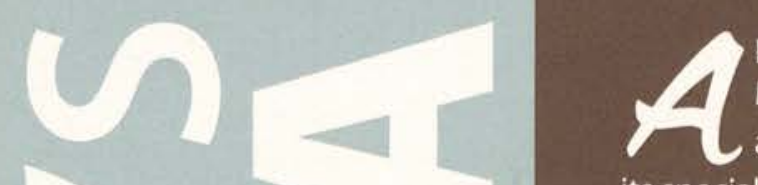

$A$ popular sell among students this past year was the re-release of t-shirts saying "Chuck's - Ain't Mama's Cookin."' Depending on your regular cuisine back home, this was either a good thing or a bad thing. But whether the experience at Chuck's was a blessing or a curse, one couldn't deny its special place in Cedarville family life.

The cafeteria director, Chuck McKinney, did what he could to make dining at CU a pleasant experience. Chuck made himself available and was often seen surveying the dining area. Students were also free to place comments in a suggestion box, which Chuck then replied to and posted. Also, this year the SGA Campus Culinary Committee was formed to help give the students a voice about the food in the cafeteria. Sophomore member of the committee Lydia Smith commented, "So many of the students here liked to critique the food, and a great way to do that was to join the Culinary Committee to voice opinions to the man in charge." This committee was responsible for many of the theme nights held throughout the year and even helped add to the choices of food made available for students. Not only did students enjoy the annual special dinners for Thanksgiving and Christmas, but there were also events like a Tailgate dinner and Valentine's Day meal. The Culinary Committee also held Fast for Food, a fund raiser for a local food pantry, raising awareness for the poverty surrounding Cedarville.

Obviously, school cafeterias are not known for their popularity among students, but Chuck's has a lot to offer, and then again, cereal is always an alternative. "I appreciated the options," junior Emily Broersma stated. "If something didn't look good, there was always something else you could choose from. Having worked in Chuck's, I understood it took a lot of work, and they did a good job."
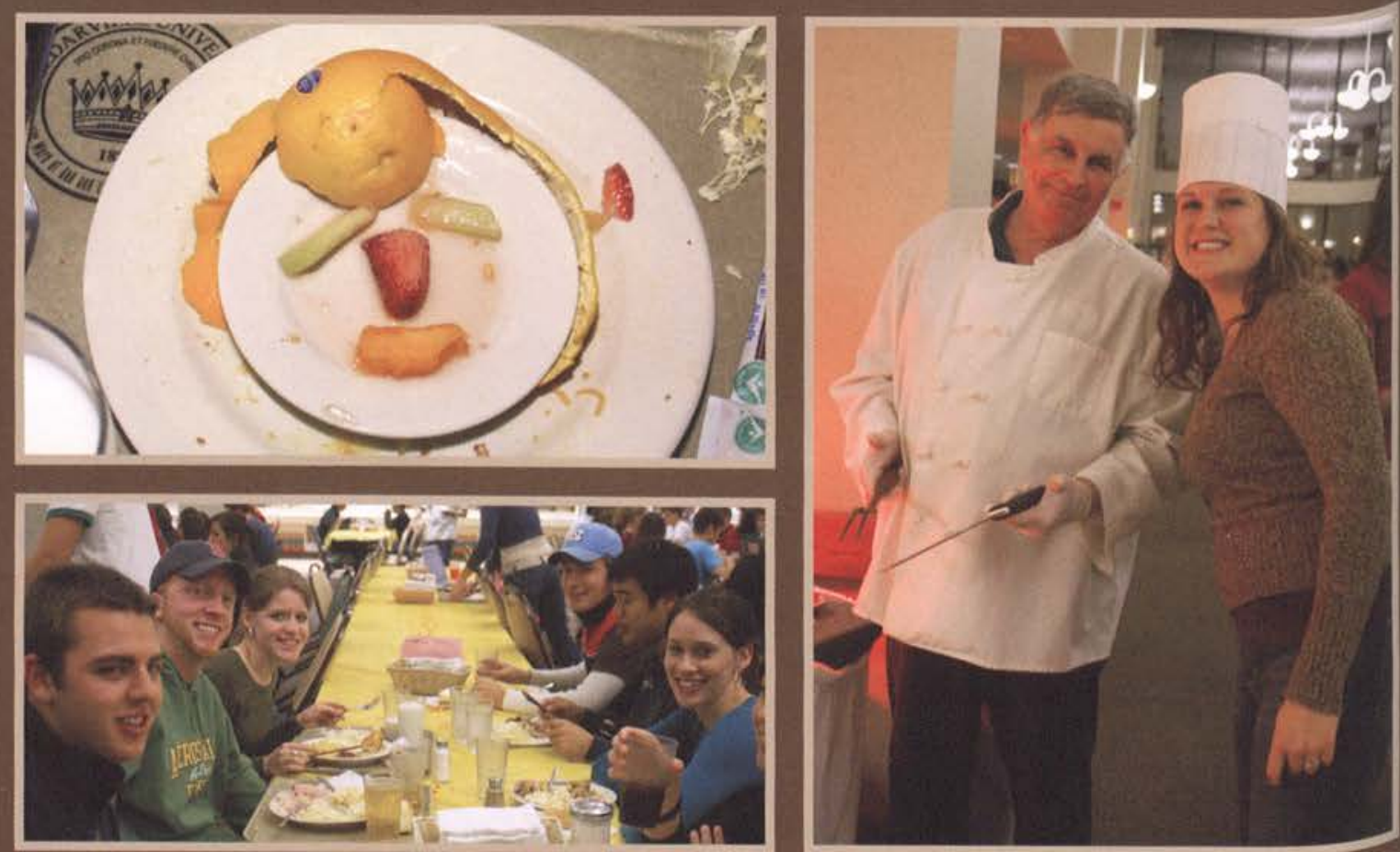

- Andrew Reep, Caleb Fletcher, Kathleen Picard (L) and Jen Gibson, Alathea Young, Neil Patnaude, and Peter Gohdes (R) enjoy the fine dining at Chuck's.

¿ Perhaps in an attempt to offer encouragement to student workers in Chuck's dish pit, a fruity image of a face is created.

- Senior Kelley Kinnard poses with a Chuck's worker carving the ham and turkey during the annual Chuck's Christmas dinner.

- Sophomore Daniel Zimmerman and freshman Nate Schirmer help themselves to the ice cream. Zimmerman noted, "The reason we had the entire carton is simple: you've got to take what you can these days".

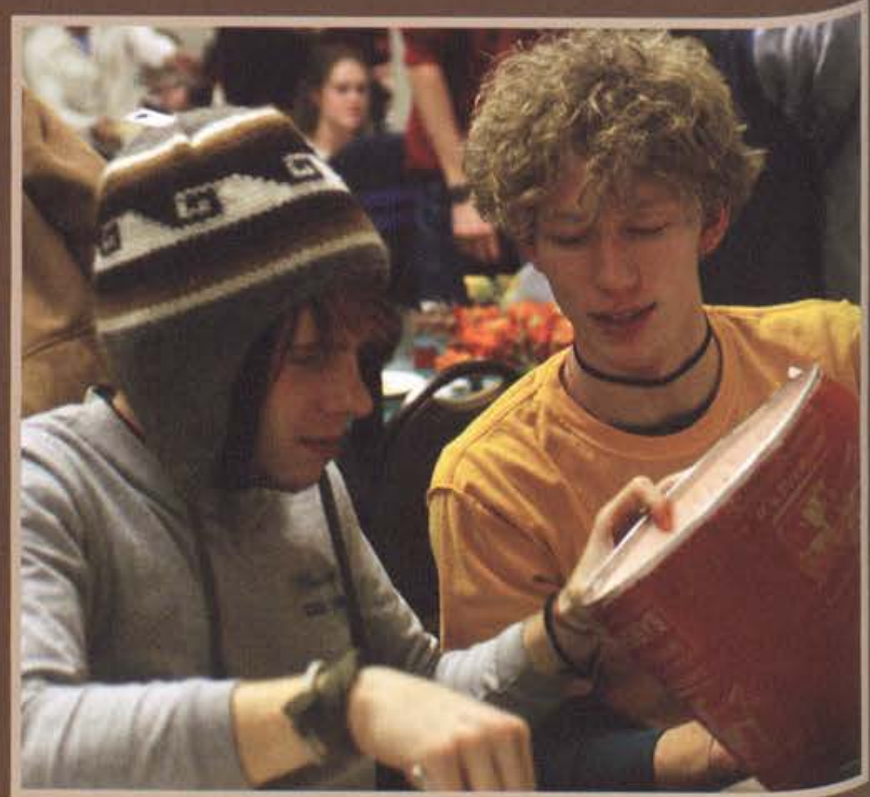



nectar of coffee before heading off to continue their busy schedule. One can grab a Stinger, the Hive's signature sandwich, and finish it off with the Killer Bee, Vecinos' caffeine kick, to keep them buzzing through the day. For senior Krissten Marr, the best part of working at the Hive is "meeting new people and having the best boss ever - we love you Dee!" Senior Hive workers Joel Wallen and Brendon Bayley enjoyed having fun while working hard with great people in a "unique atmosphere." What better way to get to know someone than over a meal and coffee-or over the coffee counter? Sophomore Josh Stephens enjoyed guessing the drinks that were ordered by repeat customers, "All to make myself a better server." Vecinos was always linked to Starbucks, but the workers always responded with "Starwho?" "The difference between Vecinos and Starbucks is the reason we exist," said senior Mindy Braun. "Vecinos exists to help support missions through Alliance World Coffees," which supports Biblical internships. Junior Teresa Keller described both the best and hardest part of working at Vecinos and the Hive in one phrase: "Remembering that I am to serve everyone every second of every day."

Every worker strove for excellence and making the students and faculty appreciate their meal or drink. One couldn't miss senior Bryan Amerine's signature phrase that he would call out from behind the Vecinos counter: "If you're not happy with your drink, I'm not happy." Cedarville proudly offered a comfy area to study and catch up with friends while listening to classic music, with a ready meal and a satisfying drink just a few steps away.
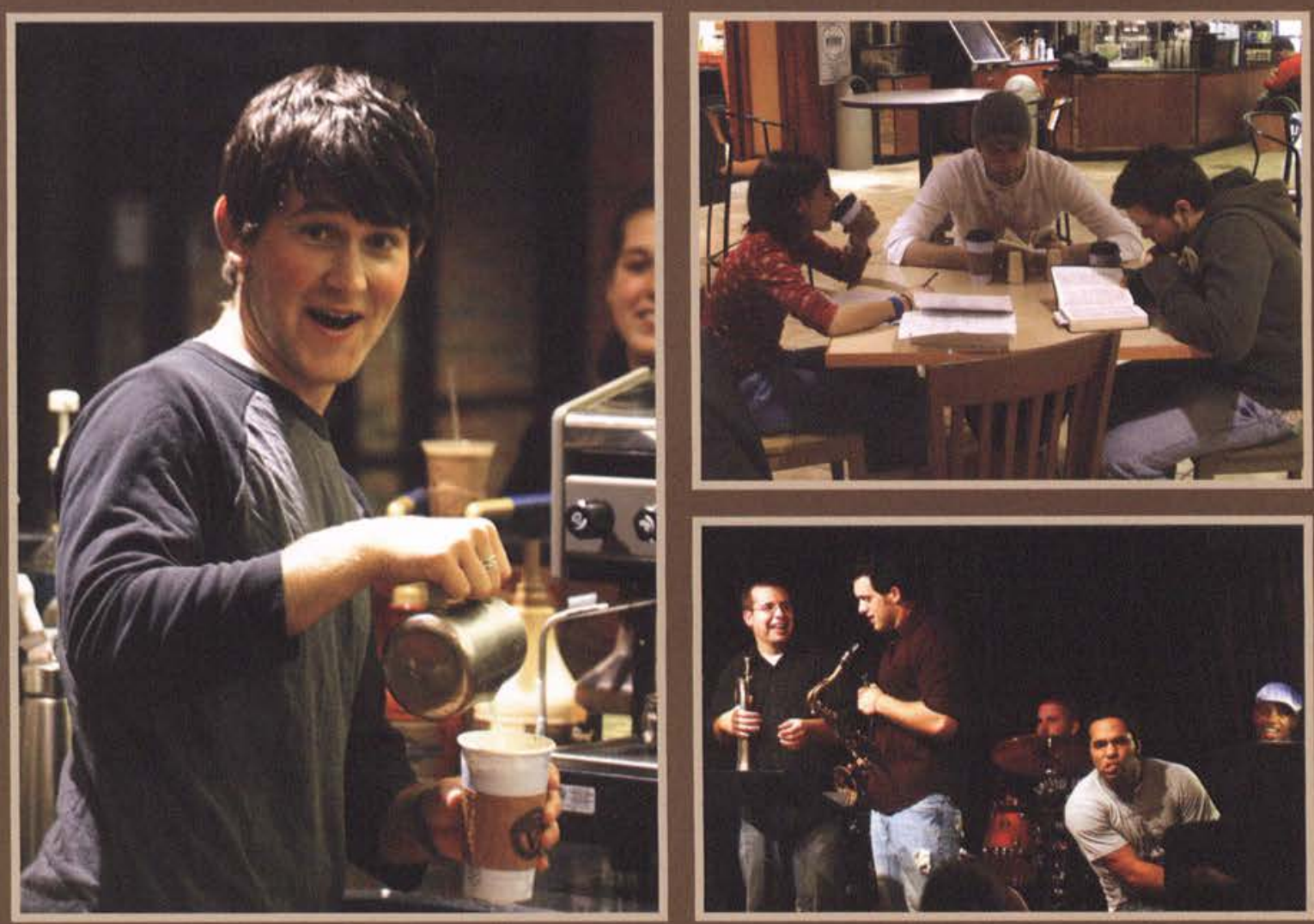
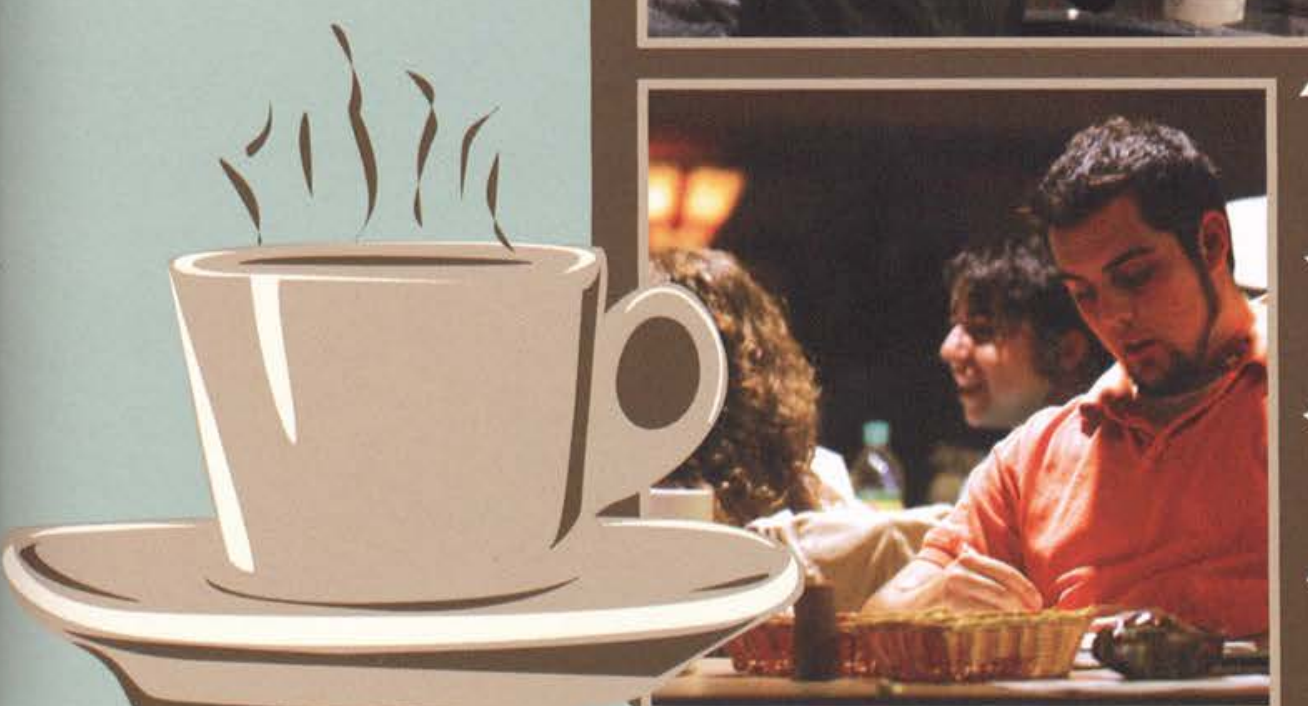

- Nate Keller makes coffee with a smile, making sure that Vecinos keeps up their reputation of service with excellence. Keller says, "Pouring drinks is child's play for [us] Vecino's workers."

- The Molby-Martin Quintet (Micah Martin, Nate Molby, Chad Winder, Robert Ried, and Stephen Sylvester) jam in the Hive, providing enjoyable music with a touch of humor for Infusion.

* Ben Kochanowski (center) hangs out with Rachel Torres and Jared Colon. Kochanowski says, "When it comes to sitting down to study, read, or just hang out, the Hive and surrounding areas offer a plethora of choices to satisfy my needs." Jordan Thayer kicks back and studies away, oblivious to the girls behind him but tapping his foot to the music of Acoustic Fire. 

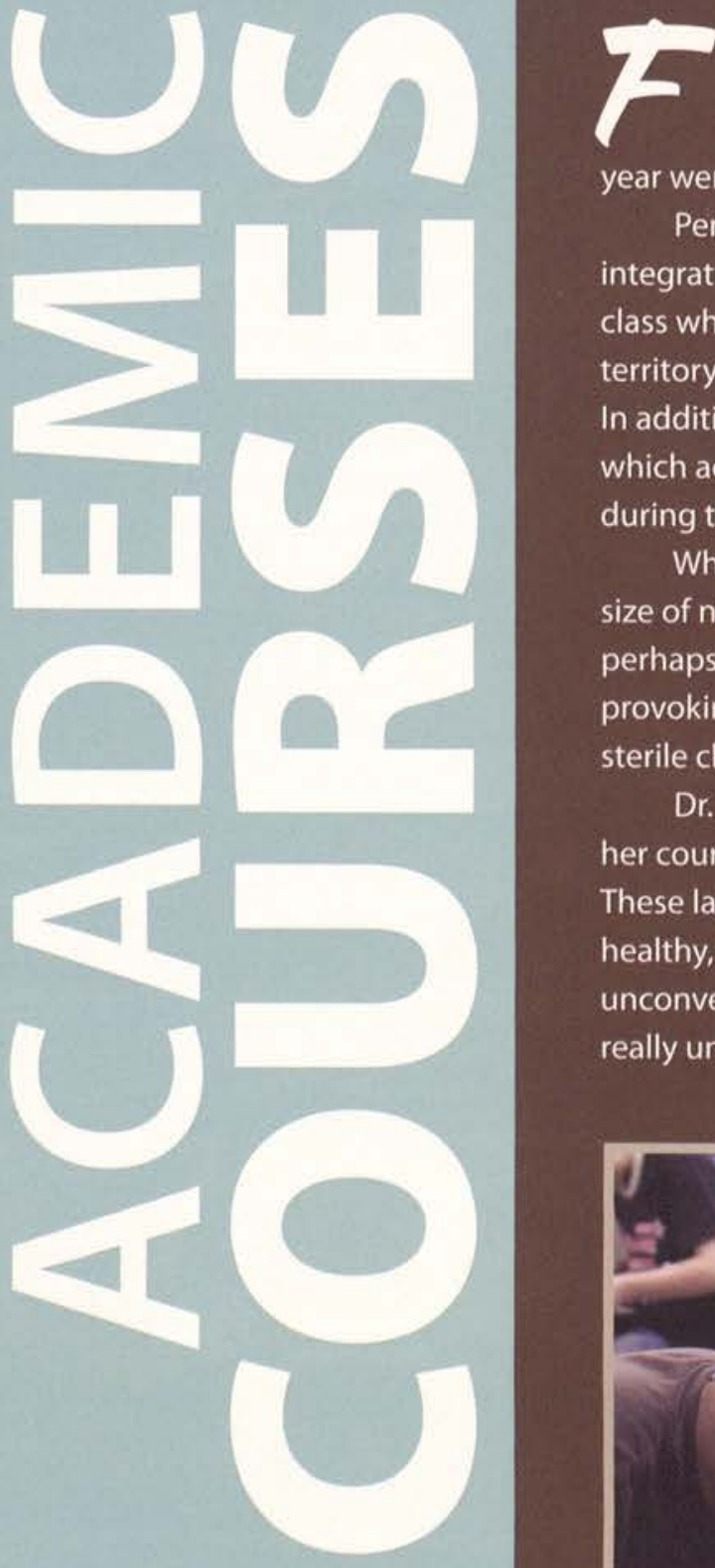

or several Cedarville students, getting stuck in the routine of everyday classes was

commonplace. This year, however, several changes to the normal class atmosphere were made to enhance learning and promote interest in certain courses. A few examples of such classes this year were Pentateuch, Milton: Poetry and Complete Prose, and Group Dynamics.

Pentateuch, taught by Drs. Blumenstock and Hoffeditz, made their class experience unique by integrating a class trip to a museum in Chicago. Junior Jennifer Dixon summarized the feelings of the class when she said, "It's about getting out of the mundane classroom, stepping into the professor's territory and watching him/her explode with excitement and spew out an endless array of knowledge." In addition to Pentateuch, Blumenstock and Hoffeditz taught Biblical Archeology during the spring, which according to Hoffeditz was "intentionally designed" for their trip to Israel and Jordan taking place during the following summer.

While Dr. Wilfong's Milton class was not subject to various trips to foreign locations, its small class size of nine allowed it to be held in Beans $n^{\prime}$ Cream. Dr. Wilfong noted that "the informal atmosphere (and perhaps the caffeine!) tends to reduce people's inhibitions and has resulted in some spirited and thought provoking discussions." Senior Justin Keller agreed, adding that the "dialogue flowed more easily than in sterile classroom setting."

Dr. Lori Hoffeditz's Group Dynamics class took place in an everyday classroom. What made her course fresh, however, was the support group-style labs that encompassed half the class time. These labs, according to Hoffeditz, were intended to confront any counseling misconceptions with "a healthy, realistic picture of the power of group counseling." Junior Kimberly Lincoln responded to this unconventional style by saying, "You can learn these [concepts] from reading a book, but I don't think yol. really understand until you get the chance to fully experience them."
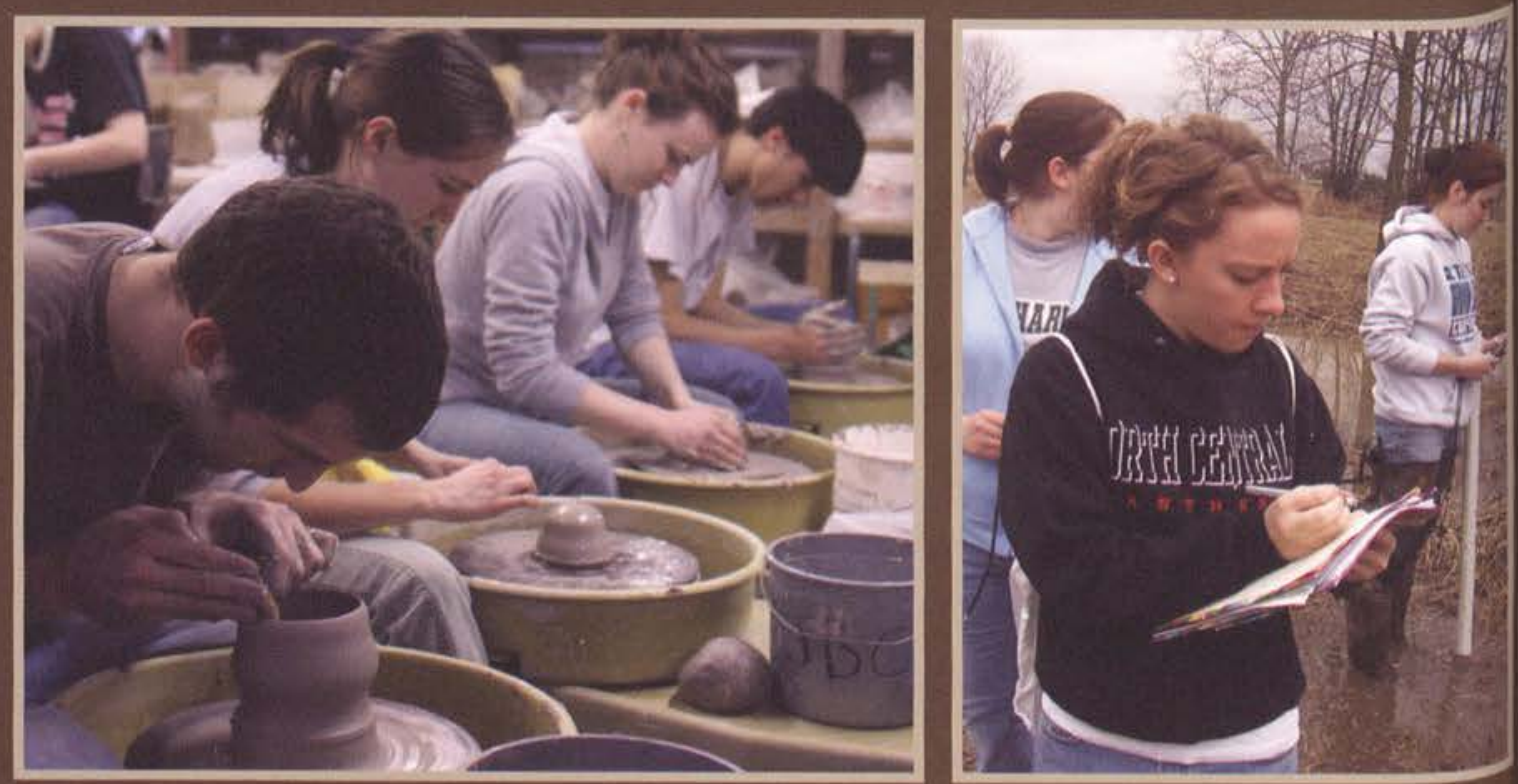

- Great concentration is required of Ricky Normandeau (front) as he works on his clay pot in ceramics class.

- Sarah Crichlow, Bethany Ziemblicki and Hannah Hindle busily document their findings as they work outside the classroom.

- At least one student is attentatively listening as Chase Baldwin gives his presentation.

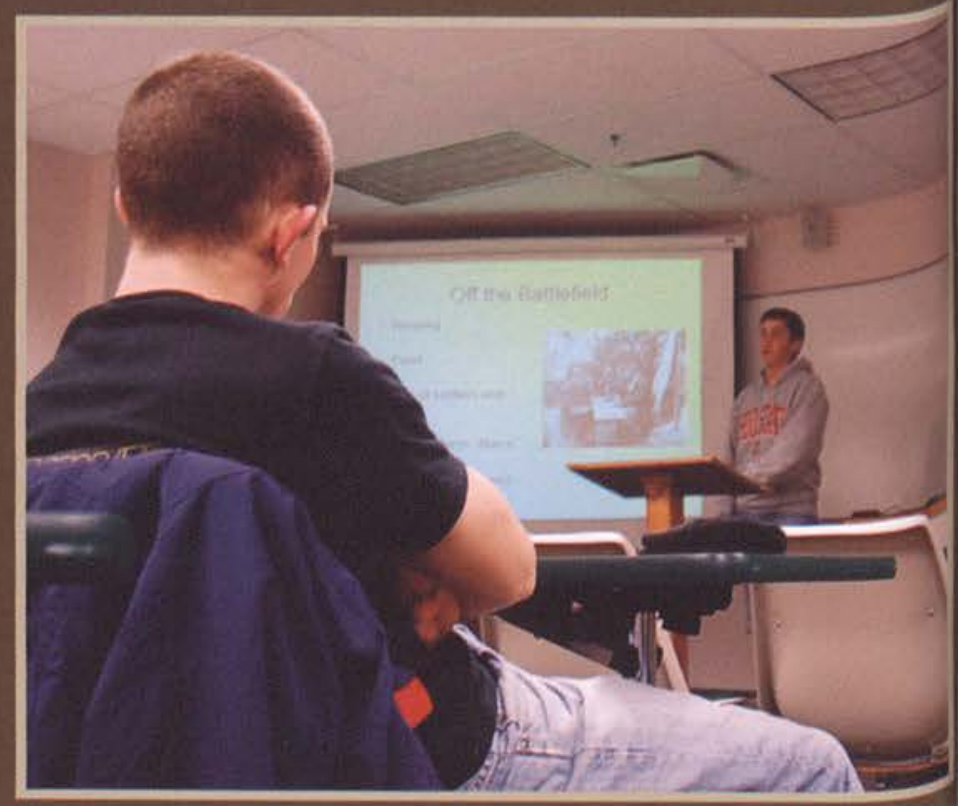




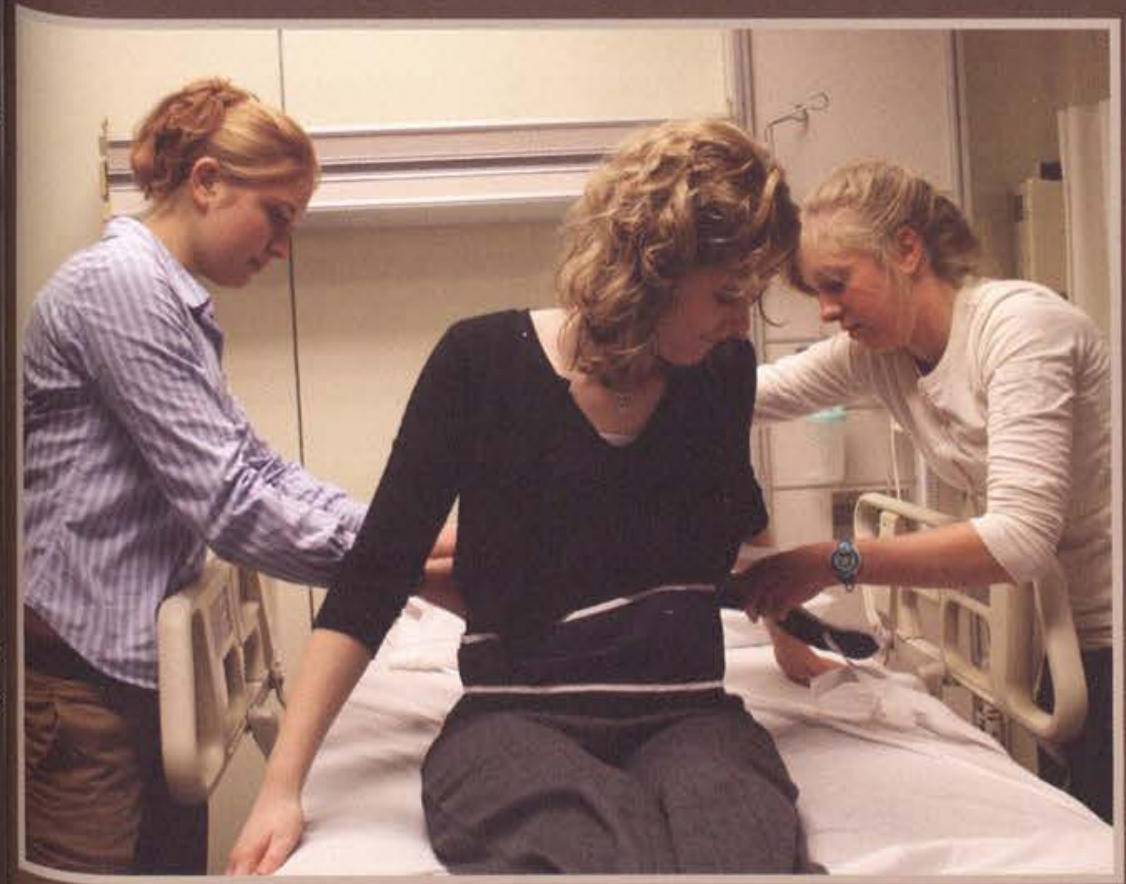

4 Nursing students are always practicing for real-life situations they will encounter later in clinicals and in their careers as nurses.

- Books open and coffee in hand is the only way to go for those early morning classes.

4 Exciting illustrations is Dr. Miller's way of helping his students remember how to "Walk Through the Bible."

Paying attention and feverishly taking notes is the way of life for college students in the classroom.

- Elizabeth Rambikur confidently gives the presentation she worked hard to prepare for.
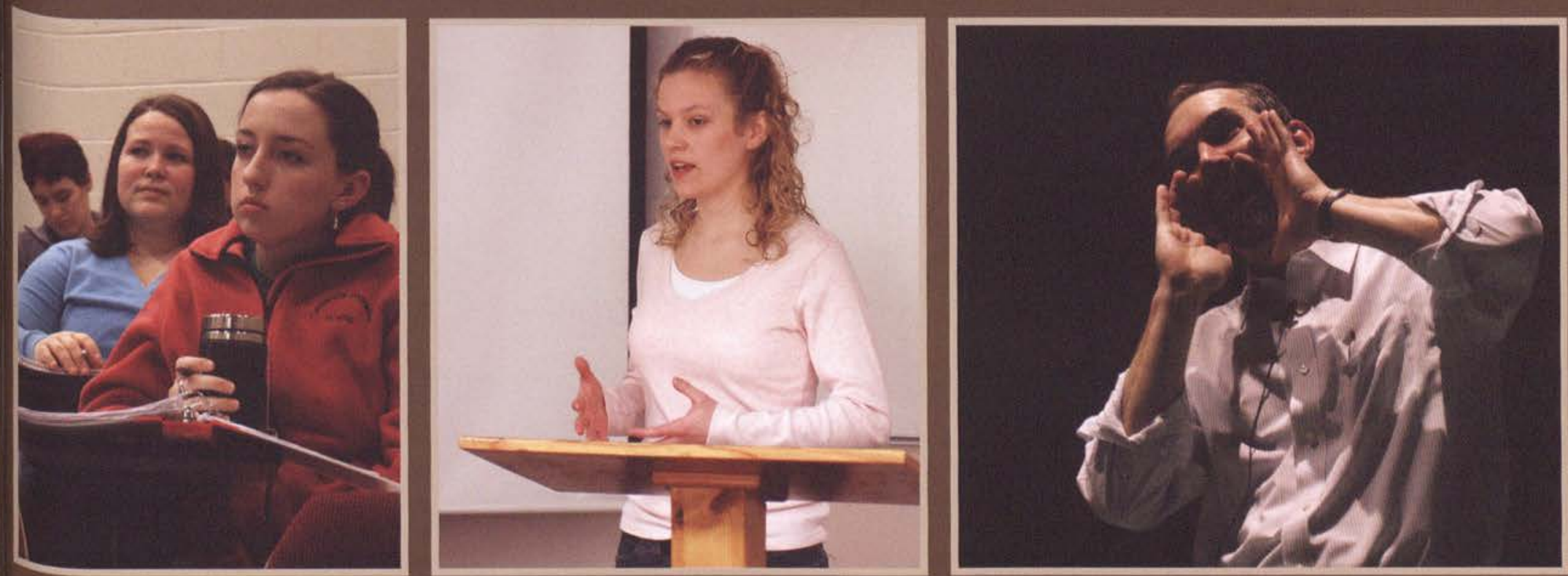

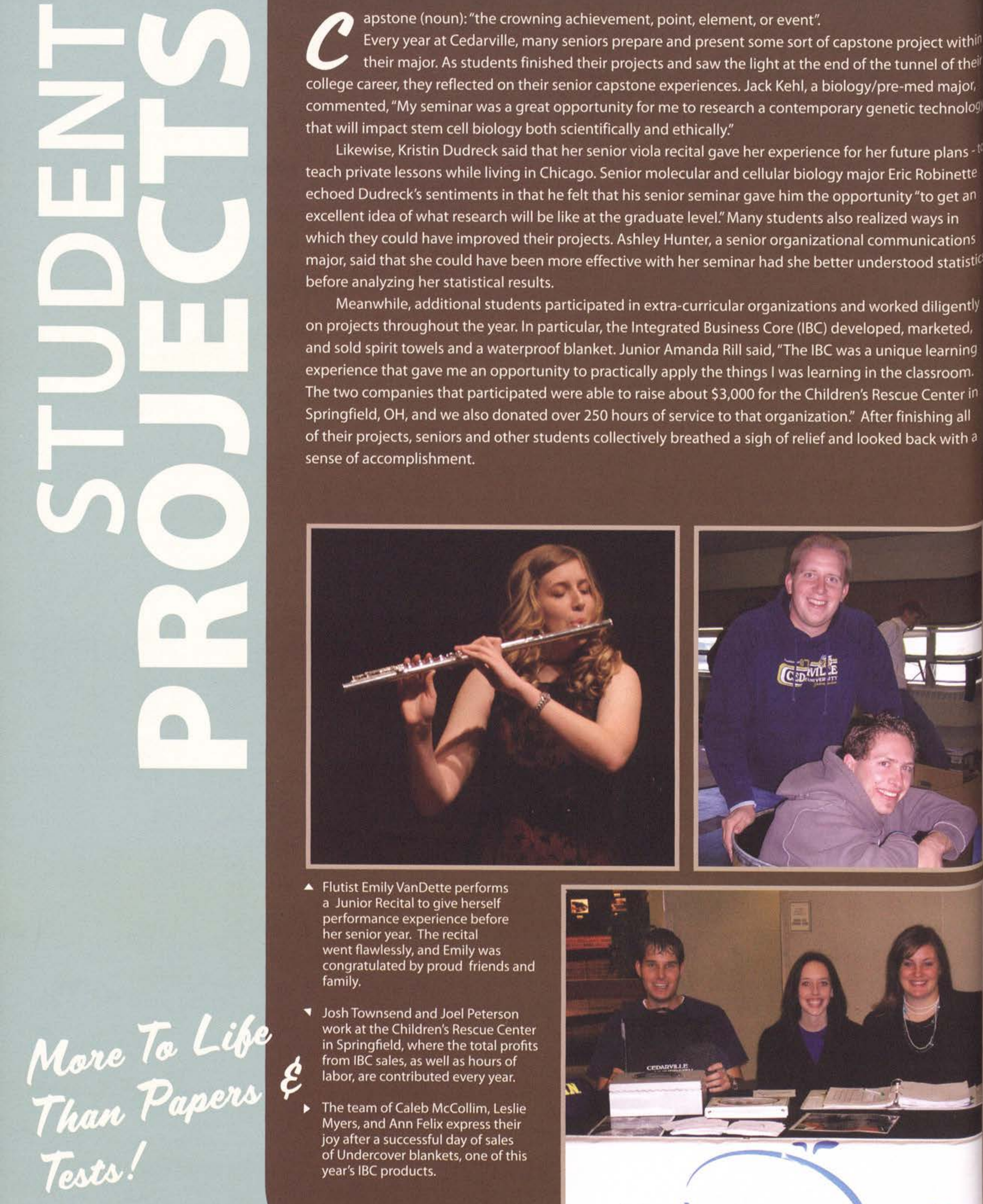

- Flutist Emily VanDette performs a Junior Recital to give herself performance experience before her senior year. The recital went flawlessly, and Emily was congratulated by proud friends and family.

- Josh Townsend and Joel Peterson work at the Children's Rescue Center in Springfield, where the total profits from IBC sales, as well as hours of labor, are contributed every year.

- The team of Caleb McCollim, Leslie Myers, and Ann Felix express their joy after a successful day of sales of Undercover blankets, one of this year's IBC products.

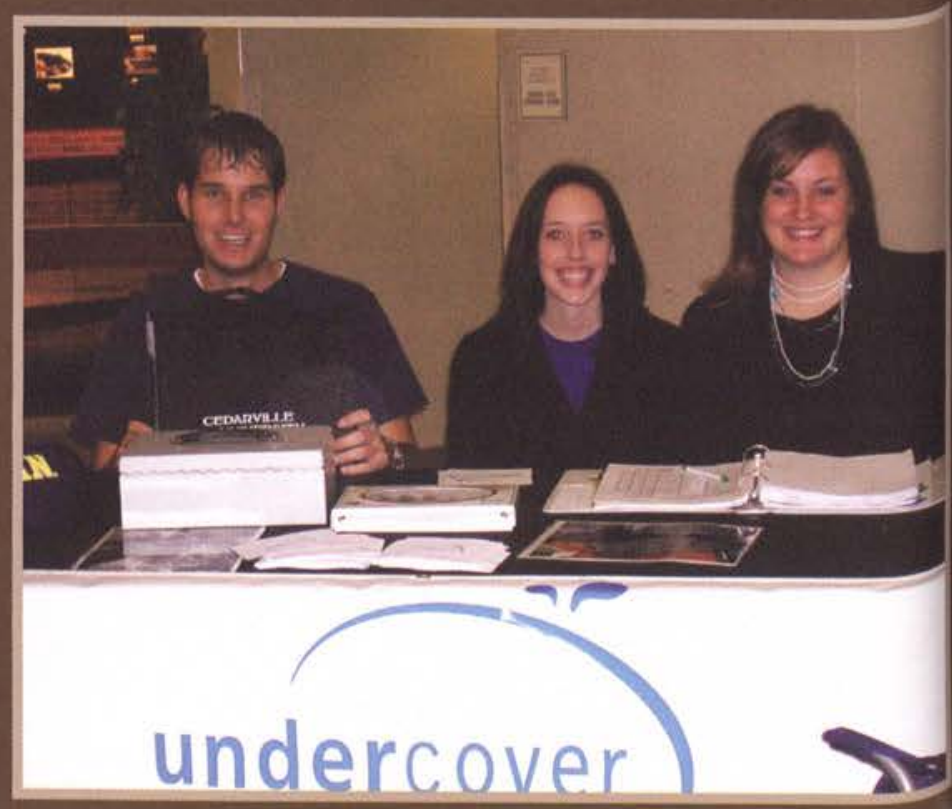




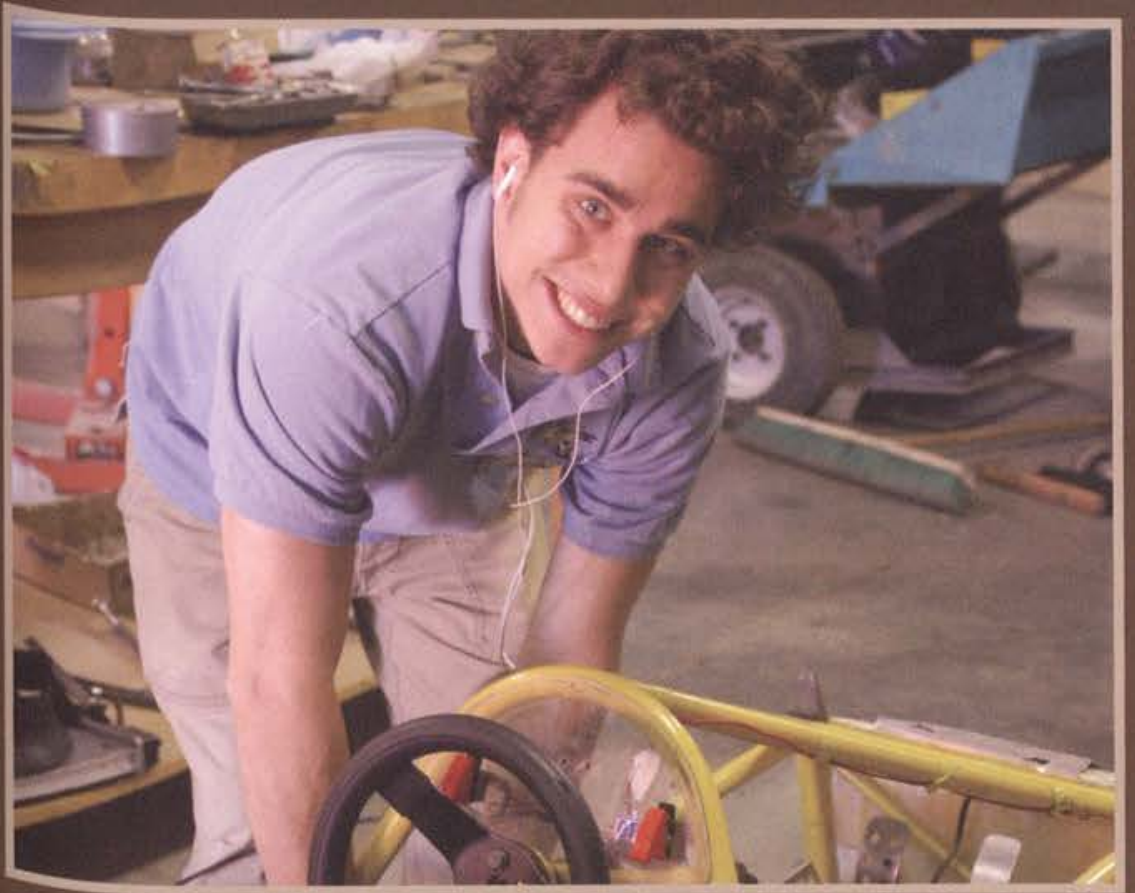

4 Engineering major Charlie Orwig demonstrates that he is an efficient multi-tasker. Apparently, engineers can work on complicated formula cars, smile for the camera, and listen to their iPod all at the same time.

- Emilee Bowman proves that practice makes perfect by beautifully performing a song during her senior music recital. As with all the other senior music majors, Emilee enjoys seeing the fruit of four years of music training at Cedarville.

4 Jonathan Daiello precisely tacks up some of his work for his senior art show. He says, "While the student art exhibition is always a source of strain for every art major...they are usually very successful in gaining interest."

- Onlookers carefully study a piece at Jennifer Krier's Senior Art Show. Krier says, "People sometimes skeptically inquire about my Studio Art major and how I will use that in life. I tell them that God has given me this gift for a unique purpose and for His glory. Now I just have to figure out what that unique purpose is!"

- Electrical Engineering seniors Dan Rogers and Joe Eisentrager work on a project that is used to collect data from various engineering project teams and send that data wirelessly to a laptop for data analysis.
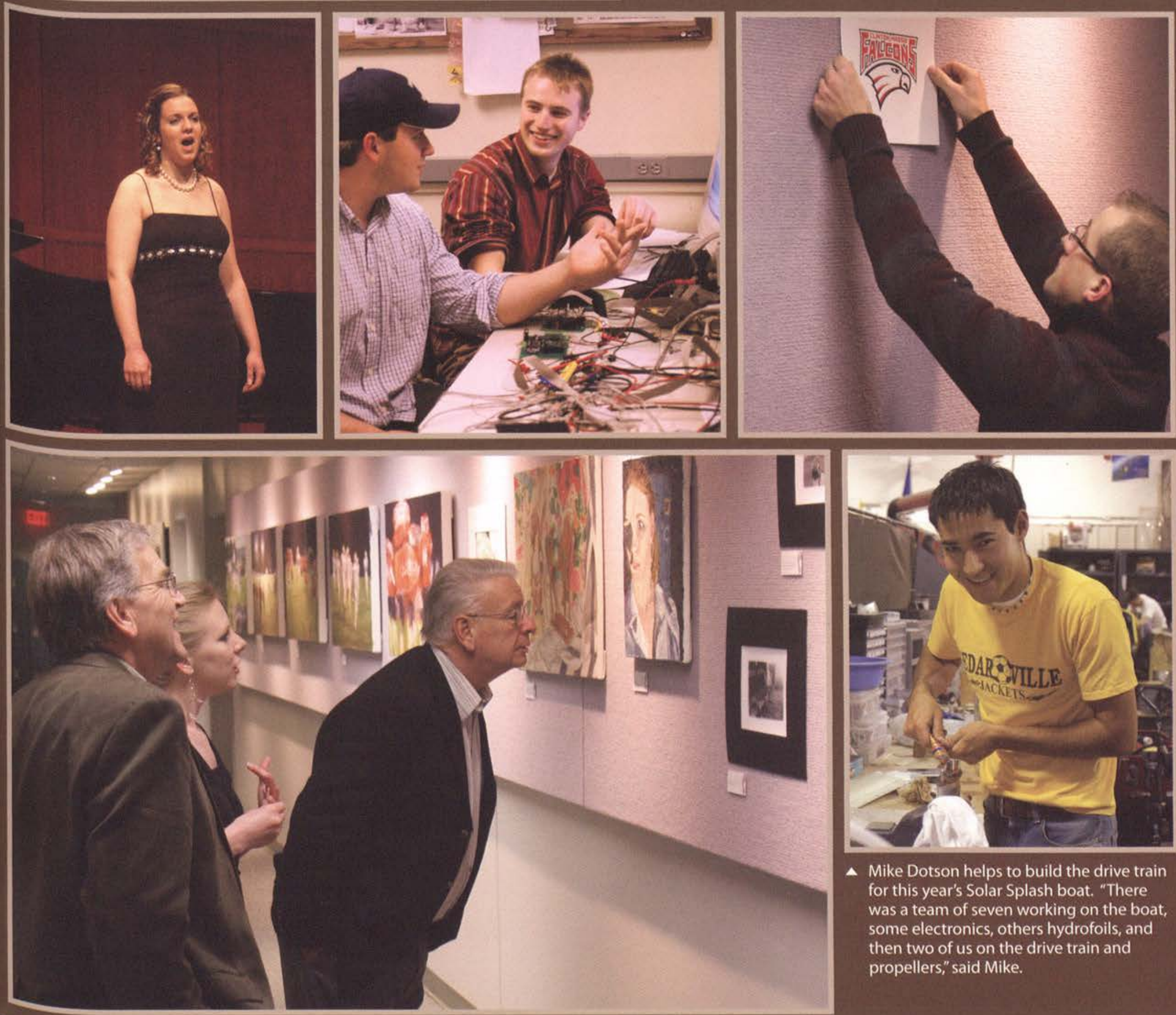

A Mike Dotson helps to build the drive train for this year's Solar Splash boat. "There was a team of seven working on the boat, some electronics, others hydrofoils, and then two of us on the drive train and propellers," said Mike. 


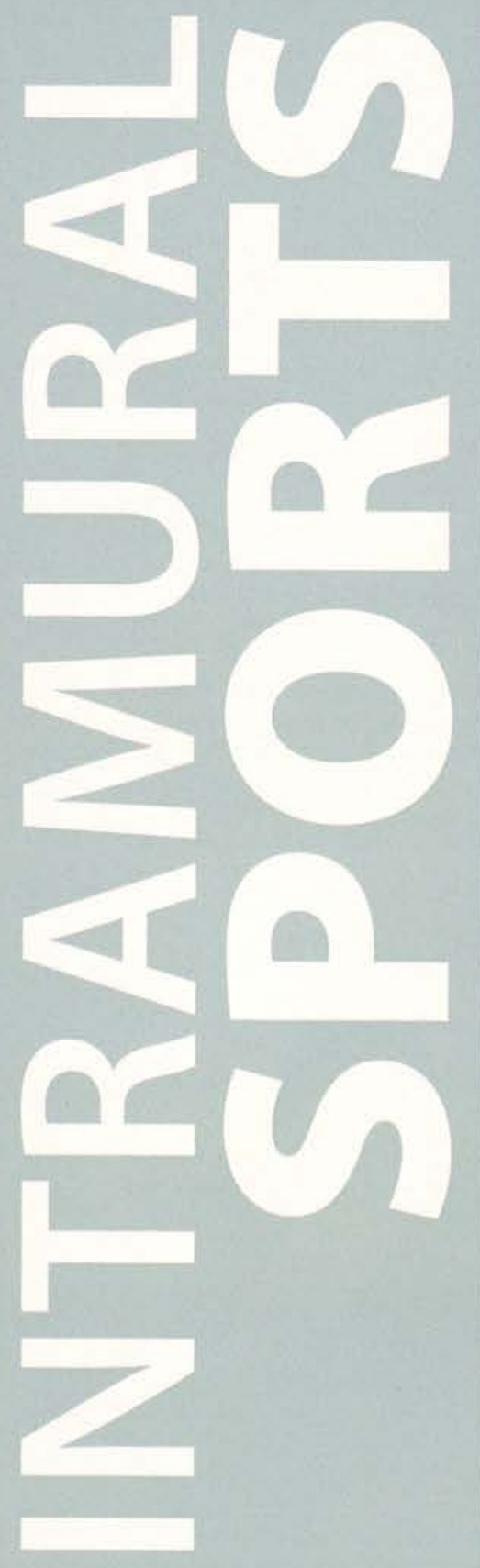

$T$ his year's Intramural Sports programs exhibited a strong combination of impressive skill and immoveable team unity. An excellent example of such an indomitable spirit is Ray Nitschke's Blind Fury, an occasionally triumphant flag football team comprised of various faculty and students in the Language and Literature department. Sophomore team member Scott Kordic explained, "The Blind Fury proved that Flag Football is certainly not for pansies," when teammate Professor Ryan Futrell dislocated his elbow during game-play.

Intramural sports were also a great way for faculty and staff to get involved in organized competition. Mark Irving, Cedarville alum, Director of Discipleship Ministries and champion of Men's Singles Racquetball, enjoyed connecting with the students. "I enjoy doing intramural sports for a couple of reasons. The exercise helps to keep my 30 -something body in shape, but first and foremost, it gives me a great contact point with students. Many of the guys that are involved with discipleship ministries on campus are guys that I've met while playing ultimate Frisbee, racquetball, or flag football."

Junior Greg Briggs, a member of the winning Ultimate Frisbee team "The DC Diskers," encapsulated what most intramural participants enjoy the most about this casual and relaxed form of competition. Briggs said, "When we start, we begin in prayer, and when we end we're shaking hands and congratulating each other. It's almost impossible to get really mad at the other team because half the time they're all good friends of mine."
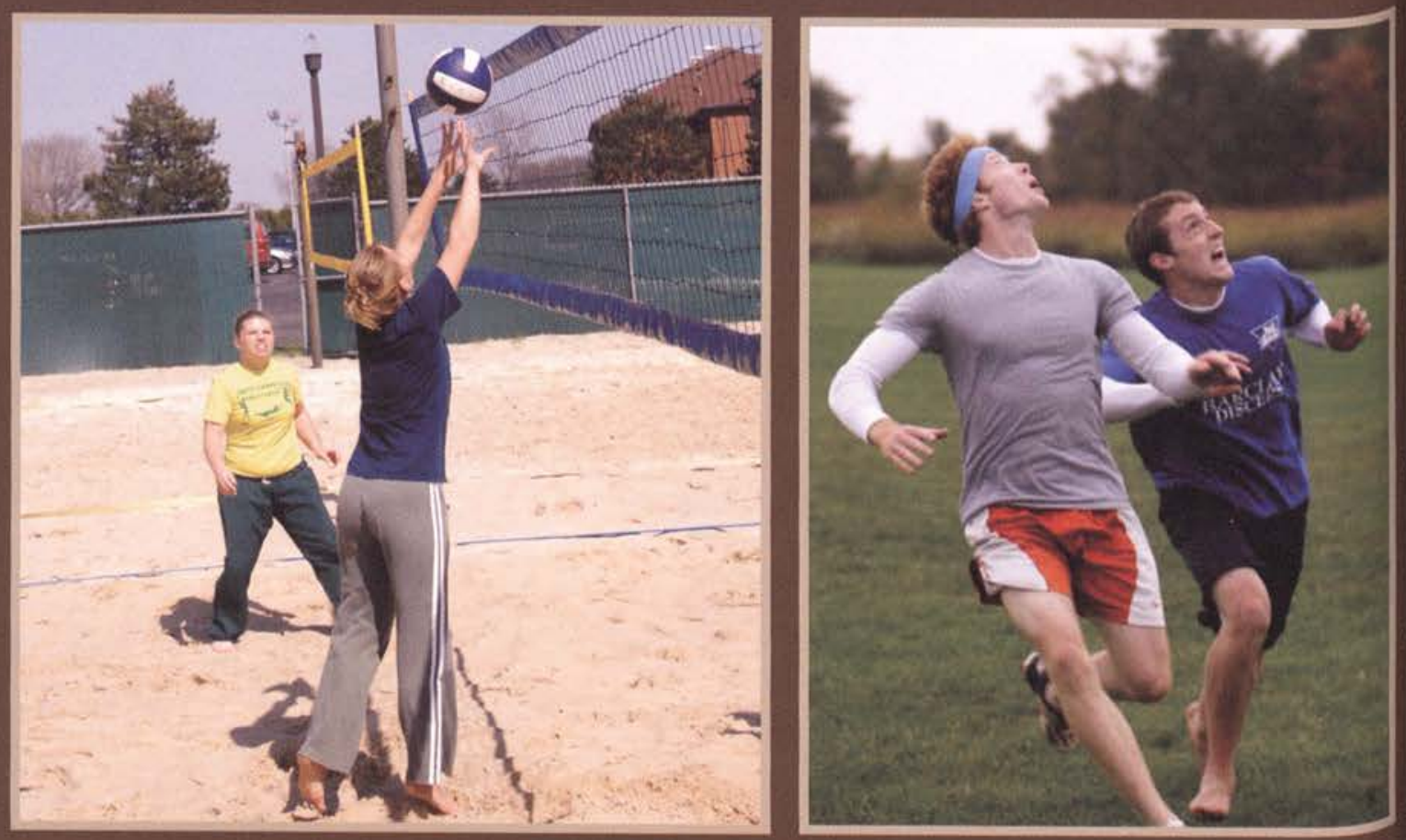

- A little sand volleyball on a sunny day is a great way to spend time with friends during a break from studies, especially when you know you could win the coveted "Intramural Championship" tee shirt.

- Sophomore Kelly Nowels fights for position as the ball comes into play.

- Josh Perrel lunges toward Josh Teekell in a rousing game of flag football as referee Ryan Hansen makes sure there is no illegal action on the play.

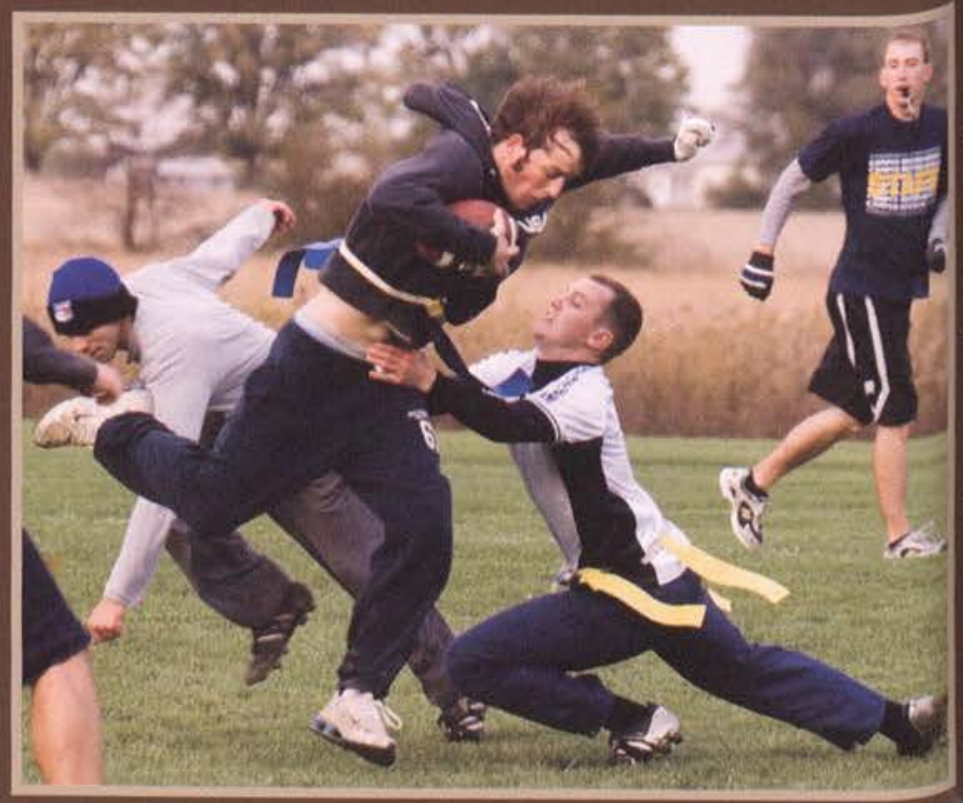




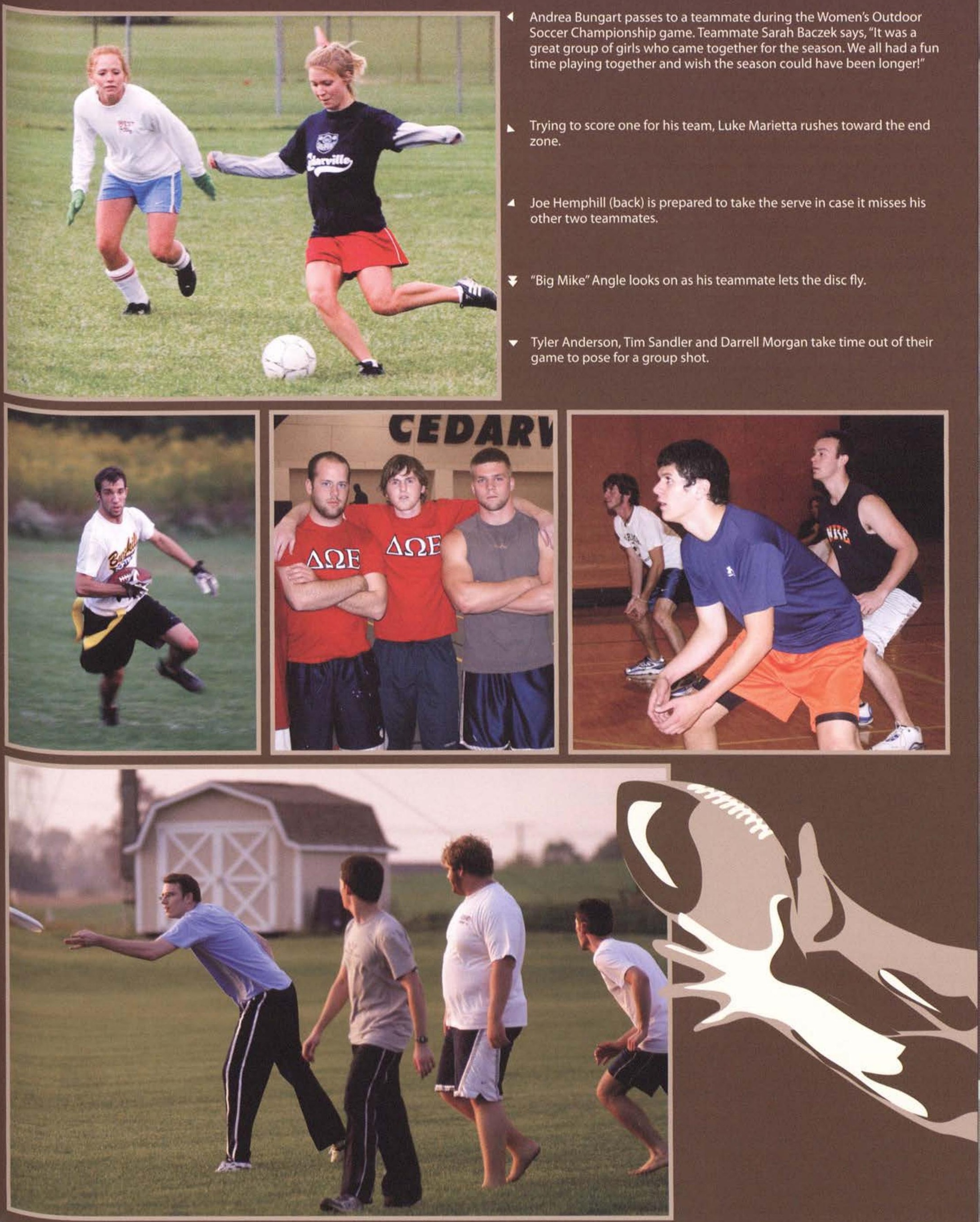

Andrea Bungart passes to a teammate during the Women's Outdoor Soccer Championship game. Teammate Sarah Baczek says, "It was a great group of girls who came together for the season. We all had a fun time playing together and wish the season could have been longer!"

Trying to score one for his team, Luke Marietta rushes toward the end zone.

Joe Hemphill (back) is prepared to take the serve in case it misses his other two teammates.

Tyler Anderson, Tim Sandler and Darrell Morgan take time out of their game to pose for a group shot. 
Theatre Department manages to draw students from all walks with the variety and quality of productions they offer every year. With its rich and rigorous curriculum, the theatre department consistently produces talented students who bring colorful, intriguing, and quite often, ruthlessly heart-wrenching stories into the everyday lives of their audiences through independent plays and senior theatre productions.

This year's fall semester began with the beautifully articulated "Less of Me....More of Him," a senior theatre project by Gretchen Riedel with the added acting talents of fellow senior Donna Sweede. This classic story used dramatic monologues to seamlessly elicit moving parallels between the lives of two radically different women - Corrie ten Boom and Lisa Beamer. The very next weekend, NeXtage and Kelsey Nelson brought "Over the River and Through the Woods" to the Cedarville stage - a contemporary telling of the tension between family ties and personal ambition.

Spring brought us "Under the Overpass," Andrew Tripp's adaptation of the book written by Mike Yankoski detailing the five months he willingly spent homeless, and the first senior film project - "Twixters" - by Grant Letizia.

For Andrew Tripp, the process was the culmination of his years at Cedarville: "Putting together a play definitely tested all aspects of my theatre experience and training - from characterization to staging to organizational and administrative responsibilities."

Grant Letizia's "Twixters" documented the tension often felt by twenty-somethings just learning to make their way in the world. In his own words, "It was incredible to be able to leave my fellows students with the encouragement that even though we may be in a time of great change, we can trust God with our futures and go out with reckless abandon into His adventure for our lives."
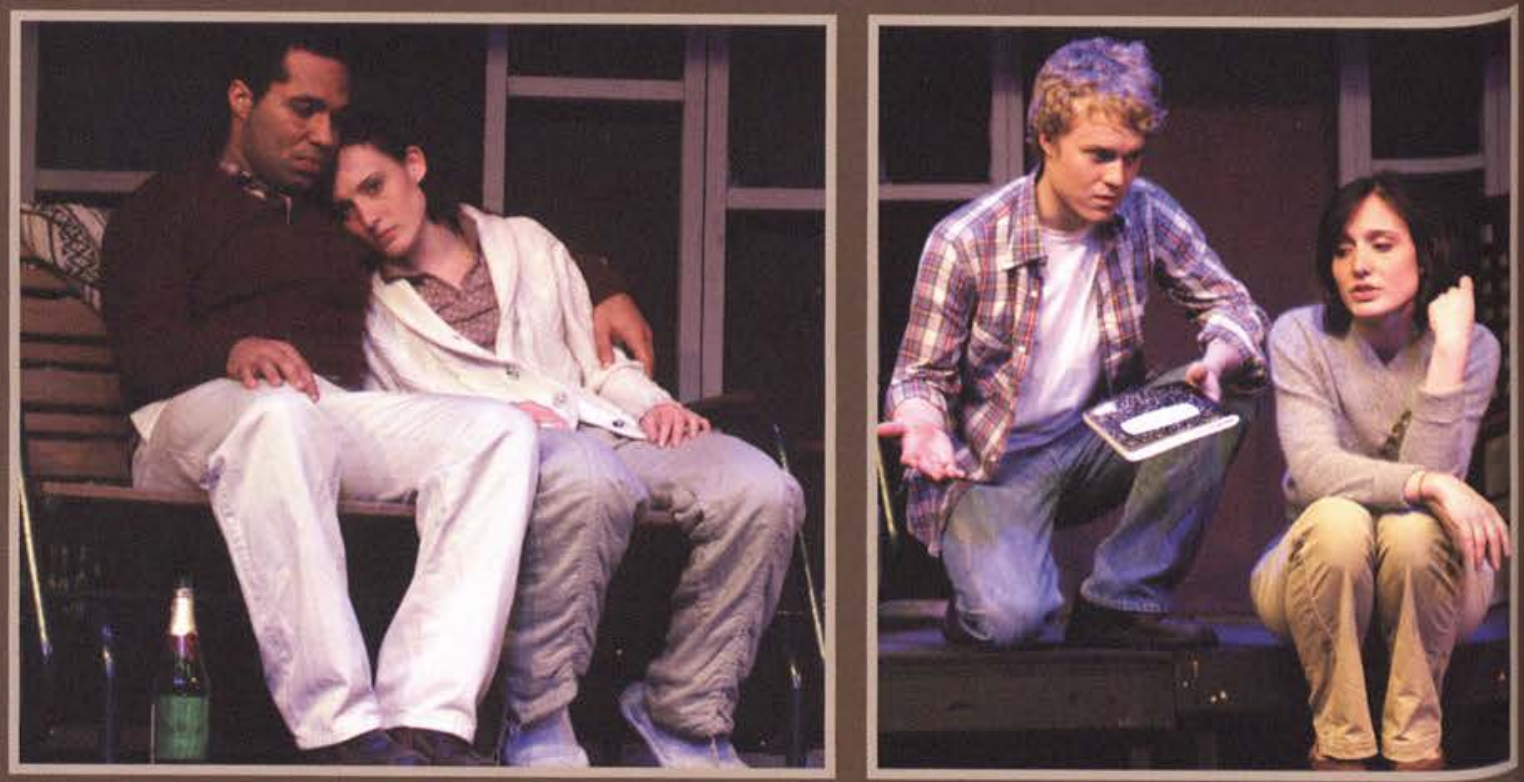

- Victoria Duff's Senior Theatre Presentation drew quite a crowd as she was known around campus for her theatrical performances in chapel, in independent theater plays, and on Cedarville's main stage.

Victoria speaks to a student of her late father's in her Senior Theater Project, titled "Proof."

- Jodi Strychalski and King Harry Rob Rasmussen discuss the fate of their daughter, Crystal Tuxhorn, in Crystal's Senior Theater Project, "The Ugly Duckling."

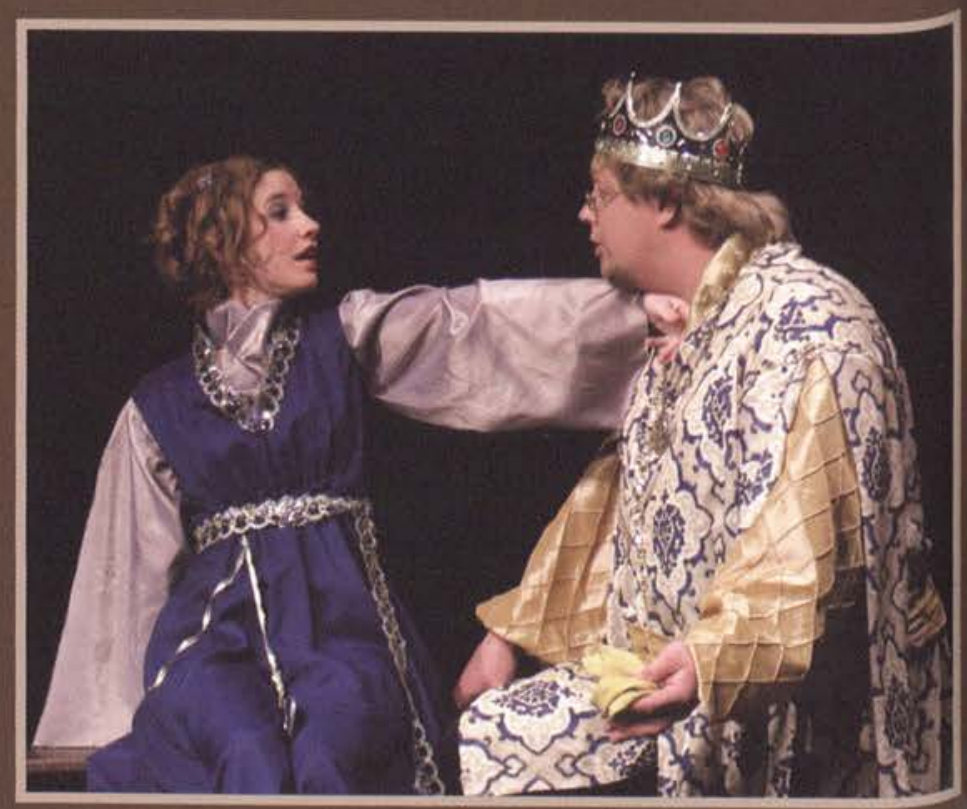




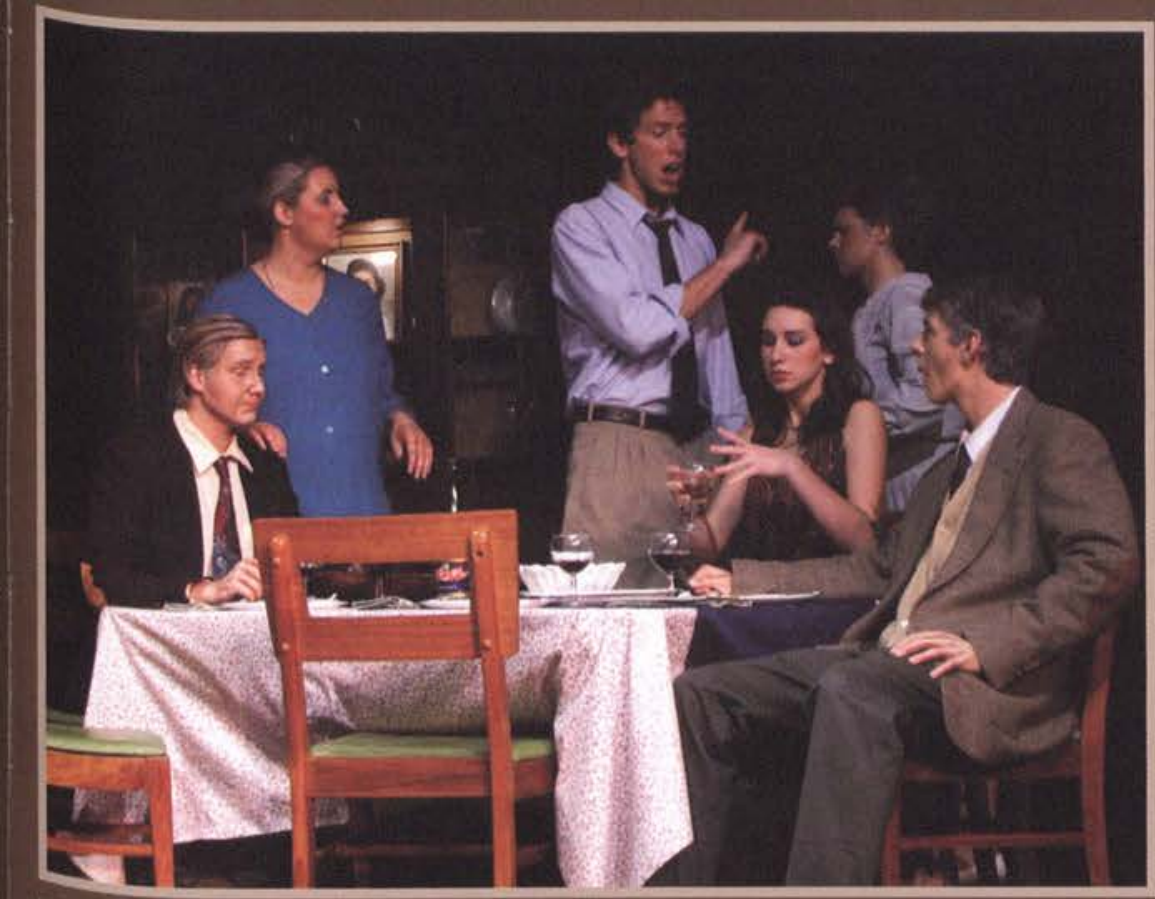

4. The cast from Alpha Phi Omega's fall production of Over the River and Through the Woods gathers to convince Nick (Brian Houchin) that he should be married to Caitlyn (Sarah Saxe).

- In the play Over the River and Through the Woods, Grandmothers Emma and Aida (Elizabeth Crowner and Kasey Rising) make sure that their grandson, Nick, is mentally okay after he lets it slip that he goes to a therapist on a regular basis.

- Colin Luther comes to the rescue of "ugly duckling" Crystal Tuxhorn in her Senior Theater Project.

4 Jess Waggoner chastises Rebecca Wolff during her Senior Theater Project "Master Class."

7 Dan Tello and Jodi Strychalski stand to one side, observing Crystal's joy of successfully finding the prince of her dreams.

- Rebecca Wolff nearly makes Gretchen Reidel cry as she peers over her shoulder during a voice lesson in "Master Class."
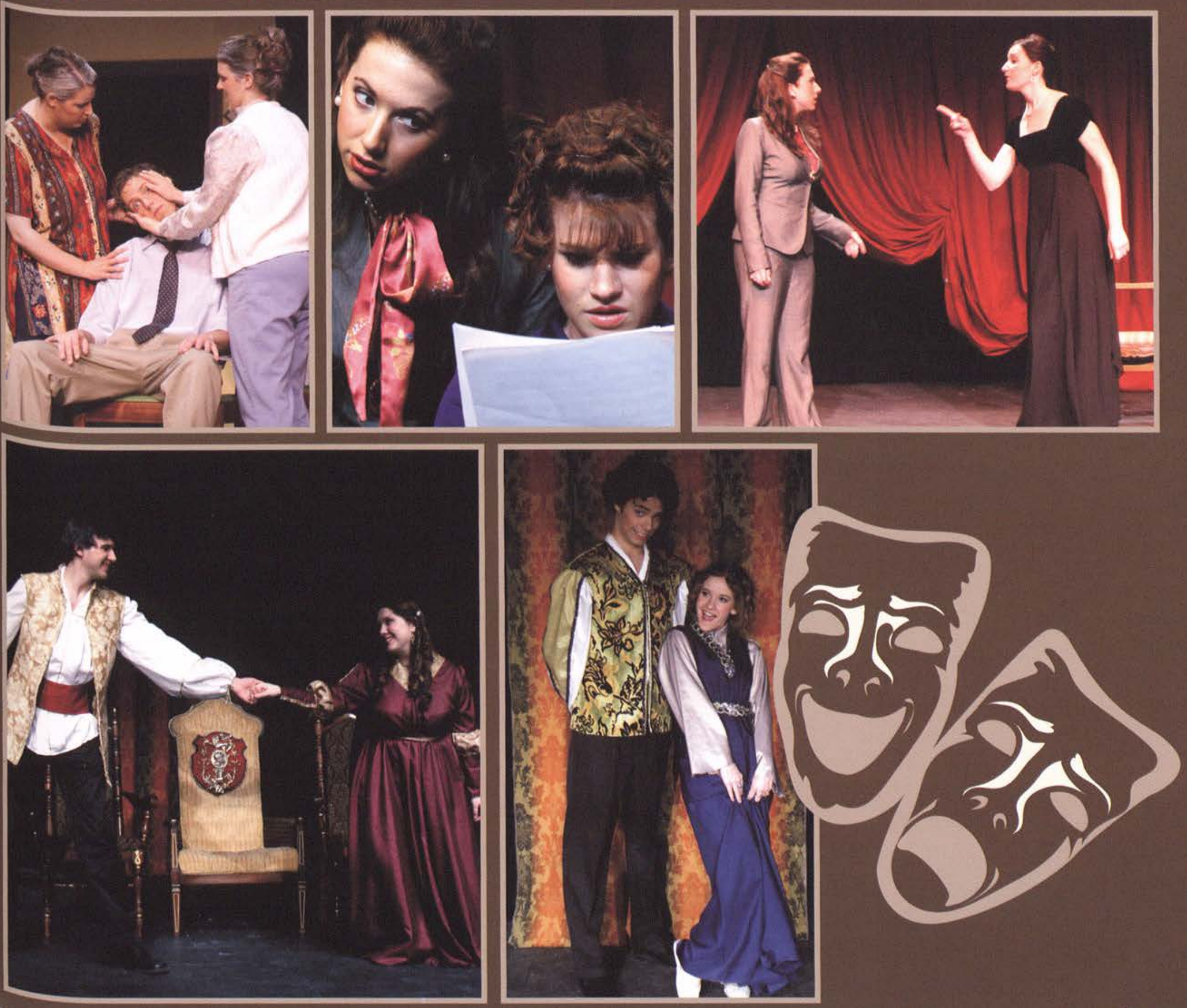

supposed to be this mysterious new event." But when SGA was left with fewer PR opportunities than expected, such as fewer announcements before chapel, Donald explained, "All we had left was word of mouth and posters."

This unexpected occurrence did not deter SGA from hosting other events, however. This year, SGA activities centered on giving, outreach, and a general awareness of need around the world. President Luke Seelye mentioned, "One of my goals this year was to encourage the student body to think beyond our 400 acres to see a world in need of redemption." This goal was realized with such activities as the Fisheni Village Project, a year-long fundraiser conducted alongside Women of Vision. Other projects included a T-shirt sale that raised funds for village goats, and a shoe drive for the needy in Iraq.

SGA also focused heavily on reaching out to other campuses. Inter-Collegiate Council Director and junior Gabe Coyle mentioned that, in addition to the activities that involved the entire student body, such as ICC bowling, the dodge ball tournament, and Battle of the Bands, Cedarville's SGA "sent Christmas gifts to SGA's from other schools and organized a picnic where SGA leaders from such school as Wilberforce, Central State, Wittenburg, Wright State, and Antioch came out." Clearly, SGA redeemed their Savvy qualities with various outreach and charity efforts.
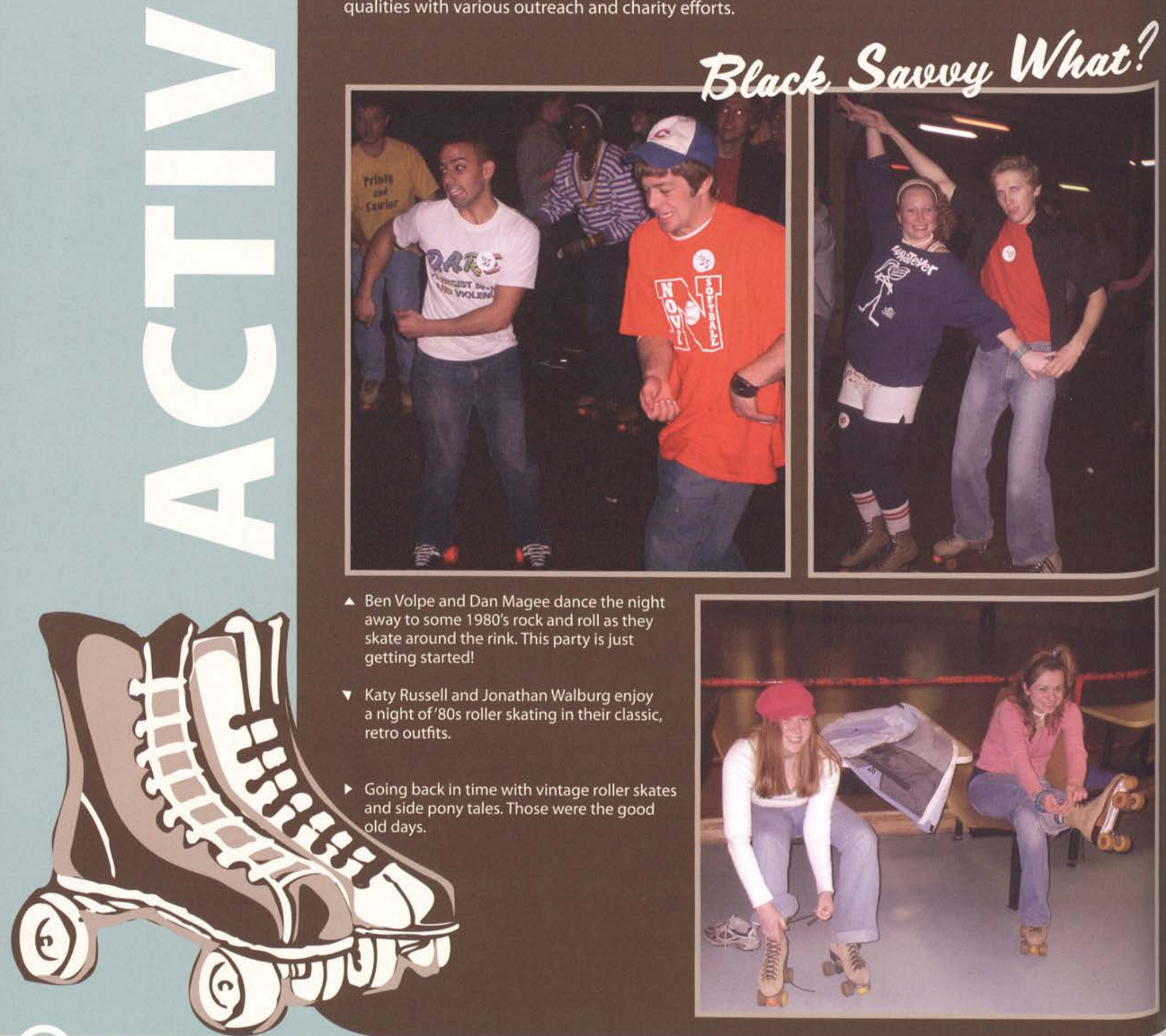

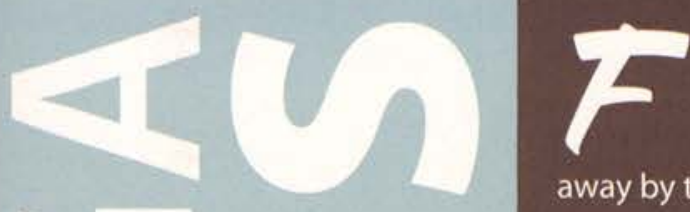

riday is the favorite day of the week for many Cedarville students, not only because it precedes a two-day break in classes, but also because it brings SGA chapel. Gabe Coyle, the worship leader, said, "This year has given me an amazing leadership opportunity, and I've been blown away by the response of the student body." In SGA chapel, students could be sure to have a good time, while still hearing a challenging message and enjoying meaningful worship.

SGA chaplain, Mark Hershey, said, "It has been exciting to see the different ways God has used chapels this year. I love the way things have turned out." Mark's creative stories and heartfelt messages touched many of the students. Sophomore Sarah Crichlow said, "I love the fact that Hershey is amusing in his random illustrations yet dedicated to the seriousness of preaching God's Word."

Chapels this year were known for Mark's cross-stitching stories, Wade's flannel shirts, and U99.5's iPod giveaways. U99.5 gave away several iPods through a series of onstage contests, including playing air guitar and batting whiffle balls into the audience. Even the school mascot had a turn at batting, but the Bee proved to be a better mascot than a player.

One of the most memorable chapels was Groundhog Day chapel. Wade stood in the cold "for hours" waiting for the groundhog to come out of its hole in the Fallen Man statue and was shocked to learn that the animal was already onstage having a discussion with Mark Hershey. The students erupted in laughter when Wade charged onstage and tackled the unsuspecting groundhog. Students appreciated SGA chapels because they had a good balance of fun and serious worship. SGA continued its tradition of leading chapels that brought the students together to worship and grow closer as a student body.
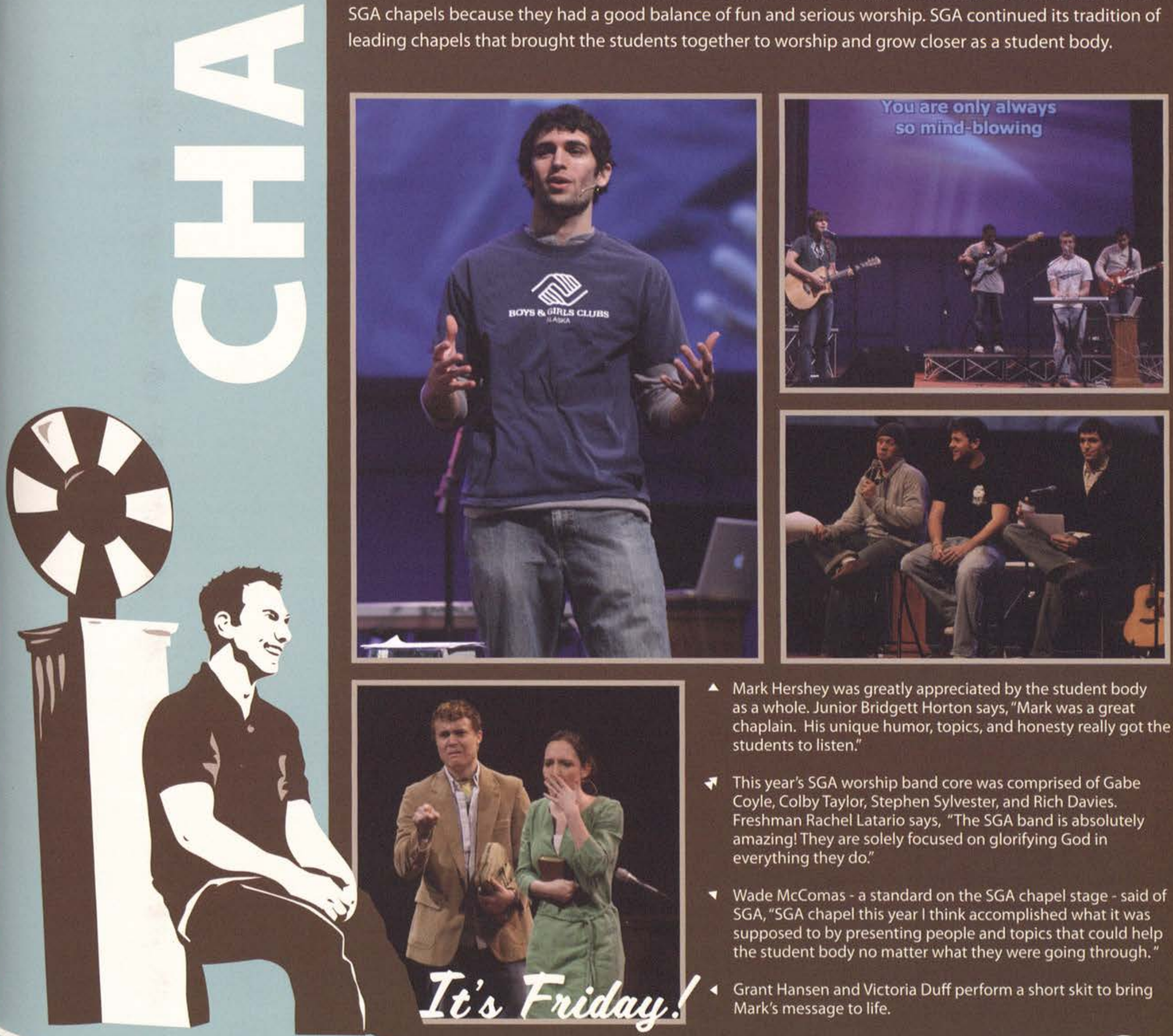

- Mark Hershey was greatly appreciated by the student body as a whole. Junior Bridgett Horton says, "Mark was a great chaplain. His unique humor, topics, and honesty really got the students to listen."

- This year's SGA worship band core was comprised of Gabe Coyle, Colby Taylor, Stephen Sylvester, and Rich Davies. Freshman Rachel Latario says, "The SGA band is absolutely amazing! They are solely focused on glorifying God in everything they do."

- Wade McComas - a standard on the SGA chapel stage - said of SGA, "SGA chapel this year I think accomplished what it was supposed to by presenting people and topics that could help the student body no matter what they were going through."

- Grant Hansen and Victoria Duff perform a short skit to bring Mark's message to life. 
This year's chapel theme was "Going and Making Disciples," challenging students to act upon Christ's final commission. President Dr. Bill Brown brought this message to our attention each Monday. His messages were often considered a highlight of the chapel schedule, and junior Jessica Daugherty noted, "I'm glad that I'm at a school where the president speaks every Monday to the student body. It really shows his involvement and interest in seeing Cedarville students grow in Christ." Dr. Brown focused on different aspects of Matthew 28:16-20 (which, according to junior Bob Thalman, "Makes it a lot easier to remember his messages"), emphasizing many highlights of the text concerning our charge to make disciples.

Other speakers also encouraged us in this aim, sharing their own experiences spreading the gospel. Steve Haas, vice president of World Vision, told of travels to Africa where many suffer from AIDS. Haas reminded us that for many of these people, the gospel of Christ is the only hope they have left to cling to. Other speakers, such as Steve Saint (son of missionary Nate Saint, killed while reaching the Waodoni tribe of the Amazon) shared not only the sufferings, but also the joys of sharing the gospel with those who've never heard.

As a Christ-centered community, time spent in chapel played a key role in encouraging the student body in their walks with Christ. Freshman Asheritah Oana commented, "Those fifty minutes of worshiping God, praying for others, and listening to the Word was the highlight of my day. During that time I could place all my other worries aside and come together with the community of believers to focus on God."
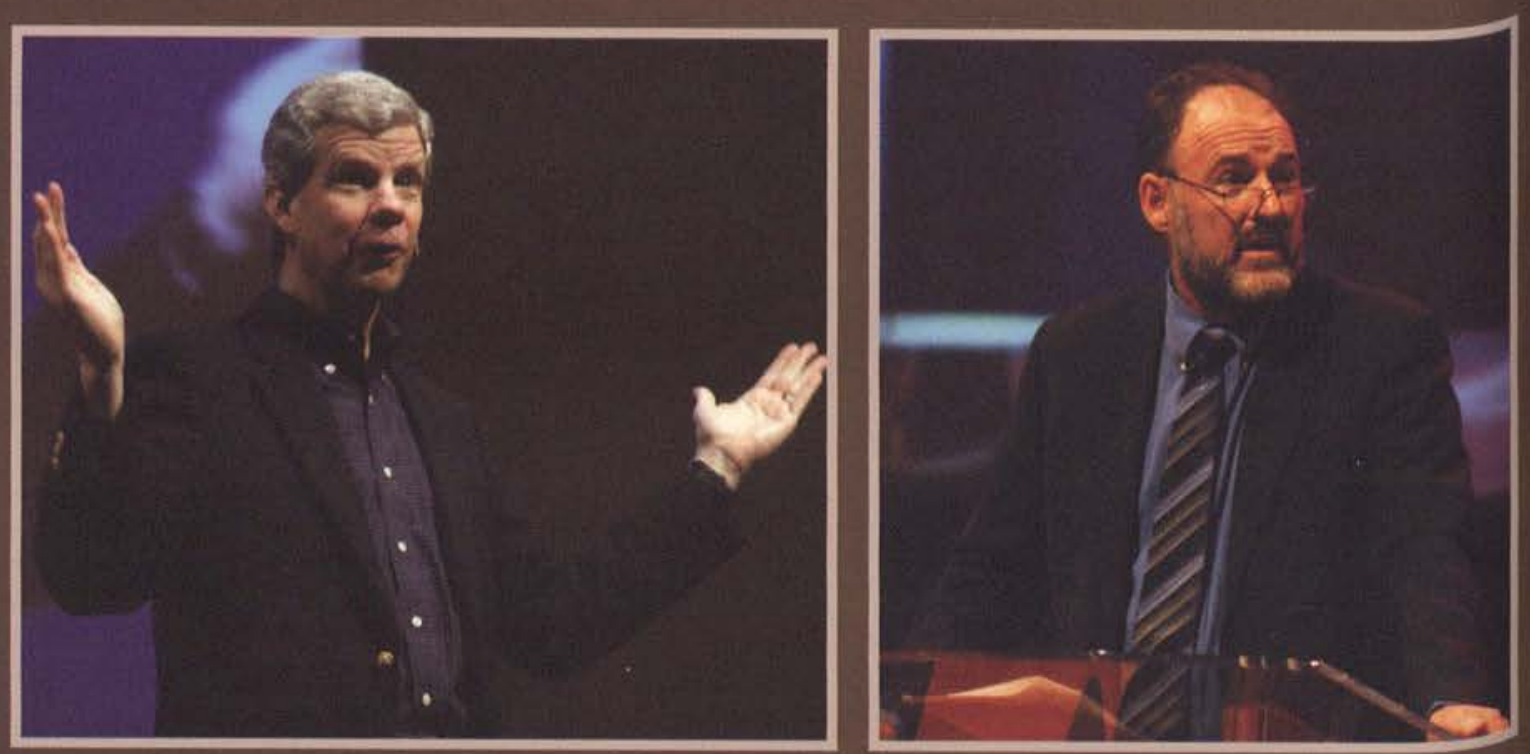

- Dr. Brown shares with the student body about going and making disciples in one of his weekly addresses.

v Dr. Robert Priest of Trinity Evangelical Divinity School speaks for this year's Staley Lecture.

Scott Lehr shares a powerful testimony, reminding us how true the song is- "My God is so big, so strong and so mighty, there's nothing my God cannot do, for you!"
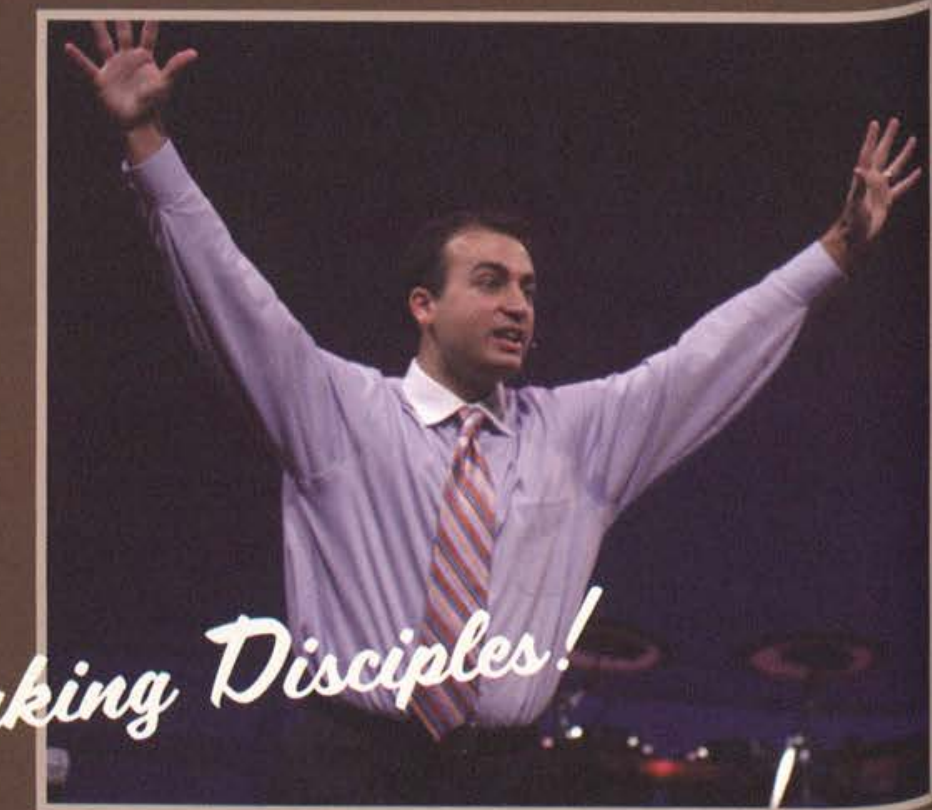

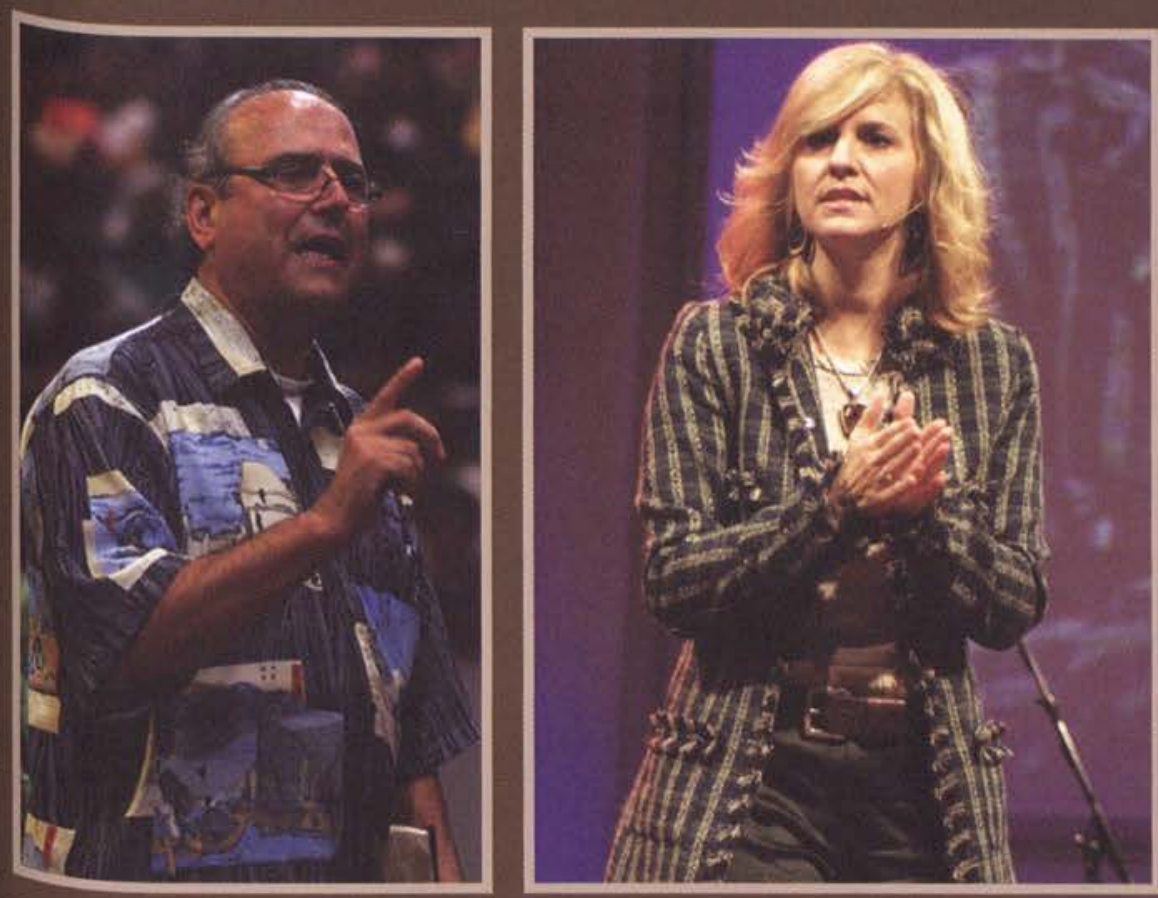

4 Dannah Gresh, author of books like And the Bride Wore White: Seven Secrets to Sexual Purity, speaks to students this year about making choices for purity and godliness.

4 Reverend Tom Mahairas shares his experiences with CitiVision, an evangelistic organization dedicated to making, motivating and equipping disciples to impact their cities with the Gospel.

- Shaunti Feldhan, author of books For Women Only and For Men Only, reminds students about the differences and particular needs of both genders.

- Alumnus Kelly Fath shares his testimony regarding the works of the Lord in his life since his own days at Cedarville.
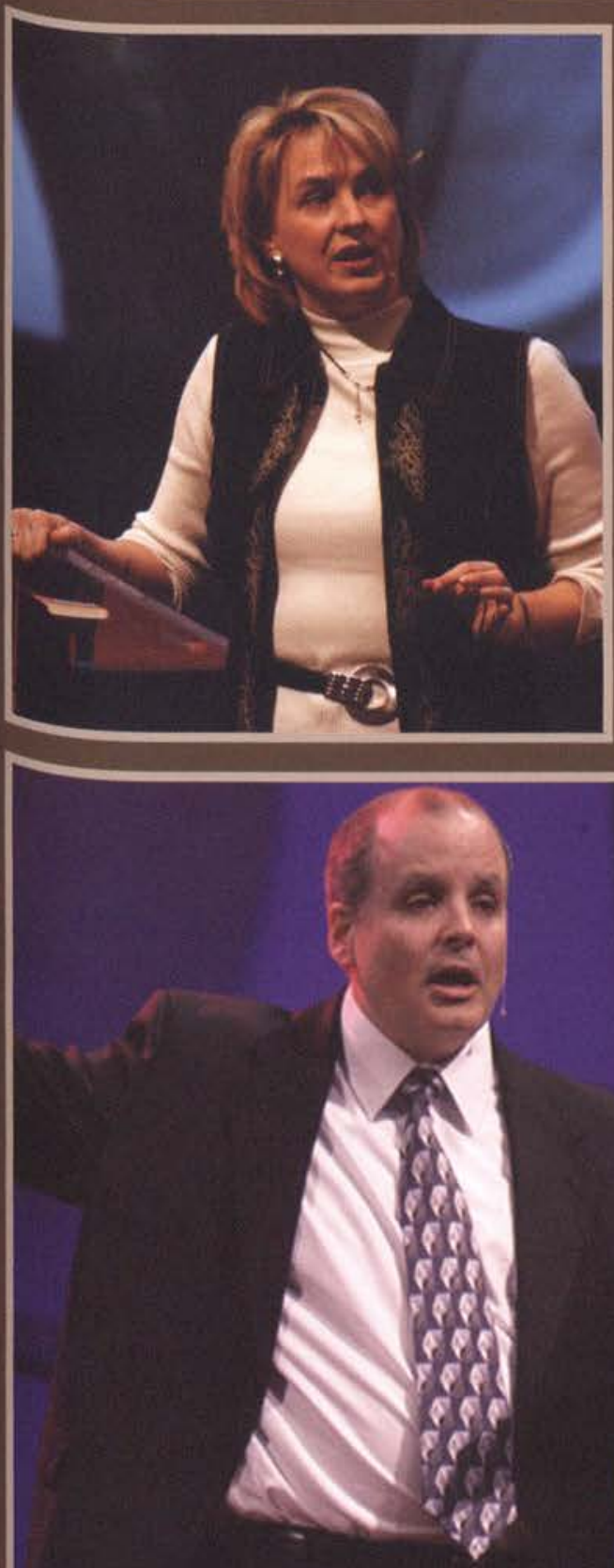
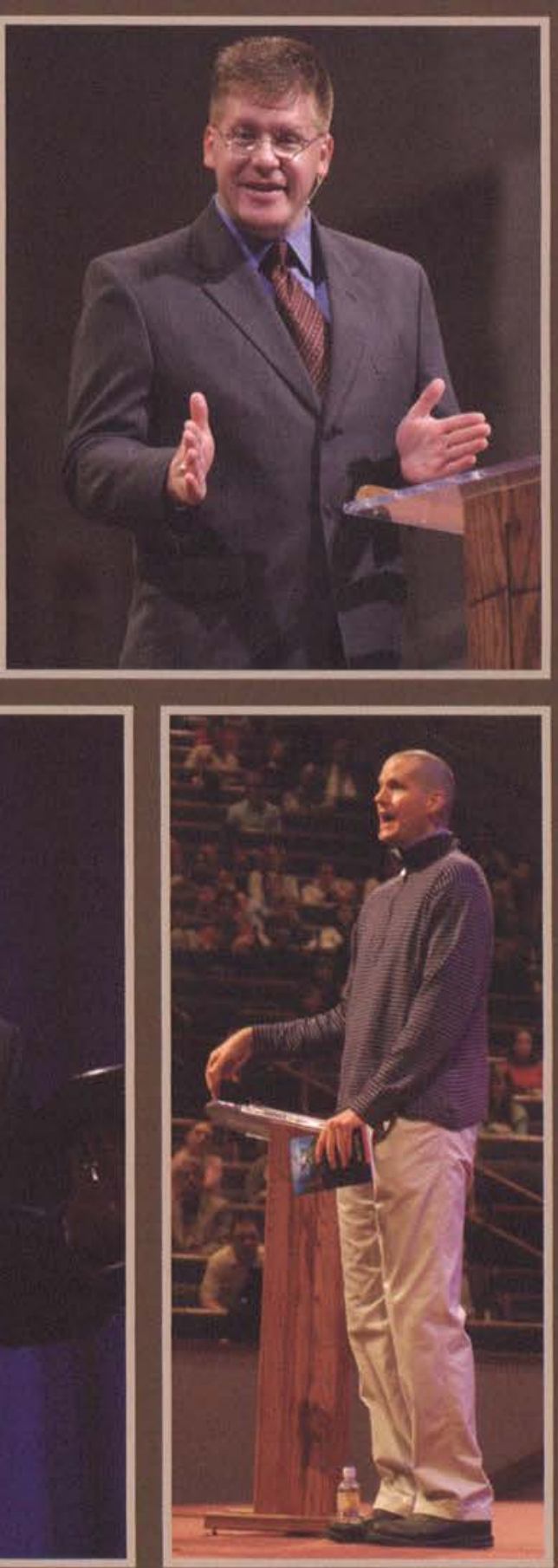

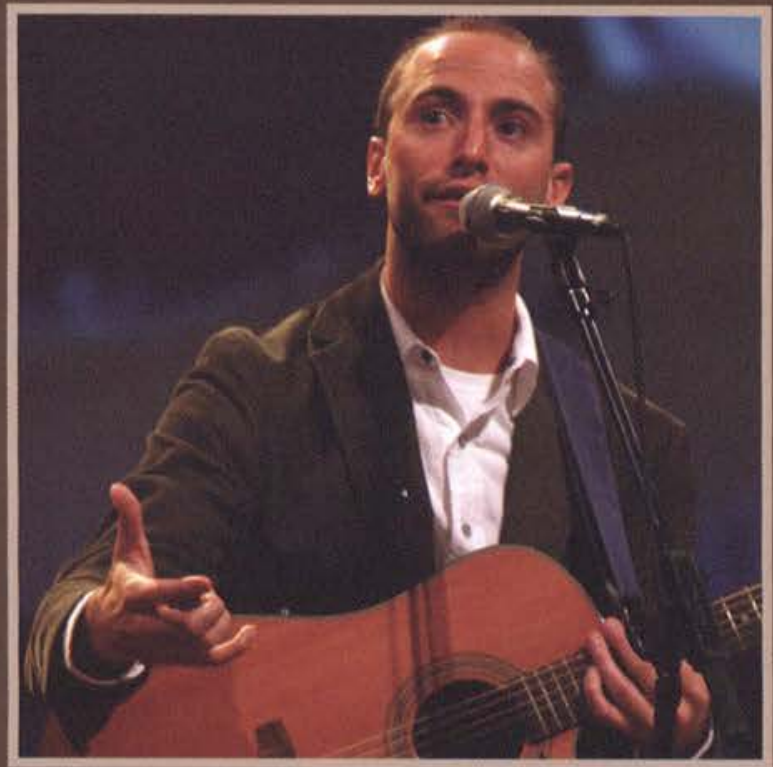

- Singer/songwriter Josh Bales leads the students in a time of worship, reflecting on the creation, fall, and redemption story of the Bible.

4 Mark Cahill, a speaker and author on evangelism, encourages students to be bold about their faith, in the knowledge of the depth of people's spiritual need.

4 Christian Pilet, a missionary serving with the Association of Baptists for World Evangelism, encourages students to cling to Jesus in all circumstances. 


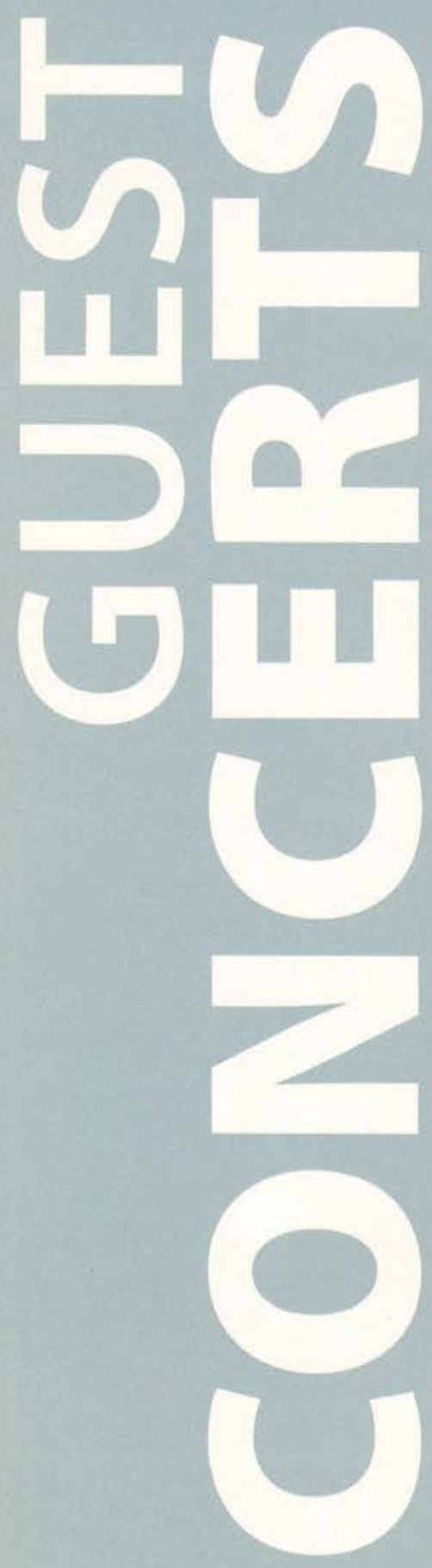

ith the help of the Student Center Activity Board, Cedarville University hosted three guest concerts throughout the year. Not only did these concerts provide weekend entertainment and study breaks, but a great opportunity for the University family to come together to praise and celebrate our amazing God.

Denver and the Mile High Orchestra stopped in again this year as a part of Campus Christmas. Blending their big-band style music with the Christmas spirit put students in the mood for the holidays and reminded them of what lay ahead, past the last crunch of papers and final exams. "Denver and the Mile High Orchestra were absolutely amazing! I loved how they integrated jazz, Christ and fun all into one concert and hope that they will come back again next year," said sophomore Matthew Hollis. This concert was a great way to close out the semester and bring the year of 2006 to a close.

The biggest concert of the year came in February with the hosting of the Newsboys" "Go" Tour, with special guests Kutless and Stellar Kart. They put on a spectacular show of music, fun, and worship that even included a revolving stage! "They were really cool and to actually have the sometimes-monotonous Cedarville way of life broken by a big name musical group was amazing," remembered freshman Jon Small.

With the semester rolling to a close, Shane and Shane, featuring Phil Wickham and Shane McDonald, came to campus in March. "Shane and Shane have such a beautiful gift of glorifying God with song. It was so fun to just sing along with my brothers and sisters in Christ and to be reminded that the body of Christ extends beyond Cedarville," said sophomore Cassie Dobutovich on the duo's visit to Cedarville. This concert also had a personal connection to the University family - a portion of the ticket sales went to the family of junior Dan Knudsen, who suffered a neck injury while on an MIS Spring Break trip to Mexico.

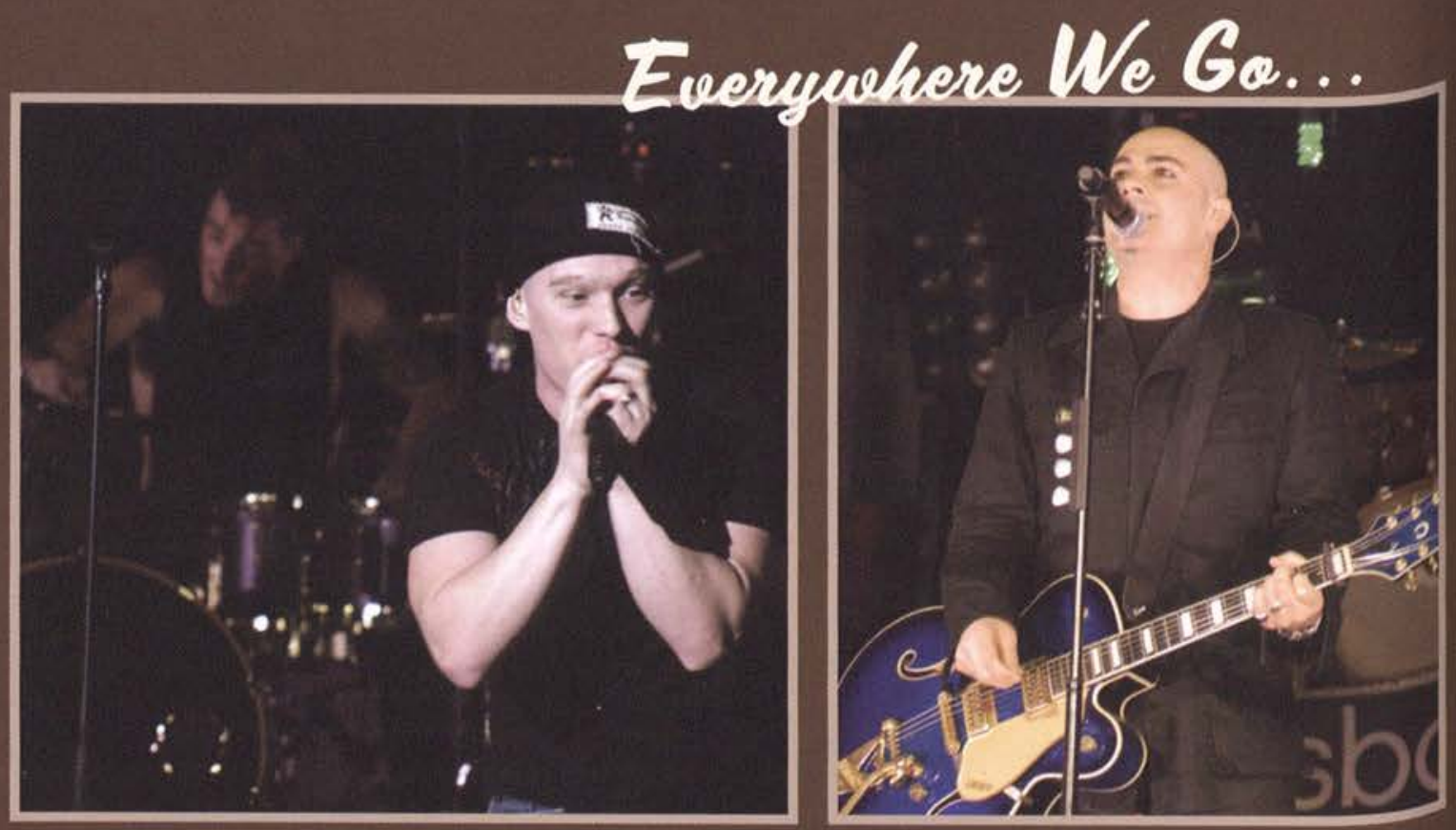

- Lead singer of Kutless, Jon Micah Sumrall, kicks off their portion of the concert.

- Peter Furler leads the audience in worship at the Newsboys "Go" Tour Concert in the Diixon Ministry Center.

- Newboys' pianist Jeff Frankenstein plays a special number. Freshman Erin Landers says, "I liked how each song was based on a story that challeged you in your Christian life."

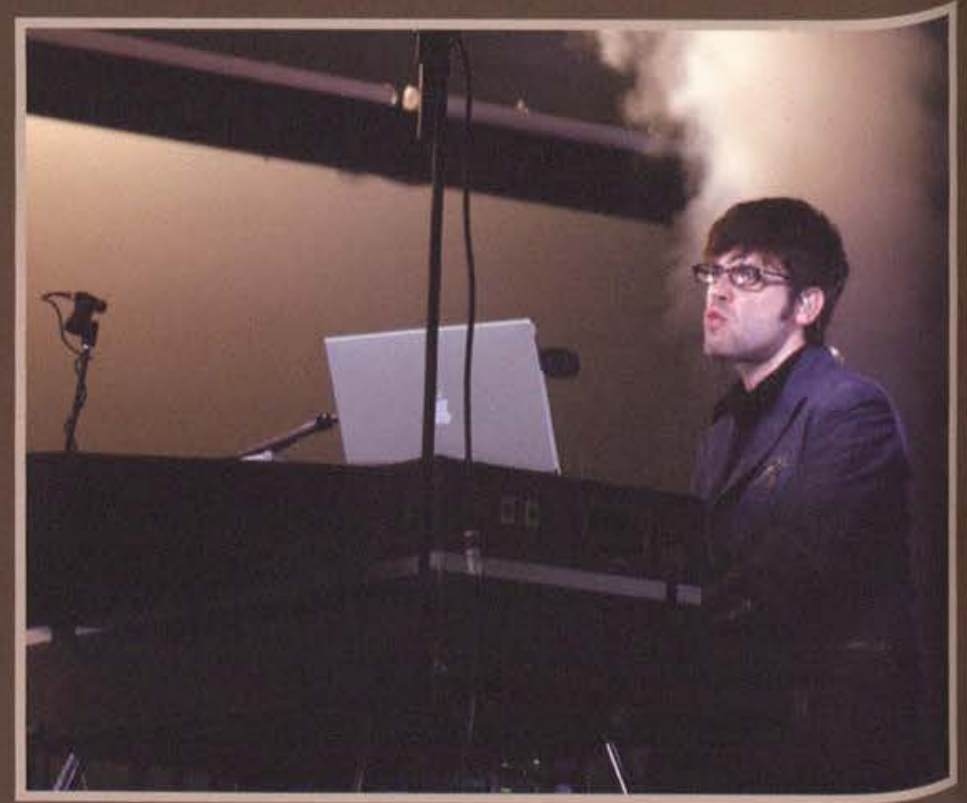




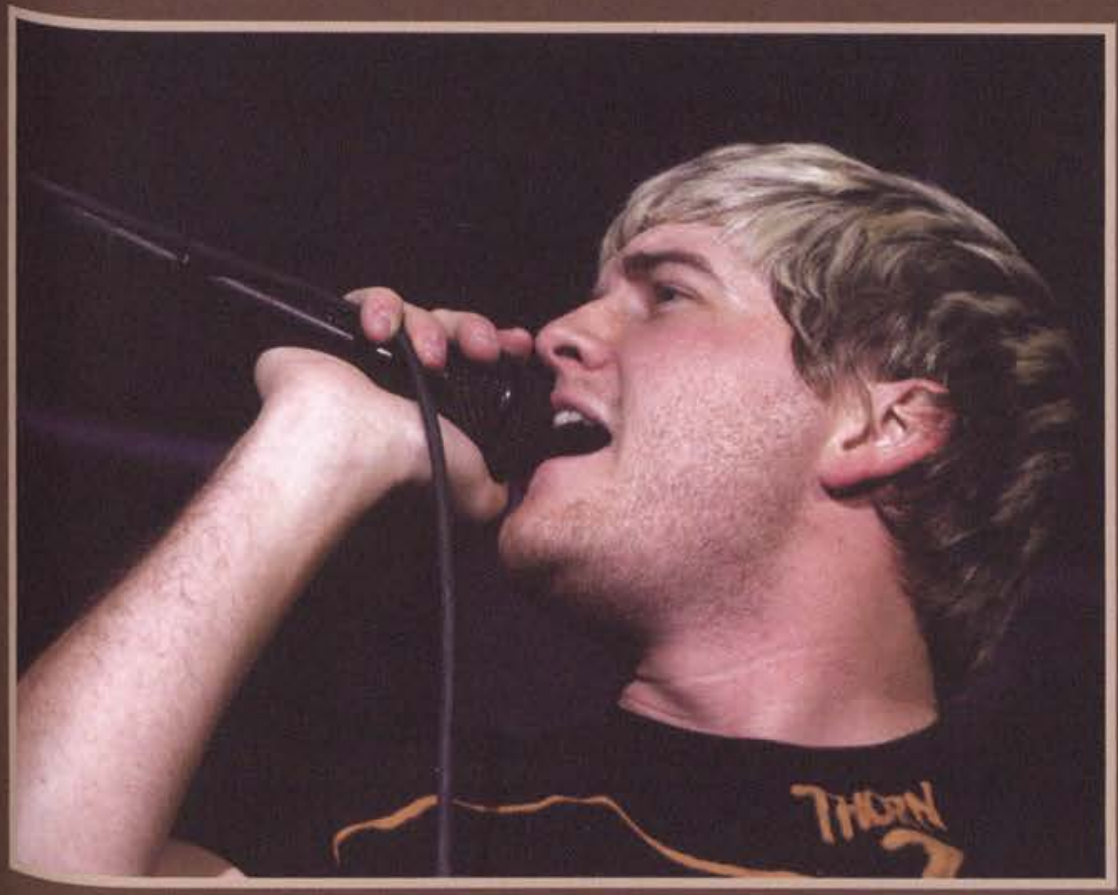

4 Stellar Kart opens up the show and gets the crowd fired up with their hit song, "Activate."

- Phil Wyckam, up-and-coming Christian artist, opens for Shane \& Shane, the spring concert which benefitted the Dan Knudson family.

4 Shane and Shane is well-known in Christian circles as a God-honoring worship band. Junior Michelle Baker-Karl says, "I enjoy listening to their worship albums. Their lyrics show a deep love for God and a compassion for their brothers and sisters in Christ.

Kutless leads the Cedarville student body in worship during their concert, illustrating their desire to impact the world for Christ through their music and lyrics.

- Denver and the Mile High Orchestra brings the holdiay spirit back to Cedarville's campus with both classics and orginial Chrismas music including a blues rendition of "Frosty the Snowman."
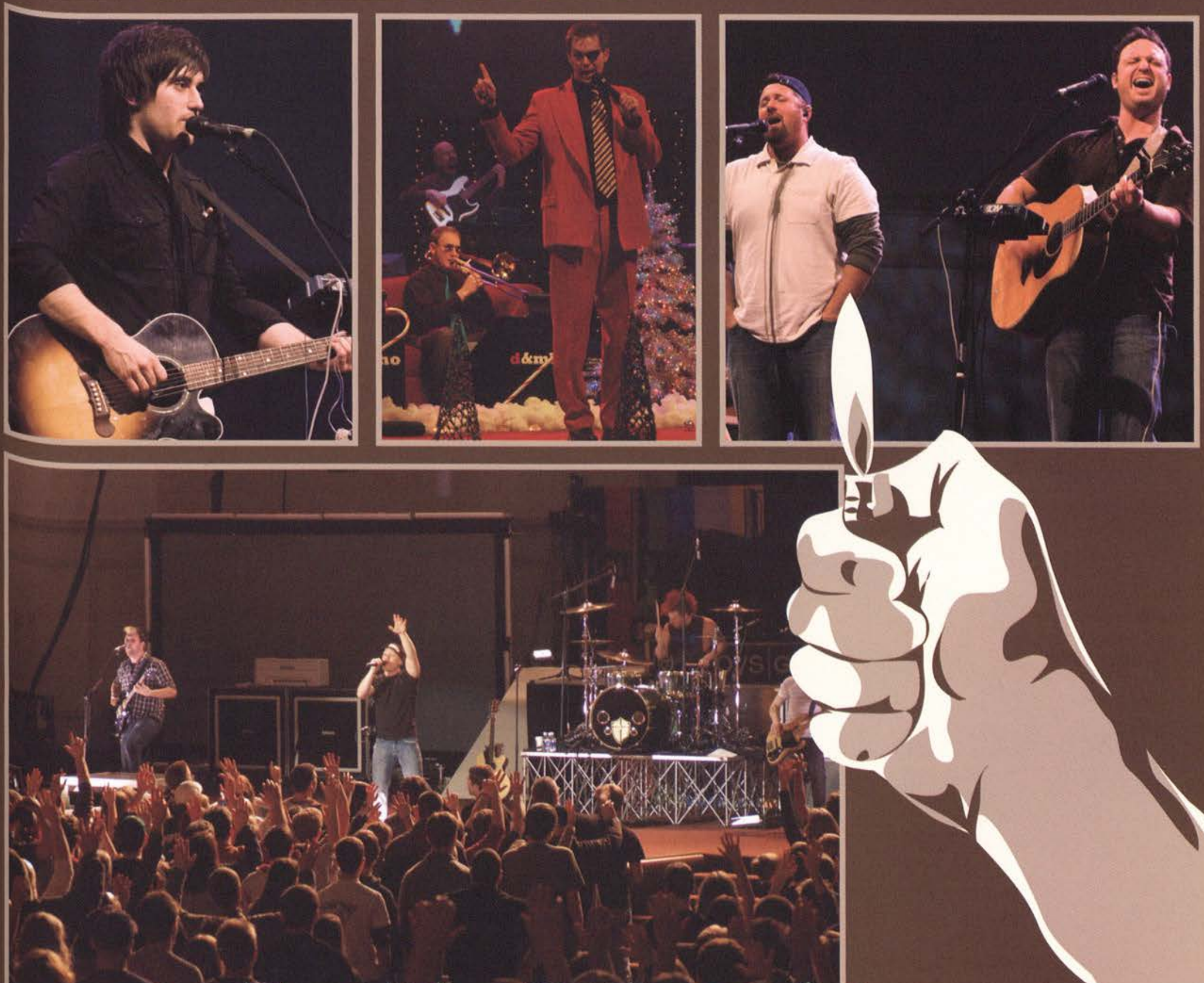


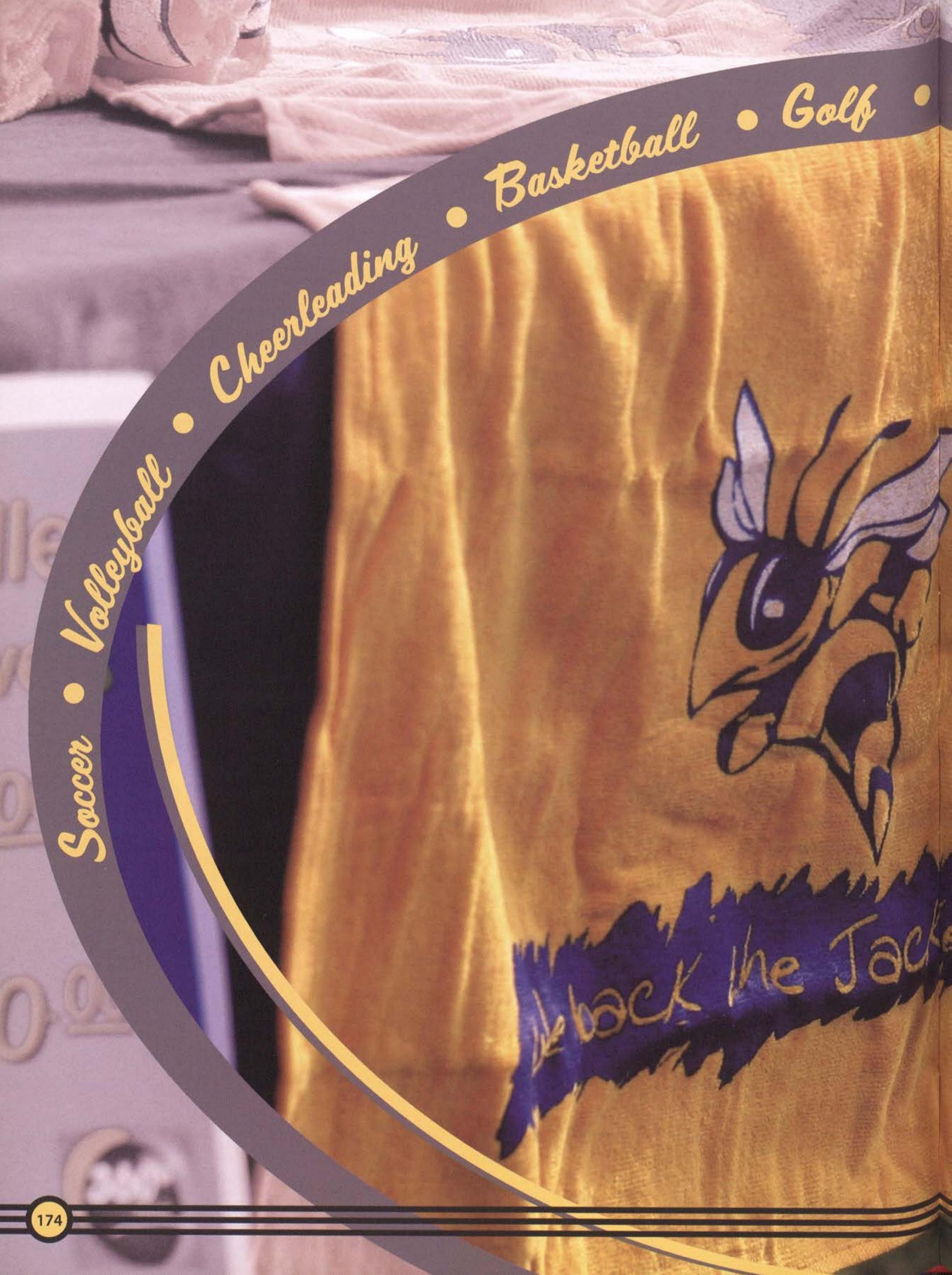





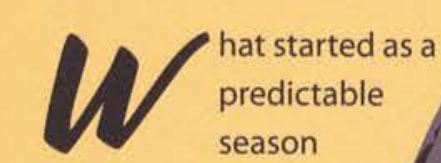

for Cedarville men's soccer became a series of incredible wins and hard, unexpected losses, but they fought their way to the NCCAA championship in Florida and finished their season 9-11-1. The team enjoyed their pre-season in North Carolina where they trained with the Charlotte Eagles. Two of the Eagles players were able to spend time with the guys and share opportunities they have with sports ministry. "We [also] decided some things we would try to do as a team for ministry," said senior Josh Gelser, number 4. Part of the ministry of the Cedarville team was to pray with the other team after every game. This was an impressive act of testimony on the guys' part, especially after a hard loss. After the home games, the opposing team then had dinner with the CU boys. "Meeting players from other schools, hearing their experiences, and sharing mine, was cool," Gelser affirmed.

The guys practiced five days a week working on possession, offense versus defense, and finishing with a scrimmage of some sort. For freshman Jason Buckley, number 24, the most challenging part of being on the team is the "time commitment year round." Another challenge for the guys, as any sports team, was moving on after a defeat. "After a hard loss we felt terrible, but had to go at it again in practice the next day and look forward to our next game," senior Grant Knight, number 8 , asserted, "there were times when I thought our season was over, but we had to keep learning perseverance." Senior Elliot Moore, number 22, added, "As seniors, we were able to unleash our excitement for the game and strengthen our love for each other during our trip to Florida for the NCCAA nationals."

\section{Fincishing The Seasan Strong!}

- "I learned a lot this season about myself and life in general," senior Grant Knight states "Even though we didn't win as much as we had wanted on the field, I believe we had ${ }^{\text {" }}$ lot of success off the field considering the lessons we had to learn."

\section{Season Results}

Siena Heights

Trinity International

Illinois Tech

Indiana Wesleyan

Bethel (Ind.) ${ }^{+}$

Houghton $^{+}$

Wittenberg

Shawnee State*

Mt. Vernon Nazarene*

Urbana*

Notre Dame (Ohio)

Rio Grande*

Tiffin*

Walsh*

Mid-Continent

T 1-1 (2ot)
W 2-1 (2ot)
L 2-3
L 1-2
L 1-2
L 0-2
L 1-2 (ot)
W 6-0
L 1-2
L 2-3
W 1-0
L 0-5
W 3-2 (ot)
W 2-1
L 0-4

Ohio Dominican*

Malone*

Spring Arbor**

Master's ${ }^{++}$

Palm Beach Atlantic ${ }^{++}$

MidAmerica Nazarene ${ }^{+}$

L 2-3

W 5-0

W 5-0

W 2-1 (ot)

W 1-0

L $0-2$

\section{9-11-1 (.452) Overall}

4-4-0 (.500) American Mideast

Conference South Division*

* American Mideast Conference South Divisio matches

+Dave Jones Memorial Classic; Cedarville

** NCCAA Midwest Regional

* NCCAA National Tournament; Kissimmee. 

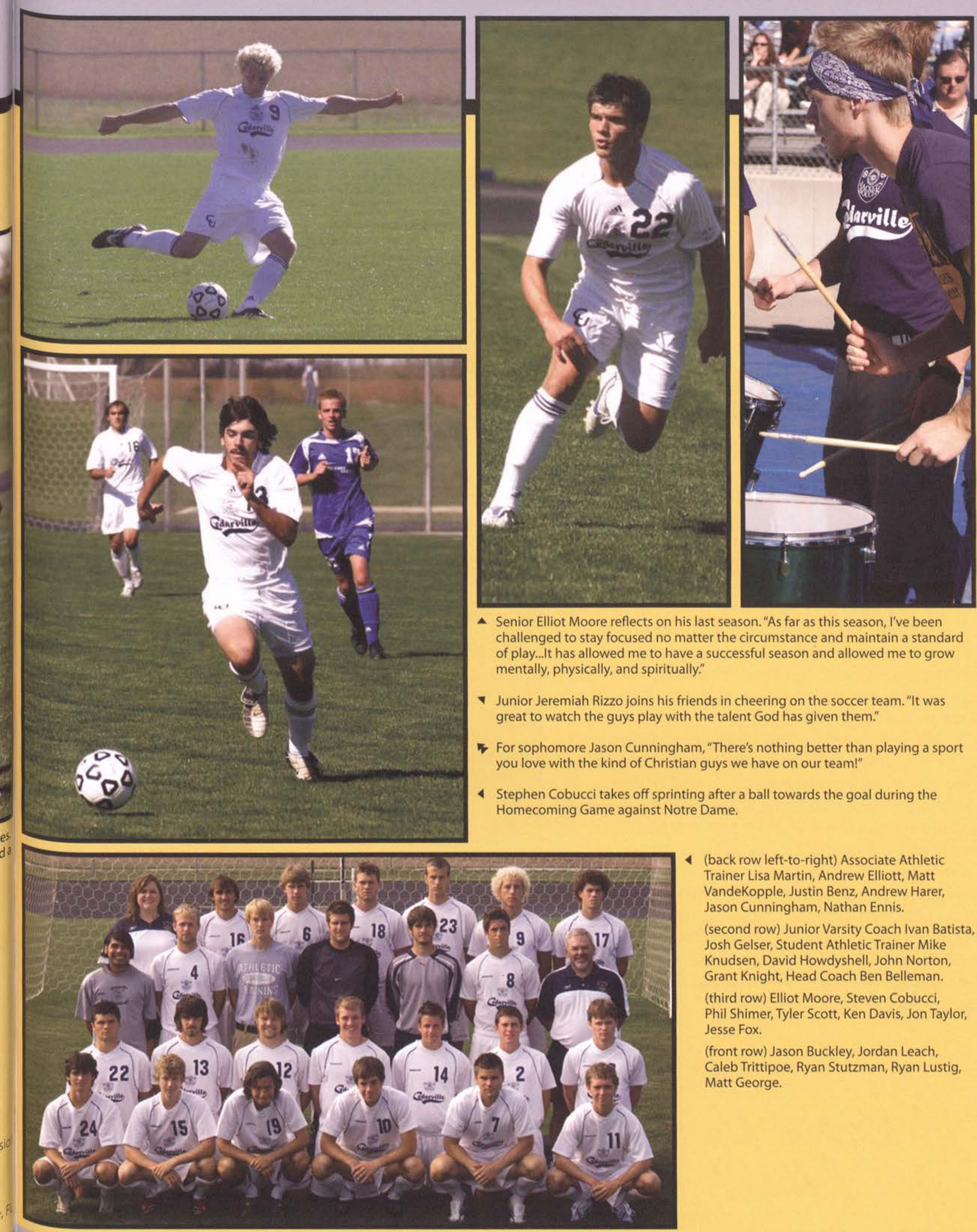

Senior Elliot Moore reflects on his last season. "As far as this season, I've been challenged to stay focused no matter the circumstance and maintain a standard of play... It has allowed me to have a successful season and allowed me to grow mentally, physically, and spiritually."

Junior Jeremiah Rizzo joins his friends in cheering on the soccer team. "It was

For sophomore Jason Cunningham, "There's nothing better than playing a sport of Christian guys we have on our team!

Stephen Cobucci takes off sprinting after a ball towards the goal during the Homecoming Game against Notre Dame.

(back row left-to-right) Associate Athletic Trainer Lisa Martin, Andrew Elliott, Matt VandeKopple, Justin Benz, Andrew Harer, Jason Cunningham, Nathan Ennis.

(second row) Junior Varsity Coach Ivan Batista, Josh Gelser, Student Athletic Trainer Mike Knudsen, David Howdyshell, John Norton, Grant Knight, Head Coach Ben Belleman.

(third row) Elliot Moore, Steven Cobucci, Phil Shimer, Tyler Scott, Ken Davis, Jon Taylor, Jesse Fox.

(front row) Jason Buckley, Jordan Leach, Caleb Trittipoe, Ryan Stutzman, Ryan Lustig, Matt George. 

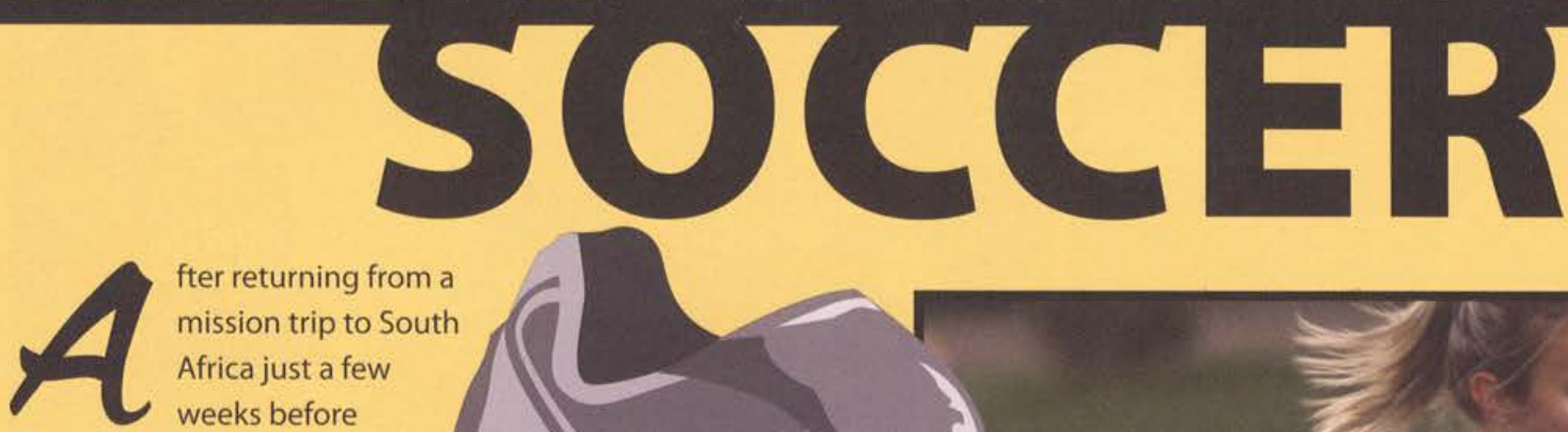

fter returning from a mission trip to South Africa just a few weeks before school started, the Cedarville Women's soccer team was pumped and ready to begin their season. When discussing their trip, senior midfielder Abby Price said, "It was a stretching and growing experience where we were able to share about God's love through the platform of soccer. It was such a great experience to be able to serve people and share about our salvation through the sport that we love." But when they arrived at Cedarville, it was time to get to work. The team started their 2006 season strong, beating Asbury, Geneva, and Campbellsville. After a loss to Georgetown, they won two in a row against Taylor and Grace. The ladies' season looked extremely promising, and many Cedarville students spent their evenings cheering their friends and classmates on to victory. Around the middle of the season, however, the team encountered a rough patch, losing or tying eight games in a row.

According to freshman goalkeeper Brianne Barnes, "Most of our losses were by $1 \mathrm{goal}$... I think if we had gotten a few different bounces we would have won some of those close games because there were times we dominated the game and just couldn't score." A few weeks later, the team beat Rio Grande and Walsh handily, but sadly could not pull out wins for their last two games. They finished with a record of 7-9-2 overall, and although they did not make the playoffs, freshman defensive player Torrie Pepper had only kind words to say about her team: "We have a great group of girls with a lot of talent... we worked so well together as a team." Overall, it was an enjoyable year for the ladies as they played soccer, but also as they developed deeper relationships with each other.

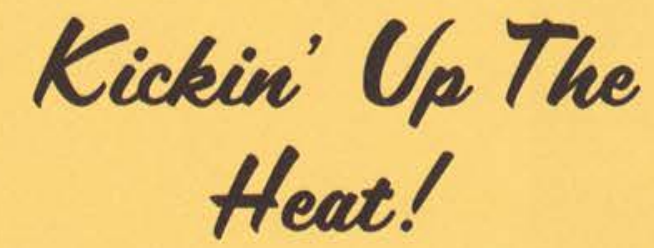

$\begin{array}{ll}\text { Asbury } & \text { W 2-0 } \\ \text { Geneva } & \text { W 4-1 } \\ \text { Campbellsville } & \text { W } 3-1 \\ \text { Georgetown } & \text { L } 0-1 \\ \text { Taylor } & \text { W } 2-1 \\ \text { Grace } & \text { W } 2-1 \\ \text { Indiana Wesleyan } & \text { L } 0-1 \\ \text { Saint Francis (Ind.) } & \text { L O-1 } \\ \text { Ohio Northern } & \text { L 1-2 } \\ \text { Wilmington } & \text { L O-2 } \\ \text { Tiffin } & \text { T 2-2 (2ot) } \\ \text { Mount Vernon Nazarene* L 0-3 } \\ \text { Shawnee State* } & \text { T 0-0 (2ot) } \\ \text { Urbana* } & \text { L 0-1 }\end{array}$

\section{Season Results}

$\begin{array}{lll}\text { W 2-0 } & \text { Rio Grande* } & \text { W } 7-0 \\ \text { W 4-1 } & \text { Walsh* } & \text { W 2-0 } \\ \text { W 3-1 } & \text { Ohio Dominican* } & \text { L 1-2 } \\ \text { L 0-1 } & \text { Malone* } & \text { L 1-2 } \\ \text { W 2-1 } & \text { Spring Arbor } & \text { L } 0-2\end{array}$

\section{7-10-2 (.421) Overall} 2-4-2 (.375) American Mideast Conference South Division*

*American Mideast Conference South Division matches

+NCCAA Midwest Regional 
Sophomore midfielder Lisa Burgman takes the ball up the line to look for a cross.

- Freshman goalkeeper Brianne Barnes says her secret to success in this Homecoming game is "Warming up...to make sure that I shut out the opposition."
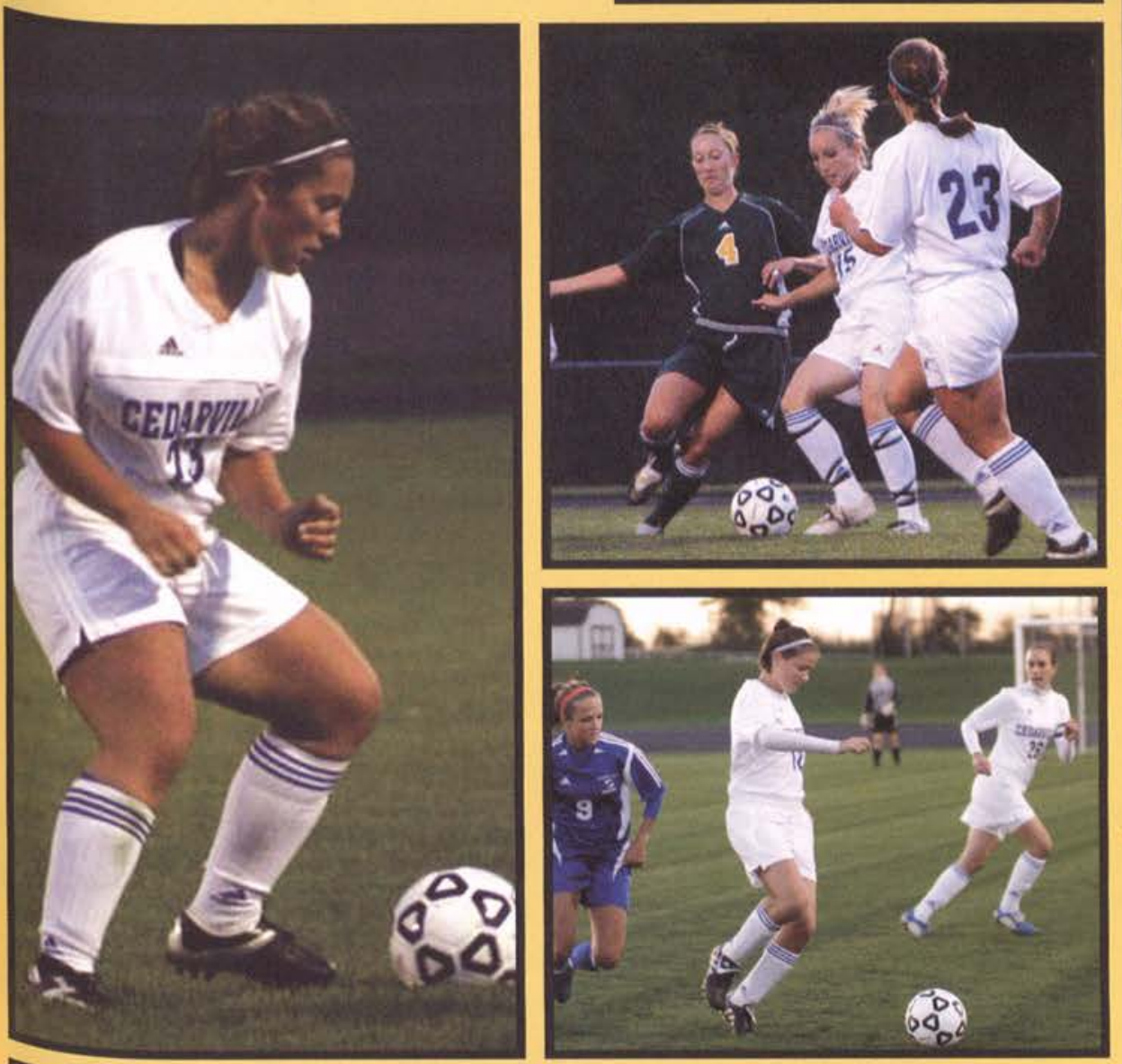

.

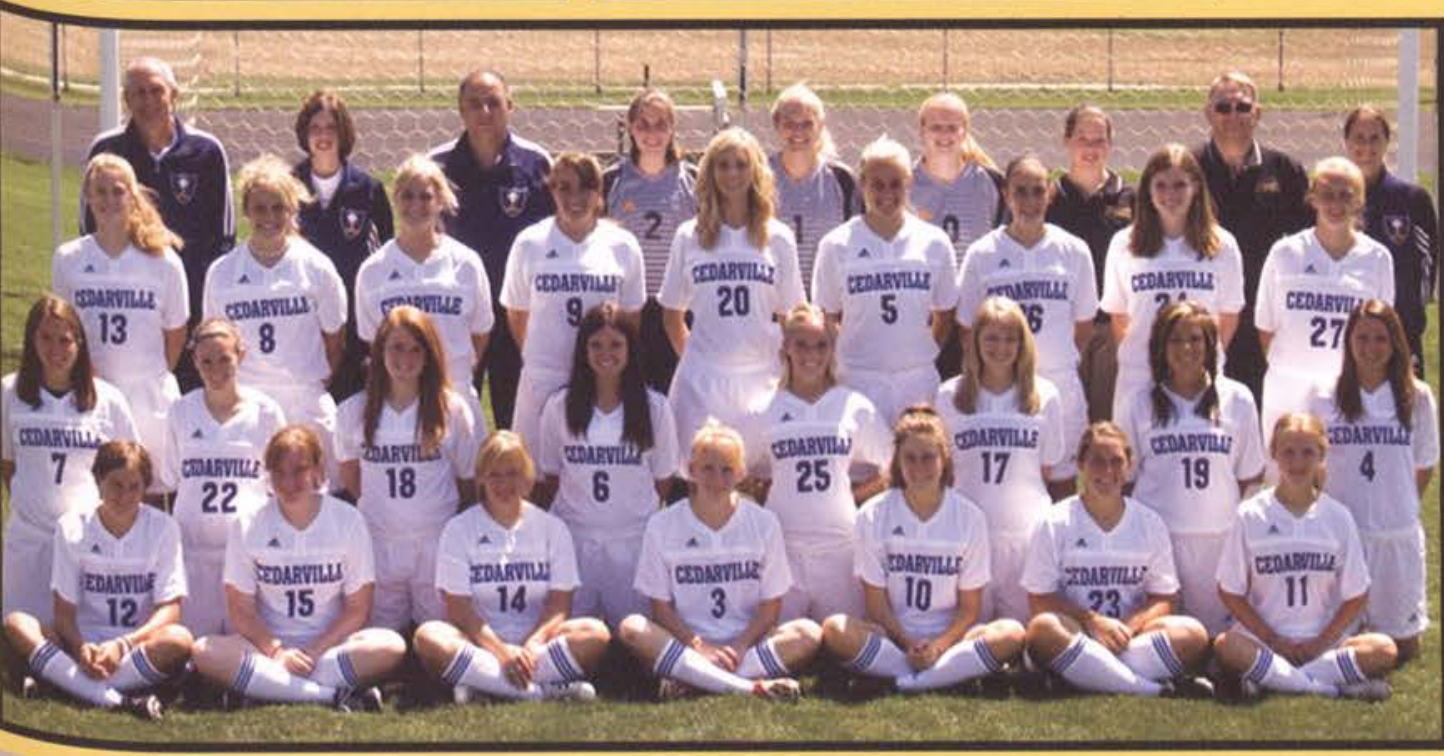

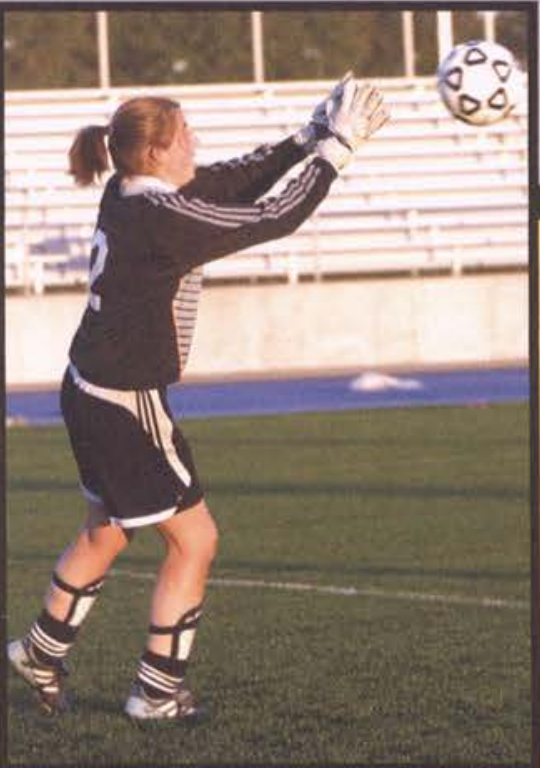
above), are loved by their younger cohorts. Freshman player Megan Walter adds, "They genuinely care about us."

Working together is extremely important for this team, and as sophomore defender Katie Koch states, "We were a lot closer and united as a team this year, and it proved to be an effective way to minister to other teams by our encouragement on the field."

Senior midfielder Colleen Derry regrets that she isn't doing anything particularly funny in the picture. "I'm just trying to get around a defender," she says sadly.

- While freshman midfielder Torrie Pepper scrambles to reach the ball, junior defenser Kristin Merkel prepares for a pass. Pepper enjoyed being on the team with Merkel. "Merkel was the first one I really got to know before we went to South Africa this summer. She made me feel welcome and very comfortable as an incoming freshman."

4 (back row left-to-right) Head Coach John McGillivray, Assistant Coach Krista Mattern, Assistant Coach Kevin Roper, Brianne Barnes, Kelly Teague, Amber Laing, Student Athletic Trainer Rebecca Phillips, Associate Athletic Trainer Bob Duchardt, Assistant Coach Kelli Zlateff.

(second row) Sarah Allison, Lisa Burgman, Jessica Thomas, Katie Koch, Sonja Storhaug, Karen Ruhlman, Kristin Merkel, Megan Walter, Sari Stroud-Lusk

(third row) Kelly Wise, Allyson Castle, Erin Landers, Evonne Fearnot, Lisa Blackburn, Lauren Haegele, Bethany Cottings, Hannah Wailes.

(front row) Torrie Pepper, Erin Hayes, Heather Merrell, Kristen Malpass, Abby Price, Colleen Derry, Jillian Losee. 

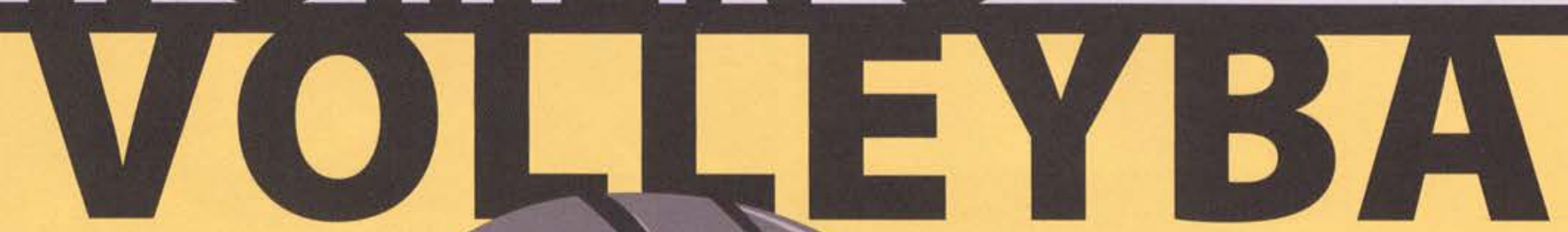

$F$ volleyball team has consistently upheld a tradition of excellence, winning countless championships and tournaments under the strong leadership of Coach Theresa Clark. However, the fall 2006 season brought an exciting change: Melissa Hartman acting as Head Coach. Hartman's skills proved to everyone that she was more than capable of maintaining this award-winning institution.

Hartman mentioned that although some areas of the position, such as "stretching the girls academically and spiritually, without crushing their spirit," was a challenge, she found that the team showed her "significant respect" and made the season an "overwhelming blessing."The blessed nature of the season was clearly evidenced in their second straight NCCAA championship win and the subsequent naming of Coach Hartman as Coach of the Year. Other achievements included Junior Sarah Zeltman being named Player of the Year by both the AMC and NAIA Region IX division and adding the consecutive home win count to 51 in a sweep against Tiffin.

Junior Melissa Parmerlee noted Coach Hartman's positive influence on the team. She said that Coach Hartman showed them that "every time we step on to the court, we do so ready to worship the God that has created, sustained, and loved us." Freshman Liz Sweeney agreed, adding, "I'm so glad that during my first year of playing volleyball at Cedarville I have had the privilege of being instructed by her." Coach Hartman shared a mutual respect for the team's character. She encapsulated the season when she said that the girls "have put their hearts on the line for each other. They have stuck together as a unit, a true team, working out differences and keeping God's Glory the main priority."

\section{Serving Far Gad's Glary!}


(back row left-to-right) Amy Garner, Justine Christiaanse, Sarah Zeltman, Julia Bradley, Anne Lohrenz, Emily Berger.

(middle row) Student Athletic Trainer Katie Tuttle, Associate Athletic Trainer Siobhan Fagan, Liz Sweeney, Kelly Theiss, Student Assistant Coach Heath Starcher, Head Coach Melissa Hartman.

(front row) Libby Short, Rachel Thompson, Melissa Parmerlee, Sarah Sheers, Maija Hampton.
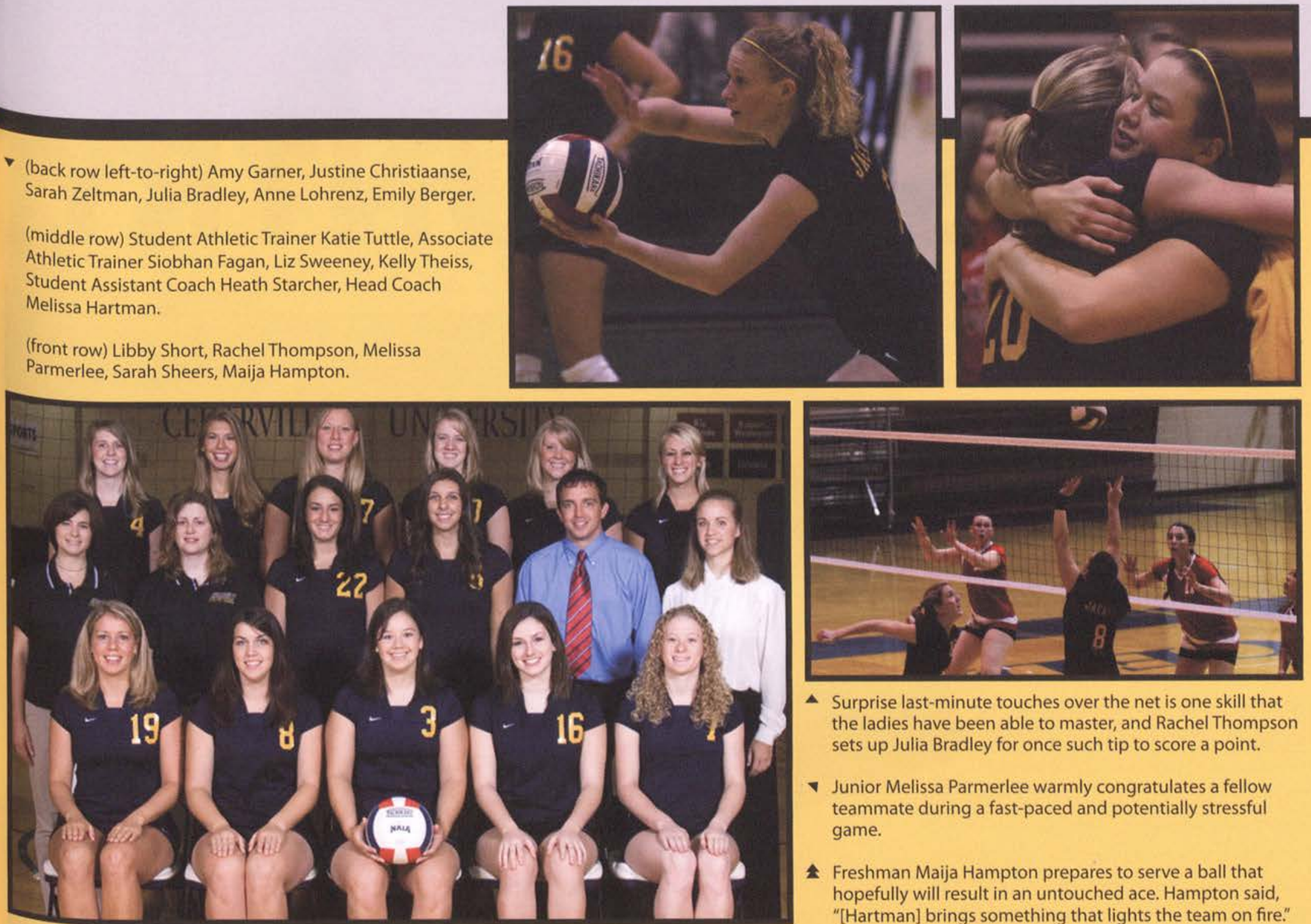

- Surprise last-minute touches over the net is one skill that the ladies have been able to master, and Rachel Thompson sets up Julia Bradley for once such tip to score a point.

- Junior Melissa Parmerlee warmly congratulates a fellow teammate during a fast-paced and potentially stressful game.

- Freshman Maija Hampton prepares to serve a ball that hopefully will result in an untouched ace. Hampton said, "[Hartman] brings something that lights the team on fire."

\section{Season Results}

Taylor-ort Wayne

Graces

Saint Francis (Ind.)*

Bethel (Ind.)"

Grace

Saint Francis (Ind.)"

Taylor

Davenport ${ }^{\&}$

Madonna\&

Rio Grande 8

Taylor:

Cumberland

Pikeville

Malone+

Rio Grande

Shawnee State

Central State

Berry"

Cornerstone

Albertson\%

Hastings ${ }^{\%}$

Urbana*

Ohio Dominican

Malone:
Mount Vernon Nazarene

Central State

Shawnee State

Ohio Dominican *

Rio Grande

Tiffin

Walsh (At Tiffin)

Urbana*

Grace $^{++}$

Spring Arbor ${ }^{++}$

Indiana Wesleyan+ ${ }^{++}$

Mount Vernon Nazarene

Malone

Walsh

Rio Grande

Faulkner"*

Hope International"

Oakland City"*

Bethel (Ind.) $)^{n *}$

Mount Vernon Nazarene ${ }^{*}$

Palm Beach Atlantic ${ }^{* \prime}$

Tiffin

Houghton"

Geneva"
Walsh"

Maine-machias ${ }^{96 \%}$

Tiffin ${ }^{96 \%}$

Walsh 996

Albertson ${ }^{\text {s }}$

California Baptist ${ }^{\text {ss }}$

Lee $^{\text {SS }}$

\section{5-10 (.818) Overall}

$12-4$ (.750) American Mideast Conference South Division*

- American Mideast Conference South Division

Matches

Grace Classic; Winona Lake, In

"19th Annual Cedarville Invitational

\& Madonna Invitational; Livonia, Mi

+ Cumberland Classic; Lebanon, Tn

\% Saint Francis Challenge; Fort Wayne, In

+ Nccaa Midwest Regional; Marion, In

" Nccaa National Tournament; St. Paul, Mn

" Amc Tournament; Rochester, Ny

${ }^{96 \%}$ Naia Region Ix-x Tournament; North Canton, Oh

ss Naia National Tournament; Columbia, Mo 
fyou don't enjoy running, it might be hard to understand why anyone would enjoy a sport that sometimes requires 8,000 meters of running at its matches, but a love for running and an even greater passion for the Lord is what brought this year's cross country team together. "We're a very close team," senior Bryan Pittman said. "We've all got something in common: we're all crazy enough to run."

Beginning the year ranked 12 th in the NAIA, the Men's Cross Country Team quickly proved themselves as noteworthy competition, placing 3rd in the Southeastern Classic where 25 teams met to race. Cedarville went on to place 2 nd overall at the AMC Championship meet, where Freshman Daniel Roberts won individual title by 29 seconds. Both Roberts and junior Justin Gutierrez earned spots on the All-AMC First Team, while Bryan Pittman and junior Justin Herbert were named Second Team Honorees.

Cedarville placed 2 nd at the 39th Annual NCCAA Cross Country Championship, with Roberts, Gutierrez, and Pittman on the NCCAA All-American Unit. Pittman and senior Matt Clark were also recognized as NCCAA All-American Scholar Athletes, maintaining a minimum $3.40 \mathrm{GPA}$.

The Yellow Jackets ended the season placed 8th overall in the NAIA. At the final NAIA National Championships, Roberts finished his rookie season placing 5 th individually, earning All-American Scholar Athlete. The team came in at 10 th place, and Cedarville had 323 points for the highest finish among American Mideast Conference teams. Matt Clark was also recognized as an NAIA All-American Scholar Athlete.

The team attributed its success to their commitment to each other and their commitment to the Lord. Beginning each run by shouting together as a team, "All For The Glory of God," and ending each meet in prayer, the men's cross country team sought to do their best for God and each other.

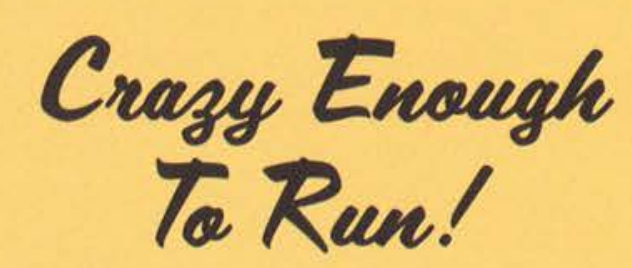
the new Elvin R. King Cross Country Course.

\section{Season Results}

Miami Invitational

Cedarville Invitational

Louisville

All-Ohio Intercollegiate

Southeastern Classic

AMC/NAIA Region IX

Championship

NCCAA NATIONALS

NAIA Nationals

NAIA Final Ranking $2^{\text {nd }}$ of 4

$1^{\text {st }}$ of 13

$14^{\text {th }}$ of 36

$6^{\text {th }}$ of 42

$3^{\text {rd }}$ of 25

$2^{\text {nd }}$ of 15

$2^{\text {nd }}$ of 22

$10^{\text {th }}$ of 28

$8^{\text {th }}$

$\mathbf{N}$ Ir RY $r$ 


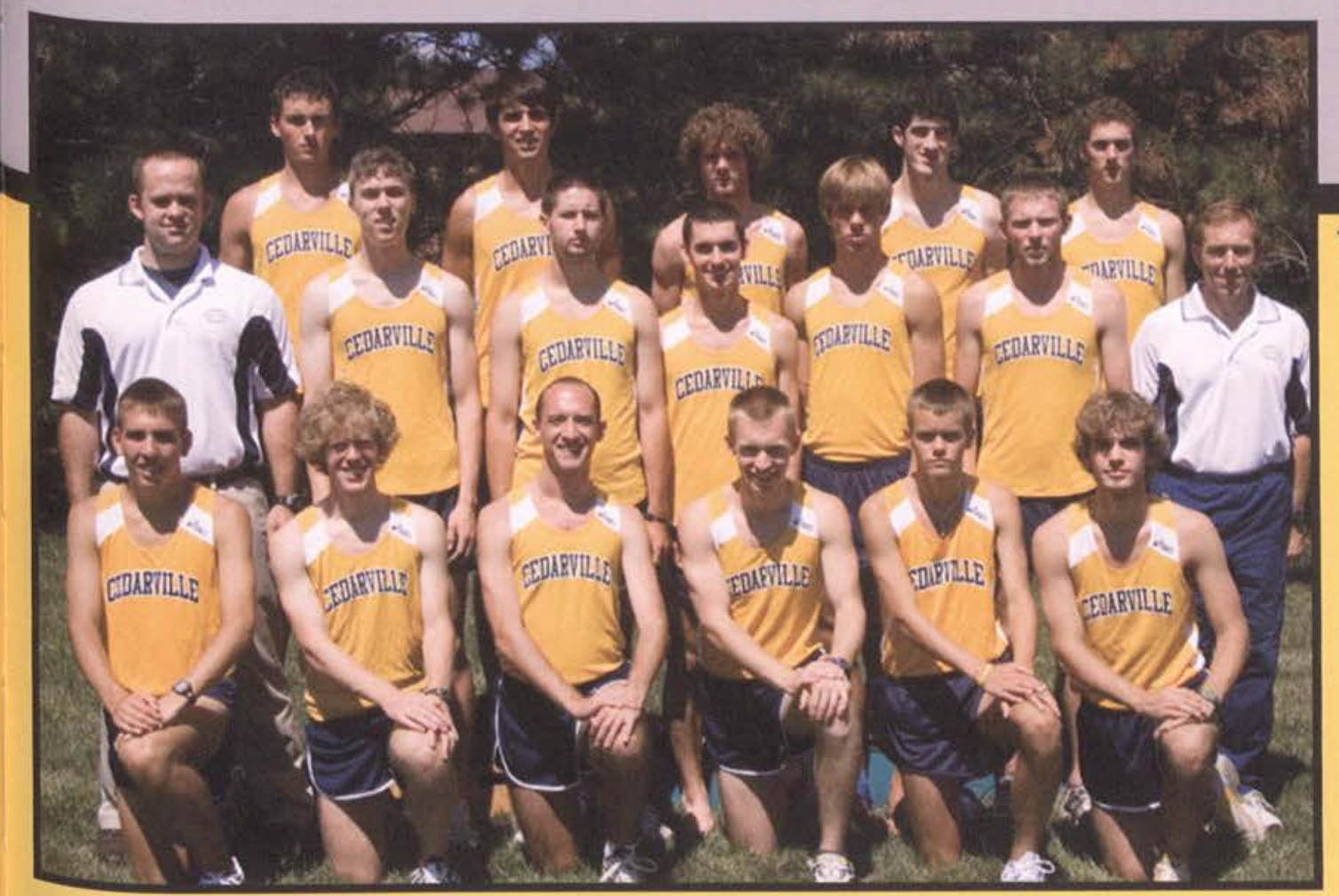

(back row left-to-right) Justin Gutierrez, Matt Silveira, Justin Herbert, Jordan Seibert, Kevin Kuhn.

(middle row) Assistant Coach Steve Powers, Rob Trennepohl, Aaron Griggs, Seth Campbell, Joshua Saunders, Jud Brooker, Head Coach Paul Orchard.

(front row) Daniel Roberts, Josh Maughon, Bryan Pittman, Matt Clark, Peter Brandt, Josh Ohms.

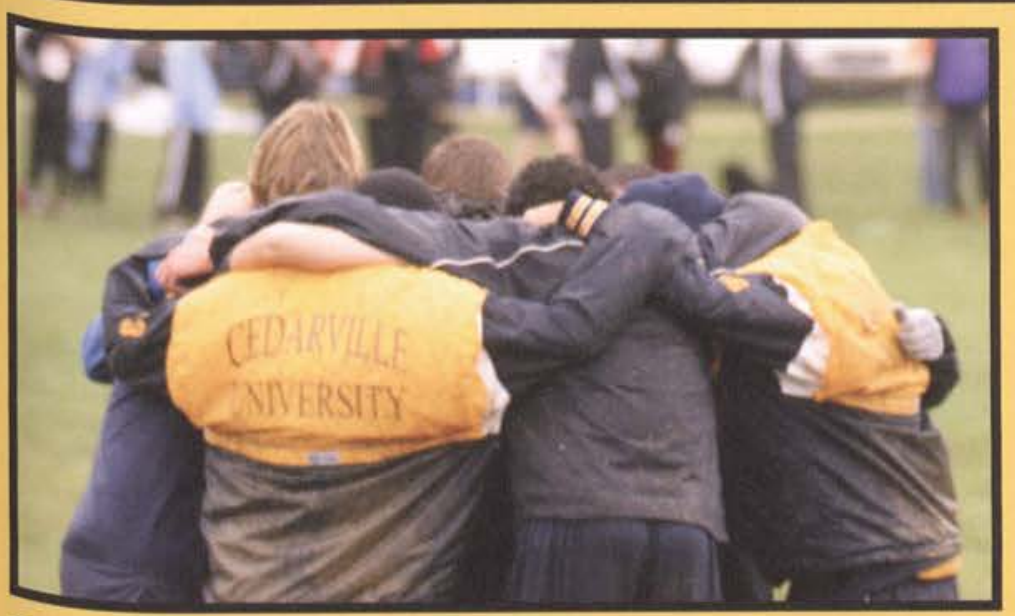

4 Team members huddle together during a meet to pray. "Our team is awesome," freshman Daniel Roberts notes. "We actually have a God-focused team, and you don't see that a lot- even in Christian schools."

- Senior Bryan Pittman runs hard during a race. He comments, "It takes a unique person to want to run like we do - but the feeling of accomplishment afterwards is great."

- "The neat thing about our team is that we're very close... everybody brings something to the team," senior Matt Clark states. "We're a deep team. If someone gets hurt, there's someone there to fill in for them."

- Sophomore Jud Brooker notes, "The pack is what keeps us up there." Senior Justin Herbert and sophomore Matt Silveira compete alongside each other.
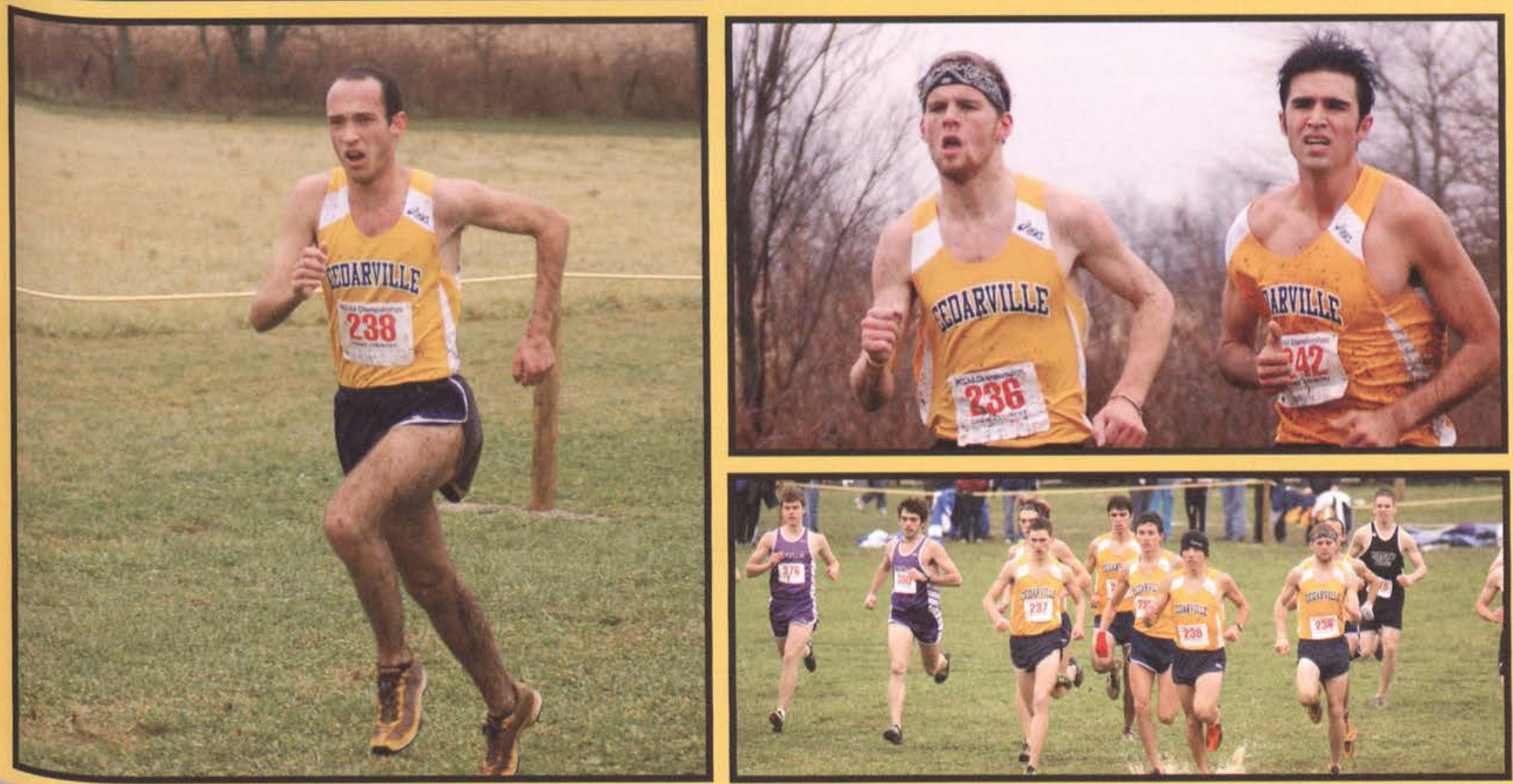


\section{WOMEN'S}

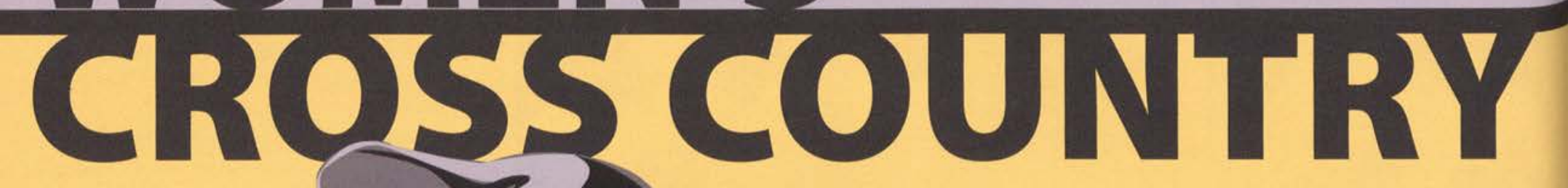

\section{7 he Lady Jackets Cross Country team started}

out the year ranked 6 th in the NAIA. Beginning what would prove to be an excellent season, the team took first place in the Southeastern Classic at Berea College for the second consecutive year. Later in the AMC Championships, Samantha Maat, Elisabeth Pyles, Lydia Wong, and Audree Goodew all contributed to another 1st place win, securing spots on the ALL-AMC first team after finishing in the top seven.

A highlight of the year was the achievement of a team goal - winning the NCCAA Cross Country Championships. This particular meet was the first to be held at the new Elvin R. King Cross Country Course, named after long-time Cedarville coach, Elvin King, and was made especially memorable by five of the ladies running All-American races. As the Lady Jacket Cross Country Coach and NCCAA Coach of the Year, this was King's 6th title.

The women's team finished 5th in the NAIA women's final cross country rating of the season. At the NAIA Nationals, the Lady Jackets came in 2nd place - the highest win since a 1st place finish in 2001. Senior Samantha Maat and juniors Leanne Crunelle and Julie Martz were honored as NAIA All-America Scholar Athletes, while Maat, Pyles, and Santos produced AllAmerica efforts at the race, placing in the top 30 finishers.

Achieving a successful cross country season was not all this team accomplished. The women also sought to reach out to others at their meets through a water bottle ministry, which included putting together water bottles, gospels of John, and contact information in order to start and maintain relationships with the other teams. Sophomore Brittany Simpson said of the ministry, "It's really good; our chaplain had been telling us we need to have a mix between boldness and sensitivity. I'm not quite as bold, so it's definitely made me get out there and share my faith." The team ran strongly and faithfully, striving continually after their motto, "Run For Him."

\section{Running For Him!}

- Sophomore Elisabeth Pyles says, "I love that our cross country team not only works hard and encourages each other to improve our running and competing, but also to 'Run for Him.'

- Sophomore Brittany Simpson runs hard at a race. She notes about the team, "Our team chemistry is awesome this yearwe're really close." 

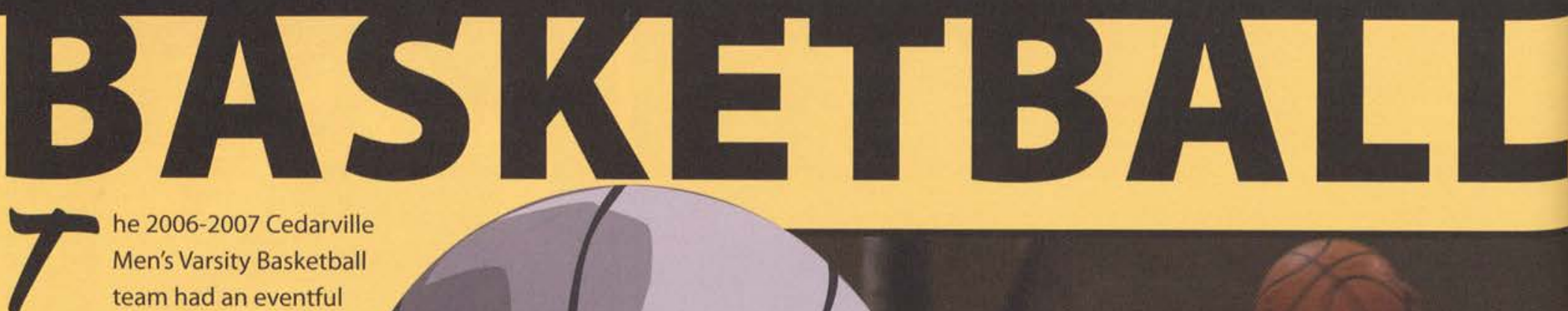

he 2006-2007 Cedarville Men's Varsity Basketball team had an eventful and exciting year. They had a successful season ending with a 23-8 record and a nationwide placing of 13 th in the official NAIA Division II rankings. They played over 30 games during their season from November to March, and they averaged a total of 83 points per game against difficult opponents such as Mount Vernon, Shawnee State, and Urbana.

With six incoming freshman players, there were many new faces on the team this year, but one thing that made Cedarville's basketball team so unique was the personal interest that the coaches and players had for each other, which led to the quick building of team unity. Freshman Haddon Anderson said, "I enjoyed being a part of the team this year because of the relationships. It was a great group of guys who became really close and sincerely cared for each other. It was unlike any other team I've ever played on because of how close we were." The five graduating seniors this year were Maicol Venter, Tyler Yoder, Guy Rathmell, Eric Leininger, and Josh Greve. Junior Ryan Reep said, "This season was very special to me, and was unlike any team I've ever been a part of. For one, I'm grateful to be a part of a team that so selflessly cared for each other. This was due in a large part to the exceptional leadership of our seniors. They led by example, showing us how to glorify God through both the wins and more importantly the losses. I really do believe that it was because of the unselfish attitude of each member of our team that the Lord chose to bless us as He did, allowing us to win the South Division of the AMC and advance to the elite eight of the national tournament." All in all, Cedarville not only had a successful year but also built valuable relationships among the players.

\section{Swoosk!}

Lindsey Wilson

Miami-Middletown

Bryan+

Daemen $^{+}$

Wilberforce*

Cincinnati Christian

Urbana*

Ohio Dominican*

Wittenberg

Walsh*

Wooster

Huntington

Mt. Vernon Nazerene*

Tiffin*

Shawnee State*

Central State

Ohio Dominican*

Malone*

Rio Grande*

\section{Season Results}

W (84-81)

W (104-80)

W (84-74)

W (76-63)

W (98-79)

W (89-65)

W (76-67)

W (63-60)

L (61-66)

W (87-76 ot)

L (95-104)

L (88-94)

L (77-84)

L (71-78)

W (93-90 ot)

W (89-85 ot)

W (77-72)

W (86-79)

W (96-72)

Walsh*

Wilberforce*

Urbana*

Tiffin*

Rio Grande*

Malone*

Evangel*-
Mount Vernon*

Shawnee State*

Point Park**

Warner Pacific +

Mayville State+.

23-8 (.742) Overall

15-3 (.833) American Mideast Conf. S Division*

*Conference South Division

+29th Annual Cedarville Invitational

* AMC Championship Game; Pittsburgh, PA

$" 16$ th Annual NAIA Division II National TournamentPoint Lookout, MO
W (90-67)

L (70-77)

W (60-57)

W (97-74)

W (88-73)

W (88-61)

W (73-70)

W (86-83)

L (83-89)

W (97-83)

W (86-75)

L (81-82) 


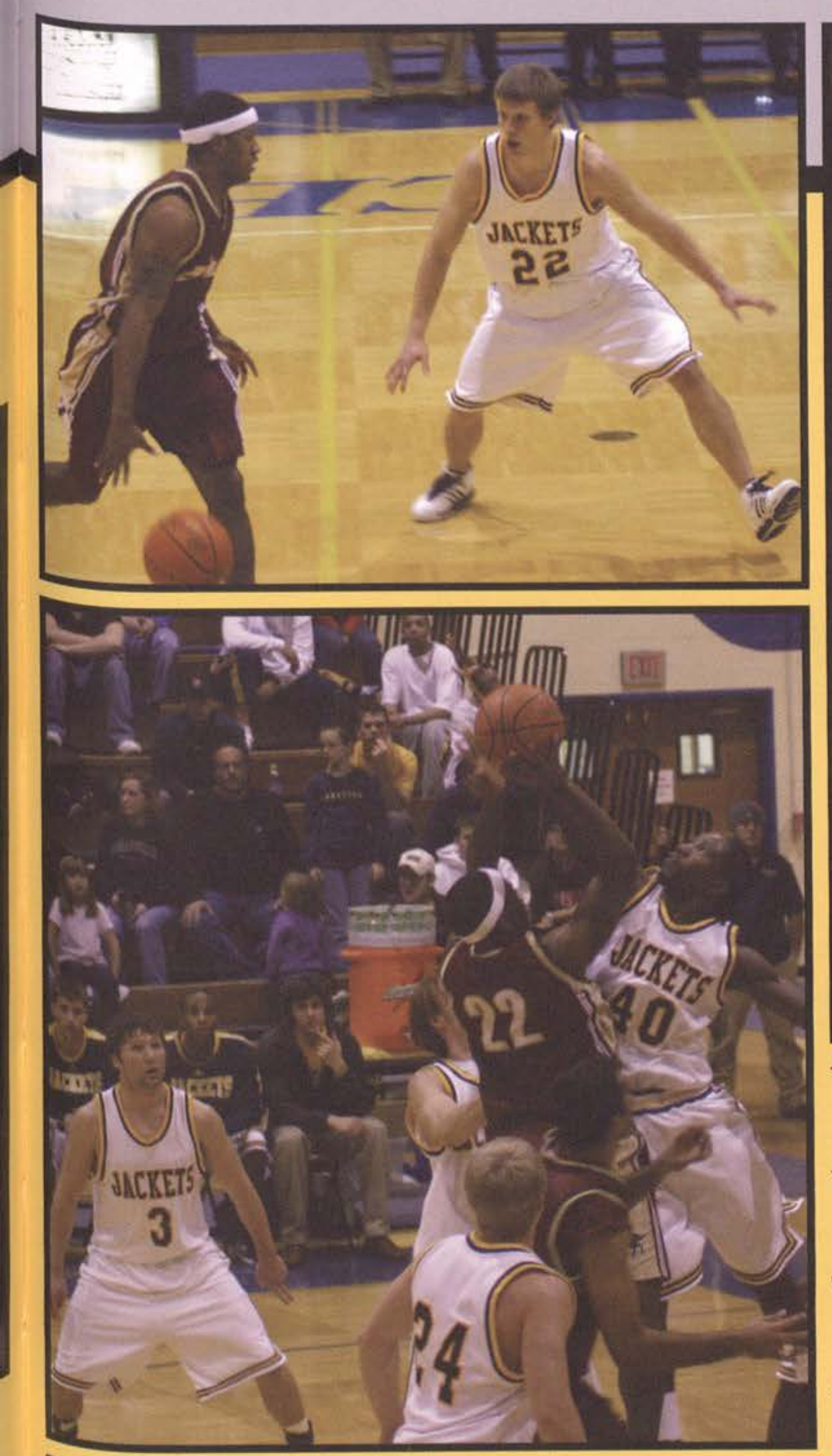

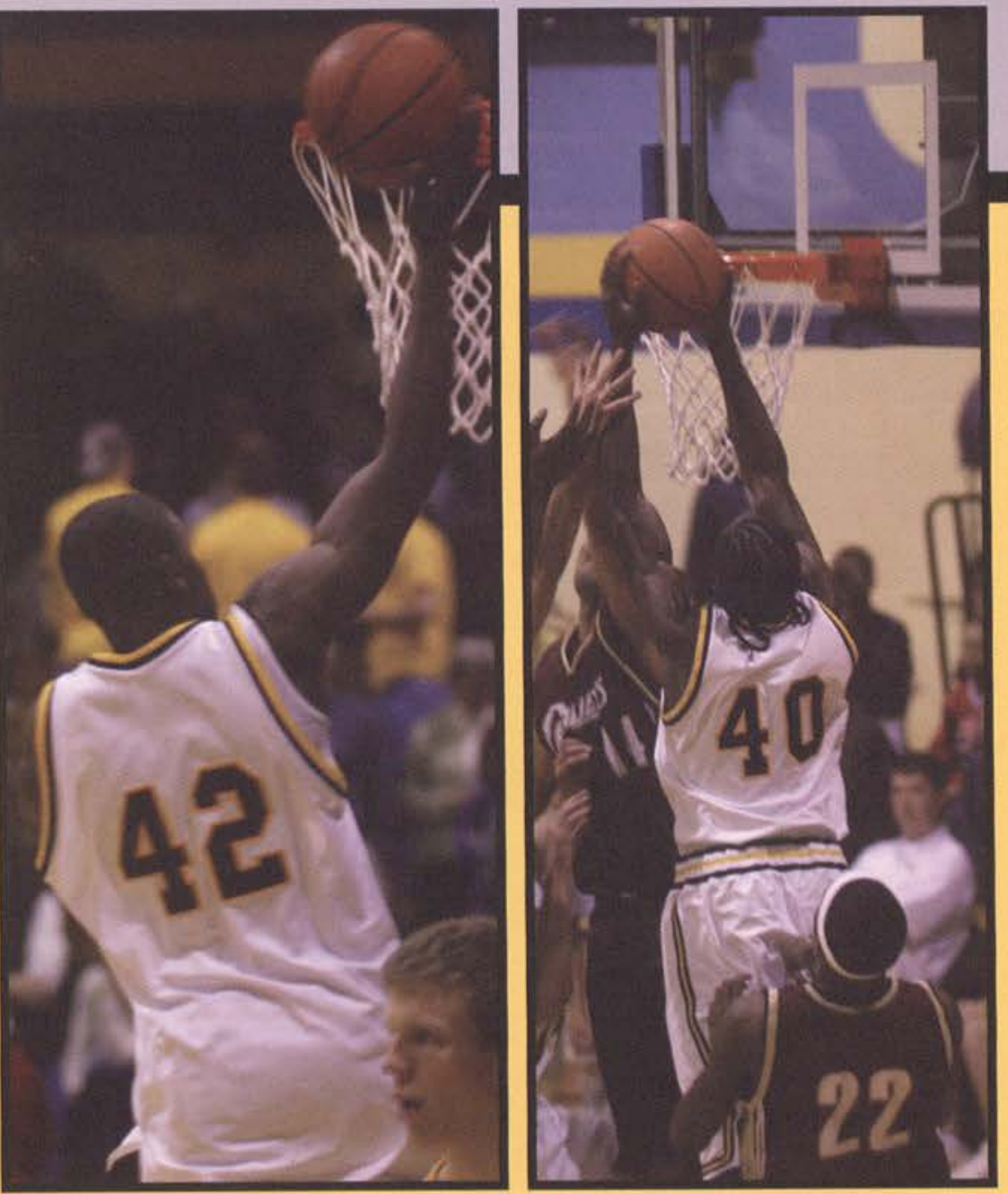

A During warm-ups before the game, Ricardo Alliman impresses the crowd with his sweet moves before a game.

- Chris Walker battles all heavy defense from the opposing team as he goes up for a slam dunk.

Ryan Short, known for his speed, maneuvers quickly to stay on top of his man.

4 Chris Walker (40), Chris Beals (3), Eric Leininger (24), and Maicol Venter (13) show the opposition the meaning of tough defense as they stuff a jump shot.

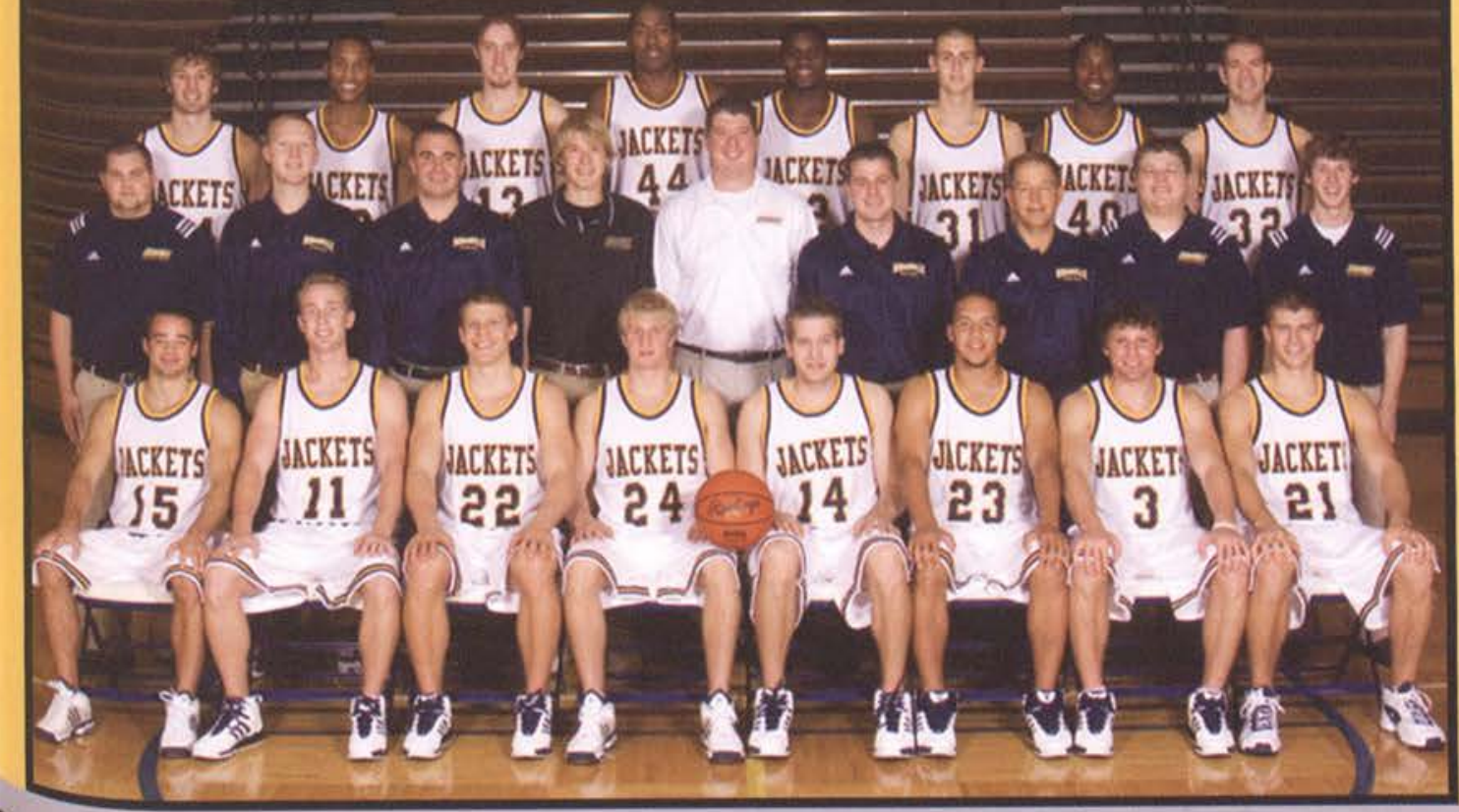

(back row left-to-right) Tyler Yoder, Mike Gause, Maicol Venter, Daniel Rose, Ricardo Alliman, Brandon Sok, Chris Walker, Josh Greve.

(middle row) Student Assistant Loren Kuhn, Assistant Coach Mark Delaney, Assistant Coach Andy Allgrim, Student Athletic Trainer Mike Knudsen, Head Athletic Trainer Chris Cross, Assistant Coach Pat Estepp, Head Coach Ray Slagle, Student Assistant Ryan Mosher, Student Assistant Ryne Oller.

(front row) Wade Rice, Grant Walker, Ryan Short, Eric Leininger, Guy Rathmell, Joel Fowler, Chris Beals, Ryan Reep. 


\section{WOMEN'S}
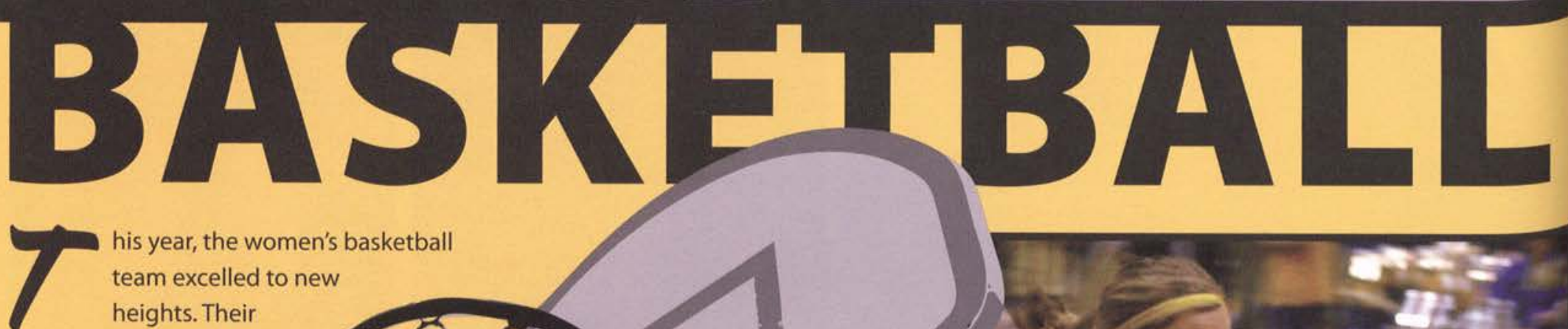

his year, the women's basketball team excelled to new heights. Their accomplishments included a 27-7 overall record, AMC South Division and American Mideast Conference Championship titles, and making it all the way to the NAIA Division II Final Four. Individually, many of the players also achieved high honors. Brittany Smart broke the record for all-time scoring at both Cedarville and the NAIA Division II, eclipsing the 3,000 point mark in her career. Smart was also named the AMC Player of the Year for the second consecutive time and also was named the NAIA Division II National Player of the Year. Her teammate Karah Walton earned a spot on the All-AMC South second team, while Kristi Beougher was named to the All-AMC South third team.

Clearly, the team's accomplishments show that they had a successful season, but their winning season wasn't what impacted them the most. According to senior guard Stacie Travis, "My favorite part of the season was our Florida trip during Christmas Break. It was fun to win both of our games, but more than that it was great to just spend time together away from the stress of school and just really get to know each other better." Junior point guard Kristine Rucker added, "It was exciting to see how God used each one of us in different ways for His purposes. It was also neat to see our team grow as we experienced trials and had to rely on the Lord to get us through each time." Both on and off the court, the Lady Jackets had a spectacular season, and the players all echoed freshman Lauren Gentene when she said, "I really enjoyed being a part of the team this year!" The 2006-2007 Lady Jackets expressed a sense of achievement and look back on the season with fond memories.

\section{We Back The Jackets Alle The Way!}

- Skill is required to play the game of basketball, and skill is something senior Brittany Smart has, as evidenced by the ease it takes for her to breeze by her opponent.

\section{Season Results}

Bryan*

Indiana Wesleyan\%

Saint Francis In $^{+}$

Spring Arbor+

Taylor

Indiana-South Bend\%

Virginia-Wise ${ }^{\%}$

Wilberforce*

Urbana*

Georgetown ${ }^{8}$

Lindsey Wilson ${ }^{8}$

Walsh*

Ohio Dominican*

Warner Southern ${ }^{5}$

Bethel (Ind.) $)^{s}$

Mount Vernon Nazarene* Tiffin*

Shawnee State*

Ohio Dominican*

Malone*

Rio Grande*

Mount Vernon Nazarene*
W 68-56

L 59-69

L $70-86$

W 72-51

W $82-77$

W $79-60$

W 81-54

W 78-51

W 101-70

L 64-92

W 90-85

W $82-56$

L 68-73

W 86-67

W $80-79$

W $85-67$

W 83-69

W 75-67

W69-59

W 58-56

L $97-100$

W 76-63
Walsh*

Wilberforce*

Shawnee State*

Urbana*

Tiffin*

Rio Grande*

Malone*

Daemen**

Union $(K y .)^{++}$

Morningside ${ }^{++}$

Taylor+

Indiana Wesleyan"+

27-7 (.794) Overall

15-3 (.833) American Mideast

*Conference South Division

*Cedarville Invitational; Callan Athletic Center

Saint Francis Classic; Fort Wayne, IN

"Shawnee State Classic; Portsmouth, $\mathrm{OH}$

${ }^{8}$ Georgetown Classic; Georgetown, KY

${ }^{5}$ Warner Southern Classic; Lake Wales, FL.

** AMC Championship Game; Amherst, NY

"16th Annual NAIA Division II National

Tournament; Sioux City, IA
W $89-84$

W $79-45$

W 88-74

W $91-58$

W $82-60$

L 69-72

W $71-62$

W 87-76

W 86-63

W 95-79

W 64-55

L. 56-93

$\mathrm{N}$
th, $\mathrm{OH}$
$\mathrm{KY}$
les, $\mathrm{FL}$
ionst, NY



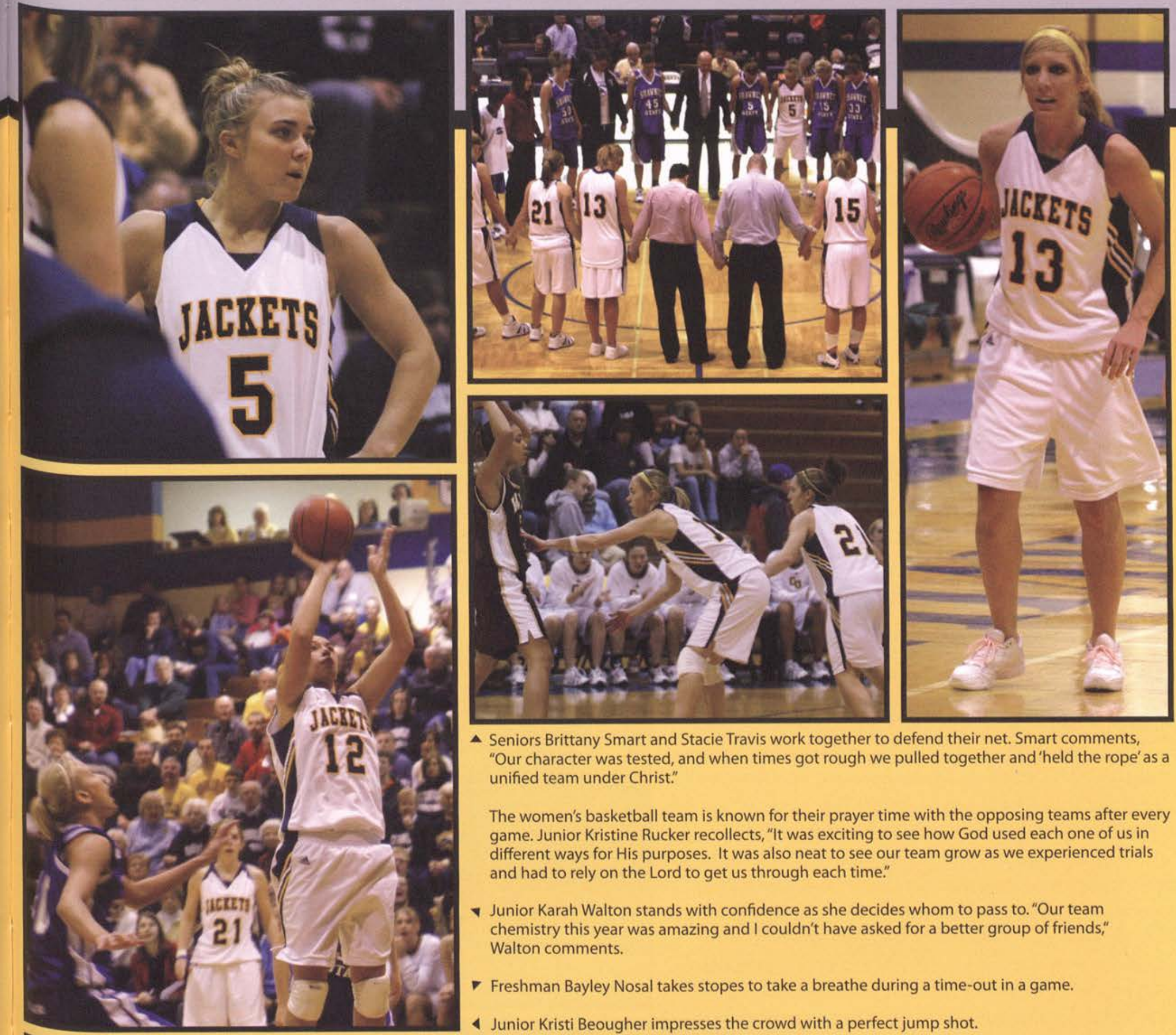

- Seniors Brittany Smart and Stacie Travis work together to defend their net. Smart comments,

"Our character was tested, and when times got rough we pulled together and 'held the rope' as a unified team under Christ."

The women's basketball team is known for their prayer time with the opposing teams after every game. Junior Kristine Rucker recollects, "It was exciting to see how God used each one of us in different ways for His purposes. It was also neat to see our team grow as we experienced trials and had to rely on the Lord to get us through each time."

- Junior Karah Walton stands with confidence as she decides whom to pass to. "Our team chemistry this year was amazing and I couldn't have asked for a better group of friends,"

Walton comments.

V Freshman Bayley Nosal takes stopes to take a breathe during a time-out in a game.

4 Junior Kristi Beougher impresses the crowd with a perfect jump shot.

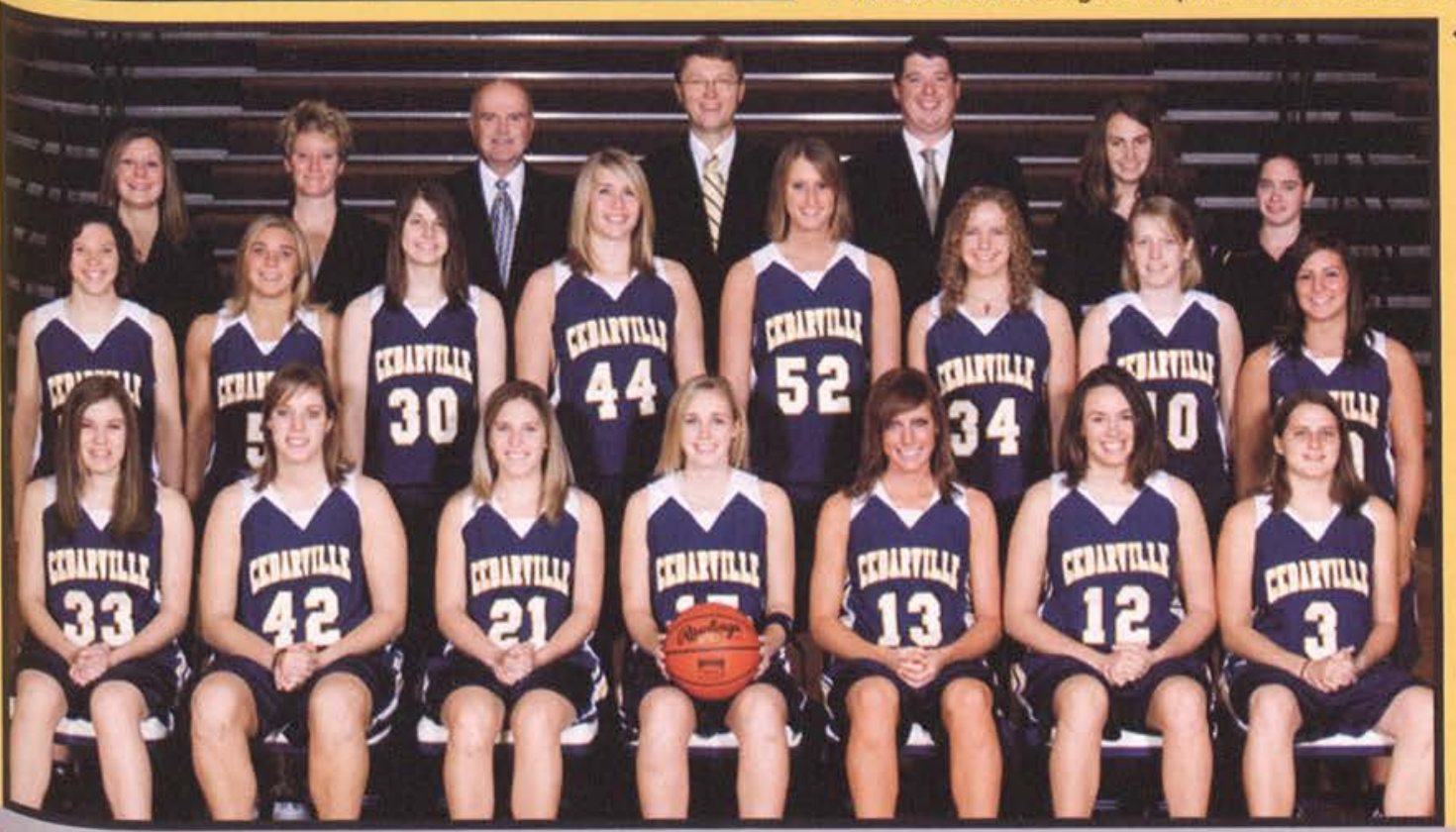

(back row left-to-right) Assistant Coach Lynsey Fabian, Assistant Coach Lori Huckaby, Assistant Coach Dan Schetter, Head Coach Kirk Martin Head Athletic Trainer Chris Cross, Student Assistant Christina Weathers, Student Athletic Trainer Hannah Haynes

(middle row) Lauren Gentene, Bayley Nosal, Stefanie Rodgers, Emily Noble, Alisha Lee, Stephanie Latham, Alison Lemon, Lacie Condon.

(front row) Kristine Rucker, Mary Stockdale, Stacie Travis, Brittany Smart, Karah Walton, Kristi Beougher, Heather Grooms. 


\section{GOLF}

\section{W}

hile the Cedarville Men's Golf team was very successful in both their Fall and Spring seasons, the guys on the team would rather people know about their deep friendships with each other and the awesome times they spent together. Sure, they placed first at the Ohio Dominican Classic, the Mt. Vernon Nazarene Invitational, and the Cedarville Invitational (for the first time since 1982 and by a record 23 shots), and received second place at the Tiffin, Wittenberg, and Shawnee State Invitationals, but the team realized that while success is often fleeting, friendships last a lifetime.

Sophomore Brendan Ojala said that his favorite part of being on the golf team was "being able to be a part of a team and hang out with the guys. Golf is such a social sport so we spent a lot more time talking to each other than most other sports." Adam Schlappi, a senior, also said that he loved being on the golf team at Cedarville because of "the friendships that I have made with all of these guys. They are so awesome." When talking about his teammates individually, senior Scott Aker said that his favorite player was, "Brendan Ojala, for reasons not all that connected to golf. He has become my best friend on campus and challenged me spiritually on a consistent basis." Freshman Matt Krogstad boasted that his favorite player was definitely Trevor Bowman. "The man has style and can hit the ball farther than I can imagine," said Matt. Other players found the best part of the

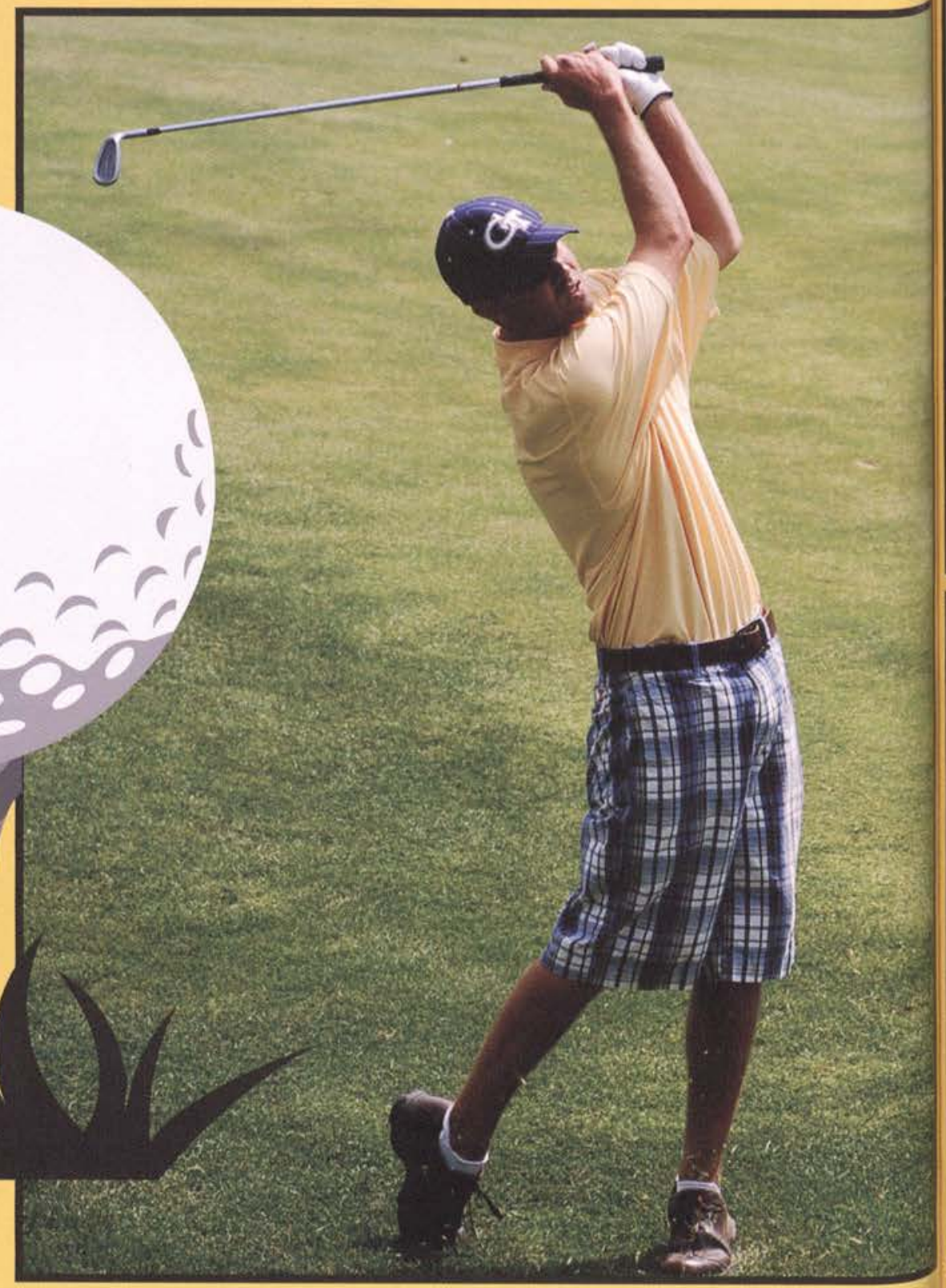

- Senior Adam Schlappi tees off with the swing he's been working to perfect for years. In looking back on his favotire memories, Schlappi says, "My favorite moment of the season was when we shot a 285 at our invitational. We finally all played well together. season to be times such as hitting golf balls off of Mountain Dew bottles or receiving unlimited transfer meals at the Hive. Overall though, the team emphasized that throughout the year, they tried to keep golf in perspective and to give God the glory for everything.

\section{Strive For The Birdie!}

\section{Season Results}

Tiffin Invitational

Wittenberg Invitational

Ohio Dominican Classic

NCCAA Nationals

Berry Invitational

Shawnee State Inv.

Mt. Vernon Nazarene Inv.

Malone Invitational

Cedarville Invitational

AMC/NAIA Region IX $2^{\text {nd }}$ of 14

$2^{\text {nd }}$ of 7

$1^{\text {st }}$ of 9

$T 3^{\text {rd }}$ of 25

$8^{\text {th }}$ of 15

$2^{\text {nd }}$ of 8

$1^{\text {st }}$ of 10

$6^{\text {th }}$ of 9

$1^{\text {st }}$ of 9

$3^{\text {rd }}$ of 12 


\section{M
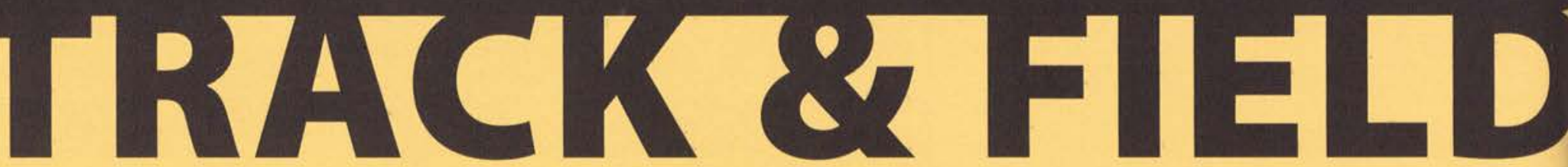

66

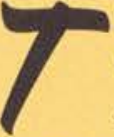

rack is not just something you do from the beginning of the school year until the end; it's a learning experience in relationships, hard work, perseverance, and dedication," said freshman Eric Kempton. The Men's Track Team invested a lot of hard work this year, as shown by its achievements. The team finished tenth at the NAIA Indoor Championships and earned its second straight NCCAA National Championship. The team also boasted some outstanding individuals. Daniel Roberts made a new school record time of 4:10.13 in the mile, and Ben Michaud earned a school record throw of 57-7 in the shot put and was given All-American status. Jason Scott, now a threetime national pole vault champion, broke his own school record at the NAIA pole vault competition, clearing 17-8.5.

The track team relied heavily on its coaches, not only for their knowledge and leadership, but also for their love of the team. "Coach Bitsko and I have this little rivalry, and we always seem to end up wrestling or trying to one-up each other. He usually wins, of course. I guess age before beauty means something," said sophomore Travis Smith. Freshman Titus Goodew said, "Since we don't have coaches for every event, Coach Scott would step in and coach three different events some days. It was awesome to have him sacrificing so much for the team."

The Men's Track Team did an outstanding job athletically this year, but some of the team's favorite memories are about time spent with each other. Sophomore Eric Andersen said, "Some of the best memories I've had are seeing how many people we can fit in the bathrooms on the bus rides." The efforts of the team and coaches were worthwhile not only because of their achievements, but also because of the relationships that were created this year.

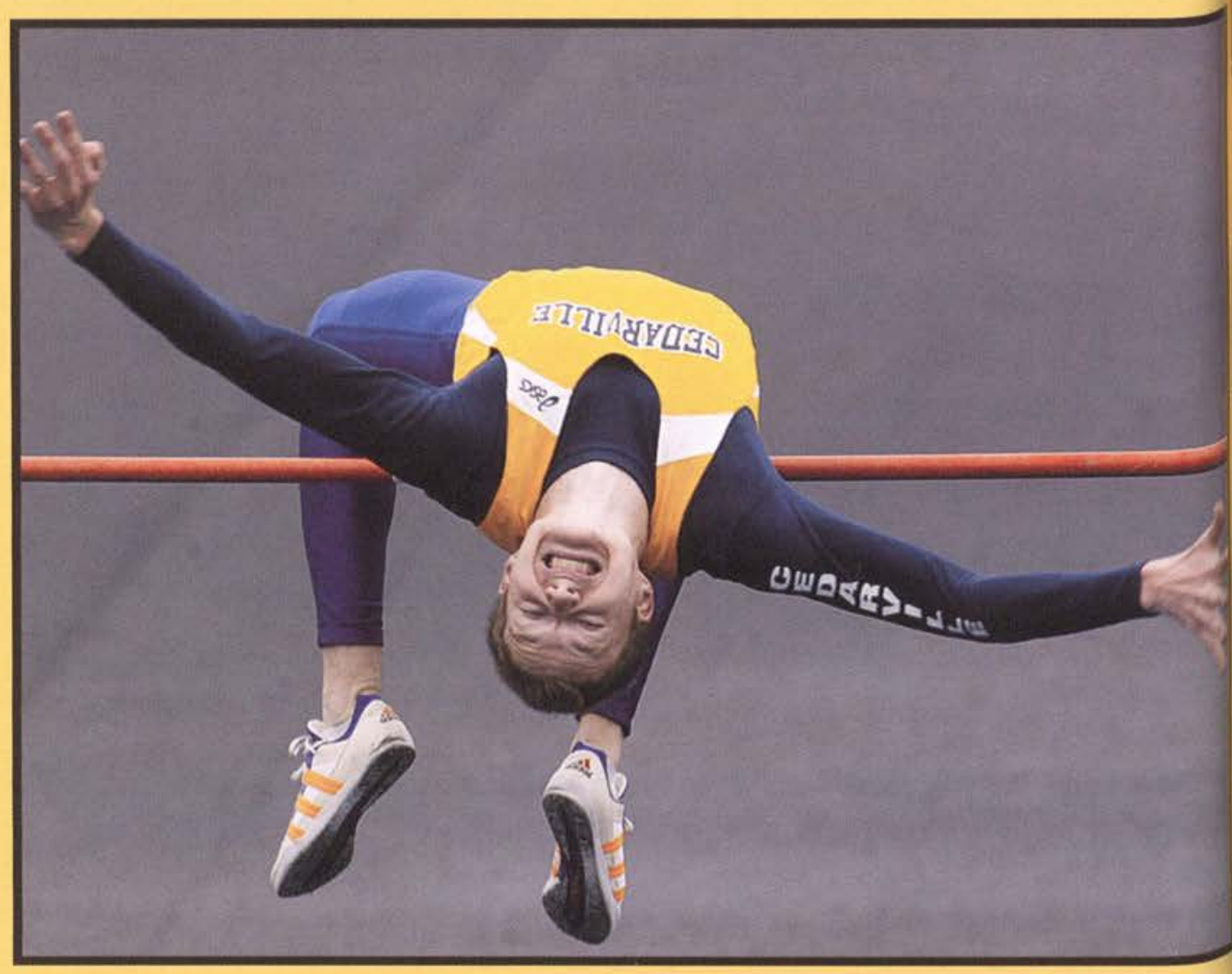

- Jim Sawin reaches his best jump thus far at 6' $9^{\prime \prime}$ at the Cedarville University Open on March 31 . Sawin says, "God has blessed me with the ability to train and use the talents He has given me to jump high this season."

\section{Indoor Season Results}

Ohio Northern

Cedarville Invitational

Indiana Relays

Findlay Open

Ohio Northern

NCCAA Indoor Championship

NAIA Indoor Championship $3^{\text {rd }}$ of 7

$1^{\text {st }}$ of 15

Non-Scoring

Non-Scoring

$2^{\text {nd }}$ of 7

$1^{\text {st }}$ of 12

$10^{\text {th }}$ of 90

\section{Outdoor Season Results}

\author{
Baylor Invitational \\ Wittenberg Invitational \\ Cedarville Open \\ Miami Invitational \\ AMC Championship \\ NCCAA Outdoor Championship Upland \\ Cedarville Qualifier \\ NAIA Outdoor Championship
}




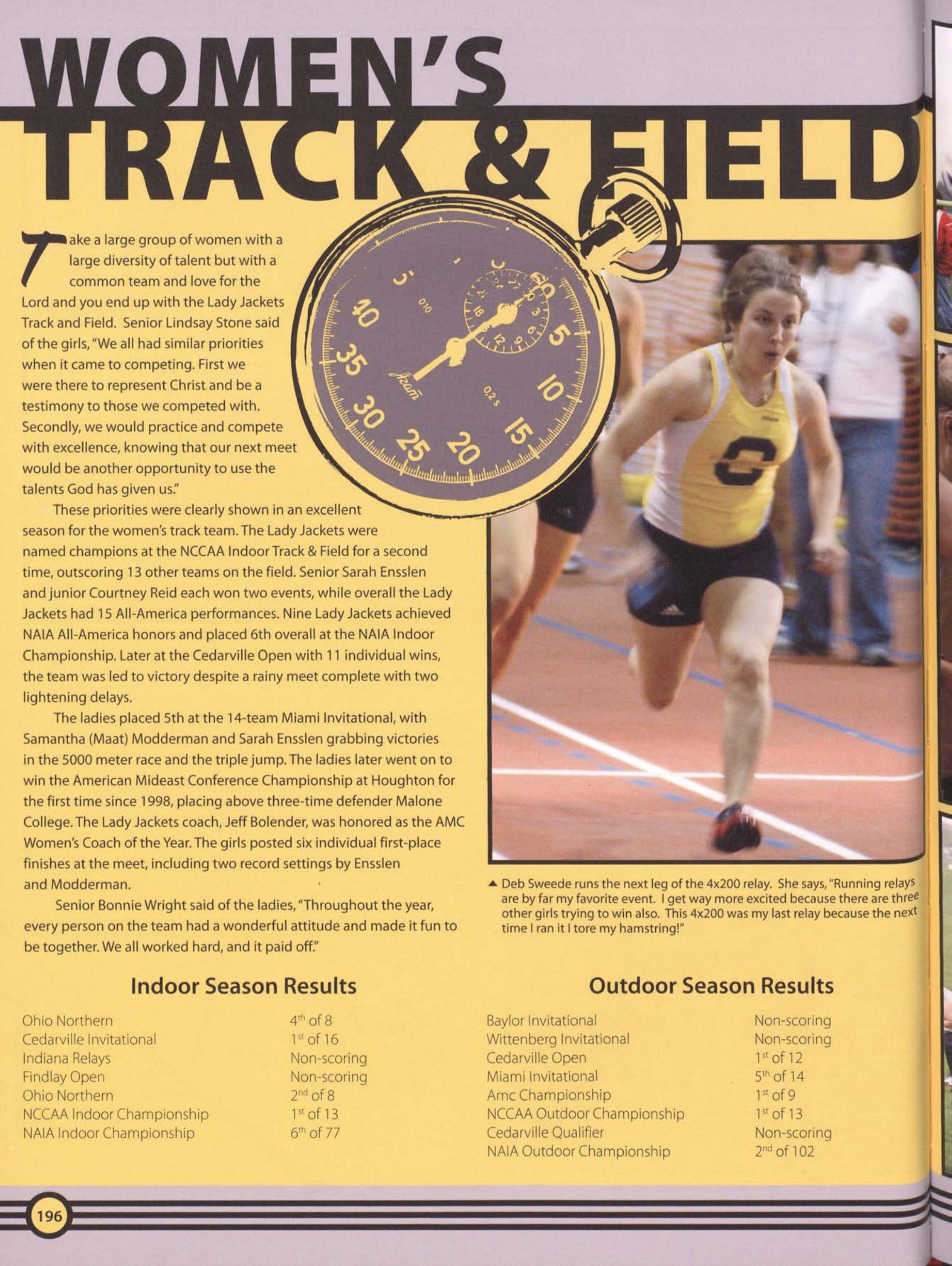




\section{f the 06-07 tennis season}

had to be summed up in one word, it would most likely be relationship. This year's team had an impressive amount of rebuilding to do. After losing four integral players at the end of last year's season and adding three more, the team had to essentially rebuild and develop new strategies. Thankfully, this drastic change was a success. Senior Jake Bezeck, as a third-year player, witnessed the adjustments the team experienced this year. He explained, "Teamwork has been the biggest reason for the team's success this year. We are not as deep or talented as past years but everyone works hard and looks to benefit the team any way they can, which has helped everyone improve throughout the year. It has also helped us win some close matches."

Sophomore Brent Martin was a first-time team member this year and attributed his smooth integration into the team to upperclassmen like Bezeck. He said, "We have a senior and two juniors with tons of varsity experience which has led to our success. They are able to help the newer kids with technique and our mental game. They provide good leadership."

This new team setup seemed to serve the tennis team nicely, as the Jackets delivered several strong wins throughout the season. Junior Kevin Furst had his initial doubts about the success of this year's team, but he explained that his mind has since been changed. He said, "I think the team has done much better this year than I expected us to. We lost a number of good players last year, but we are filling in the gaps nicely and our hard work has pushed us to the level where we can still compete and be one of the best teams in our conference."

\section{Double The Fun!}

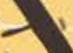

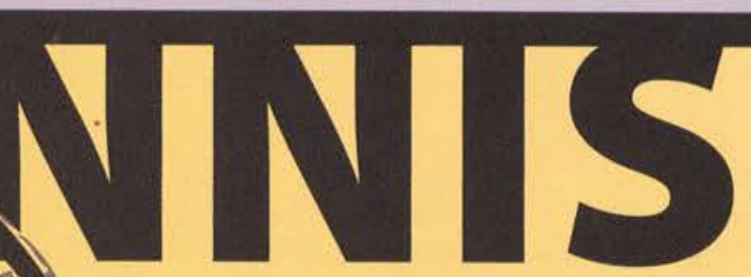

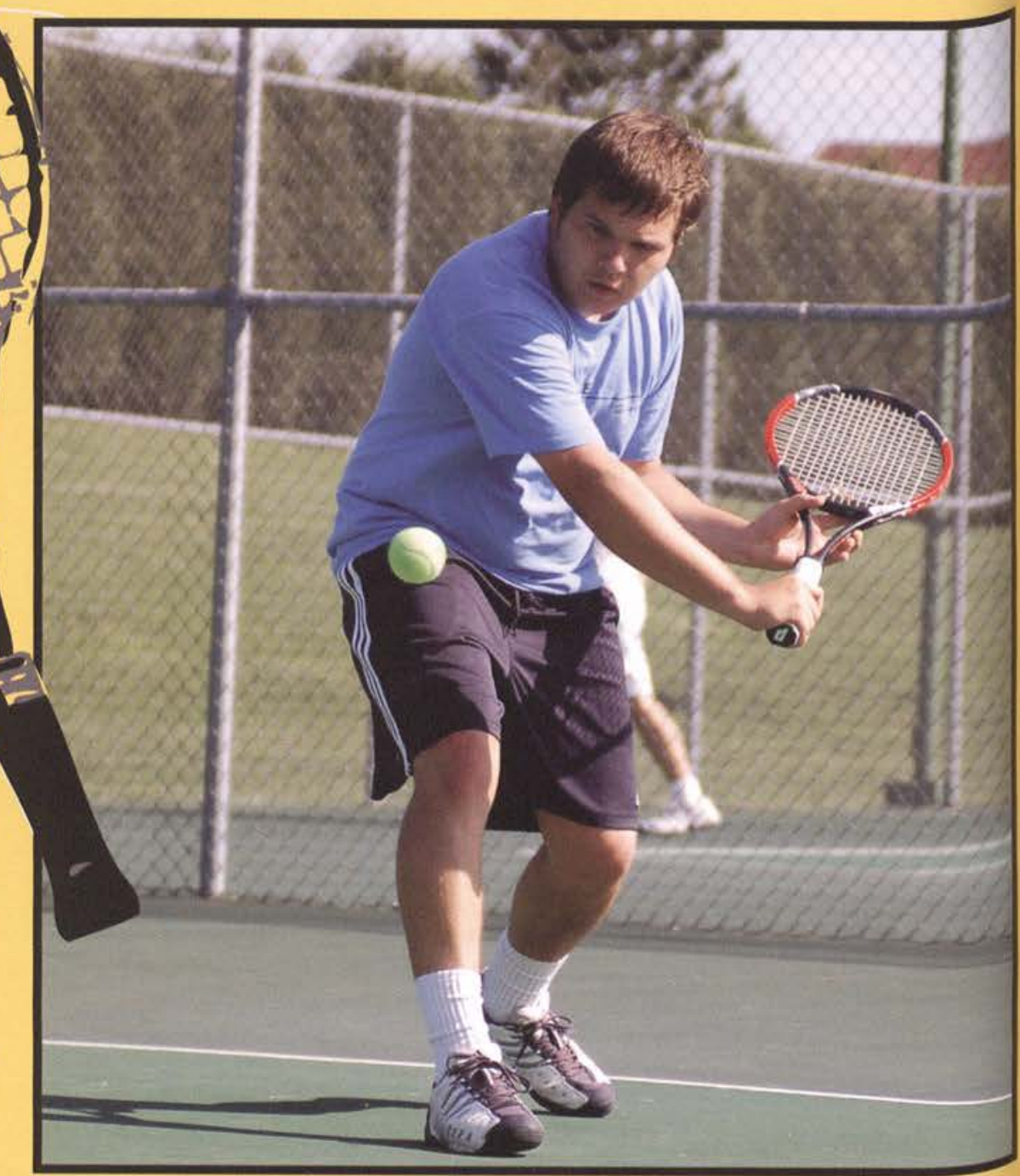

- Freshman Caleb Speicher attributes the team's success to "super, solid play from Jake [Bezeck] and the tone it sets for the rest of our matches."

\section{Season Results}

Emmanuel

Maryville

Carson-newman

Wilmington

Bellarmine

Georgetown

Wittenberg

Ohio Dominican*

Bethel (Ind.)

Walsh*

Notre Dame*

Asbury

Malone*

W 8-1
W 7-2
L 2-7
W 9-0
L 3-6
L 1-8
W 9-0
W 5-4
W 7-2
L 1-8
W 9-0
W 9-0
W 6-3

W 8-1

$2-7$

W 9-0

L 3-6

L 1-8

W 9-0

W 5-4

W $7-2$

L 1-8

W 9-0

W6-3
Seton Hill

Tiffin*

Roberts Wesleyan*

Tiffin ${ }^{+}$

Palm Beach Atlantic"

Spring Arbor"

Oklahoma Wesleyan"

W 8-1

L 3-6

W $5-4$

L 3-6

LO -5

L $0-5$

L 2-5

\section{1-9 (.550) Overall}

4-2 (.667) American Mideast Conference*

+ NAIA Region IX Tournament; Cedarville "NCCAA National Tournament; Greer, SC 

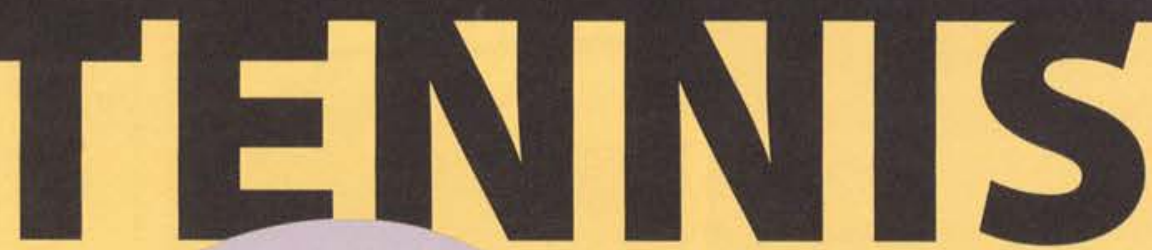

he Cedarville Women's Tennis team aced the beginning of their season, winning their first eight matches handily. Then, in mid-April, the ladies suffered a setback, losing five matches in a row. Number one singles player Alisa Rutt, however, lost only twice at singles throughout the regular season, bringing her career singles record to $32-13$. The second doubles team of Laura McCully and Betsy Verwys also played well and finished with an $8-2$ record.

A major change that these ladies underwent throughout their season was the changing of head coaches. Pam Johnson, the former head coach, stepped down due to her deanship responsibilities at the university. Dee Morris was then named the head coach after serving as the assistant coach since 1977. While the girls were sad to see Coach Johnson leave, they knew they still had her support and would be well taken care of by Coach Morris.

Another memorable event of the season involved the mission trip that the team took to the Dominican Republic over Spring Break. All of the team members echoed junior player Jade Burley when she said, "My favorite memories are definitely from the Dominican Republic! It was great seeing each girl in her element... We were stretched, and from that we were able to encourage and challenge each other. $t$ was able to get to know each of the girls better...my respect and love for them grew." When looking back on the season, junior Melinda Workman said, "We started out strong. Toward the second half of our season we had a little difficulty keeping up our intensity as we faced some difficult matches, but we tried our best to pull it together at the end and finish strong." In the end, freshman Carissa Parmerlee said, "We played strong and had fun doing it," and that was all that mattered.

\section{A Team O\& Aces!}




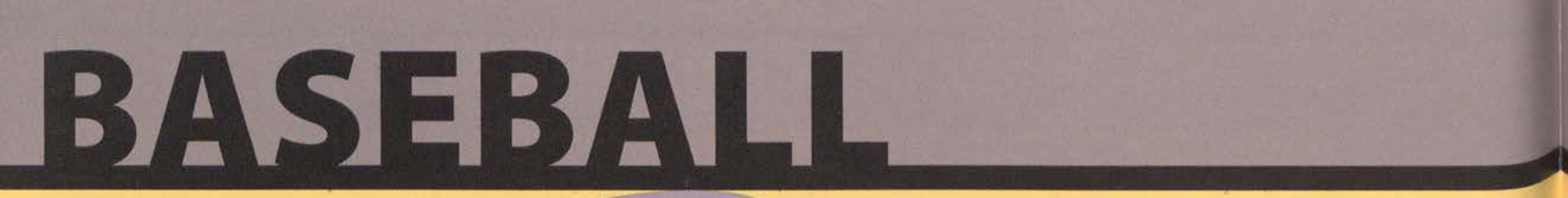

$I$

$I^{\prime}$

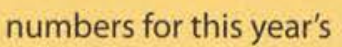
Men's Baseball season, it would appear that the team struggled to secure wins. However, a closer look into the individual efforts of the players and the overall attitude of the team revealed a positive morale and an encouraging challenge to continue the following year. The first of the four total wins occurred close to the beginning of the season, which was a win against Grace at the Clearwater Invitational. After this initial victory, the Jackets baseball team still faced tough opponents throughout the year. However, they still triumphed over Concordia, Myers, and Earlham.

Several key players on the Jackets men's baseball team encouraged fellow members through their exceptional achievements and positive attitudes. Seniors Richie Reeder, Phil Buben, Dan White, and Tim Hubler led their team with skill and character. Reeder, at season's end, was a .300+ hitter. Tim Hubler was a top-three run scorer for the club. Dan White was one of four players to play all four years at Cedarville, and Phil Buben was one of the athletes selected to be declared as scholar-athletes by both NAIA and NCCAA.

Juniors Pete Kraus and Paul Wilson were also impressive in stats and performance, giving the Jackets hope for strong leadership for next year. This, including the addition of Trinity International University former head coach Mike Manes, allows this year's team to look forward to consistently improving future seasons.

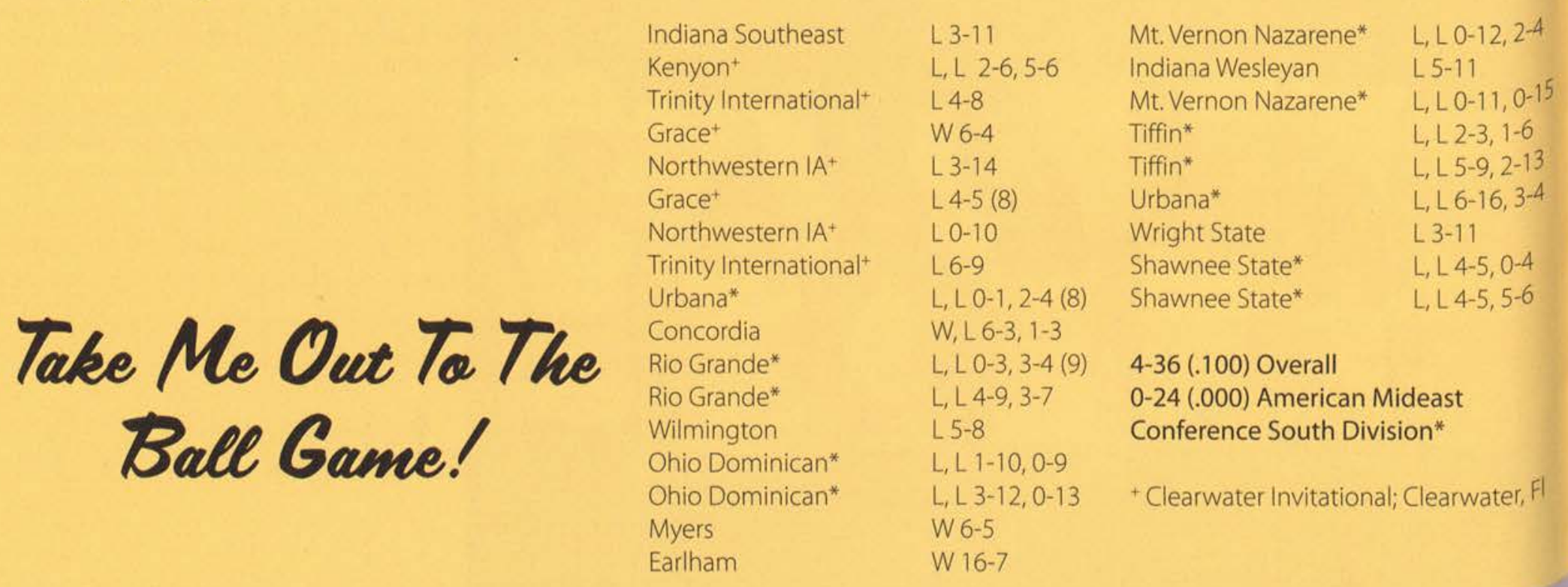

Sliding into home is what the game of baseball is all about, and that is exactly what every Cedarville player knows through and through!

\section{Season Results}

Mt. Vernon Nazarene* Indiana Wesleyan

Tiffin*

Urbana*

Wright State

Shawnee State*

4-36 (.100) Overall

$0-24$ (.000) American Mideast

+ Clearwater Invitational; Clearwater, F 


\section{SOET}

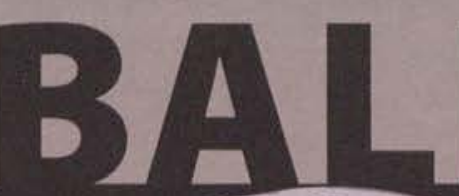

L

T

mats had a rebuilding year this season, having lost three seniors and gained seven new teammates. The team also welcomed new head coach Kathy Freese, a veteran coach who has worked with teams at Cedarville since 1991.

The girls had some exciting wins and disappointing losses but ended their overall season with a 12-27 record. The season began with a loss to Siena Heights, but the team was then able to shut out Cornerstone 5-0 with junior pitcher Kandis Armstrong tossing a two-hitter with five strikeouts and two walks. After a disappointing string of losses, the team again came out on top over Cornerstone at the Gene Cusic Classic in Florida, where they also won games against Siena Heights and Geneva. The team won a double header against Findlay and later produced a season high of 15 hits for a win against Malone.

At the NCCAA Midwest Regional, the girls achieved a win against Spring Arbor and accepted an invitation to the NCCAA Nationals in Indiana, where the girls closed their season with a 3-2 loss against Judson.

In the midst of wins and losses, the team sought to encourage the girls they played against by writing each player of the opposing team a note of encouragement. This outreach often presented an opportunity to share their love for Christ and open a door for friendship and ministry. Sophomore Charissa Rowe said of the team, "Since I went to a public high school, I never had the opportunity to play with very many Christians. In my opinion, nothing compares to the awesome experience of playing with girls that desire to glorify $\mathrm{Him}$ with their ability. I believe the best thing that happened this season was watching my teammates grow in Christ, strive to serve Him, and challenge me to do the same."

\section{Buy Me Some Peanuts And Cracker Jacks!}

Siena Heights ${ }^{+}$

Cornerstone $^{+}$

Indiana Tech+

Indiana Tech+

Cornerstone

St. Mary of the Woods ${ }^{+}$

Cornerstone

Siena Heights ${ }^{+}$

Cornerstone

Geneva+

Siena Heights

Geneva"

Madonna"

Findlay

Rio Grande*

Tiffin

Ohio Dominican*

Mt. Vernon Nazarene*

Walsh*

\section{Season Results}

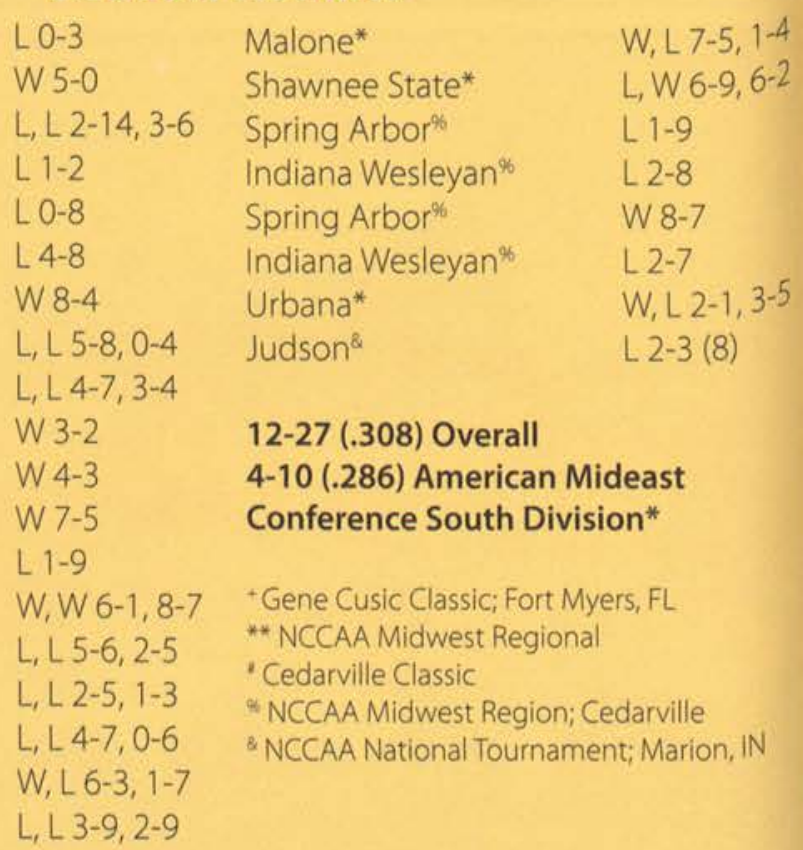



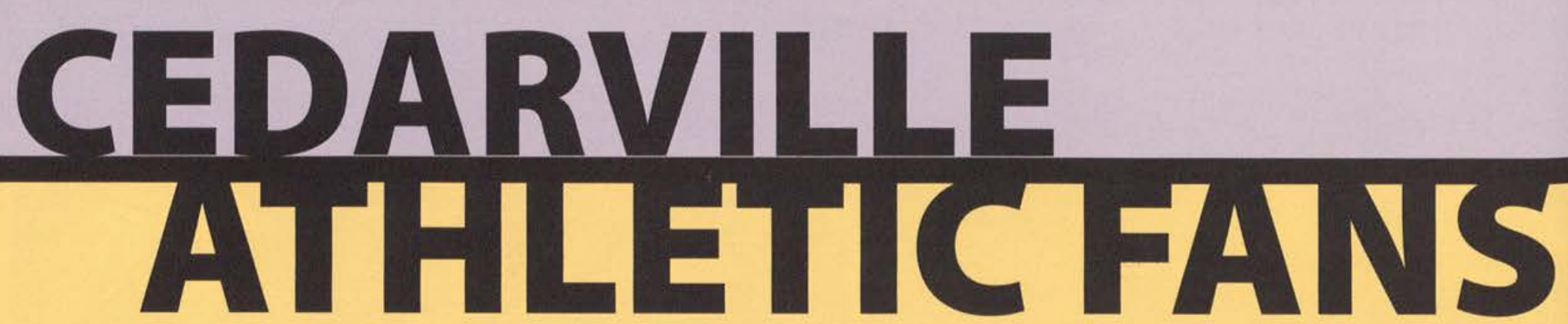

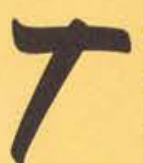

he Jackets were blessed with plenty of enthusiastic fans this year. Joel Logan, one of the Scottish warriors from Braveheart night, said, "My goal as a fan of Cedarville is to make visiting teams fearful of playing in the Callan Athletic Center, not because we curse at them and are nasty to them, but because we act like a sixth man playing defense for a team that is giving their all for God's glory."

The fans showed their dedication this year through a series of themes that included Scottish warriors, ninjas, and schoolteachers. Junior Ben Summers said, "Our sacred theme nights reached an all new high this year as the world witnessed our hard work and dedication for Braveheart night through the medium of Sports Illustrated." Braveheart night was certainly the favorite theme for most of the fans. Over fifty students participated with homemade kilts, cardboard weaponry, and blue faces. The group was led into the gym by the sound of bagpipes playing the Scottish anthem. "The climax of this entire event was right before tip-off. With solemn faces and fiery hearts, the men rallied together to blast forth in unprecedented fortitude a unified 'FREEDOM,', said Andrew Elliott.

The Jackets' fans were extremely dedicated and proved it with their creative support of our teams. "There are a lot of state schools that would love to have the kind of energy that we bring to home basketball games. There's just an air of energy and excitement that I think a lot of teams would be envious for," said senior Ben Collins. Cedarville will never forget the impact of this year's committed fans. As junior Daniel Lagan said, "Hearing the gym shake with the roar of fifty Scotsmen crying 'Freedom!' in unison will live on in Cedarville lore for ages."

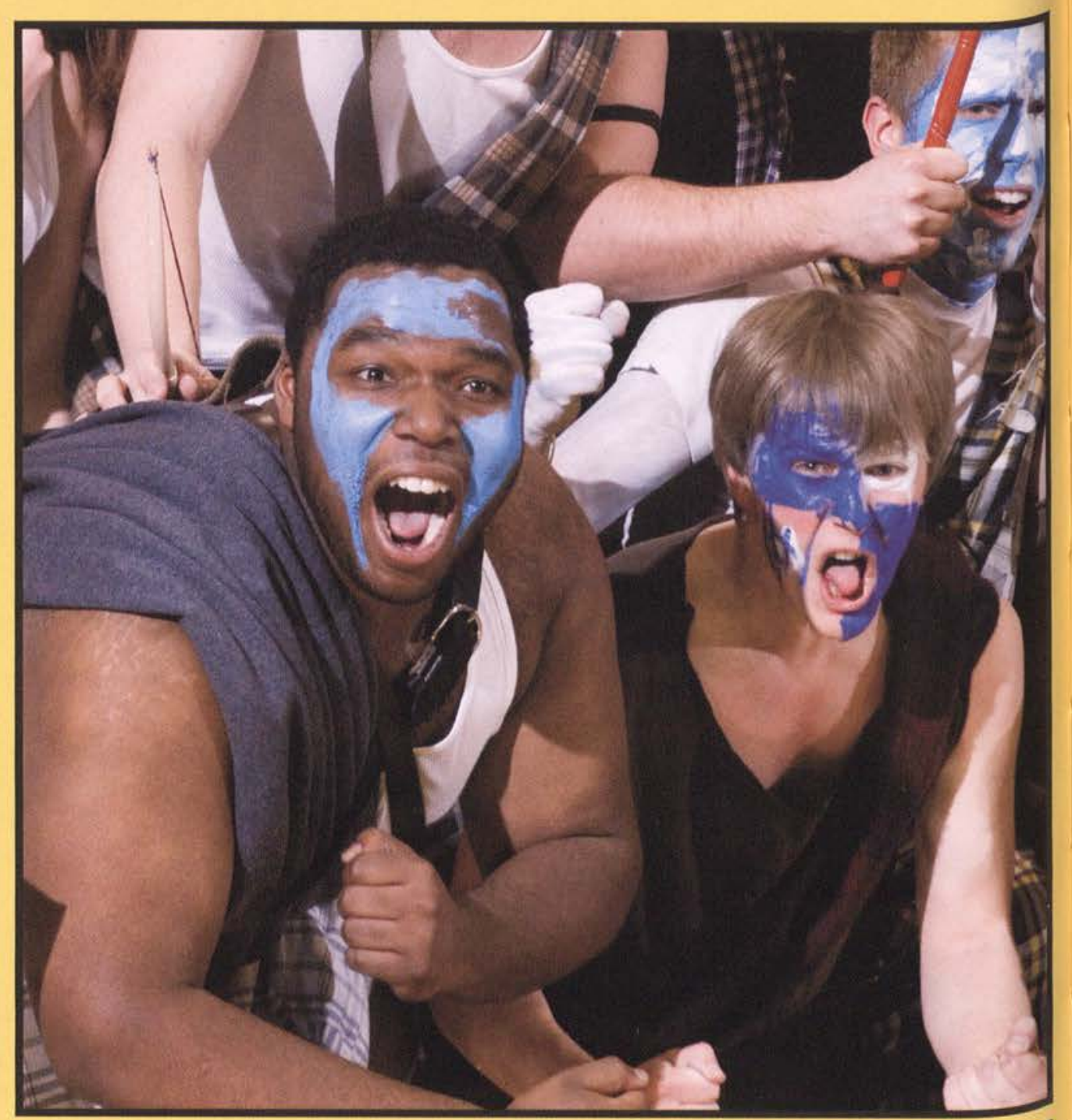

- Rod Bradley, pictured here with Andrew Bruder, said of the evening, "It is a privelege to be involved, as a fan, for our basketball games. I believe that the Cedarville fans have the most fun, out of any fans, at basketball games."

- Jackets are number one! CU fans always find a reason to celebrate our award-winning sports teams.

\section{Frecedocom!}

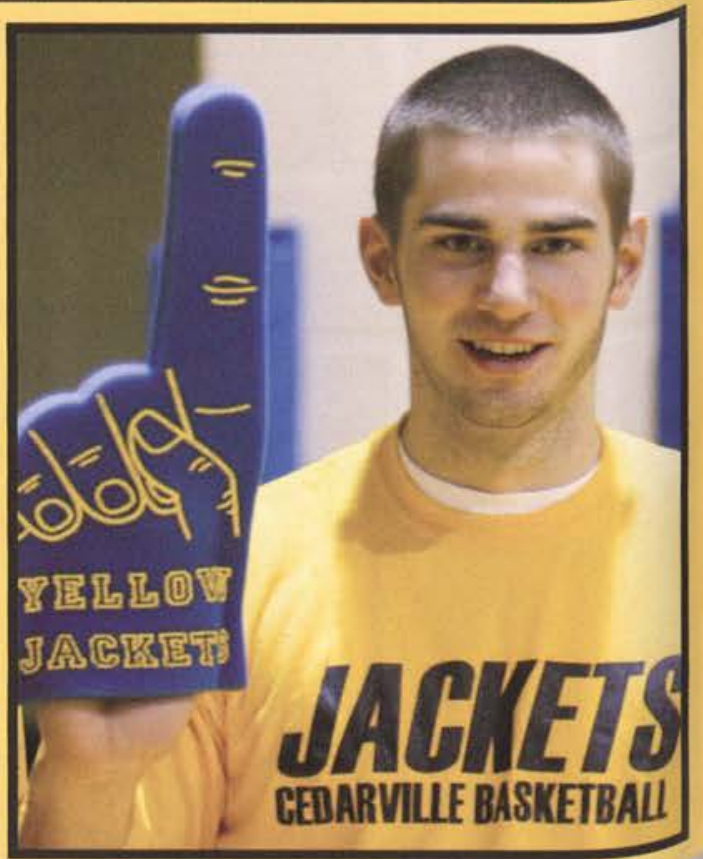



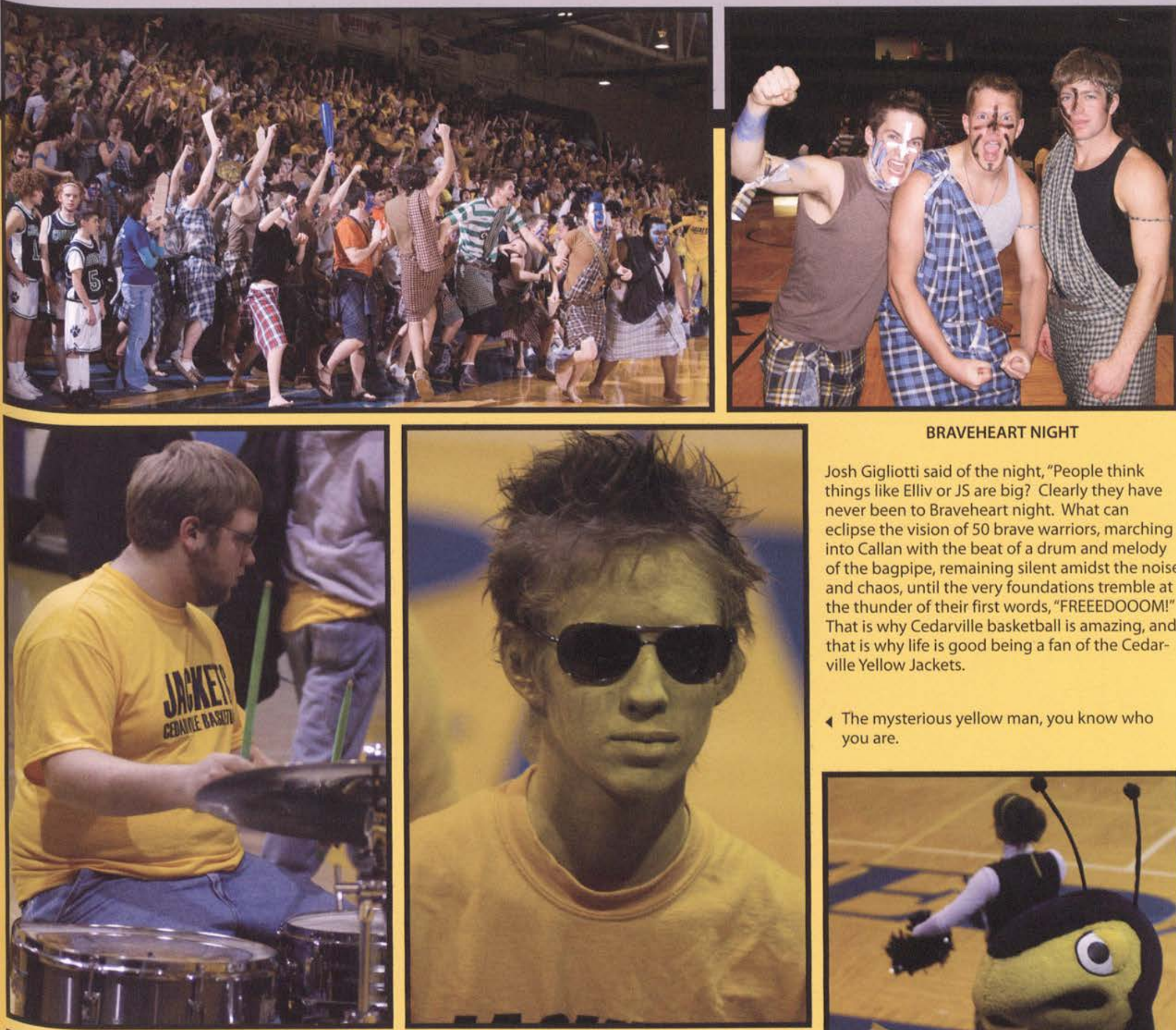

BRAVEHEART NIGHT

Josh Gigliotti said of the night, "People think things like Elliv or JS are big? Clearly they have never been to Braveheart night. What can eclipse the vision of 50 brave warriors, marching into Callan with the beat of a drum and melody of the bagpipe, remaining silent amidst the noise and chaos, until the very foundations tremble at the thunder of their first words, "FREEEDOOOM!" That is why Cedarville basketball is amazing, and that is why life is good being a fan of the Cedarville Yellow Jackets.

The mysterious yellow man, you know who you are.

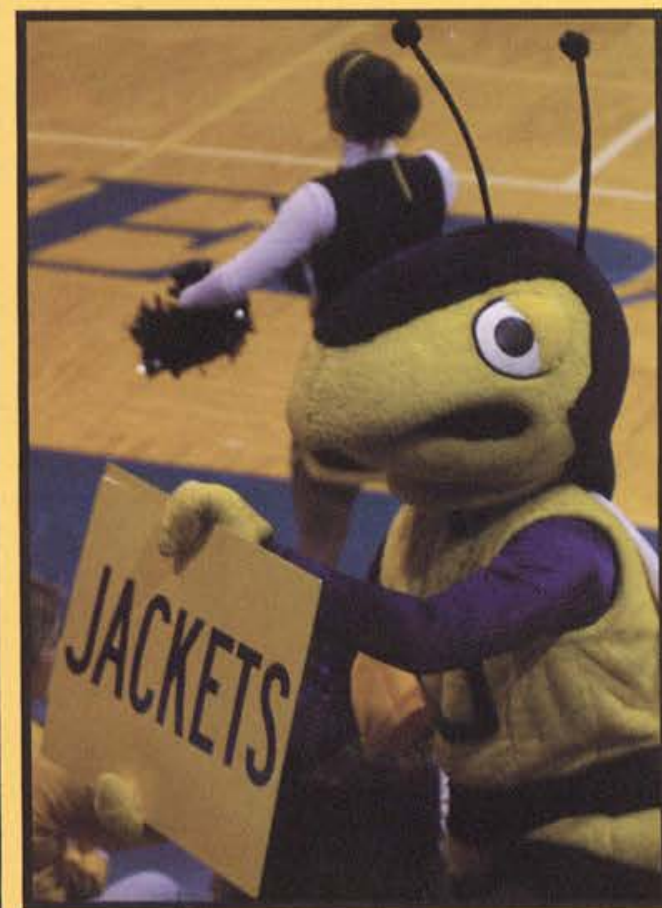

- No basketball game is the same without our beloved bee. The questions remain, "Who is the bee, and is that head really hot inside?"

1 We love soccer, yes we do, we love soccer how about you?

Sophomore John Carey says, "Playing in the pep band at Cedarville has been a great experience. It is really neat to see the crowd excited and enjoying the atmosphere at the basketball games!" 


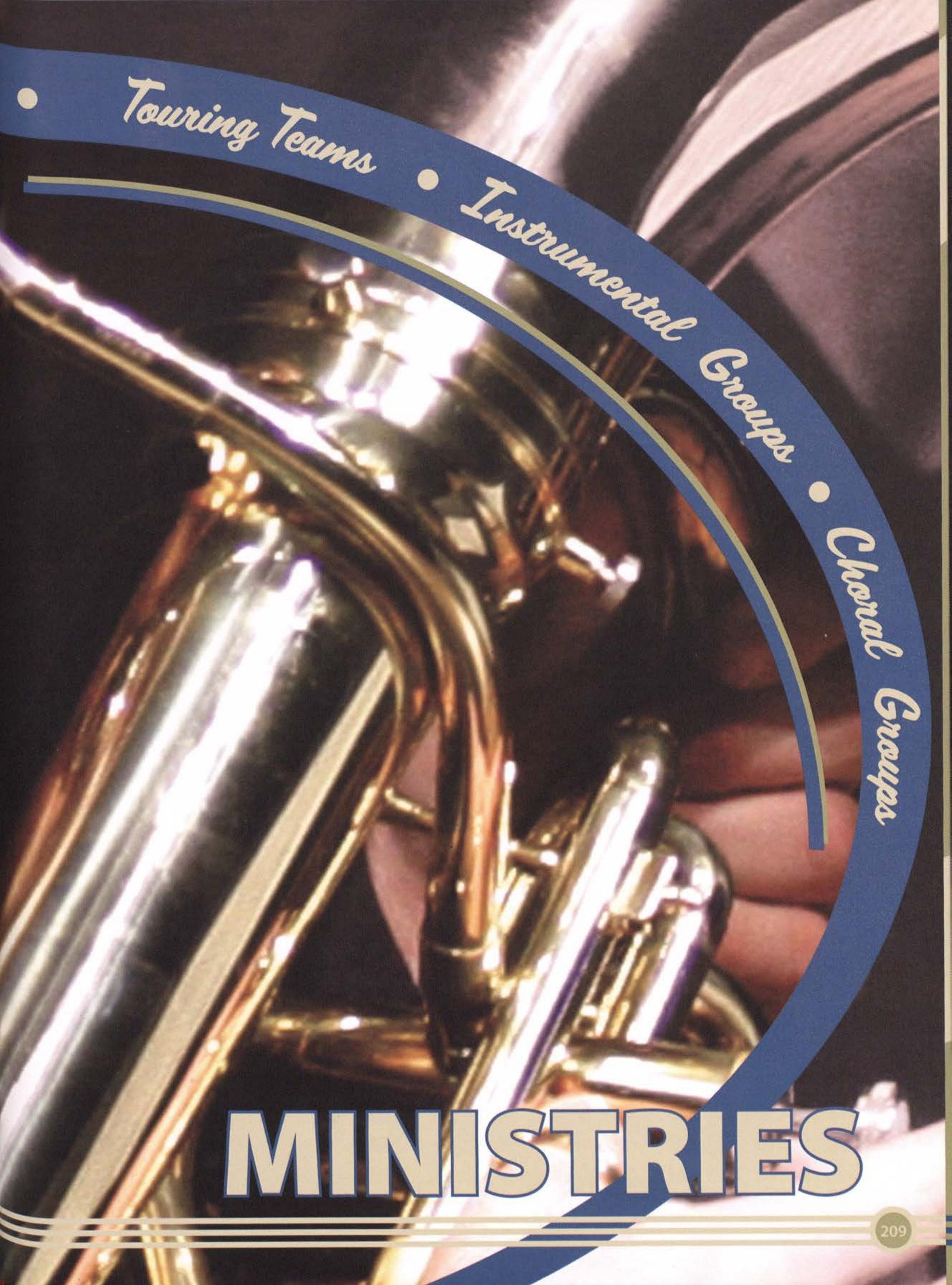


obody needed to look long or hard for a ministry to get involved with here at Cedarville. With more than fifty different ministries of all kinds to choose from, Cedarville students sought to carry out Christ's command to "Go and make disciples."

Students worked with all age groups from many different backgrounds. Junior Amanda Stephens participated in Adopt-A-Grandparent, a one-on-one ministry with the elderly. She said of the experience, "I visited Mrs. Mitchell in Cedarville. One of the first times that I went over there she ended our time by asking to pray for me, which was humbling. I enjoyed hearing her stories - it amazed me to see God's hand in placing us together this year."The organizations with which students became involved not only touched others' lives, but blessed the students' as well.

A large portion of ministries this year centered on reaching children. Senior Mike Hubenthal, student leader of Adopt-A-Block, noted, "This year we focused on ministering to the kids on the block by having a kid's club. There we played games, sang songs, and had a Bible lesson. These kids did not get much positive attention and role modeling for the most part, so it was a privilege to share God's love with them by investing in them. It was challenging but very worthwhile."

Sophomore Serenity Gingrich had the opportunity to visit the Greene County Juvenile Detention center to visit the youth staying there. She said of the experience, "Once, I had been close to not going, but I was so thankful that I did because it was such a blessing to be able to minister to a teen that had just hit rock bottom and needed encouragement and hope. I hope that what I've said to the teens will be used to plant a seed and show them that they are cared about even when they didn't feel like it."

Zachary Eagle keeps things in line with AWANA. He comments, "I would say the greatest part of helping with AWANA is being able to see the kids grow in Christ and see them learn more about their Savior...getting the chance to be the best postive role model as possible is amazing."

Senior Hilary Bromley says, "Visiting the residents at Hillside was often the highlight of my week. Each resident welcomed our group as family, and we got to know each person as an individual. Without fail, we left each week laughing."

Bekah Taylor, Brianna Mottashed, and Rachel Geiger get a chance to help needy kids at the Dayton Gospel Mission through a program called "More Than Rubies." Bri notes that "it was amazing to work with children coming right off the street. It really forced my eyes open to the fact that people our age and older are not the only ones who

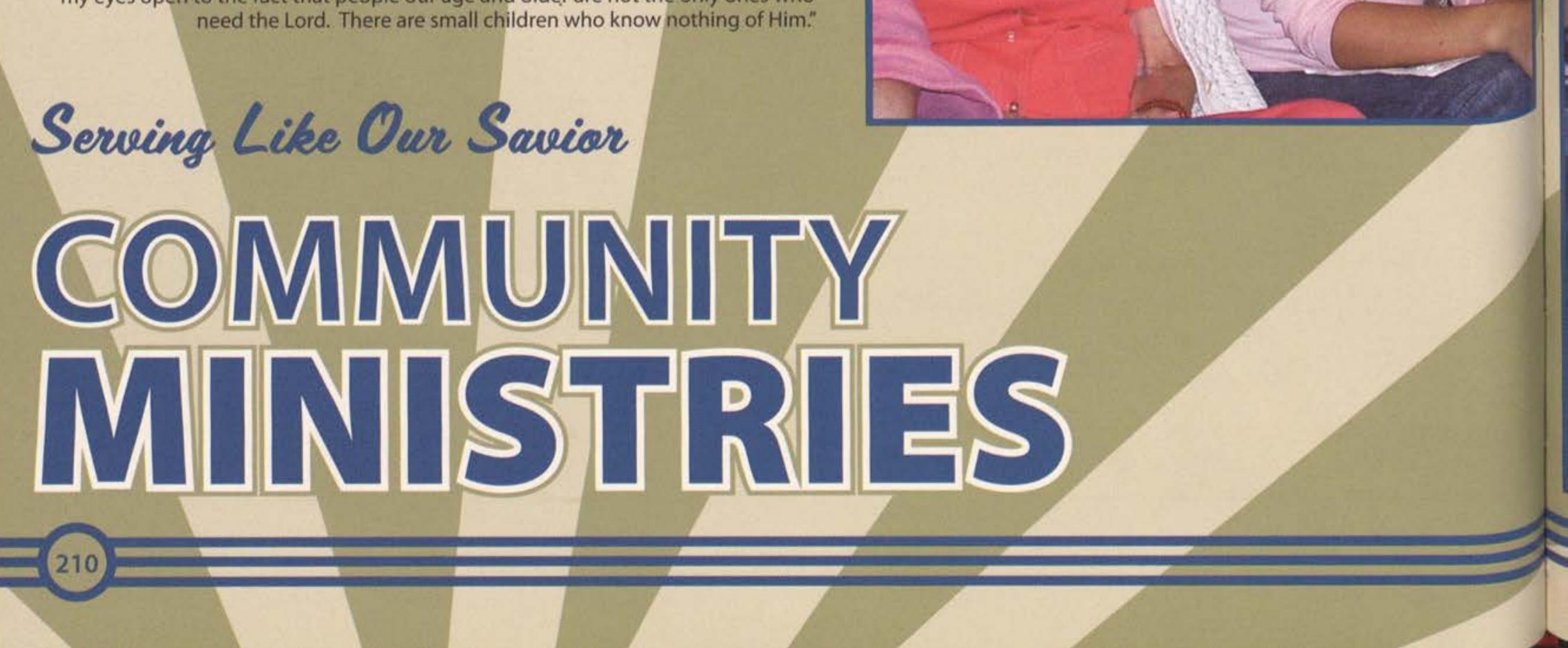



week off to serve God in missions. From Mexico to Chicago, MIS teams scoured the globe spreading the gospel with effective words and compassionate actions.

Just because Eleuthra, located in the Bahamas, has sandy beaches and balmy temperatures doesn't mean that the MIS team that traveled there lounged in the sun. Team leader Becky Hayes described their exciting trip: "We were fortunate to do assemblies in five high schools covering the entire length of the island. The theme of our high school assemblies was 'Come as You Are' and we emphasized that Jesus loves and accepts each of us just as we are." In addition to ministering to the inhabitants of the island, the Eleuthra team assisted full-time missionaries on the island by "helping with painting projects in their home and repainting the lines on a local basketball court," Hayes said.

Freshman Dori Eaves was on the Eleuthra team and noted that the trip was her first involvement in missions. "[The trip] was one of the best weeks of my life. Ministering to the kids and showing them Christ's love during that week is something I will never forget."

This year's MIS trip to Mexico encountered its fair share of challenges; however, those testing times culminated in stronger faith and a higher awareness of God's sovereignty. Junior Crystal Baldwin, MIS Mexico team member, noted that "God showed me many things that week, but I was impacted the most in the way that He is sovereign and in control of everything. Even when I am at my weakest times, $\mathrm{He}$ is strong and $\mathrm{He}$ is working in people around the world bringing them closer to Himself."

During a Spring Break trip to Chicago with Inner City Impact, Heather Flippin takes v time to snap a picture with the 2 nd grade boys who caused her so much trouble throughout the week. Flippin says, "These boys made me cards of appreciation for 'putting up' with them...Spending my Spring Break in ICI is definitely an experience I'll never forget!"

Loren Montes colors in an activity book with two kids from Chicago's Inner City Impact. This Spring Break team ministered to kids in inner city Chicago by tutoring and assisting in classrooms during the day, and putting on Bible clubs and youth activities in the evening.

Austin Engel sorts clothes at The Rescue Mission in Syracuse, NY. Among other jobs during the week they served food and ministered to the homeless

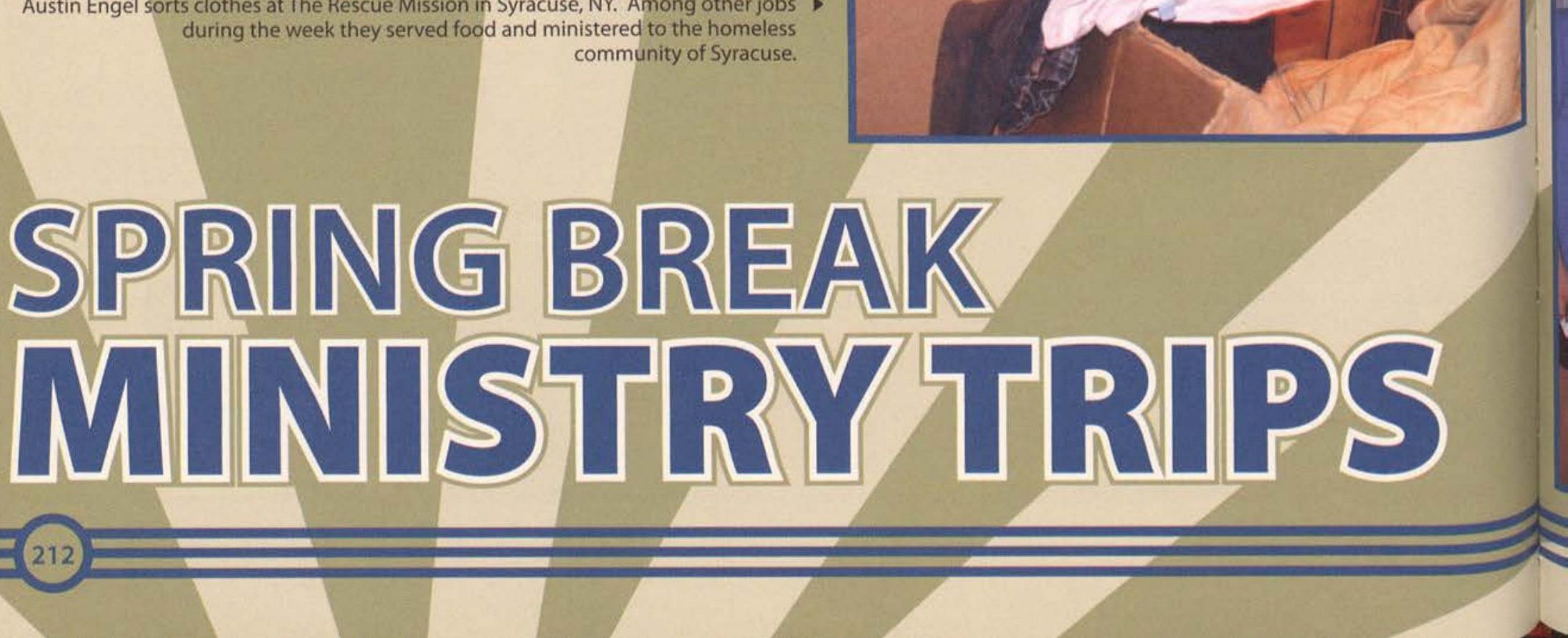



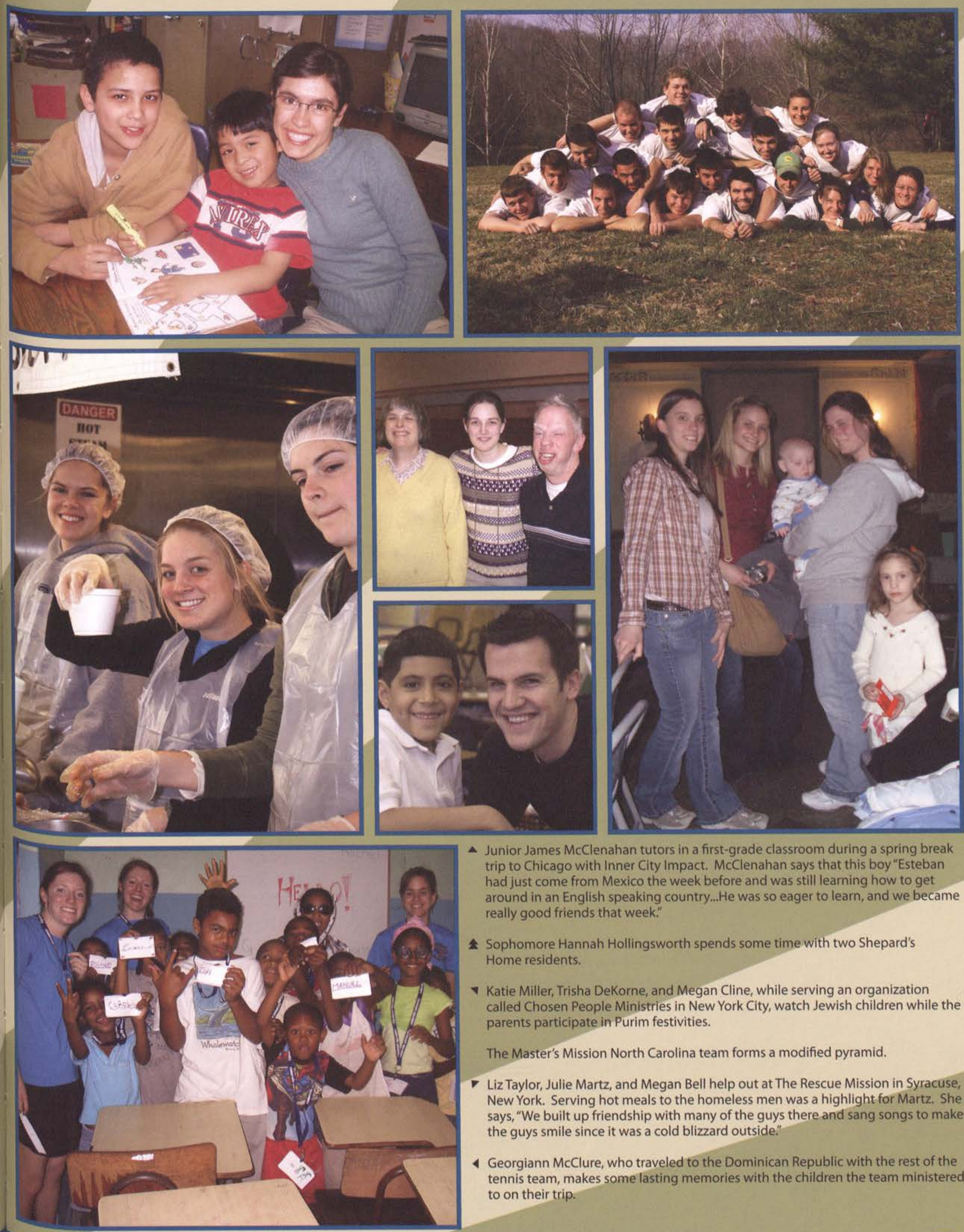

- Junior James McClenahan tutors in a first-grade classroom during a spring break trip to Chicago with Inner City Impact. McClenahan says that this boy "Esteban had just come from Mexico the week before and was still learning how to get around in an English speaking country.... He was so eager to learn, and we became really good friends that week."

A Sophomore Hannah Hollingsworth spends some time with two Shepard's Home residents.

Katie Miller, Trisha DeKorne, and Megan Cline, while serving an organization called Chosen People Ministries in New York City, watch Jewish children while the parents participate in Purim festivities.

The Master's Mission North Carolina team forms a modified pyramid.

- Liz Taylor, Julie Martz, and Megan Bell help out at The Rescue Mission in Syracuse, New York. Serving hot meals to the homeless men was a highlight for Martz. She says, "We built up friendship with many of the guys there and sang songs to make the guys smile since it was a cold blizzard outside."

4 Georgiann McClure, who traveled to the Dominican Republic with the rest of the tennis team, makes some lasting memories with the children the team ministered to on their trip. 

on missions. Students have the opportunity to participate in shortterm missions trips and ministry experiences through Missions Involvement Services (MIS), led by Brian Nester. Students participate in teams which travel to countries around the world each year during Christmas, Spring Break and throughout the summer. Since the year 1970, Cedarville MIS has sent over 5,000 participants to over 89 countries. In the 2006/2007 school year, students traveled to places like Jamaica, Hungary, Australia, and Mexico, reaching people through a variety of ministries.

Students who traveled during the summer of 2006 were given an opportunity to share their experiences during MIS week in October. This week was also a chance for both new and returning students to be exposed to missions opportunities around the world, as well as ways they could be involved during their time at Cedarville. Information about the upcoming MIS trips was also made available for students to see where God could possibly use them and lead them to on a trip in the near future.

Cedarville's MIS has played a huge role in the lives of many students over the years. "I've been able to go on the same trip for the last three years. It has been really neat to see how God works year-to-year and the impact our teams have had. It has been so amazing and life-changing for me," said Michelle Evington of her ministry at an MK camp in Mexico over Spring Break.

The purpose of MIS is not only to send students on short-term missions trip but also to broaden their interest and understanding of missions and give them exposure to how that translates into real life. Not only will this experience let them see missions for themselves, but it will also allow them to truly serve others.

To Jason Moss age does not matter as he works on building relationships with the children of Mexico.

The Eleuthra team was privileged to encourage several groups of young people with the Gospel. They left each group with a reminder of the message: a large sign scattered with their handprints. Jada Holloway and a young Eleuthran display their "painting tools" for the camera.

Mark Hershey, Josh Hueni, Anthony Rutrough, Adam Ploeg, Brian 4 Alburger, and AJ Clemans pose with a large group of Guatemalan children. Ploeg says, "We went on this trip to Guatemala with intentions of helping a remote village build a church. Not only did we do that, we also were able to bond with all these kids. It was a great experience. We went with "our" plan in mind. God, however, had other plans."

\section{Going Ta Ale The World!}
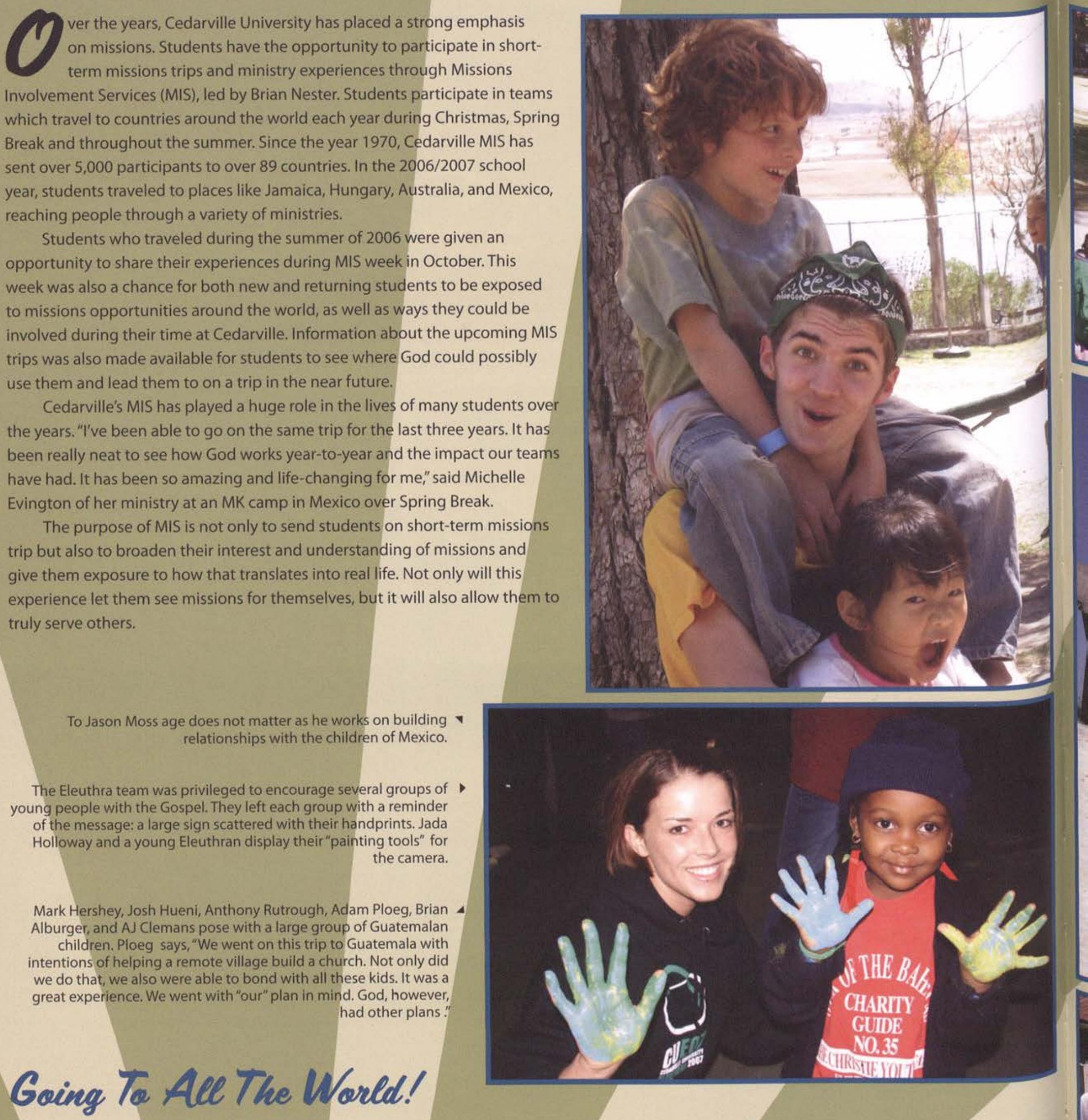

$$
\text { = }
$$

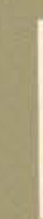

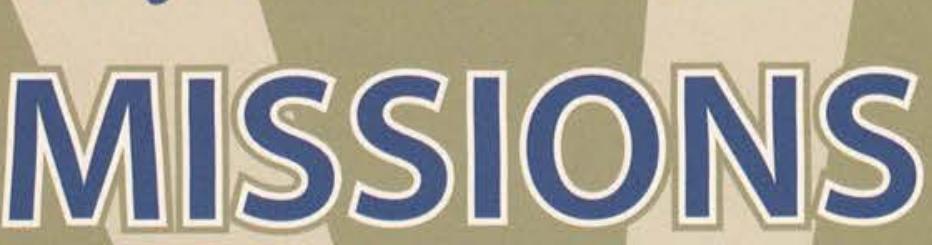
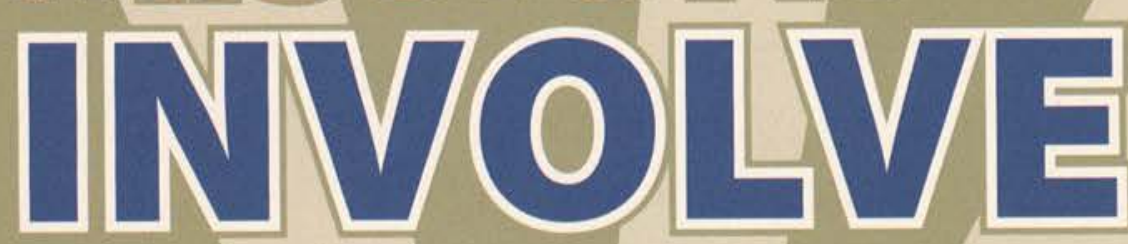

campus, but this year there seemed to be a heightened feeling of excitement and need surrounding these groups.

The women's groups focused on such topics as prayer, finding time for God, and straightforward gospel studies. And while the focus is always on the Word and how to practically apply it, the dynamics of Discipleship Groups extend even further. Junior Becky Bachman led a group that studied the book of James. In addition to learning more about this important book, she said, "I really wanted the chance to minister to other girls that deserved a chance to speak out and learn in the ways that they wanted to and expected."

On the men's side, discipleship groups delved into purity, God's love, and the examples set by great men of the Bible. Chad Eckard led group discussions and application of the book Sex and the Single Guy. Like several others, Eckard's group not only made excellent progress in their relationships with God, but also with each other. "We not only met weekly [to discuss the book], but we also formed relationships when we took extra time to hang out and just spend time with each other."

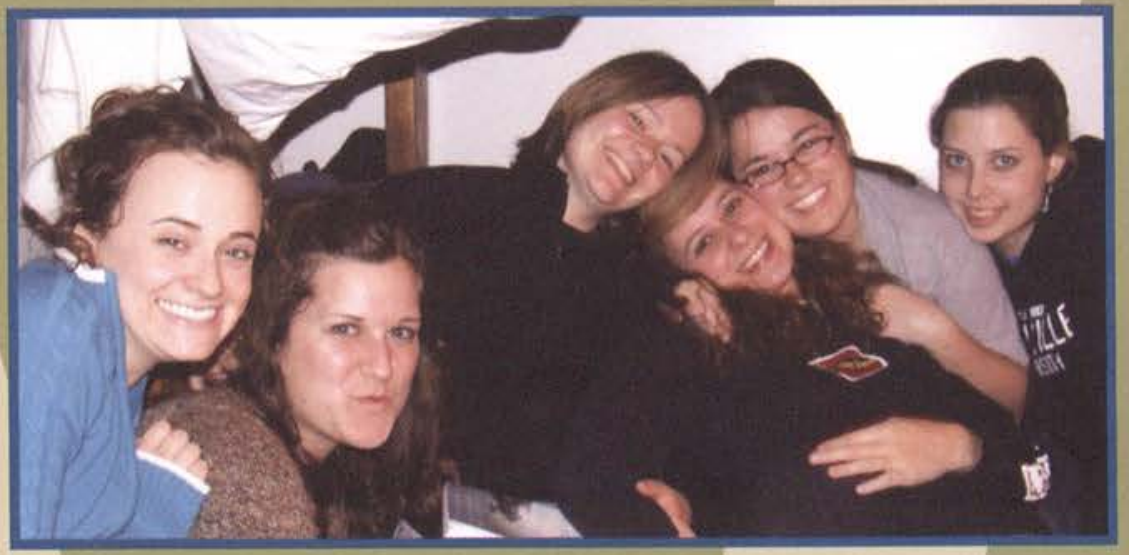

Alyssa Adams, Kelley Kinnard, Kate Philpott, Christina Taylor, Kim Brown, and Laura Robbins relax at their group meeting.

Ashley Hunter, Laura Parker, Andrea Moore, Carolyn Reiley, Jennalee Elmore, and Sarah Baczek hug a tree. Carolyn Reiley said, "It was a huge blessing to be able to study God's word, each week, with a group of girls who loved each other and were on-fire for the Lord!"

Alison Kearney, Abbey Petersen, Andrea Moore, Sarah Miller, and Sarah Crichlow create a pyramid of fun! Kearney says, "Being involved in my discipleship group was amazing! It gave me the opportunity to consistently work through a book with godly girls and get to know them on a deeper level.

Kaitlin Pankratz, Jen Davis, and Ivey Harrell brave the wind on the 4 Discipleship Council winter trip. Pankratz says, "I will always be thankful for my DC Family, how they are commited to seeking intimacy with Christ first,
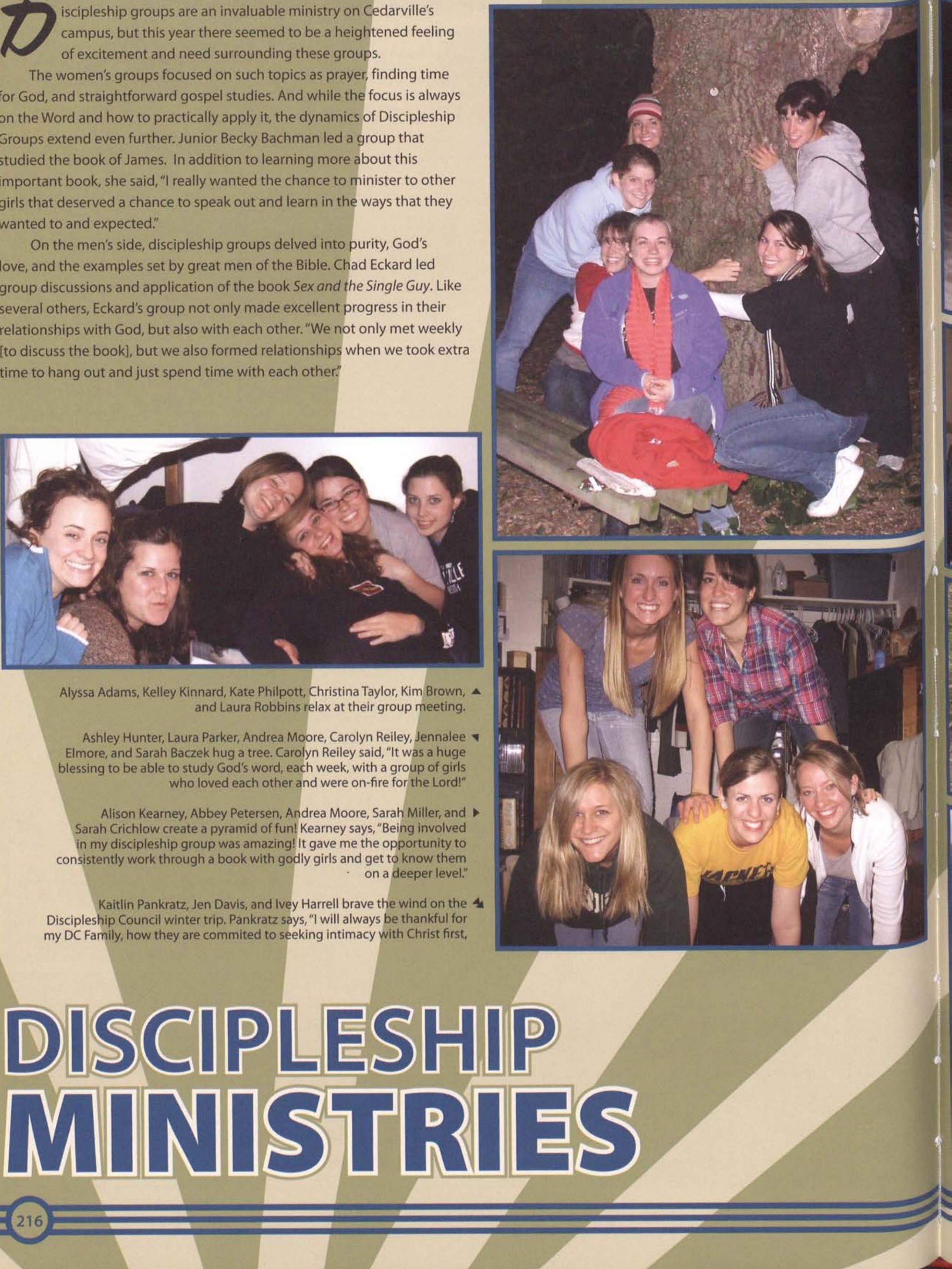

e get our hands dirty. My summer included painting cabins, scraping oatmeal out of my hair, cleaning toilets, and other

kinds of dirty cleaning. We are called and ready to serve," said HeartSong member Amy Dennis. HeartSong and the other Touring Team Ministries worked hard this year but had fun doing it. Celeste Cato said, "My favorite part of HeartSong is getting to stay with different families at every church. It's always fun getting to know people and learning about their lives."

The Master's Puppets teams also had rewarding experiences, but junior Andrew Davis remembers one event above the others. Andrew said, "During our show, a snake slithered backstage. I remember seeing two girls from my team running out from the puppet area and my team leader beating the living daylights out of something on the ground with a towel. We had quite a time getting the kids to concentrate after that." The Master's Puppets teams truly love the kids they minister to. Jill Forsberg said, "Our puppet team director always tells us, 'You impress from a distance, but you impact up close.'It is our goal to impact as many children for Christ as we can."

The Lifeline Players also made an impact, not only on their audience, but also each other. Andrew Tripp said, "I have had the privilege of serving with five teammates who make me laugh until my stomach hurts and remind me what it looks like to love and serve others." Rebecca Wolff said, "Our ministry is not only on the stage but also in the homes. It has been a blessing to meet people and learn how the Lord is working in them and through them."The Touring Teams represented the Lord wherever they went and grew closer to each other through their ministry.

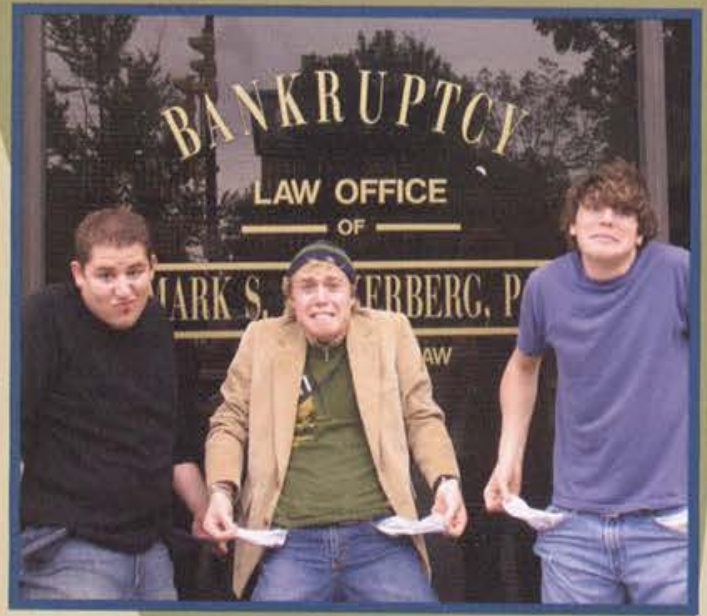

- Matt Beres, Grant Hansen, and Andrew Tripp improvise a moving scene to show the real-life financial situation of many college students.

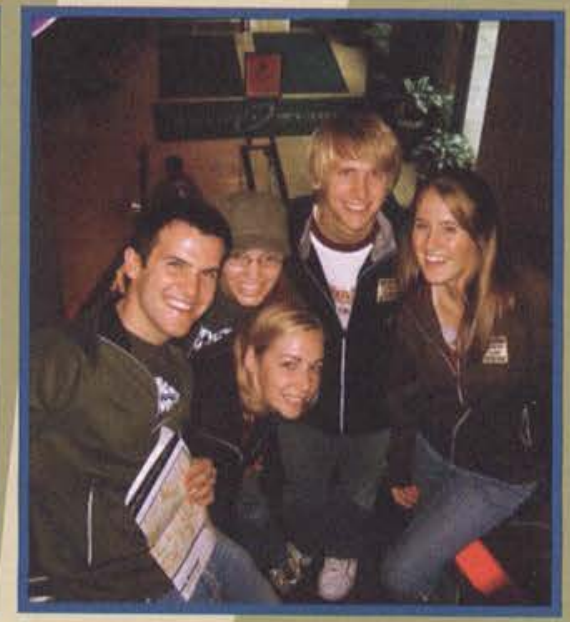

A James McClenahan, Jill Forsberg, Megan Mertus, Jonathan Walburg, and Crystal Baldwin take some time out of their busy schedules to snap a photo.
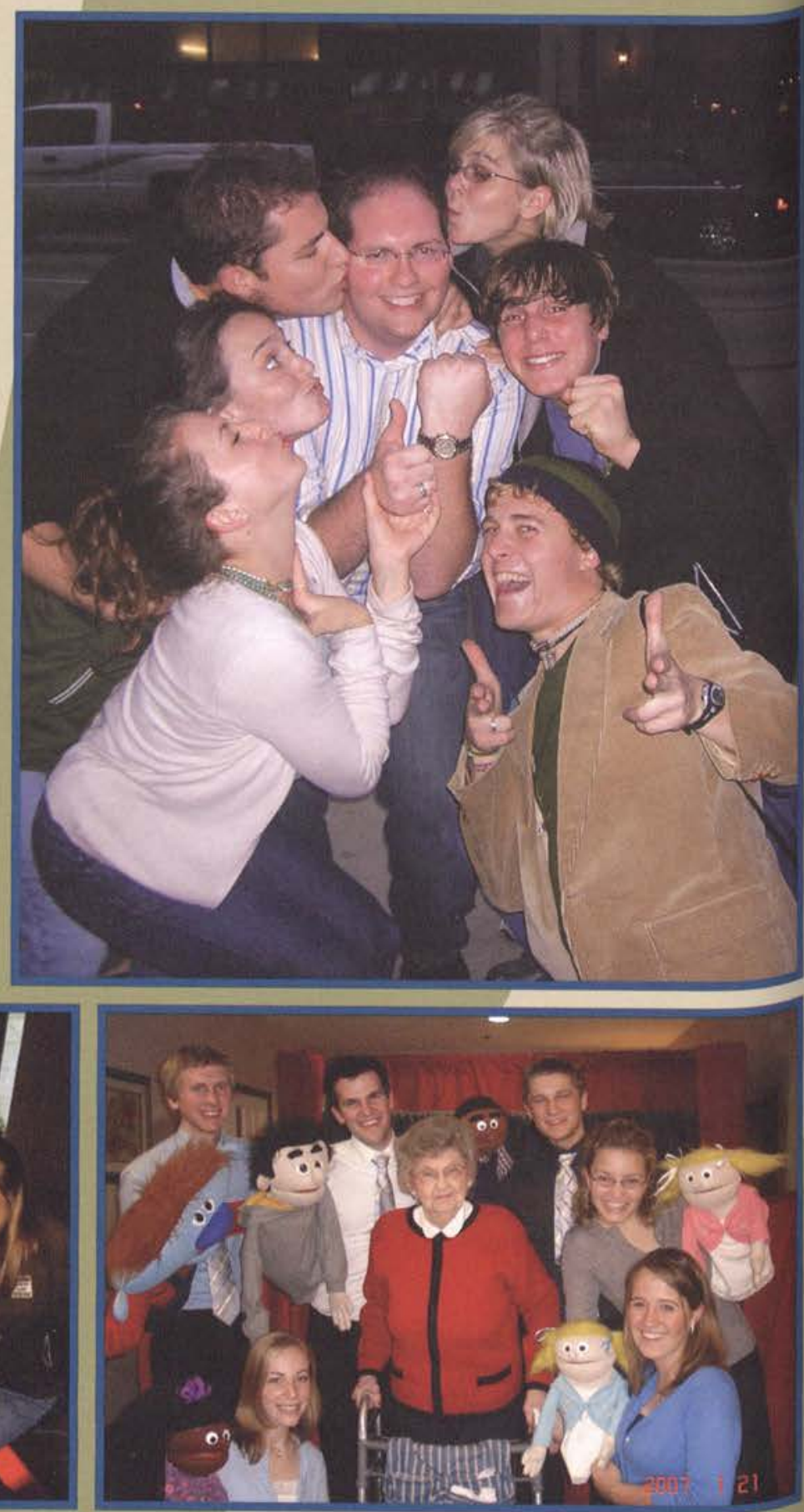

- Jonathan Walburg, Megan Mertus, James McClenahan, Zach Seelye, Jill Forsberg, and Crystal Baldwin entertain a CU alumnus at a nursing home.

4 The Lifeline Players shower Joel Tomkinson with adulation for his terrific leadership.

Impacting Lives!
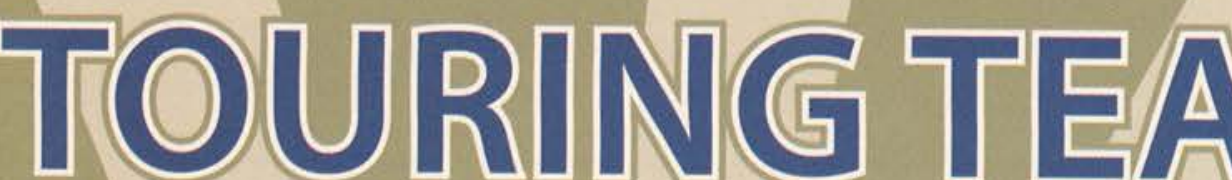

.
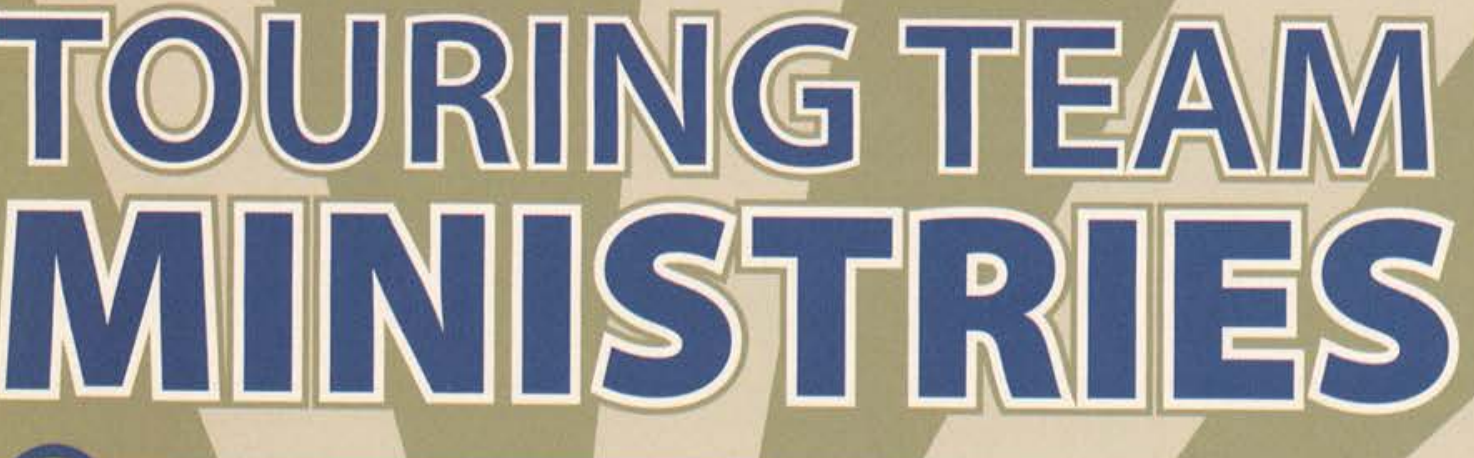


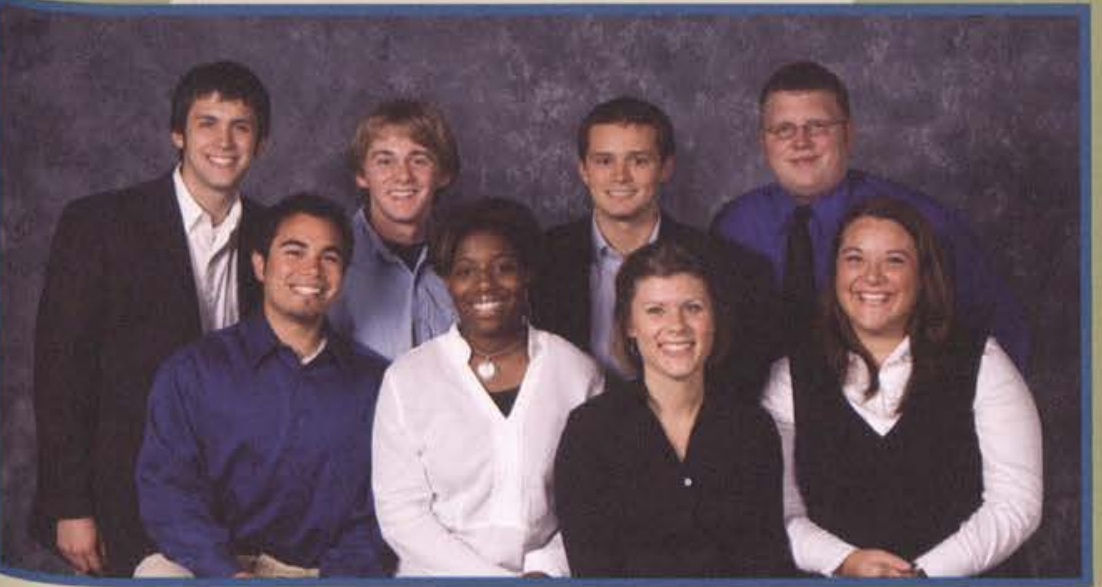

Heartsong 1 (Not Pictured:Luke Seelye)

Back Row: Seth Gibson, Matt Ward, Justin Meyers, John Carey

Front Row: Rich Davies, Jerica Ward, Cara Slaybaugh, Celeste Cato

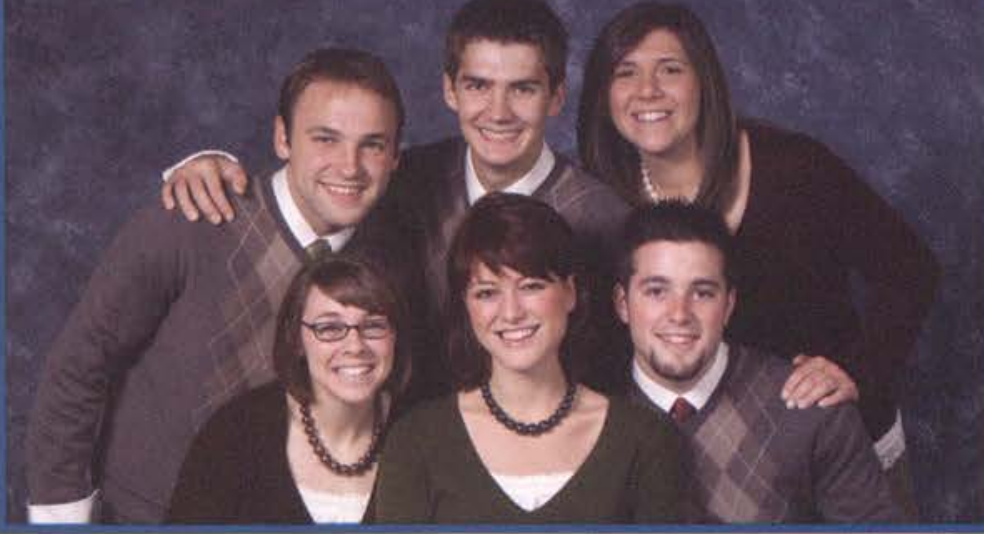

The Master's Puppets 1

Back Row: Andrew Davis, Jason Moss, Terri Schenk Front Row: Rachel Mundy, Katie Lippman, Devin Kramer

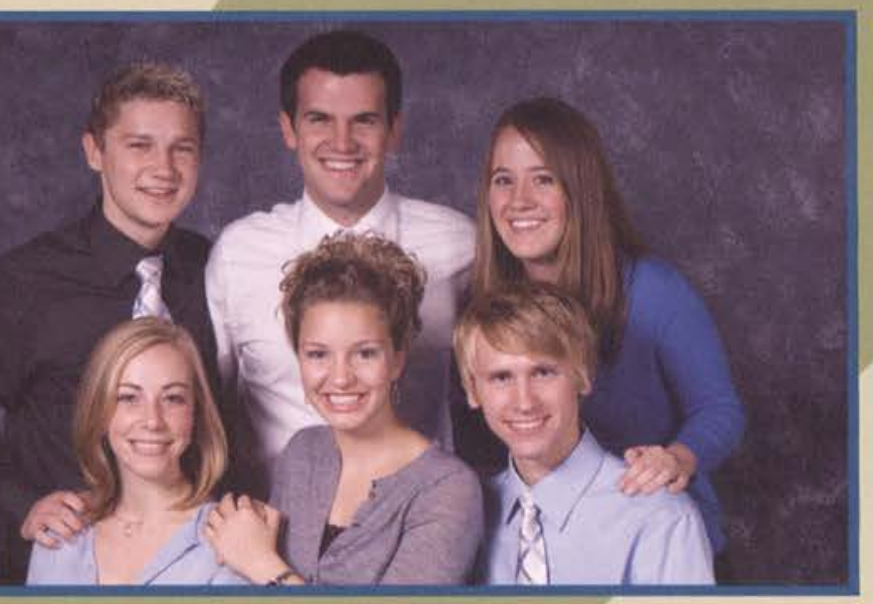

rat

The Master's Puppets 2

Back Row: Zach Seelye, James McClenahan, Crystal Baldwin Front Row: Megan Mertus, Jill Forsberg, Jonathan Walburg

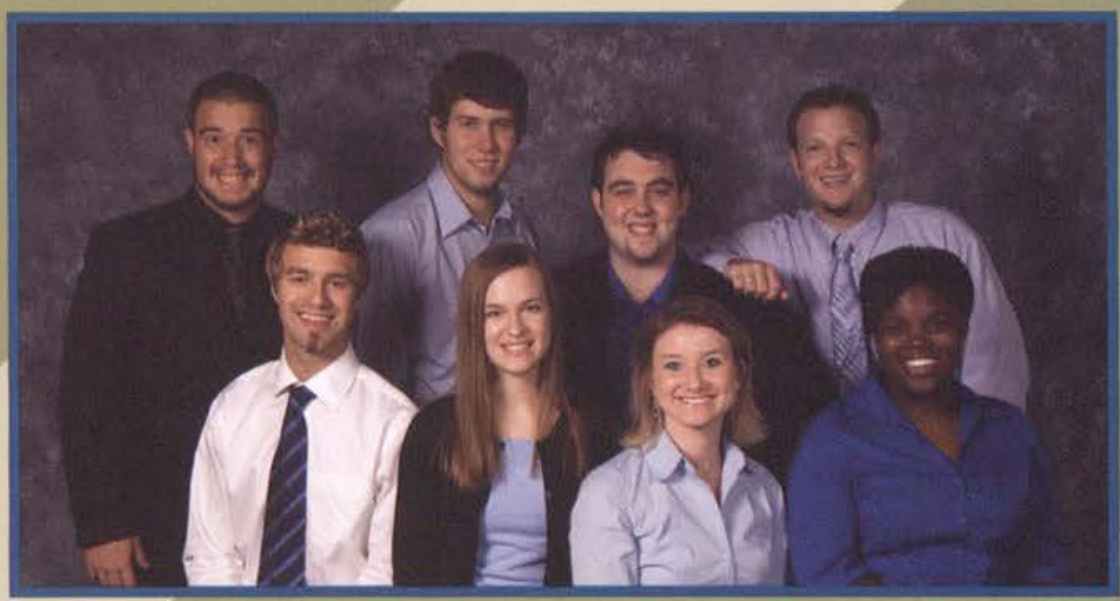

Heartsong 2

Back Row: JB Waggoner, Adam Hundley, Tony Bostic, Nathan Lane Front Row: Colby Taylor, Sarah Smoyer, Lori Crain, Charmaine Porter

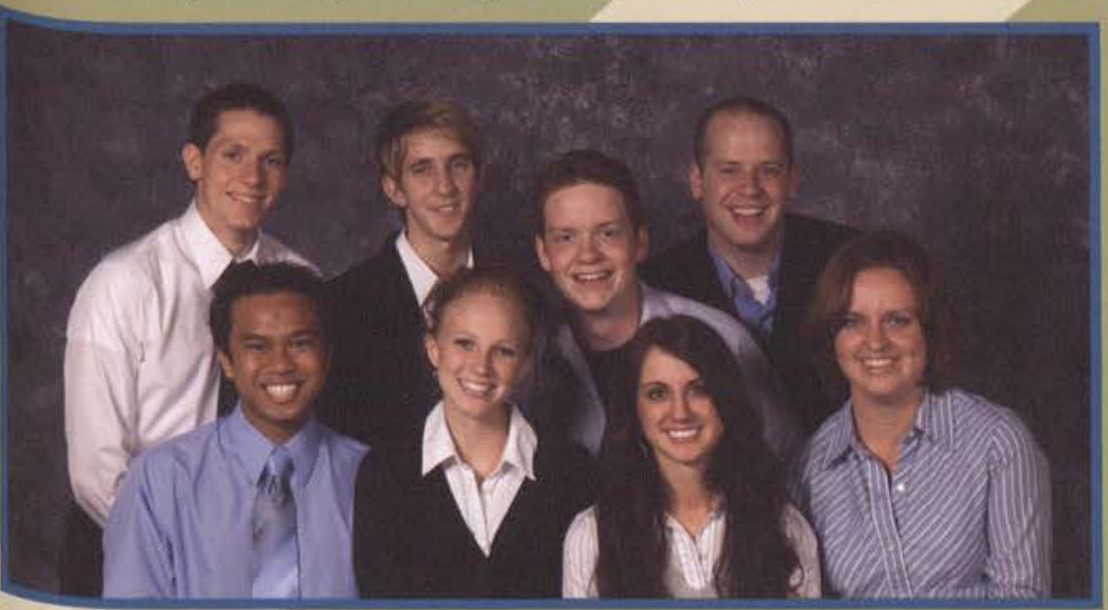

Heartsong 3

Back Row: Nate McCloskey, Keith Sider, Garrett Williams, Ty Anderson Front Row: Tim Labrado, Amy Dennis, Aubrey Womack, Dana Snyder$$
8
$$

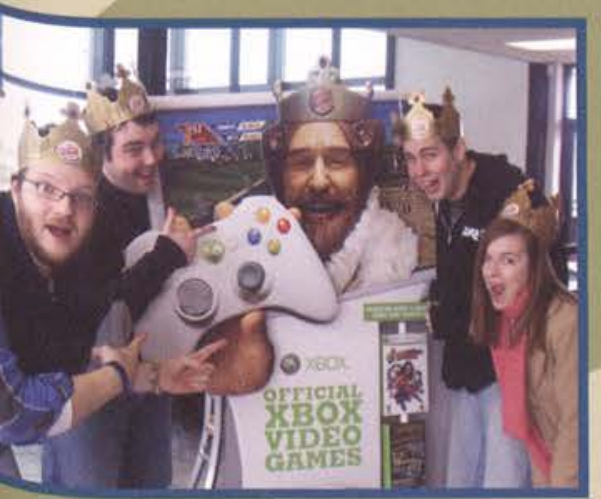

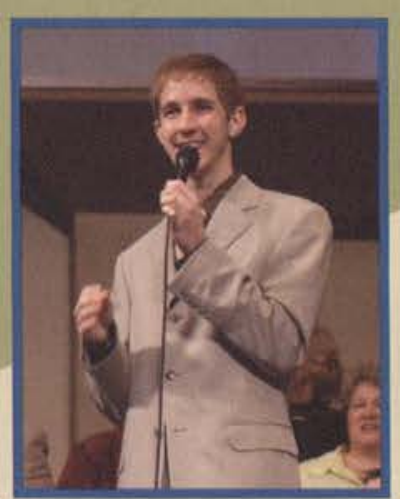

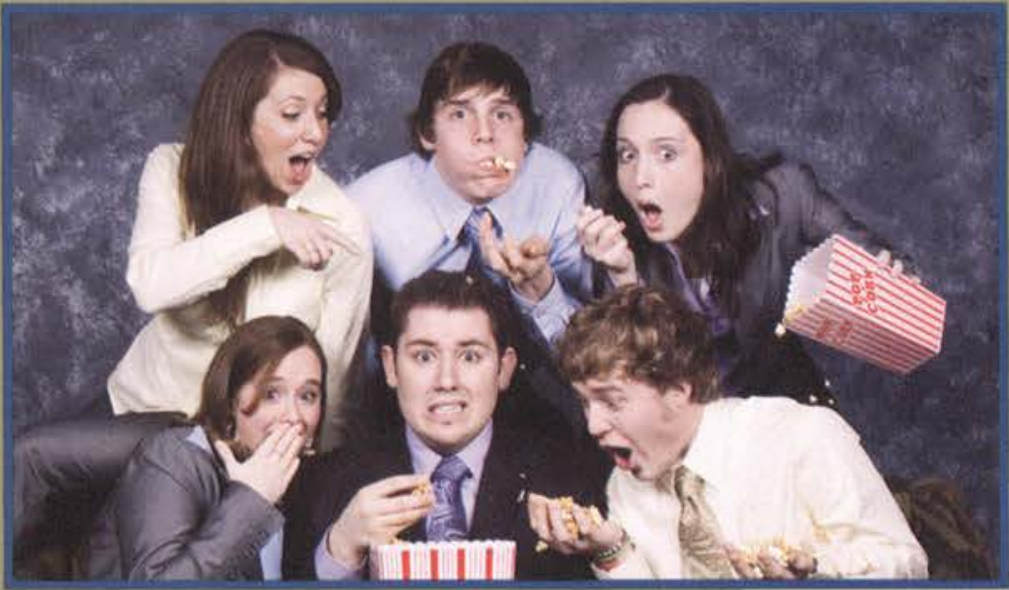

The Lifeline Players

Back Row: Rebecca Wolff, Andrew Tripp, Victoria Duff Front Row: Alexandra Clements, Matt Beres, Grant Hansen

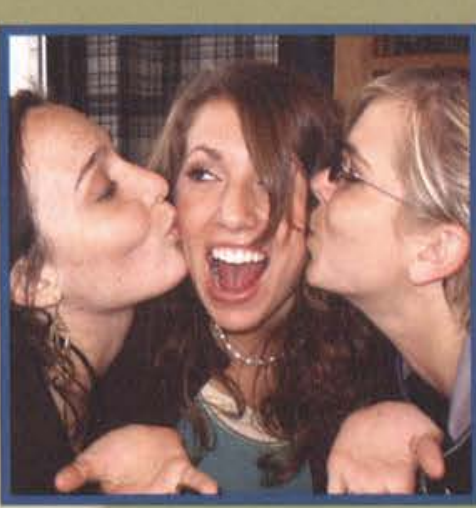

4 Victoria Duff, Rebecca Wolff, and Kelsey Nelson ham it up for the camera.

- Keith Sider, with his amazing Josh Grobanesque voice, joyfully leads a group in singing praises to the Lord.

Nathan Lane, Tony Bostic, Adam Hundley, and Sarah Smoyer "have it their way" while on tour. 


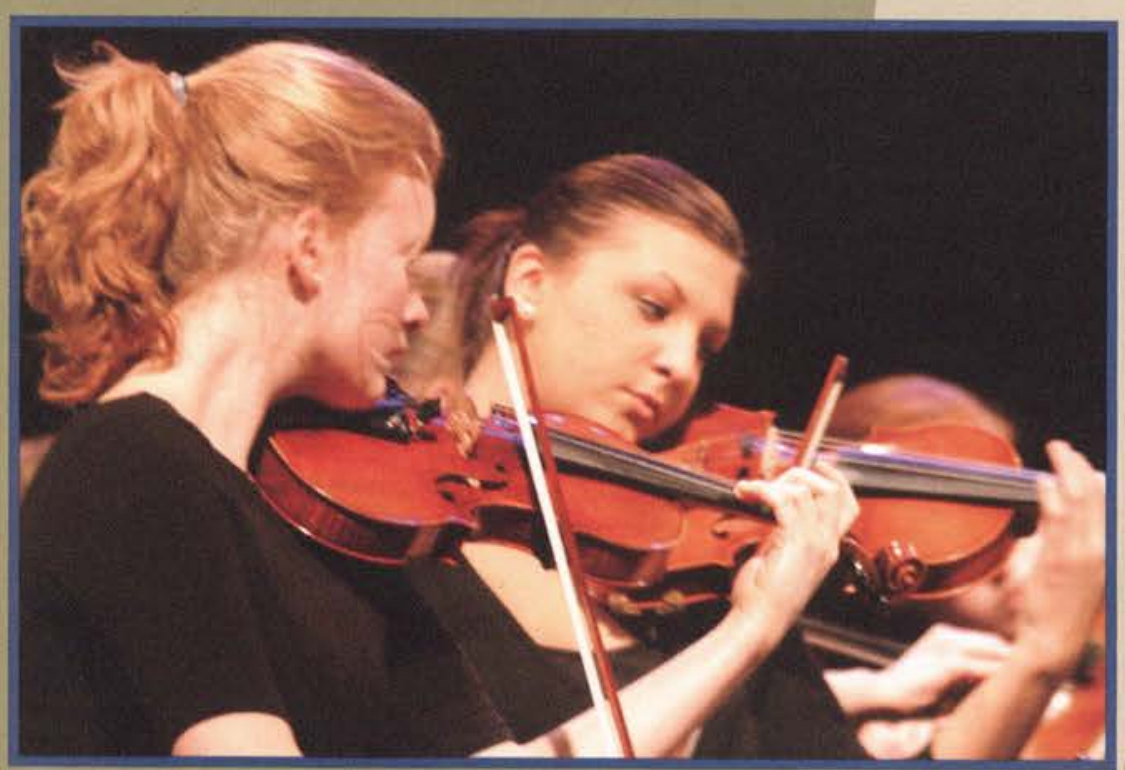

\section{A hether or not Brahms and Souza were widely played by the instrumental groups was impressive. With ensembles like the} Brass Choir, Symphonic Band, University Orchestra, Jazz Band and others, Cedarville produced fantastic music throughout the entire year.

This year the Brass choir went on a five-day tour to Washington D.C. and was a featured performer at the Gabrieli Festival hosted by Wittenberg University. They also had the opportunity to perform with nationally known composer James Curnow along with the Symphonic Band. The Symphonic Band, heard in the Vets and Charter day chapels this year, played highquality and serious literature during the year and were heard in churches throughout the area in a mini tour. Sophomore John Carey said of the group, "The experience of practicing and performing alongside my fellow student musicians here at Cedarville has been wonderful. It was incredible to see such gifted players using their talents for the glory of God."

The University Orchestra was a challenging but enjoyable group for many students. Playing Brahms' Requiem for the annual Masterworks Concert along with their showcase concerts and Pops concert, the group continued their tradition of playing high caliber music. Sophomore Cristy Hatch said of the group, "My favorite thing was playing Brahms. It was difficult music, but we felt such a sense of accomplishment after performing it well."

The Jazz band energized many listeners this year with their catchy tunes. They played for a World War II reunion, for vocalist Dave Boyer, and also had the opportunity to play with guest sax player Hal Melia. Senior Micah Martin said of the group, "The Cedarville Jazz Band was committed to playing top-notch literature, while having fun at the same time. I know that for myself, playing in Jazz Band was always one of the highlights of my day."
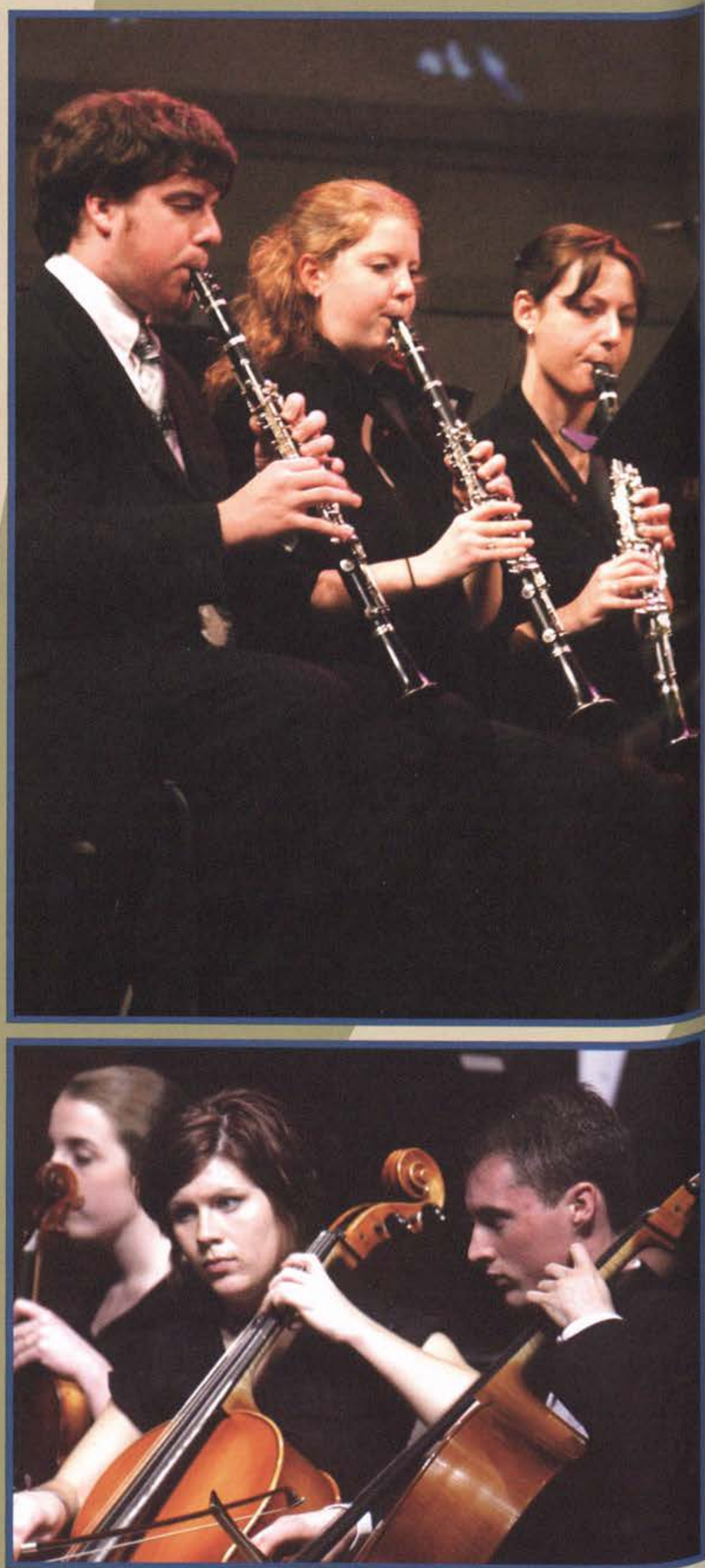

- Amanda Hamilton, Cara Slaybaugh and Randall Plate are focused on their music as they play their cellos.

Kaitlin Sattler (middle) adds to the sounds of the orchestra with her presence in the claranet section.

Everyone loves to hear the violin, but there is something special about it when

it is being played to the glory of our Lord. Here Hilary Young plays for that very reason. 
Pictured in the Jazz band trumpet section are Aaron Hollopeter, Micah Martin, Daniel Lewis, Chucky Klint and Nora Chasnov. In proclaiming his feelings about the section, Micah Martin enthusiastically gives a resounding, "Woot!"

Fairfield Commons Mall was graced with the presence of the Jazz band this 4 year for Christmas. Pictured here are Micah Martin, Daniel Lewis, Natalie Winder, and Ben Anderson.

Pictured from left to right are Tim Doenges, Steve McCune, Courtney Rice, $\mathbf{\nabla}$ and Micah Martin as they play their unique brass instruments in a familiar location, the recital hall.
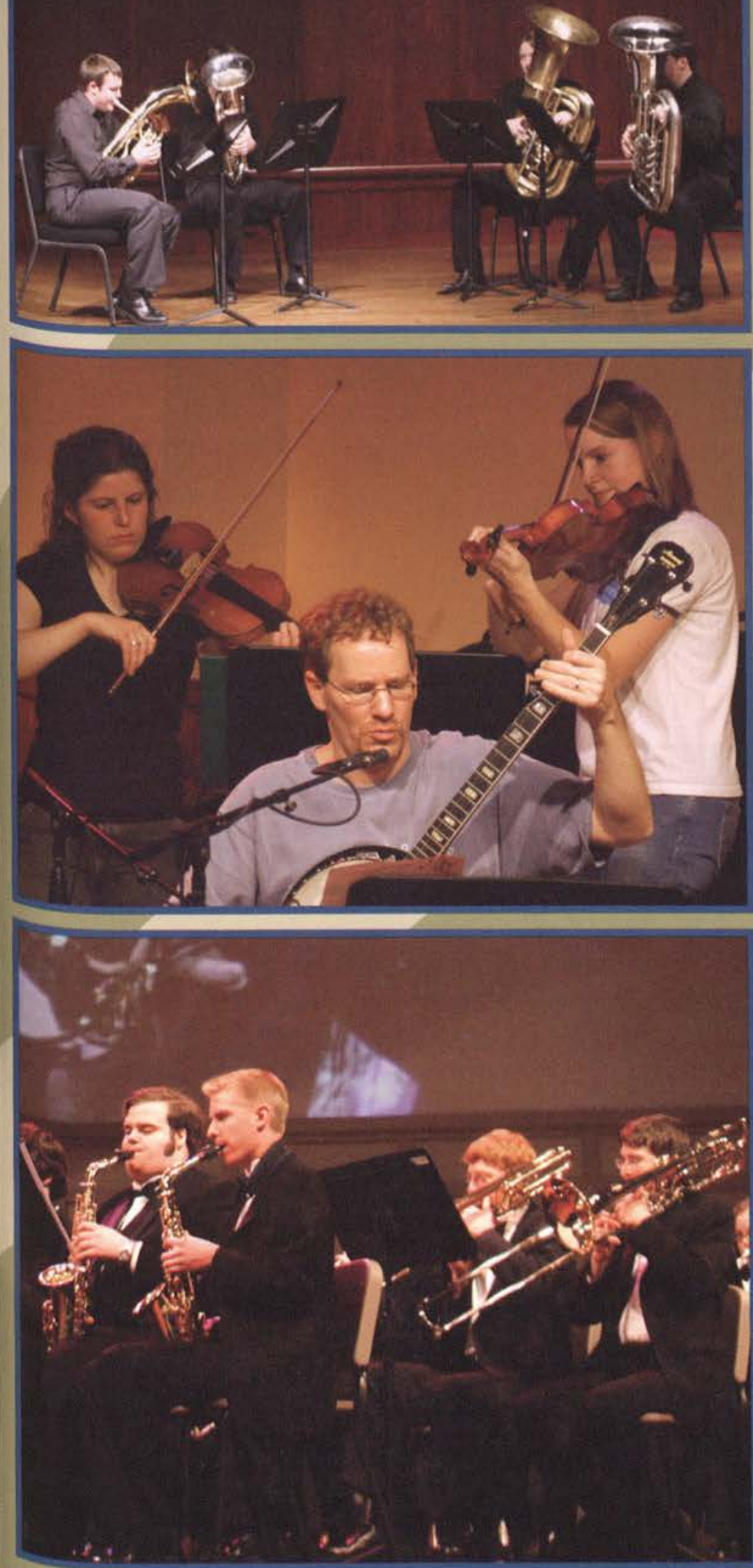
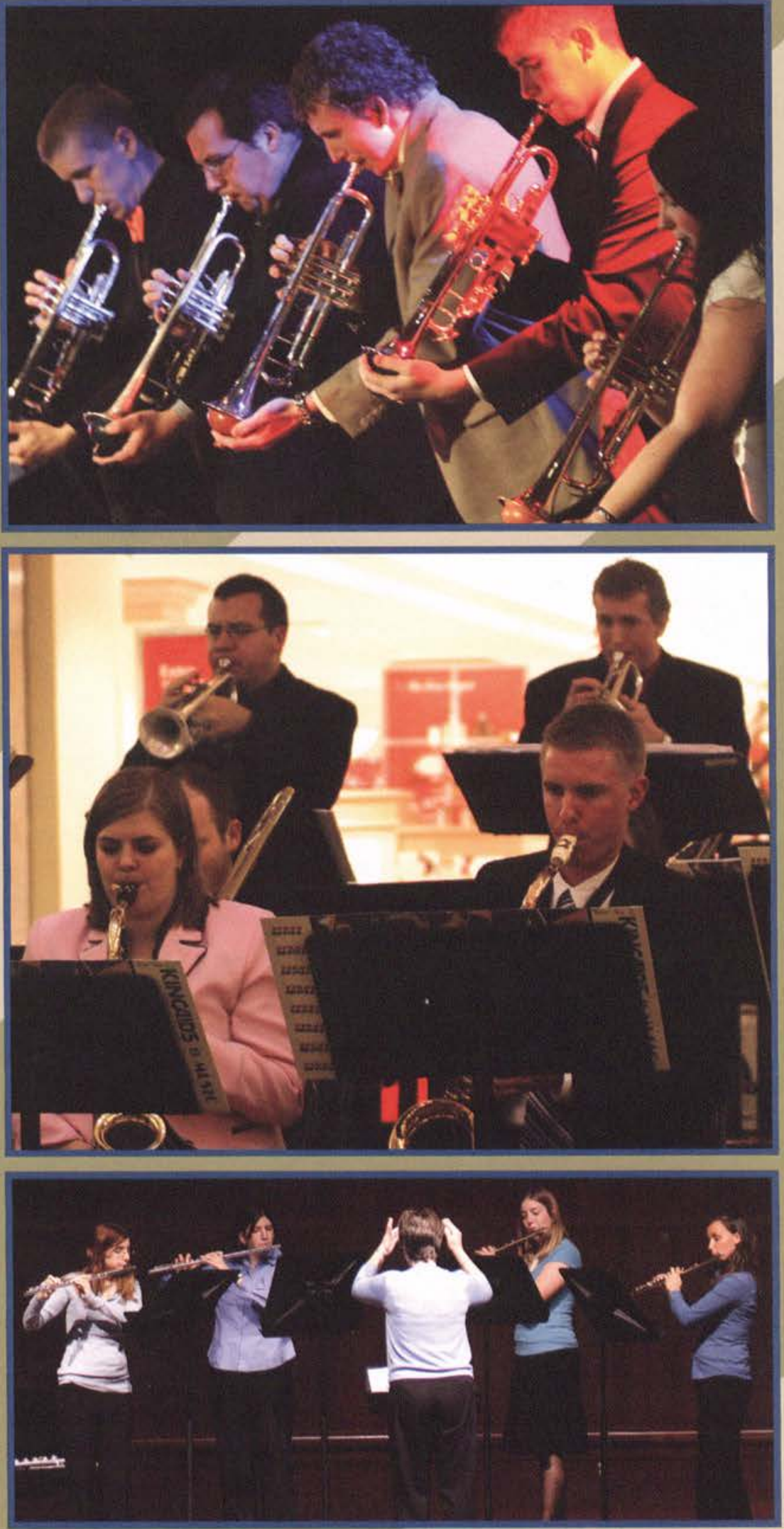

- Students can head to the recital hall to hear groups like the Flute Choir, which is always a special treat!

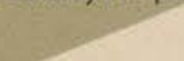

- Dr.Mortensen is always a favorite among students and faculty alike

whether he is playing the banjo or recorder. Here he is seen with two of his

fiddlechicks, Heather Hennessey and Sara Craig. "A highlight of the past year has been the Demerit's St. Patrick's Day gig in the Hivel" says fellow musician Micah Martin.

4 Brass Band members are a welcome sight on the Cedarville stage as they always bring the student body something new to hear. Lee Caterson, Carter McGriff are pictured behind Ben Anderson and Kevin Bennett. 


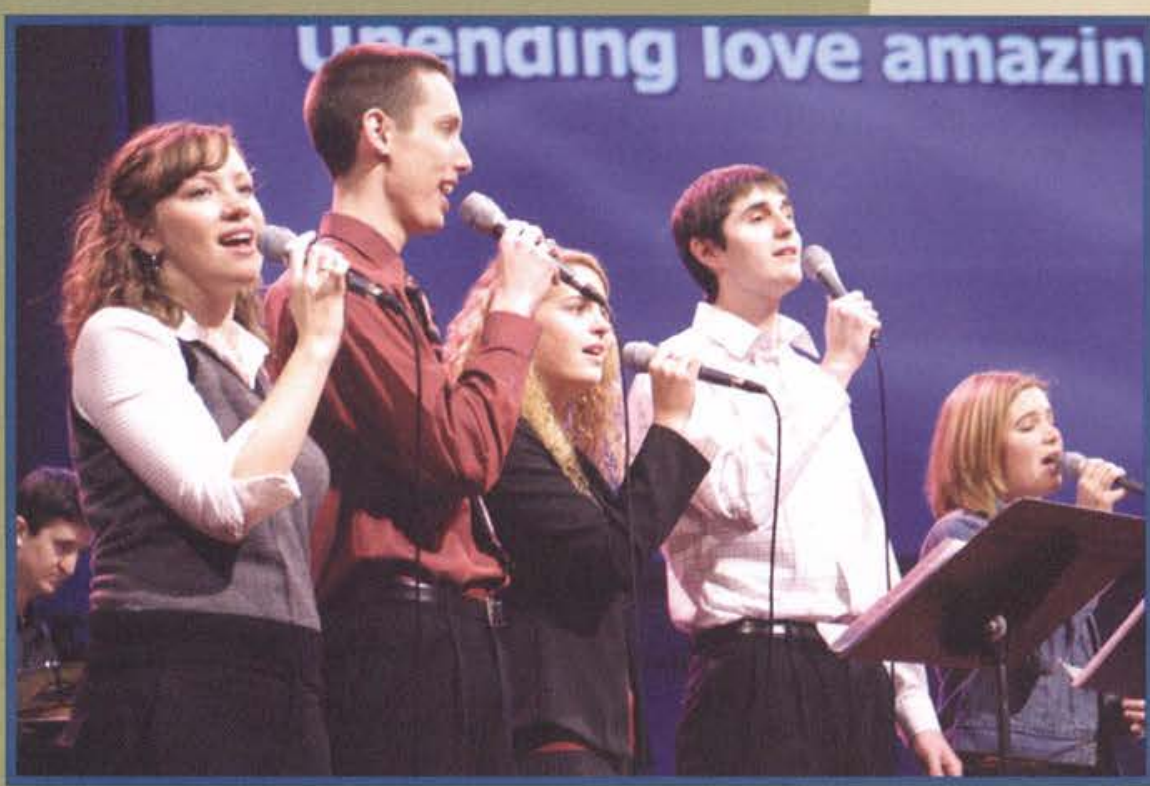

$w$ hat separated Cedarville choral groups from other schools this year was that the primary audience the students sought to please was Jesus Christ. Striving not only for excellence but also ministry, ensembles like the Concert Chorale, Men's Glee Club, Women's Choir, Jubilate, and One Voice spent many hours working to perfect the talent God gave them.

Each of the choirs spent extended weekends and weeks touring throughout various parts of the country. With tours from Indiana to New York, these students were able to both enjoy their music and reach out. Junior lan Casper of the Concert Chorale noted, "Touring was an awesome experience. It was the perfect opportunity to use the gifts God has given us to minister to and uplift others." Members of the Women's Choir, which ministered in a tour throughout Eastern Ohio and Pennsylvania, noted that during particular songs such as "My Lips Will Praise You," audience members often cried as the women not only sang but used sign language to worship the Lord.

The ensembles provided a place not only to grow as musicians, but also as Christians. Anybody involved in a music program would agree that being in an ensemble can provide an excellent group of fellowship. Jubilate ensemble member sophomore Brie Allen said, "We met twice a week to rehearse and always shared prayer requests and praises, so we bonded together as a choir really well."

The choirs all participated in the Masterworks concert, inviting guest conductor Neal Gittleman, conductor of the Dayton Philharmonic Orchestra. They sang an English translation of Brahms' German Requiem, considered one of the greatest pieces ever composed. Of the ensembles, junior Amanda Einselen said, "I loved listening to the choirs- I enjoyed the variety and the excellence for which they strived. It's neat to see people using their talents to worship the Lord."

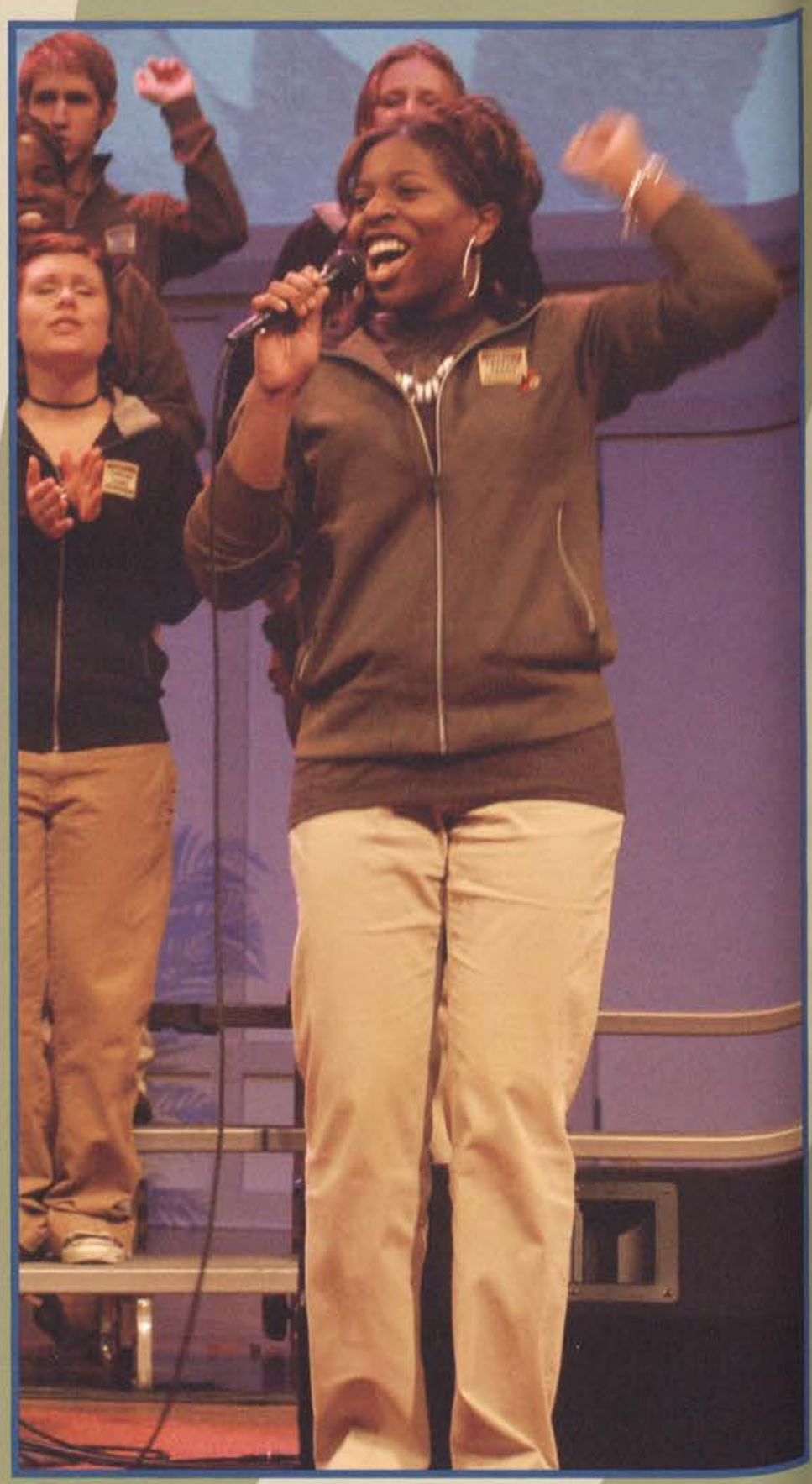

- Jerica Ward, backed by OneVoice \& Heartsong, belts out a song during a Parent's Weekend concert.

- The Jubilate Praise Team leads students in worship. Their ease with being on stage as well as their musical talent allows the rest of us to focus on the words to the songs and truly workship our God.<smiles>[C]1CCCC1</smiles>

- Women's Choir, directed by Mrs. Beth Cram Porter, is a select group of 50 girls renowned for their trips to Carnegie Hall. The Choir has traveled to Pennsylvania and Ohio this year, singing their signature piece, "My Lips Will Praise You." As junior Jen Guth says, "There is nothing like praising God through song with 50 other godly women by my side. We sing our hearts out to the Lord."

\section{$=$}

(a)

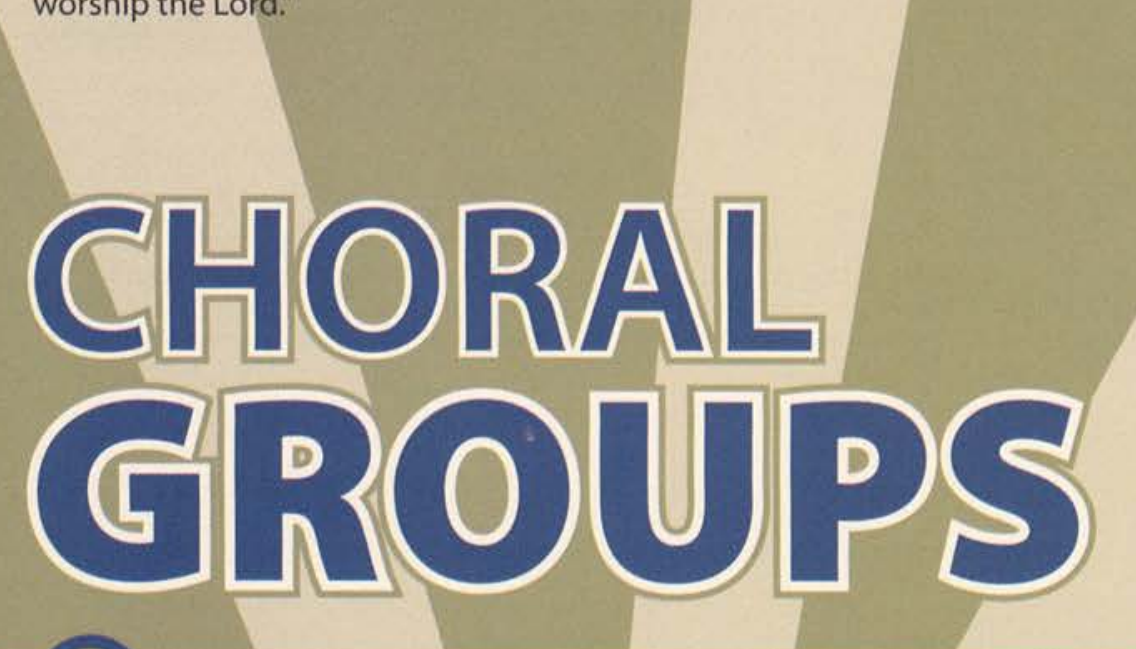




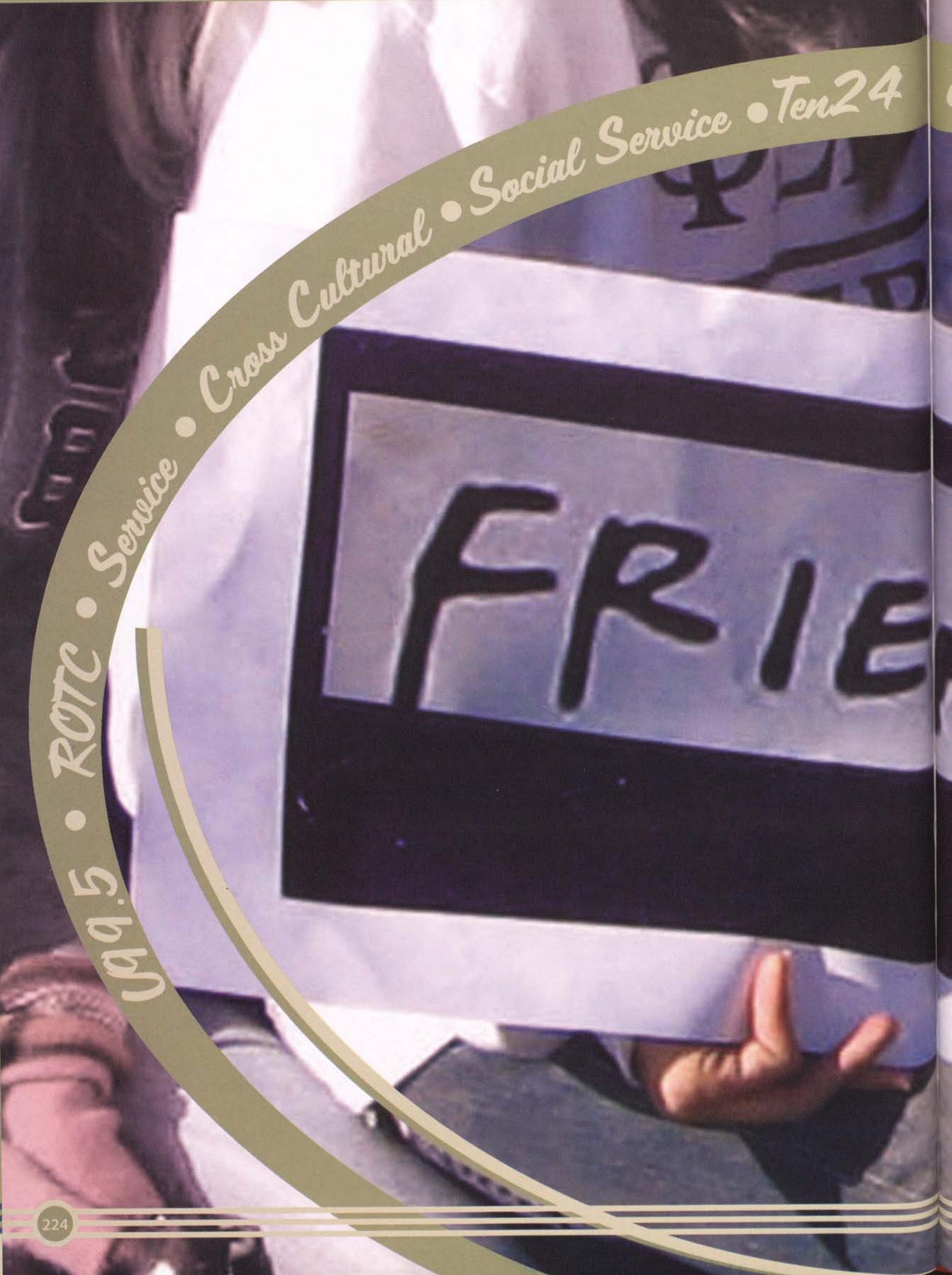


Cale - Crene SGA
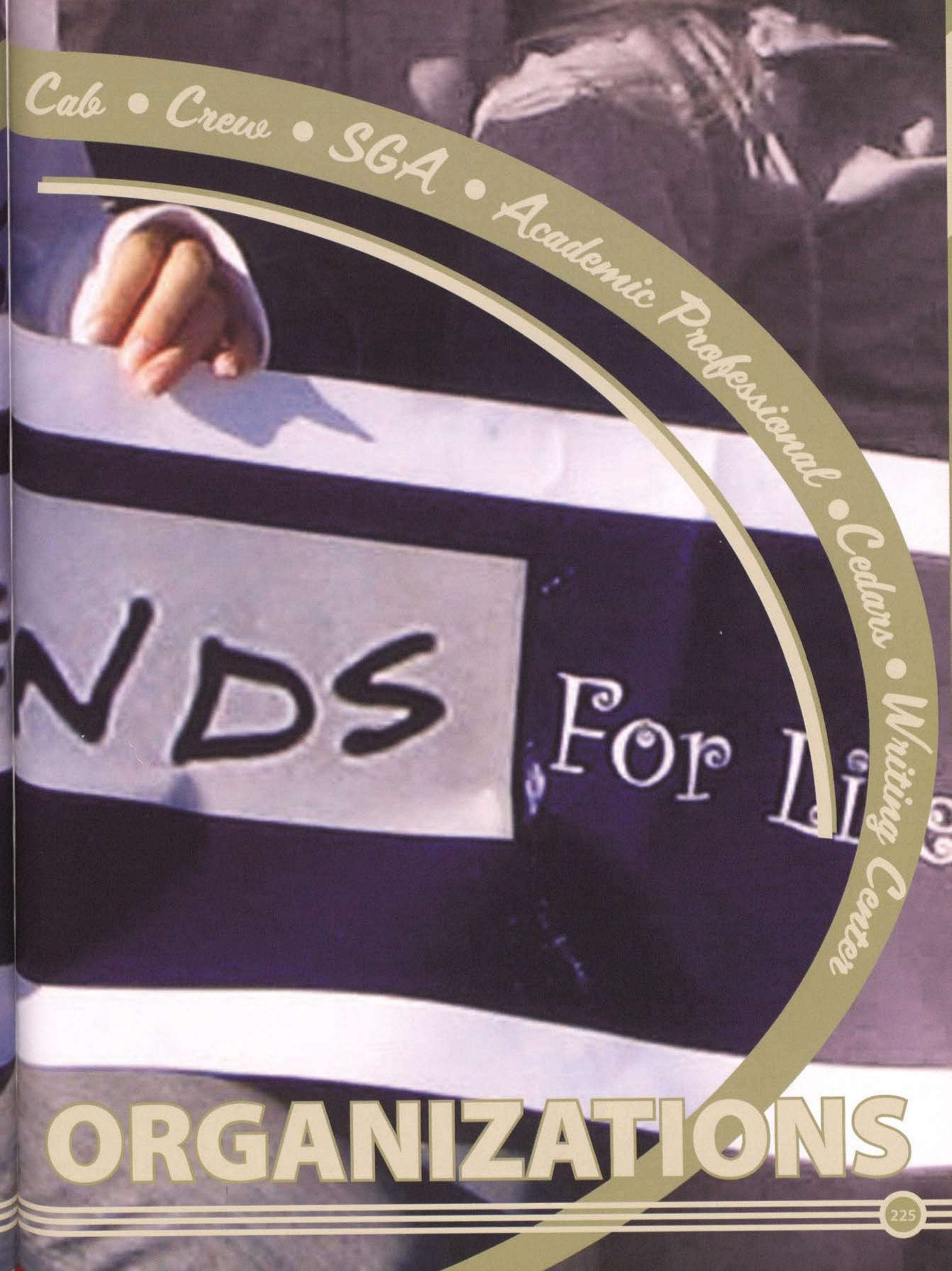

$\mathrm{FO}$

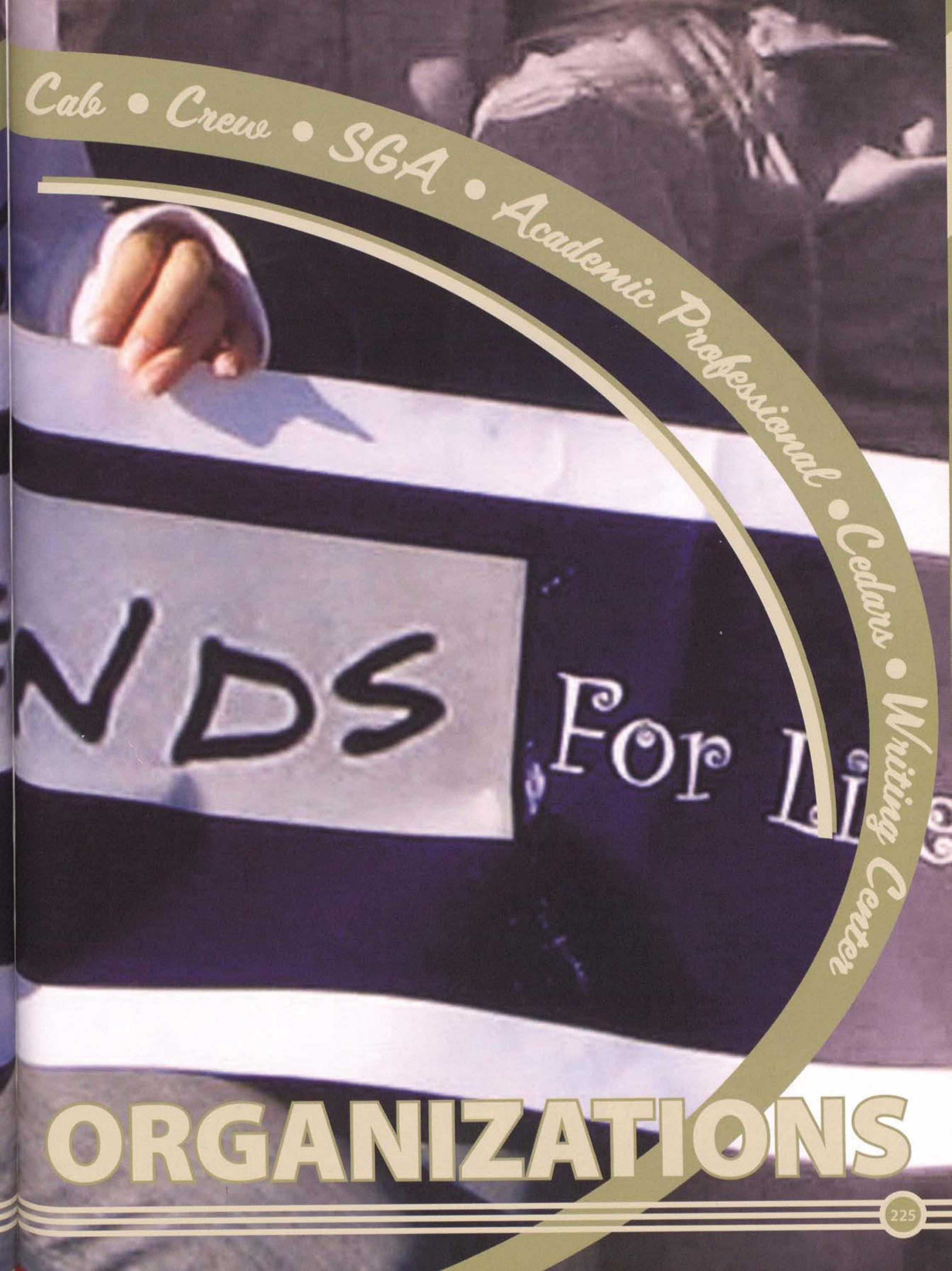


his year, Cedarville University once again proved the quality of its academics and academic organizations. From Pi Epsilon Omega to Chi Delta Epsilon to the Accounting Society, every academic field found a way to serve each other and the community outside of the classroom. Some of the memorable events produced by these orgs were the Christian Nurses Association's fun display at the Organizational Fair, Psi Kappa Theta's bonfire at Dr. Dolph's house, and the Freshman Hula Hoopla hosted by the Student CEOs. One brand new organization, Women in Business, was extremely popular among the female business students. When asked about her favorite Women in Business event, freshman Amanda Graves said, "I really enjoyed going out to lunch in Columbus and then going to see the Nutcracker. Having some girl time and meeting fellow business students was so much fun!" Meanwhile, the Sport Management Organization attended a career fair in Cleveland and assisted its members in finding internships for the summer.

In February, the Forensics Team won the recent Appalachian Swing Tournament at Ohio University in Athens, and Psi Kappa Theta members learned more about Schizophrenia through a trip to Kettering. As always, the MERCY Team provided valuable support to students with disabilities at Cedarville. Clearly, students at Cedarville regarded the use of their academic gifts and talents to help others as an extremely profitable service, and many students used the various Academic Organizations as their way to share their passion for their academic field and their faith with the rest of the world.

The Academic Professional Organizations were busy this year with their various projects and outreach programs to the community. Some of the organizations that fit under this category include the Society of Automotive Engineers, Epsilon Omega, the Health and Fitness Organization, and Omega $\mathrm{Mu}$. One group in particular, the Music Educators National Conference/ National Society of Music Educators (MENC), was extremely active during the school year. Their main event of the year was organizing the Christmas Chapel, which featured a handbell choir, Scripture reading by music faculty, special music groups comprised of music majors, and congregational carol singing. Jennifer Guth, secretary for MENC, said, "The student body enjoyed the Christmas Chapel, and MENC loved planning it! We hope to be asked to do it again sometime." In addition, Students in Free Enterprise (SIFE) had a very busy year filled with projects that reached out to both prospective and current Cedarville students as well as the community. Some of these projects included Personal Finance Day; Real Money, Real World; Ready, Sell, Dough; and Business Day. Another organization, the American Society of Mechanical Engineers, catered to Cedarville's freshmen engineering students and provided networking and social occasions for these students. Finally, the Society for Technical Communicators met regularly to hear speakers from the technical communication field and to learn valuable skills outside of a classroom setting. All of these organizations' outside experiences helped their members to gain valuable skills and to further their passion for their academic field.
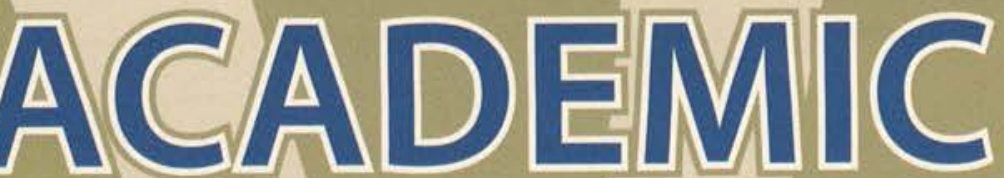

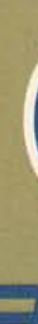
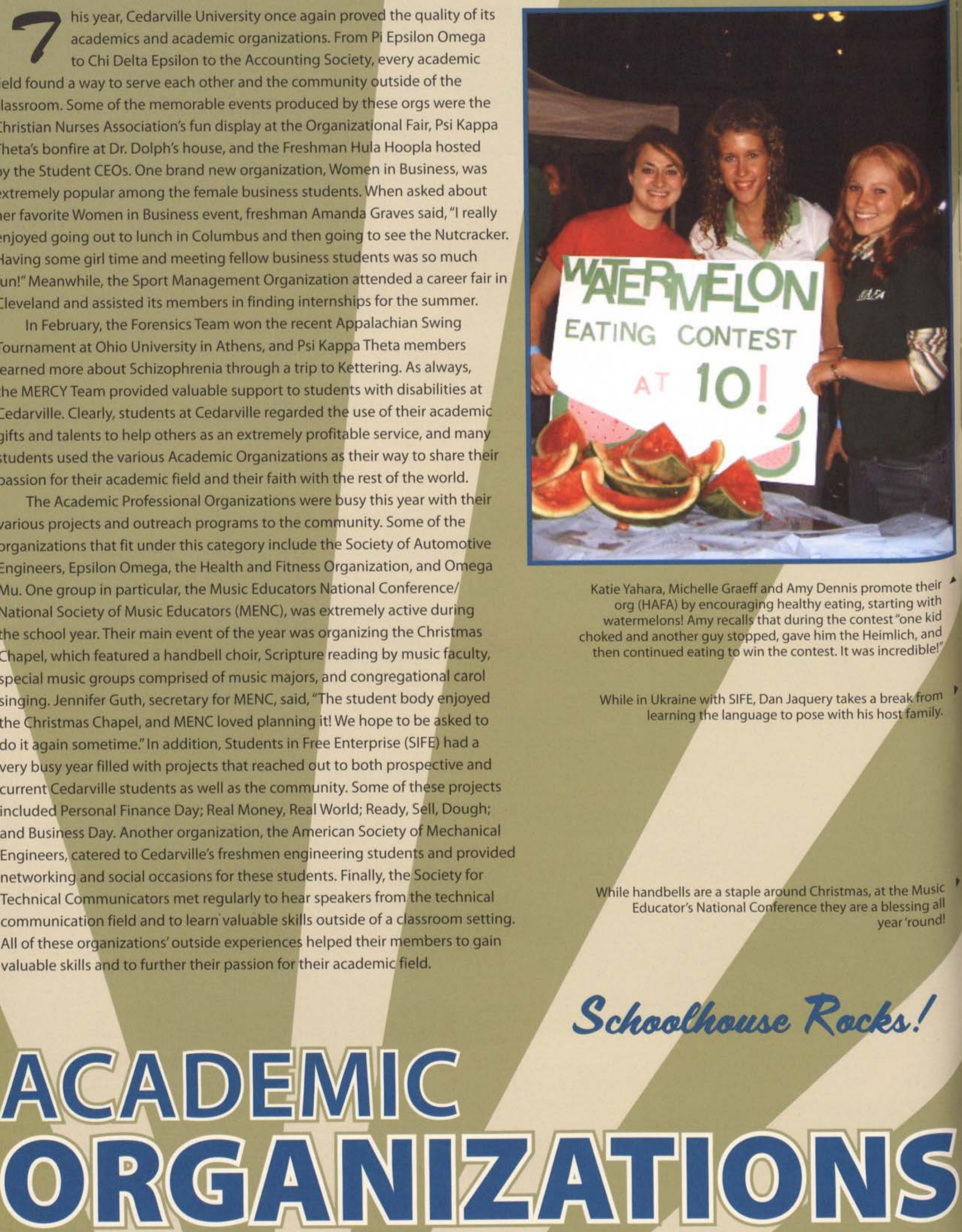

Katie Yahara, Michelle Graeff and Amy Dennis promote their org (HAFA) by encouraging healthy eating, starting with watermelons! Amy recalls that during the contest "one kid choked and another guy stopped, gave him the Heimlich, and then continued eating to win the contest. It was incredible!"

While in Ukraine with SIFE, Dan Jaquery takes a break from learning the language to pose with his host family.

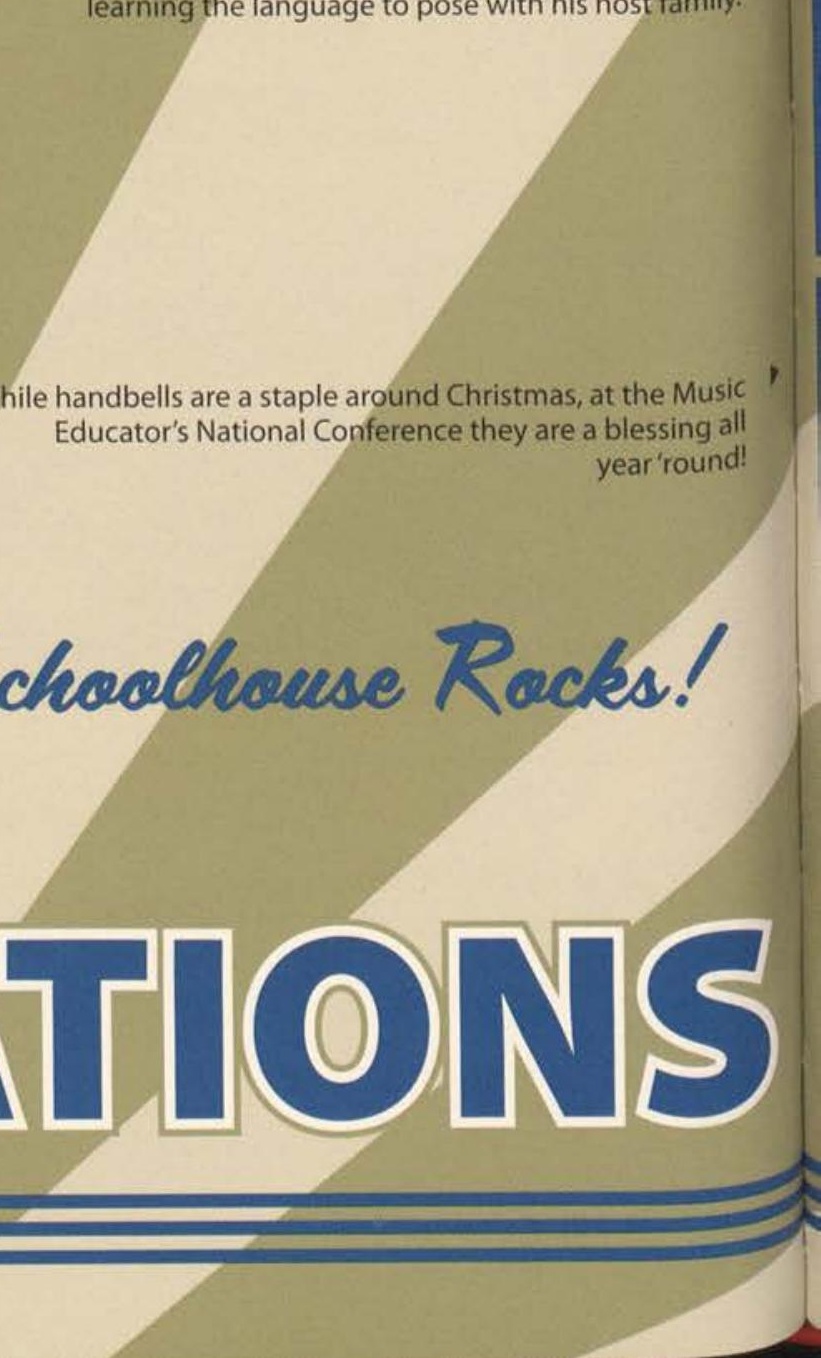



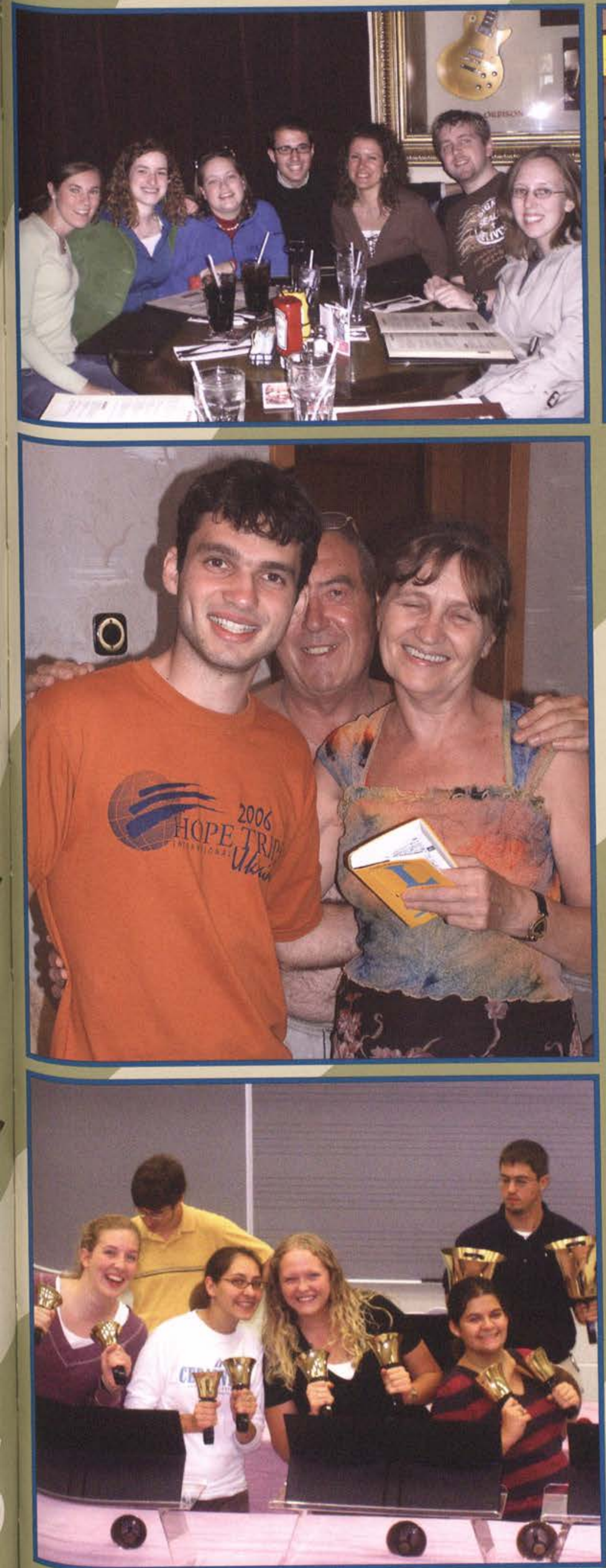

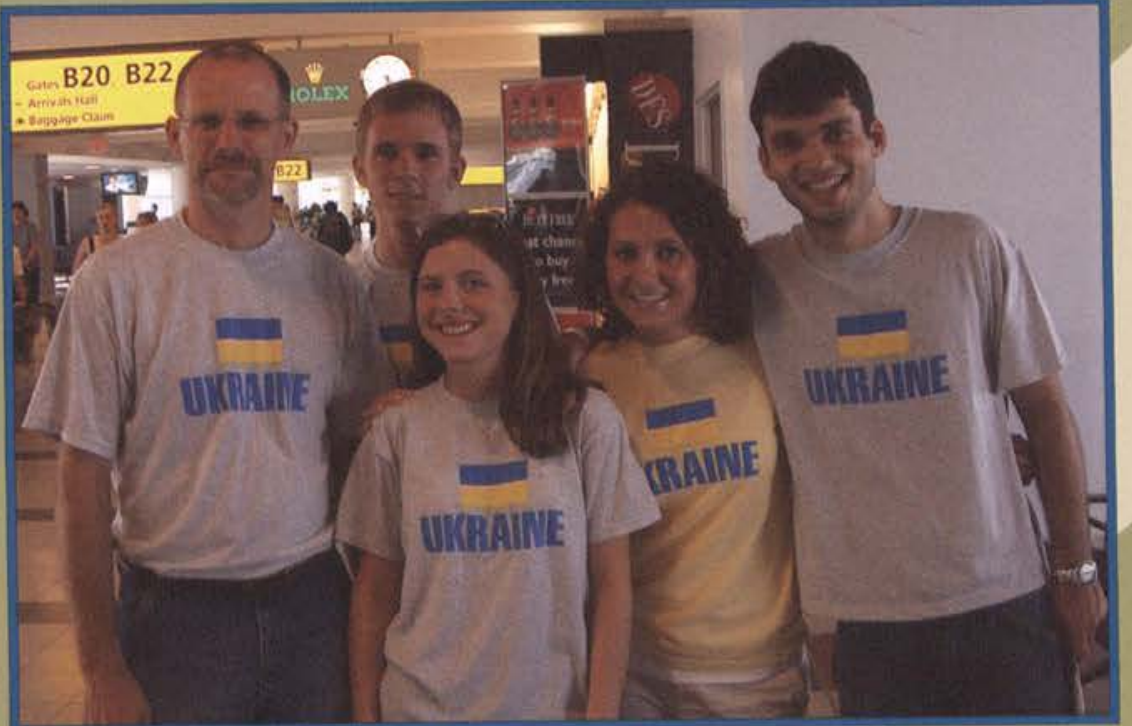

4 Professor Jeff Guernsey shows off his wonderful team headed for Ukraine. The team is comprised of members from SIFE as this trip is for business, not pleasure.. or maybe both.

- STC (Society for Technical Communication) members Lindsay Lehmann, Anna Hayes, Brynn Paine, Adam Evans, Erin SanGregory, Andrew Powell, and Katie Marburger take a break from the more techinical aspects of life to enjoy each other's company.

4 Amy Dennis and Katie Yahara display a sign for the Health and Fitness group (HAFA) at the Org Fair. Amy, who is the co-president for this new organization, says,"I know there are students who are concerned about health, and I just hope this org will continue to help and encourage students to take care of themselves. This is a great time to set habits for the rest of our lives.

7embers of MENC (Music Educators National Conference) are seen here entertaining the audience with handbells - one of their many unique musical stylings

- Lindsay Lehmann, Anna Hayes, Brynn Paine, Adam Evans, Erin SanGregory, Andrew Powell, and Katie Marburger, members of the Society for Technical Communication, also known as STC, enjoy dinner off-campus.
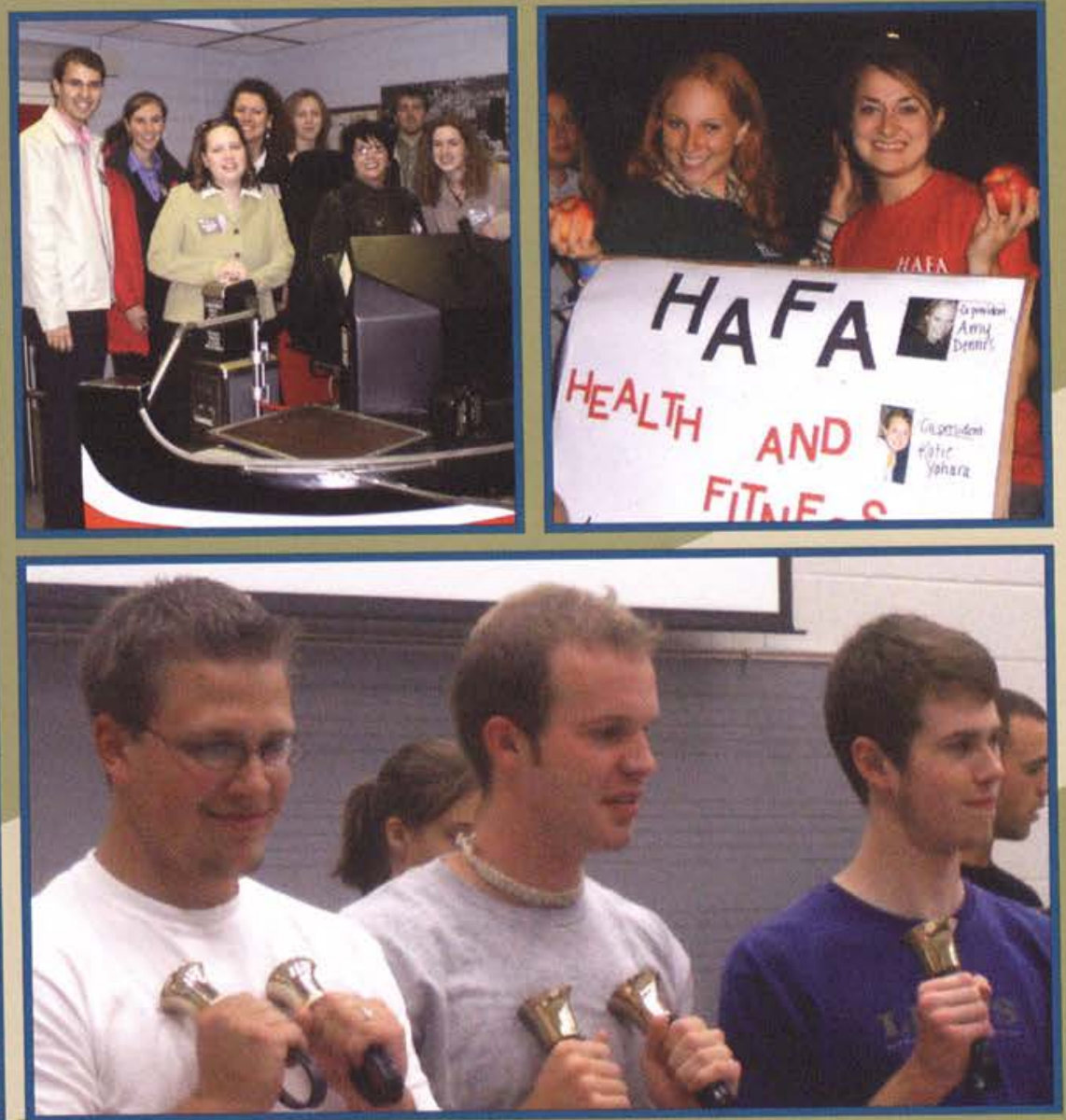
- "We are very proud of our ROTC members and what they have chosen to do for their country. It is really great to see their devotion to God, country, and home."Senior Aubrey Gries

4 Andrew Wong accepts his Air Force commission with all the dignity and honor that an office should possess.

Patrick Ederer proudly displays his leadership award at the ROTC Ball.
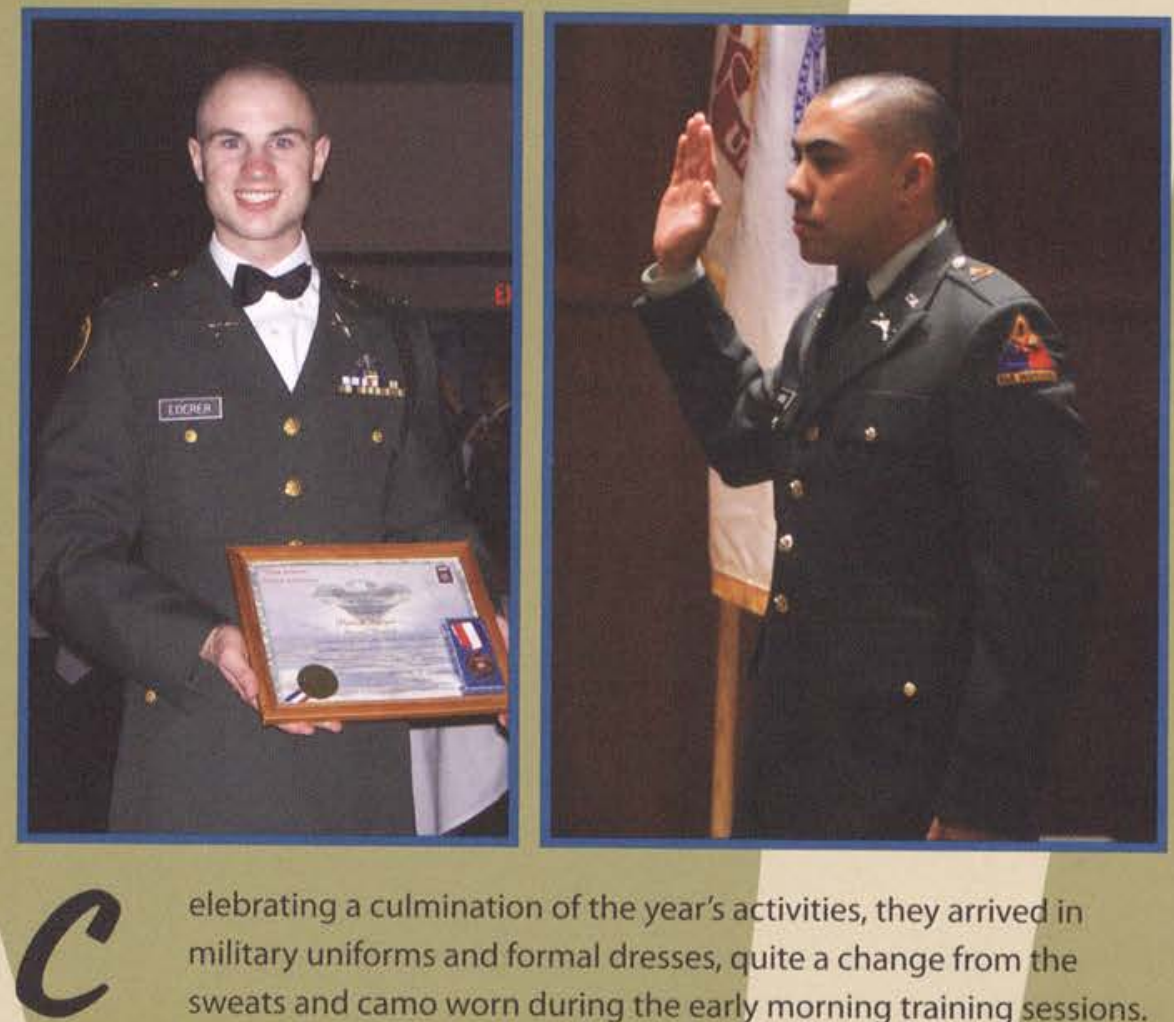

elebrating a culmination of the year's activities, they arrived in military uniforms and formal dresses, quite a change from the

sweats and camo worn during the early morning training sessions. After weeks of 6:00am PT Mondays, Wednesdays, Fridays, 6:00am Leadership Labs Thursdays, and Military Science classes on Tuesdays and Thursdays, the ROTC cadets brought their dates for a night of honoring the military legends who have gone before them. This year, Senior Patrick Ederer was the assistant Operations Officer in order to prepare him for an actual battalion position he will achieve after graduation. Junior Julia Basford reflected, "This year I have enjoyed our outings such as Field Training Exercise and Basic Rifle Marksmanship exercises. It's not always fun being up early and training all day long, but it builds camaraderie." Sophomore Corey Reeder, who is proud to be in Army ROTC, said, "My family has a rich military history that I'm proud to uphold. I definitely want to fly helicopters." This year, Air Force ROTC integrated more active duty deployment simulations in order to better prepare the cadets beyond graduation. Junior Sam Delong appreciated his challenge of leading the sophomores in order to prepare them for their Field Training. "When you are in charge of people, whether you think you have an important position or not, you directly influence, positively or negatively, those under you." The night ended with the cha-cha slide, an annual tradition at the military ball, and the cadets went on their way, the seniors one step closer to graduation and subsequent service for their country.

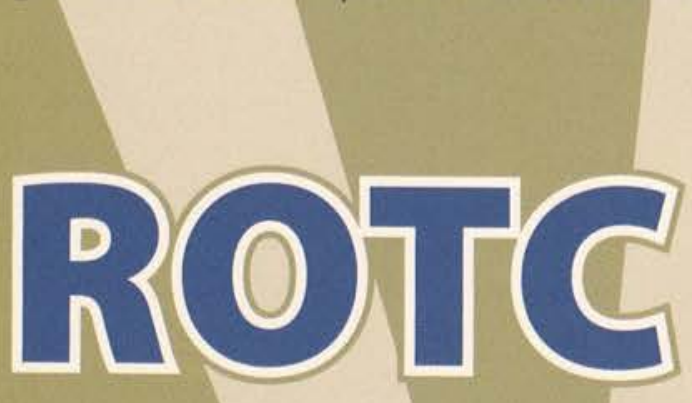

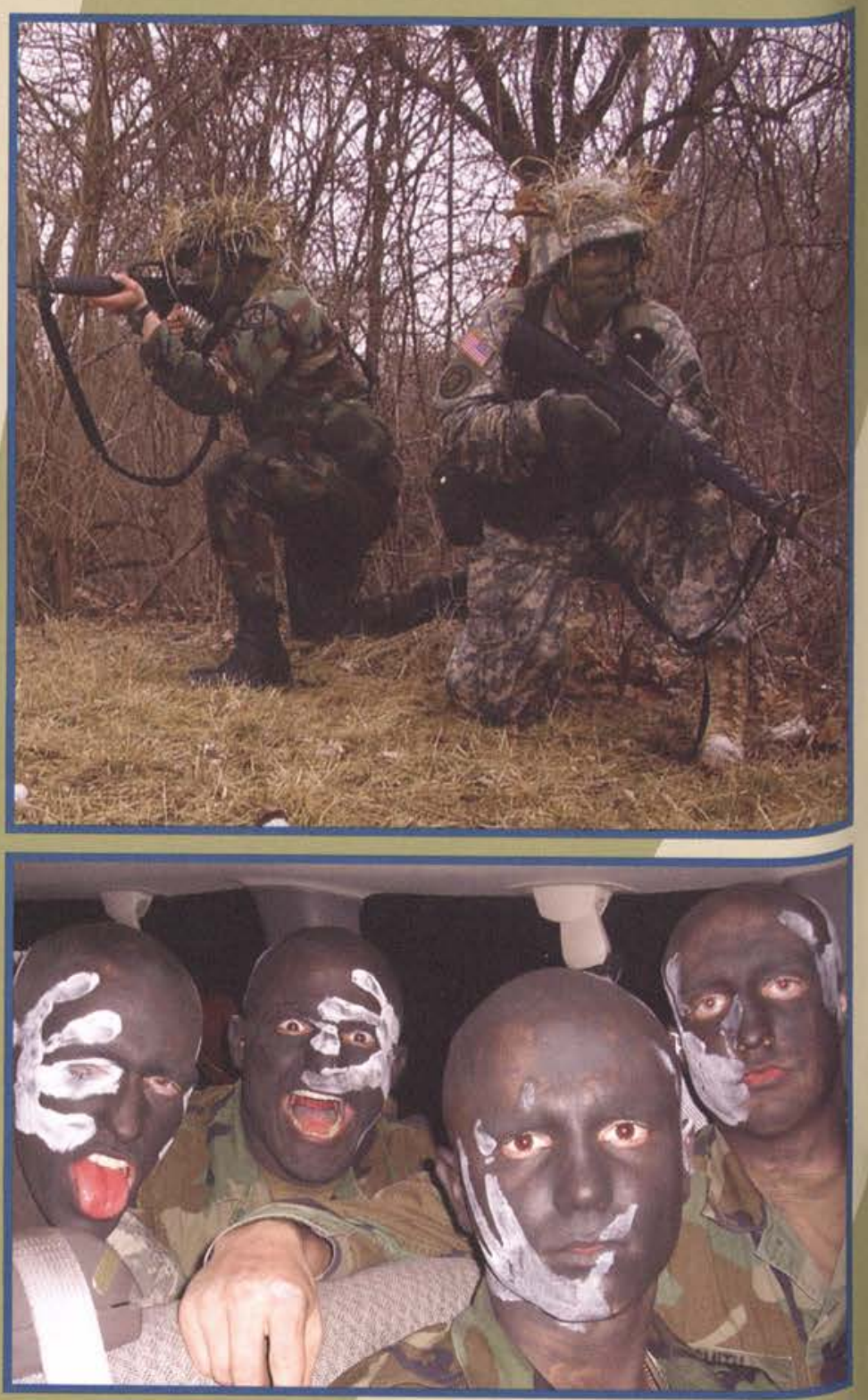

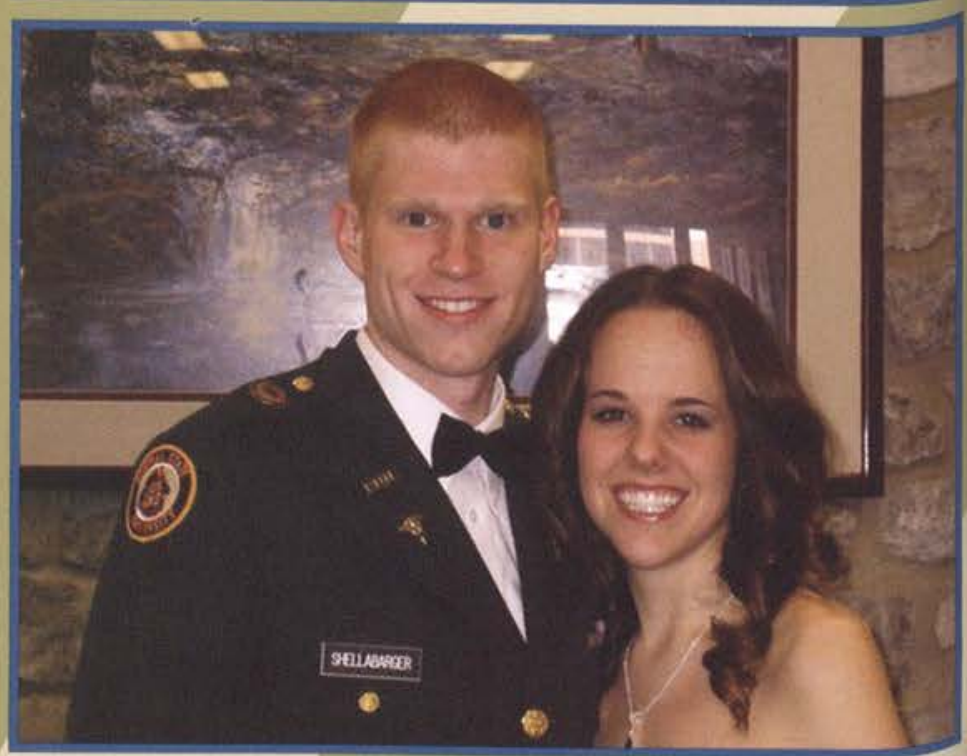

- Peter Shellaberger and girlfriend Eva Stark enjoy a night out at the ROTC Ball.

- Enthusiastic ROTC members celebrate a long day of field exercises for the 2007 Best Ranger of the Year competition. 
elect few organizations on campus this past year purposed to minister through specific services of different types. Organizations like Habitat for Humanity, Big Bird, and CU-EMS showed Christ's love by serving people throughout the community.

The Cedarville chapter of Habitat for Humanity was a student organization that was committed to serving others by working to eliminate poverty housing. Habitat sponsored a fund-raiser involving a competition between the dorms and gave the money to help rebuild a house at an Alabama Habitat affiliate on the Gulf coast. This year they participated as volunteers for the first ever Habitat project in the village of Cedarville.

Big Bird also provided service, giving assistance to area families and aiding the community with miscellaneous tasks. This past spring they put together a work week in order to help the elderly around Cedarville with raking, cleaning out the gutters and preparing flower beds. Sophomore Sarah Crichlow said of the event, "It was simply to do Whatever outdoor tasks they could not do themselves, while spreading some of the joy of Christ to our community".

CU-EMS, a student-run, 911 emergency response organization gave added assistance to the University as well as the Cedarville community. Operating the same Way as the local Fire Department and providing the same levels of care, this student Volunteer organization had 11 members ranging from Basic EMT, Intermediate EMT, and Paramedic. They were involved with other ministries, including providing cold drinks to firefighters during house fires, and praying with each of their patients at the hospital, offering them support. Senior Mikki Stanton said of the group, "The EMTs on CU-EMS were my family. They saw my good side, my bad side, and the side that rolls out of bed at three A.M. for an emergency. They will be what I miss most about Cedarville."
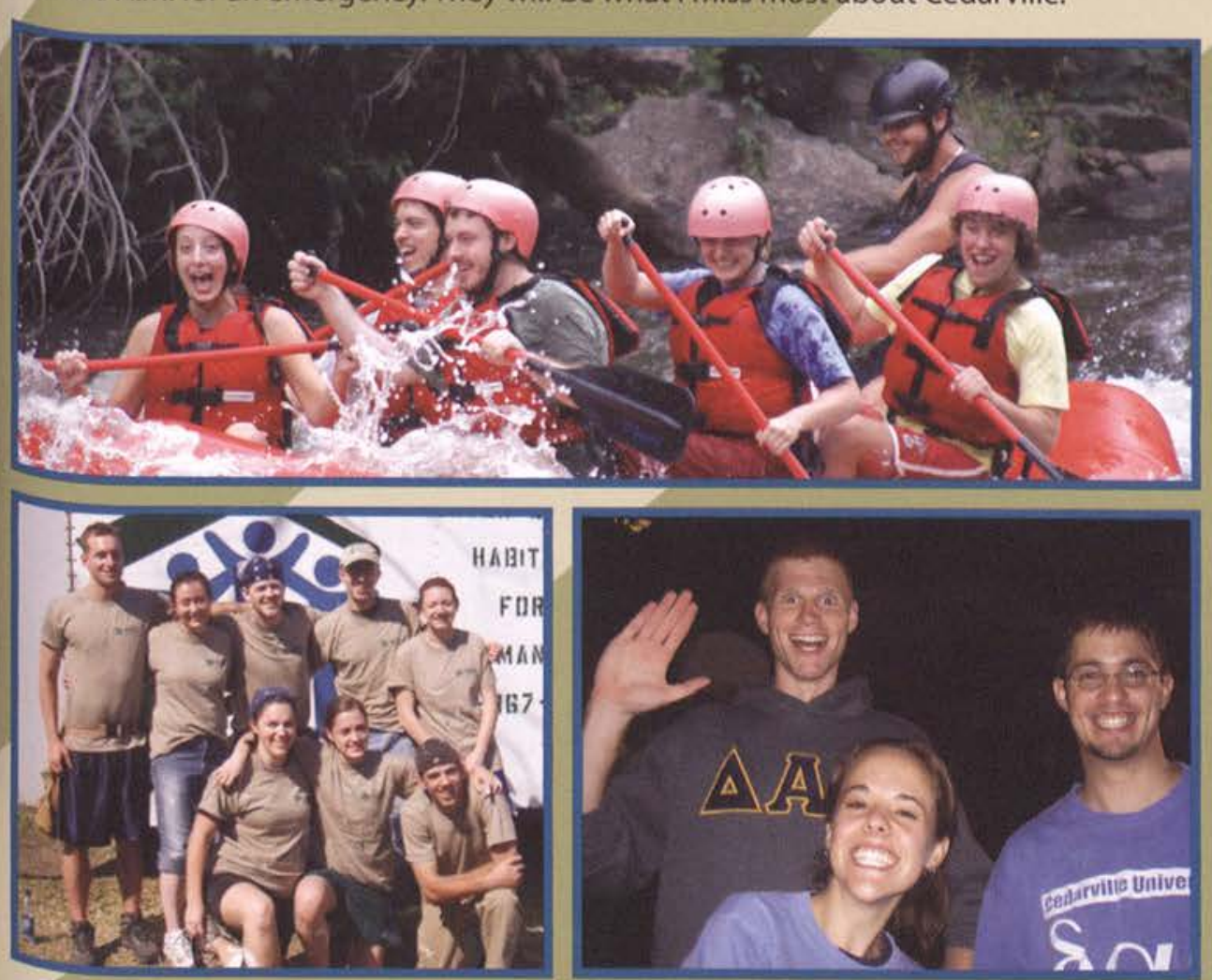

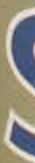

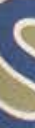
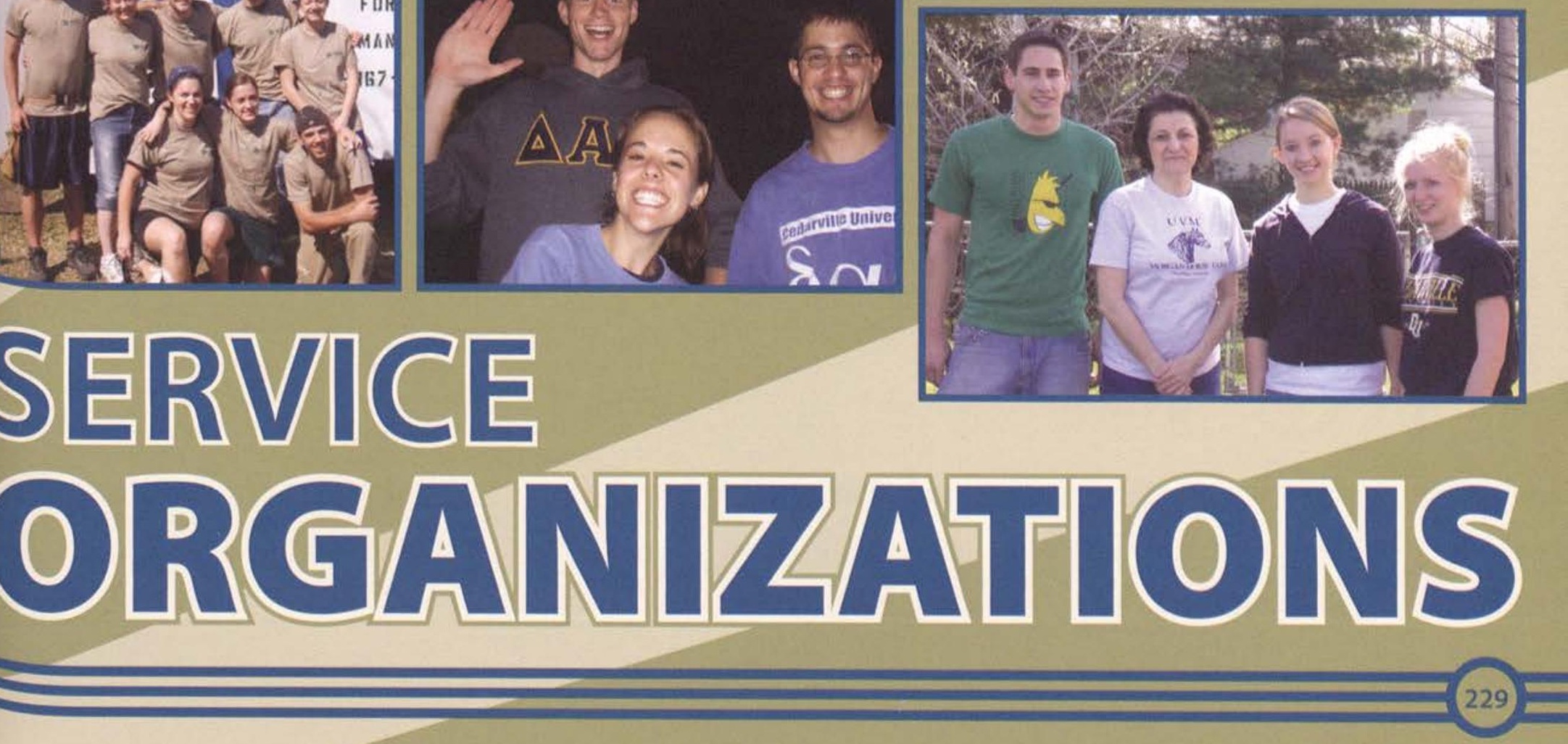

(L to R) Tau Delta Kappa members Jessica Peck, Amy Goldman, Christa Jacobs, and Allison McClain paint nails of women from the community. The event served nearly 150 women from low-income homes with a Mary Kay makeup session, manicure, and had their hair cut and styled. With lunch they were given a sermon about how God can appearance was changed on the outside.

- Members of Habitat for Humanity gather to celebrate the completion of a home built by the generous donations of students and others.

- Eva Stark, pictured with other members Peter Shellabarger and Nicholas Kohne, said of her involvement in DAE, "I love meeting alumni from around the country and getting to hear their stories of Cedarville."

4 Kelly Myers, Drew Flamm, Nicholas Fridenmaker, and Brandon Doyle a little white water fun for the DAE fall trip. Senior Daryl Sando says, "Being on DAE has taught me so much about myself and how I relate to others. I am so thankful to be able to serve along side such wonderful people, it is such a great group!"

- Members of Big Bird on their fall work week are dedicated to helping the elderly of our community with outdoor tasks such as raking leaves and cleaning gutters and such. Our hope is not only to enable them to accomplish the necessary tasks, but to extend the love of Christ through our volunteers. 


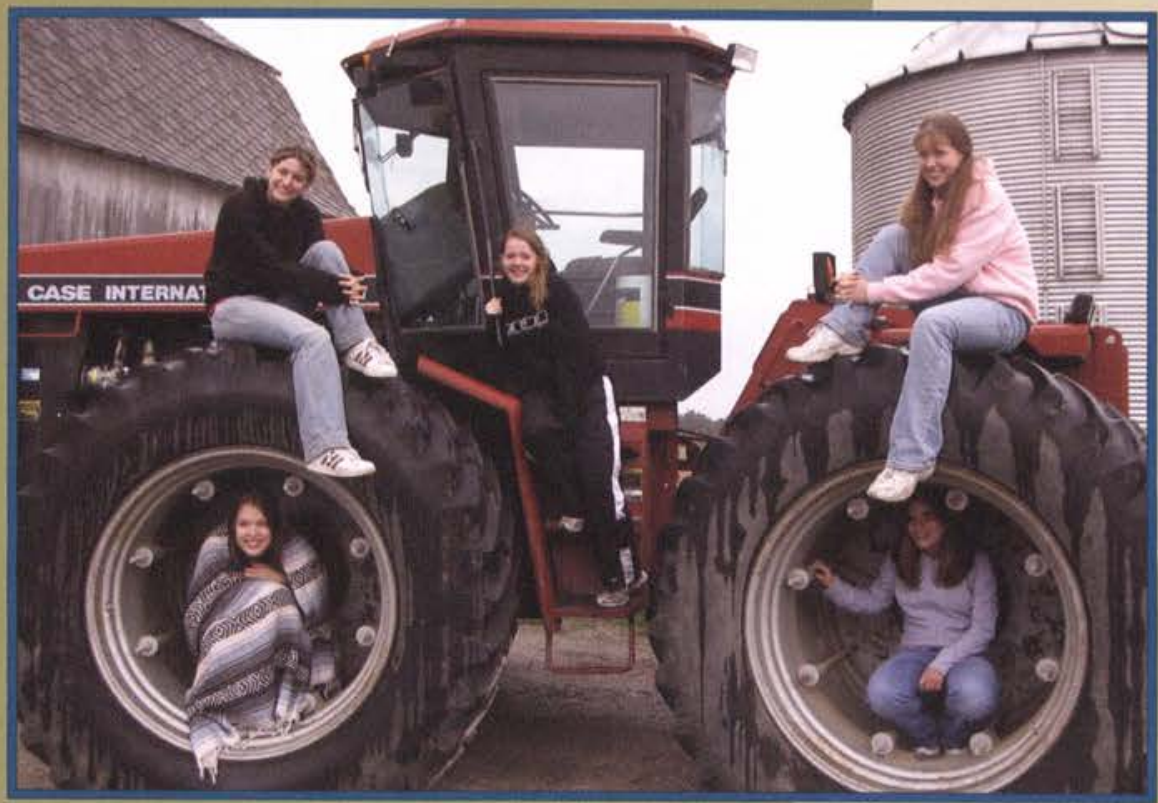

edarville's cross-cultural organizations included Beta Rho Delta, MuKappa, Spanish Club, the Societe de Francais, and the International Student Organization. Beta Rho Delta, the German Club, promoted the study of German language and culture. Its members went to Christmas parties and participated in fund-raising affairs by selling German chocolate and other ethnic foods. The Spanish Club was another ethnically-oriented organization. According to its website, the club existed to provide "opportunities to practice language skills and learn about cultures through extracurricular activities." Similarly, the Societe de Francais promoted the "use of the French language and increases the knowledge and appreciation of French culture to advance awareness of missions." The International Student Organization, however, was designed to help students from different cultural backgrounds adjust to the U.S. culture and find ways to share their uniqueness, individual abilities, and outlook on life in a meaningful way. MuKappa helped to create a comfortable atmosphere for students who have grown up with missionary parents. According to junior Katharina Stevens, "We eat together once a week, and there is also a Bible study for MKs (missionary kids) that was started this year. My best friends are MKs and they have been such an encouragement and blessing to me. It's great to be able to know others with a similar background. MuKappa organizes several activities for MKs throughout the year. The highlight of the year is Snow Camp in Indiana. MKs from several Christian schools in the mid-west get together for a weekend of worship, fellowship and games." All these organizations helped to both enrich those closely involved with the organizations and to enhance Cedarville's campus through the outreaches that made our students more culturally and socially aware.
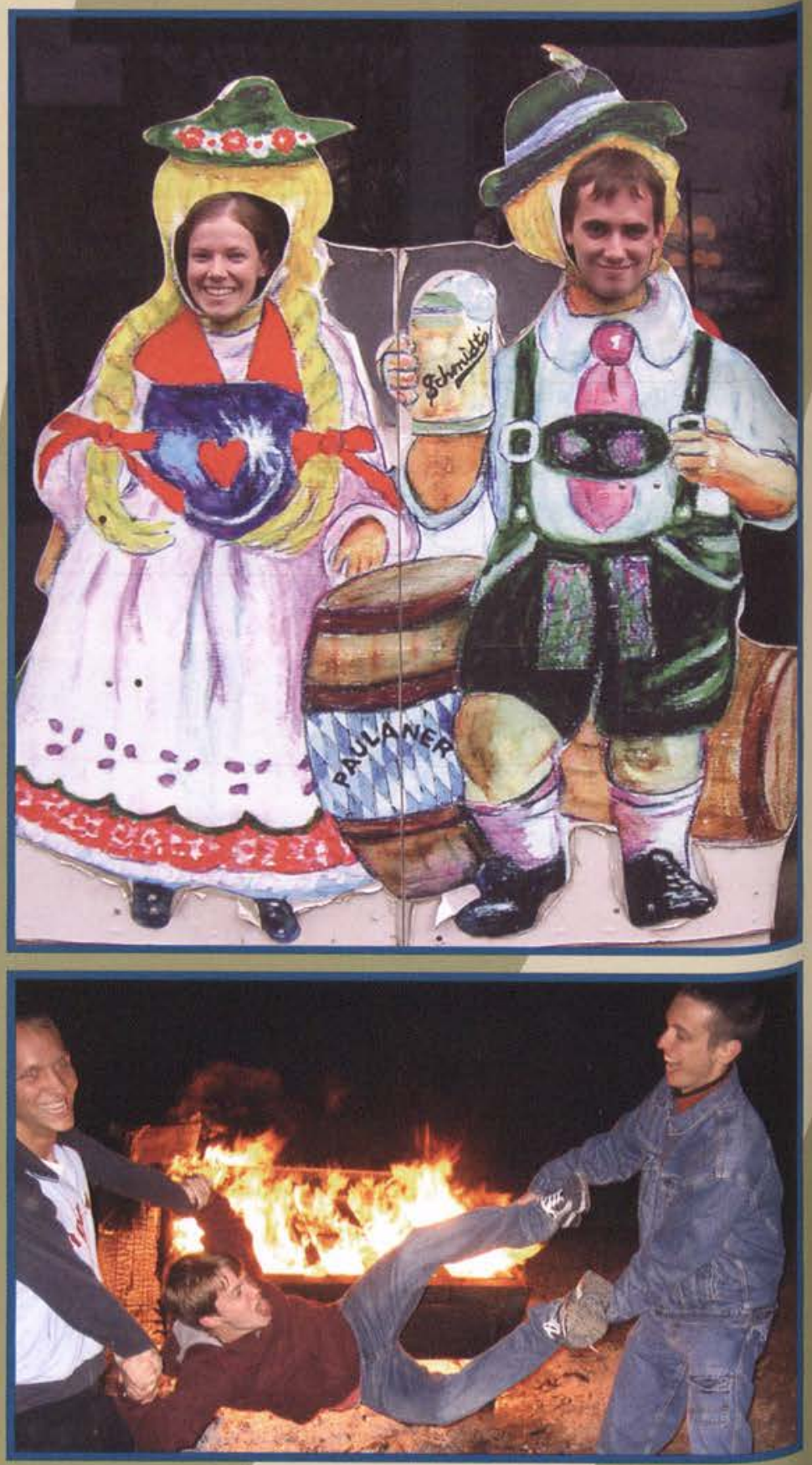

- Nathan Sheppard and Brian Sallee get a kick out of swinging Brad Hoganson close to a flaming piano at MuKappa's Barn Party. The Barn Party was a chance for MKs to hang out around a campfire, toast s'mores, enjoy a hayride, and be spiritually challenged by a devotion from chaplain Dan Martin.

\& Siblings Rebekah and Josh Nearhoof pose outside Schmidt's Sausage Haus in Columbus' German Village during a German Club outting. Rebekah says, "We went there to soak up the culture and to learn mor about the German people who settled in the area."

- MuKappa members Jane Reiner, Kendra Mattson, Julia Mattson, Elizabeth Martin, and Karie Pepe enjoy Fall Barn Party. Julia says that MuKappa is "a great support group for the MKs as we return to the states and need to adjust to American culture. I would love to see us reach out more to the American students and help them understand other cultures, while learning from them about America."

\section{(}
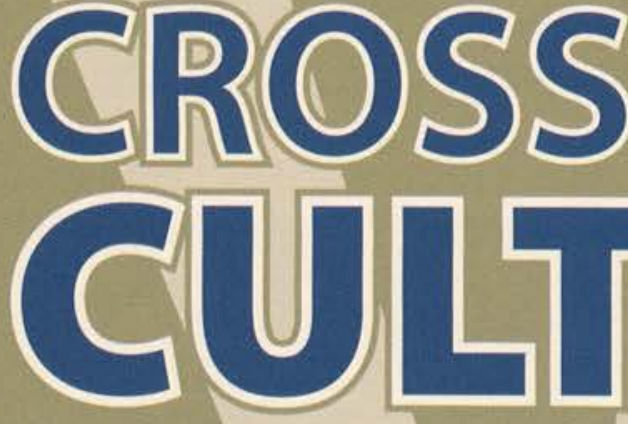

\section{Itis AS}


The Debate and Forensics teams spent this year developing skills in speaking, critical thinking, and discipline.

The Debate team sent students to eight tournaments this year, with the entire team in attendance at five. At three of those tournaments (Western Kentucky, Berea, and Wheaton), Cedarville took $1 \mathrm{st}$ place in team sweepstakes, and five individual students were also able to earn other 1st place finishes. This year the Debate team accomplished a team goal by achieving their best national ranking Yet, placing 11 th in the National Parliamentary Debate Association. At the NPDA tournament, the team was awarded 18th place,

finishing in the top 20 for the first time in Cedarville history. Debate challenged and encouraged the students involved. Senior Emily Van Vilet noted, "My participation in debate has forced me to analyze assumptions that I have and helped me to better understand why I believe what I believe, and also to see the world from other people's Perspectives."

The Forensics team, which competed in 11 different speaking events in the areas of public address, oral interpretation, and limited preparation, continued to push students to develop their talents. The team placed second in the Holiday Frolic tournament at Ohio State, a competition including 35 schools and approximately 250 student competitors. The team also finished in third place at the State Championships of the Ohio Forensics Association, making them 3 rd in the state of Ohio. The team made their aim ministry, reaching out to many who were unsaved in their competitions. Third year Competitor Eric Mishne noted, "We were able to establish some good relationships with other teams that allowed us to be encouraging to them and also to demonstrate the power of Christ's love."

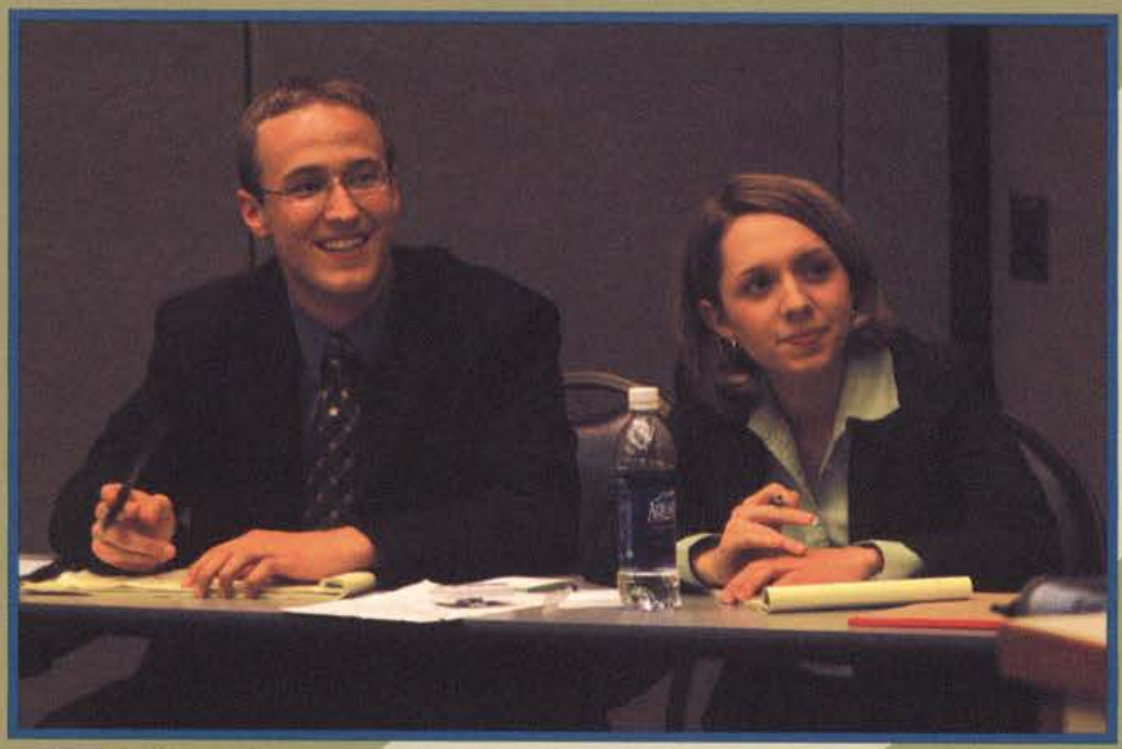

- Mark Miller and Courtney Edmonds are forced to think quickly as they receive their topic only 15 minutes before the debate begins. Mark states that "the debate team has had great success the past few years, but it has been a blessing of God. Success ultimately comes from God, and so we debate to worship and glorify him, in our losses and our wins."

- Emily Van Vilet and Joe Dugan combine their knowledge to present an effective debate. "Every year I am amazed to see how such a diverse combination of personalities works together to compete successfully," Emily says of the debate team, which attracts a variety of majors ranging from engineering, finance, and biology to philosophy, English, and pre-seminary.

4 Shawn Green has fun giving a presentation for the forensics team. Cedarville's team was respected by other Ohio schools for its standard of competitive excellence.

- Michael Domeny aims to give others a glimpse of his Savior through forensics. "This year's team has had an especially sensitive heart for other competitors," he says. "It seemed like we were returning home after every tournament with another story of how at least one of us had the chance to talk to someone on a spiritual level."
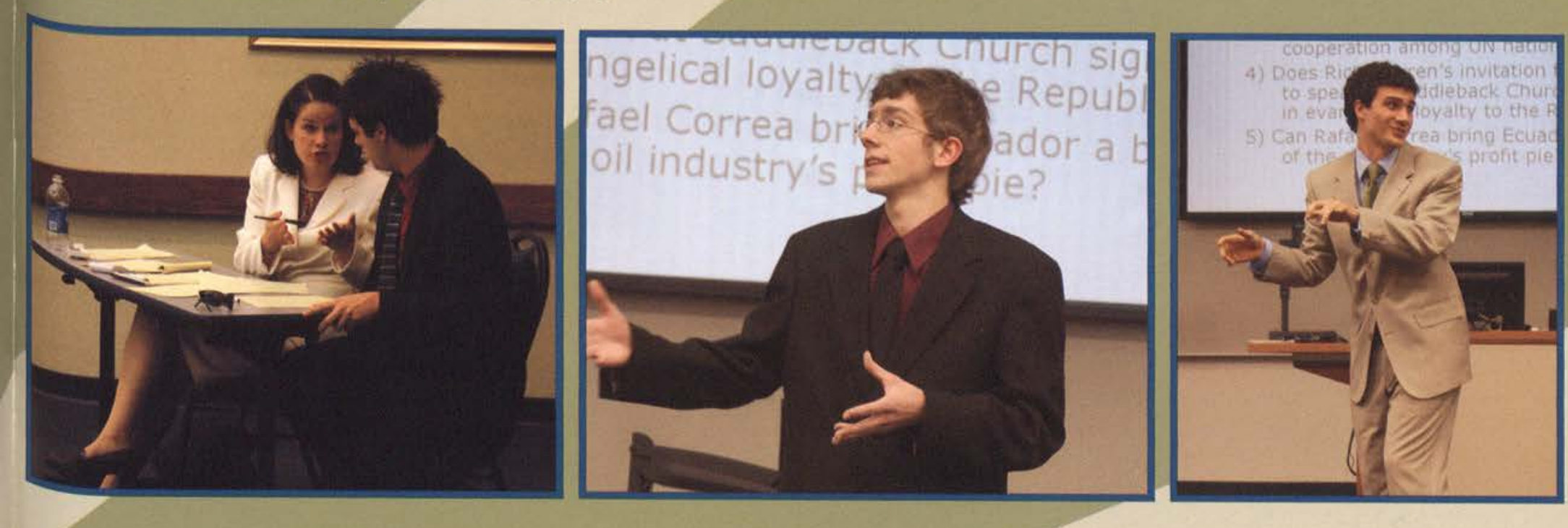

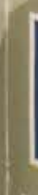
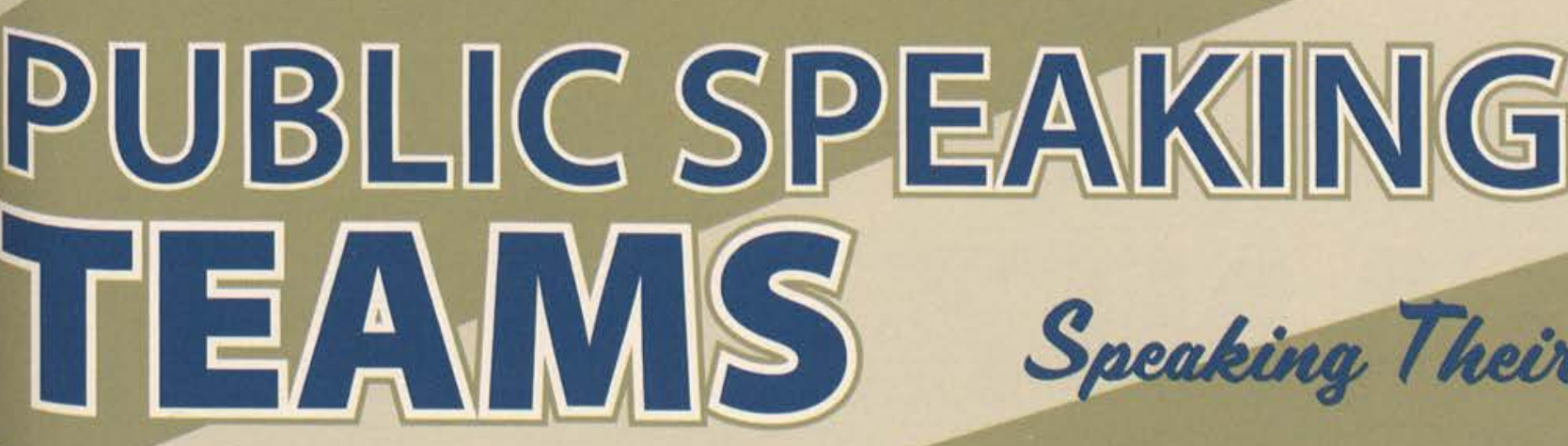

organizations, especially for those that were just starting out. Of all the three-lettered organizations that coordinated activities for students, Alpha Delta Gamma was a definite favorite on campus this year. A major social event this year was the 80's Babies Roller-skating, an ADGrun fundraiser. Also, the "Daisy for your Swayze" fundraiser relieved some pent-up Valentine's Day pressure.

These events were fun-filled, but more importantly helped fund the organization's ministries, such as an expectant mother prison ministry, a junior high mentoring program, and single women's ministry. Other organizations, such as Phi Epsilon Beta and Delta Pi Sigma, invested their time and money in ministries such as a missions trip to Jamaica and delivering food on Thanksgiving, respectively.

While these organizations focused on local ministry, Women of Vision and Students for Life specialized in world issues involvement. Women of Vision, winner of the Best Campus Organization Award at this year's Elliv, spearheaded the Fisheni Project, which both offered a glimpse into life in AIDS-laden Zambia and raised $\$ 24,000$ toward the Fisheni Village. Students for Life had their sights set on the inhumanity of Darfur. In addition to raising awareness of the genocide occurring there, Students for Life also tackled the issue of abortion in our own country.

PEB girls Cali Galloway, Jenny Boulet, Holly Foreman, and Abby Ellis show off $\checkmark$ the muscles they used to serve others in Jamaica. Holly Foreman says, "Our trip to Jamaica was so awesome! Not only did we get to meet and serve some amazing people while we were there, but we also grew closer as a group through working on the CCCD campus and praising God together."

Rachel Sherwood and Janet Bauernschmitt, DPS members, coordinate on a service project with Adopt-A-Block. Janet comments, "Working with Adopt-

A-Block was a great experience for all of us girls, and we hope to get more involved with those ministries next year."

\section{Service For Eternity}

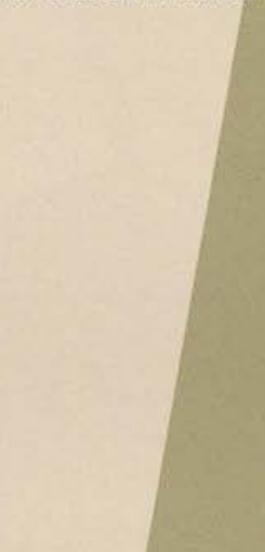

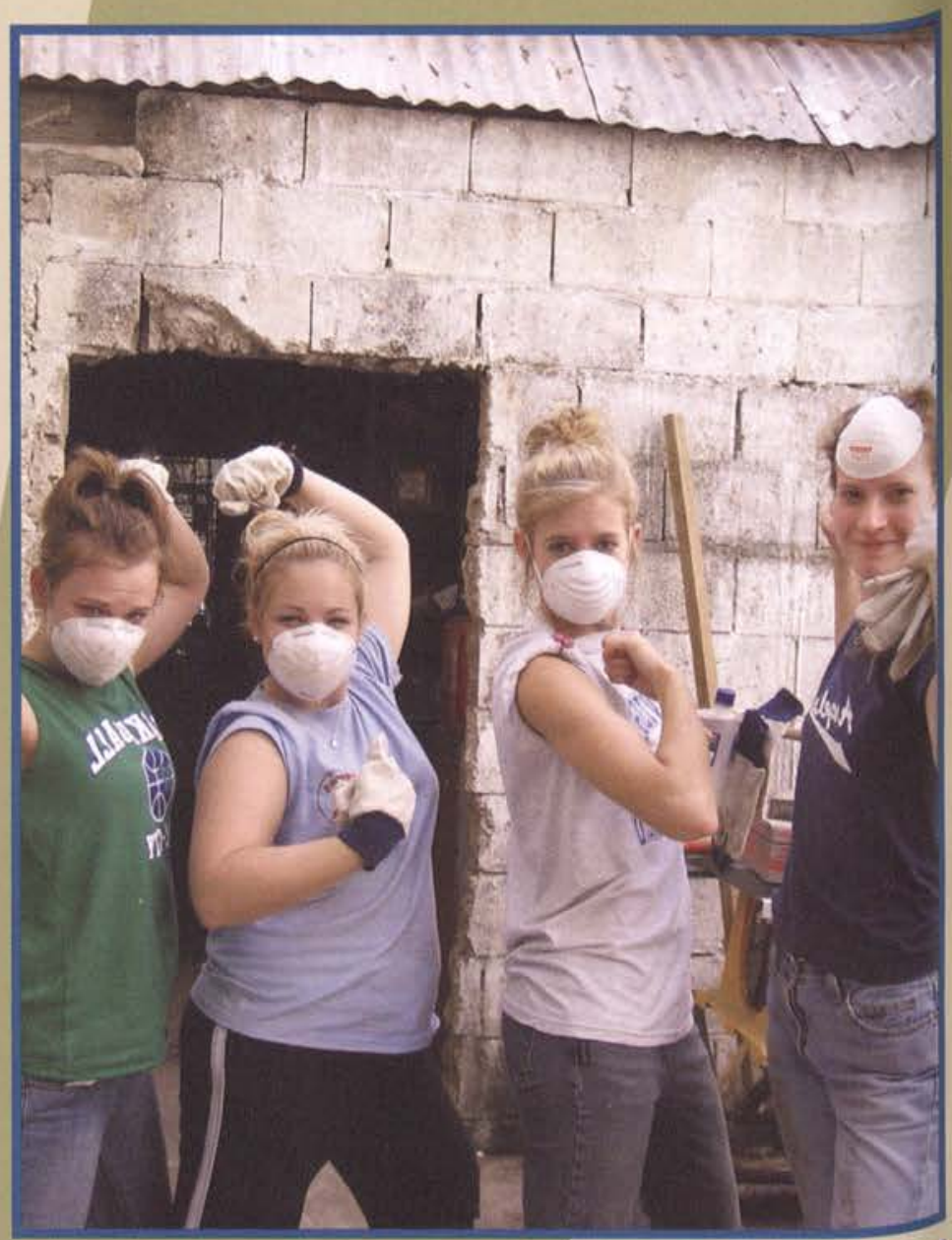
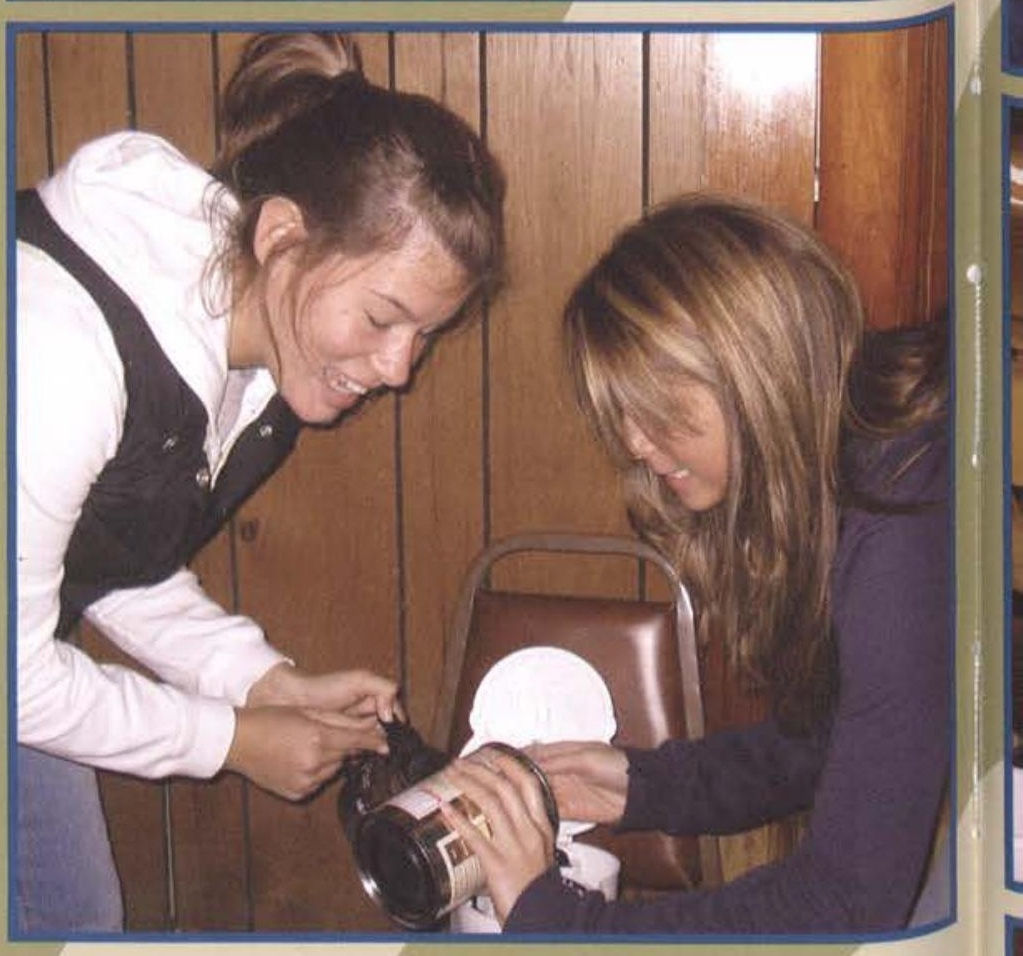

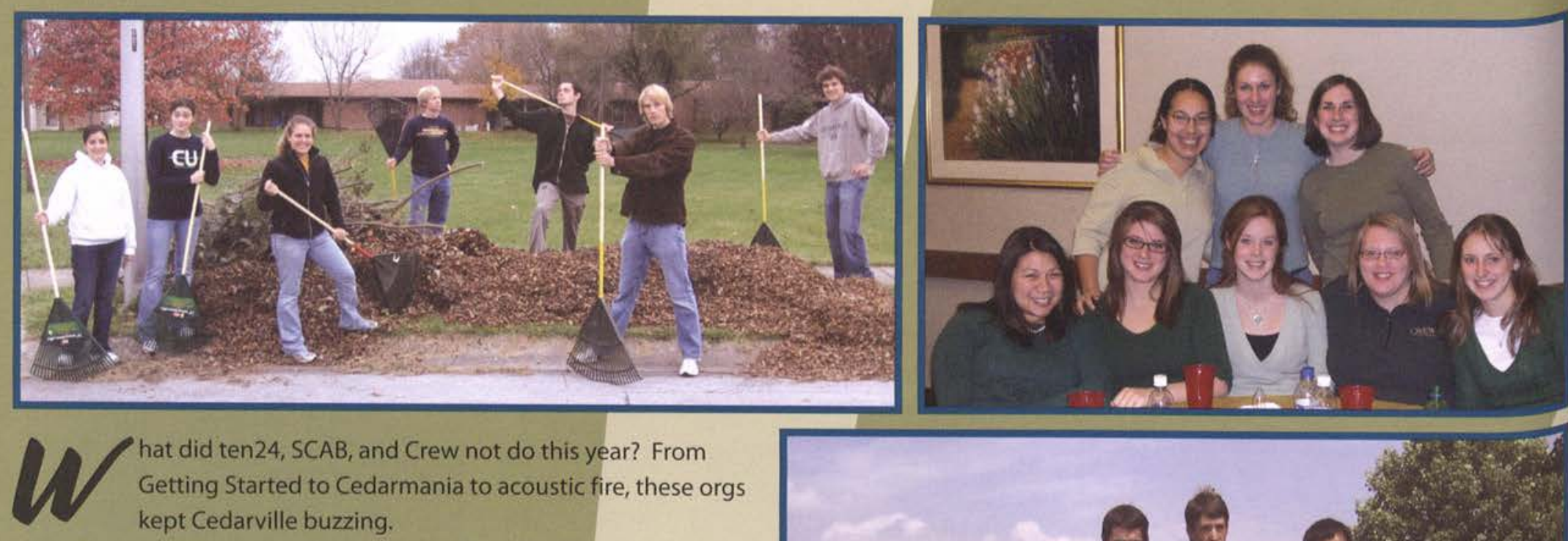

This year CAB was transformed to ten 24 , taken from Hebrews 10:24. "The mission of ten 24 is founded on service," junior Melissa Lewis explained. "We exist to serve the student body as well as faculty and staff." Junior Kimberly Loosa loved helping ten 24 with Getting Started Weekend. "It was awesome to help the freshmen move in and get to know each other and made for a great start to the year!"

Senior Bryan Amerine stated that the SCAB's focus for the year was "to bring activities to campus that would be both exciting and Christ-honoring." One new thing SCAB was able to do this year was to serve at Camp Patmos. "It was a wonderful ministry in serving those people," Amerine related, "The poison ivy was rough, but what are you going to do?"

CREW certainly had their hands full too with events like Cedarmania, which happened to be junior Dan Parrott's favorite event because, as he said, "It is all night with junior high kids who have more energy then any other age group." Working on CREW has been a growing experience for sophomore Kena Mena. "It is awesome to be able to give the children and teens that come to the events well planned activities and see them have a great time while at Cedarville."

One of ten 24 's responsibilities is taking care of huge events, such as Getting Started Weekend. Members Tiffany Inskeep, Bethany Hochsteatter, Andrew Staab, Steve McCune, Neil Patnaude, Caleb McCollim and Terri Schenk pose next to their handiwork on "The Rock.

Kena Mena, Amy Brand, Jade Burley, Sarah Coleman, Katlyn Hiteshew, Jessica Coudriet, Lisa Crowder, and Ashleigh Laswell, part of CREW, gather together for a time of fun and fellowship.

Ten24 members Ashley Bueller, Melissa Lewis, Courtney Brott, Derek Kreider, Andrew Staab, Dan Knudsen, and Andrew Clemans serve Cedarville by raking leaves.

SCAB members Amanda Kirby, Bryan Amerine, Jessica Glass, Stacy Alexander, Ashley Gochenaur, Mike Pritts and Joel Green have fun with the famous Yellow Jacket costume, better known as "The Bee."
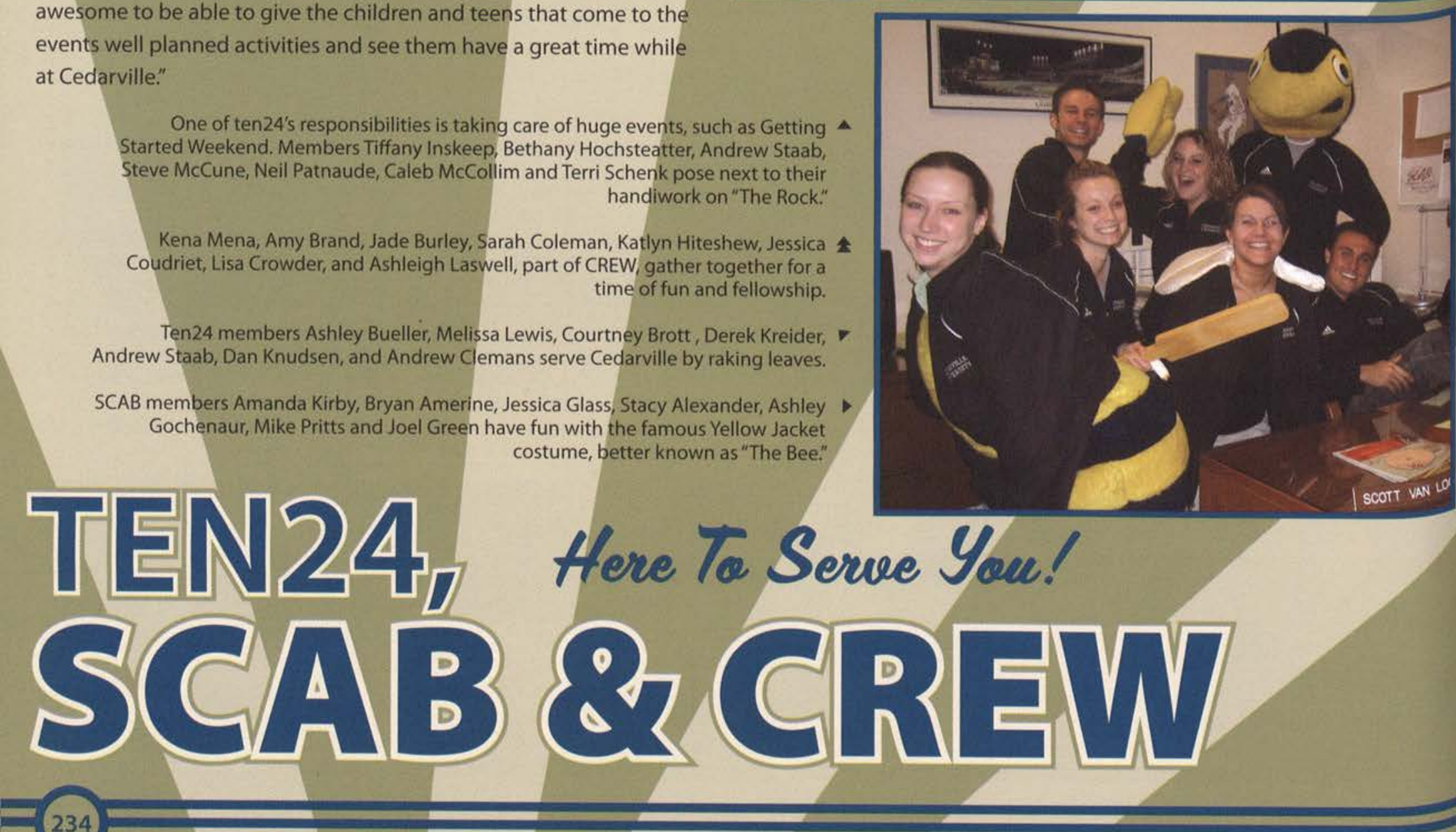

Here Ta Serve You!!
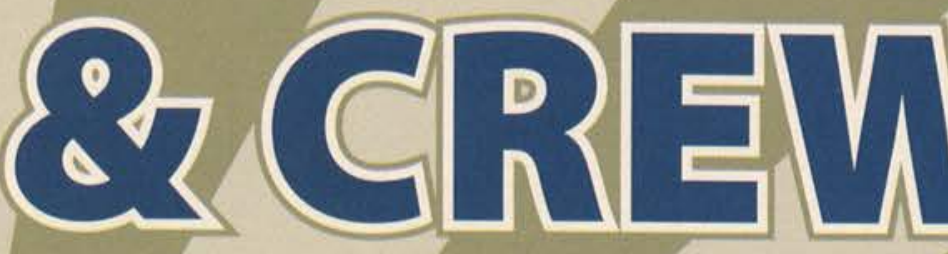

and what it strove for, but also the officers that made it all happen. Whether out in front of the student body or working behind the scenes, the SGA officers worked to represent the Cedarville Community in the greatest capacity possible.

This year's SGA officers worked to unite the student body and make the association available and personable to the students. Through weekly poll questions and the SGA mail bag, students were able to voice their own opinions and questions. The SGA office continued to become a place where students could interact with the officers and break down the barriers between SGA and the student body.

Each year, SGA is faced with the challenge of bringing new and exciting issues to an ever-changing student body. A focus this year Was placed on improving what was already in place. Forums ranging from dorm renovations, campus safety, dress code, and everything in between gave students an opportunity to voice their concerns and ideas and interact with the faculty and staff to continually improve student life at Cedarville.

No organization can function without a dedicated and hardWorking team of leaders. The 2006-2007 SGA officers led not only their own organization but the whole student body towards Christcentered community.
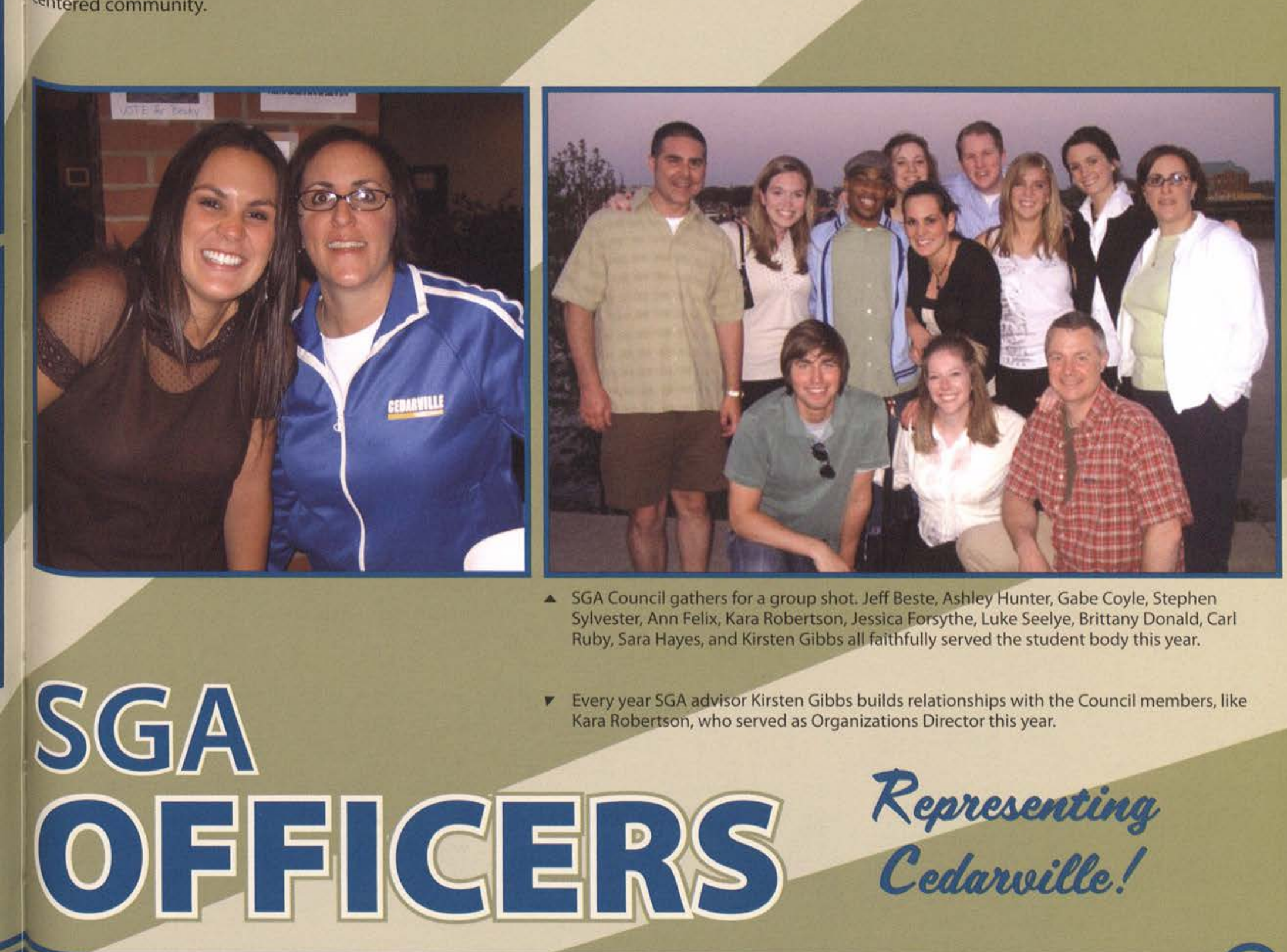

- SGA Council gathers for a group shot. Jeff Beste, Ashley Hunter, Gabe Coyle, Stephen Sylvester, Ann Felix, Kara Robertson, Jessica Forsythe, Luke Seelye, Brittany Donald, Carl Ruby, Sara Hayes, and Kirsten Gibbs all faithfully served the student body this year.

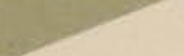

Mark Hershey, this year's SGA student body chaplain, dives into a vat of chocolate ice cream. Either the ice cream was very hard or else he was a bit confused. Maybe Mark should stick to knitting.

Brittany Donald, SGA Yice President, uses all her might to hold down an ice crean put on in the lower-level SSC.
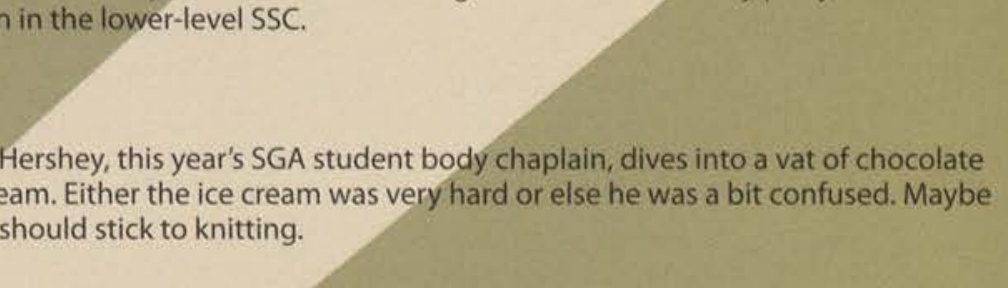

- Every year SGA advisor Kirsten Gibbs builds relationships with the Council members, like Kara Robertson, who served as Organizations Director this year.
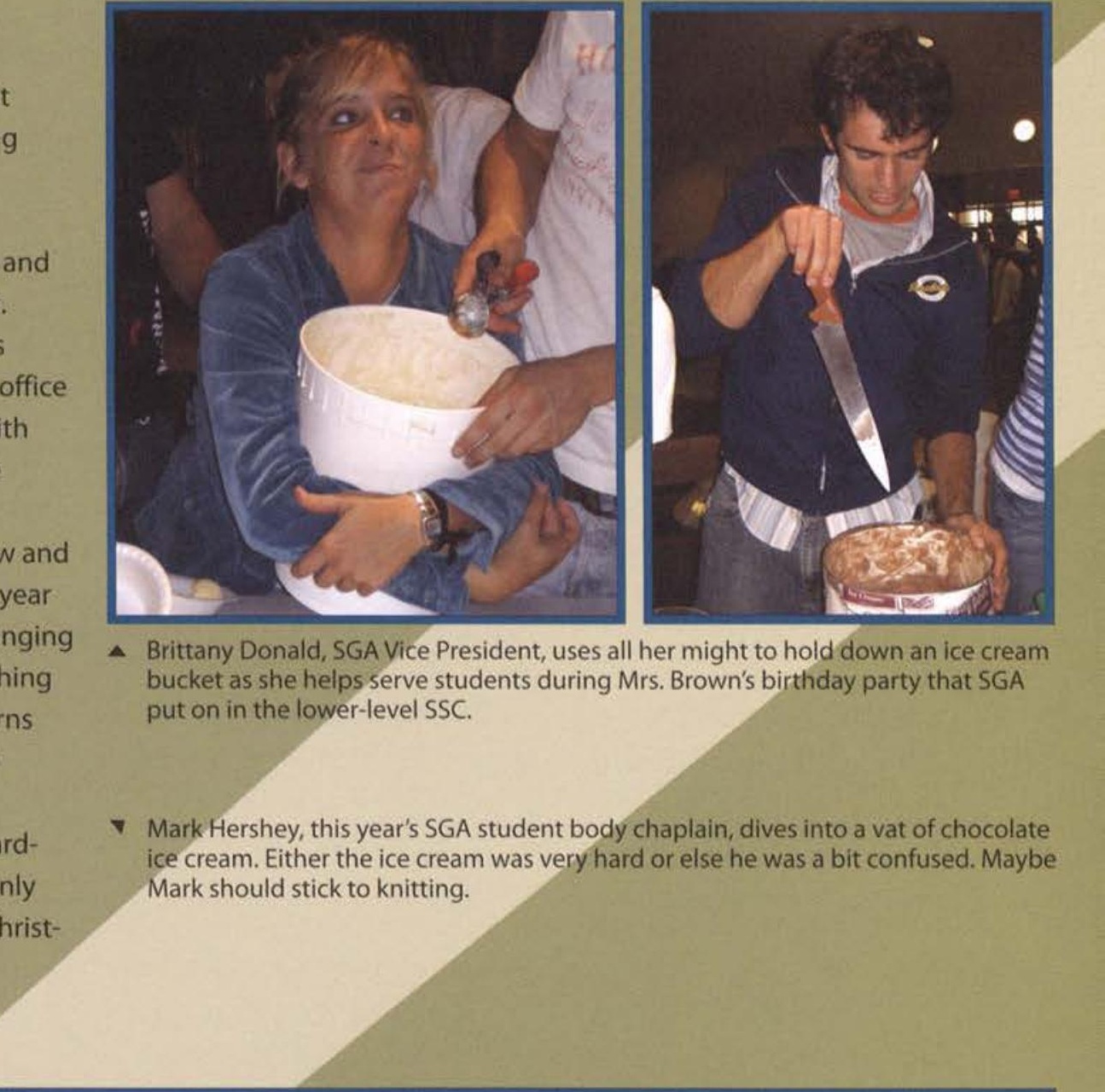

young men and women met weekly to play chess. Mordecai Veldt, an excited member of the Cedarville Chess Club says this of the year: "Usually about every other week we would have refreshments, but mostly we just had fun. Chess club isn't just about being a good chess player-it's about having fun while playing chess." The club sponsored a Chess Tournament spring semester with hefty entrance fees, half of which were awarded to the winner.

Another equally enthused group who also had an exciting spring semester was the Cedarville University College Republicans. During spring break in March 2007, the group traveled to Washington D.C. to attend the Conservative Political Action Conference. Josh Gigliotti, a member of the group, recalls the experience: "It was a great conference this year, as we were able to hear firsthand from all the republican presidential candidates. We heard from the most respected names in conservative politics... Sean Hannity, Ann Coulter..."The group was also able to have dinner with several Cedarville grads in the D.C. area who now work in politics or with the federal government.

Finally, a small group of writers and readers met weekly at Beans \& Cream every Tuesday night, calling themselves the Icarus Collective. The group was Cedarville's poetry organization. The meetings consisted of philosophical discussions about literature, art, faith in art, and at times the group work shopped each other's poems. During spring semester, a highlight was taking the opportunity to go the Cedarville University's observatory and look at Saturn through the high-powered telescope. Each member wrote a poem about the experience.

Freshman Sarah Jones and junior Amanda Roller, members of Women of Vision, help sort donated clothes at a thrift store in Kettering. Krista Kowatch, another member of WOV, says, "WOV is about directing attention to needy people and taking action with everyone who wants to join."

Ashley Coale and Nikki Rose spend time with with a small group of junior high girls from a Sanctify Purity Conference. Rebecca Phillips, a fellow member of Sanctified, says, "Ashley and Nikki were amazing blessings to the team! They bonded so well with the girls and really did a great job of showing them how much God loves them."

Members of Amplified - Ryan Ernst, Jackie McKeon, Joel Wallen, Sarah Miller, Bekah Taylor, Carla Brenneman, and Beckie Perry - traveled to City Mission in Detroit for a weekend. Ryan Ernst says, "We helped organize and make shelves in their pantry."
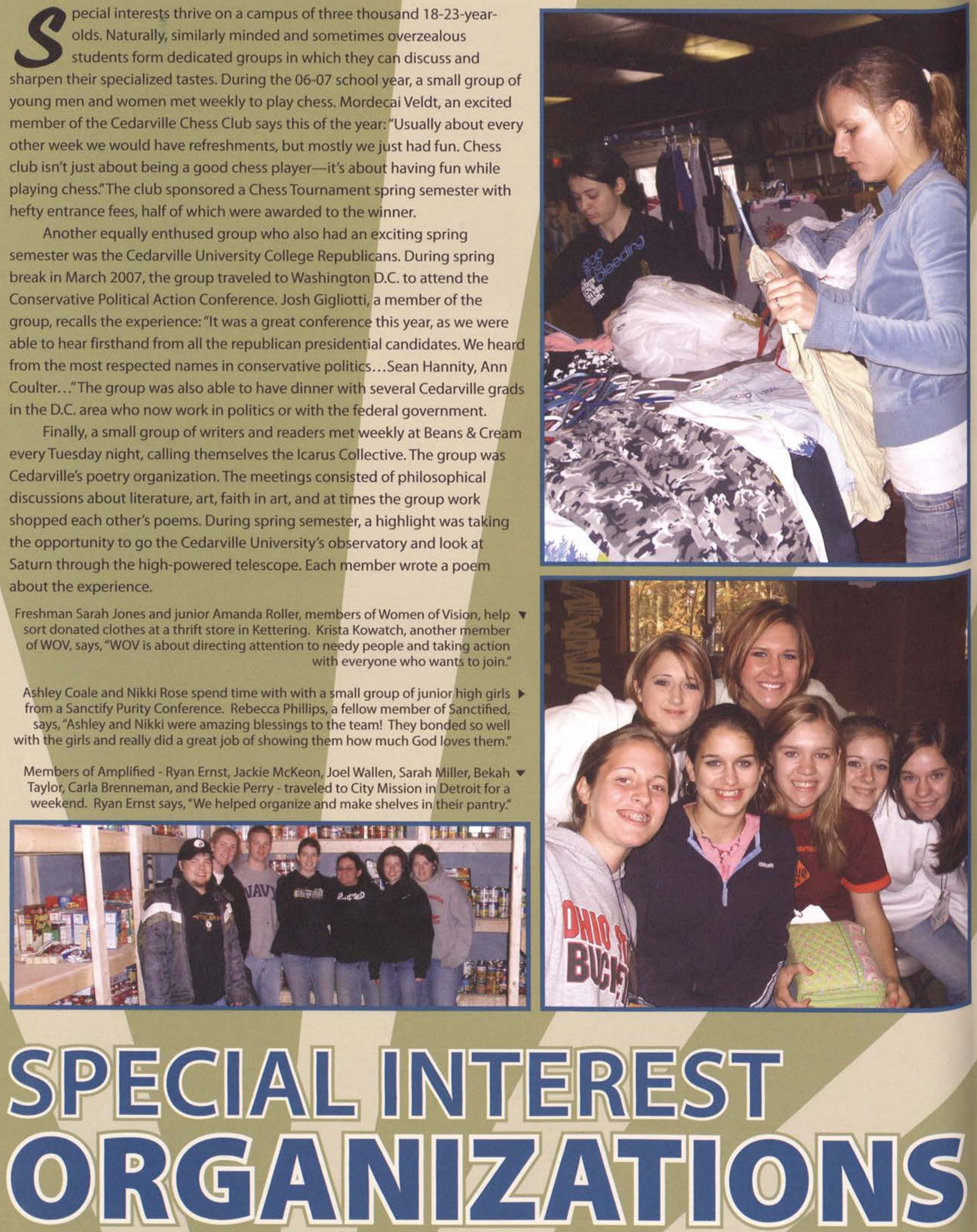

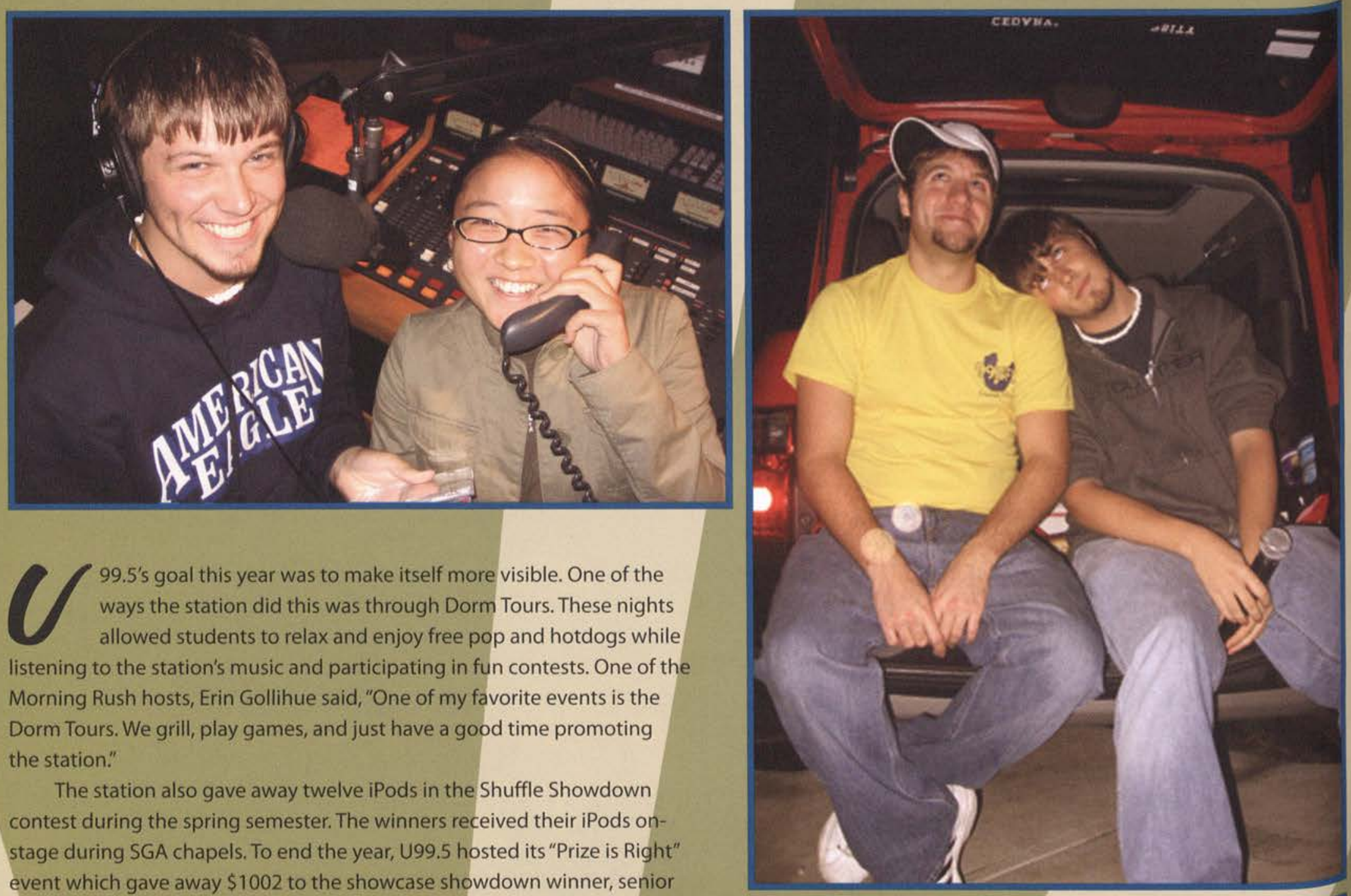

99.5 's goal this year was to make itself more visible. One of the ways the station did this was through Dorm Tours. These nights allowed students to relax and enjoy free pop and hotdogs while listening to the station's music and participating in fun contests. One of the Morning Rush hosts, Erin Gollihue said, "One of my favorite events is the Dorm Tours. We grill, play games, and just have a good time promoting the station."

The station also gave away twelve iPods in the Shuffle Showdown contest during the spring semester. The winners received their iPods onstage during SGA chapels. To end the year, U99.5 hosted its "Prize is Right" event which gave away $\$ 1002$ to the showcase showdown winner, senior Paul Hale. Melinda Workman said, "We enjoyed being able to connect with our listeners and reward them for their support. I really enjoyed the experience of working in the station. It gave me the opportunity to work on my teamwork skills and to learn how to overcome challenges that can arise in a professional environment."

U99.5 does more than play music. It prepares students for life after graduation. Station Manager Craig Vinson said, "I would never be as prepared for getting a job I love without working at U99.5FM." David Peterson agreed, saying, "The last two years U99.5FM has given away a thousand dollars in cash, and this year we gave away over a thousand more in prizes. I've had interviews with people who have said that the things our station does are unheard of for college radio stations. It's exactly the type of experience employers look for." The station certainly achieved its goal of becoming more visible. This year, U99.5 has shown its "passion for God, passion for music, passion for you," and had a good time doing it.
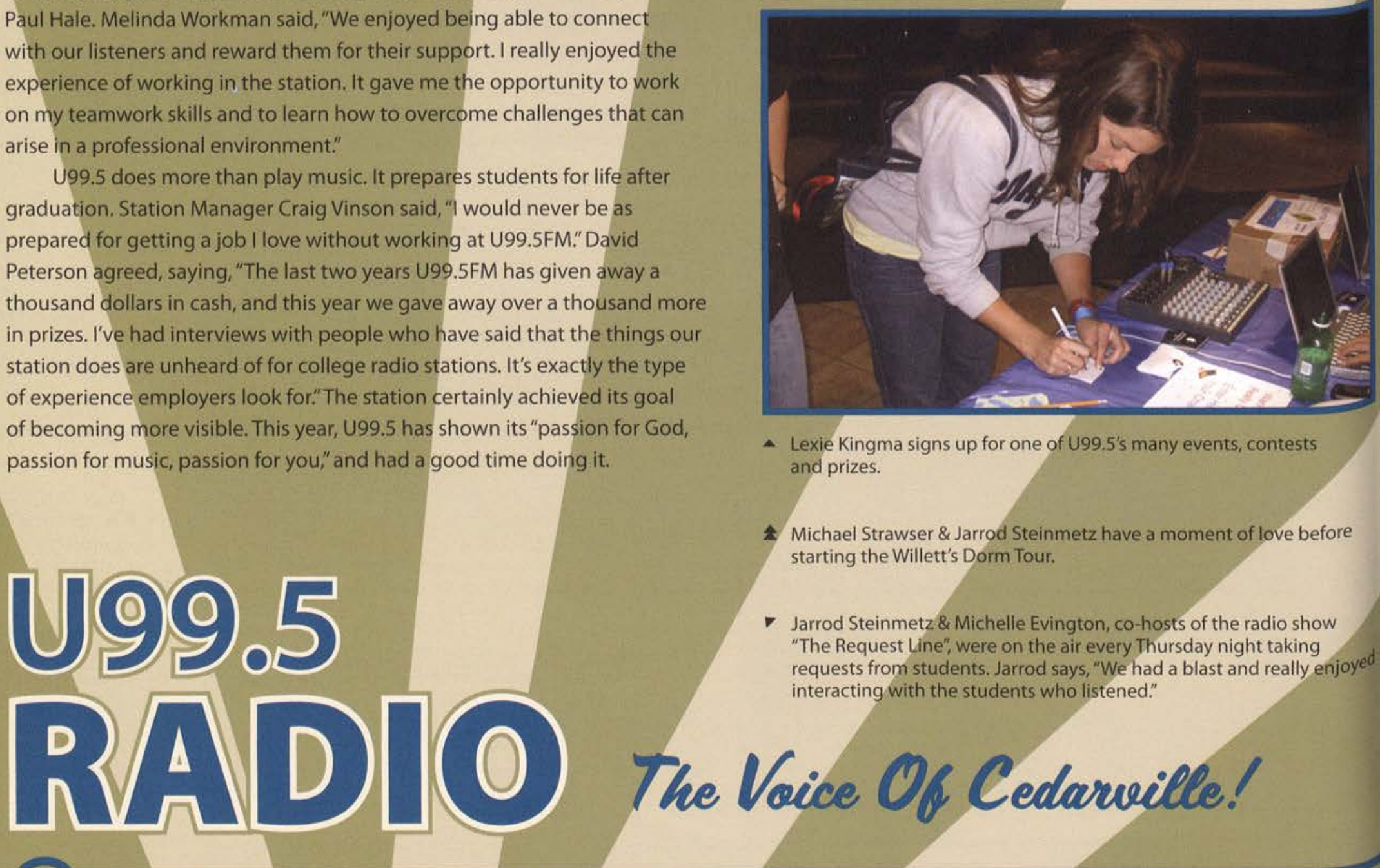

- Lexie Kingma signs up for one of U99.5's many events, contests and prizes.

A Michael Strawser \& Jarrod Steinmetz have a moment of love before starting the Willett's Dorm Tour.

V Jarrod Steinmetz \& Michelle Evington, co-hosts of the radio show "The Request Line", were on the air every Thursday night taking requests from students. Jarrod says, "We had a blast and really enjoyed interacting with the students who listened." 

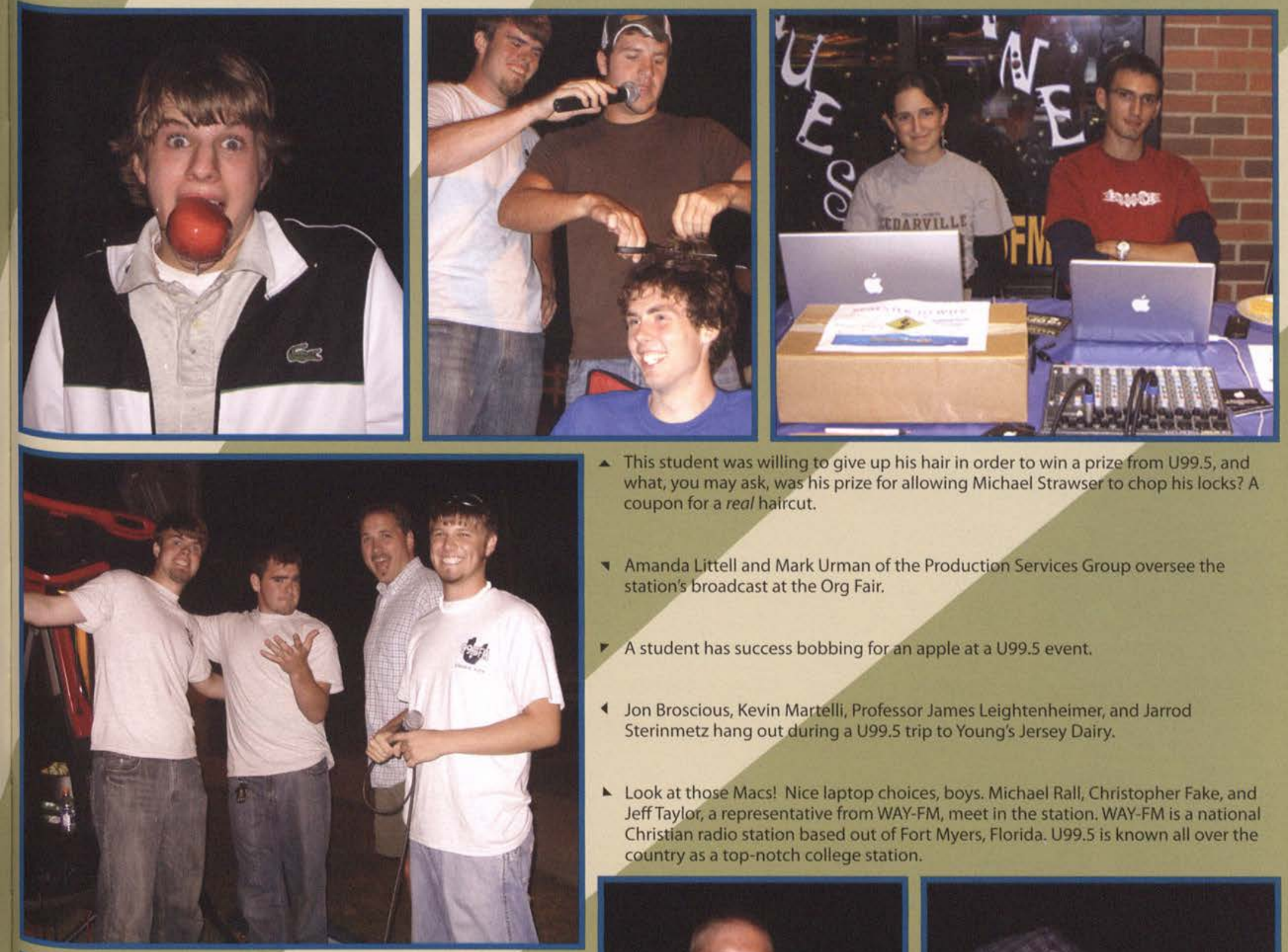

- This student was willing to give up his hair in order to win a prize from U99.5, and what, you may ask, was his prize for allowing Michael Strawser to chop his locks? A coupon for a real haircut.

v Amanda Littell and Mark Urman of the Production Services Group oversee the station's broadcast at the Org Fair.

- A student has success bobbing for an apple at a U99.5 event.

4 Jon Broscious, Kevin Martelli, Professor James Leightenheimer, and Jarrod Sterinmetz hang out during a U99.5 trip to Young's Jersey Dairy.

- Look at those Macs! Nice laptop choices, boys. Michael Rall, Christopher Fake, and Jeff Taylor, a representative from WAY-FM, meet in the station. WAY-FM is a national Christian radio station based out of Fort Myers, Florida. U99.5 is known all over the country as a top-notch college station.

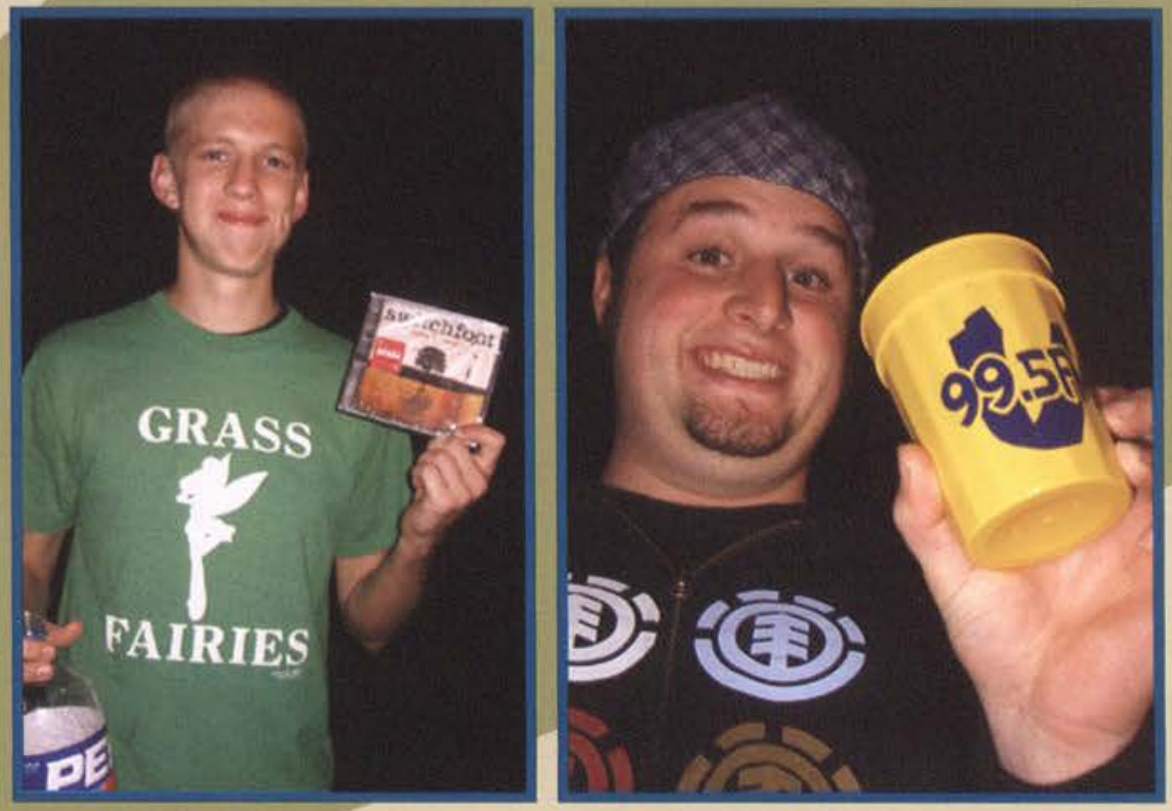

- A student displays his free Switchfoot cd complements of U99.5. As Kevin Martelli, one of the hosts of the Morning Rush, says, "U99.5 FM is Rocktastic!"

- Christopher Fake shows off the station's promotional cup. hot dogs during the Dorm Tour at Faith Hall. 


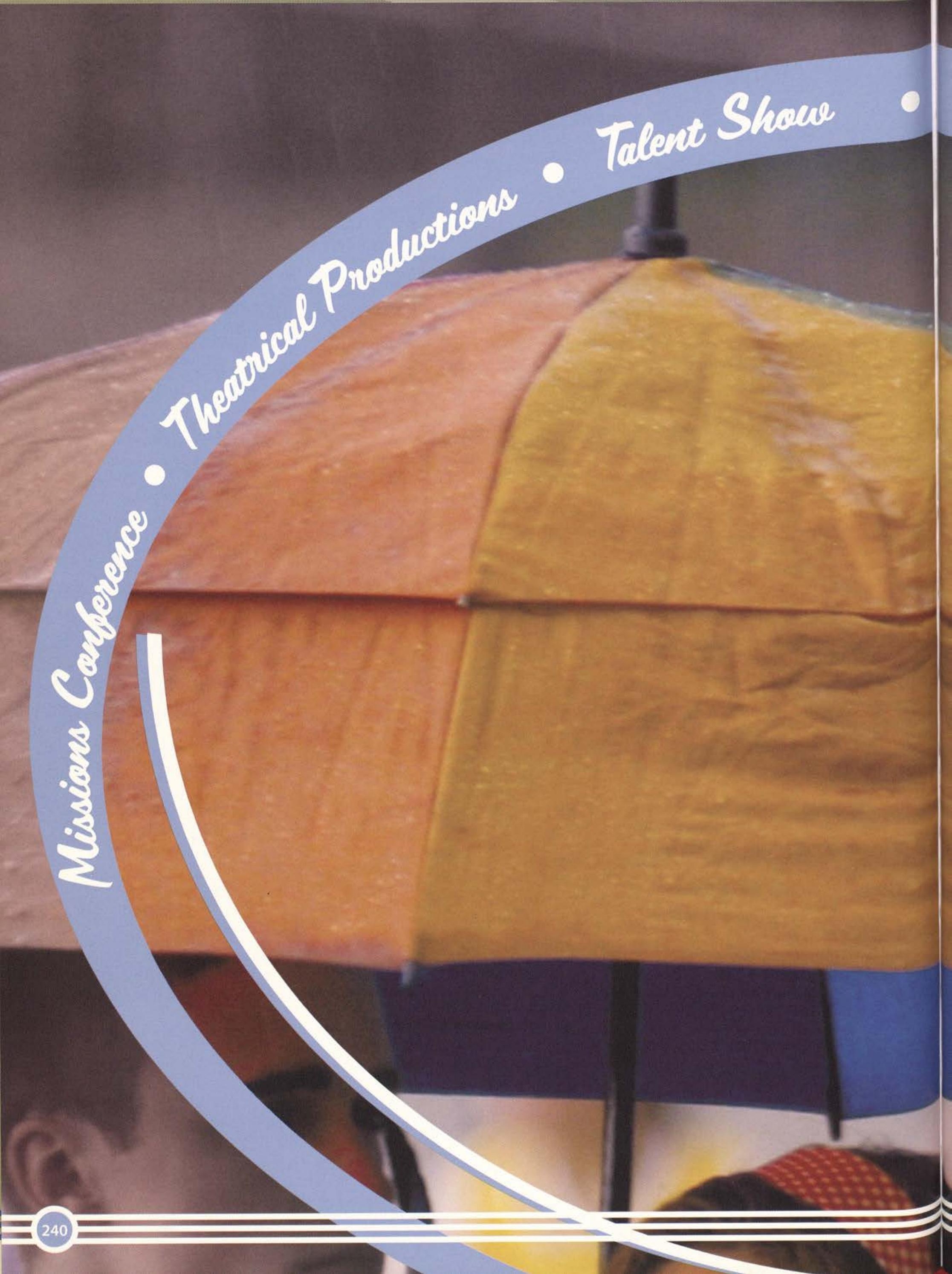




\section{Lie' Sibes.}



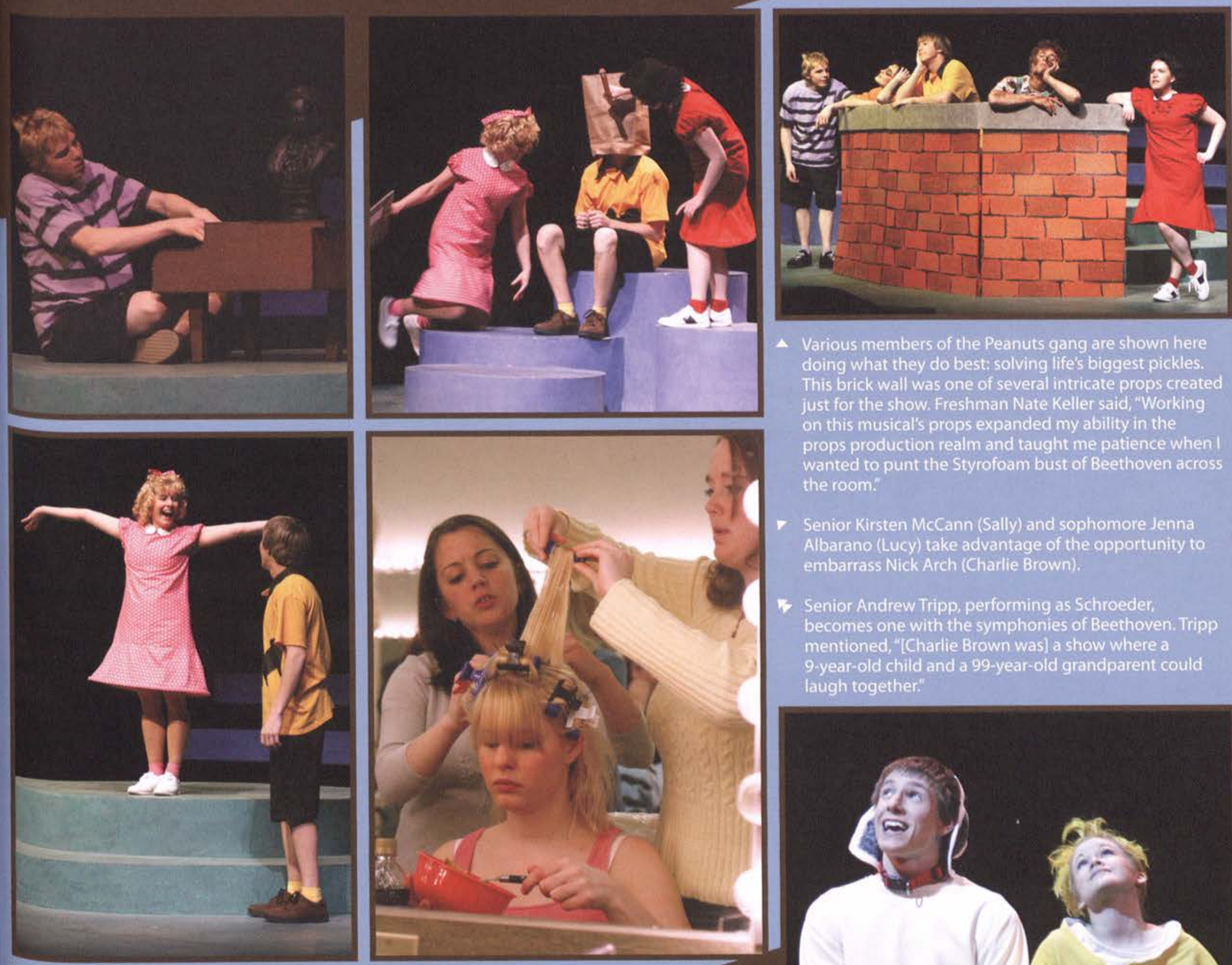

A Various members of the Peanuts gang are shown here

doing what they do best: solving life's biggest pickles.

This brick wall was one of several intricate props created

just for the show. Freshman Nate Keller said, "Working

on this musical's props expanded my ability in the

props production realm and taught me patience when I wanted to punt the Styrofoam bust of Beethoven across

the room:

- Senior Kirsten McCann (Sally) and sophomore Jenna Albarano (Lucy) take advantage of the opportunity to embarrass Nick Arch (Charlie Brown).

7. Senior Andrew Tripp, performing as Schroeder, becomes one with the symphonies of Beethoven. Tripp mentioned, "[Charlie Brown was] a show where a 9-year-old child and a 99-year-old grandparent could laugh together:

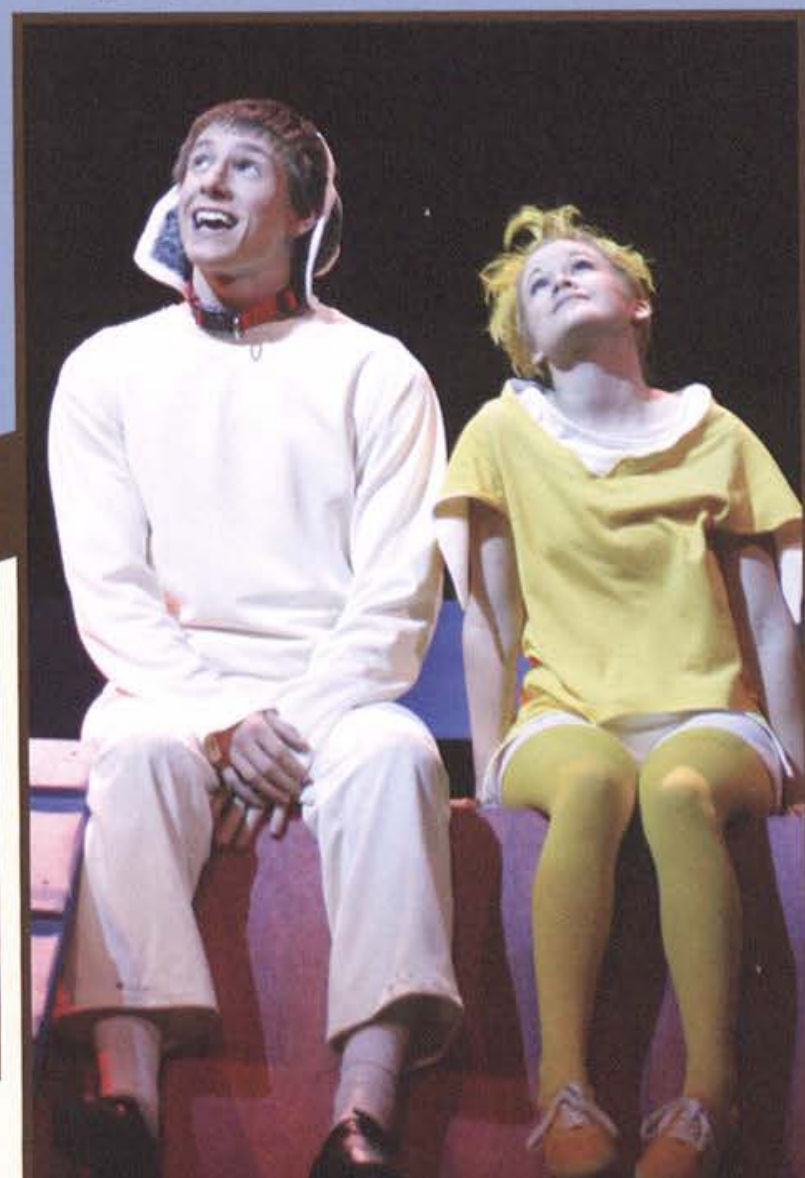

- Senior Kirsten McCann, playing Charlie Brown's sister Sally, puts passion and childlike intensity in her solo "My New Philosophy." Fellow cast member and freshman Annalise Hickok said, "Everyone remembers Peanuts, and everyone enjoys a little trip down memory lane. What a better time of life to remember than childhood?"

- Senior Kristin McCann slowly transforms into Sally Brown, Charlie's annoying little sister. Throughout the play, she constantly antagonizes Charlie, but finally firmly states, "You're a good man, Charlie Brown!"

- Snoopy (freshman Zach Hill) and Woodstock (sophomore Marisa Baker) relax on top of Snoopy's doghouse, contemplating life and admiring the stars. 


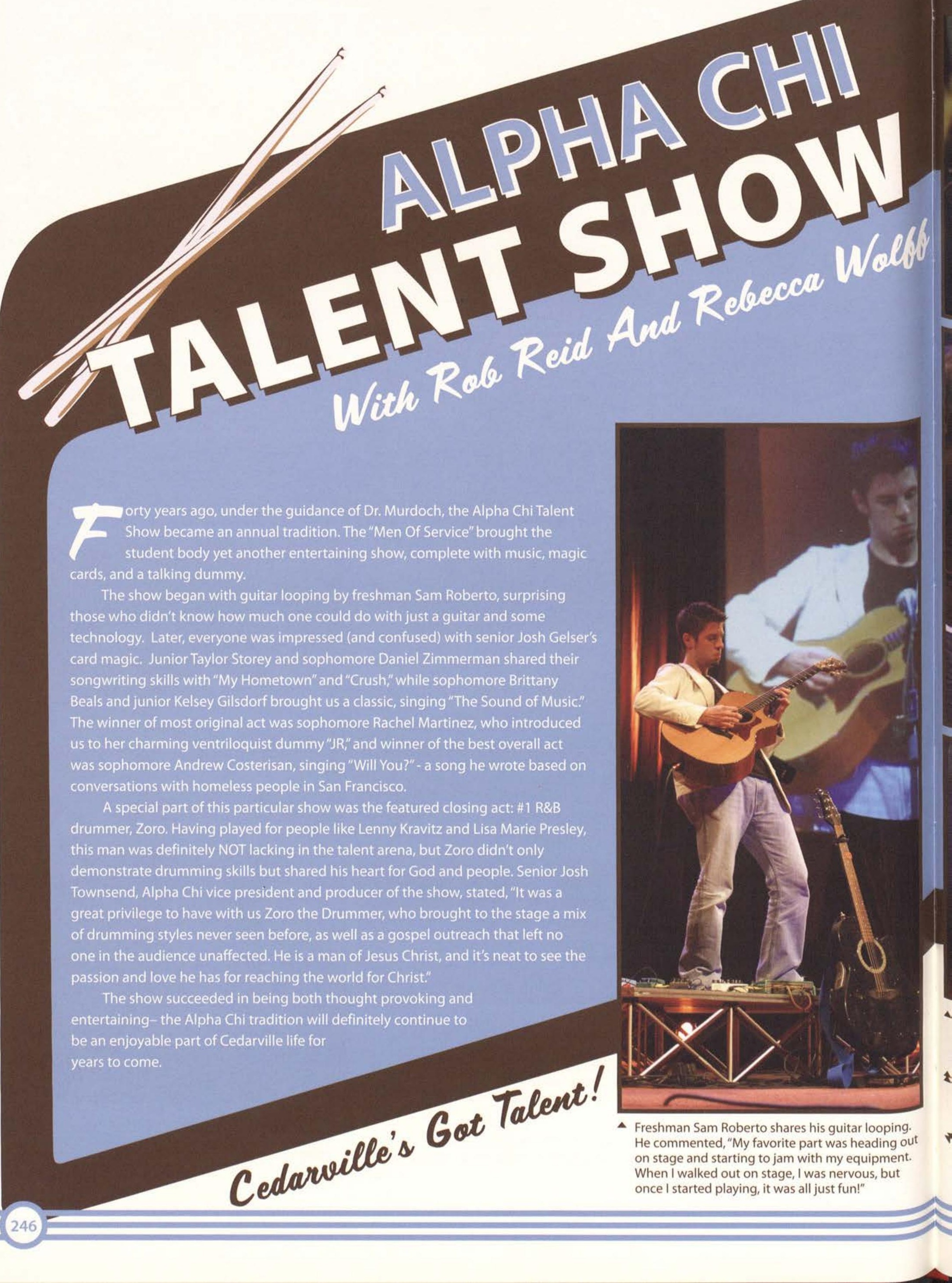




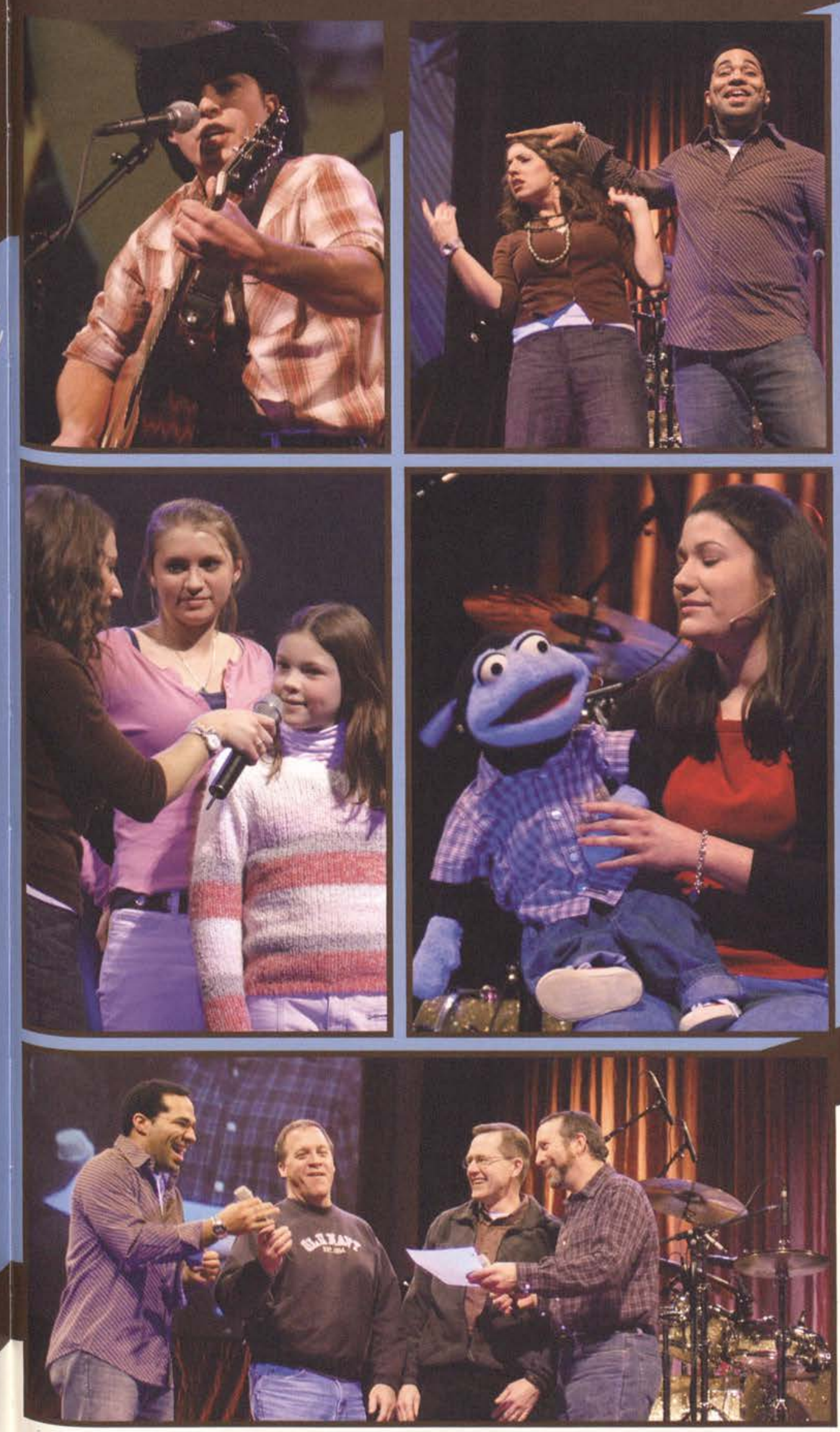

Robert Reid persuaded a few unsuspecting fathers visiting during Li'I Sibs weekend to participate in an activity between some of the talent acts. During this activity they were compelled to sing Disney's "A Whole New World."

Rebecca Wolff interviews a student's sibling visiting for Li'l Sibs weekend. The AX show involved different games between acts, giving audience members a chance to win various gift certificates and prizes.

"Rachel Martinez with "J.R." This was her second year participating in the AX show, and she notes, "My favorite part about the show this year was the interaction behind stage. All of the performers talked and joked around with each other, which made the show more enjoyable. We were there to put forth our best and we had a great time doing it!"
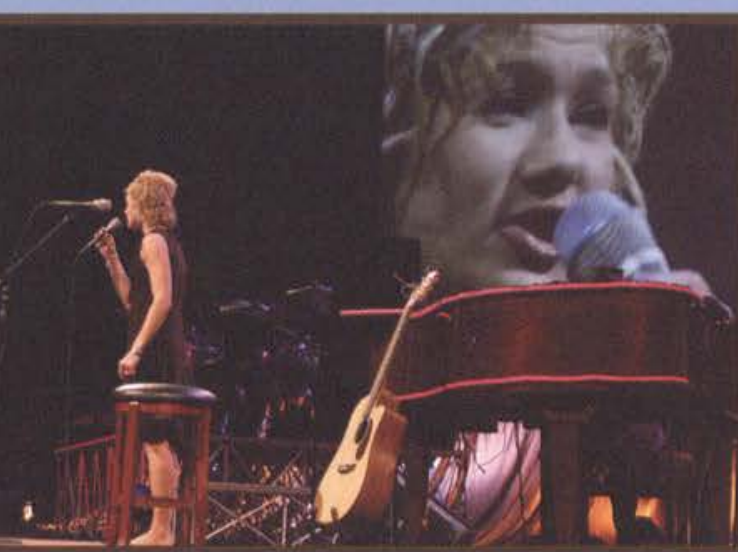

- "The Sound of Music" fills the chapel as sophomore Brittany Beals sings while her friend Kelsey Gilsdorf accompanies on the piano.

V. Rebecca Wolff and Robert Reid are this year's hosts, contributing to the humor and entertainment of the show. Junior Zachary Scott noted of the two, "As hosts, Robert and Rebecca were wonderful. They had a certain chemistry that just worked, almost as if they were engaged." Oh wait - they are!

7. Sophomore Daniel Zimmerman sings "Crush"-- a song he wrote about a teacher. He commented, "The show was so much fun. I could tell the audience loved the song I had written - I had lots of people coming up to me afterwards telling me they loved it and asking me if it was really true."

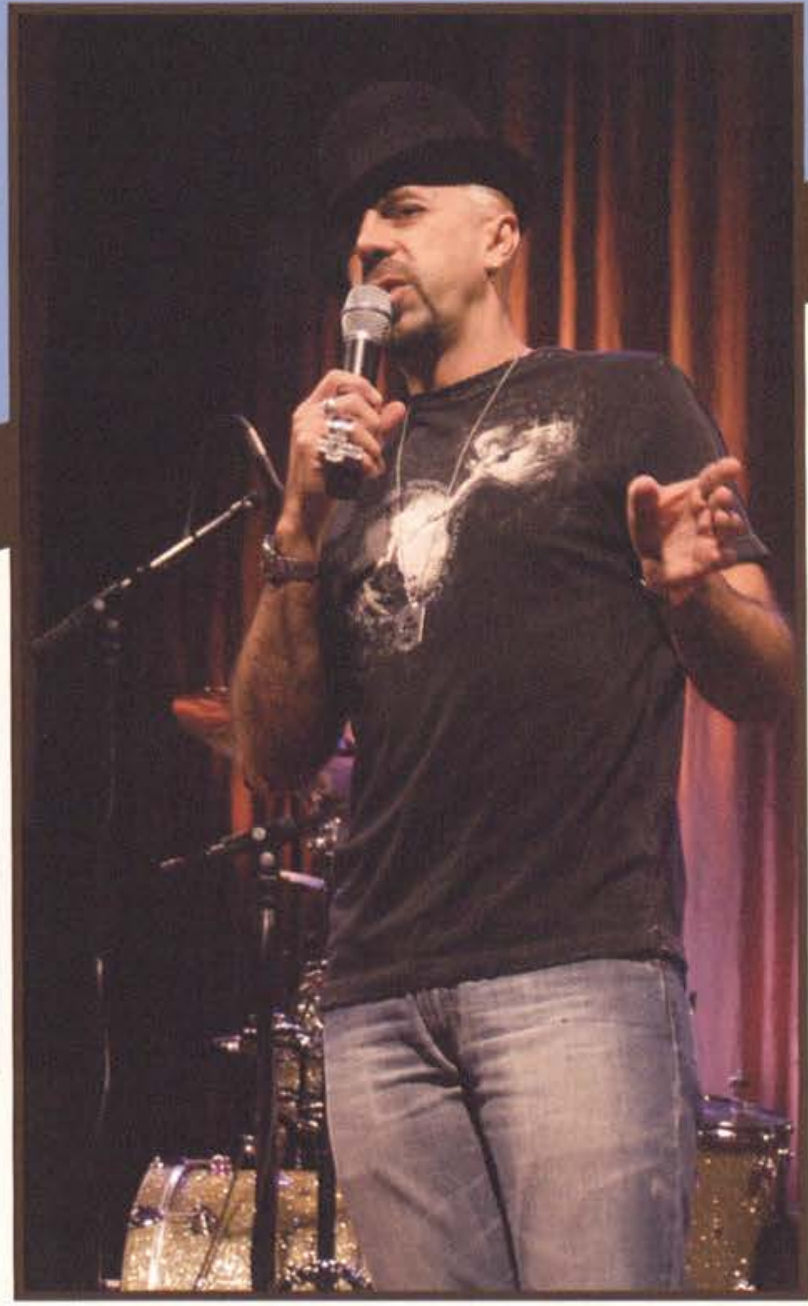

- Zoro the Drummer not only shared his drumming talent, but also his heart for God and people. Sophomore Elizabeth Studebaker commented, "I really enjoyed the fact that the talent show was fun and entertaining- without overlooking the spiritual side of life." 


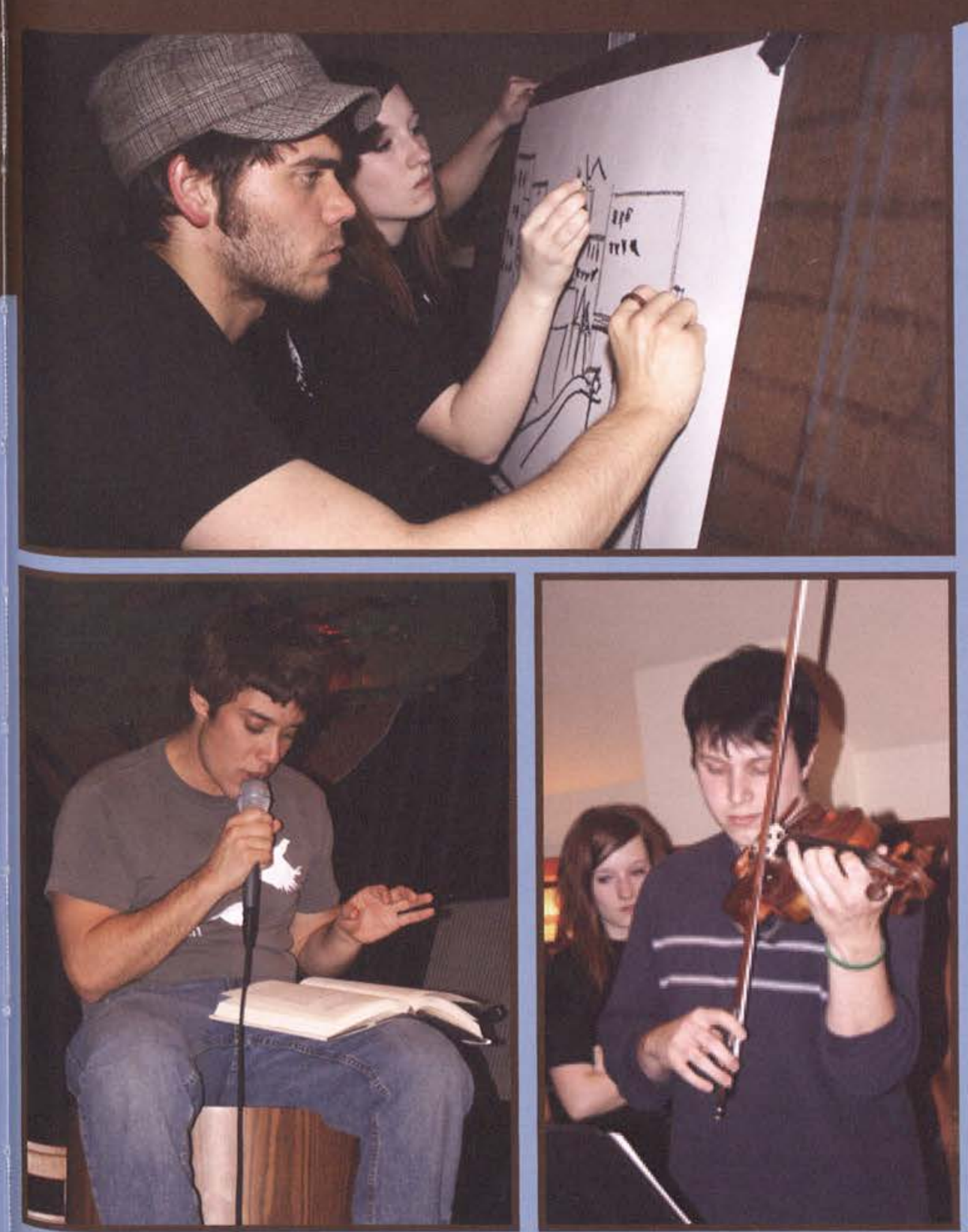

provided gifted musicians, painters, sculptors, and poets a venue to worship God in their own way.

Junior John Hawkins, coordinator of Mosaic

described the order of events and their specific intended purposes: "We organized the acts thematically, moving from innocence to fallenness and then out of despair into joy. Sets by junior David Sizemore included paper trees that were stripped of their branches when the fall came, and a cardboard city that was destroyed and then rebuilt at the moment of redemption that came during one of the songs:"

Hawkins also estimated approximately 200 people in attendance at the Hive the night of Mosaic. He mentioned, "About half were fiercely involved - they danced to the music, made construction paper animals, sang along, and banged on pots, pans, cowbells, pieces of drum kits, and ratchets. The majority of the others was respectful and engaged, participating in their own way."

Senior Sarah Baczek heard similar praises surrounding Mosaic. She attributed it to the fact that the event "was something Cedarville isn't accustomed to doing because it was very different from the Cedarville norm. It was fresh and real and very artistically driven." With such a gripping worship style and strong student following, this year's Mosaic may have been the beginning of an honored Cedarville tradition.

4 John Hawkins reads about the Shapes of Love, an orginal piece describing his view of communal love, love between lovers and, ultimately, God's perfect love.

Katie Klimek and Jonathan McGill create a work of art in black and white during a time of despair. As hope is restored, coloris added to the piece.

A Andrew Ralon plays his violin as Katie Klimek prepares to paint during a-poKlimek preps
etry reading
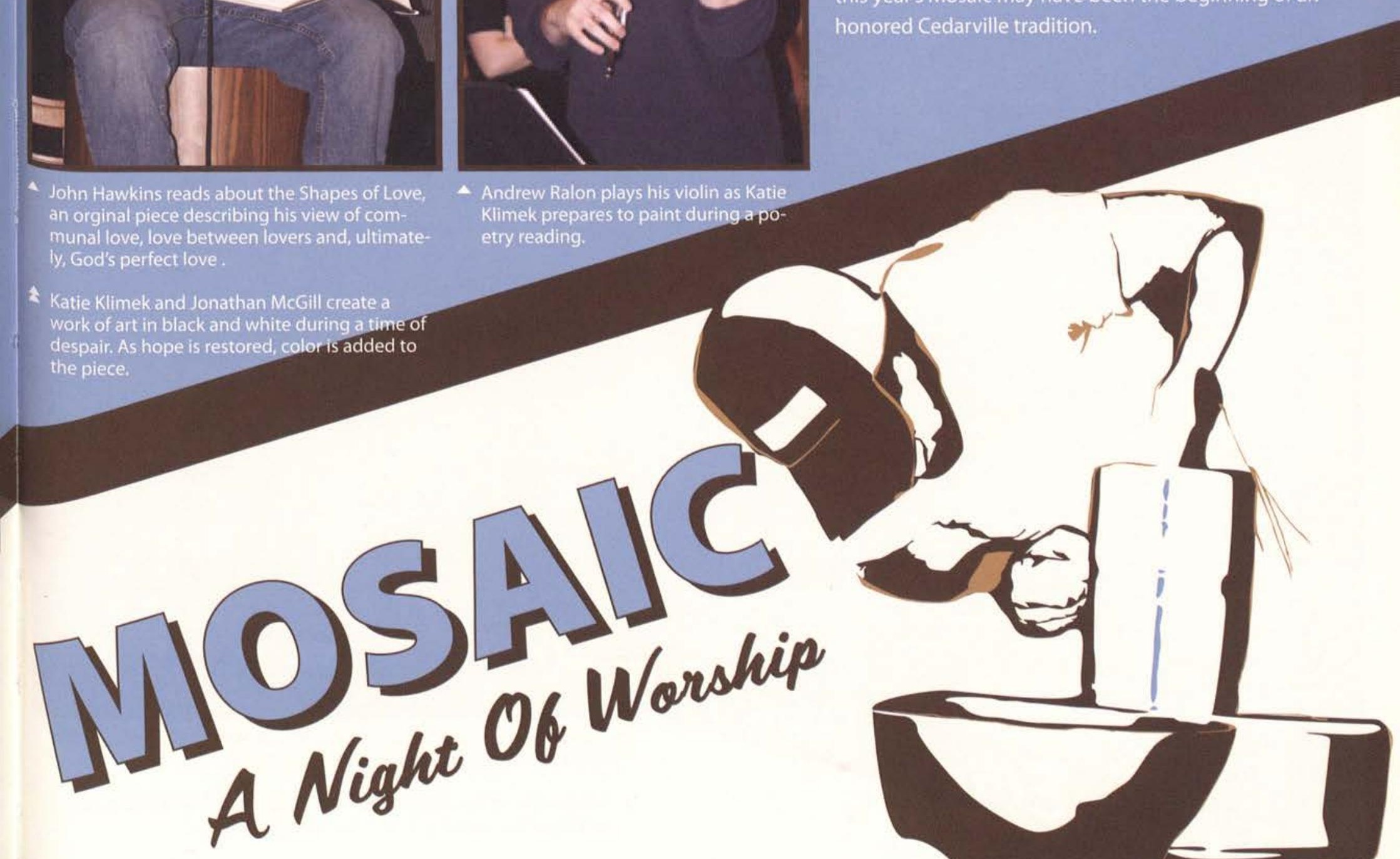


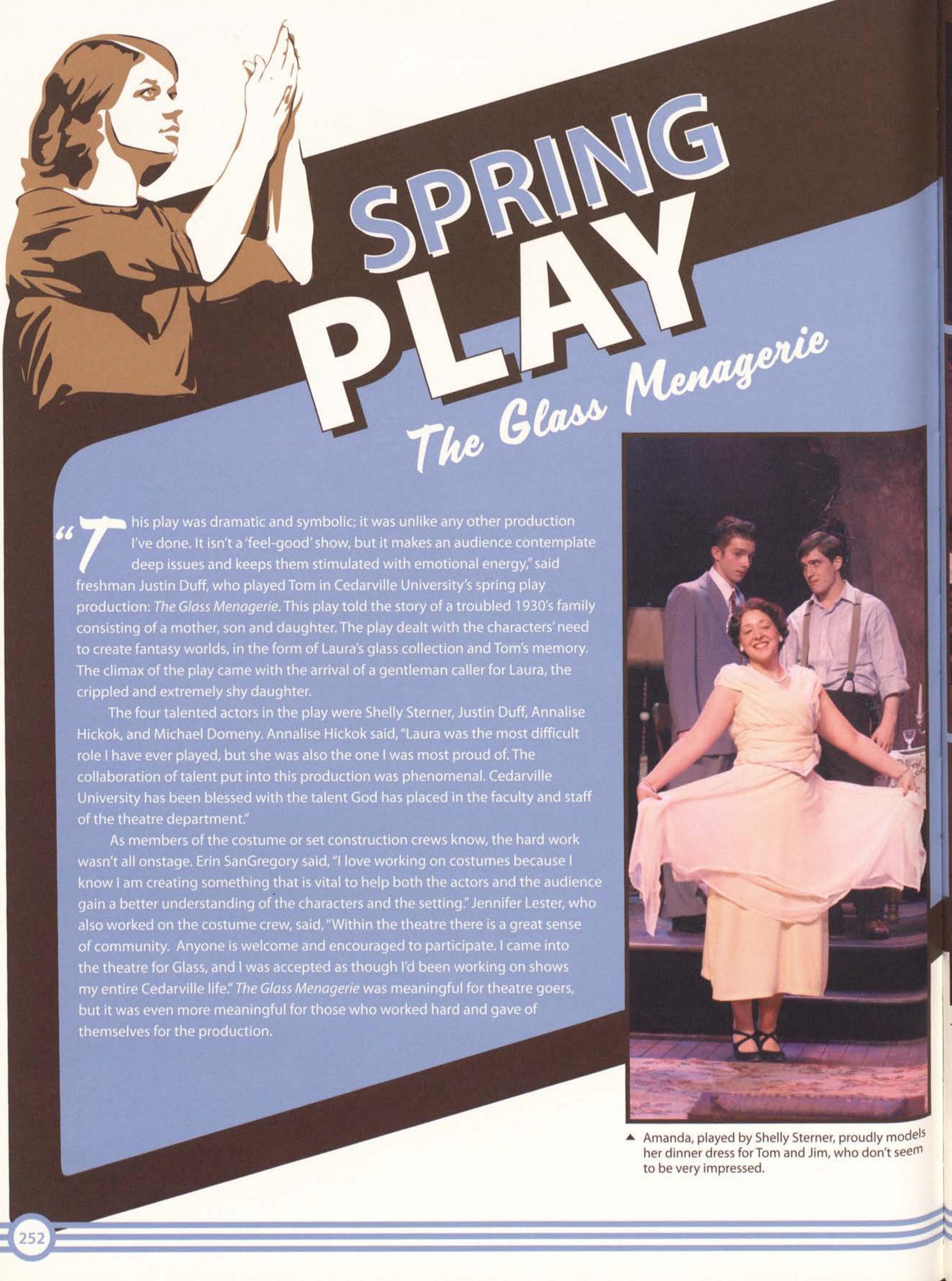



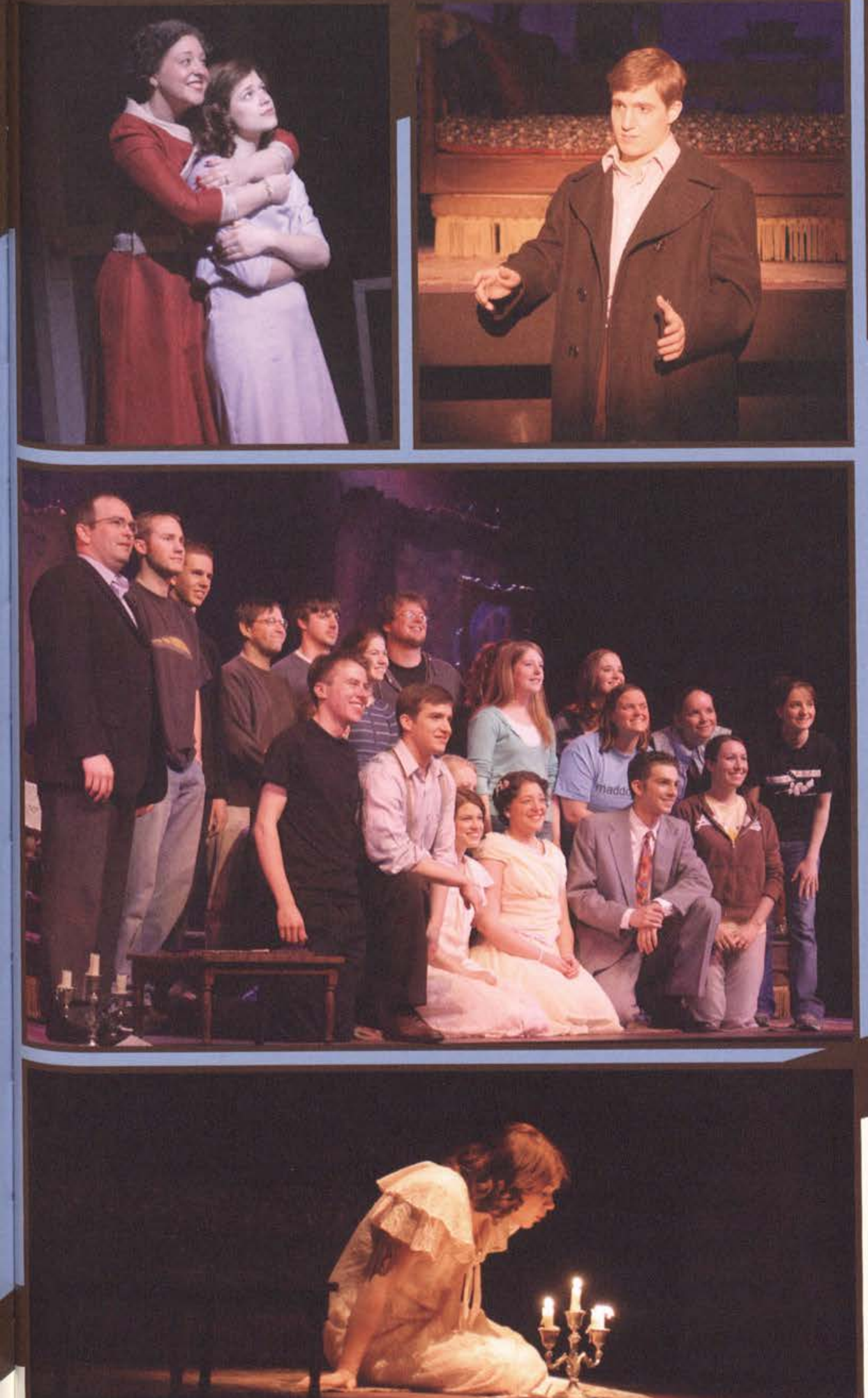

4 "This was a heartbreaking, beautiful play. Even though it was so sad, the actors' performances entranced me so that I just couldn't be depressed afterwards," said junior Anna Cummings.

- The comraderie between the cast members was amazing to watch. With such a small cast, all agreed that it was a great opportunity to really get to know one another, which added depth to the final production.

- Although the relationship between Amanda and Tom is usually tense, mother and son share a rare moment of tenderness on the porch.

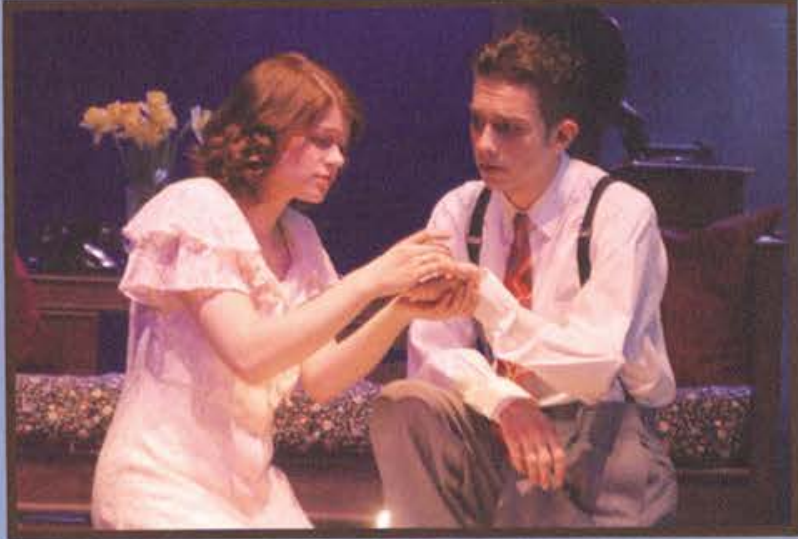

Although she is painfully shy, Laura opens up to Jim and introduces him to her beloved glass collection. This scene is an audience favorite as their hopes for Laura's happiness rise.

V "TThe play] stretched me as an actor and really opened my eyes to the strengths of college productions," said Justin Duff, who played Tom and served as the play's narrator:

5. Amanda cradles Laura, assuring her that everything will work out when her "gentleman caller" comes to dinner.

4 The backstage crew gathers for a picture. "It's fun working behind the scenes.... You're there to witness the funny bloopers and happy accidents," said costume crew member Kari Forson.

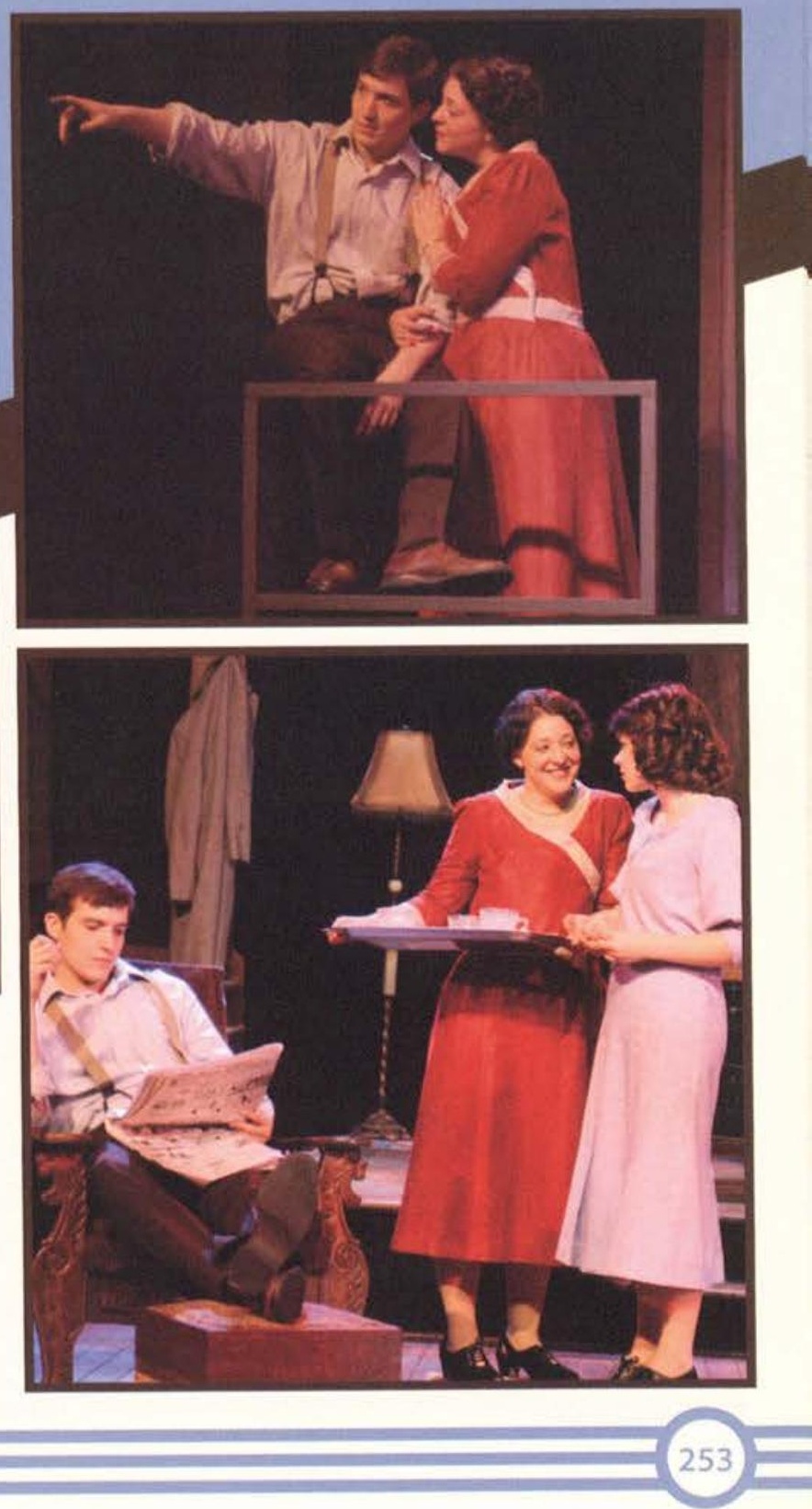



his year's student-selected Faculty and Staff of the Year awards chapel carried with it a general feeling of surprise. Professor and former coach Teresa Clark, Faculty of the year cipient, explained her surprise. She said, "Shocked would be a good description since I had no idea. It was funny that while the descriptors were being read, I thought to myself, wow - it's someone that I went to school with during my days at Cedarville as a student. I was wondering Who it was not even thinking it could be mel" Clark's complete disregard of her receiving this honor is a strong indicator of her integrity and
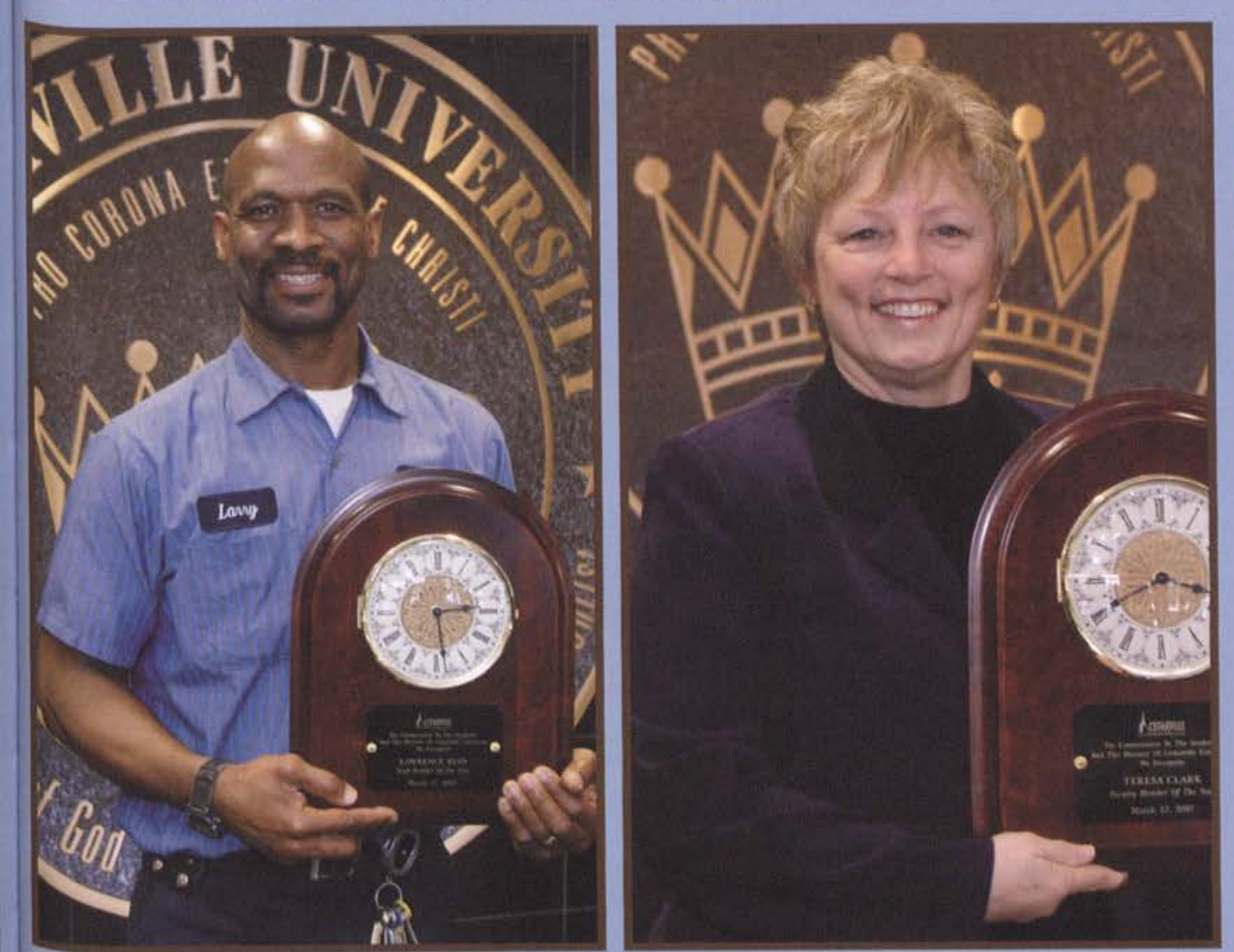

modesty. Clark also served as an excellent professor and coach. As Head Coach of the women's volleyball team, Clark led her girls to countless victories and championship wins.

\section{Just like Clark, Door Hardware Specialist and Staff of the Year} recipient Larry Reid didn't see the honor coming. According to daughter and Cedarville junior Courtney Reid, "[my Dad] had been saying a half an hour earlier to my mom on the phone that he knew it couldn't be him because they would have told him to bring nicer clothes." Larry Reid, while completely thrown off-guard by the award, explained how important it was to him. He recalled being "anxious, because I realized I had to go on stage, blessed because my family was there, and humbled by being showered with God's grace all these years." Clearly, the Cedarville student body made two strong decisions for the Faculty and Staff of the Year awards: individuals that exhibit both humility and excellence in their God-given duties.

4 Teresa Clark values her relationships with students and hopes that she is "encouraging them to train up the next generation to love our Lord:

4 Mr. Larry Reid says that his years at Cedarville have been marked by the way he has "been stretched and challenged spiritually amongst peers and coworkers." Ried is a man whose humility and godly servanthood are evident to students from the first minute they come in contact with him.

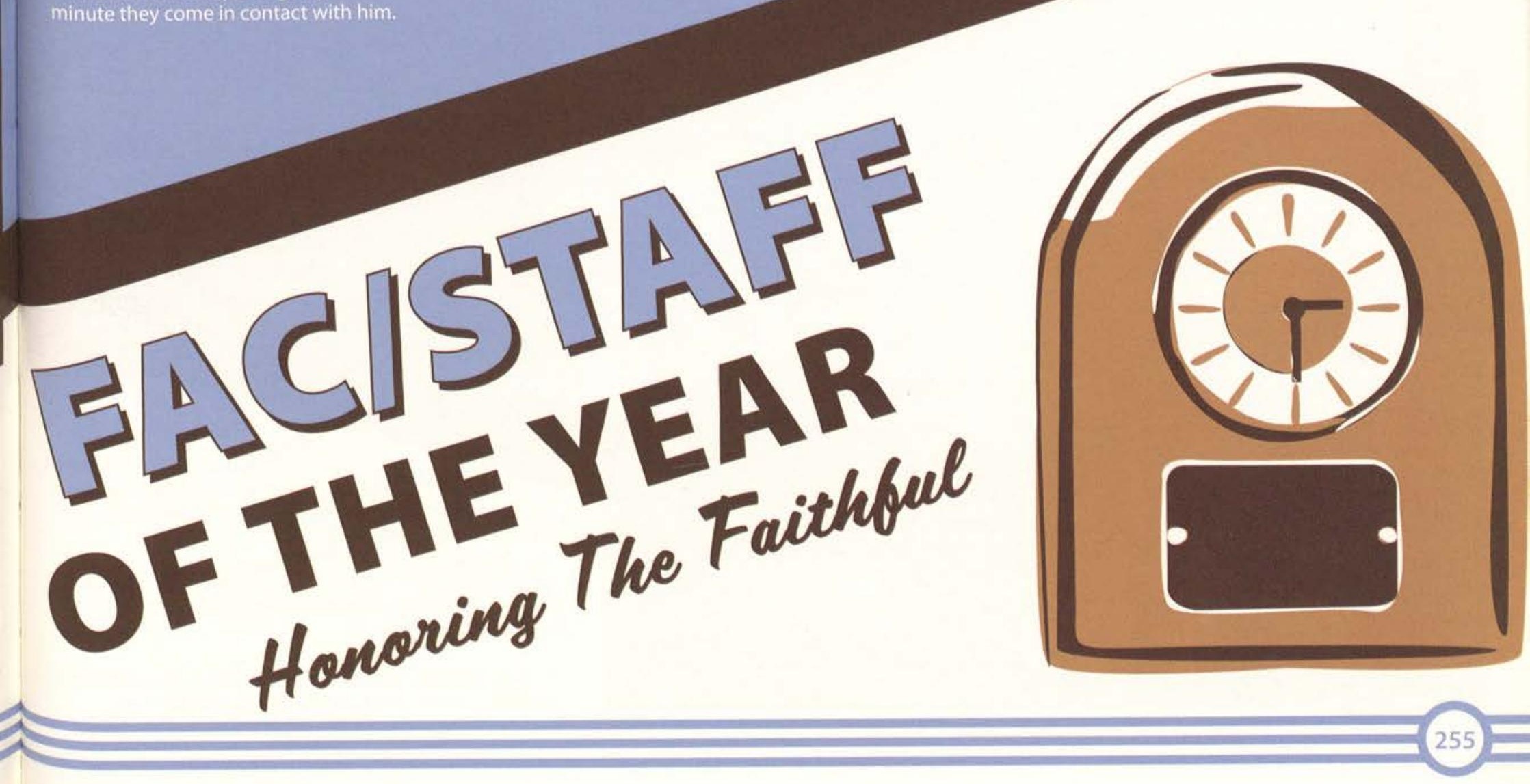




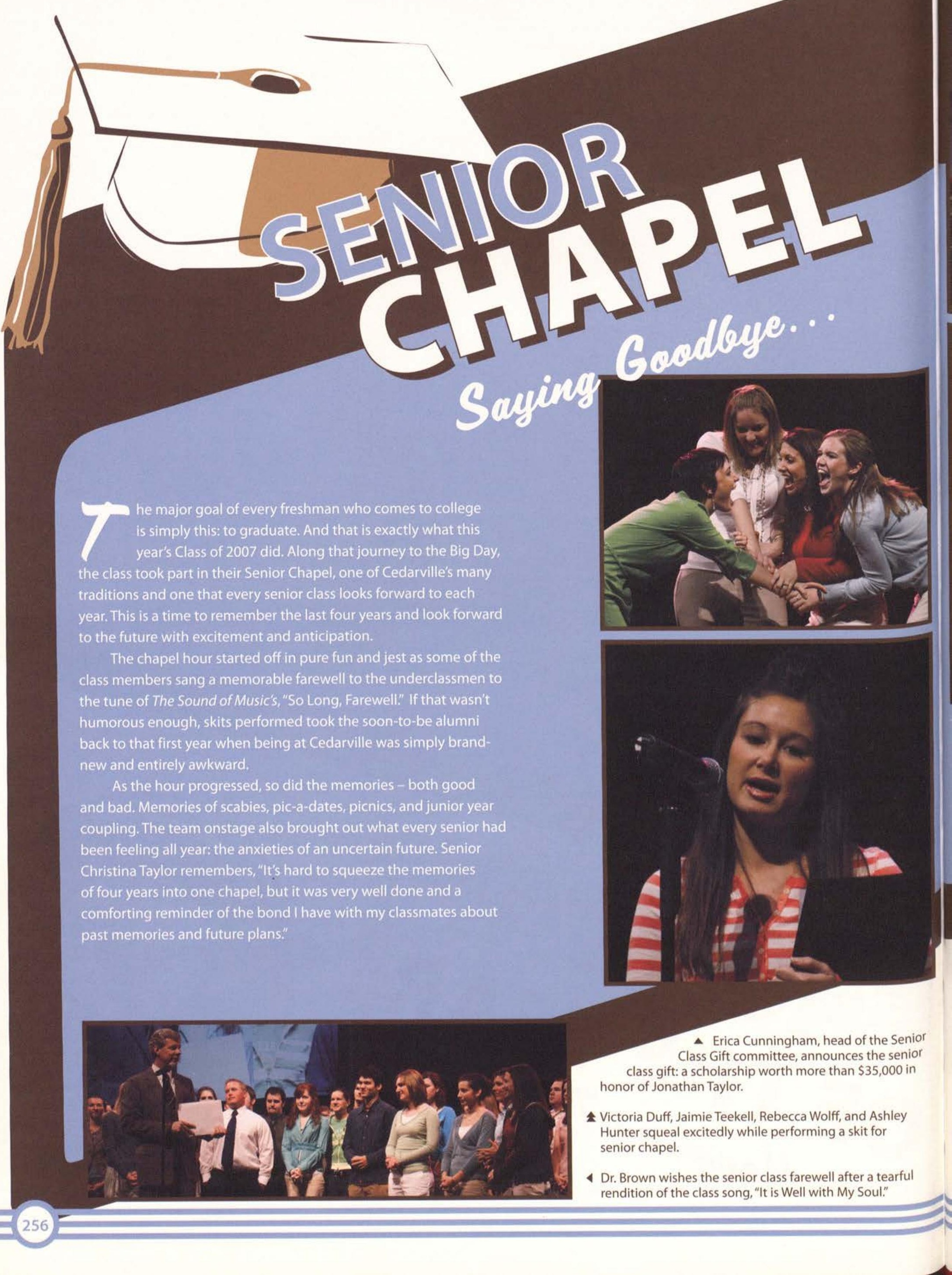



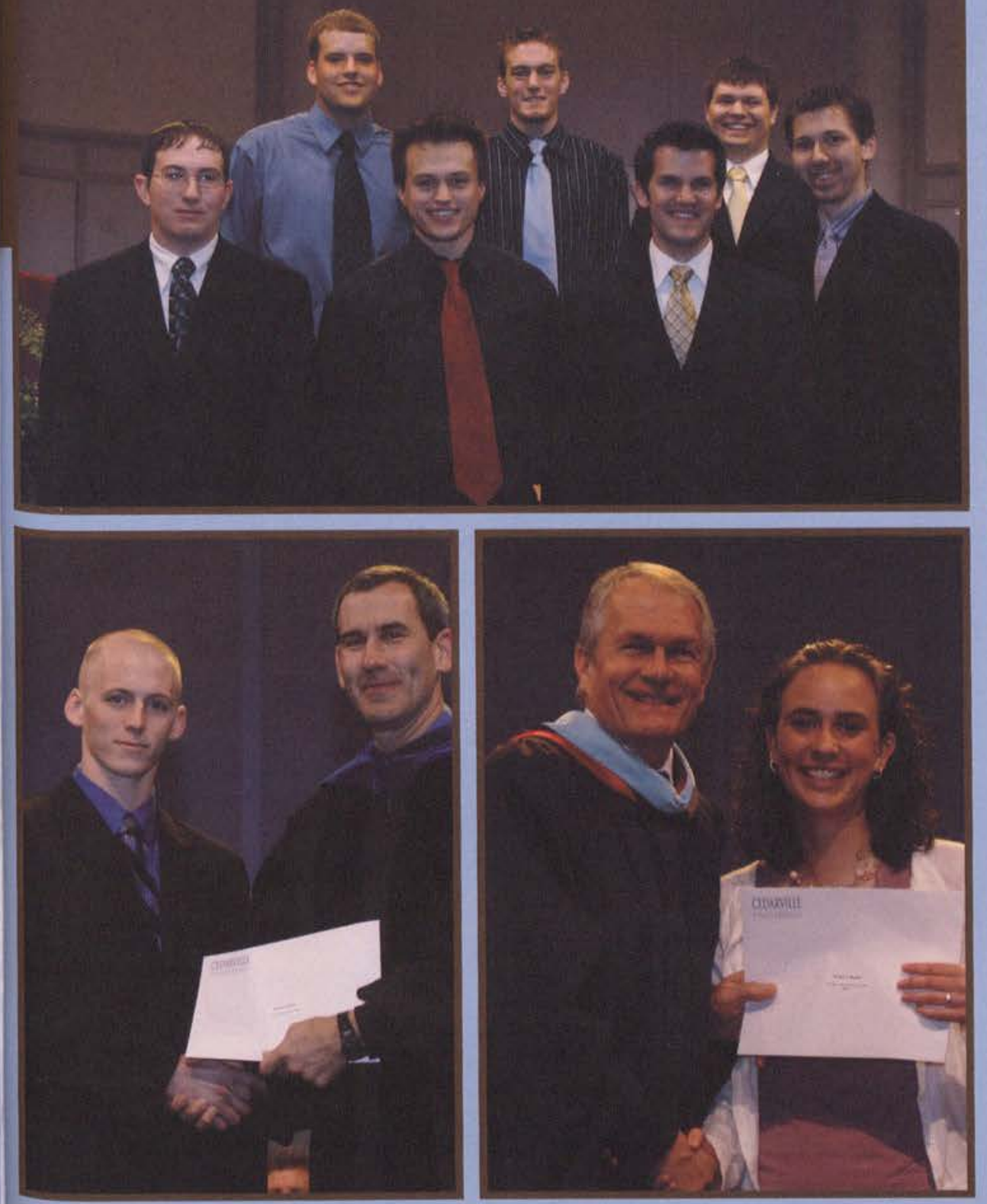

4 Senior Scott Norman receives the AFCEA Scholarship from Dr. SanGregory, Chair of the Engineering Department.

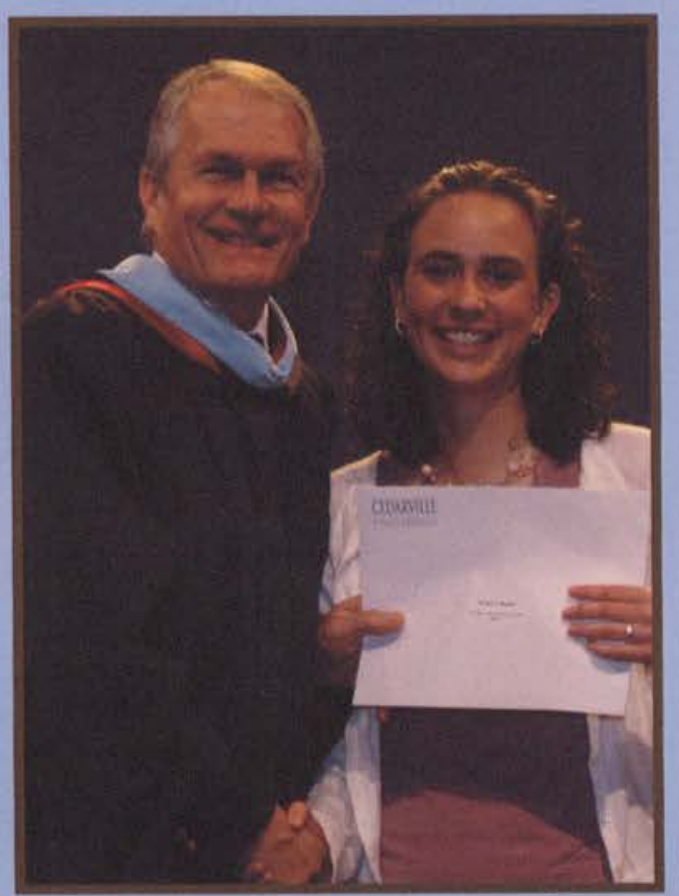

A Junior Kristin Merkel is awarded the Dave Jones Memorial Soccer Award by Coach John McGillivary.

7 yan Rotman, upon receiving awards A the infamous Honors Day Chapel Music Contribution Award' and the 'Charles

Ellington Church Music and Worship Scholarship I was shocked and surprised. I never saw myself winning an award like the Charles Ellington Scholarship. As far as the Music Contribution Award' it felt good to have all of my hard work recognized:"

Having hard work recognized was a common sentiment at this years' Honors Chapel. From 4.0 students to alumni scholarships to the Ruby Jeremiah Academic Scholarship, students were acknowledged for their hard work in academics, Christian service, and leadership.

However, beyond having the students' hard work recognized was the satisfaction of knowing that they were using their talents to the best of their abilities, and in doing so, honoring God.

A Juniors Charles Kraus, Craig Houser, Justin Dodson, Adam Sheldon, Jason Zastrow, James McClenahan, and Jonathan Lawler are this year's recipiants of the John W. Bickett Heritage Alumni Scholarship, which is given to students preparing for pastoral or missionary service.

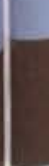

I 150 Class Top Of The

Class

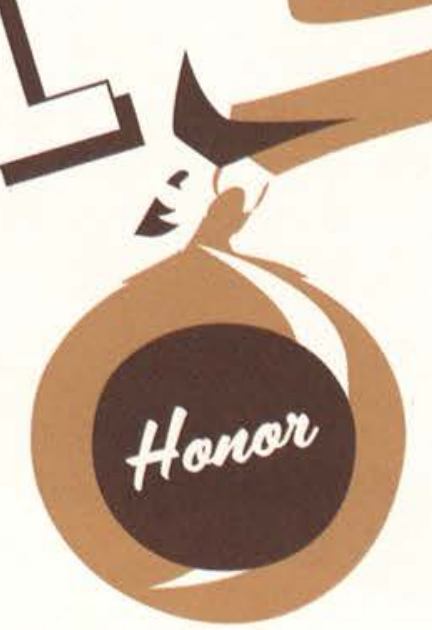




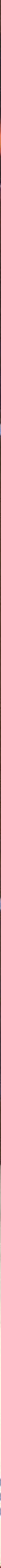



CONGRATULATIONS!

ne of the last weekends in the school year, seniors congregated

in the parking lot of the SSC, waiting for the vans that would

take them to the much talked-about reception at Dr. and Mrs.

Brown's house. Conversations ranged from what the house would look

like, to what it would be like to spend time in conversation with Dr. Brown,

to graduation, not too far in the future.

An annual tradition started by Dr. and Mrs. Dixon, those graduating in May always enjoyed this reception given exclusively for seniors. A time to relax with friends, enjoy food catered by the banquet staff at Chuck's, meet Dr. and Mrs. Brown, and get a personal tour of the President's house.

Some students lounged outside on the patio swing, on benches, chairs, and other patio furniture; while others dragged chairs into the shade, under large lawn umbrellas, or headed inside to escape the hot afternoon sun.

The afternoon wrapped up slowly, with students reluctantly heading back to their respective dorm rooms and houses to finish up homework and begin studying for finals, their time spent relaxing with the Browns a welcome respite in the midst of the hecticness of finishing out their last school year.
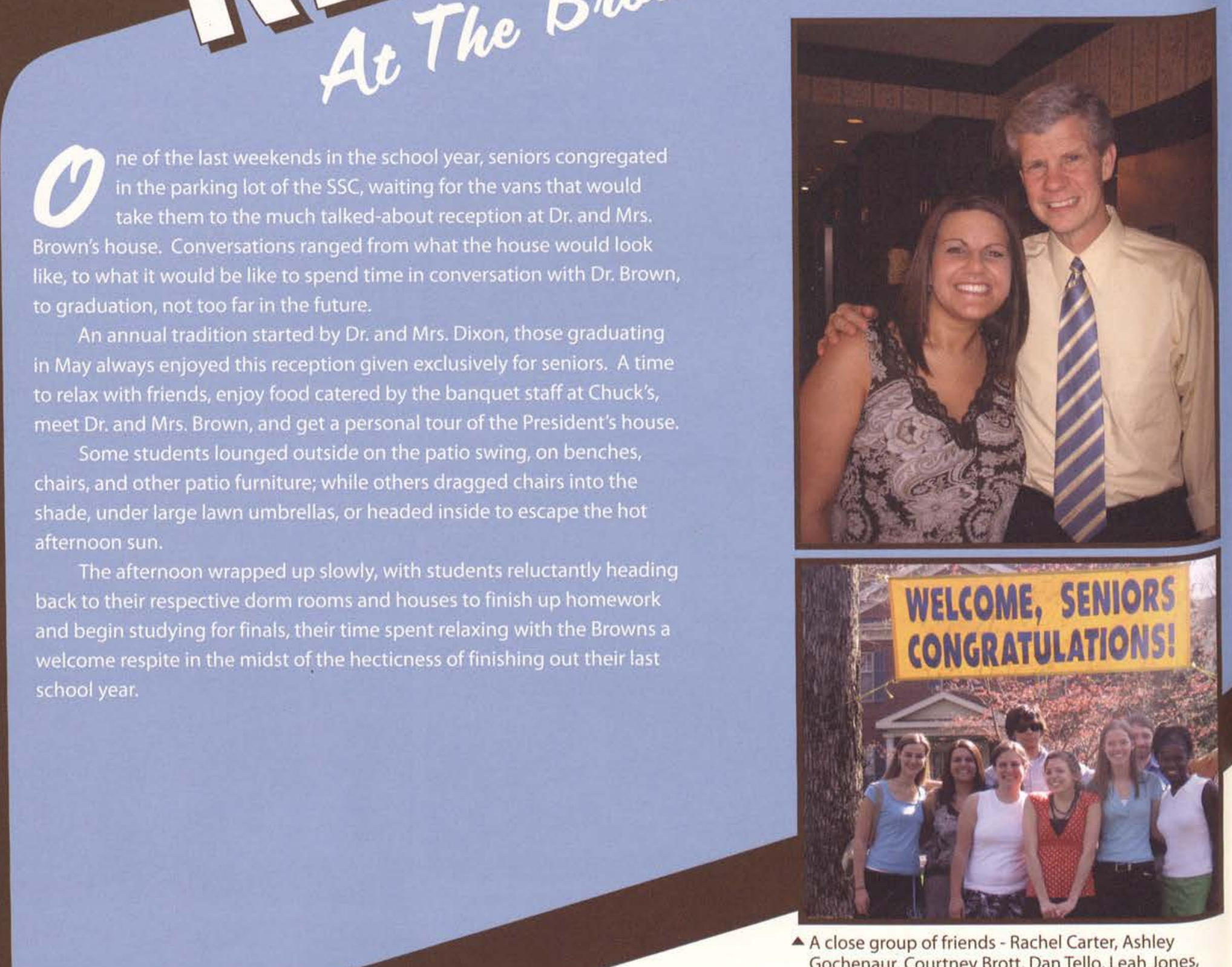

A close group of friends - Rachel Carter, Ashley Gochenaur, Courtney Brott, Dan Tello, Leah Jones, Rebekah Nearhoof, Jim Ellis, Vashti Mensah, Travis Knipple - goof off in front of the Browns' house, waiting for the van to take them back to school.

- Ashley Gochenaur poses for a picture with Dr. Brown. She says, "Dr. Brown and his wife are so gracious to open their home every year for graduating seniors." 

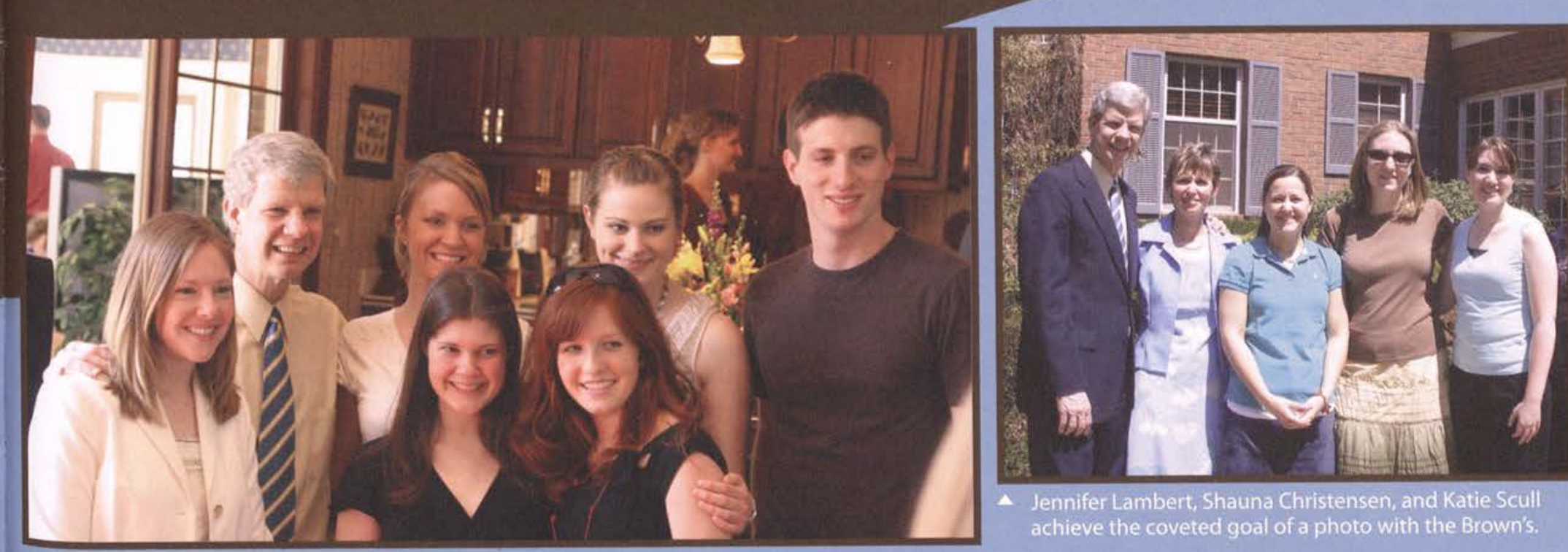

- Jennifer Lambert, Shauna Christensen, and Katie Scull achieve the coveted goal of a photo with the Brown's.
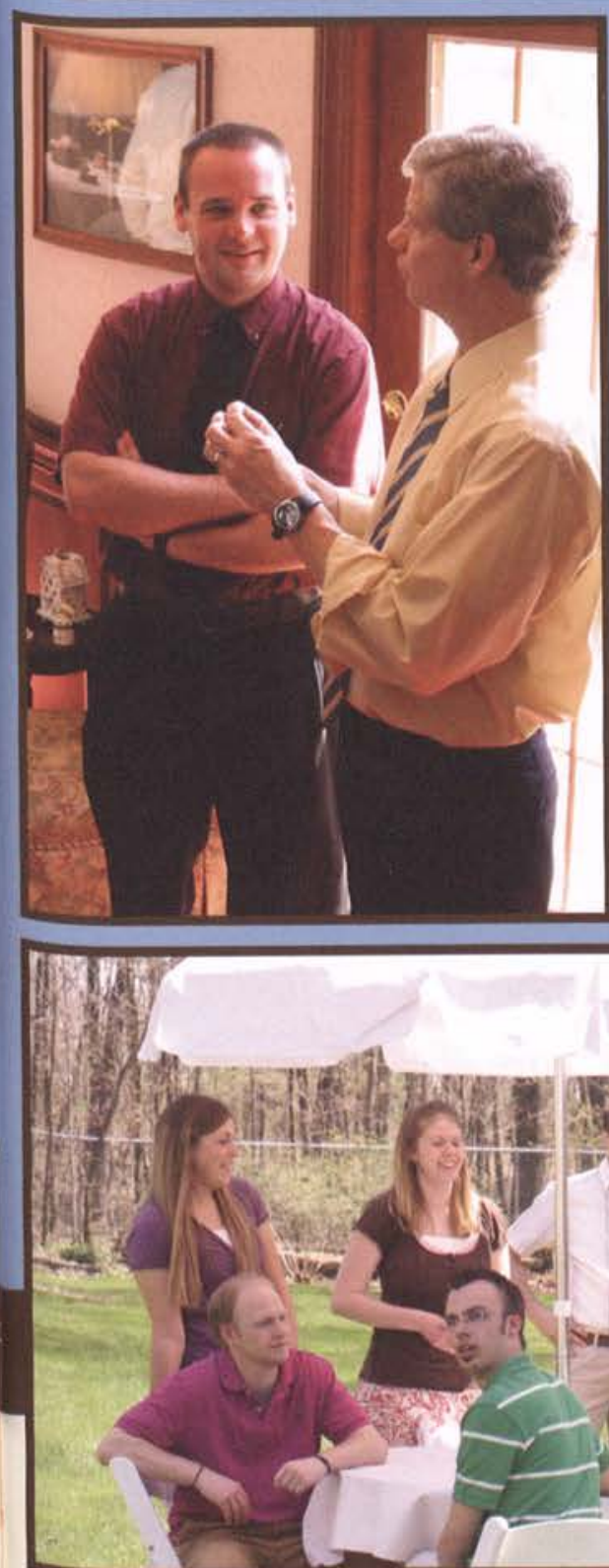

- Jen Fansler, Eric Johnson, Jason Myers, Lisa Blackburn, Ashley Morgan, Steve McCune, Kyle Gunn, Luke Snider, and Beth Winner spend some final moments together before beginning their lives away from Cedarville.

ش Michael Francis gets some good ol' Dr. Brown-style advice.
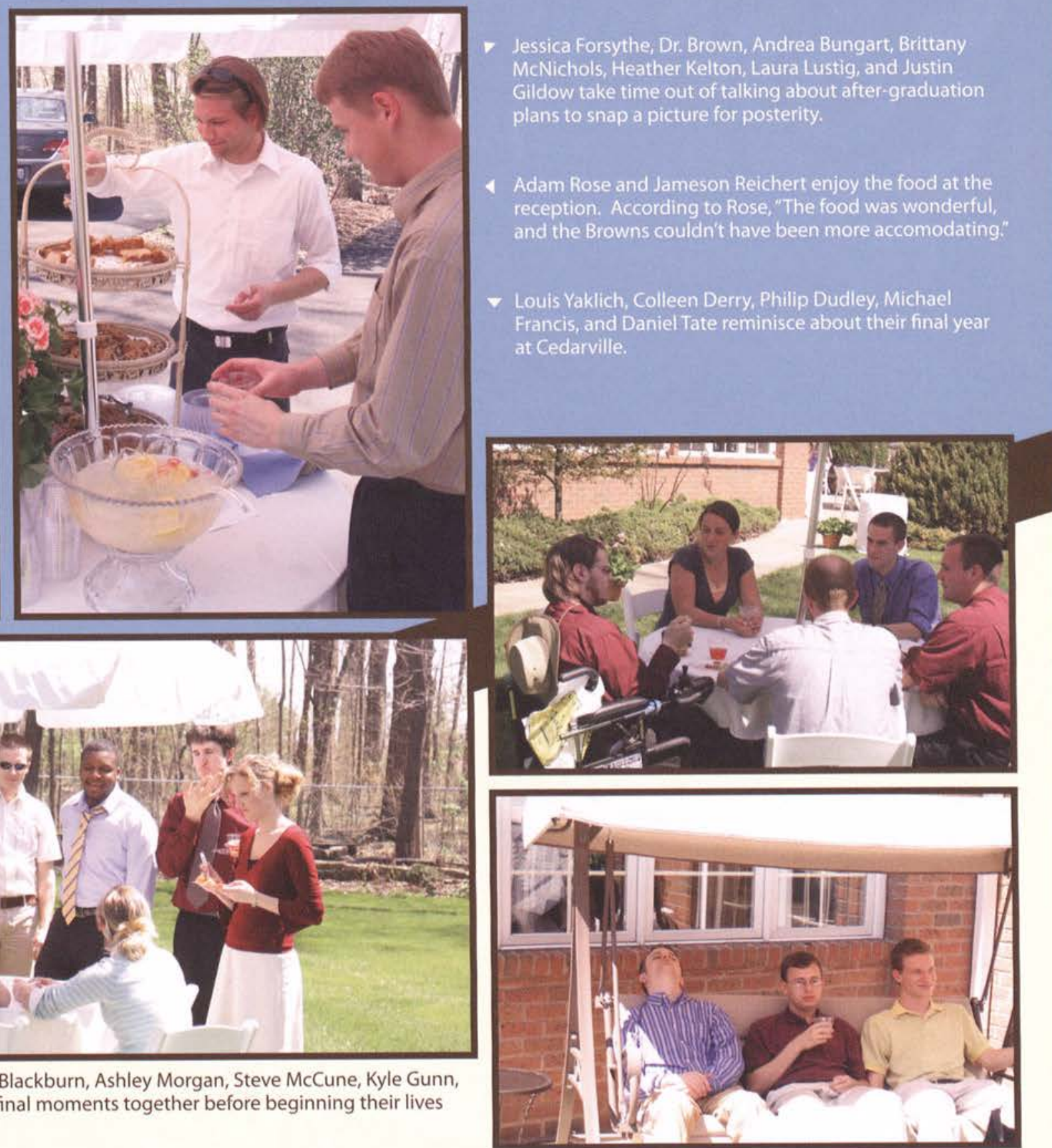

- Jeremy Conyers, Eric Robinette, and David Kindt relax at Dr. Browns' house for their study break from finals week. Conyers says, "We took advantage of it. This was the only break we really got all week." 


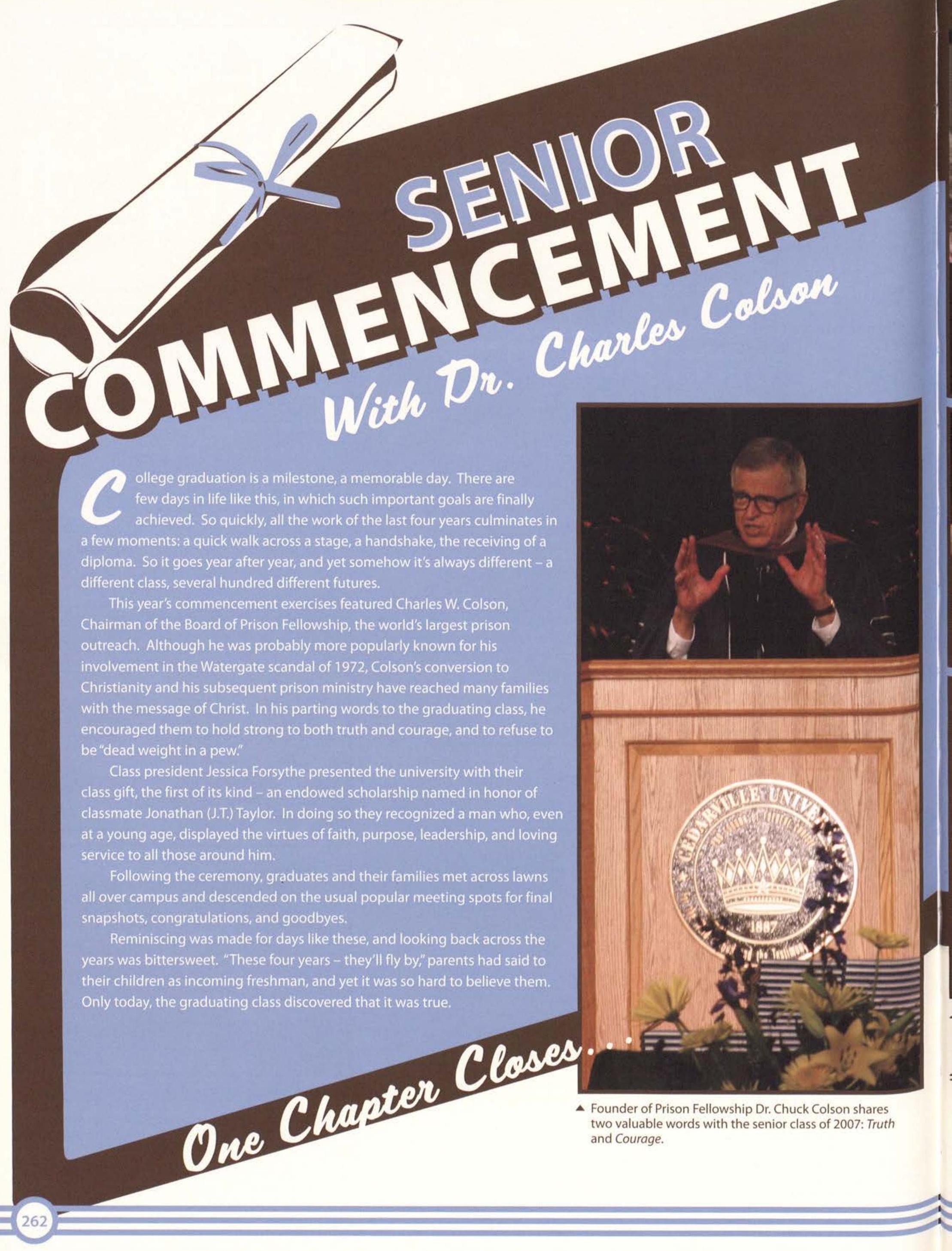


Kelly German said of the day, "Graduation was a special day to reflect on the past four years here at Cedarville. It was filled with excitement and confidence in what the Lord will do in the lives of myself and others in the future,"

Joel Green, pictured here with Ashley Gochenaur, said, "The guys in my hall 4 said that I was 'dating Cedarville.' Obviously it was hard for me to leave, but I have no doubt that I will keep up with lots of my friends. Thank goodness for facebook!"
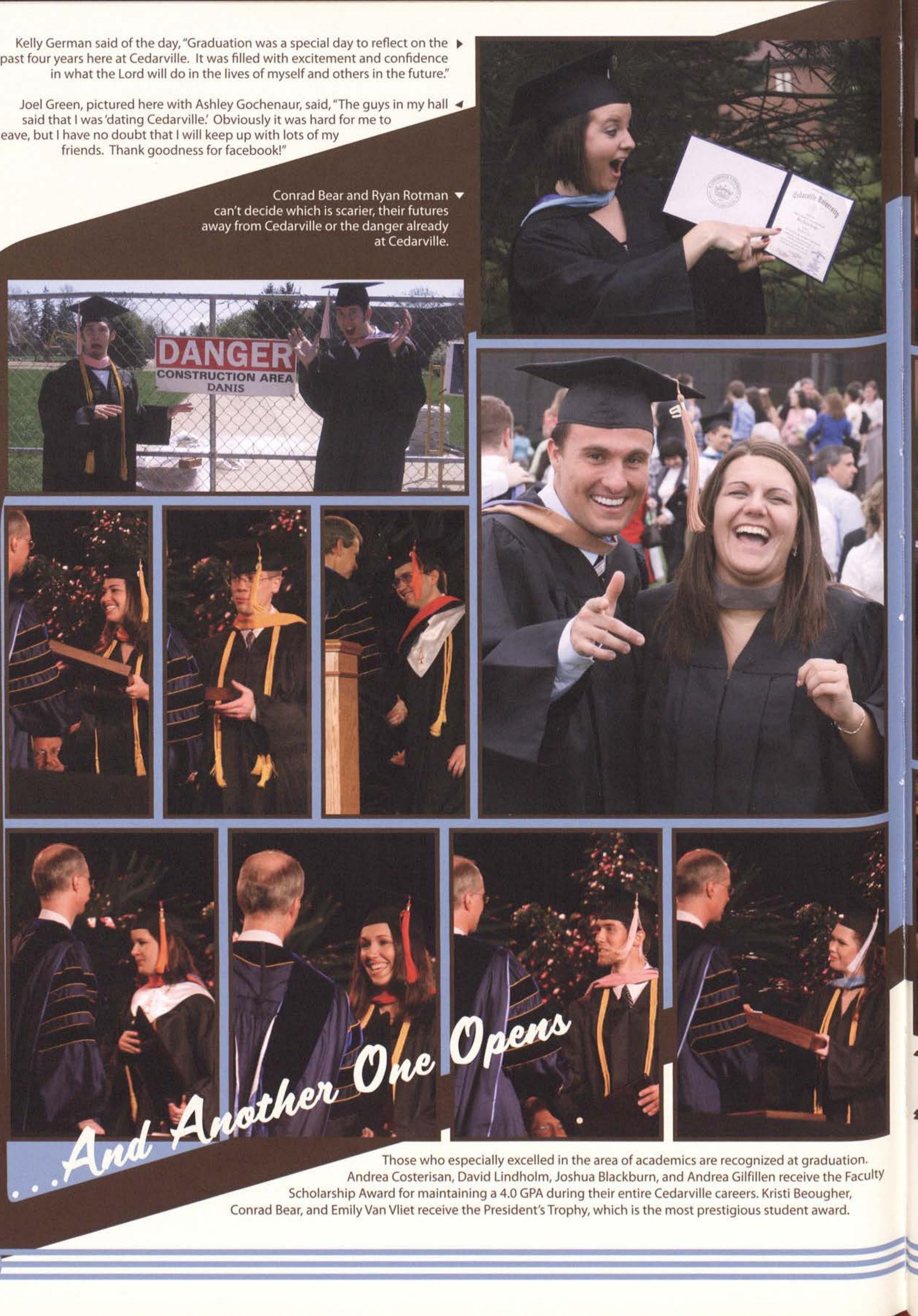


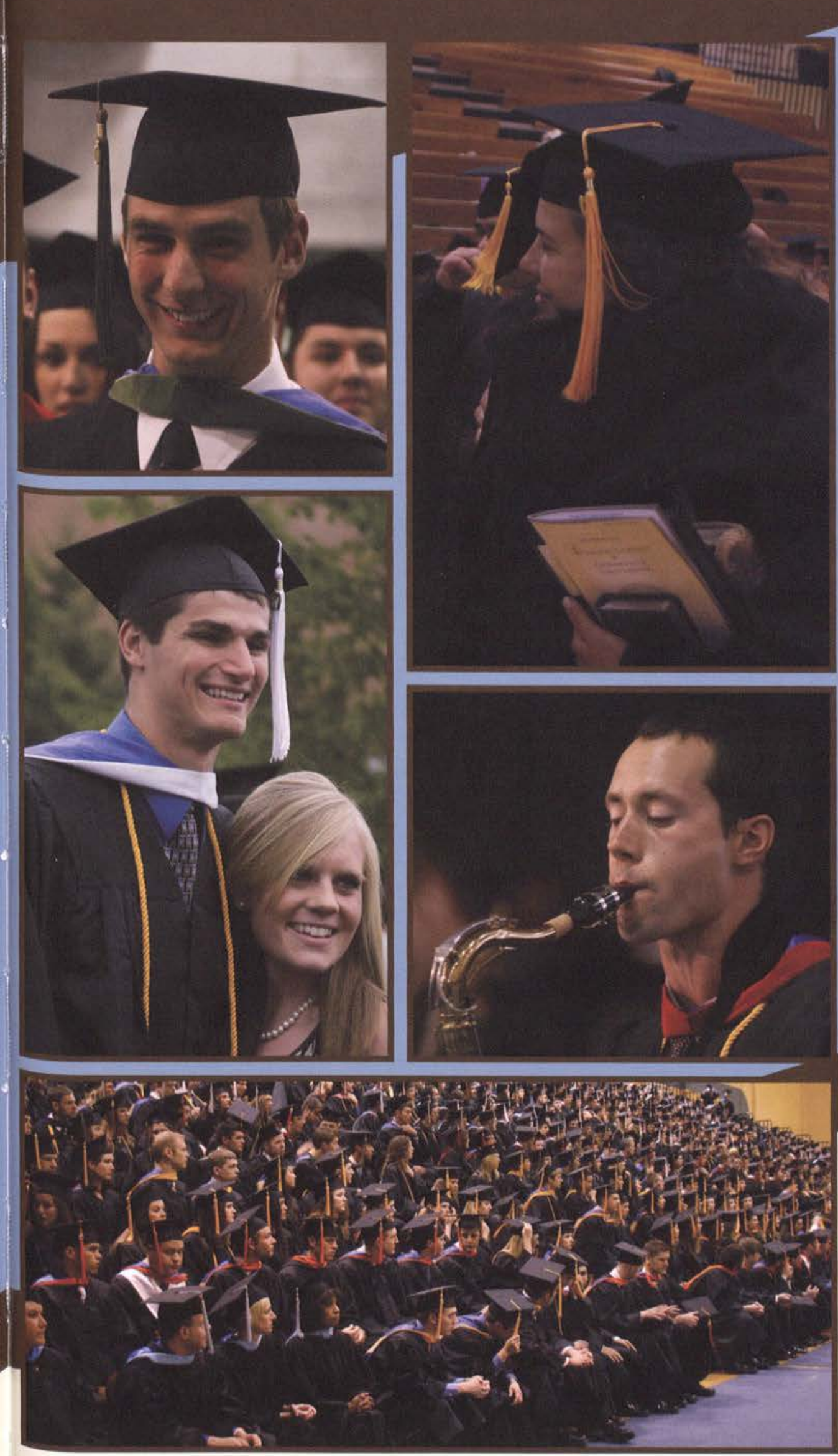

- "Aren't we all so cute! It was such an exciting day. Siting and waiting was really hard, but what was harder was believing that we actually did it. We graduated from college!" stated Daryl Sando.

Matthew Potter and Cathryn Braswell pose for one of the thousands of pictures taken after the ceremony. The excitement was just radiating through the crowds as students wandered attempting to find friends and professors to take pictures with.

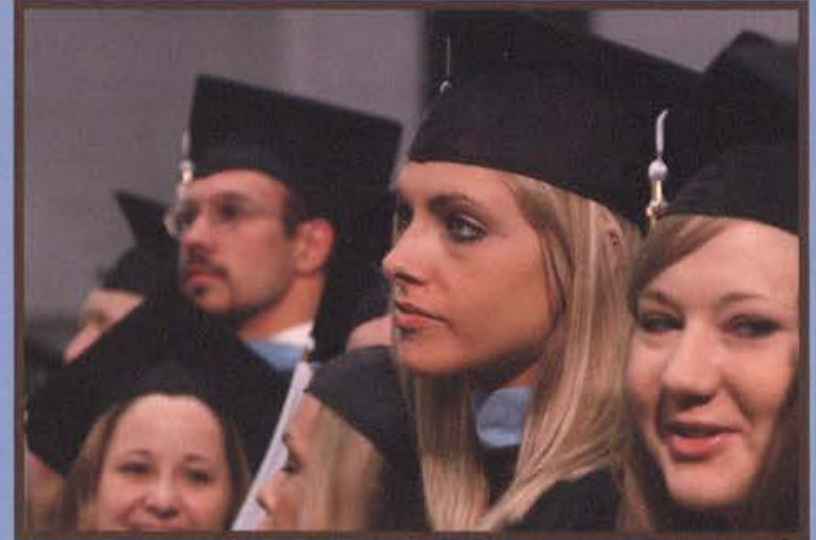

A It is so hard to contain the excitement. Almost graduated CU students stand and wait for their name to be called, signifying the end of a long road encompassing four years of many friends, smiles, and tears.

- Graduates realize the importance of taking a few more moments with friends before their adventures begin again.

7. John Hummitzsch said of the day, "Commencement was the day we all had been waiting for since we stepped on campus as freshmen. When the day finally came, l almost wished it had not come so fast. I realized it was time to say goodbye to some close friends and welcome a whole chapter in my life."

4 Phil Buben plays the his sax as over 7,000 people praised our Lord with the words of the 2007 class song, "It Is Well With My Soul."
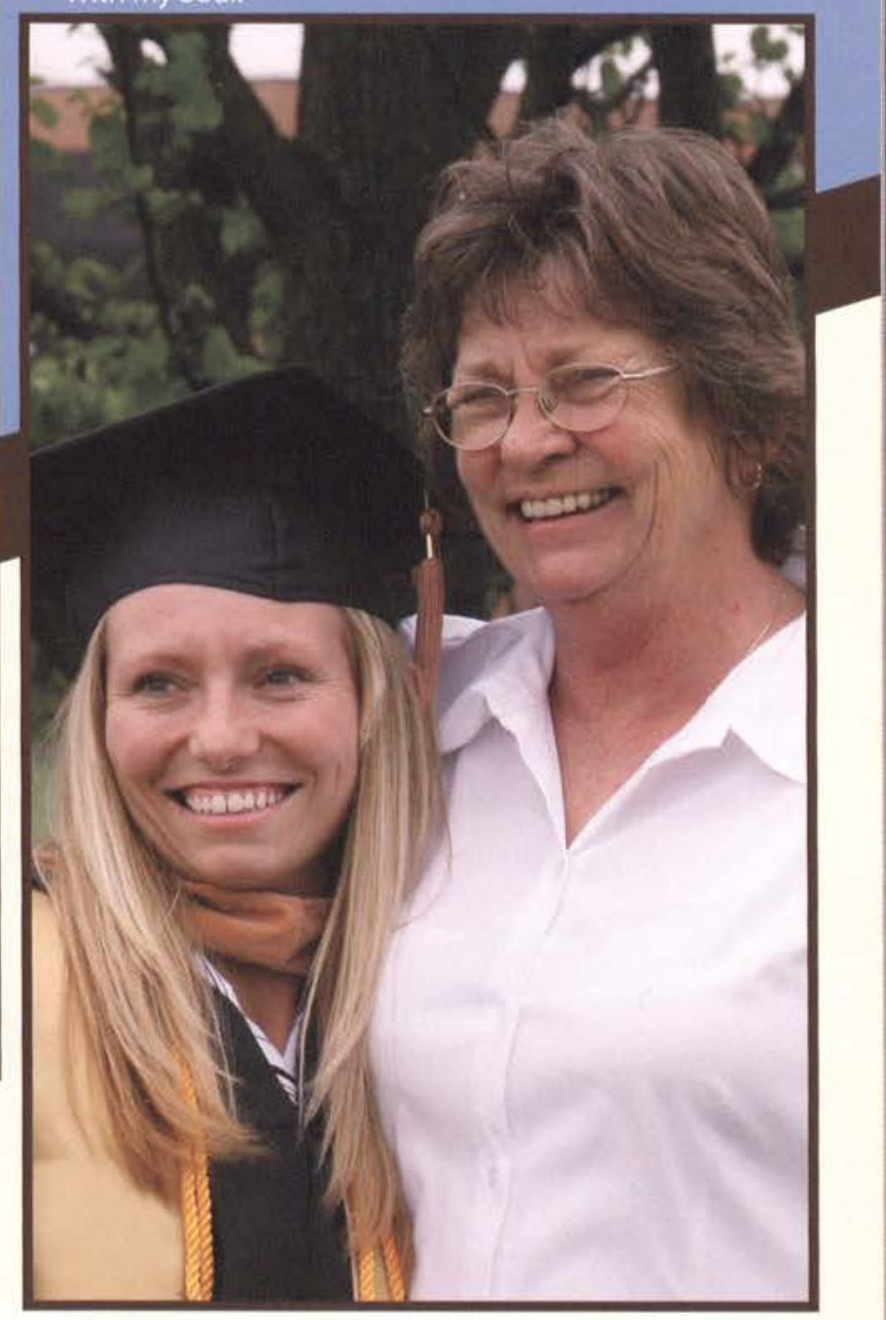

- Stephanie Langley and her mother celebrate her great acheivement of graduating from Cedarville with high honor and a member of Delta Mu Delta. 
Co-worker Mark Mazelin remembers, "Gabe worked diligently at his job and cared deeply for his family. He left behind a legacy of being a hard worker, faithful father and husband, and consistent God-follower."

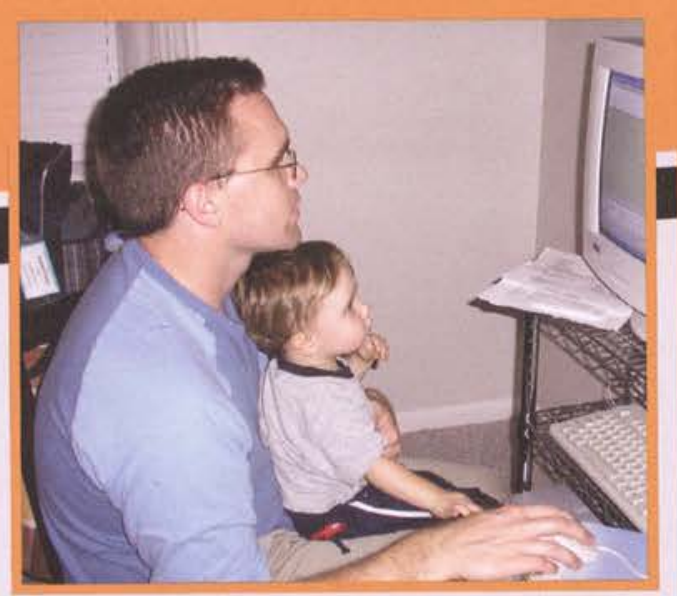

0 ctober 21, 2006, marked the tragic loss of Gabriel David Custer, a beloved husband, father, colleague, and friend. He worked at Cedarville University for eight years in the Computer Services department.

Among other strong, God-honoring attributes, Gabe will be remembered most vividly for his compassion. Many co-workers noted his dedication to all aspects of his life: professional, spiritual, and household. Colleague and friend Scott Howder said, "Gabe was careful and deliberate in the things he did. You could see this in the way he planned his career, in the way he managed his family's finances, and in the way he worked... He was constantly thinking of ways to show his love towards Katy, his wife. One interesting thing he did in this regard was every Wednesday he would spend a good portion of this lunch hour talking to Katy on the phone."

His love for his family was noticed by several other co-workers as well. Associate Vice President for Technology David Rotman hired Custer in 1998 and observed his non-stop dedication to both career and family throughout his time in Computer Services. "I was especially challenged by Gabe's devotion to his wife and two sons. He never lost that "first love" look as he talked about Katy, Sammy, and Seth," said Rotman.

It was clear that one did not have to be well acquainted with Gabe to see his kind heart and diligent work ethic. Circulation Services Manager Becky Hayes was impressed with his professional and interpersonal skill." He was one of those people who really took time to listen to what the problem was and didn't make you feel silly or stupid if it was a simple issue. He was friendly to everyone and always had a smile on his face."

Senior Analyst Ryan Bowen worked closely with Gabe and also realized his intense love for his family. "He loved them so much. He was always sharing what his kids were doing and was really proud of them. He even took one of Sammy's finger paintings, framed it, and displayed it in his office for everyone to see." Gabe Custer left an impressive legacy during his time here at Cedarville, a legacy that inspires not only diligence and kind professionalism, but also a deeply profound love for God and family.
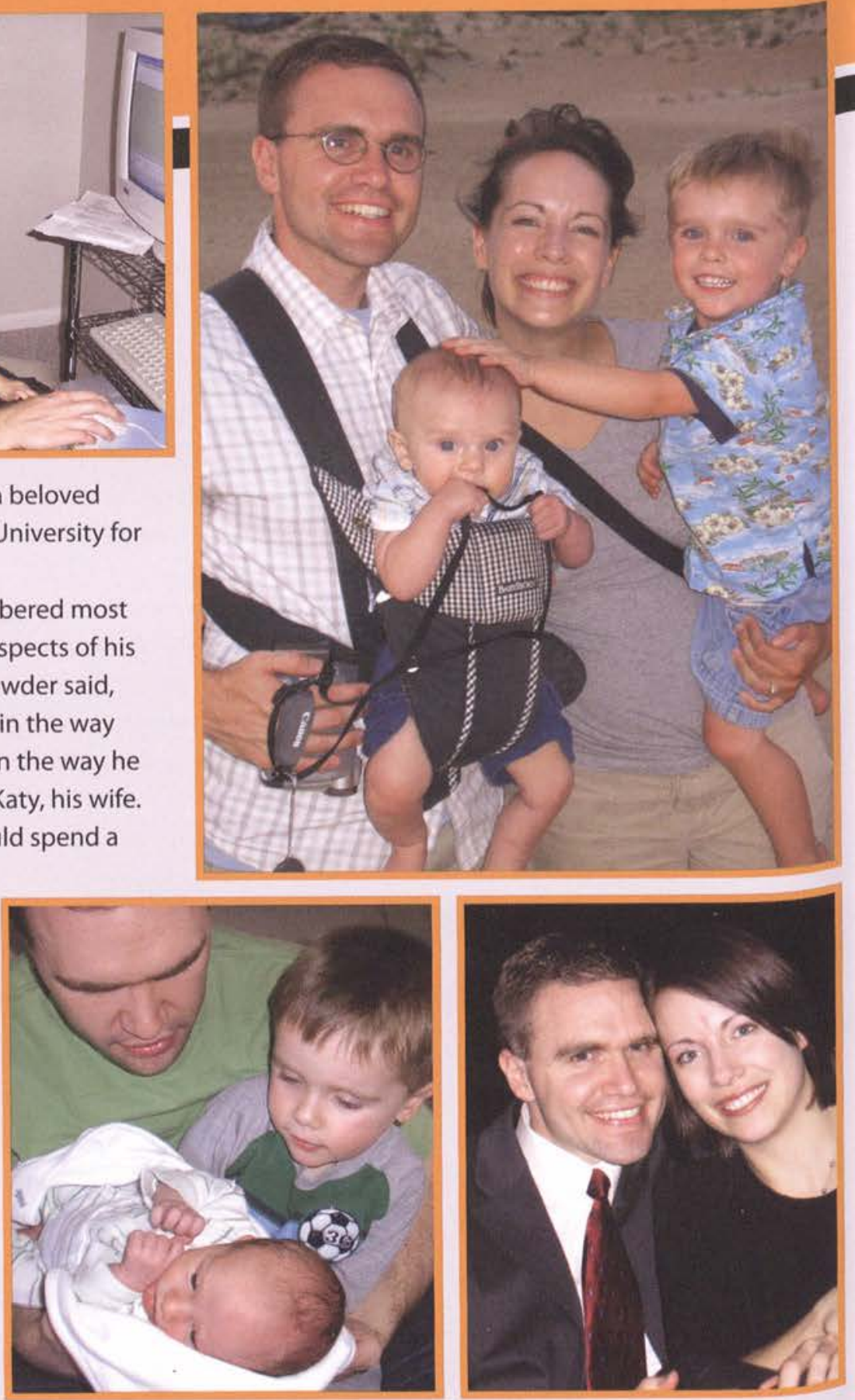

- Gabe hugs his wife Katy. Friend and colleague Scott Howder says "Gabe wanted to please God in everything he did. This desire spilled over into his love for his family, his personal life, and his work."

- Gabe shows his son Samuel how to hold his new baby brother Seth.

A Gabe, Katy, and their two beautiful boys, Seth and Sammy, take a walk in the great outdoors together.

\section{In Loving Mamory OB}




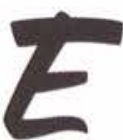

ach year that l've spent at Cedarville has been amazing for different reasons. There is one common thread in all of them though: God Almighty and the amazing work He constantly does in the lives of the Cedarville family. Thank you to every Cedarville faculty \& staff member, student, chapel speaker, and campus guest that I've come in contact with over these last four years. God seriously used each of you to mold me into a very different person than I was as an incoming freshman. I can't wait to see how He continues to use each of you in the future. Our God is good. Praise His Name that He allows us to experience that goodness!

To the Cedarville Family: I hope you enjoy this book. Let it remind of you of the amazingly joyful memories, the difficult times, the wonderful friendships, and the lessons God taught you this year. God bless!

\section{To the Yearbook Advisors:}

Cyndi: Thanks for putting up with our chaotic Wednesday morning meetings and for providing us with solid advice concerning the yearbook. Thank you also for sharing your beautiful family and house with us. Those are among my favorite yearbook memories.

Mr. Chamberlain: Thank you for your excellent guidance on the layout designs. That was a huge help. In addition, thanks for being a quality teacher and role model in my four years of art classes. I respect you more than I can say.

To the Yearbook Editors: I've enjoyed getting to know all of you better. You were a perfect staff, and I'm going to miss you. I pray that God blesses your futures and continually draws you closer to Himself in your new careers, families, and homes. Congratulations! We made it successfully through college!

Amy: Thank you so much for keeping track of Cedarville's endless amount of events and getting photographers to each of them. You did a great job hiring the right people and keeping the photos organized.

Daryl: I honestly cannot thank you enough for all the details that you took care of without the rest of us even knowing. I appreciate your willingness to organize and always pick up the loose ends. The yearbook would have fallen to pieces without you. ....And we would have run out of Goldfish.

Kara: I don't know what we would have done without you here in the fall to get us started. Thanks for answering my endless questions, helping with our initial layouts, and keeping me entertained in the office. You know I respect and love you like crazy. Hey, Time to Dance...

Mary: I have a deeper appreciation for the copy aspect of yearbook after watching you work this year. Thank you for keeping that entire part of the yearbook organized. I never once had to worry about whether a story or a page of captions would be done well and on time. In addition to your copy skills, l've been blown away by your servant-like attitude and amazing patience. Thank you for everything!

Sarah: You and I both know that your position carried an incredible amount of work. Thank you for spending the extra time it has taken to make the book a great one. Thanks for putting up with my massive, rambling emails and for taking my pickiness in stride. You did an amazing job, and I crown you the resident queen of cheesy taglines! In your future career, don't forget our biggest lesson: Delegation is key! And maybe someday one of us will be able to incorporate a dustcover poster or scratch'n'sniff into one of our graphic design projects...

To the Yearbook Staff: I didn't get a chance to interact with all of you this year, but for those of you that I did get to know, you were wonderful to work with. Thank you for taking care of your individual contribution to this project. You did amazing work! Thanks for being willing to cover assignments at the drop of a hat or even during stressful times, like finals week. I appreciate each of you!

To the Future Editors: I'm SO glad you all were willing to take these positions because I can already tell you're going to do a wonderful job. I can't wait to see what you come up with for next year's book. Yearbook is way more work than you might think, but I'm more than confident that it's going to be one of the best yearbooks yet. Have fun with it!

To the Printy 18 \& 20 girls, my 3 roommates, the Sophomore Year "Family", \& the Grey House girls: I love you. I'm going to miss you all so much. I can't even express the amount of respect and appreciation I have for you. Thanks for the prayers, conviction, encouragement, laughs, home videos, bike rides, de-stressing dances, long chats, and tons of other amazing memories. Keep discovering our King, and keep me informed on what He does in your lives!

To the Jones clan: Who am I kidding? There's nothing I could say in a couple sentences that would convey the love, appreciation, respect, and admiration that I have for my four best friends. Thank you for pointing me to Jesus Christ since the day I was born. I'm so glad that the end of my college career doesn't mean that I'm saying goodbye to you too! Hey, do you have...glashes on?

\section{Thanks! Leak Tones}



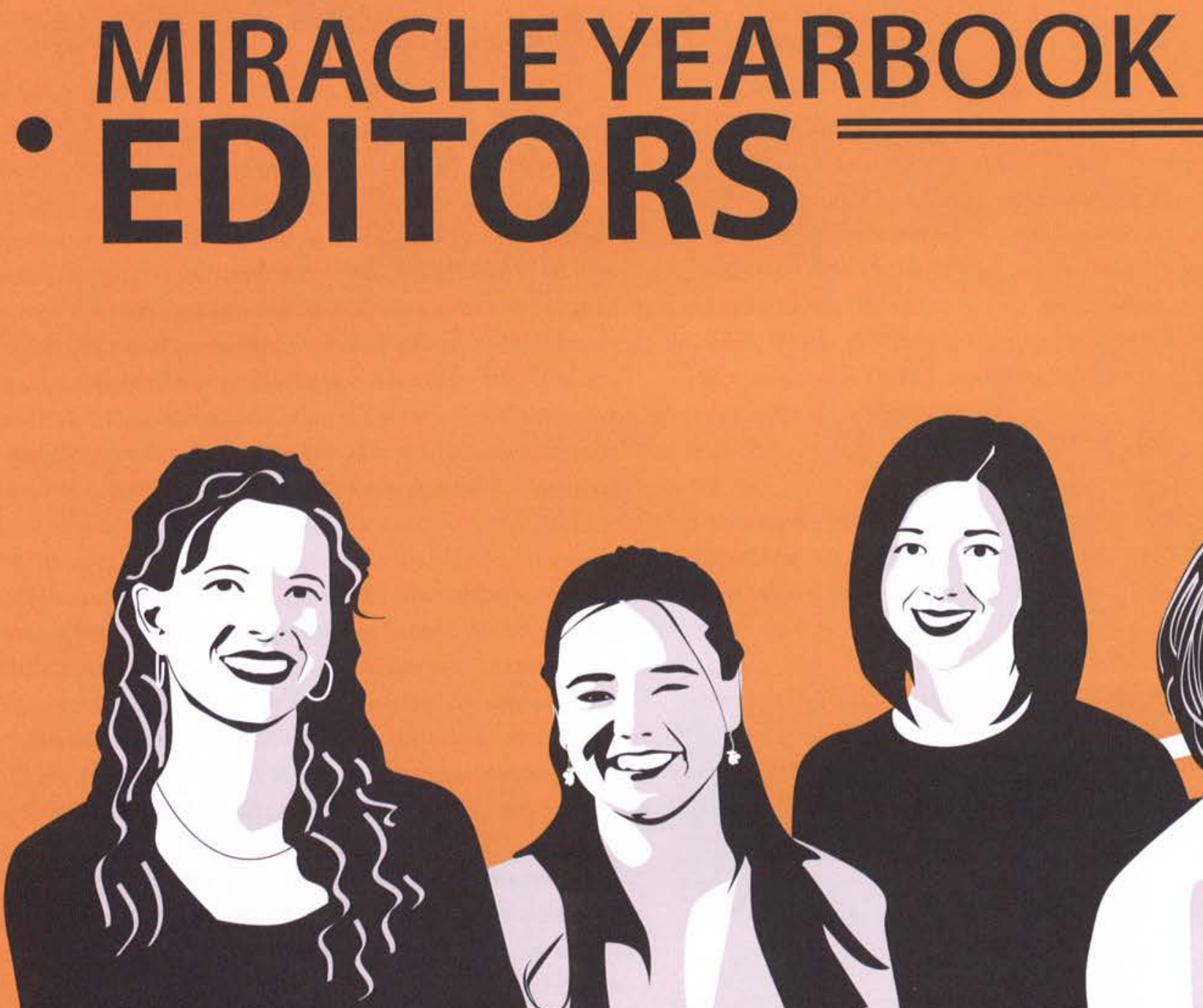

\section{Leak Jones}

Editor-in-Chief

Senior Graphic Design Major
Mary Thenguale Copy Editor

Senior Communications Major
Kara Merrich Creative Design Editor

Senior Graphic Design Major
Darge Sando Administrative Assistant Senior Global Economics \& International Business Major 


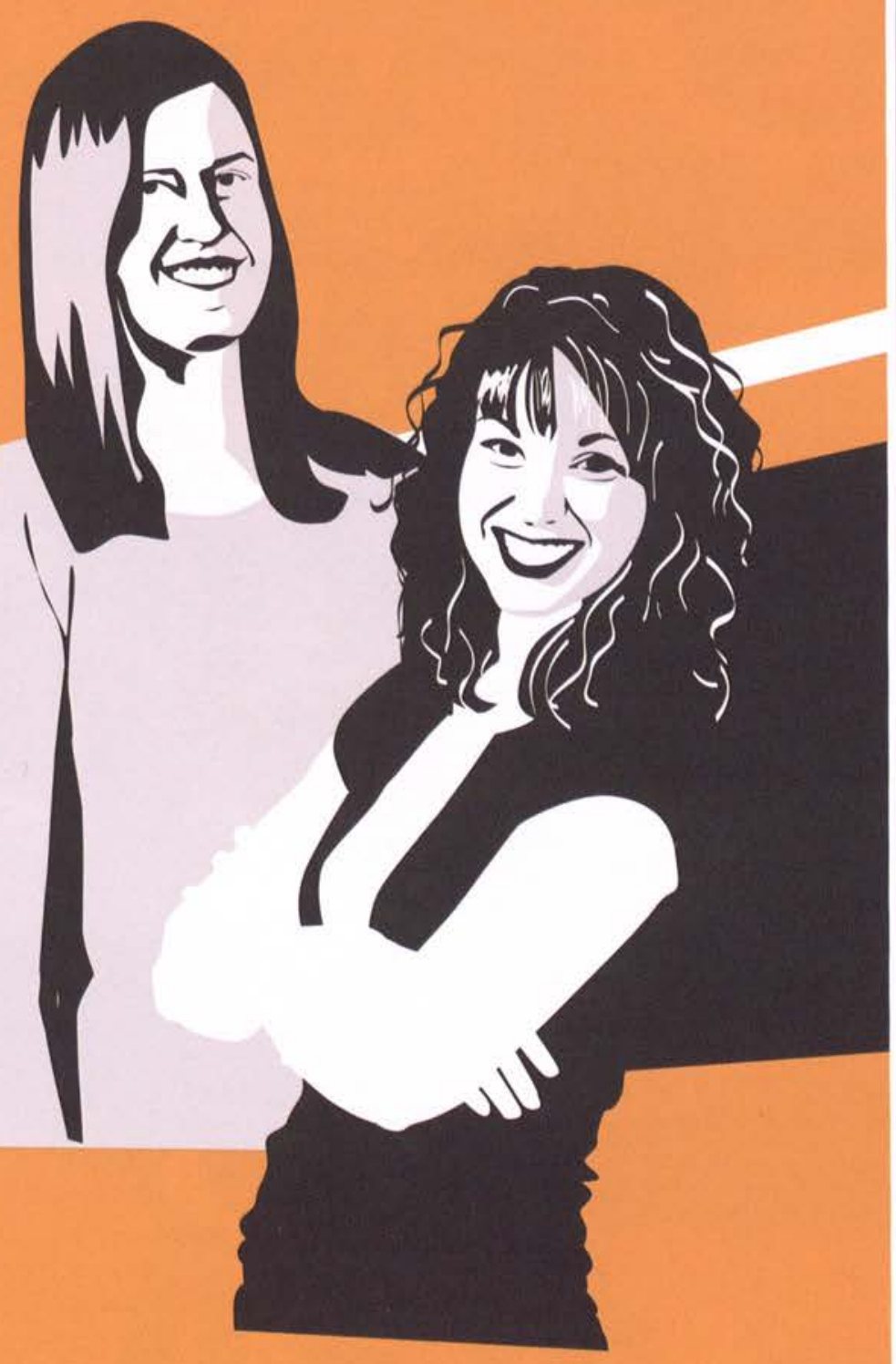

Aney Masterson Sarala Baczed Photography Editor Layout Editor Senior Graphic Design Major $\quad$ Senior Graphic Design Major

They're Tops!

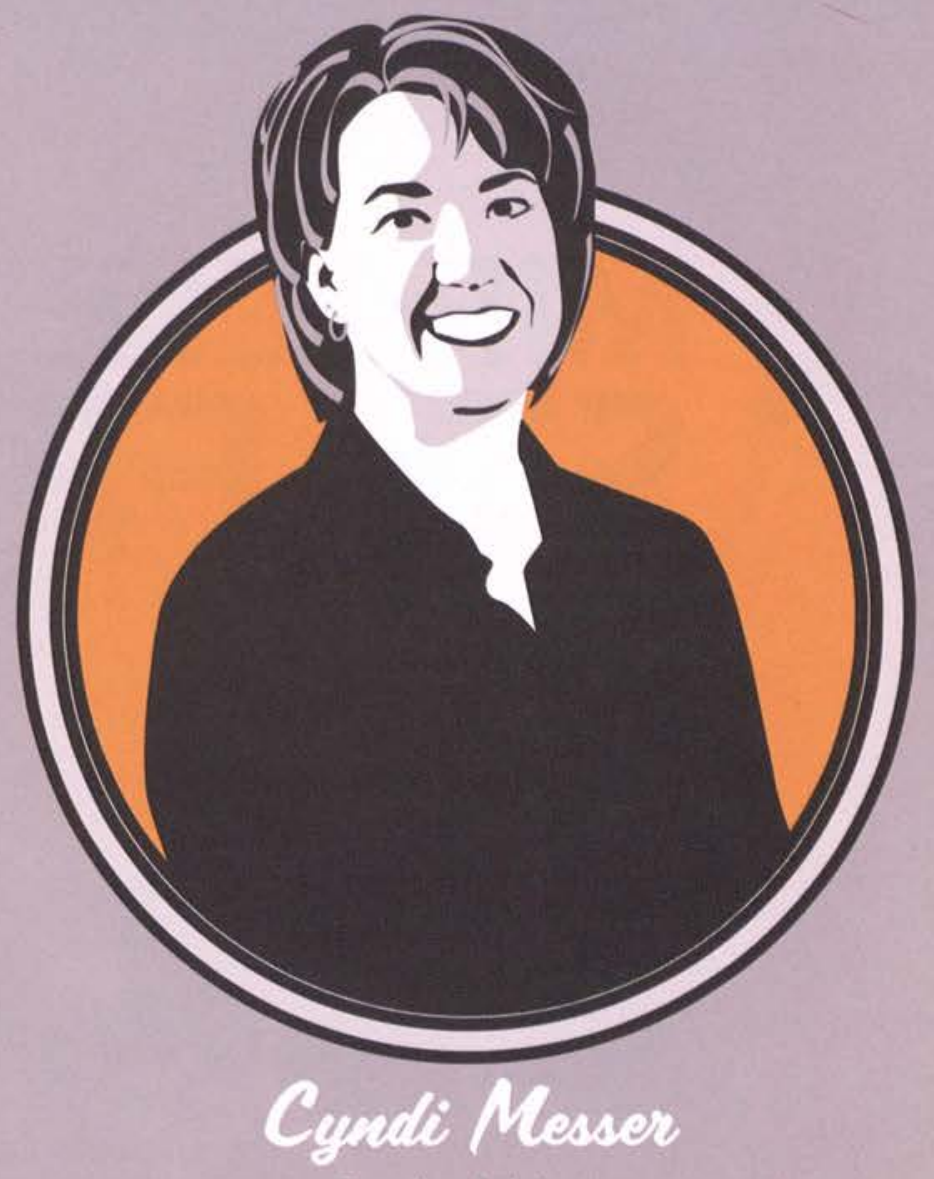

Faculty Advisor

Assistant Professor of English

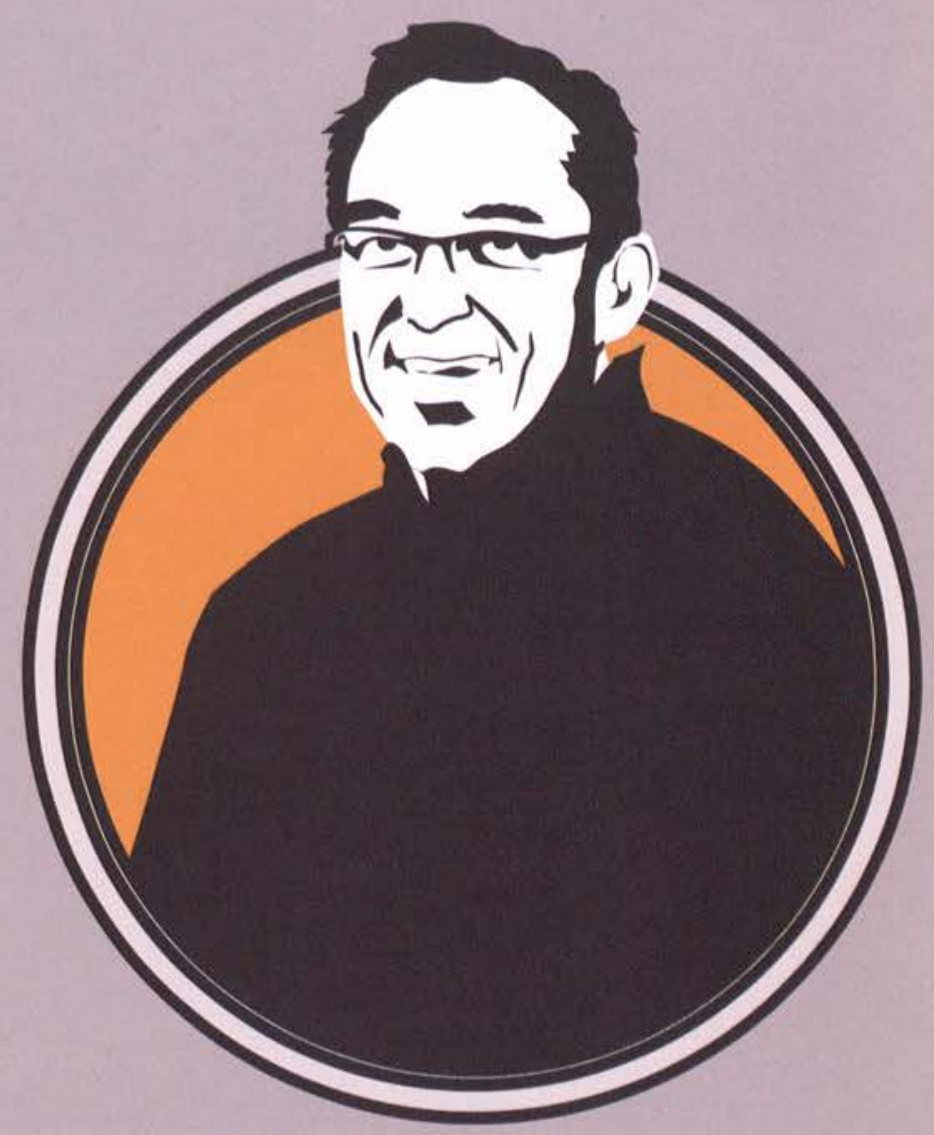

Terry, Chamberdaris

Faculty Advisor

Associate Professor of Art 


\section{MIRACLE YEARBOOK}

- STAFF

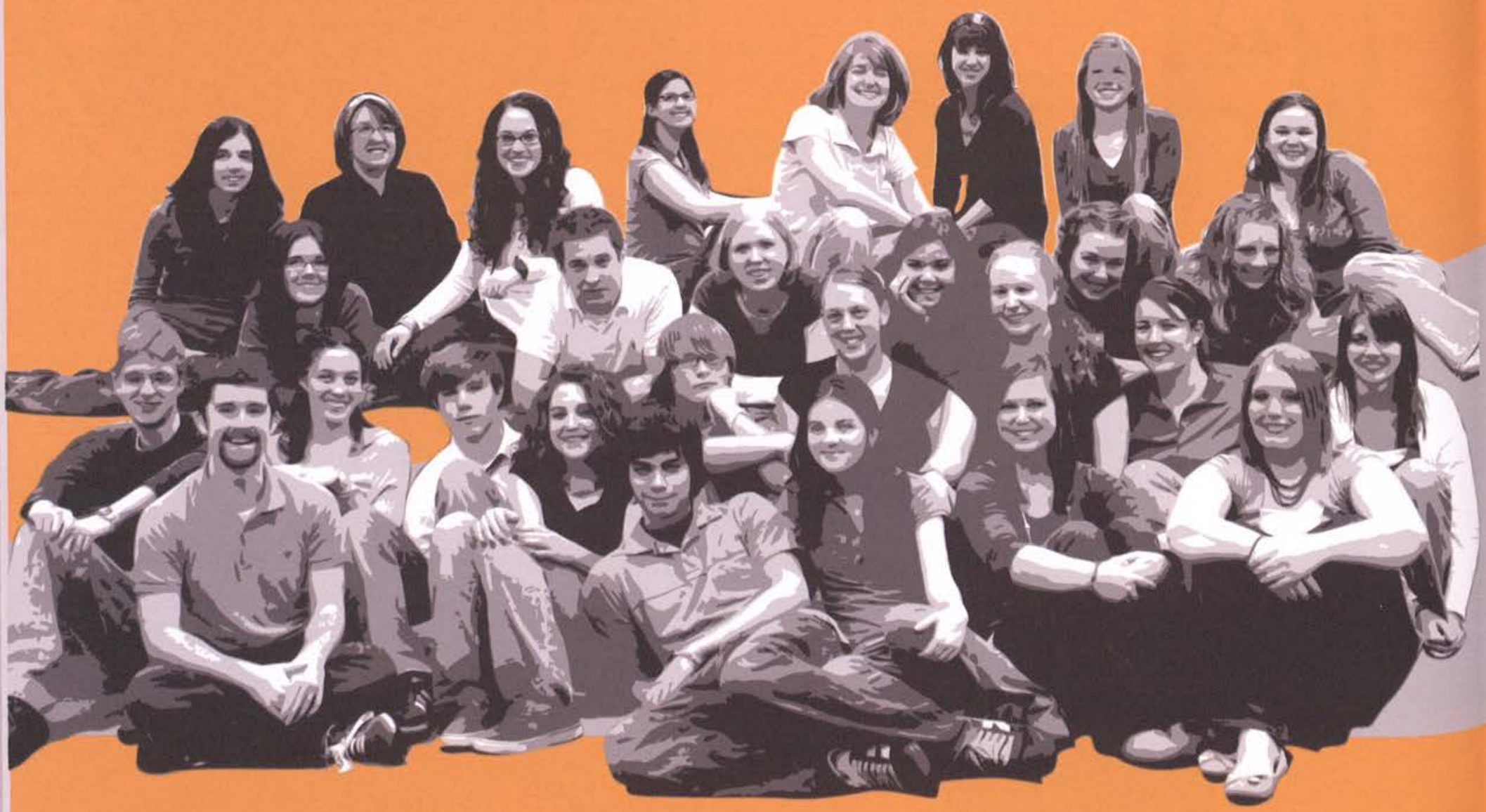

Thanks for all the hard work you guys put in this year. You made my life easier by having stories in on time and fabulously written... hardly needing any editing at all! Thanks for the fun times we had and the fantastic yearbook you helped create: - Mary Thenguadl (Copy Edbor $)$

To my wonderful layout staff, you have all been amazing throughout this whole year! Thank you so much for putting up with my scattered brain and sticking with each and every little thing that I threw your way. Without you I think I would have gone crazy trying to do everything by myself, and for that I have to give all the credit over to you for this amazing book! You're my favorites! Sarah Bacgeb (Layout Edrior)

To my photographers, Thanks so much for all the time and work you put in this year. You all did an amazing job. This yearbook wouldn't look half as good with out your hard work and amazing picture-taking skills:-Any Mastersone (Photography Edteor)

\section{A Swell Stabs!}



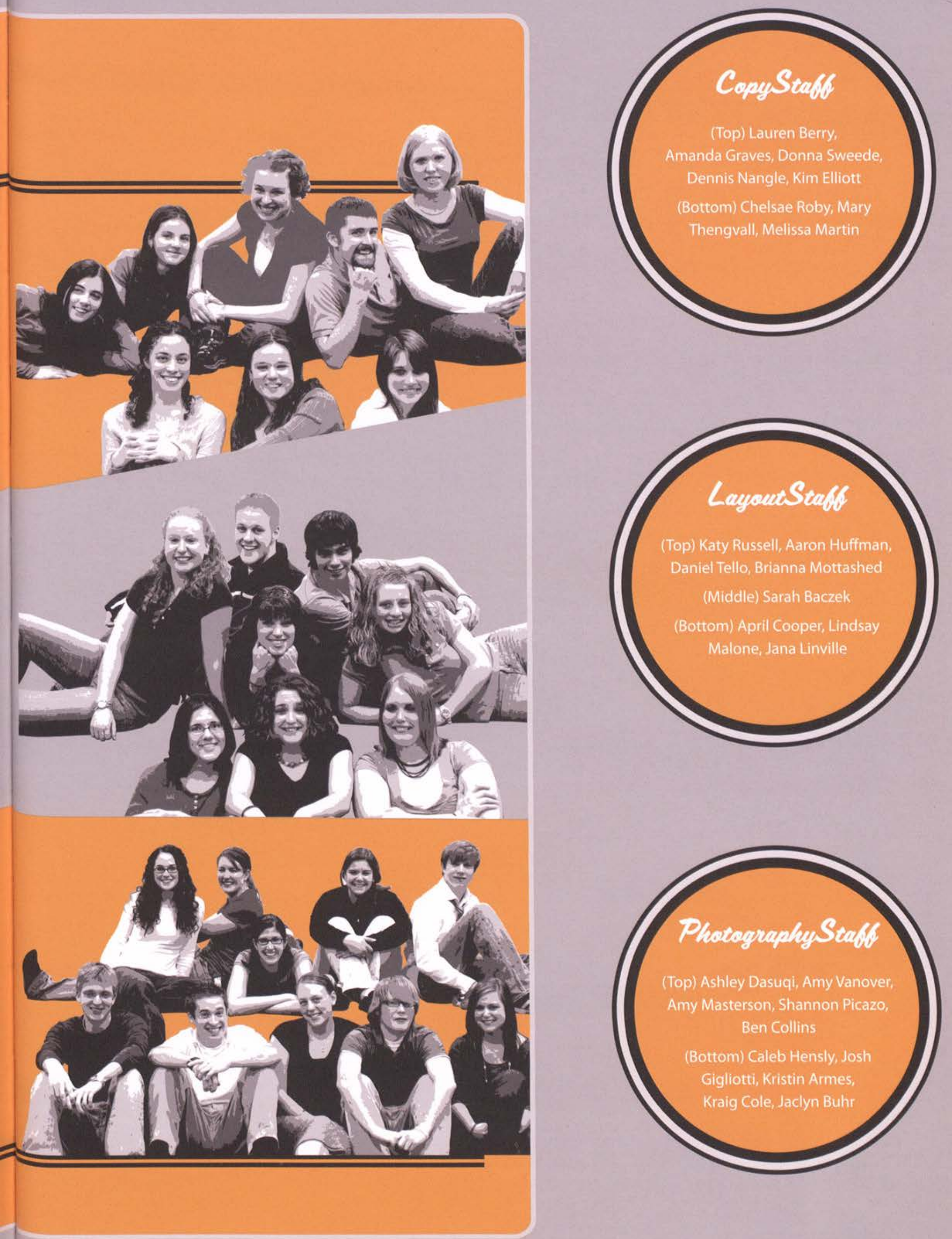


\section{Miracle Yearbecks}

\section{Publisher}

The 2007 Miracle was published by Jostens, Inc. It was printed at their plant located in State College, PA. The Jostens representative was Tille Billheimer, and plant coordinator was Crystal Gault.

\section{Cover}

The cover was a custom screened using a design by Leah Jones. It was rounded, sewn, and bound.

\section{Paper Stock}

The 2007 Miracle's 288 pages were printed on Matte 80\# paper.

Color

The 2007 Miracle was printed with four color process ink.

\section{Design}

The cover, opening, dedication, dividers, section layouts, and closing were designed by Sarah Baczek and Leah Jones with help from the layout staff.

Theme

The 2005 Miracle theme was selected by the editorial staff at the beginning of the school year. It was our hope to show the faces and to tell the stories that represent all of us here at Cedarville this year.

\section{Production}

The 2007 Miracle was produced on both IBM and Macintosh computers. Layouts were produced using Adobe InDesign CS. Photos were cropped, resized, and touched up in Adobe Photoshop CS \& CS2. Illustrations were created using Adobe Illustrator CS. Copy was typed into Microsoft Word and then imported into InDesign.

Typography

There were two main fonts used in the printing of this book: Myriad Pro and Scriptorama Tradeshow. The stories are 10 point Myriad Pro Regular. The Captions and Class Picture Names are 9 point Myriad Pro Regular. Titles are various sizes of Myraid Pro, Semibold and Bold weight. Page numbers are 11 point Myriad Pro Semibold. The taglines are various sizes of Scriptorama Tradeshow.

\section{Photography}

The pictures in the 2007 Miracle were mainly taken by Amy Masterson and her photography staff. We also accepted student submissions for candids. Our Da Vor representative for portraits was Jim Rainey. Some of the sports pictures, team pictures, and other candids were provided by Scott Huck, Photographer for Cedarville University, Public Relations.

Inquiries

The Miracle Yearbook office may be contacted for additional information about this book's production at:

Miracle, Cedarville University

251 North Main Street

Cedarville, $\mathrm{OH} 45314$

(937) 766-4995

miracle@cedarville.edu

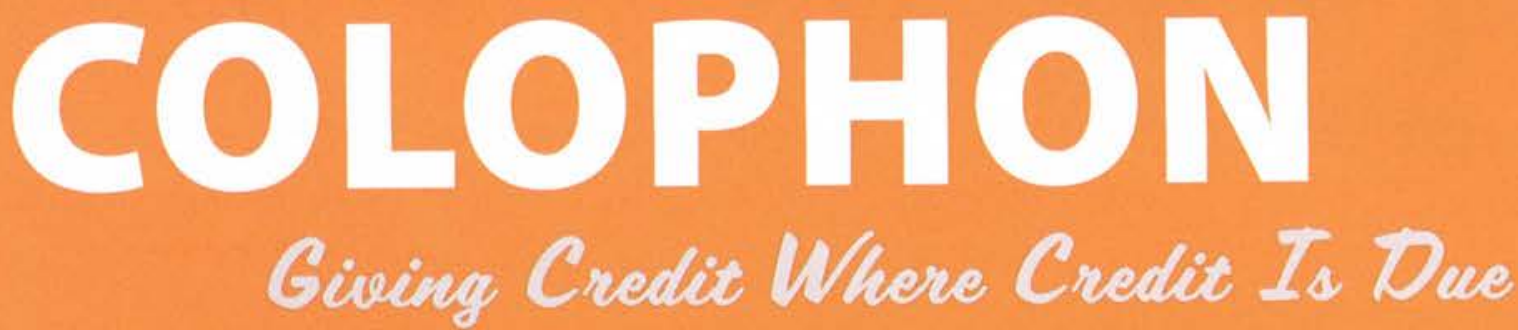




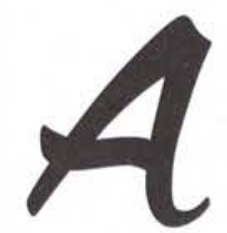

Abbey, Rebekah 114 Abel, Timothy 62 Abernathy, Joshua 114 Abraham, Mary 132 Abraham, Matthew 100

Acker, Amanda $62 \quad 3$

Adams, Alyssa 100, Anderson, Haddon 186

216

Adams, Christin 62

Adams, Rebekah 114

Adams, Theodore 62

Adkins, Kristin 132

191

Ager, Merlin $\quad 45$

Agler, Jonathan 100

Ahern, Ellen 62

Ahlgrim, Kim 48

Aho, Michael 132

Aker, Scott

192, 193

Albarano, Jenna $\quad 244$

245

Albert, Dannielle 114

Alburger, Brian 151

214,251

Alburger, Brian

214,251

Alexander, Anne

Alexander, Emily

Alexander, Stacy

234

Alfieri, Jennifer 132

Allard, Tyler

Allen, Brietta

222, 250

Allen, Kevin

Allgrim, Andy 187

Alliman, Ricardo 132,

147,187

Allison, Sarah

179

Althouse, Andrew 62

Alyn, Irene

40 ,

58

Amarante, Holly 114,

250

Amerine, Bryan 62 ,

$159,232,234,251$

\section{Andersen, Eric 114, Awabdy, Judy \\ 194 \\ Andersen, Sarah Brittny 156 \\ Andersen, Sarah Elaine 114, 201 \\ Anderson, Benjamin

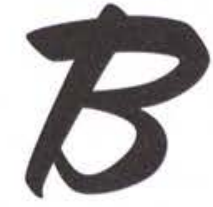

52

114,221

Anderson, Bradley 100, 223

Anderson, Brittany 62 Anderson, Connie 15,

Anderson, Harry 44

Anderson, Jamie 62

Anderson, Lyle $\quad 37$

Anderson, Melissa 100, 114

Anderson, Robert 114

Anderson, Tyler 62 ,

219

Andrews, Fran $\quad 50$

Anfang, Laura 132

Anfang, Michael 62

Angelini, Tammy 62

Anglea, John 30 ,

31

Annable, Jessica $\quad 62$,

157

Annanie, Christopher 100

Antioho, Jeffrey 132

150

Arch, Nicholas $\quad 114$

233, 244, 245

Armes, Kristin

132 ,

271

Armstrong, Kandis 204,

205

Armstrong, Kathryn

258

Arndt, Joshua 100

Arnold, Daniel 62

Arrieta, Rachel 132

Arthur, Rachel 201

Atkeson, Daniel 132

193

Aultman, Hana 128 .

217

Austin, Jon $\quad 44$

Austin, Wendy 100

Averback, Matthew

62

Averbeck, Nathan 100

Barton, Tyler 63
Babbitt, Allison 132

Babbitt, Amy $\quad 100$

Babcock, Devin 132

Bacon, Angela 62

Baczek, Sarah 27,

$216,267,269,270,271$,

272

Baczek, Stanley 40 ,

58

Bailey, Benjamin 132

Bailey, Tonya $\quad 53$

Baker, Elizabeth 132

Baker, J.Wesley 34

Baker, Lois

Baker, Marisa $\quad 114$

245

Baker, Rebecca $\quad 34$

Baker, Sue $\quad 45$

Baker-Karl, Michelle 114, 173

Balch, Maria

100

185

Baldwin, Chase $\quad 25$,

132, 160, 223

Baldwin, Crystal 215,

218, 219

Ballard, Daniel 100, Bell, Megan

199

Banks, Jonathan 100

Banz, Jared 132

Baranowski, Brittany 114

Baranski, Jacob 114

Barber, Andrew 100

Bardakjy, John 150

Barham, Nathanael 114

Barkas, Elizabeth 132, 237

Barnard, Rachel 114

Barnes, Brianne 132

178, 179

Barron, Esther 114

Bartlett, Betty 114

Barton, Caleb $\quad 114$

Basford, Diana 132
Basford, Julie $\quad 100$,

228

Basham, Daniel 63

Bashore, Jessica 156

Bassett, Kayla 63

Bastress, Dave $\quad 34$

Batista, Ivan $\quad 177$

Bauer, Ashley 63

Bauernschmitt, Janet

232

Bauman, Eddie 45

Baumann, Donald 42

Baumer, Leanna 132

Bayley, Brendon 63,

159

Beal, Danielle 63

Beals, Brittany 246

247

Beals, Christian 187

Bear, Conrad

264

Bear, Conrad 264

Beatty, Melanie

Becker, Ron

Becknell, Mark 114

Becknell, Milt 46

Beesley, Amanda 114

Behlert, Joy $\quad 114$

Behn, Sarah

155

Bell, Eric 132

Bell, Katie

233

213

Bell, Thomas

Belleman, Ben

177

Belleman, Chelsea 132, 153, 211

Belleman, Lee 54

Bender, Charles 114

Benner, Daniel 114

Bennett, David 100

Bennett, Kevin 221

Benson, David

114

Bentley, Robert 132

Benz, Erin

191

Benz, Justin

177

Beougher, Kristi

$188,189,264$

63,
0, Beres, Matthew

$97,218,219$

Berger, Emily $\quad 181$

Bernard, Stacey 132

Bernhard, William 32

Berry, Lauren 63

271

Bertsche, Benjamin 151

Bertsche, Eva $\quad 114$

Bertsche, John 132

Besaw, Laura $\quad 114$

Beste, Jeff

235, 254

Betke, Brian

Bezeck, Jacob

198, 199

Bidwell, Jennifer $\quad 115$ 157

Biehl, Andrew

Biehl, Kathryn

153, 156

Bielek, Amy

Biga, Jordan

Bigler, Brett

193

Billhartz, Kathryn 115

Billman, Steven 115

Bingham, Stephanie

132

Birch, Debra

Bisset, Deborah 34

Bjornstad, James 35

Black, Andrew 42

Blackburn, Joshua 64 , 264

Blackburn, Kelly 115

Blackburn, Lisa 64 ,

179, 261

Blackburn, Melody 115

Blacklidge, Sara 132

Blackstone, Valerie 133

Blake, Jack $\quad 100$

Bliss, Katie $\quad 133$

Blocher, Brian 133

Blocker, Hugh 115

Blodgett, John 32

Bloem, Brant 17,

64

Blumenstock, Helen

Blumenstock, Richard

35

Blythe, Rita

191

Boche, Jonathan 100

Bochman, Heidi 115

Boland, Bachar 64

Bolander, Benjamin

64

Bolander, Joseph 115

Bole, Rebekah $\quad 64$

Bolender, Jeff 39 ,

196

Boles, Stephen $\quad 100$

Bollenbacher, Karissa

115

Bollman, Stephanie

100

Bolthouse, William 32

Bonardi, Jordana 100

Bonde, Renae 100

Bonenberger, Omer

45

Bonsell, Emily 56

Boone, Tarah 64

Borck Jr., Thomas 100 


\begin{tabular}{|c|c|c|c|c|c|c|c|c|c|c|c|}
\hline Brahler, Sarah & 64 & \multirow{2}{*}{\multicolumn{2}{|c|}{$\begin{array}{l}\text { Broscious, Jonathan } \\
101,239\end{array}$}} & Burbury, Dina & 101 & Carlson, Alex & 146 & Chiu, Evan & 101 & Cole, Kraig & 116 \\
\hline Braithwaite, Edwin & 42 & & & Burch, Whitney & 133 & Carlson, Carolyn & 40 & \multirow{2}{*}{\multicolumn{2}{|c|}{$\begin{array}{l}\text { Christensen, Shauna } \\
67,237,259,261\end{array}$}} & 259,271 & \\
\hline Brand, Amy & 115 & Brott, Courtney & 19 & Burdette, Anthony & 66 & Carlson, Justin & 147 & & & Coleman, Sarah & 116 \\
\hline 234 & & & & Burgess, Dianna & 66 & \multicolumn{2}{|l|}{ Carpenter, Benjamin } & \multirow{2}{*}{\multicolumn{2}{|c|}{$\begin{array}{l}\text { Christiaanse, Justine } \\
133,181\end{array}$}} & 234 & \\
\hline Brandes, Michael & 115 & Brown, Alexis & 115 & Burgman, Lisa & 179 & 116 & & & & \multirow{2}{*}{\multicolumn{2}{|c|}{$\begin{array}{l}\text { Coleman, Stephanie } \\
116\end{array}$}} \\
\hline Brandon, Carl & 50 & Brown, Amy & 101, & Burk, Elyse & 101 & Carrier, Darrin & 13 & Christian, Angela & 134 & & \\
\hline $\begin{array}{l}\text { Brandt, Julie } \\
190,191\end{array}$ & 115 & $\begin{array}{l}244 \\
\text { Brown, Andrew }\end{array}$ & & 157 & & Carrion, Alberto & 13 & Christiansen, Heidi & i 116 & $\begin{array}{l}\text { Colgain, Janet } \\
243\end{array}$ & 67 \\
\hline Brandt, Sarah & 133 & Brown, David & 65 & $\begin{array}{l}\text { Burkett, Deborah } \\
\text { Burkett, Elizabeth }\end{array}$ & $\begin{array}{l}116 \\
66\end{array}$ & $\begin{array}{l}21,66,95 \\
\text { Carsey, Jeremiah }\end{array}$ & 101 & $\begin{array}{l}\text { Chrystal, Linda } \\
\text { Cimba, Michael }\end{array}$ & $\begin{array}{l}47 \\
67\end{array}$ & $\begin{array}{l}\text { Collins, Jon } \\
53\end{array}$ & 52 \\
\hline $\begin{array}{l}\text { Braswell, Cathryn } \\
265\end{array}$ & 115 & $\begin{array}{l}\text { Brown, Emily } \\
129\end{array}$ & 115, & $\begin{array}{l}95 \\
\text { Burks, Naomi }\end{array}$ & 66 & $\begin{array}{l}\text { Carter, Ashley } \\
260\end{array}$ & 116 & $\begin{array}{l}\text { Ciolek, Nathan } \\
116\end{array}$ & 18 & Collins, Kristine & 67 \\
\hline $\begin{array}{l}\text { Braun, Cameron } \\
95\end{array}$ & 65 & $\begin{array}{l}\text { Brown, Gerry } \\
\text { Brown, Gracia }\end{array}$ & $\begin{array}{l}41 \\
115\end{array}$ & $\begin{array}{l}259 \\
\text { Burley, Jade }\end{array}$ & 101, & $\begin{array}{l}\text { Carter, Evan } \\
217\end{array}$ & 150 & $\begin{array}{l}\text { Clark, Daniel } \\
\text { Clark, Jim39 }\end{array}$ & 36 & $\begin{array}{l}\text { Colman, Kristin } \\
\text { Colon, Brooke }\end{array}$ & $\begin{array}{l}134 \\
57\end{array}$ \\
\hline $\begin{array}{l}\text { Braun, Mindy } \\
95,215\end{array}$ & 65 & $\begin{array}{l}\text { Brown, Jessica } \\
\text { Brown, Kevin }\end{array}$ & $\begin{array}{l}133 \\
65\end{array}$ & $\begin{array}{l}200,201,215,234 \\
\text { Burnett, Ashley }\end{array}$ & 66 & $\begin{array}{l}\text { Carter, Rachel } \\
260\end{array}$ & 67 & Clark, Katie & 67 & $\begin{array}{l}\text { Colon, Jared } \\
147,159\end{array}$ & 134 \\
\hline Bravick, Gena & 65 & Brown, Kimberley & 65 & Burns, Lindsey & 66 & Carter, Timothy & 133 & $\begin{array}{l}\text { Clark, Matthew } \\
182,183\end{array}$ & 67 & Comers, Luke & 134 \\
\hline Brazalovich, Jessica & & 216,217 & & Burrichter, Margare & & Cary, Thomas & 116 & Clark, Teresa & 39 & Comers, Terry & 45 \\
\hline 133 & & Brown, Lauren & 115 & 54 & & Cary, Tiffany & 67 & 255 & & Compitello, Everett & t116 \\
\hline Brenneman, Carla & 65 & 156 & & Burris, Emily & 133 & Casper, Brett & 67 & Clawson, Brian & 101 & Compton, Bethany & 101 \\
\hline 236 & & Brown, Rebekah & 115 & 185 & & Casper, Ian & 116 & Clawson, Melissa & 116 & Condit, Katherine & 101 \\
\hline Bresson, Sarah & 133 & Brown, Tyler & 111 & Bushre, Stephen & 101 & 222,223 & & Cleek, Stephanie & 134 & Condon, Lacie & 134 \\
\hline Brewer, Bethany & 133 & Browning, Suzanne & & Butler, Siera & 116 & Castle, Allyson & 179 & Clemans, Andrew & 2 & & \\
\hline Brewer, Kaitlin & 133 & 65 & & Butters, Sarah & 66 & Caterson, Lee & 221 & 67,234 & & Conkling, Elizabeth & h116 \\
\hline Brewin, Nicole & 115 & Brownlee, Carol & 65 & Butz, Anthony & 116 & Cato, Celeste & 218 & Clements, Alexandre & & Connolly, Laura & 67 \\
\hline Brewster, Susan & 65 & Bruder, Andrew & 66 & Butz, Gregory & 66 & 219 & & $23,116,157,219$ & & Conway, Douglas & 68 \\
\hline Bricker, Richard & 27 & 206 & & Butz, Kayla & 133 & Cato, Jim 57 & & Clements, Robert & 34 & Conway, Janet & 40 \\
\hline 115,150 & & Bruekner, Gilbert & 32 & Byers, Bryant & 101 & Cato, Melody & 54 & Clemons Jr., Stevene & 67 & Conyers, Jeremy & 68, \\
\hline Briddick, Joshua & 115 & Brumbaugh, Amanc & da & Byers, Rhonda & 101 & Caudle, Catherine & 133 & Clevenger, Chuck & 37 & 261 & \\
\hline Briggs, Charles & 65 & 101 & & Byus, Chris & 101 & Cave, Linda & 40 & Cline, Megan & 134 & Conyers, Michael & 18 \\
\hline Briggs, Greg & 100 & Brummel, Joel & $\begin{array}{l}115 \\
115\end{array}$ & & & Cernetic, Faith & 133 & 213 & & & \\
\hline Briggs, Greg & 100 & Brummett, Evan & 115 & & & Chamberlain, Betha & any & Clinton, Patrick & 67 & Cook, Aaron & 68 \\
\hline $\begin{array}{l}\text { Briggs, Jason } \\
17,65,217\end{array}$ & 16, & $\begin{array}{l}\text { Bryant, lain } \\
251\end{array}$ & 250 & & & 26,116 & & Clore, Jamie & 67 & Cook, Felicia & 134 \\
\hline $\begin{array}{l}17,65,217 \\
\text { Britton, Sarah }\end{array}$ & & Bubacz, Christin & 115 & & & Chamberlain, Terry L & & Clouse, Adam & 134 & Cook, Jazmine & 134 \\
\hline $\begin{array}{l}\text { Britton, Sarah } \\
\text { Brochu, Zach }\end{array}$ & $\begin{array}{l}65 \\
100\end{array}$ & Buben, Philip & 66 & & & $37,267,269$ & & Clutcher, Alissa & 101 & Cook, Jeff & 35 \\
\hline $\begin{array}{l}\text { Brochu, Zach } \\
\text { Brock, Lynn }\end{array}$ & $\begin{array}{l}100 \\
48\end{array}$ & 203,265 & & & & Chamberlin, Joshua & & Clymer, Kaydrie & 116 & Cook, Kaitlyn & 134 \\
\hline $\begin{array}{l}\text { Brock, Lynn } \\
\text { Brock, Peter }\end{array}$ & $\begin{array}{l}48 \\
115\end{array}$ & Buchheit, Andrew & $i 15$ & Cady, Benjamin & & 203 & & Coakley, Nicole & 67 & Cook, Lisa & 134 \\
\hline $\begin{array}{l}\text { Brock, Peter } \\
\text { Brockmeyer, Kevin }\end{array}$ & $\begin{array}{l}115 \\
133\end{array}$ & Buchheit, Sarah & 66 & $\begin{array}{l}\text { Cady, Benjamin } \\
\text { Cafferty, Megan }\end{array}$ & 66 & Chamberlin, Kyle & 133 & Coale, Ashley & 21 & Cook, Matthew & 101 \\
\hline $\begin{array}{l}\text { Brockmeyer, Kevin } \\
\text { Broersma, Emily }\end{array}$ & $\begin{array}{l}133 \\
100\end{array}$ & Buck, Hali & 116 & $\begin{array}{l}\text { Cafferty, Megan } \\
\text { Cahilly, Erin }\end{array}$ & 133 & Chambers, Micah & 101 & $191,217,236$ & & Cook, Robert & 68 \\
\hline & 100 & Buckley, Jason & 16 & $\begin{array}{l}\text { Cahilly, Erin } \\
157\end{array}$ & 101 & Chandler, Katherine & & Cobucci, Stephen & 177 & Cookson, Shannon & 68 \\
\hline Broesder, Kimberly & & $133,176,177$ & & $\begin{array}{l}157 \\
\text { Cahilly, Peter }\end{array}$ & & 133 & & 250,251 & & Cookson, Shaun & 134 \\
\hline Bromer, Pam & 35 & Buckwalter, Ahna & 101 & $\begin{array}{l}\text { Cahilly, Peter } \\
\text { Cain, Emily }\end{array}$ & 116 & Chaney, Ryan & 133 & Cochran Jr., Timothy & & Cooper, April & 101 \\
\hline Bromley, Hilary & 65 & 251 & & $\begin{array}{l}\text { Cain, Emily } \\
\text { Calderon, Olivia }\end{array}$ & 66 & Charlton, Heidi & 156 & 116 & & 271 & \\
\hline 210 & & Buehler, Ashley & 66 & $\begin{array}{l}\text { Calderon, Olivia } \\
\text { Calhoun, Scott }\end{array}$ & $\begin{array}{l}133 \\
36\end{array}$ & $\begin{array}{l}\text { Chasnov, Nora } \\
221\end{array}$ & 133, & $\begin{array}{l}\text { Cody Jr., John } \\
\text { Cofer, Naomi }\end{array}$ & $\begin{array}{l}67 \\
67\end{array}$ & $\begin{array}{l}\text { Cooper, John } \\
\text { Copeland, Daniel }\end{array}$ & $\begin{array}{l}134 \\
116\end{array}$ \\
\hline Brooker, Judson & 183 & Buenz, Bridget & 133 & Callahan, Morgan & 133 & Chaznov, Robert & 41 & Coffelt, Joanna & 101 & Corbin, Jessica & 68 \\
\hline Brooks, Anna & 115 & Buhr, Jaclyn & 101 & Callan, Karen & 40 & Cheatham, Sarah & 116 & Coffill, Samantha & 134 & Corley, Ruthie & 68 \\
\hline Brooks, Janelle & 115 & Buhr, Jeffrey & 101 & Campbell, Fran & 47 & Cherland, Melanie & 133 & Coffindaffer, Kari & 116 & 97 & \\
\hline Brooks, Natalie & 133 & Bundenthal, Ryan & 66 & Campbell, Julie & 66 & 211 & & Cohoon, Michelle & 101 & Cornell, Laura & 116 \\
\hline 146 & & 195 & 00, & Campbell, Nathan & 66 & Chester, Nathan & 150 & Colas, Bradley & 134 & Costerisan, Aaron & 42 \\
\hline $\begin{array}{l}\text { Brorein, Melissa } \\
156\end{array}$ & 65 & $\begin{array}{l}\text { Bungart, Andrea } \\
261\end{array}$ & 66, & $\begin{array}{l}\text { Campbell, Seth } \\
\text { Carey, John }\end{array}$ & $\begin{array}{l}183 \\
116\end{array}$ & $\begin{array}{l}\text { Chesterman, Andrev } \\
133\end{array}$ & ew & $\begin{array}{l}\text { Cole, Amanda } \\
101\end{array}$ & 18 & $\begin{array}{l}\text { Costerisan, Andrea } \\
264\end{array}$ & 68 \\
\hline & & $\begin{array}{l}261 \\
\text { Burban, Pete }\end{array}$ & 41 & $207,219,220$ & & Childs, Robert & 101 & Cole, Esther & 101 & & \\
\hline
\end{tabular}


Costerisan, Andrew

$116,150,246,250,284$

Cottings, Bethany 116 , 128,179

Coudriet, Jessica 101, 234

Coulter, Christi 68

Coulter, Jonathan 203

Couser, Gregory 35

Cowell, Kimberly 68

Cowell, Lauren 101

Cox, Amanda $\quad 116$

Cox, Chad $\quad 134$

Coyle, Gabriel 101,

$168,169,235$

Cozad, Hannah 101

Craft, Gregory 134

Craig, Sara 116,

221

Crain, Lori

219

Crandall, Katrina 116

Craycraft, Abigail 101

Crichlow, Sarah 117 ,

160, 169, 216, 229

Crissman, Matthew134

Crommett, April 39

Cronshaw, Christina 68

Cross, Chris 38,

187,189

Croston, Ryan $\quad 117$

Crowder, Lisa 117 ,

234

Crowner, Elizabeth 117, 167

Crunelle, Leanne 102, $184,185,197$

Cughan, Richard 55

Culver, Jenna 117

Cummings, Anna 102,

253

Cummings, Lisbeth

134

Cummins, Daniel 117

Cunliffe, Evelyn 134

Cunningham, Erica 68 ,

94,256

Cunningham, Erin 68 , 94

Cunningham, Jason

177

Curlette, Bruce 37

Curtis, Andrew 102
Curtis
191

Cushman, Seth

Custer, Gabe

266

Cuthbert, Andrea 233

Cyrus, Amanda 117

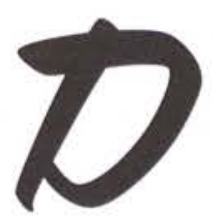

Daeschner, Katherine 134

Daiello, Jonathan 68 , 94,163

Dallas, Natalie

237

Daniels, Cathy 68 ,

95

Dankert, Andrea 68

Dasuqi, Ashley 134

271

Daughenbaugh,

Danielle 102

Daugherty, Jessica 170 , 237

Daugherty, Kelli 68

Davey, Nicholas 134

David, Priscilla 117

217

Davidson, Robert 117

Davies, Bethany 68 ,

117, 197

Davies, Richard $\quad 102$

219

Davis, Adam $\quad 117$

Davis, Andrew 117,

217, 218, 219

Davis, Courtney 134

Davis, Cynthia 47

Davis, Ellen

Davis, Jennifer 102 ,

216, 217

Davis, Kenneth 102

177

Davis, Matthew 102

Davis, Michelle 102

Davis, Nathanael 69 ,

154

Davison, Dan 129

Day, Melanie

Find Your Friends!

Derry, Colleen 69 ,

179,261

Deschaine, Joshua 134

Deseno, Kimberly 135

Develbiss, Jenna 135

de Waal Malefyt,

Matthew 151, 117

Dewhurst, Gwendolyn

117

Dewhurst, Timothy 41

Di Donato, Lindsay 102

DiCuirci, Mike $\quad 37$

Dickhoner, Barbara 135

Dixon, Jennifer 102

160

Dixon, Paul 33

Dixon, Scott 35

Dobrinoff, Ashley 117

Dobutovich, Cassandra

$117,172,211$

Doden, Daryle 32

Dodson, Justin 102

154,257

Doenges, Timothy 221

Doles, Kristen 155

Dolph, Olivia 201

Domeny, Michael 231, 252
Donald, Brittany 102 , 168,235

Donaldson, Ericka 117

Donohue, Kelly 135

Doot, Emily 102

Dorsey, Arielle 102

Dotson, Michael 69 ,

163,237

Douglas, Abby 27 ,

117

Douglas, Sara 69

Doyle, Brandon $\quad 102$,

229

Draxler, Hannah 69

Dremann, Rachel 69 .

263

Driscoll, Emily 135

Driscoll, Stephanie 135

Drullinger, David 35

Drum, Rebekah 102

Duarte, David 135

Duarte, Rachel 135

Duchardt, Bob 203

Dudley, Philip 69,

261

Dudreck, Kristin 69 ,

162

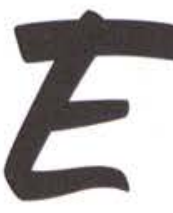

Eagle, Zachary $\quad 70$,

210

Earls, Christopher 117

Early, Sarah

12 ,

135

Eaton, Courtney 117

Eaton, Seth 70

Eaves, Dorianne 135

Eberle, Matthew 117

Eby, Jenny $\quad 70$

Eckard, Chad 216

Eddy, Megan $\quad 70$

Edem, Vicki 44

Eder, Christopher 70

Eder, Sarah $\quad 70$

Ederer, Patrick 228

Edgar, Julie $\quad 70$

Edlund, Alan 199

Edmonds, Courtney

102,231

Edwards, Meredith 135

Egenreider, Sarah 135

Ehlers, Kimberly 70 , 156

Duerrwaechter, David

102

Duff, Justin $\quad 252$,

253

Duff, Victoria

69 ,

$166,219,256$

Duffell, Monica 135 ,

185

Dugan, Joseph 231

Duncan, Bethany 117

Duncan, Nicholas 117

Dungan, Benjamin 69

Dunnevant, Kaitlin 14,

117

Dunning, John 135

Dunteman, Deborah

70

Durchardt, Bob 38

Durkee, John 70,

96

Eimers, Lee $\quad 42$

Einselen, Amanda 222

Eisentrager, Joseph

70, 163

Eldridge, Jacob $\quad 135$

Eldridge, Kaleb $\quad 117$

Elgena, Jennifer 70

Elgersma, James 117

Elgersma, William 70

Ellington, Charles 70

Elliott, Andrew 70 ,

177, 206

Elliott, Ashley $\quad 117$

Elliott, Charles 34

Elliott, Kimberly 117,

271

Elliott, Sara $\quad 135$

Ellis, Abby

156

Ellis, James

Elmore, Annamarie 135,

146

Elmore, Jennalee 117, 216

Enderle, Jordan
Endres, Hannah 135

Engel, Austin 118 ,

212

Engelmann, James 32

England, Jessica 118, 191

England, Valerie 118

Englebert, Samuel 118

Ennis, Nathan $\quad 177$

Ensminger, Kristen 118

Ensslen, Sarah 14,

70, 196

Entner, Tim 56

Ernst, Caleb $\quad 102$

Ernst, Ryan 70,

236

Erspamer, Tiffany 102

Erwin, Nichole $\quad 118$

Erwin, Rebecca 102 ,

157

Eslick, Stephen 25

Estepp, Pat 39,

187

Estes, Carol $\quad 45$

Estes, Dan 35

Eustace, Pamela 102

Evans, Adam 227

Evans, Jonathan 102

Everett, Abby 102

Everswick, Shannon

102

Evington, Michelle 70, $154,212,214,215,238$

Ewers, Lacy

118

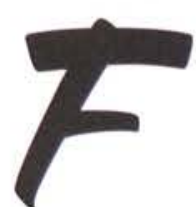

Fabian, Lynsey

39, 189

Fagan, Joy R. $\quad 35$

Fagan, Siobhan 38,

181

Fake, Christopher 239

Fallin, Dea $\quad 118$

Fang, Vicky 41

Fannor, Elizabeth 71

Fansler, Jennifer 71, 261

Fansler, Megan 118

Farnham, Amy 135 
Farrar, Katelyn $\quad 102$, 157

Farrell, Katherine 102

Faulkner, Chrissy 53

Fawcett, Jeffrey 44

Fawcett, Tonya 50

Fearnot, Evonne 179

Febo, Kristie $\quad 102$

Feczer, Jillian 118,

217

Feiler, Katherine 118

Feiler, Kimberly 71

Feiler, Michael 71,

263

Feiler, Sarah

Felix, Ann

162

Felmet, Evan 135

Ferguson, Jenna 103

Ferguson, Kristen 118

Ferkaluk, Brian 71

Ferranti, Taylor 37

Ferrell, Talitha 118

Feucht, Elisabeth 135

Few, Christopher 71

Few, David $\quad 71$

Fillion, Molly 135

Fiorelli, Victoria 71

Firmin, Michael 46,

58

Fish, Jessica $\quad 135$

Fisher, Mallory 103

Fisher, Susan 135

Fladda, Steven $\quad 71$

Flamm, Stephanie 52

Flannery, Shane 151

Flatt, Justin 135

Fleetwood, Jonathan

118

Flenar, Adam $\quad 71$

Flentge, Dennis R. 42

Fletcher, Caleb 71,

158

Flippin, Crystal 118

Flippin, Heather 135,

212

Flippin, Pam 53

Floren, Daniel 135

Flow, Elizabeth $\quad 118$

Flow, Martha 135

Focht, Matthew 118

Foley, Ann $\quad 103$

Foo, Jamie
Foote, Matthew 135

Ford, Connie

Ford, Timothy

Foreman, Holly

232

Forsberg, Jill 135,

218, 219

Forson, Kari

253

Forsythe, Jessica 71,

97, 235, 261, 262

Foster, Janna 71

Fowler, Joel $\quad 187$

Fox, Jenna 205

Fox, Jesse

71

177

Fox, Jesse

$\begin{array}{ll}\text { Fox, Matthew } & 233 \\ \text { Foy, Jannette } & 71\end{array}$

Frageorgia, Melanie

$$
71
$$

Fraley, Dara $\quad 49$

Frame, Timothy 37

Francis, Justin $\quad 118$

Francis, Michael B 71, 261

Frank, Dwayne 45

Franke, Annamarie 103

Fraser, Heidi $\quad 103$

Fraser, Samuel 20

Frasier, Kristie 135

Frederick, Josh 111

Freed, Richard $\quad 118$

Freese, Dave 205

Freese, Kathy 39 ,

204, 205

French, Kaitlyn $\quad 118$

Freswick, Rachel 72

Frey, Darrin $\quad 42$

Frichtl, Johanna 118

Fridenmaker, Nicholas

229

Friend, Michal 72 ,

155

Fugate, Brent 135

Fulmer, Jeffrey 51

Fuls, David $\quad 72$

Fuls, Rachael $\quad 135$

Fultz, Dalen 135

Funke, Christa 20,

103

Funtik, Lynne $\quad 50$

Furst, Kevin 198,

199

Futrell, Ryan

Fye, Elyse 118

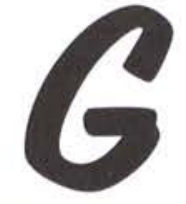

Gainer, Mark 72

Gallagher, David M.

41

Gallas, Jessica $\quad 72$

Galloway, Cali 136

232

Gamache, Natalie 72

Gandolfi, Franco 44

Gannon, Kari $\quad 72$

Garling, Joshua 103

Garner, Amy 136,

181

Garrett, Ross 136

Garrison, Jacob 118

Gathany, Paul 56

Gause, Michael 187

Gebert, Clara $\quad 136$

Gebert, Julia 72

Gehman, Christopher

136

Geiger, Rachel $\quad 210$

Geist, Alan $\quad 39$

Gelser, Joshua 103,

176, 177, 246

Gentene, Lauren 188, 189

Genter, Christina 72

George, Carol $\quad 47$

George, Matthew 177

George, Stefanie 103

George, Tricia 136

Geringer, Amber 118

Geringer, Brian 72

German, Kelly 72,

\section{4}

Gerringer, Michael 72

Gibbs, Kirsten 50,

235

Gibbs, Lew 16,

$$
50
$$

Gibson, Jennifer 158

Gibson, Leah 136

Gibson, Rachel 118

Gibson, Rebekah 72

Gibson, Sarah $\quad 72$

Gibson, Zachary 72

Gier, Megan 103

Giglio, Ben $\quad 128$
Gigliotti, Josh

207, 237, 271

Gilbert, Shelley

Gilchrist, Sarah

Gildow, Justin

261

Giles, Daniel 72

Gilgillen, April

Gilin, Rachel

153

Gillesby, Sean 136

Gillette, Jacinda 103

Gilmore, Michelle 147

Gilmour, Bethany 72

Gilsdorf, Kelsey 118,

246, 247

Gilsdorf, Kevin 73

Gingrich, Serenity 210

Gingrich, Valerie 136

Ginter, Cori 73

Girouard, Earl $\quad 73$

Glass, Jessica $\quad 73$,

234

Gleichauf, Ashley 118

Gneiser, Sarah 136,

191

Gochenaur, Ashley 73,

234, 260, 264

Godinez, Monica 73

Godwin, Bethany 136

Goecke, Erica 136

Gohdes, Peter 73,

158

Goldman, Amy 136,

229

Gole, Robert $\quad 103$

Gollihue, Erin 25,

$146,236,238,254$

Gollmer, Steven 42

Gombis, Timothy 35

Gooch, Catherine 73

Goodew, Audree 184,

185, 197

Goodew, Titus 194,

195

Goodman, Terri 118

Goodrow, Erika 136

Gorrell, Jonathan 136

Gosser, Aaron $\quad 37$

Gouch, Emilie $\quad 118$

Gower, David 32

Graeff, Michelle 118,

226, 237
Graff, Stephanie $\quad 119$,
185

Graham, David 32

Graham, Evan 136

Grant, Jennifer 119

Graves, Amanda 136,

226, 271

Gray, John

Gray, Lisa 136

Gray, Nicholas

Gredy, John

31

Green, Derek 34

Green, Erin $\quad 73$

Green, Harold R. 53

Green, Joel 73,

$155,156,234,264$

Green, Margaret 53

Green, Matthew 103

Green, Shawn 73,

231

Greenberg, Leah-Ruth 103

Greenberg, Sarah 119, 129

Greenfield, Amy 119, 157

Greer, Clark

Greer, Jennifer

136

Greer, Kimberly 103

Gregg, Rebecca 136

Gregory, Jedediah 73

Greve, Gabriel $\quad 119$

Greve, Joshua 73,

186,187

Grewe, Jessica $\quad 103$

Gries, Aubrey 103,

228, 263

Griest, Jared

203

Griffith, Joshua $\quad 119$

Griggs, Aaron 183

Grigorenko, Donald

35

Grist, Stephen 205

Griswold, Kelsey 119

Groce, Ashley 136

Groeber, Michael 119

Groen, Deborah 136

Groff, Adam $\quad 119$

Grollimund, Julie 103,

152

Gromacki, Bob 35

Groman, Kurt 136

Grooms, Heather 189

Groseclose, Daniel 73

Gruber, Steve $\quad 45$

Grudda, David 103

Gruenberg, Stephen

119

Guenther, Kathleen

119

Guernsey, Jeff 44,

227

Guiler, Abigail $\quad 73$

Guisleman, Amy-Hope 36

Gulley, Kevin 24

Gumm, Carrie 73

Gumprecht, Michael 


\section{Find Your Friends!}

Hamilton, John 119 Hamilton, Nathan 14, 74

Hamminga, Kristin 136 Hampton, Maija 119 , 180,181

Hancock, Charity 119 Hand, Jacob $\quad 136$

Hand, Jamie 199

Hand Jr., Michael 74

Hanes, Megan 74

Haney, Emily 136

Hanna, Amy 119

Hanna, Zachary 119

Hansell, Tim 44

Hansen, Aleece 137

Hansen, Grant 22,

$23,119,218,219,251$

Hansen, Ryan 103,

164

Hanson, Cody 137 ,

146

Harding, Micah $\quad 74$

Harer, Andrew 137,

177

Haring, Kyle $\quad 137$

Harmon, Christopher

74, 95

Harner, Sandi 36

Harpest, Cassie 119

Harrell, Ivey $\quad 74$,

216, 217

Harriman, Janie 74

Harris, Sonya 137

Harrison, Autumn 119

Harrison, Erin 74

Harrison II, John 103

Harro, Nathan 74

Hartman, Charles 44

Hartman, Melissa 39,

180, 181, 201

Hasselbach, Kortni 12

137

Hatch, Alison 119,

217

Hatch, Cristina $\quad 119$

220

Hatch, Joseph 137

Hattenfield, Rebecca

137

Hattenfield, Zachary

119

Hause, Eli119

Hauser, Aaron

137
Hauser, Chelsey 137

Hawkins, Garrett 137

Hawkins, Jaime 53

Hawkins, John 15,

249

Hawkins, Rebecca 137

Hawkins, Taylor 74

Hay, Nathan $\quad 48$

Hayes, Anna

217, 227

Hayes, Becky

266

Hayes, Bethany

191

Hayes, Erin

179

Hayes, Heather

179, 191

Hayes, Jordan

Hayes, Sara

235

Haynes, Hannah 103 ,

189

Haynes, Joshua 137

Haynes, Rachel 103

Hays, Elizabeth 104

Hays, John

Hazen, Alicia

32

104

Heagy, Heather 104

Heath, Kevin 36

Heaton, Tim 45

Heffield, Rachel 51

Hegarty, Beth 119

Hegna, Harwood 41,

42

Heinig, Elizabeth 74

Heinrich, Josh $\quad 49$

Heiss, Leslie $\quad 104$

Hejl, Anna $\quad 119$

Hekel, Daniel $\quad 74$

Hellwig, Evan 38

Hellwig, Kelly 36

Helmick, Larry 42

Hendricks, Nicholas

119

Henker, Angela 74

Henness, Stacey $\quad 6,74$

Hennessey, Ethan 74

Henning, Nelson L. 44

Henricks, Andrew 104

Hensley, Caleb 13,

104, 270, 271

Herbert, Justin $\quad 182$,

183, 195
Herbolt, Joshua 104

Herbolt, Rachel 119

Herbst, Brittany 104,

156

Hershey, Mark 75,

$151,169,214,235$

Hess, Alison $\quad 75$

Hess, John 46

Hess, Kyle

151, 248

Hessler, Kristin 75

Hickok, Annalise 137,

245, 252

Higginbotham, Kim

40

Higgins, Caitlin 119

Hilderbrand, Jaclyn

137

Hilerio, Stephanie 104,

233

Hill, Leann 51

Hill, Zachary 245

Hilty, Keri 185, 197

Hindle, Hannah 120 ,

128,160

Hine, Tricia $\quad 75$

Hines, Vicky L. $\quad 57$

Hinkle, Jessica 137

Hinks, Elizabeth 75

Hinks, Nathan 104

Hinshaw, Jordan 137

Hitchcock, Melanie 75

Hitchcock, Melissa 120

Hiteshew, Katlyn 120,

234

Hobbes, Seth $\quad 120$

Hobbs, Justin $\quad 137$

Hobby, Lindsay 104

Hochstaetter, Bethany

75

Hodges, Megan 137

Hofert, Suzanne 120

Hoffeditz, David M. 35 ,

36

Hoffeditz, Lori 51,

160

Hoffman, Chelsie 104

Hoffman, Crystal 120

Hoffman, Joel 75

Hoffman, Sarah 120 ,

205

Hoganson, Bradley 120,

230
Hogue, Shannah 35 ,

36

Hokuf, Ryan 137

Holderby, Amy 52

Holecko, Joseph 6

Hollingsworth, Hannah

120, 213

Hollis, Matthew 104,

172

Hollopeter, Aaron 27,

75, 221

Holloway, Jada 104,

154,214

Homan, Laurie 104

Hoover, Sara $\quad 75$

Horning, Angela 137

Horosz, Victoria 137

Horst, Ragen $\quad 104$

Horton, Bridgett 242

Horton, Kristen 104

Hosan, Sarah $\quad 120$

Hose, Emily $\quad 75$

Hosford, Leighana 75

Hostetter, Derek 24,

104, 199

Houchin, Brian 120 ,

167

Houchin, Matthew 203

Houghton, Benjamin

75

Houk, Katherine 104

Hounshell, Maxwell

137

Houser, Craig 104,

257

Houser, Dale $\quad 104$

Hovestol, Christina 75

Howard, Angela 137

Howard, Chelsea 120

Howard, Christie 104

Howard, Mary 32

Howard, Megan 104

Howdyshell, David 177

Howdyshell, Samuel

120

Howell, Julie $\quad 137$

Hoyt, Timothy 120

Huang, Chu-Yu 41

Hubbard, Caleb 137

Hubenthal, Michael

75,210

Huber, Jessica $\quad 137$

Huber, Trish $\quad 55$

Hubin, Anthony 104
Hubin, Rachael 137

Hubler, Timothy 75 ,

203

Huck, Scott

97,

272

Huckaby, Lori J. $\quad 39$

189

Hueni, Joshua $\quad 151$,

214,251

Huff, George $\quad 44$

Huff, Virginia 45

Huffman, Aaron 75 ,

94,271

Hufhand, Kari $\quad 120$

128,129

Hughes, Greg 203

Hull, Caitlin 137

Hull, Julie75, 259

Hume, Ashley $\quad 76$

Hummitzsch, John 76,

$154,155,263,265$

Humphrey Jr., William 120

Hundley, Phillip 104

Hunt, Ashley 76,

155, 162, 216, 235, 256

Hunter, Ashley 76 ,

$155,162,216,235,256$

Hutchinson, Aaron 42

Hutchinson, Mandy

50

Hutchinson, Thomas

35

Hynes, Jacob 137

Hyun, Jung Ah $\quad 104$

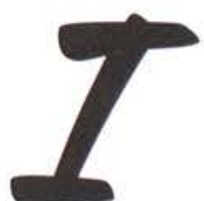

Ikehara, Aaron

104

Infante, Eben 104,

191,233

Inskeep, Tiffany 104,

234

Irish, Cheryl

Irving, Mark

46

57

Hubin, Anthony 104

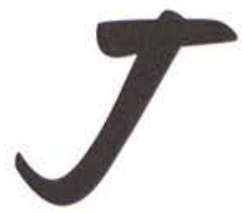

Jackson, Brent 104

Jackson, Brittany 120

Jackson, Chad 52

Jackson, Clarissa 120

Jackson, Jennifer 104

Jackson, Tonisia 137

Jacobs, Amy $\quad 120$

Jacobs, Christa 137 ,

229

Jacobs, Jack 32

Jacobsen, Jordan 76

Jacobson, Sarah 138

Janssen, Steve $\quad 35$,

36

Jaquery, Daniel 27,

$76,97,217,226,233$,

251

Jasper, Natalie $\quad 120$ 
Johnson, Sharon 45

Johnson, Steven 105

Johnson, Yukiko 52 ,

53

Jolly, Amanda 105,

237

Jolly, Laura $\quad 46$

Jones, Allison 120

Jones, Amber $\quad 120$

Jones, Grace $\quad 120$

Jones, Leah 76,

$156,242,260,267,268$, 272

Jones, Sarah 236

Jones, William I. 42

Jordan, Lindsay 138 ,

147

Joswig, Amanda-Jo76

Judd, Isaac $\quad 76$

Judd, Rachel 190,

191

Juhl, Jonathan 120

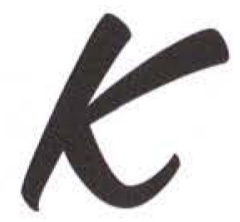

Kaemmerling, Claire 105, 244

Kaercher, Martha 51

Kahila, Annemarie 147

Kapp, Amanda $\quad 120$

Kauffman, Caleb 76

Kearney, Alison 138,

216,259

Keeports, Christine 138

Kehl, Anna

120 ,

129, 191

Kehl III, Jack $\quad 76$

Keith, Nathaniel 138

Keller, Abram 105

Keller, Amy 105

Keller, Elaine 51

Keller, Justin 23 ,

77, 160

Keller, Nathan

$138,159,245$

Keller, Stacey

185

Keller, Teresa

159, 233, 259

Kelton, Heather

$77,190,191,261$
Kempton, Eric

147.

194

Kempton, Ruth 32

Kennedy, Zachary 138

Kennelly, Rachel 77

Kent, Matthew 138

Kern, Benjamin $\quad 138$

Keslar, Dustin 77 ,

263

Kibelbek, Andrew 77

Kidd, Brian

121

Kim, Jun 37

Kim, Kyu-Bum $\quad 77$

Kindt, David 77

261

King, Elvin

184, 185

King, Erin 77

King, Joyce

185

King, Shannen 138

King, Toi 46

Kinman, Diane $\quad 77$

Kinnard, Kelley 77 ,

158, 216

Kinsinger, Jay $\quad 42$

Kirby, Amanda 77 ,

234

Kirby, Kelsey $\quad 77$

Kirby, Marie $\quad 121$

Kirby, Rebecca $\quad 77$

Kirkwood, James 138

Kiser, Andrea 121

Kitchen, Jeremiah 138

Klein, Katherine 105

Kleis, Benjamin 121

Klimek, Kathryn 121 ,

233, 249

Klimek, Mark $\quad 4 T$

Kline, Courtney 121

Klinger, Lance 138

Klint, Charles 138,

151, 221

Rebecca 121

Kloosterman, Daniel

105

Knable, Jane 77

$$
156
$$

Knauff, Nancy 50

Knaus, Susan 77

Knickerbocker, Jeremy

77

24, Knight, Grant 176 ,

21, $\quad 177$

Knight, Jonathan 138
Knilans, Joshua 105 Knipple, Travis 77 . 260

Knoedler, Brittany 121

Knott, Christine 121

Knudsen, Daniel 105,

111,172

Knudsen, Michael 105,

$111,187,251$

Kober, Jillian $\quad 121$

Kobialka, Jaime 77

Koch, Kathryn 121

179

Kochanowski, Benjamin

138,159

Kochersperger, Ryan

121

Koepke, Sara 205

Kohne, Nicholas 105,

223, 229

Kolmerten, Dezirae 121

Komarek, Lauren 138

Kooiman, Viki 105

Koranek, Daniel 105

Kordic, Scott 164

242

Korthals, Jessica 138

Kouba, Benjamin 78

Kowatch, Krista 236

Kraft, Emily $\quad 121$

Kragel, James 35

Kragel, Jonathan 78

Kraker, Justin 105

Kramer, Devin 138,

219

Kraus, Charles 105

257

Kraus, Pete 203

Krebs, Melissa 138

Kreeger, Carol 105

Kreider, Derek 78,

150, 234

Krey, Alicia

Krier, Jennifer

121

163

Krikke, Mark 19

Kriss, Lauren $\quad 138$

Krizo, Kathryn 138

Krogstad, Matthew 13 ,

$111,138,192,193$

Kroll, Michelle 121

Kropf, Michelle 15 ,

121

Krupka, Devin 78
Kuhn, Becky

39

183

195

Kuhn, Loren

187

Kuntz, Amanda 78

Kuvshinikov, Bonnie

50, 51

Kwast, Mark $\quad 78$

Kwok, Jack

33

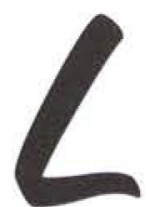

LaPorte, Jessica 78

LaRose, Jennifer 121

Labrado, Timothy 219

Lagan, Daniel 121,

154, 206

Lagan, David 78

Lagan, Joel

233

Lagar, Natalie

Laing, Amber

179

Lakomy, Kathryn 78

Lamb, Juliann 138

Lambert, Jennifer 78 ,

157, 261

Lambright, Rhoda 105

Landers, Erin

172,179

Landers, Jim

138 ,

Landis, Nathan

42

Lane, Deforia

78

Lane, Nathan

33

219

Lang, Meredith 105

Langley, Stephanie 78,

215, 265

Lanham, Esther $\quad 44$

Lanphier, Renee 138

Lansford, Jesse 105

Lansford, Joshua 78

Lapata, Eric $\quad 121$

Laramore, Robert 42

Largent, Lisa 105

Larsen, Sandra 121

Laswell, Alyssa $\quad 138$

Laswell, Ashleigh 121, 234

Latario, Rachel 139
Latham, Stephanie 189

Laura, Ramey 96

Lawler, Jonathan 105 ,

257

Lawrence, Jessica 105

Lawrence, Joel 121

Lawson, Jonathan 78

LeBlanc, Jeanie 55

LeBlanc, John 45

LeMaster, Laura 49

Leach, Jordan 177

Leach, Sarah $\quad 139$

Leafever, Suzanne 41

Leaman, Tara 139

Lear, Jesse $\quad 121$

Leary, Rebecca 121

Lease, Alicia $\quad 121$

Lee, Alisha 105,

189

Lee, Evan 78

Lee, Laura

Leeds, Andrew 78

Leffel, Jordan 121

Lehmann, Lindsay 105 ,

227

Lehr, Sharon $\quad 139$

Leininger, Eric 186,

187

Lemon, Alison 139

189

Lempa, Dale 139

Lengefeld, Philip 105

Lentz, Jason $\quad 139$

Leonard, Christina 121

157

Lester, Jennifer 105,

237, 252

Lester, Kristy $\quad 52$

Lethbridge, Tiffany 139

Letizia, Grant 78,

166

Lewis, Daniel

27 ,

121, 221

Lewis, Jacob

Lewis, Melissa

232, 234

Liggett, Kelly

211

Lincoln, Kimberly 105 , 160

Lincoln, Michael 106

Linden, Kyle

Lindholm, David

264

Linebaugh, Patricia 139

Link, Jordan 121

Linn, Faith $\quad 56$

Linville, Jana $\quad 271$

Lippman, Katherine

106, 219

Littell, Amanda $\quad 121$, 239

Liu, Annette $\quad 121$

Livengood, Kevin 121

LoCicero, Steven 139 
Lustig, Laura

95, 261

Lustig, Ryan

177

Luther, Colin

$79,156,167$

Lutz, Bob 50, 51, 155

Lutz, Kathryn $\quad 122$

Lynch, Shannon 217

Lyon, Justin

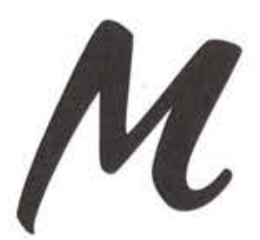

Maat, Christian 122

Maat, Samantha 79 ,

184,185

Mable, Kourtney 139

MacNeil, Kathleen 79

Mach, Tom 44

Magaha, Jessica 146

Magee, Daniel 106,

168,237

Magin, Jennifer 79

Mahl, Aaron $\quad 52$

Mahl, Teressa $\quad 47$

Malik, Larissa 122

Malmstrom, Jacquelyn 139

Malone, Lindsay 271

Malpass, Kristen 79 ,

179

Manchester, Jonathan 79

Manchester, Marcus

122

Mansker, Adrienne 80

Manzer, Stefanie 139

Mappes, David 36

Marburger, Katie 139 , 227

Marcum, Jenna 106

Mariani, Katherine 17

Marietta, Brittany 80

Marker, Larinda 106

Marnitz, Stephanie 80

Marquardt, Todd 80 , 287

Marr, Krissten 159,

237

Marshburn, Justin 122

\section{Find Your Friends!}

Martelli, Kevin 122, May, Hannah $\quad 80$, McGillivray, Bonnie54 Meyers, Sterling 123 129, 239

Martello, Stefan 139 Mayer, Michele 106

Martin, Amanda 122

80

Martin, Arleigh

Martin, Brent 20, McCarty, Devon 48

106, 198, 199 McCay, Kathryn 80 ,

Martin, Denise $\quad 41 \quad 97$

Martin, Elizabeth 106, McClain, Allison 139, McInturf, Callie 139

230

Martin, Greg $50 \quad$ McClain, Mark $\quad 42$

Martin, Jessica 139, McClenahan, James

205

Martin, Kirk

189

Martin, Lisa

177,205

Martin, Melissa

McCloskey, Joel 80

38, McCloskey, Nathan 122,

271

Martin, Micah 106, McClure, John 106

220, 221

Martindale, Mary 54

Martindale, Nathan

80

Martindale, Rachel 80

Martinez, Rachel 139,

233, 246, 247

Martinez, Richard 45

Martz, Julie

$184,185,213$

Mast, Bethany 122

Masterson, Amy 80,

$94,156,267,269,270$,

271, 272

Mathai, Sushila 139

Mathews, Lisa $\quad 80$

Mathews, Melissa 106

Mathisen, Joy $\quad 80$

Matney, Sarah 122

Matson, Dave $\quad 37$

Mattern, Krista 179

Matthews, Mark 39

Matthews, Nicholas

139

Mattson, Julia $\quad 139$,

230

Mattson, Kendra 106,

230

Mattson, Lee 55

Maughon, Joshua 183

Maupin, Meridith 80 ,

95
95, 263

Mayeux, Isaac $\quad 122$

Maynard, Elizabeth 80

McCann, Kirsten 80,

$106,151,213,218,219$,

257 219

McClure, Laura $\quad 80$

McCollim, Amanda 106

McComas, Wade 25,

106, 150

McCormack, Amy 80,

263

McCoy, Justin 106,

111, 154

McCoy, Michael 122

McCrorey, Elizabeth

81

McCue, Jamin 122

McCullough, Alicia 122

McCully, Laura 81,

200, 201

McCune, Adam T. 50, 51

McCune, David 81, 96

McCune, Michelle 56

McCune, Stephen 81 221, 234, 261

McDaniel, Bethany 81

McDaniel, Kaitlin 81

McDivitt, Naomi 81

McDonald, Jennifer

122

McElveen, Merilee 122

McFarlane, Kay 46

McGee, Heather 122

McGee, Miles 139

McGill, Jonathan 249,

257
McGillivray, John 39 ,

179

McGrew, Lauren 81

McGriff, Kelly 139

McGunnigal, Kristine

122

McHugh, Courtney 139

McIntosh, Mischelle

35

McKanna, Nathan 122

McKee, Kate 122

McKellar, Jennifer 81

McKinley, Amanda 81, 106

McLeese, Nathan 122

McLeod, Philip 23

McNichols, Brittany

81,261

McPherson, Victoria

140

Michael, Ryan 140

Michaud, Benjamin

194

Micucci, Derek 81

Midorikawa, Aya 81

Mikolon, Marguerite

106

Milbourne, Laura 140

Miles, Brittany 123,

129

Millar, Becky $\quad 123$

Millay, Emily 123,

205

Miller, Bethany 123

Miller, Chris 35 ,

36

Miller, Christina $\quad 233$

Miller, Jeremy 106

Miller, Katherine 140 ,

213

Miller, Kelly

123

McQueen, Julie 106

McRae, Benjamin 106

Meadows, Donetta 140

Mehra, Anjuli 122

Meier, Allyson $\quad 140$

Meissner, Tiffanie 81

Meitzler, Faith 122

Melendez, Angeline

122

Mellum, Lynn $\quad 122$

Mena, Kena

232, 234

Mensah, Vashti 81

260, 263

Merchant, Diane 35

Merkel, Kristin 106,

179, 257

Merkh, Michelle 81

Merrell, Heather 122

179

Merrick, Kara

267, 268

Merrin, Alise

Merritt, Fred

Mertus, Megan

218, 219

Messer, Cynthia

36, 267, 269

Meyer, David

Meyer, Marilyn

Meyers, Griffin

151

233

Miller, Mark

123

231

Miller, Melissa $\quad 140$

Miller, Melody 153

Miller, Pam $\quad 37$

Miller, Sarah Lanette

236

Miller, Sarah Lynn 140 ,

147, 216, 236

Miller, Scott

106

233

Miller, Steve

56

Milliman, Bob 30

31,58

Milliman, John $\quad 140$

Millington, Karisa 107

Mills, David $\quad 36$

Mingonet, Hannah 82,

211, 217

Minor, Amanda 82

Mishne, Eric 82 ,

231

Mitchell, Peter $\quad 140$

Mitchener, Scott 107

Miura, Amy 82

Moad, William 123

217

Moeller, Andrea 82

Mohler, Emily

107
Mohler, Joshua

233

Mohn, Kristina 123

Mol, Alexander 140

Molby, Nathan 159

Montes, Loren $\quad 82$,

212

Moody, Joel $\quad 123$

Moon, Robert $\quad 107$

Moore, Andrea 123

216

Moore, Bryan 46

Moore, Daniel 82

Moore, Elliot 16,

$24,82,176,177$

Moore, Julie 36

Moore, Katelyn 140

Moore, Kenneth 107

Moore, Matt 35

Moore, Timothy 140

Moran, Julia 107,

153, 156

Moran, Stan

123,

Miller, Laura 106, 223

Modderman, Samantha

Moreland, Kurt 35

Moreland, Sarah 140

Morgan, Ashley 82 ,

261

Morgan, Danielle 107

Morgan, Darrell 111

Morgan, Kristina 82

Moriarty, Jennifer 107 
Munson, Aubree 204, 205

Muntz Jr., Wesley 123

Murdoch, Murray 44

Murphy, Alison 140, 146

Murphy, Sarah 140, $248,249,256,257,258$, 260

Murschel, Cristin 140

Musselman, Brittany 140

Myers, Janelle $\quad 140$

Myers, Jason $\quad 82$,

261

Myers, Kelly

229

Myers, Leslie 162

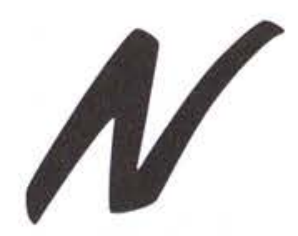

Nangle, Dennis 271

Napp, Brian 82

Neal, Kristen $\quad 107$

Nearhoof, Joshua 230

Nearhoof, Rebekah82, 260

Neetz, Tim

111, 263

Neetz, Timothy 82 , 154,263

Nelson, Annalise 123

Nelson, Brian 107 ,

150

Nelson, Jennifer 140

Nelson, Kelsey 107 , 166

Nelson, Megan 123

Nesbitt, Rebecca 140

Neuhart, Micaela 123

New-Day, Danielle 123

New-Day, Holly 146

Newfield, Allison 107

Newfield, Matthew 140

Newman, Justin 140

Newman, Sarah 24

Ney, Jason 52

Ney, Sara 123

Neyman, Rebecka 140

Nicholas, Luann 50
Nicholl, Kathryn 82 Nickum, Tiffany 82

Niday, Hannah 140

Niedermayer, Sarah

107

Nielsen, Lauren 141

Niemeyer, John 141

Niemi, Katie $\quad 123$

Nikitin, Daniel $\quad 82$

Niknam, Jonathan 107

Niznick, Aaron 82

Noble, Amberly 83

Noble, Emily 123,

189

Noble, Garrett $\quad 123$

Noden, Rondi 83

Nooney, Jessica 141

Norman, Bryan $\quad 107$

Norman, Timothy 42

Normandeau, Nicole

141, 217

Normandeau, Ricky

$83,156,160$

Norris, Linnea $\quad 18$

Norton, John $\quad 177$

Nosal, Bayley $\quad 189$

Nowels, Kelly 123,

164

Nuss, Lauren 83

Nutter, Shelly 43

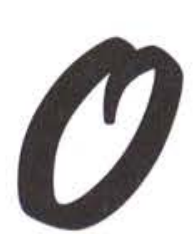

O'Callaghan, Daniel 83

O'Callaghan, Kristin 83

O'Connor, David 83

O'Donnell, John 83

O'Donnell, Timothy

83

O'Keefe, Erin 83

O'Neel, Roger $\quad 37$

Oana, Asheritah 170

Obaker, William 83

Ohlin, Bethany 141

Ohms, Josh $\quad 183$

Ohms, Joshua 107

Ojala, Brendan 192,

193
Okoro, Stephen 13, Parmerlee, Melissa 180,

141

Olin, Gary $\quad 48 \quad$ Parr, Robert G. $\quad 44$

Oliver, Amanda $\quad 27, \quad$ Parrill, Rachel 41

83,95

Oliver, Patrick $44 \quad 46 \quad$ Picazo, Shannon 270,

Oller, Ryne 141, Parrott, Daniel 232, 271

146, 187

Olmstead, Hannah 141

Olmstead, Holly 83

Olmstead, Laura 83

Olney, Lucy

123 ,

185

Olsen, Erin

141

Olson, Toryn

123,

129

Oosterhouse, Whitney

107

Orchard, Paul 39,

183

Organ, Paul $\quad 107$

Ormsbee, Dave 30,

31

Ort, Brandon 83 Peletis, Cassandra 123

Orwig, Charles 83,

163

Osborn, Jared

107

Otis, Julie123

Overly, Nicole

Owens, Matt

203

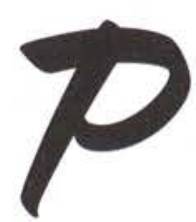

Pagnard, Charles 37, 254

Paine, Brynn

215, 217, 227

Palmer, Claire 83

Palmer, Hallee $\quad 141$

Palmer, Steven 141

Pankratz, Kaitlin 84

216, 217, 233, 263

Pankratz, Kelly 141

Paoloni, Krista 123

Parido, Michael 84

Parker, Laura $\quad 123$

216

Parks, Charles $\quad 84$

Parmerlee, Carissa 200,

201

Philpott, Katherine 216

Phipps, Jim

Phipps, Timothy 35

Picard, Kathleen 84

158, 233

Pierce, Amanda 124

Piette, Timothy 107

Pasma, Erin $\quad 107$

Patnaude, Neil $\quad 84$,

158,234

Patten, Randall 33

Patterson, Paige 33

Patterson, Rory 50

Paulsen, Donna 52

Paulson, Julianne 84,

215

Peacock, Emily 84

Peak, Rebecca $\quad 107$

Peak, Sarah 141

Peck, Jessica 141

229, 248, 249, 256, 257,

258, 260

Peletis, Cassandra 123
Pelon, Katherine $\quad 107$

237,285

Pepe, Karie

230

141 Pepper, Torrie 141,

178, 179

Percival, Michelle 84 , 259

Perrel, Joshua $\quad 164$

Perrel, Kelly $\quad 107$

Perrien, Laura $\quad 84$

Perry, Rebecca 123

Peters, Benjamin 123

Peters, Daniel 124

Peters, Trisha $\quad 124$

Petersen, Abigail 141,

147,216

Peterson, Bethany 84

Peterson, David 107,

236, 238, 239

Peterson, Joel 162

Petry, Jared 124

Petry, Megan 141

Pfoutz, Tabitha 84

Phillips, Andrea 124

Phillips, Maribeth 84 , 95

Phillips, Rebecca 84, 179, 236

Phillips, Stacy

141
Pifer, Bethany 84

Pilgrim, Hannah 84

Pittman, Bryan 84,

182, 183

Pitts, Lindsey 14

Plasterer, Ruth 141,

147

Ploeg, Adam

$151,214,251$

84,

Plume, Christopher84

Plumley, Trevor 141

Poch, Karen

Poelzer, Joseph $\quad 84$, 155

Polsdorfer, Jonathan 107

Polston, Laura $\quad 124$

Port, Katherine 141

Porter, Beth 37

Porter, Charmaine 141, 219, 251

Potter, Matthew 265

Powell, Andrew 107 , 227

Powell, Jennifer $\quad 27$, 124

Power, Karen

Powers, Steve

Pratt, Jordan

Presley, Joshua

Preston, Heather

Prether, Steve

203

Price, Abigail

178,179

Price, Amy

Price, April

Price, Christen

Price, Clinton

Price, Elisabeth

Price, Tara

Pride, Christina
Potter, John

Prins, Lisa

124

Prins, Ryan

18,

19, 85

Pritts, Kathleen $\quad 107$

Pritts, Michael 85 ,

156, 234

Proctor, Pamela 124

Prokop, Daniel 85

Prokop, Rebecca 141, 205

Provo, Renee $\quad 107$

Pruzaniec, Danielle 141

Pupillo, Christina 107

Purple, Jon 50

Putman, Nichole 85

Putman, Sarah 85

Putnam, Nicole 96

Pyles, Elisabeth 124 ,

184, 185

Pyles, Jackie 57

Pyles, Jonathan 141

Pyles, Sarah

85

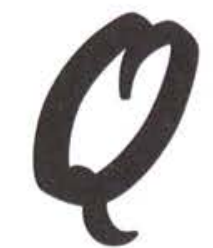

Quaid, Jessica

141

153

Quick, Laura

141

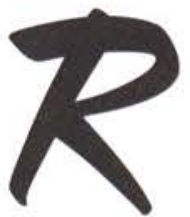

Rafoss, Jonn

107 


\section{Find Your Friends!}

Randolph, Danielle 108

Ranger, Nancy 38

Rathmell, Guy 186 ,

187

Ratliff, Mark

129

Ray, Krista

124 ,

141

Recupero, Kristen 142

Reecer, Heather 142

Reed, Nathan 142

Reed, Rachel 124

Reeder, Corey 14,

124,228

Reeder, Stacey 108

Reeder III, Richard 85, 203

Reep, Andrew 85,

158

Reep, Jeff50

Reep, Kenneth

142

Reep, Ryan

186,187

Reichert, Jameson 85, 261

Reid, Courtney 124

$196,197,251,255$

Reid, Larry 255

Reid, Robert 52 ,

$246,247,254$

Reiley, Carolyn 85,

216

Reilmann, Krista 124

Reinacher, Ina 124

Reiner, Jane 142

230

Reiskytl, Anna 108

Reke, David 142

Rempel, RosaLinda 108

Renke, Andrew 124

Rettig, Caleb 108

Reuther, Katelyn 142

Rex, Jessica 124,

129

Reyes, Jessica 185,

205

Rhoads, Heather 108

Rhoads, Michael 86

Ricci, LaVaughn 52

Rice, Courtney 86 ,

221

Rice, Heather 142

Rice, Keith

Rice, Marla

197
Rice, Melissa

108
86

Rice, Regan

86

Rice, Wade

187

Rich, Anne

45

Richard, Meredith 142

Richardson, Lindsey

142, 146

Richardson, Noah 124

Richter, Lachelle 108,

233

Rieck, Karen 86,

156

Rill, Amanda

108

162

Rill, Angela $\quad 124$

Rintelman, Kristin 142

Rising, Kasey 108,

167, 191

Risner, Ashley $\quad 86$

Ritchey, Timothy 86

Riznick, Aaron 86

Rizzo, Jeremiah 177

Roach, Travis 193

Roark, Janna 124

Robbins, Laura 108,

216

Robbins, Sarah 142

Roberto, E. Samuel 142

Roberts, Amanda 142

Roberts, Daniel 182 ,

183, 194

Roberts, John C. 142

Roberts, John F. 142

Roberts, Rachel 108

Robertson, Danielle

124

Robertson, Kara $\quad 86$,

235

Robey, Beverly 51

Robinette, Eric 86 ,

162, 261

Robinette, Keith 142

Robinette, Megan 124

Robinson, Emmett 86

Robison, Laura 108

Roby, Chelsae 108 ,

\section{1}

Rodgers, Erin $\quad 108$

Rodgers, Lydia 124

Rodgers, Stefanie 142, 189

108 Rodriguez, Rachel 50,

124, 154

Roebuck, Amanda 125
Roelli, Matt

Rohm, Bob

31

Rohm, Lynn

Roller, Amanda

191, 236

Ronan, Kristina

Root, Jordan

Roper, Kevin

Rose, Adam

261

Rose, Daniel

187

Rose, Nicole

217, 236

Rosner, Nevin

205

Ross, Ashley

Ross, Courtney

Ross, Justine

128

Ross, Rachel

205

Rost, Jennifer

Rost, Zachary

Rothacker, Lisa

Rotman, David

266

Rotman, Ryan

264

Rotroff, Ken $53 \quad$ Sanders, Stephanie217

205

Rowlands, Kimberly

108,155

Rozelle, Cristine 86

Ruby, Carl 30,

$31,235,250$

Rucker, Kristine 188,

189

Rudd, Stacie $\quad 142$

Rudd, William 33

Ruegsegger, Tyler 108

Ruffner, Emily 142

Ruhlman, Karen 86 ,

179

Russell, Katy

168, 271

Russell, Richard 86

Rustine, Steven 108

Rutrough, Gary $\quad 86$

Rutt, Alisa $\quad 108$,

200, 201

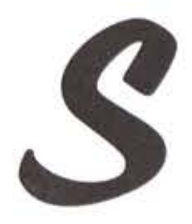

Sakaguchi, Roslyn 201

Salisbury, Kelsey 87

Salladay, Susan 41

Sallee, Brian 87 ,

230

Salsbury, Brandon 142, 203

Sammons, Emily 125

Sammons, Jessica 87

Sampson, Jeffrey 87

Samuelsen, Aubrey 87

Samuelsen, Ryan 108

SanGregory, Angela

142

227, 252

SanGregory, Samuel 41

Sanborn, Ethan 108

Sandberg, Janaye 142

Sandberg, Julianne 108

Sandefur, Karyn 142

Sanders, Angela 125,

237

Sandlund, Emily 125
Sandlund, Timothy 87

Sando, Daryl 9,

$87,229,251,259,265$,

267, 268

Sands, Kaitlin $\quad 87$

Sanford, Amy $\quad 108$

Sanford, Timothy 142

Santos, Nicole 125,

185

Sartori, Jessica $\quad 108$

Sattler, Kaitlin 125,

220

Saunders, Joshua 183,

197

Sawdon, Katherine 87

Sawin, James $\quad 87$

Saxe, Sarah $\quad 167$

Scarpuzzi, Joanna 108

Schanely, Karen 87

Scharnberg, Lorne 33

Schaszberger, Sarah

142
Scull, Kathleen

237, 259, 261

Scull, Peter 143

Seals, Dianne $\quad 49$

Searles, Sarah $\quad 88$

Seawell, Bethany 109

Secor, Jennifer $\quad 43$

Secor, Jennifer 43

Schenk, Terri 111,

219, 234

Schermer, Matthew

87

Schetter, Dan 189

Schimmenti, Jessica

109

Schlabach, Grant 203

Schlappi, Adam 87,

192, 193

Schlener, Jessica $\quad 125$,

217

Schlindwein, Ann 143

Schlueter, Tiffany 125

Schmanke, Benjamin

143

Schmidt, Alison 143

Schmidt, Dave 45

Schmidt, Frances 211

Schmidt, Matthew 109

Schmidt, Sarah 143

Schmidt, Teresa 87

Schmoyer, Bradley 87

Schnepp, Rachael 125

Schoen, David 109

Schoonover, Michael

143

Schrank, Rebecca 87

Schroeder, Karah 88 ,

259

Schumacher, Marie 88

Schumacher, Tyler 88

Scott, Brittny $\quad 88$

Scott, Cayla $\quad 143$

Scott, Dawn 50

Scott, Jason 20,

$88,194,195$

Scott, Jessica $\quad 125$

Scott, Kathryn 143 ,

146

Scott, Michael 109

Scott, Todd $\quad 88$

Scott, Tyler $\quad 177$

Scott, Zachary 109 ,

247

Seelye, Lukas 24 ,

$88,168,219,235,250$

Seelye, Zachary 25 ,

218, 219

Seibert, Jordan 183, 195

Seifert, Samantha 143

Self, Tara 109

Sergent, Andrea 143

Servi, Daniel 193

Shackelford, Kelly 143

Shaeffer, Jason $\quad 88$

Shank, Stephanie 38 , 88

Shanks, Catherine 125

Sharp, David $\quad 41$ 
Short, Libby
181

Short, Ryan

Shorts, Mikal

\section{5,}

Smith, Amie

Smith, Amy

Shortt, D. Jeffrey 42

Shortt, Sandra $\quad 41$

Shriber, Andrew 109

Shumacher, Robert M.

43

Shumaker, Jordan 203

Shumaker, Timothy 143

Shupe, Cheryl 57

Sibbitt, Bethany $\quad 6,88$

Sider, Keith

219

Sides, Justin $\quad 143$

Siefkes, Jordan 203

Siegrist, Jennifer 109

Sietman, Daniel 88

Sietman, Rebecca 35

Silveira, Matthew 183

Silvius, John 43

Simon, Ashley 109,

157

Simon, Trenton 109

Simonaire, Eric $\quad 88$

Simons, Tasha $\quad 88$

Simpson, Brittany 125 , 184, 185, 197

Simpson, Jared 143

Sims, Grace $\quad 125$

Sims, Kevin 43

Sims, Paul David 109

Sims, Sarah 233

Sipe, Jessica $\quad 143$

Sizemore, David 109,

154

Sjoquist, Megan 125

Skelton, Julia 143

Skinner, Jessica 143,

147

Slagh, Rachel 143

Slagle, Ray $\quad 187$

Slaybaugh, Cara $\quad 24$

$89,219,220$

Sloan, Timothy 125

Slone, Tammy 205

Small, Elisabeth 125

Small, Jonathan $\quad 143$,

172

Small, Stephanie $\quad 125$,

237

Smart, Brittany

188, 189

Smith, Amanda

198, 199
Smith, Anna

155

Smith, Ben

Smith, Brandon

Smith, Brennan

Smith, Brett

Smith, Candace

237

Smith, Clifford

Smith, Elizabeth

Smith, Galen

Smith, Jacob

128

Smith, Josh

Smith, Kyle

Smith, Lydia

158

Smith, Mark

217

Smith, Matthew 109

Smith, Nathaniel 109

Smith, Paul M 143

Smith, Rhonda $\quad 41$

Smith, Roscoe 52

Smith, Sara $\quad 143$

Smith, Sarah $\quad 45$

Smith, Timothy S. 125 ,

143

Smith, Timothy W. 125 , 128

Smith, Travis 194

Smith Jr., Ricky 125

Smoyer, Sarah 126,

219

Snavely, Rachelle 109

Sneed, Kara $\quad 126$

Snider, Luke 89 ,

261

Snyder, Dana

219

Sok, Brandon

89,

Solari, Erica

Solomon, Ashley 143

Sosey, Lauren $\quad 144$

Spahr, Eric

Speicher, Caleb

Spencer, Edward 17 ,

37

Spencer, Mark 37

Spivey, Kyle

144
Spurlock, Pat

205

Squires, Paul

89

St Paul, Kettia

126

Stahl, Robert

89

Stahl, Stephanie 144

Stambaugh, Derek 89

Standish, Erica $\quad 89$

Stands, Joshua 144

Stanton, Charlene 89

Stanton, Mikki 229

Starbuck, Joy 126

Starcher, Heath 181

Stark, Eva109, 228, 229

Starr, Samuel 144

States, Crystal 144,

205

Stauffer, Aaron $\quad 126$

Steckel, Jennifer 144

Steckel, Jeremy 126

Steele, Meghan 126

Steele, Michael 126

Steinbach, Elisabeth

109

Steinbach, Mary 144

Steinmetz, Jarrod 126, 238

Steinmetz, Jessica 126

Stephens, Amanda 109,

210

Stephens, David 126,

242

Stephens, Debby 33

Stephens, Joshua 159

Stephens, Justin 203

Sterling, Levi 144

Sterner, Shelly 89 ,

252

Sternsher, Daniel 37

Steury, Andrew 89

Stevens, Albert 33

Stevens, Jennifer 126

Stevens, Katharina 109 230

Stevenson, John 151, 248

Stewart, Ashleigh 126

Stewart, Caroline 109

Stock, Amanda 126,

217

Stock, Christin $\quad 89$

Stockdale, Mary 109 ,

189

Stockwell, Derek 25,

144
217

Stoltzfus, Colby

Stone, Lindsay

196

Storey, Taylor

246

Storhaug, Sonja

179

Stout, Heather

Stout, Jacob

223

Stowers, Becky

51

Straton, Macy 126

Strawser, Michael 109, 238, 239

Strayhorn, Anna 126,

201

Stream, Kerry $\quad 48$

Stream, Samantha 109

Streitmatter, Amy 144

Strickland, Latice 16 ,

90

Stringfellow, Michelle

144

Strode, Elizabeth 144

Stroud-Lusk, Sari 144,

179

Stroup, Jessica 144

Strowd, Elizabeth 109

Strutton, Deborah 109

Studebaker, Elizabeth

126, 247

Studebaker, Nathaniel

144

Sturgis, Rachel 90,

155

Stutzman, Ryan 90,

177

Suchorzewski, Artur

90

Sudlow, Elisabeth 109

Sue, David $\quad 109$

Sugg, Amanda 144

Suhn, Jessica $\quad 109$

Sullivan, Dennis 43

Sullivan, Dennis 58

Summers, Benjamin

206

Surant, Zachary 144

Sutphen, Jeremy 90

Svendsen, Caleb 126

Swaney, Abby 144

Swayze, Jessica 109

Sweede, Deborah 38,
90, 196

Sweede, Donna 90, 271

Sweeney, Elizabeth 144 , 181

Sweeten, Phillip 90

Sweigard, Tom $\quad 46$

Sylvester, Brittany 144 215

Sylvester, Stephen 159, 235

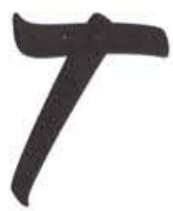

Taft, Ashley $\quad 109$

Talbott, Katelyn 217

Talbott, Valerie 144

Tasker, Catherine 110

Tate, Daniel 90,

261

Taylor, Bekah 236

Taylor, Bethany 90

Taylor, Christina 90, 216

Taylor, Hannah 90, 96

Taylor, Jonathan A 177

Taylor, Jonathan D. 96, 256

Teague, Kelly $\quad 126$,

179

Teague, Laura 90

Teat, Rachel 90,

259

Teekell, Jaimie $\quad 22$,

23, 90, 256

Teekell, Joshua 90

Tello, Daniel 90,

156, 167, 260, 271

Terkelsen, Susan 45

Terra, Nathan 144

Thacker, Benjamin 144

Thalman, Robert 110

Thayer, Jordan $\quad 159$

Thayer, Laura 144

Theiss, Kelly $\quad 181$

Thengvall, Mary 90,

267, 268, 270, 271

Thomas, Daniel 144

Thomas, lan 110,

254

Thomas, Jessica 91, 179

Thomas, Katie 144, 179

Thomas, Robert 33

Thompson, Crystal 91 , 233, 263

Thompson, Katie 263 Thompson, Michaela 126

Thompson, Rachel 110 , 


\section{U}

Unis, Alexander 110 Unis, Robert $\quad 110$ Urman, Mark 91, 239

Utz, Julie 145

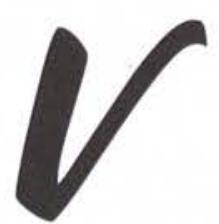

Van Dyke, Jennifer 91 Van Matre IV, Howard 91

Van Schepen, David 91

Van Schepen, Megan 145

Van Vliet, Anna 126

Van Vliet, Emily 91 , 231, 264

van der Wal, John 126 VanDette, Emily 126, 162

VanDette, Ethan 126

VanDyke, Scott 110

VanMatre, Emily 126

Vande Lune,

Christopher 91

VandeKopple, Matthew 177

Vander Zouwen,

Shandra 110

VanderSchuur, Christina 91

Vanlier, Matthew 91

Vanover, Amy 271

Vasser, Donald 110

Vaughan, Alexander

127

Vaughn, Jillian 145

Vawser, Tyler $\quad 110$

Veldhuizen, Sherri 110 , 157

Veldt, Mordecai 127

Venter, Maicol 21,

$91,186,187$

Verlander, Lynn 127 129

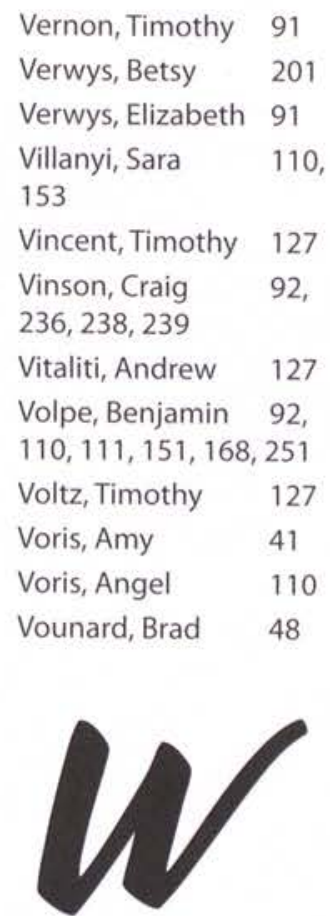

Wadlington Jr., Lee 127 Waggoner, Jessica 57 , $167,251,254$

Wagner, Grace 127 Wagner, Jeffrey 92

Wagner, Jonathan 110

Wailes, Andrew 92

Wailes, Hannah 127 ,

179

Walach, Laura $\quad 110$

Walburg, Jonathan 25,

$145,168,218,219$

Waldo, Shawna 92

Walker, Andrea 110 ,

205

Walker, Christopher

20,21, 187

Walker, Dick 51

Walker, Grant 187

Walker, Paul 92,

263

Walker, Ron

45

Walker, Tricia $\quad 50$

Walkley, Elizabeth 127

Wallen, Joel 92,

159, 236

Walter, Eric

145

Walter, Megan 145,

152, 179

Walter, Nathaniel 156

Walter, Rebecca 92

Walther, Joshua 110

\section{Find Your Friends!}

Walton, Karah

$92,188,189$

Waltz, Sharon

242

Ward, Jerica

219, 222, 251

Wight, Abigal 127

$219 \quad$ Wilcox, Joshua 92

Ward, Stephen 127 Wildman, John 127

Warndahl, Katie 92 Wiley, Benjamin 127

Warren, David $33 \quad$ Wilfong, Peggy 37

Warren, Timothy $92 \quad$ Wilgus, Geneva 145

Wasem, Robert 203

Watson, Ashley 92

Watson, Matthew 92

Waugh, Dakotah 145

Weathers, Christina

110,189

Weaver, Alyssa 145

Weaver, Marcus 110

Webber, Christi 52

Weeks, Matthew 127

Wegman, Tricia 110

Weideman, Cassie 110

Weidman, Amanda 145

Weix, Elizabeth 145

Welch, Sarah 92

Welker, Kendall 110 205

Wells, Gina $\quad 92$

West, Brittany 127

West, Justin $\quad 127$

West, Susan 50 ,

51

West, Tyler $\quad 145$

Westenbarger, Philip

92, 203

Westerhof, Rebecca 145

Weston, John 13

Westrum, Zach 146

Wexler, Nathan $\quad 110$

Weyandt, Jean 42

Wheeler, Katie 92

Wheeler, Margaret

Stowell 35

Whitacre, Amy 127

Whitacre, Jeffrey 127

Whitaker, Brittany 145

White, Daniel 92,

203

White, Mallory $\quad 145$,

205

White, Sara
Whited, Lynette 127

Whitman, Alicia 110

Whitmore, John 43

Wicker, Hayes 33

Wiest, Christopher 145

Wilhelm, Matthew 127

Wilkinson, Zachary 127

Willaman, Katie 92

Willetts, Joshua 110

Willetts, Justin 25

Williams, Christopher

$145,248,249,256,257$.

258,260

Williams, Drew 203

Williams, Garrett 111, 219

Williams, Jordan 93

Williams, Joy 47

Williams, Mark 145

Williams, Michelle 111

Williams, Pamela 145

Williams, Sara 93

Williams, Valerie 127

Williamson, Rebekah

93

Wills, Kristen 93

Wilson, Eric 203

Wilson, Joan 52 ,

53

Wilson, Kayla $\quad 145$

Wilson, Micah 127,

203

Wilson, Paul 203

Wilson, Suzanne 111

Winchester, Jamie 93

Winder, Natalie 93,

221

Winey, Steve 53

Wingert, Cynthia 43

Winner, Elizabeth 93

Winslow, Cessna 53

Winslow, Kevin 46

Winter, Edward 127

Winteregg, Steven 37

Wirt, Lindsay 93

Wirt, Lisa 127

Wise, Kelly

153,179
Wise, Tiffany 93

Wiseman, Andrew 37

Witte, Kelly 93

Wolff, Rebecca 22 ,

$23,93,167,218,219$,

246, 247, 256

Woloshyn, Derek 203

Woltman, Thomas 111

Wolverton, Jonathan

145

Womack, Amber 93

Womack, Aubrey 219

Womack, Jay 54

Womack, Mark 39

Wong, Andrew 93,

156, 228

Wong, Lydia

184,185

Wood, Duane $\quad 58$

Wood, Duane 47

Wood, Jordan M 223

Wood, Michelle 37

Wood, Nicholas 111 ,

223

Wood, Sherrie 34

Wood, Valerie $\quad 145$

Wood, Whitny 129

Woodbury, Philip 93

Woods, Kurtis 145

Woodwyk, Alissa 111

Workman, Brady 203

Workman, Melinda 111,

200, 201, 236, 238

Wright, Amy 93

Wright, Bonnie 22,

93, 196

Wright, Craig 111

Wuenstel, Leslie 127

Wuobio, Heather 127

Wurz, Darren 145

Wyant, Tiffany 93 ,

259

Wynalda, Robert 145

Wyne, Cara

Wynn, Steven 94

Wysong, Melissa 94

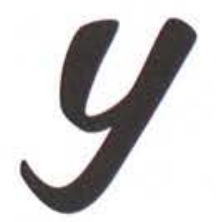

Yahara, Katie

226, 227, 237

Yaklich III, Louis 94

Yanega, Elisa $\quad 111$

Yecke, Alex $\quad 145$

Yoakam, Andrew 145

Yoder, Jo Lyn 127

Yoder, Lauren $\quad 127$

Yoder, Rachel 127

Yoder, Tyler 94,

186, 187

York, Caitlyn 254

York, Sarah 94

Yosinski, Kati 111

Young, Alathea 94,

158

Young, Bethany C. 128

Young, Bethany J. 128

Young, Brandon 203

Young, Deborah 94

Young, Hilary 220

Young, Linnea 145

Young, Rachel 94

Young, Richard 111

Young, Sarah 55

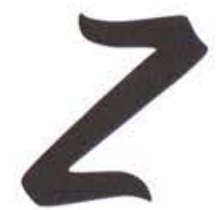

Zastrow, Jason $\quad 111$ 257

Zavodney, Lawrence D. 42

Zeiset, Ryan 146

Zellner, Matthew 128

Zeltman, Sarah 111 ,

180,181

Zerminski, Michelle

128

Zhang, Bingjia 111

Ziegenfus, Laura 111

Ziemblicki, Bethany

128,160

Zike, Christy

13,

146

Zimmerman, Daniel

Edward 22, 146, 246,

247

Zimmerman, Daniel

Eugene 23, 158, 246, 247

Zion, Kristen 94

Zlateff, Kelli $\quad 179$

Zumbo, Kristina 111 

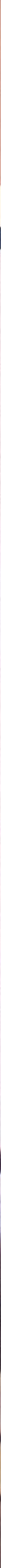


\section{Finale!}

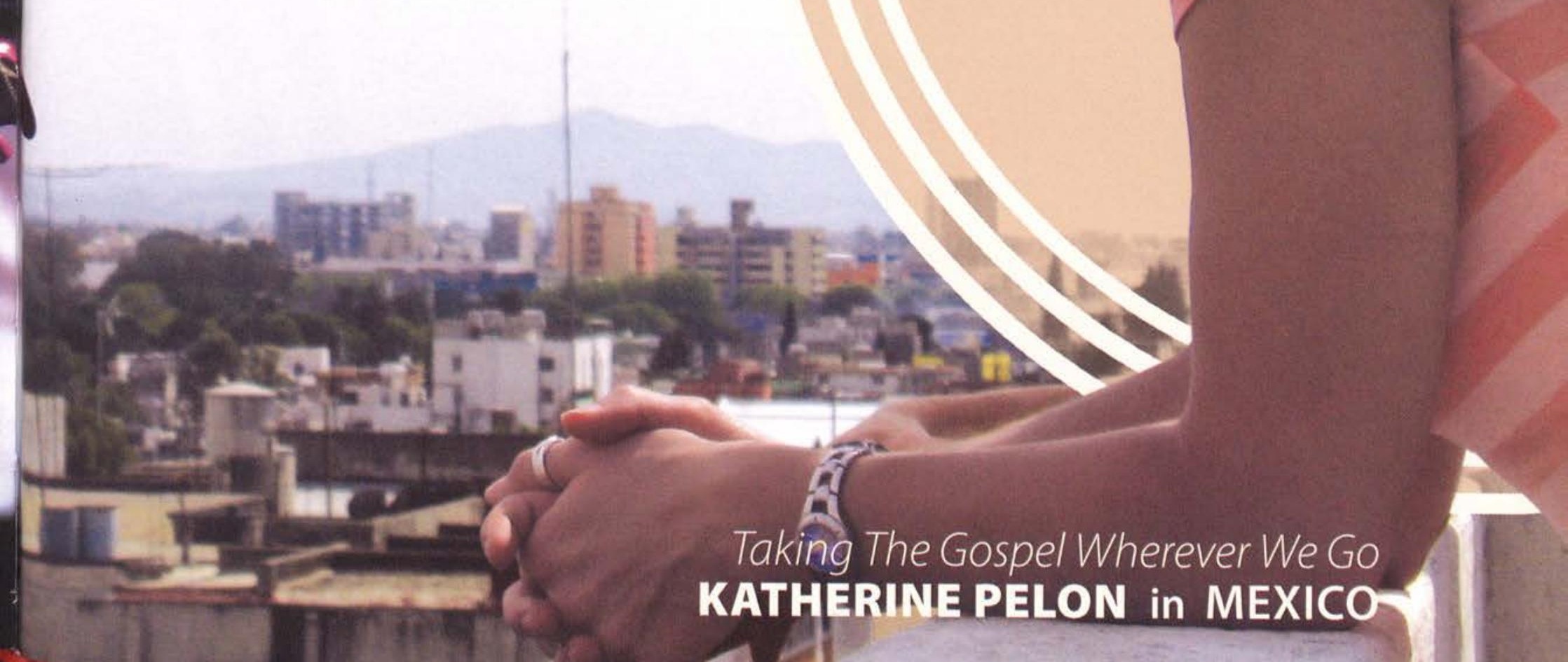




\section{Stoviting \& Ending}

\section{Elvin R. King}


ar a Good Wote!

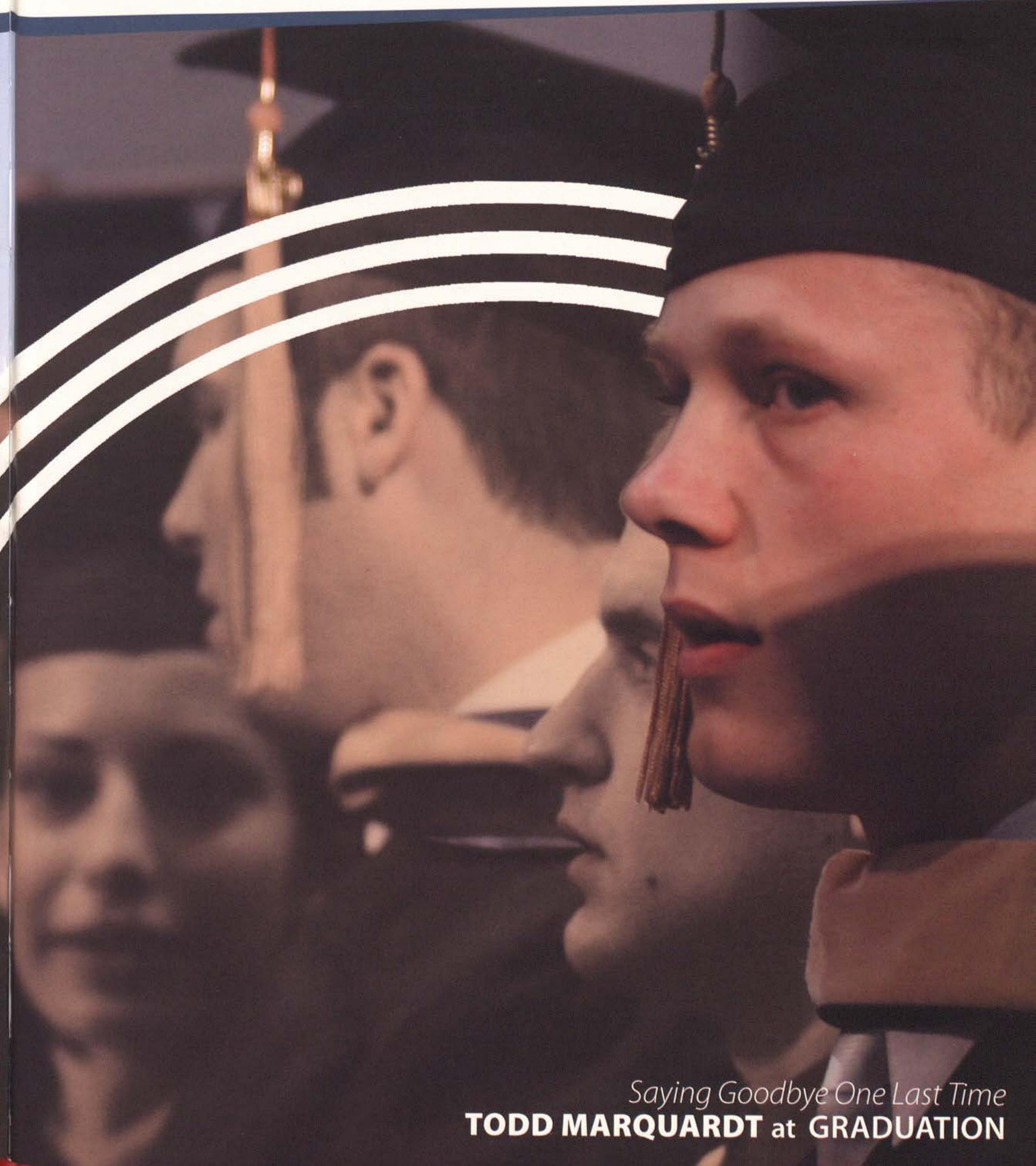




\section{The Enal!}

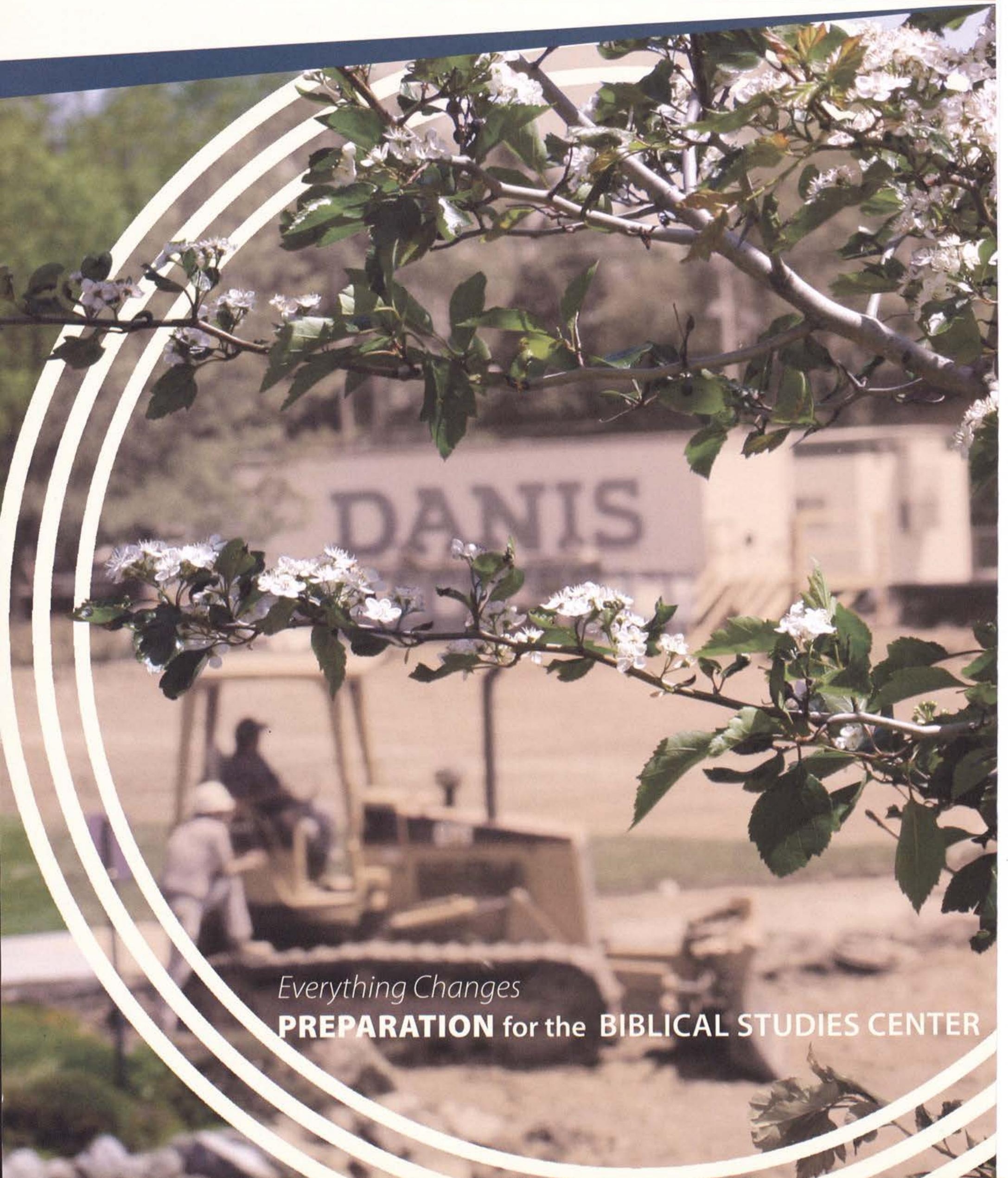




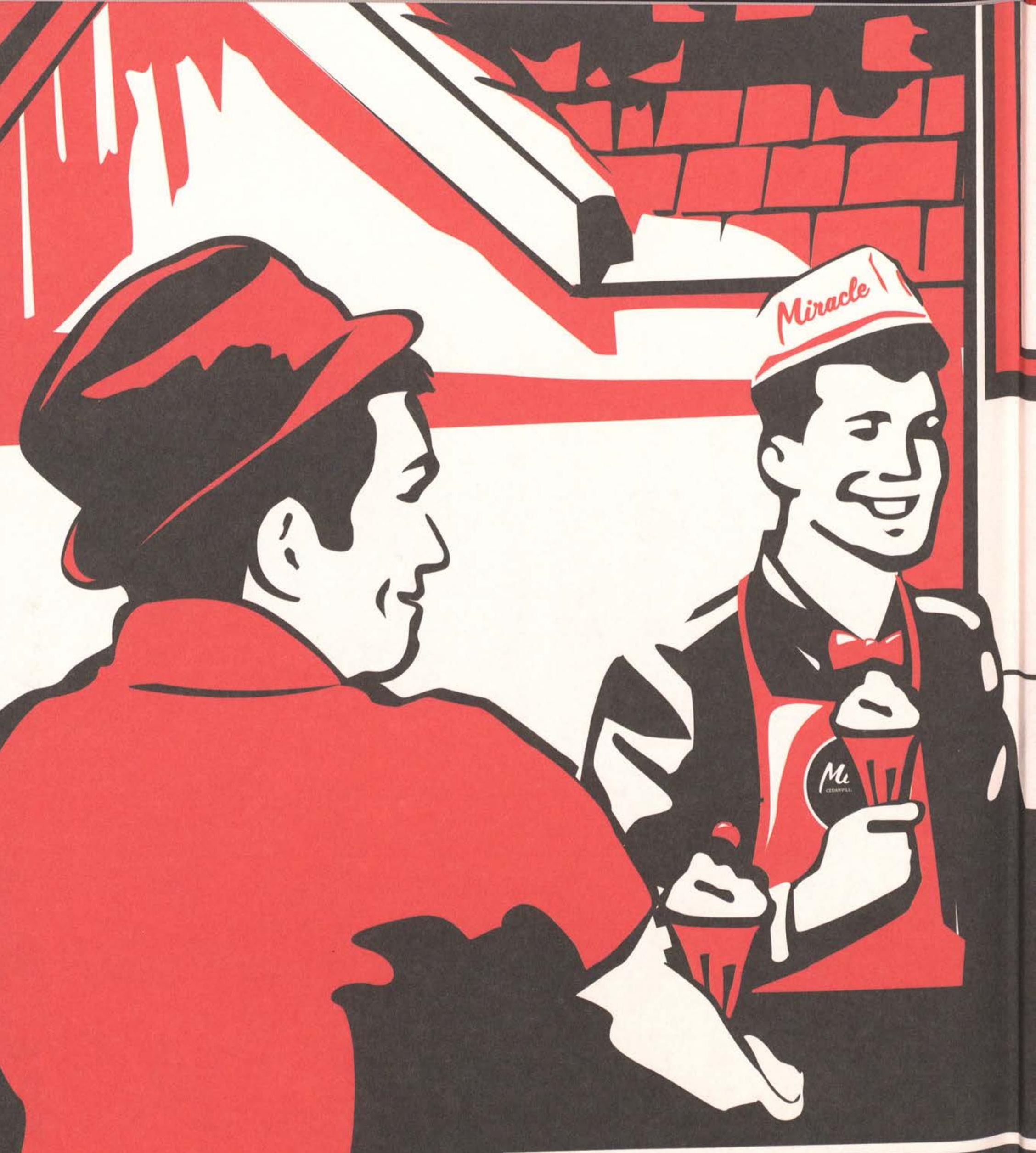



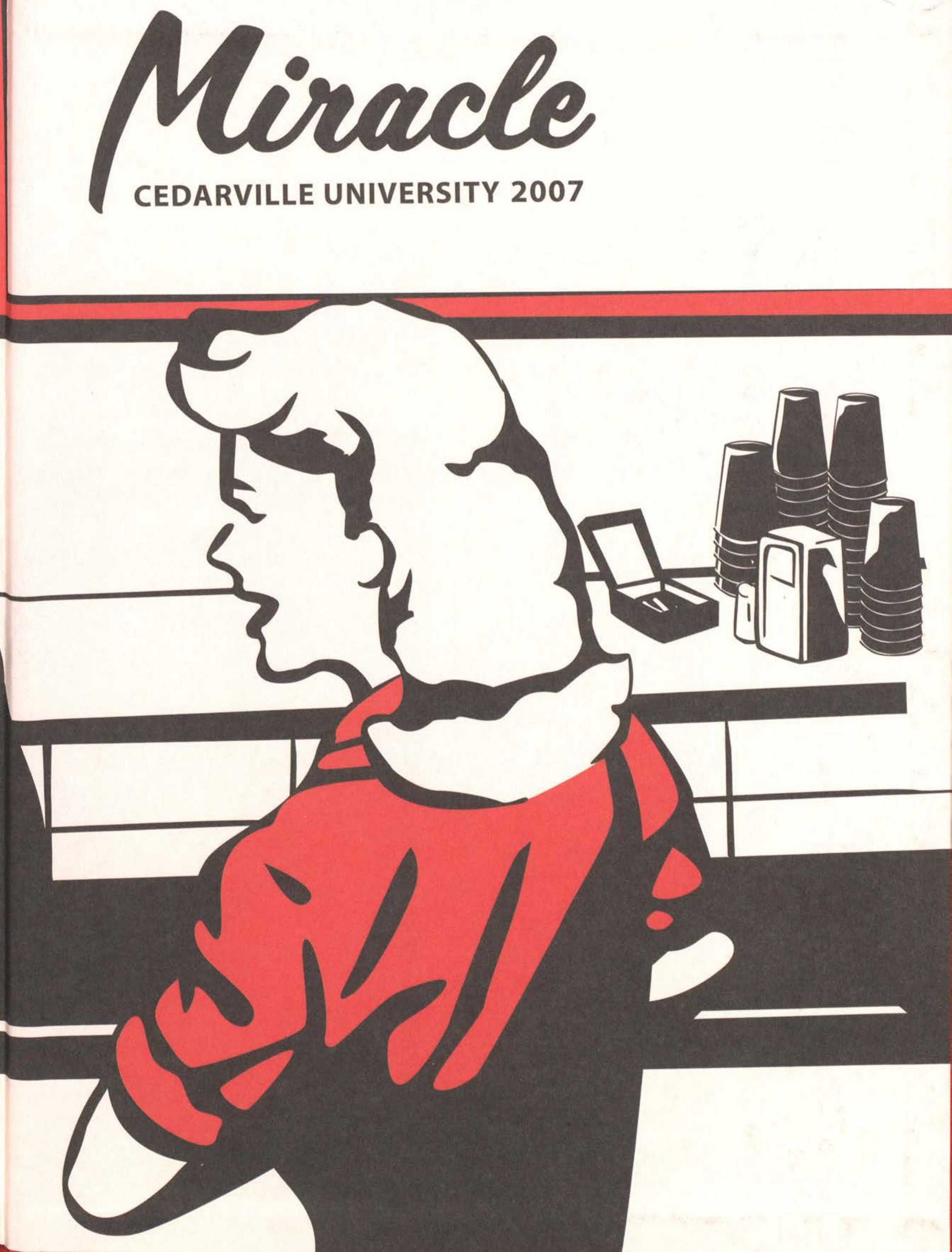
